

A ICasibivinghim

\section{A}

Avigonine National Laboratony

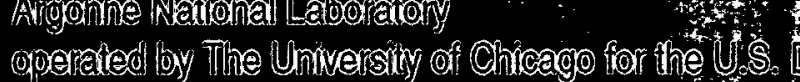

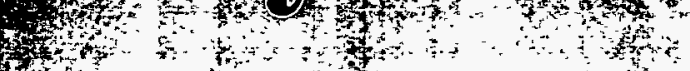

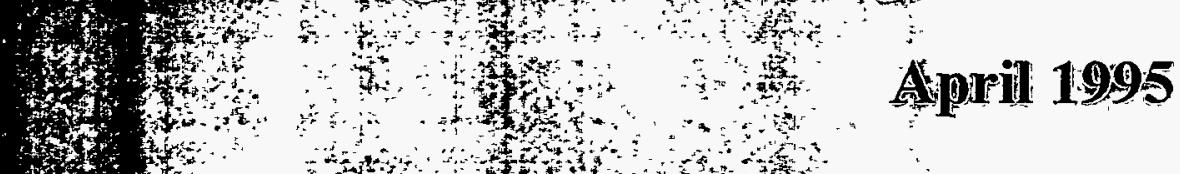

Hond

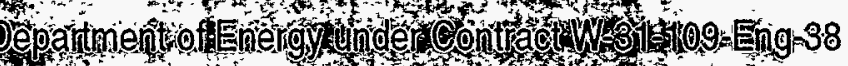

If

Fis. 
- 
Argonne National Laboratory, with facilities in the states of Illinois and Idaho, is owned by the United States government, and operated by The University of Chicago under the provisions of a contract with the Department of Energy.

Publishing support services provided by Argonne National Laboratory's Information and Publishing Division.

\section{DISCLAIMER}

This report was prepared as an account of work sponsored by an agency of the United States Government. Neither the United States Government nor any agency thereof, nor any of their employees, makes any warranty, express or implied, or assumes any legal liability or responsibility for the accuracy, completeness, or usefulness of any information, apparatus, product, or process disclosed, or represents that its use would not infringe privately owned rights. Reference herein to any specific commercial product, process, or service by trade name, trademark, manufacturer, or otherwise, does not necessarily constitute or imply its endorsement, recommendation, or favoring by the United States Government or any agency thereof. The views and opinions of authors expressed herein do not necessarily state or reflect those of the United States Government or any agency thereof. 
RECEIVED

MAY O 91996

OSTI 
Distribution Category: General Energy Research (UC-400)

\author{
ANL-95/13
}

\title{
IPNS Upgrade: A Feasibility Study
}

\author{
ARGONNE NATIONAL LABORATORY \\ 9700 South Cass Avenue \\ Argonne, Illinois 60439-4801
}

April 1995

Work sponsored by

U.S. DEPARTMENT OF ENERGY Office of Energy Research 


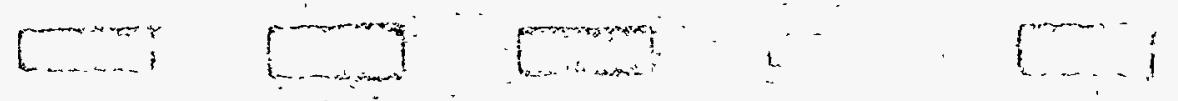

$\cdots$ 
Many of Argonne National Laboratory's (ANL's) scientific staff members were very active in R\&D work related to accelerator-based spallation sources in the 1970s and early 1980s. In 1984, the Seitz/Eastman Panel of the National Academy of Sciences reviewed U.S. materials science research facilities. One of the recommendations of this panel was that the United States build a reactor-based steady-state source, the Advanced Neutron Source (ANS), at Oak Ridge National Laboratory. Subsequently, R\&D activities related to the design of an accelerator-based source assumed a lower priority.

The resumption of pulsed-source studies in this country started simultaneously with design activities in Europe aimed at the European Spallation Source (ESS). The European Community funded a workshop in September 1991 to define the parameters of the ESS. Participants in this workshop included both accelerator builders and neutron source users. A consortium of European countries has proposed to build a 5-MW pulsed source, and a feasibility study is currently under way. Soon after the birth of the ESS, a small group at ANL set about bringing themselves up to date on pulsed-source information since 1984 and studied the feasibility of upgrading ANL's Intense Pulsed Neutron Source (IPNS) to 1 MW by means of a rapidly cycling synchrotron that could be housed, along with its support facilities, in existing buildings.

Coincidentally, in 1992, the U.S. Department of Energy asked its Basic Energy Science Advisory Committee (BESAC) to review the nation's neutron science facility needs. A BESAC subpanel, chaired by Walter Kohn, was appointed to carry out the review. Preliminary results of ANL's IPNS upgrade study were presented at the subpanel meetings, along with Los Alamos National Laboratory's plan for a 1-MW facility based around the Los Alamos linac.

In early 1993, the Kohn panel recommended that (1) design and construction of the ANS should be completed according to the proposed project schedule and (2) development of competitive proposals for cost-effective design and construction of a 1-MW pulsed spallation source should be authorized immediately. The panel urged that evaluation of these proposals be done as soon as possible, leading to a construction timetable that would not interfere with rapid completion of the ANS.

In response to these encouraging Kohn-panel recommendations, the ANL study group carried out a complete feasibility study of a 1-MW pulsed source. This document, together with three separate supplements, contains the results of the feasibility study.

Personnel from several ANL divisions contributed to this study. Y. Cho provided the overall leadership and integration for the study. Work related to the accelerator systems was performed by Y.-C. Chae, Y. Cho, E. Crosbie, H. Friedsam, K. Harkay, D. Horan, S. Kim, R. Kustom, E. Lessner, W. McDowell, D. McGhee, F. Mills, H. Moe, R. Nielsen, A. Rauchas, K. Symon, K. Thompson, D. Warner, and M. White, all from the Advanced Photon Source Project (APS). The linear accelerator feasibility study was performed by AccSys Technology, Inc., of Pleasanton, California, under the guidance of the study group. 
J. Carpenter (IPNS) led the target station design effort. A concept development group comprising J. Carpenter and R.K. Crawford (IPNS), R. Kleb (Materials Science Division), and A. Knox (Technology Development Division) worked out the basic target station design. Providing engineering, analytical, management, and documentation assistance were J. Bailey, P. Calahan, L. Carlson, T. Fornek, A. Knox, and R. Smith (TD); T. Kassner and J. Routbort (Energy Technology Division); R. Kleb (MSD); I. Bresof, D. Jerng, and W. Ruzicka (IPNS); and T. Broome of ISIS (U.K.).

R.K. Crawford led the compilation of neutron scattering instrument requirements and the documentation of instrument designs. Instrumentation requirements and designs for specific neutron scattering instruments and components, and instrument performance evaluations, were provided by R. Birtcher, J.E. Epperson, G. Felcher, J. Jorgensen, R. Kleb, R. Osborn, and D. Price (MSD); W. Dozier, C.-K. Loong, F. Rotella, A. Schultz, P. Thiyagarajan, F. Trouw, and T. Worlton (IPNS); J. Haumann (Electronics and Computing Technologies Division); and G. Kearley of the Institut Laue-Langevin (Grenoble, France).

Conventional facilities work was performed by engineers and architects from the Plant Facilities and Services Division. Coordination and interfacing between technical and conventional facilities personnel were performed by G. Norek (PFS) and K. Peterson (APS). Costs of the conventional facilities were estimated by Hanscomb and Associates of Chicago, Illinois.

The costs and schedules are a result of work performed by Y. Cho; with management information support from A. Rauchas.

The editorial tasks of integrating and assembling the document were performed by M. White, with support from M. Addison, D. Haid, and A. Owens (APS); F. Bennett and staff from the Technical Communication Services Department (Information and Publishing Division); and B. Salbego and staff from the Document Processing and Control Department (IPD).

The Kohn panel report also included the following statement: "If the ANS is not built, a 5-MW pulsed spallation source would be needed to basically cover its capacities in neutron scattering." A feasibility study is currently under way for a future upgrade from the 1-MW source described in this report to a 5-MW facility; the 1-MW accelerator would be used as the injector for the 5-MW accelerator. 


\section{CHAPTER I BACKGROUND AND OVERVIEW}

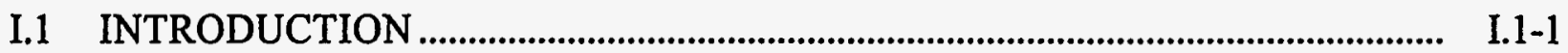

I.1.1 BACKGROUND............................................................................... I.1-1

I.1.2 ACCELERATOR AND TARGET STATION PARAMETERS ..................... I. I.1-4

I.2 DESIGN SPECIFICATIONS AND PERFORMANCE............................................. I.2-1

I.2.1 ACCELERATOR SYSTEM............................................................... I.2-1

I.2.2 NEUTRON SCATTERING CAPABILITIES............................................. I.2-3

I.2.3 OTHER EXPERIMENTAL FACILITIES ................................................. I.2-11

I.3 PROJECT DESCRIPTION ........................................................................... I.3-1

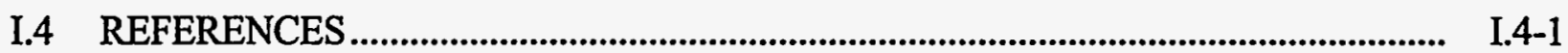

\section{CHAPTER II SYNCHROTRON}

II.1 INTRODUCTION ................................................................................. II.1-1

II.1.1 CHOICE OF MACHINE TYPE................................................................. II.1-1

II.1.2 CHOICE OF BEAM ENERGY .......................................................... II.1-2

II.1.3 CHOICE OF REPETITION RATE OF THE RCS ..................................... II. I-3

II.1.4 NEGATIVE HYDROGEN ION INJECTION .............................................. II. $1-3$

II.1.5 CHOICE OF INJECTION ENERGY ..................................................... II.

II.1.6 REFERENCE .............................................................................. II. $1-5$

II.2 BEAM OPTICS................................................................................... II.2-1

II.2.1 SYNCHROTRON OPTICS .............................................................. II.2-1

II.2.1.1 Magnet Lattice ............................................................................ II.2-1

II.2.1.2 Synchrotron Magnet Excitation and Repetition Rate ....................... II.2-7

II.2.1.3 Beam Emittance and Synchrotron Acceptance ............................... II.2-7

II.2.1.3.1 Space Charge Limit and Choice of Linac Energy........... II. I. 2-7

II.2.1.3.2 Beam Emittance and Machine Acceptance .................. II.2-12

II.2.1.4 Single-Particle Instabilities ........................................................... II.2-14

II.2.1.5 Choice of Working Point............................................................... II.2-17

II.2.1.6 Transverse Tracking Studies ......................................................... II.2-22

II.2.1.6.1 Closed-Orbit Correction.................................................. II.2-23

II.2.1.6.2 Chromaticity Correction ............................................. II.2-24

II.2.1.6.3 Dynamic Aperture .................................................... II.2-24

II.2.2 INJECTION OPTICS...................................................................... II.2-34

II.2.2.1 $\mathrm{H}^{-}$Injection ............................................................................. II.2-34

II.2.2.2 $\mathrm{H}^{0}$ Catcher........................................................................ II.2-46 
II.2.3 LOW-ENERGY TRANSFER LINE....................................................... II.2-48

II.2.4 BEAM EXTRACTION …............................................................. II.2-56

II.2.5 HIGH-ENERGY TRANSFER LINE .................................................... II.2-60

II.2.6 REFERENCES..................................................................................... II.2-63

II.3 MAGNET AND KICKER SYSTEM................................................................. II.3-1

II.3.1 SYNCHROTRON MAGNETS ............................................................... II.3-1

II.3.1.1 Synchrotron Dipole Magnets........................................................ II.3-4

II.3.1.2 Synchrotron Quadrupole Magnets.................................................... II.3-4

II.3.1.3 Synchrotron Sextupole Magnets................................................... II.3-8

II.3.1.4 Synchrotron Correction Magnets...................................................... II.3-8

II.3.2 SYNCHROTRON MAGNET POWER SUPPLIES...................................... II.3-12

II.3.2.1 Introduction.................................................................................. II.3-12

II.3.2.1.1 Power Supply Description ........................................... II.3-12

II.3.2.1.2 Control System and Control Electronics....................... II.3-12

II.3.2.1.3 Grounding..................................................................... II.3-15

II.3.2.1.4 Lockout/Tagout of Magnet Power Supplies................. II.3-16

II.3.2.2 Dipole Magnet Power Supplies....................................................... II.3-18

II.3.2.2.1 General Considerations .............................................. II.3-18

II.3.2.2.2 Dual-Frequency Magnet Circuit .................................. II.3-18

II.3.2.2.3 Dipole Circuit Loss Budget and Parameters ................. II.3-22

II.3.2.3 Quadrupole Magnet Power Supplies............................................. II.3-25

II.3.2.3.1 General Considerations ................................................ II.3-25

II.3.2.3.2 Quadrupole Circuit Loss Budget and Parameters ......... II.3-25

II.3.2.4 Sextupole Magnet Power Supplies ................................................... II.3-25

II.3.2.5 Dipole Corrector Magnet Power Supplies...................................... II.3-25

II.3.3 BEAM TRANSFER LINE MAGNETS AND POWER SUPPLIES ............ II.3-30

II.3.3.1 Low-Energy Transport Line ........................................................... II.3-30

II.3.3.1.1 Magnets......................................................................... II.3-30

II.3.3.1.2 Power Supplies ............................................................... II.3-30

II.3.3.2 High-Energy Transport .................................................................. II.3-33

II.3.3.2.1 Magnets..................................................................... II.3-33

II.3.3.2.2 Power Supplies .............................................................. II.3-33

II.3.4 BUMPER AND KICKER MAGNETS AND POWER SUPPLIES............. II.3-36

II.3.4.1 Introduction............................................................................ II.3-36

II.3.4.2 Injection Bumper Magnets........................................................... II.3-36

II.3.4.3 Injection-Bumper-Magnet Power Supplies ..................................... II.3-36

II.3.4.4 Extraction Kicker System................................................................... II.3-40

II.3.5 SEPTUM MAGNETS AND POWER SUPPLIES...................................... II.3-45

II.3.5.1 Introduction........................................................................................ II.3-45

II.3.5.2 Injection System......................................................................... II.3-45

II.3.5.3 Extraction System ..................................................................... II.3-45

II.3.5.4 Septum-Magnet Power Supplies ..................................................... II.3-49 
II.3.6 MAGNET SUPPORTS................................................................. II.3-56

II.3.6.1 Synchrotron..................................................................................... II.3-56

II.3.6.2 Transport Lines............................................................................... II.3-56

II.3.7 REFERENCES...................................................................................... II.3-58

II.4 ACCELERATING SYSTEM..................................................................... II.4-1

II.4.1 RF VOLTAGE PROGRAM............................................................. II.4-1

II.4.1.1 Introduction............................................................................... II.4-1

II.4.1.2 Longitudinal Tracking Studies ........................................................... II.4-3

II.4.1.3 RF Voltage Program for Capture and Acceleration.......................... II.4-6

II.4.2 BEAM LOADING................................................................................. II.4-15

II.4.2.1 Introduction................................................................................. II.4-15

II.4.2.2 Stability of the Beam-Cavity Interaction....................................... II.4-16

II.4.2.3 Transient Beam Loading................................................................. II.4-21

II.4.2.4 Summary and Conclusions ............................................................. II.4-22

II.4.3 RADIO-FREQUENCY SYSTEM...................................................... II.4-22

II.4.3.1 Introduction........................................................................ II.4-22

II.4.3.2 Synchrotron Cavities........................................................................ II.4-23

II.4.3.3 RF Amplifiers .............................................................................. II.4-29

II.4.4 REFERENCES.............................................................................................. II.4-38

II.5 COLLECTIVE BEAM INSTABILITIES ....................................................... II.5-1

II.5.1 INTRODUCTION .................................................................................... II.5-1

II.5.2 COUPLING IMPEDANCE....................................................................... II.5-1

II.5.2.1 Space Charge Impedance.................................................................... II.5-3

II.5.2.2 Other Contributions ......................................................................... II.5-6

II.5.3 LONGITUDINAL INSTABILITIES........................................................ II.5-10

II.5.4 TRANSVERSE INSTABILITIES …................................................... II.5-17

II.5.5 ELECTRON-PROTON INSTABILITY................................................. II.5-23

II.5.6 REFERENCES............................................................................... II.5-24

II.6 VACUUM SYSTEM AND BEAM PIPE .............................................................. II.6-1

II.6.1 INTRODUCTION ........................................................................... II.6-2

II.6.2 VACUUM CHAMBER DESIGN ...................................................... II.6-2

II.6.3 VACUUM PUMPING SYSTEM.............................................................. II.6-8

II.6.4 REFERENCES......................................................................................... II.6-15

II.7 BEAM DIAGNOSTIC INSTRUMENTATION …….............................................. II.7-1

II.7.1 INTRODUCTION .................................................................................. II.

II.7.2 RCS DIAGNOSTICS ..................................................................... II.7-1

II.7.2.1 RCS Beam Position Monitors.......................................................... II. I 
II.7.2.2 RCS Current Transformers ......................................................... II.7-1

II.7.2.3 RCS Beam Loss Monitors ........................................................... II.7-1

II.7.2.4 Special Beam Studies Instrumentation............................................. II.7-3

II.7.2.5 Injection Diagnostics........................................................................ II.7-3

II.7.3 TRANSPORT LINE DIAGNOSTIC INSTRUMENTATION ..................... II. I.

II.7.3.1 General Instrumentation................................................................ II.7-3

II.7.3.2 Target Protection Monitors ................................................................. II.

II.8 CONTROL SYSTEM......................................................................................... II.8-1

II.8.1 INTRODUCTION AND PHILOSOPHY .................................................. II.8-1

II.8.2 EPICS OVERVIEW ................................................................................ II.8-1

II.8.2.1 Specific EPICS Features......................................................................... II.8-3

II.8.2.2 EPICS Core Software .................................................................. II.8-3

II.8.2.3 Channel Access ......................................................................... II.8-3

II.8.2.4 Database................................................................................. II.8-4

II.8.2.5 Database Scanning........................................................................... II.8-4

II.8.2.6 EPICS Tools.............................................................................. II.8-4

II.8.3 CONTROL SYSTEM HARDWARE ........................................................ II.8-6

II.8.3.1 Console Computers............................................................................. II. $\quad$ II. $8-6$

II.8.3.2 Front End Systems...................................................................... II.8-10

II.8.3.3 Subnets..................................................................................... II.8-11

II.8.3.4 Networks and Protocols..................................................................... II.8-11

II.8.4 APPLICATION SOFTWARE.............................................................. II.8-1.1

II.8.5 EPICS APPLIED TO IPNS UPGRADE................................................... II.8-14

II.8.6 OTHER SERVICES.............................................................................. II.8-14

II.8.6.1 Timing System ......................................................................... II.8-14

II.8.6.2 Event System ................................................................................ • II.8-16

II.8.6.3 Analog Signal System ................................................................ II.8-16

II.8.6.4 Computer-Controlled Video Signal System .................................... II.8-16

II.8.6.4 Network Access from Offices and Laboratories and User Housing Facility .................................................................... II.8-16

II.8.7 CONCLUSION................................................................................ II.8-16

II.8.8 REFERENCES................................................................................ II.8-16

II.9 SHIELDING AND ACTIVATION ......................................................................... II.9-1

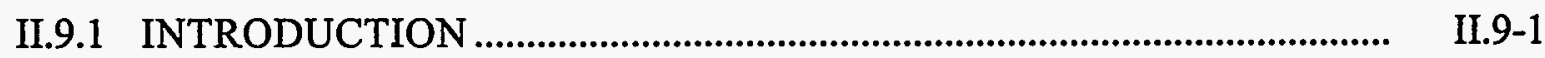

II.9.2 SHIELDING DESIGN OBJECTIVES .................................................. II.9-1

II.9.2.1 Radiation Attenuation Lengths .................................................... II.9-1

II.9.2.2 Bulk Shielding Calculations.............................................................. II.9-5

II.9.2.3 Radiation Dose Equivalent Factors............................................... II.9-7

II.9.2.4 Beam Loss Assumptions and Shielding Requirements ................... II.9-8

II.9.2.5 $\mathrm{H}^{0}$ Beam Catcher, Beam Stop, and Collimators............................. II.9-8 
II.9.3 ACTIVATION ............................................................................. II.9-10

II.9.4 REFERENCES ................................................................................. II.9-11

II.10 SURVEY AND ALIGNMENT ................................................................ II.10-1

II.10.1 INTRODUCTION ...........................................................................10-1

II.10.2 GEODETIC REFERENCE SYSTEM.................................................. II.10-1

II.10.3 ALIGNMENT TOLERANCES........................................................ II.10-1

II.10.4 ALIGNMENT CONCEPT .............................................................. II.10-1

II.10.5 CONTROL NETWORK SIMULATIONS AND

DATA HANDLING ........................................................................ II.10-3

II.10.6 INJECTOR ALIGNMENT.............................................................. II.10-8

II.10.7 REFERENCES .................................................................................. II.

CHAPTER III INJECTOR SYSTEM

III.1 SUMMARY

III.1-1

III.2 INTRODUCTION ........................................................................................ III.2-1

III.3 PHYSICS DESIGN .......................................................................................... III.3-1

III.4 LINAC SYSTEM DESCRIPTION ..................................................................... III.4-1

III.4.1 INTRODUCTION .......................................................................... III.4-1

III.4.2 LINAC SUBSYSTEMS ................................................................... III.4-1

III.4.3 LINAC INJECTOR SUBSYSTEM.................................................... III.4-2

III.4.3.1 H-Ion Source....................................................................... III.4-2

III.4.3.2 Chopper .................................................................................... III.4-6

III.4.3.3 Low-Energy Beam Transport....................................................... III.4-6

III.4.3.4 Ion Source, Chopper, and LEBT Electronics................................... III.4-6

III.4.3.5 Radio-Frequency Quadrupole........................................................ III.4-8

III.4.3.6 Ramped-Gradient Drift Tube Linac.............................................. III.4-8

III.4.3.7 Radio-Frequency System for the RFQ and RGDTL.................... III.4-12

III.4.3.8 Vacuum and Auxiliary Systems .................................................... III.4-15

III.4.4 DRIFT TUBE LINAC...................................................................... III.4-15

III.4.4.1 Overview............................................................................... III.4-15

III.4.4.2 Radio-Frequency and Vacuum Vessel.......................................... III.4-20

III.4.4.3 Drift Tubes and Post-Stabilizer Assemblies.................................. III.4-20

III.4.4.4 Vacuum and Cooling......................................................................... III.4-21

III.4.4.5 Support Stand ................................................................................ III.4-21

III.4.5 MATCHING SECTION................................................................... III.4-21

III.4.5.1 Introduction.......................................................................... III.4-21

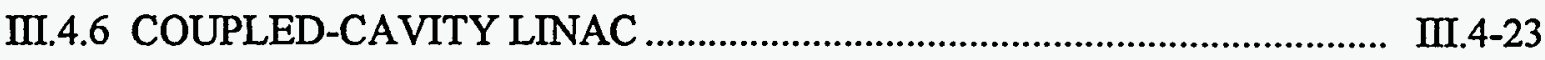

III.4.6.1 Overview................................................................................ III.4-23 
III.4.6.2 Radio-Frequency Structure Fabrication

III.4-23

III.4.6.3 Support Systems and Mechanical Design Features..

III. $4-26$

III.4.6.4 Vacuum System.

III. $4-26$

III.4.7 KLYSTRON POWER SYSTEMS.

III.4-28

III.4.7.1 Overview

III. $4-28$

III.4.7.2 Klystrons.

III. $4-28$

III.4.7.3 Modulators.

III.4-32

III.4.8 BEAM DIAGNOSTICS.

III. $4-32$

III.4.8.1 Overview

III. $4-32$

III.4.8.2 Microstrip Beam Diagnostics

III.4-34

III.4.8.3 Beam Loss Monitors.

III.4-34

III.4.8.4 Other Diagnostics.

III. $4-34$

III.4.9 CONTROL SYSTEM.

III.4-35

III.4.9.1 Overview.

III.4-35

III.4.9.2 Data Acquisition and Control

III.4-35

III.4.9.3 Radio-Frequency Reference Signal Distribution and Low-Level System.

III.4-39

III.4.9.4 Timing Signal Distribution

III.4-39

III.5 LINAC FACILITY REQUIREMENTS.

III.5-1

III.5.1 DIMENSIONED FACILITY LAYOUT

III.5-1

III.5.2 UTILITY REQUIREMENTS

III.5-1

III.5.3 REFERENCES

III.5-5

\section{CHAPTER IV TARGET STATIONS}

IV.1 INTRODUCTION

IV.1-1

IV.1.1 GENERAL

IV.1-1

IV.1.2 OVERVIEW OF THE DESIGN.

IV.1-2

IV.1.3 REFERENCES

IV.1-18

IV.2 TARGET

IV.2-1

IV.2.1 TARGET GEOMETRY AND MATERIAL

IV.2-1

IV.2.2 NEUTRONICS AND HEAT GENERATION

IV.2-3

IV.2.2.1 Calculational Model

IV.2-3

IV.2.2.2 Heat Deposition in the Target and Surroundings.

IV.2-6

IV.2.2.3 High-Energy Neutron Distribution around the Target.

IV.2.2.4 Activation Analyses.

IV.2-17

IV.2-23

IV.2.3 THERMAL-HYDRAULIC ANALYSES

IV.2-29

IV.2.4 STRESS ANALYSIS.

IV.2-47

IV.2.5 TARGET METALLURGY AND CORROSION.

IV.2-55

IV.2.5.1 Introduction.

IV.2-55 
IV.2.5.2 Mechanical Properties.................................................................... IV.2-55

IV.2.5.3 Corrosion................................................................................. IV.2-58

IV.2.5.4 Hydrogen Embrittlement................................................................ IV.2-58

IV.2.5.5 Afterheat ............................................................................... IV.2-58

IV.2.5.6 Other Properties.......................................................................... IV.2-62

IV.2.5.7 Target Material Selection ................................................................ IV.2-62

IV.2.6 TARGET COOLING SYSTEM ................................................................ IV.2-62

IV.2.7 POTENTIALLY HAZARDOUS INCIDENTS ............................................ IV.2-66

IV.2.7.1 Loss of Coolant Analyses.............................................................. IV.2-72

IV.2.8 REFERENCES........................................................................................ IV.2-74

IV.3 MODERATORS AND REFLECTORS ............................................................ IV.3-1

IV.3.1 MODERATOR AND REFLECTOR GEOMETRY

AND MATERIALS ................................................................................ IV.3-1

IV.3.2 MODERATOR NEUTRONICS.................................................................. IV.3-4

IV.3.2.1 Monte Carlo Calculations for Room-Temperature

Water Moderators.......................................................................... IV.3-4

IV.3.2.2 The Effects of Premoderators ......................................................... IV.3-7

IV.3.2.3 Monte Carlo Calculations for Liquid Hydrogen Moderators ......... IV.3-9

IV.3.2.4 Neutron Beam Currents at $1 \mathrm{eV}$...................................................... IV.3-15

IV.3.2.5 Neutron Spectra and Pulse-Widths below $1 \mathrm{eV}$.............................. IV.3-17

IV.3.2.5.1 Neutron Spectra .......................................................... IV.3-17

IV.3.2.5.2 Neutron Pulse-Widths................................................... IV.3-19

IV.3.3 RADIOLYSIS

IV.3-19

IV.3.3.1 Production of Hydrogen................................................................... IV.3-19

IV.3.3.2 Buildup of Higher Hydrocarbons in the Liquid

Methane Moderator ....................................................................... IV.3-21

IV.3.4 COOLING

IV.3.4.1 Component Layouts for Water Cooling Systems.

IV.3-24

IV.3-24

IV.3.4.2 Room-Temperature Water Moderators and Premoderators

IV.3-24

IV.3.4.3 Liquid Methane Moderators

IV.3-30

IV.3.4.4 Liquid Hydrogen Moderators

IV.3-32

IV.3.4.5 Reflector.

IV.3-39

IV.3.4.6 Biological Shield

IV.3-41

IV.3.4.7 Target Tank.

IV.3-41

IV.3.4.8 Proton HET Line Vacuum Window Insert...................................... IV.3-44

IV.3.5 REFERENCES.

IV.3-46

IV.4-1

IV.4 SHIELDING

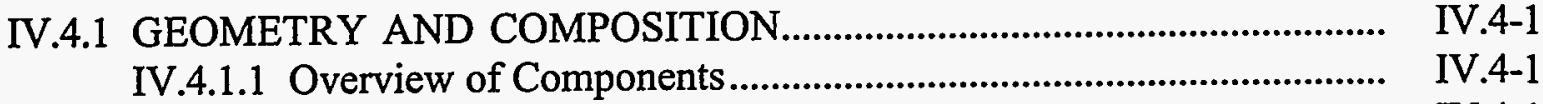

IV.4.1.2 Steel Shielding......

IV.4-1 
IV.4.1.3 Concrete Shielding.

IV.4-5

IV.4.1.4 Hot Cell

IV.4-5

IV.4.2 SUPPORT STRUCTURE.

IV.4-5

IV.4.3 SHIELDING EFFECTIVENESS.

IV.4-7

IV.4.3.1 Design Objectives

IV.4-7

IV.4.3.2 Shielding Parameters.

IV.4-7

IV.4.3.3 Shielding Methodology

IV. $4-8$

IV.4.4 REFERENCES

IV.4-8

IV.5 REMOTE HANDLING.

IV.5-1

IV.5.1 HOT CELL

IV.5-1

IV.5.2 PLUMBING CONNECTIONS

IV.5-1

IV.5.3 TARGET TRAIN REMOVAL AND DISASSEMBLY

IV.5-4

IV.5.4 HANDLING OF SPENT COMPONENTS

IV.5.5 REFERENCES.

IV.5-4

IV.5-10

IV.6 CONTAINMENT

IV.6-1

IV.6.1 CONTAINMENT BARRIERS.

IV.6-1

IV.6.2 GAS EXHAUST SYSTEMS.

IV.6-2

IV.6.2.1 Target Coolant Gases.

IV.6-2

IV.6.2.2 Moderator Gases.

IV.6-2

IV.6.2.3 Shield, Reflector, and Other Coolant Gases.

IV.6-2

IV.6.2.4 Neutron Beamline Exhaust.

IV.6-4

IV.6.2.5 Irradiation Tube Exhaust.

IV.6-4

IV.6.2.6 Purge and Exhaust Systems for Target Tank, Shielding Tank, and Hot Cell

IV.6-4

IV.6-6

IV.6.3 GAS HOLDUP TANKS

IV.6-6

IV.6-7

IV.6.5 LIQUID DRAIN AND RETENTION-TANK SYSTEM.

IV.6.6 RADIOACTIVE EFFLUENTS.

IV.6-9

IV.6.6.1 Gaseous Effluents

IV.6-9

IV.6.6.2 Target Cooling Water System Tritium Level

IV.6.6.3 Cooling Water System Piping Dose Rates

IV.6-10

IV.6-10

IV.6.7 REFERENCES IV.6-12

IV.7 INSTRUMENTATION, CONTROL, AND PROTECTION.

IV.7-1

IV.7.1 PERSONNEL AND FACILITY PROTECTION SYSTEMS

IV.7-1

IV.7.1.1 Overview.

IV.7-1

IV.7.1.2 Facility Shutdown Interface

IV.7-1

IV.7.1.3 Radiation

IV.7-2

IV.7.1.4 Cooling Systems..

IV.7-2 
IV.7.1.5 Containment Systems ................................................................. IV.7-3

IV.7.1.6 Mechanical Systems........................................................................ IV.7-4

IV.7.2 CONTROL AND MONITORING SYSTEMS................................................ IV.7-4

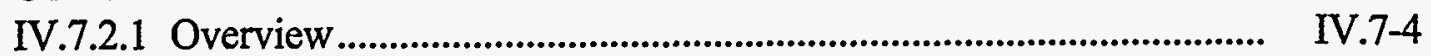

IV.7.2.2 Control System Interface ................................................................... IV.7-4

IV.7.2.3 Radiation Monitoring....................................................................... IV.7-5

IV.7.2.3.1 Exhaust Monitoring....................................................... IV.7-5

IV.7.2.3.2 Personnel Monitoring.................................................. IV.7-6

IV.7.2.4 Cooling Systems......................................................................... IV.7-6

IV.7.2.5 Mechanical Systems......................................................................... IV.7-6

IV.7.3 CONTROL ROOM.................................................................................... IV.7-7

IV.7.4 REFERENCES............................................................................................ IV.7-7

IV.8 NEUTRON BEAM PORTS ......................................................................... IV.8-1

IV.8.1 BIOLOGICAL SHIELDING PENETRATIONS ........................................ IV.8-1

IV.8.2 BEAM GATES............................................................................... IV.8-1

IV.8.3 NEUTRON BEAM GUIDES AND COLLIMATORS. ............................. IV.8-4

IV.8.4 CHOPPER CAVITIES FOR CHOPPERS AND FILTERS .......................... IV.8-7

IV.8.5 INTERFACE TO NEUTRON BEAMLINE SHIELDING........................... IV. IV.8-8

IV.8.6 ATMOSPHERE CONTROL ....................................................................... IV.8-8

IV.8.7 REFERENCES........................................................................................... IV.8-8

\section{CHAPTER V EXPERIMENTAL FACILITIES}

V.1 INTRODUCTION ............................................................................................ V.1-1

V.1.1 OVERVIEW OF NEUTRON BEAM INSTRUMENTATION ................... V.. V.1

V.1.1.1 Instrument Discussions and Reference Set of Instruments............. V. V.1-1

V.1.1.2 Details of Instrument Components................................................. V.. V.1-2

V.1.1.3 Utilization of the Remaining 12 Beam Ports.................................... V.. V.1-2

V.1.2 OVERVIEW OF OTHER EXPERIMENTAL FACILITIES ......................... V. V.1-2

V.2 CONCEPTS FOR SPECIFIC INSTRUMENTS ................................................. V.. V.2-1

V.2.1 POWDER DIFFRACTOMETERS.............................................................. V.. V-1

V.2.1.1 Transfer of Existing IPNS Instruments.......................................... V.. V.2-1

V.2.1.1.1 SEPD - High-Intensity Powder Diffractometer .......... V.2-1

V.2.1.1.2 GPPD - Medium-Resolution Powder

Diffractometer............................................................ V... V.2-1

V.2.1.2 New Reference Set Instruments................................................. V.. V.2-5

V.2.1.2.1 HRPD - High-Resolution Powder Diffractometer ..... V. V.2-5

V.2.1.2.2 RSD - Residual Stress Diffractometer........................ V.2-5

V.2.1.2.3 VSPD - Very-Small-Sample Powder

Diffractometer.

V.2-11 
V.2.1.3 Other Possibilities

V.2-11

V.2.1.3.1 LFHRPD - Low-Frequency High-Resolution Powder Diffractometer.

V.2-11

V.2.1.3.2 LWPD - Long-Wavelength Powder Diffractometer.

V.2.1.3.3 PNPD - Polarized-Neutron Powder Diffractometer.

V.2-12

V.2.1.3.4 UHPD - Ultra-High-Pressure Diffractometer V.2-12

V.2.1.3.5 UHRPD - Ultra-High-Resolution Powder Diffractometer.

V.2.1.4 Powder Diffractometer Summary V.2-12

V.2.2 SMALL-ANGLE DIFFRACTOMETERS

V.2-14

V.2.2.1 Transfer of Existing IPNS Instruments

V.2-14

V.2.2.1.1 SAND - General-Purpose Small-Angle Neutron Diffractometer.

V.2.2.2 New Reference Set Instruments V.2-17

V.2.2.2.1 HRSAND - High-Resolution Small-Angle Neutron Diffractometer.

V.2.2.2.2 SPSAND - Special-Purpose Small-Angle Neutron Diffractometer.

V.2-20

V.2.2.3 Other Possibilities V.2-20

V.2.2.3.1 QSSAND - Quasi-Steady-State Small-Angle Neutron Diffractometer

V.2.2.3.2 DCSAND - Double-Perfect-Crystal Bonse-Hart Small-Angle Neutron Diffractometer .

V.2.2.4 Small-Angle Diffractometer Summary

V.2.3 OTHER DIFFRACTOMETERS

V.2-23

V.2.3.1.1 GLAD - Glasses, Liquids, and Amorphous Materials Diffractometer.

V.2.3.1.2 SCD - General-Purpose Single-Crystal Diffractometer

V.2.3.2 New Reference Set Instruments V.2-30

V.2.3.2.1 HQSCD - High-Q Single-Crystal Diffractometer....... V.2-30

V.2.3.3 Other Possibilities

V.2-32

V.2.3.3.1 PCSCD - Single-Crystal Diffractometer for

Protein Crystallography

V.2-32

V.2.3.4 Summary of Other Diffractometers V.2-32 V.2-34

V.2.4 REFLECTOMETERS V.2-34

V.2.4.1 Transfer of Existing IPNS

V.2.4.1.1 POSY-I - Polarized-Neutron Reflectometer V.2-34

V.2.4.1.2 POSY-II - General-Purpose Reflectometer

V.2.4.2 New Reference Set Instruments V.2-36

V.2.4.2.1 HIREF - High-Intensity Reflectometer. 
V.2.4.2.2 GREF - Reflectometer for Grazing-Incidence Scattering

V.2.4.2.3 REFD - Reflectometry Development......................... V.2-36

V.2.4.3 Reflectometer Summary.

V.2-41

V.2.5 CHOPPER INELASTIC-SCATTERING INSTRUMENTS

V.2-41

V.2.5.1 Transfer of Existing IPNS Instruments

V.2-41

V.2.5.1.1 HRMECS - High-Resolution Chopper

Spectrometer .

V.2-41

V.2.5.1.2 LRMECS - High-Intensity General-Purpose Chopper Spectrometer.

V.2-46

V.2.5.2 New Reference Set Instruments

V.2-46

V.2.5.2.1 CNCS - Cold-Neutron Chopper Spectrometer .......... V.2-46

V.2.5.2.2 SCCS - Chopper Spectrometer for Excitations in

V.2.5.3 Other Possibilities

Single Crystals and Low-Dimensional Systems............. V.2-49

V.2.5.3.1 BSCS - Brillouin Scattering Chopper

Spectrometer

V.2.5.4 Chopper Spectrometer Summary

V.2-51

V.2-51

V.2.6 CRYSTAL-ANALYZER INELASTIC-SCATTERING

INSTRUMENTS

V.2-51

V.2.6.1 Transfer of Existing IPNS Instruments

V.2-51

V.2.6.1.1 QENS - Medium-Resolution Crystal-Analyzer

Spectrometer

V.2-51

V.2.6.2 New Reference Set Instruments

V.2-53

V.2.6.2.1 HRBS - High-Resolution Backscattering

Spectrometer

V.2-53

V.2.6.2.2 TFCA - Time-Focused Crystal-Analyzer

Spectrometer for Chemical Spectroscopy..................... V.2-59

V.2.6.2.3 QSTAXC - Cold-Neutron Quasi-Steady-State

Triple-Axis Spectrometer

V.2.6.2.4 MICAS - Multiple-Independent-Crystal-Analyzer

Spectrometer for Coherent Excitations

V.2-59

V.2-62

V.2-64

V.2.6.3 Other Possibilities

V.2.6.3.1 QSTAXT - Thermal-Neutron Quasi-Steady-State

Triple-Axis Spectrometer.

V.2-64

V.2-64

V.2.6.4 Summary of Crystal-Analyzer Spectrometers...

V.2-66

V.2.7 OTHER INELASTIC-SCATTERING INSTRUMENTS.

V.2-66

V.2.7.1.1 TOFNSE - Time-of-Flight Neutron Spin-Echo

Spectrometer.

V.2-66

V.2-67

V.2.8 OTHER NEUTRON BEAM FACILITIES

V.2-67

V.2.8.1.1 DEVEL - Instrument and Component

Development.

V.2-67 
V.2.8.2 Other Possible Beamline Facilities

V.2-67

V.2.8.2.1 RADIO - Neutron Radiography/Tomography........... V.2-67

V.2.8.2.2 NDP/NAA - Neutron Depth Profiling and Neutron Activation Analysis

V.2-70

V.2.8.2.3 UCN - Ultra-Cold Neutron Facility........................... V.2-70

V.2.8.2.4 NINT - Neutron Interferometer ................................ V.2-70

V.2.9 REFERENCE BEAM PORT ASSIGNMENTS

V.2-70

V.2.10 REFERENCES

V.2-75

V.3 NEUTRON SCATTERING INSTRUMENT COMPONENTS

V.3-1

V.3.1 CHOPPERS

V.3-1

V.3.1.1 Chopper Fundamentals

V.3-1

V.3.1.2 Survey of Chopper Requirements..................................................... V.3-5

V.3.1.3 Specific Chopper Designs ................................................................ V.. V.3-5

V.3.1.3.1 Fermi E $0_{0}$ Choppers..................................................... V... V.5

V.3.1.3.2 Narrow-Band to Choppers ......................................... V.3-9

V.3.1.3.3 Broad-Band to Choppers............................................ V.3-11

V.3.1.3.4 Pulse-Eliminating to Choppers ..................................... V.3-11

V.3.1.3.5 Bandwidth-Selecting Choppers..................................... V.3-13

V.3.1.4 Chopper Control.................................................................... V.3

V.3.2 NEUTRON GUIDES ..................................................................................... V.3-16

V.3.2.1 Important Considerations .......................................................... V.. V.16

V.3.2.2 Survey of Guide Requirements .................................................. V.3-19

V.3.3 BEAMLINE SHIELDING AND BEAMSTOPS ........................................ V.3-22

V.3.3.1 Beamline Shielding - Neutrons from Moderator............................. V.3-22

V.3.3.2 Beamline Shielding - "Direct" Neutrons from the Target .............. V.3-28

V.3.3.3 Beamstops.......................................................................... V.3-30

V.3.3.4 Shielding Interlocks ................................................................... V.3-32

V.3.3.5 Summary of Neutron Beamline Shielding Requirements.................. V.3-32

V.3.4 NEUTRON POLARIZATION.............................................................. V.3-33

V.3.5 DETECTORS ............................................................................ V.3-33

V.3.5.1 Discrete Detectors.................................................................................. V.3-33

V.3.5.2 Position-Sensitive Detectors....................................................... V.3-35

V.3.5.3 Beam Monitors ..................................................................... V.3-36

V.3.6 DATA ACQUISITION.............................................................. V...3-36

V.3.7 DATA ANALYSIS................................................................................. V.3-41

V.3.8 ANCILLARY EQUIPMENT.................................................................. V.3-41

V.3.8.1 General-Use Items................................................................... V.3-41

V.3.8.2 Specialized Items....................................................................... V.3-41

V.3.8.2.1 Rotating-Crystal Insert for Diffractometers ................. V.3-41

V.3.8.2.2 Control of Sample Atmosphere ..................................... V.3-42

V.3.8.2.3 Pulsed Sample Environments......................................... V.3-42 


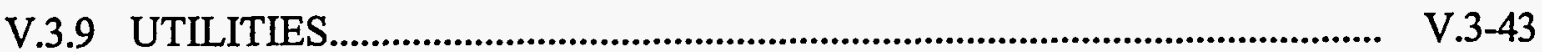

V.3.9.1 Electrical Power............................................................................... V... V

V.3.9.2 Other Utilities .................................................................................. V.3-43

V.3.10 EXPERIMENTAL SUPPORT FACILITIES............................................... V.3-43

V.3.11 REFERENCES................................................................................... V.3-45

V.4 IRRADIATION FACILITIES ........................................................................ V.4-1

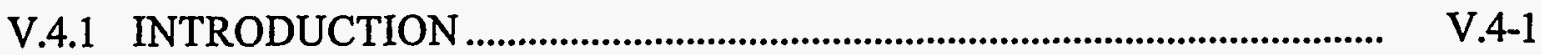

V.4.2 ANTICIPATED USES OF THE FACILITIES .............................................. V.4.1

V.4.2.1 Radiation Effects...................................................................... V.4-1

V.4.2.2 Isotope Production......................................................................... V.4-1

V.4.2.3 Activation Analysis ..................................................................... V... V.4

V.4.3 OVERVIEW OF THE IRRADIATION FACILITIES .................................. V. V.4-2

V.4.4 IRRADIATION SUPPORT FACILITIES ................................................... V.. V.4-5

V.4.4.1 Radiation Effects.................................................................... V.4-5

V.4.4.2 Isotope Production............................................................................ V.4.5

V.4.4.3 Activation Analysis ........................................................................... V.. V.4

\section{CHAPTER VI CONVENTIONAL FACILITIES}

VI.1 INTRODUCTION ...................................................................................... VI.1-1

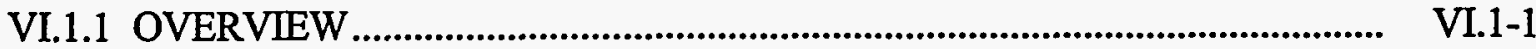

VI.1.2 APPLICABLE CODES AND STANDARDS ............................................ VI.1-8

VI.1.2.1 Architectural/Structural Codes and Standards................................... VI.1-8

VI.1.2.2 Heating, Ventilation, and Air Conditioning Codes

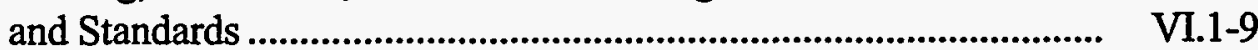

VI.1.2.3 Plumbing Codes and Standards ..................................................... VI.1-10

VI.1.2.4 Fire Protection Codes and Standards ............................................ VI.1-11

VI.1.2.5 Electrical...................................................................................... VI.1-11

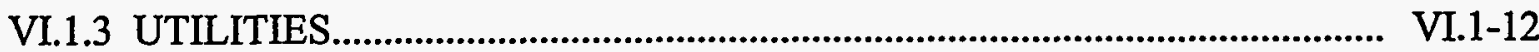

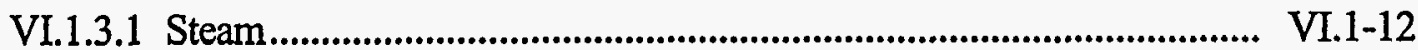

VI.1.3.2 Natural Gas .......................................................................... VI.1-12

VI.1.3.3 General Water Supply....................................................................... VI.1-12

VI.1.3.4 Canal Water.......................................................................................... VI.1-13

VI.1.3.5 Storm Sewers............................................................................ VI.1-13

VI.1.3.6 Sanitary Sewers.......................................................................... VI.1-13

VI.1.3.7 Chilled Water Systems...................................................................... VI.1-13

VI.1.3.8 Electric Power Distribution................................................................ VI.1-13

VI.1.3.9 Telecommunication Systems...................................................... VI.1-13 
VI.2.1 LINAC BUILDING (BUILDINGS 394 AND 394A) ................................. VI.2-1

VI.2.1.1 Architecture (Buildings 394 and 394A) ....................................... VI.2-5

VI.2.1.2 Structure (Buildings 394 and 394A)............................................. VI.2-5

VI.2.1.3 Mechanical Systems (Buildings 394 and 394A) ............................ V. V.2-6

VI.2.1.4 Electrical Systems (Buildings 394 and 394A)............................... VI.2-7

VI.2.2 RING BUILDING (EXISTING BUILDING 365)...................................... VI.2-9

VI.2.2.1 Architecture (Building 365)......................................................... VI.2-9

VI.2.2.2 Structure (Building 365) ............................................................... VI.2-12

VI.2.2.3 Mechanical Systems (Building 365)................................................ VI.2-12

VI.2.2.4 Electrical Systems (Building 365) .................................................. VI.2-14

VI.2.3 CENTER BUILDING (EXISTING BUILDING 364) ................................ VI.2-16

VI.2.3.1 Architecture (Building 364)........................................................... VI.2-16

VI.2.3.2 Structure (Building 364)................................................................... VI.2-20

VI.2.3.3 Mechanical Systems (Building 364)................................................. VI.2-24

VI.2.3.4 Electrical Systems (Building 364) ................................................. VI.2-25

VI.2.4 POWER SUPPLY BUILDING (EXISTING BUILDING 376) ................... VI.2-25

VI.2.4.1 Architecture (Building 376)........................................................ VI.2-25

VI.2.4.2 Structure (Building 376)........................................................... VI.2-28

VI.2.4.3 Mechanical Systems (Building 376)............................................. VI.2-28

VI.2.4.4 Electrical Systems (Building 376) .................................................. VI.2-30

VI.2.5 10-Hz TARGET BUILDING (EXISTING BUILDING 369) ...................... VI.2-32

VI.2.5.1 Architecture (Building 369).......................................................... VI.2-32

VI.2.5.2 Structure (Building 369) .................................................................. VI.2-32

VI.2.5.3 Mechanical Systems (Building 369).............................................. VI.2-35

VI.2.5.4 Electrical Systems (Building 369) ................................................ VI.2-35

VI.2.6 10-Hz TARGET SUPPORT BUILDING

(EXISTING BUILDING 367).......................................................... VI.2-37

VI.2.6.1 Architecture (Building 367).............................................................. VI.2-37

VI.2.6.2 Structure (Building 367)............................................................ VI.2-40

VI.2.6.3 Mechanical Systems (Building 367).............................................. VI.2-40

VI.2.6.4 Electrical Systems (Building 367) ................................................. VI.2-40

VI.2.7 30-Hz TARGET BUILDING (EXISTING BUILDING 370) ..................... VI.2-41

VI.2.7.1 Architecture (Building 370)........................................................ VI.2-41

VI.2.7.2 Structure (Building 370)........................................................... VI.2-41

VI.2.7.3 Mechanical Systems (Building 370)................................................ VI.2-44

VI.2.7.4 Electrical Systems (Building 370) .................................................. VI.2-45

VI.2.8 30-Hz TARGET SUPPORT BUILDING

(EXISTING BUILDING 371)............................................................ VI.2-47

VI.2.8.1 Architecture (Building 371) ........................................................... VI.2-47

VI.2.8.2 Structure (Building 371) ......................................................... VI.2-47

VI.2.8.3 Mechanical Systems (Building 371)................................................. VI.2-50

VI.2.8.4 Electrical Systems (Building 371) .............................................. VI.2-50 
VI.2.9 PREPARATION LABORATORIES

(EXISTING BUILDING 368)......................................................... VI.2-50

VI.2.9.1 Architecture (Building 368)...................................................... VI.2-51

VI.2.9.2 Structure (Building 368) ............................................................ VI.2-51

VI.2.9.3 Mechanical Systems (Building 368)............................................ VI.2-51

VI.2.9.4 Electrical Systems (Building 368) .............................................. VI.2-53

VI.2.10 LABORATORY AND OFFICE BUILDING I

(EXISTING BUILDING 360)................................................................. VI.2-53

VI.2.10.1 Architecture (Building 360)........................................................... VI.2-53

VI.2.10.2 Structure (Building 360).............................................................. VI.2-55

VI.2.10.3 Mechanical Systems (Building 360)............................................. VI.2-55

VI.2.10.4 Electrical Systems (Building 360) ................................................. VI.2-55

VI.2.11 LABORATORY AND OFFICE BUILDING II

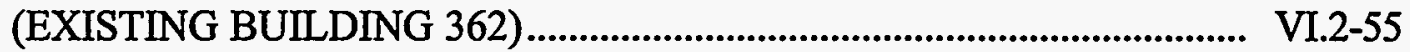

VI.2.11.1 Architecture (Building 362)........................................................ VI.2-57

VI.2.11.2 Structure (Building 362).......................................................... VI.2-57

VI.2.11.3 Mechanical Systems (Building 362)............................................. VI.2-58

VI.2.11.4 Electrical Systems (Building 362) .................................................. VI.2-58

VI.2.12 UTILITIES AND SITE WORK .............................................................. VI.2-58

VI.2.12.1 Site Mechanical Systems............................................................. VI.2-58

VI.2.12.2 Site Power Distribution System................................................... VI.2-61

VI.2.12.3 Site Work........................................................................................... VI.2-61

\section{CHAPTER VII COST AND SCHEDULE}

VII.1 INTRODUCTION ...................................................................................... VII.1-1

VII.2 COST ESTIMATE ........................................................................................ VII.2-1

VII.3 TIME SCHEDULE..................................................................................... VII.3-1

VII.4 COST SCHEDULE............................................................................................ VII.4-1

APPENDIX A: PARAMETER LIST......................................................................... A-1

APPENDIX B: PRECONSTRUCTION RESEARCH AND

DEVELOPMENT PLAN ................................................................... B-1

APPENDIX C: POSSIBLE FUTURE EXPERIMENTAL FACILITIES ........................ $\quad$ C-1

APPENDIX D: GREENFIELD OPTION ............................................................... D-1

APPENDIX E: NOTATION ........................................................................... E-1 


\section{FIGURES}

I.1.2-1 Variation of the Thermal Power Density and Neutron Production Density as a Function of Axial Position in Targets Irradiated by Protons of Various Energies.

I.1.2-2 Spatial Distribution of Neutrons Emerging from theTarget as a Function of Axial Position

I.1.2-3 Spatial Distribution of Thermal Power Density in the Target as a Function of Axial Position

I.2.2-1a Performance of the Different Moderators at IPNS Upgrade: Intensities, Normalized to 1 at $1 \mathrm{eV}$

I.2.2-1b Performance of the Different Moderators at IPNS Upgrade: Pulse Widths....

I.2.2-2 Layout of the Experiment Halls, Showing the Locations of the

Target Stations and Neutron Scattering Instruments

I.3.-1 General View of the IPNS Upgrade

II.2.1-1 Lattice Functions of the Normal Cell.

II.2.1-2 Lattice Functions through the Dispersion-Suppressor and

Dispersion-Free Cells

II.2.1-3 Lattice Functions for One-Half of a Super-Period

II.2.1-3b Overview of the RCS

II.2.-8

II.2.1-4 Average Current from Space Charge Limit $\Delta v_{\mathrm{y}}=0.15, \varepsilon_{\mathrm{y}}=\varepsilon_{\mathrm{x}}=375 \pi \times 10^{-6} \mathrm{~m}$.

II.2.1-5 Time Variation of the Bunching Factor and Time Variation of the Space Charge Tune Shift for the First $6 \mathrm{msec}$

II.2.1-6 Quadrupole Vacuum Chamber Cross Section Showing BeamStay-Clear and Beam Envelope

II.2.1-7 Sum and Difference Resonances Shown up to Fourth Order in the Region Bounded by $6 \leq v_{x} \leq 8$ and $5 \leq v_{y} \leq 7$ 


\section{FIGURES (Cont.)}

II.2.1-8 Structure Resonances up to Fourth Order Shown for the

Region Bounded by $6<v_{x}<7$ and $5<v_{y}<6$.

II. $2-20$

II.2.1-9 Phase Space Motion with $v_{x}=6 \frac{2}{3}$ without (a) and with

(b) Harmonic Correction Sextupoles.

II.2-21

II.2.1-10 Amplification Factors of the RCS.

II.2.1-11 Closed-Orbit Distortions before (a) and after (b) Correction for Quadrupole Misalignments $(\delta \mathrm{z})_{\mathrm{Q}}=0.4 \mathrm{~mm}$, Dipole Field Deviations $(\triangle \mathrm{B} / \mathrm{B})_{\mathrm{B}}=0.001$, and Dipole Roll Angles $(\delta \theta)_{\mathrm{B}}=1.0 \mathrm{mrad}$

II.2.1-12 Required Corrector Strengths to Correct the Closed-Orbit Distortion Shown in Figure II.2.1-11

II.2.1-13 Dynamic Apertures in the Presence of Quadrupole Misalignments of $0.4 \mathrm{~mm}$, Dipole Field Deviations of 0.001, and Dipole Roll Angle Misalignments of $1.0 \mathrm{mrad}$ Plotted before and after Corrections of the Closed Orbit to $0.1 \mathrm{~mm}$ Root Mean Square.

II.2.1-14 Dynamic Aperture Reductions Caused by Multipoles at Four Times the Values Listed in Table II.2.1-3.

II.2.1-15 Dynamic Aperture Reductions Caused by Misalignment and Multipoles, Plotted before and after Corrections of the Closed Orbit

II.2.2-1 Bumped Orbit in the Injection Straight Section II.2-35

II.2.2-2 Injection Details in Two Scales

II.2-37

II.2.2-3 Bending Angle of the Four Bumper Magnets as a Function of Injected Turn Number.

II.2.2-4 Location of the 500 Turns, at the End of Injection, in the Normalized Horizontal Phase Space at the Stripper Location

II.2.2-5 Location of the 500 Turns, at the End of Injection, in the Normalized Vertical Phase Space at the Stripper Location.

II.2.2-6 Time Dependence of the Injected Horizontal and Vertical Amplitudes

II.2-43 


\section{FIGURES (Cont.)}

II.2.2-7 Spatial Distribution of the 500 Turns at the End of Injection ......................... II.2-44

II.2.2-8 Calculated Equilibrium Temperature Distribution on the Stripping Foil.......... II.2-45

II.2.2-9 Direction of Neutral Particles Created in the Stripper...................................... II.2-47

II.2.3-1 Overview of Linac and LET ................................................................... II.2-49

II.2.3-2 Injection System Linac and LET .............................................................. II.2-50

II.2.3-3 Lattice Functions for the $72^{\circ}$ Bend Section of the LET (Region-A)................. II.2-51

II.2.3-4 Envelope Functions for the $72^{\circ}$ Bend Section of the LET (Region-A)............. II.2-52

II.2.3-5 Lattice Functions for Region-B ................................................................... II.2-53

II.2.3-6 Lattice Functions for Region-C..................................................................... II.2-55

II.2.4-1 Extraction Systems and HET Lines ............................................................. II.2-57

II.2.4-2 Extraction Trajectory from the Kicker through the Septum Magnet................. II.2-58

II.2.5-1 Beam Envelope for the 30-Hz Target HET Line ............................................... II.2-61

II.2.5-2 Beam Envelope for the 10-Hz Target HET Line ............................................ II.2-62

II.3.1-1 End View of the Synchrotron Dipole Magnet ................................................... II. I.

II.3.1-2 Contours of Uniformity at Injection for Synchrotron Dipole along with the Calculated Beam Envelope......................................................................... II.3-6

II.3.1-3 End View of the Synchrotron Quadrupole Magnet .......................................... II.3-7

II.3.1-4 Contours of Field Gradient Uniformity at Injection for an F-Quadrupole, along with the Calculated Beam Envelope ........................................................ II.3-9

II.3.1-5 End View of the Synchrotron Sextupole Magnet............................................. II. II-10

II.3.1-6 End View of the Synchrotron Vertical Correction Dipole Magnet................... II.3-11

II.3.2-1 Resonant Circuit Dipole Magnet Power Supply .............................................. II.3-13 


\section{FIGURES (Cont)}

II.3.2-2 Resonant Circuit Quadrupole Magnet Power Supply ...................................... II.3-14

II.3.2-3 Magnet Power Supply Input ac Distribution................................................. II.3-17

II.3.2-4 Power Supply for One Feed Point..................................................................... II.3-19

II.3.2-5 Dipole Magnet Current and Voltage Waveforms for Dual-Frequency Operation .................................................................................................... II.3-20

II.3.2-6 Sextupole Magnet Power Supply ............................................................ II.3-28

II.3.2-7 Bipolar Power Supply that Powers the Dipole Corrector Magnets.................. II.3-29

II.3.4-1 Cross Section of an Injection Bumper Magnet ................................................ II.3-38

II.3.4-2 Circuit Diagram for the Bumper-Magnet Power Supply.................................... II.3-39

II.3.4-3 Cross Section at Upstream End of Extraction Kicker A................................... II.3-42

II.3.4-4 Kicker Waveform ..................................................................................... II.3-43

II.3.4-5 Schematic of an Extraction Kicker Power Supply and Magnet ......................... II.3-44

II.3.5-1 Cross Section of Injection Septum A............................................................... II.3-47

II.3.5-2 Cross Section of Injection Septum B .......................................................... II.

II.3.5-3 Cross Section of Extraction Septum C......................................................... II.3-51

II.3.5-4 Cross Section of Extraction Septum D.......................................................... II.3-52

II.3.5-5 Cross Section of Extraction Septum E ............................................................. II.3-53

II.3.5-6 Basic Septum Magnet Power Supply …........................................................... II..3-55

II.3.6-1 Supports for the RCS Ring Magnets ........................................................... II.3-57

II.4.1-1 (a) The rf frequency of the RCS from Injection to Extraction and

(b) Energy Increase per Revolution of the Synchronous Particle ....................... II.4-2

II.4.1-2 Radio-Frequency Voltage Program for the Injection and Acceleration Cycles. 


\section{FIGURES (Cont.)}

II.4.1-3 Bucket and Bunch Areas, Obtained from the Simulation under the rf Voltage Program, Plotted as a Function of Time.

II.4.1-4 Particle Distribution of the First Injected Turn

II.4-10

II.4.1-5 (a) Half-Width of the Full Momentum Spread of the Bunch Obtained from the Simulation as a Function of Time; (b) Bucket and Bunch Height Growth from Injection to Extraction

II.4.1-6 Synchronous Phase (a) and Synchrotron Frequency (b) Variations from Injection to Extraction

II.4.1-7 Phase Space Distributions from the Simulation, from the Middle of Injection to $4 \mathrm{msec}$ in the Acceleration Cycle.

II.4.1-8 Phase Space Distributions from the Simulation, from $8 \mathrm{msec}$ in the Acceleration Cycle to Extraction.

II.4.2-1 Circuit Representation of Amplifier, Cavity and Beam Loading Current. II.4-17

II.4.2-2 RF Phasor Diagram at Injection Currents Referenced to Gap Voltage II.4-20

II.4.3-1 Quarter Sector of Single-Ended RF Cavity with Four Ferrite Subassemblies

II.4.3-2 Ferrite Ring Assembly Details. II.4-26

II.4.3-3 Radio-Frequency Relative Permeability versus dc Bias. II.4-27

II.4.3-4 8973 Tetrode Outline. II.4-31

II.4.3-5 RF Amplifier Circuit Diagram. II.4-32

II.4.3-6 Building 365, Operating Floor, RF Amplifier Plan, Showing 10 Amplifiers and Cavities.

II.4.3-7 RF Amplifier System Layout II.4-35

II.4.3-8 Building 364, Roof, RF Power Supply Layout II.4-36

II.4.3-9 Center Building 364, Third Floor, RF Control Layout II.4-37 


\section{FIGURES (Cont.)}

II.5.2-1 Ceramic Vacuum Chamber Cross Section in the Focusing Quadrupole Magnet, Showing Placement of the rf Shield Wires at the BSC Aperture

II.5.2-2 Impedance due to Space Charge Plotted as a Function of Time.

II.5.3-1 Variation of the (a) Peak Current and (b) Bunching Factor, $B_{f}=I / I_{p k}$. II.5-12

II.5.3-2 Longitudinal Instability Threshold According to the K-S Criterion Plotted Together with the Momentum Spread Obtained from Tracking.

II.5.3-3 Longitudinal Stability Boundary (a) for a Quartic Momentum Distribution (b)

II.5.3-4 Dependence of the Longitudinal Stability Boundary (a) on Momentum Distribution, Using the Distribution in (b)

II.6.2-1 Dipole Chamber Cross Section and Quadrupole/Sextupole Chamber Cross Section

II.6.2-2 Alignment System for Glass Fusion Bonding of Alumina Sections.

II.6.2-3 Ceramic Vacuum Chamber Joint Detail

II.6.2-4 Typical Quadrupole/Sextupole Ceramic Chamber Cell.

II.6.2-5 Wire Liner, Which Acts as a Radio-Frequency Shield and Is Supported on Ceramic Ribs.

II.6.2-6 Vacuum Chamber Cell-to-Cell Capacitive Coupling Layout.

II.6.2-7 Radio-Frequency Finger Layout

II.6.3-1 Ion Pump Locations around One Quarter of the Ring II.6-11

II.6.3-2 RCS Sector Vacuum Profile.

II.7.3-1 Target Protection Monitor That Uses Secondary Emission Monitors as Detectors, Shown from the Side (a) and from the Beam's Perspective (b).......

II.8.1-1 IPNS Upgrade Control System 


\section{FIGURES (Cont.)}

II.8.2-2 Alarm Handler Console Readout........................................................................ II.8-7

II.8.2-3 Graphical Database Configuration Tool............................................................ II.8-8

II.8.2-4 Knob Manager..................................................................................... II.8-9

II.8.3-1 IPNS Facility Network ....................................................................... II.8-12

II.8.3-2 IPNS Upgrade Local Control Network ................................................................ II.8-13

II.8.4-1 MEDM Control Panel .................................................................................... II.8-15

II.9.2-1 Geometry for Calculating the Transverse Dose Due to Secondary Particles Attenuated by a Shield

II.9.2-2 Cross Section of the Zero Gradient Synchrotron Ring and Center Buildings.................................................................................................. II.9-6

II.10.2-1 Example of an RCS Survey and Alignment Control Network Design .............. II.10-2

II.10.4-1 Target Ball Receptor at the Control Point ........................................................... II.10-4

II.10.4-2 Explosion and Assembly Views of Floor Mounting Instrument Adapter........ II.10-5

II.10.4-3 Monopod Instrument Adapter, Extending the Floor Reference Point to a Height of $1.4 \mathrm{~m}$................................................................................................. II.10-6

II.10.5-1 Error Ellipses of an RCS Survey Network Design Simulation........................... II.10-7

III.1-1 IPNS Upgrade Injector Linac Layout.............................................................. III.1-2

III.3-1 Block Diagram of the IPNS Upgrade Injector Linac ............................................ III.3-3

III.3-2 LEBT Optics...................................................................................... III.3-4

III.3-3 Calculated Beam Dynamics through the RFQ ..................................................... III.3-5

III.3-4 Output Beam Phase Space Projections from the RFQ .................................... III.3-6

III.3-5 Calculated Beam Dynamics through the DTL .................................................... III.3-7

III.3-6 Output Beam Phase Space Projections from the DTL ...................................... III.3-8 


\section{FIGURES (Cont)}

III.3-7 Calculated Beam Optics through the DTL/CCL Matching Section

III.3-10

III.3-8 Calculated Beam Dynamics through the CCL.

III.3-11

III.3-9 Output Phase Space Projections at $400 \mathrm{MeV}$

III.3-12

III.4.3-1 Linac Injector Subsystem.

III.4-3

III.4.3-2 $\quad \mathrm{H}^{-}$Ion Source.

III.4-4

III.4.3-3 Schematic of Ion Source Electrical Connections..

III.4-7

III.4.3-4 Cross-Sectional View of the RFQ.

III.4-9

III.4.3-5 Photograph of the RFQ.

III. $4-11$

III.4.3-6 Planar Triode if Amplifier Schematic.

III.4-14

III.4.4-1 Side View of a Typical DTL Tank.

III.4-17

III.4.4-2 Cross Section of a Typical DTL Tank.

III.4-18

III.4.4.-3 Photograph of the Cross Section of a Typical DTL Tank

III.4-19

III.4.6-1 Side View of CCL with Vacuum Manifold and Bridge Couplers.

III. $4-25$

III.4.6-2 Assembly View of CCL with Support Stand.

III.4-27

III.4.9-1 Control System Diagram. III.4-36

III.5.1-1 Section through Linac Facility Showing Building and Tunnel Configuration.

III.5-2

III.5.1-2 Plan View of the Linac Showing Locations of Utility Requirements.

III.5-3

IV.1.2-1 Floor Plans for the 30-Hz Target Building (370), 10-Hz Target Building (369), Sample Preparation Laboratories (368), and Cooling System Buildings $(367,371)$

IV.1.2-2 Schematic View of the Target and Moderator System. IV.1-4

IV.1.2-3 View of the IPNS Upgrade Integral Split Target. 


\section{FIGURES (Cont.)}

IV.1.2-4 Six Moderators Arranged around the Target

IV.1-8

IV.1.2-5 Bottom Reflector, Showing Neutron Beam Ports, Coolant Passages, Proton Duct, and Target and Moderator Cavities

IV.1.2-6 Exploded View of the Target-Train Assembly

IV.1.2-7 Sealed Target Tank into Which the Target Train Is Inserted for Operation.

IV.1.2-8a Target Train Assembly Inserted into the Target Tank IV.1-13

IV.1.2-8b Target Train Assembly Inserted into the Target Tank, Showing Internal Details

IV.1.2-9 Plan, Elevation, and Cross-Sectional Views of the Biological Shield and Hot Cell

IV.1.2-10 Proton Beam Port and Window Insert Assembly, Which Transports the Proton Beam through the Biological Shield to the Target.

IV.2.1-1 Exploded View of the Upstream Target Section

IV.2.1-2 Exploded View of the Downstream Target Section

IV.2.2-1 Validation of IPNS Upgrade Calculational Model for Heat Deposition in Targets.

IV.2.2-2 Validation of IPNS Upgrade Calculational Model for Neutron Generation Rate.

IV.2.2-3 Isometric View of the Target Station Model

IV.2-10

IV.2.2-4 Plan View of the Modeled Target Station

IV.2.2-5 Isometric View of the Modeled Target and Moderator Arrangement.

IV.2-12

IV.2.2-6 Modeled Configurations of the Reflector and Shield

IV.2.2-7 Cross Section of the Modeled Target Sections 


\section{FIGURES (Cont)}

IV.2.2-9 Power Density Distribution in the Target along the Proton Beam Direction. IV.2-19

IV.2.2-10 Radial Power Distribution in the Disks in the Upstream Target Section IV.2-21

IV.2.2-11 Power Distribution in the Reflector and Shield IV.2-22

IV.2.2-12 High-Energy Neutron Yield around the Target. IV.2-24

IV.2.2-13 High-Energy Neutron Spectra around the Target. IV.2-25

IV.2.3-1 Geometry for Three-Dimensional Thermal-Hydraulic Model of IPNS Upgrade Target Disk

IV.2.3-2 Geometry for Two-Dimensional Thermal-Hydraulic Model IV.2-31

IV.2.3-3 Power Density Distribution vs. Radius for Four Axial Positions in the Upstream Target Section

IV.2.3-4 Centerline, Surface, and Coolant Exit Temperature vs. Coolant Velocity for Disk at Position 3

IV.2.3-5 Centerline, Surface, and Coolant Exit Temperature vs. Disk Thickness for Disk at Position 1

IV.2.3-6 Mid-Thickness Temperature Distribution in Flow Direction for Four Axial Positions in the Upstream Target Section

IV.2.3-7 Surface-Temperature Distribution in Flow Direction for Two Axial Positions in the Upstream Target Section

IV.2.3-8 Upstream Target Section Configuration IV.2-37

IV.2.3-9 Mid-Thickness Temperature Distribution in Flow Direction for Three Axial Positions in the Downstream Target Section

IV.2.3-10 Surface Temperature Distribution in the Flow Direction for Three Axial Positions in the Downstream Target Section

IV.2.3-11 Downstream Target Section Configuration IV. $2-40$ 


\section{FIGURES (Cont.)}

IV.2.3-12 Peak Centerline and Surface Temperature vs. Axial Position, Upstream and Downstream Target Sections.

IV.2-41

IV.2.3-13 Variation of the Peak Temperature in the First Disk in the Upstream Target Section for Three Modes of Operation.

IV.2-42

IV.2.3-14 Radial Temperature-Time Variation in a Disk at Position 1 in the Upstream Target Section, Operated in Mode 1 or 2.

IV.2.3-15 Radial Temperature-Time Variation in a Disk at Position 1 in the Upstream Target Section, Operated in Mode 3

IV.2.3-16 Radial Temperature-Time Variation in a Disk at Position 4 in the Upstream Target Section, Operated in Mode 1

IV.2.3-17 Surface Temperature of Target Vessel Window IV.2-48

IV.2.4-1 ANSYS Model for 2-D Thermal Stress IV.2-50

IV.2.4-2 Mechanical Properties of Tungsten IV.2-51

IV.2.4-3 Stress Intensity in Disks at Two Positions in the Upstream Target Section Due to Radial Gradient Only

IV.2.4-4 Stress Intensity in Disks at Three Positions in the Downstream Target Section Due to Radial Temperature Gradient Only.

IV.2.4-5 Tangential Stress Distribution in Last Disk of the Upstream Target Section Using 2-D Temperature Distribution

IV.2.5-1 Yield Stress Measured at a Strain Rate, $\dot{\varepsilon} \approx 1 \times 10^{-4} \mathrm{~s}^{-1}$ for Pure Ta, Pure W, and T-111

IV.2.5-2. Variation of the Yield Stress and Percent Elongation at $400^{\circ} \mathrm{C}$ for Tungsten

IV.2.5-3 Variation of Brittleness with Hydrogen Concentration for Various Tantalum Alloys

IV.2.6-1 Target Coolant Flow Circuit IV.2-65

IV.2.6-2 Arrangement of the 30-Hz Target Cooling System in Building 371 


\section{FIGURES (Cont)}

IV.2.6-2a Sectional View of the $30-\mathrm{Hz}$ Target Cooling System in Building 371

IV.2-68

IV.2.6-3 Arrangement of the 10- $\mathrm{Hz}$ Target Cooling System in Building 367

IV.2-69

IV.2.6-3a Sectional View of the 10-Hz Target Cooling System in Building 367

IV.2-70

IV.3.1-1 Schematic Representation of a Typical Cryogenic Moderator Assembly for IPNS Upgrade

IV.3.1-2 Typical Dimensions of a Coupled Liquid Hydrogen Moderator Assembly in Wing Geometry at IPNS Upgrade

IV.3.2-1 Neutron Beam Spectra at the Ambient Water Moderator Surfaces.

IV.3-6

IV.3.2-2 Calculated Power Distribution in Moderators and Premoderators

IV.3.2-3 Comparison of Neutron Spectra from the Models with and without Shield

IV.3.2-4 Effects of the Premoderator on the Neutron Beam Spectrum. IV.3-11

IV.3.2-5 Effects of the Decoupler on the Neutron Beam Spectrum. IV.3-12

IV.3.2-6 Comparison of Neutron Beam Spectra for Water and Liquid Hydrogen Moderators at Room Temperature

IV.3.2-7 Effects of the Decoupler IV.3-14

IV.3.2-8 Pulse-Widths for the Moderators Listed in Table IV.3.2-2 ............................. IV.3-20

IV.3.4-1a Layout of Skid-Mounted Cooling Systems in Building 367, for the $10-\mathrm{Hz}$ Target

IV.3.4-1b Sectional Elevation of Skid-Mounted Cooling Systems in Building 367, for the $10-\mathrm{Hz}$ Target.

IV.3.4-2a Layout of Skid-Mounted Cooling Systems in Building 371, for the $30-\mathrm{Hz}$ Target

IV.3.4-2b Sectional Elevation of Skid-Mounted Cooling Systems in Building 371, for the $30-\mathrm{Hz}$ Target 


\section{FIGURES (Cont.)}

IV.3.4-3 Cooling Circuit Schematic for Water Moderators and Premoderators

IV.3.4-4 Liquid Methane Moderator Cooling Circuit Schematic, Shown for Three Moderators

IV.3.4-5a Plan View of One of the Shielded Areas Housing the Liquid Methane and Liquid Hydrogen Cooling Systems.

IV.3.4-5b Sectional View A-A of One of the Shielded Areas Housing the Liquid Methane and Liquid Hydrogen Cooling Systems.

IV.3.4-5c Sectional View B-B of One of the Shielded Areas Housing the Liquid Methane and Liquid Hydrogen Cooling Systems.

IV.3.4-6 Schematic Diagram of the Liquid Hydrogen Moderator Cooling System, Shown for Two Hydrogen Moderators. IV.3-37

IV.3.4-7 Schematic Diagram of the Reflector Cooling System IV.3-40

IV.3.4-8 Schematic Diagram of the Biological Shield Cooling System IV.3-42

IV.3.4-9 Schematic Diagram of the Target Tank Cooling System. IV.3-43

IV.3.4-10 Schematic Diagram of the Proton HET Line Vacuum Window Cooling System

IV.4.1-1 Locations of the Biological Shields for the 30-Hz and $10-\mathrm{Hz}$ Target Stations

IV.4.1-2 Layout of a Target Station, Showing the Biological Shield and the Remote-Handling Hot Cell

IV.4.1-3 Cross Section through the Biological Shield, Centered on Neutron Beam Ports B-2 and E-2 of IV.4.1-2

IV.4.2-1 Foundation Plan and Section Views

IV.5.1-1 Target-Train Assembly Shown in the Operating Position.

IV.5.2-1 Details of the Target-Train Assembly

IV.5.2-2 Arrangement of Target-Train Fluid Connections behind the Seal Sheet. 


\section{FIGURES (Cont.)}

IV.5.2-3 Outside View of the Target Seal Sheet

IV.5-6

IV.5.2-4 Arrangement of Target Cooling Water Connections behind the Seal Sheet.

IV.5.2-5 Typical Junction Box for Cryogenic Moderator Fluid Connections

IV.5-8

IV.5.3-1 Sequence of Operations to Remove the Target-Train Assembly into the Remote-Handling Cell.

IV.6.2-1 Common Holdup and Exhaust System Used for Most Gaseous Exhausts.

IV.6.2-2 Purge System for Target Tank, Shielding Tank, and Hot Cell

IV.6.5-1 Radioactive Waste Retention Tank for the 10-Hz Target Station, Located in the Vault near Building 367

IV.8.1-1 Longitudinal Section through a Typical Neutron Beam Port

IV.8-2

IV.8.1-2 Horizontal Slice through the Reflector at the Midplane of the Upper Moderators, Showing the Cutouts for the Neutron Beams

IV.8.2-1 Cross-Sectional Views of the Biological Shield, Showing Details of the Neutron Beam Gates and Gate Actuators

IV.8.2-2 Details of a Neutron Beam Gate and Actuator

IV.8-6

V.2.1-1 Plan View of the SEPD Scattering Flight Path at IPNS Upgrade.

V.2.1-2 GPPD Configuration at IPNS Upgrade, Showing Increased $90^{\circ}$ Scattering Path Length.

V.2.1-3 Schematic Representation of the Detector Positioning for the HRPD

V.2.1-4 Schematic Representation of the RSD, Showing the 12-m and 25-m Sample Positions.

V.2.2-1 Plan View of the SAND at IPNS Upgrade

V.2.2-2 Schematic Representation of the HRSAND 


\section{FIGURES (Cont)}

V.2.2-3 Schematic Representation of the DCSAND ............................................... V.. V.2-22

V.2.3-1 Plan View of the GLAD........................................................................ V.2-26

V.2.3-2 Schematic Representation of the SCD ....................................................... V..2-28

V.2.4-1 Configurations of the POSY-I and POSY-II at IPNS....................................... V.2-35

V.2.4-2 Schematic Repqwresentation of the Multiple-Beam Geometry Used for the HIREF.

V.2.4-3 Schematic Indication of the Geometry Used in the GREF Reflectometer........ V.2-39

V.2.5.1 Plan View of the HRMECS at IPNS............................................................ V.2-43

V.2.5-2 Plan View of the HRMECS at IPNS Upgrade............................................... V.2-45

V.2.5-3 Plan View of the LRMECS........................................................................ V... V.

V.2.5-4 Schematic Representation of the SCCS......................................................... V.. V.2-50

V.2.6-1 Unmodified Configuration of the QENS.......................................................... V.2-54

V.2.6-2 Schematic Representation of the HRBS........................................................ V.. V.2-57

V.2.6-3 Schematic Representation of the TFCA .......................................................... V.2-60

V.2.6-4 Schematic Representation of the MICAS ......................................................... V.2-63

V.2.7-1 Schematic Representation of the TOFNSE.................................................... V.2-69

V.2.9-1 Location of the Reference Set of Instruments on the Neutron Beamlines ........ V.2-74

V.3.1-1 Schematic Representations of (a) Fermi, (b) Drum, and

(c) Disk Choppers.

V.3.1-2 The IPNS Fermi $E_{0}$ Chopper Design ............................................................ V... V.7

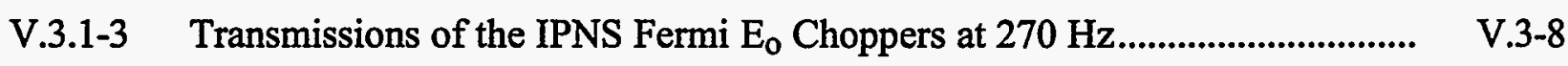

V.3.1-4 The IPNS Narrow-Band $t_{0}$ Choppers.......................................................... V.3-10 


\section{FIGURES (Cont.)}

V.3.1-5 The ISIS Broad-Band $t_{0}$ Chopper.

V.3-12

V.3.3-1 Neutron and Gamma-Ray Dose-Rate-to-Flux Conversions

V.3-24

V.3.3-2 Target-Moderator-Beamline Geometries: (a) Wing Geometry with Proton Beam Direction Normal to the Page and (b) Flux-Trap Geometry with Proton Beam Direction as Shown.

V.3.3-3 Neutron-Beam Spectra from the IPNS Upgrade Moderators.

V.3.3-4 Dose Rate $30 \mathrm{~cm}$ from the Surface of a Cylindrical Iron Beamline Shield of Radius R, Calculated for a Distance of $6 \mathrm{~m}$ from the Moderator

V.3.3-5 (a) Geometry for Shielding against High-Energy Neutrons from the Target; (b) Transition Shield at the Beamline Interface with the Biological Shield

V.3.6-1 Schematic Configuration of a Typical Instrument DAS

V.4.3-1 Locations of the Irradiation Tubes.

VI.1.1-1 ANL Site Plan

VI.1.1-2 360/ZGS Area Site Plan

VI.1-3

VI.1.1-3 IPNS Upgrade Site Photo

VI.1.1-4 IPNS Upgrade Area Plan

VI.2.1-1 Linac Building 394 Operating Floor.

VI.2-2

VI.2.1-2 Section through Linac Facility and LET Tunnel into Ring Building (top); Section A through Linac Building Showing Klystron Gallery and Linac Tunnel (bottom).

VI.2.1-3 Section B-2 Personnel Stairs and Bridge over LET Berm (top right); Section B-4 LET at Wide Area next to Building 370 (top right);

Section B-1 Old Bluff Road Crossing over LET (bottom left);

Section B-3 LET Crossing Utility Tunnel at Buildings 376 and 370 (bottom right).

VI.2.1-4 Building 394 Service Layout 


\section{FIGURES (Cont.)}

VI.2.2-1 Vertical Cross Section of the Ring Building and Center Building Showing the Original ZGS.

VI.2-10

VI.2.2-2 Operating Floor Building 365

VI.2-11

VI.2.2-3 Center Building and Ring Building Section, Buildings 364/365

VI.2-13

VI.2.2-4 Building 364 and 365 Service Layout.

VI.2-15

VI.2.3-1 Center Building 364 Service Floor Plan

VI.2-17

VI.2.3-2 Center Building 364 First Floor Plan

VI.2-18

VI.2.3-3 Center Building 364 Second Floor Plan

VI.2-19

VI.2.3-4 Center Building 364 Third Floor Plan

VI.2-21

VI.2.3-5 Center Building 364 Fourth Floor Plan

VI.2-22

VI.2.3-6 Building 364 and 365 Roof/Roadway Plan

VI.2-23

VI.2.4-1 Power Supply Building 376 Service Floor Plan

VI.2-26

VI.2.4-2 Power Supply Building 376 First Floor Plan.

VI.2-27

VI.2.4-3 Section A-A through Building 376

VI.2-29

VI.2.4-4 Building 376 Utility Substation

VI.2-31

VI.2.5-1 $\quad 10-\mathrm{Hz}$ Target Building 369 First Floor Plan

VI.2-33

VI.2.5-2 Section A-A through Building 369

VI.2-34

VI.2.5-3 Building 367 and 369 Utility Substation. VI.2-36

VI.2.6-1 $10-\mathrm{Hz}$ Target Support Building 367 First Floor Plan VI.2-38

VI.2.6-2 Section A-A through Building 367 with Underground Adjacent Vault VI.2-39

VI.2.7-1 30-Hz Target Building 370 First Floor Plan VI.2-42

VI.2.7-2 Section A-A through Building 370 VI.2-43 


\section{FIGURES (Cont.)}

VI.2.7-3 Building 370 and 371 Utility Substation.

VI.2-46

VI.2-8-1 30-Hz Target Support Building 371 First Floor Plan.

VI.2-48

VI.2.8-2 Section A-A through Building 371 with Underground Adjacent Vault.

VI.2-49

VI.2.9-1 Target Labs/Preparation Building 368 First Floor Plan

VI.2-52

VI.2.10-1 Lab/Office Building 360 First Floor Plan.

VI.2-54

VI.2.11-1 Lab/Office Building 362 First Floor Plan

VI.2-56

VI.2.12-1 360/ZGS Mechanical Site Plan.

VI.2-59

VI.2.12-2 377 Area Cooling Tower Plan

VI.2-60

VI.2.12-3 360/ZGS Electrical Site Plan

VI.2-62

VII.3-1 Conventional Facilities Schedule.

VII.3-3

VII.3-2a Linac System Schedule.

VII.3-4

VII.3-2b Synchrotron Magnet Schedule.

VII.3-5

VII.3-2c Synchrotron Magnets - Power Supplies Schedule.

VII.3-6

VII.3-2d Special Magnets and Power Supplies Schedule.

VII.3-7

VII.3-2e Synchrotron rf System Schedule.

VII.3-8

VII.3-2f Synchrotron Vacuum System Schedule.

VII.3-9

VII.3-2g Diagnostics System Schedule.

VII.3-10

VII.3-2h Controls and Computing System Schedule.

VII.3-11

VII.3-2i Transfer Lines Schedule.

VII.3-12

VII.3-2j Neutron Target Stations Schedule.

VII.3-13

VII.3-2k Instrumentation Schedule. 


\section{FIGURES (Cont)}

VII.3-3 Project Critical Path. VII.3-15

VII.4-1 Construction Project Person-Effort Distribution. VII.4-2

VII.4-2 Budget Authority Distribution. VII.4-4

VII.4-3 Cumulative BA Distribution. VII.4-5

VII.4-4 Comparison between Cumulative BA and BO VII.4-8

B.1-1 Preconstruction R\&D Schedule.

C.1-1 Layout of the ISIS Pulsed Muon Facilities

C.1-2 IPNS Upgrade Experiment Halls, Showing a Muon Facility in One Possible Location

D.1.1-1 ANL Site Plan

D-2

D.1.1-2 IPNS Upgrade Greenfield 800-Site

D-3 


\section{TABLES}

I.2.1-1 Accelerator Parameters

I.2.2-1 IPNS and IPNS Upgrade Moderators............................................................ I...

I.2.2-2 Reference Set of Diffractometers and Reflectometers........................................ I.2-7

I.2.2-3 Reference Set of Inelastic Scattering Instruments and

Component Development Instruments.

II.1.5-1 Design Goal Parameters ..................................................................................... II.1-4

II.2.1-1 Normal Cell Parameters............................................................................... II.2-3

II.2.1-2 Synchrotron Parameters....................................................................... II.2-6

II.2.1-3 Multipole Coefficients of Synchrotron Magnets............................................. II.2-30

II.2.4-1 Extraction Kicker and Septum Magnet Parameters............................................ II.2-59

II.3.1-1 Synchrotron Ring Magnet Field Strengths and Aperture Requirements........... II.3-2

II.3.1-2 2.0-GeV Parameters for the Synchrotron Ring Magnets ..................................... II.

II.3.2-1 Loss Budget for the Dipole Dual-Frequency Resonant Circuit at $2.2 \mathrm{GeV} . . . . . \quad$ II.3-23

II.3.2-2 Parameters for the Dipole Dual-Frequency Resonant Circuit at $2.2 \mathrm{GeV}$........ II.3-24

II.3.2-3 Loss Budget for the Quadrupole Dual-Frequency Resonant Circuit of One Family at $2.2 \mathrm{GeV}$

II.3.2-4 Parameters for the Quadrupole Dual-Frequency Resonant Circuit of One Family at $2.2 \mathrm{GeV}$

II.3.3-1 Parameters for LET Magnets............................................................................ II.3-31

II.3.3-2 LET Power-Supply Parameters at $10 \%$ over $400 \mathrm{MeV}$.................................... II.3-32

II.3.3-3 Parameters for HET Magnets ...................................................................... II.3-34

II.3.3-4 HET Power-Supply Parameters at $2.2 \mathrm{GeV}$.................................................... II..3-35

II.3.4-1 Parameters for Synchrotron Bumper Magnets ................................................. II.3-37 


\section{TABLES (Cont.)}

II.3.4-2 Parameters for Extraction Kicker Magnets

II. $3-41$

II.3.5-1 Parameters for Synchrotron Injection Septum Magnets..................................... II..3-46

II.3.5-2 Parameters for Synchrotron Extraction Septum Magnets................................... II.3-50

II.3.5-3 Septum Magnet Power Supply Parameters for 2.2-GeV Operation ................. II.3-54

II.4.3-1 Physical Parameters of Cavity ....................................................................... II.4-25

II.4.3-2 Operating Characteristics of Cavity for Nominal Operation............................. II. II.4-28

II.4.3-3 Eimac 8973 Tetrode, Class-B Operation .......................................................... II.4-30

II.4.3-4 Final Amplifier Characteristics for Single-Ended Grounding Cathode............... II.4-33

II.5.2-1 RCS Impedance Estimation................................................................................ II.5-2

II.5.2-2 Ratio of Shunt Impedance and Q of First Few HOMs for an rf Cavity........... II.5-9

II.5.4-1 Head-Tail Instability Growth Rate vs. Chromaticity in the Vertical Plane at the End of Injection

II.5.4-2 Threshold Tune Spread According to the Coasting Beam Stability Criterion

II.6.1-1 Vacuum Chamber Dimensions ...................................................................... II.6-2

II.6.3-1 Input Data for Vacuum Finite-Element-Analysis Computer Program ............. II. II.13

II.7.1-1 Beam Diagnostic Instrumentation ................................................................. II.

II.9.2-1 Transverse Attenuation Lengths for High-Energy Neutrons.............................. II.9-4

II.9.2-2 Shielding Requirements for the IPNS Upgrade .............................................. II.9-9

III.2-1 IPNS Upgrade Injector Linac Performance Requirements ................................. III.2-2

III.3-1 Summary of IPNS Upgrade Injector Linac Physics Design Parameters ........... III.3-2

III.4.3-1 Ion Source Operating Parameters...................................................................... III.4-5 


\section{TABLES (Cont.)}

III.4.3-2 Main RFQ Parameters .............................................................................. III.4-10

III.4.3-3 RGDTL Parameters .................................................................................... III.4-13

III.4.4-1 DTL Parameters............................................................................................ III.4-16

III.4.5-1 Matching Section Parameters...................................................................... III.4-22

III.4.6-1 CCL Parameters .......................................................................................... III.4-24

III.4.7-1 Klystron Operating Parameters Common to the Entire Injector ........................ III.4-29

III.4.7-2 Klystron Operating Parameters for Linac Subsystems..................................... III.4-30

III.4.7-3 Varian Klystron System Parameters..................................................................... III.4-31

III.4.7-4 Klystron Modulator Specifications by Subsystem ......................................... III.4-33

III.4.9-1 Number of Data Channels Required by Linac Subsystems ............................... III.4-37

III.4.9-2 Number of VXI Components Required by Linac Subsystems.......................... III.4-38

III.5.2-1 Power and Water Cooling Requirements for the Injector Linac System........... III.5-4

IV.2.2-1 Geometrical and Material Features of the Target Station Model........................ IV.2-9

IV.2.2-2 Power Deposition in the Target Station for Proton Beam Power

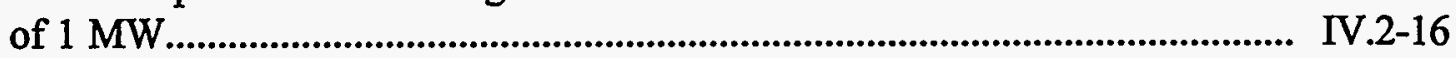

IV.2.2-3 Power Distribution in the Target Sections for Proton Beam Power of $1 \mathrm{MW}$........................................................................................................... IV.2-18

IV.2.2-4 Radial Power Distribution in the Disks of the Upstream Target Section for Proton Beam Power of 1 MW........................................................................ IV.2-20

IV.2.2-5 Buildup of Activity and Afterheat for the IPNS Upgrade Target .................... IV.2-26

IV.2.2-6 Decay of Afterheat Power for the IPNS Upgrade Target................................. IV.2-27

IV.2.2-7 Decay of Activity for the IPNS Upgrade Target............................................. IV.2-28

IV.2.4-1 Summary of Stresses.................................................................................... IV.2-54 


\section{TABLES (Cont.)}

IV.2.5-1 Properties to Consider in Selection of Target Material.

IV.2-57

IV.2.5-2 Acceptable Candidate Materials in Order of Preference, Compared

to the Desired Prioritized Properties of a Target Material .

IV.2-63

IV.2.7-1 Peak LOCA Transient Temperatures in the Target, Target Vessel, and Reflector.

IV.3.2-1 Neutron Beam Current at $1 \mathrm{eV}$ from Moderator.

IV.3-16

IV.3.2-2 Maxwellian Parameters for Several Moderators

IV.3-18

IV.3.3-1 Lifetimes of Liquid Methane Moderators

IV.3-23

IV.6.6-1 Annual Predicted Dose from 1-MW IPNS Upgrade to an Individual at the Nearest ANL Site Boundary.

V.2.1-1 SEPD and GPPD Parameters at IPNS Upgrade.

V.2.1-2 HRPD Parameters at IPNS Upgrade.

V.2.1-3 Parameters for the RSD.

V.2-10

V.2.1-4 Reference Set of TOF Powder Diffractometers at IPNS Upgrade.

V.2-13

V.2.2-1 Parameters for the SAND

V.2-16

V.2.2-2 Parameters for the HRSAND..

V.2-19

V.2.2-3 Reference Set of TOF Small-Angle Diffractometers at IPNS Upgrade.

V.2-24

V.2.3-1 GLAD Parameters at IPNS Upgrade

V.2.3-2 SCD Parameters at IPNS Upgrade

V.2.3-3 Parameters for the HQSCD.

V.2.3-4 Reference Set of Additional TOF Diffractometers at IPNS Upgrade. V.2-33

V.2.4-1 Parameters for the HIREF. V.2-38

V.2.4-2 Parameters for the GREF. V.2-40 


\section{TABLES (Cont.)}

V.2.4-3 Reference Set of TOF Reflectometers at IPNS Upgrade ................................... V.2-42

V.2.5-1 HRMECS and LRMECS Parameters at IPNS Upgrade .................................. V. V.2-44

V.2.5-2 CNCS Parameters at IPNS Upgrade ........................................................... V.2-48

V.2.5-3 Reference Set of TOF Chopper Spectrometers at IPNS Upgrade..................... V.2-52

V.2.6-1 QENS Parameters at IPNS Upgrade ............................................................. V.2-55

V.2.6-2 Parameters for the HRBS......................................................................... V.2-58

V.2.6-3 Parameters for the TFCA...................................................................... V.2-61

V.2.6-4 Reference Set of Crystal-Analyzer Spectrometers at IPNS Upgrade................ V.2-65

V.2.7-1 TOFNSE Parameters at IPNS Upgrade .......................................................... V.2-68

V.2.9-1 Beamline Requirements for Instruments..................................................... V.2-72

V.2.9-2 Moderator Types......................................................................................... V...2-73

V.3.1-1 Required Chopper Types .............................................................................. V.3-6

V.3.1-2 Parameters for Bandwidth-Limiting Choppers................................................. V.3-14

V.3.2-1 Neutron Guide Assessment ............................................................................. V.3-20

V.3.2-2 Neutron Guide Requirements......................................................................... V.3-21

V.3.3-1 Neutron Mean-Free-Paths in Shielding Materials............................................... V.3-25

V.3.5-1 Discrete Detector Requirements ....................................................................... V... V.34

V.3.5-2 Position-Sensitive Detector Requirements...................................................... V.3.3.-37

V.3.5-3 Position-Sensitive Detectors Requiring Development......................................... V.3-38

V.3.9-1 Utility Requirements for the Instruments at IPNS Upgrade ............................ V.3-44

V.4.3-1 Characteristics of IPNS Upgrade Neutron Irradiation Tubes at 1-MW Proton Beam Power 


\section{TABLES (Cont.)}

VII.1-1 Complete Work Breakdown Structure ............................................................. VII.1-2

VII.2-1 Craft Code Rate for 1995 ............................................................................. VII.2-2

VII.2-2 Escalation Rates .......................................................................................... VII.2-3

VII.2-3 Project Cost Breakdown by Major Categories ................................................. VII.2-4

VII.2-4 Project Cost Breakdown by WBS Code ........................................................... VII.2-6

VII.4-1 Budget Authority Distribution .......................................................................... VII.4-3

VII.4-2 TEC Breakdown by Major Category............................................................... VII.4-6

VII.4-3 Budget Outlay Distribution ............................................................................. VII.4-7

B.13-1 Costs for Preconstruction R\&D..................................................................... B-15

D.2-1 Relationships between 360 Area and 800 Area Buildings ................................ D-5 
I

Background and Overview 



\subsection{BACKGROUND}

Over the past 40 years, research based on slow neutron beams has been crucial to advances in fundamental science, technology, and medicine. Achievements in neutron scattering research have attracted many awards in recent years, including the highest when, last year, two of the early workers, Clifford G. Shull and Bertram N. Brockhouse, shared the 1994 Nobel Prize in Physics for their pioneering work in developing neutron scattering methods. Discussions and summaries of advances in this field appear regularly. 1.4

Neutrons provide critical information that is impossible to acquire by any other means. Furthermore, for many purposes, they provide a necessary complement to $x$-rays, so that the parallel development of both neutron sources and $\mathrm{x}$-ray synchrotron facilities is essential.

In the past 10 years, neutron studies have provided information critical to the understanding of new materials that have emerged and have had a singular impact in materials science. One can cite the following specific examples of key contributions of neutrons in fundamental science:

- Structure and excitations of high- $\mathrm{T}_{\mathrm{c}}$ superconductors,

- Polymer conformations and interactions,

- Structure and dynamics of new-generation catalysts,

- Interfacial structure of polymeric and magnetic layers,

- Spin dynamics in highly correlated metals,

- Structure and phase transformations of fullerenes ("buckyballs"), and

- Condensate fraction in superfluid helium.

Important contributions to technological advances have paralleled the major developments in fundamental sciences cited above:

- Residual stress in metals and ceramics,

- Radiography of aircraft and energy-production components,

- Near-surface impurities and deposits in semiconductors,

- Giant magneto-resistant multilayers,

- Cavitation and embrittlement of structural alloys, 
- Adhesion of polymer laminates,

- Materials irradiation for fission and fusion power programs, and

- Sintering processes of ceramics.

Over the last 20 years, the United States has fallen alarmingly behind the European scientific community in the availability of up-to-date neutron sources and instrumentation. HFBR and HFIR, which are the major research reactors of the U.S. Department of Energy (DOE), were built more than 25 years ago, and their remaining lifetimes of a decade or so are uncertain; the status of HFIR appears especially precarious. In June 1992, Dr. W. Happer, Director of DOE's Office of Energy Research, requested the formation of a panel to report on key issues concerning possible new sources, emphasizing especially the comparison of reactors and pulsed spallation neutron sources. The Panel, chaired by Professor Walter Kohn (University of California, Santa Barbara), had substantial representation from universities, industry, and government laboratories, and it included both neutron specialists and other condensed matter scientists. In addition to visiting the sites of the four DOE laboratories with interests in constructing future sources, the Kohn Panel organized a broad Review of Neutron Sources and Applications, with the participation of 70 national and international experts, in Oak Brook, Illinois. Separate working groups were assembled to (1) review the status of advanced research reactors and spallation sources and (2) provide an update on scientific, technological, and medical applications, including neutron scattering research in a number of disciplines, isotope production, materials irradiation, and other important uses of neutrons. The Kohn Panel's report was published in January $1993^{1}$ and findings and conclusions of the working groups involved in the Review were published in January 1994.4

After reviewing the capability and cost-effectiveness of different alternatives, the Kohn Panel concluded that as a technologically leading nation, the United States urgently needs to construct a complementary pair of neutron sources: a next-generation research reactor and a powerful pulsed spallation source (PSS). These facilities are essential to maintain or reestablish U.S. leadership in broad areas of physical, biological, and materials sciences, in radiomedicine, and in associated technologies. Although the required investment is substantial, the payoff - in terms of both directly associated jobs and enhancement of the nation's technological and economic power - will be much greater and will extend far into the next century.

Recommendation 1: Complete the design and construction of the Advanced Neutron Source (ANS) according to the schedule proposed by the project.

Recommendation 2: Immediately authorize the development of competitive proposals for the cost-effective design and construction of a 1-MW PSS. Evaluation of these proposals should be done as soon as possible, leading to a construction timetable that does not interfere with rapid completion of the ANS. 
Subsequently, two workshops took place within the PSS community. One workshop further evaluated accelerator prospects (Santa Fe, February 1993) 5 ; the other addressed technological and scientific applications of a 1-MW PSS (Argonne, May 1993). ${ }^{6}$ The accelerator workshop addressed the different possible methods of supplying 1-MW proton beams to spallation targets, while the technology and science workshop documented the great variety of problems that could be solved with a 1-MW pulsed source, ranging from fundamental science to engineering materials and technology.

The President's budget for fiscal year (FY) 1996, which was announced on February 6, 1995, calls for cancellation of the ANS project. This situation puts even more emphasis on the development of PSSs for the nation's future. This feasibility study represents the response of Argonne National Laboratory (ANL) to the Kohn Panel's recommendation for a cost-effective design and construction of a dedicated 1-MW PSS.

Argonne comes to this proposal with 12 years of successful experience in operating the Intense Pulsed Neutron Source (IPNS). The hallmarks of the IPNS experience are its very high reliability, continuous sequence of source and instrumentation developments, strong user program, and the highest scientific productivity per unit cost of operation, by any measure, of all the DOE neutron scattering facilities. The reliable operation of the facility has its origin in ANL's tradition of excellence in accelerator science and technology.

The present IPNS synchrotron was originally designed and built in the mid-1970s as the booster for a high-energy physics proton accelerator, the 12-GeV Zero Gradient Synchrotron (ZGS). The ZGS was used for the nation's high-energy physics program during the 1960s and 1970s. Notable achievements in accelerator science by ZGS personnel include the first-ever acceleration of polarized protons to the multi-GeV energy range ${ }^{7}$ and the first demonstration of negative-hydrogen-ion charge exchange injection into a circular accelerator. ${ }^{8}$ Existing pulsed source facilities, as well as those planned for the future, all use charge exchange injection.

From 1982 to 1983, accelerator scientists who formerly worked at the ANL ZGS designed a synchrotron option for Germany's SNQ (Spallations Neutronenquelle). 9,10 Many of the design concepts employed in this report originated in the SNQ studies. This group also studied a pulsed-source option using the fixed field alternating gradient (FFAG) ${ }^{11}$ synchrotron concept. The FFAG proposal was presented to the Seitz-Eastman Panel in 1984, and it was designated as the fourth priority for new materials research facilities.

These same ZGS accelerator scientists planned and designed the Advanced Photon Source (APS) ${ }^{12}$ from 1984 to 1987 and obtained approval for its construction in 1988. The construction of the APS at ANL is nearly complete; in the process, ANL accelerator personnel have accumulated a tremendous wealth of current information on the construction and procurement of accelerator components.

The proposal put forward here uses all buildings and infrastructure of the former ZGS facility. The ZGS Ring Building can house a synchrotron about 200 meters in circumference and has enough radiation shielding to accommodate a 1-MW accelerator system. Two of the former 
high-energy physics experimental buildings can house two neutron-generating target stations and associated neutron beamlines. It is estimated that the use of existing buildings saves about $\$ 175$ million in construction costs.

The IPNS Upgrade will be the fourth in a series of pulsed spallation sources constructed at Argonne. The first was the ZGS Intense Neutron Generator Prototype (ZING-P). This facility was built in 1973 and was the world's first test of the spallation neutron source concept. It used the ZGS $200-\mathrm{MeV}$ prototype booster synchrotron and achieved a beam power of $100 \mathrm{~W}$. ZING-P', a second prototype that used the present IPNS accelerator, operated very productively from 1977 through 1980. It achieved $2 \mathrm{~kW}$ of beam power. The IPNS, operational since 1981, achieves a beam power of $7 \mathrm{~kW}$ and is a scientifically prolific and efficient research installation that has led in the development of pulsed source technology and of pulsed source, time-of-flight neutron scattering instrumentation. The IPNS has set the standard for PSS operational reliability, being available $95 \%$ of the scheduled time since it started operation in 1981. From the outset, the IPNS has hosted a productive and highly cost-effective user program, producing more than 250 scientific publications and serving more than 200 users each year.

Argonne is conveniently located near Chicago, providing ready access from around the country and the world. Argonne has established an international reputation for neutron source and instrumentation development. The community of neutron scattering specialists, both residents and long-term users from other institutions, has consistently produced high-quality results. One of Argonne's major activities is materials research, which will provide important support to the research program at the IPNS Upgrade. As has been found at several other installations (for example, Brookhaven, Grenoble, and KEK), the collocation of a neutron source with a synchrotron radiation facility provides significant scientific synergism. The accelerator design and construction groups come from a series of successful programs that lead conveniently into the IPNS Upgrade project. Close collaboration is maintained with other installations around the world through frequent visits and cooperation and through the International Collaboration on Advanced Neutron Sources (ICANS), scheduled to hold its 13th meeting in October 1995 at the Paul Scherrer Institut (PSI) in Villigen, Switzerland.

\subsection{ACCELERATOR AND TARGET STATION PARAMETERS}

The IPNS Upgrade is a dedicated source for slow neutron scattering that uses a proton accelerator delivering a time-average beam power of $1 \mathrm{MW}$.

The choice of proton beam energy is determined by several factors, including neutron yield, heat removal from the target, ease of machine construction and serviceability, and available space in the ZGS Ring Building. Efficient production of neutrons by spallation requires proton 
energies above $500 \mathrm{MeV}$, so that protons interact principally with nuclei in the target. The neutron yield (number of neutrons per proton) for thick targets is given by

$$
\mathrm{Y}(\mathrm{E}, \mathrm{A})= \begin{cases}0.1\left(\mathrm{E}_{\mathrm{GeV}} \pm 0.120\right)(\mathrm{A}+20), & \text { except fissionable materials } \\ 50\left(\mathrm{E}_{\mathrm{GeV}} \pm 0.120\right), & { }^{238} \mathrm{U}\end{cases}
$$

where $\mathrm{E}_{\mathrm{GeV}}$ is the proton beam energy in $\mathrm{GeV}$ and $\mathrm{A}$ is the atomic number of the target material. For energies above the apparent threshold of $0.12 \mathrm{GeV}$, the neutron yield is roughly proportional to energy. The neutron production rate is proportional to the beam power.

The design is also influenced by the distribution of the deposited heat and the distribution of produced neutrons. Most experience to date has been confined to energies below $1 \mathrm{GeV}$ and confirms Equation I.1.2-1 above. Monte Carlo studies show that the power density distribution, neutron production distribution, and total yield of neutrons favor energies higher than $1 \mathrm{GeV}$, as shown in Figure I.1.2-1, 13 at least up to about $3 \mathrm{GeV}$, the code-imposed limit of the study. The calculations confirm that the neutron production rate for constant beam power is approximately constant and that the neutron production rate is approximately proportional to the proton beam power within the range of proton energies studied.

Figure I.1.2-2 shows the variation of the emerging neutron current as a function of axial position in a target (tallied at a radius of $6.2 \mathrm{~cm}$ ), and Figure I.1.2-3 shows the variation of the thermal power density (averaged over radius). Higher energies are preferable due to the lower peak power density at the upstream end of the target for a given beam power. These calculations indicate that proton energies above $1.5 \mathrm{GeV}$ are equivalent with respect to the thermal and neutronic design of the target and moderator systems.

The choice of $30 \mathrm{~Hz}$ as the source repetition frequency was based upon a survey of scientific requirements and an evaluation of preliminary designs for neutron scattering instruments for a 1-MW pulsed source. Two categories of instruments emerged from the study of scattering instruments, those for which $30 \mathrm{~Hz}$ is preferable, and those that require a lower frequency, for which $10 \mathrm{~Hz}$ is satisfactory. Few of the instruments we considered could effeciently use frequencies higher than $30 \mathrm{~Hz}$. Two targets that operate at different frequencies provide the flexibility to serve both classes of instruments and double the available number of beamlines and instruments. The accelerator delivers one out of three pulses to the low-frequency station at $10 \mathrm{~Hz}$, and the high-frequency station receives the remaining two pulses of the $30-\mathrm{Hz}$ pulse train. The high-frequency target is capable of using the full $1 \mathrm{MW}$ of beam power, or all pulses of the $30-\mathrm{Hz}$ pulse train.

Our preference is to have the highest possible energy and lowest possible current. This preference does not affect the neutron production rate, since the rate is proportional to the proton beam power. High-energy protons can be obtained by acceleration through a linear accelerator or through a circular machine. A single-pass machine such as a linac tends to have high construction and operating costs; therefore, proton linacs tend to be lower-energy machines. 


\section{1-MW Proton Beam}

on $10 \mathrm{~cm} \times 100 \mathrm{~cm}$ target

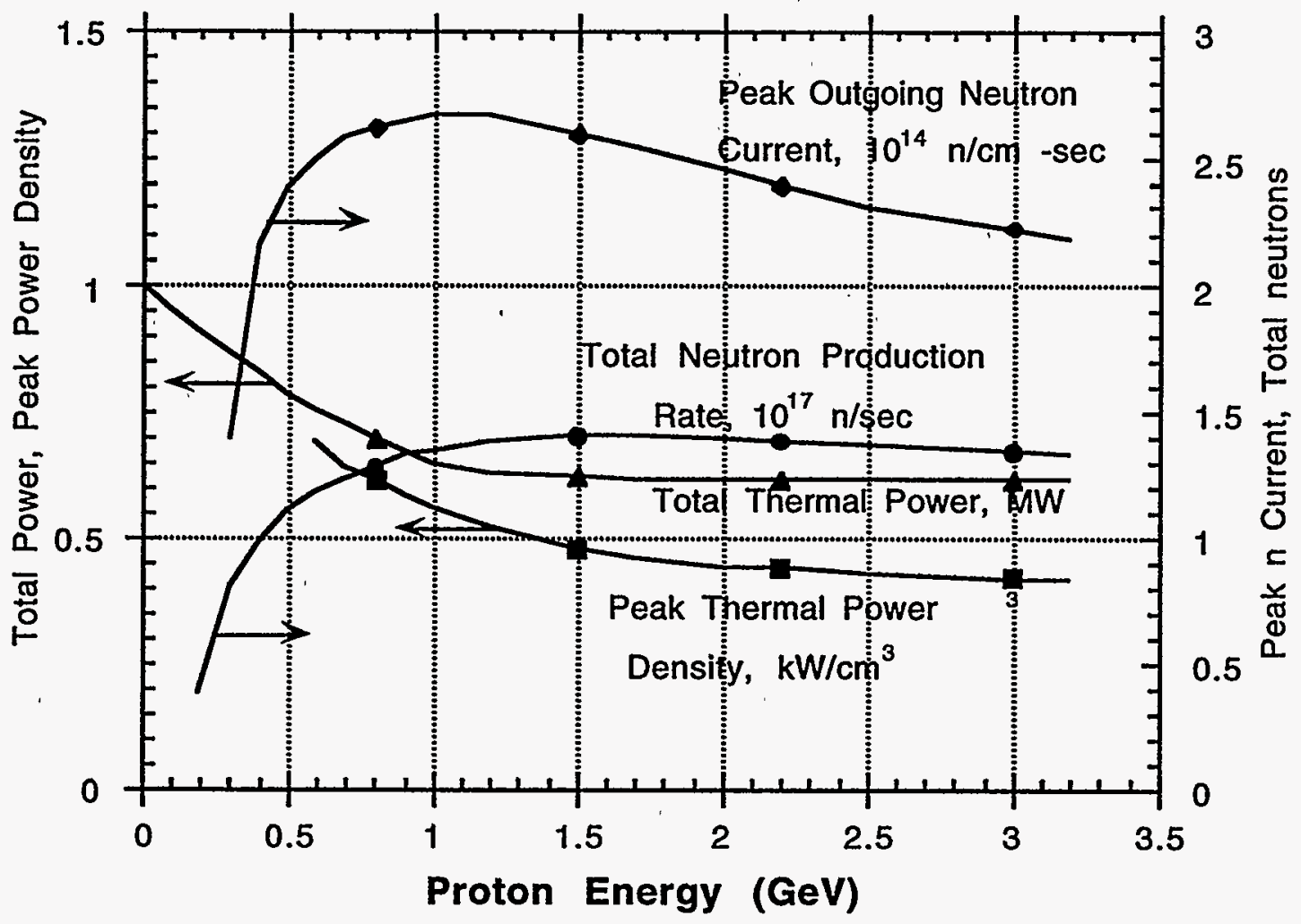

Figure 1.1.2-1 Variation of the Thermal Power Density and Neutron Production Density as a Function of Axial Position in Targets Irradiated by Protons of Various Energies. 


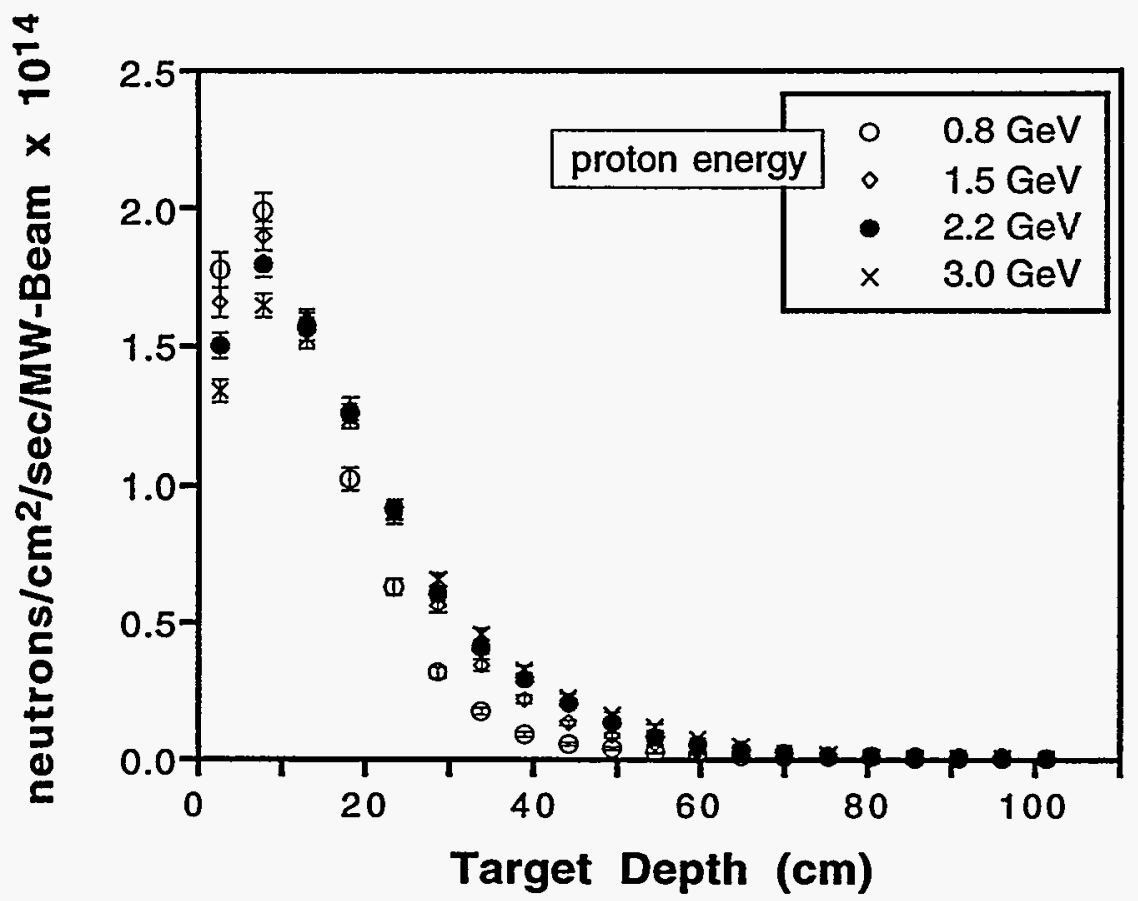

Figure I.1.2-2 Spatial Distribution of Neutrons Emerging from the Target as a Function of Axial Position.

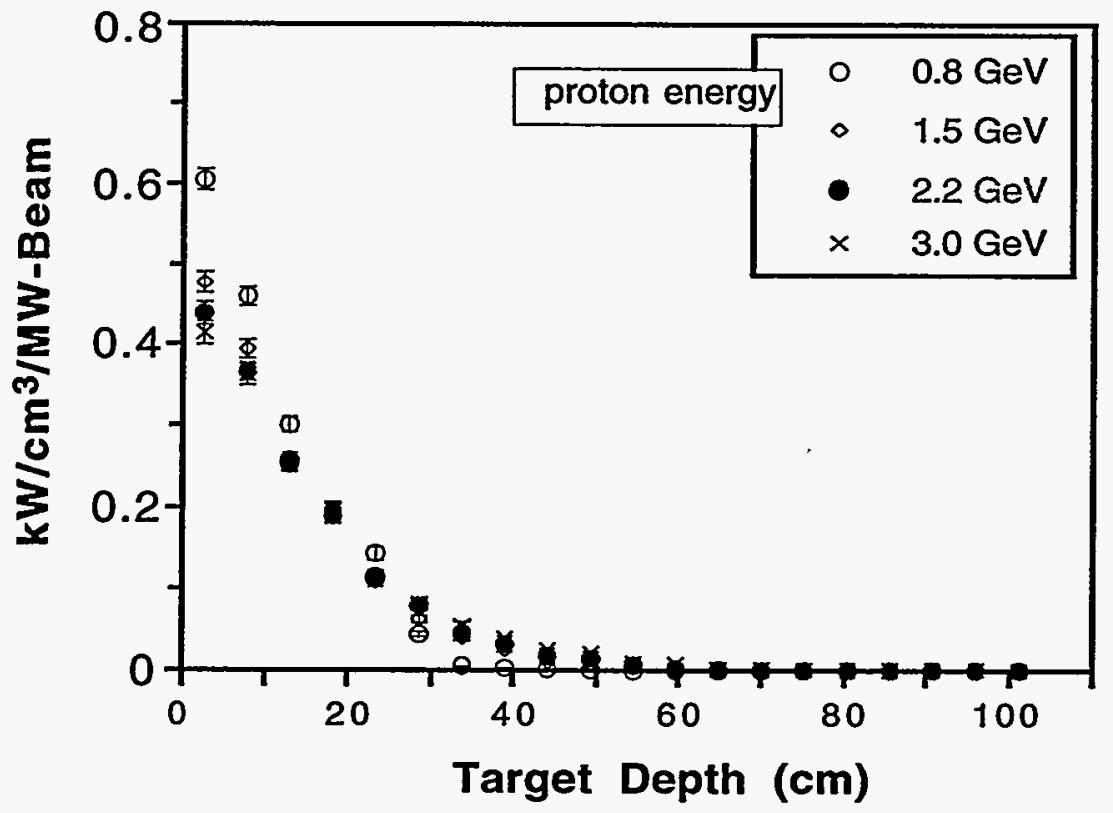

Figure 1.1.2-3 Spatial Distribution of Thermal Power Density in the Target as a Function of Axial Position. 
For this reason, the highest-energy proton linac is the $800-\mathrm{MeV}$ Los Alamos linac. Because a circular machine takes advantage of multiple traversals of the particles, the machine energy can be very high. Construction and operating costs for circular machines are substantially lower than those for linacs of similar energy. Circular machines can accelerate a time-averaged current of $0.5 \mathrm{~mA}$ to $1.0 \mathrm{~mA}$ to several $\mathrm{GeV}$. We chose to use a linac to provide a $400-\mathrm{MeV} \mathrm{H}^{-}$beam for injection into a rapid cycling synchrotron (RCS). The RCS is designed to accelerate an average proton beam current of $0.5 \mathrm{~mA}$ to $2 \mathrm{GeV}$. 


\subsection{ACCELERATOR SYSTEM}

The accelerator system delivers a proton beam of 1-MW power at a repetition rate of $30 \mathrm{~Hz}$. The required beam power is achieved by using a rapid cycling synchrotron (RCS). Its beam is extracted into two separate extraction lines. There are two targets, a low-frequency target and a high-frequency target operating at $10 \mathrm{~Hz}$ and $30 \mathrm{~Hz}$, respectively. One target receives one pulse out of three from the accelerator; the other target receives the remaining two pulses.

The injector system consists of a negative hydrogen $\left(\mathrm{H}^{-}\right)$ion source similar to that of ISIS, 14 a beam chopper system, a low-energy beam transport, a $2-\mathrm{MeV}$ radio-frequency (rf) quadrupole, a $70-\mathrm{MeV}$ drift-tube linac, and a $330-\mathrm{MeV}$ coupled-cavity linac. The linac design is similar to that of the Superconducting Super Collider linac and utilizes existing technology. The $400-\mathrm{MeV}$ injector linac system delivers $\mathrm{H}^{-}$ions to the synchrotron, where they are injected after stripping by a thin carbon foil. The RCS accelerates a time-averaged current of $0.5 \mathrm{~mA}$ of protons, corresponding to $1.04 \times 10^{14}$ protons per pulse at a $30-\mathrm{Hz}$ repetition rate, to $2 \mathrm{GeV}$.

The decision to design the RCS for the highest possible extraction energy and the lowest possible injection energy was driven by beam loss considerations. The injection energy of the RCS was determined on the basis of the space charge tune shift and the ring aperture. Experience with high-intensity proton accelerator operation shows that beam losses are usually associated with the injection and capture processes and that very few losses occur during the acceleration processes. Low-energy injection is preferable because of beam loss considerations, since there is less activation when beam is lost at low energy. Therefore, the design goal was to inject into the RCS at the lowest possible energy. A stacked-beam emittance of $375 \times 10^{-6} \pi \mathrm{m}$ in both transverse planes and an injection energy of $400 \mathrm{MeV}$ give a space charge limit of $1.04 \times 10^{14}$ protons per pulse. The beam-stay-clear region for a $375 \times 10^{-6} \pi \mathrm{m}$ emittance provides an RCS aperture similar to that of the ISIS. To reach the desired beam power, an appropriate amount of beam current is injected at $400 \mathrm{MeV}$ and accelerated to the final energy of $2 \mathrm{GeV}$.

The synchrotron operates in the first harmonic mode with single-turn extraction. It delivers pulses of approximately $200-n s e c$ duration and cycles at $30 \mathrm{~Hz}$. The machine has four long sides, with four straight sections per side. Two of the straight sections are used to extract beam to the two target areas; one is used for the injection system, and the others are reserved for . the rf system.

Design performance parameters of the accelerator system are summarized in Table I.2.1-1. Detailed descriptions are presented in Chapter II for the RCS and in Chapter III for the $\mathrm{H}^{-}$injector linac.

There is about $55,000 \mathrm{~m}^{2}$ of space available in the ZGS complex, and this space is used for the 1-MW source. The RCS must fit into the existing 200-m-circumference Ring Building. The locations of the two former experiment halls that house the two targets require construction of two separate extraction lines. These were the only geometrical constraints in designing this 1-MW source. 
Table 1.2.1-1 Accelerator Parameters

\begin{tabular}{lll}
\hline \multicolumn{1}{c}{ Parameters } & Values & Units \\
& & \\
\hline & & $\mathrm{GeV}$ \\
Beam energy at extraction & 2.2 & $\mathrm{GeV}$ \\
Maximum beam energy attainable & 400 & $\mathrm{MeV}$ \\
Beam energy at injection & 0.5 & $\mathrm{~mA}$ \\
Beam average current & 1.0 & $\mathrm{MW}$ \\
Beam power & $<1$ & $\mu \mathrm{sec}$ \\
Beam pulse length & 30 & $\mathrm{~Hz}$ \\
Synchrotron repetition rate & -200 & $\mathrm{~m}$ \\
Synchrotron circumference & 2 & - \\
Number of external proton beams & 2 & - \\
Number of extraction lines & & \\
& 400 & $\mathrm{MeV}$ \\
Linac beam energy & \pm 2.5 & $\mathrm{MeV}$ \\
Linac energy spread (95\%) & 30 & $\mathrm{~Hz}$ \\
Linac repetition rate & 44 & $\mathrm{~mA}$ \\
Linac pulse current & 0.5 & $\mathrm{msec}$ \\
Linac pulse length & $1.0 \times 10^{-6} \pi$ & $\mathrm{m}$ \\
Linac emittance (rms normalized) & $\mathrm{m}$ \\
\hline
\end{tabular}

a $\mathrm{rms}=$ root mean square. 
The existing Ring Building for the $12-\mathrm{GeV}$ ZGS is adequate to house the 2-GeV RCS described in this report. The use of a $2-\mathrm{GeV}$ beam reduces the maximum power density deposited in the target by about $25 \%$ from its value at $0.8 \mathrm{GeV}$, which is a significant advantage for target design.

\subsection{NEUTRON SCATTERING CAPABILTTES}

Shielded proton beam transport lines carry two extracted proton beams to two target stations, multiplexed so that the $30-\mathrm{Hz}$ station receives two of the $30-\mathrm{Hz}$ pulses while every third pulse goes to the $10-\mathrm{Hz}$ target. Each station contains a neutron-producing target consisting of water-cooled tungsten plates in two sections. Moderators positioned close to each target slow down neutrons from the primary source energies (about $1 \mathrm{MeV}$ ) to useful energies (less than about $10 \mathrm{eV}$ ). Water, liquid methane, and liquid hydrogen moderators are used. Reflectors of beryllium metal or of beryllium and heavy metal surround the moderators to enhance the intensities of the neutron beams. Decouplers and heterogeneous poisons within the moderatorreflector system tailor the spectra and pulse characteristics of the neutron beams. Massive steel and concrete shields surround the targets and moderators and provide multiple levels of confinement of radioactive materials within. Because some components of the target have finite lifetimes and because changes need to be made over the years to accommodate changing demands of the instruments, each target station is equipped with a hot cell and remote handling equipment and is designed to facilitate moving and servicing the internal components.

Irradiation facilities for neutron activation and fast neutron materials irradiation applications are arranged close to the targets. These irradiation facilities do not interfere with the neutron scattering application. The irradiation facilities are accessible during operation.

The IPNS Upgrade provides 36 beam ports for neutron scattering instruments, 18 each at the $30-\mathrm{Hz}$ and $10-\mathrm{Hz}$ target stations. Neutron beams for more than one instrument are extracted from a single beam port in some cases, so more than 36 neutron scattering instruments can be supported at this facility. There are a total of 12 moderators, one for every three beam ports, so the moderator characteristics can be optimized to the requirements of the individual instruments. Table I.2.2-1 lists the IPNS Upgrade moderators, and Figure 1.2.2-1 indicates the range of intensities and pulse widths available. The present IPNS moderators are also listed in the table for comparison.

The IPNS Upgrade provides 27 instruments for 24 of these 36 beam ports. The remaining 12 uninstrumented beam ports are available for later development and installation of new state-of-the-art instruments, as well as for the installation of specialized instruments developed by Participating Research Teams (PRTs). Chapter V of this document outlines a reference set of 27 instruments that provide a well-balanced initial instrumentation complement. Tables I.2.2-2 and I.2.2-3 briefly describe this reference set of instruments and indicate the wide range of neutron scattering science possible. This reference set of instruments has been used to 
Table 1.2.2-1 IPNS and IPNS Upgrade Moderators

Designation

Composition, Size

Temperature (K)

Present IPNS Moderators

"H"

"C*

Solid $\mathrm{CH}_{4}$, poisoned, $10 \times 10 \mathrm{~cm}^{2}$

25

Solid $\mathrm{CH}_{4}$, grooved, $10 \times 10 \mathrm{~cm}^{2}$

25

" $F$ "

Liquid $\mathrm{CH}_{4}$, poisoned, $10 \times 10 \mathrm{~cm}^{2}$

100

IPNS Upgrade Moderators

10- $\mathrm{Hz}$ Target $(25 \times$ IPNS)

Upstream top, "F"

Upstream bottom, "A"

Flux trap right, " $E$ "

Flux trap left, "B"

Downstream top, " $D$ "

Downstream bottom, " $C$ "

Liquid $\mathrm{H}_{2}, 10 \times 10 \mathrm{~cm}^{2}$

18

Liquid $\mathrm{H}_{2}$, coupled, $10 \times 10 \mathrm{~cm}^{2}$

18

Liquid $\mathrm{H}_{2}$, coupled, $10 \times 20 \mathrm{~cm}^{2}$

18

Undetermined

Undetermined

Liquid $\mathrm{H}_{2}$, Poisoned, $10 \times 10 \mathrm{~cm}^{2}$

$-$

30-Hz Target (50 $\times$ IPNS)

Upstream top, " $F$ "

Liquid $\mathrm{CH}_{4}$, poisoned, $10 \times 10 \mathrm{~cm}^{2}$

95

Upstream bottom, "A"

Liquid $\mathrm{CH}_{4}$, poisoned, $10 \times 10 \mathrm{~cm}^{2}$

95

Flux trap right, " $E$ "

Liquid $\mathrm{H}_{2} \mathrm{O}$, poisoned, $10 \times 20 \mathrm{~cm}^{2}$

320

Flux trap left, " $B$ "

Liquid $\mathrm{H}_{2} \mathrm{O}$, poisoned, $10 \times 20 \mathrm{~cm}^{2}$

320

Downstream top, " $D$ "

Liquid $\mathrm{H}_{2}$, coupled, $10 \times 10 \mathrm{~cm}^{2}$

18

Downstream bottom, " $\mathrm{C}$ "

Liquid $\mathrm{CH}_{4}$, poisoned, $10 \times 10 \mathrm{~cm}^{2}$

95 


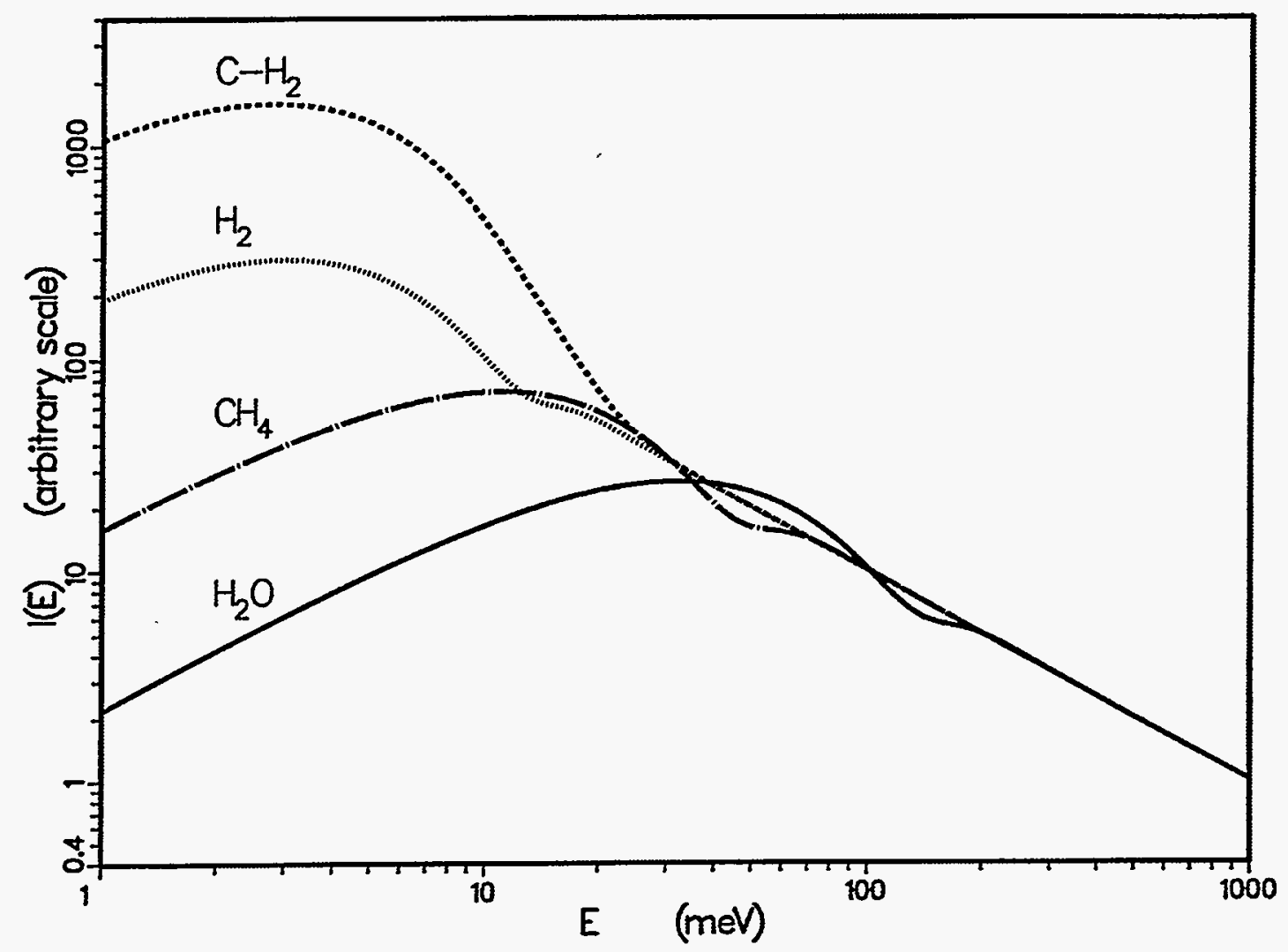

Figure I.2.2-1a Performance of the Different Moderators at IPNS Upgrade: Intensities, Normalized to 1 at $1 \mathrm{eV}$. 


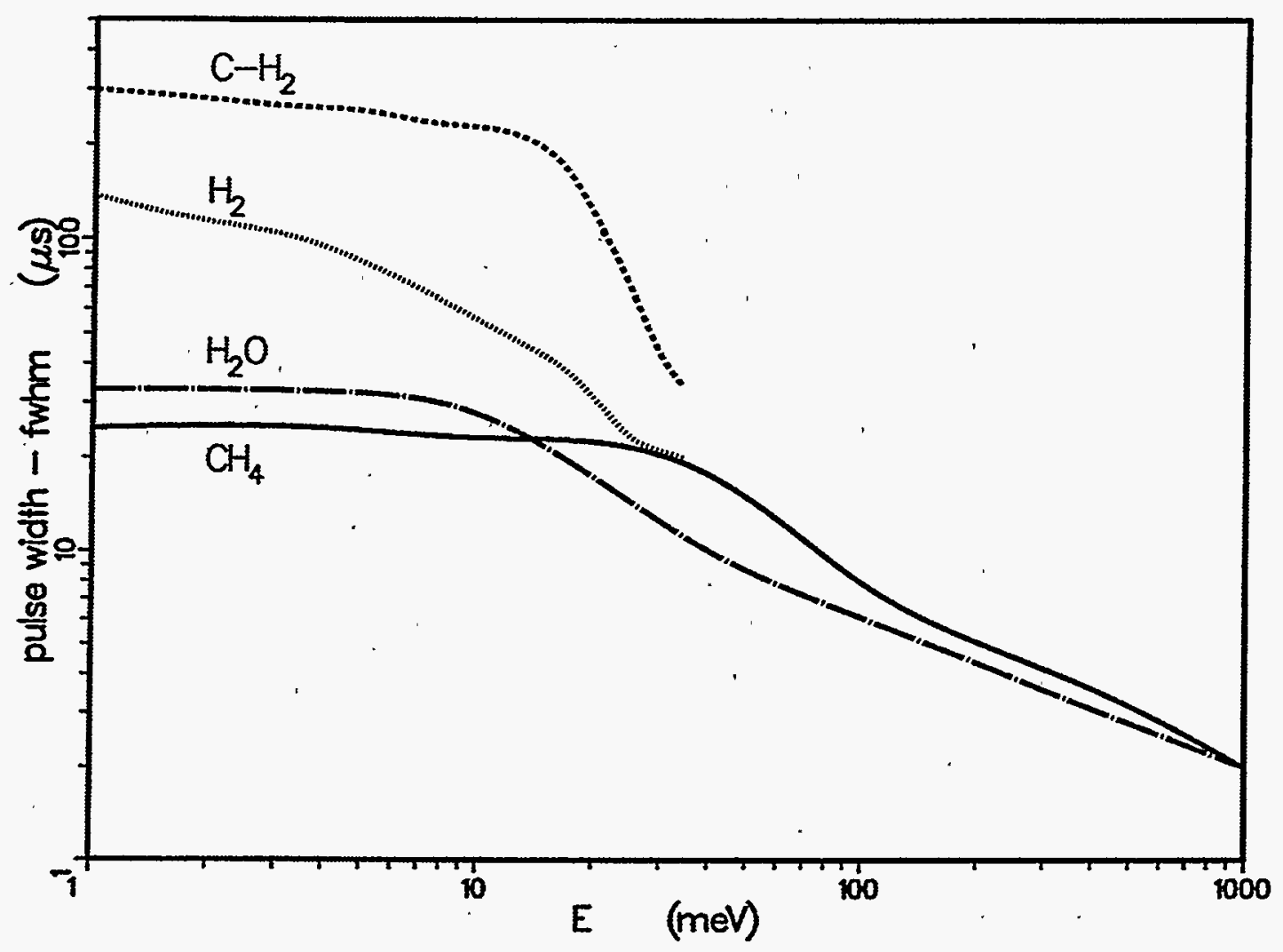

Figure I.2.2-1b Performance of the Different Moderators at IPNS Upgrade: Pulse Widths. (Moderators are water at $\sim 320 \mathrm{~K}\left(\mathrm{H}_{2} \mathrm{O}\right)$, liquid methane at $\sim 95 \mathrm{~K}\left[\mathrm{CH}_{4}\right]$, decoupled liquid hydrogen at $\sim 18 \mathrm{~K}\left[\mathrm{H}_{2}\right]$, and coupled liquid hydrogen at $\sim 18 \mathrm{~K}\left[\mathrm{C}-\mathrm{H}_{2}\right]$ ). 
Table 1.2.2-2 Reference Set of Diffractometers and Reflectometers

\begin{tabular}{|c|c|c|c|c|c|}
\hline & Powder Diffractometers & Target & Moderator ${ }^{a}$ & $\begin{array}{l}\text { Range for } \\
\text { d }(\AA)\end{array}$ & $\begin{array}{c}\text { Best } \\
\Delta d / d \\
(\%)\end{array}$ \\
\hline VSPD & Very small samples (10-100 mg) & $30-\mathrm{Hz}$ & $\mathrm{CH}_{4}$ & $0.2-17$ & 0.35 \\
\hline SEPD & High intensity & $30-\mathrm{Hz}$ & $\mathrm{H}_{2} \mathrm{O}$ & $0.2-17$ & 0.35 \\
\hline GPPDC & Medium resolution (excellent at $90^{\circ}$ ) & $30-\mathrm{Hz}$ & $\mathrm{H}_{2} \mathrm{O}$ & $0.2-9$ & 0.2 \\
\hline HRPD & High resolution & $30-\mathrm{Hz}$ & $\mathrm{H}_{2} \mathrm{O}$ & $0.2-5$ & 0.08 \\
\hline \multirow[t]{3}{*}{ RSD } & Residual stress (12-m position) & $30-\mathrm{Hz}$ & $\mathrm{H}_{2} \mathrm{O}$ & $0.3-6$ & 0.55 \\
\hline & (25-m position) & $30-\mathrm{Hz}$ & $\mathrm{H}_{2} \mathrm{O}$ & $0.2-3$ & 0.30 \\
\hline & Small-Angle Diffractometers & Target & Moderatora & $\frac{Q_{\min }}{\left(A^{-1}\right)}$ & $\begin{array}{l}Q_{\max } \\
\left(\AA^{-1}\right)\end{array}$ \\
\hline SAND & General purpose (wide $Q$ range) & $10-\mathrm{Hz}$ & $\mathrm{C}-\mathrm{H}_{2}$ & 0.002 & 2 \\
\hline HRSAND & High resolution & $10-\mathrm{Hz}$ & $\mathrm{C}-\mathrm{H}_{2}$ & 0.0005 & 0.4 \\
\hline \multirow[t]{2}{*}{ SPSAND } & Reconfigurable for special purposes & $10-\mathrm{Hz}$ & $\mathrm{C}-\mathrm{H}_{2}$ & Variable & Variable \\
\hline & norphous Materials Diffractometer & Target & Moderator ${ }^{a}$ & $\begin{array}{l}\text { Range for } \\
Q\left(\AA^{-1}\right)\end{array}$ & $\Delta Q / Q(\%)$ \\
\hline \multirow[t]{2}{*}{$G L A D^{b}$} & Liquids and glasses & $30-\mathrm{Hz}$ & $\mathrm{CH}_{4}$ & $0.07-120$ & $1.2-10$ \\
\hline & Single-Crystal Diffractometers & Target & Moderatora & $\begin{array}{l}\text { Range for } \\
Q\left(\AA^{-1}\right)\end{array}$ & $\Delta Q / Q(\%)$ \\
\hline$S C D^{b}$ & General purpose & $30-\mathrm{Hz}$ & $\mathrm{CH}_{4}$ & $0.9-17$ & $0.6-0.9$ \\
\hline \multirow[t]{2}{*}{ HQSCD } & High real-space resolution & $30-\mathrm{Hz}$ & $\mathrm{H}_{2} \mathrm{O}$ & $2-30$ & 0.4 \\
\hline & Reflectometers & Target & Moderator ${ }^{\mathbf{a}}$ & Sample & $\begin{array}{c}\text { Minimum } \\
\text { Reflectivity }\end{array}$ \\
\hline POSY-1c & Polarized neutrons & $10-\mathrm{Hz}$ & $\mathrm{H}_{2}$ & Vertical & $10^{-6}$ \\
\hline POSY-IIc & General purpose & $10-\mathrm{Hz}$ & $\mathrm{H}_{2}$ & Horizontal & $10^{-7}$ \\
\hline HIREF & High intensity & $10-\mathrm{Hz}$ & $\mathrm{H}_{2}$ & Horizontal & $10^{-4}$ \\
\hline GREF & Grazing incidence & $10-\mathrm{Hz}$ & $\mathrm{H}_{2}$ & Horizontal & $10^{-7}$ \\
\hline
\end{tabular}

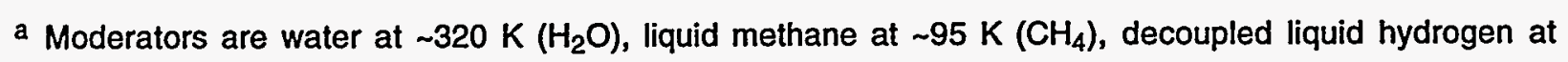
$\sim 18 \mathrm{~K}\left(\mathrm{H}_{2}\right)$, and coupled liquid hydrogen at $\sim 18 \mathrm{~K}\left(\mathrm{C}-\mathrm{H}_{2}\right)$. Moderator performance is shown in Figure 1.2.2-1.

b Transferred from IPNS with little change.

c Transferred from IPNS with some modification. 
Table 1.2.2-3 Reference Set of Inelastic Scattering Instruments and Component Development Instruments

\begin{tabular}{|c|c|c|c|c|c|}
\hline & Chopper Spectrometers & Target & Moderatora & $\begin{array}{l}\text { Range for } \\
E_{\text {inc }}(m e V)\end{array}$ & $\Delta E / E_{i}(\%)$ \\
\hline HRMECS $^{b}$ & High-resolution general purpose & $30-\mathrm{Hz}$ & $\mathrm{CH}_{4}$ & $4-2000$ & $2-4$ \\
\hline LRMECS ${ }^{C}$ & Low-resolution general purpose & $30-\mathrm{Hz}$ & $\mathrm{CH}_{4}$ & $3-2000$ & $4-7$ \\
\hline CNCS & High-resolution low energy & $10-\mathrm{Hz}$ & $\mathrm{H}_{2}$ & $0.3-20$ & $<1$ \\
\hline \multirow[t]{2}{*}{ sccs } & Excitations in single crystals & $30-\mathrm{Hz}$ & $\mathrm{H}_{2} \mathrm{O}$ & $50-2000$ & $\sim 1$ \\
\hline & stal-Analyzer Spectrometers & Target & Moderator ${ }^{a}$ & $\begin{array}{l}\text { Range for } \\
E(\mathrm{meV})\end{array}$ & $\Delta E(m e V)$ \\
\hline TFCA & General purpose & $30-\mathrm{Hz}$ & $\mathrm{CH}_{4}$ & $0-1000$ & $0.5-30$ \\
\hline QENSC & Quasielastic, medium resolution & $30-\mathrm{Hz}$ & $\mathrm{CH}_{4}$ & $0-150$ & $0.05-3$ \\
\hline HRBS & Microvolt resolution & $10-\mathrm{Hz}$ & $\mathrm{H}_{2}$ & $0-10$ & $0.005-0.06$ \\
\hline MICAS & Survey of single-crystal excitations & $30-\mathrm{Hz}$ & $\mathrm{CH}_{4}$ & $0-20$ & variable \\
\hline \multirow[t]{2}{*}{ QSTAXC } & Cold-neutron triple axis (QSS) ${ }^{d}$ & $30-\mathrm{Hz}$ & C- $\mathrm{H}_{2}$ & variable & variable \\
\hline & Spin-Echo Spectrometer & Target & Moderator ${ }^{a}$ & $\begin{array}{l}\text { Resolution } \\
\text { (meV) }\end{array}$ & $\Delta Q / Q(\%)$ \\
\hline TOFNSE & TOF spin-echo, cylindrical geometry & $10-\mathrm{Hz}$ & $\mathrm{C}-\mathrm{H}_{2}$ & $10^{-6}$ to $10^{-1}$ & 1.5 \\
\hline \multicolumn{2}{|c|}{ Component Development Instruments } & Target & Moderatora & & \\
\hline REFD & Reflectometer development & $10-\mathrm{Hz}$ & $\mathrm{H}_{2}$ & & \\
\hline DEVEL & General purpose development & $10-\mathrm{Hz}$ & $\mathrm{H}_{2}$ & & \\
\hline
\end{tabular}

a Moderators are water at $\sim 320 \mathrm{~K}\left(\mathrm{H}_{2} \mathrm{O}\right)$, liquid methane at $\sim 95 \mathrm{~K}\left(\mathrm{CH}_{4}\right)$, decoupled liquid hydrogen at $-18 \mathrm{~K}\left(\mathrm{H}_{2}\right)$, and coupled liquid hydrogen at $\sim 18 \mathrm{~K}\left(\mathrm{C}-\mathrm{H}_{2}\right)$. Moderator performance is shown in Figure 1.2.2-1.

b Transferred from IPNS with some modification.

c Transferred from IPNS with little change.

d Quasi-steady-state. 
select the target station parameters, lay out the locations of the target stations within the experiment halls, and provide the cost estimates. Up to 10 of the instruments that are presently operating at the IPNS would be refurbished and transferred to the IPNS Upgrade as part of this initial instrument complement. This approach provides a core of proven instruments ready for operation at startup. The neutron scattering instruments are located in existing experiment halls that provide internal space for beamlines up to $50 \mathrm{~m}$ long, as shown in Figure I.2.2-2. Longer beamlines can be extended outside the buildings if necessary.

Most of the neutron scattering instruments use the neutron time-of-flight (TOF) principle for determination of neutron wavelengths. Of these instruments, the majority require good wavelength resolution. For such instruments, the IPNS Upgrade target stations provide 25-50 times the intensity available at the IPNS. However, for those instruments that do not require good wavelength resolution (for example, small-angle-scattering instruments), the coupled liquid hydrogen moderators shown in Figure I.2.2-1 are used to provide 100-200 times the intensity available at the IPNS. include:

These high fluxes open up a number of new and exciting scientific possibilities. These

- Rapid measurements of structure or dynamics, which make it possible to follow the evolution of sample properties or chemical processes in real time on a scale of minutes or even seconds.

- Structural or dynamic measurements on very small samples, opening up neutron scattering to entire classes of materials that have previously been inaccessible.

- Measurements to much higher resolution than previously available.

The upgraded source is sufficiently intense that it is no longer necessary that all instruments be based on TOF techniques. With moderators optimized for total neutron output rather than for sharp neutron pulse structure, time-averaged thermal or cold neutron fluxes are equivalent to those at a medium flux reactor. The reactor-equivalent cold neutron flux has been calculated to be $\sim 5 \times 10^{13} \mathrm{n} / \mathrm{cm}^{2}$-sec for one of the liquid hydrogen moderators at the upgraded source. Thus, any instrument that works at a medium-flux reactor can be made to work at least as well at the IPNS Upgrade. Furthermore, even a moderator optimized for high time-averaged flux has a pulse width of less than one millisecond, and so has a duty factor of 1:30 or less at the IPNS Upgrade. This time structure can be used to significant advantage even on nominally steady-state instruments; for example, it can be used to reduce background and eliminate unwanted orders from crystal monochromators. A cold-neutron triple-axis spectrometer operating in this "quasi-steady-state" (QSS) mode is proposed as one instrument for the initial complement of IPNS Upgrade instruments. 


\section{IPNS UPGRADE I.2 DESIGN SPECIFICATIONS AND PERFORMANCE}

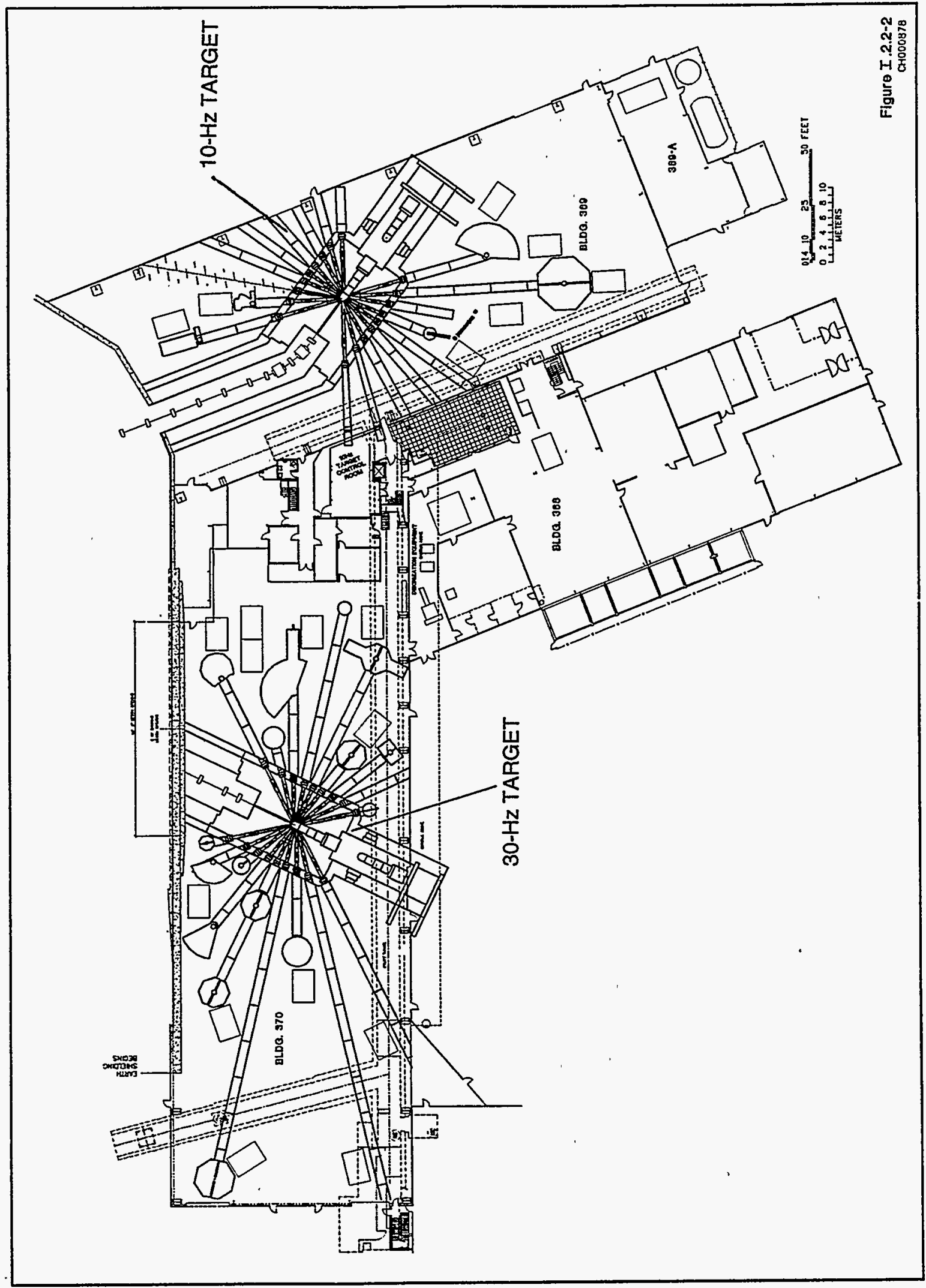

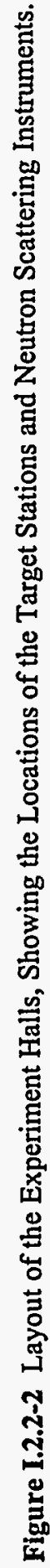




\subsection{OTHER EXPERIMENT FACHLITIES}

The IPNS Upgrade facilities can be expanded to allow for a number of other research opportunities - including radiation damage studies, isotope production, neutron activation analysis, neutron radiography and tomography, muon spin rotation studies for materials science research, and the study of some aspects of neutrino physics - with minimum interference to operation as a dedicated neutron scattering source. These possibilities are discussed briefly in Chapter V and in Appendix C. Facilities for radiation damage studies, isotope production, and neutron activation analysis are included within the present scope of the IPNS Upgrade project. 
The major components of the IPNS Upgrade facility described here are an accelerator system, two target stations, and neutron scattering instruments. The accelerator system consists of a new, $400-\mathrm{MeV} \mathrm{H}^{-}$linac and a new, 2-GeV rapid-cycling proton synchrotron (RCS). The RCS has a circumference of about $200 \mathrm{~m}$ and delivers a time-averaged proton current of $0.5 \mathrm{~mA}$, for a total time-averaged power of 1.0 MW. Figure I.3-1 shows a general view of the installation.

The 400-MeV injector linac, along with its associated technical equipment, is housed in a new building and tunnel. The $400-\mathrm{MeV}$ linac beam is transported by a low-energy transport (LET) line to the RCS. The linac tunnel and klystron gallery are the only new buildings to be built for the IPNS Upgrade project. An extensive study was undertaken to choose a location for the new injector that would avoid interruption of present IPNS operation. There will be an interruption of IPNS operation to move the instruments to the IPNS Upgrade facility.

All other technical components are housed in approximately $55,000 \mathrm{~m}^{2}$ of the former ZGS-complex space and use existing infrastructure. The synchrotron is located in the existing ZGS Ring Building. The power supplies for the RCS magnets, if system, and beam transport systems are housed in the Center Building and other adjacent buildings. The target stations and instruments are located in existing large buildings that previously housed high-energy experiments at the ZGS, a use very similar to the new use. System control rooms, maintenance facilities, and a considerable amount of laboratory and office space for resident personnel and visitors are provided in existing buildings. The project uses water systems, cooling towers, main power and transformer systems, roads, sewers, and general infrastructure items that already exist. There is little need for constructing new conventional facilities. The cost estimate includes an allowance for refurbishing these existing buildings and facilities. Use of these existing buildings results in a very large savings in cost and construction time.

We expect that there is no need for land-use studies, such as wetlands accommodation and archaeological investigations, because construction takes place within existing buildings or in areas such as parking lots that are already graded. Altogether, these factors effect considerable cost and time savings.

If the project is initiated in FY 1997, the total estimated cost (TEC) is $\$ 559$ million. The facility commissioning is to start at the beginning of FY 2001. Construction of new components and refurbishment of existing facilities will not interfere with the present IPNS operations, except for reworking and relocation of the scattering instruments. An interval as short as a few months will be required to rework the scattering instruments and install them in their new locations. The accelerator commissioning period will be followed by commissioning of the target stations and instruments.

A detailed discussion of the cost and schedule is presented in Chapter VII. 


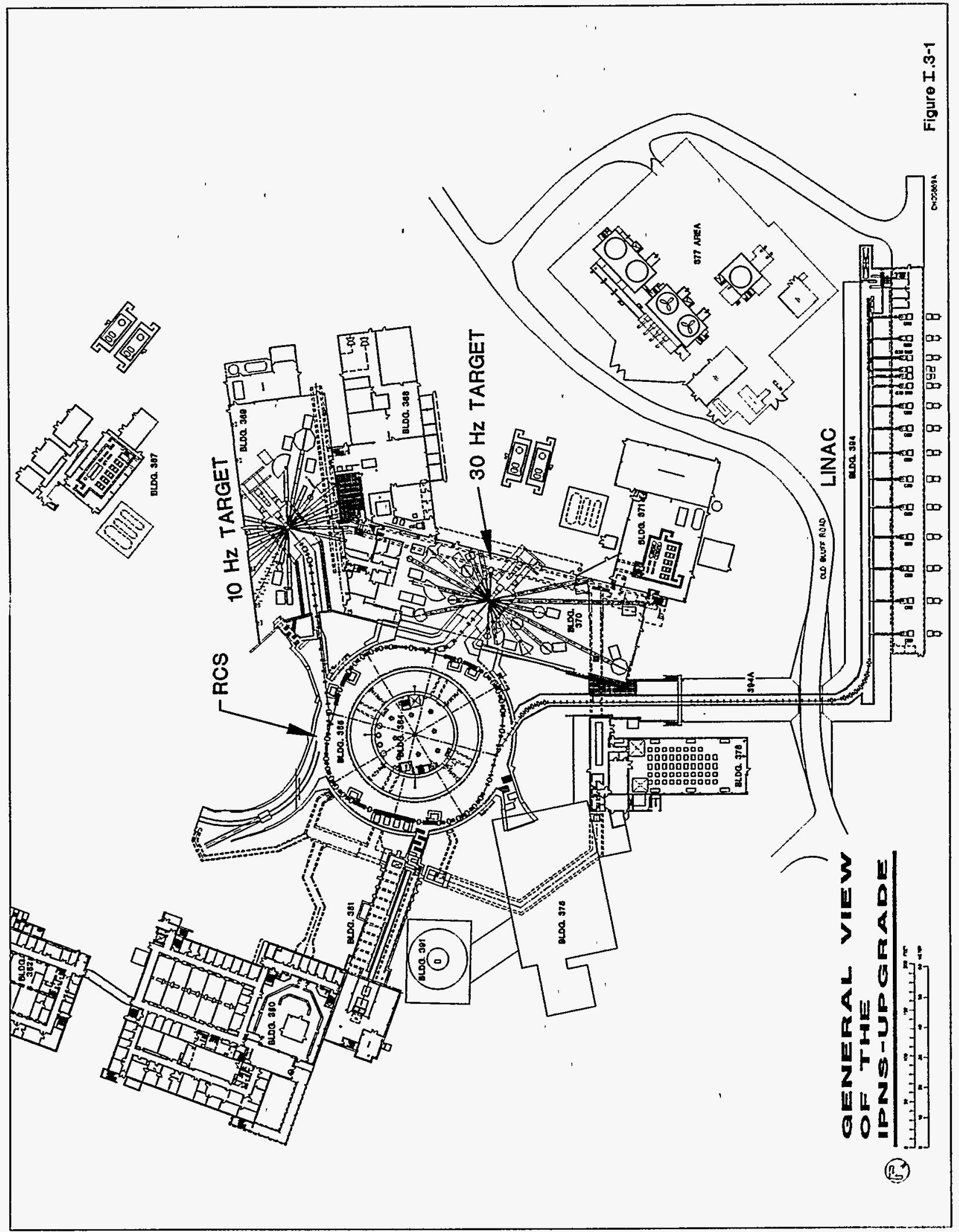


1. Neutron Sources for America's Future, Report of the DOE Basic Energy Sciences Advisory Committee Panel on Neutron Sources, chaired by W. Kohn, U.S. Department of Energy Report ER-0576P (Jan. 1993).

2. Major Facilities for Materials Research and Related Disciplines, F. Seitz and D. Eastman, Panel of the National Research Council, NTIS Report PB85-108850 (1985).

3. G.H. Lander and V.J. Emery, editors, Scientific Opportunities with Advanced Facilities for Neutron Scattering, Report of a Workshop held at Shelter Island, N.Y., Oct. 23-26, 1984, NTIS Report CONF-8410256 (1985).

4. J.J. Rush and D.L. Price, editors, Neutron Sources and Applications, Report of a review held at Oak Brook, Ill., Sept. 8-10, 1992, U.S. Department of Energy Report ER-0607P (Jan. 1994).

5. R.J. Macek and H.A. Thiessen, editors, Proceedings of the Workshop on Accelerators for Future Spallation Neutron Sources (Santa Fe, N.M., Feb. 16-20, 1993), Los Alamos National Laboratory Report LA-UR-93-1356 (1993).

6. G. Aeppli and B.S. Brown, editors, Technology and Science at a High Power Spallation Source, Proceedings of a Workshop held at Argonne National Laboratory, May 13-16, 1993, Argonne National Laboratory Internal Report (Feb. 1994).

7. Y. Cho, R.L. Martin, E.F. Parker, C.W. Potts, L. Ratner, J. Gareyte, C. Johnson, P. LeFevre, D. Mohl, and A.D. Krisch, "Phenomena Associated with the Acceleration of Polarized Protons in Circular Accelerators, " AIP Proceedings of the High Energy Physics with Polarized Beams and Targets, No. 35, Particles and Fields Subseries No. 12, p. 396 (1976).

8. E. Crosbie, A. Gorka, E. Parker, C. Potts, and L. Ratner, "Injection and acceleration of protons in the ZGS by stripping $\mathrm{H}^{-}$ions," IEEE Trans. Nucl. Sci. NS-22, 1056 (1975).

9. Conceptual Design of a Rapid Cycling Synchrotron for the KFA - Jülich Spallation Neutron Source, Argonne National Laboratory Report ANL-83-13 (Jan. 1983).

10. Working Papers on the Conceptual Design of a Rapid Cycling Synchrotron for the KFA Jülich Spallation Neutron Source, Argonne National Laboratory Report ANL-83-13Addendum (Jan. 1983).

11. T.K. Khoe and R.L. Kustom, "ASPUN, design for an Argonne super intense pulsed neutron source," IEEE Trans. Nucl. Sci. NS-30, 2086 (1983).

12. 7-GeV Advanced Photon Source Conceptual Design Report, Argonne National Laboratory Report ANL-87-15 (Apr. 1987). 
13. D.W. Jerng and J.M. Carpenter, "Heat generation and neutron beam characteristics in a high power pulsed spallation neutron source," submitted to IEEE Trans. Nucl. Sci. (1994).

14. L. Schroeder, K.N. Leung, and J. Alonso (editors), Proceedings of the Workshop on Ion Source Issues Relevant to a Pulsed Neutron Source (Lawrence Berkeley Laboratory, Berkeley, California, Oct. 24-26, 1994), Lawrence Berkeley Laboratory Report No. LBL-36347 (1994). 
Synchrotron 



\subsection{CHOICE OF MACHINE TYPE}

The accelerator for a pulsed spallation neutron source is required to produce a high-power (1-MW) proton beam with a short $(<1-\mu \mathrm{sec})$ pulse length and a reasonable pulse repetition rate. Two commonly used accelerator configurations produce beams of the desired quality. One of these is a full-energy linac, which usually has a pulse length on the order of milliseconds, coupled with a pulse-compressor ring, which accepts the millisecond pulse and compresses it to the order of $1 \mu \mathrm{sec}$. In this scheme, the linac provides full power to the protons. The other configuration employs a linac of substantially lower energy that injects into a Rapid Cycling Synchrotron (RCS), and the RCS provides the major portion of beam power.

The present Intense Pulsed Neutron - Source (IPNS) accelerator system consists of a $30-\mathrm{Hz}, 500-\mathrm{MeV}$ RCS coupled with a $50-\mathrm{MeV}$ proton injector linac, and it provides $8 \mathrm{~kW}$ of beam power. The proposed IPNS Upgrade accelerator system provides $1 \mathrm{MW}$ of beam power by using a $400-\mathrm{MeV}$ injector linac, followed by a $2-\mathrm{GeV}$ RCS. The RCS described here is, therefore, the centerpiece of the accelerator facility. The decision to use the RCS configuration to provide the beam power rather than to use other accelerator configurations, such as a linac pulsecompressor ring, was made after extensive comparisons between the two schemes.

Since the full-energy linac is a single-pass device, costs associated with its construction and operation are higher than those associated with a synchrotron, which is a multiple-pass device. Therefore, for a given beam power ( $1 \mathrm{MW}$ ), a pulsed spallation source using an RCS concept can have higher beam energy $(2 \mathrm{GeV})$ and less beam current $(0.5 \mathrm{~mA})$ than a pulsed source based on the linac/pulse-compressor scheme, which necessarily has to have lower energy $(0.8 \mathrm{GeV})$ and higher beam current $(1.25 \mathrm{~mA})$. Here, the numbers in parentheses are used to compare a 2-GeV RCS concept to an $800-\mathrm{MeV}$ accumulator concept for a 1-MW pulsed source.

An important consideration with respect to the beam energy and current is that beam losses must be minimized or eliminated during the injection, acceleration, and extraction processes. Past experience shows that the beam loss, if any, usually occurs during injection and capture, not during acceleration and extraction. It is preferable to have beam losses occur at the lowest possible beam energy and least amount of beam current, another advantage that is offered by the RCS. Lower particle energy implies lower production of residual radioactivity, and lower beam current implies lower losses for the same fractional losses.

This concept of lower-energy injection at lower beam current has additional advantages with respect to the construction and operation of the facility. One advantage of having a lower current is that the existing ion source technology is capable of providing the required current without extensive development. ${ }^{1}$ Another advantage is that injecting a lower-energy beam is easier than injecting a high-energy beam.

Present-day proton machines of this type are equipped with $\mathrm{H}^{-}$injection systems, which enable the transverse phase space distributions of the injected beam to be controlled. This controlled populating of phase space uses the irreversible-charge-exchange injection process 
known as phase space painting. The charge-exchange from $\mathrm{H}^{-}$ions to protons is achieved by passing the $\mathrm{H}^{-}$beam through a thin foil that strips the electrons.

State-of-the-art $\mathrm{H}^{-}$ion sources provide the required $0.5-\mathrm{mA}$ time-averaged current without further research and development (R\&D) effort. The ion source required for the IPNS Upgrade can be a copy of the ISIS ion source, which has a proven record of reliability. Substantial R\&D work is required to improve the peak beam current and pulse length performance and reliability for any source that produces more than a $0.5-\mathrm{mA}$ average current.

The decision to use an RCS scheme for the IPNS Upgrade is made on the basis of the considerations described above. It is preferable to have the lowest possible beam current and highest possible beam energy at extraction. Fifty years of experience have shown that achieving higher energy by using a synchrotron is much easier than achieving high intensity. This implies that a higher-energy, lower-current machine can readily achieve the design goal.

An additional item that influenced the choice of machine parameters was the availability of existing buildings and infrastructure. Several buildings and accelerator enclosures that were formerly used by the high-energy physics program have been available for accelerator use since 1979. This underutilized area is the former Zero Gradient Synchrotron (ZGS) complex. The existing structures match the design requirements closely enough that very little compromise is needed in the design of the facility.

Moreover, Argonne National Laboratory (ANL) personnel have more than 20 years of experience in the development and operation of several rapid cycling synchrotrons, and in particular in neutron scattering.

The proposed plan utilizes the existing ZGS complex to house a 1-MW pulsed source.

\subsection{CHOICE OF BEAM ENERGY}

It has been shown that the neutron yield is roughly proportional to proton beam energy from several hundred $\mathrm{MeV}$ to about $10 \mathrm{GeV}$. There are no firm experimental data on the neutron yield from an operational, optimally coupled, heavy-metal target and moderator, in which practical necessities such as cooling have been incorporated into the target construction. At present, designers of neutron-generating targets are comfortable with a beam energy of 1 to $3 \mathrm{GeV}$.

The proposed RCS operates with a beam energy of $2 \mathrm{GeV}$. This energy was chosen because the ZGS Ring Building can accommodate a circular machine with a circumference of about 200 meters. A detailed optimization has shown that the ZGS Ring Building can house a $30-\mathrm{Hz}$ separated-function RCS with a peak dipole field of $1.5 \mathrm{~T}$. This configuration corresponds to a beam energy of $2.2 \mathrm{GeV}$. All proposed items of accelerator hardware are designed to be able to operate at up to $2.2 \mathrm{GeV}$. The accelerator operates at a nominal energy of $2 \mathrm{GeV}$, thus 
providing built-in reliability from the beginning. The time-averaged beam current must be $0.5 \mathrm{~mA}$ for a $2-\mathrm{GeV}$ beam.

\subsection{CHOICE OF REPETITION RATE OF THE RCS}

The choice of synchrotron repetition rate dictates several machine parameters, such as the number of protons per pulse to be accelerated to achieve the time-averaged current, and also the peak radio-frequency (rf) voltage. The repetition rate also influences the data-taking process of the scientific research program.

Scientific users prefer a lower repetition rate to avoid data pile-up and accidental counting of cvents from two consecutive machine pulses. Consultation with IPNS users indicates that a $30-\mathrm{Hz}$ repetition rate is a desirable goal.

\subsection{NEGATIVE HYDROGEN ION INJECTION}

During the past decades, almost all proton synchrotrons have been converted from $\mathrm{H}^{+}$to $\mathrm{H}^{-}$ion injection, because $\mathrm{H}^{-}$charge exchange injection can be used to manipulate the transverse phase space during the injection process. The use of this process is essential to achieve a highintensity beam with very little beam loss. An $\mathrm{H}^{-}$linac is used as the injector for the IPNS Upgrade RCS.

\subsection{CHOICE OF INJECTION ENERGY}

The injection energy is determined by the space charge tune shift, RCS repetition rate, and time-averaged current. A machine operating at $30 \mathrm{~Hz}$ with a $0.5-\mathrm{mA}$ average current requires an acceleration of $1.04 \times 10^{14}$ protons per pulse.

As described in detail in Section M.2.1.3, the space charge tune shift due to the self-field of particles is proportional to the number of protons in a pulse and inversely proportional to the acceptance of the machine and to $\beta^{2} \gamma^{3}$ of the protons, where $\beta$ and $\gamma$ are the standard relativistic parameters.

The complete discussion of space charge and injection energy is given in Section II.2.1.3; after this discussion, the acceptance of the machine is discussed. A $400-\mathrm{MeV}$ injection would provide a tune shift of 0.15 , which is quite comfortable for reliable operation.

The high-level design goal parameters are summarized in Table II.1.5-1. The magnetic lattice for the RCS is designed to meet those performance requirements. 
Table II.1.5-1 Design Goal Parameters

\begin{tabular}{|c|c|c|}
\hline Parameter & Value & Units \\
\hline Beam energy at extraction & 2 & $\mathrm{GeV}$ \\
\hline Beam maximum attainable energy & 2.2 & $\mathrm{GeV}$ \\
\hline Beam energy at injection & 400 & $\mathrm{MeV}$ \\
\hline Beam repetition rate & 30 & $\mathrm{~Hz}$ \\
\hline Beam average current & 0.5 & $\mathrm{~mA}$ \\
\hline Beam power & 1.0 & MW \\
\hline Linac beam energy & 400 & $\mathrm{MeV}$ \\
\hline Linac energy spread (95\%) & \pm 2.5 & $\mathrm{MeV}$ \\
\hline Linac repetition rate & 30 & $\mathrm{~Hz}$ \\
\hline Linac pulse current & 44 & $m A$ \\
\hline Linac pulse length & 0.5 & msec \\
\hline Linac emittance (rms normalized) ${ }^{a}$ & $1.0 \times 10^{-6}$ & $\pi \mathrm{m}$ \\
\hline
\end{tabular}

a $\mathrm{rms}=$ root mean square. 


\subsection{REFERENCE}

1. L. Schroeder, K.N. Leung, and J. Alonso (editors), Proceedings of the Workshop on Ion Source Issues Relevant to a Pulsed Neutron Source (Lawrence Berkeley Laboratory, Berkeley, California, Oct. 24-26, 1994), Lawrence Berkeley Laboratory Report No. LBL-36347 (1994). 
II.1-6 


\subsection{SYNCHROTRON OPTICS}

\subsubsection{Magnet Lattice} include:

The requirements and desirable features for a high-intensity proton machine lattice

1. Sufficient straight sections for radio-frequency (rf) cavities and injection/ extraction hardware,

2. Dispersion-free straight sections for injection,

3. Dispersion-free straight sections for $\mathrm{rf}$ cavities,

4. Largest possible transition energy of the machine, and

5. Peak field of the dipole magnet less than $1.5 \mathrm{~T}$ to avoid saturation effects.

A large transition energy, $\gamma_{t}$, has two implications. One has to do with the ease of manipulating synchrotron motions of the particles, and the other with beam instability thresholds due to high-intensity phenomena. The instability threshold current is proportional to the parameter $\eta=1 / \gamma_{t}^{2}-1 / \gamma^{2}$. In order to make $|\eta|$ large for a machine below transition energy, $\gamma_{t}$ should be large. This means the lattice should have a large horizontal tune, $v_{x}$.

To implement all these features in the lattice, a FODO cell of $\sim 90^{\circ}$ phase advance in each transverse phase space was chosen for the normal cells that make up the arc of the ring. The desired dispersion suppression was achieved by removing one of the dipoles from a normal cell. The dispersion-free straight sections are formed by adding empty cells (normal cell with both dipoles removed) to the lattice after the dispersion-suppressor cell. A super-period is constructed by arranging normal cells, dispersion-suppressor cells, and empty cells followed by their mirror images.

The super-periodicity can be made to equal 3 or 4 . This accelerator requires a substantial number of $\mathrm{rf}$ cavities, thus necessitating a large number of straight sections. A super-periodicity of 4 was chosen in order to take optimal advantage of space in the circular Zero Gradient Synchrotron (ZGS) tunnel.

Figure II.2.1-1 shows lattice functions of the normal cell, and Table II.2.1-1 lists cell parameters of each element in the normal cell. Figure II.2.1-2 shows the lattice functions through the dispersion-suppressor and dispersion-free cells. The S1 sextupole shown in Figure II.2.1-2 is for harmonic correction and is discussed in Section II.2.1.5. This segment of the lattice constitutes the straight section area. Figure II.2.1-3 shows the lattice functions for one-half of a super-period. 

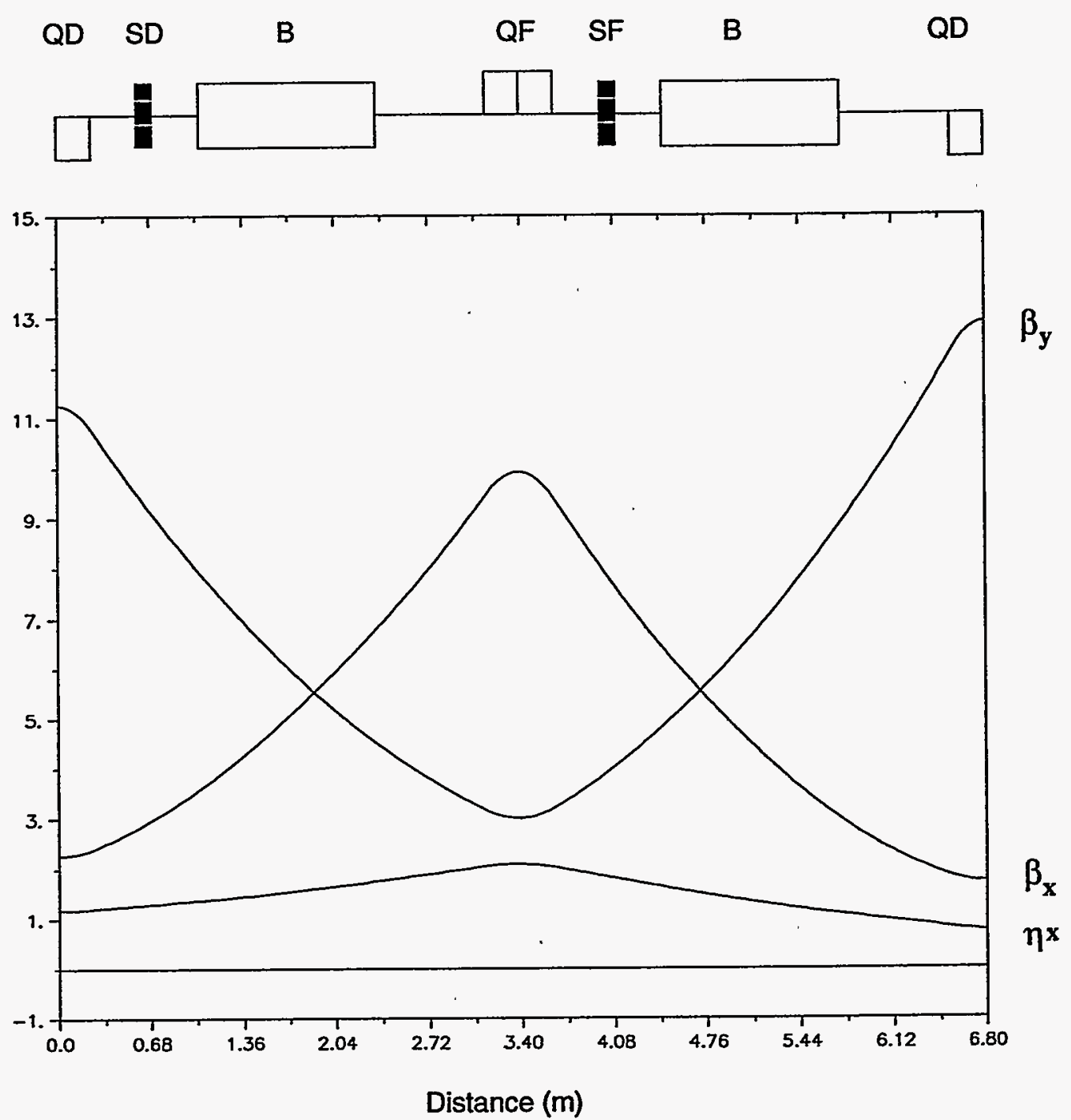

Figure II.2.1-1 Lattice Functions of the Normal Cell. 
Table II.2.1-1 Normal Cell Parameters $(2.2 \mathrm{GeV}, \mathrm{Bp}=9.989 \mathrm{~T} \cdot \mathrm{m})$

\begin{tabular}{llll}
\hline Elements & Length $(\mathrm{m})^{\mathrm{a}}$ & Strength & Units \\
\hline QD & 0.25 & -7.276 & $\mathrm{~T} / \mathrm{m}$ \\
D1 & 0.3 & & \\
SD & 0.2 & -0.843 & $\mathrm{~m}^{-2}$ \\
D1 & 0.3 & & \\
B & 1.3 & 1.5088 & $\mathrm{~T}$ \\
D2 & 0.8 & & \\
QF & 0.5 & 8.267 & $\mathrm{~T} / \mathrm{m}$ \\
D1 & 0.3 & & \\
SF & 0.2 & 0.612 & $\mathrm{~m}^{-2}$ \\
D1 & 0.3 & & \\
B & 1.3 & 1.5088 & $\mathrm{~T}$ \\
D2 & 0.8 & & \\
QD & 0.25 & -7.276 & $\mathrm{~T} / \mathrm{m}$ \\
\hline
\end{tabular}

a Total cell length $=6.8 \mathrm{~m}$. 

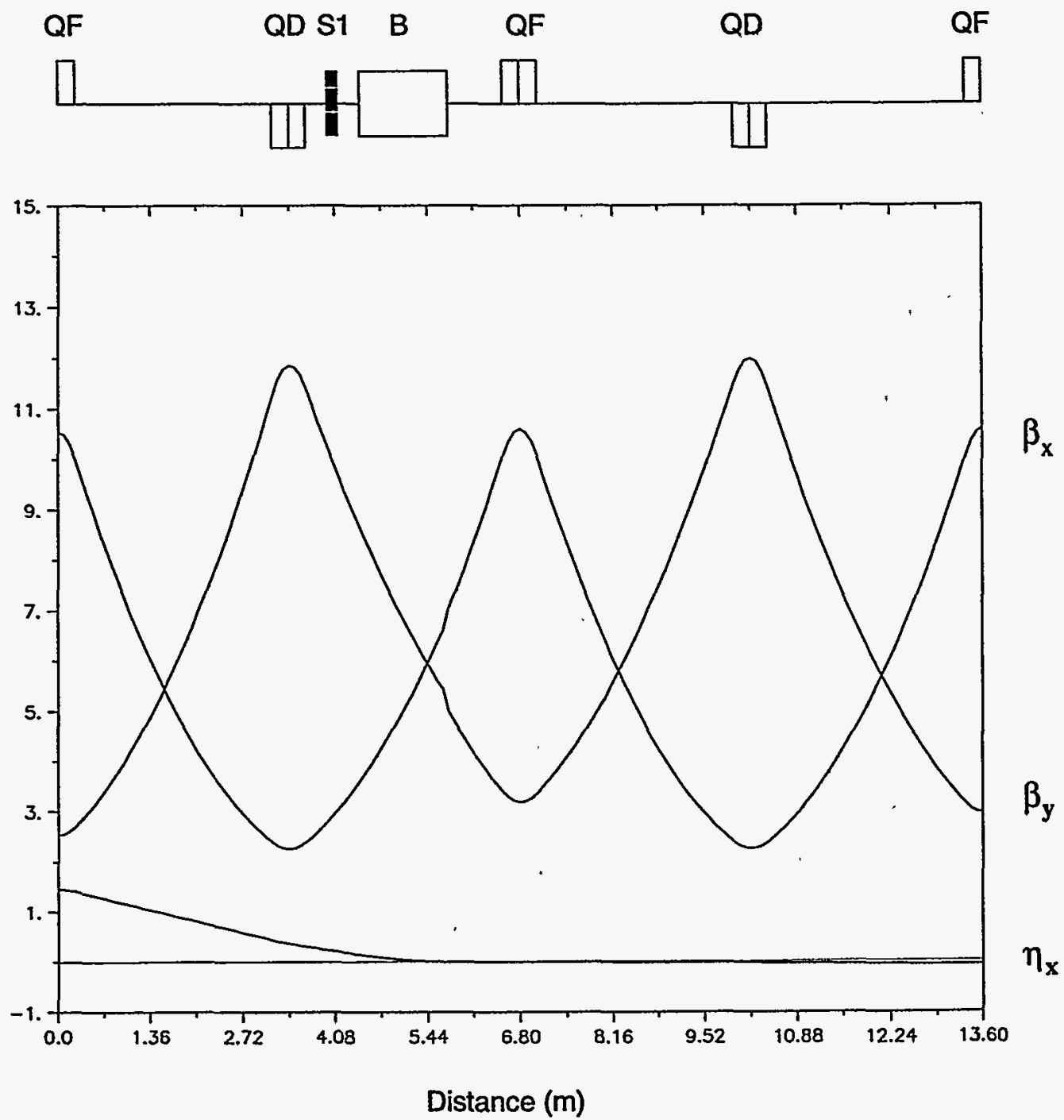

Figure II.2.1-2 Lattice Functions through the Dispersion-Suppressor and Dispersion-Free Cells. 


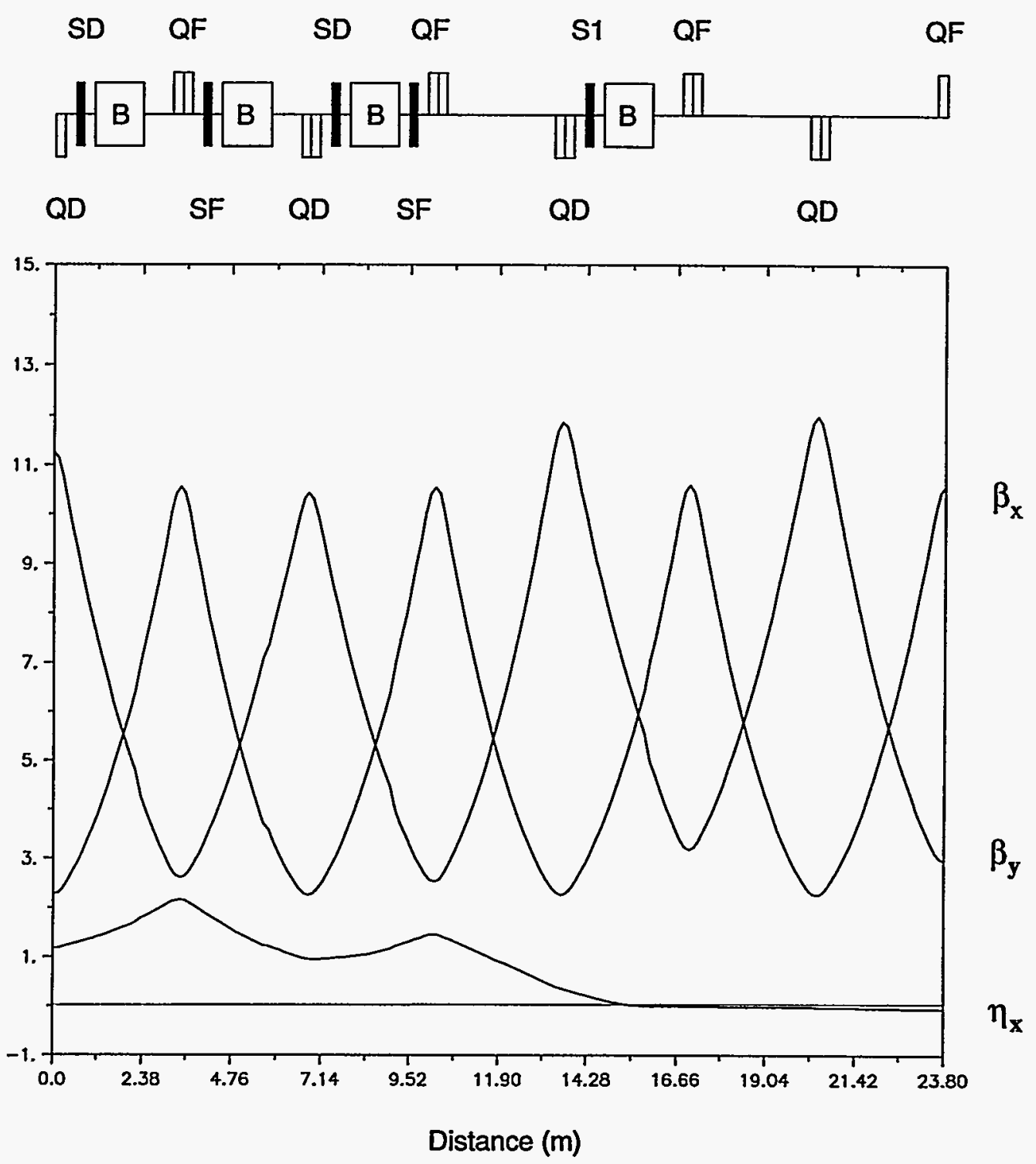

Figure II.2.1-3 Lattice Functions for One-Half of a Super-Period. 
Table II.2.1-2 Synchrotron Parameters

\begin{tabular}{|c|c|c|}
\hline Parameters & Values & Units \\
\hline Circumference & 190.4 & m \\
\hline Super-periodicity & 4 & - \\
\hline Total number of cells & 28 & - \\
\hline Number of normal cells & 12 & - \\
\hline Number of dispersion suppressor cells & 8 & - \\
\hline Number of straight section cells & 8 & - \\
\hline Nominal length of straight section & 2.9 & m \\
\hline Injection energy & 400 & $\mathrm{MeV}$ \\
\hline Injection field & 0.48 & $\mathrm{~T}$ \\
\hline Nominal extraction energy & 2.0 & GeV \\
\hline Maximum design energy & 2.2 & GeV \\
\hline Dipole field at $2.2 \mathrm{GeV}$ & 1.5088 & $\mathrm{~T}$ \\
\hline Bending radius & 6.6207 & m \\
\hline Dipole length & 1.3 & m \\
\hline Dipole good field region & 0.45 & m \\
\hline Dipole gap height & 0.182 & m \\
\hline Number of dipoles & 32 & - \\
\hline Number of quadrupoles & 56 & - \\
\hline Quadrupole length & 0.5 & m \\
\hline Quadrupole aperture radius & 0.1185 & $\mathrm{~m}$ \\
\hline Maximum quadrupole gradient & 8.8 & $\mathrm{~T} / \mathrm{m}$ \\
\hline Number of sextupoles (F) & 16 & - \\
\hline Number of sextupoles (D) & 16 & - \\
\hline Number of harmonic sextupoles & 8 & - \\
\hline Maximum sextupole strength & 1.2 & $m^{-2}$ \\
\hline Sextupole length & 0.2 & m \\
\hline Sextupole aperture radius & 0.13 & m \\
\hline Horizontal tune, $v_{x}$ & 6.821 & - \\
\hline Vertical tune, $v_{y}$ & 5.731 & - \\
\hline Normalized transition energy, $\gamma_{t}$ & 5.40 & - \\
\hline Natural chromaticity, $\xi_{x}=(\Delta v / v)_{x} /(\Delta p / p)$ & -1.06 & - \\
\hline Natural chromaticity, $\xi_{y}=(\Delta v / v)_{y} /(\Delta p / p)$ & -1.20 & - \\
\hline Maximum $\beta$ function & 12 & $\mathrm{~m}$ \\
\hline Minimum $\beta$ function & 2.2 & $m$ \\
\hline Maximum $\eta$ function & 2.2 & $\mathrm{~m}$ \\
\hline Minimum $\eta$ function & -0.06 & $\mathrm{~m}$ \\
\hline Revolution time at injection & 890.1 & nsec \\
\hline Revolution time at extraction & 665.1 & nsec \\
\hline$\dot{\mathrm{B}}_{\max }$ & 64.5 & T/sec \\
\hline Maximum energy gain/turn & 81.4 & $\mathrm{keV}$ \\
\hline
\end{tabular}


The lattice is symmetrical about either end; the entire RCS is shown in Figure II.2.1-3b. Some parameters, such as the machine acceptance and chromaticity corrections, are discussed in Section II.2.1.6, while Table II.2.1-2 summarizes the pertinent synchrotron parameters.

\subsubsection{Synchrotron Magnet Excitation and Repetition Rate}

As noted in Section II.1.3, the synchrotron operates at a repetition rate of $30 \mathrm{~Hz}$. It is customary that this kind of rapidly cycling synchrotron magnet system be energized by a resonant circuit power supply generating a sinusoidal wave form. This results in a time-varying field of the ring magnet, $B(t)$, described by

$$
B(t)=B_{0}-B_{1} \cos (2 \pi f t),
$$

where $B_{0}$ and $B_{1}$ are the $d c$ and ac components of the synchrotron magnetic field, respectively, and $f$ is the repetition rate.

Particle energy gain is proportional to $d B / d t$, and $d B / d t$ sets the first-order if voltage required to accelerate the beam. From the above equation, $d B / d t$ is $2 \pi f B_{1} \sin (2 \pi f t)$. This means that the required if voltage can be lowered by energizing the magnets at a lower frequency and de-energizing them at a higher frequency, keeping the overall repetition rate constant.

The synchrotron magnet system is energized with a dual resonant power supply system, which uses a $20-\mathrm{Hz}$ magnet excitation rate and a $60-\mathrm{Hz}$ magnet reset rate while keeping the overall $30-\mathrm{Hz}$ rate. This reduces the required rf voltage by $1 / 3$.

\subsubsection{Beam Emittance and Synchrotron Acceptance}

\subsubsection{Space Charge Limit and Choice of Linac Energy}

It is customary to use the Laslett tune shift due to space charge to calculate the number of protons that can be accelerated by a synchrotron. Knowledge of the longitudinal and transverse beam distributions is required to make these calculations. These distributions come either from assumptions based on previous experience or from tracking studies. The initial estimate of the space charge limit is obtained by using the elliptic beam distributions and a bunching factor obtained from assumptions. A refined study of the space charge limit was performed after a longitudinal tracking study from injection to full-energy acceleration was made.

We now discuss the initial estimate of the space charge limit and choice of the injection energy. The discussion presented here closely follows that described in Report of the ISIS Project Group. ${ }^{1}$ It is reasonable to assume the local elliptic energy distribution of Hofmann and Pederson $^{2}$ for longitudinal motion of protons in the bunch. For this distribution, self-forces 


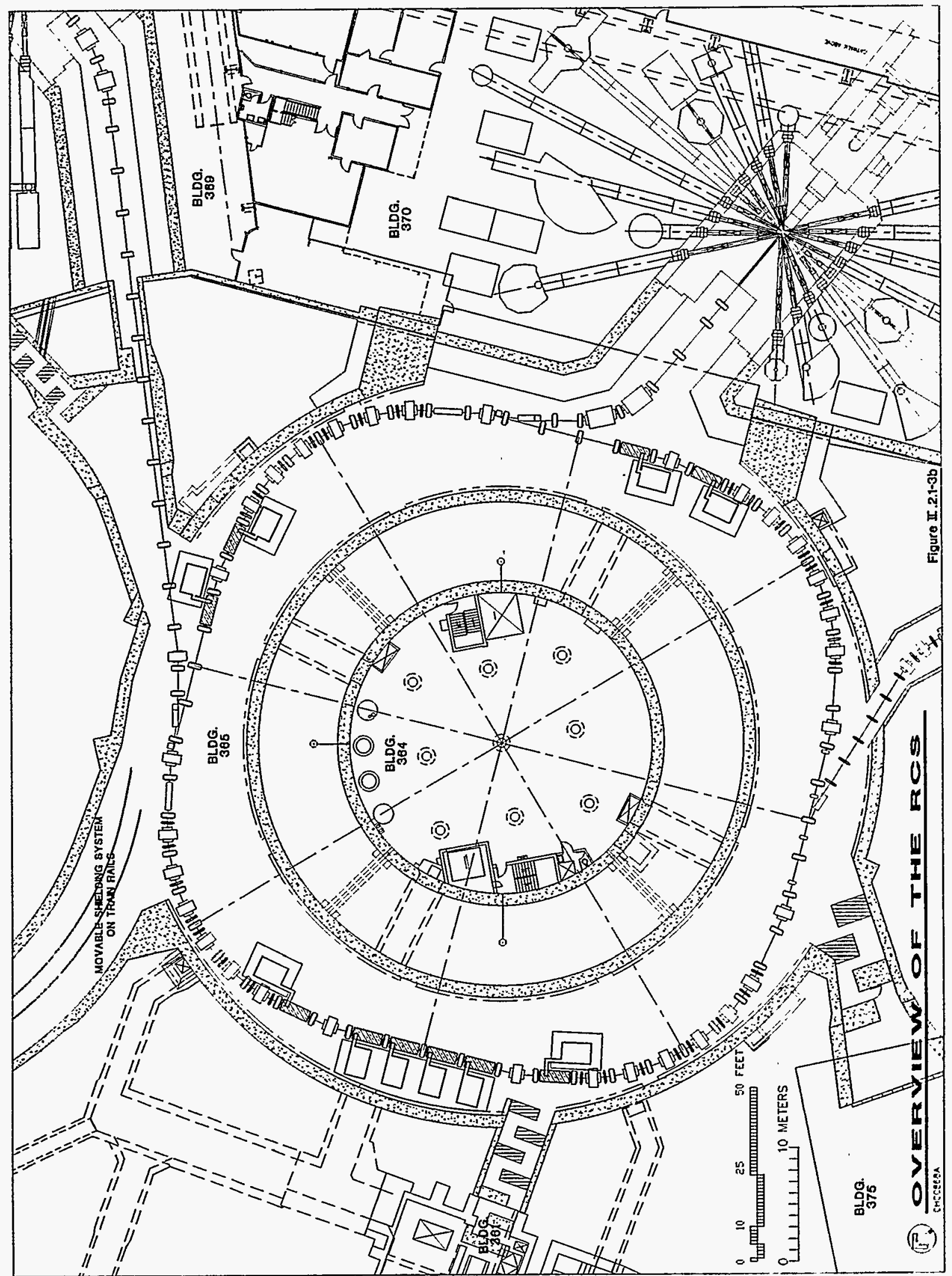


caused by longitudinal space charges are directly proportional to the external focusing forces. This allows bunching factors and if bucket area reductions to be calculated analytically. The bunching factor, $\mathrm{B}_{\mathrm{f}}$, is defined to be the ratio of mean to peak number of particles per unit length, given by

$$
\mathrm{B}_{\mathrm{f}}=\frac{1}{2 \pi} \frac{\sin \phi_{2}-\sin \phi_{1}-\left(\phi_{2}-\phi_{1}\right) \cos \phi_{2}-0.5\left(\phi_{2}-\phi_{1}\right)^{2} \sin \phi_{\mathrm{s}}}{\cos \phi_{\mathrm{s}}-\cos \phi_{2}+\left(\phi_{\mathrm{s}}-\phi_{2}\right) \sin \phi_{\mathrm{s}}}
$$

where $\phi_{2}$ is the maximum angle, $\phi_{1}$ is the minimum angle, and $\phi_{\mathrm{s}}$ is the synchronous angle.

If we assume a small synchronous phase angle of $5^{\circ}$ for early acceleration, the bunching factor from Equation II.2.1-1 is about 0.4. For the transverse charge distribution, it is reasonable to assume that the protons are distributed in an elliptical geometry of semi-axes a (horizontal) and $b$ (vertical) with the normalized two-dimensional elliptic density distribution, $\rho(x, y)$ :

$$
\rho(x, y)=\frac{3}{2 \pi \mathrm{ab}}\left[1-\left(\frac{x}{a}\right)^{2}-\left(\frac{y}{b}\right)^{2}\right]^{1 / 2} .
$$

The density at the center of such a distribution is 1.5 times that of a beam with a uniformfilled, elliptical cross section. It is believed that the density at the core of the beam soon after the injection process will not exceed the assumed elliptic distribution. The Laslett transverse space charge limit, $\mathrm{N}$, in a circular machine is directly proportional to the transverse emittance and inversely proportional to the central density. Therefore, the space charge limit for an elliptic distribution is 1.5 times less than that of a uniformly distributed beam of similar geometry.

The Laslett incoherent transverse space charge limit can be written

$$
\mathrm{N}=-\Delta v_{\mathrm{y}} \varepsilon_{\mathrm{y}}\left(1+\frac{\mathrm{a}}{\mathrm{b}}\right) \beta^{2} \gamma^{3} \frac{\mathrm{B}_{\mathrm{f}}}{\left(\mathrm{r}_{\mathrm{p}} \mathrm{G}_{\mathrm{v}} \mathrm{F}_{\mathrm{v}}\right)}
$$

where

$$
F_{v}=1+\left(1+\frac{a}{b}\right)\left(\varepsilon_{1} \frac{b^{2}}{h^{2}}+\beta^{2} \gamma^{2} B_{f}\left[\varepsilon_{1} \frac{b^{2}}{h^{2}}+\varepsilon_{2} \frac{b^{2}}{g^{2}} \frac{\rho_{m}}{R}\right]\right) / G_{v},
$$

$\mathrm{h}=$ vacuum chamber half-height;

$\mathrm{g}=$ bending magnet gap half-height;

$\varepsilon_{1}=$ electrostatic image coefficient $=0.1$;

$\varepsilon_{2}=$ magnetostatic image coefficient $=0.411$; 


$$
\begin{aligned}
\rho_{\mathrm{m}}= & \text { bending radius of the machine; } \\
\mathrm{R}= & \text { average radius of the machine; } \\
\mathrm{r}_{\mathrm{p}}= & \text { classical radius of proton }=1.5347 \times 10^{-18} \mathrm{~m} ; \\
\Delta v_{\mathrm{y}}= & \text { allowed vertical tune shift due to the space charge; } \\
\mathrm{G}_{\mathrm{v}}= & \text { form factor }=1.2 \text { for elliptic distributions and } 1.0 \text { for uniform } \\
& \text { distributions; and } \\
\varepsilon_{\mathrm{y}}= & \begin{array}{l}
\text { vertical emittance of injected beam, or acceptance of the machine not } \\
\end{array}
\end{aligned}
$$

As noted above, the space charge limit is proportional to the phase space area of the stacked beam. Therefore, the phase space area must be decided while taking into account its implications for such machine parameters as magnet apertures and vacuum chamber dimensions. It is prudent to iterate the values of the choices of vertical and horizontal emittances, $\varepsilon_{\mathrm{y}}$ and $\varepsilon_{\mathrm{x}}$, with consideration given to other parameters.

The study led to the following conclusions:

1. Although it is customary to have $\varepsilon_{\mathrm{y}}$ smaller than $\varepsilon_{\mathrm{x}}$, so that the ring dipole magnet gap height can be made smaller, a very high-intensity machine like the one being described may not benefit from having $\varepsilon_{\mathrm{y}}<\varepsilon_{\mathrm{x}}$. The important point is to minimize the beam losses and to control them. There are some advantages to having the two emittances equal, because coupling resonances can be used to stack the beam in a Kapchinskij-Vladimirskij (K-V)-like distribution. ${ }^{3}$ Having equal acceptances in both planes alleviates concerns over the coupling resonances.

2. Using Equation II.2.1-2 and assuming stacked beam emittances of $375 \pi \times 10^{-6} \mathrm{~m}$ rad in each plane, the space charge limit of the machine under these conditions gives the number of particles per pulse as a function of the injection energy. This number can be converted to the time-averaged beam current at a repetition rate of $30 \mathrm{~Hz}$. The allowed tune shift, $\Delta v_{y}$, is 0.15 . Figure II.2.1-4 shows the average current versus the injection energy.

From this figure, one can conclude that an injector linac of $400 \mathrm{MeV}$ allows $0.5-\mathrm{mA}$ operation of the synchrotron. The current of $0.5 \mathrm{~mA}$ at a $30-\mathrm{Hz}$ repetition rate implies that the number of protons per pulse is $1.04 \times 10^{14}$. 


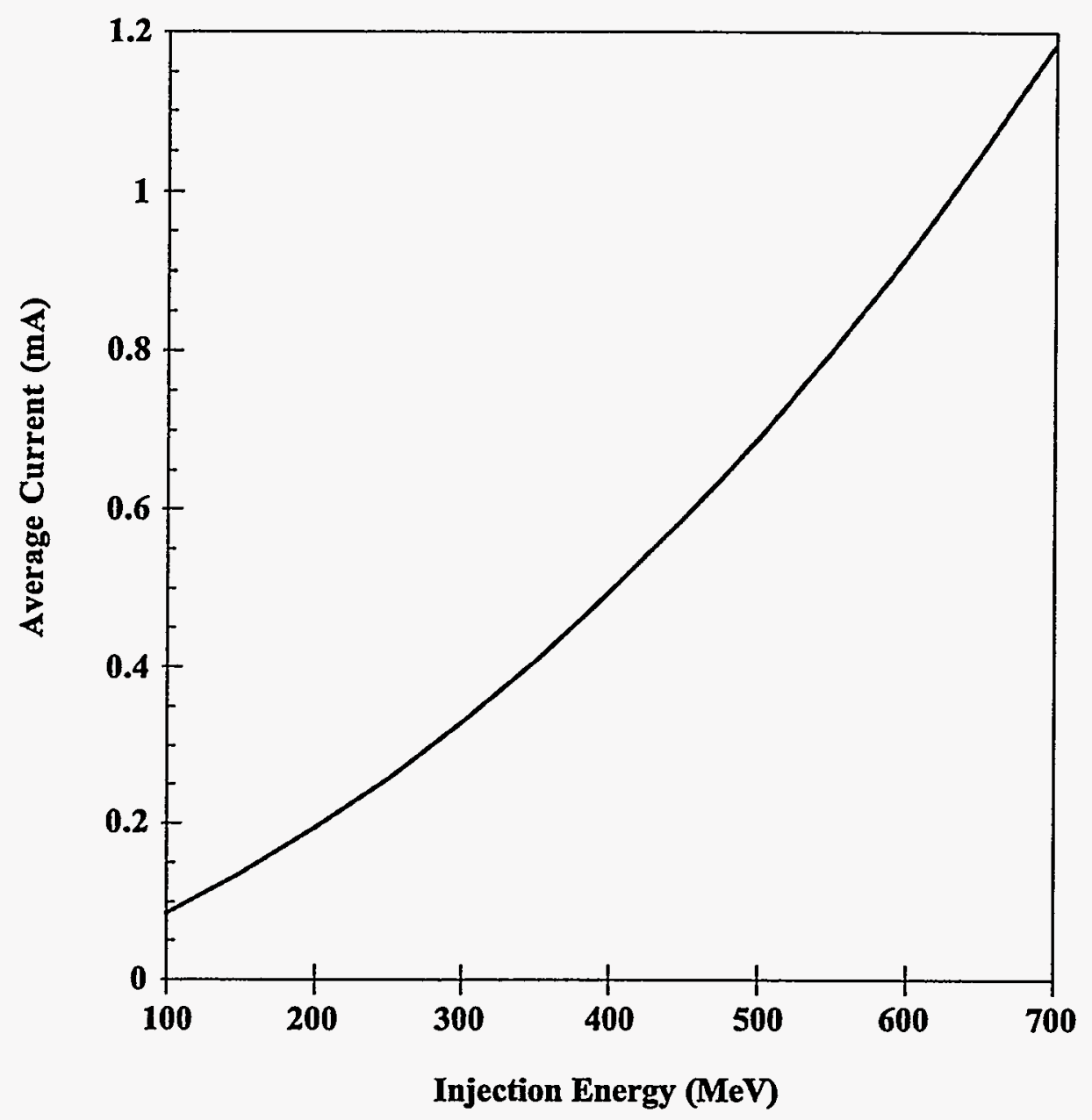

Figure II.2.1-4 Average Current from Space Charge Limit $\Delta v_{y}=0.15, \varepsilon_{y}=\varepsilon_{x}=375 \pi \times 10^{-6} \mathrm{~m}$. 
The beam emittance of $\varepsilon=375 \pi \times 10^{-6} \mathrm{~m}$ can be compared with the emittances of other machines. LANCSE-II uses $420 \pi \times 10^{-6} \mathrm{~m}$, the ESS uses $525 \pi \times 10^{-6} \mathrm{~m}$, and the BNL proposal uses $320 \pi \times 10^{-6} \mathrm{~m}$. Here, it is implied that the beam emittance contains $100 \%$ of the beam. Unlike electron beams, proton beams do not have Gaussian density distributions; instead, they tend to have cosine-squared distributions. For this reason, usage of rms quantities is avoided. The other often-used convention is to define the $100 \%$ emittance as six times the rms emittance.

In this preliminary estimate of the space charge limit, the use of $\Delta v=0.15$ is quite conservative, and there is a safety margin associated with the assumption. However, the calculation was based on the assumption of an elliptic charge distribution. In order to obtain refined information on the particle distribution, an extensive tracking study was performed to simulate both the capture and acceleration processes and to minimize beam loss during these processes. A detailed discussion of the tracking is given in Section II.4.1. The longitudinal charge distribution as a function of time from injection to extraction is obtained from the tracking study. This gives the time-varying bunching factor.

Because the goal is to accelerate $1.04 \times 10^{14}$ protons per pulse, we use the time-varying bunching factor, the emittance of $375 \pi \times 10^{-6} \mathrm{~m}$, and Equation II.2.1-2 to obtain a time-varying, incoherent, space charge tune shift. Figure II.2.1-5 shows both the time-varying bunching factor and the space charge tune shift during the first $6 \mathrm{msec}$ of the acceleration cycle.

Note that the space charge tune shift is maximal around 1 to $2 \mathrm{msec}$ after injection, and the maximum tune shift is about 0.19 .

\subsubsection{Beam Emittance and Machine Acceptance}

On the basis of these studies, it was decided to use a beam emittance after injection of $375 \pi \times 10^{-6} \mathrm{~m}$ in both planes and an injection energy of $400 \mathrm{MeV}$. Since the maximum and minimum $\beta$-functions in the transverse planes are $12 \mathrm{~m}$ and $2 \mathrm{~m}$, respectively, the largest and smallest beam sizes due to the betatron motions are $6.7 \mathrm{~cm}$ and $2.7 \mathrm{~cm}$, respectively.

For the synchrotron aperture, it is customary to add the maximum beam size due to betatron motion and synchrotron motion, plus some alignment tolerances, in order to obtain the dipole, quadrupole, and sextupole apertures. One of the design features is to have the smallest beam loss possible, so the aperture was chosen based on the following arguments. Clearly, the larger the aperture, the easier it is to prevent beam loss and to implement a lost beam catcher system. On the other hand, larger aperture results in higher construction and operating costs. The decision was made to define the beam-stay-clear (BSC) region as that calculated from the lattice $\beta$-functions and an acceptance of $750 \pi \times 10^{-6} \mathrm{~m}$. The acceptance is twice as large as the 


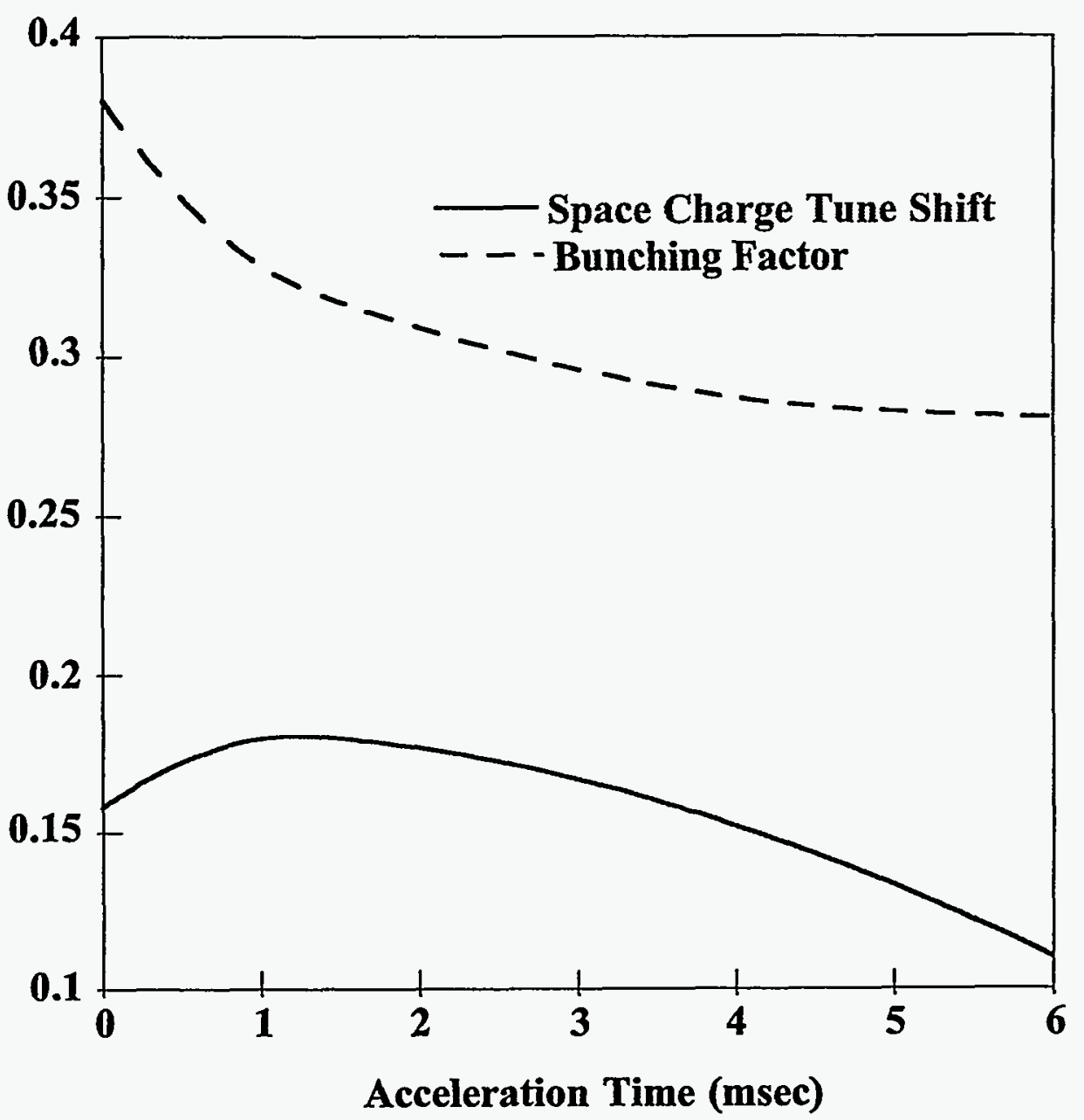

Figure II.2.1-5 Time Variation of the Bunching Factor (top curve) and Time Variation of the Space Charge Tune Shift (bottom curve) for the First $6 \mathrm{msec}$. 
stacked beam emittance. In other words, the half-width of the $\mathrm{BSC}$ region, $\mathrm{A}_{\mathrm{h}}$ is derived from the following formula:

$$
\mathrm{A}_{\mathbf{h}}=\sqrt{\frac{2 \beta \varepsilon}{\pi}}+\left|\eta_{\max }(\Delta \mathrm{p} / \mathrm{p})_{\max }\right|+\mathrm{COD}
$$

where COD, the expected closed-orbit distortion after the corrections are performed, is expected to be on the order of $0.1 \mathrm{~cm}$. The horizontal and vertical emittances are six times the $\mathrm{rms}$ emittance, $\varepsilon_{\mathrm{x}}=\varepsilon_{\mathrm{y}}=6 \varepsilon_{\mathrm{rms}}$, and are $375 \pi \times 10^{-6} \mathrm{~m}$. The maximum values of the dispersion function and relative momentum spread are given by $\eta_{\max }$ and $(\Delta \mathrm{p} / \mathrm{p})_{\max }$, respectively. The maximum $\Delta \mathrm{p} / \mathrm{p}$ used for this case is $1 \%$, which is about twice as large as the stacked beam momentum bite. This implies that

1. The acceptance is 12 times the rms emittance and

2. The vacuum chamber opening is 1.4 times larger than the beam size.

The design of the vacuum chamber system uses $750 \pi \times 10^{-6} \mathrm{~m}$ acceptances in both planes and the beam size due to the momentum spread. The resulting chamber cross-sectional dimensions are quite similar to those of the ISIS synchrotron. Figure II.2.1-6 shows the chamber cross section in the quadrupole region, and the details of the chamber are discussed in Section II.6.

\subsubsection{Single-Particle Instabilities}

Single-particle instabilities arise through resonances between the natural oscillations of the particles and components of the magnetic guiding and if accelerating fields. These resonances can be intrinsic to the fields or can be driven by imperfections in the fields. They are controlled through the if and magnet lattice designs and the setting of appropriate tolerances on the magnetic field quality.

Betatron instabilities can arise when there is a resonance between the transverse oscillations of the particles and the harmonic components of the lattice magnetic fields. This relation can be expressed as

$$
\mathrm{k} v_{\mathrm{x}} \pm l v_{\mathrm{y}}=\mathrm{p}
$$




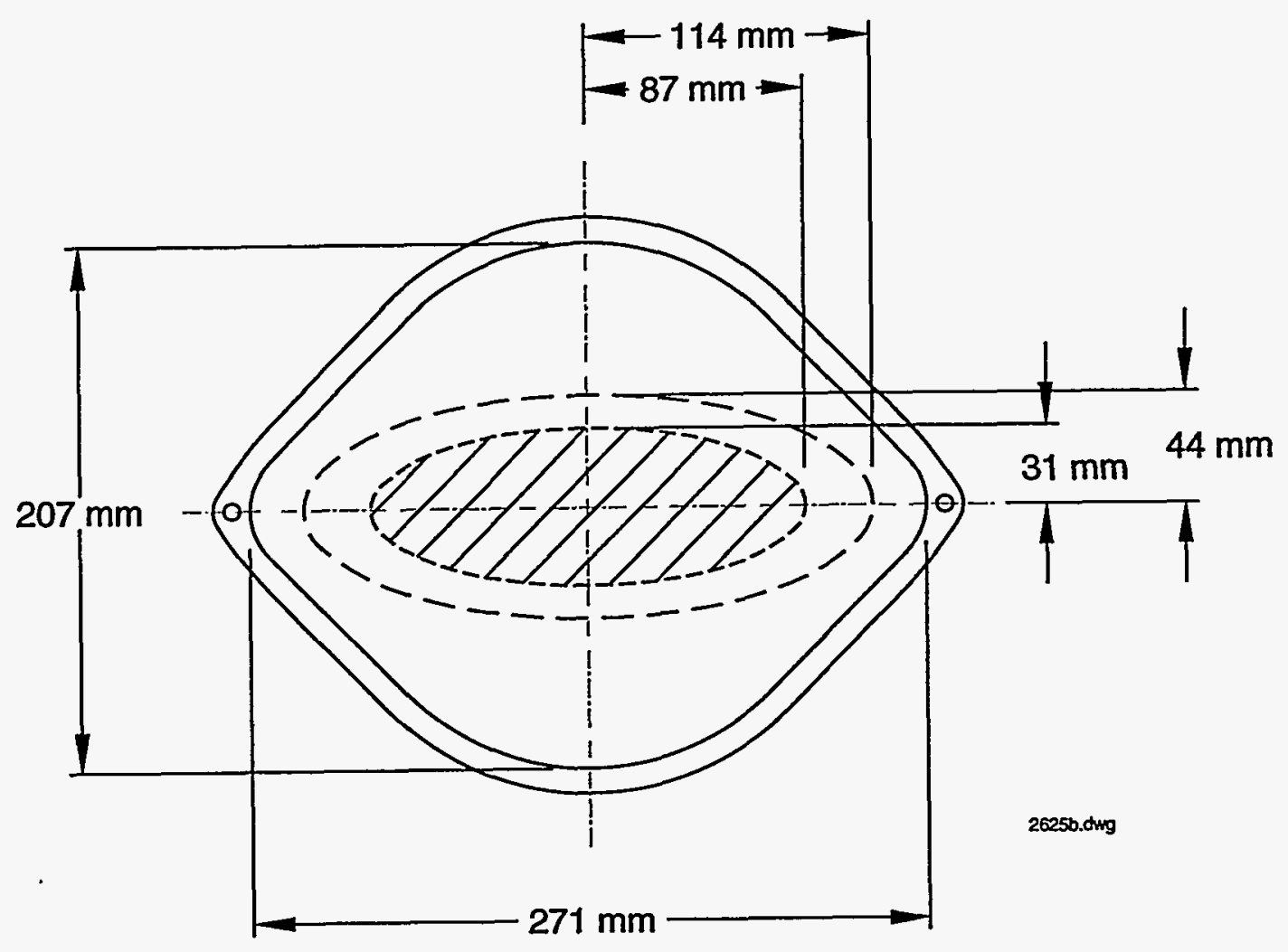

Figure II.2.1-6 Quadrupole Vacuum Chamber Cross Section Showing Beam-Stay-Clear (dashed line) and Beam Envelope (area containing diagonal lines). 
where $v_{\mathrm{x}}$ and $v_{\mathrm{y}}$ are the horizontal and vertical betatron tunes, and $\mathrm{k}, l$, and $\mathrm{p}$ are positive integers. The integer $p$ refers to the driving harmonic of the magnetic structure originating from imperfections in the construction. In general, these imperfection resonances are weak and have no detrimental effects on the beam except when $p$ is an integer multiple of some lattice structural numbers, such as the super-periodicity, and the total number of cells. In this latter case, the beam is subject to a potentially dangerous structural resonance. Because the resonance excitation force depends on the $(\mathrm{k}+l-1)$ th derivative of the magnetic field, resonances of order $(\mathrm{k}+l)$ must be considered. Since higher-order resonances are less harmful, lower-order multipole components are considered. The effects of the multipoles are discussed in Sections II.2.1.5 and II.2.1.6.

The other single-particle resonances that need to be considered are the synchro-betatron resonances, which couple the longitudinal and transverse oscillations through the relation:

$$
\mathrm{k} v_{\mathrm{x}} \pm W_{\mathrm{y}} \pm \mathrm{m} v_{\mathrm{s}}=\mathrm{p}
$$

where $m$ is another integer and $v_{s}$ is the synchrotron tune. In proton machines, the synchrotron tune is much smaller than the betatron tunes, and for the RCS machine, $v_{s}$ is at least three orders of magnitude smaller than $v_{x}$ or $v_{y}$. In such a case, synchro-betatron resonances $(m \geq 1)$ manifest as satellites of the $m=0$ betatron resonances. The resonance condition is

$$
v_{x, y} \pm m v_{s}=p
$$

Here, the \pm signs refer to the sum and difference resonances, respectively. These are driven by the position-dependent energy gains from the accelerating fields. A primary cause is the dispersion at if cavity locations.

The RCS has 16 dispersion-free straight sections and eight straight sections with a dispersion of approximately $1 \mathrm{~m}$. Six of ten $\mathrm{rf}$ cavities are located in the dispersion-free area, and the remainder are in the non-dispersion-free area. The decision to use the latter area for the If cavities is made on the basis of following argument:

According to T. Suzuki, ${ }^{4}$ when if cavities are placed in non-dispersion-free straight sections, the maximum growth of the energy spread per revolution, $\delta(\Delta \mathrm{E} / \mathrm{E})$, and the maximum betatron amplitude change per revolution, $\delta x_{\max }$, are given by:

$$
\begin{aligned}
& \delta\left(\frac{\Delta E}{E}\right)=\frac{1}{2^{m}(m-1) !} \frac{\beta^{3}\left|\alpha_{m}\right| \phi_{\max }^{m} x}{c p R \beta_{x}} \frac{v_{s} A_{\eta}}{|\eta|(\Delta E / E)} \quad \text { and } \\
& \delta x_{\max }=\frac{1}{2^{m} m !} \frac{\left|\alpha_{m}\right| \phi_{\max }^{m}}{c p \beta} A_{\eta},
\end{aligned}
$$


where

$$
\begin{aligned}
\beta & =\text { relativistic factor, } \\
\beta_{\mathrm{x}} & =\beta \text {-function at the cavities, } \\
\mathrm{c} & =\text { velocity of light, } \\
\mathrm{p} & =\text { momentum of the particle, } \\
\mathrm{R} & =\text { average radius of the ring, } \\
\eta & =\text { slip factor, } \\
\phi_{\max } & =\text { maximum synchrotron oscillation phase, } \\
\alpha_{\mathrm{m}} & =\text { Taylor series expansion coefficient, less than or equal to } 1, \text { and } \\
\mathrm{A}_{\eta} & =\text { parameter that depends on the rf voltage and dispersion function. }
\end{aligned}
$$

The parameter $A_{\eta}$, calculated using the Suzuki formulation, is $\sim 50 \mathrm{keV} \mathrm{m}$ for the RCS. 5 The largest $v_{\mathrm{s}}$ is 0.003 , which occurs $4 \mathrm{msec}$ into the acceleration period. From Equation II.2.1-3 together with $v_{s}, v_{x}$, and $v_{y}$, the minimum values of $m$ are about 60 for a sum resonance and about 250 for a difference resonance. The estimated growth rates for these $m$ values are vanishingly small for the synchro-betatron coupling resonance. 5 Therefore, the decision to place rf cavities in the non-dispersion-free straight area is acceptable.

\subsubsection{Choice of Working Point}

In addition to the single-particle instabilities discussed in the previous section and the Laslett space charge tune shift described in Section II.2.1.3, the considerations discussed below are taken into account in choosing the working point.

For a very-high-intensity machine, high tune values have several advantages, as discussed in Section II.2.1.1. One advantage is a relatively small space charge tune shift, and another is larger $\gamma_{\mathrm{t}}$. The latter has implications for the collective instability threshold and the rf capability. Higher tunes are achieved by using a $90^{\circ}$ phase advance cell as the base of the lattice. In addition, the phase advances in the horizontal and vertical planes are chosen to achieve the dispersion suppression with the removal of a single dipole from a normal cell. This is accomplished by choosing the vertical phase advance to be slightly less than $90^{\circ}$. The standard $90^{\circ}$ cell dispersionsuppressor requires placement of a half-dipole in place of a full dipole. 
This small difference in the phase advances between the two planes results in a $v_{x}$ that is larger than $v_{y}$ by one unit when the complete lattice is formed from the cells. The tune can be raised or lowered by 0.5 unit while keeping a difference of one unit between $v_{x}$ and $v_{y}$ and keeping the dispersion function through straight-section cells small $(\sim 10 \mathrm{~cm})$. Therefore, the working point is not located in the vicinity of the traditional $v_{x}-v_{y}=0$ line.

The next consideration for choosing the working point is placing it on the tune diagram. Figure II.2.1-7 shows the tune diagram in the region $6 \leq v_{x} \leq 8$ and $5 \leq v_{y} \leq 7$, with the sum resonances (solid lines) and the difference resonances (dashed lines) up to fourth order. The line thickness indicates the relative strength of the sum resonances. The working point of the bare machine (i.e., the machine without space charge), is indicated in the figure. It should be noted that it is not necessary to avoid all resonances for rapidly cycling machines, because the beam spends a relatively short time at the resonance lines. Thus, it is rarely necessary to consider resonances above fourth order.

The next item to be investigated is the spread in the working point due to space charge. The working point becomes diamond-shaped, as shown in Figure II.2.1-8. As discussed in Section II.2.1.3, this tune shift depends on the charge distribution in the machine and can differ by a factor of 1.5 between a uniform and an elliptic charge distribution. In the figure, the point $P$ is the tune point of the bare machine. The point $Q$ in the figure is the working point for a $K-V$ distribution at $2 \mathrm{msec}$ after injection, when the space charge tune shift is maximal. The diamondshaped pattern is for the case in which the charge distribution is elliptic. The injection mechanism to achieve a K-V-like distribution is described in detail in Section I.2.2.

Figure II.2.1-8 also shows that the tune spread around the working point can cross the nonlinear structural resonances of $3 v_{x}=20$ and $v_{x}+3 v_{y}=24$. These are important because 20 and 24 are divisible by 4 , the super-periodicity.

The $v_{x}+3 v_{y}=24$ resonance can be driven by skew-octupole fields. Such terms arise only from multipole errors and have little effect on the dynamic aperture, as discussed in Section II.2.1.6.

The $3 v_{x}=20$ resonance near the full space charge tune can be driven by the chromaticity-correcting sextupoles and can have a deleterious effect on the circulating beam. However, the 20th harmonic of the sextupole component is suppressed by placing harmoniccorrecting sextupoles in the dispersion-free sections. Sextupoles with integrated strengths of $0.4 \mathrm{~m}^{-2}$ placed in each of the eight dispersion-suppressor cells eliminate the 20th harmonic. Figure II.2.1-9 shows the phase space motion with and without the harmonic correction for the particles oscillating with a tune of $v_{x}=6{ }^{2} / 3$. This is a standard technique used for a ChasmanGreen lattice in third-generation synchrotron light sources. 




Figure II.2.1-7 Sum (solid lines) and Difference (dashed lines) Resonances Shown up to Fourth Order in the Region Bounded by $6 \leq v_{x} \leq 8$ and $5 \leq v_{y} \leq 7$. (Working point for the bare machine is indicated.) 


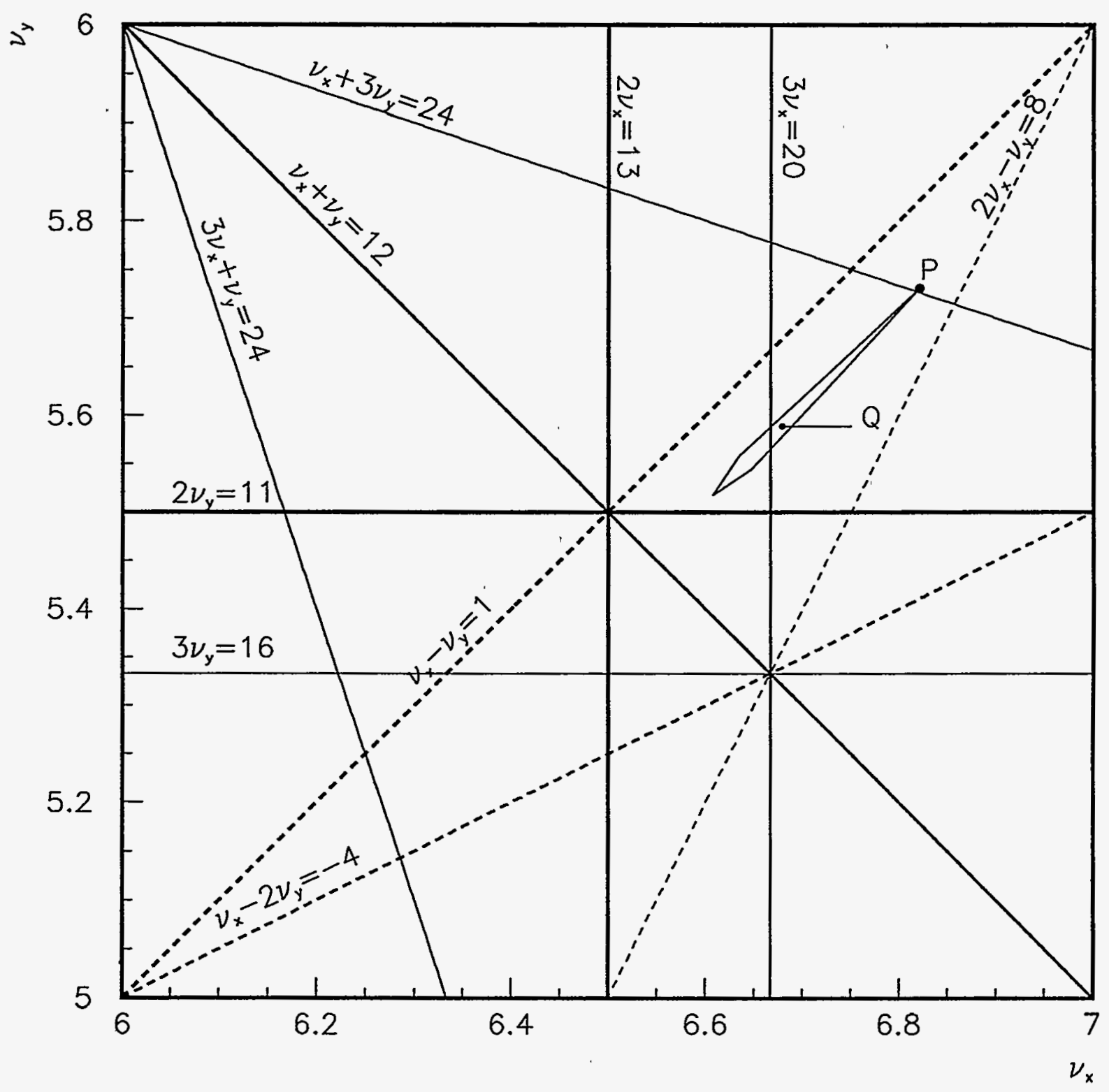

Figure II.2.1-8 Structure Resonances up to Fourth Order Shown for the Region Bounded by $6<v_{x}<7$ and $5<v_{y}<6$. The working point for the bare RCS machine is labeled $P$. The maximum space charge tune shift for a uniform, K-V distribution is labeled $Q$. The diamond-shaped area bounds the space charge tune shifts of individual particles in an elliptic distribution. 


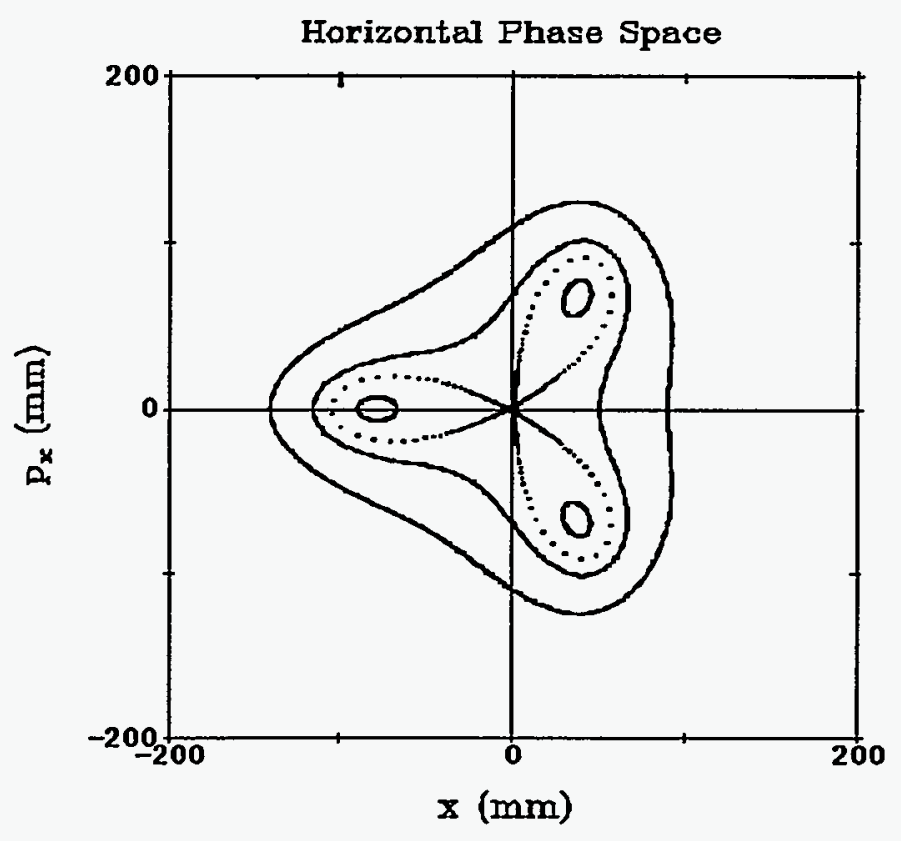

(a)

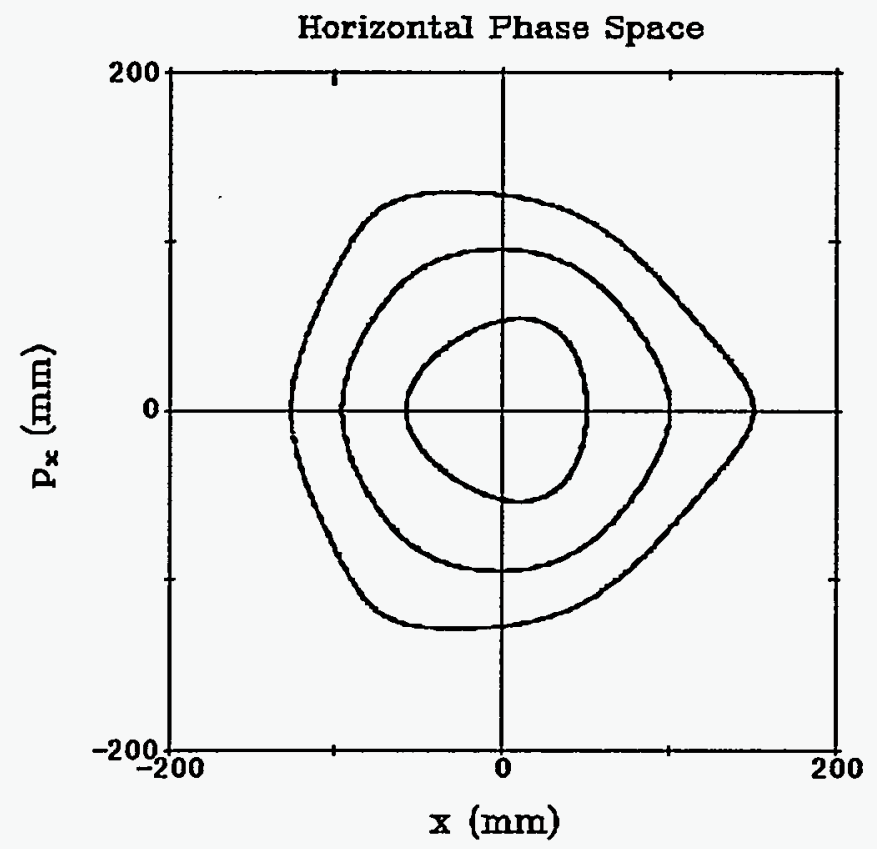

(b)

Figure II.2.1-9 Phase Space Motion with $v_{x}=6 \frac{2}{3}$ without (a) and with (b) Harmonic Correction Sextupoles. (The variable $p_{x}$ is defined to be $\left[p_{x}=\beta_{x} x^{\prime}+\alpha_{x} x\right]$.) 


\subsubsection{Transverse Tracking Studies}

The key design feature of the RCS is the prevention of beam loss during the injection, capture, and acceleration processes. Beam loss prevention in the transverse phase space is achieved by providing large dynamic apertures in both transverse planes. We have performed tracking studies to ensure that the RCS has a dynamic aperture larger than the physical aperture of the ring vacuum chamber, taking into account magnetic field imperfections due to fabrication tolerances and misalignments caused by surveying tolerances.

The results of tracking studies of closed-orbit distortions and dynamic aperture reduction in the RCS are discussed below.

Closed-orbit distortions are caused by:

- Deviations of the vertical field value of dipole magnets, which introduce horizontal orbit distortions;

- Roll-angle misalignments of dipole magnets, which cause vertical orbit distortions; and

- Misalignments of quadrupole magnets, which produce extraneous dipole fields that distort the orbit.

Design specifications for the dipole corrector magnets are obtained from tracking studies.

The natural normalized chromaticities of the lattice described in Section II.2.1.1 are $\xi_{\mathrm{x}}=-106$ and $\xi_{\mathrm{y}}=-1.20$. Chromaticity is corrected by adding two families of sextupoles in the normal cells of the lattice.

The dynamic aperture is limited primarily by these chromaticity-correcting sextupoles. With the sextupoles energized to the values required to adjust the chromaticities to zero, we studied further reductions of the dynamic aperture caused by field deviations and misalignments, as cited above, and multipole components arising from magnet fabrication imperfections.

Dynamic aperture reduction caused by closed-orbit distortion is recovered by orbit corrections. The dynamic aperture is insensitive to reasonable levels of multipole components, up to four times the levels achieved in the APS Synchrotron magnets. 


\subsubsection{Closed-Orbit Correction}

Closed-orbit distortions due to quadrupole misalignments and dipole field deviations can be estimated by using the following formula:

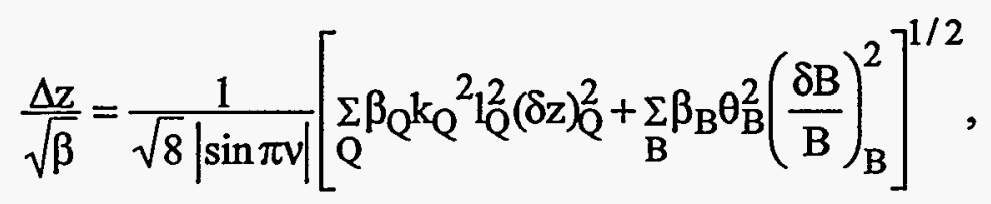

where the subscripts $Q$ and $B$ denote the quadrupole and dipole magnets, respectively, and

$$
\begin{aligned}
\Delta z & =\text { rms value of the COD in either the } x \text { - or } y \text {-plane, } \\
\beta & =\text { amplitude function, } \\
v & =\text { tune of the lattice, } \\
k_{Q} & =\text { quadrupole strength, } \\
l_{Q} & =\text { quadrupole length, } \\
(\delta z)_{Q} & =\text { rms quadrupole misalignment, } \\
\theta_{B} & =\text { bending angle, and } \\
(\delta B / B)_{B} & =\text { rms field deviation in dipole magnet. }
\end{aligned}
$$

For the RCS, we find that

$$
\begin{aligned}
& \frac{\Delta \mathrm{x}}{\sqrt{\beta_{\mathrm{x}}}}=\left[5.05^{2}(\delta \mathrm{x})_{\mathrm{Q}}^{2}+1.73^{2}(\delta \mathrm{B} / \mathrm{B})_{\mathrm{B}}^{2}\right]^{1 / 2} \text { and } \\
& \frac{\Delta \mathrm{y}}{\sqrt{\beta_{\mathrm{y}}}}=\left[3.49^{2}(\delta \mathrm{y})_{\mathrm{Q}}^{2}+1.32^{2}(\delta \mathrm{B} / \mathrm{B})_{\mathrm{B}}^{2}\right]^{1 / 2},
\end{aligned}
$$

where $(\delta B / B)_{B}$ in the $y$-plane represents the dipole roll angle, and all lengths are in meters.

Thus, the amplification factors for quadrupole misalignments, $A_{x, y}$, defined as the ratio of the maximum $\mathrm{COD}$ in the ring and the rms quadrupole misalignments, are

$$
A_{x}=32.88 \text { at } \beta_{x}=10.60 \mathrm{~m} \text { and } A_{y}=24.12 \text { at } \beta_{y}=11.94 m \text {, }
$$


where we used $(\Delta z)_{\max }=2(\Delta z)$, corresponding to $95 \%$ probability. These analytical values are compared with $\mathrm{MAD}^{6}$ simulations. The results are shown in Figure II.2.1-10, where the horizontal axis represents 30 different machine simulations.

The APS Booster Synchrotron quadrupoles were aligned to an ms accuracy of $(\delta \mathrm{z})_{\mathrm{Q}}=0.2 \mathrm{~mm} .{ }^{7}$ The RCS dynamic aperture is fairly insensitive to misalignment. For computational purposes, an alignment accuracy of $0.4 \mathrm{~mm}$ was used to estimate the maximum COD. Similarly, a value of $(\delta B / B)_{B}=0.001$, corresponding to a dipole roll angle of $1.0 \mathrm{mrad}$, was used in the computations. This is a factor of three larger than the field deviations achieved at APS. These levels are expected to produce maximum orbit distortions of $18 \mathrm{~mm}$ in the horizontal plane and $14 \mathrm{~mm}$ in the vertical plane before correction.

The MICADO algorithm ${ }^{8}$ included in the MAD program was used to simulate orbit corrections. Horizontal and vertical BPMs are located in the focusing and defocusing quadrupoles, respectively. Similarly, horizontal and vertical correctors are located near the focusing and defocusing quadrupoles. There are a total of 28 BPMs and 28 correctors in each plane. Horizontal and vertical corrector strengths of up to 85 and $71 \mathrm{G} \mathrm{m}$, respectively, permit correction of the orbit to within the rms BPM accuracy of $0.1 \mathrm{~mm}$. The maximum dipole corrector strength is specified as $110 \mathrm{G} \mathrm{m}$. Typical CODs before and after correction are shown in Figure II.2.1-11. The corresponding corrector strengths are shown in Figure II.2.1-12.

\subsubsection{Chromaticity Correction}

The natural chromaticities of the lattice described in Section II.2.1.1 are $\xi_{\mathrm{x}}=-1.06$ and $\xi_{\mathrm{y}}=-1.20$, where $\xi=(\Delta v / v) /(\Delta \mathrm{p} / \mathrm{p})$. Chromaticity is corrected by the addition of sextupoles in the normal cells of the lattice. The sextupole strengths, $\mathrm{B}^{\prime \prime} l / \mathrm{B} \rho$, required to make the chromaticities zero are $0.627 / \mathrm{m}^{2}$ for each horizontal focusing sextupole (SF) and $0.863 / \mathrm{m}^{2}$ for each horizontal defocusing sextupole (SD). There are $16 \mathrm{SF}$ and $16 \mathrm{SD}$ magnets.

The effects of chromaticity on the beam are discussed in Section II.5.4 in connection with transverse beam stability. We expect this lattice to operate with a chromaticity between zero and its natural chromaticity.

\subsubsection{Dynamic Aperture}

The dynamic aperture is reduced by the presence of chromaticity-correcting sextupoles as well as misalignments and magnet fabrication imperfections. Dynamic aperture limitations were simulated by using the tracking program RACETRACK. ${ }^{9}$ The sextupoles were energized to the values required to adjust the chromaticities to zero. 

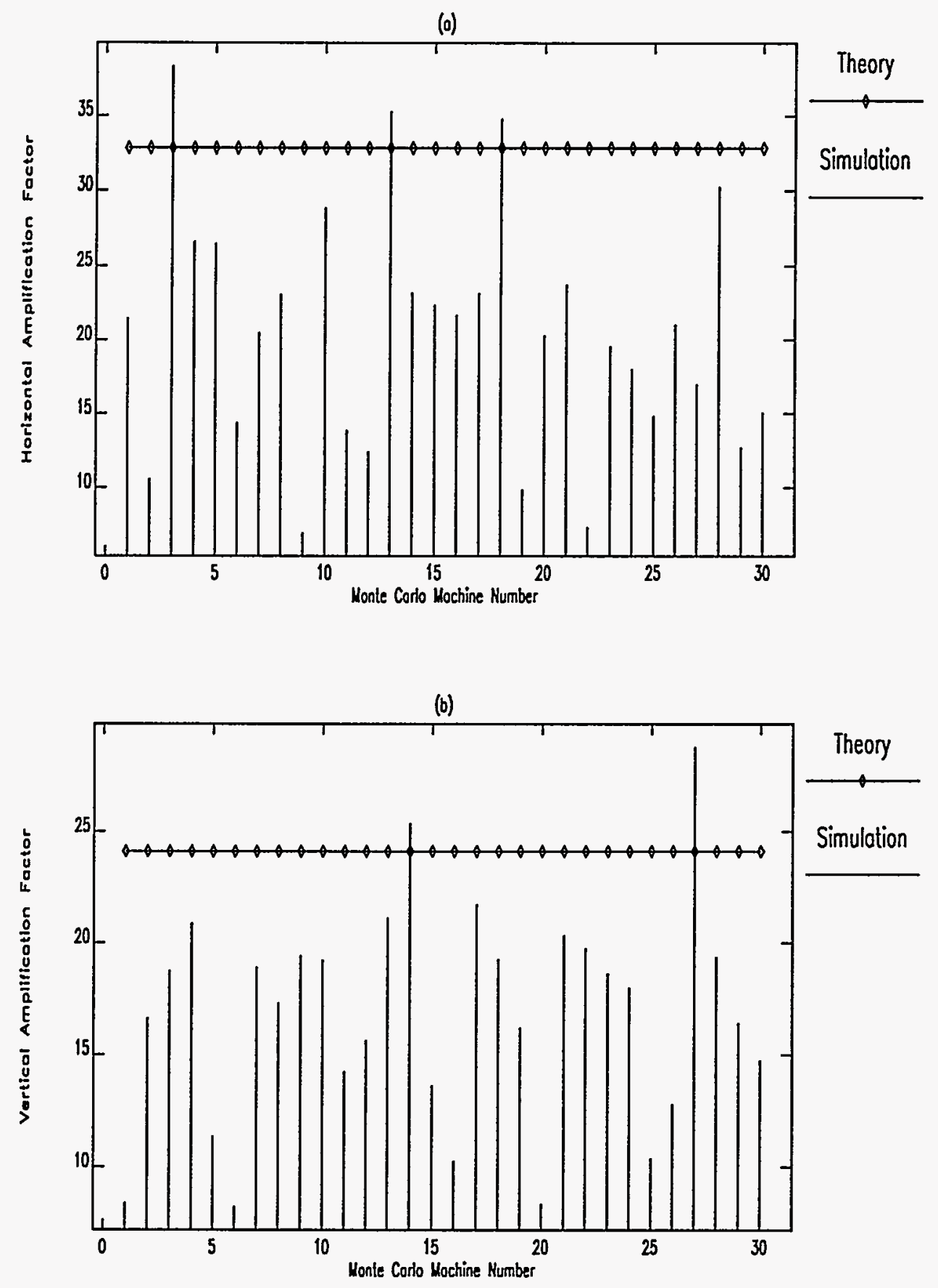

Figure II.2.1-10 Amplification Factors of the RCS. (Thirty different machines are simulated and compared with the theoretical estimation.) 


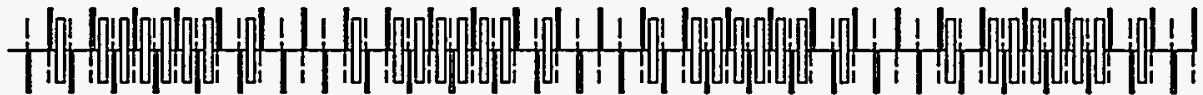

(a)

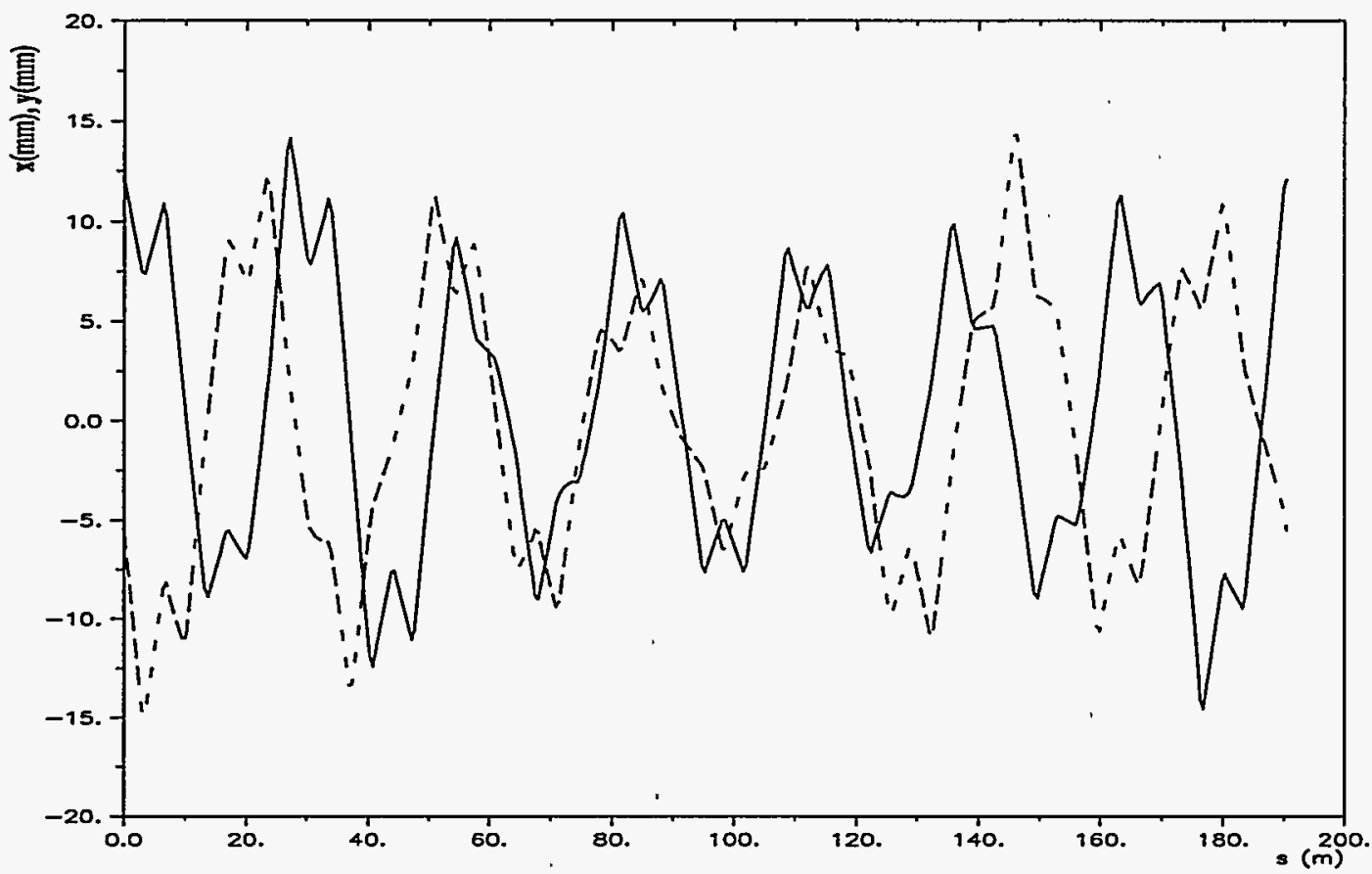

(b)

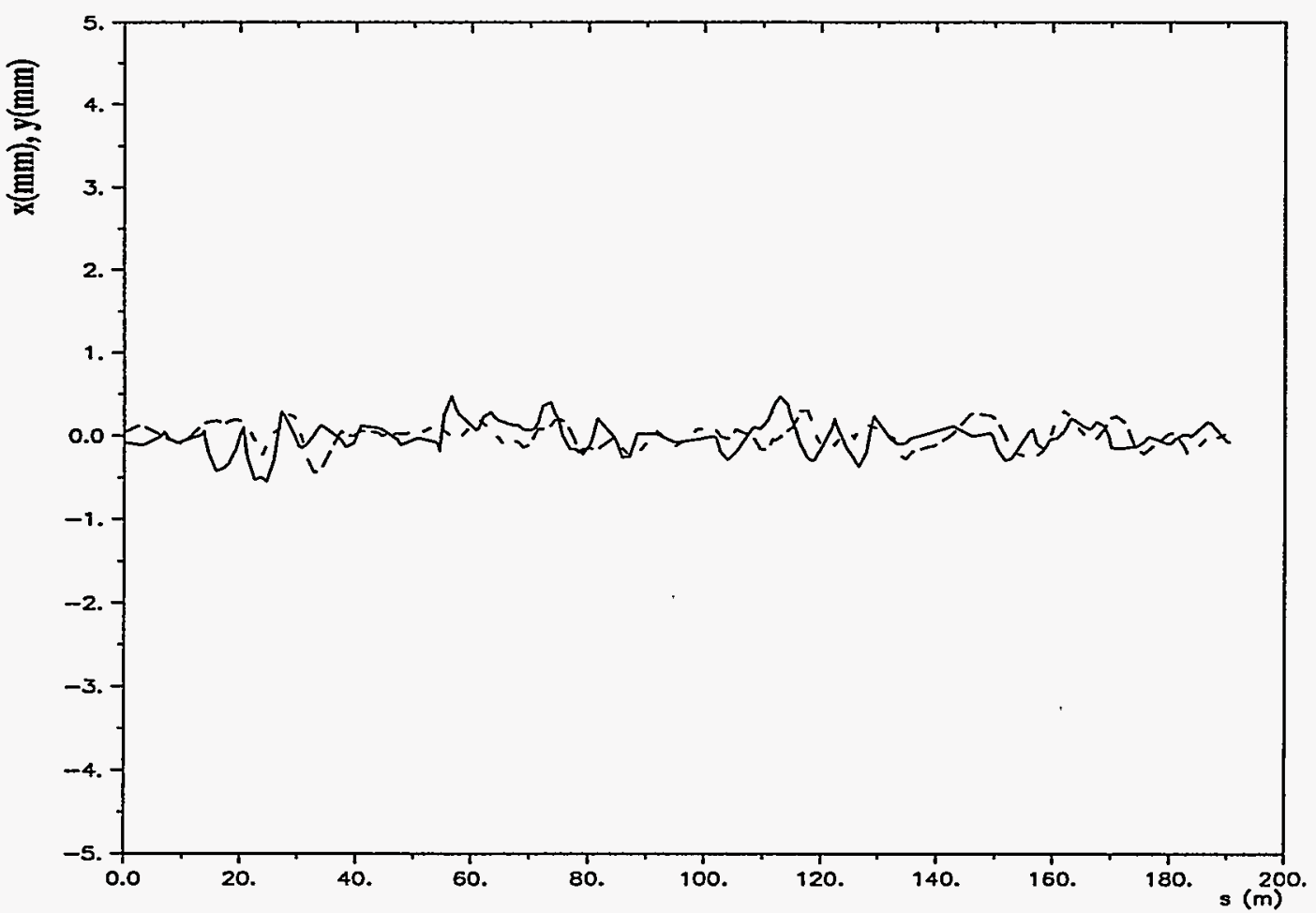

Figure II.2.1-11 Closed-Orbit Distortions before (a) and after (b) Correction for Quadrupole Misalignments $(\delta \mathrm{z})_{\mathrm{Q}}=0.4 \mathrm{~mm}$, Dipole Field Deviations $(\Delta \mathrm{B} / \mathrm{B})_{\mathrm{B}}=0.001$, and Dipole Roll Angles $(\delta \theta)_{\mathrm{B}}=1.0 \mathrm{mrad}$.

(Solid/dashed lines represent the horizontal/vertical orbit distortions, respectively.) 

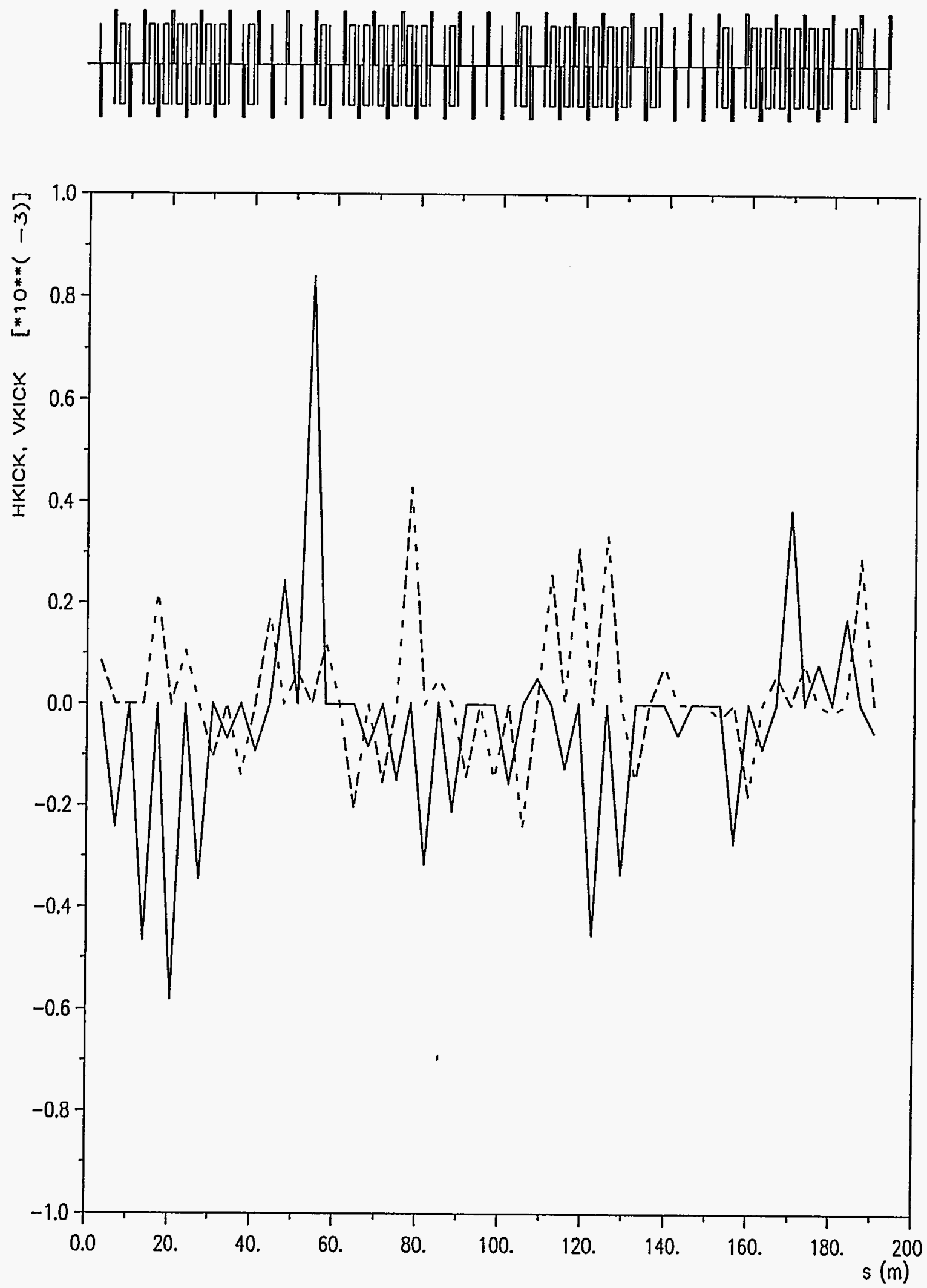

Figure II.2.1-12 Required Corrector Strengths to Correct the Closed-Orbit Distortion Shown in Figure II.2.1-11. (Solid/dashed lines represent the horizontal/vertical corrector strengths, respectively.) 
The dynamic aperture was searched along five directions of the $x-y$ plane. First the positive and negative horizontal directions along the $y=0$ axis and the positive vertical direction along the $x=0$ axis were searched. Then the diagonal directions $y= \pm x$ were searched. The dynamic aperture was defined as the limiting stable betatron oscillation amplitude of a particle that survived 2,000 turns in a static field.

The following discussion is limited to reductions caused by random misalignments and random multipole components arising from magnet imperfections. The distribution of errors was assumed to be Gaussian, with a cutoff of $\pm 5 \sigma$. The simulations were performed for 10 different machines. The effects resulting from systematic errors were found to be smaller than those resulting from random errors and are not discussed.

First the dynamic aperture reductions caused by closed-orbit distortions arising from the alignment tolerances discussed in Section II.2.1.6.1 were investigated. Recovery of the dynamic aperture after orbit correction was also verified. Second, reductions caused by higher-order multipoles were investigated. Finally, dynamic aperture reduction due to alignment tolerances and higher-order multipoles was calculated. These calculations were performed with and without closed-orbit correction. It is particularly important to verify that the dynamic aperture is adequate to permit beam injection prior to initial startup of the machine.

Dynamic aperture reductions resulting from alignment tolerances, with and without closed-orbit corrections, are shown in Figure II.2.1-13. The lines in the figure are the averages and the error bars are the standard deviations for the 10 machines. The dynamic apertures are displayed at the focusing quadrupole. The dynamic aperture of the perfect machine and BSC are also shown in the figure. The closed orbit was corrected to $0.1 \mathrm{~mm} \mathrm{mms}$, as described in Section II.2.1.6.1. Before orbit correction, the dynamic aperture is sufficient to permit beam injection, as seen in Figure II.2.1-13. Correction of the orbit restores the dynamic aperture to that of the perfect machine.

Dynamic aperture reductions due to magnetic field imperfections were studied by using the multipole coefficients, $a_{n}$ and $b_{n}$, shown in Table II.2.1-3. The coefficient values were obtained by scaling the measured data of the APS Booster Synchrotron magnets to the RCS magnets. The magnetic field expressed in terms of these coefficients is

$$
B=B_{0} \sum_{n=0}^{\infty}\left(b_{n}+i a_{n}\right)(x+i y)^{n},
$$

where $b_{n}$ and $a_{n}$ are the normal and skew coefficients in units of $\mathrm{cm}^{-n}$.

There were no appreciable effects on the dynamic aperture for multipoles up to twice the values depicted in Table II.2.1-3. Some reduction was observed for multipoles at four times those values, as shown in Figure II.2.1-14. 


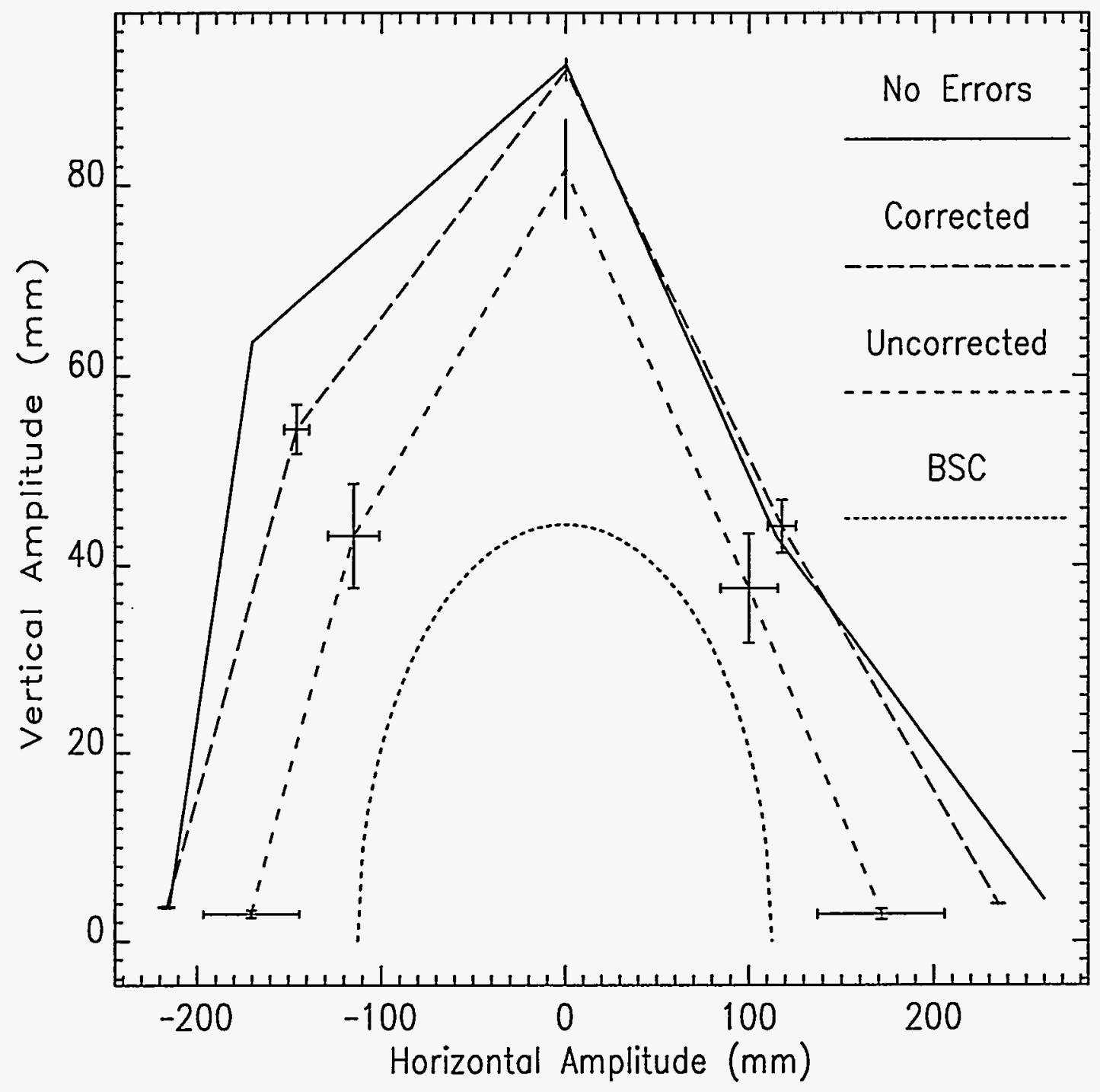

Figure II.2.1-13 Dynamic Apertures in the Presence of Quadrupole Misalignments of $0.4 \mathrm{~mm}$, Dipole Field Deviations of 0.001 , and Dipole Roll Angle Misalignments of $1.0 \mathrm{mrad}$ Plotted before and after Corrections of the Closed Orbit to 0.1-mm Root Mean Square (ms). (Dynamic aperture for the perfect machine is shown for comparison. The ellipse represents the BSC region at the focusing quadrupole.) 
Table II.2.1-3 Multipole Coefficients of Synchrotron Magnets

\begin{tabular}{ccr}
\hline Multipole ${ }^{\mathrm{a}}$ & $\begin{array}{c}\text { Random } \\
\left(\mathrm{cm}^{-n}\right)\end{array}$ & \multicolumn{1}{c}{$\begin{array}{c}\text { Systematic } \\
\left(\mathrm{cm}^{-n}\right)\end{array}$} \\
\hline$b_{10}$ & $2.60 \times 10^{-6}$ & $-7.10 \times 10^{-6}$ \\
$b_{2 D}$ & $3.90 \times 10^{-8}$ & $-0.70 \times 10^{-6}$ \\
$b_{1 Q}$ & $4.90 \times 10^{-4}$ & $2.50 \times 10^{-4}$ \\
$a_{1 Q}$ & $1.70 \times 10^{-4}$ & $-0.40 \times 10^{-4}$ \\
$b_{2 Q}$ & $4.80 \times 10^{-6}$ & $-1.20 \times 10^{-6}$ \\
$a_{2 Q}$ & $0.13 \times 10^{-4}$ & $0.28 \times 10^{-6}$ \\
$b_{3 Q}$ & $1.20 \times 10^{-6}$ & $-5.50 \times 10^{-6}$ \\
$a_{3 Q}$ & $0.62 \times 10^{-6}$ & $0.22 \times 10^{-6}$ \\
$b_{4 Q}$ & $3.60 \times 10^{-8}$ & $0.30 \times 10^{-8}$ \\
$a_{4 Q}$ & $4.50 \times 10^{-8}$ & $-0.80 \times 10^{-8}$ \\
$b_{5 Q}$ & $0.24 \times 10^{-8}$ & $1.95 \times 10^{-8}$ \\
$b_{2 S}$ & $1.36 \times 10^{-3}$ & $5.00 \times 10^{-4}$ \\
$a_{2 S}$ & $3.30 \times 10^{-4}$ & $-4.90 \times 10^{-4}$ \\
$b_{3 S}$ & $0.17 \times 10^{-4}$ & $-2.40 \times 10^{-6}$ \\
$a_{3 S}$ & $0.61 \times 10^{-4}$ & $3.20 \times 10^{-6}$ \\
\hline
\end{tabular}

a $\mathrm{D}=$ dipole; $\mathrm{Q}=$ quadrupole; $\mathrm{S}=$ sextupole. 


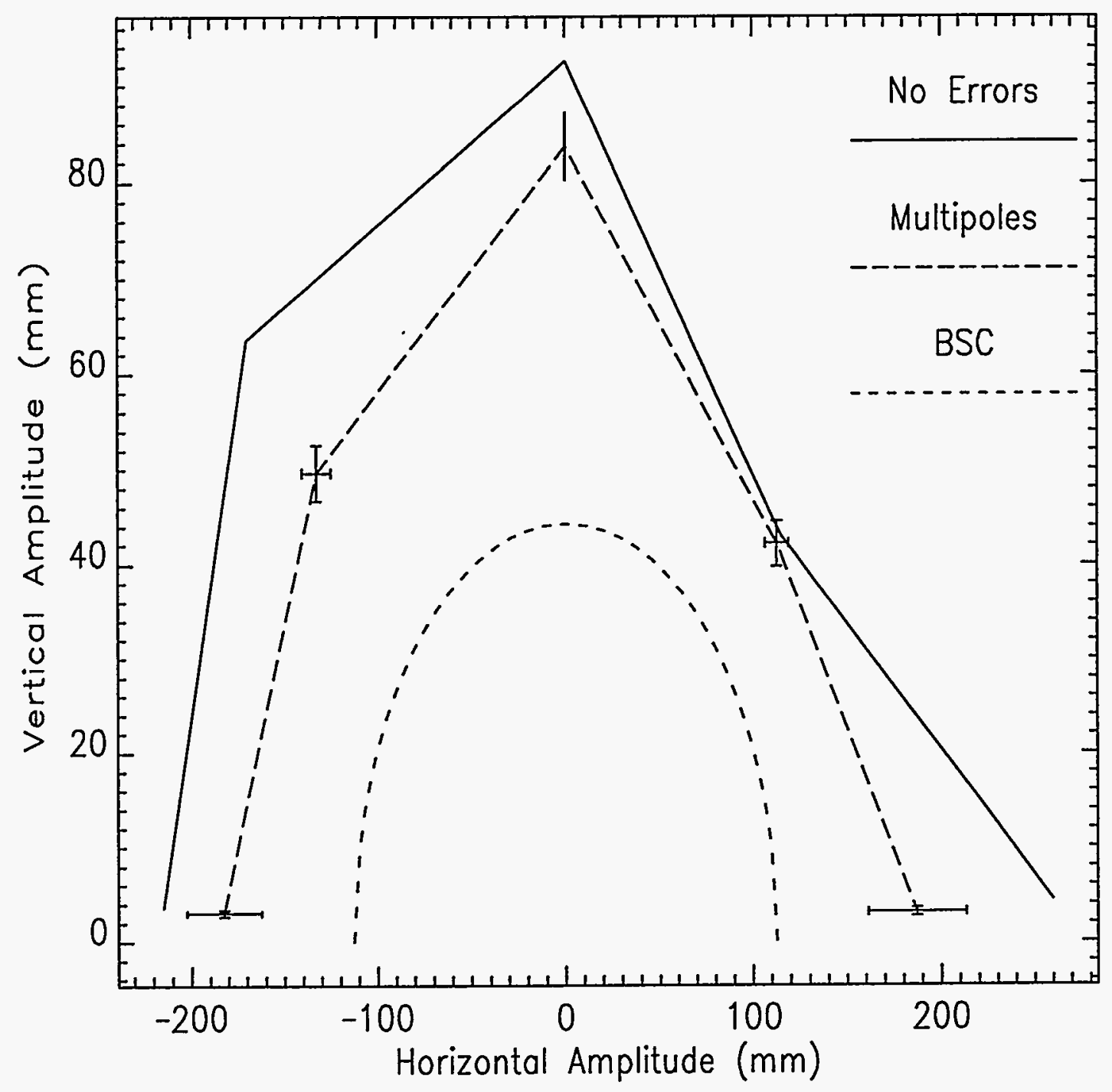

Figure II.2.1-14 Dynamic Aperture Reductions Caused by Multipoles at Four Times the Values Listed in Table II.2.1-3. 
Finally, alignment tolerances were included along with multipole components. Reductions, both before and after closed-orbit correction, were studied. The results are shown in Figure II.2.1-15. The dynamic aperture before COD is still as large as the BSC, thus permitting the establishment of a closed-orbit solution at injection. The dynamic aperture after correction of the closed orbit is very close to that shown in Figure II.2.1-14, which only included multipole components. 


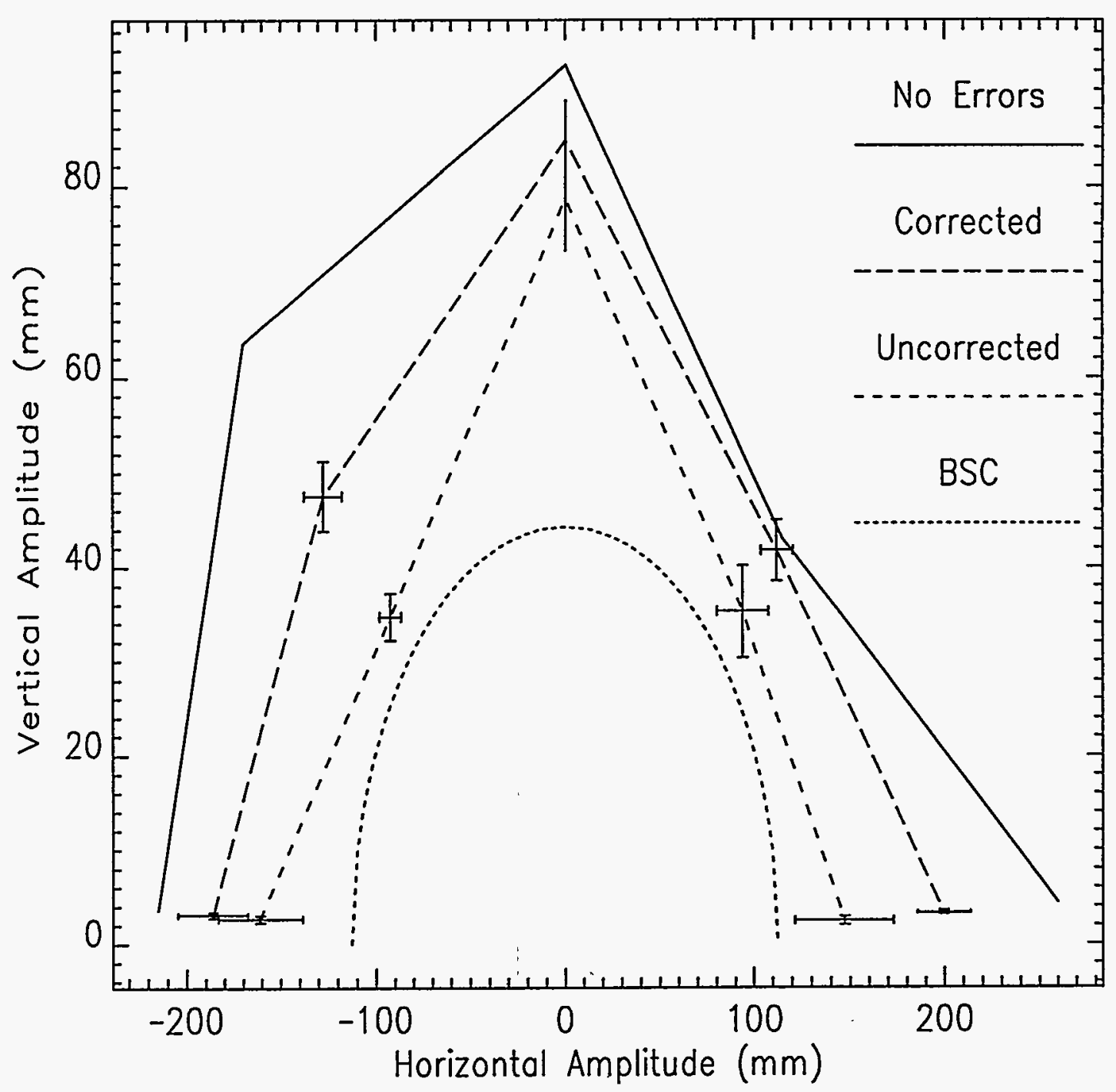

Figure II.2.1-15 Dynamic Aperture Reductions Caused by Misalignment and Multipoles, Plotted before and after Corrections of the Closed Orbit. 


\subsection{INJECTION OPTICS}

\subsection{1 $\mathrm{H}^{-}$Injection}

A total of $1.04 \times 10^{14}$ protons are injected into the synchrotron in $\sim 500$ turns by stripping negative hydrogen ions. The stripping foil is located $10 \mathrm{~cm}$ downstream of the central horizontal focusing quadrupole in one of the four dispersion-free straight sections, as shown in Figure II.2.2-1. The betatron functions at the injection point are $\beta_{x}=9.6279 \mathrm{~m}, \alpha_{x}=2.0491$, $\beta_{\mathrm{y}}=3.2871 \mathrm{~m}$, and $\alpha_{\mathrm{y}}=-0.7735$.

The separation of the $\mathrm{H}^{-}$injected ions and the circulating protons is achieved in the quadrupole magnet upstream of the stripping foil. To prevent partially stripped $\mathrm{H}^{0}$ neutral particles from entering the downstream bending magnet, the injection angle at the stripper is chosen so that these particles can be collected at an inside aperture stop upstream of the magnet. Stripping of the $\mathrm{H}^{0}$ in the bending magnet would lead to an undesirable halo of the circulating proton beam. Additional details are discussed in Section II.2.2.2.

The horizontal betatron oscillation amplitude of the injected protons is controlled by a local bump of the orbit, as shown in Figure II.2.2-1. Two magnets, $B_{1}$ and $B_{2}$, placed upstream of the injection point are used to adjust the position, $X_{b}$, and angle, $X_{b}{ }^{\prime}$, of the orbit at the stripper, which is located $10 \mathrm{~cm}$ from the central orbit. Two more magnets, $B_{3}$ and $B_{4}$, placed downstream of the injection point are required to restore the closed orbit.

Let

$$
\Delta \mathrm{x}=\mathrm{X}_{0}-\mathrm{X}_{\mathrm{b}}
$$

and

$$
\Delta \mathrm{x}^{\prime}=\mathrm{X}_{0^{\prime}}-\mathrm{X}_{\mathrm{b}^{\prime}}
$$

where $\mathrm{X}_{0}$ and $\mathrm{X}_{0}$ ' are the position and angle of $\mathrm{H}^{-}$ions at the stripper. The horizontal oscillation amplitude is

$$
A_{x}=\sqrt{\Delta x^{2}+\left(\beta_{x} \Delta x^{\prime}+\alpha_{x} \Delta x\right)^{2}}
$$




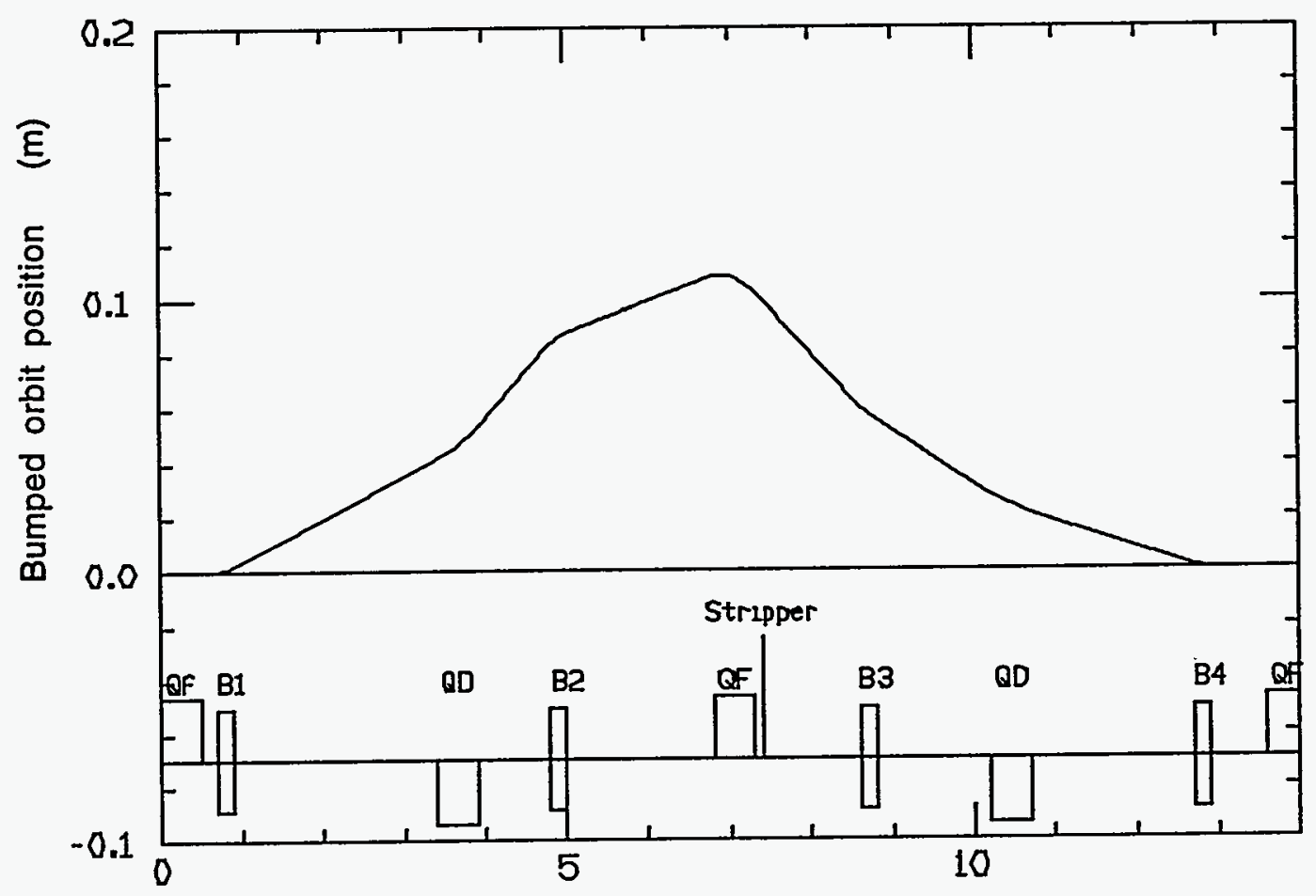

(m)

Figure II.2.2-1 Bumped Orbit in the Injection Straight Section. 
To minimize the number of passes through the stripping foil by the circulating protons, one injects them at the maximum of the oscillation amplitude:

$$
\beta_{\mathrm{x}} \Delta \mathrm{x}^{\prime}+\alpha_{\mathrm{x}} \Delta \mathrm{x}=0
$$

and

$$
A_{x}=\Delta x
$$

This condition is usually achieved by adjusting the injection angle, $\mathrm{X}_{0^{\prime}}$, such that

$$
\beta_{\mathrm{X}} \mathrm{X}_{0^{\prime}}+\alpha_{\mathrm{X}} \mathrm{X}_{0}=0
$$

and by controlling the bumped orbit to give

$$
\beta_{\mathrm{X}} \mathrm{X}_{\mathrm{b}}{ }^{\prime}+\alpha_{\mathrm{x}} \mathrm{X}_{\mathrm{b}}=0
$$

Under this condition, the bends required at all four bumper magnets are proportional to $\mathrm{X}_{\mathrm{b}}$.

With the injection point at $10 \mathrm{~cm}$, these conditions result in an injection angle of $-21.3 \mathrm{mrad}$. This injection angle, however, is too shallow to prevent the neutral particles from entering the downstream bending magnets. The required angle is $-33.0 \mathrm{mrad}$. Injection at the maximum of the betatron oscillation amplitude now gives the condition:

$$
\mathrm{X}_{\mathrm{b}^{\prime}}=\mathrm{X}_{0^{\prime}}+\alpha_{\mathrm{x}}\left(\mathrm{X}_{0}-\mathrm{X}_{\mathrm{b}}\right) / \beta_{\mathrm{X}}
$$

The bends at the four bumper magnets have a constant term in addition to the normal $\mathrm{X}_{\mathrm{b}}$ dependence.

Figure II.2.2-2 shows the injection details. The $\mathrm{H}^{-}$ion beam enters at $84.0 \mathrm{~cm}$ and has an angle of $-333 \mathrm{mrad}$ with respect to the central orbit. Two injection septum magnets, $1.2 \mathrm{-m}$ long and $0.3-\mathrm{m}$ long, produce outward bends of $228 \mathrm{mrad}$ and $24 \mathrm{mrad}$, respectively. The quadrupole upstream of the stripper produces an additional outward bend and separates the $\mathrm{H}^{-}$ions from the circulating protons. The upstream $1.2-\mathrm{m}$ septum magnet has a field index of -20 to compensate for the defocusing effect of the quadrupole on negative ions. The phase-space distribution of the $\mathrm{H}^{-}$beam is approximated by a Gaussian distribution with standard deviation $\sigma$. The emittance is defined as $\varepsilon=6 \pi \sigma^{2} / \beta$, which contains $99 \%$ of the beam. According to this definition, the emittance of the $\mathrm{H}^{-}$beam is $6 \pi \mathrm{mm} \mathrm{mrad}$. The horizontal size of the injected beam shown in Figure II.2.2-2 is $\sqrt{6} \sigma$. 

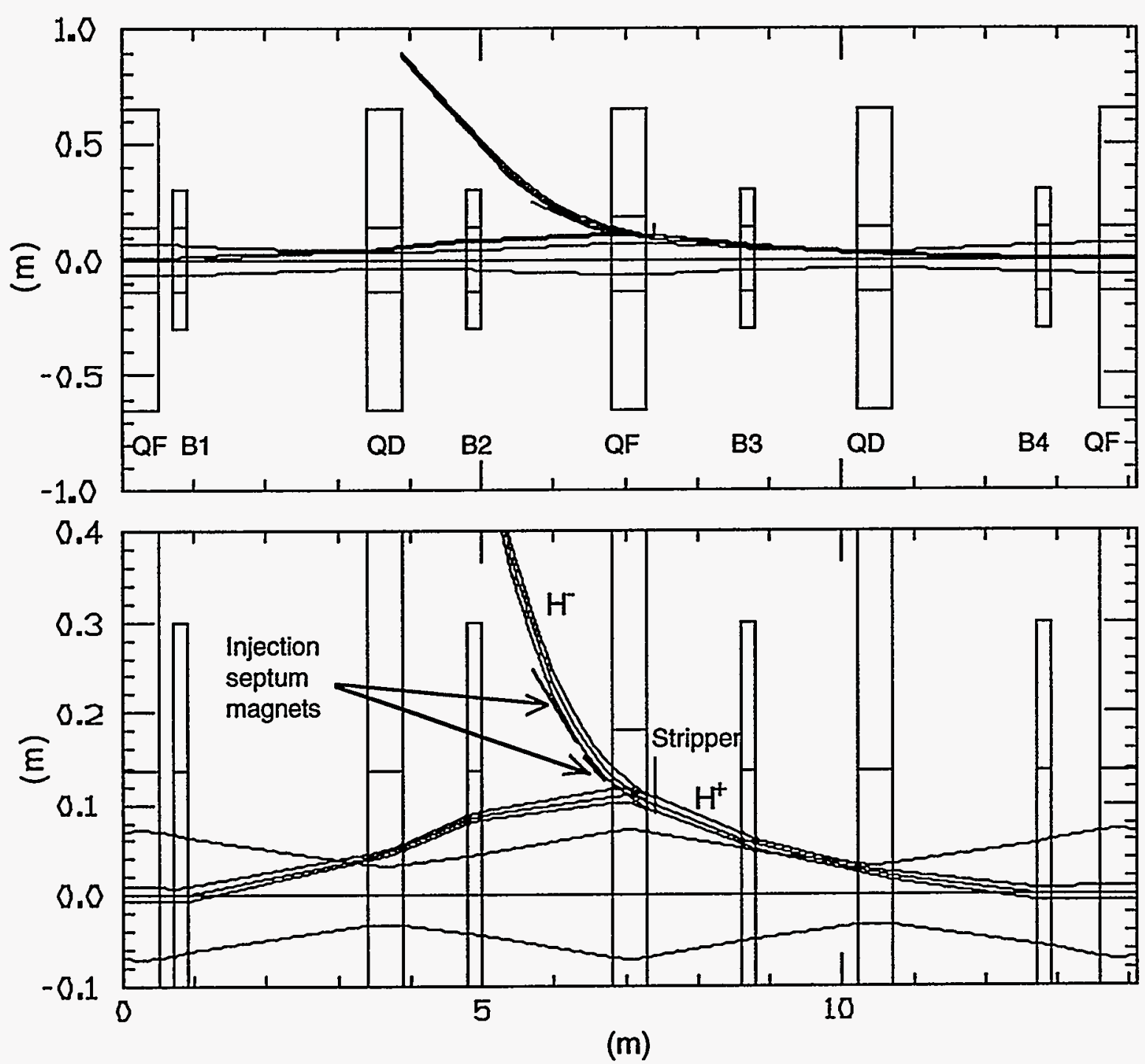

Figure I1.2.2-2 Injection Details in Two Scales. (The horizontal size of the circulating beam after filling is also shown.) 
The total phase space area occupied by the protons after injection is $375 \pi \mathrm{mm}$ mrad in each plane. Thus the horizontal and vertical sizes of the proton beam at the stripper location are $12.0 \mathrm{~cm}$ and $7.0 \mathrm{~cm}$, respectively. Figure II $2.2-2$ also shows the horizontal circulating beam size in the injection straight section. Figure II 2.2-3 shows the time dependence of the bends required at the four bumper magnets.

To produce amplitude-independent tune shifts, the protons are injected in such a manner as to satisfy the $\mathrm{K}-\mathrm{V}$ distribution function. ${ }^{3}$ This requires that the horizontal and vertical amplitudes, $A_{x}$ and $A_{y}$, lie on an ellipse, i.e.,

$$
\frac{A_{x}^{2}}{A_{x}^{2}(\max )}+\frac{A^{2} y}{A_{y}^{2}(\max )}=1 .
$$

At the stripper locations, $A_{x}(\max )$ and $A_{y}(\max )$ are $60 \mathrm{~mm}$ and $35 \mathrm{~mm}$, respectively.

One way to satisfy the $\mathrm{K}-\mathrm{V}$ distribution is to inject the protons so as to satisfy the following time relations:

and

$$
A_{x}(t)=A_{x}(\max ) \sqrt{\frac{t}{t_{f}}}
$$

$$
A_{y}(t)=A_{y}(\max ) \sqrt{\frac{t_{f}-t}{t_{f}}},
$$

where $\mathrm{t}_{\mathrm{f}}=500 \times 0.89=445 \mathrm{msec}$.

In the horizontal plane, the bumper magnets are timed to cause the bumped orbit to pull away from the injection position according to the relation

$$
X_{b}(t)=X_{0}-A_{x}(t)
$$

This timing sequence produces the horizontal phase space and density distributions shown in Figure II.2.2-4. The figure shows the locations of each of the 500 turns at the end of injection. The circles are 1-sigma contours of the incoming linac beam. 


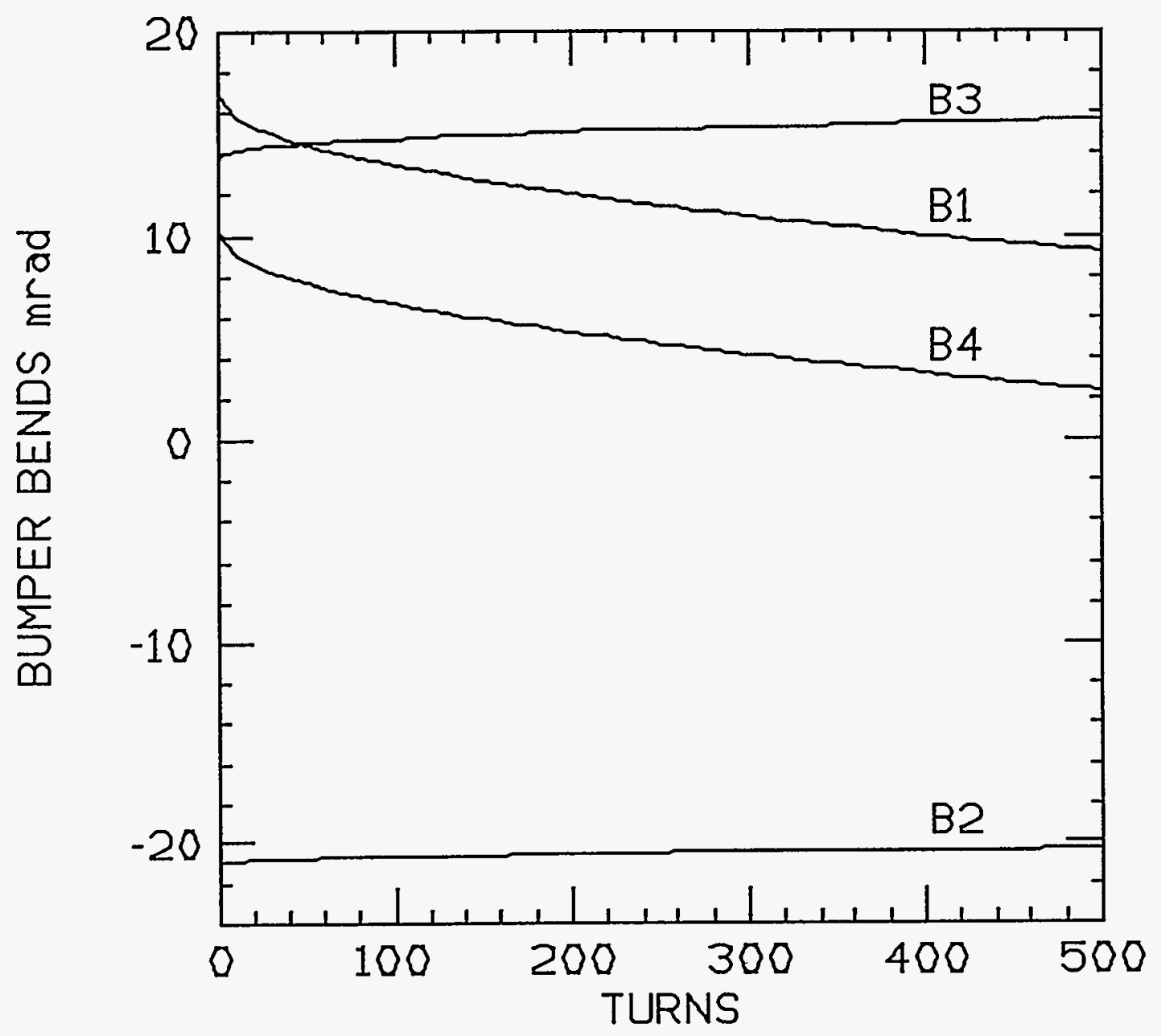

Figure II.2.2-3 Bending Angle of the Four Bumper Magnets as a Function of Injected Tum Number. 


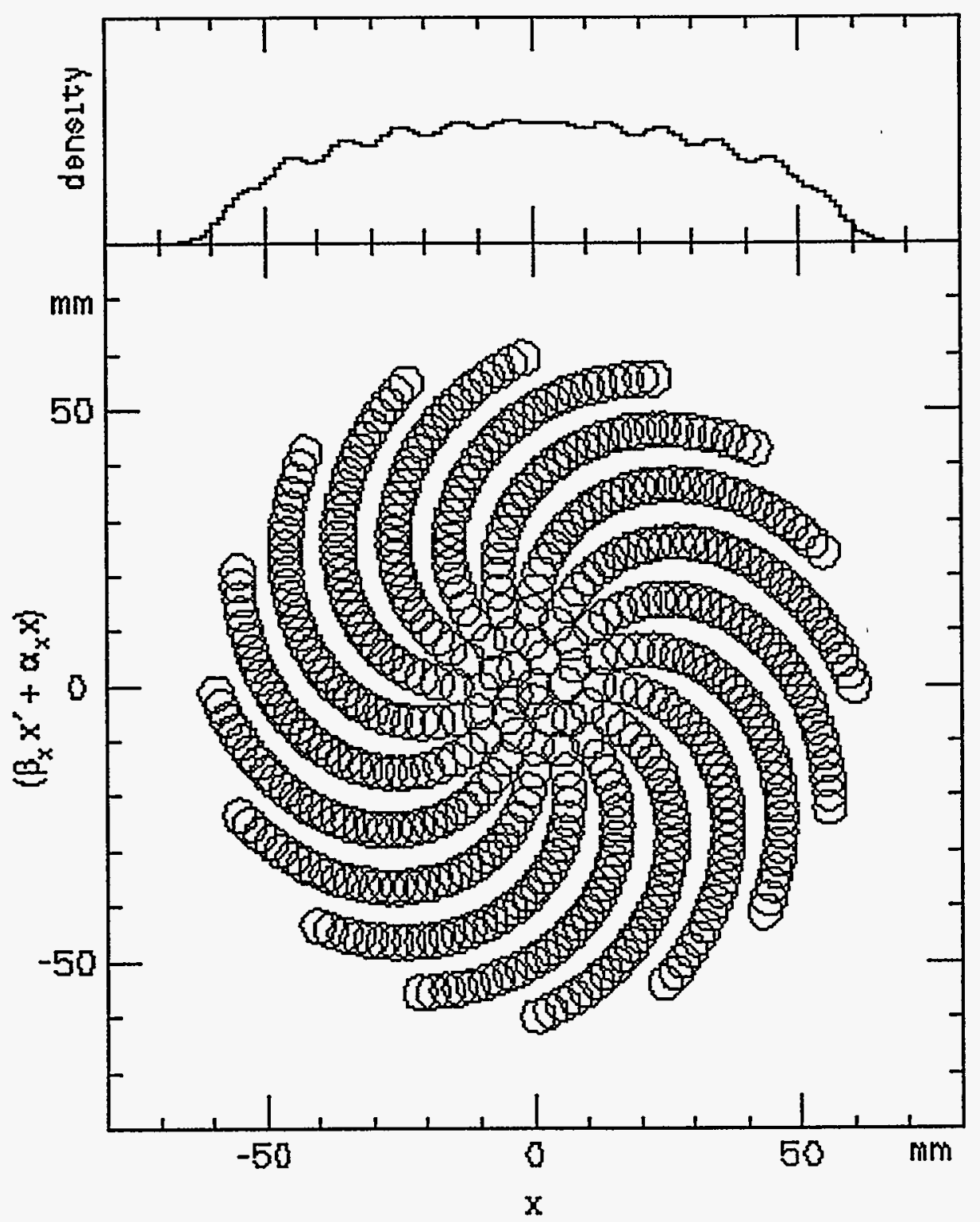

Figure II.2.2-4 Location of the 500 Turns, at the End of Injection, in the Normalized $\left(x, \beta_{x} x^{\prime}+\alpha_{x} x\right)$ Horizontal Phase Space at the Stripper

Location. (Circles are 1-sigma contours of the incoming linac beam. The horizontal density distribution is shown at the top.) 
Vertical phase space painting is achieved by using a vertical steering magnet placed $4.7 \mathrm{~m}$ upstream of the stripper in the $\mathrm{H}^{-}$injection line. Taking into account the intervening focusing elements, a kick of $\theta$ produces the following results at the stripper:

$$
\begin{gathered}
\mathrm{y}=4.48 \theta, \\
\mathrm{y}^{\prime}=-0.188 \theta, \\
\beta_{\mathrm{y}} \mathrm{y}^{\prime}+\alpha_{\mathrm{y}} \mathrm{y}=-3.92 \theta,
\end{gathered}
$$

and

$$
A_{y}(t)=5.95 \theta(t),
$$

where dimensions are in meters.

The timing sequence described above produces the final vertical phase space distribution and space density shown in Figure II.2.2-5. Figure II.2.2-6 shows the time dependence of $A_{x}$ and $A_{y}$.

The final spatial distribution of the 500 turns, after a minor adjustment of the horizontal and vertical tunes to eliminate undesirable Lissajous patterns that arise from integer tune ratios, is shown in Figure. II.2.2-7.

Assuming a stripping foil with full vertical aperture, the average number of passes per injected proton through the stripping foil is 16 . Actually, a foil with only a little more than half a vertical aperture is needed for the injection process described above. The rms scattering angle for passage through a $250-\mu \mathrm{g} / \mathrm{cm}^{2}$ foil is $0.026 \mathrm{mrad}$. The horizontal and vertical divergences for the injected beam are $\sigma_{x^{\prime}}=0.32 \mathrm{mrad}$ and $\sigma_{y^{\prime}}=0.55 \mathrm{mrad}$, respectively. The 16 foil passes for each turn produce only a $10 \%$ increase in the horizontal emittance and a $3 \%$ increase in the vertical emittance. Therefore, there is no need to use a half-aperture stripping foil.

Figure II.2.2-8 shows the equilibrium heating contours on the stripping foil caused by injection of the $\mathrm{H}^{-}$and multiple passes of the circulating protons. The temperatures are calculated assuming 500 injected turns, $2 \times 10^{11}$ protons per turn, a foil thickness of $250 \mu \mathrm{g} / \mathrm{cm}^{2}$, and a $30-\mathrm{Hz}$ repetition rate. The vertical movement of the injected $\mathrm{H}^{-}$beam and average of 16 foil passes for each injected proton produce a maximum equilibrium temperature of $700 \mathrm{~K}$. 


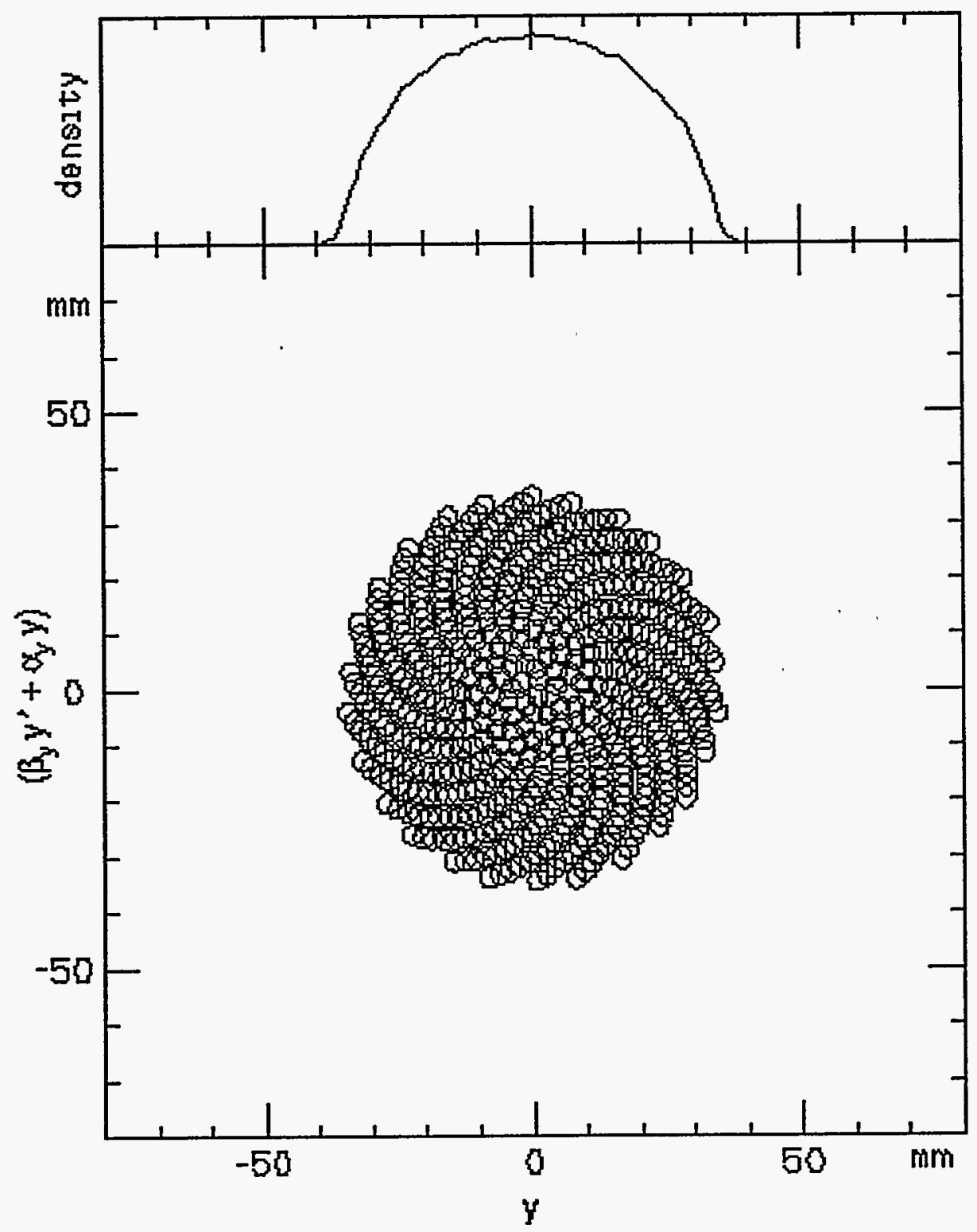

Figure 1I.2.2-5 Location of the 500 Turns, at the End of Injection, in the Normalized $\left(y, \beta_{y} y^{\prime}+\alpha_{y} y\right)$ Vertical Phase Space at the Stripper Location. (Circles are 1-sigma contours of the incoming linac beam. The vertical density distribution is shown at the top.) 


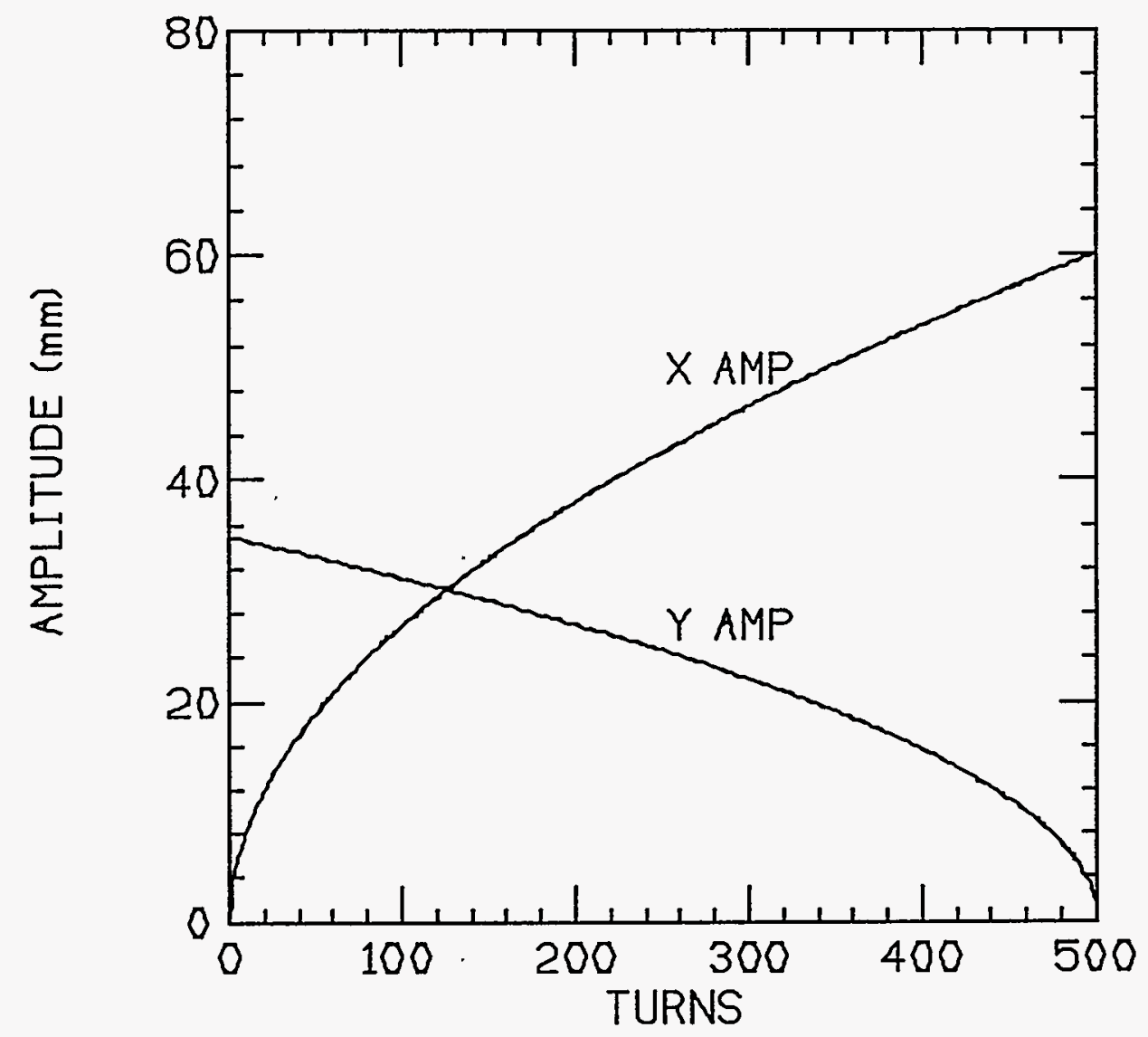

Figure II.2.2-6 Time Dependence of the Injected Horizontal and Vertical Amplitudes. 


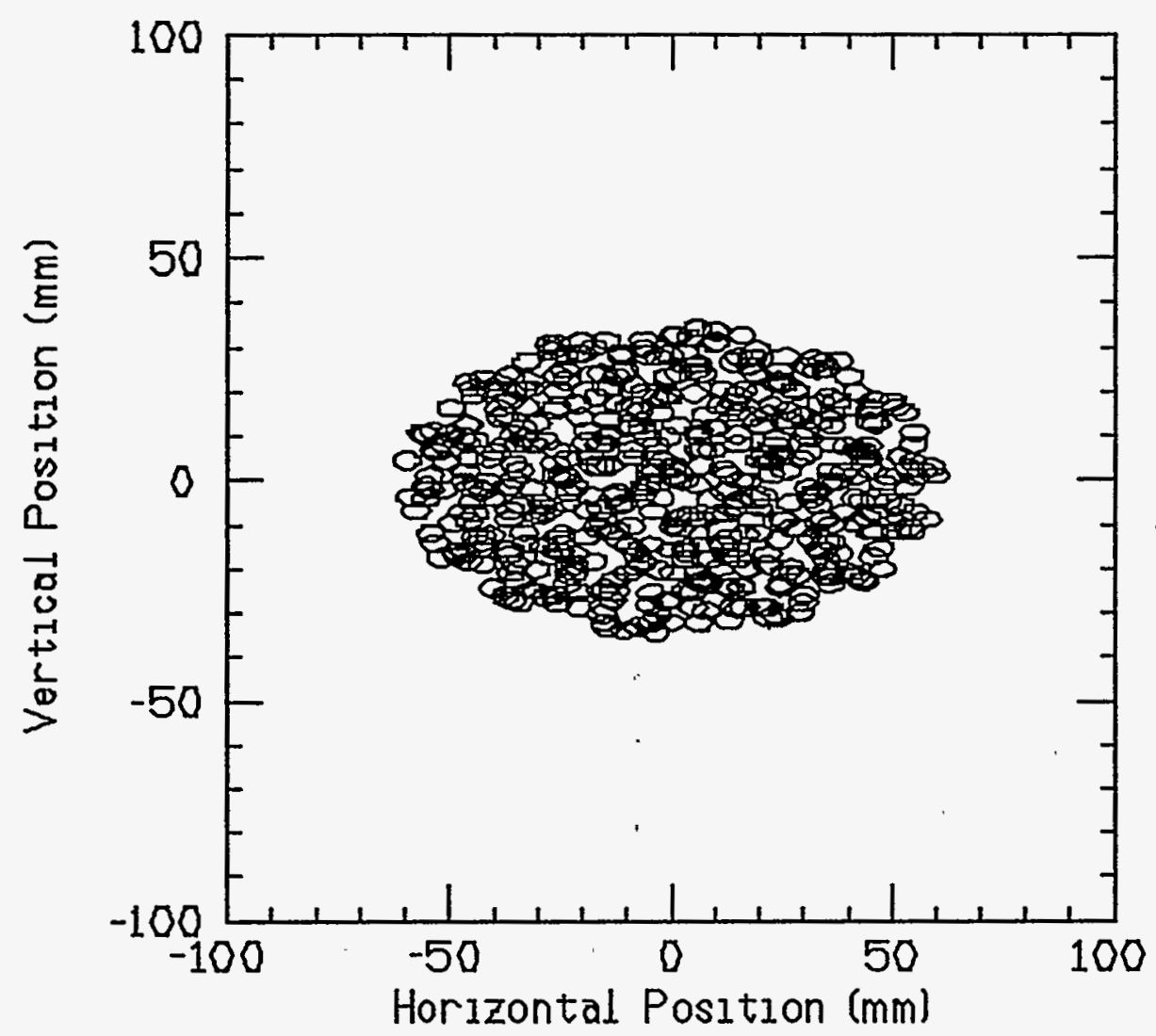

Figure II.2.2-7 Spatial Distribution of the 500 Turns at the End of Injection. 


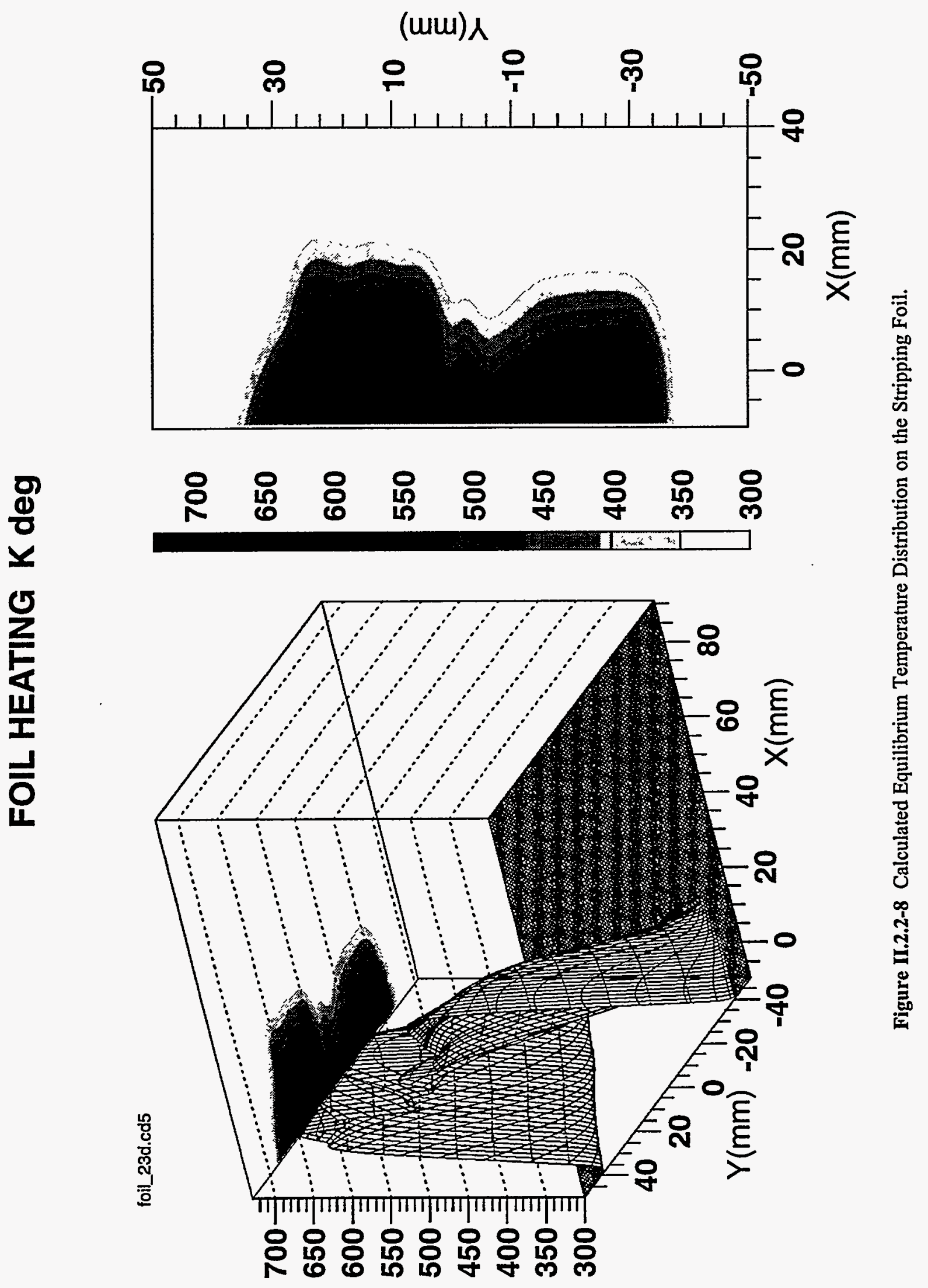




\subsection{2 $\mathrm{H}^{0}$ Catcher}

With a $250-\mu \mathrm{g} / \mathrm{cm}^{2}$ stripper foil, about $0.54 \%$ of the $500-\mu \mathrm{A} \mathrm{H} \mathrm{H}^{-}$beam emerges from the foil as partially stripped neutral hydrogen atoms, some of which are in the ground state and some of which are in an excited state. If these particles are allowed to enter a normal bending magnet field, they will become completely stripped and either hit the vacuum chamber wall or, if not lost, form a halo of large betatron oscillations around the normal proton beam. When the injection process and angle are as described in Section II.2.2.1, these neutrals can be collected before they enter strong magnetic fields.

Figure II.2.2-9 shows that the neutrals pass through the center of one quadrupole and enter the next quadrupole at $-11 \mathrm{~cm}$, where the field is $0.3 \mathrm{~T}$. This field over a length of $0.5 \mathrm{~m}$ is enough to strip all electrons with $n \geq 5$, or about $20 \%$ of the $\mathrm{H}^{0}$ beam. ${ }^{10}$ The catcher, therefore, is placed upstream of this quadrupole. 


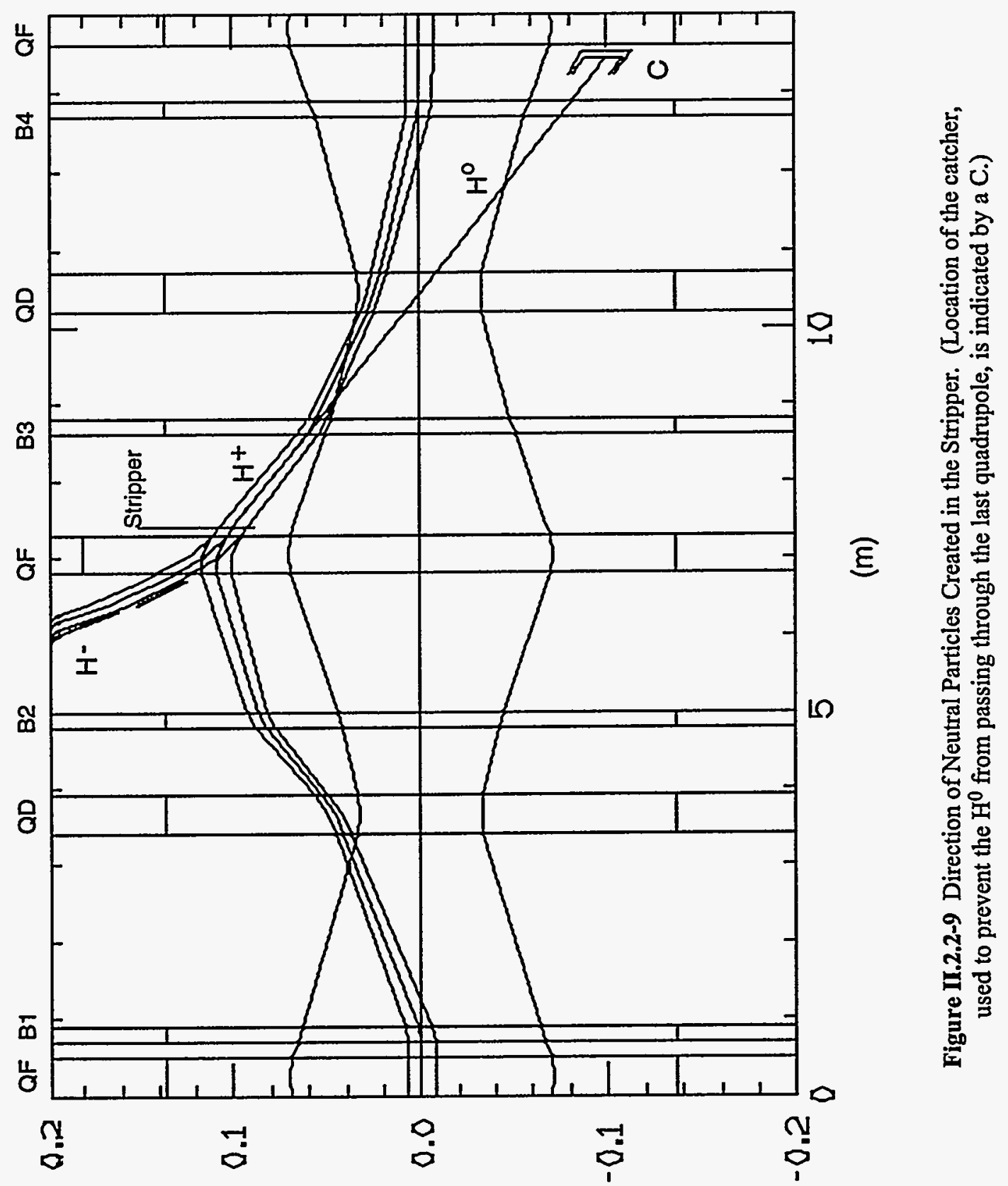

(ur) 


\subsection{LOW-ENERGY TRANSFER LINE (LET)}

The LET transports the 400-MeV beam from the linac to the RCS and matches the optics between the two accelerators. Figure II.2.3-1 shows the IPNS Upgrade accelerator layout, including the linac and LET line.

It is essential to match the beam optics between the linac and the RCS to obtain the best possible performance. Matching is performed from the downstream end of the RCS focusing quadrupole (F-quad), which combines the trajectories of the $\mathrm{H}^{-}$and $\mathrm{H}^{+}$beams. This RCS F-quad functions as a defocusing quadrupole (D-quad) for the incoming $\mathrm{H}^{-}$ions because of their opposite polarity.

An advantage of this defocusing action by the RCS quadrupole is that the incoming beam receives an extra kick; therefore, the LET has a large angular separation from the RCS in the interface region between the RCS and the LET. A drawback of having an effective D-quad is that the horizontal $\beta$-function is large. The $\beta$-function can be reduced by introducing a magnetic field gradient in the injection septum magnet.

The 157.36-m-long LET has three important regions: (1) Region-A is a $72^{\circ}$ horizontal bend near the RCS, (2) Region-B is a $3.05 \mathrm{~m}$ vertical translation from the linac elevation to the RCS elevation, and (3) Region-C is a $90^{\circ}$ horizontal bend near the linac. The $\beta$-function matching is performed for each of above three regions sequentially, and the dispersion function is suppressed at the ends of each horizontal bend and the vertical displacement. Figure II.2.3-2 shows the layout of the LET; the three regions are indicated. As the figure shows, the three regions are connected by a string of FODO cells.

The lattice functions of the $72^{\circ}$ bend section are shown in Figure II.2.3-3. The first element, QD, shown in the figure is the F-quad of the RCS, which defocuses the $\mathrm{H}^{-}$beam. The S1 septum has a field gradient of $n=-20$ to compensate for the defocusing effect of the QD. The maximum value of the dispersion function along this segment of the line is $5 \mathrm{~m}$, and the effect is shown in the envelope functions in Figure II.2.3-4. A momentum-tail scraper and a wireseptum halo catcher can be placed in this region to take advantage of the large dispersion function.

The set of Twiss parameters from Region-A is back-tracked to Region-B by using the FODO cell optics.

Figure II.2.3-5 shows the beam optics in Region-B. The beam elevation change of $3.05 \mathrm{~m}$ is accomplished by two bending magnets. The phase advance between two bending magnets is required to be $360^{\circ}$ to suppress the dispersion introduced by the dipoles. Also note in the figure that there are five matching quadrupoles preceding Dipole B. 


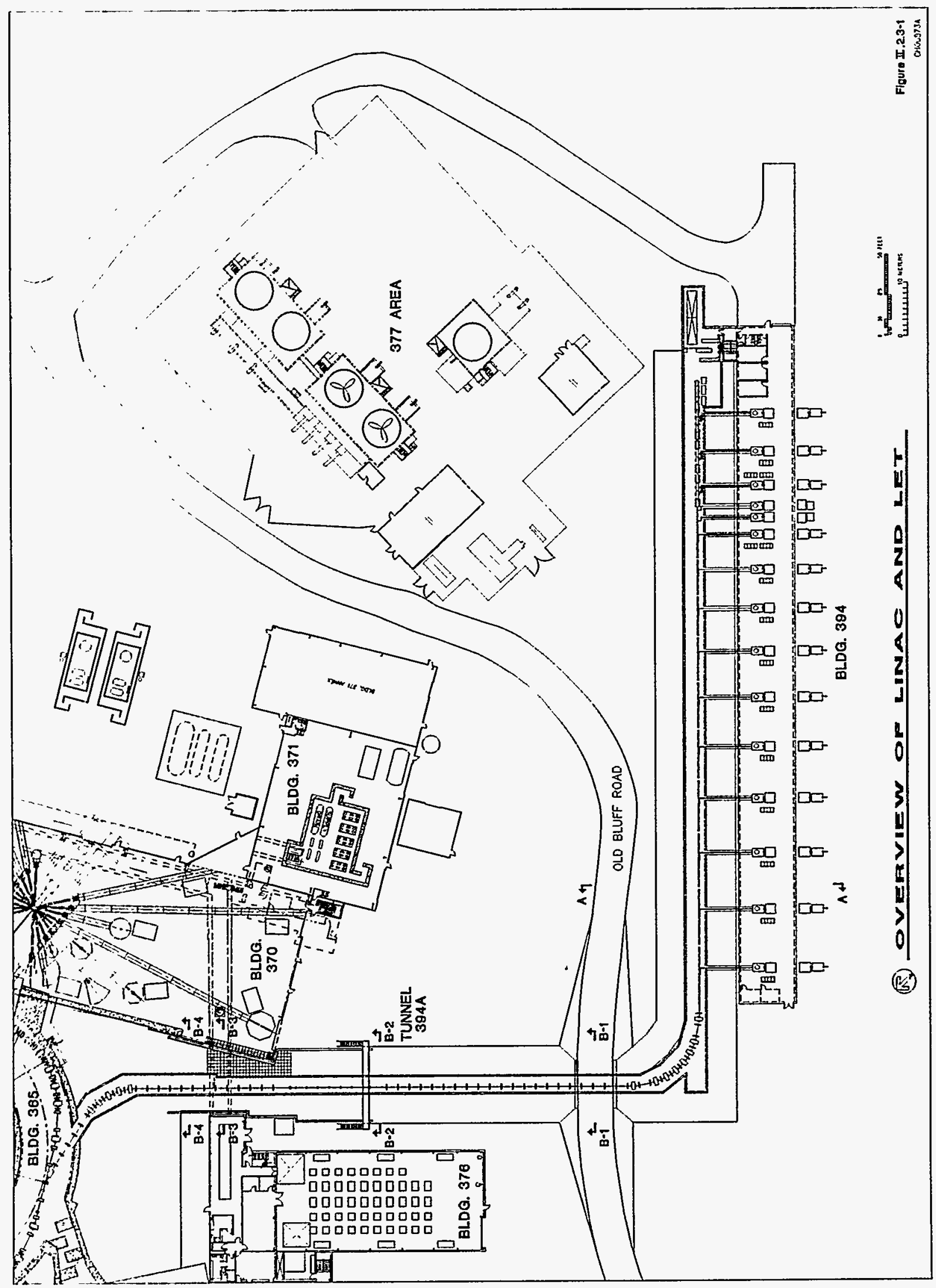




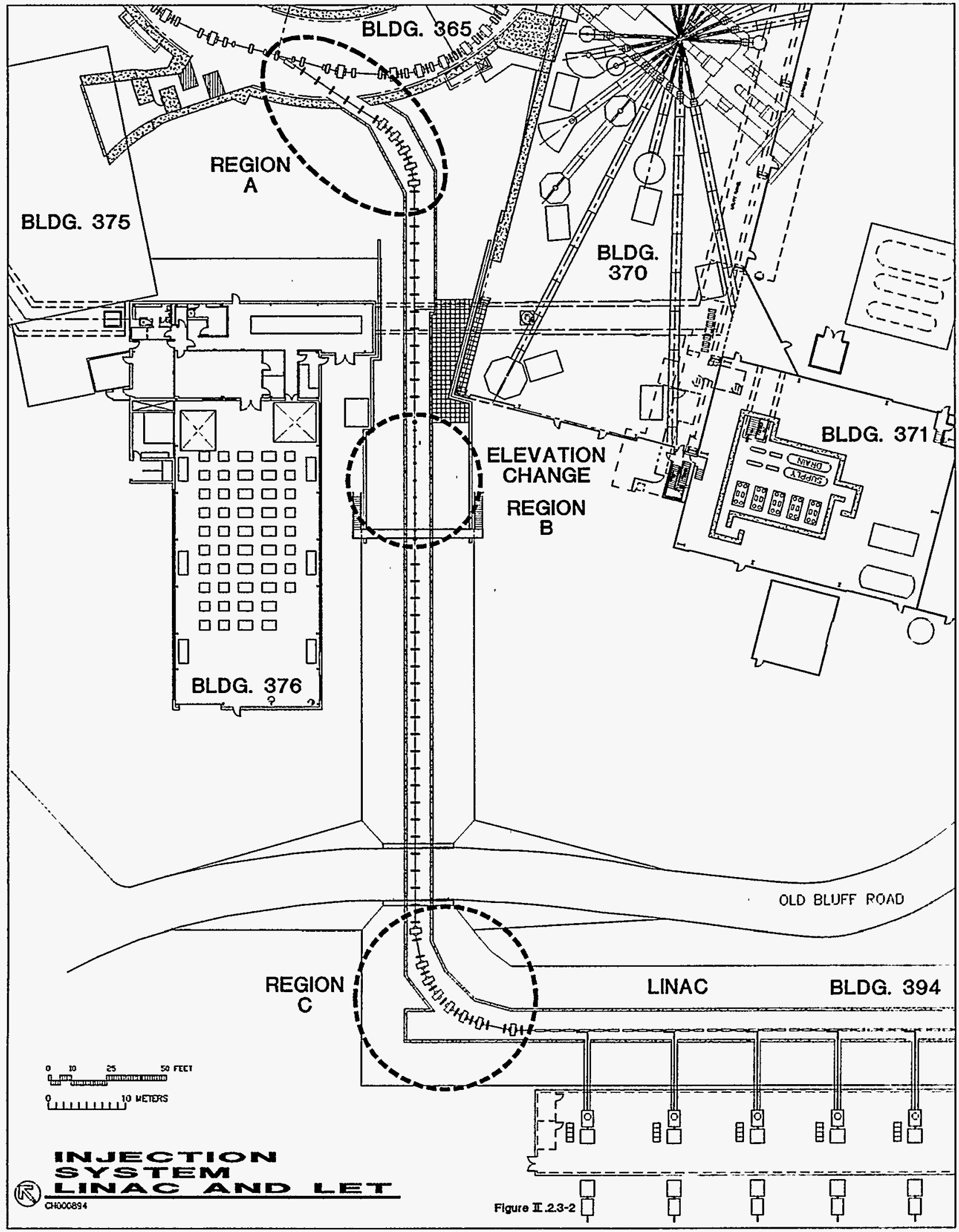


INJACHDECS

Beta $X$, Beta $Y$, Eta $X$ for 1 Porlod

28 Mar 1995

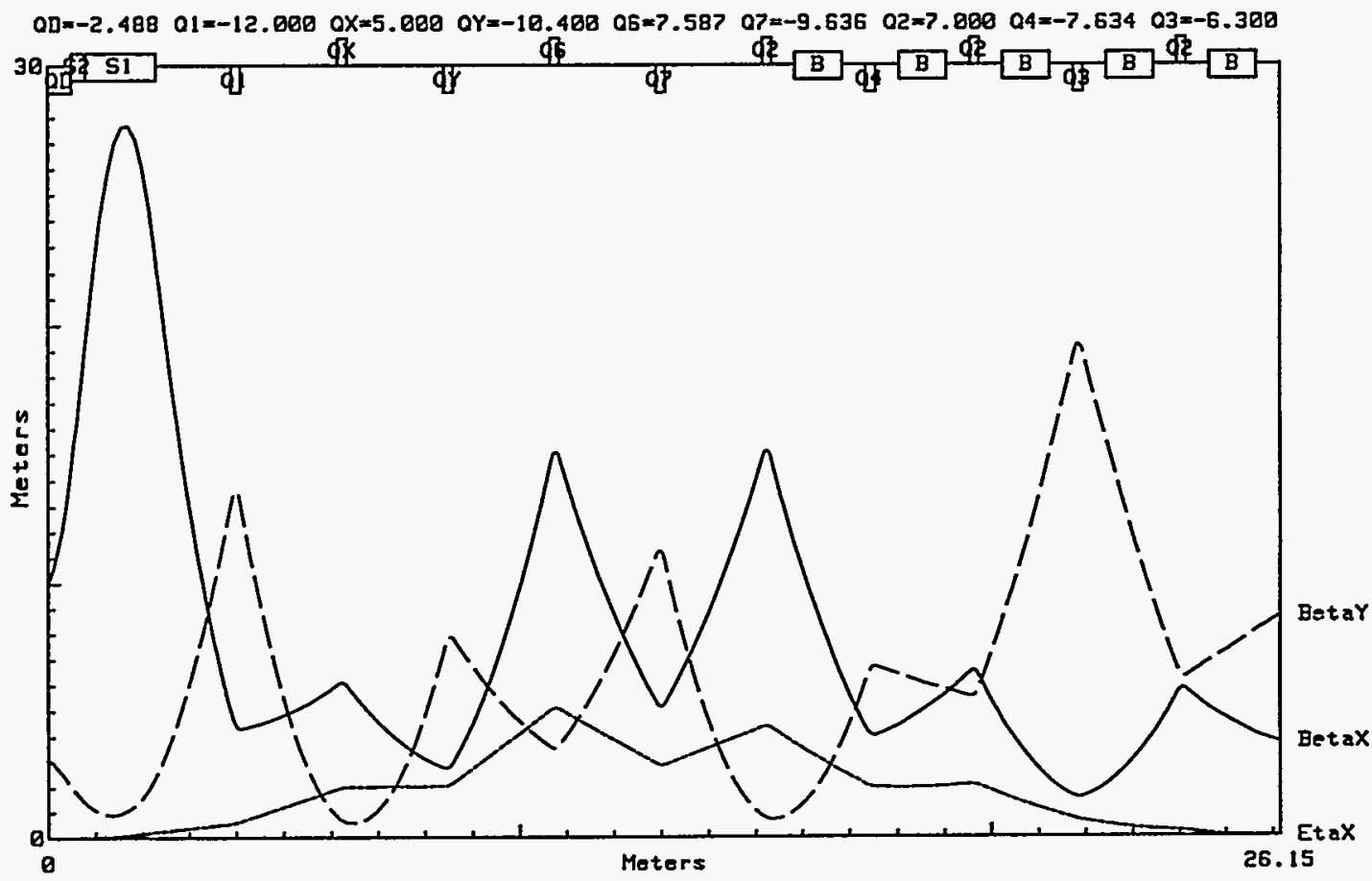

Figure II.2.3-3 Lattice Functions for the $72^{\circ}$ Bend Section of the LET (Region-A). 
INJACHDEC9

$X$ and $Y$ Envelopes for 1 Period

28 Mar 1995

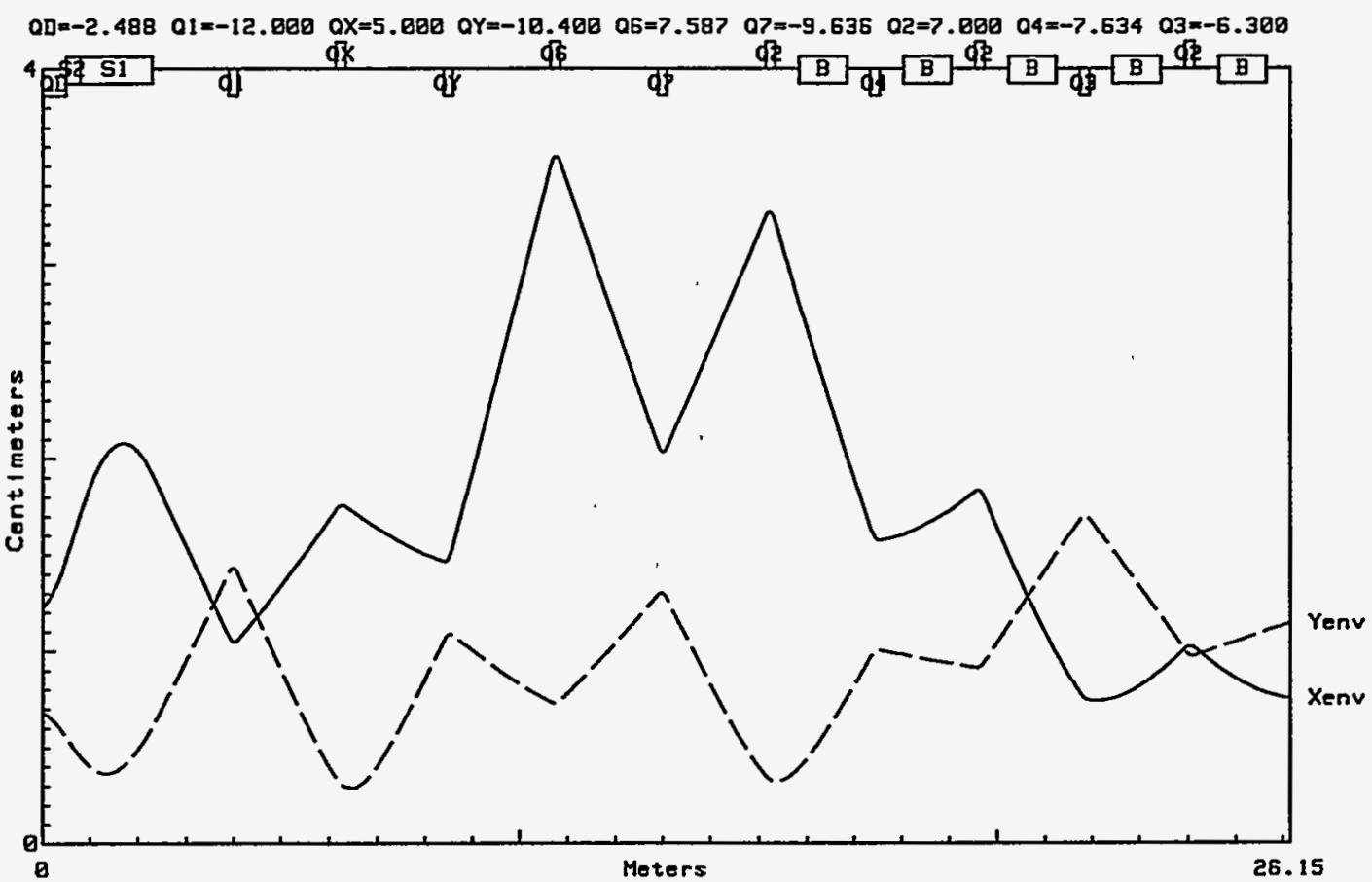

Figure II.2.3-4 Envelope Functions for the $72^{\circ}$ Bend Section of the LET (Region-A). (The solid line is the horizontal envelope, and the dashed line is the vertical envelope.) 


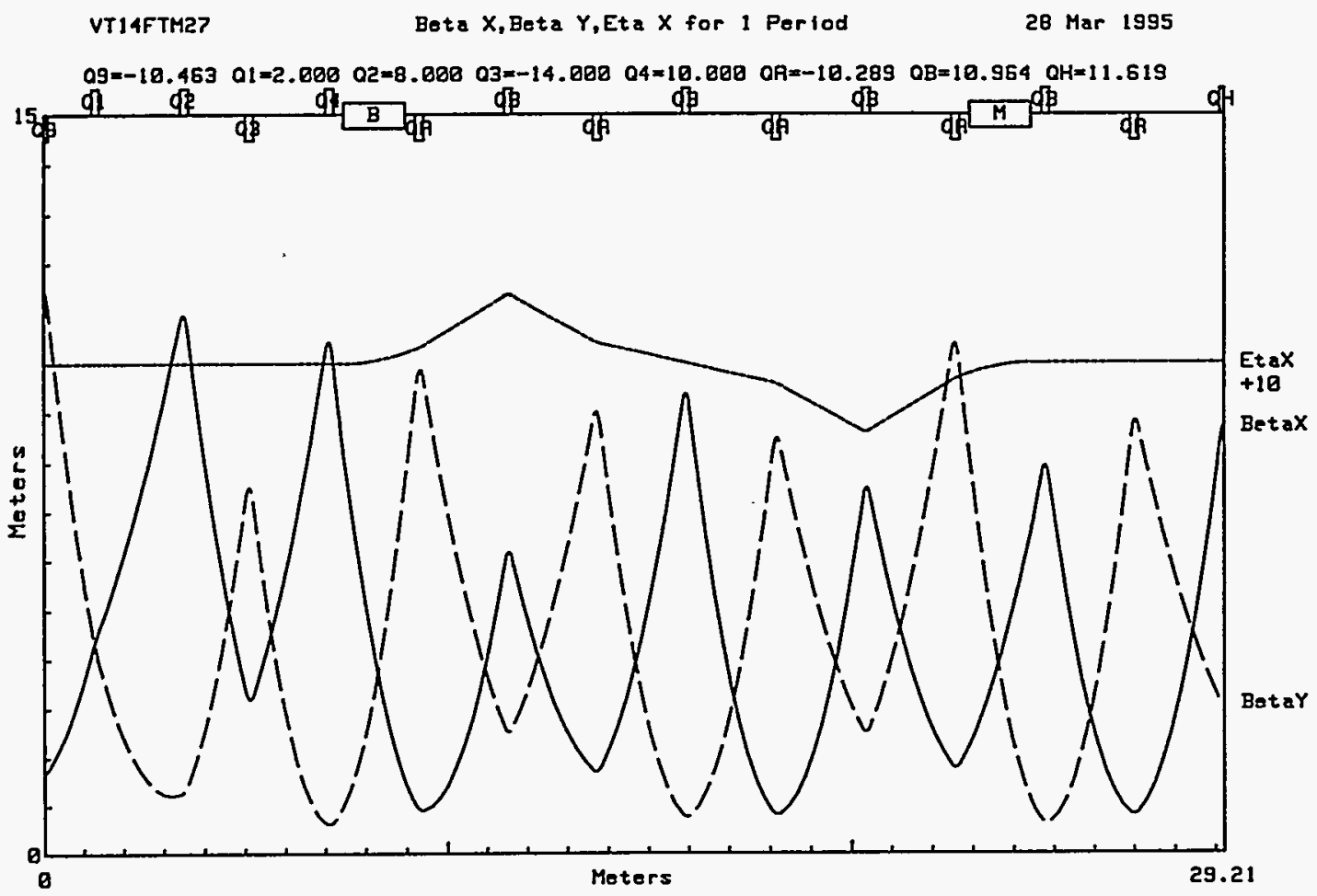

Figure II.2.3-5 Lattice Functions for Region-B. (The $\eta$-function is displaced by $10 \mathrm{~m}$ for clarity. Note that in this figure, $x$ refers to the vertical direction, and $y$ refers to the horizontal direction.) 
A long string of FODO cells between regions $\mathrm{B}$ and $\mathrm{C}$ facilitates matching between those two regions.

The $90^{\circ}$ bend of the linac beam in Region-C is accomplished using five FBDB cells. Each cell has a phase advance of $90^{\circ}$. An achromatic bend is obtained by removing a dipole from the next to the last half-cell at each end, as shown in Figure II.2.3-6. The figure also shows the lattice functions of the bend.

In this section, we have described the optics matching through the LET, particularly the dispersion matching. Matching between the linac and the LET has not yet been performed, but we do not expect any difficulty in the matching. 


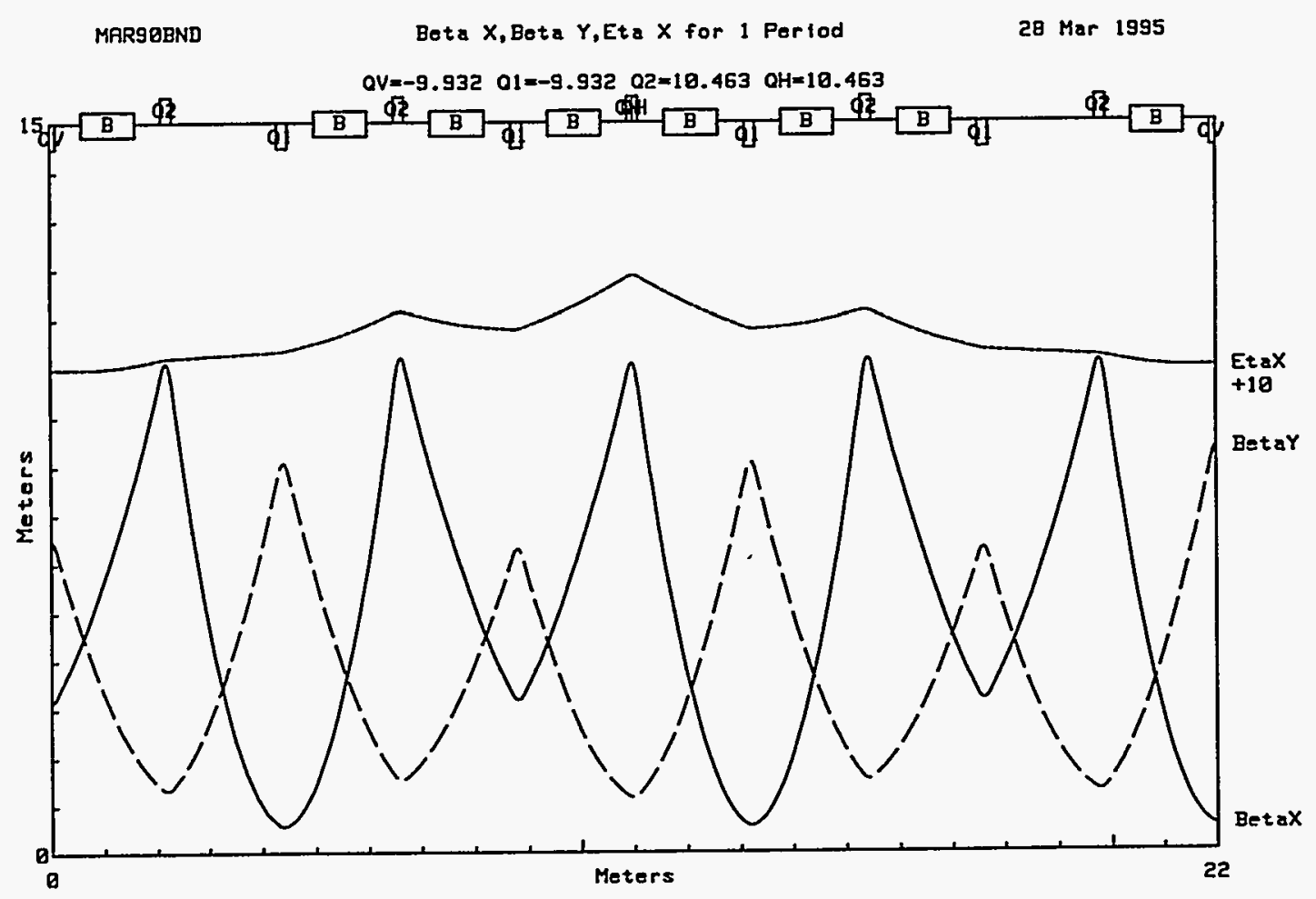

Figure II.2.3-6 Lattice Functions for Region-C. (The $\eta$-function is displaced by $10 \mathrm{~m}$ for clarity.) 


\subsection{BEAM EXTRACTION}

Extraction kickers are placed in the dispersion suppressor cell where a dipole is missing, and the extraction septum magnets are placed at $90^{\circ}$ phase advance relative to the kickers. The physical layout of the two extraction channels and their HETs is shown in Figure II.2.4-1.

The extraction trajectory, the associated horizontal envelope of the damped beam, and the BSC envelope of the injected beam are shown in Figure II.2.4-2. Since the extracted beam has to pass through an F-quad as shown in the figure, a short septum magnet $S 1$ is placed just upstream of the F-quad magnet to compensate for the inward bending by the quadrupole.

The extraction process starts by moving the $2-\mathrm{GeV}$ beam outward against the edge of the acceptance area inside the F-quad just upstream of the extraction kicker. This is done with a closed orbit bump around each extraction point. The horizontal displacement of this bump from the central orbit is $43.8 \mathrm{~mm}$. This closed-orbit bump was introduced to reduce the required kicker strength. The kicker produces an integrated bend of $14 \mathrm{mrad}$. This places the beam at a radial position of $134 \mathrm{~mm}$ from the central orbit at the first septum. The beam then passes through an F-quad in the RCS lattice and enters the last two septa. The three septum magnets produce an integrated bend of $22.4^{\circ}$. This bend places the inside edge of the beam at a radial distance of $690 \mathrm{~mm}$ from the central orbit adjacent to the leading edge of the downstream quadrupole in the RCS lattice. This allows the beam to clear the quadrupole magnet that has a half-width of $664 \mathrm{~mm}$.

The extraction kicker system is segmented into 3 independently powered kicker sections assembled around a shared ceramic vacuum chamber. This segmentation is used to reduce the impedance of each kicker to a manageable level. The aperture of each section is designed to accommodate the BSC of the injected beam and allows space for passage of the extracted beam. The field strength, effective length, and aperture dimensions were derived for each section through an iterative process while taking into account peak-current and voltage limits.

The required parameters for the three kicker sections and septum magnets are listed in Table II.2.4-1. A detailed description of the kicker magnets is given in Section II.3.4, and the septum magnets are described in Section II.3.5. 


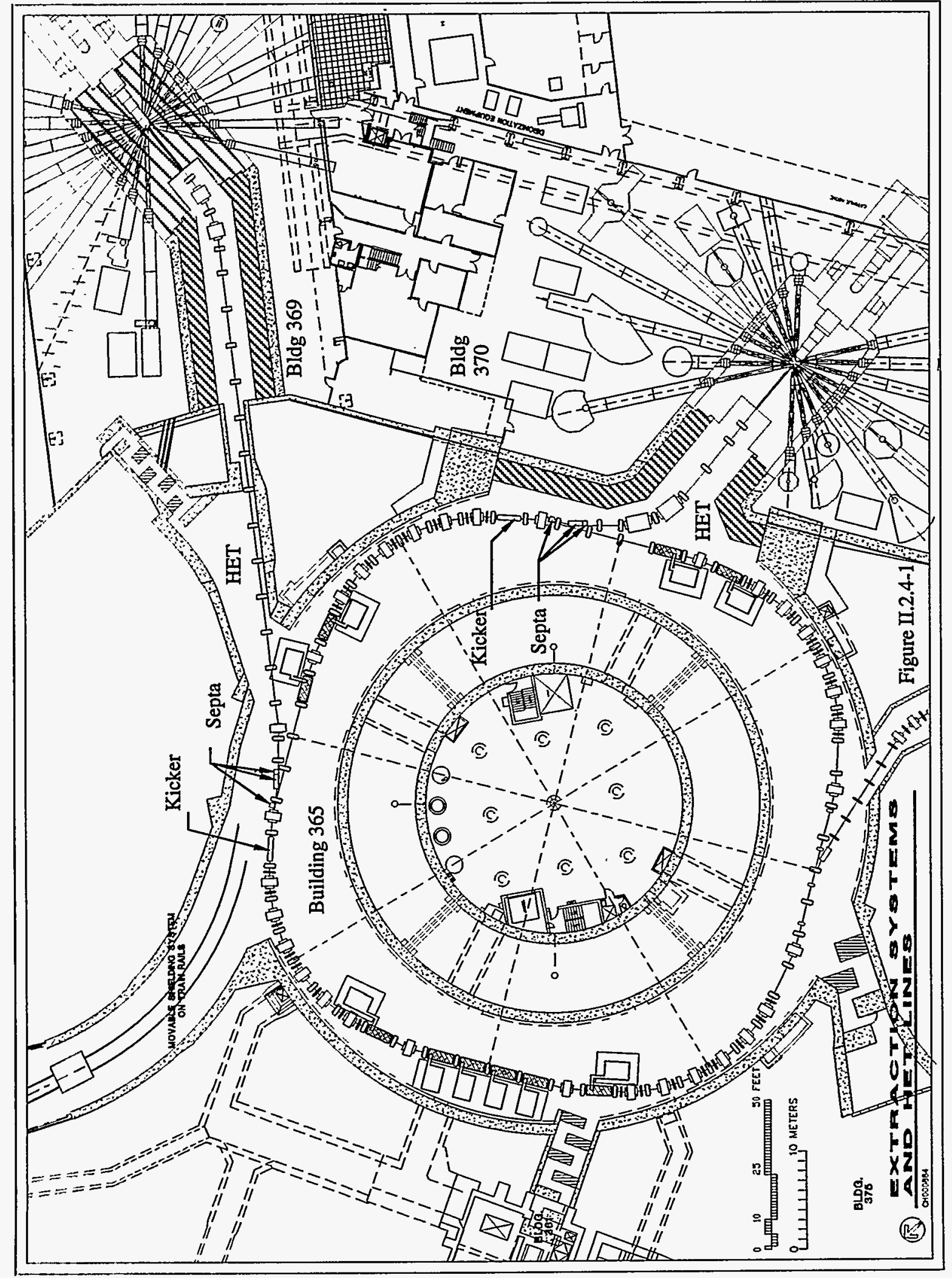




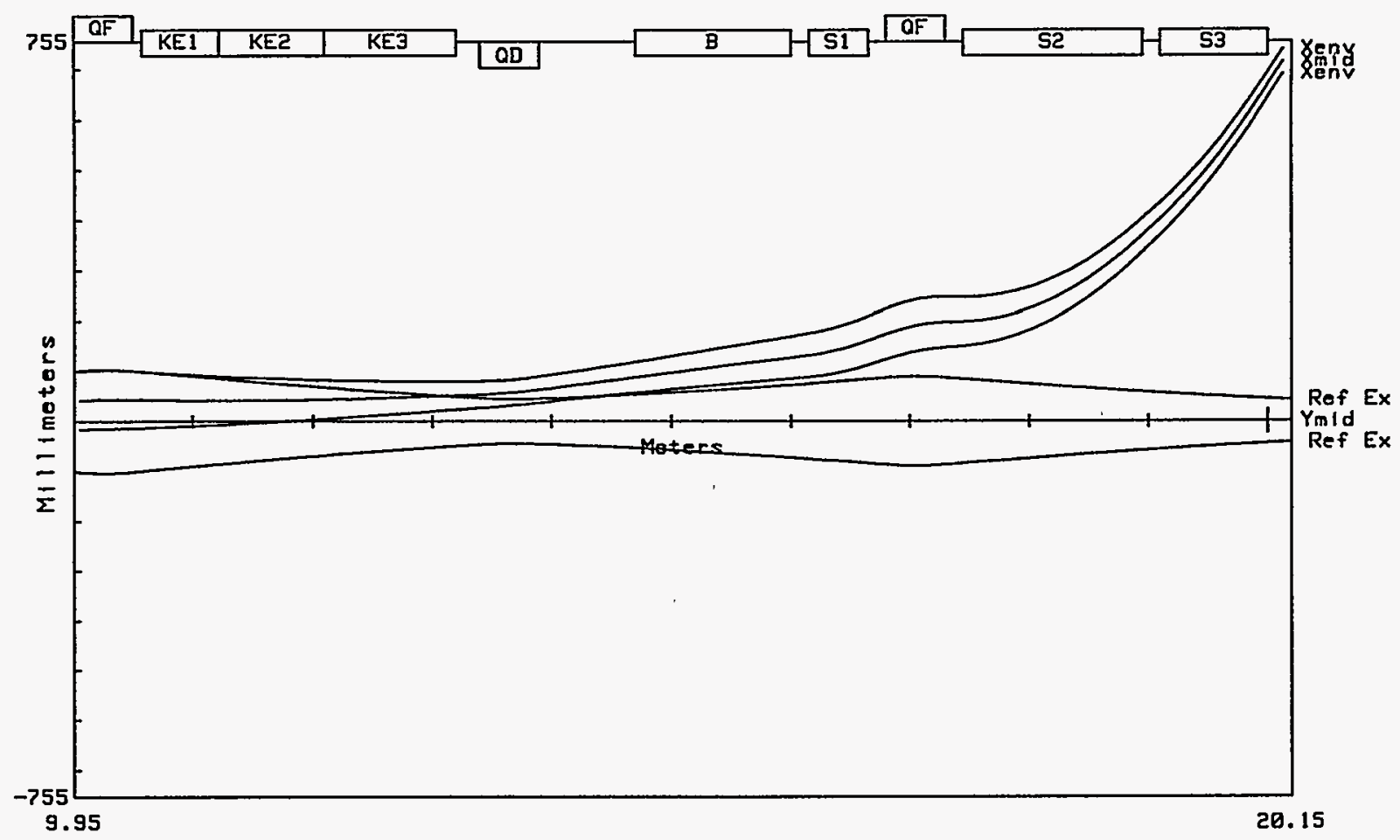

Figure II.2.4-2 Extraction Trajectory from the Kicker through the Septum Magnet. (The horizontal envelopes of the injected and extracted beam are shown.) 
Table II.2.4-1 Extraction Kicker and Septum Magnet Parameters

\begin{tabular}{lcccccc}
\hline $\begin{array}{c}\text { Magnet } \\
\text { Type }\end{array}$ & $\begin{array}{c}\text { Bend Angle } \\
(\mathrm{mrad})\end{array}$ & $\begin{array}{c}\text { Effective } \\
\text { Length } \\
(\mathrm{m})\end{array}$ & $\begin{array}{c}\text { Strength } \\
2-\mathrm{GeV} \\
(\mathrm{T})\end{array}$ & $\begin{array}{c}\text { Aperture } \\
\text { Height } \\
(\mathrm{mm})\end{array}$ & $\begin{array}{c}\text { Aperture } \\
\text { Width } \\
(\mathrm{mm})\end{array}$ & $\begin{array}{c}\text { Septum } \\
\text { Thickness } \\
(\mathrm{mm})\end{array}$ \\
\hline & & & & & & \\
Kicker A & 4.37 & 0.65 & 0.0618 & 107.4 & 195 & - \\
Kicker B & 4.79 & 0.87 & 0.0512 & 134.2 & 170 & - \\
Kicker C & 4.84 & 1.105 & 0.0408 & 175 & 145 & - \\
Septum C & 55.1 & 0.5 & 1.02 & 70 & 122 & 13 \\
Septum D & 210 & 1.5 & 1.3 & 84 & 97 & 66 \\
Septum E & 126 & 0.9 & 1.3 & 103 & 65 & 309 \\
\hline
\end{tabular}

a Septum thickness in Table II.2.4-1 is the allowable minimum thickness of the septum. 


\subsection{HIGH-ENERGY TRANSFER LINE (HET)}

Two HET lines transport the beam to the $10-\mathrm{Hz}$ Target in Building 369 and to the $30-\mathrm{Hz}$ Target in Building 370. These HET lines are shown in Figure II.2.4-1. Both HET lines have $45^{\circ}$ bends near the targets in order to prevent backstreaming neutrons from irradiating the transport enclosures. Bending of the beam near the targets makes dispersion suppression difficult, and it is not planned.

The beam envelope functions for the both HET lines are shown in Figures II.2.5-1 and II.2.5-2. To achieve the required 5-cm-radius beam size at the target containing $100 \%$ of the beam, the quadrupoles near the targets must have large apertures and must be made of radiationhard materials. 


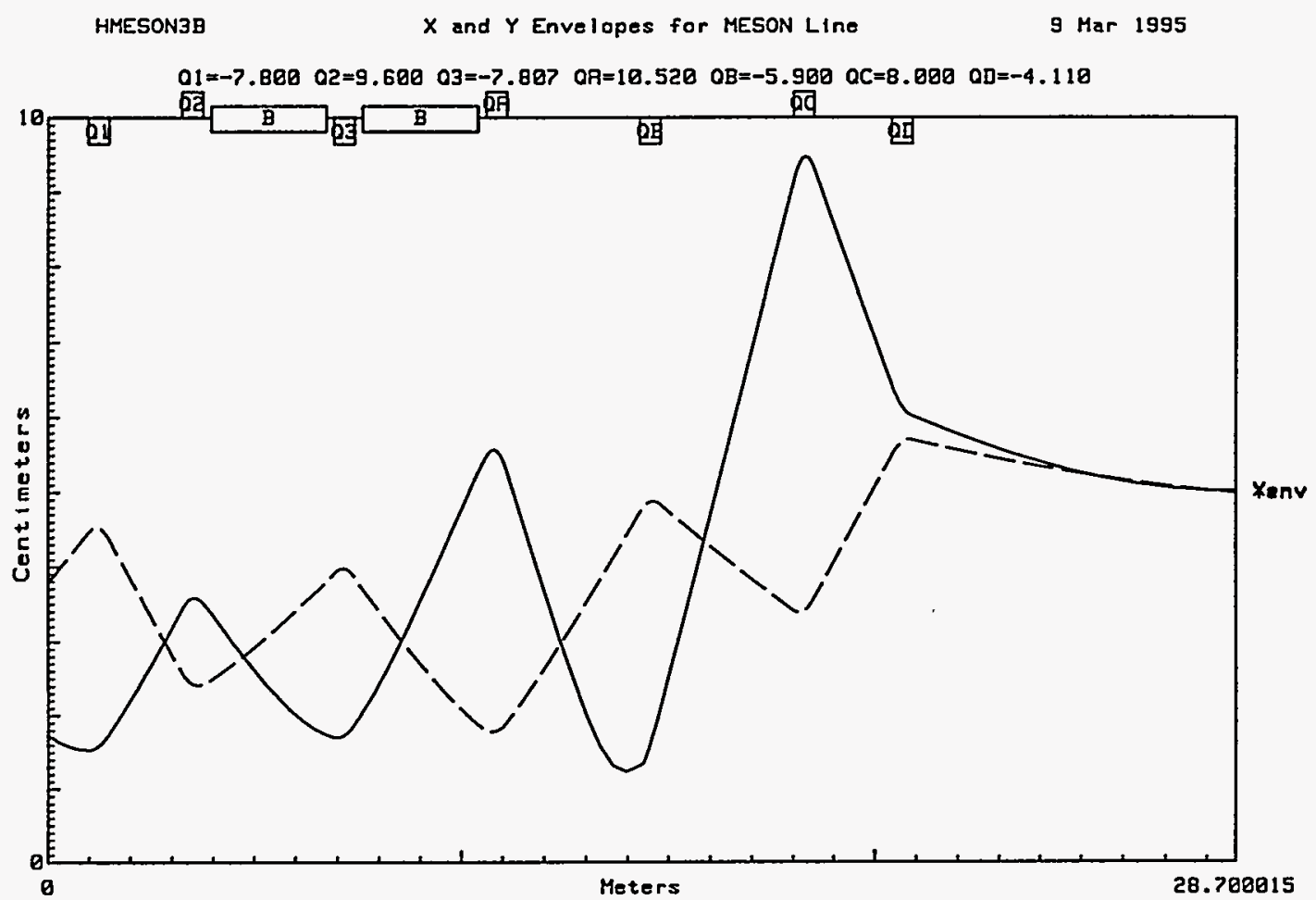

Figure II.2.5-1 Beam Envelope for the 30-Hz Target HET Line. 
EPB1F

$X$ and $Y$ Envelopes for EPBI Line

15 Mar 1995

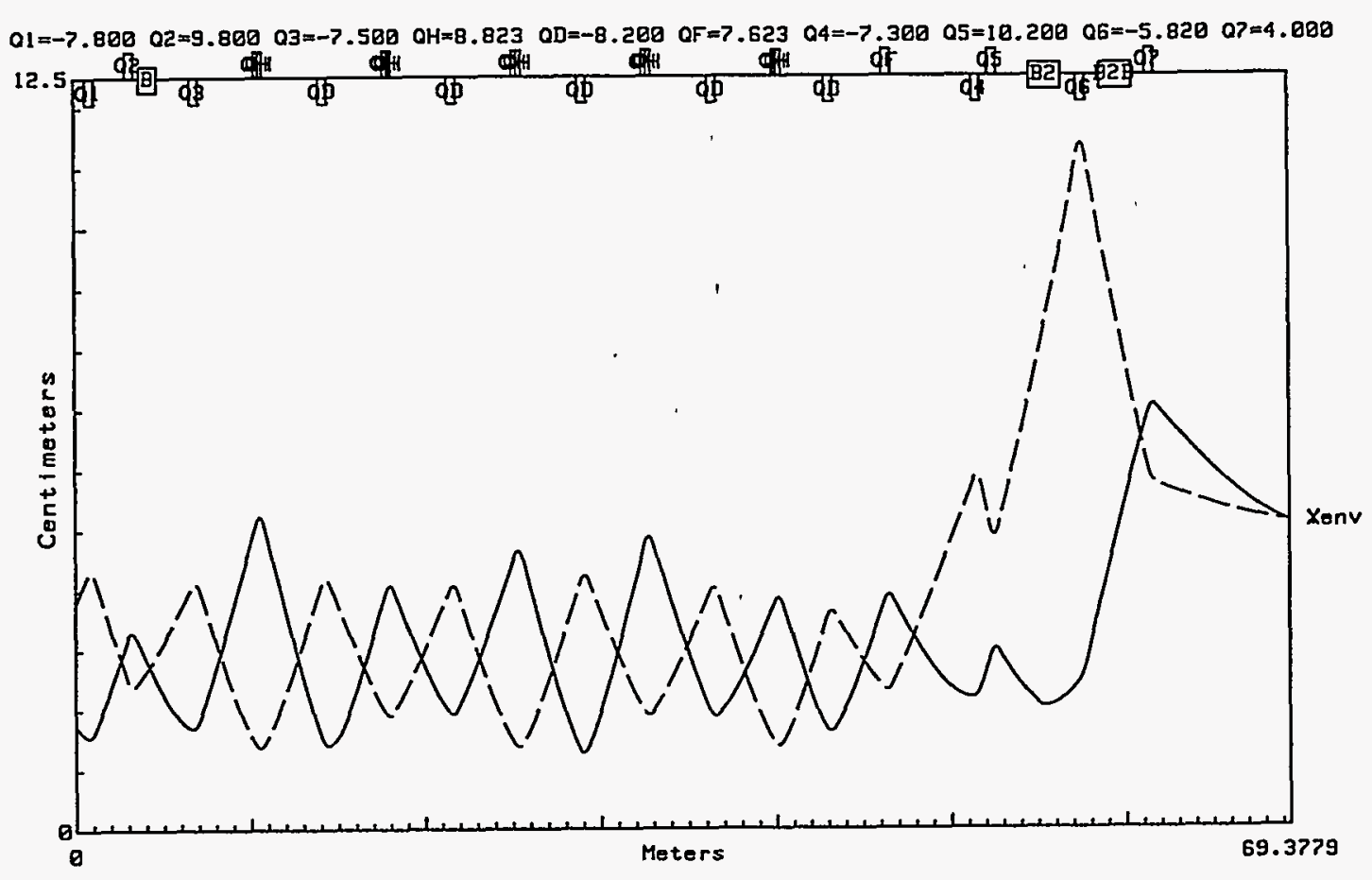

Figure 11.2.5-2 Beam Envelope for the 10-Hz Target HET Line. 


\subsection{REFERENCES}

1. Report of the ISIS Project Group, Rutherford Laboratory (November 1986).

2. A. Hofmann and F. Pederson, "Bunches with local elliptic energy distributions," IEEE Trans. Nucl. Sci. NS-26 (3), 3526 (1979).

3. I.M. Kapchinskij and V.V. Vladimirskij, "Limitations of Proton Beam Current in a Strong Focusing Linear Accelerator Associated with the Beam Space Charge, " in Proceedings of the CERN Symposium on High Energy Accelerators (1959).

4. T. Suzuki, "Synchro-betatron resonances driven by dispersion in $\mathrm{Rf}$ cavities," in Particle Accelerators Vol. 18 (1985).

5. K.C. Harkay, Study of Synchro-betatron Coupling in the IPNS-II RCS, Argonne National Laboratory Report No. NSA-95-3 (March 1995).

6. H. Grote and F.C. Iselin, The MAD Program, User's Reference Manual, CERN Report No. CERN/SL/90-13 (AP), Rev. 4 (1994).

7. H. Friedsam, "A New Accelerator Alignment Concept Using Laser Trackers," in Proceedings of the European Particle Conference 3, 2570-2572 (1994).

8. B. Autin and Y. Marti, Closed Orbit Correction of Alternating Gradient Machines Using a Small Number of Magnets, CERN Report No. CERN/ISR-MA/73-17 (1973).

9. A. Wrulich, RACETRACK: A Computer Code for the Simulation of Nonlinear Particle Motion in Accelerators, Report No. DESY 84-026 (1984).

10. Y.C. Chae, Study of Field Ionization in $\mathrm{H}^{-}$Charge Exchange Injection, Argonne National Laboratory Report No. NSA-94-2 (April 1994). 


\subsection{SYNCHROTRON MAGNETS}

The synchrotron ring lattice contains 128 main magnets consisting of 32 dipoles, 56 quadrupoles, and 40 sextupoles. An additional 56 correction magnets will serve to adjust the dipole field. The primary design features of the ring magnets are described in this section. The required field strengths and aperture dimensions for the ring magnets are listed in Table II.3.1-1.

All magnets are designed for a nominal beam energy of $2.0 \mathrm{GeV}$ but are capable of handling a maximum value of $2.2 \mathrm{GeV}$. Two-dimensional static magnetic field calculations at $2.2 \mathrm{GeV}$ were made with OPERA-2 $\mathrm{d}^{1}$ for the main magnets. Results of these calculations enable core saturation and the associated low magnetic efficiencies to be avoided. They also indicate that the higher harmonic coefficients of the fields can be reduced to appropriate levels. The design goal of the relative harmonic coefficients was $6.0 \times 10^{-4}$ for points within the calculated beam envelopes previously defined in Section II.2.1.3. Detailed magnetic calculations were performed at $2.2 \mathrm{GeV}$, but magnetic field parameters were also studied as a function of magnetic field at lower values. The results satisfy design goals throughout the entire operating range of the machine. Eddy current effects are not expected to change the magnetic fields significantly.

Two-dimensional calculations do not take end-effects into account. Appropriate endshapes and shims, similar to those successfully used at the Advanced Photon Source (APS), ${ }^{2}$ are used here to reduce field imperfections introduced by the ends to acceptable levels.

The major engineering parameters for each of the synchrotron ring magnets were determined with the program MADEST ${ }^{3}$ and are summarized in Table II.3.1-2. Dipoles, quadrupoles, sextupoles, and correction magnets are described in detail in the following sections, and design features common to all are described below.

The cores are parallel stacks of low-silicon steel laminations. The alloy, M45, is used because of its high saturation field values. The thickness of the laminations is $0.36 \mathrm{~mm}$, which is commonly used in $60-\mathrm{Hz}$ applications. This thickness is appropriate because the excitation frequency during the reset of the main fields is $60 \mathrm{~Hz}$. The cores consist of two or three sections, depending on the magnet type, to allow assembly of the magnets around the ceramic vacuum chamber. Both ends of each section have bonded end-packs. These packs contain the special contouring required to control the field shapes at the ends. Each core section is held under compression by welded parts on the exterior surfaces and by insulated tie-rods that extend through the core. The pole taper-angles were selected to keep the calculated flux densities on the pole axes to less than $2.0 \mathrm{~T}$ at $2.2 \mathrm{GeV}$. Similarly, the yoke thicknesses result in average flux densities between 1.0 and 1.2 T. Keeping the flux density values low helps to control the core losses. Similar techniques were used in the cores of the storage ring magnets for the APS. ${ }^{4}$

The coils for the three main magnet types are made with hollow copper conductor. Estimates of the power losses due to the eddy currents in the coils were derived with the aid of OPERA-2d, and the results are included in the calculated power losses for each magnet. The insulation for each coil consists of polyimide film, fiberglass tape, mica tape, and 
Table II.3.1-1 Synchrotron Ring Magnet Field Strengths and Aperture Requirements

\begin{tabular}{|c|c|c|c|c|c|c|}
\hline \multirow[b]{2}{*}{$\begin{array}{l}\text { Type of } \\
\text { Magnet }\end{array}$} & \multirow[b]{2}{*}{$\begin{array}{l}\text { Number } \\
\text { Required }\end{array}$} & \multirow[b]{2}{*}{$\begin{array}{l}L_{\text {eff }} \\
\text { (m) }\end{array}$} & \multirow[b]{2}{*}{$\begin{array}{c}\text { Strength at } \\
2.0 \mathrm{GeV}\end{array}$} & \multicolumn{3}{|c|}{ Full Bore Dimension (mm) } \\
\hline & & & & Height & Width & Diameter \\
\hline Dipole & 32 & 1.3 & $1.40 \mathrm{~T}$ & 182.0 & 450 & $-a$ \\
\hline F-Quadrupole & 28 & 0.5 & $7.73 \mathrm{~T} / \mathrm{m}$ & - & - & 237 \\
\hline D-Quadrupole & 28 & 0.5 & $6.77 \mathrm{~T} / \mathrm{m}$ & - & - & 237 \\
\hline Sextupole & 40 & 0.2 & $52 \mathrm{~T} / \mathrm{m}^{2}$ & - & - & 260 \\
\hline Corrector & 56 & 0.15 & $0.070 \mathrm{~T}$ & 300 & 300 & - \\
\hline
\end{tabular}

a - = not applicable. 
Table II.3.1-2 2.0-GeV Parameters for the Synchrotron Ring Magnets

\begin{tabular}{|c|c|c|c|c|c|}
\hline Parameters & Dipoles & Quadrupoles & Sextupoles & Correctors & Units \\
\hline Number required & 32 & 56 & 40 & 56 & - \\
\hline Peak strength & $1.40 \mathrm{~T}$ & $7.73 \mathrm{~T} / \mathrm{m}$ & $52 \mathrm{~T} / \mathrm{m}^{2}$ & $0.070 \mathrm{~T}$ & - \\
\hline Effective length & 1.3 & 0.50 & 0.20 & 0.15 & $\mathrm{~m}$ \\
\hline Gap height or diameter & 182 & 237 & 260 & 300 & $\mathrm{~mm}$ \\
\hline Bending radius & 6.62 & & & & $\mathrm{~m}$ \\
\hline Magnet mass & 21,200 & 3,607 & 550 & 460 & $\mathrm{~kg}$ \\
\hline Coils per pole & 1 & 1 & 1 & 1 & - \\
\hline \multicolumn{6}{|l|}{ Conductor } \\
\hline Height & 8.9 & 8.9 & 7.3 & 5.8 & $\mathrm{~mm}$ \\
\hline Width & 8.9 & 8.9 & 7.3 & 5.8 & $\mathrm{~mm}$ \\
\hline Hole diameter & 4.9 & 5.3 & 4.1 & 3.2 & $\mathrm{~mm}$ \\
\hline Number of turns per pole & 12 & 24 & 32 & 140 & - \\
\hline Number of parallel conductors & 48 & 4 & 2 & 1 & - \\
\hline Inductance & 2.8 & 10.0 & 16 & 3.6 & $\mathrm{mH}$ \\
\hline Resistance & 0.66 & 12.4 & 28 & 243 & $\mathrm{~m} \Omega$ \\
\hline Time constant & 4,242 & 806 & 571 & 15 & msec \\
\hline \multicolumn{6}{|l|}{ Supply current } \\
\hline dc & 5,733 & 1,267 & $498^{a}$ & $136^{a}$ & A \\
\hline ac peak & 2,806 & 620 & $171^{b}$ & $0^{\mathrm{b}}$ & A \\
\hline $\mathrm{rms}^{c}$ & 6,067 & 1,341 & 354 & 66 & A \\
\hline $\mathrm{rms}^{\mathrm{c}}$ current density in coil & 1.0 & 3.0 & 2.5 & 1.4 & $\mathrm{~A} / \mathrm{mm}^{2}$ \\
\hline Peak voltage ${ }^{\mathrm{d}}$ & 13,448 & 11,064 & 1,100 & 111 & v \\
\hline Power losses & 47.9 & 24.7 & 3.8 & 1.1 & $k W$ \\
\hline Cooling circuits/magnet & 48 & 8 & 6 & 4 & - \\
\hline Total water flow & 20.8 & 5.2 & 2.6 & 0.6 & $\mathrm{gal} / \mathrm{min}$ \\
\hline Water pressure drop & 100 & 100 & 100 & 100 & psi \\
\hline Water temperature rise & 9 & 18 & 5 & 7 & ${ }^{\circ} \mathrm{C}$ \\
\hline
\end{tabular}

a Maximum arbitrary function value.

b Minimum arbitrary function value.

c rms $=$ root mean square.

d See Section II.3.2. 
vacuum-impregnated, heat-cured epoxy. Although the insulating materials are radiation resistant, the potential of the coils to be exposed to radiation is further reduced by placing the coils well away from the midplanes. Parallel electrical paths are used for the coils in the dipoles, quadrupoles, and sextupoles. This procedure keeps the peak voltages across a series-connected group of magnets at manageable levels. The parallel circuits extend around pairs of poles to equalize the impedances of all paths for a given magnet.

The cooling water for the coils is provided by a deionized water system able to supply water at a temperature of $32^{\circ} \mathrm{C}$ and pressure drop or gradient of at least $100 \mathrm{psi}$ across the magnets. The maximum temperature rise in the water is in the quadrupole magnets and is less than $18^{\circ} \mathrm{C}$ at $2.0 \mathrm{GeV}$. It is still less than $22^{\circ} \mathrm{C}$ at $2.2 \mathrm{GeV}$.

\subsubsection{Synchrotron Dipole Magnets}

An end view of the dipole, which is assembled from two halves, is shown in Figure II.3.1-1. The ceramic vacuum chamber is also shown for reference. There is at least a 3-mm clearance between the chamber and each of the poles.

The dipole magnet is curved with a radius of $6.62 \mathrm{~m}$ to minimize the inductance. The pole width is 1.5 -gap-heights wider than the maximum beam envelope. The pole sides are tapered out by another 0.25 gap height on each side to reduce the saturation of the pole-root. The core has parallel ends, which results in a small edge-focusing effect but makes the magnet easy to construct. The curvature is formed by forcing the laminations against an appropriately curved surface in the stacking fixture. There are steel plates against the four long outside surfaces, which are welded to each other and to the laminations. These weldments stabilize the curvature and maintain the core compression. The coil on each pole consists of 24 layers, with 24 turns per layer.

The calculated deviation from uniformity of the static dipole field, $\left|\Delta \mathrm{B} / \mathrm{B}_{0}\right|$, is less than $5 \times 10^{-4}$ within the calculated beam envelope at injection for energies between $400 \mathrm{MeV}$ and $2.2 \mathrm{GeV}$. Contours of the field uniformity at injection, along with the calculated beam envelope, are shown in Figure II.3.1-2.

\subsubsection{Synchrotron Quadrupole Magnets}

All ring quadrupoles have the same design and are assembled from two halves. An end view of the quadrupole is shown in Figure II.3.1-3. The ceramic vacuum chamber is also shown for reference. There is a 5-mm clearance between the chamber and each of the poles. The taperangle of the pole reduces saturation, resulting in a calculated magnetic efficiency of $95 \%$ at $2.2 \mathrm{GeV}$. The coils have a diamond cross section to allow the parallel connections and to enable installation of the coils onto the poles of a half-core. This shape also allows the coils to be 


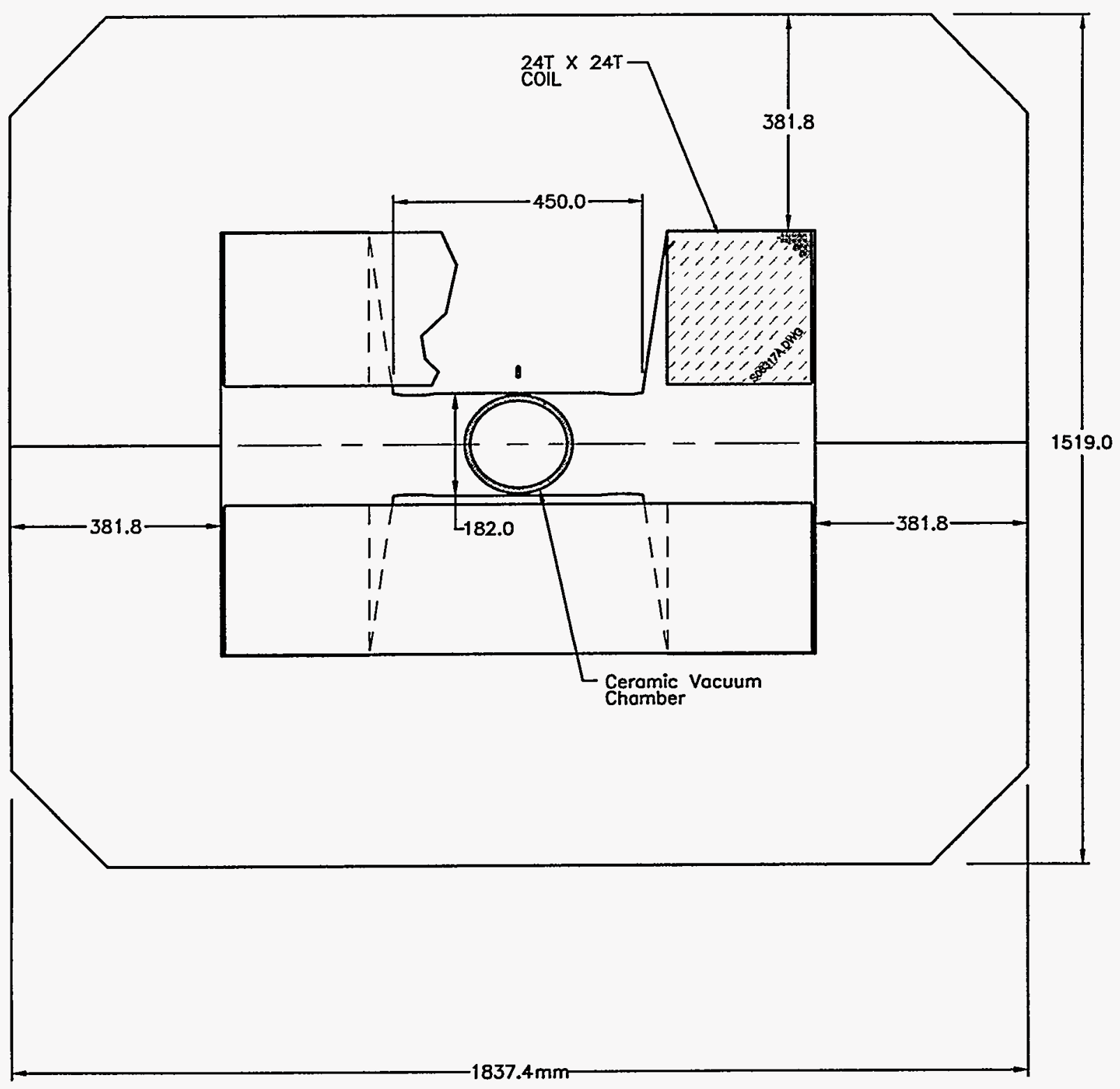

Figure II.3.1-1 End View of the Synchrotron Dipole Magnet. 


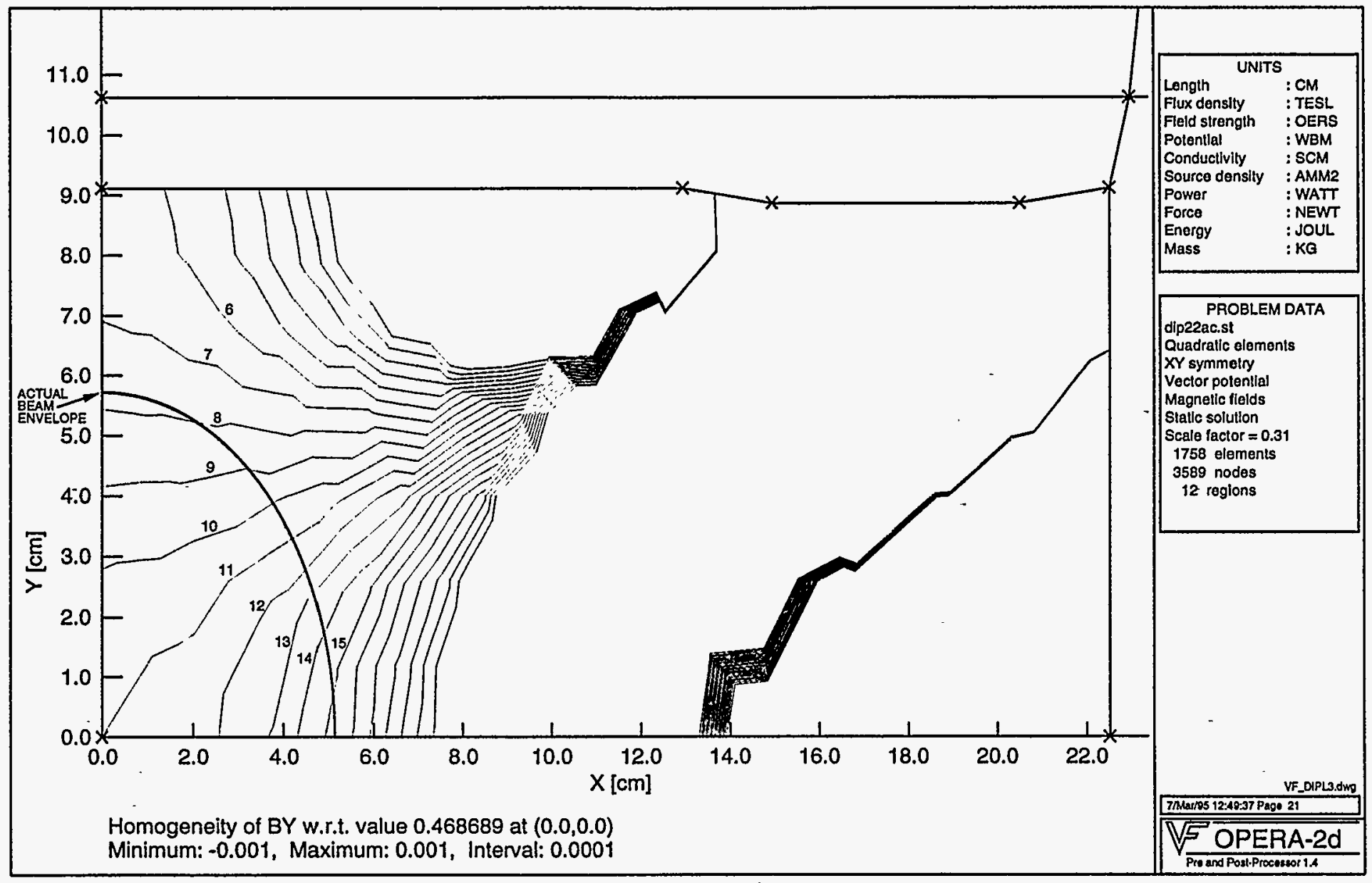

Figure II.3.1-2 Contours of Uniformity at Injection for Synchrotron Dipole along with the Calculated Beam Envelope. (Contour spacing is $0.01 \%$ and contour $\# 11$ is $0 \%$.) 


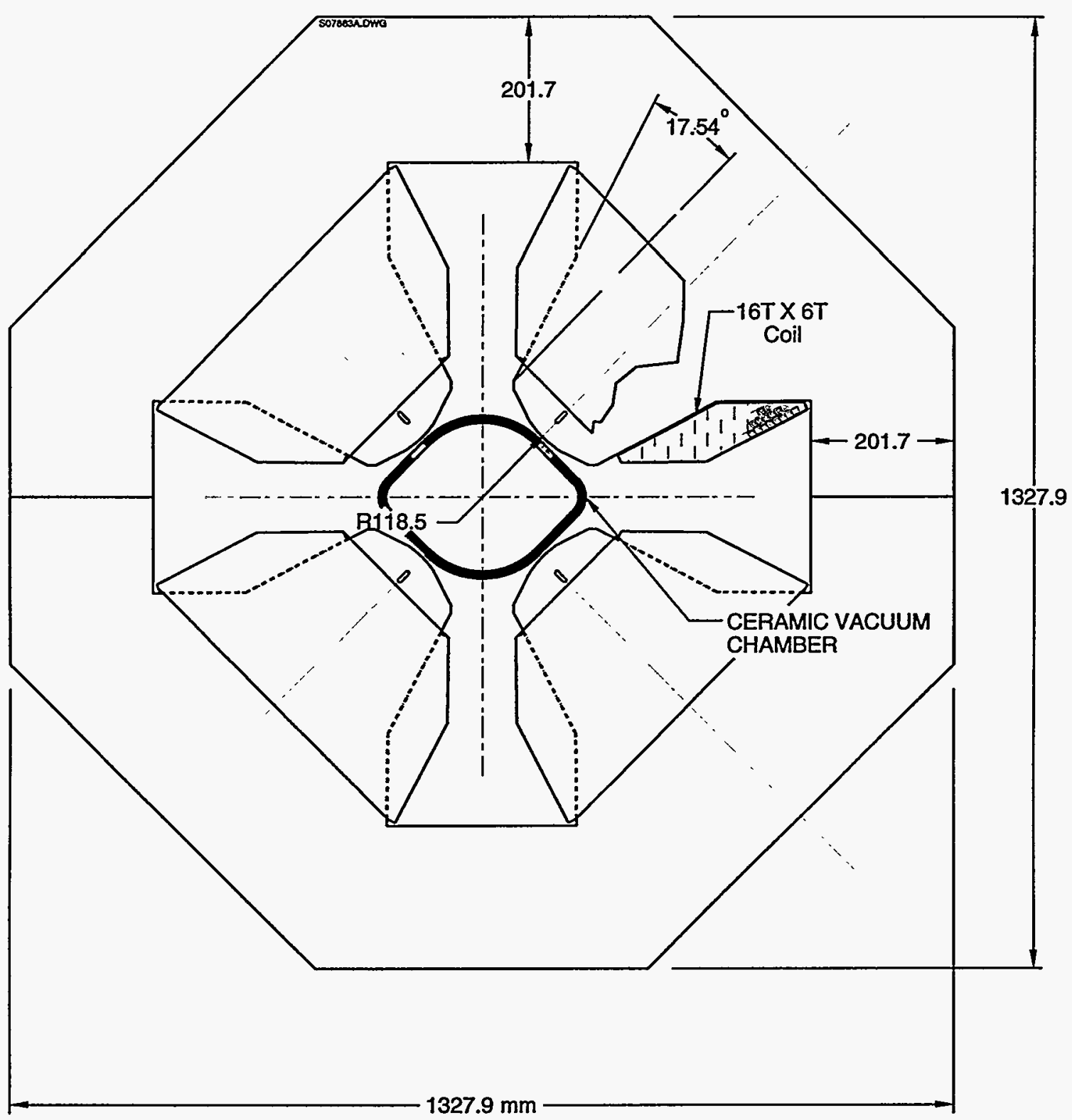

Figure II.3.1-3 End View of the Synchrotron Quadrupole Magnet. 
mounted closely to the pole faces and to be well away from the midplanes. The coil on each pole consists of six layers, with 16 turns per layer.

The calculated deviation from uniformity of the quadrupole field gradient, $\left|\Delta \mathrm{B}^{\prime} / \mathrm{B}_{0}^{\prime}\right|$, is less than $3 \times 10^{-4}$ within the calculated beam envelope for energies between $400 \mathrm{MeV}$ and $2.2 \mathrm{GeV}$. Contours of the field uniformity at injection, along with the calculated beam envelope, are shown in Figure II.3.1-4.

\subsubsection{Synchrotron Sextupole Magnets}

All ring sextupoles have the same design and are assembled from three identical sections, each of which contains two poles. The magnetic field values are low enough to avoid core saturation. The sextupole bore diameter is slightly larger than that of the quadrupole. The same ceramic vacuum chamber is used in both magnets, and the extra space is required to accommodate the sloping sides of the chamber.

An end view of the sextupole is shown in Figure II.3.1-5. The ceramic vacuum chamber is also shown for reference. There is at least a $5-\mathrm{mm}$ clearance between the chamber and each of the poles. The coil size shown allows the coils to be inserted between the two poles of each core section. The coil on each pole consists of four layers, with 16 turns per layer.

The calculated deviation from uniformity of the second derivative of the sextupole field, $\left|\Delta \mathrm{B}^{\prime \prime} / \mathrm{B}_{0}^{\prime \prime}\right|$, for the geometry shown is less than $7.0 \times 10^{-3}$ within the calculated beam envelope for energies between $400 \mathrm{MeV}$ and $2.2 \mathrm{GeV}$.

\subsubsection{Synchrotron Correction Magnets}

A horizontal correction dipole is located near each focusing quadrupole, and a vertical correction dipole is located near each defocusing quadrupole. These magnets have $\mathrm{C}$-cores, which are manufactured from one-piece laminations of low-silicon electrical steel that are $0.36-\mathrm{mm}$ thick. The core is bonded by a preapplied, B-staged epoxy coating on the laminations, which also serves as the electrical insulation between laminations. A single design provides both horizontal and vertical corrections, depending on the orientation of the magnet. These magnets can be installed over the vacuum chamber without being disassembled. The coils are made with hollow copper conductor and are insulated with the same materials as the main magnets. The coil on each pole consists of 10 layers, with 14 turns per layer. An end view of a vertical corrector and its vacuum chamber is shown in Figure II.3.1-6. The associated parameters are listed in Table II.3.1-2. 


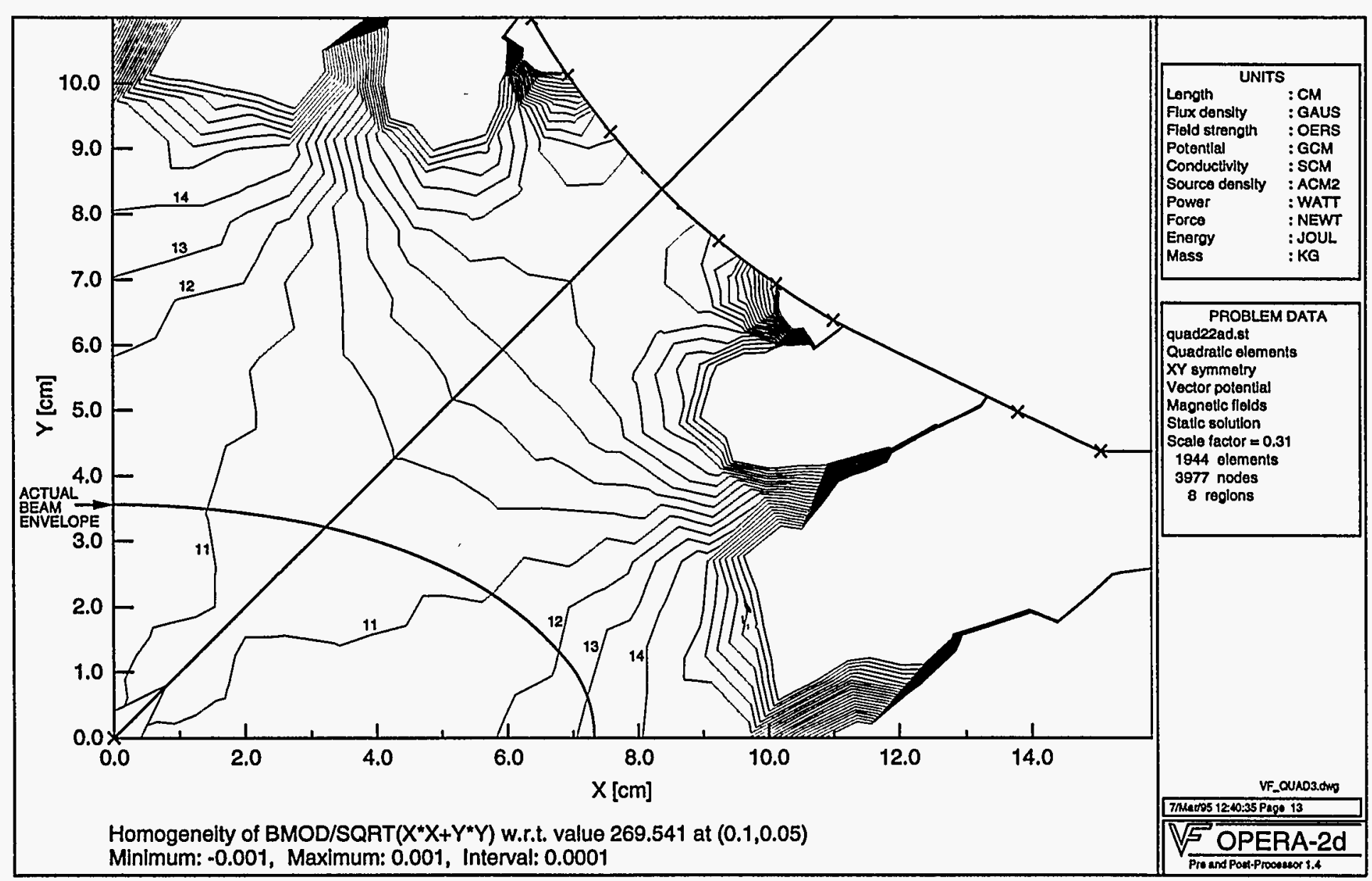

Figure II.3.1-4 Contours of Field Gradient Uniformity at Injection for an F-Quadrupole, along with the Calculated Beam Envelope. (Contour spacing is $0.01 \%$, and contour $\# 11$ is $0 \%$.) 


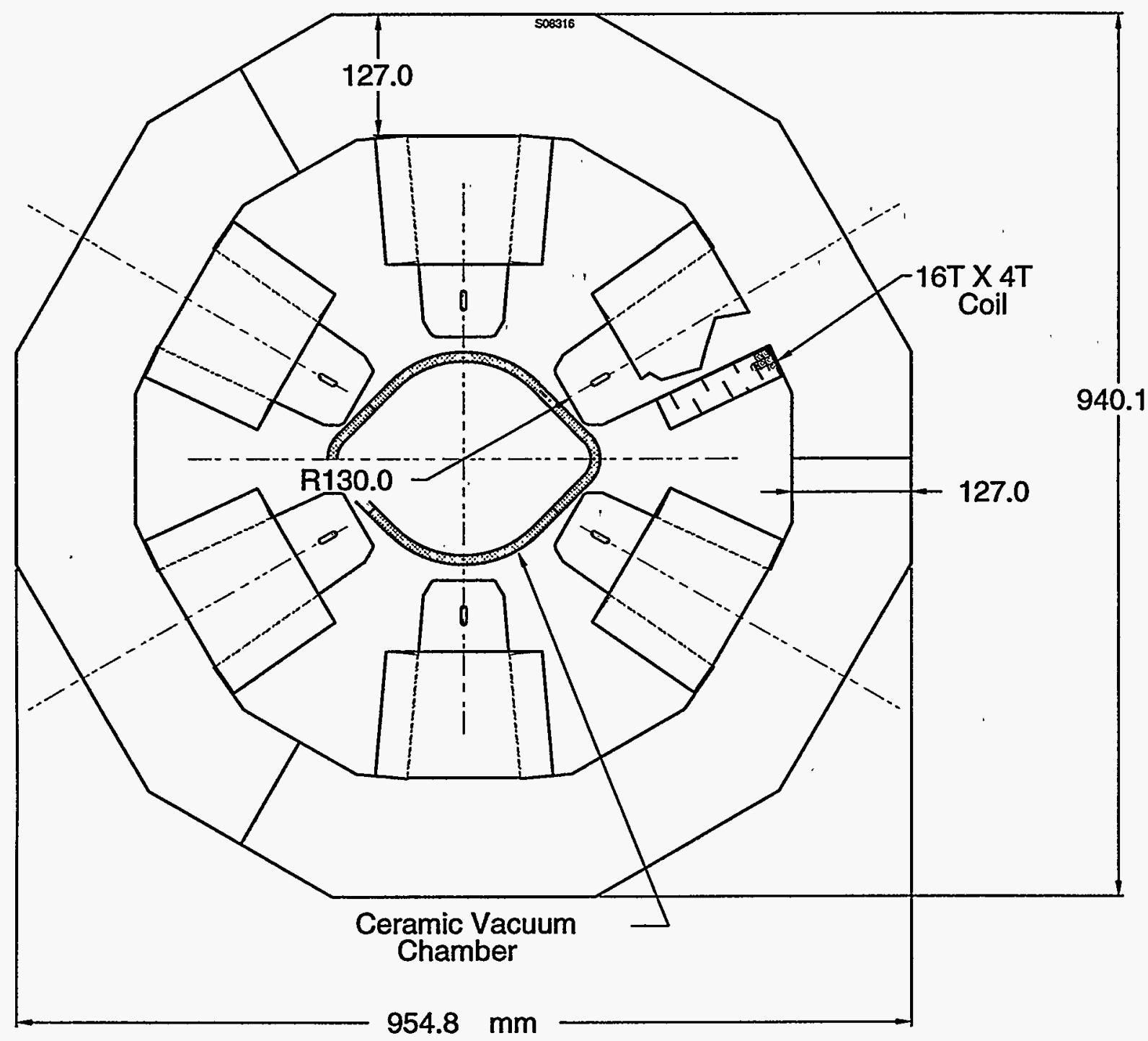

Figure II.3.1-5 End View of the Synchrotron Sextupole Magnet. 


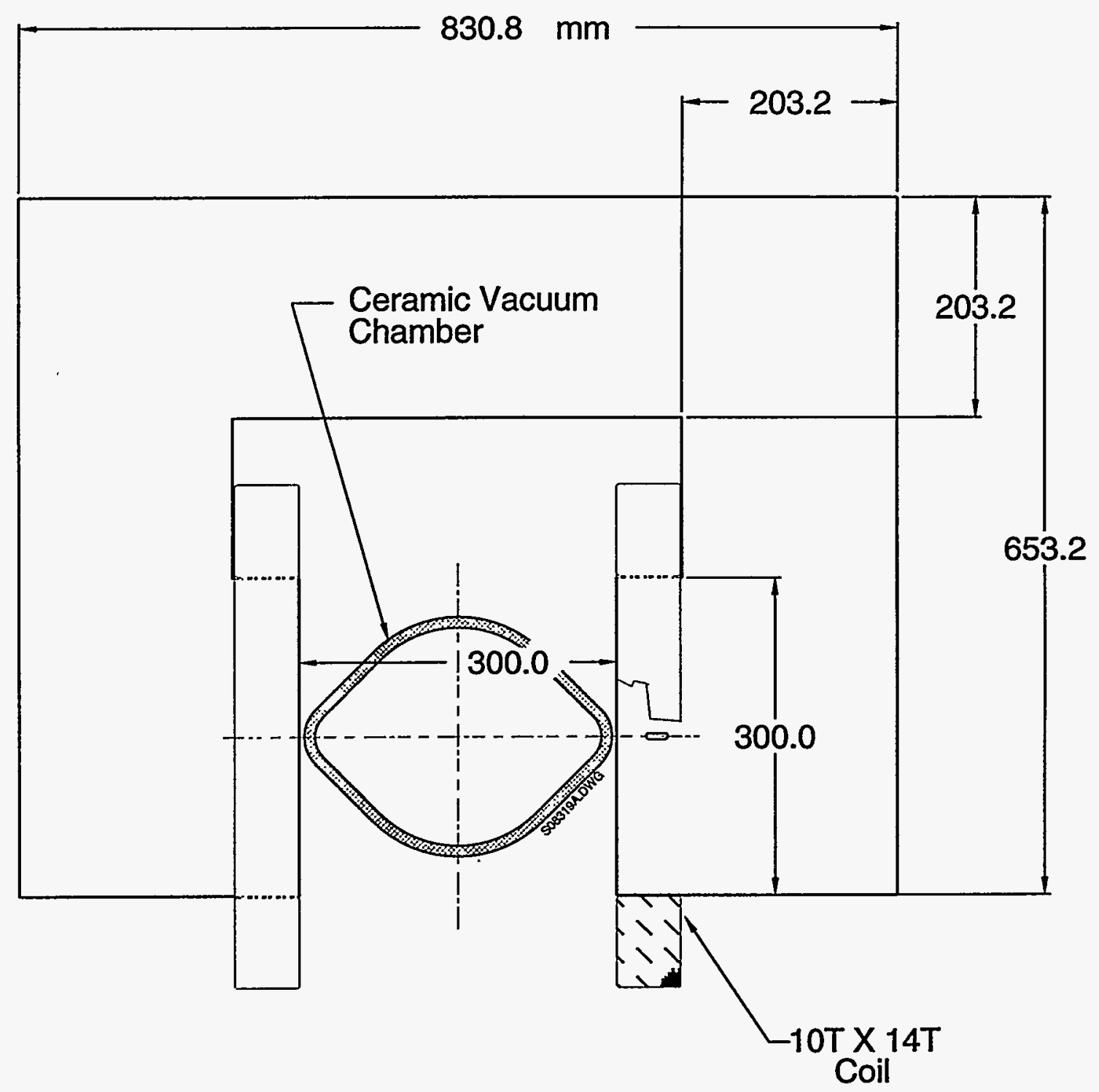

Figure II.3.1-6 End View of the Synchrotron Vertical Correction Dipole Magnet. 


\subsection{SYNCHROTRON MAGNET POWER SUPPLIES}

\subsubsection{Introduction}

\subsubsection{Power Supply Description}

The synchrotron has a repetition rate of $30 \mathrm{~Hz}$. A stability of $\pm 100 \mathrm{ppm}$ for the main bending and focusing magnet power supplies (dipoles and quadrupoles) is the design goal to achieve an overall magnetic stability of $\pm 200 \mathrm{ppm}$. The requirements for the dipole corrector magnets are less stringent. These requirements range from $\pm 300 \mathrm{ppm}$ for sextupoles to $\pm 400 \mathrm{ppm}$ for dipole corrector magnets. The nominal operating values correspond to $2.0 \mathrm{GeV}$ for all power supplies, and their maximum design values correspond to $2.2 \mathrm{GeV}$. The main dipole magnets are connected in series with eight parallel-resonant-cells and eight feed points, as shown in Figure II.3.2-1. The power circuits in this design are a resonant cycloconverter/rectifier and are similar to those used in the 500-MeV RCS. The energy lost in the resonant circuit is resupplied by rectifier operation of the circuit. The combination of power supplies used for continuous excitation have a rectifier ripple frequency of $2,160 \mathrm{~Hz}$ (36-pulse) and a total power rating of $3.7 \mathrm{MW}$. The quadrupole magnets are divided into two families and are connected in the same manner as the dipoles, except that they have seven feed points per family, as shown in Figure II.3.2-2. The total power supply rating for the combination of the focusing and defocusing quadrupoles is $3.8 \mathrm{MW}$. The sextupole magnets are individually powered by 40 identical pulse-width-modulated (PWM) power supplies operating at a frequency of 15 to $20 \mathrm{kHz}$. They respond to an arbitrary function during the acceleration ramp, and the remaining stored energy after losses is returned to a capacitor bank for use during the next cycle. The peak sextupole power supply voltage rating is sufficiently high to provide twice the normalized rate of current change as that provided by the dipole power supply during acceleration. The regulator reference-voltage is supplied by a digital-to-analog converter (DAC) that is serially counted up and down, thereby creating the desired arbitrary current shape between zero and the maximum current in each cycle. Fifty-six independently controlled 6-kW bipolar power supplies are provided for the horizontal and vertical dipole corrector magnets. The need for smooth control of the dipole corrector magnet current through the zero point dictates the use of bipolar output stages.

\subsubsection{Control System and Control Electronics}

The power supplies require regulation and control to a resolution better than $100 \mathrm{ppm}$. The critical DAC and analog-to-digital conversion (ADC) devices, along with their regulator circuits, are located in a shielded enclosure near the analog signal source, generally in the power supply cabinet. One regulator is required for each group of ac-to-dc power converters for the resonant magnet circuits. Therefore, there are eight regulators for the dipole magnet system. 


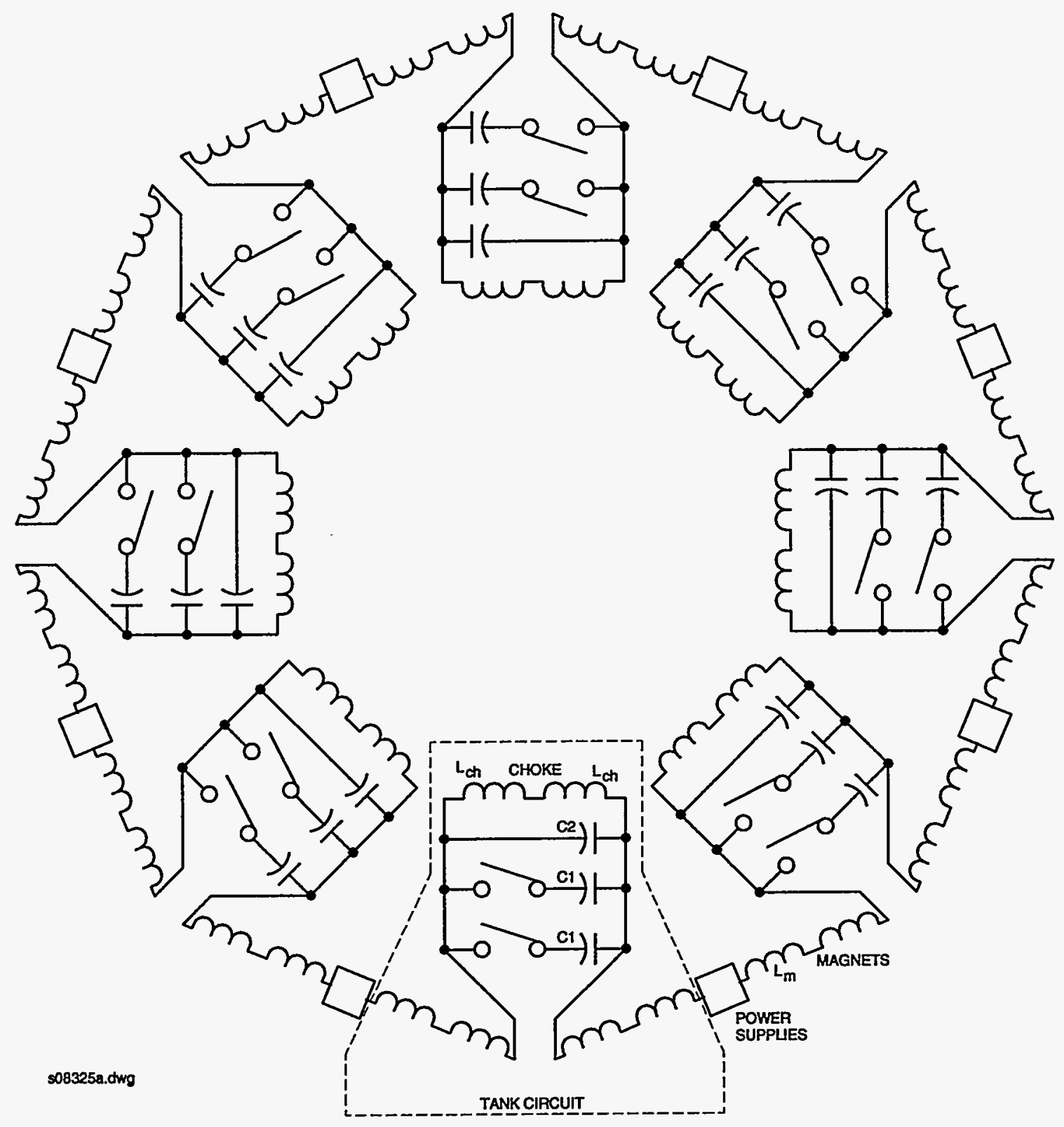

Figure II.3.2-1 Resonant Circuit Dipole Magnet Power Supply. 


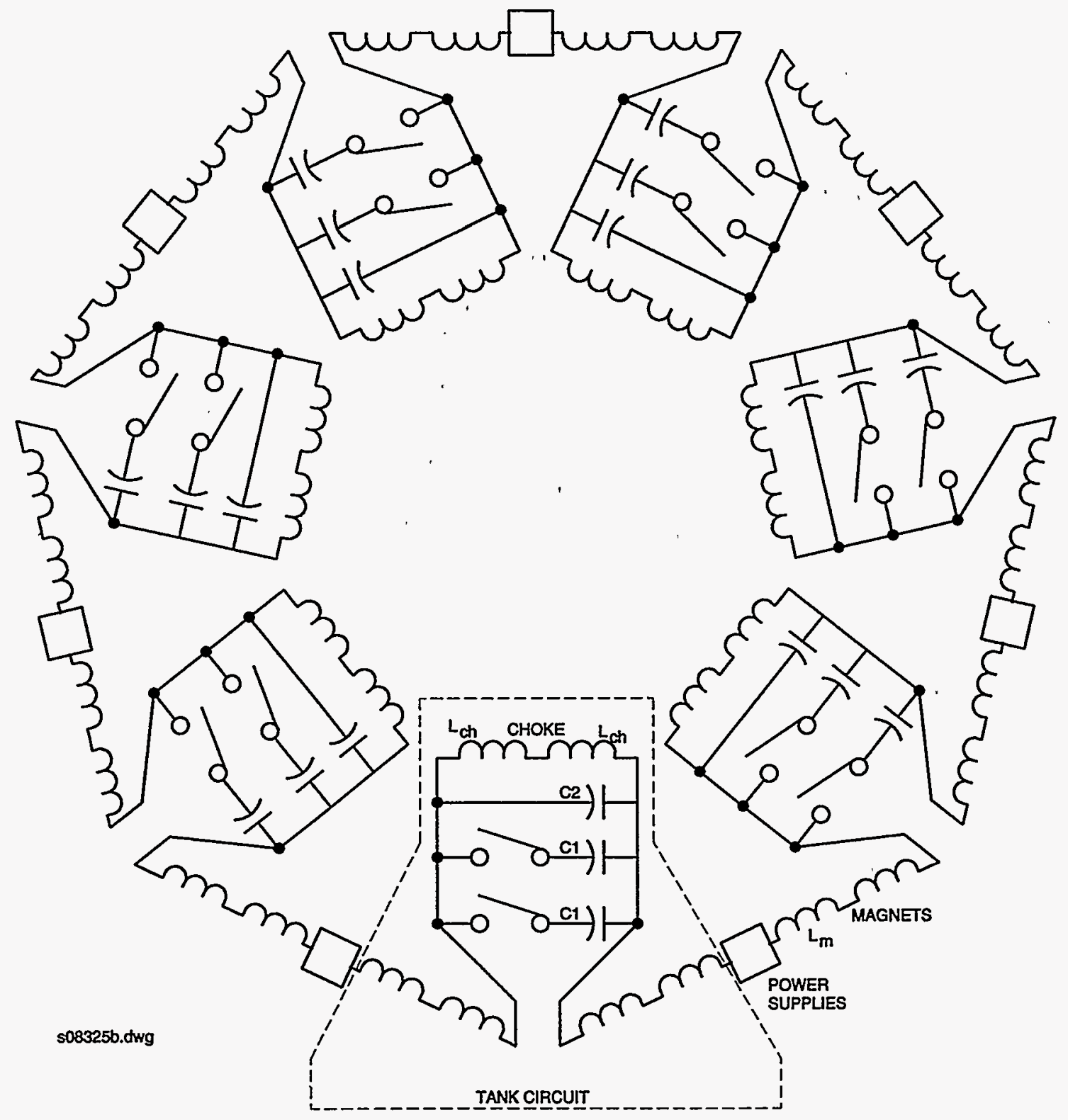

Figure II.3.2-2 Resonant Circuit Quadrupole Magnet Power Supply. 
Communication with these regulator units is established by means of digital signals through optical cables, or at least optical couplers, to remove possible ground loops and reduce electrical noise. A multidrop-cable local network is used and is described in Section II.8.

A local microprocessor that uses digital signal processing techniques is used to keep the tank circuits tuned and balanced. It also controls, monitors, and balances the regulators.

Local control of the regulator group is by means of a local control console (a PC Notebook). This local console is able to emulate all local network actions while displaying regulator responses and status. This same system of local control and testing is used in the construction phase to bench-check the regulator systems; no other control system components are required.

\subsubsection{Grounding}

Most of the problems encountered in magnet power supply applications originate from inappropriate grounding practices, which can be explained by two different meanings attributed to the term "ground."

"Ground" is synonymous with "earth" in the context of an electrical utility or power grounding. Its function is to provide a low-impedance path for return of fault currents to the generator. This low-impedance path serves a safety role by limiting voltage differences during system faults and by allowing fault currents of sufficient magnitude to assure prompt opening of the source circuit breakers.

"Ground" is synonymous with "chassis" in electronics and data processing equipment. It can be a metal equipment enclosure or a single metal pad at the bottom of the cabinet, to which all connections to "ground" are routed. Data processing circuitry involves analog or pulse voltage signals that are generally less than $15 \mathrm{~V}$ and/or current loops of a few milliamperes. These signals can be transferred in a balanced, ungrounded circuit or a single-ended circuit with a common reference. Balanced circuits are isolated from ground, but the voltage with respect to ground (common mode) is still significant to the operation and potential susceptibility of the circuits. Single-ended circuits carry signals referred to a common potential, usually that of the equipment's internal chassis and generally called "reference." The concern in either case is that the referencing system might carry signal currents that can become polluted by spurious ground currents, either through common connection points with the "earthed" system or by common-mode noise coupling.

Because of the high frequencies associated with electronic and switching circuits, the inductance between the circuit and the reference should be kept to a minimum. Therefore, large existing copper cables, suitable for earthing, are not suitable for referencing. A ground reference grid providing a large number of different path-links for the various noise frequencies is incorporated into the system. For power supply applications, there is concern for personnel and equipment safety and for noise. Safe operation of the electrical or electronic system depends on 
the integrity and low impedance of its "earth" system. Undisturbed operation of the same electrical or electronic system depends on the geometry and intrinsic low impedance of its reference grid, regardless of the impedance between the reference and the earth.

Electric and magnetic field coupling between circuits must be considered in addition to the noise voltages coupled to equipment by ground currents flowing through a common ground bus.

In summary, power supply monitoring and control circuits are designed to:

- Generate as little noise as practicable,

- Be affected by noise as little as practicable,

- Keep electromagnetic interference to a minimum,

- Keep conductive coupling to a minimum, and

- Keep common impedance coupling to a minimum.

Argonne National Laboratory (ANL) has a long and successful history of both conventional and digital computer-based control and monitoring of large, complex power systems. Solutions to problems associated with high and low signal levels in areas with high electromagnetic noise and in otherwise hostile environments have been found. These include both conventional and rapid cycling synchrotrons, bubble chamber magnets, large magnetohydrodynamic (MHD) magnets, and the fusion power devices APEX, FELIX, and ALEX.

\subsubsection{Lockout/Tagout (LOTO) of Magnet Power Supplies}

All magnet power supplies are powered from one 138-kV three-phase ac line through a $138-\mathrm{kV}$ to $13.2-\mathrm{kV}$ transformer. The $13.2-\mathrm{kV}$ to $480-\mathrm{V}$ distribution transformers used as the main source of power to the magnet power supplies have no other equipment connected to them. LOTO of the main power to the magnet power supplies is accomplished (see Figure II.3.2-3) in one of the following ways:

- One LOTO on the incoming $138-\mathrm{kV}$ ac line breaker. This locks out all magnet power supplies.

- One LOTO on each of the $13.2-\mathrm{kV}$ ac input line breakers for the $13.2-\mathrm{kV}$ to $480-\mathrm{V}$ distribution transformers used with the magnet power supplies. This can be used to lock out subsets of all major types of accelerator power supplies. 


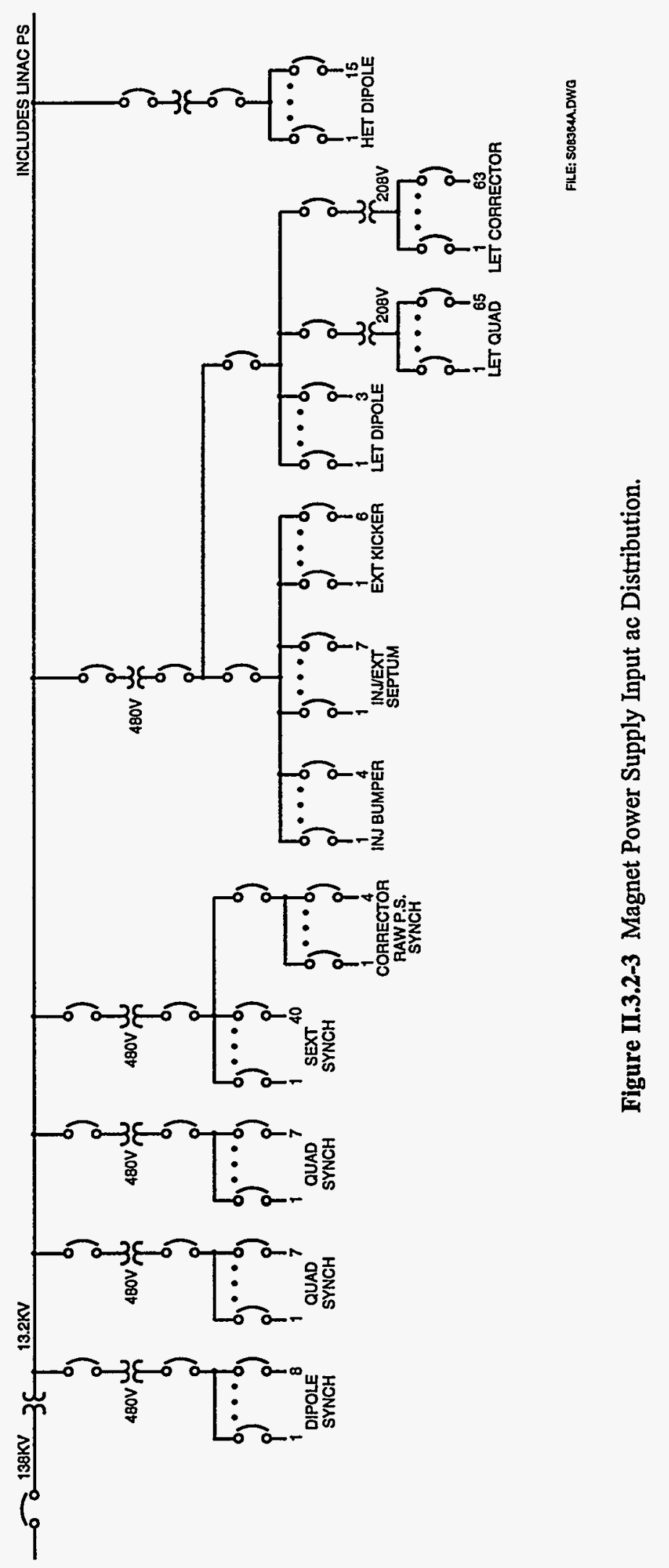


- One LOTO on each of the $480-\mathrm{V}$ ac main transformer output breakers for the $13.2-\mathrm{kV}$ to $480-\mathrm{V}$ distribution transformers used with the magnet power supplies. This can be used to lock out individual major power supplies or groups of smaller power supplies.

- One LOTO on each of the 480-V distribution breakers used shown in Figure II.3.2-3. This can be used to lock out any individual power supply.

The 110-V ac control power for the power supplies is sometimes supplied from another source and must be locked out separately, if required.

\subsubsection{Dipole Magnet Power Supplies}

\subsubsection{General Considerations}

The RCS dipoles and quadrupoles are excited by dc-biased sinusoidal currents. Capacitors and chokes resonate with the magnets to save energy. A modulated multiphase dc power supply provides both $\mathrm{dc}$ and ac excitation from one source. The energy losses during each cycle are resupplied by the rectifier action of the supply. It operates at $20 \mathrm{~Hz}$ and $60 \mathrm{~Hz}$ and is readily phase-locked to the line or to a stable oscillator. Figure II.3.2-4 illustrates one of the ac-dc power sources, which uses three identical 12-pulse dc power supplies connected in series. Each has its three-phase ac inputs phase-shifted by 10 electrical degrees with respect to the others. The phase shifting is done by an autotransformer. This combination provides a 36-pulse, phase-controlled, dc power supply generating the desired wave shape. A slightly underdamped filter is connected to the power supply to attenuate ripple above the operating frequency. This configuration is repeated eight times around the ring to reduce the peak voltages. The capacitors are switched to achieve dual-frequency operation. The acceleration ramp is at $20 \mathrm{~Hz}$, and the reset is at $60 \mathrm{~Hz}$, which reduces the radio-frequency (rf) voltage required per turn compared with continuous $30-\mathrm{Hz}$.

\subsubsection{Dual-Frequency Magnet Circuit}

A dual-frequency resonant magnet circuit is used for the dipole magnets. The $30-\mathrm{Hz}$ repetition rate is maintained for the RCS, while a $20-\mathrm{Hz}$ magnet guide field is used during acceleration, and a $60-\mathrm{Hz}$ reset field is used when no beam is present. The result is a longer accelerating-field rise time and shorter fall time; the duty factor is improved as a result of the longer acceleration time. The maximum $\dot{B}$ is reduced by $33 \%$, reducing the maximum of voltage by $33 \%$. Figure II.3.2-5 shows current and voltage waveforms for dual-frequency operation. 


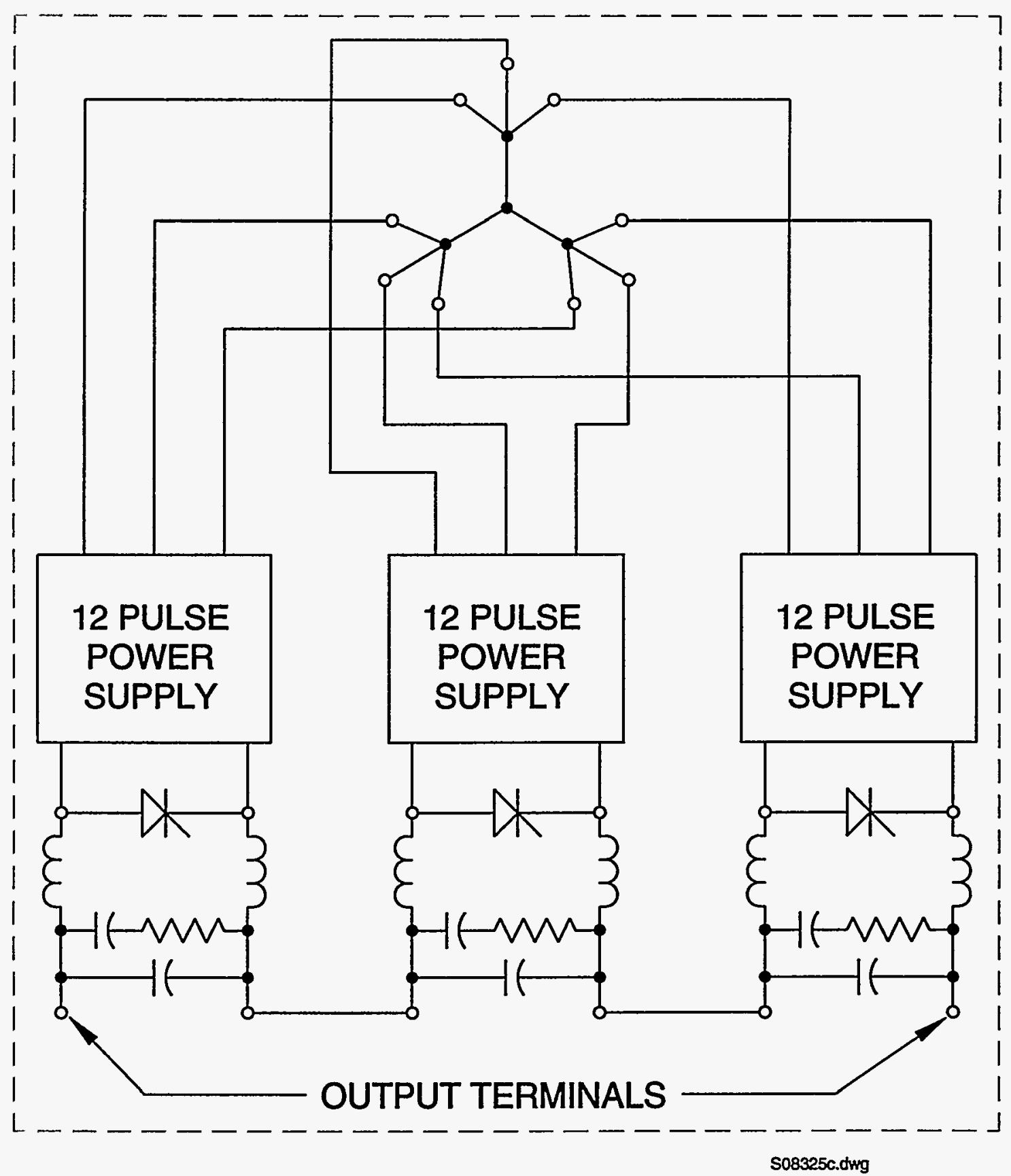

Figure II.3.2-4 Power Supply for One Feed Point. 


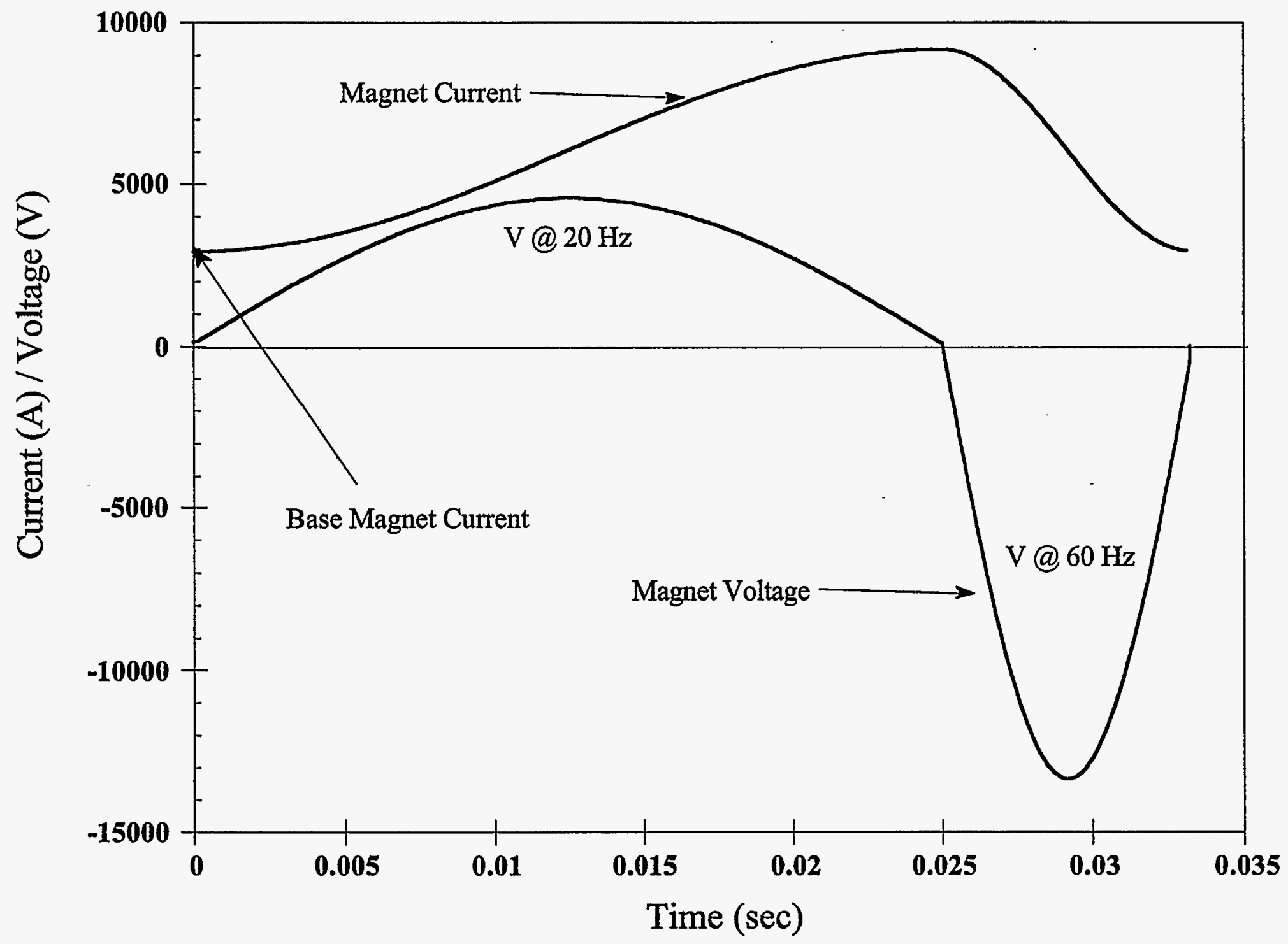

Figure II.3.2-5 Dipole Magnet Current and Voltage Waveforms for Dual-Frequency Operation. 
When the frequency during acceleration is denoted by $f_{1}$ and the frequency during magnet reset is denoted by $f_{2}$, the effective RCS repetition rate, $f_{0}$, is given by:

$$
\mathrm{f}_{0}=\frac{2 \times \mathrm{f}_{1} \times \mathrm{f}_{2}}{\mathrm{f}_{1}+\mathrm{f}_{2}}
$$

The peak magnet voltage during reset is proportional to $f_{2}$, provided $f_{2} \geq f_{1}$. The capacitance of $C_{1}$ in Figure II.3.2-1 increases and the voltage across the $C_{1}$ capacitor decreases as the ratio $f_{0} / f_{1}$ increases. The capacitance of $C_{2}$ decreases and the voltage across the $C_{2}$ capacitor increases as the ratio $f_{0} / f_{1}$ increases.

For the baseline design, $f_{1}=20 \mathrm{~Hz}, f_{2}=60 \mathrm{~Hz}$, and $f_{0}=30 \mathrm{~Hz}$. These values can be changed in the future but are limited by the maximum tolerable voltage.

The magnet and choke currents are $180^{\circ}$ out of phase. The stored energy is transferred back and forth between the choke and the magnet through the resonant capacitor $\mathrm{C}_{1}$ during the acceleration ramp and through the $\mathrm{C}_{2}$ capacitor during the resets. The choke inductance, $\mathrm{L}_{\mathrm{ch}}$, is selected to be equal to the magnet inductance, $\mathrm{L}_{\mathrm{m}}$, to optimize the voltage across the magnets. The resonant frequency, $\omega_{0}$, of the circuit is given by

$$
\omega_{0}=\sqrt{\frac{L_{m}+L_{c h}}{L_{m} \times L_{c h} \times C}},
$$

where

$\mathrm{L}_{\mathrm{m}}=$ sum of the inductances of the magnets in one tank circuit,

$\mathrm{L}_{\mathrm{ch}}=$ sum of the inductances of the choke in one tank circuit, and

$C=$ capacitance value of one tank circuit at $f_{0}$.

When $L_{c h}=L_{m}$, then the capacitance of the circuit becomes

$$
\mathrm{C}=\frac{2}{\mathrm{~L}_{\mathrm{m}} \times \omega_{0}^{2}} .
$$

The time-variation of the magnet current during acceleration, $i_{\text {magnets }}$ when saturation effects are neglected, is given by

$$
\mathrm{i}_{\text {magnet }}=\mathrm{I}_{\mathrm{dc}}-\mathrm{I}_{\mathrm{ac}} \times \cos \omega_{1} \mathrm{t} .
$$


The choke and capacitor currents, $i_{\text {choke }}$ and $i_{\text {capacitor, }}$, are given by

$$
\mathrm{i}_{\text {choke }}=\mathrm{I}_{\mathrm{dc}}+\mathrm{I}_{\mathrm{ac}} \times \cos \omega_{1} \mathrm{t}
$$

and

$$
\mathrm{i}_{\text {capacitor }}=\mathrm{i}_{\text {magnet }}-\mathrm{i}_{\text {choke }}=-2 \mathrm{I}_{\mathrm{ac}} \times \cos \omega_{1} \mathrm{t} \text {. }
$$

The capacitor and magnet voltages, $e_{c}$ and $e_{m}$ are given by

$$
\begin{gathered}
e_{c}=\frac{2 I_{a c}}{\omega_{1} C_{1}} \sin \omega_{1} t \\
e_{m}=E_{d c}+e_{c},
\end{gathered}
$$

where

$$
\begin{aligned}
\mathrm{I}_{\mathrm{dc}} & =\mathrm{dc} \text { magnet or choke current, } \\
\mathrm{I}_{\mathrm{ac}} & =\text { ac magnet or choke current peak, } \\
\mathrm{E}_{\mathrm{dc}} & =\mathrm{dc} \text { magnet voltage, } \\
\mathrm{i}_{\text {magnet }} & =\text { instantaneous magnet current, } \\
\mathrm{i}_{\text {choke }} & =\text { instantaneous choke current, } \\
\mathrm{i}_{\text {capacitor }} & =\text { instantaneous capacitor bank current, } \\
\mathrm{e}_{\mathrm{c}} & =\text { instantaneous capacitor bank voltage, and } \\
\mathrm{e}_{\mathrm{m}} & =\text { instantaneous magnet voltage. }
\end{aligned}
$$

\subsubsection{Dipole Circuit Loss Budget and Parameters}

A loss budget for the dipole resonant circuit shown in Figure II.3.2-1 is given in Table II.3.2-1. Table II.3.2-2 lists the parameters for the dipole resonant circuit. 
Table II.3.2-1 Loss Budget for the Dipole Dual-Frequency Resonant Circuit at $2.2 \mathrm{GeV}^{\mathrm{a}}$

\begin{tabular}{lllllllll}
\hline & $\begin{array}{c}\text { Ring } \\
\text { Dipole } \\
\text { Losses }\end{array}$ & Magnets & Chokes & Capacitors & $\begin{array}{c}\text { Inter- } \\
\text { Connects }\end{array}$ & Filters & $\begin{array}{c}\text { Power } \\
\text { Supplies }\end{array}$ & Units \\
\hline & & & & & & & \\
Percent of total & 46 & 19 & 5 & 10 & 5 & 15 & $\%$ \\
$\begin{array}{l}\text { Per magnet } \\
\text { Per tank circuit }\end{array}$ & 52.7 & 21.5 & 5.7 & 11.5 & 5.7 & 17.2 & kW \\
Full circuit & $1,684.9$ & 86.0 & 22.9 & 45.8 & 22.9 & 68.7 & kW \\
& & 688.0 & 183.1 & 366.3 & 183.1 & 549.4 & kW \\
\hline
\end{tabular}

a There are eight resonant tank circuits with eight feed points operating at $2.2 \mathrm{GeV}$. Each of the eight groups of three power supplies is required to make up $457 \mathrm{~kW}$ lost per feed point. Total system losses are 3,655 kW. 
Table II.3.2-2 Parameters for the Dipole Dual-Frequency Resonant Circuit at $2.2 \mathrm{GeV}$

\section{Parameters}

Values Units

Magnet inductance

Magnet inductance of $1 / 8$ of the total magnets

Magnet dc current

Magnet peak ac current

Magnet rms current

Capacitor bank peak ac current

6,056

3,129

6,448

$-6,258$

$\mathrm{mH}$

Number of feed points

Total number of power supplies

Number of magnet families

Total number of magnets

ac loss per:

Magnet

Choke

Capacitors $(1 / 32$ of the total)

Interconnections $(1 / 32$ of the total)

Filters (1/32 of the total)

Power supplies (1/32 of the total)

ac total loss

8

24

1

32

25.3

8.2

3.9

3.9

2.0

5.9

$1,575.5$

27.4

13.3

0

7.9

3.9

11.8

$2,057.6$

11.110

1.234

Capacitance (1/8 of total) for $20-\mathrm{Hz}$ operation

Capacitance ( $1 / 8$ of total) for $60-\mathrm{Hz}$ operation

One $185-\mathrm{kW}$ power supply ( $\mathrm{kW}$ value was selected to include $10 \%$ for low

line and $10 \%$ for regulation)

Voltage rms $\mathrm{s}^{\mathrm{a}}$ of one power supply based on $\mathrm{kW}$ value selected

Voltage dc of one power supply based on $\mathrm{kW}$ value selected

Voltage ac peak of one power supply based on $\mathrm{kW}$ value selected

Voltage peak of one power supply based on power supply selected

ac voltage peak across the tank circuit for $20-\mathrm{Hz}$ operation

ac voltage peak across the tank circuit for $60-\mathrm{Hz}$ operation
184.7

28.7

17.5

13.0

30.6

$-4,483$

$-13,448$ $m F$

$k W$

$k W$

$k W$

$k W$

kW

$k W$

$k W$

$k W$

kW

$k W$

$k W$

$k W$

kW

$k W$

$\mathrm{mF}$

$k W$

V

V

V

V

V

V

\footnotetext{
a $\mathrm{rms}=$ root mean square.
} 


\subsubsection{Quadrupole Magnet Power Supplies}

\subsubsection{General Considerations}

The quadrupole magnets are divided into two families, with seven feed points per family, as shown in Figure II.3.2-2. They have a total power rating of 3.8 MW and operate as a dualfrequency resonant circuit. Details are similar to those for the dipole circuit. The cells are repeated seven times per family. The design curves shown in Figure II.3.2-5 for the main dipole circuit also apply to the quadrupole circuits, but with different amplitudes.

\subsubsection{Quadrupole Circuit Loss Budget and Parameters}

A loss budget for the quadrupole resonant circuit shown in Figure II.3.2-2 is given in Table II.3.2-3. Table II.3.2-4 lists the parameters for the quadrupole resonant circuit.

\subsubsection{Sextupole Magnet Power Supplies}

The sextupole magnets are individually powered by 40 identical unipolar pulse-widthmodulated (PWM) power supplies operating at a frequency of 15 to $20 \mathrm{kHz}$, as shown in Figure II.3.2-6. During the acceleration cycle, the power supply is operated by closing switch $\mathrm{AA}$ and pulse-width-modulating switch $\mathrm{A}$. When switch $\mathrm{A}$ is closed, energy is transferred from the capacitor to the magnet. When switch $\mathrm{A}$ is open, the magnet freewheels through switch AA and diode D3, thereby losing energy. The regulator reference voltage is supplied by a DAC that is serially counted up and down, thus creating an arbitrary ramp. At the end of the acceleration ramp, the DAC is reset to zero, and switches $\mathrm{A}$ and $\mathrm{AA}$ are both opened. This procedure forces the remaining energy stored in the magnet to be returned to the capacitor bank through diodes D2 and D3 for use during the next cycle.

The peak sextupole power supply voltage rating is sufficiently high to provide twice the normalized rate of current change of the dipole magnet power supply during acceleration. The sextupole power supplies have a current stability of $\pm 300 \mathrm{ppm}$.

\subsubsection{Dipole Corrector Magnet Power Supplies}

A total of 56 independently controlled bipolar power supplies are provided for the horizontal and vertical dipole corrector magnets. They are rated at $6 \mathrm{~kW}$ each. The bipolar power supplies provide smooth control of the current through zero. Figure II.3.2-7 shows a simplified circuit of a bipolar power supply. The topology is a PWM full-bridge converter operating at $20 \mathrm{kHz}$. The switches are insulated-gate bipolar-transistors (IGBTs), and the antiparallel diodes are included within them. $L_{c}$ and $C_{c}$ represent the inductance and capacitance 
Table II.3.2-3 Loss Budget for the Quadrupole Dual-Frequency Resonant Circuit of One Family at 2.2 GeVa

\begin{tabular}{|c|c|c|c|c|c|c|c|}
\hline Losses & $\begin{array}{c}\text { Ring } \\
\text { Quad } \\
\text { Magnets }\end{array}$ & Chokes & Capacitors & $\begin{array}{c}\text { Inter- } \\
\text { Connects }\end{array}$ & Filters & $\begin{array}{l}\text { Power } \\
\text { Supplies }\end{array}$ & Units \\
\hline $\begin{array}{l}\text { Percent of total } \\
\text { Per magnet }\end{array}$ & $\begin{array}{l}44 \\
30.0\end{array}$ & $\begin{array}{l}22 \\
15.0\end{array}$ & $\begin{array}{l}5 \\
3.3\end{array}$ & $\begin{array}{l}10 \\
6.5\end{array}$ & $\begin{array}{l}5 \\
3.3\end{array}$ & $\begin{array}{l}15 \\
9.8\end{array}$ & $\begin{array}{l}\% \\
k W\end{array}$ \\
\hline Per tank circuit & 120.1 & 60.2 & 13.1 & 26.1 & 13.1 & 39.2 & $k W$ \\
\hline Full circuit & 840.7 & 421.1 & 91.4 & 182.8 & 91.4 & 274.2 & $k W$ \\
\hline
\end{tabular}

a There are seven resonant tank circuits with seven feed points operating at $2.2 \mathrm{GeV}$. Each of the seven groups of three power supplies is required to make up $271.7 \mathrm{~kW}$ lost per feed point. Total system losses are 1,901.6 kW. 
Table II.3.2-4 Parameters for the Quadrupole Dual-Frequency Resonant Circuit of One Family at $2.2 \mathrm{GeV}$

\begin{tabular}{|c|c|c|}
\hline Parameters & Values & Units \\
\hline Magnet inductance & 10 & $\mathrm{mH}$ \\
\hline Magnet inductance of $1 / 7$ of the total magnets per family & 40 & $\mathrm{mH}$ \\
\hline Magnet dc current & 1,420 & A \\
\hline Magnet peak ac current & 733.7 & A \\
\hline Magnet rms current & 1,512 & A \\
\hline Capacitor bank peak ac current & $-1,467.4$ & A \\
\hline Number of feed points & 7 & - \\
\hline Total number of power supplies & 42 & - \\
\hline Number of magnet families & 2 & - \\
\hline Total number of magnets & 56 & - \\
\hline \multicolumn{3}{|l|}{ ac loss per: } \\
\hline Magnet & 2.6 & kW \\
\hline Choke & 0.9 & kW \\
\hline Capacitors (1/28 of the total) & 3.3 & kW \\
\hline Interconnections ( $1 / 28$ of the total) & 2.2 & $\mathrm{~kW}$ \\
\hline Filters $(1 / 28$ of the total) & 1.1 & kW \\
\hline Power supplies ( $1 / 28$ of the total) & 3.3 & $\mathrm{~kW}$ \\
\hline ac total loss & 53.4 & $\mathrm{~kW}$ \\
\hline \multicolumn{3}{|l|}{ dc loss per: } \\
\hline Magnet & 27.4 & kW \\
\hline Choke & 14.2 & $\mathrm{~kW}$ \\
\hline Capacitors ( $1 / 28$ of the total) & 0 & kW \\
\hline Interconnections ( $1 / 28$ of the total) & 4.3 & kW \\
\hline Filters $(1 / 28$ of the total) & 2.2 & $\mathrm{~kW}$ \\
\hline Power supplies ( $1 / 28$ of the total) & 6.6 & kW \\
\hline dc total loss: & 272.3 & $\mathrm{~kW}$ \\
\hline Capacitance $(1 / 7$ of total) for $20-\mathrm{Hz}$ operation & 3.166 & $\mathrm{mF}$ \\
\hline Capacitance $(1 / 7$ of total) for $60-\mathrm{Hz}$ operation & 0.352 & $\mathrm{mF}$ \\
\hline \multicolumn{3}{|l|}{ One $110-k W$ power supply ( $k W$ value was selected to include $10 \%$ for low } \\
\hline line and $10 \%$ for regulation) & 108.9 & kW \\
\hline Voltage rms ${ }^{\mathrm{a}}$ of one power supply based on the $\mathrm{kW}$ value selected & 72.8 & $\mathrm{~V}$ \\
\hline Voltage dc of one power supply based on the $\mathrm{kW}$ value selected & 62.3 & V \\
\hline Voltage ac peak of one power supply based on the $\mathrm{kW}$ value selected & 15.2 & v \\
\hline Voltage peak of one power supply based on power supply selected & 77.5 & v \\
\hline ac voltage peak across the tank circuit for $20-\mathrm{Hz}$ operation & $-3,688$ & v \\
\hline ac voltage peak across the tank circuit for $60-\mathrm{Hz}$ operation & $-11,064$ & v \\
\hline
\end{tabular}

a $\mathrm{rms}=$ root mean square. 


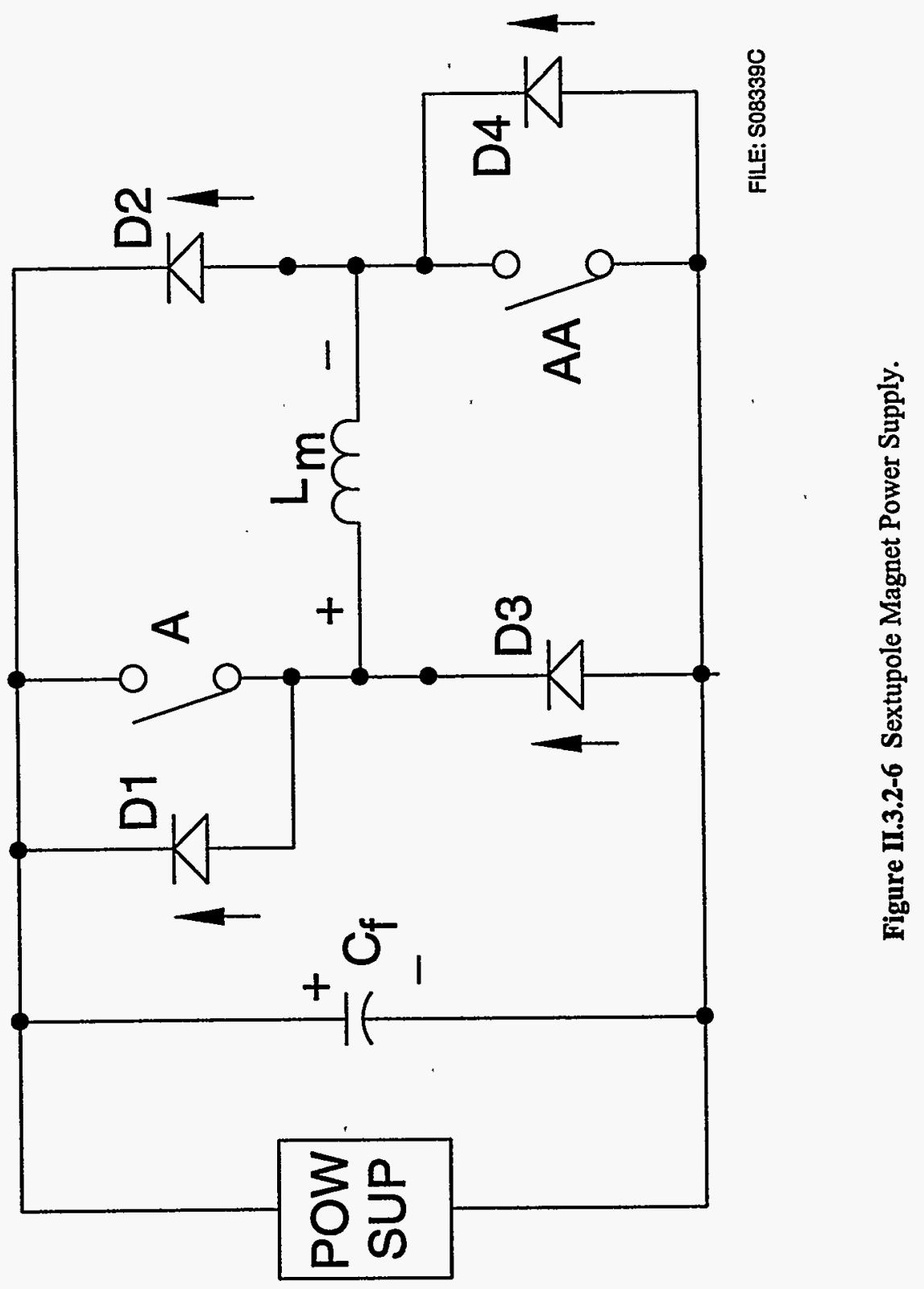




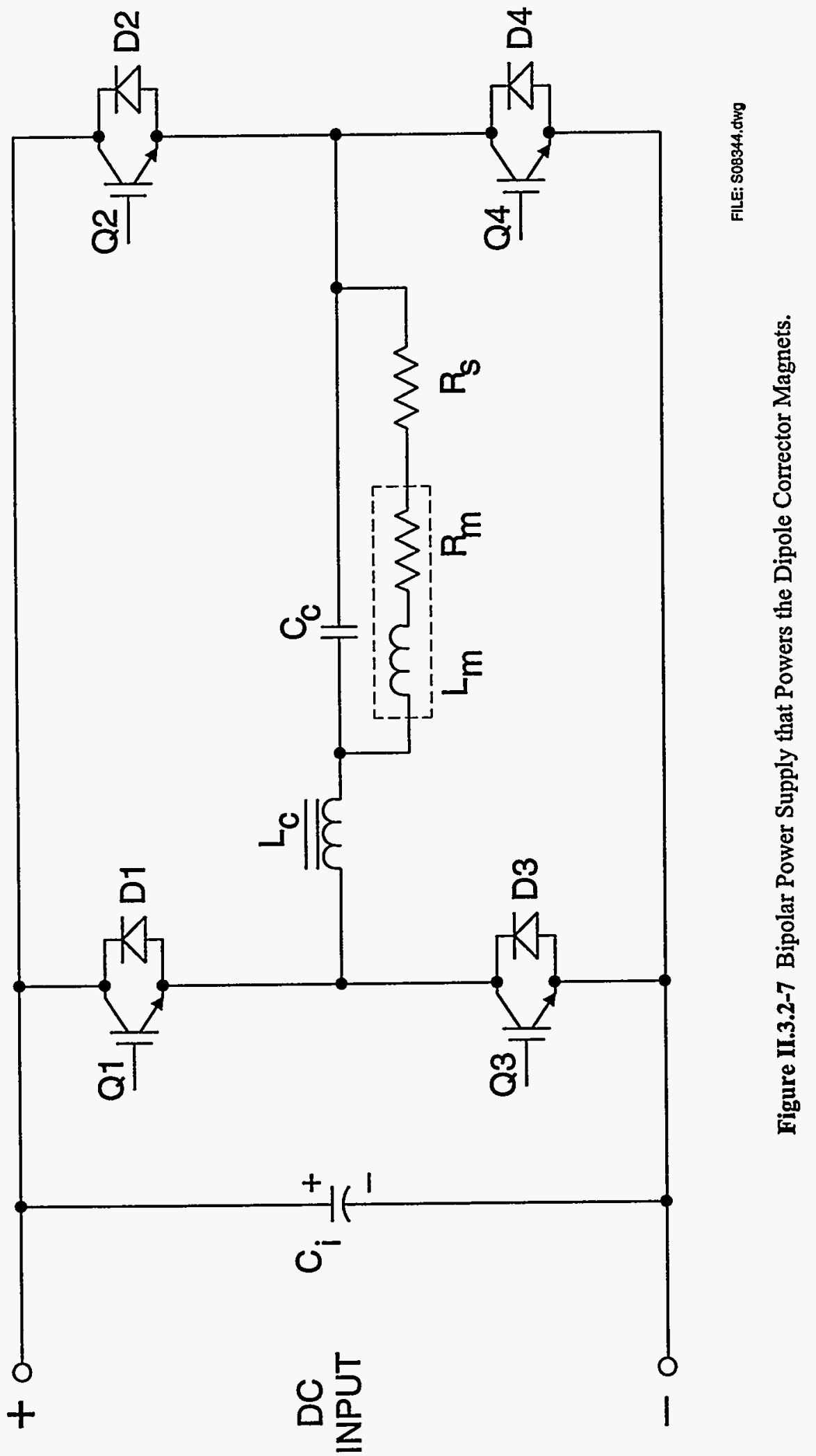


of the output filter; $L_{m}$ and $R_{m}$ are the inductance and resistance of the magnet; and $R_{s}$ is the resistance of a current-sensing device. The peak output current and voltage are $\pm 149.2 \mathrm{~A}$ and $\pm 111 \mathrm{~V}$, respectively, for $2.2 \mathrm{GeV}$. The current stability of the bipolar power supplies is $\pm 400 \mathrm{ppm}$.

\subsection{BEAM TRANSFER LINE MAGNETS AND POWER SUPPLIES}

\subsubsection{Low-Energy Transport Line (LET)}

\subsubsection{Magnets}

The LET has 15 dipole, 65 quadrupole, and 16 corrector magnets to transport the beam between the linac and the synchrotron injection septum magnets. All LET magnets are commercially supplied, and they have laminated cores constructed from 1.5-mm-thick lowcarbon steel. The coils are made with copper conductors that are insulated with fiberglass and vacuum-impregnated, heat-cured epoxy. The parameters for the four LET magnet types are listed in Table II.3.3-1.

All LET dipoles are of the same design, using hollow water-cooled conductor. Each can be split into two halves for installation around the vacuum chamber.

There are 62 quadrupoles with 53-mm-diameter apertures (quad A) and three 72-mmdiameter-aperture quadrupoles (quad B) in the LET. Each quadrupole has coils made with hollow water-cooled conductor and is assembled from four quadrants. Each can be split into halves for installation around the vacuum chamber.

There are 16 small correction dipoles in the LET. The correctors are made with one-piece $\mathrm{C}$-cores to facilitate assembly and installation around the vacuum chambers. Each has coils made with solid air-cooled conductor.

\subsubsection{Power Supplies}

The LET has 15 dipole, 65 quadrupole, and 16 dipole corrector magnets. All dipole corrector and quadrupole magnets are individually powered by air-cooled commercial dc power supplies. Current-regulator circuitry is added to the dipole corrector and quadrupole supplies. The dipoles are powered by three identical dc power supplies, which are water cooled and are built to a performance specification. Eight dipoles are powered by one supply, five dipoles are powered by the second supply, and two dipoles are powered by the third supply. All parameters are shown in Table II.3.3-2. 
Table II.3.3-1 Parameters for LET Magnets

\begin{tabular}{|c|c|c|c|c|c|}
\hline Parameters & Dipole A & Quad A & Quad B & $\begin{array}{l}\text { LET } \\
\text { Corrector }\end{array}$ & Units \\
\hline Number required & 15 & 62 & 3 & 16 & - \\
\hline Peak strength at $400 \mathrm{MeV}$ & $0.63 \mathrm{~T}$ & $14.0 \mathrm{~T} / \mathrm{m}$ & $12.0 \mathrm{~T} / \mathrm{m}$ & $0.064 \mathrm{~T}$ & - \\
\hline $\begin{array}{l}\text { Effective length } \\
\text { Gap }\end{array}$ & 1.0 & 0.20 & 0.20 & 0.10 & $\mathrm{~m}$ \\
\hline Height or diameter & 40 & 53 & 72 & 53 & $\mathrm{~mm}$ \\
\hline Width & 140 & - & - & 135 & $\mathrm{~mm}$ \\
\hline $\begin{array}{l}\text { Magnet mass } \\
\text { Conductor }\end{array}$ & 2,000 & 110 & 210 & 55 & $\mathrm{~kg}$ \\
\hline Height & 8.3 & 4.2 & 5.8 & $1.6 \phi$ & $\mathrm{mm}$ \\
\hline Width & 8.3 & 4.2 & 5.8 & - & $\mathrm{mm}$ \\
\hline Hole diameter & 4.6 & 2.4 & 3.2 & Solid & $\mathrm{mm}$ \\
\hline Number of turns per pole & 96 & 26 & 26 & 720 & - \\
\hline Inductance & 200 & 10 & 9 & 888 & $\mathrm{mH}$ \\
\hline Resistance & 190 & 94 & 48 & 8684 & $\mathrm{~m} \Omega$ \\
\hline Time constant & 1,052 & 106 & 188 & 102 & $\mathrm{~ms}$ \\
\hline Maximum supply current & 106 & 154 & 243 & 1.9 & A \\
\hline Maximum current density in coil & 1.2 & 5.6 & 5.2 & 0.4 & $\mathrm{~A} / \mathrm{mm}^{2}$ \\
\hline Peak voltage & 20 & 16 & 14 & 19 & V \\
\hline Power losses & 2.1 & 2.2 & 2.8 & 0.03 & $k W$ \\
\hline Cooling circuits/magnet & 4 & 4 & 4 & - & - \\
\hline Total water flow & 1.3 & 0.7 & 1.5 & - & $\mathrm{gal} / \mathrm{min}$ \\
\hline Water pressure drop & 100 & 100 & 100 & - & psi \\
\hline Water temperature rise & 6 & 11 & 7 & $36^{a}$ & ${ }^{\circ} \mathrm{C}$ \\
\hline
\end{tabular}

a Maximum estimated internal temperature for the coils. 
Table II.3.3-2 LET Power-Supply Parameters at $10 \%$ over $400 \mathrm{MeV}$

\begin{tabular}{|c|c|c|c|c|c|}
\hline \multirow[b]{2}{*}{ Parameters } & \multicolumn{4}{|c|}{ Magnet Power Supply } & \multirow[b]{2}{*}{ Units } \\
\hline & Dipole A & Quad A & Quad B & $\begin{array}{l}\text { Dipole } \\
\text { Corrector }\end{array}$ & \\
\hline Number of power supplies & 3 & 62 & 3 & 16 & - \\
\hline Number of magnets & 15 & 62 & 3 & 16 & - \\
\hline Inductance & 200 & 10 & 9 & 888 & $\mathrm{mH}$ \\
\hline Resistance & 190 & 94 & 48 & 8,684 & $\mathrm{~m} \Omega$ \\
\hline Maximum supply current & 169,4 & 169.4 & 267.3 & 2.09 & A \\
\hline Peak voltage & 31.9 & 15.4 & 13.2 & 18.7 & v \\
\hline Stability $( \pm)$ & $1 \times 10^{-4}$ & $2 \times 10^{-4}$ & $2 \times 10^{-4}$ & $3 \times 10^{-4}$ & $\Delta \mathrm{i} / \mathrm{Imax}$ \\
\hline Current reproducibility $( \pm)$ & $2 \times 10^{-4}$ & $4 \times 10^{-4}$ & $4 \times 10^{-4}$ & $6 \times 10^{-4}$ & $\Delta \mathrm{i} / \mathrm{Imax}$ \\
\hline Current ripple $( \pm)$ & $5 \times 10^{-4}$ & $6 \times 10^{-4}$ & $6 \times 10^{-4}$ & $1 \times 10^{-3}$ & $\Delta \mathrm{i} / \mathrm{Imax}$ \\
\hline Tracking error $( \pm)$ & $5 \times 10^{-4}$ & $6 \times 10^{-4}$ & $6 \times 10^{-4}$ & $4 \times 10^{-3}$ & $\Delta \mathrm{i} / \mathrm{Imax}$ \\
\hline Resolution bits & 14 & 14 & 14 & 12 & $\Delta \mathbf{i} / \mathrm{Imax}$ \\
\hline
\end{tabular}




\subsubsection{High-Energy Transport Line (HET)}

\subsubsection{Magnets}

The two HET lines contain a total of 5 dipole, 25 quadrupole, and 16 corrector magnets to transport the beam from the synchrotron extraction septum magnets to the two external neutron targets. All HET magnets are commercially supplied, and they have laminated cores constructed from 1.5-mm-thick laminations of low-carbon steel. The coils are made with hollow copper conductors that are insulated with fiberglass and vacuum-impregnated, heat-cured epoxy. The parameters for the six HET magnet types are listed in Table II.3.3-3.

All dipole cores are made with the same lamination shape as that used for the synchrotron dipoles. Three different dipole lengths are required for the HET lines; these three are referred to as dipole $\mathrm{B}$, dipole $\mathrm{C}$, and dipole $\mathrm{D}$ in Table II.3.3-3.

There are 17 HET quadrupoles, quad $\mathrm{C}$, which have a bore diameter of $170 \mathrm{~mm}$, and $8 \mathrm{HET}$ quad D magnets with a bore diameter of $264 \mathrm{~mm}$. The four quadrupole magnets closest to the neutron target in each of the two HET lines are manufactured with radiation-hard insulation.

The 16 HET correction dipoles have cores identical to those used in the synchrotron correctors. The coils are also similar, but they use larger conductors.

\subsubsection{Power Supplies}

There are 5 dipole, 25 quadrupole, and 16 dipole corrector magnets in the HET lines to the $10-\mathrm{Hz}$ and $30-\mathrm{Hz}$ targets. All magnets are individually powered. The dipole dc power supplies are built to a performance specification and are water cooled. The quadrupoles are powered by air-cooled commercial dc power supplies to which current-regulator circuitry has been added. The dipole correctors are powered with bipolar pulse-width modulated (PWM) power supplies built to a performance specification. All parameters are shown in Table II.3.3-4. 
Table II.3.3-3 Parameters for HET Magnets

\begin{tabular}{|c|c|c|c|c|c|c|c|}
\hline Parameters & Dipole B & Dipole C & Dipole D & Quad C & Quad D & $\begin{array}{c}\text { HET } \\
\text { Corrector }\end{array}$ & Units \\
\hline Number required & 1 & 2 & 2 & 17 & 8 & 16 & - \\
\hline Peak strength at $2.0 \mathrm{GeV}$ & $1.20 \mathrm{~T}$ & $1.43 \mathrm{~T}$ & $1.36 \mathrm{~T}$ & $9.1 \mathrm{~T} / \mathrm{m}$ & $7.3 \mathrm{~T} / \mathrm{m}$ & $0.127 \mathrm{~T}$ & - \\
\hline $\begin{array}{l}\text { Effective length } \\
\text { Gap }\end{array}$ & 1.0 & 1.8 & 2.8 & 0.50 & 0.50 & 0.15 & $\mathrm{~m}$ \\
\hline Height or Diameter & 182 & 182 & 182 & 170 & 264 & 300 & $\mathrm{~mm}$ \\
\hline Width & 450 & 450 & 450 & - & - & 300 & $\mathrm{~mm}$ \\
\hline $\begin{array}{l}\text { Magnet mass } \\
\text { Conductor }\end{array}$ & 19,000 & 36,600 & 58,200 & 5,100 & 6,900 & 410 & $\mathrm{~kg}$ \\
\hline Height & 12.7 & 12.7 & 12.7 & 12.7 & 15.2 & 5.8 & $\mathrm{~mm}$ \\
\hline Width & 12.7 & 12.7 & 12.7 & 12.7 & 15.2 & 5.8 & $\mathrm{~mm}$ \\
\hline Hole diameter & 7.0 & 7.0 & 7.0 & 7.0 & 8.4 & 3.2 & $\mathrm{~mm}$ \\
\hline Number of turns per pole & 306 & 306 & 306 & 113 & 129 & 140 & - \\
\hline Inductance & 1,424 & 2,564 & 3,988 & 314 & 317 & 3.6 & $\mathrm{mH}$ \\
\hline Resistance & 325 & 481 & 677 & 115 & 94 & 250 & $\mathrm{~m} \Omega$ \\
\hline Time constant & 4,382 & 5,331 & 5,891 & 2,730 & 3,372 & 15 & $\mathrm{~ms}$ \\
\hline Maximum supply current & 266 & 318 & 303 & 219 & 371 & 252 & A \\
\hline $\begin{array}{l}\text { Maximum current density } \\
\text { in coil }\end{array}$ & 1.3 & 1.5 & 1.5 & 1.2 & 1.4 & 5.9 & $\mathrm{~A} / \mathrm{mm}^{2}$ \\
\hline Peak voltage & 100 & 178 & 236 & 29 & 40 & 70 & V \\
\hline Power losses & 26.7 & 56.1 & 71.8 & 6.4 & 14.9 & 17.7 & $k W$ \\
\hline Cooling circuits/magnet & 18 & 18 & 18 & 4 & 4 & 10 & - \\
\hline Total water flow & 18.2 & 14.9 & 12.5 & 3.1 & 4.7 & 2.7 & $\mathrm{gal} / \mathrm{min}$ \\
\hline Water pressure drop & 100 & 100 & 100 & 100 & 100 & 100 & psi \\
\hline Water temperature rise & 6 & 14 & 22 & 8 & 12 & 25 & ${ }^{\circ} \mathrm{C}$ \\
\hline
\end{tabular}


Table II.3.3-4 HET Power-Supply Parameters at $2.2 \mathrm{GeV}$

\begin{tabular}{|c|c|c|c|c|c|c|c|}
\hline \multirow[b]{2}{*}{ Parameters } & \multicolumn{6}{|c|}{ Magnet Power Supply } & \multirow[b]{2}{*}{ Units } \\
\hline & Dipole B & Dipole C & Dipole D & Quad C & Quad D & $\begin{array}{l}\text { Dipole } \\
\text { Corrector }\end{array}$ & \\
\hline Number of power supplies & 1 & 2 & 2 & 17 & 8 & 16 & - \\
\hline Number of magnets & 1 & 2 & 2 & 17 & 8 & 16 & - \\
\hline Inductance & 1,424 & 2,564 & 3,988 & 314 & 317 & 3.6 & $\mathrm{mH}$ \\
\hline Resistance & 325 & 481 & 677 & 115 & 94 & 240 & $\mathrm{~m} \Omega$ \\
\hline Maximum supply current & 286 & 342 & 326 & 236 & 399 & \pm 271 & $A$ \\
\hline Peak voltage & 112 & 197 & 264 & 32 & 44 & \pm 65 & v \\
\hline Stability ( \pm ) & $1 \times 10^{-4}$ & $1 \times 10^{-4}$ & $1 \times 10^{-4}$ & $2 \times 10^{-4}$ & $2 \times 10^{-4}$ & $3 \times 10^{-4}$ & $\Delta \mathrm{i} / \mathrm{Imax}$ \\
\hline Current reproducibility ( $( \pm)$ & $2 \times 10^{-4}$ & $2 \times 10^{-4}$ & $2 \times 10^{-4}$ & $4 \times 10^{-4}$ & $4 \times 10^{-4}$ & $6 \times 10^{-4}$ & $\Delta \mathrm{i} / \mathrm{Imax}$ \\
\hline Current ripple $( \pm)$ & $5 \times 10^{-4}$ & $5 \times 10.4$ & $5 \times 10^{-4}$ & $6 \times 10^{-4}$ & $6 \times 10^{-4}$ & $1 \times 10^{-3}$ & $\Delta \mathrm{i} / \mathrm{Imax}$ \\
\hline Tracking error ( $( \pm)$ & $5 \times 10^{-4}$ & $5 \times 10^{-4}$ & $5 \times 10^{-4}$ & $6 \times 10^{-4}$ & $6 \times 10^{-4}$ & $4 \times 10^{-3}$ & $\Delta \mathrm{i} / \mathrm{Imax}$ \\
\hline Resolution bits & 14 & 14 & 14 & 14 & 14 & 12 & $\Delta \mathrm{I} / \mathrm{I} \max$ \\
\hline
\end{tabular}




\subsection{BUMPER AND KICKER MAGNETS AND POWER SUPPLIES}

\subsubsection{Introduction}

The synchrotron bumper and kicker magnets have large gaps to accommodate the beamstay-clear (BSC) requirements. Four bumper magnets are used to move the circulating synchrotron beam close to the injection septum during injection of the beam from the linac to the synchrotron. The bumpers are of conventional design without stringent requirements, but the extraction kicker requirements are more demanding.

\subsubsection{Injection Bumper Magnets}

All four bumper magnets have the same apertures and coil configurations. The bumper magnet parameters for a maximum bend angle of $20 \mathrm{mrad}$ are summarized in Table II.3.4-1. A cross section of an injection bumper magnet is shown in Figure II.3.4-1. The pole faces are machined in uncut cores to assure parallelism of the pole faces and dimensional stability of the air gaps. The coils are made with hollow copper conductor and are insulated with mica-glass tape and vacuum-impregnated heat-cured epoxy. After the coils are cured, the outer surfaces are coated with an electrically conducting paint to define the ground planes. This coating prevents corona formation at the sharp corners and edges of the grounded core laminations.

Tape-wound grain-oriented silicon steel cores that are $0.3 \mathrm{~mm}$ thick are used for all of the bumper magnets. A peak field of $0.212 \mathrm{~T}$ is generated in a pulse period of $1 \mathrm{msec}$. Detailed pulse requirements are described in Section II.2.2.

\subsubsection{Injection-Bumper-Magnet Power Supplies}

The four bumper magnets are individually powered. The circuit diagram is shown in Figure II.3.4-2. The four 0.3-m-long bumper magnets are energized to move the closed orbit closer to the injection septum when the incoming bunch and the bucket are synchronized. Switch S1 in Figure II.3.4-2 is closed, causing capacitor C to discharge in $500 \mu \mathrm{sec}$ into the bumper magnets. The peak current is determined by the charge on C. All four bumper magnets are capable of peak fields of $0.212 \mathrm{~T}$. The end of the pulse is shaped by closing switches $\mathrm{S} 2$ and S3, and by opening switch S1, which allows the current to decay through R1 to approximately two-thirds of its peak in $500 \mu \mathrm{sec}$. The final pulse shape is controlled by opening switch S3, putting R1 and R2 in series, and driving the current to zero in $50 \mu \mathrm{sec}$.

The transformer T1 is used to match the current and voltage ratings of the switching circuit components to the magnet. The regulation of the charging power supply and control of the switch timing keep the peak current repeatable to within $\pm 0.05 \%$. 
Table II.3.4-1 Parameters for Synchrotron Bumper Magnets

\begin{tabular}{|c|c|c|}
\hline Parameters & Bumper & Units \\
\hline Bend angle & 20 & mrad \\
\hline Peak central field at $400 \mathrm{MeV}$ & 0.212 & $T$ \\
\hline Effective length & 0.3 & $m$ \\
\hline \multicolumn{3}{|l|}{ Gap } \\
\hline Height & 241.5 & $\mathrm{~mm}$ \\
\hline Width & 300 & $\mathrm{~mm}$ \\
\hline \multicolumn{3}{|l|}{ Conductor } \\
\hline Height & 7.3 & $\mathrm{~mm}$ \\
\hline Width & 7.3 & $\mathrm{~mm}$ \\
\hline Hole diameter & 4.1 & $\mathrm{~mm}$ \\
\hline Number of turns & 28 & - \\
\hline Number of parallel conductors & 2 & - \\
\hline Inductance & 0.13 & $\mathrm{mH}$ \\
\hline Resistance & 10 & $\mathrm{~m} \Omega$ \\
\hline \multicolumn{3}{|l|}{ Supply current } \\
\hline Maximum & 2,427 & A \\
\hline$r m s^{a}$ & 475 & A \\
\hline Peak voltage & 680 & v \\
\hline Power losses & 2.3 & $k W$ \\
\hline Cooling circuits/magnet & 2 & - \\
\hline Total water flow & 0.8 & $\mathrm{gal} / \mathrm{min}$ \\
\hline Water pressure drop & 100 & psi \\
\hline Water temperature rise & 11 & ${ }^{\circ} \mathrm{C}$ \\
\hline
\end{tabular}

a $\mathrm{rms}=$ root mean square. 


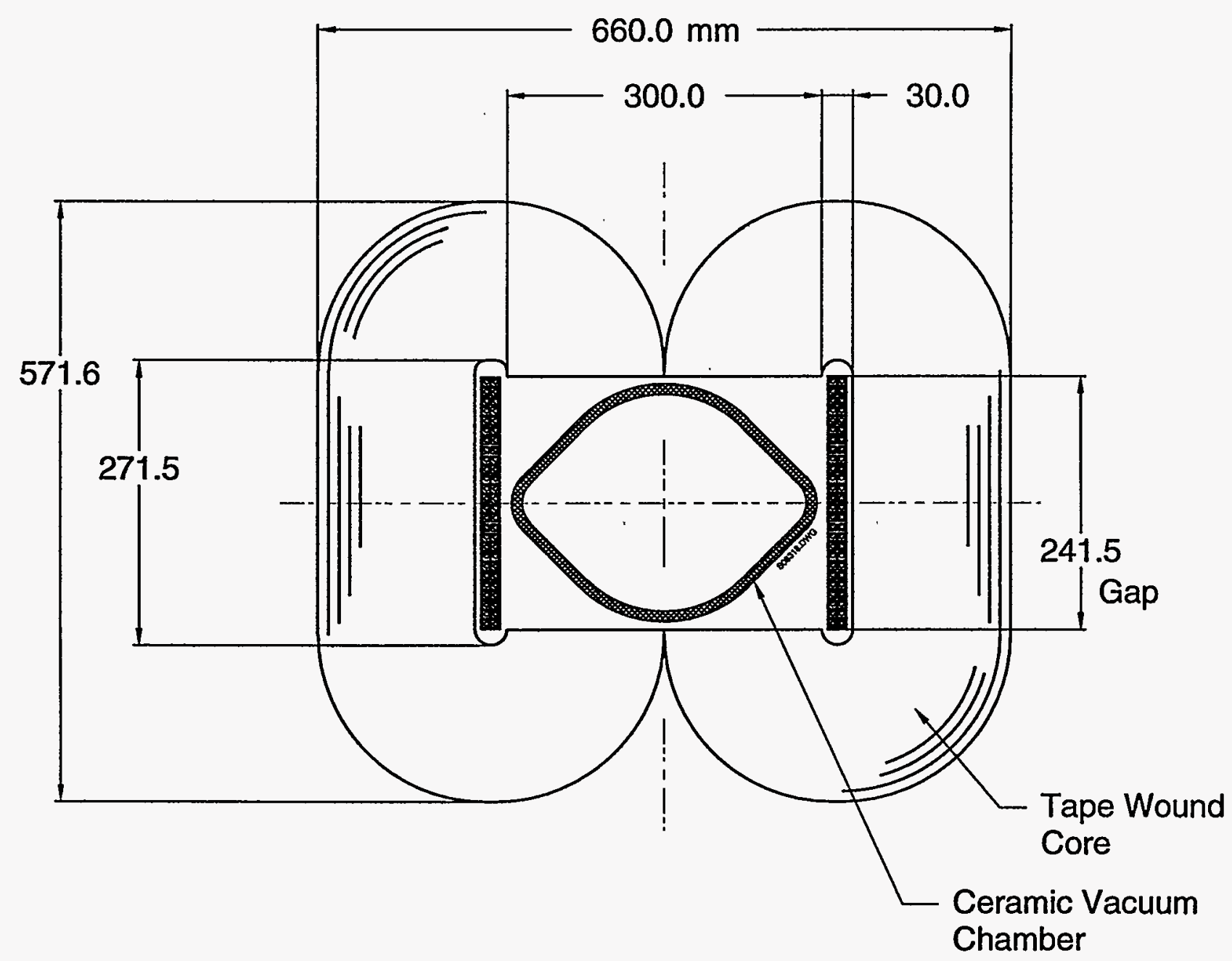

Figure I1.3.4-1 Cross Section of an Injection Bumper Magnet. 


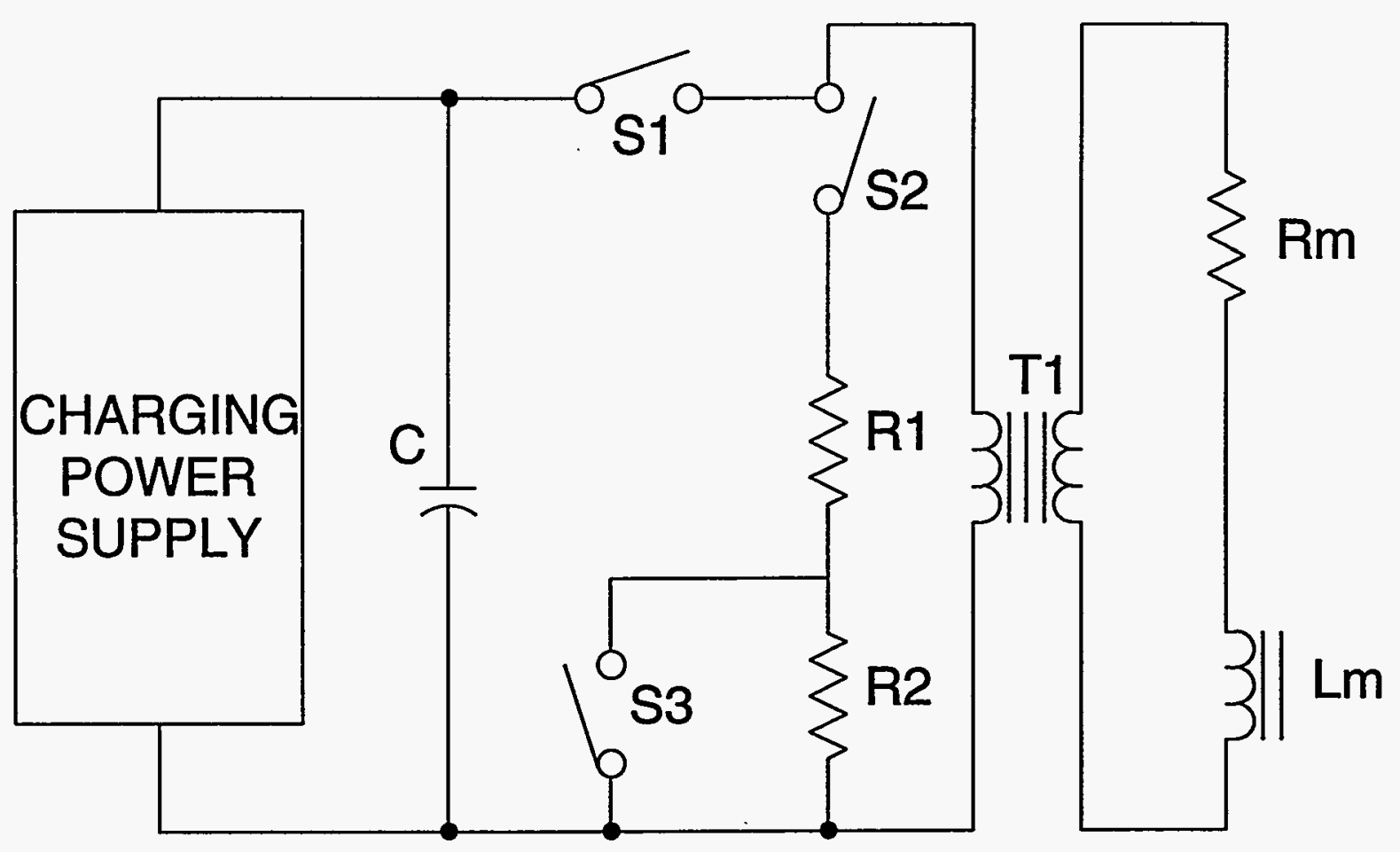

FILE: S08339F

Figure II.3.4-2 Circuit Diagram for the Bumper-Magnet Power Supply. 


\subsubsection{Extraction Kicker System}

At extraction, the beam is kicked from the normal orbit into the aperture of the first septum magnet. Each of the two extraction kicker systems fits into a 2.9-m straight section. Each system is made up of three separate magnets, designated $A, B$, and $C$, to reduce the selfinductance. Thus, the required 350 -nsec rise time is achieved with a commercially available $50-\mathrm{kV}$ power supply. Each kicker magnet is individually powered. Extraction kicker parameters are listed in Table II.3.4-2. The aperture of each kicker magnet is large enough to match the BSC size in both height and width, to allow space for a ceramic vacuum chamber with 10 -mm-thick walls and to provide $2.5-\mathrm{mm}$ clearance on all sides. Each kicker magnet is also matched to the $15-\mathrm{kA}$ peak current of the chosen thyratron and to the $50-\mathrm{kV}$ peak power supply voltage.

The kicker cores are constructed of 102-mm-thick ferrite blocks of type CMD5005. Picture-frame ferrite cores are used in each kicker. Each kicker magnet is made of two half-turn distributed transmission lines powered in parallel and magnetically isolated from each other by a copper sheet. An equivalent impedance of $3.28 \Omega$ is achieved by dividing the magnet core into longitudinal segments separated by matching capacitors and damping resistors. A cross section of kicker $A$ and the vacuum chamber at the upstream end is shown in Figure II.3.4-3. This ceramic vacuum chamber tapers in height and in width as it extends through all three kickers. The inside surface of this chamber is metalized to minimize the impedance to the beam.

Each extraction-kicker power supply provides pulses with rise times of $350 \mathrm{nsec}$, flattops of $315 \mathrm{nsec}$, and fall times about the same as the rise times, as shown in Figure II.3.4-4. The fall time must be less than $4.1 \mathrm{msec}$, to allow the supply to charge for the next pulse. Each of these supplies has a high-voltage dc (HVDC) power supply, two pulse-forming networks (PFNs), and a thyratron switch. The HVDC power supply is a constant-current charging supply, which eliminates the need for a charging control circuit. Each PFN is made of five 52 -m-long, 16.4- $\Omega$ coaxial cables connected in parallel to achieve an equivalent impedance of $3.28 \Omega$. The total capacitance of two PFNs for one magnet is $0.2 \mu \mathrm{F}$. The stored energy in each PFN at $50 \mathrm{kV}$ is $250 \mathrm{~J}$. The power rating of the HVDC power supply at $30 \mathrm{~Hz}$ is $7.5 \mathrm{~kW}$.

The fall time of the magnetic field for each of these kicker systems is about the same as the rise time. The current wave form has a trapezoidal shape, as shown in Figure II.3.4-4. The switching device is a commercially available thyratron that is rated for a maximum voltage of $70 \mathrm{kV}$, a peak current of $15 \mathrm{kA}$, and an average current of $5 \mathrm{~A}$.

A schematic diagram of an extraction-kicker power supply and magnet section is shown in Figure II.3.4-5. 
Table II.3.4-2 Parameters for Extraction Kicker Magnets

\begin{tabular}{|c|c|c|c|c|}
\hline Parameters & Kicker A & Kicker B & Kicker C & Units \\
\hline Bend angle & 4.37 & 4.79 & 4.84 & mrad \\
\hline Peak central field at $2.0 \mathrm{GeV}$ & 0.0618 & 0.0512 & 0.0408 & $\mathrm{~T}$ \\
\hline Effective length & 650 & 870 & 1,105 & $\mathrm{~mm}$ \\
\hline \multicolumn{5}{|l|}{ Gap } \\
\hline Height & 132.4 & 159.2 & 200.0 & $\mathrm{~mm}$ \\
\hline Width & 220.0 & 195.0 & 170.0 & $\mathrm{~mm}$ \\
\hline \multicolumn{5}{|l|}{ Core } \\
\hline Length & 500 & 720 & 995 & $\mathrm{~mm}$ \\
\hline Yoke thickness & 102 & 102 & 102 & $\mathrm{~mm}$ \\
\hline Inductance ${ }^{a}$ & 1.35 & 1.34 & 1.19 & $\mu \mathrm{H}$ \\
\hline Resistance $^{a}$ & 0.10 & 0.10 & 0.09 & $\mathrm{~m} \Omega$ \\
\hline \multicolumn{5}{|l|}{ Supply current } \\
\hline Maximuma & 6,648 & 6,611 & 6,626 & A \\
\hline $\mathrm{rms}^{\mathrm{a}, \mathrm{b}}$ & 24 & 24 & 24 & A \\
\hline Average $^{a}$ & 0.13 & 0.13 & 0.13 & A \\
\hline Peak voltage & 50,000 & 50,000 & 50,000 & v \\
\hline Power losses & 0.16 & 0.09 & 0.06 & $k W$ \\
\hline
\end{tabular}

\footnotetext{
a Parameter values are for series-connected coils.

b $r m s=$ root mean square.
} 


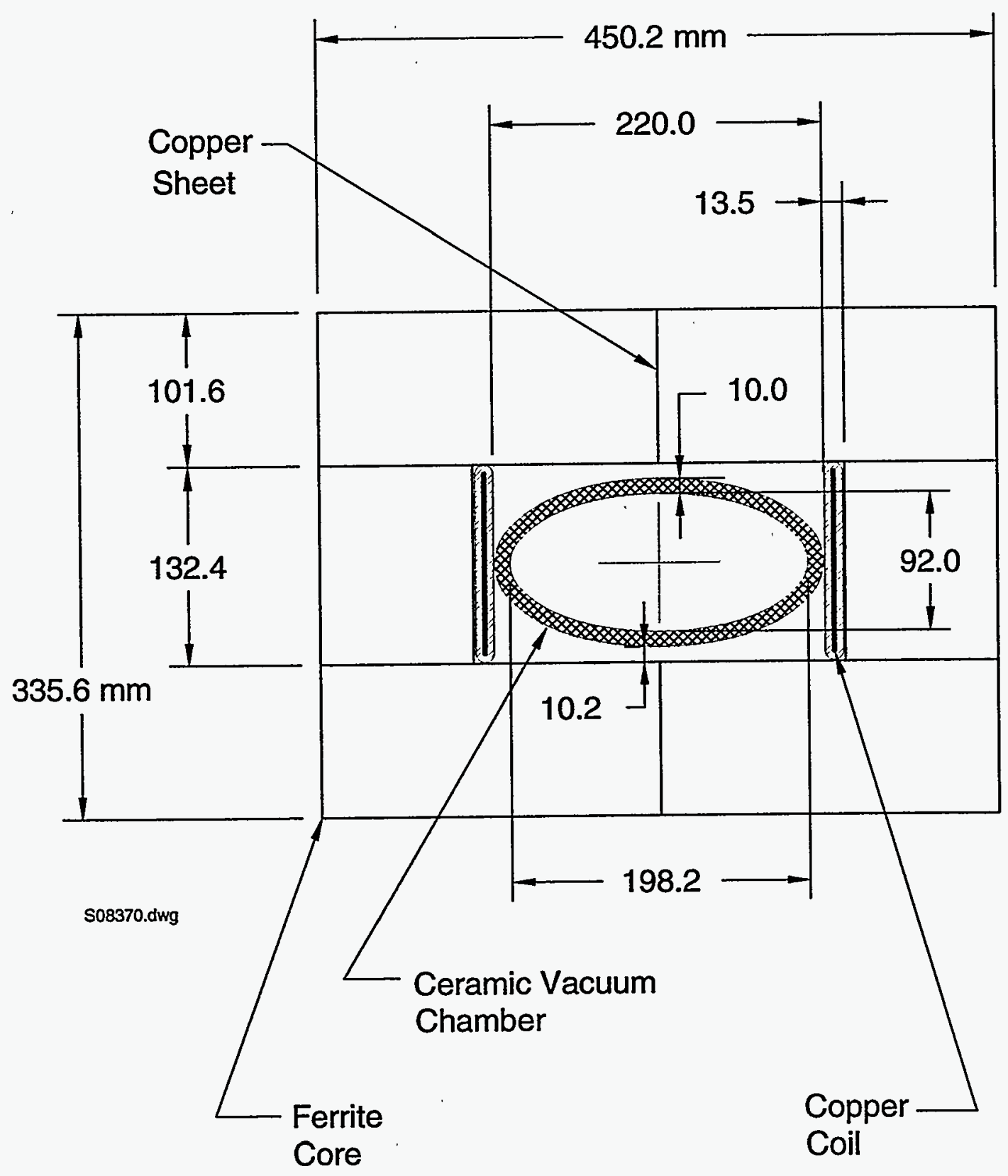

Figure II.3.4-3 Cross Section at Upstream End of Extraction Kicker A. 


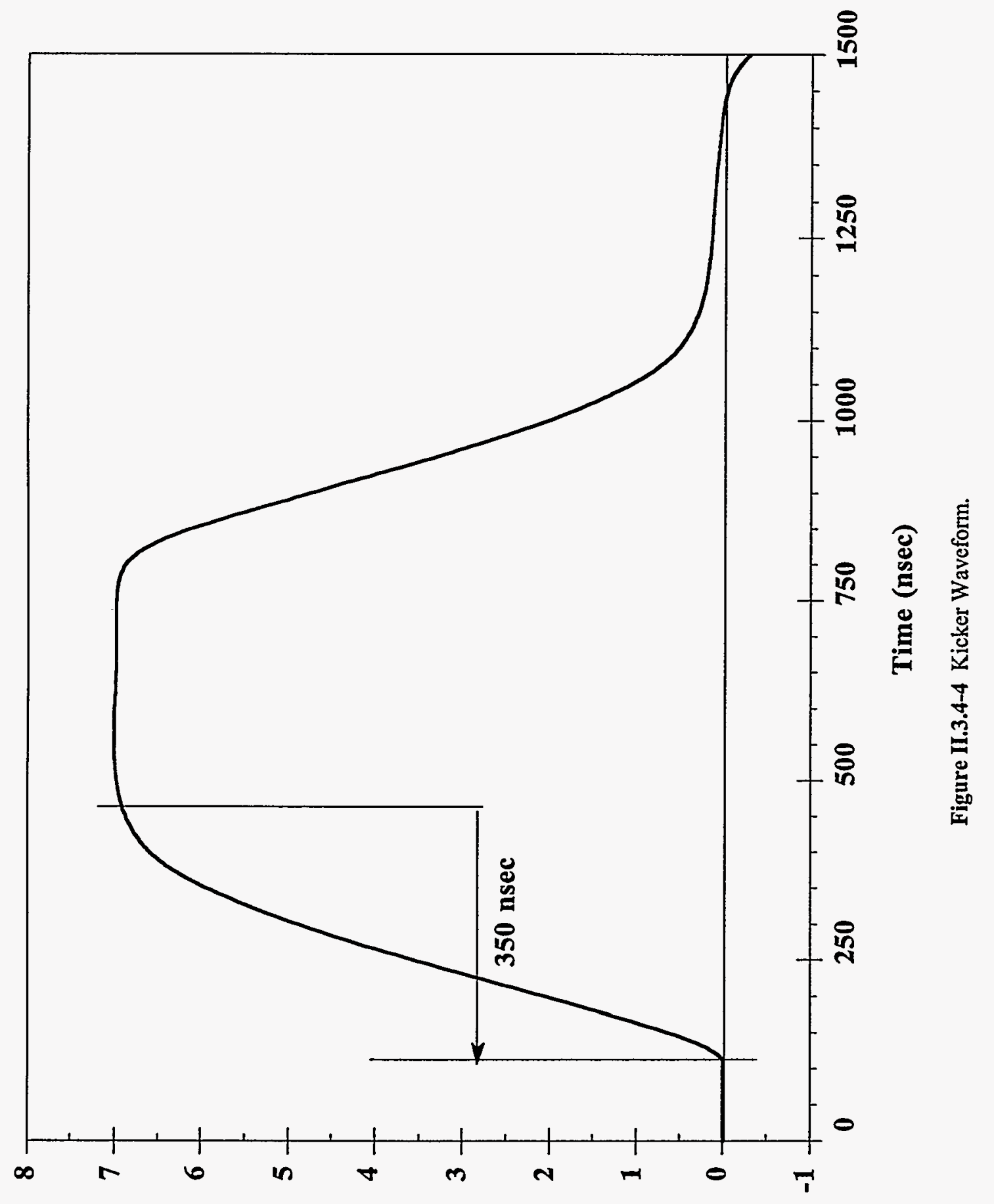

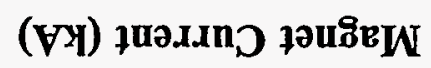




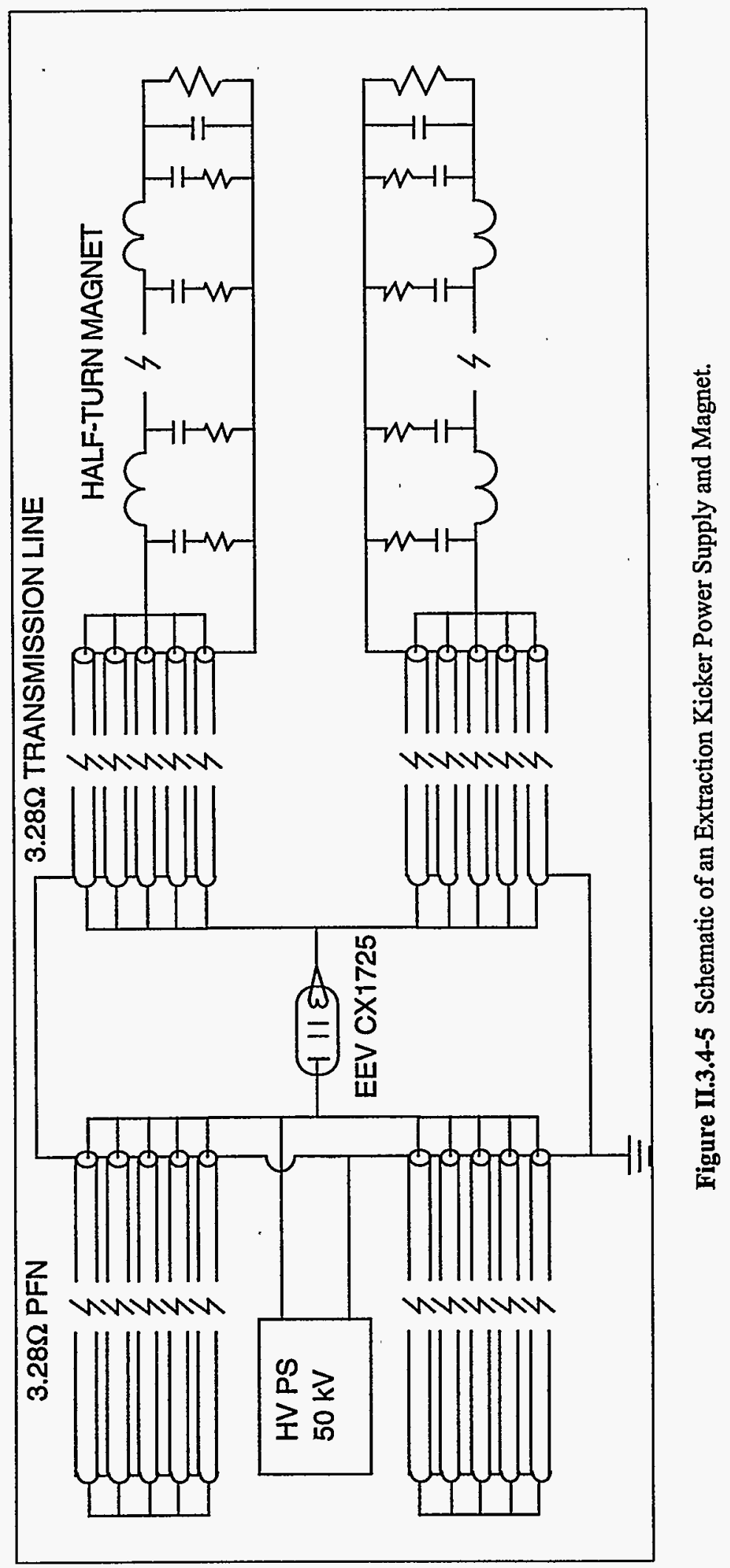




\subsection{SEPTUM MAGNETS AND POWER SUPPLIES}

\subsubsection{Introduction}

Septum magnets are those dipole magnets that form the interfaces between the three beam transport lines and the RCS. Injection septa are required to place the incoming beam on orbit. Extraction septa accept the beam from the kicker system and bend it in order to clear the adjacent quadrupole, which requires a displacement of $0.664 \mathrm{~m}$. The last injection septum element and the first extraction septum element are thin-pulsed septa. All septa are pulsed to reduce electrical power losses and to ease the associated cooling requirements. The required magnet apertures for injection and extraction are defined in Sections II.2.3 and II.2.4, respectively. The thin-septum magnets are located inside the straight-section vacuum chambers. There are thin Inconel vacuum chambers inside the apertures of the other magnets.

\subsubsection{Injection System}

The magnets septum $A$ and septum $B$ are used for injection into the synchrotron. Septum $A$ is 1.2-m long, has a field index $n=-20$, and has a curved axis to keep the beam trajectory centered in the gap. Septum B is 0.3-m long, has no field index, and is straight. Septum A and septum B are both excited with a single half-sine-wave pulse of $60 \mathrm{~Hz}$. Septum B has a reset pulse of similar shape. The parameters for these two magnets are listed in Table II.3.5-1. The cross sections of septum magnets A and B are shown in Figures II.3.5-1 and II.3.5-2, respectively.

Septum $A$ is a gradient dipole and is made with $0.36-\mathrm{mm}$-thick silicon steel laminations. The coil is made with a single size of hollow copper conductor that is insulated with mica-glass tape and vacuum-impregnated, heat-cured, radiation-resistant epoxy.

Septum B is a transformer type, with a single-turn secondary and a four-turn primary winding. The minimum total thickness of the septum is $3 \mathrm{~mm}$, but the septum gradually thickens along its length to improve the magnetic, mechanical, and thermal characteristics. The design of this magnet is similar to that of the pulsed septum magnet ${ }^{5}$ used to extract beam from the existing IPNS synchrotron. This design has proven performance and durability, $>2$ billion pulses at $30 \mathrm{~Hz}$, and peak central fields of $1 \mathrm{~T}$. The core is made with $0.36-\mathrm{mm}$-thick silicon steel laminations. The primary coil is made of hollow copper conductor that is insulated with micaglass tape and vacuum-impregnated, heat-cured, radiation-resistant epoxy.

\subsubsection{Extraction System}

The septum magnets used for extraction from the synchrotron consist of a fast-pulsed septum magnet, septum $C$, followed by two slower-pulsed magnets, septum D and septum $E$. 
Table II.3.5-1 Parameters for Synchrotron Injection Septum Magnets

\begin{tabular}{|c|c|c|c|}
\hline Parameters & Septum A & Septum B & Units \\
\hline Peak strength at $400 \mathrm{MeV}$ & 0.56 & 0.52 & $T$ \\
\hline Field index & -20 & 0.0 & - \\
\hline Effective length & 1.2 & 0.3 & $\mathrm{~m}$ \\
\hline \multicolumn{4}{|l|}{ Gap } \\
\hline Height & 20 & 24 & $\mathrm{~mm}$ \\
\hline Width & 51 & 40 & $\mathrm{~mm}$ \\
\hline Minimum septum thickness & 33.4 & 3.0 & $\mathrm{~mm}$ \\
\hline \multicolumn{4}{|l|}{ Conductor } \\
\hline Height & 4.2 & 5.0 & $\mathrm{~mm}$ \\
\hline Width & 4.2 & 5.0 & $\mathrm{~mm}$ \\
\hline Hole diameter & 2.4 & 4.9 & $\mathrm{~mm}$ \\
\hline Number of turns per magnet & 16 & 4 & $\therefore$ \\
\hline Inductance & 1.1 & 0.023 & $\mathrm{mH}$ \\
\hline Resistance & 74 & 3.4 & $\mathrm{~m} \Omega$ \\
\hline Pulse width (half-sine-wave shape) & 8.3 & 8.3 & msec \\
\hline Repetition rate & 30 & 30 & $\mathrm{~Hz}$ \\
\hline Reset pulse width & - & 8.3 & msec \\
\hline \multicolumn{4}{|l|}{ Supply current } \\
\hline Maximum & 568 & 2,533 & A \\
\hline $\mathrm{rms}^{\mathrm{a}}$ & 201 & 1,264 & A \\
\hline rms current density in coil & 6.2 & 38 & $\mathrm{~A} / \mathrm{mm}^{2}$ \\
\hline Peak voltage & 240 & 306 & V \\
\hline Power losses & 2.6 & 5.5 & $k W$ \\
\hline Cooling circuits/magnet & 4 & 2 & - \\
\hline Total water flow & 1.0 & 1.8 & $\mathrm{gal} / \mathrm{min}$ \\
\hline Water pressure drop & 100 & 100 & psi \\
\hline Water temperature rise & 11 & 12 & ${ }^{\circ} \mathrm{C}$ \\
\hline
\end{tabular}

a $\mathrm{rms}=$ root mean square. 


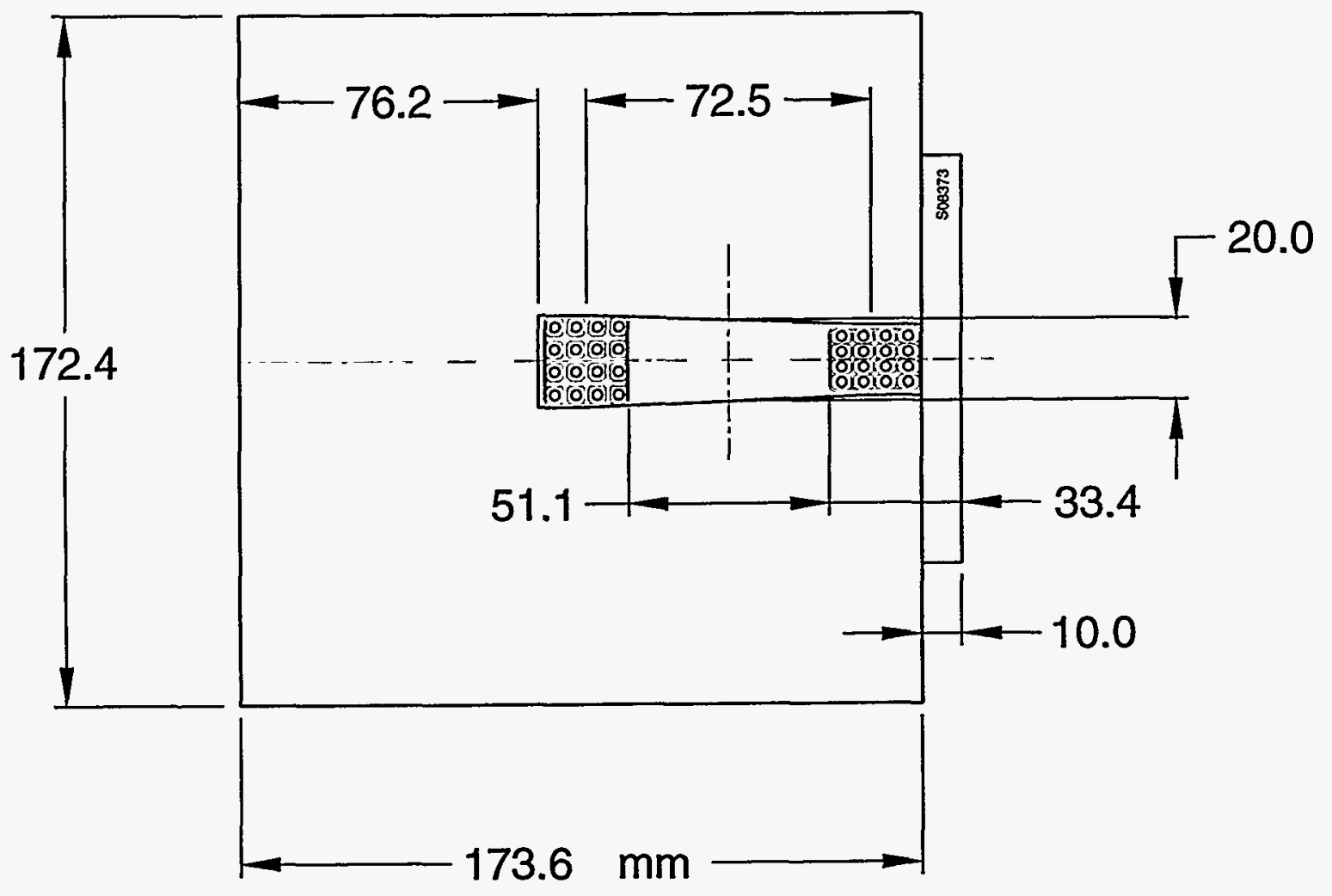

Figure II.3.5-1 Cross Section of Injection Septum A. 


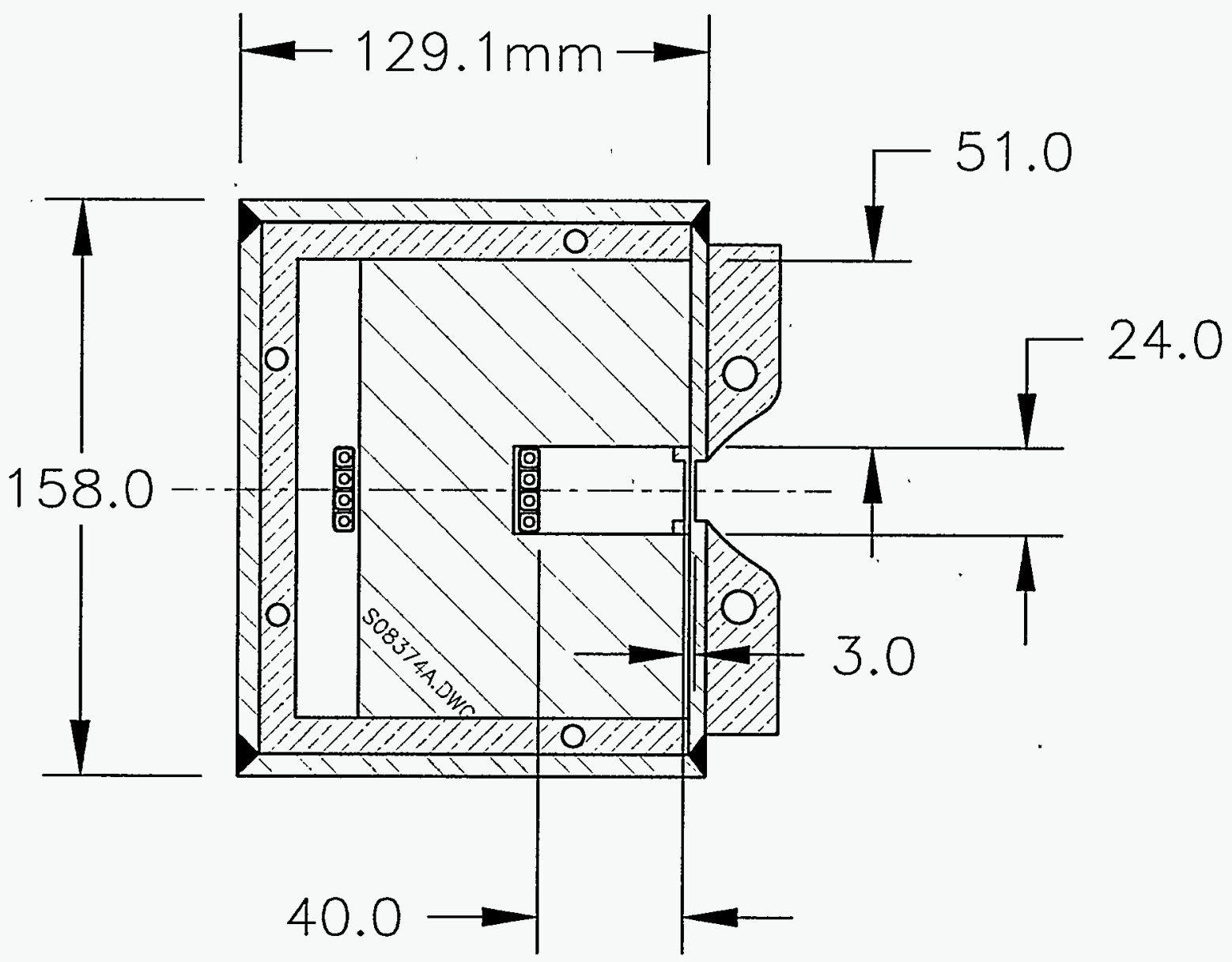

COPPER

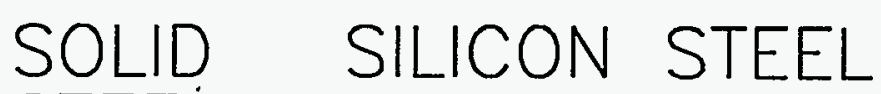
STEEL LAMINATIONS
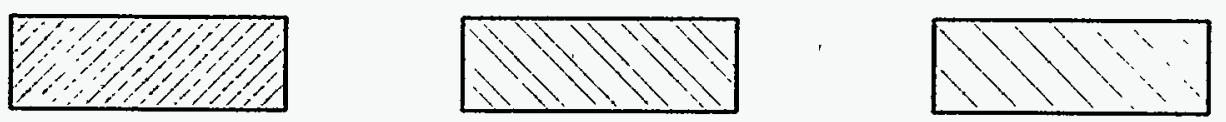

Figure II.3.5-2 Cross Section of Injection Septum B. 
The axis of septum $C$ is straight. The axes of septum $D$ and septum $E$ are curved to keep the magnet apertures as small as possible and to allow for thicker septa. The parameters for these three magnets are listed in Table II.3.5-2. The cross sections of septum C, septum D, and septum E are shown in Figures II.3.5-3, II.3.5-4, and II.3.5-5, respectively.

\subsubsection{Septum-Magnet Power Supplies}

There are eight septum magnets, as listed in Table II.3.5-3. Septum A and septum B are used for injection from the LET into the RCS; septum C, septum D, and septum E are used for extraction into the HET lines. All eight septum power supplies are designed for $30-\mathrm{Hz}$ operation. They have the same components except for the charging-power supplies, the output capacitor banks, the chokes used for energy recovery from the five thick septa, and the impedance-matching transformers that are used with the three thin septa. Using the same components minimizes the spare parts count. The basic capacitor discharge power supply is shown in Figure II.3.5-6.

All magnets have a half-sine-wave discharge pulse width of $8.3 \mathrm{msec}$, except for septum C, which has a 1-msec discharge pulse. All septum power supplies use energy recovery. The thin septa get the correct polarity back on the discharge capacitor bank by means of a reset pulse through the magnets. The thick septa get the correct polarity back on the discharge capacitor bank by means of a reset pulse using a choke in place of the magnet. The peak current in all septa is repeatable within $\pm 0.05 \%$. The magnet and power supply parameters are given in Table II.3.5-3. 
Table II.3.5-2 Parameters for Synchrotron Extraction Septum Magnets

\begin{tabular}{|c|c|c|c|c|}
\hline Parameters & Septum C & Septum D & Septum E & Units \\
\hline Peak strength at $2.0 \mathrm{GeV}$ & 1.02 & 1.30 & 1.30 & $\mathbf{T}$ \\
\hline Effective length & 0.5 & 1.5 & 0.9 & m \\
\hline \multicolumn{5}{|l|}{ Gap } \\
\hline Height & 70 & 90 & 110 & $\mathrm{~mm}$ \\
\hline Width & 122 & 102 & 70 & $\mathrm{~mm}$ \\
\hline Minimum septum thickness & 6.0 & 57.1 & 227.7 & $\mathrm{~mm}$ \\
\hline \multicolumn{5}{|l|}{ Conductor } \\
\hline Height & 7.3 & $7.9 / 12.0$ & 12.3 & $\mathrm{~mm}$ \\
\hline Width & 7.3 & $7.9 / 12.0$ & 12.3 & $\mathrm{~mm}$ \\
\hline Hole diameter & 4.1 & $4.4 / 6.6$ & 6.8 & $\mathrm{~mm}$ \\
\hline Number of turns per magnet & 8 & 48 & 64 & - \\
\hline Number of parallel conductors & 1 & 1 & 2 & - \\
\hline Inductance & 0.072 & 9.2 & 6.4 & $\mathrm{mH}$ \\
\hline Resistance & 6.1 & 47 & 15 & $\mathrm{~m} \Omega$ \\
\hline Pulse width (half-sine-wave shape) & 1 & 8.33 & 8.33 & msec \\
\hline Repetition rate & 30 & 30 & 30 & $\mathrm{~Hz}$ \\
\hline Reset pulse width & 1 & - & - & msec \\
\hline \multicolumn{5}{|l|}{ Supply current } \\
\hline Maximum & 7,268 & 1,982 & 1,817 & A \\
\hline rms & 1,259 & 699 & 641 & A \\
\hline rms current density in coil & 18 & $8.7 / 3.6$ & 1.8 & $\mathrm{~A} / \mathrm{mm}^{2}$ \\
\hline Peak voltage & 1,688 & 3,679 & 5,083 & V \\
\hline Power losses & 12.2 & 35.8 & 12.6 & $\mathrm{~kW}$ \\
\hline Cooling circuits/magnet & 4 & 8 & 4 & - \\
\hline Total water flow & 7.0 & 9.0 & 4.3 & $\mathrm{gal} / \mathrm{min}$ \\
\hline Water pressure drop & 100 & 100 & 100 & psi \\
\hline Water temperature rise & 7 & 15 & 11 & ${ }^{\circ} \mathrm{C}$ \\
\hline
\end{tabular}

a $\mathrm{rms}=$ root mean square. 


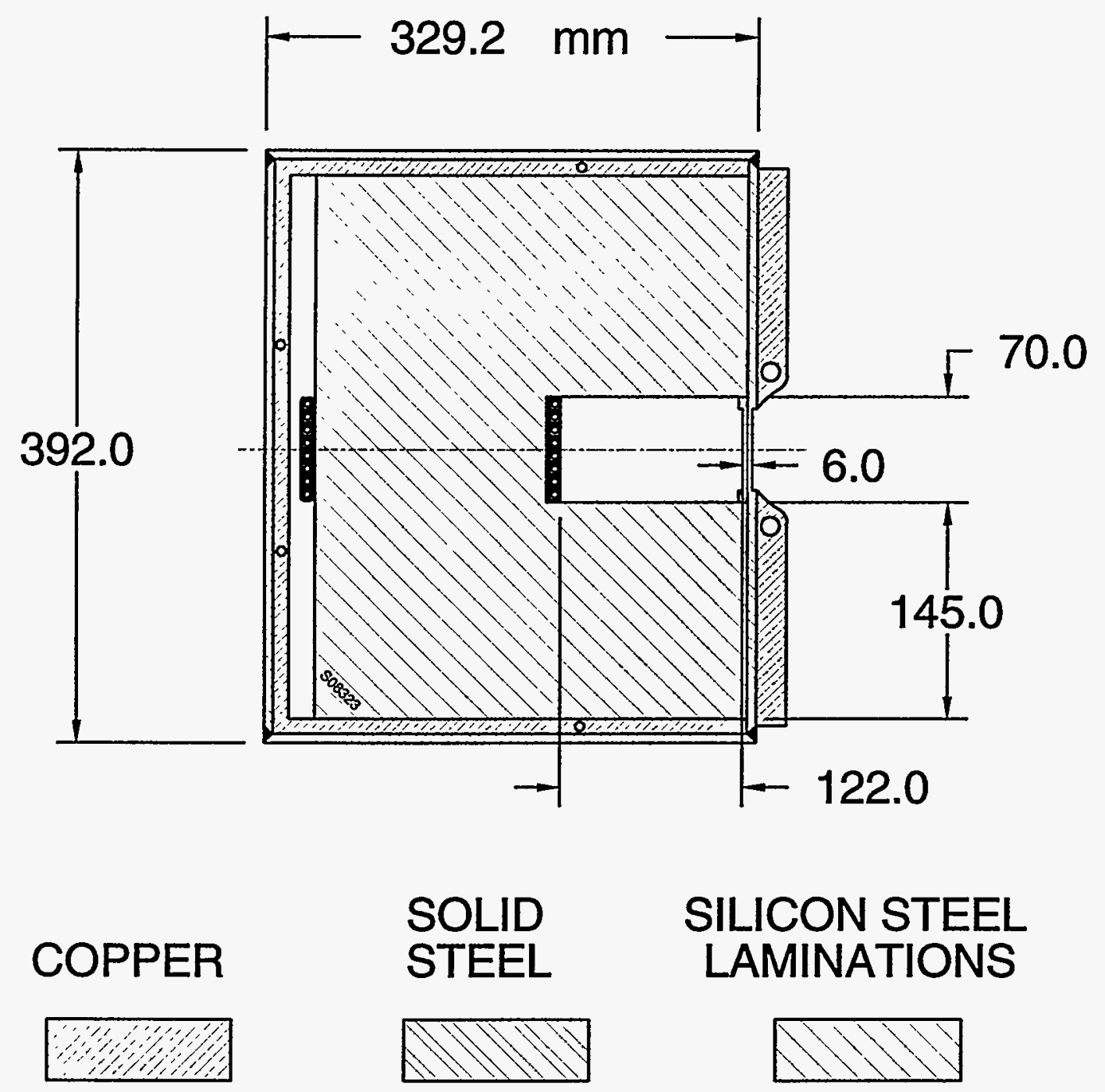

Figure II.3.5-3 Cross Section of Extraction Septum C. 


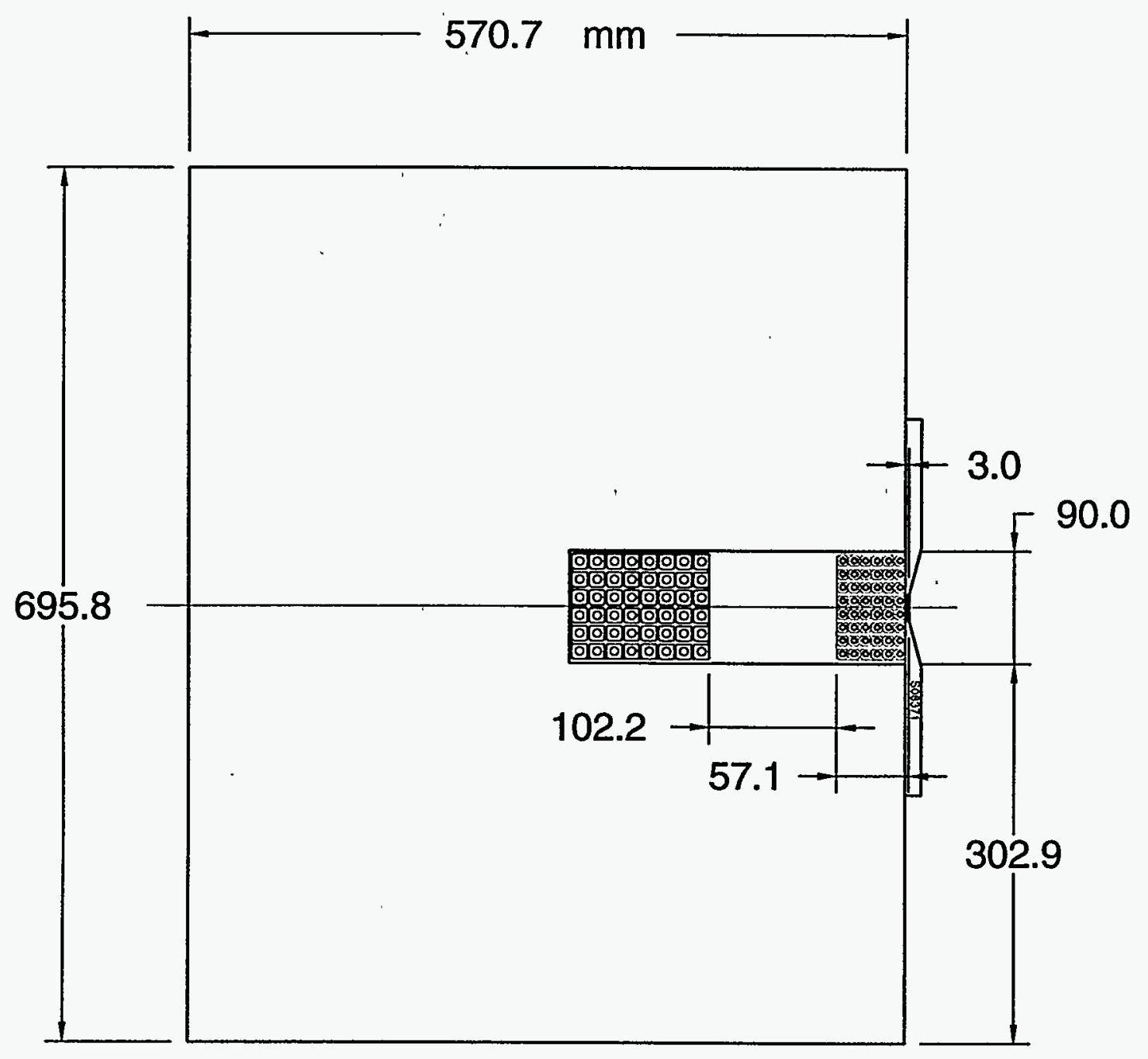

Figure II.3.5-4 Cross Section of Extraction Septum D. 


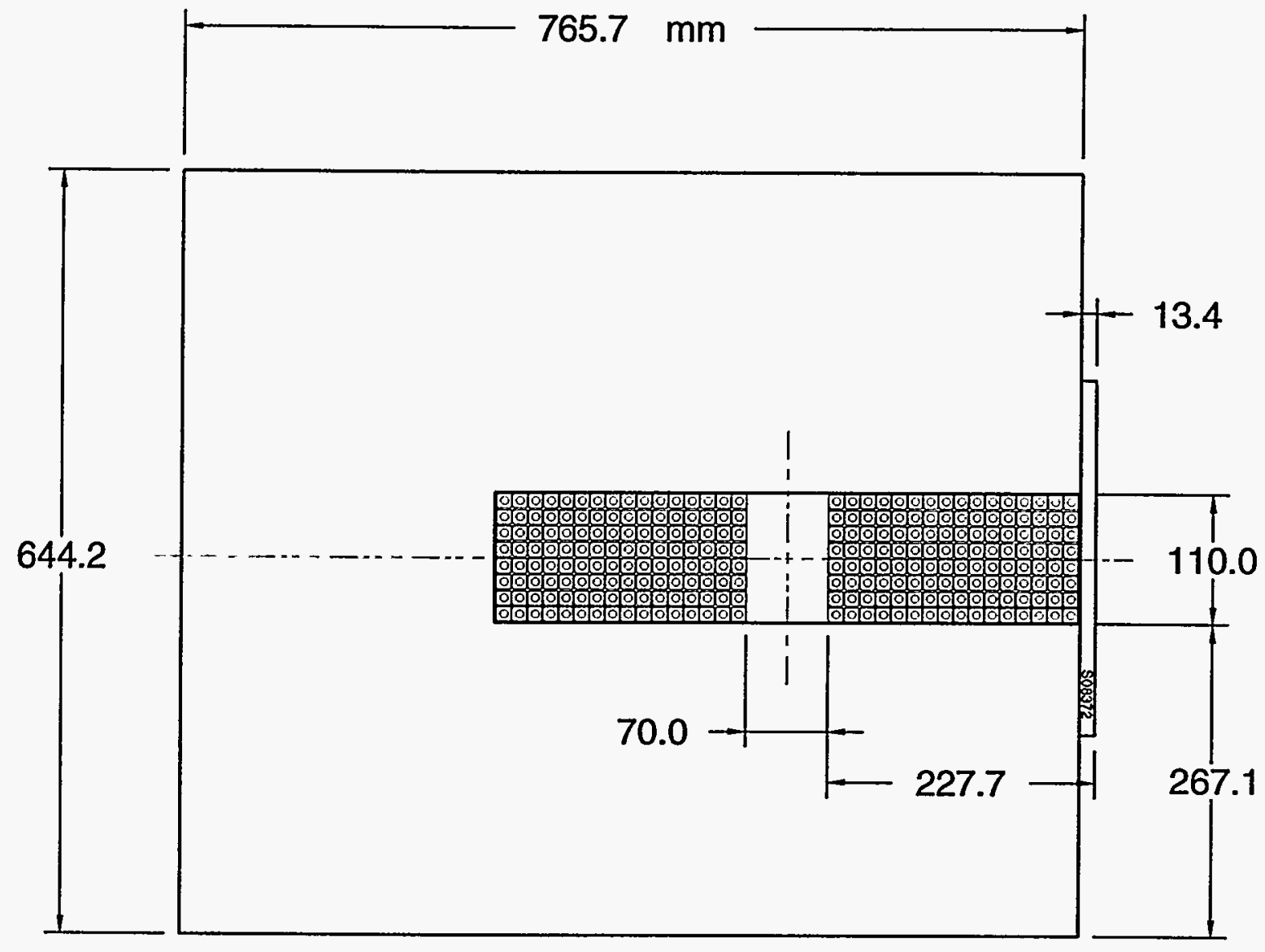

Figure II.3.5-5 Cross Section of Extraction Septum E. 
Table II.3.5-3 Septum Magnet Power Supply Parameters for 2.2-GeV Operation

\begin{tabular}{|c|c|c|c|c|c|c|}
\hline Location & LET & LET & HET & HET & HET & Units \\
\hline Septum & A & B & C & $D$ & $E$ & - \\
\hline Type & Thick & Thin & Thin & Thick & Thick & - \\
\hline Number of magnets & 1 & 1 & 2 & 2 & 2 & - \\
\hline Pulse width (half-sine wave) & 8.3 & 8.3 & 1.0 & 8.3 & 8.3 & msec \\
\hline Repetition rate & 30 & 30 & 30 & 30 & 30 & $\mathrm{~Hz}$ \\
\hline PS $^{a}$ peak current & 625 & 2,786 & 8,484 & 1,958 & 1,579 & A \\
\hline PS rms current & 258 & 267 & 1,470 & 691 & 557 & A \\
\hline PS peak voltage & 240 & 306 & 1,688 & 3,679 & 5,083 & V \\
\hline Magnet resistance & 74 & 3.4 & 6.1 & 47 & 15 & $\mathrm{~m} \Omega$ \\
\hline Magnet inductance & 1.1 & 0.023 & 0.072 & 9.2 & 6.4 & $\mathrm{mH}$ \\
\hline
\end{tabular}

a PS = power supply. 


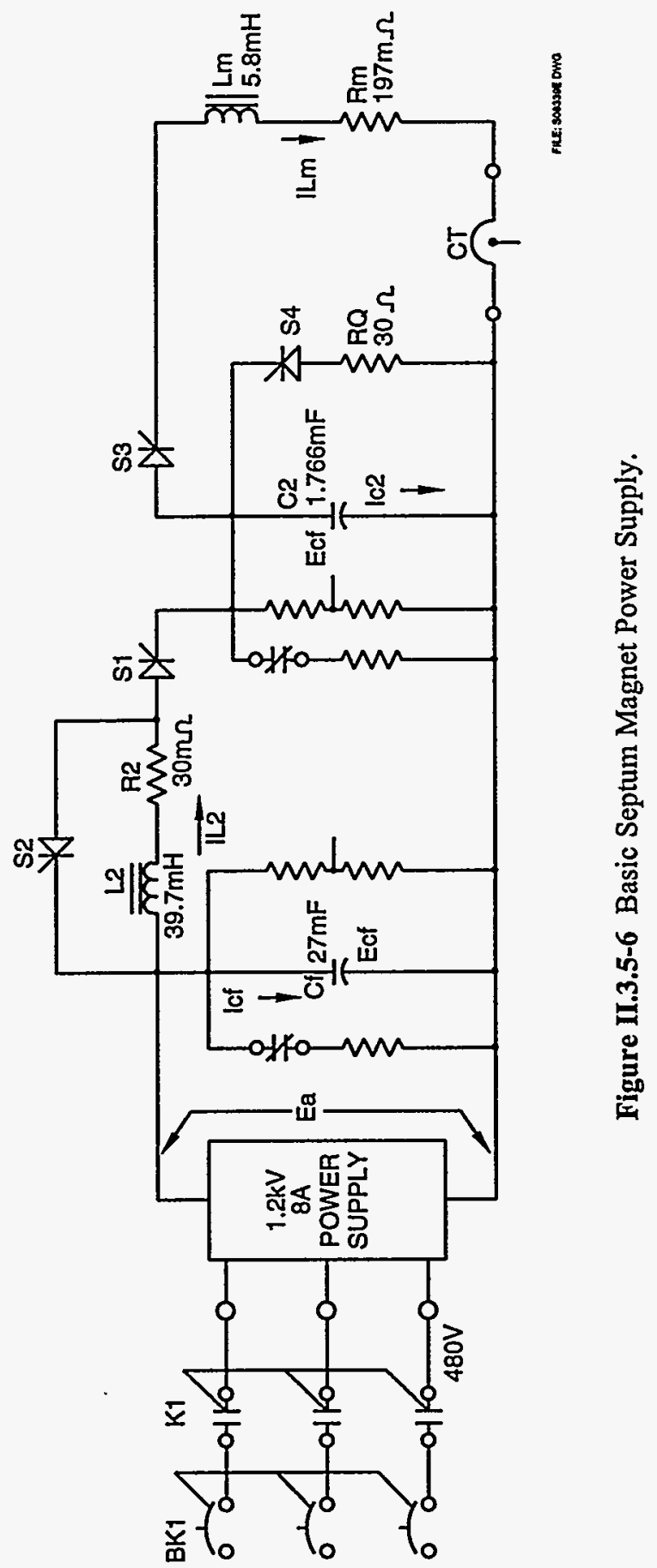




\subsection{MAGNET SUPPORTS}

\subsubsection{Synchrotron}

The synchrotron beam height is located $1.4 \mathrm{~m}$ above the floor. A structure that allows for both linear adjustments of $\pm 25 \mathrm{~mm}$ in all three directions and angular adjustments of more than $50 \mathrm{mrad}$ around all three axes is located between the bottom of the dipole and the floor. The dipole support shown in Figure II.3.6-1 includes an X-Y adjustor that is secured to the floor and a $\mathrm{Z}$ adjustor that is attached to the movable member. The $\mathrm{Z}$ adjustor incorporates a three-point support using fine-thread screws with spherical heads at each point, each of which can be adjusted and locked.

The quadrupole is held in place by a support structure similar to that for the dipole, but there is an additional weldment extending between the $\mathrm{X}-\mathrm{Y}-\mathrm{Z}$ adjustor on the floor and the bottom of the magnet. This support is shown in Figure II.3.6-1. There are outline representations of a sextupole and a horizontal correction dipole to show relative sizes and real estate usage. These smaller magnets have support hardware extending down to the quadrupole weldment, allowing them to be adjusted relative to the quadrupole.

\subsubsection{Transport Lines}

All of the transport-line dipoles have separate support stands and $X-Y-Z$ adjustors. Quadrupoles and nearby correction dipoles are supported by a single stand and adjustor. The correctors are mounted to the associated quadrupole. 


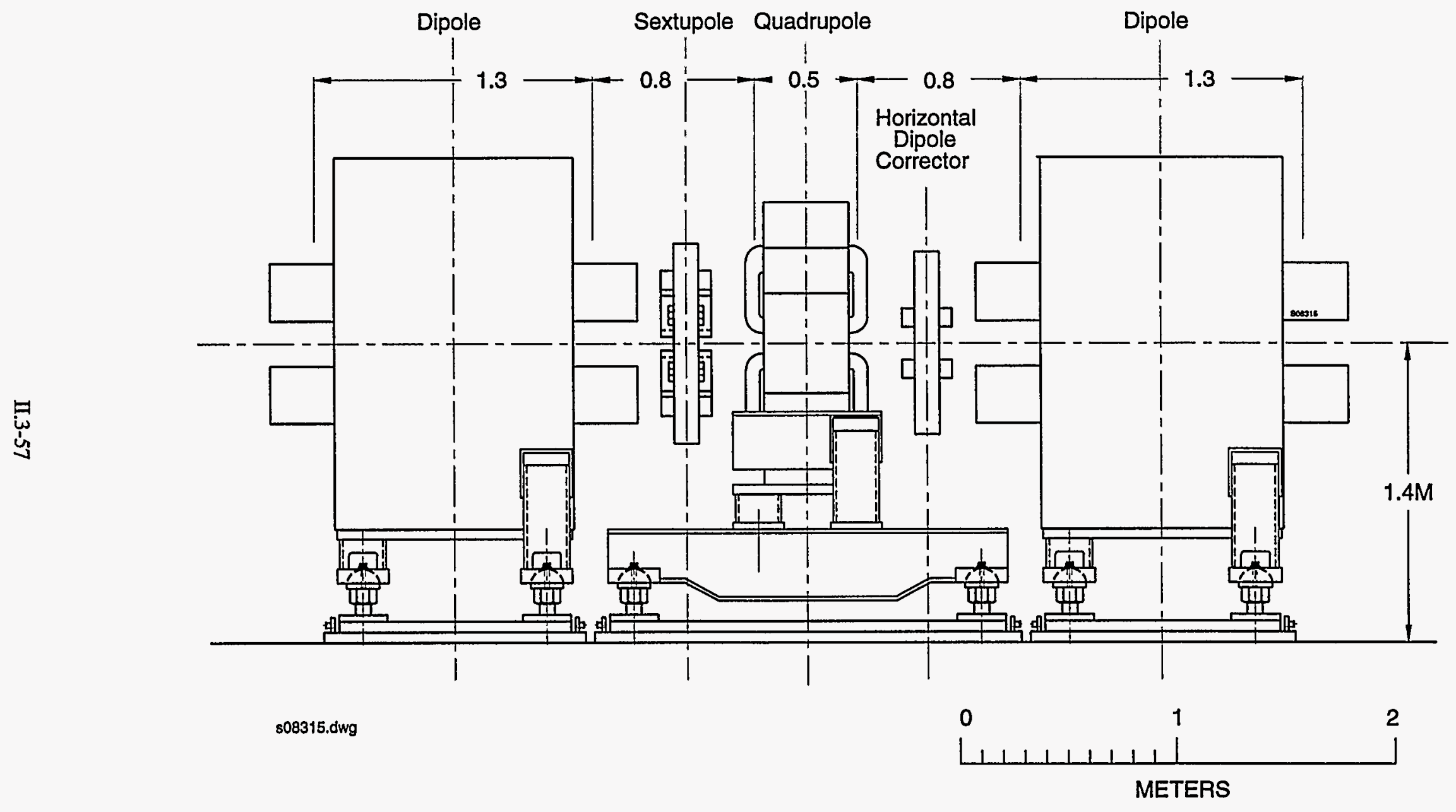

Figure II.3.6-1 Supports for the RCS Ring Magnets. (Dimensions are effective lengths.) 


\subsection{REFERENCES}

1. Code OPERA-2d (formerly referred to as PE2D) is available from Vector Fields, Inc., Aurora, Illinois.

2. K. Kim et al., "Design and tests of the injector synchrotron magnets for the 7-GeV Advanced Photon Source," in Proceedings of the 1993 IEEE Particle Accelerator Conference, p. 2808 (1993).

3. K.M. Thompson, "An interactive computer program for the design and costing of magnets," in Proceedings of the 8th International Conference on Magnet Technology (Grenoble, France, 1983).

4. 7-GeV Advanced Photon Source: Conceptual Design Report, Argonne National Laboratory Report No. ANL-87-15 (Apr. 1987).

5. M. Foss, K. Thompson, and W. Praeg, "A transformer septum magnet," IEEE Trans. Nucl. Sci. NS-26, 4024 (1979). 


\subsection{RF VOLTAGE PROGRAM}

\subsubsection{Introduction}

The key design feature of the RCS is the prevention of beam loss during the injection, capture, and acceleration processes. Beam loss is prevented by providing large dynamic apertures in both transverse planes and by tailoring the injected beam bunch and the if bucket areas to facilitate longitudinal motion without losses. In this section, we describe the if voltage programming for beam capture and acceleration.

The rf system of the RCS has a harmonic number equal to one, which provides a time gap in the accelerated beam that facilitates fast kicker operation for clean extraction. The if system has a modest frequency swing of 1.12-1.50 MHz, as shown in Figure II.4.1-1a. The maximum energy gain per turn is $81.4 \mathrm{keV}$, occurring at $\dot{\mathrm{B}}_{\text {max }}$, where $\mathrm{B}$ is the sinusoidal ring magnet dipole field discussed in Section II.2.1.2. The energy gain per turn is shown in Figure II.4.1-1b. The $\dot{\mathrm{B}}_{\max }$ occurs at $12.5 \mathrm{msec}$ into the acceleration cycle. The peak voltage requirement is estimated on the basis of $\dot{\mathrm{B}}_{\max }$ and the bucket acceptance.

The incoming linac beam energy spread ${ }^{1}$ is expected to be about $\pm 2.5 \mathrm{MeV}$. The circumference of the RCS is equivalent to $890 \mathrm{nsec}$ of revolution time at injection. Therefore, a circulating dc beam has a bunch area of about $4.5 \mathrm{eV} \mathrm{sec}$. This number is the starting point for bucket area considerations, which also take into account a dilution factor from bunch to bucket and chopped beam injection from the linac to tailor the bunch.

Simulation studies have shown that implementation of adiabatic capture, ${ }^{2}$ which captures the entire linac macro-pulse, is impractical, due to the lack of a long "flat-bottom field" in the RCS. Another way to achieve $100 \%$ capture is to inject chopped linac macro-pulses into a waiting bucket. Our studies show that the best capture efficiency is achieved with $25 \%$ chopping. The disadvantage is that $25 \%$ of the linac beam is discarded, so better ion source performance is required.

Under the best circumstances, the adiabatic capture process provides a phase space dilution factor of $\pi / 2$. Using that as a guide, we decided to allow a factor of about two in phase space dilution during the capture process, which makes the bucket area of the RCS equal to $9 \mathrm{eV} \mathrm{sec} \mathrm{soon}$ after injection. This bucket area is sufficient to contain the beam during the initial stages of acceleration, when the space charge forces are largest. The space charge self-fields in the longitudinal direction vary as the inverse of the energy squared. In the later stages of the acceleration period, the bucket area is adjusted to increase the momentum spread of the bunch, to prevent instabilities.

In the following sections, we discuss the rf voltage programming that was obtained from tracking simulation studies. These studies take into account space charge effects and have been used to achieve low loss conditions during injection, capture, and acceleration, while maintaining a 

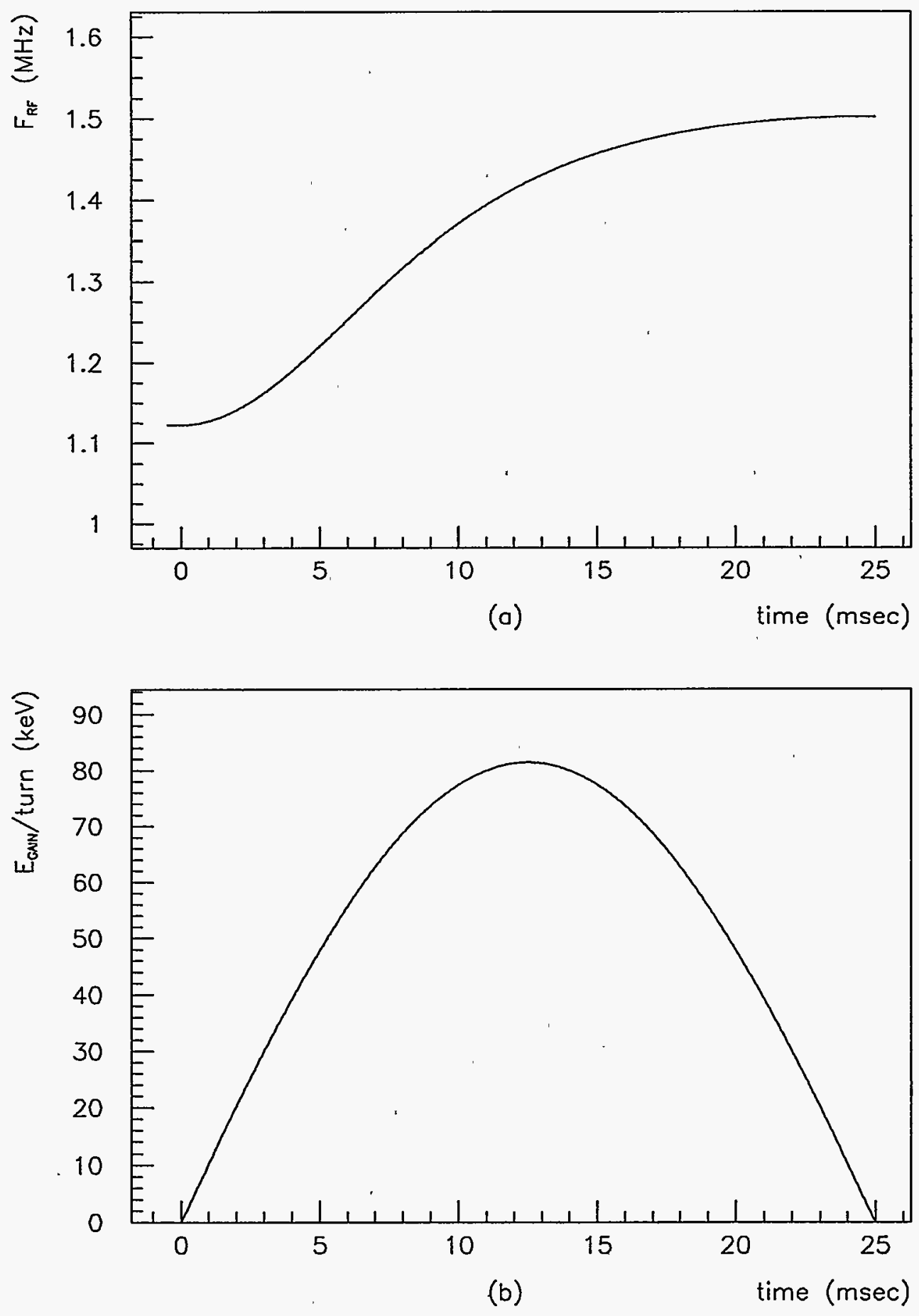

Figure II.4.1-1 (a) The If Frequency of the RCS from Injection to Extraction and (b) Energy Increase per Revolution of the Synchronous Particle. (Beginning of the accelerator clock is at $t=0$.) 
large momentum spread to avoid the microwave instability. We discuss the algorithm used in the tracking studies and then present the if voltage programming for the capture and acceleration processes that best meets the requirements.

\subsubsection{Longitudinal Tracking Studies}

The tracking studies are carried out using a Monte Carlo method according to the following algorithm:

- The incoming linac beam particle is represented by its energy deviation from the central energy, $\Delta \mathrm{E}$, and its phase coordinate, $\phi$. The value of $\phi$ can range from $-180^{\circ}$ to $180^{\circ}$ for unchopped beam injection or from $-135^{\circ}$ to $135^{\circ}$ for $25 \%$ chopped beam injection.

- The accumulation of 561 turns during the $0.5-\mathrm{msec}$ injection period is simulated turn-by-turn in order to understand the dynamics during accumulation with the rf voltage on.

- Macro-particle representation is used to describe the total charge in the bunch.

- The initial coordinates of each macro-particle, $\Delta \mathrm{E}$ and $\phi$, and the injectionturn number are retained throughout the calculation. Knowledge of the injection conditions of lost particles is used to minimize beam loss. This information is useful in designing a chopping system.

- At any point in time, the energy deviation and phase of all particles can be projected to the energy and phase coordinate axes to show the distributions of these quantities. The $\Delta \mathrm{E}$ and $\phi$ projections give the momentum spread and length of the bunch, respectively.

- A set of finite-difference recurrence equations suitable for numerical calculations is used to track the particles.

- The recurrence relations follow a time-stepping leapfrog method. When the particle motion is slowly varying, a large time-step is used to expedite the calculation. The recurrence relations are recalculated with this larger step size.

We assume that there is a synchronous particle that remains on the equilibrium orbit whose energy is denoted by $E_{s}$. The Hamiltonian, $H$, for longitudinal motion in a synchrotron, ${ }^{3}$ including space charge forces, for a particle of energy $E$ and phase $\phi$ is

$\mathrm{H}=\frac{\mathrm{h} \eta_{\mathrm{s}}}{\beta_{\mathrm{s}}^{2}} \frac{\omega_{\mathrm{s}}^{2}}{\mathrm{E}_{\mathrm{s}}} \mathrm{W}^{2}+\frac{\mathrm{d} \phi_{\mathrm{s}}}{\mathrm{dt}} \mathrm{W}+\frac{\mathrm{eV}}{2 \pi}\left(\cos \phi+\phi \sin \phi_{\mathrm{s}}-\cos \phi_{\mathrm{s}}-\phi_{\mathrm{s}} \sin \phi_{\mathrm{s}}\right)-\frac{\mathrm{e}^{2} \mathrm{~g}_{0} \mathrm{~h}^{2}}{4 \pi \varepsilon_{0} \gamma_{\mathrm{s}}^{2} \mathrm{R}} \lambda(\phi)$, 
where the subscript $\mathrm{s}$ refers to the synchronous particle and

$$
\begin{aligned}
\mathrm{h} & =\text { harmonic number, } \\
\eta_{\mathrm{s}} & =1 / \gamma_{\mathrm{t}}^{2}-1 / \gamma_{\mathrm{s}}^{2} \\
\omega_{\mathrm{s}} & =\text { angular revolution frequency, } \\
\beta_{\mathrm{s}}, \gamma_{\mathrm{s}} & =\text { relativistic factors, } \\
\gamma_{\mathrm{t}} & =\text { transition energy } \\
\phi_{\mathrm{s}} & =\text { rf phase of the synchronous particle, } \\
\mathrm{e} & =\text { electric charge, } \\
\mathrm{t} & =\text { time, } \\
\mathrm{V} & =\text { peak } \mathrm{ff} \text { voltage, } \\
\varepsilon_{0} & =\text { vacuum permittivity, } \\
\mathrm{R} & =\text { average ring radius, } \\
\lambda & =\text { linear particle density, and } \\
\mathrm{g}_{0} & =\text { capacitive geometrical factor }=1+2 \ln (\mathrm{b} / \mathrm{a}), \text { where } \mathrm{a} \text { is the beam radius }
\end{aligned}
$$

We define $W=\left(E-E_{s}\right) / \omega_{s}$, such that $d W \cdot d \phi$ is conserved. The last term in Equation Il.4.1-1 represents the space charge potential. The sign of the space charge term is opposite to that of the rf potential; thus, the space charge reduces the restoring force and changes the bucket shape.

The differential equations of motion derived from this Hamiltonian describe the timeevolution of a particle subject to an accelerating force that depends on the rf phase and to the space charge forces that act on the particle. These differential equations of motion are solved numerically by using leapfrog difference equations. ${ }^{3}$ The leapfrog algorithm advances the coordinates of each particle, $W$ and $\phi$, by a specified time-step, $\tau$, according to

$$
\phi_{n+1}=\phi_{n}+h \tau\left(\frac{\eta_{s} \omega_{s}^{2}}{\beta_{s}^{2} E_{s}} W\right)_{n+\frac{1}{2}}+\phi_{s, n+\frac{1}{2}}-\phi_{s, n-\frac{1}{2}}
$$

and 


$$
\mathrm{W}_{\mathrm{n}+\frac{1}{2}}=\mathrm{W}_{\mathrm{n}-\frac{1}{2}}+\frac{\mathrm{eV} \tau}{2 \pi}\left(\sin \phi_{\mathrm{n}}-\sin \phi_{\mathrm{s}, \mathrm{n}}\right)+\frac{\mathrm{e}^{2} \mathrm{~g}_{0} \mathrm{~h}^{2} \tau}{4 \pi \varepsilon_{0} \mathrm{R} \gamma_{\mathrm{s}}^{2}}\left(\frac{\mathrm{d} \lambda(\phi)}{\mathrm{d} \phi}\right)_{\mathrm{n}}
$$

where the subscript $n$ indicates that a quantity is to be calculated at a time $t=n \tau$. The values of the $\phi$ coordinates are calculated at integer time-steps, and the values of $W$ are calculated at halfinteger time-steps. The last two terms in Equation II.4.1-2 account for the changes in $\phi_{\mathrm{S}}$.

The numerical simulation of the injection, capture, and acceleration processes follows an ensemble of macro-particles, whose initial coordinates in phase and energy are chosen according to a specified distribution. A total of $10^{4}$ macro-particles is used to represent the $10^{14}$ protons in the bunch.

The turn-by-turn injection process is simulated by randomly placing $\mathrm{N}_{\mathrm{p}} / \mathrm{N}_{\mathrm{t}}$ macroparticles into the machine. Here, $N_{p}$ is the total number of macro-particles, and $N_{t}$ is the total number of injection turns. The particles are distributed uniformly in phase within the maximum and minimum values of the chopped beam. The energy distribution follows a cosine function from $-\pi / 2$ to $\pi / 2$, where the base is equal to $\pm 2.5 \mathrm{MeV}$. The particle distribution rapidly becomes governed by the synchrotron oscillations and is fairly insensitive to the initial energy distribution chosen.

During injection, the time-step size is equal to the revolution period. At each turn, the $\mathrm{W}$ coordinates of the $\mathrm{N}_{\mathrm{p}} / \mathrm{N}_{\mathrm{t}}$ injected particles are advanced by a half-step. In the first half-step of each injection turn, the space charge forces due to the incoming particles are small and are neglected. In the iteration process, all the particles, including the most recently injected, are advanced in $\phi$ by a time-step according to Equation II.4.1-2. The space charge forces due to all particles present in the ring are then calculated, and their contribution is used in Equation II.4.1-3 to advance the $\mathrm{W}$ coordinates by a time-step.

During the acceleration process, the time-step size can be increased to reduce computer time, but it must be kept smaller than the synchrotron oscillation period, $\tau_{\mathrm{s}}$. In the simulations, the time-step size is kept smaller than $\tau_{\mathrm{s}} / 30$.

The initial phase space coordinates for each particle are saved. The analysis of lost particles using these parameters permits the design of a more efficient injection scheme by depopulating the undesirable phase space. The vacuum chamber wall is represented by a momentum cutoff of $\Delta \mathrm{p} / \mathrm{p}=0.05$. A particle outside the separatrix at a given time, but with momentum $\Delta p / p<0.05$, contributes to the space charge self-field and may be recaptured later in the cycle. The particle can also hit the vacuum chamber wall. In that case, the particle is removed from the calculations, so it cannot contribute to the space charge forces. In our tests, an average delay of $2 \mathrm{msec}$ is observed between the time a particle leaves the separatrix and the time it hits the vacuum chamber wall. 
To reduce the statistical fluctuations due to the relatively coarse ensemble of macroparticles used in the simulations, the projected phase distribution is binned by using the cloud-incell method. For each particle, a weighted contribution is assigned to the two closest grid points, according to how far the particle is from these points. The cell grid is chosen fine enough to describe the structure of the particle distribution in a bunch, yet coarse enough to attenuate the short wavelength noise due to the statistical fluctuations in a cell. The data are then fast-Fouriertransformed and filtered to reduce the Gibbs phenomenon. Our studies show that for the bunch lengths considered, cutoff frequencies ranging from the 9 th to 11 th harmonics reduce noise. The underestimation of the peak and overestimation of the bunch ends, introduced by the filtering process, are acceptable. As the bunch length shortens during the cycle, higher cutoff frequencies are used.

The simulation code was tested extensively. The code was checked for the correct stationary and moving buckets in the absence of space charge forces. The program was run above the transition energy by reversing the sign of the slip factor, $\eta_{\mathrm{s}}$, to produce the negative mass instability. The if voltage was turned off and space charge forces were included to verify that the negative mass instability appears with the correct threshold and correct growth rate. The simulation result for the negative mass instability threshold energy spread is $1.15 \mathrm{MeV}$, compared to the analytical prediction of $1.12 \mathrm{MeV}$. The growth rates for several perturbed density distributions were also in good agreement with the analytical values. For a beam with an initial energy spread of $\pm 1 \mathrm{MeV}$, perturbed by a density modulation of harmonic 2 and amplitude $0.1 \mathrm{MeV}$, a growth rate of $0.042 \mathrm{msec}$ was obtained, compared to the analytical value of $0.040 \mathrm{msec}$.

The capture efficiency was studied for several scenarios of if programming, initial phase space distribution of the injected particles, and chopping of the linac beam. ${ }^{4}$ For each case, the momentum spread of the beam was tested against the theoretical microwave instability threshold at different times in the cycle. These studies led to the establishment of the rf programming and injected beam parameters that best meet the requirements of low loss and high microwave instability threshold. The rf programming is presented in the next section.

\subsubsection{RF Voltage Program for Capture and Acceleration}

The rf voltage program for the injection and acceleration cycles is depicted in Figure II.4.1-2. The time variation of the bucket and bunch areas is shown in Figure II.4.1-3. Details of the rf program and the bucket and bunch area variations during the injection period are shown in the inserts in these figures. The beginning of the acceleration clock is indicated by $t=0$.

A beam with $\pm 2.5 \mathrm{MeV}$ energy spread $\left(\Delta \mathrm{p} / \mathrm{p}=3.7 \times 10^{-3}\right)$, occupying $75 \%$ of the ring, is injected in 561 turns, which corresponds to an injection time of $0.5 \mathrm{msec}$. Since the variation of the magnetic field is negligible during that period, $\phi_{S}$ is assumed to be zero during injection. The bunch area of the injected beam is $3.3 \mathrm{eV} \mathrm{sec}$. 


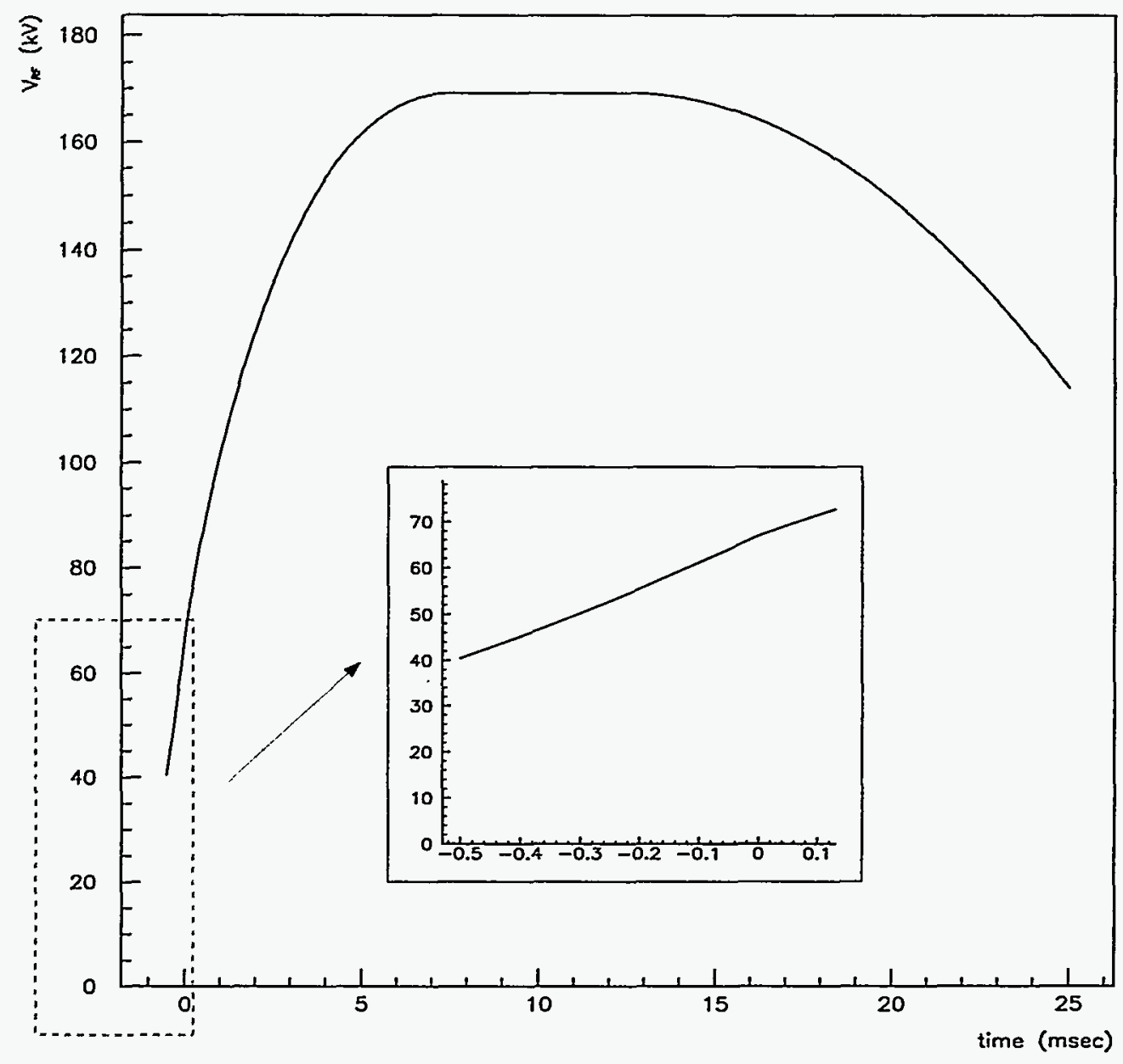

Figure II.4.1-2 Radio-Frequency Voltage Program for the Injection and Acceleration Cycles.

(The beginning of the acceleration clock is at $t=0$. The insert shows the details of the voltage program during injection. The voltage is chosen to maintain a constant bucket area from the end of injection to $7.5 \mathrm{msec}$. The voltage for the remainder of the cycle is adjusted to keep the beam below the longitudinal microwave instability threshold.) 


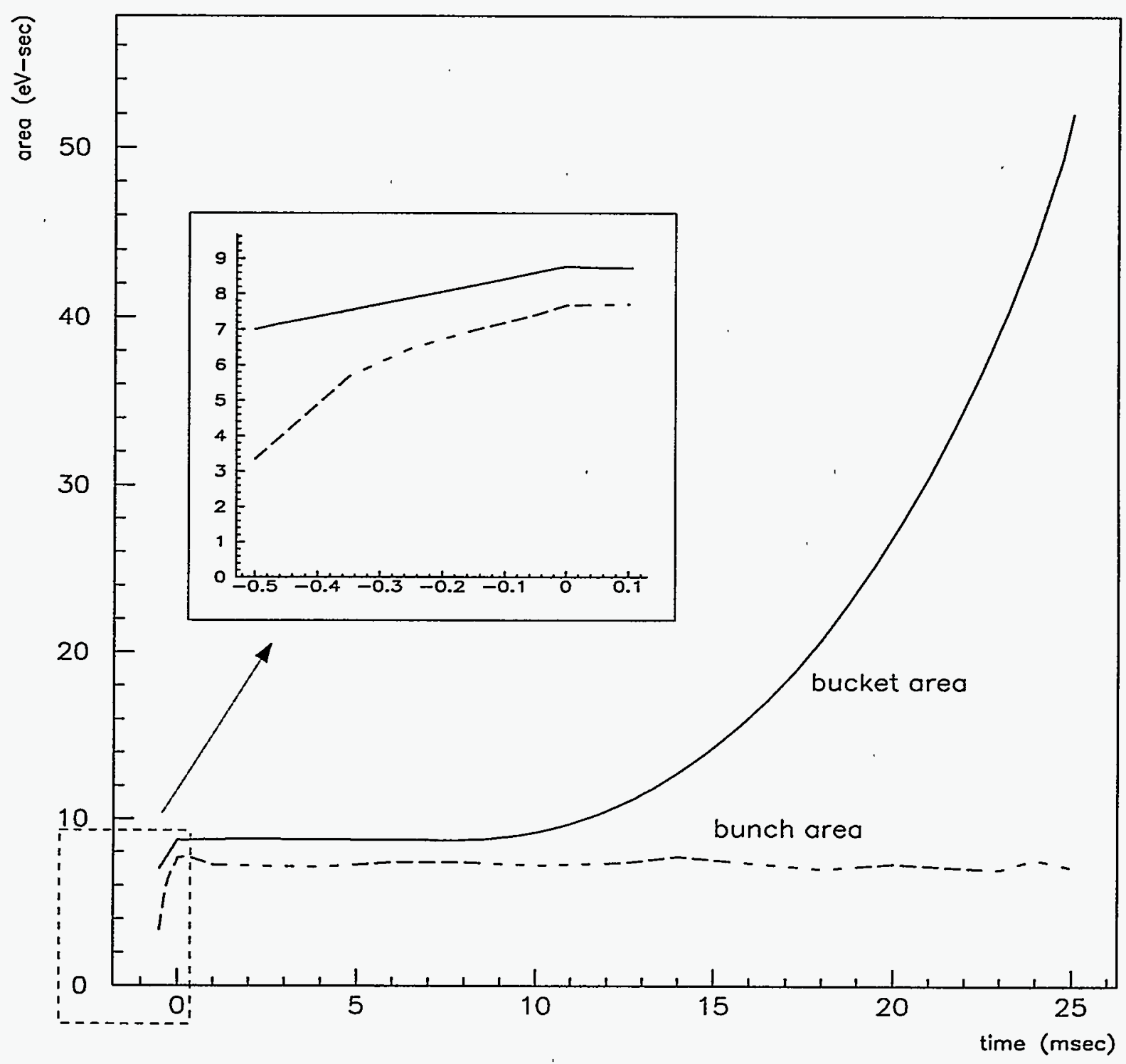

Figure II.4.1-3 Bucket and Bunch Areas, Obtained from the Simulation under the rf Voltage Program, Plotted as a Function of Time. (The injected beam has a $\pm 2.5 \mathrm{MeV}$ energy spread and occupies $75 \%$ of the ring. Details of the variations during injection are shown in the insert. The small variations in the bunch area are due to the Monte Carlo statistics.) 
To prevent unnecessary dilution of the beam and thus avoid large voltages at later stages, the initial voltage was set as low as possible. The minimum voltage required to contain the beam during the initial injection is $40.5 \mathrm{kV}$. Figure II.4.1-4 depicts the bucket area formed by this voltage and the particle distribution of the first injected turn. The figure shows that $40.5 \mathrm{kV}$ provides the necessary bucket height at the edges of the $\pm 2.5 \mathrm{MeV}$ beam chopped by $25 \%$. The corresponding bucket area of $7 \mathrm{eV} \mathrm{sec}$ allows an initial phase space dilution factor of two. During injection, the voltage must be increased from $40.5 \mathrm{kV}$ to $66.9 \mathrm{kV}$ to prevent beam loss. This rapid voltage rise is crucial to overcome space charge forces. The space charge forces increase with increasing particle density, which can lead to a reduction of as much as $50 \%$ in the bucket area. The bucket area changes from 7 to $9 \mathrm{eV}$ sec during the injection period, as can be seen in Figure II.4.1-3. The bunch area grows from its initial value of $3.3 \mathrm{eV} \mathrm{sec}$ to $7.3 \mathrm{eV} \mathrm{sec}$ over the injection cycle, giving a dilution factor of 2.2. The bunch has undergone about 1.4 synchrotron oscillations during this period. The bunch area stays at $7.3 \mathrm{eV} \mathrm{sec}$ for the remainder of the cycle.

The $9 \mathrm{eV} \mathrm{sec}$ bucket area is maintained constant during the first $7.5 \mathrm{msec}$ of the acceleration period. The voltage reaches a maximum of $169.2 \mathrm{kV}$ at the end of this period. It should be noted that the maximum voltage does not occur at $12.5 \mathrm{msec}$, when $\dot{B}$ is at a maximum, but occurs at $7.5 \mathrm{msec}$, because $\eta_{\mathrm{s}}$ varies during acceleration.

In the remaining part of the cycle, the energy spread of the beam is controlled by manipulating the bucket area. As detailed in Section II.5.3, the $\Delta \mathrm{p} / \mathrm{p}$ requirement for longitudinal stability is met by making the bucket area large. Figure II.4.1-5a shows the $\Delta \mathrm{p} / \mathrm{p}$ variation from injection to extraction. The value of $\Delta \mathrm{p} / \mathrm{p}$ varies rapidly during injection, from $0.4 \%$ to $1.0 \%$ in $0.5 \mathrm{msec}$. It reaches a maximum of $1.2 \%$ at $2 \mathrm{msec}$ of acceleration and decreases to a value of $0.8 \%$ at the end of the cycle. The bucket and bunch heights are shown in Figure II.4.1-5b.

From $7.5 \mathrm{msec}$ to $12.5 \mathrm{msec}$, the bucket area increases from 9.0 to $11.3 \mathrm{eV} \mathrm{sec}$ while the voltage is maintained at $169.2 \mathrm{kV}$. The voltage is then decreased from $169.2 \mathrm{kV}$ to $113.9 \mathrm{kV}$ at extraction. The rf voltage for the latter part of the cycle is kept high, which causes the bucket area to increase, as shown in Figure II.4.1-3. The need to keep the voltage high is a consequence of the following phenomena: The value of $\dot{B}$ decreases rapidly and becomes zero at the peak field. Therefore, the synchronous phase angle also rapidly approaches $0^{\circ}$ at the same time, as shown in Figure II.4.1-6a. In order for the particles in the bunch to follow this rapidly changing $\phi_{\mathrm{s}}$, they must have a synchrotron motion fast enough to follow the change in stable phase angle. A synchrotron frequency of about $1 \mathrm{kHz}$ is required. Figure $11.4 .1-6 \mathrm{~b}$ shows the synchrotron frequency through the acceleration cycle.

The phase space distributions from this simulation study are shown in Figures II.4.1-7 and II.4.1-8. The plots in Figure II.4.1-7 show the distributions at the earlier stages of injection and acceleration, while those in Figure II.4.1-8 show the distributions at later stages. 


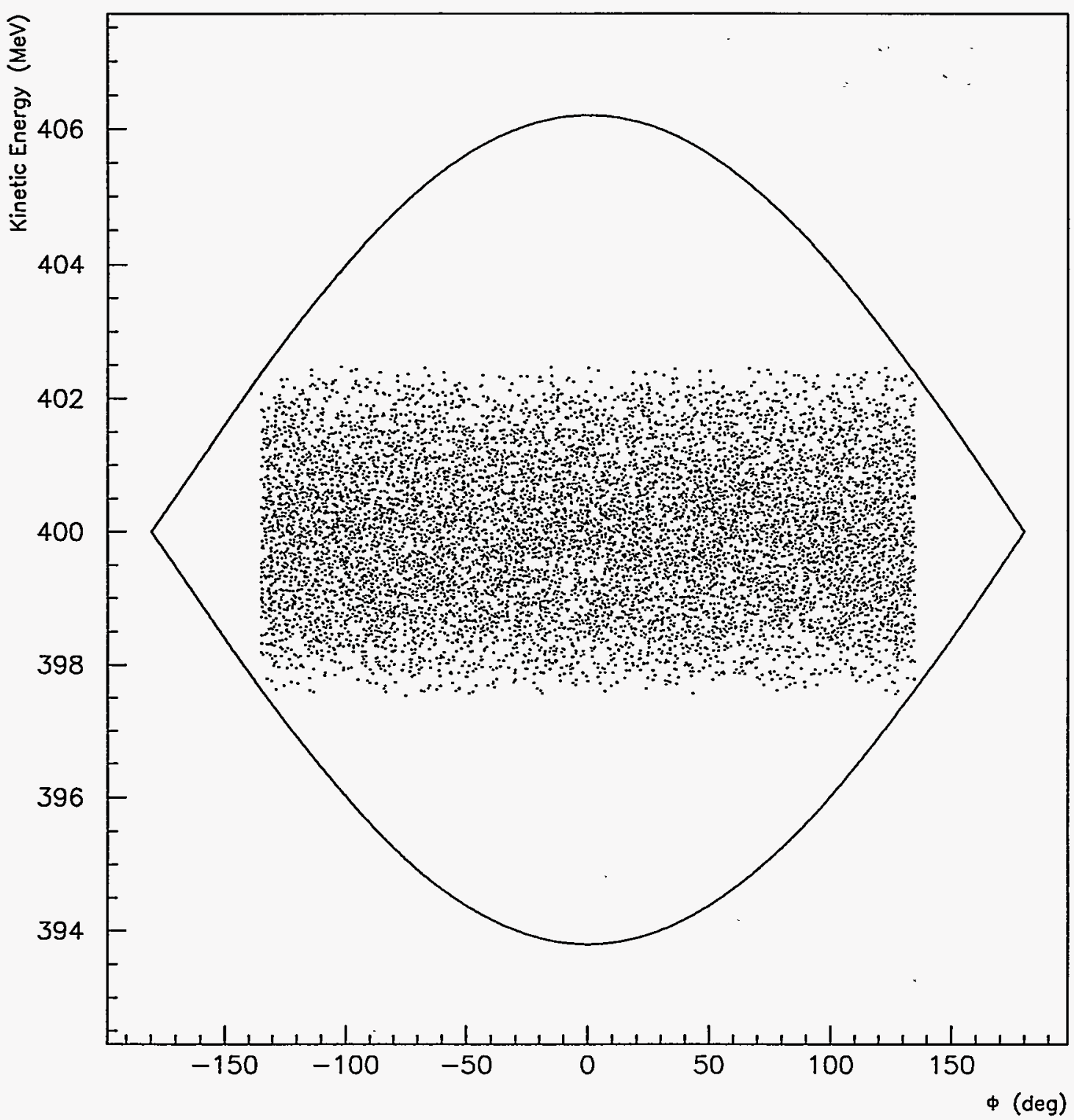

Figure II.4.1-4 Particle Distribution of the First Injected Turn. (The rf voltage of $40.5 \mathrm{kV}$ provides the necessary bucket height to contain the $\pm 2.5 \mathrm{MeV}$ beam chopped at $25 \%$. The bucket area is $7 \mathrm{eV} \mathrm{sec}$ and the bunch area is $3.3 \mathrm{eV} \mathrm{sec}$.) 

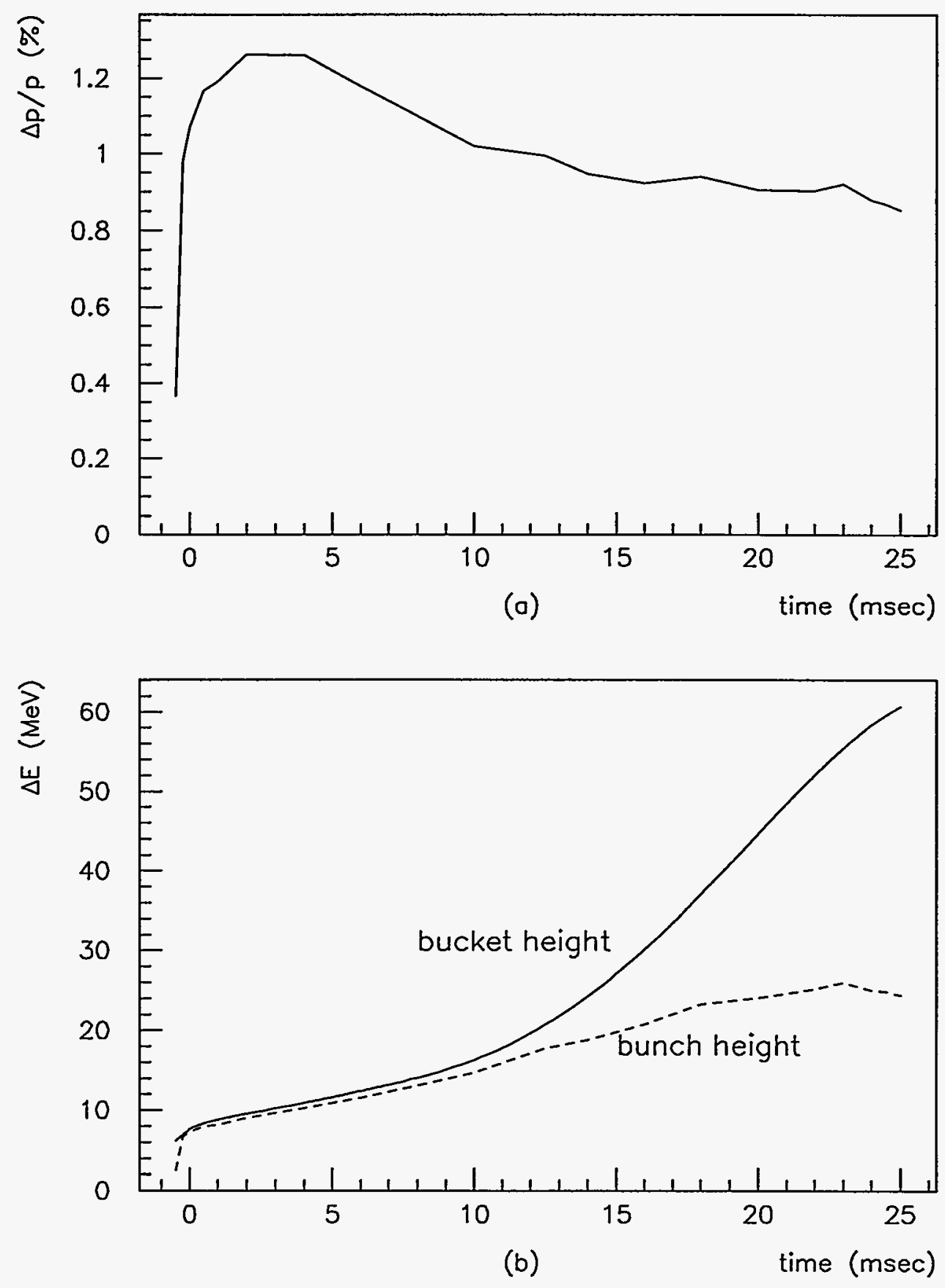

Figure II.4.1-5 (a) Half-Width of the Full Momentum Spread of the Bunch Obtained from the Simulation as a Function of Time; (b) Bucket and Bunch Height Growth from Injection to Extraction. 

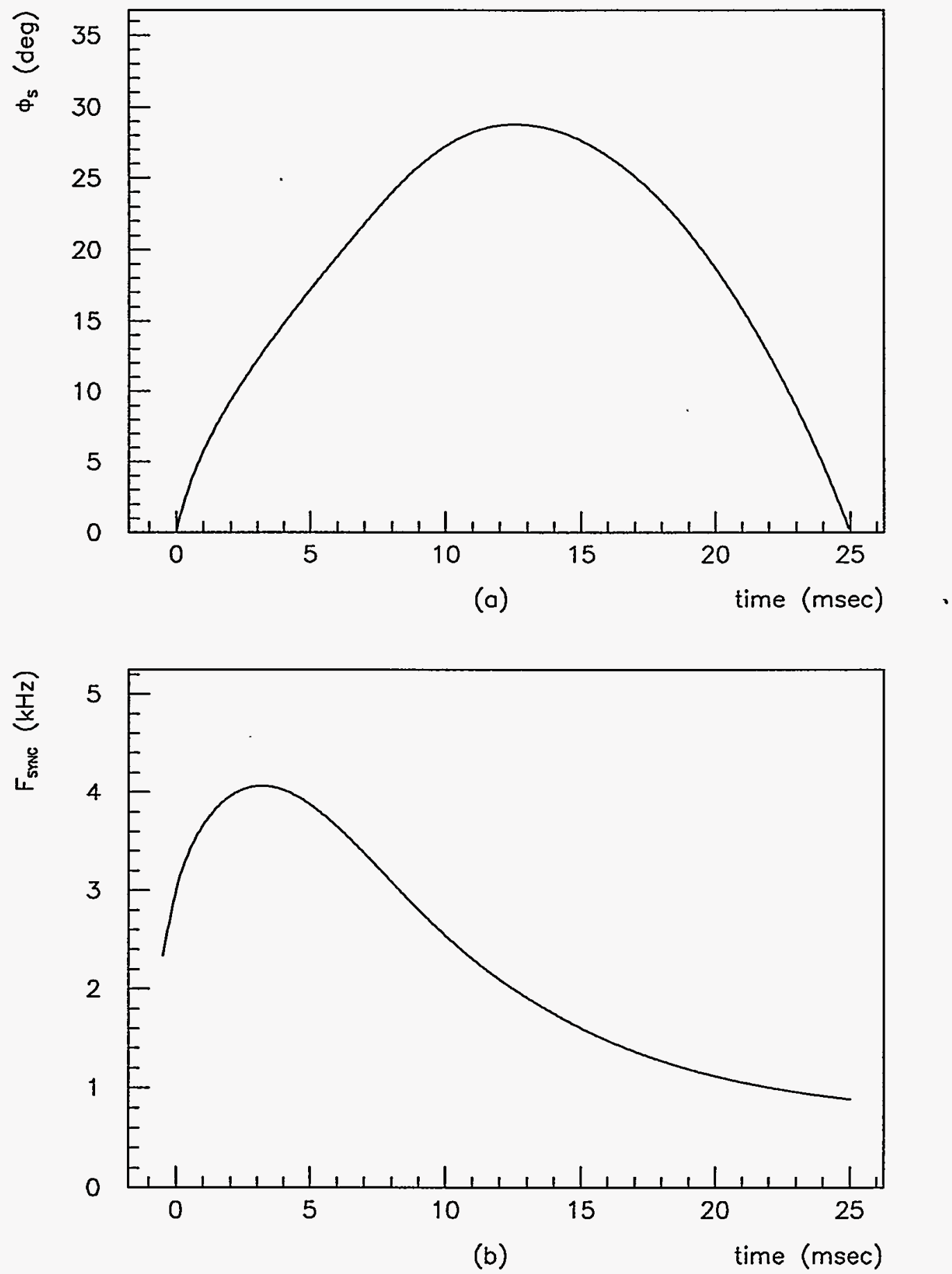

Figure II.4.1-6 Synchronous Phase (a) and Synchrotron Frequency (b) Variations from Injection to Extraction 


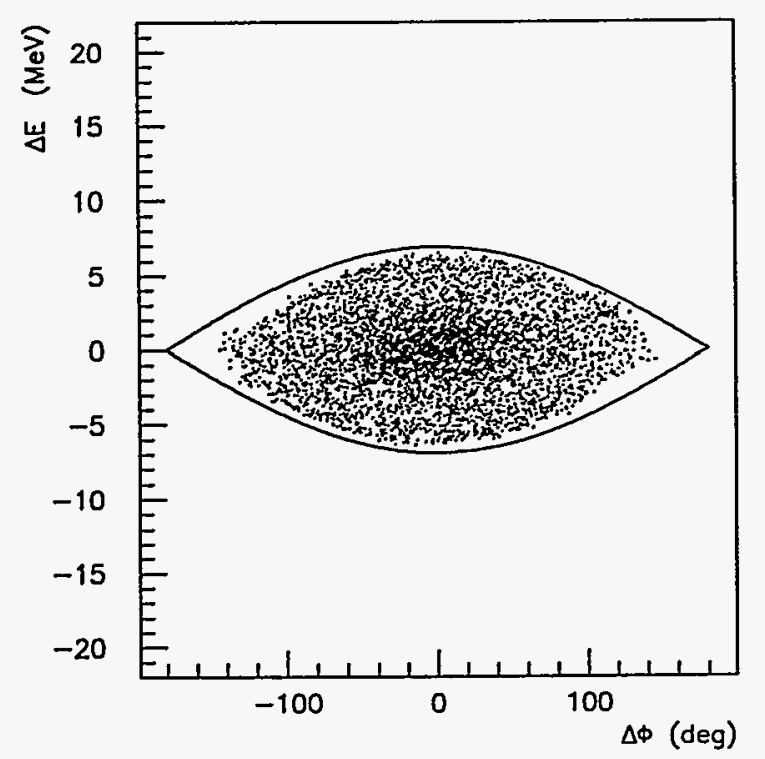

(a) $t=-0.25 \mathrm{msec}, V_{R F}=52.9 \mathrm{kV}$

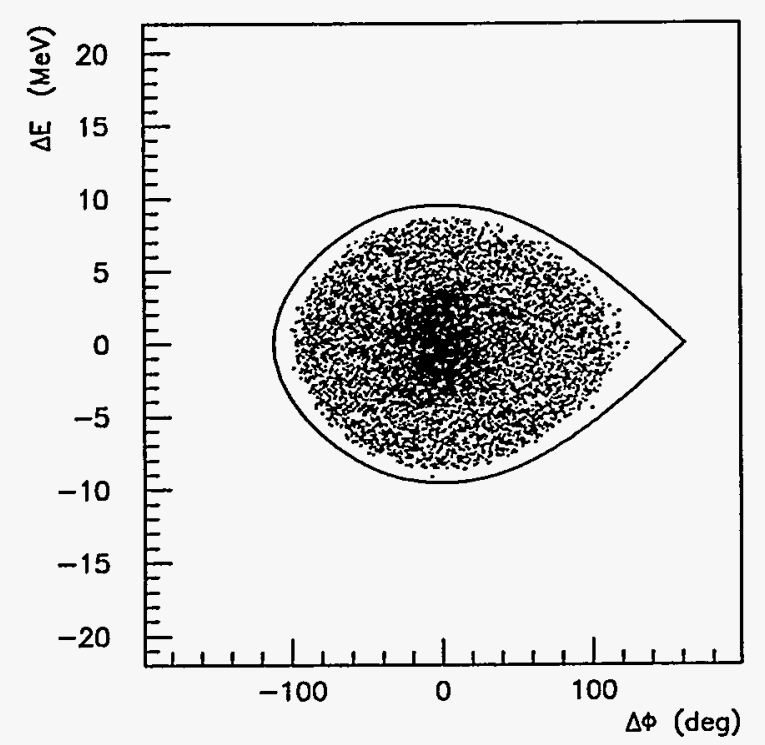

(c) $\mathrm{t}=2 \mathrm{msec}, \mathrm{V}_{\mathrm{RF}}=124.6 \mathrm{kV}$

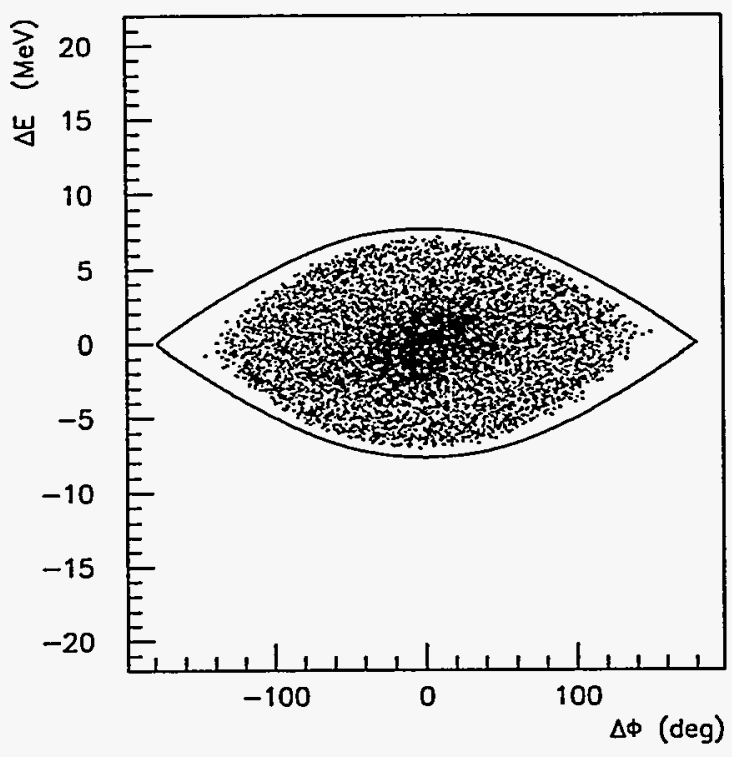

(b) $\mathrm{t}=0 \mathrm{msec}, \mathrm{V}_{\mathrm{RF}}=66.9 \mathrm{kV}$

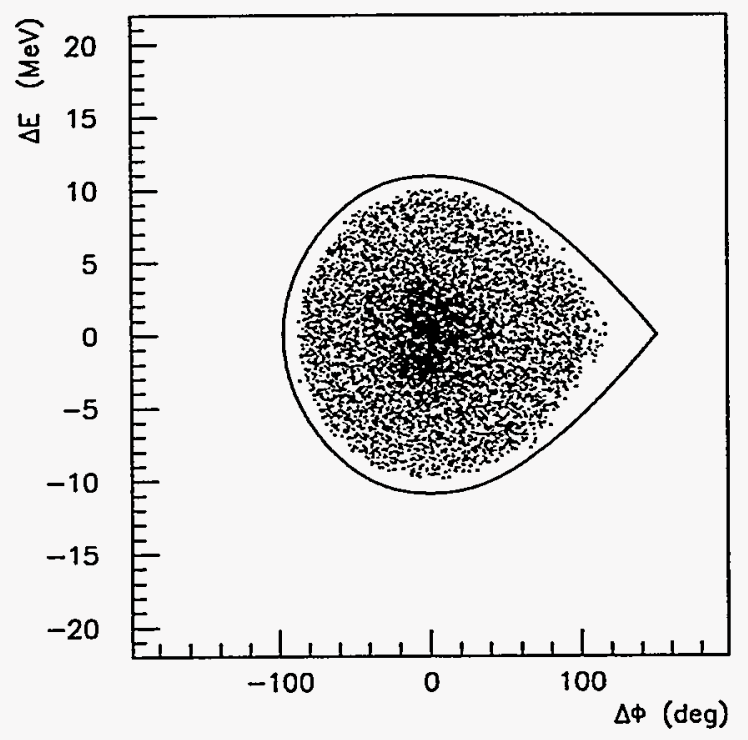

(d) $t=4 \mathrm{msec}, V_{\mathrm{RF}}=153.5 \mathrm{kV}$

Figure II.4.1-7 Phase Space Distributions from the Simulation, from the Middle of Injection to $4 \mathrm{msec}$ in the Acceleration Cycle. 


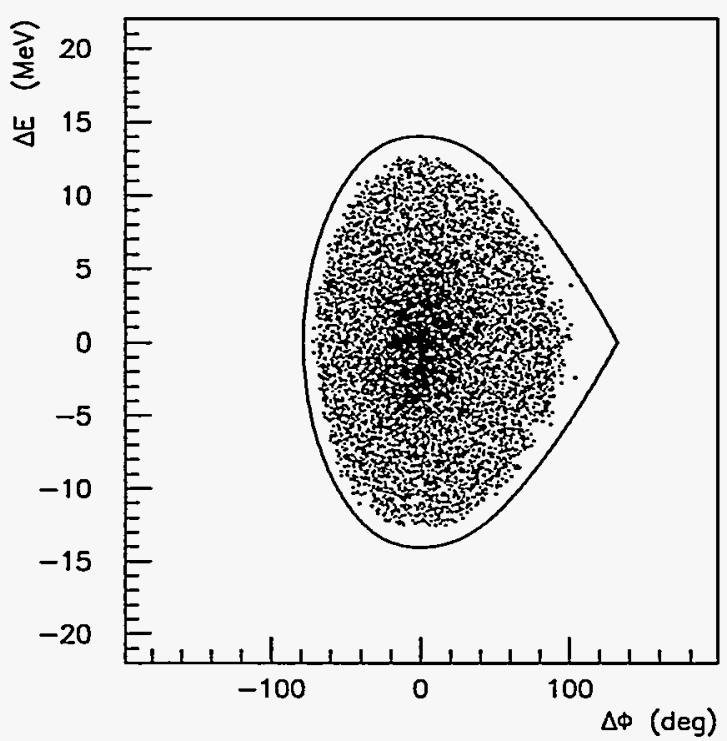

(a) $\mathrm{t}=8 \mathrm{msec}, \mathrm{V}_{\mathrm{RF}}=169.2 \mathrm{kV}$

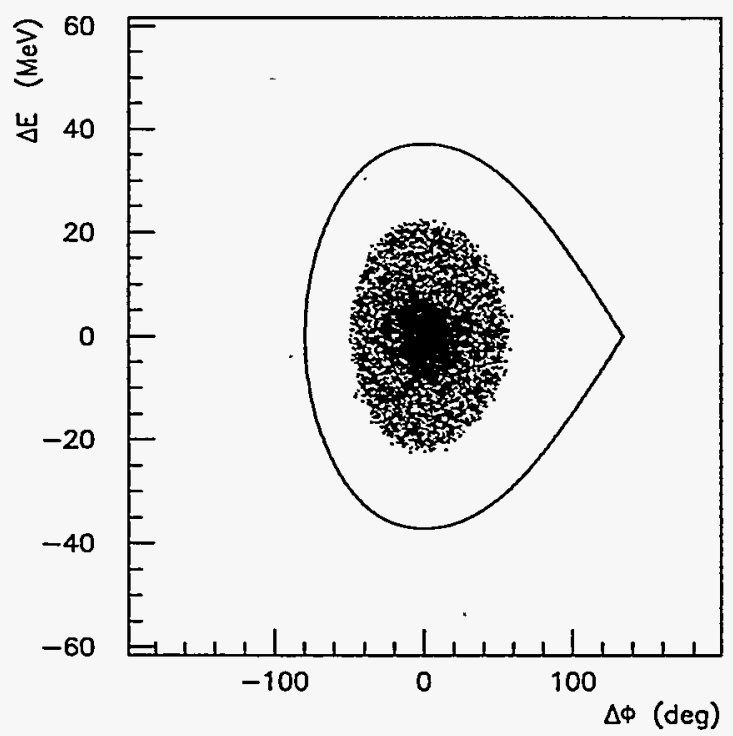

(c) $t=18 \mathrm{msec}, V_{\mathrm{RF}}=158.5 \mathrm{kV}$

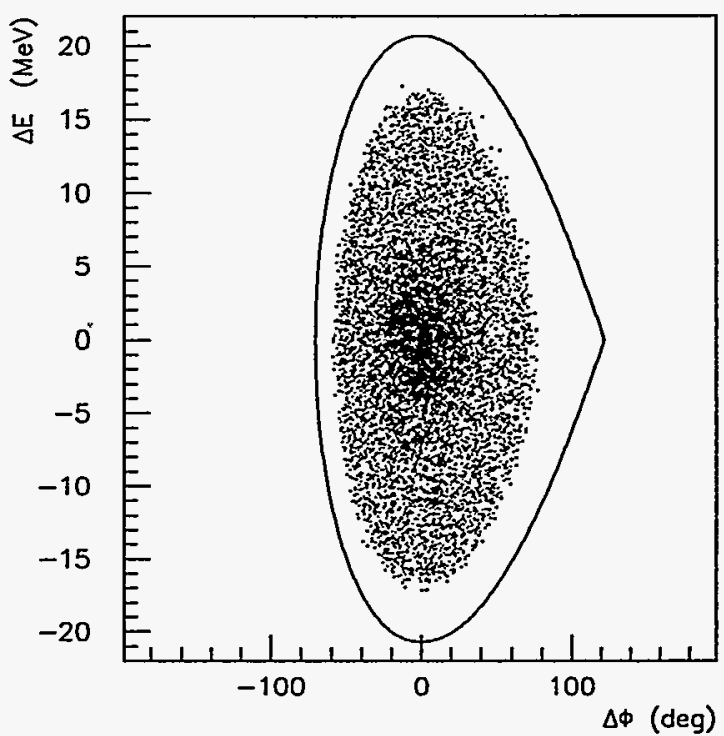

(b) $t=12.5 \mathrm{msec}, V_{\mathrm{RF}}=169.2 \mathrm{kV}$

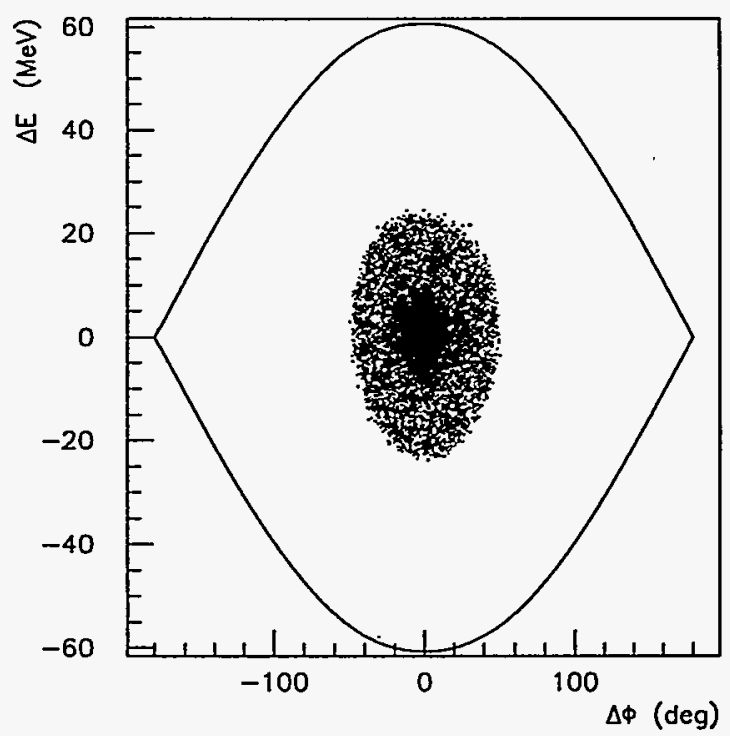

(d) $t=25 \mathrm{msec}, V_{R F}=113.9 \mathrm{kV}$

Figure II.4.1-8 Phase Space Distributions from the Simulation, from $8 \mathrm{msec}$ in the Acceleration Cycle to Extraction. 
Figure II.4.1-7a depicts the distribution at $\mathrm{t}=-0.25 \mathrm{msec}$. At this time, half of the beam, or 281 turns, has been injected. The voltage is $52.9 \mathrm{kV}$, and the bucket area is $8 \mathrm{eV} \mathrm{sec}$. The distribution at the end of injection is plotted in Figure II.4.1-7b. The voltage is $66.9 \mathrm{kV}$, producing a bucket area of $9 \mathrm{eV} \mathrm{sec}$. The bunch has an area of $7.3 \mathrm{eV} \mathrm{sec}$ and fills $80 \%$ of the bucket. Figures II.4.1-7c and II.4.1-7d are plotted at $\mathrm{t}=2 \mathrm{msec}$ and $4 \mathrm{msec}$, respectively. At these times, the bucket area remains at $9 \mathrm{eV} \mathrm{sec}$ and the bunch area remains at $7.3 \mathrm{eV} \mathrm{sec}$. The bunch is well-matched to the bucket.

The plots in Figure II.4.1-8 show the distributions at $\mathrm{t}=8,12.5,18$, and $25 \mathrm{msec}$. In Figures II.4.1-8a and II.4.1-8b, the voltage is constant at $169.2 \mathrm{kV}$. During the interval from 8 to $12.5 \mathrm{msec}$, the bucket area grows from 9 to $11 \mathrm{eV} \mathrm{sec}$. The distribution at $\mathrm{t}=18 \mathrm{msec}$ is plotted in Figure II.4.1-8c, when the voltage is $158.5 \mathrm{kV}$ and the bucket area is $21 \mathrm{eV} \mathrm{sec}$. Figure II.4.1-8d depicts the distribution at the end of the cycle, when the voltage is $113.9 \mathrm{kV}$. The bucket area grows from 21 to $51 \mathrm{eV} \mathrm{sec}$ during the last $7 \mathrm{msec}$ of acceleration. The if program ensures that the synchrotron frequency is adequate to keep the bunch centered in the bucket.

We have shown that the rf voltage program described in this section is able to capture and accelerate $10^{14}$ protons per pulse with no loss of particles during the injection, capture, and acceleration processes. The engineering design of the rf system provides $180 \mathrm{kV}$ peak voltage to satisfy the required voltage program.

\subsection{BEAM LOADING}

\subsubsection{Introduction}

The rf systems are able to handle the heavy beam loading that occurs in the RCS. Injection is the most demanding period for amplifier stability. The average circulating current at injection is about $18 \mathrm{~A}$ for $1.04 \times 10^{14}$ protons. The total voltage per turn during injection varies from 40.5 to $66.9 \mathrm{kV}$. Since there are 10 cavities in the RCS, the gap voltage on each cavity varies between 4 and $6.69 \mathrm{kV}$. The voltage on each cavity needed at injection is about $4 \mathrm{kV}$. The effective shunt impedance of the cavities varies from 5 to $8 \mathrm{k} \Omega$, which means that the beam can induce more than 20 times the accelerating voltage at injection. Although the beam current increases by as much as $40 \%$ during the acceleration cycle, the net beam loading effect decreases, because the demand for voltage increases to a peak value of $16.9 \mathrm{kV}$ per cavity and remains relatively high, even up to extraction. Several techniques are used to handle the heavy beam loading: closed-loop feedback, which effectively lowers the impedance seen by the beam; 5 swamping resistors to lower the effective shunt impedance of the cavity; 6 and feed-forward techniques to compensate for the voltage and phase changes induced by the beam. ${ }^{5}$ Another approach to heavy beam loading that has been proposed, but not yet used for acceleration, is to connect the power tube in a cathode-follower topology.7,8,9 
The IPNS Upgrade baseline design uses a tetrode in a grounded-cathode topology, with feedback and feed-forward techniques to handle beam loading. The cathode follower is an option, dependent on the success of continuing research and development.

\subsubsection{Stability of the Beam-Cavity Interaction}

The analysis of the beam loading interaction presented in this section follows the conceptual development used by the TRIUMF-Kaon project team. ${ }^{10}$ The tetrode amplifier acts like a current source, $i_{g}$; the cavity acts like an inductance-capacitance-resistance (LCR) parallel network; and the beam acts like a second current source, $\mathrm{i}_{\mathrm{b}}$, connected across the cavity gap. This circuit model is shown in Figure II.4.2-1. When the if frequency is denoted by $f_{r}$, the resonant frequency of the cavity is denoted by $f_{0}$, and the difference between the rf frequency and the resonant frequency is denoted by $\Delta \mathrm{f}$, the currents and voltages are as follows:

$$
\begin{aligned}
& i_{g}=I_{g} e^{j\left(\phi_{g}+\omega_{r} t\right)}, \\
& i_{b}=-j I_{b} e^{-j\left(\phi_{b}-\omega_{r} t\right)}, \\
& i_{t}=I_{t} e^{-j\left(\phi_{t}-\omega_{r} t\right)}=i_{g}+i_{b},
\end{aligned}
$$

and

$$
V=V_{m} e^{j\left(\phi_{v}+\omega_{r} t\right)}
$$

where $\mathrm{j}=\sqrt{-1} ; \omega_{\mathrm{r}}=2 \pi f_{\mathrm{r}} ; \mathrm{I}_{\mathrm{g}}, \mathrm{I}_{\mathrm{b}}, \mathrm{I}_{\mathrm{t}}$, and $\mathrm{V}_{\mathrm{m}}$ are constants denoting peak magnitudes; and $\phi_{\mathrm{g}}, \phi_{\mathrm{b}}$, $\phi_{t}$, and $\phi_{\mathrm{V}}$ are constants denoting relative phase.

The sum of the generator current and beam current is equal to the total cavity current:

$$
\begin{aligned}
I_{t} e^{-j\left(\phi_{t}-\omega_{r} t\right)} & =C \frac{d V}{d t}+\frac{1}{L} \int V d t+\frac{V}{R}, \\
I_{t} e^{-j \phi_{t}} & =\left(j \omega_{r} C+\frac{1}{j \omega_{r} L}+\frac{1}{R}\right) V_{m},
\end{aligned}
$$




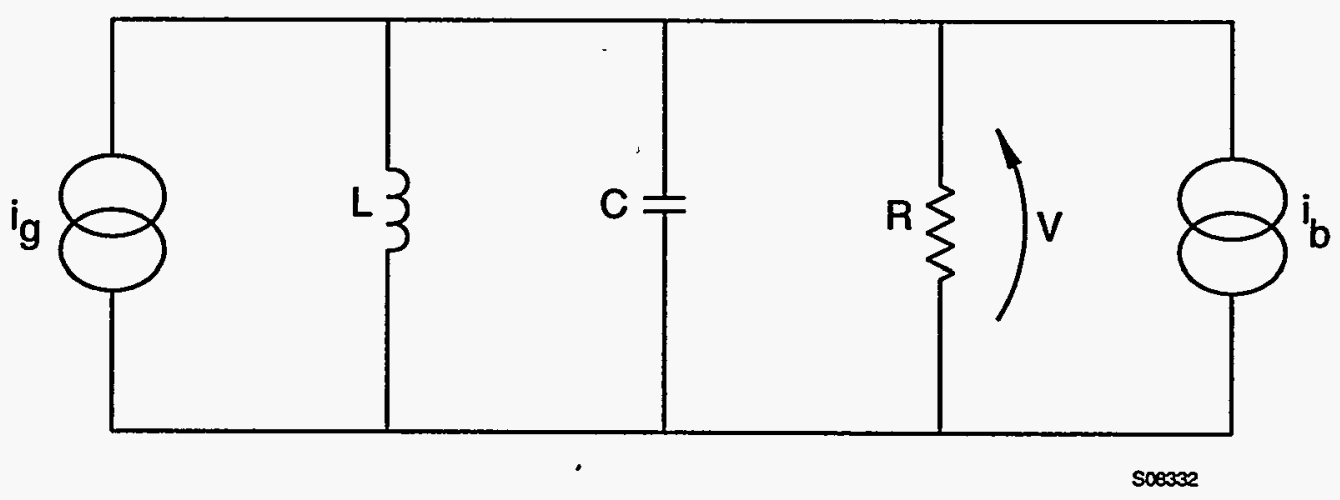

Figure II.4.2-1 Circuit Representation of Amplifier, Cavity and Beam Loading Current. 
where $\mathrm{C}$ is the capacitance; $\mathrm{L}$ is the inductance; $\mathrm{R}$ is the resistance; and the voltage, $\mathrm{V}$, is selected to be the reference value for all phasors (i.e., $\phi_{\mathrm{V}}=0$ ):

$$
I_{t} e^{-j \phi_{t}}=\left(j\left(\omega_{o}+\Delta \omega\right) C+\frac{1}{j\left(\omega_{o}+\Delta \omega\right) L}+\frac{1}{R}\right) V_{m},
$$

and because $\omega_{0}^{2}=1 / \mathrm{LC}$,

$$
I_{t} e^{-j \phi_{t}} \approx\left[j \Delta \omega C+j \frac{\Delta \omega}{\omega_{o}}\left(\frac{1}{\omega_{0} L}\right)+\frac{1}{R}\right] V_{m}
$$

For a parallel resonant circuit, the quality factor, $Q$, is equal to $R / \omega_{0} L$ and is also equal to $R \omega_{0}$ C. Then, Equation II. $4.2-3$ becomes

$$
\mathrm{I}_{\mathrm{t}} \mathrm{e}^{-\mathrm{j} \phi_{\mathrm{t}}} \approx \frac{\mathrm{V}_{\mathrm{m}}}{\mathrm{R}}\left(1+\mathrm{j} 2 \mathrm{Q} \frac{\Delta \omega}{\omega_{\mathrm{o}}}\right)
$$

The term $\left(1+\mathrm{j} 2 \mathrm{Q} \Delta \omega / \omega_{0}\right)$ converted to exponential form is

$$
1+\mathrm{j} 2 \mathrm{Q} \frac{\Delta \omega}{\omega_{0}}=\sqrt{1+\left(2 \mathrm{Q} \frac{\Delta \omega}{\omega_{0}}\right)^{2}} \mathrm{e}^{\mathrm{j} \Psi},
$$

where $\tan \Psi=2 \mathrm{Q} \Delta \omega / \omega_{0}$. The square root term of Equation II.4.2-5 reduces to $1 / \cos \Psi$. Thus, the cavity voltage is

$$
V_{m}=R \cos \Psi e^{-j \Psi} I_{t} e^{-j \phi_{t}}
$$

Normally the cavity resonance is adjusted to compensate for the reactive term of the beam current. The condition is met by the phase angles $\phi_{\mathrm{g}}=\phi_{\mathrm{V}}=0$ and $\phi_{\mathrm{b}}=\Psi$. The phasor diagram is shown in Figure II.4.2-2. The following expressions result:

$$
\begin{aligned}
& -j I_{b} e^{-j \phi_{b}}=-j I_{b}\left(\cos \phi_{b}-j \sin \phi_{b}\right), \\
& -j I_{b} e^{-j \phi_{b}}=-j I_{b} \cos \phi_{b}-I_{b} \sin \phi_{b},
\end{aligned}
$$


and $I_{g}=V_{m} / R+I_{b} \sin \phi_{b}$.

The magnitude of the reactive part of the beam current equals the reactive part of the total current:

$$
\begin{aligned}
\operatorname{Im}\left(i_{t}\right) & =\frac{V_{m}}{R}\left(2 Q \frac{\Delta \omega}{\omega_{0}}\right) \\
& =I_{o}\left(2 Q \frac{\Delta \omega}{\omega_{0}}\right),
\end{aligned}
$$

where $I_{0}=V_{m} / R$. From Equation II.4.2-8,

$$
\mathrm{I}_{\mathrm{b}} \cos \phi_{\mathrm{b}}=\mathrm{I}_{\mathrm{o}}\left(2 \mathrm{Q} \frac{\Delta \omega}{\omega_{\mathrm{o}}}\right)
$$

As shown by Robinson ${ }^{11}$ and developed by the TRIUMF-Kaon staff, stability below the transition energy requires

$$
\frac{\mathrm{I}_{\mathrm{b}}}{\mathrm{I}_{\mathrm{o}}}<\frac{2 \cos \phi_{\mathrm{b}}}{\sin 2 \Psi}
$$

Since from Equation II.4.2-10,

$$
\begin{gathered}
\cos \phi_{b}=\frac{I_{o}}{I_{b}}\left(2 Q \frac{\Delta \omega}{\omega_{o}}\right), \\
\cos \phi_{b}=\frac{I_{o}}{I_{b}} \tan \Psi,
\end{gathered}
$$

Equation II.4.2-11 for stability becomes

$$
\left(\frac{\mathrm{I}_{\mathrm{b}}}{\mathrm{I}_{\mathrm{o}}}\right)^{2}<\frac{2 \tan \Psi}{\sin 2 \Psi}
$$

The parameter $\Psi$, called the detuning angle, is the angle by which the cavity phase is changed from no load to the beam loaded condition. 


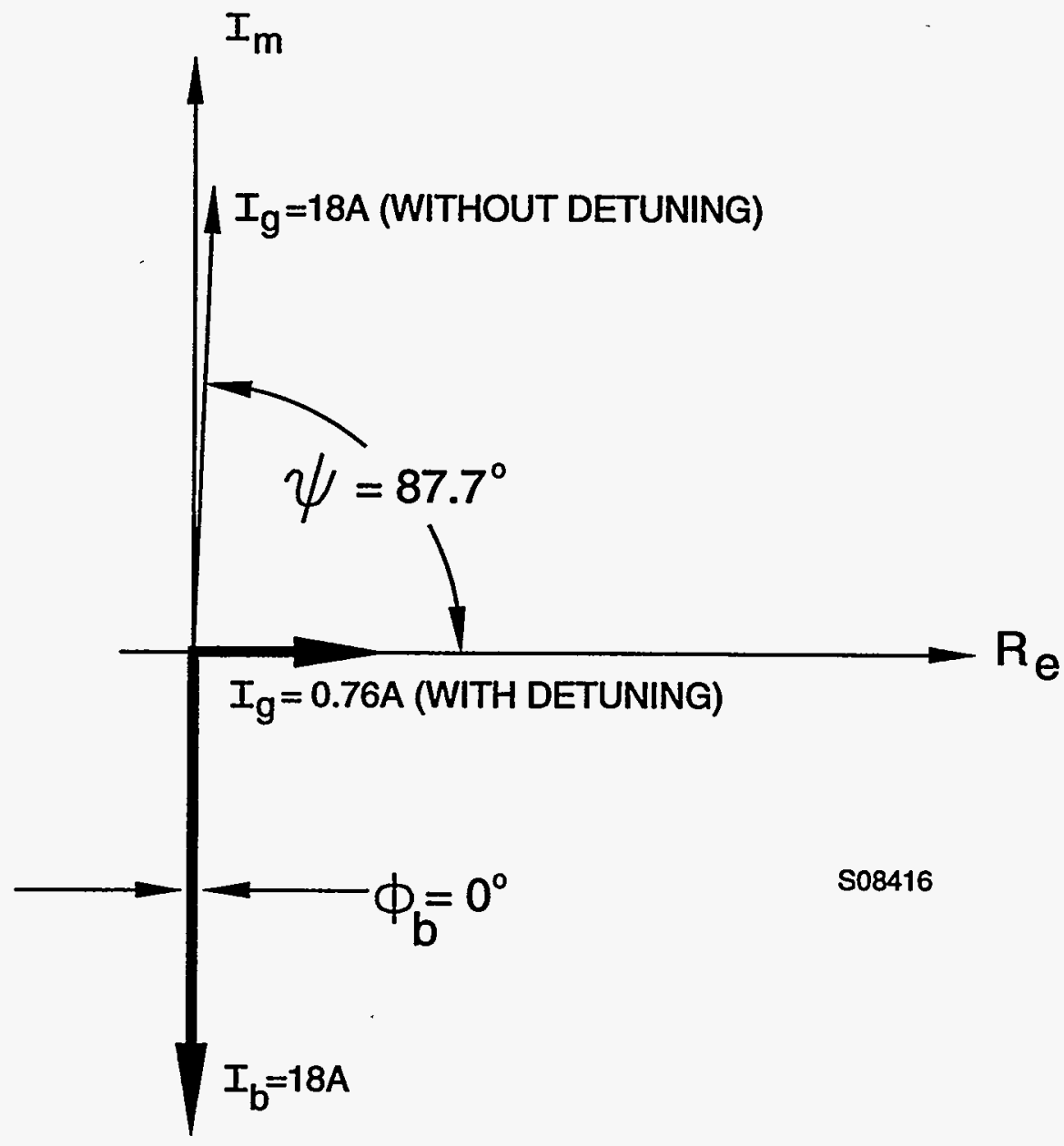

Figure II.4.2-2 RF Phasor Diagram at Injection Currents Referenced to Gap Voltage. (Not to scale.) 
For the cavity parameters described in Section II.4.3, the value of $Q$ at injection is 33.2, the value of $\mathrm{I}_{\mathrm{o}}$ is only $0.76 \mathrm{~A}$, and the circulating beam current is about $18 \mathrm{~A}$ for $1.04 \times 10^{14}$ protons. The ratio of $\mathrm{I}_{b} / \mathrm{I}_{\mathrm{o}}$ is about 23.6 , and $\tan \Psi$ is 24.9 , to compensate for the reactive beam current. At extraction, the $Q$ of the cavity is 63.3 , the shunt impedance is $8,108 \Omega$, the value of $I_{0}$ is $1.406 \mathrm{~A}$, and the beam current is $24.6 \mathrm{~A}$. The ratio of $\mathrm{I}_{\mathrm{b}} / \mathrm{I}_{0}$ is 17.5 , and $\tan \Psi$ is 17.4 , to compensate for the reactive beam current.

Because these values of $\tan \Psi$ are large, it is proposed to place a resistance across the cavity gap to lower the cavity $Q$, thus reducing this term, as discussed in Section 4.2.3.

\subsubsection{Transient Beam Loading}

The most severe beam loading conditions exist at injection, when the cavity phase in the absence of detuning is changed by $87.7^{\circ}$, as seen in Figure II.4.2-2. The feedback loop that maintains the generator phase at $0^{\circ}$ detunes the cavity by $38 \%$ over the injection period. It is unlikely that the magnetic field in the ferrite can be changed this rapidly. In order to overcome this problem, a resistance is connected across the accelerating gap to lower the cavity $Q$ and fast feedback is used. Copper sulfate resistors were successfully used at ISIS 6 to lower the Q. Resistors with values between 8 and $9 \mathrm{k} \Omega$ can decrease the detuning range by almost a factor of two. In addition, fast feedback, as proposed by Boussard, ${ }^{5}$ further reduces the beam loading effect. A signal proportional to the gap voltage is fed back into the reference signal for the amplifier chain. The effective resistance of the cavity seen by the beam is reduced by a factor of $(1+\mathrm{H})$, where $\mathrm{H}$ is the loop gain. The limitation on achievable loop gain is the loop delay. According to Boussard, 5

$$
\mathrm{H} \leq \frac{\mathrm{Q}}{2 \pi \mathrm{f}_{\mathrm{r}} \mathrm{T}},
$$

where $\mathrm{T}$ is the delay in the feedback chain. This requirement forces the amplifiers to be located inside the ring tunnel close to the cavities. Therefore, the amplifiers are located within $6 \mathrm{~m}$ of the cavities, so that the cable lengths only impose about $40 \mathrm{nsec}$ of delay in the feedback loop. It should be possible to achieve a total loop delay of no more than about 50 to $75 \mathrm{nsec}$, in which case, $H \leq 66$. The value of $H$ must be greater than $\sqrt{2} \tan \Psi$ for stability, i.e., at least 35.2 at injection, giving a factor of two in the safety margin. This, coupled with the use of copper sulfate resistors, should be sufficient to achieve amplifier stability at the fundamental frequency.

The harmonic content of the circulating beam current can also be a problem, especially the second harmonic, which is as large as $37 \%$ to $40 \%$ of the peak value of the fundamental current. The cavity impedance is capacitive and has a value of $116 \Omega$ at the second harmonic frequency. This value results in a second harmonic voltage of as much as 2,500 volts being induced on the 
cavity. The loop gain at the second harmonic frequency is down to only about 10 or 20 , but it should be large enough to reduce the second harmonic voltage to an acceptable level. Moreover, to ensure that the longitudinal beam loading is kept sufficiently low, fast feed-forward is planned with tuned filters and digital delay lines for some of the other harmonics.

These compensation techniques are used throughout the entire acceleration cycle. Stability is easier to achieve during the acceleration cycle than at injection.

\subsubsection{Summary and Conclusions}

Despite the heavy loading on the RCS cavities and the heavy demand put on the amplifier system, longitudinal stability is achievable. The use of swamping resistors is needed to complement the fast-feedback loops, especially at injection. Fast feedback is needed throughout the acceleration cycle. Feed-forward at the fundamental frequency and at some of the higher harmonics is used throughout the acceleration cycle.

The compensation schemes appear to provide a factor of three or more safety margin in amplifier stability for a beam of $1.04 \times 10^{14}$ protons per pulse. Care is to be taken to ensure that the beam bunching factor and the delays in the feedback networks do not become too large, so as to avoid amplifier instability.

\subsection{RADIO-FREQUENCY SYSTEM}

\subsubsection{Introduction}

The RCS rf system has 10 single-ended, ferrite-loaded cavities to generate the required $180 \mathrm{kV}$. There is one cavity per straight section, and a total of ten straight sections dedicated for the installation of the cavities. A change in resonant frequency from $1.1235 \mathrm{MHz}$ at injection to $1.5035 \mathrm{MHz}$ at extraction is accomplished by changing the dc current in the bias windings that are wound around the ferrite. The design choice for the ferrite type is Philips $8 \mathrm{C} 12$, which is the same as that used in the GSI Heavy Ion Synchrotron ${ }^{12}$ and for which extensive performance data are available.

Each cavity is driven by a Class- $B$ tetrode in grounded-cathode configuration. The entire amplifier chain is located in the Ring Building (Building 365), close to the cavities. This close proximity keeps the loop delay down, in order to achieve high gain in the fast-feedback loops. The final amplifier is driven by a grounded-grid triode Class- $\mathrm{C}$ amplifier. The amplifier circuit is discussed in detail in Section II.4.3.3. 


\subsubsection{Synchrotron Cavities}

Each cavity delivers $18 \mathrm{kV}$ across a single accelerating gap. The physical length of a cavity is $2.59 \mathrm{~m}$ from flange face to flange face, with the accelerating gap at one end. There are 15 ferrite rings per subassembly and 4 ferrite subassemblies in each cavity. Four bias windings are wound in the typical figure-eight configuration ${ }^{13}$ around each pair of ferrite subassemblies, so that the if induction cancels at the input terminals of the bias supply. The cavity layout is shown in Figure II.4.3-1, and the physical parameters are listed in Table II.4.3-1.

The ferrite rings are made of $0.0254-\mathrm{cm}$-thick Philips $8 \mathrm{C} 12$ material, or equivalent. The inner radius of each ring is $0.125 \mathrm{~m}$, and the outer radius is $0.25 \mathrm{~m}$. Copper cooling plates, each $3 \mathrm{~mm}$ thick, separate the ferrite rings. A cooling-water tube is brazed along the outer circumference of the exposed copper fins of the cooling plates. This arrangement is shown in Figure II.4.3-2.

A ferrite subassembly consists of 15 ferrite rings, 16 cooling plates, and aluminum oxide clamping plates at the end of each ferrite stack. The cooling plate surfaces are coated with a heatconducting compound to make good thermal contact with the ferrite rings. The entire assembly is put into compression by means of threaded copper rods extending from one end of the stack to the other. These rods are also part of the bias windings.

The cavity fabrication plan is to assemble each stack of 15 ferrite rings, cooling plates, and bias windings separately. Each assembly is slid over the vacuum pipe and locked into position. The final bias and water connections are completed from access panels on the top of the cavity, after all the ferrite assemblies are in place on the vacuum pipe.

The relative if permeability of the ferrite, $\mu_{\mathrm{r}}$, at the injection and extraction operating points, with and without beam loading, is shown in Figure II.4.3-3. With four bias windings, the dc current varies from $80 \mathrm{~A}$ at injection to $115 \mathrm{~A}$ at extraction, when the cavity is unloaded, which results in a change in relative if permeability from 116 to 68 . In order to cancel the inductive portion of the beam current, the cavity resonant frequency is operated above the if frequency by the amount given by Equation II.4.2-5; i.e., the resonant frequency needs to be 1.137 times the rf frequency at extraction. Resonant operation at this frequency requires a higher bias current, $142 \mathrm{~A}$, to lower the relative if permeability to 52 . An $8-\mathrm{kW}(50 \mathrm{~V}$ at $160 \mathrm{~A})$ programmable dc bias supply is required to drive the four-turn winding over $0.5 \mathrm{~T}$ in $8.33 \mathrm{msec}$.

The maximum if field during acceleration reaches $0.0106 \mathrm{~T}$, which is less than the field at which the Q-loss effect was observed by the GSI team. The Q-loss effect occurred at GSI when the product of field and frequency was equal to $18 \mathrm{mT} \mathrm{MHz}$. For our design, the threshold for Q-loss is about 1.5 times higher than the maximum if field. The operating characteristics for the cavities under nominal conditions are listed in Table II.4.3-2. 


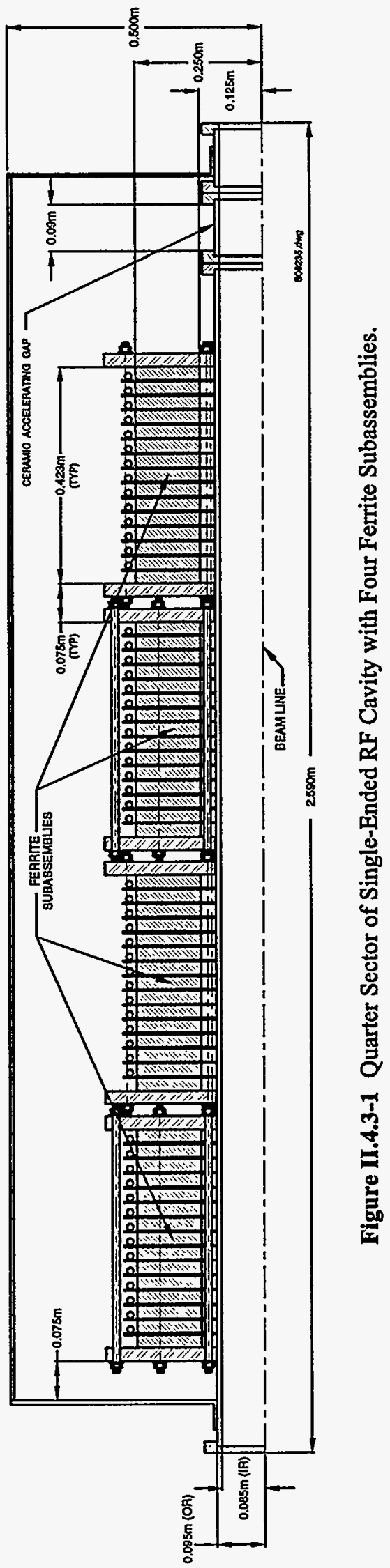


Table II.4.3-1 Physical Parameters of Cavity

\begin{tabular}{lll}
\hline \multicolumn{1}{c}{ Parameters } & \multicolumn{1}{c}{ Values } & Units \\
\hline & & \\
Physical length of cavity, flange-to-flange & 2.59 & $\mathrm{~m}$ \\
Inside length of cavity & 2.39 & $\mathrm{~m}$ \\
Length of accelerating gap & 0.09 & $\mathrm{~m}$ \\
Outer radius of cavity (inside dimension) & 0.50 & $\mathrm{~m}$ \\
Inner/outer radii of beam tube & $0.085 / 0.095$ & $\mathrm{~m}$ \\
Type of ferrite & Philips $8 \mathrm{C} 12$ & - \\
$\mu_{\mathrm{r}} \mathrm{Q}$ of ferrite & 6,000 & - \\
& & \\
Ferrite ring dimensions: & & $\mathrm{m}$ \\
Inner radius & 0.125 & $\mathrm{~m}$ \\
Outer radius & 0.250 & $\mathrm{~m}$ \\
Thickness & 0.0254 & \\
& & - \\
Single-ended single-gap cavity: & & $\mathrm{m}^{3}$ \\
Number of ferrite rings/cavity & 60 & $\mathrm{~m}$ \\
Volume of ferrite/cavity & 0.224 & - \\
Thickness of copper cooling plates & 0.003 & \\
Number of turns on bias winding & 4 & \\
\hline
\end{tabular}




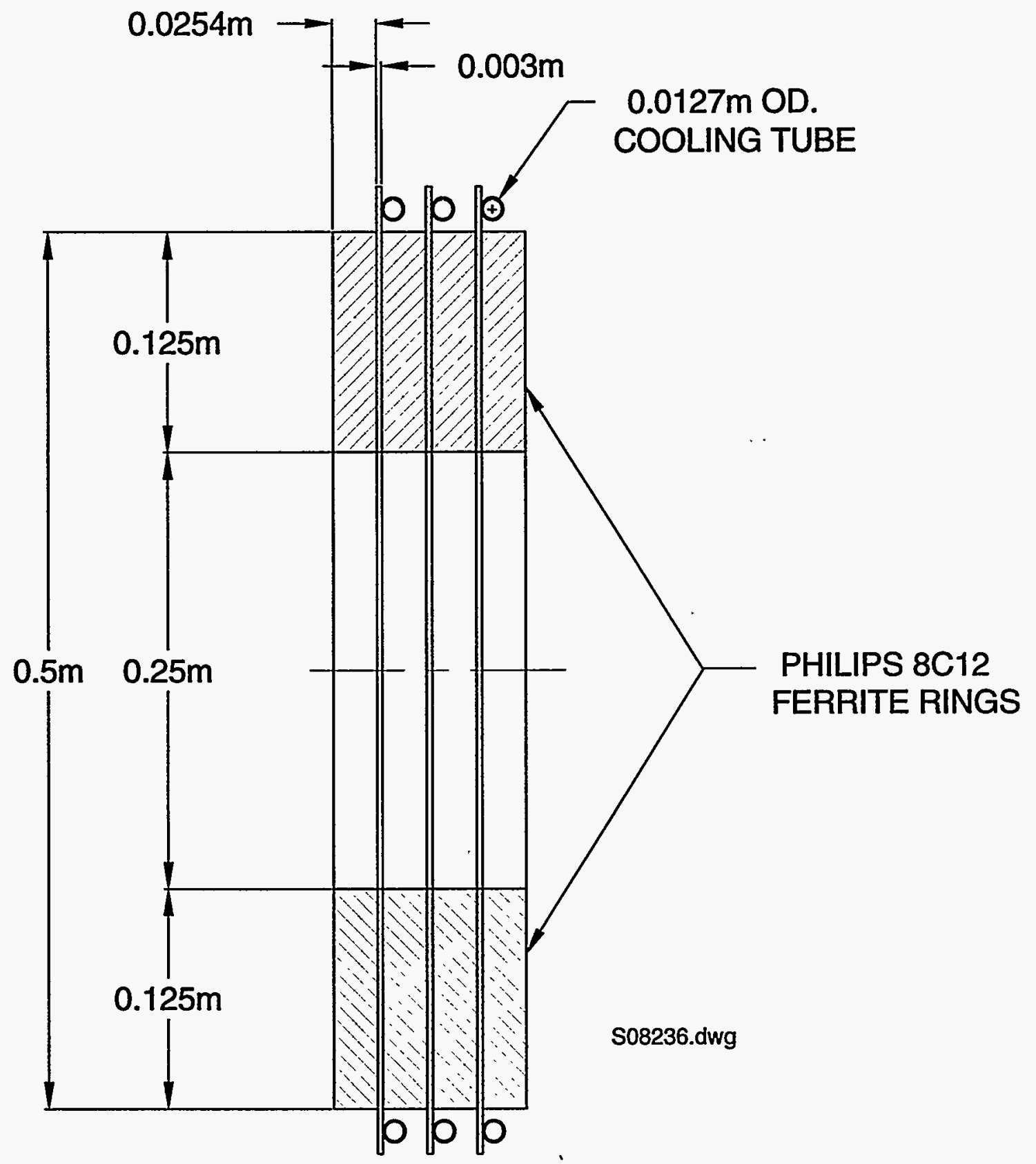

Figure II.4.3-2 Ferrite Ring Assembly Details. 


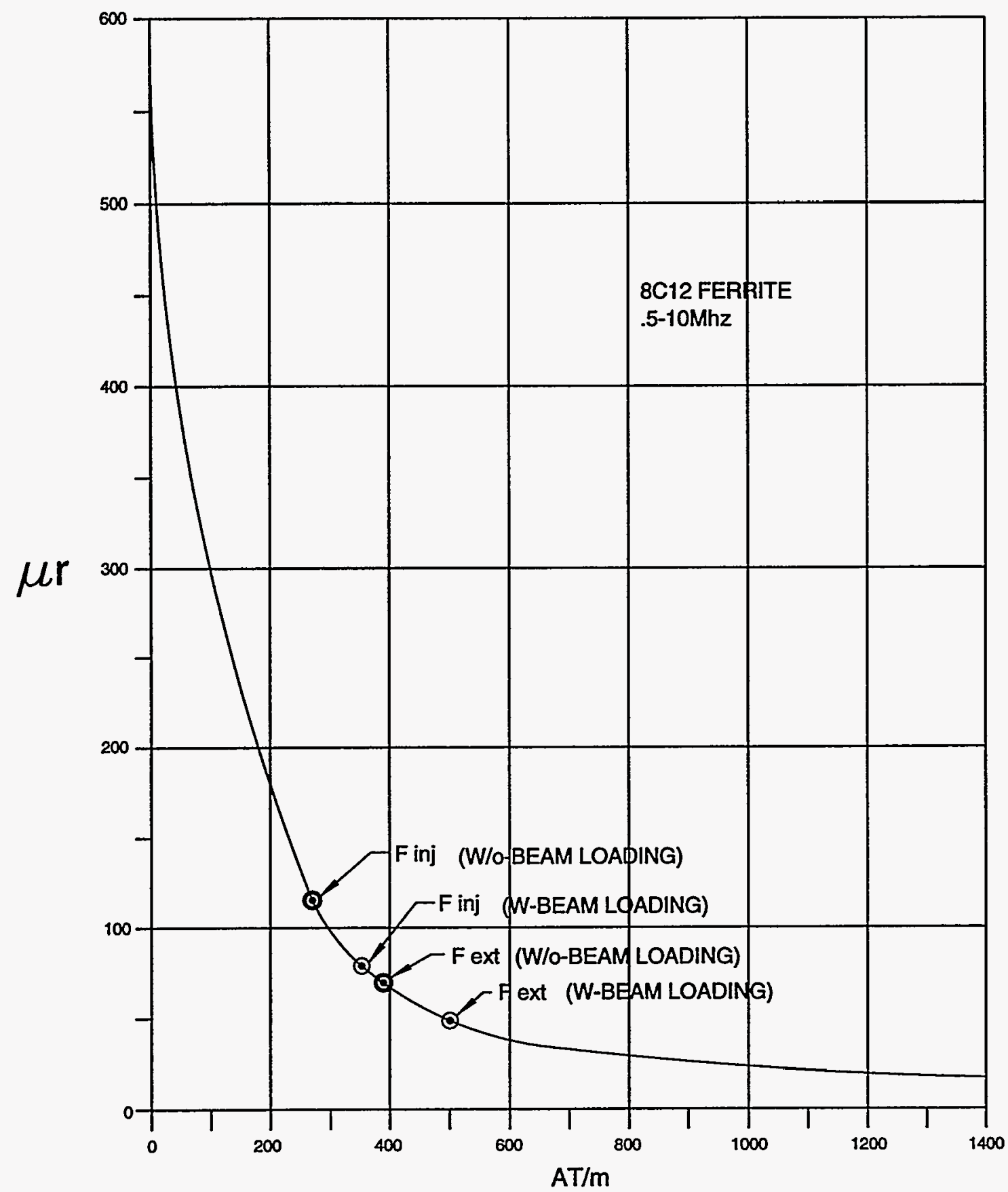

Poo344.dwg

Figure II.4.3-3 Rf Relative Permeability versus dc Bias. (Bias is expressed in ampere-turns per meter.) 
Table II.4.3-2 Operating Characteristics of Cavity for Nominal Operation

\begin{tabular}{|c|c|c|}
\hline Parameters & Values & Units \\
\hline Maximum flux density & 0.0106 & $\mathrm{~T}$ \\
\hline Maximum voltage per cavity & 18.0 & kV \\
\hline Peak power in ferrite & 75.4 & $\mathrm{~kW} / \mathrm{m}^{3}$ \\
\hline Average power in ferrite & 28.2 & $\mathrm{~kW} / \mathrm{m}^{3}$ \\
\hline Average power in ferrite per cavity & 6.3 & kW \\
\hline Peak power to copper & 7.2 & kW \\
\hline Average power to copper & 2.7 & kW \\
\hline dc coercive force at injection, without beam loading & 271 & $\mathrm{AT} / \mathrm{m}$ \\
\hline dc current at injection, without beam loading & 80 & A \\
\hline Relative permeability at injection, without beam loading & 116 & - \\
\hline dc coercive force at injection with beam loading & 360 & $A T / m$ \\
\hline dc current at injection with beam loading & 106 & A \\
\hline Relative permeability at injection with beam loading & 80 & - \\
\hline dc coercive force at extraction, without beam loading & 390 & $A T / m$ \\
\hline $\begin{array}{l}\text { dc current at extraction, without beam loading } \\
\text { Relative permeability at extraction, without beam loading }\end{array}$ & $\begin{array}{l}115 \\
68\end{array}$ & A \\
\hline dc coercive force at extraction, with beam loading & 484 & $A T / m$ \\
\hline dc current at extraction, with beam loading & 142 & A \\
\hline Relative if permeability at extraction, with beam loading & 52 & - \\
\hline
\end{tabular}




\subsubsection{RF Amplifiers}

The final amplifier is a Class- $B$, grounded-cathode type, using a single Eimac 8973 tetrode, or equivalent. The tube characteristics are given in Table II.4.3-3, and the tube outline is shown in Figure II.4.3-4.

The amplifier provides $18 \mathrm{kV}$ to the gap and is able to deliver at least $90 \mathrm{~A}$ to compensate for the instantaneous beam current, deliver enough current to excite the resonant cavity, and deliver the necessary power to the beam. The Eimac 8973 is capable of delivering considerably more current than is required.

The basic circuit diagram of the amplifier, with the predriver, driver, and the final stage, is shown in Figure II.4.3-5. The plate supply voltage is $22 \mathrm{kV}$, and the screen voltage is $+1500 \mathrm{~V}$. The dc bias on the control grid is $-700 \mathrm{~V}$. The final amplifier characteristics are listed in Table II.4.3-4. The grid of the Eimac 8973 is driven by a Class-B grounded-grid amplifier using an Eimac 3CW20000A7, or equivalent. The 3CW20000A7 is driven by a $1-\mathrm{kW}$ Eimac $4-400$ tetrode, operating in Class- $\mathrm{AB}_{1}$ common-cathode configuration.

The low-level rf system consists of four major elements: (1) a dc bias control system to keep the cavity on resonance and synchronous with the changing magnetic field, (2) an automatic gain control to maintain amplitude at the prescribed reference level for voltage on the accelerating gap, (3) a phase-control system that measures the beam position relative to the cavity phase and makes incremental changes in frequency to keep the beam at the phase-stable angle, and (4) an adaptive feed-forward system that uses the difference between the instantaneous beam current and the difference signal between the instantaneous amplifier current and the circulating current in the cavity to generate a change in the AGC reference to further correct for beam loading.

All amplifiers are located in the Ring Building (Building 365), close to the rf cavities to keep the loop delays to a minimum. A plan view is shown in Figure II.4.3-6 and a view looking down the beam axis is shown in Figure II.4.3-7. The amplifier cabinets are surrounded by a walkway on the platform for servicing. The main transformers and dc power supplies are located outdoors, on the roof of Building 364. A single dc supply provides high-voltage dc power for two complete amplifier systems. The plan view of the power supply layout is shown in Figure II.4.3-8. Much of the control equipment, bias supplies, etc., are located on the third floor of Building 364, as shown in Figure II.4.3-9. 
Table II.4.3-3 Eimac 8973 Tetrode, Class-B Operation

\begin{tabular}{lll}
\multicolumn{1}{c}{ Parameters } & Values & Units \\
& & \\
\hline & & \\
Plate voltage (maximum) & 22.5 & $\mathrm{kV}$ \\
Plate current (maximum) & 110 & $\mathrm{~A}$ \\
Plate dissipation (maximum) & 1,000 & $\mathrm{~kW}$ \\
Grid \#1 dissipation (maximum) & 2 & $\mathrm{~kW}$ \\
$\mu\left(\mathrm{G}_{1}-\mathrm{G}_{2}\right)$ & 4.5 & - \\
Plate resistance & $\sim 90$ & $\Omega$ \\
Plate rf power output (maximum) & $\sim 750$ & $\mathrm{~kW}$ \\
& & \\
\hline
\end{tabular}




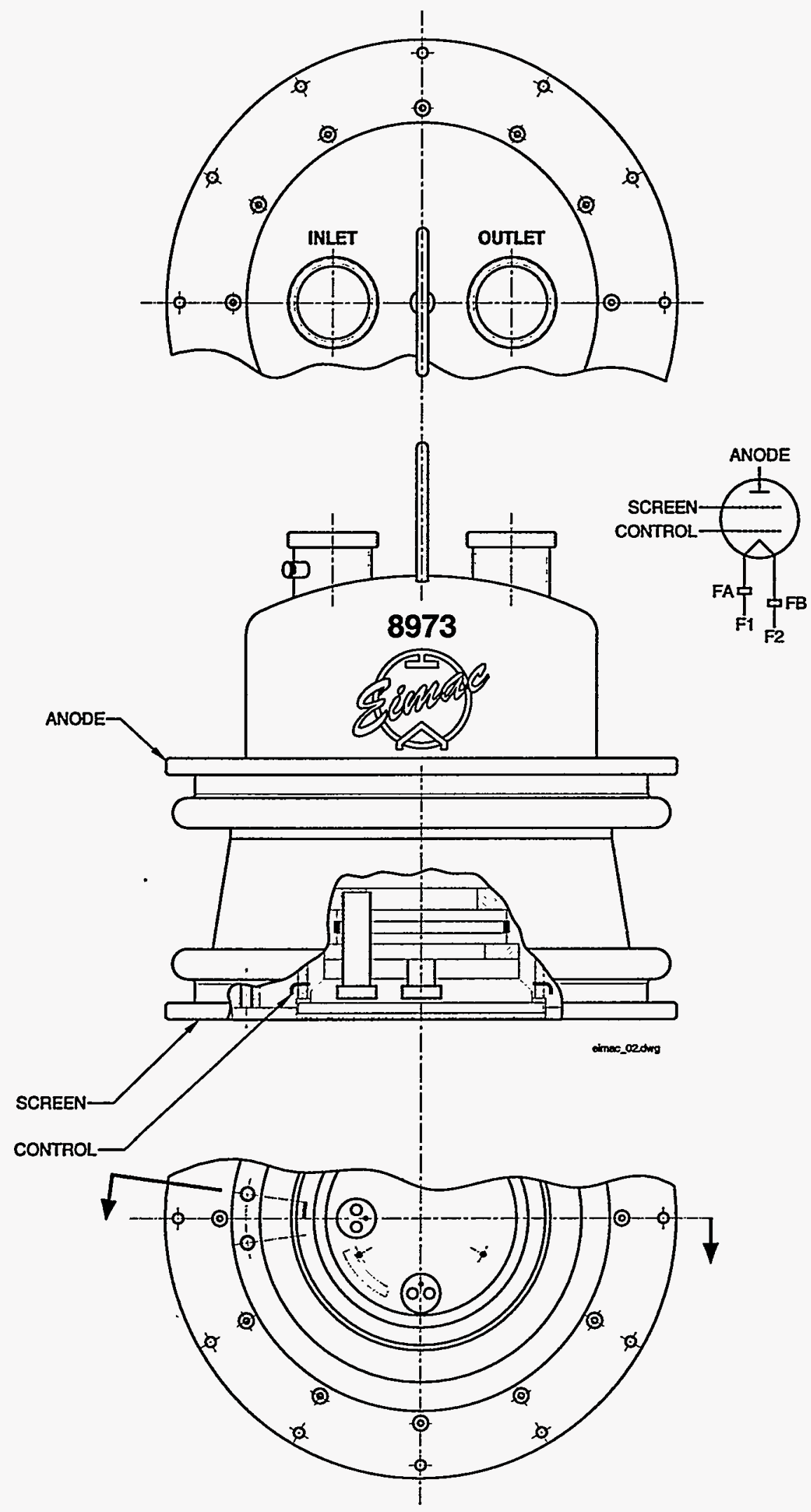

Figure II.4.3-4 8973 Tetrode Outline. 


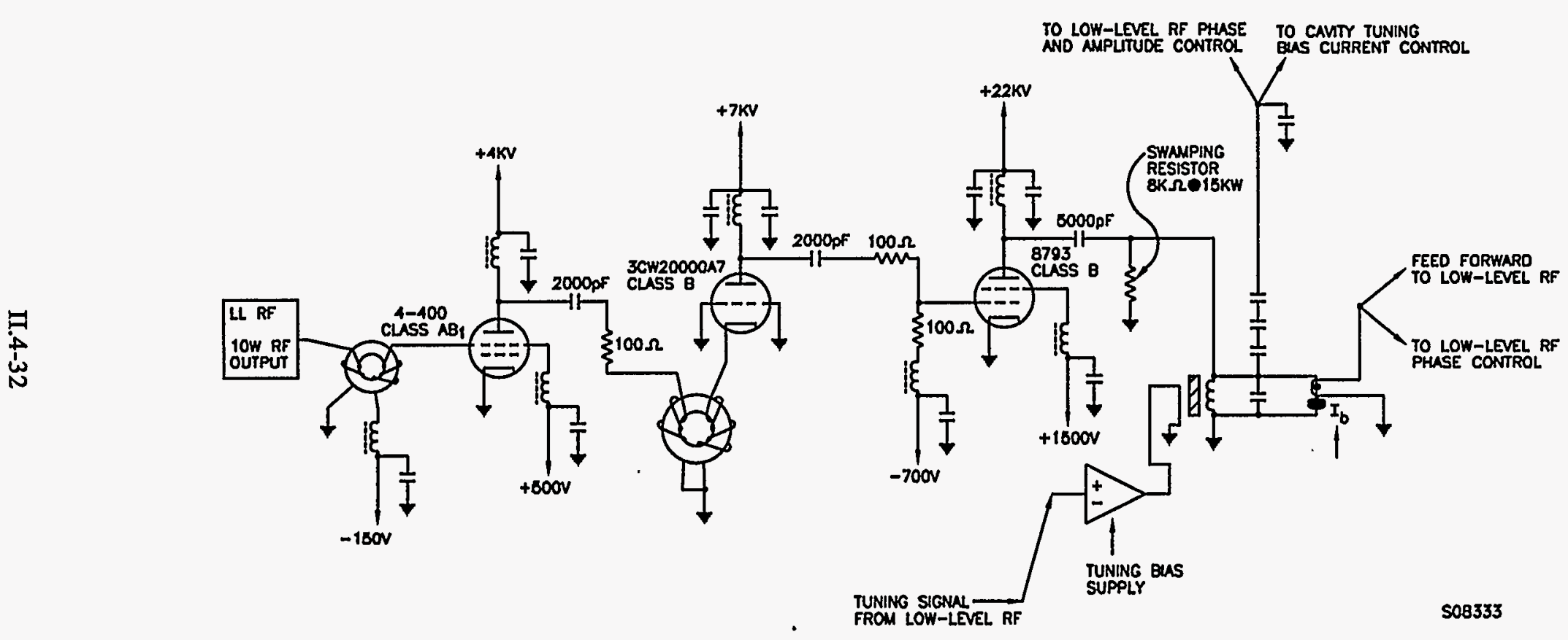

Figure II.4.3-5 RF Amplifier Circuit Diagram. 
Table II.4.3-4 Final Amplifier Characteristics for Single-Ended Grounding Cathode

\begin{tabular}{|c|c|c|}
\hline Parameters & Values & Units \\
\hline Tube type & Eimac 8973 & - \\
\hline Plate voltage & 22.0 & kV \\
\hline Maximum gap voltage & 18.0 & kV \\
\hline Peak plate current to beam & 61.0 & A \\
\hline Peak plate current from amplifier & -80.0 & A \\
\hline Peak power to beam per amplifier & 237.9 & $k W$ \\
\hline Peak power to ferrite per cavity & 16.9 & $\mathrm{~kW}$ \\
\hline Peak power to copper per cavity & 7.2 & kW \\
\hline Average if power from amplifier & 99.0 & $k W$ \\
\hline Approximate dc power to amplifier & 141.0 & $k W$ \\
\hline Approximate ac power to amplifier & 176.0 & $k W$ \\
\hline
\end{tabular}




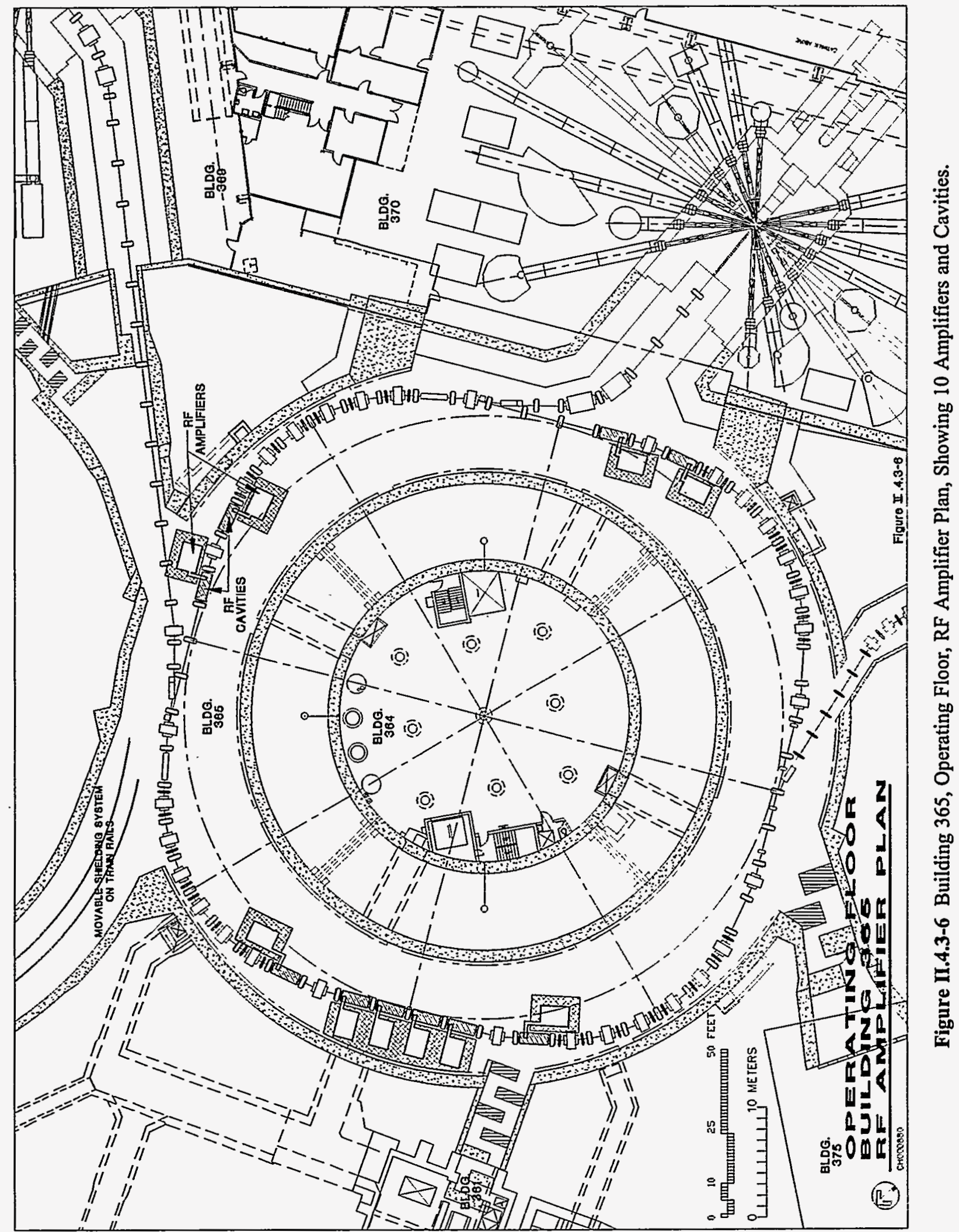




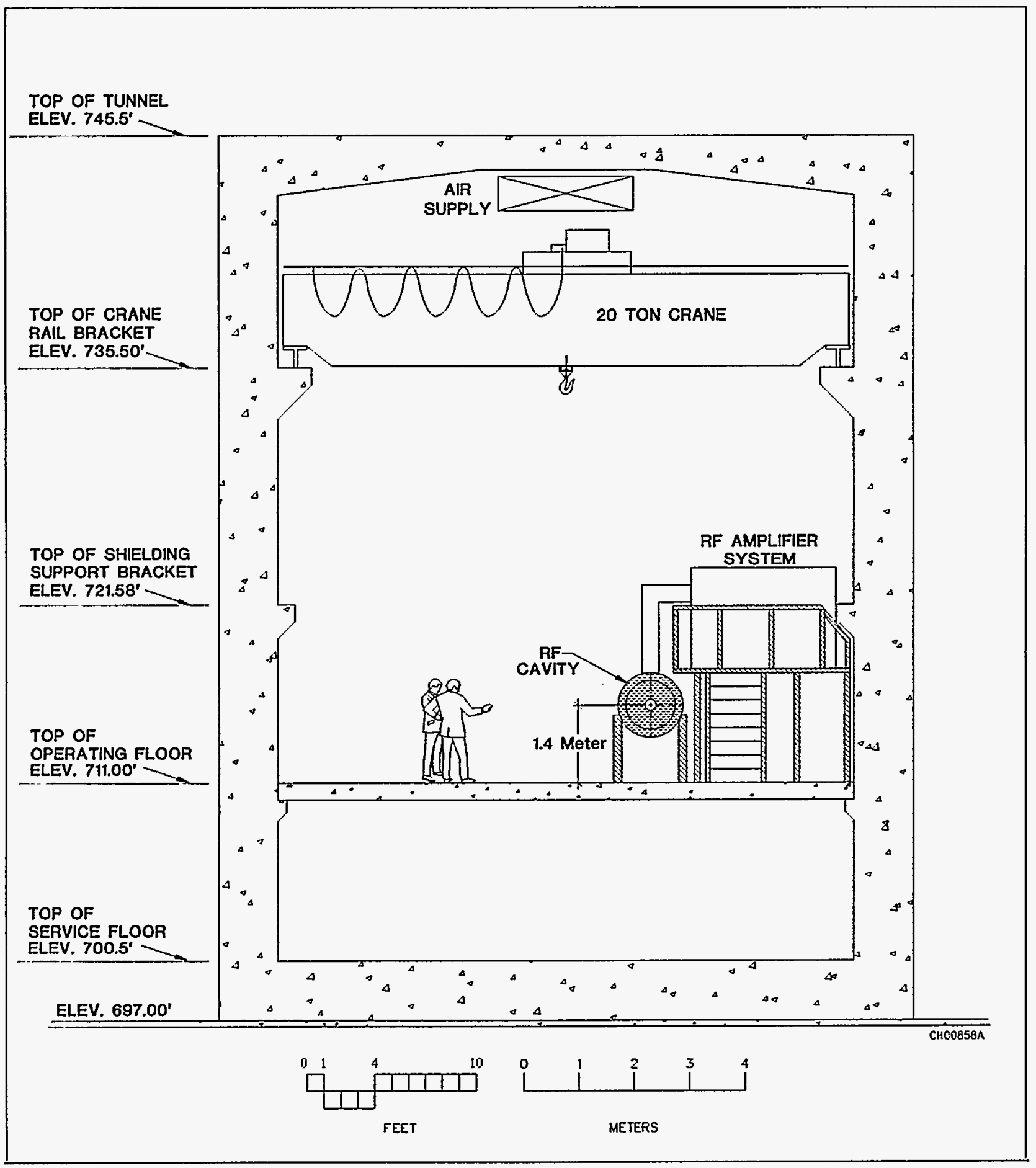

Figure I.4.3-7 RF Amplifier System Layout. 


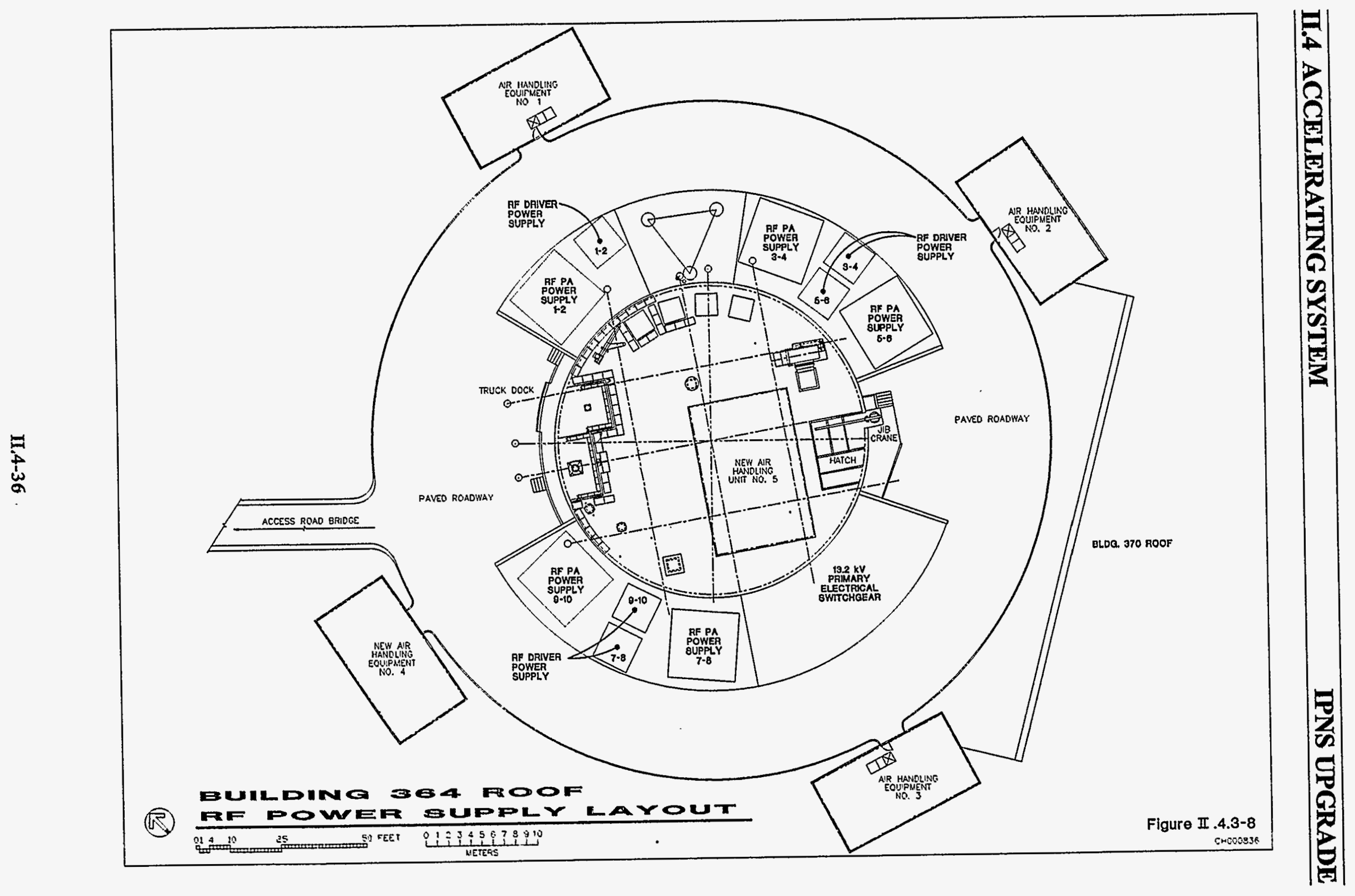




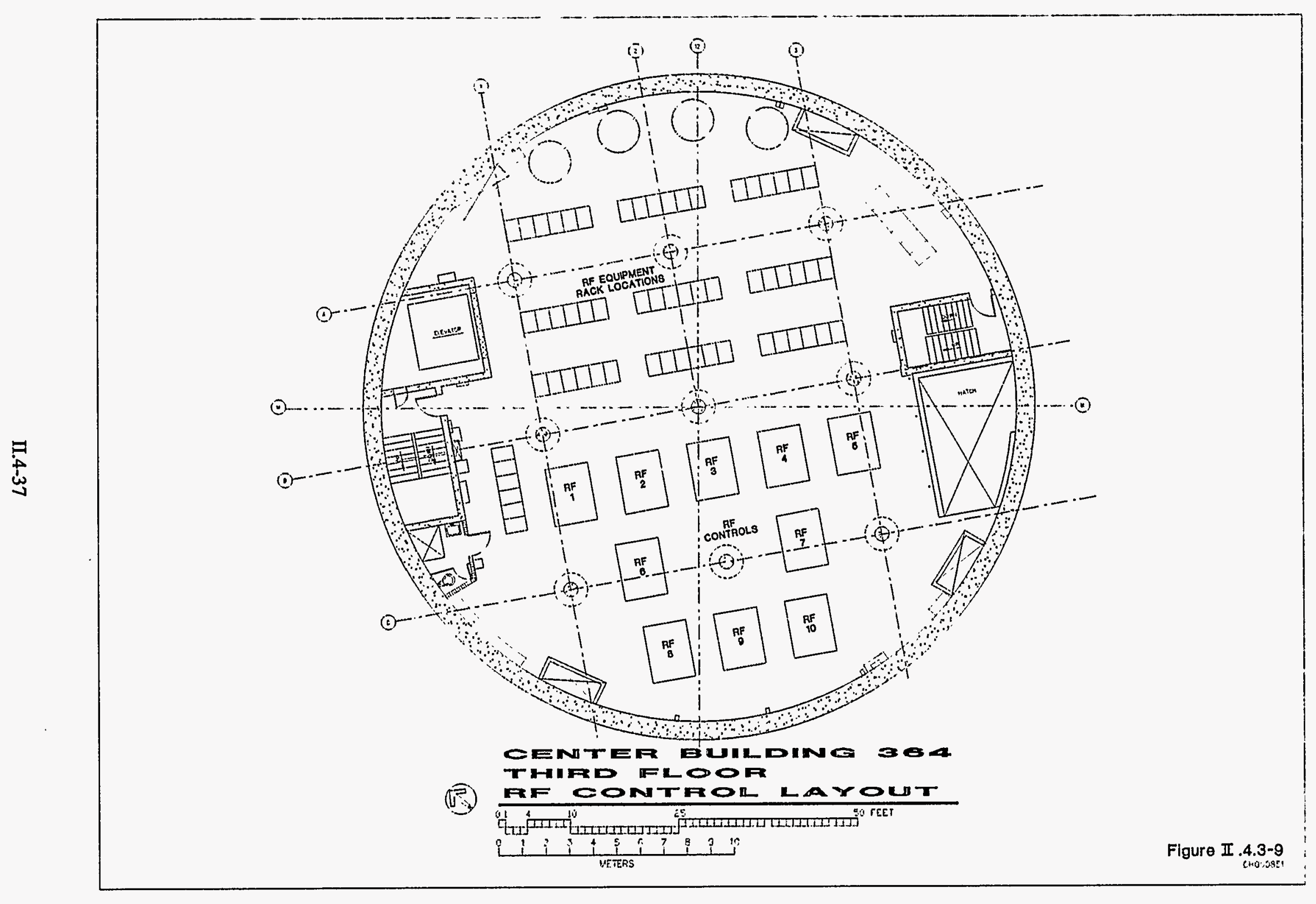




\subsection{REFERENCES}

1. G. Hemmie (compiler), Catalogue of High Energy Accelerators, XVth International Conference on High Energy Accelerators (Hamburg, Germany, Nov. 1992).

2. E. S. Lessner and Y. Cho, "RF capture studies for injection into a synchrotron," in Proceedings of the 1993 Particle Accelerator Conference, p. 399 (1993).

3. K. Symon, Synchrotron Motion with Space Charge, Argonne National Laboratory Report No. NSA-94-3 (1994).

4. Y. Cho, E. Lessner, and K. Symon, "Injection and capture simulations for a high intensity proton synchrotron," in Proceedings of the European Particle Accelerator Conference, p. 1228 (1994).

5. D. Boussard, "Control of cavities with high beam loading," IEEE Trans. Nucl. Sci. NS-32(5) (1985).

6. G. H. Rees, "Status report on ISIS," presented at 1987 IEEE Particle Accelerator Conference (March 16-19, 1987), IEEE Catalog No. 87CH2387-9 (1987).

7. T. W. Hardek and W. E. Chyna, "Common anode amplifier development," IEEE Trans. Nucl. Sci. NS-26(3) (1979).

8. S. Giordano and M. Puglisi, "A cathode follower power amplifier," IEEE Trans. Nucl. Sci. NS-30(4) (1983).

9. Y. Irie, "Very low output impedance RF system for high intensity accelerators," in Proceedings of the 12th Meeting of the International Collaboration on Advanced Neutron Sources, p. A131 (May 1993).

10. Kaon Factory Study, Accelerator Design Report (TRIUMF, Vancouver, British Columbia, Canada, undated).

11. K. W. Robinson, Cambridge Electron Accelerator Report No. CEAL-1010 (1964).

12. K. Kasper and M. Emmerling, "Development of the if accelerating system for the GSI heavy ion synchrotron," IEEE Trans. Nucl. Sci. NS-28, 3028 (1981).

13. M. Plotkin, "Isabelle cavity gap assemblies," IEEE Trans. Nucl. Sci. NS-28(3), 2752 (1981). 


\subsection{INTRODUCTION}

Collective instabilities are an important consideration in the RCS. These instabilities are intensity-dependent effects that arise as a result of the electromagnetic wake fields generated by the beam as it interacts with its surroundings. The interactions are characterized by the coupling impedance. In the circulating current, small density fluctuations driven by wake fields of sufficient magnitude and the proper phase can grow, leading to emittance growth and/or beam loss.

The machine coupling impedance is described first, followed by discussion of collective instabilities, which must be controlled in a high-intensity machine of this type. Longitudinal, transverse, and electron-proton instabilities are discussed separately. Passive control is included as an integral part of the discussion because it is preferred, where possible, in mitigating collective instabilities. This passive control includes minimizing the coupling impedance and making use of Landau damping by introducing a tune spread. It is expected that active control of instabilities through beam feedback can be avoided.

\subsection{COUPLING IMPEDANCE}

The coupling impedance is estimated by using the standard, approximate models. ${ }^{1-5}$ The results for the longitudinal impedance, $Z_{\|}$, and the transverse impedance, $Z_{\perp}$, are summarized in Table II.5.2-1, and detailed calculations follow in the next two sections. The longitudinal impedance is normalized by the mode numbers, $n=\omega / \omega_{0}$, where $\omega_{0}$ is the revolution frequency. For the longitudinal case, the coupling impedance of interest corresponds to the mode numbers $\mathrm{n} \leq 500$, using the cutoff frequency $\omega_{\mathrm{c}}=\mathrm{c} / \mathrm{b}$, where $\mathrm{b}$ is the average if shield radius, and $\mathrm{c}$ is the velocity of light. For the transverse case, $\omega=(p+v) \omega_{0}$, where $p$ is a negative integer near $v$, the horizontal or vertical tune.

The impedance is dominated by the space charge self-field. The beam position monitors (BPMs), extraction kickers, if cavities, and the rf shield also contribute to the impedance. The impedance due to other components, such as vacuum ports and bellows, is expected to be negligible, because these are isolated from the beam by the rf shield. The components of the coupling impedance depend on time-varying machine parameters. The results in the table correspond to injection, unless otherwise noted. The time-dependence of the other components is discussed in Section II.5.2.2. The space charge impedance is capacitive, while the others are inductive at the frequencies of interest and include a resistive term. 
Table II.5.2-1 RCS Impedance Estimation

\begin{tabular}{|c|c|c|c|c|}
\hline & $\begin{array}{c}\operatorname{Re}\left(Z_{\|} / n\right) \\
(\Omega)\end{array}$ & $\begin{array}{c}\operatorname{Im}\left(Z_{\|} / n\right) \\
(\Omega)\end{array}$ & $\begin{array}{l}\operatorname{Re}\left(Z_{\perp}\right) \\
(k \Omega / m)\end{array}$ & $\begin{array}{l}\operatorname{Im}\left(Z_{\perp}\right) \\
(k \Omega / m)\end{array}$ \\
\hline Space charge (injection) & $-a$ & -220 & - & $-2,800$ \\
\hline Space charge (extraction) & - & -50 & - & $-1,500$ \\
\hline rf shield & $0.2 / \sqrt{n}$ & $0.2 / \sqrt{\mathrm{n}}$ & $11 / \sqrt{\omega}^{b}$ & $11 / \sqrt{\omega}^{b}$ \\
\hline Extraction kickers & - & - & $30^{c}$ & 50 \\
\hline If cavities & $14^{d}$ & e & - & - \\
\hline BPMs ${ }^{f}$ & $10^{-3} n$ & 0.06 & $10^{-2} \omega^{b}$ & 110 \\
\hline
\end{tabular}

$a_{-}=$negligible or not applicable.

b $\omega$ has units of $10^{6} \mathrm{rad} / \mathrm{sec}$.

c Maximum value, at $1 \mathrm{MHz}$, decreasing thereafter as $\omega^{-1}$.

d Maximum value, at $\omega_{c}$, decreasing to zero at $\omega \ll \omega_{c}$ and $\omega \gg \omega_{c}$.

e Zero at $\omega_{c}$, inductive for $\omega<\omega_{c}$, capacitive for $\omega>\omega_{c}$.

Valid up to $125 \mathrm{MHz}$. 


\subsubsection{Space Charge Impedance}

The impedance due to the self-field of a charged beam in a vacuum chamber, commonly called the space charge impedance, is given by ${ }^{1,3}$

$$
\frac{Z_{\|}}{n}=-j \frac{Z_{0}}{2 \beta \gamma^{2}} g_{0} \quad Z_{\perp}=-j \frac{R Z_{0}}{\beta^{2} \gamma^{2}} g_{\perp}
$$

where $Z_{0}=\mu_{0} c=377 \Omega, R$ is the average radius of the synchrotron, and $\beta$ and $\gamma$ are the standard relativistic factors, which are time-varying because of the acceleration. The beam has an elliptical cross section, its major axis alternating between the horizontal and vertical planes. However, for the calculations, a uniform, round, unbunched beam of radius a in a vacuum chamber of radius $b$ is assumed in evaluating the geometrical factors $g_{0}$ and $g_{\perp}$ :

$$
\mathrm{g}_{0}=1+2 \ln \left(\frac{\mathrm{b}}{\mathrm{a}}\right), \quad \mathrm{g}_{\perp}=\frac{1}{\mathrm{a}^{2}}-\frac{1}{\mathrm{~b}^{2}} .
$$

The transverse beam radius, a, decreases during the acceleration cycle.

The vacuum chamber is constructed with a special rf shield, shown in Figure II.5.2-1, to minimize the impedance due to space charge. The shield follows the beam envelope at an aperture equal to the BSC, as discussed in Section II.2.1.3. Details of the design are discussed in Sections II.5.2.2 and II.6.2.

The impedance is estimated by using the average beam and $\mathrm{rf}$ shield radii, which are $5 \mathrm{~cm}$ and $7 \mathrm{~cm}$, respectively, at injection. The results for the space charge impedance during the acceleration cycle are shown in Figure II.5.2-2 for both the longitudinal and transverse planes. The difference between calculating $Z_{\|}$by using the average values of $a$ and $b$, and calculating the average value of $Z_{\|}$directly, is no more than $1 \%$ for the longitudinal plane and $10 \%$ for the transverse plane. Compared with a fixed-radius rf shield, this contour-following scheme reduces the longitudinal space charge impedance by $30 \%$ at injection and $20 \%$ at extraction. The transverse impedance is reduced by $35 \%$ at injection and $10 \%$ at extraction.

A number of corrections to the geometrical factors have been proposed to account for the wires and the more realistic elliptical beam cross section. The electrostatic fields produced by a uniform beam propagating inside a round, if-screening wire cage have been derived by T. Wang. 6 A second correction, derived by $\mathrm{H}$. Okamoto, takes into account the varying elliptical shape of the beam in a smooth, metallized vacuum chamber without wires and with a fixed radius. ${ }^{7}$ These 


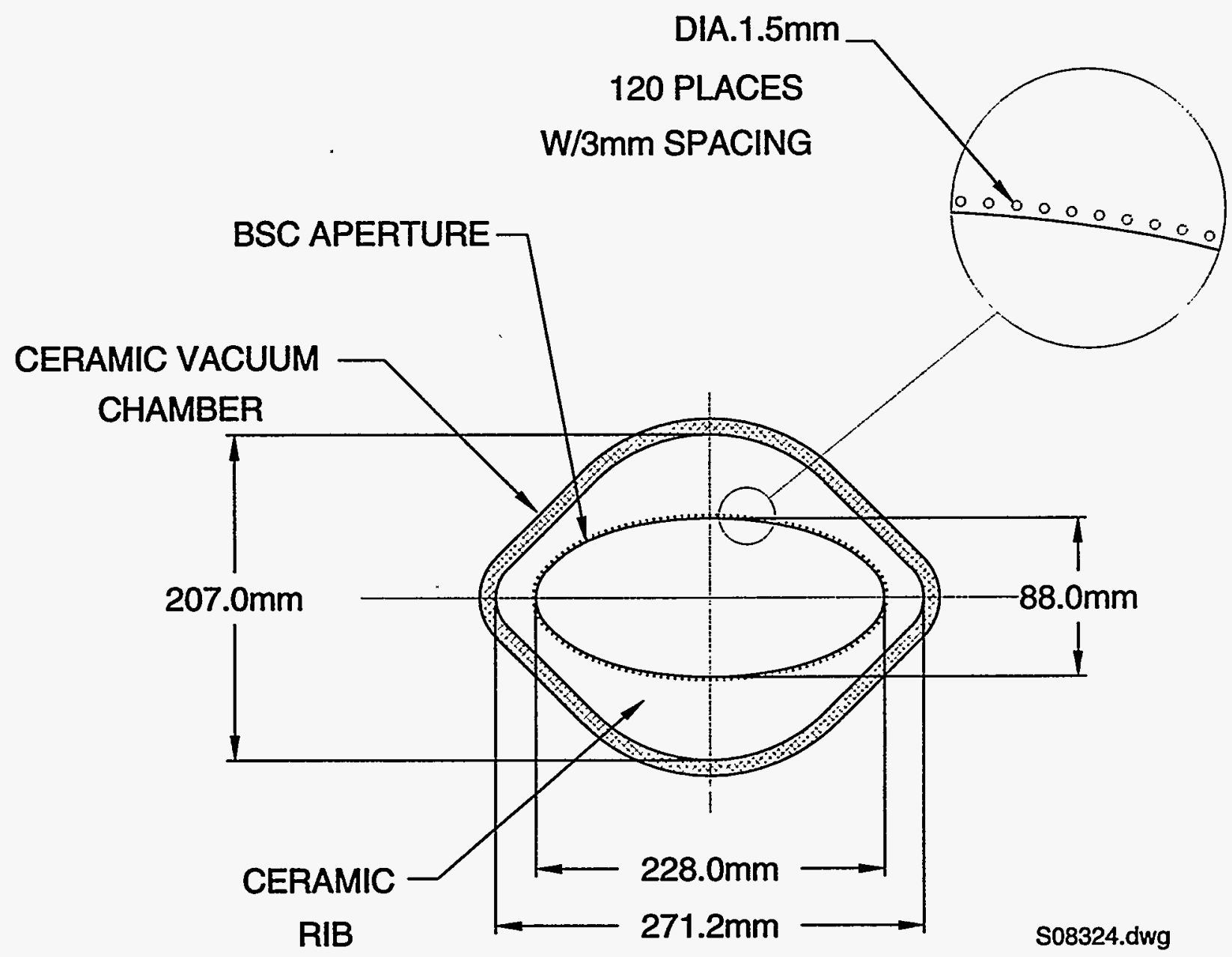

Figure II.5.2-1 Ceramic Vacuum Chamber Cross Section in the Focusing Quadrupole Magnet, Showing Placement of the if Shield Wires at the BSC Aperture. 

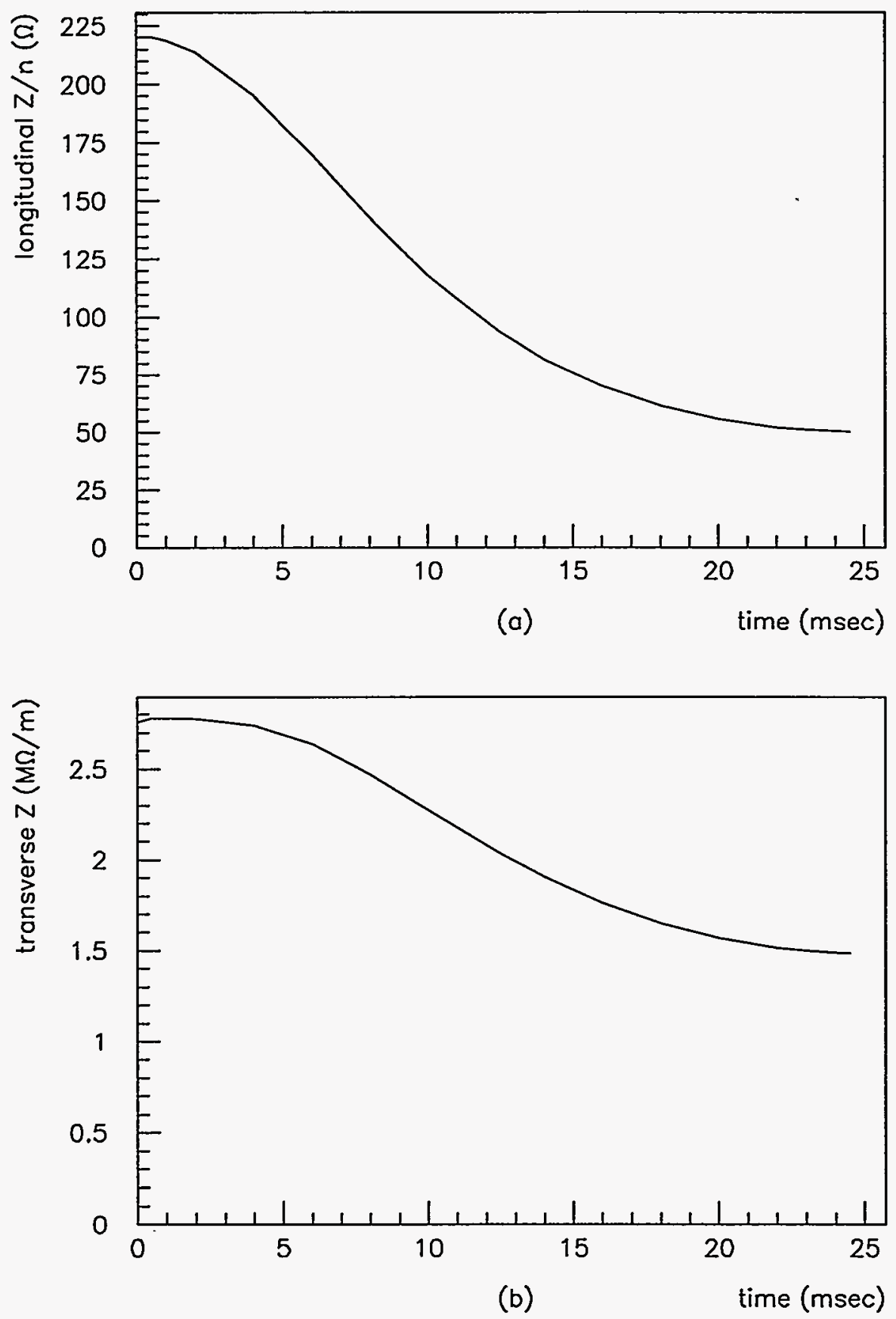

Figure II.5.2-2 Impedance due to Space Charge Plotted as a Function of Time. (Longitudinal impedance is shown in the top plot, a; transverse impedance is shown in the bottom plot, b.) 
corrections result in a difference of less than $2 \%$ in the longitudinal impedance. Wang's method results in a $+15 \%$ correction in the transverse impedance, and Okamoto's method results in a $-20 \%$ correction. 8 Until a rigorous derivation is performed for a contour-following wire if shield, we retain the averaged result and treat the above corrections as an uncertainty.

\subsubsection{Other Contributions}

Contributions to the coupling impedance from the rf shield, kickers, if cavities, and BPMs are evaluated in this section. Unless otherwise noted, the formulae are derived in Reference 4.

The RCS has a ceramic vacuum chamber to avoid eddy current losses and distortions of the magnetic field. The if shield is designed to make good contact between neighboring chambers to reduce the resistive impedance and to provide a return path for the image current. It shields the beam current and allows penetration of the slowly varying magnetic fields. About 120 equally spaced beryllium-copper wires, with an electrical conductivity of $\sigma=14 \times 10^{7} \Omega^{-1} \mathrm{~m}^{-1}, 9$ are mounted axially inside the chamber, as shown in Figure II.5.2-1. Formulae for a smooth, cylindrical, conducting beam pipe are used to estimate the impedance due to the rf shield at frequency $\omega$ :

$$
\left(\frac{Z_{\| l}}{n}\right)_{\text {wall }}=(1+j) \frac{R}{\sigma \delta b} \frac{\alpha}{n} \quad\left(Z_{\perp}\right)_{\text {wall }}=\frac{2 c}{\omega b^{2}}\left(Z_{\| l}\right)_{\text {wall }},
$$

where $\delta=\sqrt{2 / \mu \sigma \omega}$ is the skin depth, $\alpha=\left(1+\mathrm{e}^{-2(1-\mathrm{j}) \mathrm{d} / \delta}\right) /\left(1-\mathrm{e}^{-2(1-\mathrm{j}) \mathrm{d} / \delta}\right)$ is a multiplier, and $d$ is the thickness of the conducting surface. The multiplier becomes unity when the thickness of the conducting surface is greater than the skin depth, or $d>>$. The lowest possible frequencies occur at the values $n=1$ in the longitudinal case and $|p+v| \approx 0.2$ in the transverse case. The skin depth is $0.28 \mathrm{~mm}$ for the lower frequency, $200 \mathrm{kHz}$, compared with a wire diameter of $1.5 \mathrm{~mm}$. Therefore, the thick-wall approximation is used. $Z_{\|} / \mathrm{n}$ scales in time with $\sqrt{\omega_{0}}$ and is $16 \%$ higher at extraction than at injection for a given $n$. $Z_{\perp}$ scales with $1 / \sqrt{\omega_{0}}$ and is $16 \%$ lower at extraction for a given $\mathbf{p}$. 
The coupling impedance due to the six extraction kicker magnets is estimated by following the approach of Nassibian and Sacherer. 5 For no eddy current losses and infinite permeability in the ferrite core, the impedance due to a window-frame magnet is given by

$$
\left(\frac{\mathrm{z}_{\|}}{\mathrm{n}}\right)=\frac{\omega^{2} \mu_{0}^{2} \mathrm{x}_{0}^{2} l^{2}}{4 \mathrm{w}^{2} \mathrm{Z}_{\mathrm{k}}} \frac{1}{\mathrm{n}} \quad\left(\mathrm{z}_{\perp}\right)=\frac{\mathrm{c} \omega \mu_{0}^{2} l^{2}}{4 \mathrm{w}^{2} \mathrm{Z}_{\mathrm{k}}},
$$

where $l$ and $\mathrm{w}$ are the length and half-width of the magnet, respectively. The maximum horizontal displacement, $\mathrm{x}_{0}$, of the corrected beam centroid is $1.0 \mathrm{~mm}$. The kickers are travelingwave structures terminated into a matched load; therefore, the magnet circuit impedance is $Z_{k}=2 Z_{s}$, where $Z_{s}$ is the characteristic impedance of the cables that feed the magnet. When transit-time effects are included, the impedance due to the kickers becomes 4

$$
\begin{array}{ll}
\operatorname{Re}\left(\frac{Z_{\|}}{\mathrm{n}}\right)=\frac{Z_{\mathrm{s}}}{2} \frac{\mathrm{x}_{0}^{2}}{\mathrm{ng}^{2}} \sin ^{2}\left(\frac{\mathrm{k} l}{2}\right), & \operatorname{Im}\left(\frac{\mathrm{Z}_{\|}}{\mathrm{n}}\right)=\frac{\mathrm{Z}_{\mathrm{s}}}{4} \frac{\mathrm{x}_{0}^{2}}{\mathrm{ng}^{2}}(\mathrm{k} l-\sin \mathrm{k} l), \\
\operatorname{Re} Z_{\perp}=\frac{\mathrm{Z}_{0} l}{4 \mathrm{wg} k} \frac{1}{\mathrm{k} l}(1-\cos \mathrm{k} l), & \operatorname{Im} Z_{\perp}=\frac{Z_{0} l}{4 \mathrm{wg}} \frac{1}{\mathrm{k} l}(\mathrm{k} l-\sin \mathrm{k} l),
\end{array}
$$

where $\mathrm{g}$ is the gap half-height and $\mathrm{k} l=\frac{\omega \mathrm{Z}_{0} \mathrm{~g}}{\mathrm{cZ}_{\mathrm{s}} \mathrm{w}} l$ is the electrical length of the kicker winding. The magnet parameters are given in Table II.3.4-2. The longitudinal impedance is negligible. The real part of the transverse impedance is peaked around $1 \mathrm{MHz}$.

The aggregate effect of all the if cavity higher-order modes (HOMs) on the bunch is described by an rf-equivalent broadband impedance. This is represented by a single, $Q=1$ resonator centered at the cutoff frequency $\omega_{c}$ and is calculated by using 10

$$
\left(\frac{Z_{\|}}{n}\right)_{\text {HOM }}=N_{\text {rf }} \sum_{q=2}^{M}\left(\frac{R_{\text {sh }}}{Q}\right)_{q} \frac{\omega_{0}}{\omega_{\text {res q }}},
$$

where $R_{\text {sh }}$ is the shunt resistance of cavity mode $q, \omega_{\text {res }}$ is the resonant frequency, $Q$ is the quality factor, and $\mathrm{N}_{\mathrm{rf}}=10$ is the total number of cavities. The fundamental mode is excluded from the sum. Modal analysis of the rf cavities described in Section II.4.3 was performed by using URMEL-T, 11 and the resulting mode frequencies and ratio $R_{S h} / Q$ are summarized in Table II.5.2-2. These solutions correspond to a cavity tuned to the fundamental frequency of 1.3 MHz about midway through the acceleration cycle and for which $R_{s h} / Q$ is equal to 105 . The 
mode at $5.6 \mathrm{MHz}$ is the first quarter-wave resonance. At the fundamental frequency, the gap capacitance and ferrite inductance appear as lumped elements.

Modes where $\mathrm{q}$ is greater than 4 do not contribute significantly. The impedance in Table II.5.2-1 is obtained by using the values from Table II.5.2-2 and Equation II.5.2-3. The deflecting modes, corresponding to transverse impedance, lie considerably above the fundamental frequency and do not couple well to the beam. The lowest transverse mode occurs at $34 \mathrm{MHz}$, with an $R_{\perp} / Q$ of $0.03 \Omega / \mathrm{m}$, and is negligible, as indicated in Table II.5.2-1.

There are a total of 56 two-electrode, stripline BPMs, one in each quadrupole. Half of these are horizontal pickups, and half are vertical. The impedance of each BPM is calculated by using

$$
\left(\frac{\mathrm{Z}_{\|}}{\mathrm{n}}\right)_{\mathrm{BPM}}=\left(\frac{\omega l}{\mathrm{c}}+\mathrm{j}\right)\left(\frac{\varphi}{2 \pi}\right)^{2} \frac{\omega_{0} l}{\mathrm{c}} \mathrm{Z}_{\mathrm{s}} \quad\left(\mathrm{Z}_{\perp}\right)_{\mathrm{BPM}}=\frac{\mathrm{c}}{\omega \mathrm{b}^{2}}\left(\frac{4}{\varphi}\right)^{2} \sin ^{2}\left(\frac{\varphi}{2}\right)\left(\mathrm{Z}_{\|}\right)_{\mathrm{BPM}},
$$

where it is assumed that $Z_{s}=50 \Omega$ is the characteristic impedance, $l=0.6 \mathrm{~m}$ is the length, and $\varphi=0.25 \mathrm{rad}$ is the angle subtended by one $2.5-\mathrm{cm}$-wide stripline. The longitudinal impedance is evaluated at $\omega=n \omega_{0}$. These expressions are valid up to the frequency $\omega=\pi c /(2 l)$, or $125 \mathrm{MHz} . \operatorname{Re}\left(\mathrm{Z}_{\|} / \mathrm{n}\right)$ increases by $80 \%$ at extraction, and $\operatorname{Im}\left(\mathrm{Z}_{\|} / \mathrm{n}\right)$ increases by $34 \% . \operatorname{Re}\left(\mathrm{Z}_{\perp}\right)$ increases by $15 \%$ at extraction, and $\operatorname{Im}\left(Z_{\perp}\right)$ is constant.

The impedance due to other components, such as vacuum ports and bellows, is expected to be negligible, because these components are isolated from the beam by the rf shield.

The impedances obtained from these calculations are summarized in Table II.5.2-1. 
Table II.5.2-2 Ratio of Shunt Impedance and $Q$ of First Few HOMs for an if Cavity

\begin{tabular}{cc}
$\begin{array}{c}\omega_{\text {res }} / 2 \pi \\
(\mathrm{MHz})\end{array}$ & $\begin{array}{c}\mathrm{R}_{\text {st }} / \mathrm{Q} \\
(\Omega)\end{array}$ \\
\hline & \\
5.6 & 5.5 \\
10.5 & 0.8 \\
$14.8^{\mathrm{a}}$ & 0.2 \\
\hline
\end{tabular}

a Extrapolation of ferrite properties beyond normal operating range of 0.5 to $10 \mathrm{MHz}$. 


\subsection{LONGITUDINAL INSTABILITIES}

Of the several known longitudinal instabilities, only the microwave instability due to the coupling impedance between the beam and its surroundings needs to be considered for the RCS. This instability should not occur in a machine operating below transition energy if the coupling impedance for the ring is purely capacitive. In the RCS, the impedance is largely capacitive, and the instability is not expected to occur; however, the instability could occur when there are resistive components. The instability growth rate then depends on the momentum distribution of the beam, particularly the shape of the distribution tails. A rigorous analysis of this instability requires knowledge not only of the coupling impedance seen by the beam, but also of the peak current and energy spread of the beam. At this stage, this beam information was obtained through Monte Carlo simulations.

The following steps are taken to design a conservative system to prevent the onset of instability:

- The impedance contributions from the various RCS components are estimated.

- The Keil-Schnell criterion, derived for a coasting beam, is applied to bunched beams; this is a commonly accepted practice.

- Although the Keil-Schnell criterion overestimates the severity of the instability below transition, this criterion is used to obtain the required $\Delta p / p$ to raise the threshold current.

- By using the peak current and energy spread of the circulating beam obtained from the longitudinal tracking studies described in Section II.4.1.3, a detailed analysis of the stability diagram is made.

A simplified criterion for the longitudinal stability threshold, independent of the details of the particle phase space distribution and applicable to bunched beams, is written as 1,2

$$
\left|\frac{Z_{\text {il }}}{n}\right| \leq F \frac{|\eta| \beta^{2} E / e}{I_{p k}}\left(\frac{\Delta p_{f w h m}}{p}\right)^{2}
$$

where local values of the current and momentum are used. Equation II.5.3-1 is commonly referred to as the Keil-Schnell $(K-S)$ criterion. Here, $F \approx 1$ is a form factor, $I_{p k}$ is the peak current, and $\eta=1 / \gamma_{t}^{2}-1 / \gamma^{2}$, where $\gamma_{t}$ is the transition energy. 
The K-S criterion imposes a lower bound on the ratio $(\Delta \mathrm{p})^{2} / \mathrm{I}_{\mathrm{pk}}$. This requirement drives the peak If voltage, because the momentum spread and peak current both depend on the if voltage. Since the K-S criterion depends quadratically on the momentum spread and is inversely proportional to the peak current, increasing the momentum spread is more effective in achieving stability than is lowering the peak current. The optimal rf voltage profile over the cycle is obtained through simulation studies, as discussed in Section II.4.1.3. The peak current is obtained from the simulation by searching for the maximum of the particle distribution projected on the phase axis. The time-varying rf voltage and peak current are shown in Figure II.5.3-1a, and the bunching factor, $\mathrm{B}_{\mathrm{f}}=\mathrm{I} / \mathrm{I}_{\mathrm{pk}}$, is shown in Figure II.5.3-1b. The energy spread is obtained from the projection of the particle distribution on the energy axis.

The momentum spread corresponding to the threshold for the microwave instability is computed by substituting the impedance listed in Table II.5.2-1 and the peak current from Figure II.5.3-1a into Equation II.5.3-1. The time variation in the longitudinal instability threshold is plotted together with the momentum spread obtained from tracking in Figure II.5.3-2. The beam remains in the stable region throughout the cycle, within the assumptions of the theory and the impedance estimate.

Below transition energy, it is possible to operate the machine with a large capacitive impedance outside the K-S boundary and preserve stability. The stable region is dependent on both the resistive component of the impedance and the shape of the distribution tails. The stable region can be seen in Figures II.5.3-3a and II.5.3-4a for two different momentum distributions; the details are discussed below.

The response of a beam to a periodic perturbation, $\exp (j(\Omega t-n \varphi))$, is given by the Vlasov equation, where $\varphi$ is the azimuthal phase coordinate and $\Omega$ is the driving frequency of the perturbation. The solution to the small-amplitude Vlasov equation leads to a dispersion relation, which, for a coasting beam, is given by 12

$$
1=-\frac{2}{\pi(\mathrm{E} / \mathrm{e}) \beta^{2}|\eta|(\Delta \mathrm{p} / \mathrm{p})^{2}} \frac{\mathrm{Z}}{\mathrm{n}} \mathrm{I}_{\mathrm{D}}^{\prime} .
$$

$\mathrm{I}_{\mathfrak{D}}^{\prime}$ is the normalized dispersion integral, given by

$$
I_{D}^{\prime}=j \int \frac{\partial f / \partial x}{x-x_{1}} d x
$$



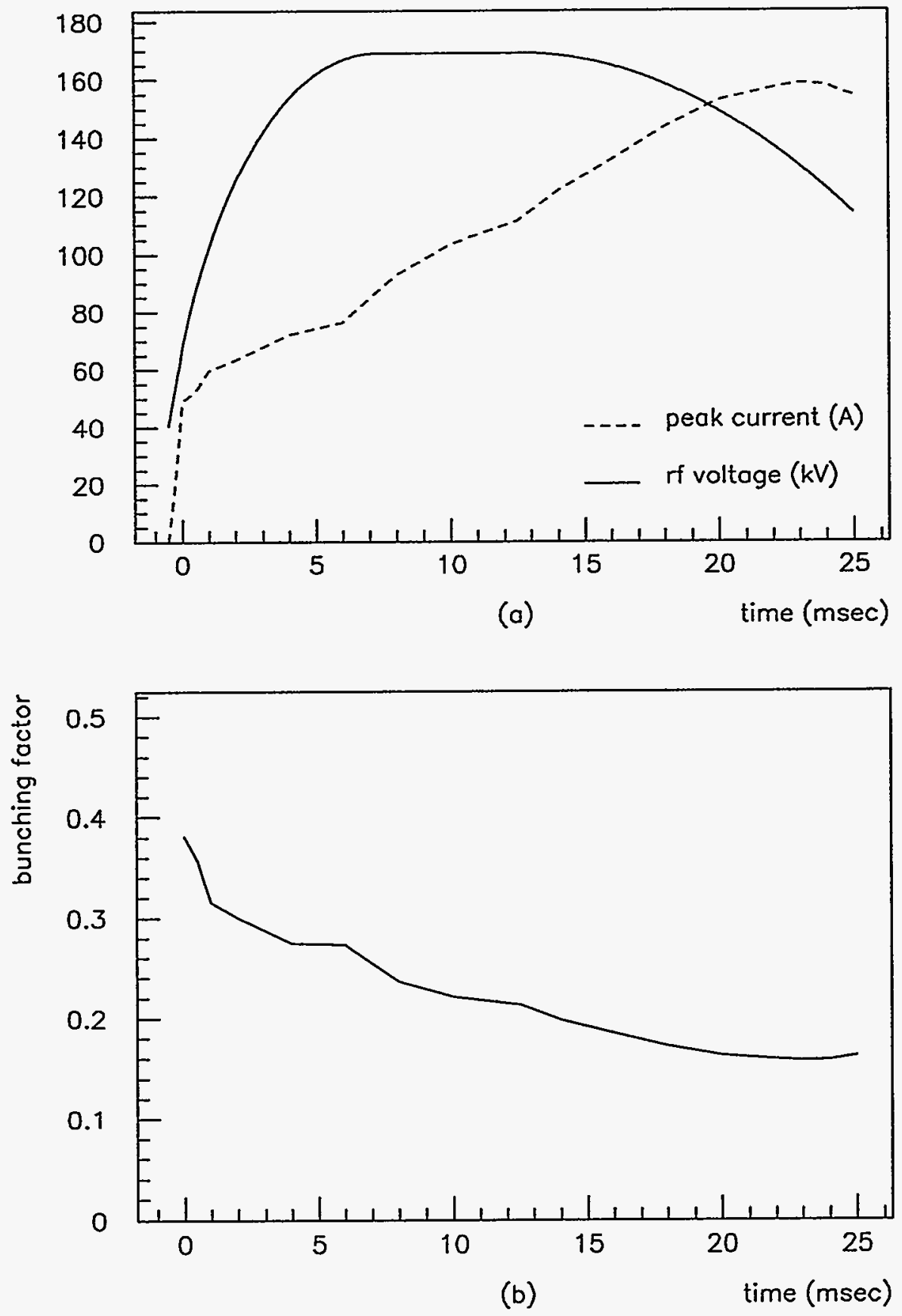

Figure II.5.3-1 Variation of the (a) Peak Current and (b) Bunching Factor, $B_{f}=I / I_{p k}$. (The if voltage program is superimposed in the top graph, a. The peak current is obtained from the longitudinal tracking, and the small fluctuations are due to Monte Carlo statistics.) 


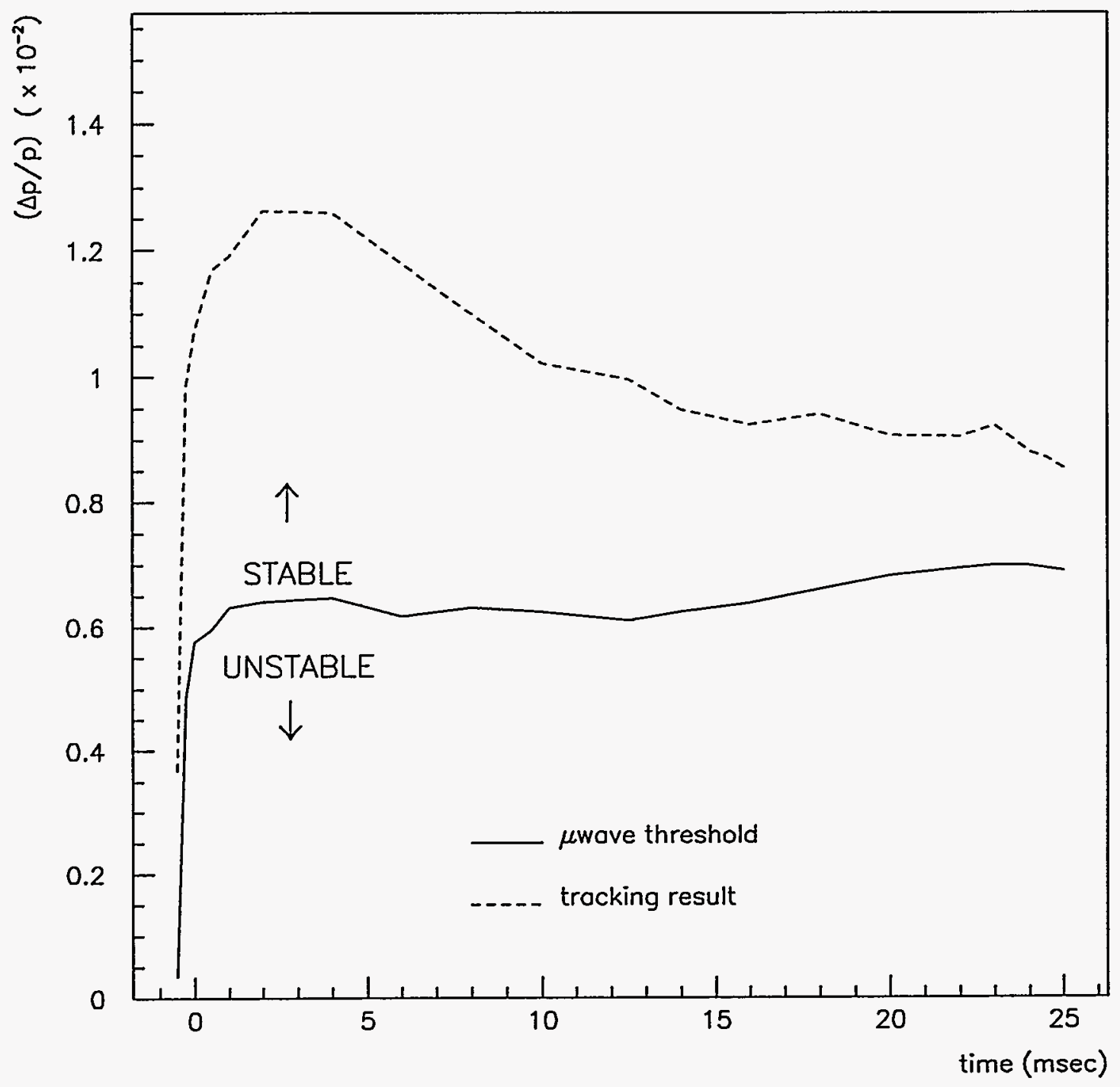

Figure II.5.3-2 Longitudinal Instability Threshold According to the K-S Criterion (lower curve) Plotted Together with the Momentum Spread Obtained from Tracking. (The small fluctuations are due to the Monte Carlo statistics. The beam remains in the stable region throughout the cycle.) 

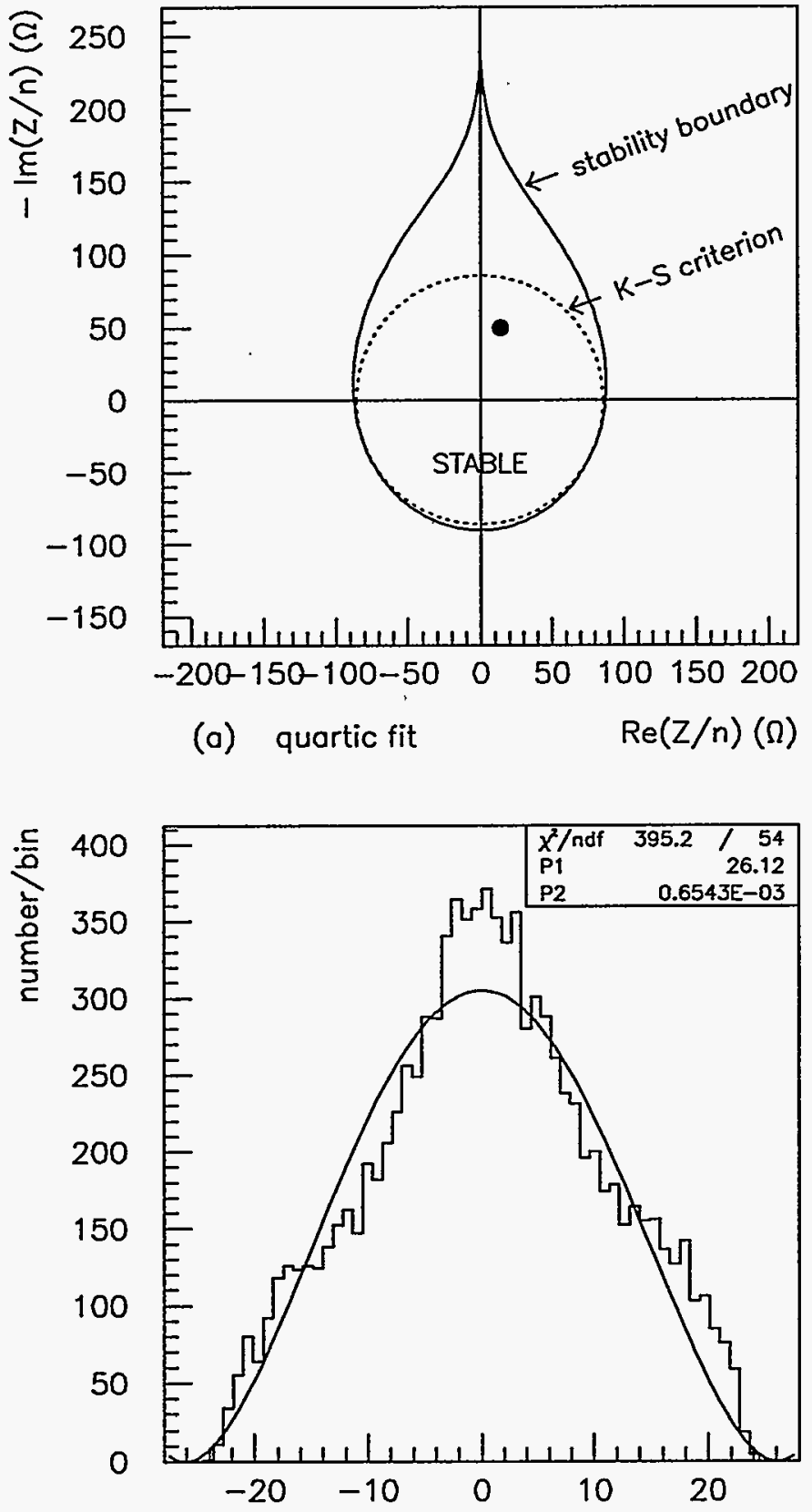

(b) distrib $\Delta E(M e V)$ : quartic fit $p 2 *\left(p 1^{2}-x^{2}\right)^{2}$

Figure II.5.3-3 Longitudinal Stability Boundary (a) for a Quartic Momentum Distribution (b). (The particle distribution is obtained from the tracking. The region in the top plot, a, that includes the origin is stable.

Plot a also shows the stable region that satisfies the K-S criterion. The value of the longitudinal coupling impedance from Table II.5.2-1 is indicated by a dot near the vertical axis.) 


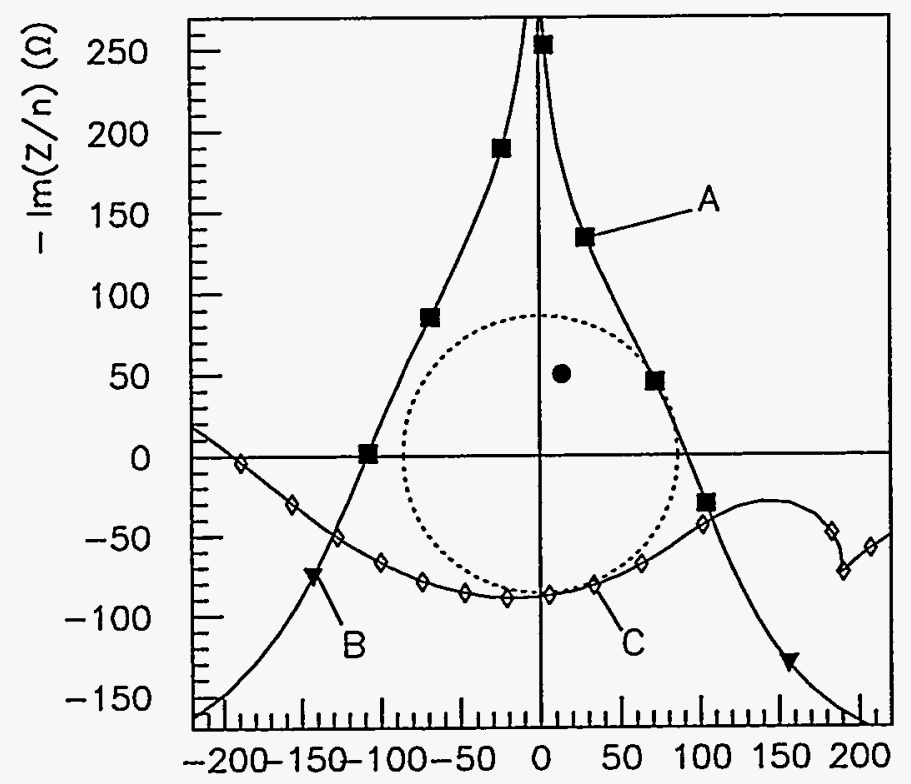

(a) smooth fit $\operatorname{Re}(\mathrm{Z} / \mathrm{n})(\Omega)$

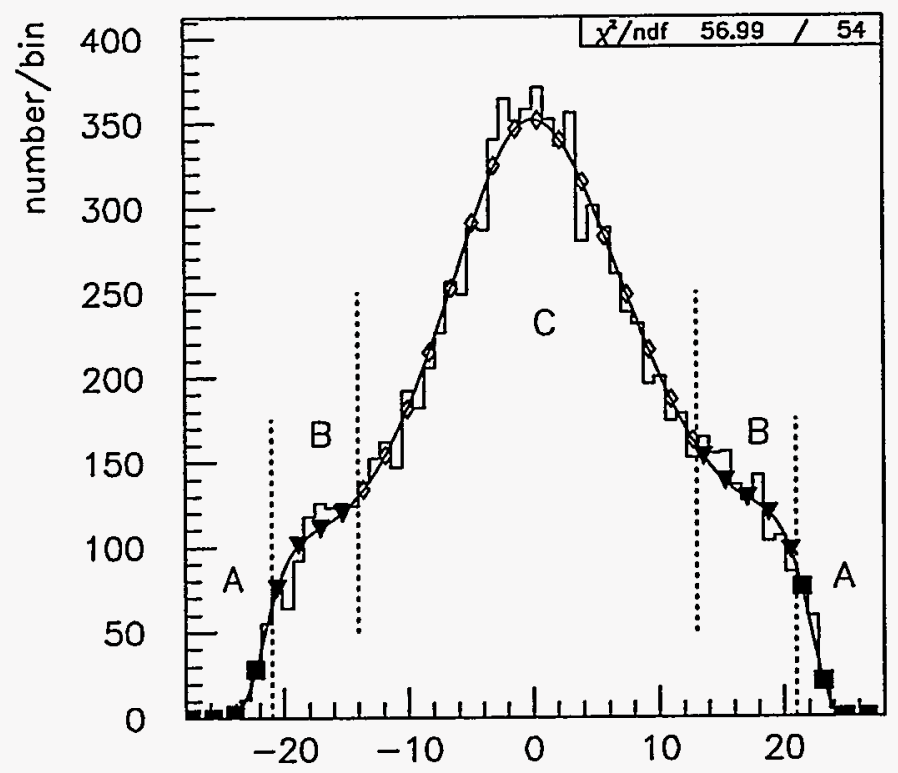

(b) distrib $\triangle \mathrm{E}(\mathrm{MeV})$ : smooth algorithm $353 \mathrm{QH}$

Figure II.5.3-4 Dependence of the Longitudinal Stability Boundary (a) on Momentum Distribution, Using the Distribution in (b). (The particle distribution in the lower plot, b, is a smooth fit of the results from the tracking. Sections of the stability diagram are labeled with the corresponding sections of the distribution. The upper portion of the stable region is entirely determined by the tails of the distribution.) 
where

$$
\begin{aligned}
& \mathrm{x}=\left(n \omega-n \omega_{0}\right) /(\mathrm{nS}) \text { describes the frequencies of the particles in the beam, } \\
& \mathrm{x}_{1}=\left(\Omega-n \omega_{0}\right) /(\mathrm{nS}) \text { is related to the driving frequency of the perturbation, } \\
& 2 \mathrm{~S}=-\eta \omega_{0}(\Delta \mathrm{p} / \mathrm{p}) \text { is the full spread at half maximum of the revolution frequency } \\
& \text { distribution, and }
\end{aligned}
$$$$
f(x)=\text { normalized beam density distribution in frequency space. }
$$

The real part of the driving frequency, $\Omega$, is related to an energy shift, while the imaginary part leads to growth of the perturbation.

A finite spread in the revolution frequency or, equivalently, in momentum, gives rise to Landau damping through $I_{D}^{\prime}$. The factor in Equation II.5.3-2 in front of $I_{D}^{\prime}$ is defined as

$$
\left(V^{\prime}+j U^{\prime}\right)=-\frac{2}{\pi(E / e) \beta^{2} \mid \eta(\Delta p / p)^{2}} \frac{\left(\operatorname{ReZ}_{\|}+j \operatorname{Im} Z_{\|}\right)}{n}
$$

Therefore, the dispersion relation can be rewritten as

$$
1=\left(V^{\prime}+j U^{\prime}\right) I_{D}^{\prime}
$$

This leads to the $\left(U^{\prime}-V^{\prime}\right)$ stability diagram, where the stability boundary is obtained when the dispersion integral is evaluated with $\operatorname{Im} \Omega=0$. The stability diagram can be plotted in the $Z_{\|} / \mathrm{n}$ impedance plane by using Equations II.5.3-3 and II.5.3-2.

The stability diagram for the RCS at $2 \mathrm{GeV}$ is plotted in the impedance plane in Figure II.5.3-3a for the quartic momentum spread shown in Figure II.5.3-3b. Also shown in Figure II.5.3-3a is the stability region that satisfies the K-S criterion. The positive vertical axis denotes capacitive impedance, and the longitudinal coupling impedance listed in Table II.5.2-1 is plotted as a large dot. The particle momentum distribution in Figure II.5.3-3b is obtained from the simulation results.

The stability boundary, which depends specifically on the momentum distribution, can give a higher limit than the $\mathrm{K}-\mathrm{S}$ criterion. However, the upper portion of the stability boundary, which corresponds to the tails of the distribution, is highly sensitive to the beam distribution. This can be seen in Figure II.5.3-4a, which shows the stability boundary obtained by using a 
different distribution, shown in Figure II.5.3-4b. The latter is obtained by using the $353 \mathrm{QH}$ smoothing algorithm. ${ }^{13}$ Sections of the stability diagram are marked according to the corresponding section of the momentum distribution. Experience shows that in measured data, the tails in the line density can be rather unpredictable.

The machine is dominated by the capacitive space charge impedance, which then determines the resistive $\operatorname{Re}\left(Z_{\|} / n\right)$ limit for stability. The stable boundary satisfying the K-S criterion in Figure II.5.3-3a suggests that the beam is stable to a threshold $\operatorname{Re}\left(Z_{\|} / \mathrm{n}\right)$ of $70 \Omega$. This can readily be achieved in the RCS. A similar analysis at $400 \mathrm{MeV}$ gives a resistive limit of $550 \Omega$. The stability criterion is most critical near extraction because the peak current increases by a factor of five, while the momentum spread decreases by $30 \%$. The result is that the righthand side of Equation II.5.3-1 decreases by a factor of ten, while the impedance decreases by a factor of four.

\subsection{TRANSVERSE INSTABחITIES}

A transversely displaced beam generates an imbalance in the image current, which in turn perturbs the circulating beam. A non-zero resistivity in the coupling impedance causes a phase delay in the wake field, which can amplify the perturbations and cause them to grow. The headtail instability is discussed first using Sacherer's bunched beam formalism. ${ }^{14}$ Further discussion of damping transverse instabilities considers the coasting beam stability criterion.

The head-tail effect has been observed at the 500-MeV IPNS, ${ }^{15}$ the KEK Booster, ${ }^{16}$ and ISIS. 17 Therefore, it is analyzed in some detail for the RCS. In the absence of a tune spread, the growth rate of the head-tail instability, assuming a time dependence of $\exp \left(j \Omega_{m} t\right)$, is given by the imaginary part of 14

$$
\Omega_{\mathrm{m}}=\frac{\mathrm{j}}{(1+\mathrm{m})} \frac{\mathrm{cIZ}_{\perp}\left(\omega_{\mathrm{p}}\right)}{4 \pi v(E / \mathrm{e})} \mathrm{F}_{\mathrm{m}}^{\prime},
$$

where

$$
F_{m}^{\prime}=\frac{1}{B} \frac{h_{m}\left(\omega_{p}-\omega_{\xi}\right)}{\sum_{p} h_{m}\left(\omega_{p}-\omega_{\xi}\right)}
$$

In this expression, it is assumed that the instability is dominated by the contribution from the impedance evaluated at a single frequency, $\omega_{p}=(p+v) \omega_{0}$. The total growth rate involves a convolution of this impedance with the bunch spectra, which are given by the functions $h_{m}$. The 
lowest mode numbers, $\mathrm{m}$, which denote the number of nodes along the bunch, give the highest growth rates. The chromatic frequency is $\omega_{\xi}=(\xi / \eta) v \omega_{0}$, where $\xi=(\Delta v / v) /(\Delta \mathrm{p} / \mathrm{p})$ is the normalized chromaticity. The head-to-tail phase difference in the bunch is given by $\chi=\omega_{\xi} \tau_{L}$, where $\tau_{L}$ is the bunch length in seconds. The ratio of bunch length to ring circumference is given by $B$. When $\operatorname{Re} Z_{\perp}\left(\omega_{p}\right)<0$, the instability grows; when $\operatorname{Re} Z_{\perp}\left(\omega_{p}\right)>0$, the instability is damped.

For the RCS at $400 \mathrm{MeV}$, the form factor, $\mathrm{F}_{\mathrm{m}}^{\prime}$, for $\mathrm{m}=0$ has a half-width of about $2 \mathrm{MHz}$, and the form factor for $\mathrm{m}=1$ peaks around $1 \mathrm{MHz}$. The chromaticity is generally corrected to zero to avoid a large betatron tune spread in the large-momentum particles, as discussed in Section II.2.1.6. When the chromaticity is zero, $\chi=0$ and the bunch spectra are centered at zero frequency, where the rf shield and kicker impedances are important. In the vertical plane, the largest growth due to the rf shield impedance occurs when $p=-6$, and the largest growth due to the kicker impedance occurs when $p=-7$. The BPM impedance does not contribute significantly to the instability growth. The growth rates of the first few head-tail modes are given in Table I1.5.4-1 for the corrected and uncorrected chromaticities at the end of injection. Similar results are found for the horizontal plane. The impedance and the frequency giving the largest growth are also listed; note that $\operatorname{Re} Z_{\perp}\left(\omega_{p}\right)=-\operatorname{Re} Z_{\perp}\left(-\omega_{p}\right)$. The lowest modes are stabilized at the natural chromaticity, $\xi=-1.2$. With the value of $\Delta \mathrm{p} / \mathrm{p}=1.2 \%$ obtained from the longitudinal tracking, however, the corresponding betatron tune spread at the natural chromaticity is 0.1 . Therefore, the chromaticity is adjusted to be between 0 and -1.2 to shift the bunch spectrum to higher-order head-tail modes that have lower growth rates, while at the same time ensuring that the tune spread remains inside a resonance-free working region. The available region can be seen in the tune diagram, Figure II.2.1-8. The stability of modes $m \leq 2$ is assured with $\xi \leq-0.35$. This gives a betatron tune spread of 0.024 .

Discussed next is the transverse stabilization of the beam, achieved by using a betatron tune spread or shift. An amplitude-dependent tune spread is introduced by octupoles and second-order effects in the sextupoles, leading to Landau damping. A momentum-dependent tune shift results from a finite chromaticity. The stability criterion derived for coasting beams is extended heuristically to bunched beams. According to Chao, ${ }^{18}$ there are two stability criteria that can apply to bunched beams, depending on whether the instability growth rate is much larger or much smaller than the synchrotron frequency, $\omega_{\mathrm{s}}$. As shown in Table II.5.4-1, the expected 
Table II.5.4-1 Head-Tail Instability Growth Rate vs. Chromaticity in the Vertical Plane at the End of Injection

\begin{tabular}{cccccc}
\hline & & & & \multicolumn{2}{c}{ Growth Rate (sec) $)^{-1}$} \\
\cline { 5 - 6 } $\begin{array}{c}\text { Mode } \\
\text { number, } m\end{array}$ & $\begin{array}{c}\operatorname{Re}\left(\mathrm{Z}_{\perp}\right) \\
(\mathrm{k} \Omega / \mathrm{m})\end{array}$ & $\begin{array}{c}\text { Frequency } \\
(\mathrm{MHz})\end{array}$ & $\begin{array}{c}\text { Source of } \\
\text { Impedance }\end{array}$ & $\xi=0$ & $\xi=-1.2$ \\
\hline & & & & & \\
0 & 14 & 0.3 & rf shield & 350 & 0 \\
1 & 28 & 0.3 & $\begin{array}{l}\text { kickers } \\
\text { kickers }\end{array}$ & 620 & 0 \\
2 & 28 & 1.4 & kickers & 590 & 0 \\
\hline
\end{tabular}


growth rates are of the order of the synchrotron frequency. Therefore, to be conservative, the peak current is used. The transverse stability criterion for the RCS is given by

$$
\left|\mathrm{Z}_{\perp}\right|<\frac{4 \pi v \omega_{0}(\mathrm{E} / \mathrm{e})}{\mathrm{cI}_{\mathrm{pk}}} \Delta v
$$

The stability criterion is evaluated to determine the threshold tune spread, $(\Delta v)_{\text {thresh }}$, by equating the left-and right-hand sides of the above equation:

$$
(\Delta v)_{\text {thresh }}=\frac{\mathrm{cI}_{\mathrm{pk}}\left|\mathrm{Z}_{\perp}\right|}{4 \pi v \omega_{0}(\mathrm{E} / \mathrm{e})}
$$

Because the impedance is dominated by space charge effects, this expression is equivalent to the coherent Laslett tune shift (due to image charges only.) The results are presented in Table II.5.4-2 for the vertical plane at $400 \mathrm{MeV}$ and $2 \mathrm{GeV}$. The impedance is taken from Table II.5.2-1.

The threshold tune spread determined from the stability criterion can be met by using the momentum-dependent chromatic contribution, $\Delta v_{\xi}=|(p-v) \eta+v \xi|(\Delta p / p)$, and/or the amplitudedependent octupolar contribution, $\Delta v_{\text {oct }}=\frac{\beta_{\mathrm{y}} \mathrm{a}_{\mathrm{y}}^{2}}{32 \pi} \mathrm{k}_{\mathrm{oct}}, 19$ where $\mathrm{k}_{\mathrm{oct}}=\left(\frac{\mathrm{B}^{\prime \prime \prime} l}{\mathrm{B \rho}}\right)$ is the octupole strength. The standard strategy requires that each term satisfy the stability criterion independently, if possible.

Considered first is a solution using the condition $\Delta v_{\xi} \geq \Delta v_{\text {thresh }}$, and using the chromatic term only. In the vertical plane, the value of $p$ is 6 . If the momentum spread is left as a variable and the natural chromaticity is used, simple manipulations give

$$
\frac{\Delta \mathrm{p}}{\mathrm{p}} \geq \frac{(\Delta v)_{\text {thresh }}}{|(\mathrm{p}-v) \eta+v \xi|}=\left\{\begin{array}{cc}
0.8 \% & 400 \mathrm{MeV} \\
0.6 \% & 2 \mathrm{GeV}
\end{array} .\right.
$$

This is to be compared with a momentum spread of about $1 \%$ obtained in the longitudinal tracking study. Conversely, using the momentum spread from the tracking, one obtains the required chromaticity:

$$
\xi \leq \frac{(\Delta v)_{\text {thresh }}}{v(\Delta \mathrm{p} / \mathrm{p})}-\frac{(\mathrm{p}-\mathrm{v}) \eta}{v}=\left\{\begin{array}{rr}
-0.97 & 400 \mathrm{MeV} \\
-0.66 & 2 \mathrm{GeV}
\end{array} .\right.
$$


Table II.5.4-2 Threshold Tune Spread According to the Coasting Beam Stability Criterion

\begin{tabular}{ccc}
\hline $\begin{array}{c}\text { Time in } \\
\text { Cycle } \\
\text { (msec) }\end{array}$ & $\begin{array}{c}1 \mathrm{mZ}_{\perp} \\
(\mathrm{M} \Omega / \mathrm{m})\end{array}$ & $(\Delta \mathrm{V})_{\text {thresh }}$ \\
& & \\
& & \\
0 & 2.7 & 0.06 \\
25 & 1.4 & 0.03 \\
\hline
\end{tabular}


Considered next is a solution using octupoles only and chromaticity fully corrected to zero. The required octupole tune spread is

$$
\Delta v_{\text {oct }} \geq \Delta v_{\text {thresh }}
$$

With a beta function, $\beta_{y}$, of $12 \mathrm{~m}$ and beam size, $a_{y}$, of $0.07 \mathrm{~m}$ at $400 \mathrm{MeV}$ and $0.04 \mathrm{~m}$ at $2 \mathrm{GeV}$, the integrated octupole strength per super-period required for stability is given by

$$
\mathrm{k}_{\mathrm{oct}} \geq \frac{1}{4} \frac{32 \pi}{\beta_{\mathrm{y}}} \frac{(\Delta v)_{\text {thresh }}}{\mathrm{a}_{\mathrm{y}}^{2}}=\left\{\begin{array}{cc}
24 \mathrm{~m}^{-3} & 400 \mathrm{MeV} \\
44 \mathrm{~m}^{-3} & 2 \mathrm{GeV}
\end{array} .\right.
$$

It is assumed that the octupoles are located in each of the four super-periods, at the location of each defocusing quadrupole. The required octupole strength does not degrade the dynamic aperture. It has long been observed in other low-energy accelerators that the current required in the correction octupoles is found to be less than that predicted by the theory. 16,20

Active beam feedback is an option to suppress or damp undesired beam oscillations for the head-tail instability. A typical feedback system consists of a beam position detector, processing electronics and a delay unit, a power amplifier, and a deflection kicker. The characteristics of the feedback system include specifications of the bandwidth, gain, and kicker strength. Other considerations for the system, not discussed here, include reducing noise sensitivity and maintaining synchronicity with the rapidly varying revolution frequency. The bandwidth is about $\omega_{0} / 2$, or $500 \mathrm{kHz}$. The angular deflection given by an electrostatic kicker is given by

$$
\Delta \theta=\frac{\Delta \mathrm{x}}{l}=\frac{\mathrm{eE}_{\mathrm{k}} l}{2 \beta^{2}\left(\mathrm{E}_{\text {beam }}\right)},
$$

where $E_{b e a m}$ is the beam energy, $E_{k}$ is the electric field, and $l$ is the length of the kicker. If an instability rise time of $1 \mathrm{msec}$ and a revolution period of $1 \mu \mathrm{sec}$ are assumed, the instability amplitude growth is

$$
\frac{\Delta \mathrm{x}}{\mathrm{x}_{0}}=\frac{\tau_{\mathrm{rev}}}{\tau_{\text {growth }}}=10^{-3}
$$


where $x_{0}$ is the minimum detected beam deviation, assumed to be $0.5 \mathrm{~mm}$. This ratio is the gain divided by two. Since $x^{\prime}=\left.\frac{x}{\beta_{x}}\right|_{\text {max }}$, the kicker deflection angle can be written as $\Delta \theta=\frac{\Delta x}{x_{0}} \frac{x_{0}}{\beta_{x}}$, and the electric field for the kicker becomes

$$
E_{k} \geq 10^{-3} \frac{x_{0}}{\beta_{x}} \frac{2 \beta^{2}\left(E_{\text {beam }}\right)}{e l}
$$

This becomes $230 \mathrm{~V} / \mathrm{m}$ for a $0.5-\mathrm{m}$ kicker length at $400 \mathrm{MeV}$, and $950 \mathrm{~V} / \mathrm{m}$ at $2 \mathrm{GeV}$, using the average value $\beta_{x}=6 \mathrm{~m}$. It is customary, in practice, to design in an extra factor of 5 to 10 in the electric field.

\subsection{ELECTRON-PROTON INSTABILITY}

The residual gas in the vacuum chamber is ionized by the beam due to collisional processes. The electrons liberated from the gas molecules can be trapped in the proton beam's potential well. Because the trapped electrons oscillate in the potential well, the transverse motions of electrons and protons are coupled, which can result in amplitude growth. This instability, first observed in the coasting beam at the CERN Intersecting Storage Ring (ISR), is called the electron-proton ("e-p") instability. This instability is characterized by a growth time that is comparable with the fastest coherent instabilities caused by coupling impedances. This instability should not be a concern in the RCS because the circulating beam is bunched, and it has also not been observed in similar rapid cycling machines, such as ISIS ${ }^{21}$ at Rutherford, IPNS at Argonne, and the KEK Neutron Source (KENS).

However, a fast instability known as "PSR instability," which is similar to the e-p instability, was recently observed at the Proton Storage Ring (PSR) at Los Alamos during chopped beam accumulation. 22 Whether or not this is a true e-p instability is unknown; however, prior to the onset of the instability, the gap between the bunch passages is filled by bunch leakage. The RCS rf voltage is high enough to avoid bunch leakage during the acceleration period (see Section II.4.1).

The longitudinal beam distribution in the waiting bucket during injection is sensitive both to chopper efficiency and to injection mismatch from the linac. The possibility of e-p instability during injection was investigated, assuming a coasting beam, which is the worst case. The analytical results show that the required neutralizations (electrons/protons) are between $5 \%$ and 
$10 \%$, depending on the tune and the beam size..$^{23}$ Neutralization from the residual gas satisfies the following formula: 24

$$
\eta=0.85 \mathrm{P}(\mathrm{nTorr}) \mathrm{t}(\mathrm{sec})
$$

The vacuum pressure must be less than $10^{-7}$ Torr for $t=0.5 \mathrm{msec}$ injection time. The design value is $10^{-8}$ Torr.

The stripping foil is also a source of electrons, because each $\mathrm{H}^{-}$ion produces two electrons after stripping. This is potentially dangerous, because the circulating bunch could interact with the electron cloud during the injection period of every turn. Clearing electrodes are installed around the foil.to circumvent this potentially dangerous situation.

\subsection{REFERENCES}

1. V.K. Neil and A.M. Sessler, "Longitudinal resistive instabilities of intense coasting beams in particle accelerators," Rev. Sci. Instrum. 36(4), 429 (1965).

2. E. Keil and W. Schnell, Concerning Longitudinal Stability in the ISR, CERN Report No. TH-RF/69-48 (1969).

3. L.J. Laslett, V.K. Neil, and A.M. Sessler, "Transverse resistive instabilities of intense coasting beams in particle accelerators," Rev. Sci. Instrum. 36(4), 436 (1965).

4. K.-Y. Ng, "Fields, impedances, and structures," in The Physics of Particle Accelerators, AIP Conference Proceedings 184, p. 515f (1989).

5. G. Nassibian and F. Sacherer, "Methods for measuring transverse coupling impedances in circular accelerators," Nucl. Instrum. Methods 159, 21 (1979).

6. T-S. F. Wang, Electrostatic Field of a Perturbed Beam with RF-Screening Wires, CERN Report No. PS 94-08 (DI) (1994).

7. AUSTRON Accelerator Feasibility Study, Chapter 4, p. 145 (Oct. 26, 1994).

8. K.C. Harkay, Study of Corrections to the Geometrical Factor in the Space Charge Impedance for the IPNS-II, Argonne National Laboratory Report No. NS-95-4 (Mar. 1995).

9. CRC Handbook of Physics and Chemistry, 74th edition. 
10. J.L. Laclare, "Bunched beam coherent instabilities," in Proceedings of 1985 CERN Accelerator School, CERN Report No. 87-03 (1987).

11. U. Lauströer, U. van Rienen, and T. Weiland, URMEL and URMEL-T User Guide, DESY Report No. M-87-03 (Feb. 1987).

12. A. Hoffman, "Single-beam collective phenomenon - longitudinal," in Proceedings of Theoretical Aspects of the Behavior of Beams in Accelerators and Storage Rings, CERN Report No. 77-13 (Erice, Nov. 1976).

13. J.H. Friedman, "Data analysis techniques for high energy particle physics," in Proceedings of the 1974 CERN School of Computing, CERN Report No. 74-23, p. 295 (Godoysund, Norway, Aug. 1974).

14. B. Zotter and F. Sacherer, Transverse Instabilities of Relativistic Particle Beams in Accelerators and Storage Rings, CERN Report No. 77-13 (1977).

15. Y. Cho and A.V. Rauchas, "Observations and cure of the head-tail effect in the Argonne Rapid Cycling Synchrotron," IEEE Trans. Nucl. Sci. NS-28(3), 2585 (June 1981).

16. Y. Kimura et al., "Transverse coherent instability in the KEK Booster," in Proceedings of the Xth International Conference on High Energy Accelerators, Vol. 2, p. 30 (Serpukhov, July 1977).

17. G.H. Rees, "Interpretation of the higher-order mode, head-tail motion observed on ISIS," Particle Accelerators 39, 159 (1992).

18. A. Chao, Physics of Collective Beam Instabilities in High Energy Accelerators (John Wiley and Sons, Inc., 1993).

19. E. Wilson, Nonlinearities and Resonances, CERN Report No. 85-19 (Nov. 1985).

20. D. Möhl and H. Schonauer, "Landau damping by non-linear space-charge forces and octupoles," in Proceedings of the 9 th International Conference on High Energy Accelerators, p. 380 (1974).

21. C.W. Planner, "Reaching $200 \mu \mathrm{A}$ on ISIS," in Proceedings of the 12th ICANS Workshop (Abingdon, U.K., May 1993).

22. D. Neuffer, "Transverse instability in the PSR," in Proceedings of the Workshop on Accelerators for Future Spallation Neutron Sources, LA-UR-93-1356, Vol. II B (Feb. 1993). 
23. Y.C. Chae, Electron-Proton Instability in the IPNS-Upgrade RCS: Part I, Argonne National Laboratory Report No. NSA-95-2 (Feb. 1995).

24. Y.C. Chae, Calculation of Beam Neutralization in the IPNS-Upgrade RCS, Argonne National Laboratory Report No. NSA-95-1 (Jan. 1995). 


\subsection{INTRODUCTION}

An operating pressure on the order of $10^{-8}$ Torr is achieved by using ion pumps distributed evenly around the RCS ring. The vacuum chamber is made of high-purity ceramic to avoid eddy current effects from the ramping fields. It has a wire liner, which acts as a radiofrequency (rf) shield to minimize coupling impedances, in a manner similar to that previously accomplished at ISIS. 1,2

\subsection{VACUUM CHAMBER DESIGN}

The vacuum chamber is constructed in sections made of $99.7 \%$-pure alumina ceramic. These sections span several magnets and are independently supported. Clearance is sufficient to avoid thermal and mechanical stresses. The nominal cross sections for a dipole chamber and a quadrupole/sextupole chamber are shown in Figure II.6.2-1. Vacuum chamber dimensions are listed in Table II.6.1-1.

Ceramic chambers with these cross sections are produced in standard lengths of up to $60 \mathrm{~cm}$ by means of the isostatic forming process. This process provides the most accurate dimensional control of both interior and exterior ceramic surface geometry, and it allows modest curvatures to be imparted without sacrificing dimensional accuracy. Both cross sections have been analyzed for mechanical strength at the aforementioned operating pressure with the ANSYS 5.0 finite-element-analysis software. Maximum tensile loading for both sections was calculated to be less than 4,000 pounds per square inch (psi). High-purity alumina ceramics have an ultimate tensile strength ranging from 38 to $40 \mathrm{ksi}$ for material with the normal amount of inclusions and flaws.

A typical vacuum chamber cell is longer than $60 \mathrm{~cm}$; therefore, several of the isostatically formed 60 -cm-long sections must be fused together. The doweling system shown in Figure II.6.2-2 is used to maintain chamber-to-chamber alignment during glass fusion.

At the end of each section are glass-fused ceramic flanges that mate with the metallic sections of adjoining components. A metallic $\mathrm{O}$-ring forms the vacuum seal between the ceramic and metal flanges. A clamping system uniformly compresses the seal circumferentially while keeping the ceramic flange in compression, as shown in Figure II.6.2-3. A typical cell is shown in Figure II.6.2-4. Vacuum integrity is maintained at pressures on the order of $10^{-8}$ Torr.

A semiconducting ceramic glaze consisting of conductive materials within a lead-quartz glass matrix is applied to the interior of the ceramic chamber. The coating is bonded to the ceramic at high temperature, resulting in a surface resistance of $100 \mathrm{~K} \Omega$ per square. Outgassing rates are typically between $10^{-8}$ and $10^{-14} \mathrm{Torr}-\mathrm{L} / \mathrm{sec}-\mathrm{cm}^{2}$ for such coatings. ${ }^{4,5}$ The design value for this machine is $10^{-10} \mathrm{Torr}-\mathrm{L} / \mathrm{sec}-\mathrm{cm}^{2}$. The coating provides static charge dissipation, and some if shielding. 
Table II.6.1-1 Vacuum Chamber Dimensions

\begin{tabular}{lcc}
\hline \multicolumn{1}{c}{ Parameters } & Values & Units \\
\hline Dipole chamber height & 15.6 & $\mathrm{~cm}$ \\
Dipole chamber width & 17.6 & $\mathrm{~cm}$ \\
& 20.7 & $\mathrm{~cm}$ \\
Quadrupole chamber height & 27.1 & $\mathrm{~cm}$ \\
Quadrupole chamber width & 19,040 & $\mathrm{~cm}$ \\
& 6,390 & $\mathrm{~L}$ \\
Total RCS chamber length & $1.29 \times 10^{6}$ & $\mathrm{~cm}$ \\
Total RCS chamber volume & & \\
Total RCS chamber surface area & & \\
\hline
\end{tabular}




\subsection{INTRODUCTION}

An operating pressure on the order of $10^{-8}$ Torr is achieved by using ion pumps distributed evenly around the RCS ring. The vacuum chamber is made of high-purity ceramic to avoid eddy current effects from the ramping fields. It has a wire liner, which acts as a radiofrequency (rf) shield to minimize coupling impedances, in a manner similar to that previously accomplished at ISIS. 1,2

\subsection{VACUUM CHAMBER DESIGN}

The vacuum chamber is constructed in sections made of $99.7 \%$-pure alumina ceramic. These sections span several magnets and are independently supported. Clearance is sufficient to avoid thermal and mechanical stresses. The nominal cross sections for a dipole chamber and a quadrupole/sextupole chamber are shown in Figure II.6.2-1. Vacuum chamber dimensions are listed in Table II.6.1-1.

Ceramic chambers with these cross sections are produced in standard lengths of up to $60 \mathrm{~cm}$ by means of the isostatic forming process. This process provides the most accurate dimensional control of both interior and exterior ceramic surface geometry, and it allows modest curvatures to be imparted without sacrificing dimensional accuracy. Both cross sections have been analyzed for mechanical strength at the aforementioned operating pressure with the ANSYS 5.0 finite-element-analysis software. Maximum tensile loading for both sections was calculated to be less than 4,000 pounds per square inch (psi). High-purity alumina ceramics have an ultimate tensile strength ranging from 38 to $40 \mathrm{ksi}$ for material with the normal amount of inclusions and flaws.

A typical vacuum chamber cell is longer than $60 \mathrm{~cm}$; therefore, several of the isostatically formed 60 -cm-long sections must be fused together. The doweling system shown in Figure II.6.2-2 is used to maintain chamber-to-chamber alignment during glass fusion.

At the end of each section are glass-fused ceramic flanges that mate with the metallic sections of adjoining components. A metallic O-ring forms the vacuum seal between the ceramic and metal flanges. A clamping system uniformly compresses the seal circumferentially while keeping the ceramic flange in compression, as shown in Figure II.6.2-3. A typical cell is shown in Figure II.6.2-4. Vacuum integrity is maintained at pressures on the order of $10^{-8}$ Torr.

A semiconducting ceramic glaze consisting of conductive materials within a lead-quartz glass matrix is applied to the interior of the ceramic chamber. The coating is bonded to the ceramic at high temperature, resulting in a surface resistance of $100 \mathrm{~K} \Omega$ per square. Outgassing rates are typically between $10^{-8}$ and $10^{-14} \mathrm{Torr}-\mathrm{L} / \mathrm{sec}-\mathrm{cm}^{2}$ for such coatings. 4,5 The design value for this machine is $10^{-10}$ Torr- $\mathrm{L} / \mathrm{sec}-\mathrm{cm}^{2}$. The coating provides static charge dissipation, and some rf shielding. 
Table II.6.1-1 Vacuum Chamber Dimensions

\begin{tabular}{lcc}
\hline \multicolumn{1}{c}{ Parameters } & Values & Units \\
\hline Dipole chamber height & 15.6 & $\mathrm{~cm}$ \\
Dipole chamber width & 17.6 & $\mathrm{~cm}$ \\
& 20.7 & $\mathrm{~cm}$ \\
Quadrupole chamber height & 27.1 & $\mathrm{~cm}$ \\
Quadrupole chamber width & 19,040 & $\mathrm{~cm}$ \\
& 6,390 & $\mathrm{~L}$ \\
Total RCS chamber length & $1.29 \times 10^{6}$ & $\mathrm{~cm}$ \\
Total RCS chamber volume & & \\
Total RCS chamber surface area & & \\
\hline
\end{tabular}



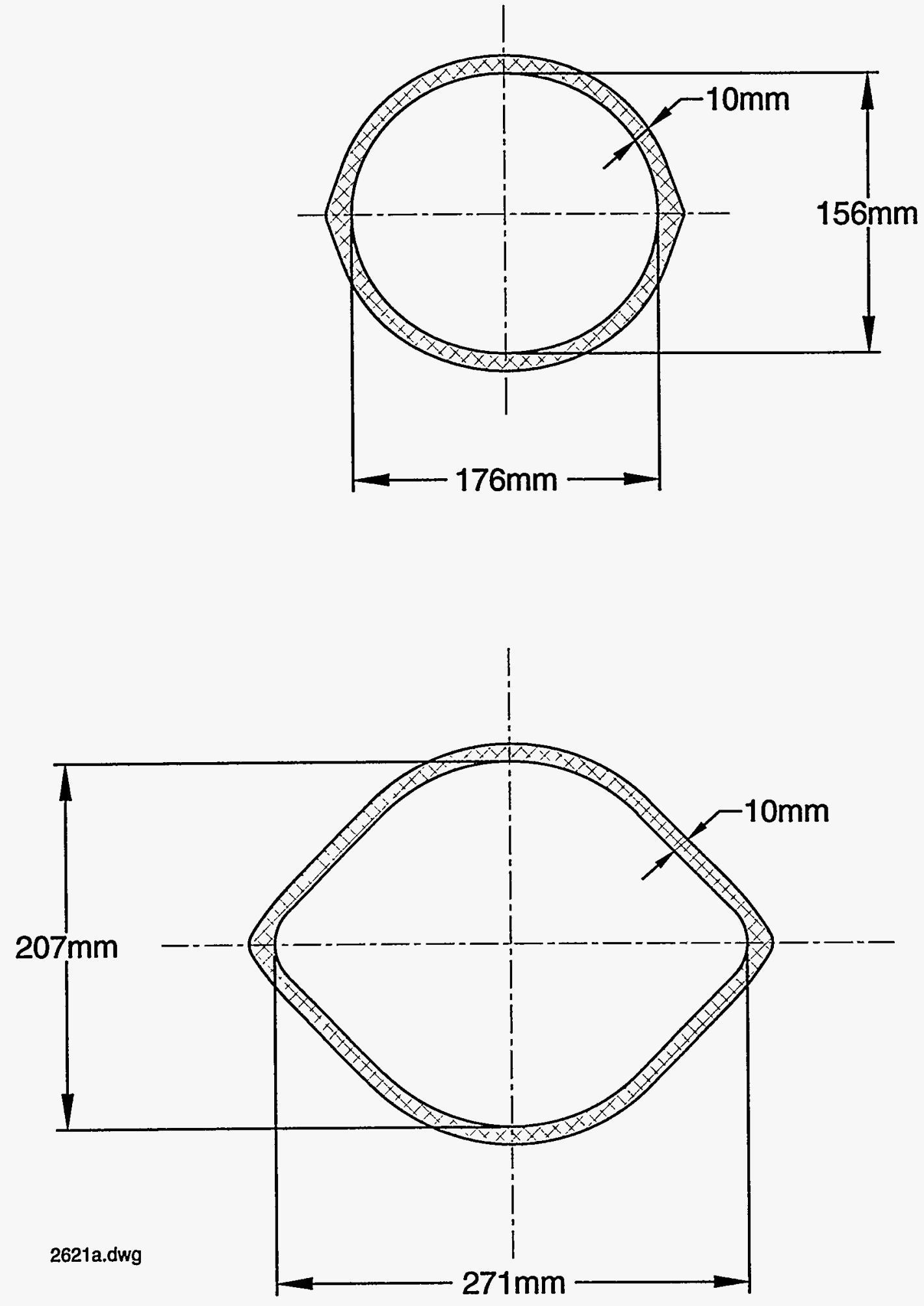

Figure II.6.2-1 Dipole Chamber Cross Section (upper) and Quadrupole/Sextupole Chamber Cross Section (lower). 


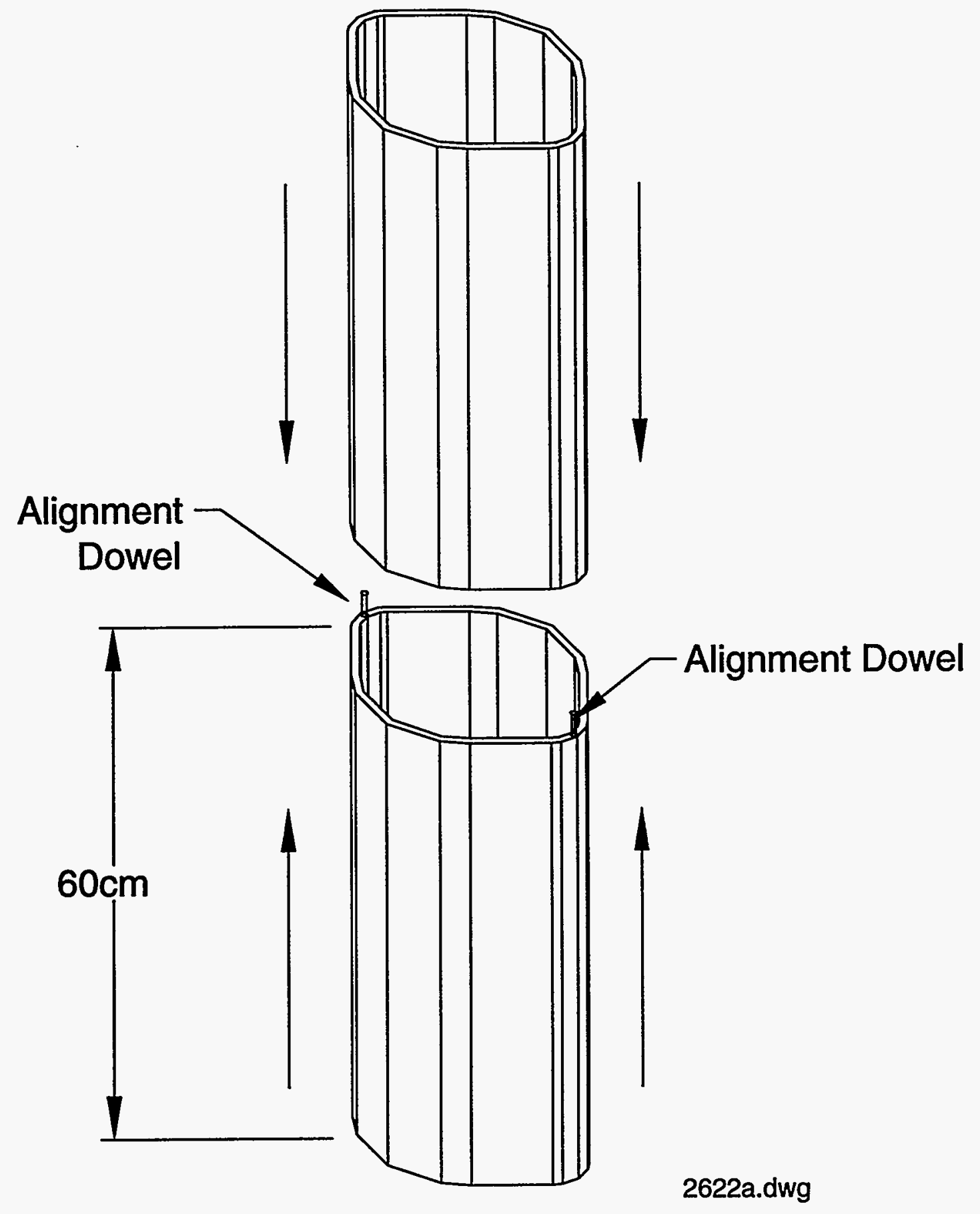

Figure II.6.2-2 Alignment System for Glass Fusion Bonding of Alumina Sections. 


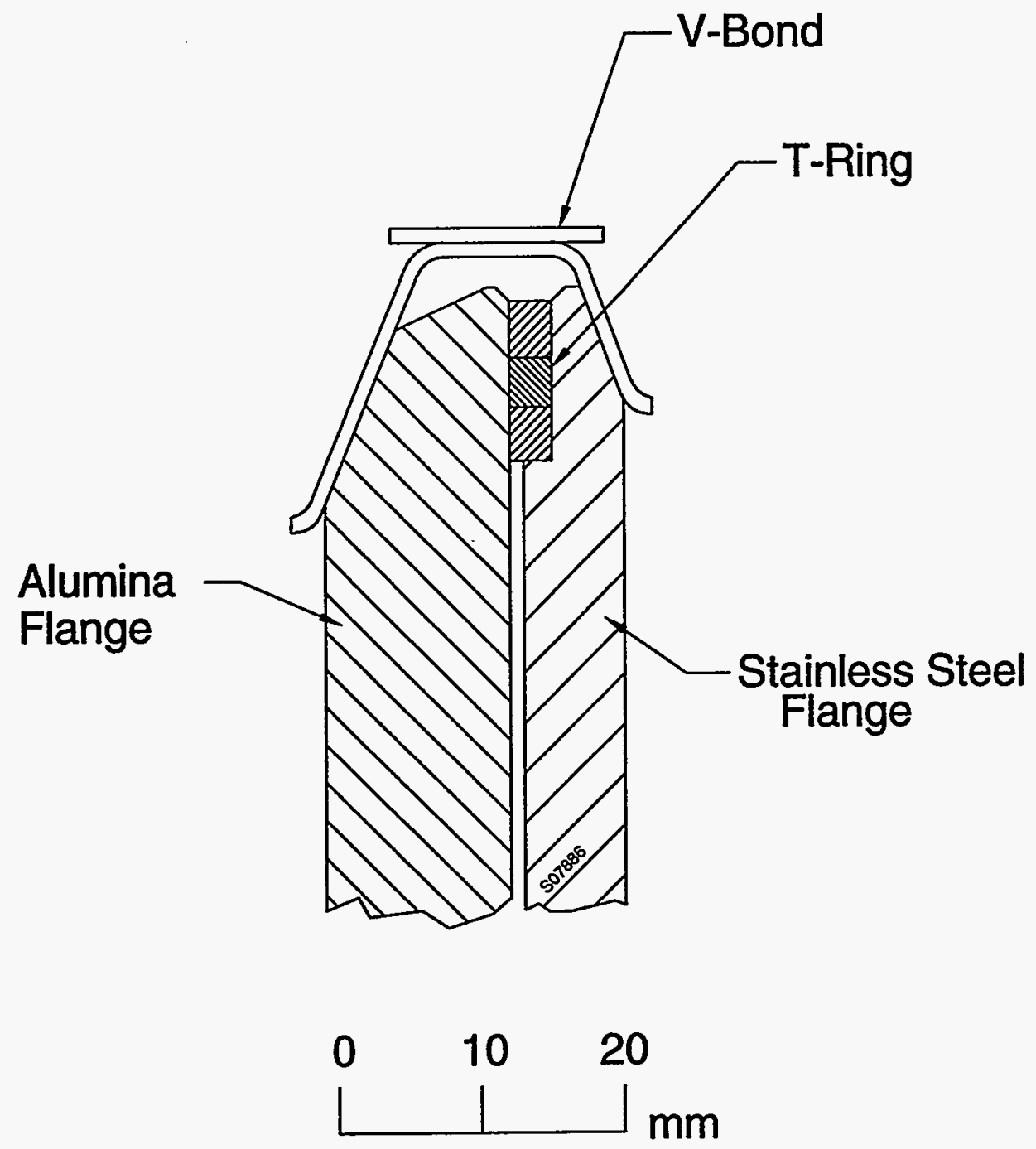

Figure II.6.2-3 Ceramic Vacuum Chamber Joint Detail. 


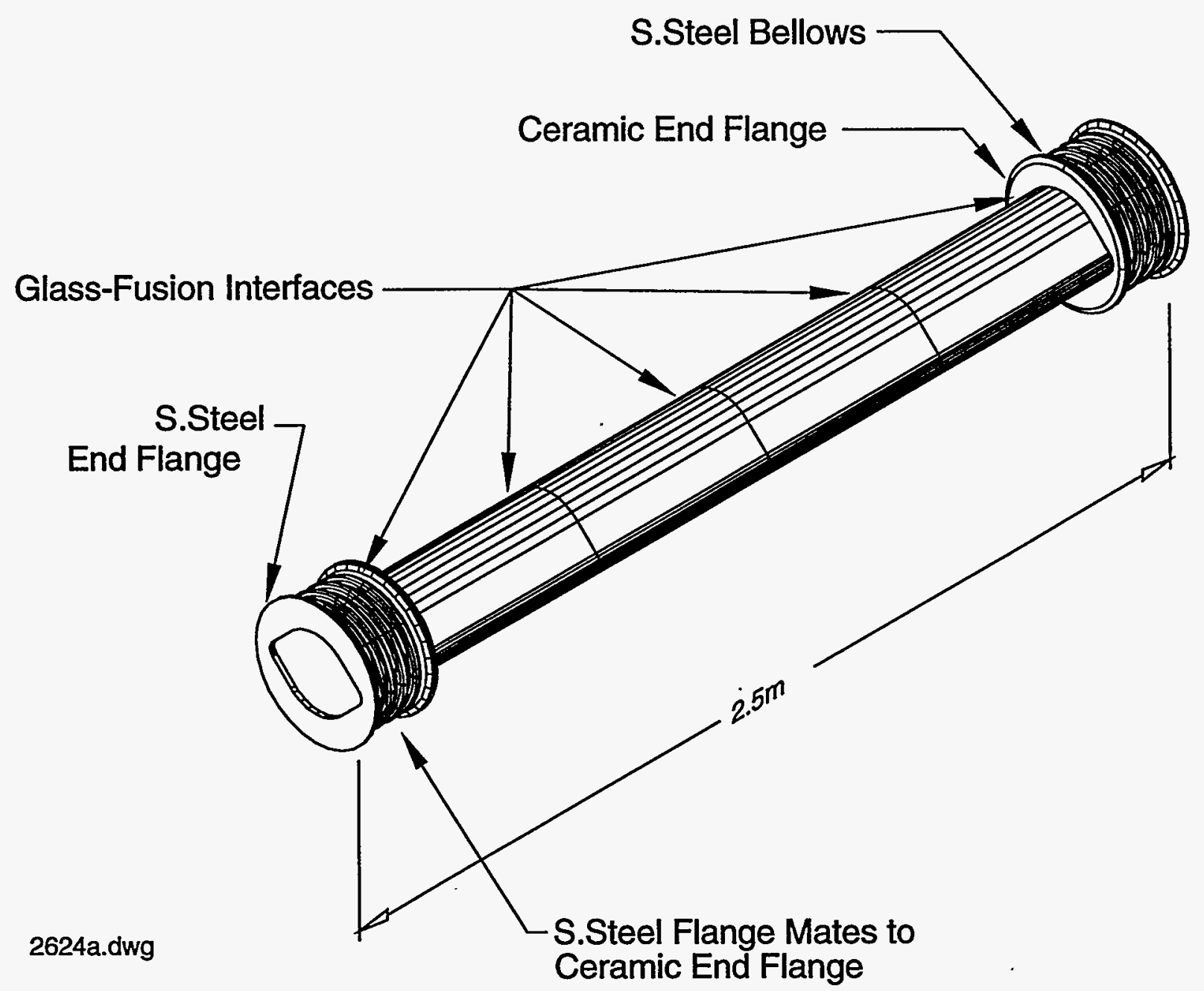

Figure II.6.2-4 Typical Quadrupole/Sextupole Ceramic Chamber Cell. 


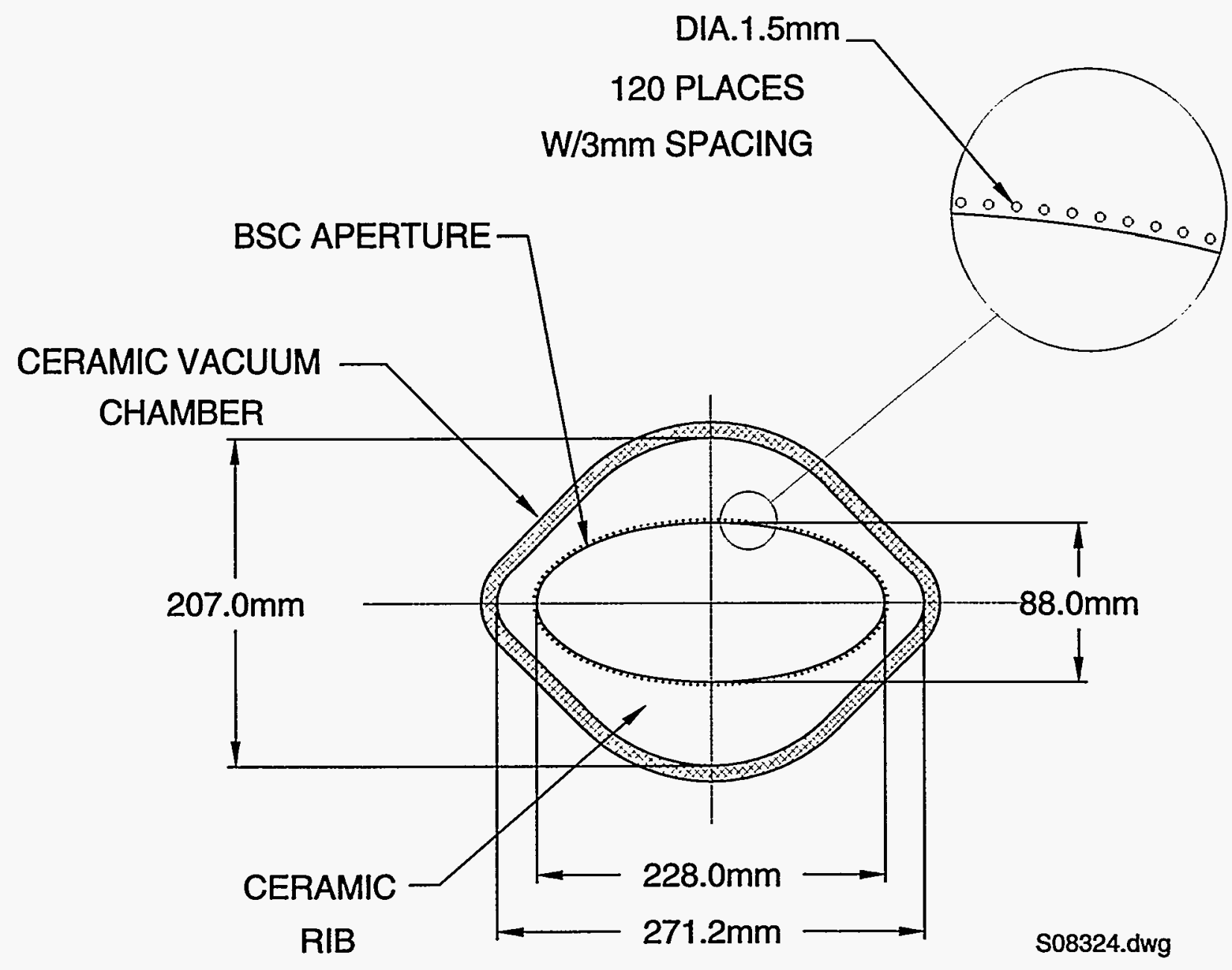

Figure II.6.2-5 Wire Liner, Which Acts as a Radio-Frequency Shield and Is Supported on Ceramic Ribs. 
The rf shield is actually a wire liner that runs the entire length of the vacuum chamber. Its cross section follows the beam envelope along the lattice, as shown in Figure II.6.2-5. It shields accelerator components from if energy induced by the beam, and it also provides a lowimpedance return path for beam image currents.

The wires are made of 1.5-mm-thick $\mathrm{Be}-\mathrm{Cu}$ and have a center-to-center spacing of $4.5 \mathrm{~mm}$ around the perimeter of the chamber. They are periodically supported on ceramic ribs. One of the ceramic ribs in a quadrupole chamber is shown in Figure II.6.2-5.

Each wire is terminated in discrete capacitors at the flanged ends, as shown in Figure II.6.2-6. The capacitors inhibit current flow around the loop formed by the if shield wires and ground.2,3

Intracell compliance, and also alignment between cells and individual special components such as kicker magnets, is achieved by the use of flanged bellows, such as those shown in Figure II.6.2-4.

Metallic finger assemblies provide electrical continuity for If shielding and maintain the vacuum chamber geometry in the bellows region, as shown in Figure II.6.2-7.

This vacuum chamber system uses existing technologies and designs and can be produced by commercial vendors. Coating materials and manufacturing techniques exist.

\subsection{VACUUM PUMPING SYSTEM}

Eight 300-L/min mechanical pumps and eight 400-L/sec turbo-molecular pumps are used for the roughing system. Rough pumping from atmospheric pressure to $10^{-3}$ Torr takes about one hour. The turbo-molecular pumps reduce the pressure to the order of $10^{-6}$ Torr in six hours.

Thirty-six 220-L/sec ion pumps plus four $60-\mathrm{L} / \mathrm{sec}$ ion pumps are used to pump the RCS down to less than $10^{-7}$ Torr.

Ion pump locations around one quarter of the ring (or one super-period) are shown in Figure II.6.3-1. The total pumpdown time from atmospheric pressure to $10^{-7}$ Torr is about 10 hours.

A vacuum finite-element-analysis computer program developed at Argonne National Laboratory has successfully modeled other vacuum systems. ${ }^{6}$ This program was used to help design the RCS vacuum system. 


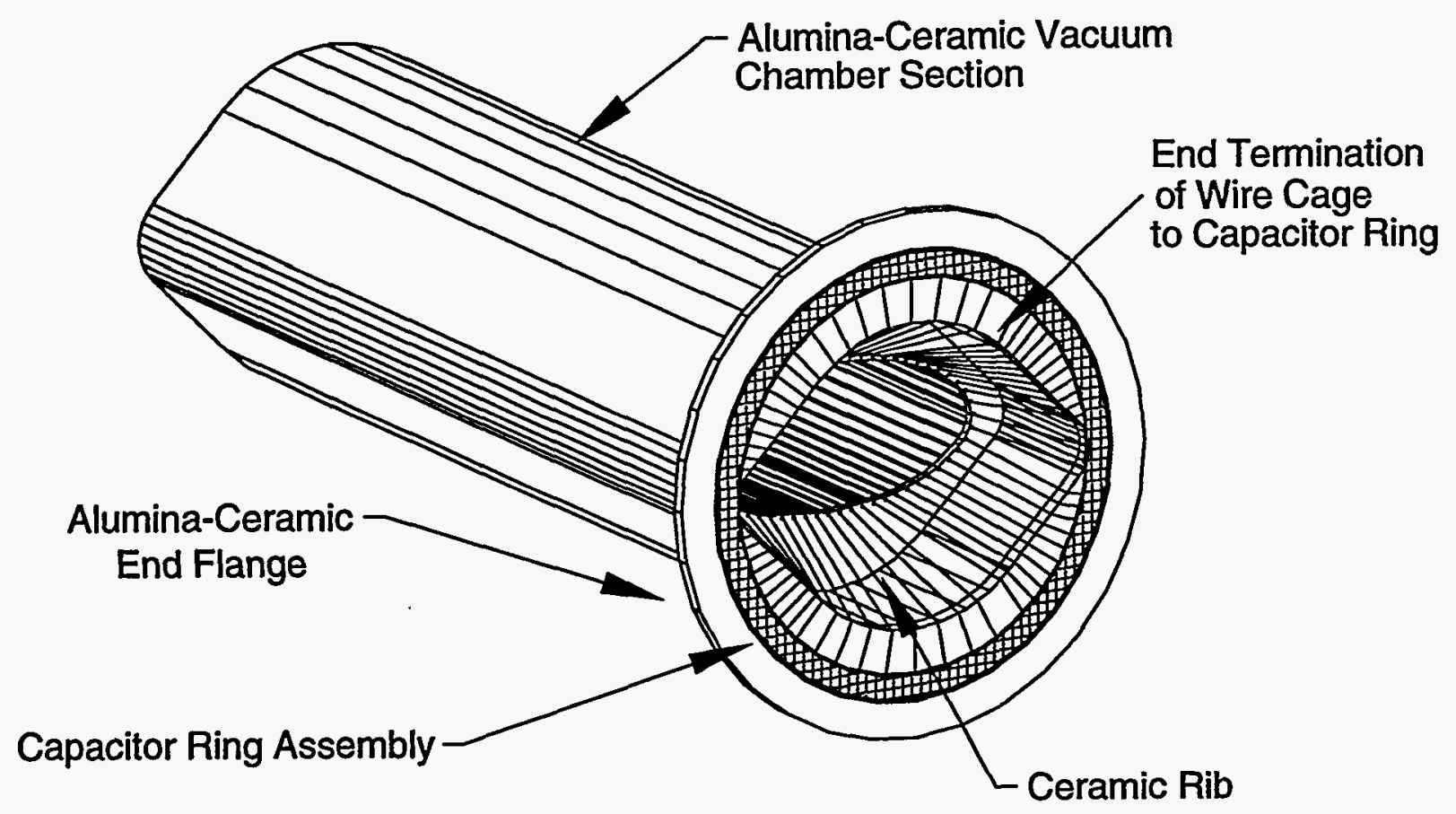

Figure II.6.2-6 Vacuum Chamber Cell-to-Cell Capacitive Coupling Layout. 


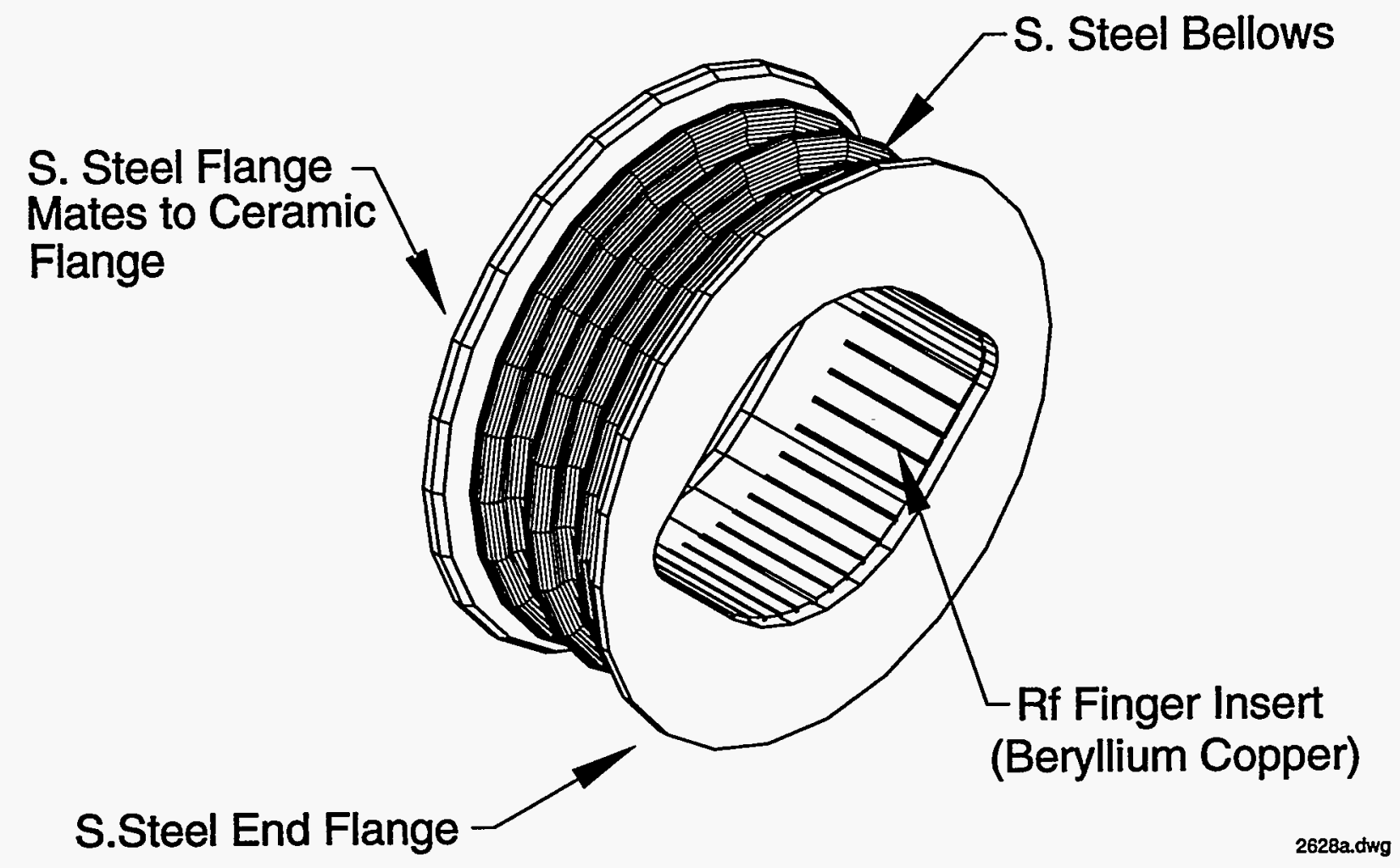

Figure II.6.2-7 Radio-Frequency Finger Layout. 


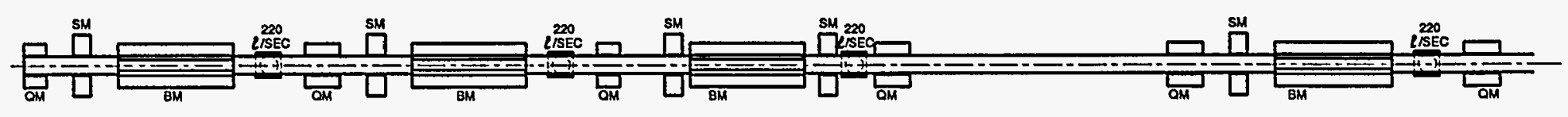

舁

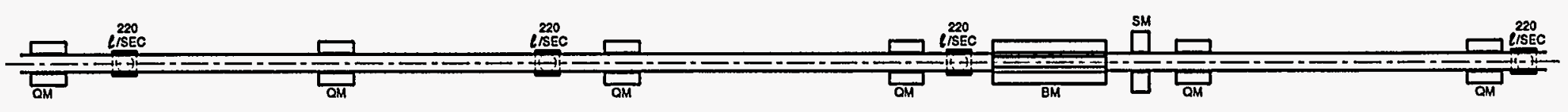

SM = SEXTUPOLE MAGNET

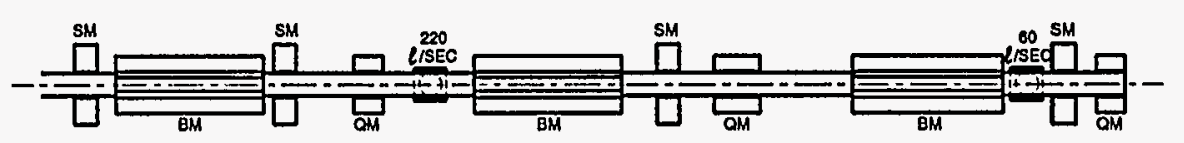
QM = QUADRUPOLE MAGNET $B M=$ BENDING MAGNET

$O=$ ION PUMPS

FLE: soesosc.owra 
A super-period is divided into 20 elements. Data on the volume, surface area, outgassing rate, pumping speed, and conductance for each element are input for the program. Table II.6.3-1 lists the input data for the program. Figure II.6.3-2 shows the calculated vacuum profile in one sector. The highest pressure is less than $3 \times 10^{-8}$ Torr. 
Table II.6.3-1 Input Data for Vacuum Finite-Element-Analysis Computer Program

\begin{tabular}{|c|c|c|c|c|}
\hline $\begin{array}{l}\text { Element } \\
\text { Number }\end{array}$ & $\begin{array}{l}\text { Volume } \\
\text { (L) }\end{array}$ & $\begin{array}{l}\text { Conductance } \\
\text { (L/sec) }\end{array}$ & $\begin{array}{l}\text { Pumping Speed } \\
\text { (L/sec) }\end{array}$ & $\begin{array}{l}\text { Thermal Desorption } \\
\text { (Torr L/sec) }\end{array}$ \\
\hline 1 & $3.99 E+01$ & $1.24 \mathrm{E}+03$ & 0 & 7.50 E- 07 \\
\hline 2 & 2.67E+01 & $4.00 E+02$ & 0 & $6.61 \mathrm{E}-07$ \\
\hline 3 & $9.54 \mathrm{E}+01$ & $6.20 \mathrm{E}+02$ & $2.00 E+02$ & 1.92E-06 \\
\hline 4 & $2.67 E+01$ & $4.00 E+02$ & 0 & 6.61E-07 \\
\hline 5 & $9.54 \mathrm{E}+01$ & $6.20 E+02$ & $2.00 E+02$ & $1.92 E-06$ \\
\hline 6 & 2.67E+01 & $4.00 E+02$ & 0 & 6.61E-07 \\
\hline 7 & $2.25 E+02$ & $3.31 E+02$ & $2.00 \mathrm{E}+02$ & 4.36E-06 \\
\hline 8 & 2.67E+01 & $4.00 E+02$ & 0 & 6.61E-07 \\
\hline 9 & $1.60 \mathrm{E}+02$ & $3.31 E+02$ & $2.00 E+02$ & $3.43 E-06$ \\
\hline 10 & $1.64 E+02$ & $3.34 E+02$ & $2.00 E+02$ & $3.21 E-06$ \\
\hline 11 & $1.60 \mathrm{E}+02$ & $3.21 \mathrm{E}+02$ & $2.00 E+02$ & $3.43 E-06$ \\
\hline 12 & $1.60 \mathrm{E}+02$ & $3.21 E+02$ & $2.00 E+02$ & $3.43 E-06$ \\
\hline 13 & $2.67 E+01$ & $4.00 E+02$ & 0 & $6.61 E-07$ \\
\hline 14 & $2.25 \mathrm{E}+02$ & 2.31E+02 & $2.00 E+02$ & 4.36E-06 \\
\hline 15 & $2.67 E+01$ & $4.00 E+02$ & 0 & $6.61 E-07$ \\
\hline 16 & $8.88 E+01$ & $6.20 \mathrm{E}+02$ & 0 & $1.76 E-06$ \\
\hline 17 & $2.67 E+01$ & $4.00 E+02$ & $2.00 E+02$ & $6.61 E-07$ \\
\hline 18 & $8.88 E+01$ & $6.20 \mathrm{E}+02$ & 0 & $1.76 \mathrm{E}-06$ \\
\hline 19 & 2.67E+01 & $4.00 E+02$ & 0 & 6.61E-07 \\
\hline 20 & $4.89 E+01$ & $1.24 E+03$ & $6.00 E+01$ & $1.00 E-06$ \\
\hline
\end{tabular}




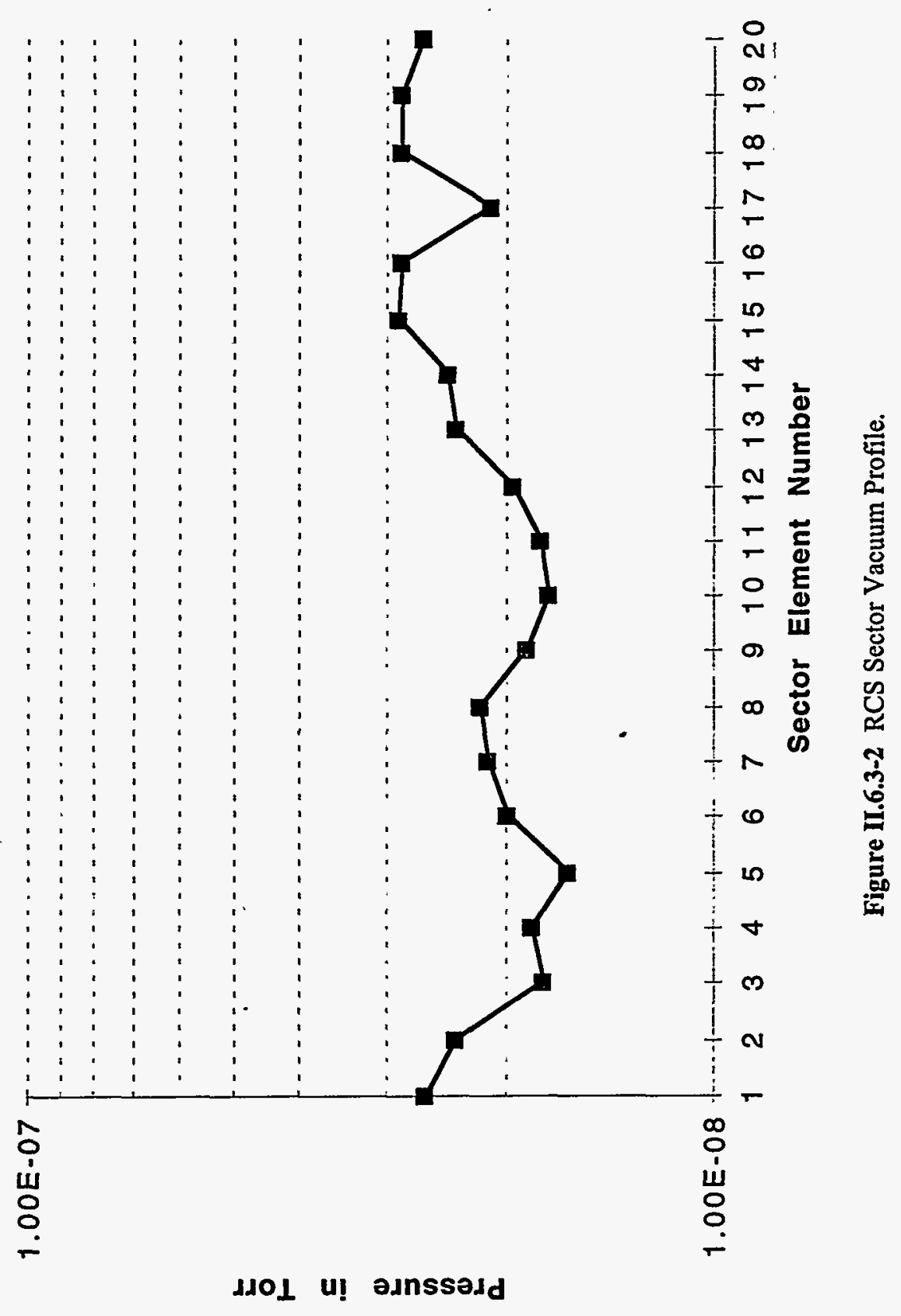




\subsection{REFERENCES}

1. G.H. Rees, "Status report on ISIS," in Proceedings of the IEEE Particle Accelerator Conference (March 16-19, 1987), IEEE Catalog No. 87CH2387-9 (1987).

2. Kaon Factory Study, Accelerator Design Report (TRUUMF, British Columbia, Canada, undated).

3. R. Cappi, "RF bypass on the proton synchrotron vacuum chamber flanges," in Proceedings of the IEEE Particle Accelerator Conference, pp. 2012-2014 (March 1989).

4. J.W. Ackley et al., 1962 Vacuum Symp. Trans., p. 380 (1963).

5. D. Alpert and R.S. Buritz, J. Appl. Phys. 25, 200 (1954).

6. 7-GeV Advanced Photon Source: Conceptual Design Report, Argonne National Laboratory Report No. ANL-87-15, pp. II.6-16 (1987). 


\subsection{INTRODUCTION}

The RCS has diagnostic instrumentation to monitor the beam position, profile, current, and losses. In addition, the high-energy transport (HET) lines and low-energy transport (LET) line leading to and from the RCS have beam diagnostics. Table II.7.1-1 lists the types and quantities of beam diagnostics provided for the RCS and transport lines. Diagnostic instrumentation provided for the linac is covered in Chapter III.

\subsection{RCS DIAGNOSTICS}

\subsubsection{RCS Beam Position Monitors}

The synchrotron's beam position monitors consist of pairs of traveling-wave (stripline) electrodes located within each of the quadrupole magnets. The angles of the pairs alternate by $90^{\circ}$, so they provide horizontal position information in horizontally focusing quadrupoles and vertical position information in vertically focusing quadrupoles. Each plane has 28 electrodes. The rf shield inside the vacuum chamber is modified near the electrodes to provide the proper characteristic matching impedance. Constant impedance and matching feedthroughs are provided at each end of the electrode.

The signals from each electrode of the pair are processed by a hybrid junction that provides the sum and difference information. This information is then heterodyned to an intermediate frequency for synchronous detection. The detected signals are digitized by 14-bit analog-to-digital converters (ADCs). Local processors normalize the beam position, and the information is provided to circuitry for orbit control feedback and to the control console for operator monitoring. The measurement accuracy of the system is better than $500 \mu \mathrm{m}$.

\subsubsection{RCS Current Transformers}

Two current transformers are installed in the RCS. A slow current transformer is used to accurately measure the intensity of the accelerated beam and to detect and quantify gross beam losses. The other current transformer has high-frequency response and is used to monitor bunch structure.

\subsubsection{RCS Beam Loss Monitors}

Beam losses in the synchrotron are detected by a gas-filled, coaxial-cable ion chamber. The ion chamber is composed of four segments that overlap, a configuration that ensures that losses can be localized to any octant of the synchrotron. The signals can be switched to a highresolution timing chassis to precisely detect the position of persistent, highly localized losses. 
Table II.7.1-1 Beam Diagnostic Instrumentation

\begin{tabular}{|c|c|c|}
\hline Location & Monitor & Quantity \\
\hline RCS & $\begin{array}{l}\text { Beam position monitor electrodes } \\
\text { Current transformers } \\
\text { Beam loss monitors } \\
\text { Pingers } \\
\text { Segmented injection-orbit Faraday cups } \\
\text { Solid first-turn Faraday cup }\end{array}$ & $\begin{array}{l}56(28 \mathrm{H}, 28 \mathrm{~V})^{\mathrm{a}} \\
2 \\
4 \text { segments } \\
2(1 \mathrm{H}, 1 \mathrm{~V}) \\
6(3 \mathrm{H}, 3 \mathrm{~V}) \\
1\end{array}$ \\
\hline LET line & $\begin{array}{l}\text { Current transformers } \\
\text { Beam position monitor electrodes } \\
\text { Profile detectors } \\
\text { Beam loss monitors }\end{array}$ & $\begin{array}{l}3 \\
8 \\
16 \\
1 \text { segment }\end{array}$ \\
\hline $\begin{array}{l}\text { HET line } \\
(30 \mathrm{~Hz})\end{array}$ & $\begin{array}{l}\text { Current transformers } \\
\text { Beam position monitor electrodes } \\
\text { Profile detectors } \\
\text { Beam loss monitors } \\
\text { Target protection monitors }\end{array}$ & $\begin{array}{l}3 \\
4 \\
4 \\
1 \text { segment } \\
1\end{array}$ \\
\hline $\begin{array}{l}\text { HET line } \\
(10 \mathrm{~Hz})\end{array}$ & $\begin{array}{l}\text { Current transformers } \\
\text { Beam position monitor electrodes } \\
\text { Profile detectors } \\
\text { Beam loss monitors } \\
\text { Target protection monitors }\end{array}$ & $\begin{array}{l}3 \\
6 \\
4 \\
1 \text { segment } \\
1\end{array}$ \\
\hline
\end{tabular}

a $\mathrm{H}=$ horizontal; $\mathrm{V}=$ vertical. 


\subsubsection{Special Beam Studies Instrumentation}

The RCS includes a set of vertical and horizontal pinger magnet systems that allow excitation of the beam to measure the betatron frequencies. The resulting betatron motion is measured by pickup electrodes. High-frequency instrumentation and high-speed digitizing circuitry are provided to allow complete analysis of the resulting signals.

\subsubsection{Injection Diagnostics}

Three segmented-Faraday-cup assemblies and one solid Faraday cup are located in the RCS. A horizontal and vertical Faraday cup are mounted on one actuator. The actuators are motorized and designed so either cup can be inserted into the beam path. Three of the cups are designed to allow half of the beam to be intercepted on the segments. The other half continues around the synchrotron. The segmented cups allow several injection orbits to be viewed. The solid Faraday cup is located just upstream of the injection region and is used to measure the firstturn beam intensity. The charge from all Faraday cup segments is digitized, and the resulting profiles are analyzed by the control system to provide operators with injection orbit information.

\subsection{TRANSPORT LINE DIAGNOSTIC INSTRUMENTATION}

\subsubsection{General Instrumentation}

Each of the two HET lines and the single LET line has three current transformers distributed along its length. The current pulse from each transformer is integrated by precision integrators and digitized by the control system to calculate transport efficiency.

Beam position in the transport lines is monitored by arrays of four traveling-wave stripline electrodes similar to the ones used in the RCS. Such monitoring allows both horizontal and vertical information to be gathered from each array. The detection system for the LET line is optimized to detect the $1,275-\mathrm{MHz}$ component of the beam. In the HET lines, the peak of the single, high-current beam pulse is detected from each of the electrodes. It is then digitized, and its position is calculated.

Moving-wire scanners are used in the LET line to measure the $\mathrm{H}^{-}$ion beam profile. Segmented-wire ion chambers, mounted in vacuum valve assemblies, are used to measure the profile in each of the HET lines. The beam profile measurement devices are not designed to be operated at full beam power. They are operated at either low currents or low repetition rates.

Beam losses in all of the transport lines are measured by gas-filled, coaxial-cable ion chambers, identical to the system used in the RCS. 


\subsubsection{Target Protection Monitors}

Maintaining a predetermined minimum beam profile and beam position at the neutron generating target is essential to the operation of the facility, because severe target damage can occur if the beam size becomes smaller than the predetermined size or if the beam drifts out of position. Target protection instrumentation that shuts off the beam in the event of changing spot size or position is necessary, but the standard beam-intercepting profile monitors cannot survive very long under full beam power. The target is instrumented with the nonintercepting target protection monitors (TPMs) described below, which provide adequate protection against fast changes or long-term drifts in beam size and position.

A TPM consists of four secondary emission monitors (SEMs), arranged as shown in Figure II.7.3-1 and positioned upstream of the target. SEM-A and SEM-B are placed at the upper and lower sides of the target, while SEM-C and SEM-D are at the left and the right sides of the target. The SEM support stands have position adjustment mechanisms, so that the position of each SEM can be precisely adjusted. The SEMs are modular, and individual units can be replaced by new ones without requiring realignment. The SEMs are placed at the positions shown in the figure and do not intercept any portion of the beam. They provide a null signal unless the beam shape or position changes. Heartbeat signals ensure that all monitor channels are functioning.

The TPM provides a signal when the beam position changes or when the beam size increases in either of the transverse planes. Since the HET lines are constructed with quadrupole focusing magnets, a decrease in the horizontal beam size would most likely occur with a corresponding increase in the vertical beam size, and vice versa. Therefore, signals from a pair of SEMs indicate a change in beam size. Any individual SEM would be able to indicate a change in beam position.

At initial start-up, the HET lines are tuned at very low beam power by using signals from beam-intercepting profile monitors. The SEMs are then brought into position and adjusted to bring them close to the beam. The beam-intercepting profile monitors are removed, and full beam power is directed onto the target. From this time on, the TPM monitors changes in the beam position and changes in the beam profile. Information from the TPM is processed together with monitored HET magnet setpoints and readback values and provides the desired beam shutdown signal.

Some research and development are required to ensure the long-term reliability and optimize the functionality of these devices. 


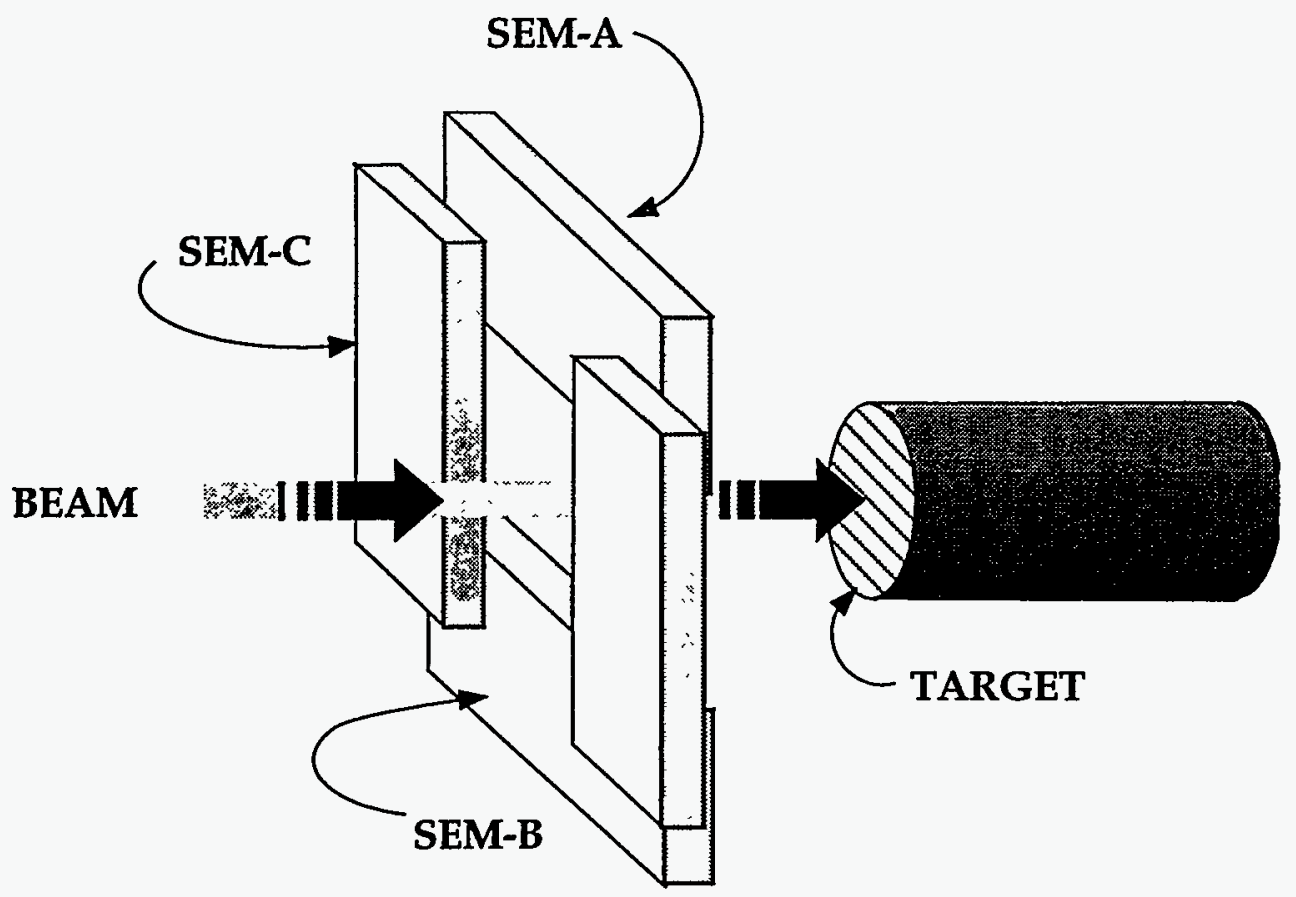

(a) Side View

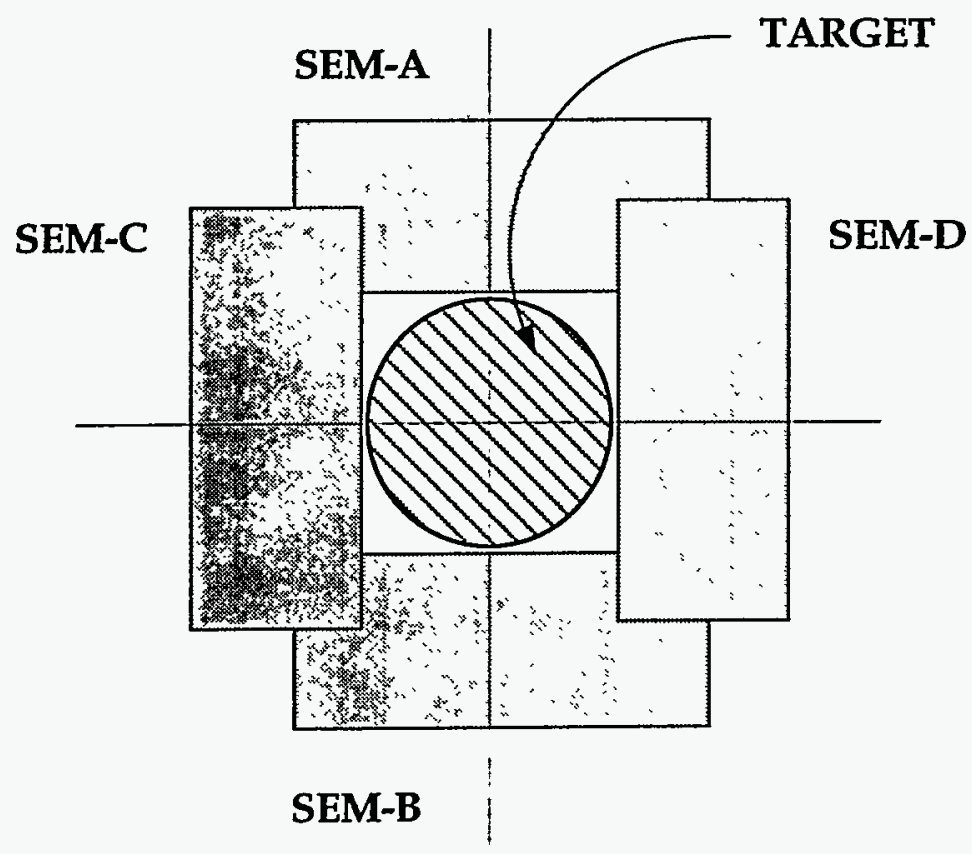

(b) Beam View

Figure II.7.3-1 Target Protection Monitor That Uses Secondary Emission Monitors as Detectors, Shown from the Side (a) and from the Beam's Perspective (b). 


\subsection{INTRODUCTION AND PHILOSOPHY}

The IPNS Upgrade control system design is based on using high-performance workstations to act as the operator consoles, distributed microprocessors to control equipment interfacing and preprocess data, and an interconnecting network. Figure II.8.1-1 shows in schematic form all major components and their relationships. The IPNS Upgrade design includes 58 distributed microprocessors and 10 workstation console systems. Some of the workstations have at least two physical screens.

The IPNS Upgrade control system is a direct application of the Experimental Physics and Industrial Control System (EPICS), ${ }^{1-4}$ created by a collaboration between the Controls and Computing group at the Argonne Advanced Photon Source (APS) and the ATO-8 group at Los Alamos National Laboratory. Other laboratories and universities, including Lawrence Berkeley Laboratory (LBL), the Continuous Electron Beam Accelerator Facility (CEBAF), the Stanford Linear Accelerator Center (SLAC), the Deutsches Elektronen Synchrotron (DESY), MIT-Bates, the Duke Free Electron Laser (FEL), and the KECK II and Gemini telescopes, are developing control systems using EPICS software tools. They are also developing additional tools that will be shared with all EPICS users. EPICS has been successfully installed on the existing IPNS, replacing its outdated computer system. Three commercial vendors now supply EPICS.

Accelerator control system designers face the following challenges:

1. Obtain system requirements and specifications from the machine and subsystem designers,

2. Protect the investment in hardware and software from rapid obsolescence, and

3. Find methods to quickly incorporate new generations of equipment and replace obsolete equipment without disrupting the existing system.

To help solve these and related problems, the control system designers are committed to the use of standards in system design and to collaboration with other laboratories when possible. This approach allows the IPNS Upgrade controls team to build a control system by using the software techniques and hardware developed at Argonne and other laboratories. This philosophy has been demonstrated to save development time and expense associated with inventing a system from first principles. EPICS and its implementation in the IPNS Upgrade control system are described in this section.

\subsection{EPICS OVERVIEW}

EPICS-based control systems include operator interfaces (OPIs) consisting of Unix workstations that use a graphical user interface (GUI) based on the X Window Motif model. The selection of a workstation implementation based on X Window for the operator interface 


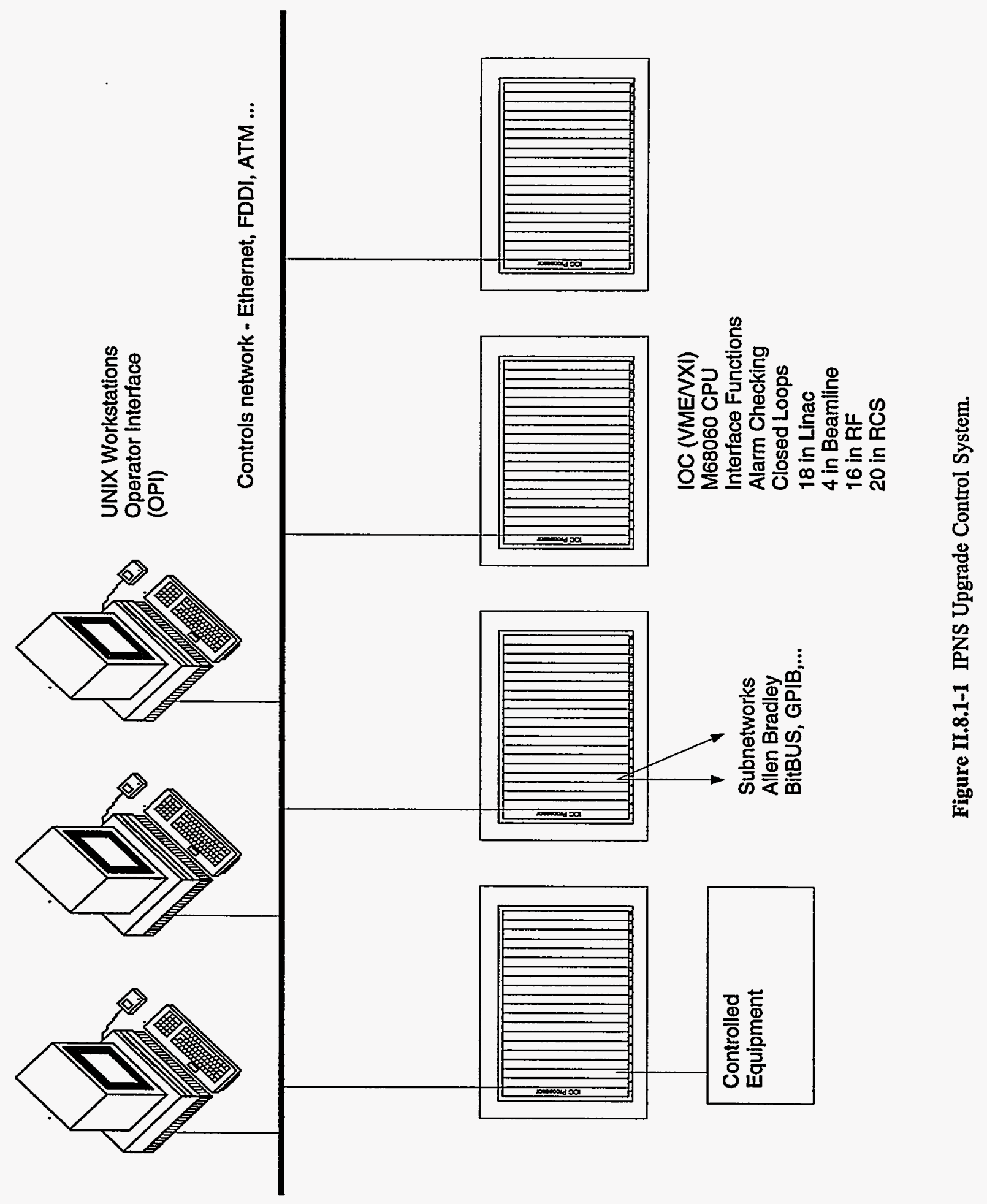


allows the software development effort to take place in the same environment as the field instruments, video tools, and the interfaces for controls and data acquisition. Consoles can be located in offices, labs, seminars, and wherever they are required in the field. The OPI includes menu-selection lists, diagrams, flow charts, and maps, as well as the more standard text-based selection menus. The workstation supports external software interfaces, including spreadsheets and commercial applications (such as IDL/PvWave and Mathematica) and application software (such as accelerator modeling codes written in Fortran, C, or other languages). Auxiliary displays using X Window terminals, as well as projection and large-screen displays in the main control room and seminar rooms, are easily implemented by means of commercial equipment. EPICS OPI runs on SUN Sparc, Hewlett-Packard PA, and Digital Equipment Alpha architecture workstations.

\subsubsection{Specific EPICS Features}

EPICS provides a number of tools for control system implementation. This minimizes the need for custom coding and helps ensure uniform operator displays. As long as the network is not saturated, an arbitrary number of input/output controllers (IOCs) and OPIs can be supported. If a single IOC becomes CPU-bound, its functions can be spread over several IOCs, or additional CPUs can be added to a single IOC. Rather than having all applications run on a single host, the applications can be spread over many OPIs.

\subsubsection{EPICS Core Software}

EPICS consists of a set of core software components and a set of optional components. The core software consists of a channel-access client and server, database scanners-monitors, and the database configuration tool (DCT). All other software components are optional.

\subsubsection{Channel Access}

Channel access is based on a client-server model and provides network-transparent access to IOC databases. Each IOC provides a channel-access server that is capable of establishing communication with an arbitrary number of clients. Channel-access client services are available on both OPIs and IOCs. A client can communicate with a number of servers, within the limits of available memory.

The central feature of both the OPI and IOC software designs is the protocol for connections between software modules for the purpose of exchanging information. This protocol is called channel access and is built on the transmission control protocol/Internet protocol (TCP/IP) standard. TCP/IP is an integral part of every Unix-based workstation and is also built into VxWorks, the real-time operating system. When an OPI application program needs to connect to a process variable located in an IOC, it issues a broadcast over the network, and the 
IOC in whose database the requested process variable resides provides a response. A socket-tosocket connection is established, and efficient two-way communication takes place. IOC-to-IOC channel access can take place to exchange inter-IOC information. The location of the desired process variable is transparent to the OPI, and only its name must be unique within a subnet.

The database that defines all IOC channel connections and properties is distributed over the IOCs and downloaded at IOC boot-time along with the IOC operating system, VxWorks, and the particular device driver software modules required by that IOC. The entire database is centrally maintained and configured.

\subsubsection{Database}

The heart of an IOC is a memory-resident database, together with various memoryresident structures describing the contents of that database. These structures are manipulated by the application designer through the use of database records. EPICS supports a large and extensible set of record types, such as ai (analog input) and ao (analog output). Each record type has a fixed set of fields. Some fields are common to all record types; others are specific to particular record types. Every record has a record name and every field has a field name. The first field of every database record holds the record name, which must be unique across all IOCs attached to the same TCP/IP subnet. A number of data structures are provided so that the database can be accessed efficiently. These structures are transparent to most software components because they access the database via database access routines.

\subsubsection{Database Scanning}

With the exception of record and device support, all access to the database is through the channel or database access routines. Database scanning is the mechanism for deciding when to process a record. Four types of scanning are possible: periodic, event, input/output (I/O) event, and passive. Periodic scanning supports a number of time intervals. Event scanning is based on the posting of an event by any IOC software component. The I/O event scanning system processes records on the basis of external hardware interrupts. Passive record processing is triggered by processing of linked records.

\subsubsection{EPICS Tools}

The EPICS tools are used by application developers to build a control system. The Motif-based editor display/manager tool (MEDM) allows designers to easily create, configure, and modify displays and then activate and connect displays to actual hardware. The display editor portion of MEDM (Figure II.8.2-1) provides process connections via an interactive display editor/control. Editing/drawing commands are used to build displays with little or no 


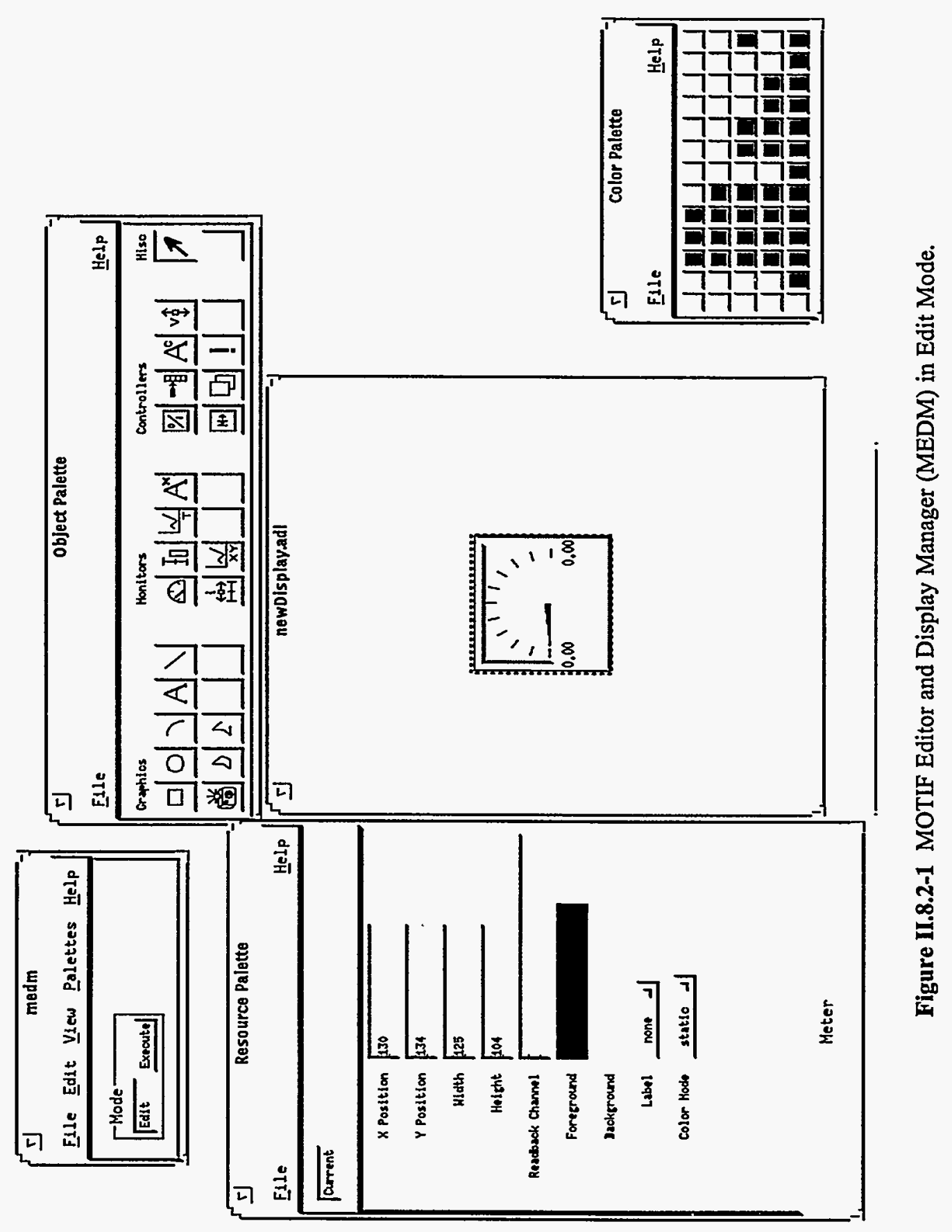


custom programming required. Process connections to the data are entered as process variable and field names. MEDM allows the designer to modify any aspect of the display element, as well as to copy, align, and group the display elements. Standard drawing tools can be used to create backgrounds, which can then be imported into MEDM by means of graphic interface format (GIF) files.

The alarm handler (Figure II.8.2-2) displays alarm messages in a hierarchical manner. The backup and restore tool (BURT) stores and retrieves data from selected channels. A graphical database configuration tool (GDCT), shown in Figure II.8.2-3, allows the application designer to configure the run-time database. The knob manager (KM) (Figure II.8.2-4) allows the operator to attach physical knobs to process variables.

\subsection{CONTROL SYSTEM HARDWARE}

The IPNS Upgrade system hardware is depicted in Figure II.8.1-1. The front-end computers or IOCs are based on Motorola M68060 processors with direct upgrade paths to the new Motorola Power PC processors as they become available. The hardware is based upon the VME/VXI family, and the choice of crate is dictated by the particular application. The OPIs run Unix software and have an X Window GUI. The workstations are connected to the network by using a single-attach fiber-distributed data interface (FDDI) for high-speed access to all data. Standard network monitoring tools, such as remote network monitoring and out-of-band hub management, are provided.

\subsubsection{Console Computers}

A computer workstation is the primary operator interface. It has a high-resolution color display and is capable of supporting several separate graphic windows simultaneously. Each OPI has access to all process variables in the IPNS Upgrade complex and, depending on system permissions, can control any of the various systems. Depending on machine mode (commissioning, operations, or system maintenance), each console is used alone or in conjunction with adjacent console systems.

In the X Window client-server paradigm, an application program is divided into the "client," which provides the computation and logic of the program, and the "server," which provides the interaction facilities for the human operator or user. Both client and server are implemented in processors at the OPI level in the IPNS Upgrade control system. The client and server need not reside in the same processor. For example, a specialized parallel processor can be running an application and that application can be accessed by a common workstation or $\mathrm{X}$ Window terminal. In this way, each OPI is able to have windows open to clients operating both locally and on other processors on the network. 
File Action View Setup

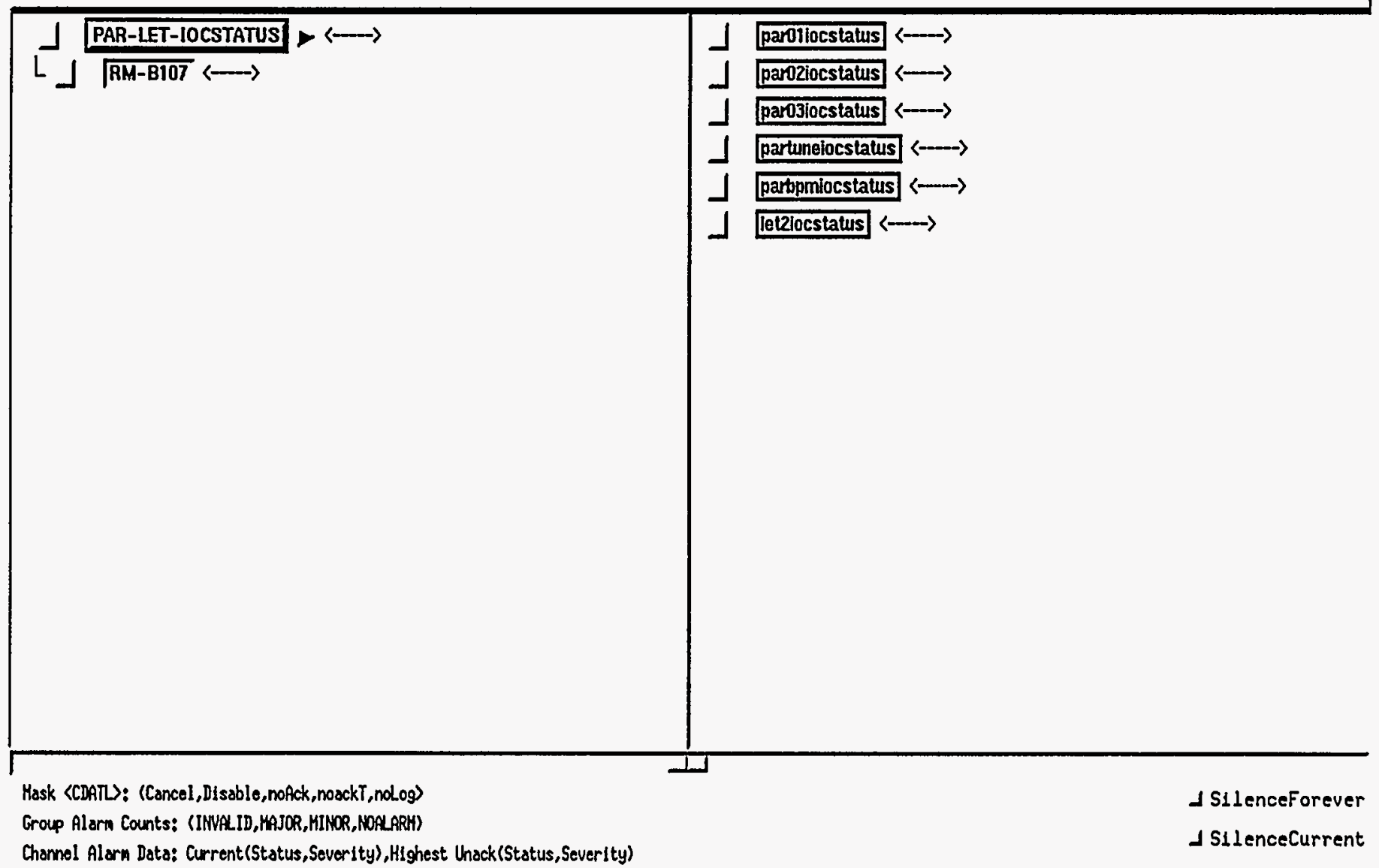




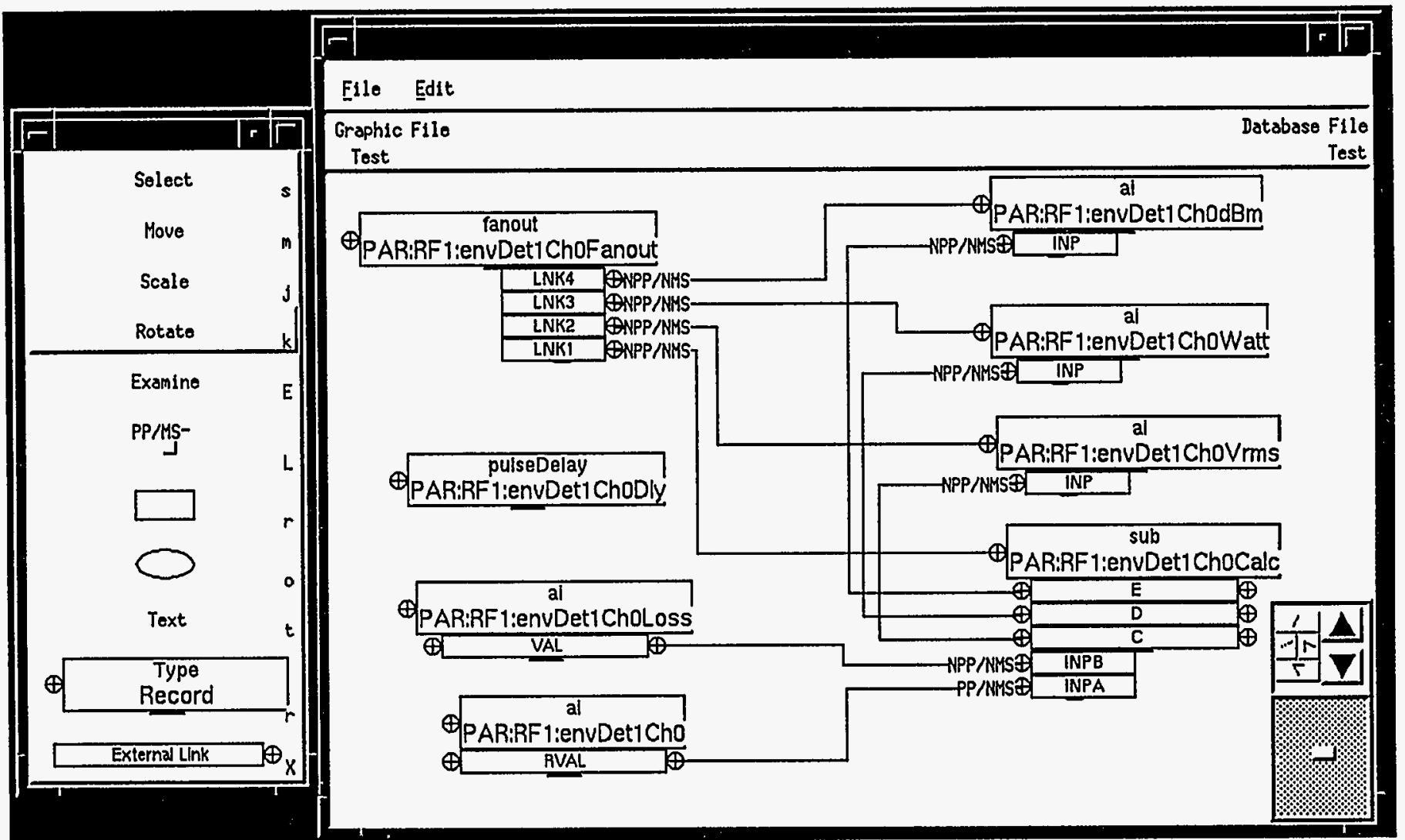

Figure II.8.2-3 Graphical Database Configuration Tool. 


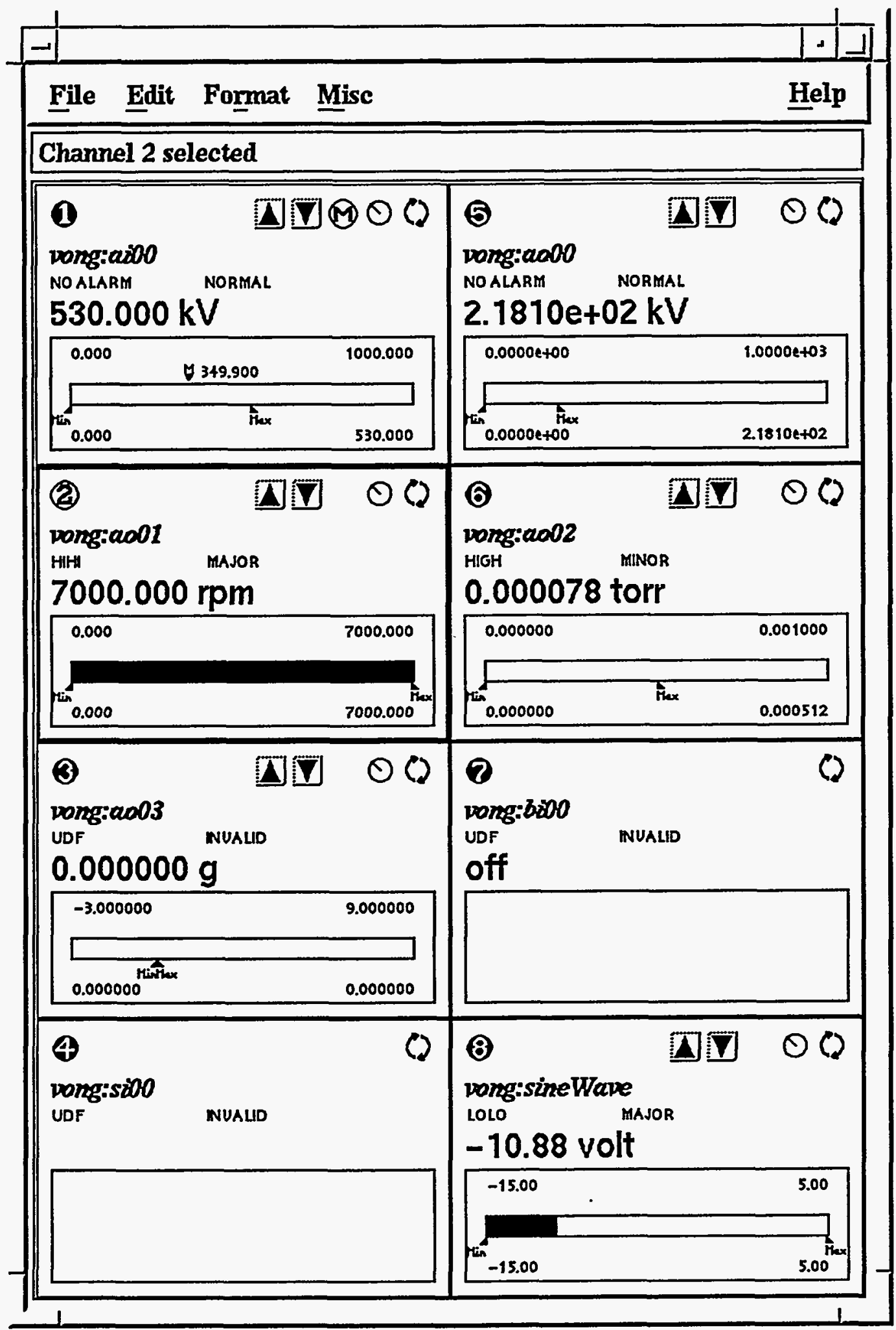

Figure II.8.2-4 Knob Manager. 


\subsubsection{Front End Systems}

The IOC is based on the VME/VXI standard and uses a Motorola 68060 microprocessor for control. A fast, real-time kernel, VxWorks, is the operating system. No local disk drive is required in this system. The software image can be downloaded from a file server or from on-board programmable read-only memory (PROM). The IOC also supports task priorities and interrupts as well as custom code. The IPNS Upgrade control system uses 58 IOC crates. More than $20 \mathrm{VME}$ input-output modules, including analog input/output, motor control, and binary input/output, are currently supported. Others will be supported as required.

Most information preprocessing is performed at the IOC level, including sequential and control-loop operations. In this way, maximum benefit is gained from the many IOC processors operating in parallel.

Hardware $\mathrm{I} / \mathrm{O}$ can be generalized into several types: analog and binary $\mathrm{V} / \mathrm{O}$, stepper motor, and subnetwork connections. Hardware records contain information for run-time processing, including scaling, engineering units, hardware address, and scanning. The IOC database contains such parameters as scan requirements, I/O address, engineering unit conversions, alarm limits, control parameters, and current values for each defined process variable. Database records can be linked together to implement complex algorithms.

The database scanner processes database records and updates the dynamic fields in the database, such as value, alarm status, and severity. Data conversions are supported. Alarm checking for limit violations, state violations, hardware communication errors, and alarm severity is supported. Binary and analog $1 / O$ are provided through the database. Analog input can be smoothed; analog output can be either incremental or positional and the output rate of change can be specified. Multi-bit (16 states) and single binary I/Os are supported.

All I/O fields can be connected to a hardware device or another database element, such as a calculator record or proportional integral derivative (PID) record, or can fan out to several devices. Timing signals, motion control, and transient digitizers are supported through the database.

Stepper motor records receive position/velocity input from user or other data, provide positional or velocity mode operation, provide position feedback through an encoder or another database record, and allow simple closed-loop position. Limit switches are supported.

General purpose calculations, a PID algorithm, and a signal selector are supported to provide closed-loop control and data filtering. The calculation record supports up to six sets of input, $\mathrm{C}$ language expressions, algebraic operations, relational operations, Boolean operations, and trigonometric functions. The PID algorithm allows user control of setpoint, proportional, integral, and derivative gains; incremental or positional outputs; and anti-windup and reset on the integral term. 


\subsubsection{Subnets}

The IPNS Upgrade control system provides for VME-based IOCs to be distributed throughout the facility and interconnected via Ethernet to one another and also to Unix-based operator interface consoles. Although this distributed architecture allows for intelligent processors near the major subsystems, $\mathrm{I} / \mathrm{O}$ subnets are frequently required to interface directly to the equipment and to communicate I/O information to the nearest IOC. The concept of a subnet is an important element in control systems in general and EPICS in particular. EPICS supports several types of subnets: Allen-Bradley, general-purpose interface bus (GPIB), and BITBUS. The Allen-Bradley $\mathrm{I} / \mathrm{O}$ subnet uses $\mathrm{AB} 1771$ series $\mathrm{I} / \mathrm{O}$ modules to provide basic binary and analog I/O support for the control system. Allen-Bradley is an inexpensive and rugged standard for industrial control systems. Copper and fiber-optic-based multidrop subnets are available for this equipment.

Laboratory test and measurement equipment often uses the IEEE-488 (GPIB) standard as an interface to an external control system. IEEE-488 subnets present some serious challenges and potential problems to the system designer. IEEE-488 has a distance limitation of $20 \mathrm{~m}$, which requires the instruments connected to the bus to be in close proximity to the IOC. In addition, the signals within the GPIB cable are not balanced and thus are susceptible to EMU/RFI noise and ground spikes. Signal transfer and isolation techniques are not part of the GPIB standard, and although commercial equipment is available to extend the distance of a GPIB interface, it is prohibitively expensive. To overcome these limitations, a BITBUS has been developed for use with EPICS to provide a method of high speed transfer of short control messages over a multidrop network. The BITBUS subnet can be used as a method for remote, single point I/O as well as for a gateway for remote IEEE-488 and RS232 signals. A differential, opto-isolated, wired subnet is the BITBUS standard; however, a BITBUS multidrop fiber-optic network has been developed for use with EPICS.

\subsubsection{Networks and Protocols}

Argonne uses FDDI as the intralaboratory network (Figure II.8.3-1). Backbone cables are in place in individual buildings, and communication between buildings is presently done via FDDI and high-speed asynchronous transfer mode (ATM) networks. The IPNS Upgrade facility uses FDDI and ATM to connect network hubs, as shown in Figure II.8.3-2. The network hubs connect to OPIs. The IOCs will use ATM, FDDI, or fiber-optic-connected Ethernet branches as performance needs dictate.

\subsection{APPLICATION SOFTWARE}

Application software is available to provide features or services not provided by the control system. For example, the software required to provide a local orbit bump is application software. These application programs can be of two general forms. The first is a control panel 


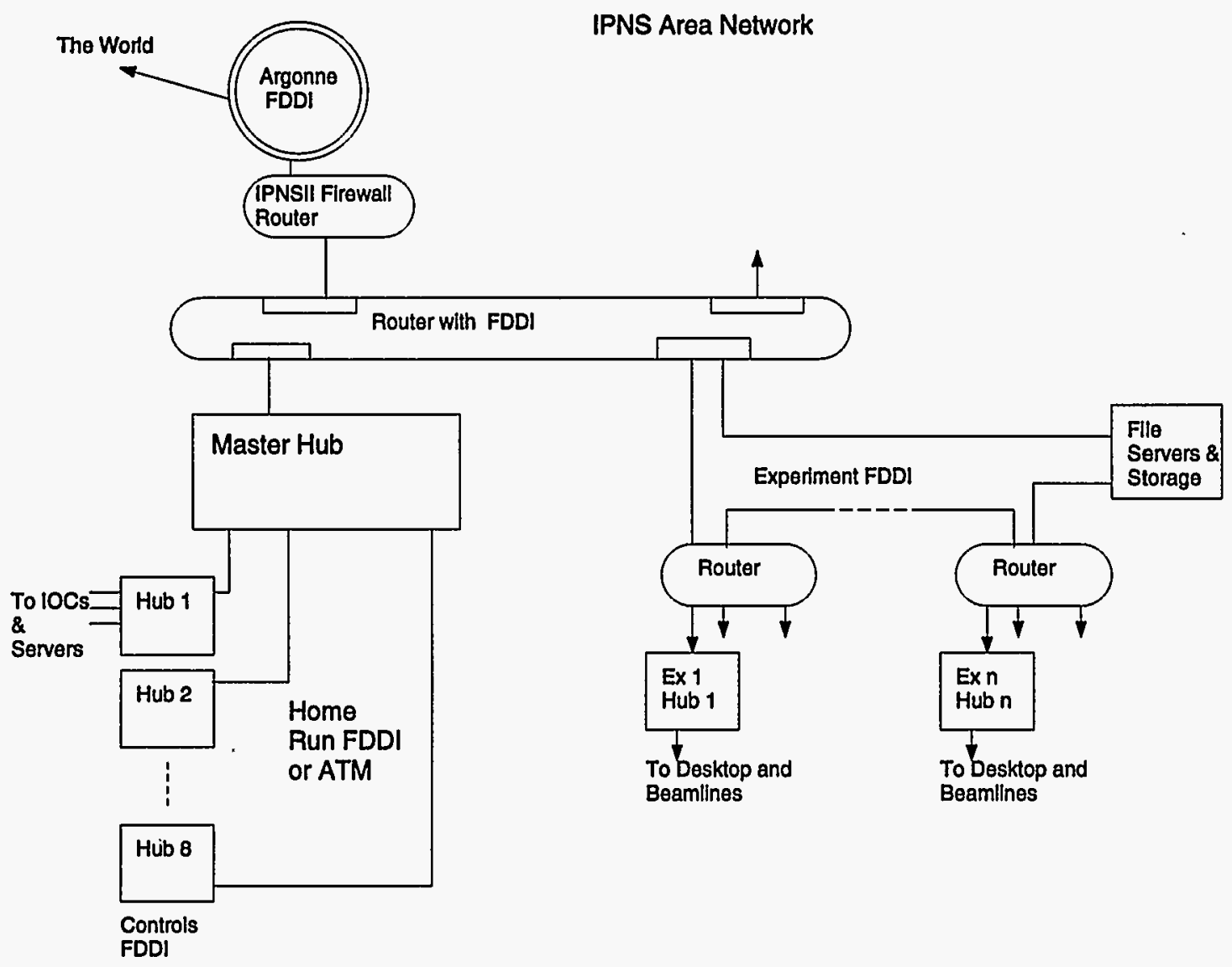

Figure II.8.3-1 IPNS Facility Network. 


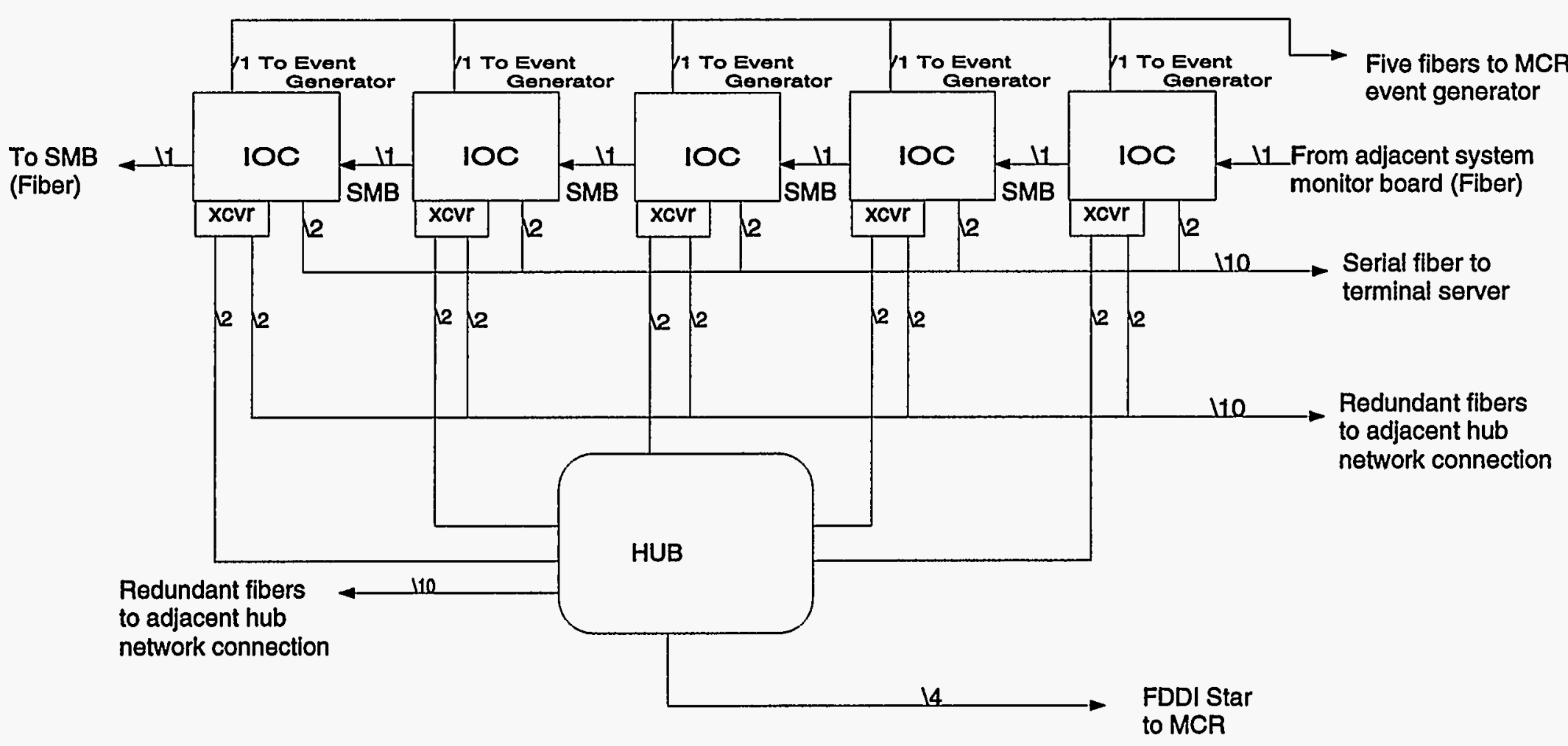

Figure II.8.3-2 IPNS Upgrade Local Control Network. 
created with MEDM, in which graphic tools such as buttons, sliders, indicator lights and meters, and graph paper are selected, attached to process variable (pv) names, and located on the panel (Figure II.8.4-1). Static entities are used to depict the physical system, such as piping diagrams, and are added where appropriate. When complete, the panel is called up for execution, and channel-access calls are made. No actual code is written, and no compilation is made.

The second form of application program generates classic in-line code. In this case, standard entry points are provided in the same manner as for the graphic and channel-access tools. When this approach is used, existing code can be adapted to EPICS by calling the channelaccess routines and displaying the results as either traditional line-by-line or graphical output.

All software is developed under Unix, including that for the IOCs. Multiple windows can be opened for software development, actual run-time applications, database configuration, and electronic mail. This capability streamlines software development, database servicing, and system troubleshooting.

\subsection{EPICS APPLIED TO IPNS UPGRADE}

Figure II.8.1-1 schematically depicts the IPNS Upgrade controls network. The IOCs are located as close as possible to the equipment to be controlled. All network connections to the IOCs are through fiber-optic cables to eliminate most sources of electrical noise.

\subsection{OTHER SERVICES}

\subsubsection{Timing System}

Two classes of timing coordination are necessary. Beam transfer events and signal sampling between the Linac and the RCS are controlled. The second class is associated with the need to control sampling of beam position in the RCS. Both systems use essentially the same method, but with different clock rates and sources. Depending on the mode of operation, various delay counts are downloaded to the microprocessor clusters and set into clock-delay modules in the VME crates. A systemwide start pulse based on the magnetic field is generated, and the delay modules count a clock source and produce a pulse output after their programmed delay. For event sequencing in the RCS, a crystal-derived clock is used; it produces real-time triggers for sampling beam parameters.

Accelerator control and monitoring tasks are performed by using the timing configuration that is generated by a magnetic-field-triggered crystal clock. 


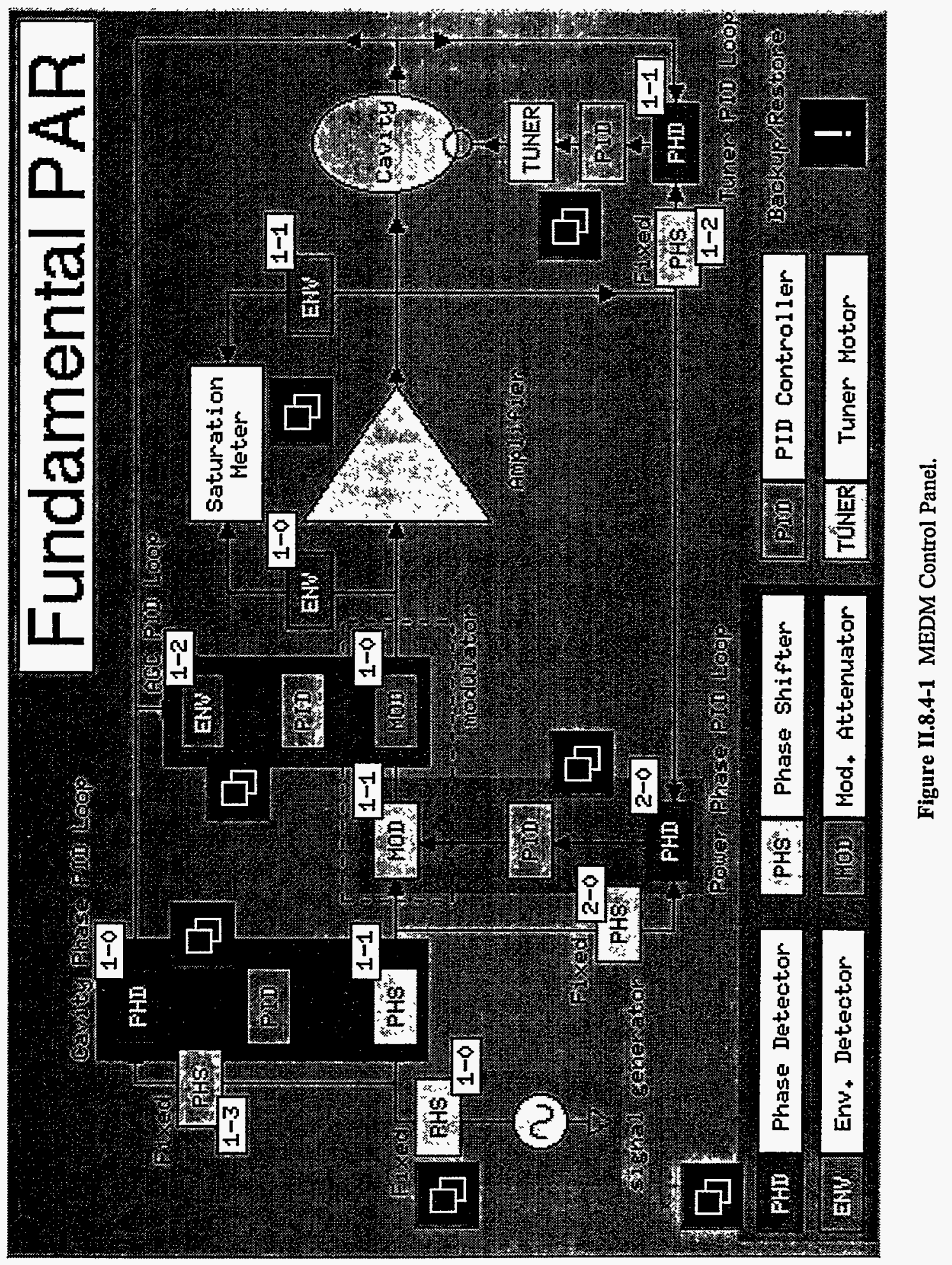




\subsubsection{Event System}

The event system provides the ability to generate signals on the basis of timing and software events. This system has been designed to work hand-in-hand with EPICS. It consists of two types of interfaces, an event generator and an event receiver. An event receiver is located at each IOC.

\subsubsection{Analog Signal System}

To convey the required number of high-bandwidth analog signals to the main control room, a signal distribution system is used. Commercial, VME-based, coaxial relay modules and commercial, high-bandwidth fiber-optic systems are used and controlled by the IOCs. At the console end, the analog signals are switched to the desired display or instrument. The choice of signal and output destination are selected by the operator from standard EPICS screens.

\subsubsection{Computer-Controlled Video Signal System}

The same method used for the analog system is used for video signals. Again, the computer system provides the control. The video sources are also delivered to frame grabbers for on-line, dynamic beam analysis. All of these techniques are in use at the APS.

\subsubsection{Network Access from Offices and Laboratories and User Housing Facility}

The distributed nature of the IPNS Upgrade control system allows the system to be monitored from the office and laboratory areas by the operational staff. This feature facilitates equipment checkout, status inquiries, and software development, which can take place on a noninterfering basis. Actual control of the various systems is highly restricted and controlled by the EPICS software on each IOC.

\subsection{CONCLUSION}

The IPNS Upgrade control system allows monitoring and control of the accelerator and experiments from offices, laboratories, and the user residence facility.

\subsection{REFERENCES}

1. M. Knott et al., "EPICS: A control system software co-development success story," Nucl. Instrum. Methods A 352, 486 (1994). 
2. L. Dalesio et al., "The experimental physics and industrial control system architecture: Past, present and future," Nucl. Instrum. Methods A 352, 179 (1994).

3. McDowell et al., "Status of the Advanced Photon Source and its accelerator control system," Nucl. Instrum. Methods A 352, 13 (1994).

4. L. Dalesio et al., "EPICS architecture," in Proceedings of the International Conference on Accelerator and Large Experimental Physics Control Systems (KEK, Tsukuba, Japan, November 11-15, 1991), pp. 278-281 (1991). 


\subsection{INTRODUCTION}

Shielding provides protection for workers and for the general public during operation of the IPNS Upgrade accelerators. The shielding limits the radiation dose for both on-site and off-site exposure to less than that allowed by the U.S. Department of Energy (DOE) guidelines.

The shielding design utilizes semi-empirical formulae with experimental dose data and measured attenuation characteristics as input parameters, when these data are available. The calculations with the semi-empirical formulae are in reasonable agreement with the results of computer simulations. The accelerator design has been carried out with the objective of limiting beam losses to less than $1 \times 10^{-3}$. Low beam losses help ensure that radiation levels in the vicinity of the equipment are not excessive during shutdown periods and that the level of activation is sufficiently low to allow hands-on maintenance.

\subsection{SHIELDING DESIGN OBJECTIVES}

The design standard ${ }^{1}$ for new facilities and major modifications to existing facilities, Article 128 of DOE/EH 0256T Revision 1 requires that individual worker dose be less than $5 \mathrm{mSv} / \mathrm{yr}(500 \mathrm{mrem} / \mathrm{yr})$ and as low as reasonably achievable (ALARA). The neutron quality factor has been doubled for design purposes; thus, the design dose rate is $1.25 \mu \mathrm{Sv} / \mathrm{h}$ for occupational exposure ( 40 hours per week, 50 weeks per year) for those situations in which neutrons dominate the dose rate. The shielding is designed to meet a more conservative value than this criterion to ensure that occupational radiation doses are ALARA. DOE Order 5400.5 limits the annual dose to members of the public ${ }^{2}$ to $1 \mathrm{mSv} / \mathrm{yr}$. A further requirement of this Order is that actual or potential doses in excess of $100 \mu \mathrm{Sv}$ in a year to any member of the public require notification of DOE Headquarters. These dose-limiting objectives and guidances are used as the basis for establishing the adequacy of the bulk shielding design and other dose-mitigation systems for normal operations of the facility.

The bulk shielding provided for the Rapid Cycling Synchrotron (RCS), high-energy transport (HET) line, and target biological shields limits the dose rate to $<1.25 \mu \mathrm{Sv} / \mathrm{h}$ at $30 \mathrm{~cm}$ from the outside of the shielding walls under normal loss conditions. This dose rate assumes a current of $500 \mu \mathrm{A}$ of protons at $2.2 \mathrm{GeV}$. The computations are based upon semi-empirical formulae and attenuation data presented in the literature. ${ }^{3-10}$

The calculations for the low-energy transport (LET) line and linac are based on $500 \mu \mathrm{A}$ of protons at $400 \mathrm{MeV}$. The calculations are based on semi-empirical formulae and attenuation data presented by Tesch. ${ }^{11}$

The methodology of Stevenson and Thomas ${ }^{12}$ was used to estimate the skyshine contribution at the nearest site boundary, which is $350 \mathrm{~m}$ away. The results of the calculations for both direct and skyshine radiation indicate that the total off-site contribution is less than $80 \mu \mathrm{Sv} / \mathrm{yr}$ for 8,000 hours of operation. 
A continuing accidental loss of the entire proton beam at one point is considered an unlikely event. However, sufficient shielding is provided to ensure that the potential dose to an individual on the outside of the shield in the vicinity of the loss is mitigated. The objective is to provide enough shielding to limit the dose, from the onset of the occurrence to its termination, to less than $1 \mathrm{mSv}$.

An assumed occupancy factor of 0.1 has been applied in order to estimate the radiation dose outside of the shield in the vicinity of the linac, injection or extraction areas, or a high-energy transport line. This factor is a conservative estimate of the time an individual may be required to be in these regions.

The dominating radiation, which determines the necessary shielding thickness for a highenergy proton beam, is the high-energy neutron component formed in nuclear cascades. ${ }^{13}$ The amount of shielding necessary is determined by the attenuation of the high-energy component in equilibrium with the slowing-down spectrum of lower energy neutrons. This high-energy component is composed of neutrons with $\mathrm{E}>150 \mathrm{MeV}$, for which the attenuation length is independent of energy; for low-energy neutrons $(<100 \mathrm{MeV})$, attenuation lengths decrease rapidly with neutron energy. Typically, in concrete, the low-energy neutron attenuation length is a factor of three less than for high-energy neutrons. Therefore, designing the shields to attenuate the high-energy neutron component automatically takes care of the low-energy neutrons because of their shorter attenuation lengths. The gamma dose outside thick shields has been estimated to be $10-16 \%$ of the neutron dose; 14,15 therefore, no additional calculations for gamma radiation are necessary.

\subsubsection{Radiation Attenuation Lengths}

Information on the attenuation of high-energy and evaporation neutrons in several shield media was obtained from the literature.3,4,8,9 For each material, the quoted values for attenuation length vary, depending upon the angle at which the radiation was measured and on assumptions made about the neutron spectrum. Much of the measured data deals only with "transverse attenuation." Transverse attenuation as used here means the attenuation of secondary particles produced when the beam hits a target. These particles are measured after passing through a shield wall, generally at $90^{\circ}$, as shown in Figure II.9.2-1. Data on longitudinal attenuation are scarce and limited mainly to published computer simulations.7,9,10,16 Table II.9.2-1 lists representative values for high-energy neutron attenuation lengths in typical shield materials, measured at $90^{\circ}$.

The attenuation length at $90^{\circ}$ in other materials can be scaled from

$$
\lambda / \lambda_{\mathrm{E}}=\left(\mathrm{A} / \mathrm{A}_{\mathrm{E}}\right)^{1 / 3},
$$




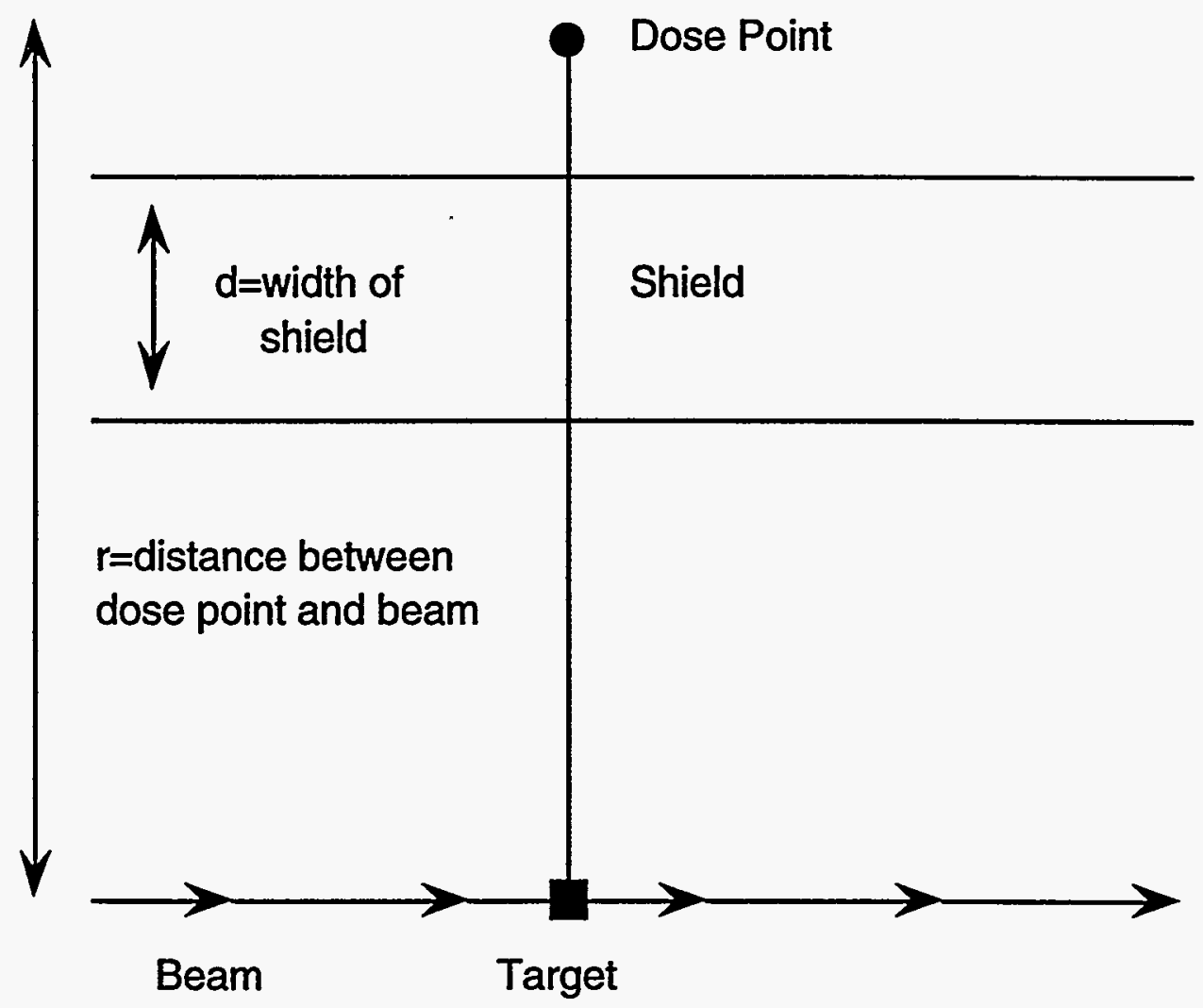

Figure II.9.2-1 Geometry for Calculating the Transverse Dose Due to Secondary Particles Attenuated by a Shield. 
Table II.9.2-1 Transverse Attenuation Lengths for High-Energy Neutrons

\begin{tabular}{lcc}
\hline \multicolumn{1}{c}{ Shielding Material } & $\begin{array}{c}\text { Density } \\
\left(\mathrm{g} / \mathrm{cm}^{3}\right)\end{array}$ & $\begin{array}{c}\text { Attenuation Length } \lambda \\
\left(\mathrm{g} / \mathrm{cm}^{2}\right)\end{array}$ \\
\hline Ordinary Concrete & 2.35 & $99-117$ \\
High Density Concrete & 3.7 & $116-134$ \\
Iron & 7.8 & $140-180$ \\
Earth & 1.6 & 117 \\
\hline
\end{tabular}


where $\lambda$ and $A$ are the attenuation length and atomic number of the shield material, normalized to $\lambda_{E}$ and $A_{E}$ of earth $\left(\lambda_{E}=117 \mathrm{~g} / \mathrm{cm}^{2}\right.$ and $\left.A_{E}=20.4\right) .{ }^{4}$ Tesch and Zazula ${ }^{3}$ give $\lambda=240 \mathrm{~g} / \mathrm{cm}^{2}$ for the attenuation length in iron at $5^{\circ}$ in the forward direction, and Dinter et al. ${ }^{10}$ give $\lambda=150 \mathrm{~g} / \mathrm{cm}^{2}$ at $40^{\circ}$ for forward-directed radiation in ordinary concrete.

\subsubsection{Bulk Shielding Calculations}

The linac features an ion source, a 2-MeV radio-frequency quadrupole (RFQ), a $25-\mathrm{m}$ long, $70-\mathrm{MeV}$ drift-tube linac, and a $92-\mathrm{m}$-long, 330-MeV coupled-cavity linac. $\mathrm{H}^{-}$ions are accelerated in pulses of $1.04 \times 10^{14}$ protons/pulse to $400 \mathrm{MeV}$ at a repetition rate of $30 \mathrm{~Hz}$. The resultant average current of $500 \mu \mathrm{A}$ is transported by the LET line and is injected into the RCS. The entire linac is housed in a tunnel with inner dimensions $3.65 \mathrm{~m}$ wide by $2.74 \mathrm{~m}$ high, which is located $3.04 \mathrm{~m}$ below grade. Beam height is $1.4 \mathrm{~m}$ above the tunnel floor. The linac shielding is adequate for limited access to the Klystron Gallery during operation at the design parameters.

The RCS is housed in the existing Zero Gradient Synchrotron (ZGS) Ring Building, which is shown in Figure II.9.2-2. The ZGS was a 12.5-GeV proton synchrotron, which accelerated more than $7 \times 10^{12}$ protons per pulse at $0.5 \mathrm{~Hz}$. The Ring Building (Building 365) is an annularshaped building approximately $83 \mathrm{~m}^{2}$ in cross section and $61 \mathrm{~m}$ in diameter. Located in the center region of the annulus is the Center Building (Building 364). This building is approximately $26.8 \mathrm{~m}$ in diameter and $24 \mathrm{~m}$ high. The Ring Building is covered with a mound of earth of minimum thickness $6.1 \mathrm{~m}$ on the side.

The RCS roof shielding amounts to about $4.9 \mathrm{~m}$ of earth on top of $0.61 \mathrm{~m}$ of reinforced concrete. The walls of the Ring Building are made of $1.07 \mathrm{~m}$ of reinforced concrete. The earth shielding thickness between the Ring Building and the Center Building is about $7 \mathrm{~m}$, and at beam height, the earth shielding thickness outward from the Ring Building is about $21.3 \mathrm{~m}$. When the ZGS was in use, beams of protons were sent to Building 369 (EPB1) and Building 370 (Meson Building). For the IPNS Upgrade, the $30-\mathrm{Hz}$ target station is housed in Building 370 and the $10-\mathrm{Hz}$ target station is housed in Building 369. The existing Ring Building shielding is sufficient for the IPNS Upgrade, as is demonstrated in the following sections.

The RCS accelerates protons from $400 \mathrm{MeV}$ to $2.0 \mathrm{GeV}$. High-energy transport lines deliver $2.0-\mathrm{GeV}$ protons to either of two neutron-generating target areas. The design goals are an average current of $500 \mu \mathrm{A}$ at $2.0 \mathrm{GeV}$ for a maximum power level of $1.0 \mathrm{MW}$. Shielding requirements for the target biological shields are discussed in Chapter IV.

The shielding achieves the design goal of $<1.25 \mu \mathrm{Sv} / \mathrm{h}$ at all normally occupied points on the outside of the shield. Access to all accelerator areas is administratively controlled. The shielding requirements are satisfied by using sufficient iron and/or concrete as a bulk shield to ensure adequate attenuation of the high-energy and evaporation neutrons. The concrete is supplemented by earth berms in some regions, including the linac, a portion of the LET, and the 


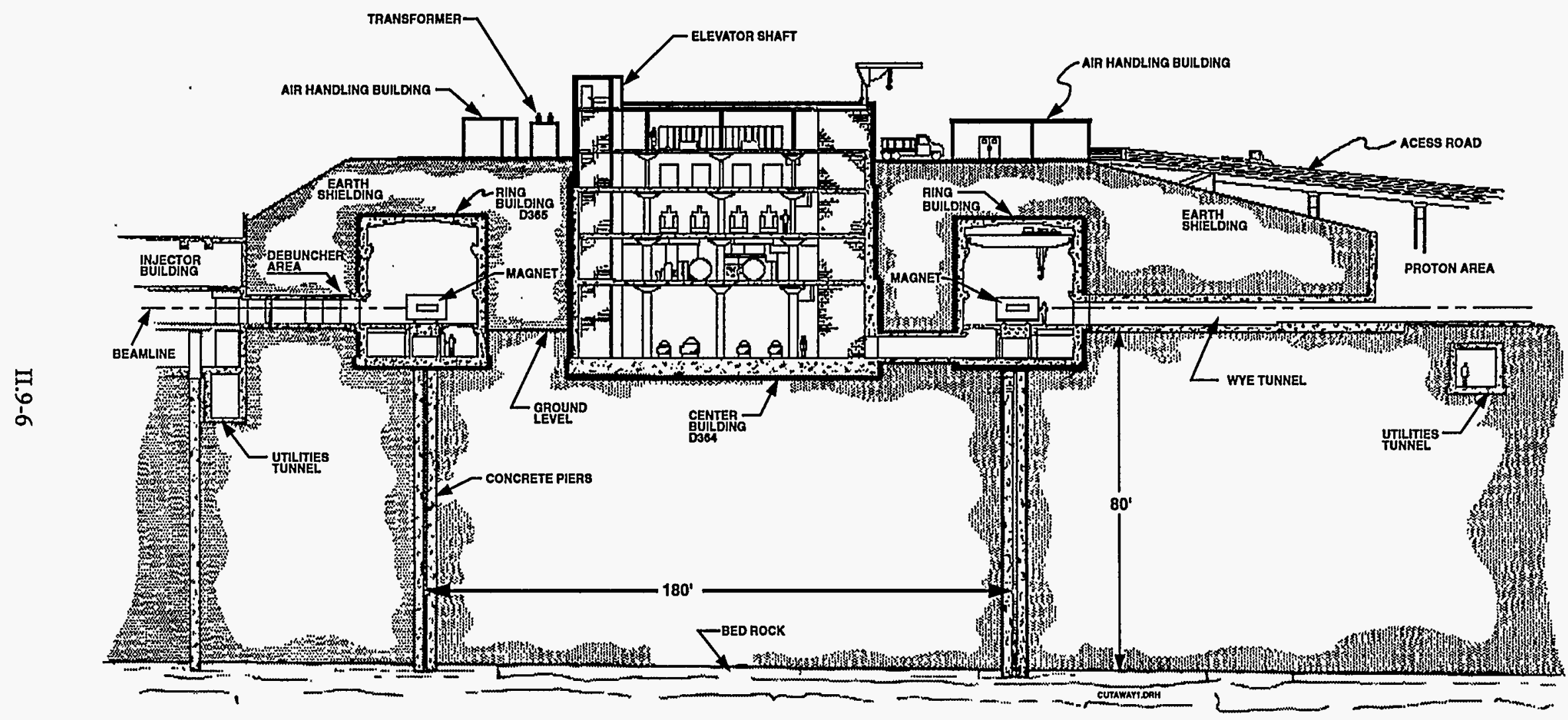

Figure I1.9.2-2 Cross Section of the Zero Gradient Synchrotron Ring and Center Buildings. 
perimeter shielding of the RCS. Lead, steel, dense polyethylene, and castable-composite shielding are used to improve the localized shielding, as required. Localized losses can occur at septum magnets and at other bending and focusing magnets during injection and extraction.

\subsubsection{Radiation Dose Equivalent Factors}

The dose equivalent in Sv per incident proton, $\mathrm{H}$, based on the Moyer model, 17 is given by $\mathrm{McCaslin},{ }^{4}$ when $E_{p}$ is the incident proton energy $(\mathrm{GeV}), d$ is the shield thickness $\left(g / \mathrm{cm}^{2}\right)$, and $r$ is the transverse distance to the beam $(\mathrm{m})$ :

$$
\mathrm{H}=\frac{\mathrm{H}_{\mathrm{o}} \mathrm{E}_{\mathrm{p}}^{0.8}}{\mathrm{r}^{2}} \mathrm{e}^{-\mathrm{d} / \lambda}
$$

$\mathrm{H}_{\mathrm{o}}$ has units of $\mathrm{Sv} \mathrm{m} / \mathrm{GeV}^{0.8}$ per proton and is the unshielded dose at $1 \mathrm{~m}$. The value of $\mathrm{H}_{\mathrm{o}}$ is dependent on the target material. Attenuation length, $\lambda$, for the relevant shield materials is found in Table II.9.2-1. For angles other than $90^{\circ}$, data in the literature were used. $3,5,11$

We use the concept of a line source to estimate dose from beam losses over an extended distance. For an assumed uniform beam loss over an extended region, the Moyer model can be numerically integrated to find the line source contribution.

The attenuation properties of iron shields backed up by concrete have been calculated by Tesch and Zazula. ${ }^{3}$ Parameters for a modified Moyer model representation are provided, including attenuation by the concrete. They suggest values of $\mathrm{H}_{\mathrm{o}}=4 \times 10^{-14}$ and $\lambda=180 \mathrm{~g} / \mathrm{cm}^{2}$ for an iron target and an iron shield, respectively. A problem in using only iron is that neutrons of approximately $25 \mathrm{keV}$ and $125 \mathrm{keV}$ tend to build up and stream through the iron after deep penetration. This effect can be handled by including $1 \mathrm{~m}$ of concrete to back up the iron. Tesch and Zazula quote an attenuation length of $30 \mathrm{~g} / \mathrm{cm}^{2}$ for low-energy neutrons and $107 \mathrm{~g} / \mathrm{cm}^{2}$ for high-energy neutrons in concrete. The $1 \mathrm{~m}$ of concrete represents about eight attenuation lengths for the low-energy neutrons and effectively removes them. The final expression for the dose equivalent rate, $\dot{\mathrm{H}}_{\text {tot }}$ for an iron shield backed up by $1 \mathrm{~m}$ of concrete is then

$$
\dot{\mathrm{H}}_{\text {tot }}=\dot{\mathrm{H}}_{\mathrm{Fe}} \mathrm{e}^{-235 / 107} \text {, }
$$

in which $\dot{\mathrm{H}}_{\mathrm{Fe}}$ is the dose equivalent rate through the iron shield. The dose equivalent through the iron shield is obtained from Equation II.9.2-2 and converted to dose equivalent rate by multiplying by the proton loss rate. 
The dose equivalent factor can be calculated using the Moyer model, or the values can be taken from Tesch and Zazula. ${ }^{3}$ At $2 \mathrm{GeV}$, the Moyer model gives a dose per proton of $1.3 \times 10^{-14} \mathrm{~Sv}$ at $1 \mathrm{~m}$, and Tesch and Zazula give a dose per proton of $4 \times 10^{-14} \mathrm{~Sv}$ at $1 \mathrm{~m}$, based on their recent computer studies. The values from Tesch and Zazula are a factor of three more conservative and are used in our calculations of the dose rates from the RCS, HET, and through the target biological shields. The calculations for $400 \mathrm{MeV}$ protons are based on Tesch, ${ }^{11}$ since his formalism is based on measured data over the energy range from 50 to $1000 \mathrm{MeV}$.

\subsubsection{Beam Loss Assumptions and Shielding Requirements}

To estimate bulk shielding needs, a distributed loss of $10^{-3}$ is assumed for each subsystem of the accelerator under normal operating conditions. This loss is distributed over the length of the subsystem for analysis as a line source. The loss rate along the linac and the LET under normal operating conditions is assumed to be a uniform $5 \mathrm{nA} / \mathrm{m}$, but the unshielded dose contribution is not uniform because of the increase in energy as the protons are accelerated. The line source contribution from the linac system was numerically integrated to determine the total dose equivalent rate at the relevant dose point. The bulk shielding requirements for the various parts of the accelerator system are summarized in Table II.9.2-2. This shielding ensures that the dose rate is less than $1.25 \mu \mathrm{Sv} / \mathrm{h}$. For point losses, the assumption was made that shutdown would occur within a few pulses of the onset of an abnormal condition. The shielding described in Table II.9.2-2 is adequate under these conditions.

\subsection{5 $\mathrm{H}^{0}$ Beam Catcher, Beam Stop, and Collimators}

The beam catcher is needed to stop the $\mathrm{H}^{0}$ atoms formed during the stripping reaction of the injected beam by the carbon stripping foil. An analysis by Chae ${ }^{18}$ showed that the stripping efficiency to protons of a $250-\mu \mathrm{g} / \mathrm{cm}^{2}$ foil is $99.46 \%$. The fraction of $\mathrm{H}^{0}$ emerging is $5.4 \times 10^{-3}$. These atoms are caught in a beam catcher before they enter the second quadrupole after the carbon stripping foil. This is discussed in Section II.2.2.2. Since the $30-\mathrm{Hz}$ injected beam contains $1 \times 10^{14} \mathrm{p} / \mathrm{pulse}$, the number of hydrogen atoms is significant. Sullivan ${ }^{19}$ gives an expression for the angular distribution of the fluence of secondary particles from a thick target for interacting protons of energy in the range 50 to $1000 \mathrm{MeV}$. This formulation takes into account the ionization losses as well as the interaction probability to predict the fluence per proton at $1 \mathrm{~m}$ at a given angle $\theta$ :

$$
\phi(\theta)=0.5\left(1-\mathrm{e}^{-\mathrm{m}}\right) /(\theta+40 / \sqrt{\mathrm{E}})^{2} \text { neutrons } / \mathrm{cm}^{2},
$$


Table II.9.2-2 Shielding Requirements for the IPNS Upgrade

\begin{tabular}{llll}
\hline & \multicolumn{3}{c}{ Shielding Requirements $(\mathrm{m})$} \\
\cline { 2 - 4 } \multicolumn{1}{c}{ Subsystem } & Earth & Concrete & Iron \\
& & & \\
& & & \\
Drift-Tube Linac & 1.5 & 0.3 & \\
Coupled-Cavity Linac & 3.2 & 0.3 & \\
LET & 2.0 & 2.4 & 2.0 \\
ACS & 8.0 & 1.07 & 2.3 \\
HET (10 Hz) & & 1.0 & \\
HET (30 Hz) & & 1.0 & \\
& & &
\end{tabular}


in which the proton energy $\mathrm{E}$ is in $\mathrm{GeV}$ and $\mathrm{m}=3.6 \times 10^{1.6}$. The $1-\mathrm{e}^{-\mathrm{m}}$ term corrects for the fractional energy lost due to ionization by the lower energy protons. Using attenuation lengths given in the same reference ${ }^{19}$ for relevant materials, the necessary shielding in the lateral and forward directions can be calculated. Assuming a shield made of iron and concrete, the inner iron core is used to completely stop the protons, whereas the outer concrete shield is used to absorb low-energy neutrons that can cause activation. The beam catcher consists of an iron core, $15.2 \mathrm{~cm}$ in diameter and $20.32 \mathrm{~cm}$ in length, surrounded by concrete $1 \mathrm{~m}$ thick on the sides and $1.3 \mathrm{~m}$ thick in the forward direction. The concrete shield overlaps the iron core on the front face by $15.2 \mathrm{~cm}$ to protect against backscattered radiation. This shield reduces the neutron fluence at $2 \mathrm{~m}$ by a factor of 100 in the longitudinal direction.

The $30-\mathrm{Hz}$ target is used to tune up the synchrotron. The tune-up is performed at $1-\mathrm{Hz}$ operation. Since the target biological shield is designed for $30-\mathrm{Hz}$ operation, this shield is also adequate for $1-\mathrm{Hz}$ operation, and resultant dose rates are less than $0.06 \mu \mathrm{Sv} / \mathrm{h}$.

Collimators are used to remove halo particles that would otherwise be lost in the RCS. Off-momentum protons are removed by collimators in the high-dispersion areas, and betatron collimation is done by collimators in the high-beta areas. There are eight collimators total, or one of each type in each of the four sectors of the RCS. These collimators cause losses to occur at specific, highly shielded regions. This shielding reduces uncontrolled losses around the ring and reduces activation of the accelerator components. The power dissipated in each of the collimators is expected to be of the order of $0.5 \mathrm{~kW}$. This amount of power dissipation necessitates collimator dimensions on the order of $1.4 \mathrm{~m}$ in diameter and $2 \mathrm{~m}$ long.

\subsection{ACTIVATION}

Activation of accelerator components by high-energy protons occurs because of spallation reactions in the accelerator materials, which generally leave the struck nucleus in an excited or radioactive state. Further radioactivation can be produced by the secondary particles released in the spallation reaction and by the evaporation neutrons that are emitted as the excited nucleus rids itself of excess energy. Finally, slowing down of evaporation neutrons and highenergy neutrons leads to activation by capture of low-energy neutrons. The overall amount of activity formed depends on the primary beam losses. The production of radionuclides in a given material depends upon the spallation cross section for high-energy hadrons and the mean free path in the target material. ${ }^{19}$ The formation of a particular radionuclide depends upon the composition of the struck material, the energy speetrum of the secondaries, and the production cross section for that radionuclide. Among the radionuclides produced in shielding and accelerator components by spallation reactions are ${ }^{22} \mathrm{Na}$ and ${ }^{24} \mathrm{Na}$ in concrete and ${ }^{54} \mathrm{Mn},{ }^{56} \mathrm{Mn}$, and ${ }^{60} \mathrm{Co}$ in steel. Thermal neutron capture produces ${ }^{64} \mathrm{Cu}$ and ${ }^{65} \mathrm{Zn}$ in copper, radioactive sodium in concrete, and radioactive manganese and cobalt in iron and steel. Other products, such as ${ }^{7} \mathrm{Be},{ }^{11} \mathrm{C},{ }^{51} \mathrm{Cr}$, ${ }^{46} \mathrm{Sc}$, and ${ }^{56} \mathrm{Co}$, are also formed.

Since a number of products are formed in the various interactions, each with a different half-life, the time variation of the radionuclide population depends upon the beam irradiation time 
and the time of decay following shutdown. Because of the different types of radiation emitted by the radionuclides, the dose rate is time-dependent. Of critical importance for the maintenance of the accelerator and the minimization of down time is the dose rate from activated accelerator components within a day or two following an accelerator run of reasonable duration. After short irradiation times, the induced activity in the system is proportional to the number of interacting protons, and the activity level decreases inversely with time. 19 "Hands-on" maintenance of the machine requires careful design attention to minimize the loss rate of protons in the various machine components.

Sullivan ${ }^{19}$ gives an expression for estimating the order of magnitude of dose rates produced by induced activity along a beam line or accelerator component. The majority of the activity remains inside the machine and is shielded by the iron in the magnets. Sullivan assumes around $10 \%$ of the activity actually contributes to the dose rate. On this basis, the dose rate (in units of $\mu \mathrm{Sv} / \mathrm{h}$ ) at $50 \mathrm{~cm}$ from the accelerator components, for protons of energy $E_{o} \mathrm{GeV}, t$ days after the beam is switched off, following beam loss of $1 \mathrm{~W} / \mathrm{m}$ for a period of $T$ days, is approximately

$$
\dot{\mathrm{H}}=30 \mathrm{E}_{\mathrm{o}}^{-0.08} 1 \mathrm{n}\left[\frac{\mathrm{T}+\mathrm{t}}{\mathrm{t}}\right] \quad \mu \mathrm{Sv} / \mathrm{h}
$$

The above expression was used to estimate dose rates from accelerator components, using the assumed loss rates in the various IPNS Upgrade accelerator systems and taking $T=30$ days and $t=1$ day. These dose rates are from $140-220 \mu \mathrm{Sv} / \mathrm{h}$ in the linac and LET, and $290 \mu \mathrm{Sv} / \mathrm{h}$ in the RCS. The dose rates are in the range that allows hands-on maintenance by workers without excessive down-time delays and without high radiation doses.

\subsection{REFERENCES}

1. U. S. Department of Energy, Radiological Control Manual, DOE/EH 0256T Revision 1 (April 1994).

2. U. S. Department of Energy, "Radiation Protection of the Public and the Environment," DOE Order 5400.5 (Feb. 8, 1990).

3. K. Tesch and J. M. Zazula, "Shielding properties of iron at high energy proton accelerators studied by a Monte Carlo code," Nucl. Inst. Meth. A300, 179-187 (1991).

4. J. B. McCaslin et al., "Moyer model approximations for point and extended beam losses," Nucl. Inst. Meth. A256, 418-426 (1987).

5. A. H. Sullivan, "The intensity distribution of secondary particles produced in high energy proton interactions," Rad. Prot. Dos. 27( 3), 189-192 (1989). 
6. R. H. Thomas and G. R. Stevenson, Radiological Safety Aspects of the Operation of Proton Accelerators, IAEA Technical Report Series No. 283, IAEA, Vienna, Austria (1988).

7. K. Tesch and J. M. Zazula, "Study of the neutron field from a hadronic cascade in iron: verification of a Monte Carlo calculational model by comparison with measured data," Nucl. Inst. Meth. A300, 164-178 (1991).

8. G. Stevenson et al., "Determination of transverse shielding for proton accelerators using the Moyer model," Health Phys. 43(1), 13-29 (1982).

9. $\mathrm{K}$. Tesch and H. Dinter, "Estimation of radiation fields at high energy proton accelerators," Rad. Prot. Dos. 15(2), 89-107 (1986).

10. H. Dinter et al., "Shielding of proton accelerators: a comparison between the Moyer model and Monte Carlo calculations," Nucl. Inst. Meth. A276, 1-7 (1989).

11. K. Tesch, "A simple estimation of the lateral shielding for proton accelerators in the energy range 50 to $1000 \mathrm{MeV}$," Rad. Prot. Dos. 11(3), 165-172 (1985).

12. G. R. Stevenson and R. H. Thomas, "A simple procedure for the estimation of neutron skyshine from proton accelerators," Health Phys. 46(1), 115-122 (1984).

13. J. T. Routti and M. H. Van de Voorde, "Dose estimates outside the shielding of the CERN intersecting storage rings by the Moyer method," Nucl. Eng. \& Des. 21, $421-434$ (1972).

14. J. M. Zazula and $\mathrm{K}$. Tesch, "Analysis of the transverse shielding problem at proton accelerators using a hadronic cascade code with low energy particle modules," Nucl. Inst. Meth. A286, 279-294 (1990).

15. H. Dinter and K. Tesch, "Determination of neutron spectra behind lateral shielding of high energy proton accelerators," Rad. Prot. Dos. 42(1), 5-10 (1992).

16. J.M. Zazula et al., "Sky- and groundshine phenomena and related radiological quantities evaluated for the environment of a high-current spallation facility," Part. Accel. 21, 28-42 (1987).

17. B. J. Moyer, "Method of calculation of the shielding enclosure for the Berkeley bevatron", presented at 1st International Conference on Shielding around High Energy Accelerators, Presses Universitaires de France, Paris 65 (1962).

18. Y. Chae, "Study of field ionization in $\mathrm{H}^{-}$charge exchange injection for the proposed neutron spallation source at Argonne," internal Neutron Source note, NSA-94-2, Argonne National Laboratory (1994). 
19. A. H. Sullivan, A Guide to Radiation and Radioactivity Levels Near High Energy Particle Accelerators, Nuclear Technology Publishing, Ashford, Kent, England (1992). 


\subsection{INTRODUCTION}

The IPNS Upgrade is a 2-GeV proton accelerator with two neutron-generating targets for condensed-matter research. The accelerator system consists of a linear accelerator section, a linac-to-RCS transfer line, the RCS, and two extraction lines to the target stations. The RCS is housed in the already existing ZGS Ring Building. Approximately 350 to 400 beam components require positioning to critical alignment tolerances. To accomplish this task efficiently and economically, state-of-the-art survey equipment and techniques are employed. For the most part, existing APS alignment equipment is utilized, resulting in significant cost savings for this project.

\subsection{GEODETIC REFERENCE SYSTEM}

The absolute positioning of the IPNS Upgrade components largely depends on the accuracy with which the position of the control network can be determined. Four preexisting, intervisible, and very stable alignment monuments, $\mathrm{P} 1-\mathrm{P} 4$, are used to establish the primary reference within the ZGS Ring Building, shown in Figure II.10.2-1. When electronic distance measurement (EDM) systems and electronic theodolites are used, an accuracy of better than $\pm 0.5 \mathrm{~mm}$ can be achieved for these control points. ${ }^{1}$ To ensure the proper location of the linac and the beam transfer line from the linac to the RCS, a gyro-theodolite is used to establish a homogeneous coordinate system for all accelerator subsystems, eliminating the need for the installation of a costly surface control network.

\subsection{ALIGNMENT TOLERANCES}

To obtain a smooth particle beam path, the vertical and radial deviations from the ideal position of one beam component to the next must be minimized. The relative alignment accuracy required to position the RCS quadrupoles and sextupoles is $\pm 0.2 \mathrm{~mm}$ in the vertical and radial positions. In the longitudinal direction, components have to be placed to within $\pm 0.5 \mathrm{~mm}$. The dipoles have to be set to $\pm 1 \mathrm{mrad}$ of roll. On a global scale, the position of each RCS beam element should not deviate from the ideal location by more than $\pm 2 \mathrm{~mm}$. The deviation of the circumference of the RCS is expected to be less than $\pm 12.6 \mathrm{~mm}$. Similar positioning tolerances have been specified and actually achieved for the alignment of the APS booster beam components.

\subsection{ALIGNMENT CONCEPT}

The alignment concept is based on the definition of a common coordinate system. The task is to determine the coordinates of the control network points and magnet fiducials by using redundant measurements and statistical analysis tools for blunder detection. ${ }^{2}$ To guarantee repeatability of the control points, the centering system used is the same as that used for the 


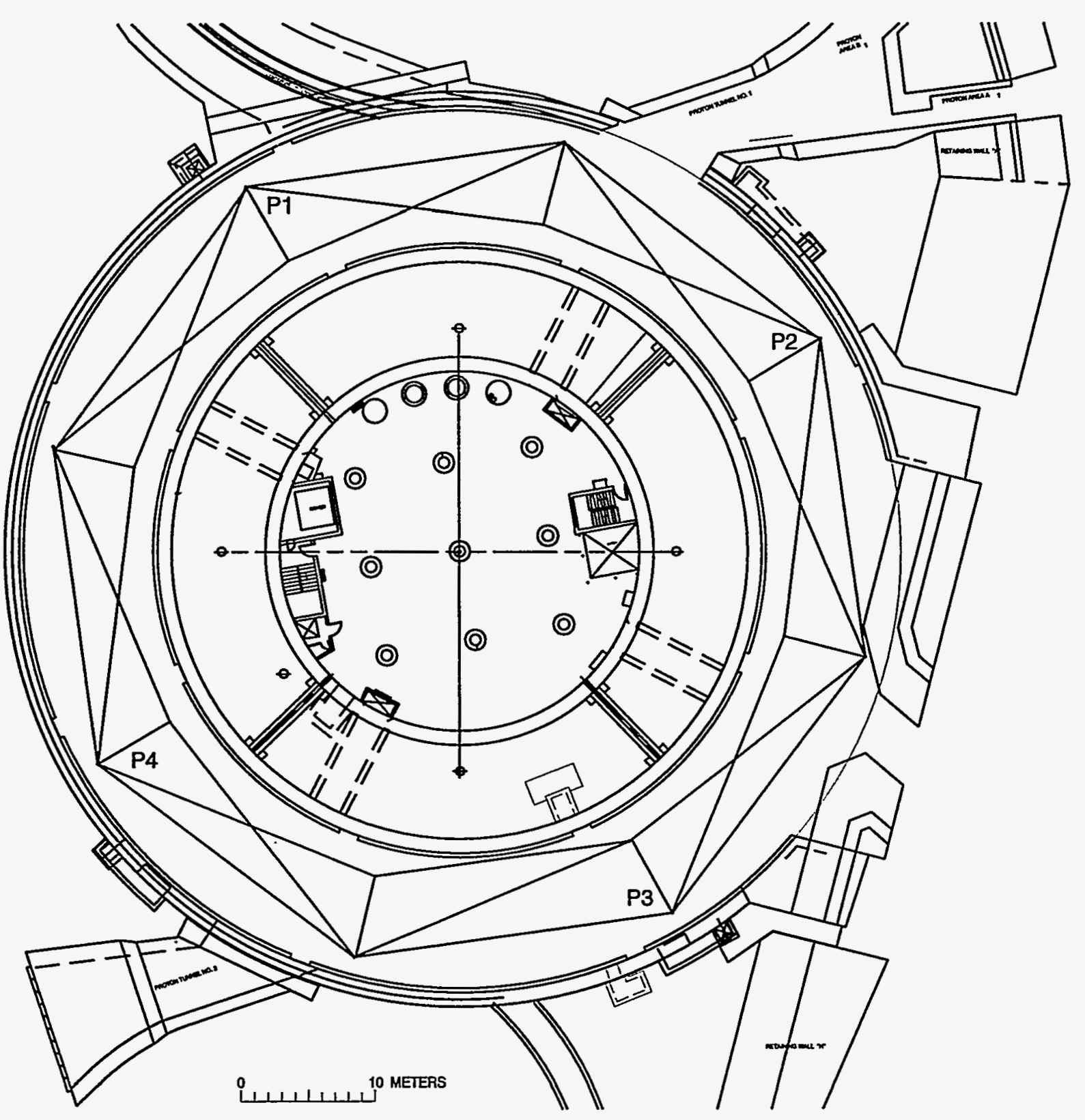

Figure II.10.2-1 Example of an RCS Survey and Alignment Control Network Design. 
alignment of the APS. This system is based on a nearly perfect fit between a conical surface and a sphere, as shown in Figure II.10.4-1. It provides the means for determining the threedimensional coordinates of each control point. Appropriate adapters to use this system have been designed and used for the APS project, as depicted in Figures II.10.4-2 and II.10.4-3.

Secondary control points are connected to the primary control network and are used to position the magnet support system and all beam components. The symmetrical lattice structure of the RCS ring lends itself to the use of templates for the purpose of laying out the beam component supports. The coordinates of the secondary control points are determined by means of a laser tracking system.

The ideal position of each beam component in the global coordinate system is calculated by using the information on each magnet fiducial cup location that was gathered during the magnet mapping process. With the use of this information and the laser tracking system, which provides data on the actual measured position of each beam component with respect to the control network, all beam elements are positioned. ${ }^{3}$ The magnet location resulting from this step is only as good as the accuracy of the control network. Considering the possible accumulation of instrumental ${ }^{4}$ and procedural errors, the accuracy of the control network for positioning beam components can only be viewed as an upper limit that, in reality, can never be reached. Therefore, a final alignment step is required. ${ }^{5}$

The final alignment step abandons the control network information for determining the position of beam elements. It serves two purposes. The first purpose is to check for blunders that may have been introduced during the previous positioning phase. The second is to ensure the quality of the alignment efforts. As a result, the radial and vertical deviations of each beam component from a smooth curve are calculated, ${ }^{6}$ thus providing a quantitative assessment of the achieved tolerances. ${ }^{3}$

The initial alignment of all RCS beam components and support structures is closely tied to the component installation schedule. The time frame for the installation of all technical RCS components is six months. The survey and alignment tasks to support this rapid installation schedule can easily be accomplished by using state-of-the-art equipment and procedures.

It is estimated that the realignment of all RCS beam elements, when necessary, can be done in three to five weeks.

\subsection{CONTROL NETWORK SIMULATIONS AND DATA HANDLING}

Before the installation of the control network takes place, simulations are performed to find the optimal network design that meets the constraints given by the ZGS Ring Building while obtaining the required control network accuracy with the least number of measurements. ${ }^{7}$ The results of such a simulation are shown in Figure II.10.5-1. It shows the estimated accuracy in the form of error ellipses around the control points for a given network geometry and observation plan. The major axes of the largest error ellipses depicted are on the order of $0.3 \mathrm{~mm}$. 


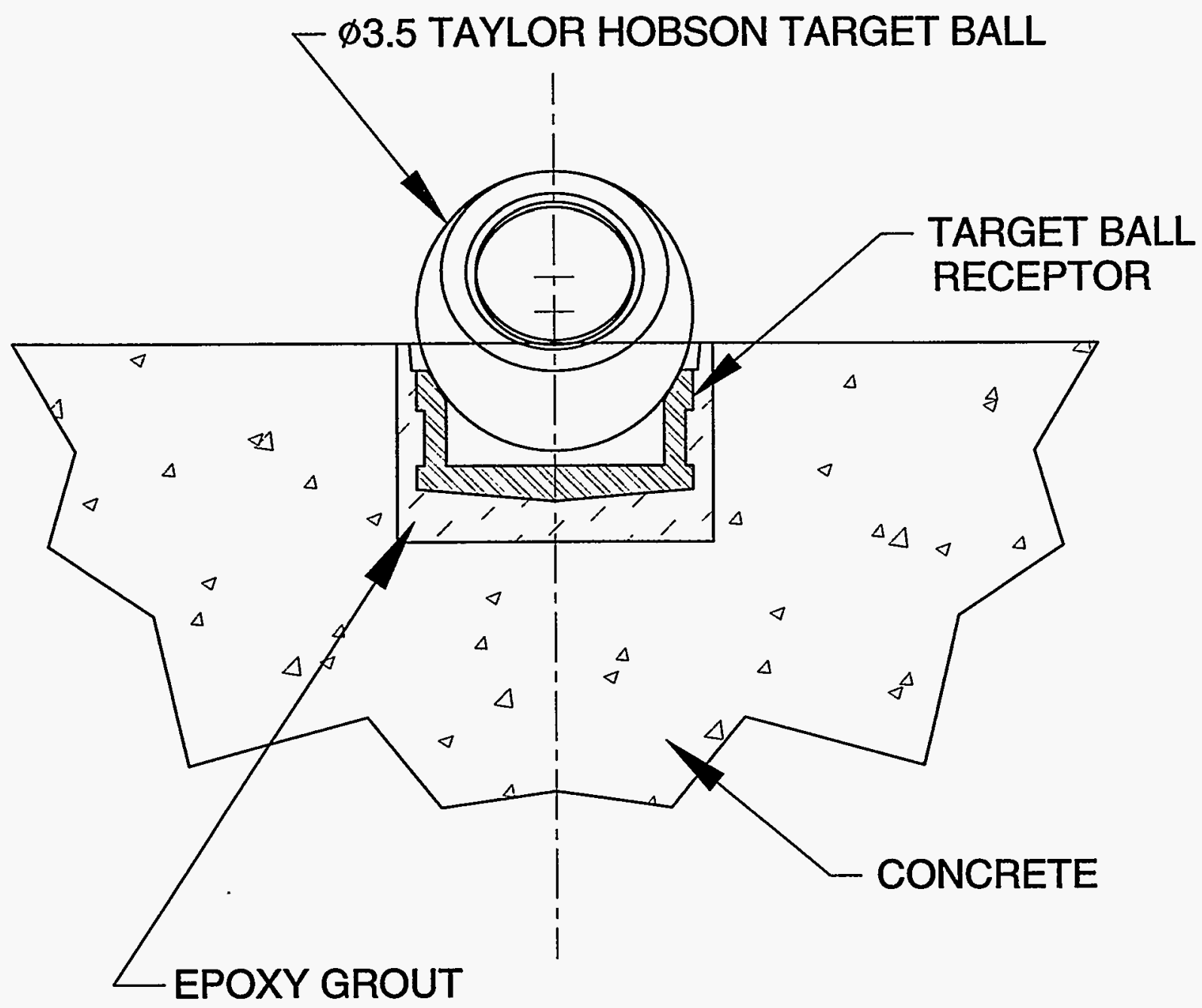

Figure II.10.4-1 Target Ball Receptor at the Control Point. 

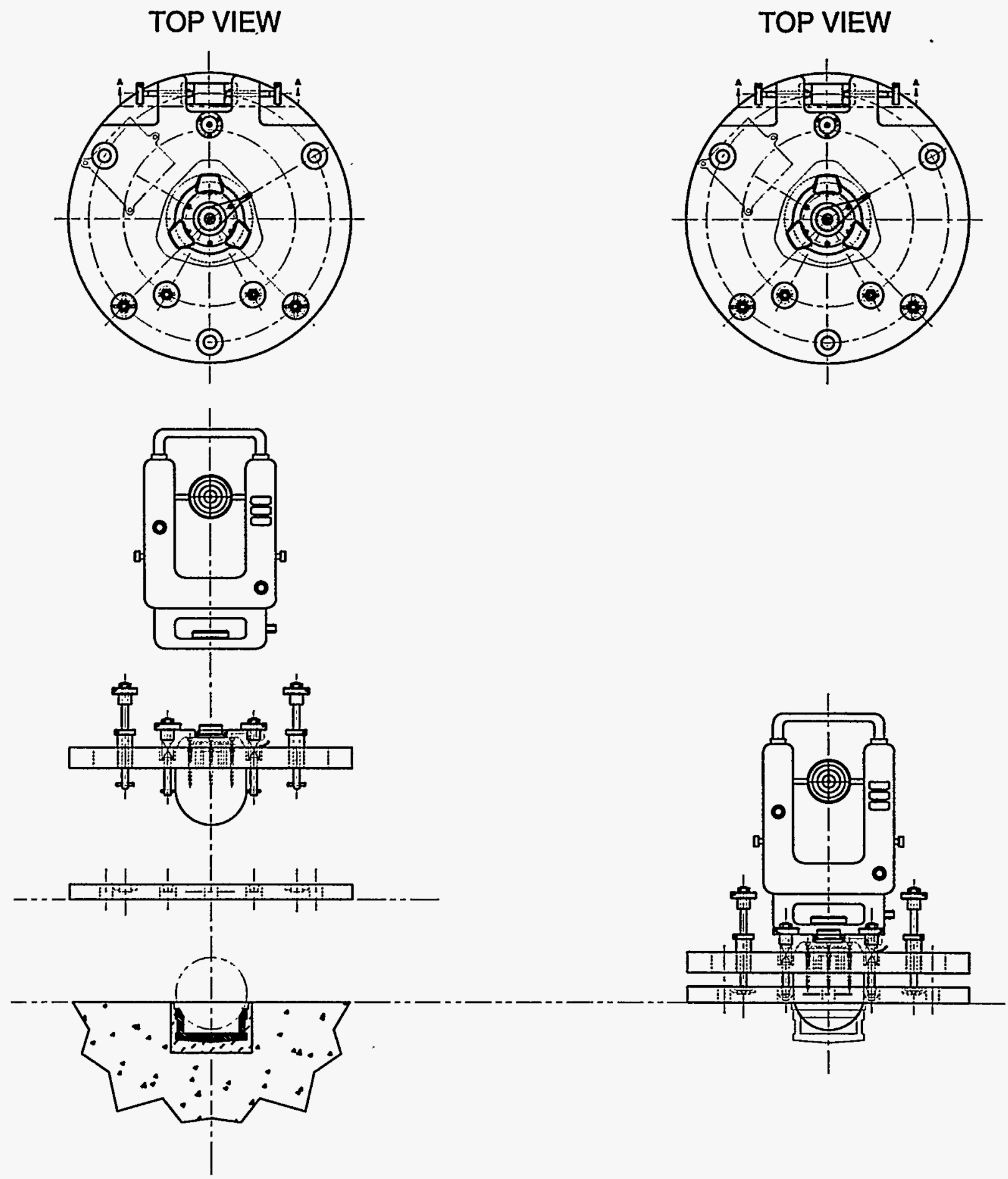

Figure II.10.4-2 Explosion (left) and Assembly (right) Views of Floor Mounting Instrument Adapter. 

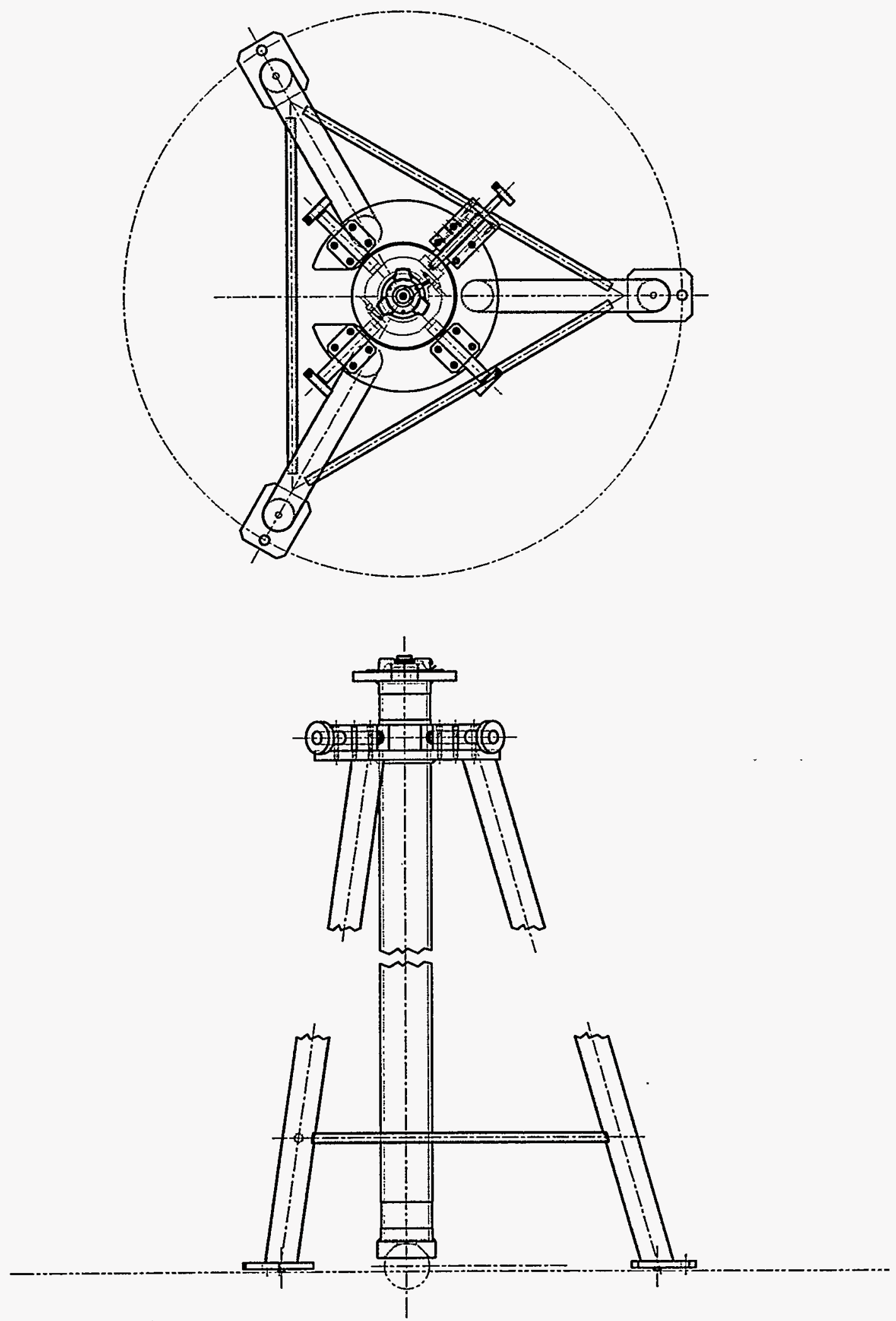

Figure II.10.4-3 Monopod Instrument Adapter, Extending the Floor Reference Point to a Height of $1.4 \mathrm{~m}$. 


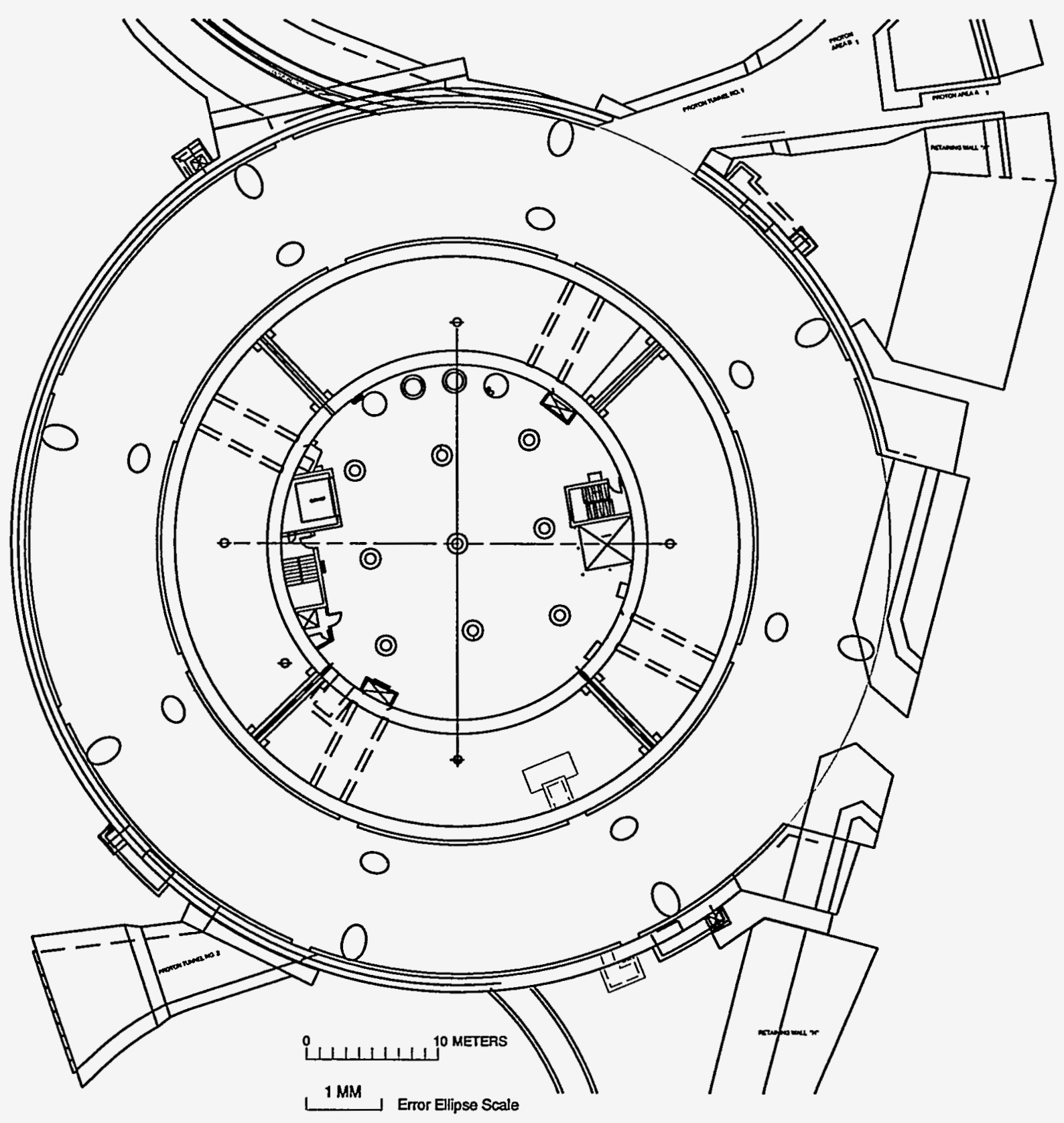

Figure II.10.5-1 Error Ellipses of an RCS Survey Network Design Simulation. (Ellipses are shown at a different magnification than the rest of the drawing.) 
Throughout all the alignment efforts, automated data flow is used. This not only improves the alignment productivity in terms of output, but also results in fewer errors when measurement data are recorded. Laptop computers are connected to electronic theodolites, electronic distance measuring systems, and automatic levels. ${ }^{8}$ The data are analyzed on a personal computer connected to the local area network. The resulting coordinates from this process are then used to supply the laser tracker system with information for beam element positioning.

\subsection{INJECTOR ALIGNMENT}

Both the linear accelerator section and the low-energy transfer line between the linac and the RCS are long straight layouts. Such layouts are ideally suited for common optical tooling techniques in conjunction with an established secondary control network. To extend the RCS control network into the linac, a $250-\mathrm{m}$-long traverse is required. To ensure the proper direction of the linac with respect to the transfer line and the proper location of the transfer line to the RCS, observations with a gyro-theodolite are necessary to support such a long open-ended traverse.

\subsection{REFERENCES}

1. H. Friedsam, J. Penicka, and S. Zhao, Survey and Alignment Report on the Primary Control Network for the APS, Light Source Note LS-220 (1993).

2. H. Friedsam, "The alignment of the Advanced Photon Source at Argonne National Laboratory," in Proceedings of the Third International Workshop on Accelerator Alignment (Annecy, France, 1993).

3. H. Friedsam, "A new accelerator alignment concept using laser trackers," in Proceedings of the Fourth European Particle Accelerator Conference (London, England, 1994).

4. H. Friedsam and J. Penicka, "A new method to position the APS dipoles with the use of a laser tracker," in Proceedings of the Fourth European Particle Accelerator Conference (London, England, 1994).

5. H. Friedsam and W. Oren, The Application of the Principal Curve Analysis Technique to Smooth Beamlines (Stanford Linear Accelerator Center, Stanford, California, 1991).

6. T. Hastie, Principal Curves and Surfaces, SLAC Report No. 276 (Stanford Linear Accelerator Center, Stanford, California, 1984).

7. I. Burstedde, Adjustments of Geodetic Networks at SLAC (1983).

8. H. Friedsam, Survey and Alignment Workshop on Data Processing Using GEONET for Accelerator Alignment (Stanford Linear Accelerator Center, Stanford, California, 1992). 
III

Injector System 
A $400-\mathrm{MeV} \mathrm{H}^{-}$linac capable of delivering a time-averaged current of $0.5 \mathrm{~mA}$ is required for the IPNS Upgrade injector. The linac system was designed by AccSys Technology, Inc. The design relies heavily on those of previous linac systems, including the injector linac for the Superconducting Super Collider (SSC), to obtain the most practical system with proven technology. Existing, available linac structure designs were used.

This linac design is shown schematically in Figure III.1-1. The linac is $120 \mathrm{~m}$ long and can accelerate an $\mathrm{H}^{-}$beam of $50-\mathrm{mA}$ peak current at a duty factor up to $1.5 \%$. The total peak radiofrequency (rf) power required for operation is $104 \mathrm{MW}$; average input power is less than 4.85 MW. The design is optimized to achieve the lowest capital cost available, consistent with routinely achievable accelerating gradients and the lowest attainable operating costs.

The $\mathrm{H}^{-}$linac has the following accelerator components:

- $\mathrm{H}^{-}$ion injector - A cesium-enhanced volume ion source produces a 50-mA $\mathrm{H}^{-}$beam at an energy of $35 \mathrm{keV}$. Alternatively, the ISIS $\mathrm{H}^{-}$source can be used without further development. ${ }^{1}$ An electrostatic deflector driven by a field effect transistor (FET) and located at the output of the ion injector electrode stack permits beam chopping at duty factors up to $75 \%$.

- Low-energy beam transport (LEBT) - The $35-\mathrm{keV} \mathrm{H}^{-}$beam from the ion injector is matched into the RFQ linac by using two large-bore solenoid lenses.

- Radio-frequency quadrupole (RFQ) - A conventional 4-vane $R F Q$, operating at $425 \mathrm{MHz}$, bunches and accelerates the $35-\mathrm{keV} \mathrm{H}^{-}$ion beam up to $2.0 \mathrm{MeV}$, while focusing the 50-mA pulsed current.

- Ramped-gradient drift tube linac (RGDTL) - A short section of a conventional DTL, also operating at $425 \mathrm{MHz}$, is close-coupled to the RFQ. The fields in it are ramped to match the beam from the RFQ into the DTL. The RGDTL accelerates the bunched 2-MeV beam from the RFQ up to 4.2 MeV for injection into the DTL.

- Drift tube linac (DTL) - A standard drift tube linac, also operating at $425 \mathrm{MHz}$, accelerates the $\mathrm{H}^{-}$beam up to $70 \mathrm{MeV}$. This 20-m-long DTL consists of three tank sections, each driven by a 4-MW klystron rf system.

- Matching section (MS) - The 70-MeV H${ }^{-}$beam from the DTL is matched into the coupled cavity linac by using a 3.2-m-long beam transport system containing two buncher tanks and ten quadrupole magnets. 


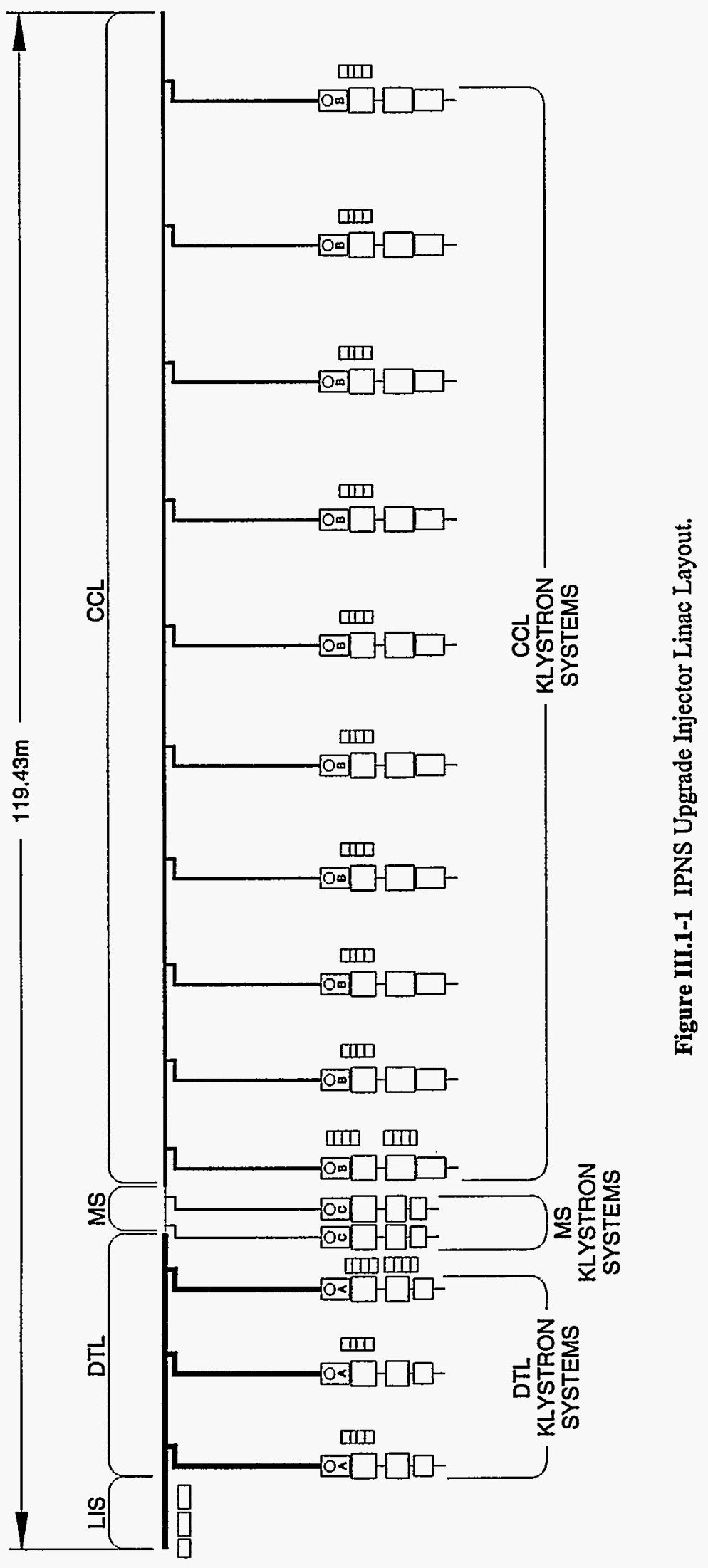


- Coupled cavity linac (CCL) - The final stage of the system is a conventional side-coupled linac (SCL) operating at 1,275 MHz. This 92-m-long structure contains 10 accelerating modules, each consisting of six short tanks connected by conventional bridge couplers, and each driven at the middle bridge coupler by a 9-MW klystron if system.

This chapter describes the background and scope of the physics design and provides a technical description of the subsystems listed above. 
III. $1-4$ 
The IPNS Upgrade requires a $400-\mathrm{MeV} \mathrm{H}^{-}$ion linac that is capable of delivering a $35-\mathrm{mA}$, unchopped peak beam current or a $44-\mathrm{mA}, 25 \%$ chopped beam current in a $500-\mu \mathrm{sec}$ pulse at a $30-\mathrm{Hz}$ repetition rate. ${ }^{1}$ The IPNS Upgrade injector linac performance requirements are shown in Table III.2-1.

The linac design is based on existing subsystems and designs from Los Alamos National Laboratory (LANL), Fermilab, and the SSC. Where possible, existing, proven components are used. The entire $\mathrm{H}^{-}$injector linac can be commercially procured. 
Table III.2-1 IPNS Upgrade Injector Linac Performance Requirements

\begin{tabular}{|c|c|c|}
\hline Parameters & Values & Units \\
\hline Accelerated ion & $\mathrm{H}$ & - \\
\hline Output kinetic energy & 400 & $\mathrm{MeV}$ \\
\hline Pulsed beam currenta & 35 & $\mathrm{~mA}$ \\
\hline Beam pulse length & 500 & $\mu \sec$ \\
\hline Beam repetition rate & 30 & $\mathrm{~Hz}$ \\
\hline Time-averaged current & 500 & $\mu \mathrm{A}$ \\
\hline Radio-frequency duty factor & 3 & $\%$ \\
\hline $\begin{array}{l}\text { Normalized rms transverse } \\
\text { emittance }^{\mathrm{b}}\end{array}$ & 0.1 & $\pi \mathrm{cm}-\mathrm{mrad}$ \\
\hline Output energy spread (fwhm)c & 2.5 & $\mathrm{MeV}$ \\
\hline
\end{tabular}

a Unchopped; the requirement for $25 \%$ chopped beam is $44 \mathrm{~mA}$.

b $\mathrm{rms}=$ root mean square.

c fwhm $=$ full width at half maximum. 
The IPNS Upgrade injector linac delivers a $400-\mathrm{MeV} \mathrm{H}^{-}$beam with pulse lengths up to $500 \mu \mathrm{sec}$ at a nominal peak current of $35 \mathrm{~mA}$ for injection into the Rapid Cycling Synchrotron (RCS). The required energy spread is less than $2.5 \mathrm{MeV}$ fwhm and the transverse normalized rms emittance is less than $0.1 \pi \mathrm{cm}$-mrad. As shown in Figure III.3-1, the linac consists of an $\mathrm{H}^{-}$ ion source, which includes a beam chopper, LEBT system, 2.0-MeV RFQ, 70-MeV DTL, and $400-\mathrm{MeV}$ CCL. The beam from the RFQ goes directly into the DTL. Matching is achieved in a short ramped-gradient tank section, while a separate matching section is used between the DTL and CCL. The dimensions of these subsystems are indicated in Figure III.3-1. Calculated if power for each subsystem is also noted in the figure.

The physics design of the IPNS Upgrade injector linac was based on several existing designs, including those of the SSC injector linac and the high-current proton linac designed by AccSys for commercial radioisotope production. The $\mathrm{H}^{-}$ion injector design is based on the Lawrence Berkeley Laboratory (LBL) rf-driven $\mathrm{H}^{-}$volume ion source, with its output energy optimized at $35 \mathrm{keV}$ for injection into the RFQ. The RFQ and DTL physics designs are based on the AccSys high-current proton linac and are very similar to the SSC design. This RFQ is designed for up to $50-\mathrm{mA}$ output current to ensure an adequate safety margin for the IPNS Upgrade injector. The 70-MeV DTL provides maximum shunt impedance while maintaining a low beam loss during acceleration. This DTL is shorter than the SSC 70-MeV DTL, primarily because of the much shorter ramped-gradient tank.

The designs of the DTL/CCL matching section and CCL configuration are similar to those of the SSC, but are optimized at a lower accelerating gradient to accommodate the much longer beam pulse and higher duty factor. This was done to minimize capital cost and lower input power consumption. The SSC design was based on if klystrons capable of 20-MW peak power each. The IPNS Upgrade design is based on 9-MW peak power klystrons, the maximum available at a $3 \%$ duty factor.

A summary table of the final physics design parameters for the linac sections is given in Table III.3-1.

End-to-end beam dynamics were calculated for output currents of 38 and $50 \mathrm{~mA}$. The ion source extraction system was designed by using the code SNOW, ${ }^{2}$ which includes space charge effects due to $\mathrm{H}^{-}$ions and the extracted electrons. The output from the ion injector was characterized in two-dimensional phase space, because it is a dc beam. The code TRACE2D ${ }^{3}$ was then used to obtain the LEBT design and to match the beam into the RFQ, as seen in Figure III.3-2. The matched beam was then put into the RFQ, and the code PARMULT ${ }^{4}$ was used to simulate the RFQ. Figure III.3-3 shows the beam size, phase, and energy profiles as the 45-mA input beam traverses the RFQ. The output beam particles from the RFQ are shown in Figure III.3.3-4. A total of 720 input particles was used. The output was used as input to the code PARMILA ${ }^{5}$ to simulate the beam dynamics in the DTL. Figure III.3-5 shows the beam size, phase and energy profiles calculated through the DTL, and Figure III.3-6 gives the output phase space projection at $70 \mathrm{MeV}$. 
Table III.3-1 Summary of IPNS Upgrade Injector Linac Physics Design Parameters

\begin{tabular}{|c|c|c|c|c|c|}
\hline Parameters & RFQ & DTL & MS & $\mathrm{CCL}$ & Units \\
\hline Input energy & 0.035 & 2.0 & 70.0 & 70.0 & $\mathrm{MeV}$ \\
\hline Output energy & 2.0 & 70.0 & 70.0 & 400.0 & $\mathrm{MeV}$ \\
\hline Output currenta & 35.0 & 35.0 & 35.0 & 35.0 & $\mathrm{~mA}$ \\
\hline $\begin{array}{l}\text { Output norm rms transverse } \\
\text { emittanceb }\end{array}$ & 0.0120 & 0.0135 & 0.0151 & 0.0194 & $\pi \mathrm{cm}$-mrad \\
\hline $\begin{array}{l}\text { Operating frequency } \\
\text { Peak surface field }\end{array}$ & $\begin{array}{l}425 \\
36\left(1.8 \times E_{k p}\right)\end{array}$ & $\begin{array}{l}425 \\
28\left(1.4 \times E_{k p}\right)\end{array}$ & $\begin{array}{l}1,275 \\
24\left(0.8 \times E_{k p}\right)\end{array}$ & $\begin{array}{l}1,275 \\
28\left(0.9 \times E_{k p}\right)\end{array}$ & $\begin{array}{l}\mathrm{MHz} \\
\mathrm{MV} / \mathrm{m}\end{array}$ \\
\hline Accelerating gradient, $E_{0} T$ & - & $2.4-4.6$ & - & $5.9-5.5$ & $\mathrm{MV} / \mathrm{m}$ \\
\hline Bore radius & $2.4-3.0$ & $5.0-9.0$ & 10.0 & $10.0-12.0$ & $\mathrm{~mm}$ \\
\hline Synchronous phase & -30 & -30 & - & -30 to -25 & deg \\
\hline Structure power & 0.22 & 6.28 & 1.2 & 52.8 & MW \\
\hline Number of tanks/modules & 1 & 4 & 2 & $6 / 10$ & - \\
\hline Structure length & 2.16 & 20.54 & 3.2 & 92.1 & $\mathrm{~m}$ \\
\hline Beam power at $40 \mathrm{~mA}$ & 0.08 & 2.72 & - & 13.2 & MW \\
\hline
\end{tabular}

a Unchopped; $25 \%$ chopped beam requirement is $44 \mathrm{~mA}$.

b $\mathrm{rms}=$ root mean square. 


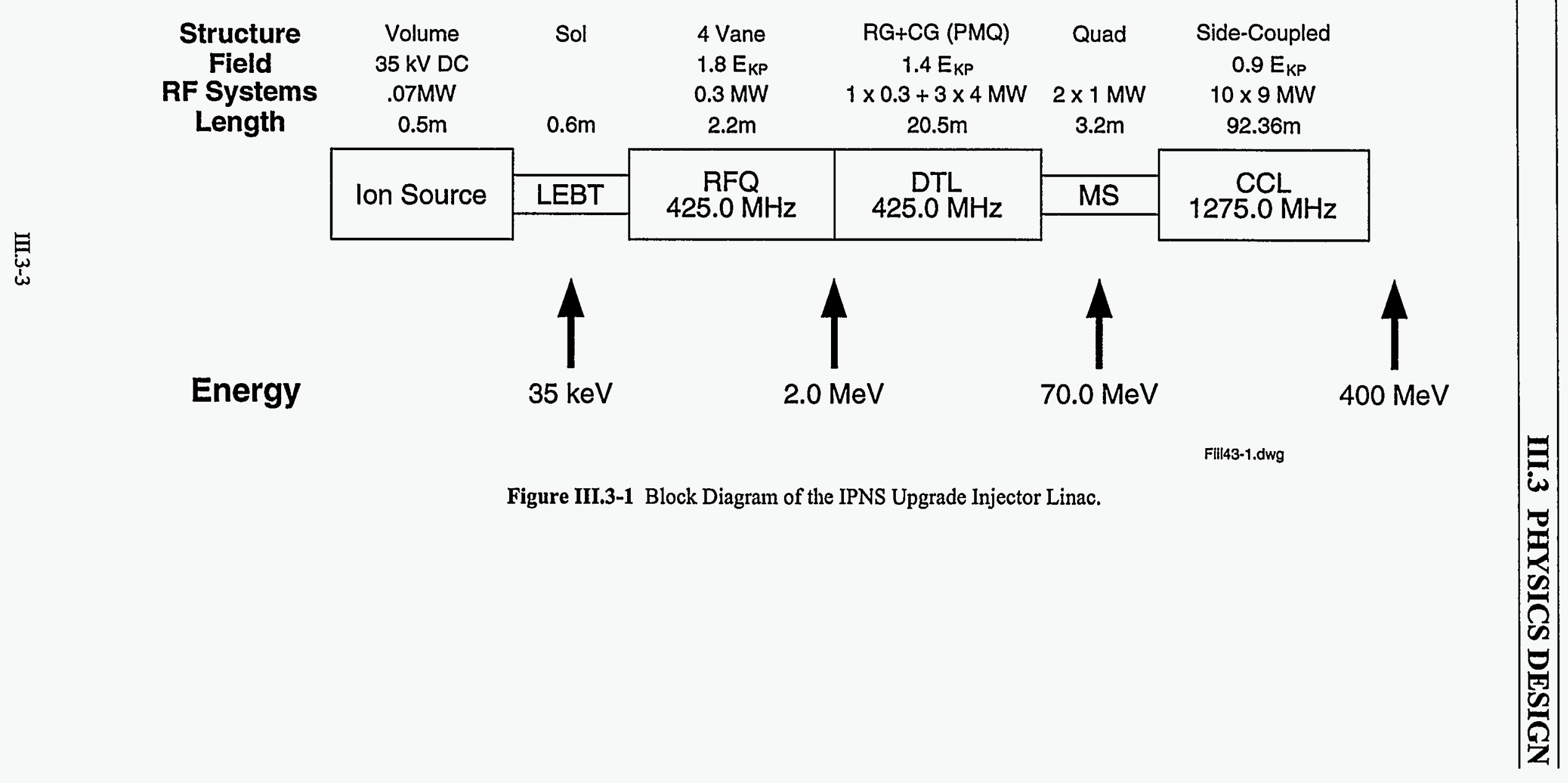




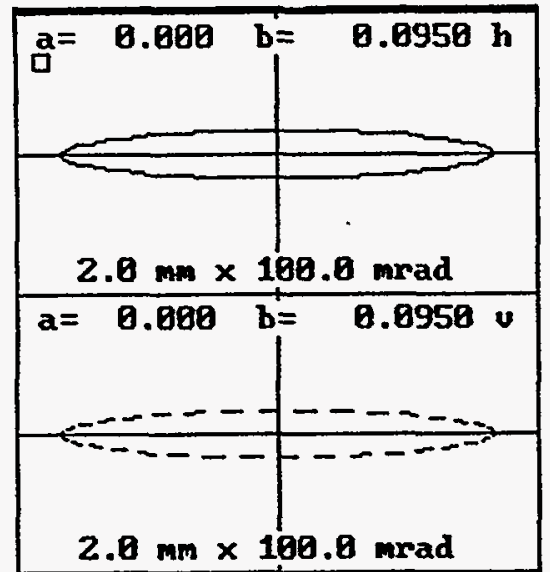

$i=\quad 50.8$
emiti= 30.08
emito= 39.98

$\begin{array}{ll}\text { emito }= & 30.68 \\ w= & 0.035\end{array}$

50.9

30.90

30.00

$$
\begin{array}{rrr}
\text { np } & \text { ne } & \text { value } \\
1 & 1 & 110.098 \\
1 & 2 & 4935.090 \\
1 & 3 & 195.090 \\
1 & 4 & 195.090 \\
1 & 5 & 5313.080 \\
1 & 6 & 66.090
\end{array}
$$
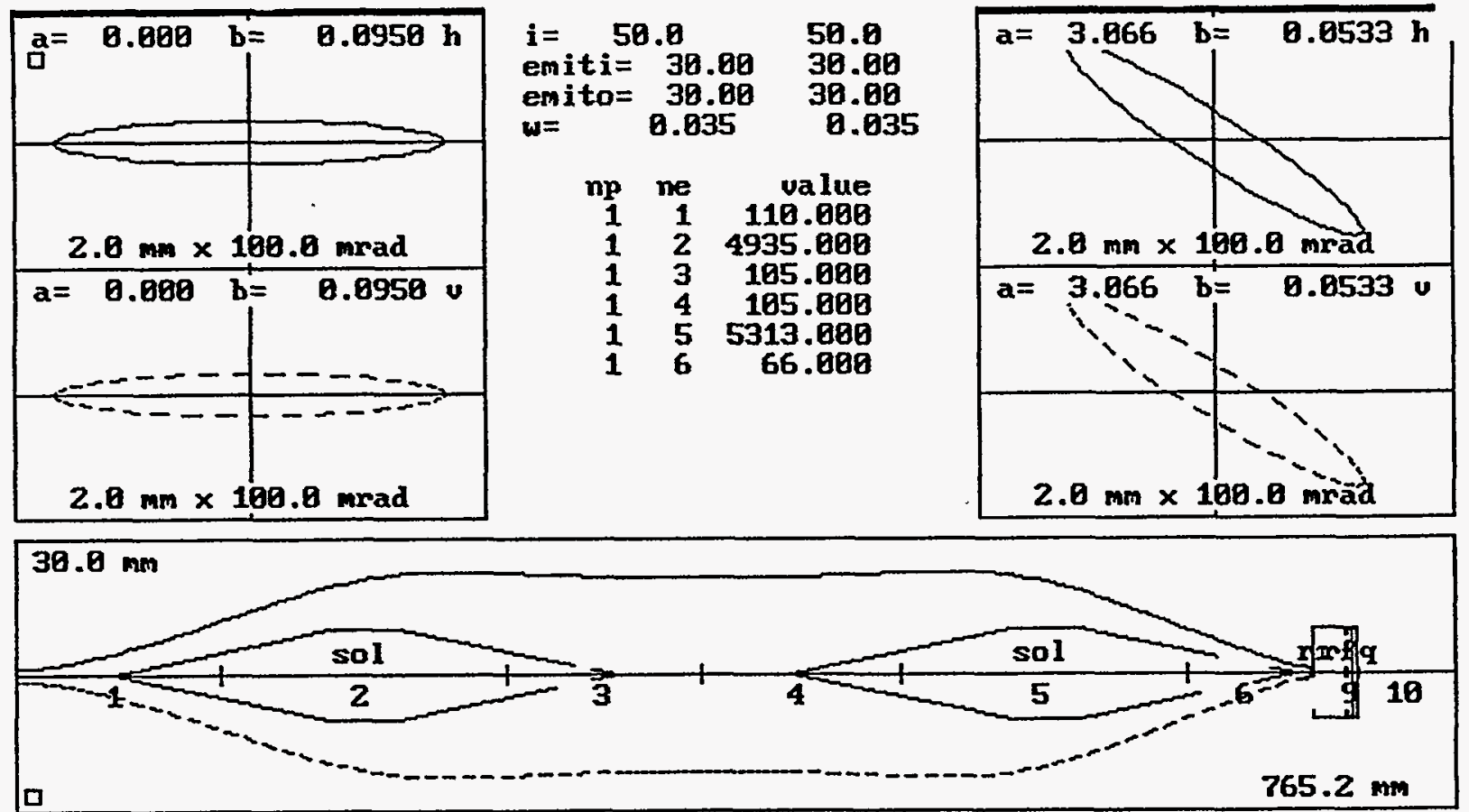

Figure III.3-2 LEBT Optics. 

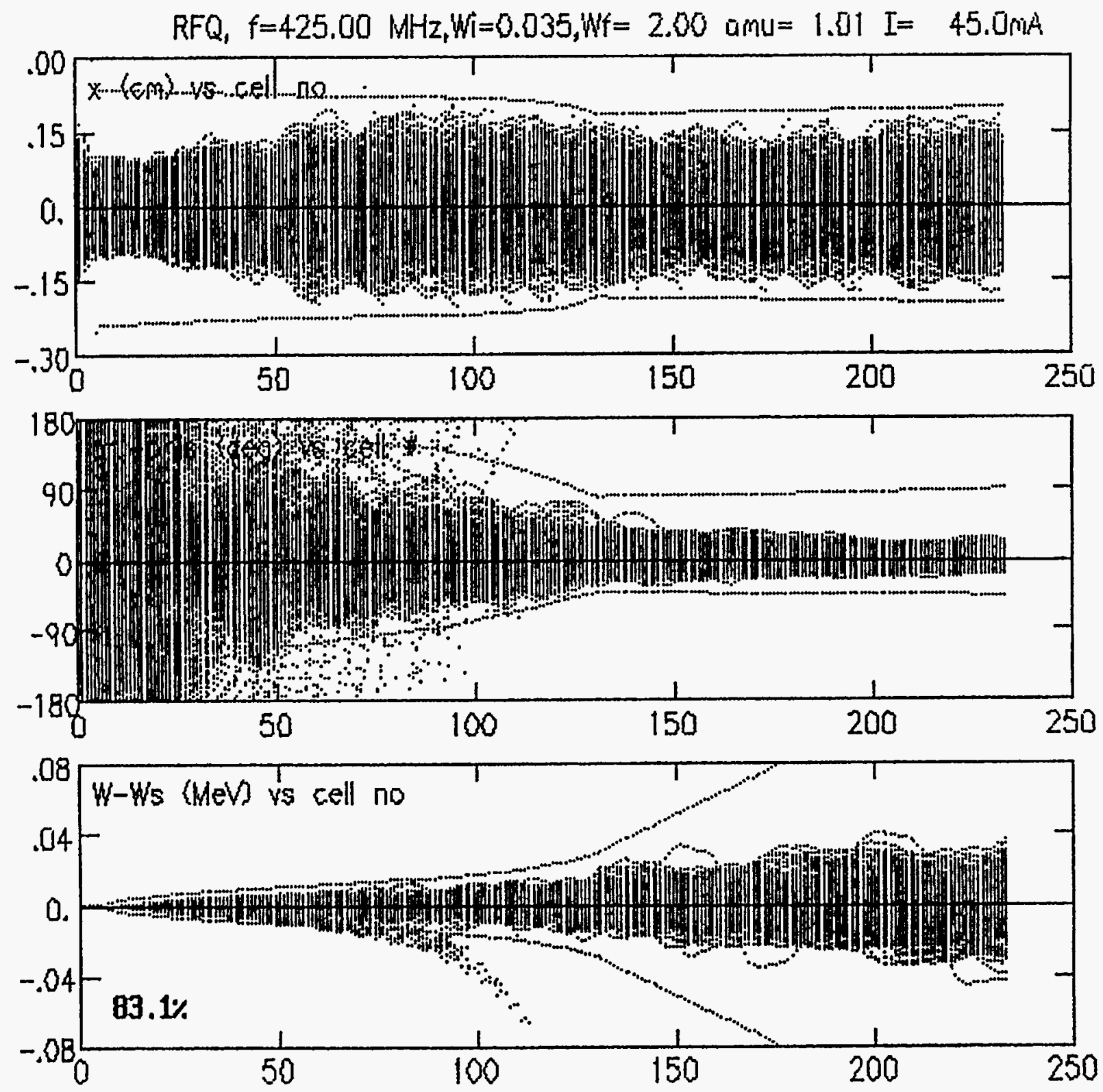

Figure III.3-3 Calculated Beam Dynamics through the RFQ. (Beam size [top], phase [center], and energy profile [bottom] are plotted as the $45-\mathrm{mA}$ beam traverses the RFQ.) 
$R F Q, f=425.00 \mathrm{MHz}, W i=0.035, W f=2.00$ amu $=1.01 \quad I=45.0 \mathrm{~mA}$
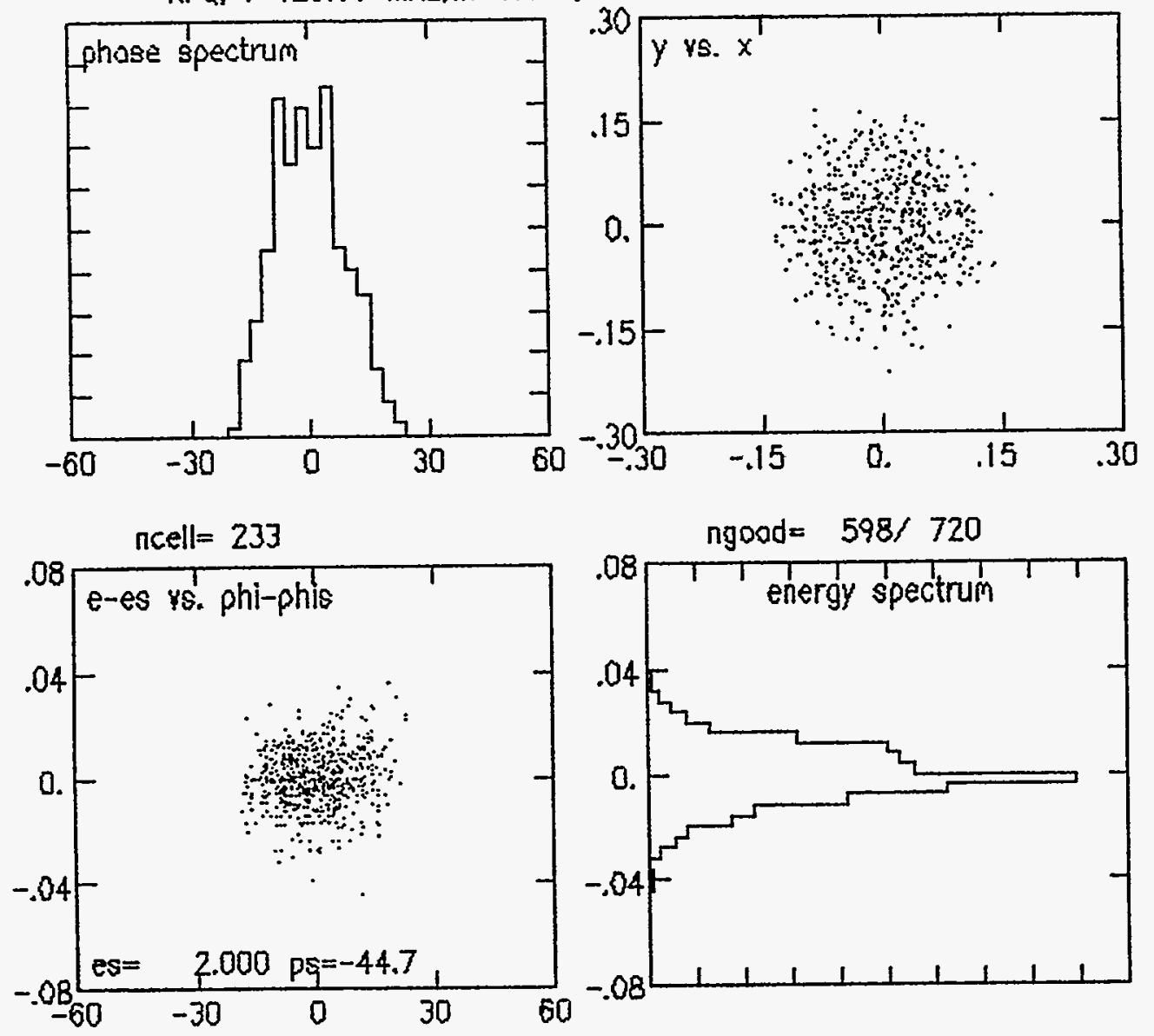

Figure III.3-4 Output Beam Phase Space Projections from the RFQ. 


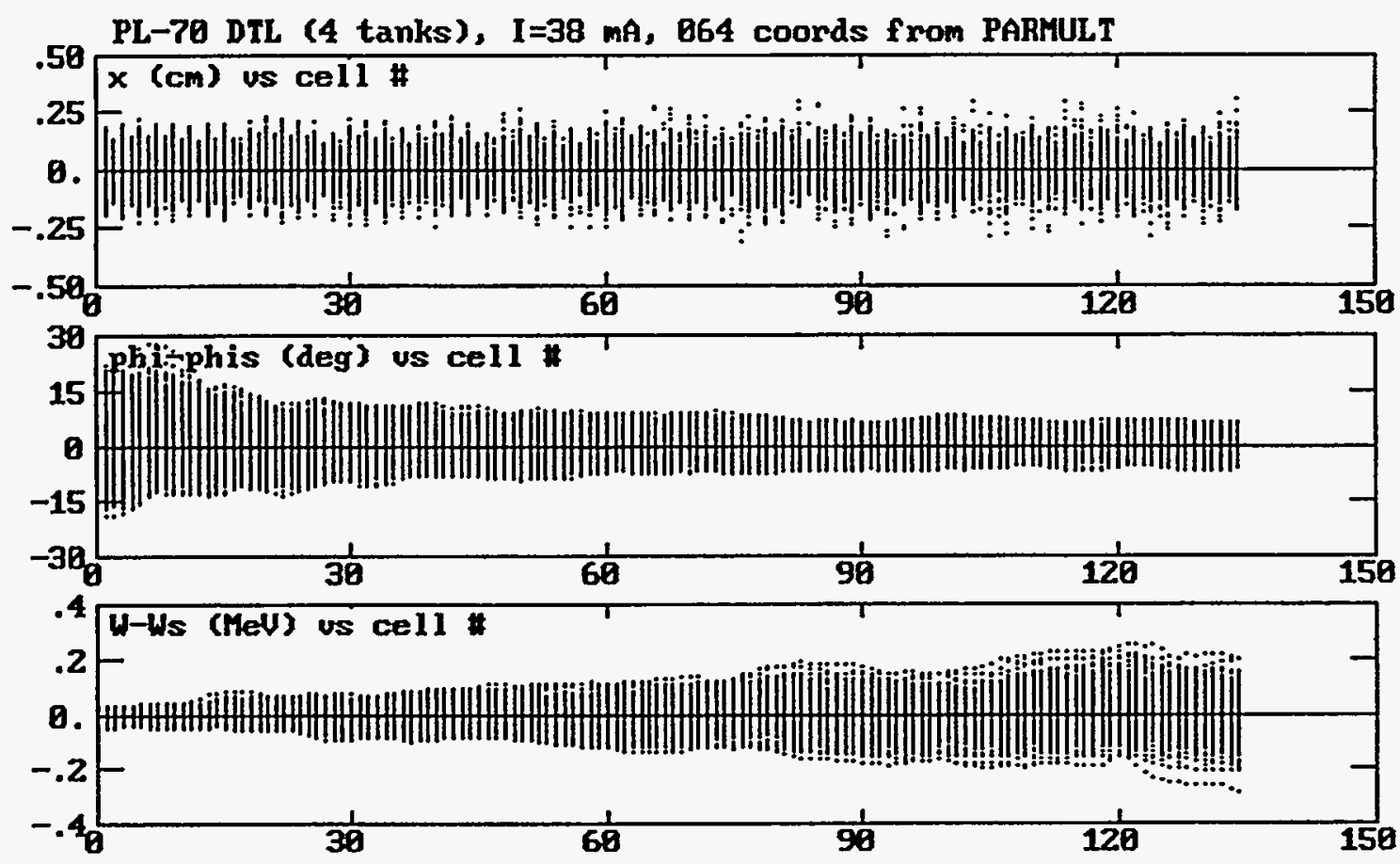

Figure III.3-5 Calculated Beam Dynamics through the DTL. (Calculated size [top], phase [center], and energy profile [bottom] are shown in the DTL.) 
PL-70 DTL (4 tanks), I=38 ma, 864 coords fram PARMULT
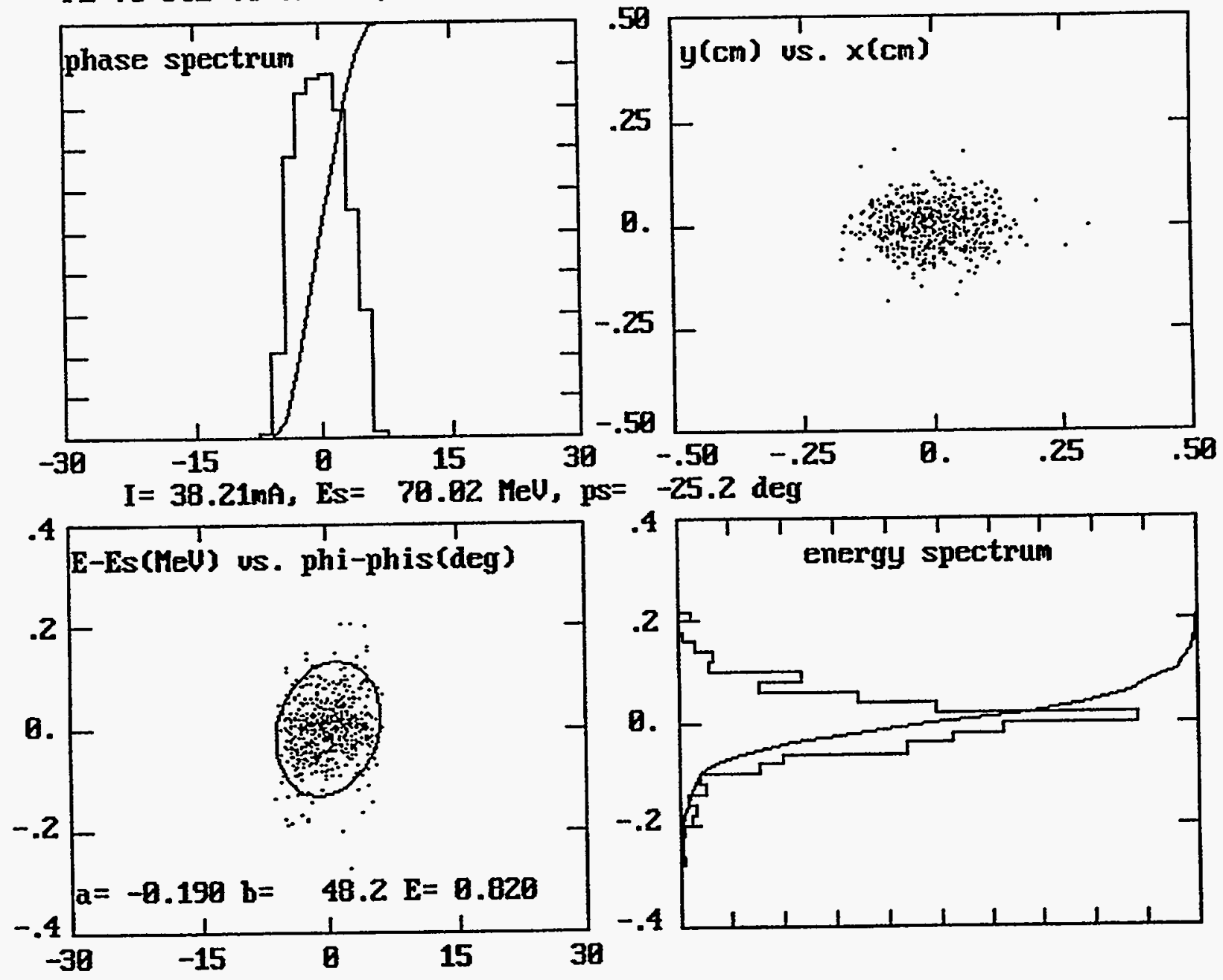

Figure III.3-6 Outpuit Beam Phase Space Projections from the DTL. 
The code TRACE-3D 6 was used with the output beam rms phase space parameters to design the DTL/CCL matching section and to determine the quadrupole magnet strengths and buncher cavity voltages. Figure III.3-7 shows the TRACE-3D beam optics through this matching section. Elements of the transport region downstream from the DTL were input to the code PARMILA to obtain the particle coordinates at the input to the CCL.

The longitudinal coordinates of the original particles that had been injected in the RFQ and followed through the RFQ, DTL, and matching section were redefined with respect to the CCL frequency. Then the particles were followed through the CCL by using CCLDYN.7 Figure III.3-8 shows the beam dynamics through the CCL. Figure III.3-9 shows the projected six-dimensional output phase space, from which the final transverse emittance in each plane and the final energy spread can be estimated.

The baseline design of the 400-MeV IPNS Upgrade injector linac provides the 33- $\mathrm{mA} \mathrm{H} \mathrm{H}^{-}$ output current required for injection into the RCS. The calculated rms transverse emittance is approximately $0.02 \pi \mathrm{cm}$-mrad, which is sufficiently below the required value of $0.1 \pi \mathrm{cm}$-mrad to leave adequate margin for realistic fabrication tolerances. The energy spread provides for a factor-of-two dilution and is not as sensitive to mechanical tolerances. 
BEAM AT NEL1- 12

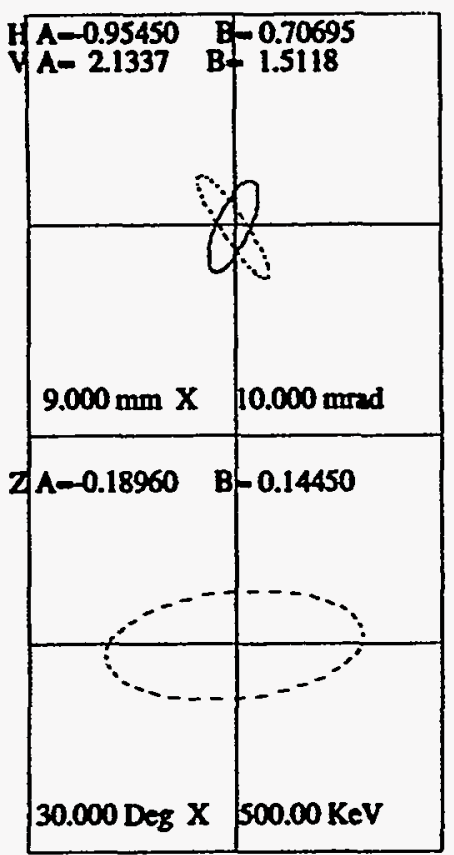

I- $114.6 \mathrm{~mA}$ $W=70.000070 .0000 \mathrm{MeV} \quad$ A- 0.44487 E -9.5938 FREO-1275.00MHz WI-235.13mm A-3.25602E-07 B- 1.7833 EMII- $1.77 \quad 1.652460 .00$ EMITO- $1.77 \quad 1.652460 .17$ N1- 12 N2- 35

PRINTOUT VALUES PPPE VALUE

116150.6578

$217-90.0000$

118150.6578

I 30196.9220

231.900000

132196.9220

MATCHING TYPE -9 DESIRED VALUES (BEAMF) alph beta

$\begin{array}{rrr}0.4451 & 9.5965 \\ y & -0.0327 & 1.7834\end{array}$

$\begin{array}{lll}0.1100 & 0.0389\end{array}$

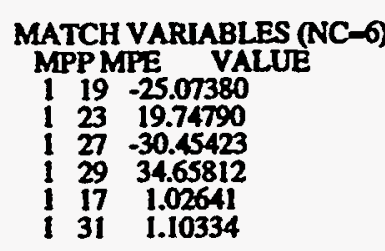

BEAM AT NE12- 35

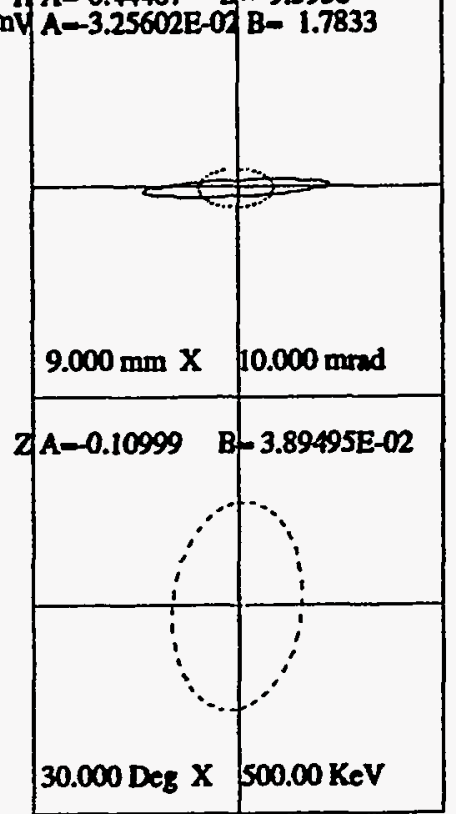

NP2 -44

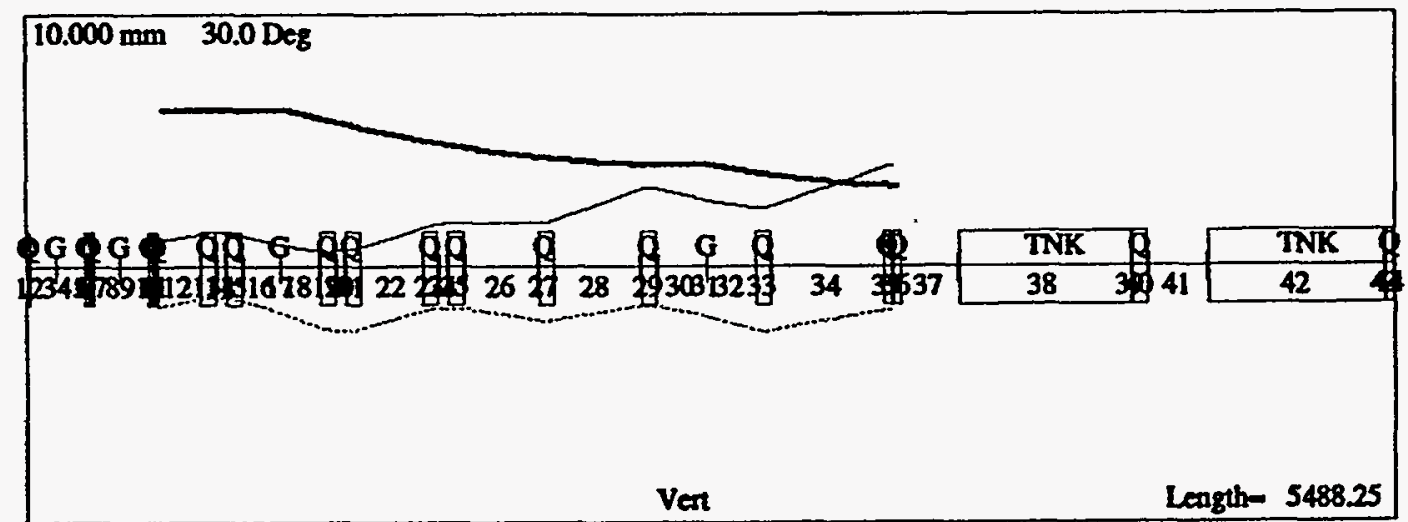

Figure III.3-7 Calculated Beam Optics through the DTL/CCL Matching Section. 


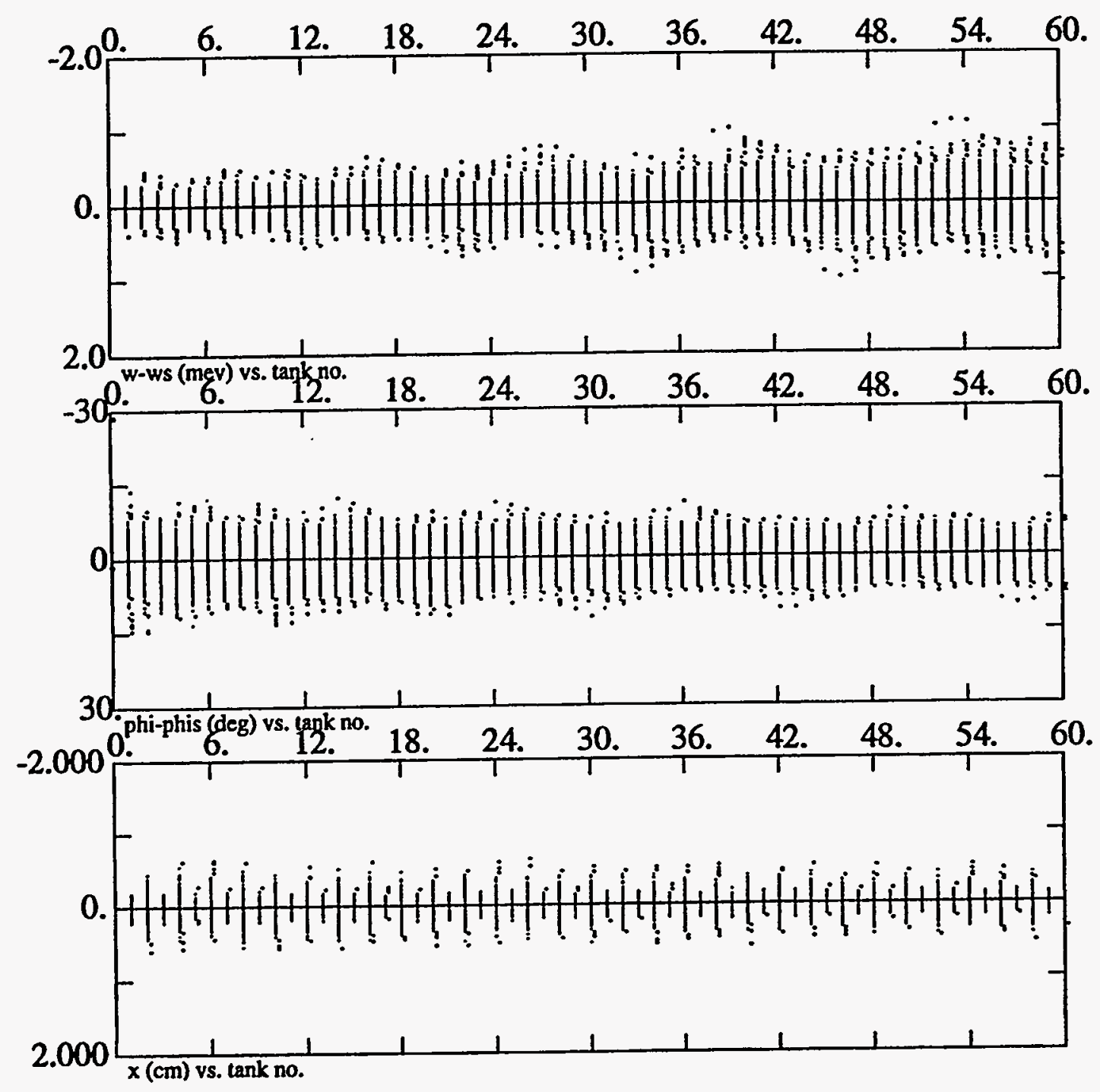

Figure III.3-8 Calculated Beam Dynamics through the CCL. 

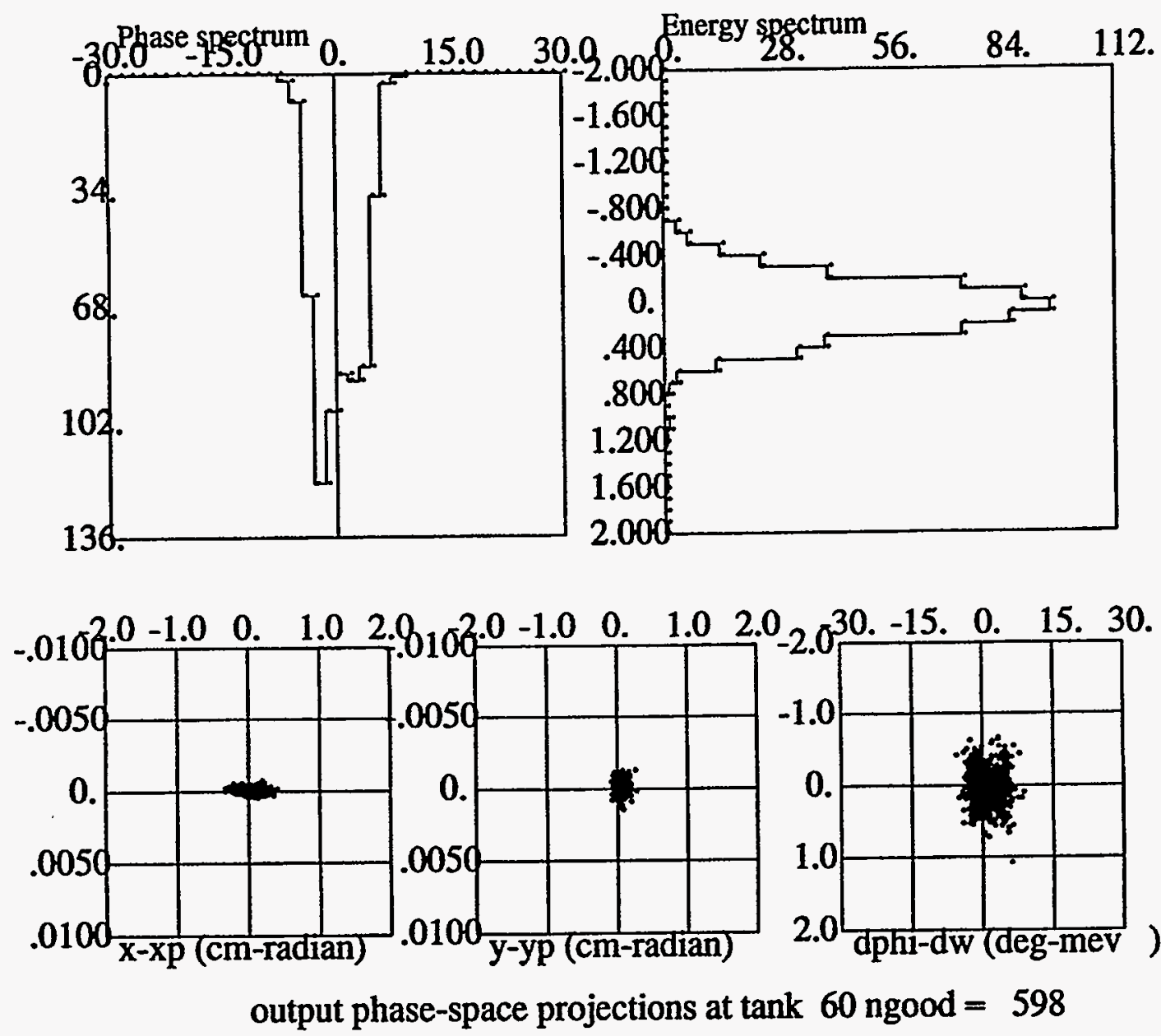

Figure III.3-9 Output Phase Space Projections at $400 \mathrm{MeV}$. 


\subsection{INTRODUCTION}

This section describes the various subsystems of the linac. The actual designs presented are those most familiar to AccSys and ones that have been used successfully in several programs at the national laboratories. The first $70 \mathrm{MeV}$ of the linac is based on the AccSys standard design for a high-duty-factor DTL, with the exceptions of the chopper and the higher current required from the $\mathrm{H}^{-}$ion source. The matching section and CCL are based on designs from LANL, Fermilab, and the SSC. Whenever possible, commercially available components have been used. When that has not been possible, components already developed at one of the national laboratories or other research centers have been used. For enhanced reliability, all components are designed for operation at a $3 \%$ system duty factor, although the linac operation is planned at a $1.6 \%$ system duty factor and a $1.5 \%$ beam duty factor.

\subsection{LINAC SUBSYSTEMS}

The linac consists of the following main subsystems:

- Linac injector subsystem (LIS) - The LIS includes the ion source, chopper, LEBT, RFQ, RGDTL, and also associated amplifiers, power supplies, and vacuum and cooling systems.

- Drift tube linac (DTL) - The DTL includes three accelerating tanks, with a total of 118 drift tubes. Each tank is made of several sections. $\mathrm{Rf}$ power is fed to each tank through a waveguide window mounted on the tank.

- Matching section (MS) - The MS includes two buncher tanks and 10 quadrupole focusing magnets.

- Coupled-cavity linac (CCL) - The CCL consists of 10 side-coupled-linac (SCL) modules, each with six tanks connected by bridge couplers. Each tank has 16 identical accelerating cavities. Rf power is fed to each module through a waveguide window into the center bridge coupler.

- Klystron/modulator systems - Three different klystron amplifier systems are used to power the linac. Three 4-MW (peak), 425-MHz klystrons are used to power the constant gradient DTL tanks. Two 1.0-MW (peak), $1,275-\mathrm{MHz}$ klystrons are used for the buncher tanks in the matching section, and ten 9-MW (peak), 1,275-MHz klystrons power the CCL tanks. These klystrons and their modulators are located in the klystron gallery and are connected to the linac by transmission waveguide.

- Control system - The Experimental Physics and Industrial Control System (EPICS) ${ }^{8}$ is used as the tool kit to design the front end control software. The hardware interface to the linac is done in VME and VXI. 


\subsection{LINAC INJECTOR SUBSYSTEM}

The LIS is shown in Figure III.4.3-1. Its main components are described in this section.

\subsection{1 $\mathrm{H}^{-}$Ion Source}

A cesium-enhanced volume ion source produces a $50-\mathrm{mA} \mathrm{H} \mathrm{H}^{-}$beam at an energy of $35 \mathrm{keV}$. Alternatively, the ISIS $\mathrm{H}^{-}$source can be used without further development. ${ }^{1}$ Only the volume source is described.

The plasma in an rf-driven volume ion source is confined by a multicusp magnetic field produced by samarium cobalt magnets. The plasma chamber is $10 \mathrm{~cm}$ in diameter. Hydrogen plasma is produced by exciting an antenna coil inside the source. The coil is driven by a 70-kW peak if power amplifier at a frequency of about $2 \mathrm{MHz}$. The hydrogen gas pressure in the ion source is controlled by a pulse-width-modulated gas control system synchronized to the linac repetition rate. The antenna coil is made of water-cooled copper tubing coated with a thin layer of porcelain to insulate the antenna from the plasma and to protect the copper tubing from plasma bombardment. A small heated tungsten filament, the igniter, provides electrons that allow plasma to ignite at lower pressures than would be possible with only the if drive. This source has two of these igniters for redundancy.

$\mathrm{NdFe}$ permanent magnets in water-cooled tubes form a magnetic filter that divides the $\mathrm{H}^{-}$ion source into two regions. A stainless steel insert defines the extraction aperture; it is mounted on the plasma electrode, along with a stainless steel collar. The collar projects back into the source to reduce the extracted electron current. Small cesium dispensers installed on the collar provide a thin cesium layer that enhances $\mathrm{H}^{-}$production. The plasma electrode is isolated from the source so that it can be biased up to $10 \mathrm{~V}$ with respect to the source body. A cross section of the $\mathrm{H}^{-}$ion source is shown in Figure III.4.3-2. The operating parameters are listed in Table III.4.3-1.

Three electrodes - the plasma electrode, extractor, and ground electrode - extract the beam from the ion source and accelerate it to $35 \mathrm{keV}$. These electrodes are as open as possible to minimize $\mathrm{H}^{-}$stripping in the background gas. Injection energy and extraction voltage can be independently adjusted to optimize beam optics. A potential difference of $20 \mathrm{kV}$ is applied between the plasma electrode and the extractor electrode to extract the beam from the source. Four permanent magnets in the electron dump create a double dipole field that bends extracted electrons into a graphite-lined cavity while deflecting the ions only slightly. A current transformer is mounted at the exit of the injector to measure current leaving the accelerating column. 


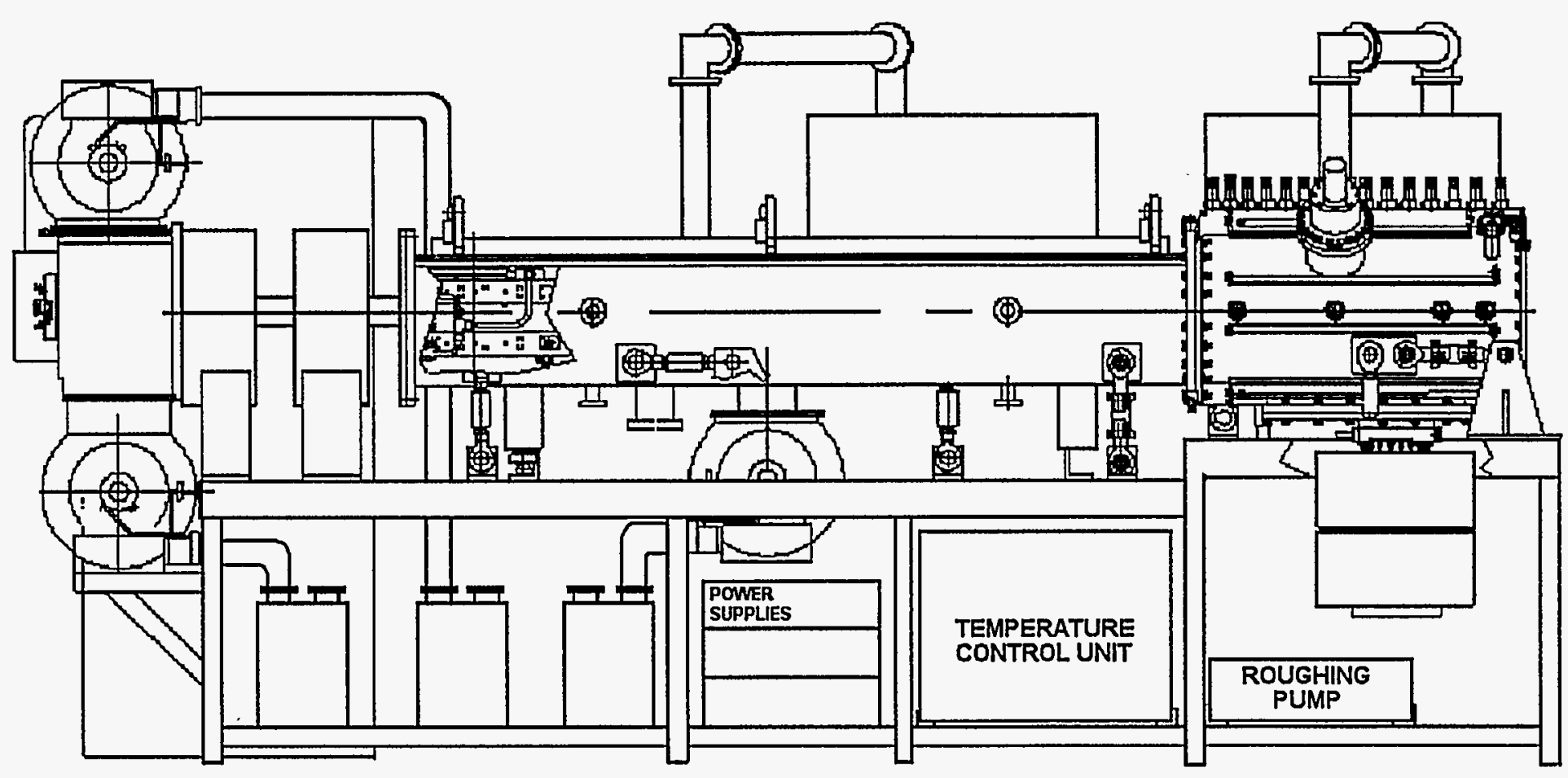

Figure III.4.3-1 Linac Injector Subsystem (LIS). 


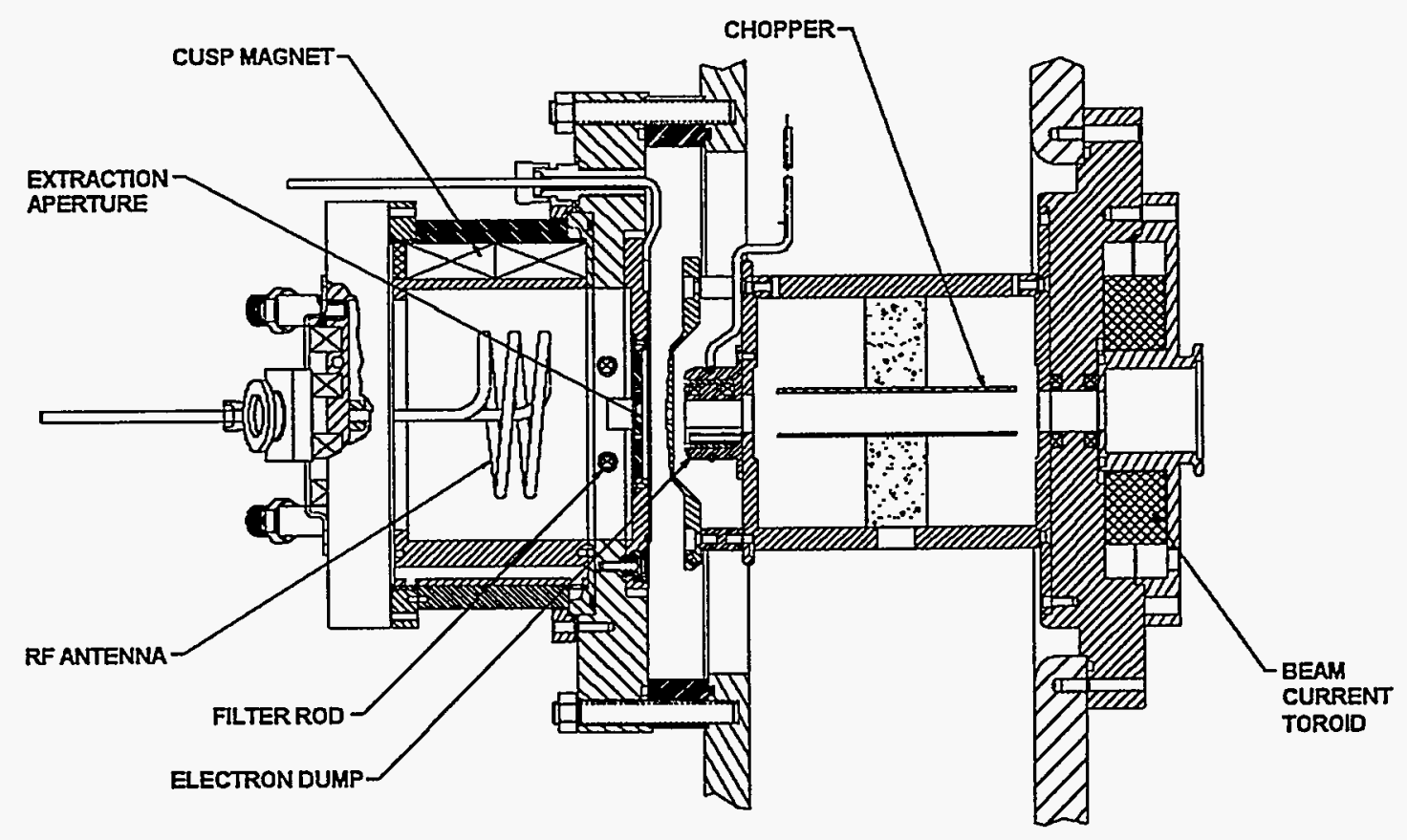

Figure III.4.3-2 $\mathrm{H}^{-}$Ion Source. 
Table III.4.3-1 Ion Source Operating Parameters

\begin{tabular}{lll}
\hline \multicolumn{1}{c}{ Parameters } & Values & \multicolumn{1}{c}{ Units } \\
& & \\
\hline Extraction voltage & 35 & $\mathrm{kV}$ \\
Extracted beam current & 60 & $\mathrm{~mA}$ \\
Hydrogen gas flow & $5-7$ & $\mathrm{~atm} \mathrm{~cm} 3 / \mathrm{min}$ \\
Norm rms beam emittance & & \\
Peak pulsed radio-frequency power & 70 & $\mathrm{~kW}$ \\
ac input power & 5 & $\mathrm{~kW}$ \\
& & \\
\hline
\end{tabular}

a $\mathrm{rms}=$ root mean square. 


\subsubsection{Chopper}

Injection into the RCS requires that the beam be chopped at about $1 \mathrm{MHz}$ and that it be on $75 \%$ of the time. An electrostatic chopper located at the exit of the ion source is used to modulate the beam intensity in response to a timing signal from the synchrotron control system. An FET pulser drives the chopper. ${ }^{9}$ A schematic diagram of the ion source electrical connections is shown in Figure III.4.3-3.

Chopping the beam has a deleterious effect on space charge compensation needed to transport a high-current $\mathrm{H}^{-}$ion beam with minimal emittance growth. ${ }^{10}$ A second magnetic double dipole, located at the exit of the injector just after the chopper, limits the extent of the perturbation. Effects still occur downstream, as the neutralization channel is displaced toward the direction of the beam deflection.

The chopper subsystem requires additional development.

\subsubsection{Low-Energy Beam Transport (LEBT)}

The beam transported from the injector is focused into the RFQ by using two large-bore solenoid lenses. There is space between these lenses for beam diagnostics.

\subsubsection{Ion Source, Chopper, and LEBT Electronics}

This electronics system includes the If driver for the source, high- and low-voltage supplies for the ion source and associated systems, power supplies for the LEBT solenoids, and monitoring, control, and interlock circuits. A schematic of the if and high-voltage circuits is shown in Figure $\amalg$. $4.3-3$.

The electronics are housed in a standard cabinet that is grounded and interlocked. The cabinet contains a commercial 2-MHz if driver and its power supplies. The driver provides up to $70 \mathrm{~kW}$ of pulsed power at $2 \mathrm{MHz}$. All components in this system are conservatively rated for long life and high reliability. The cabinet also contains power supplies and control and interface circuits. The ion source igniter and plasma electrode power supplies, valve driver, and instrumentation are isolated at $35 \mathrm{kV}$ in the injector electronics rack. Power that is fed to the ion source is shielded to reduce transient coupling to other parts of the system. The injector electronics rack contains the $35-\mathrm{kV}$ high-voltage power supply; the high-voltage isolation transformer; pulsing and timing controls; LEBT power supplies; and vacuum control and monitoring. The injector's electronics feature extensive transient protection.

The timing, monitoring, and control signals are transmitted through fiber-optic links. 


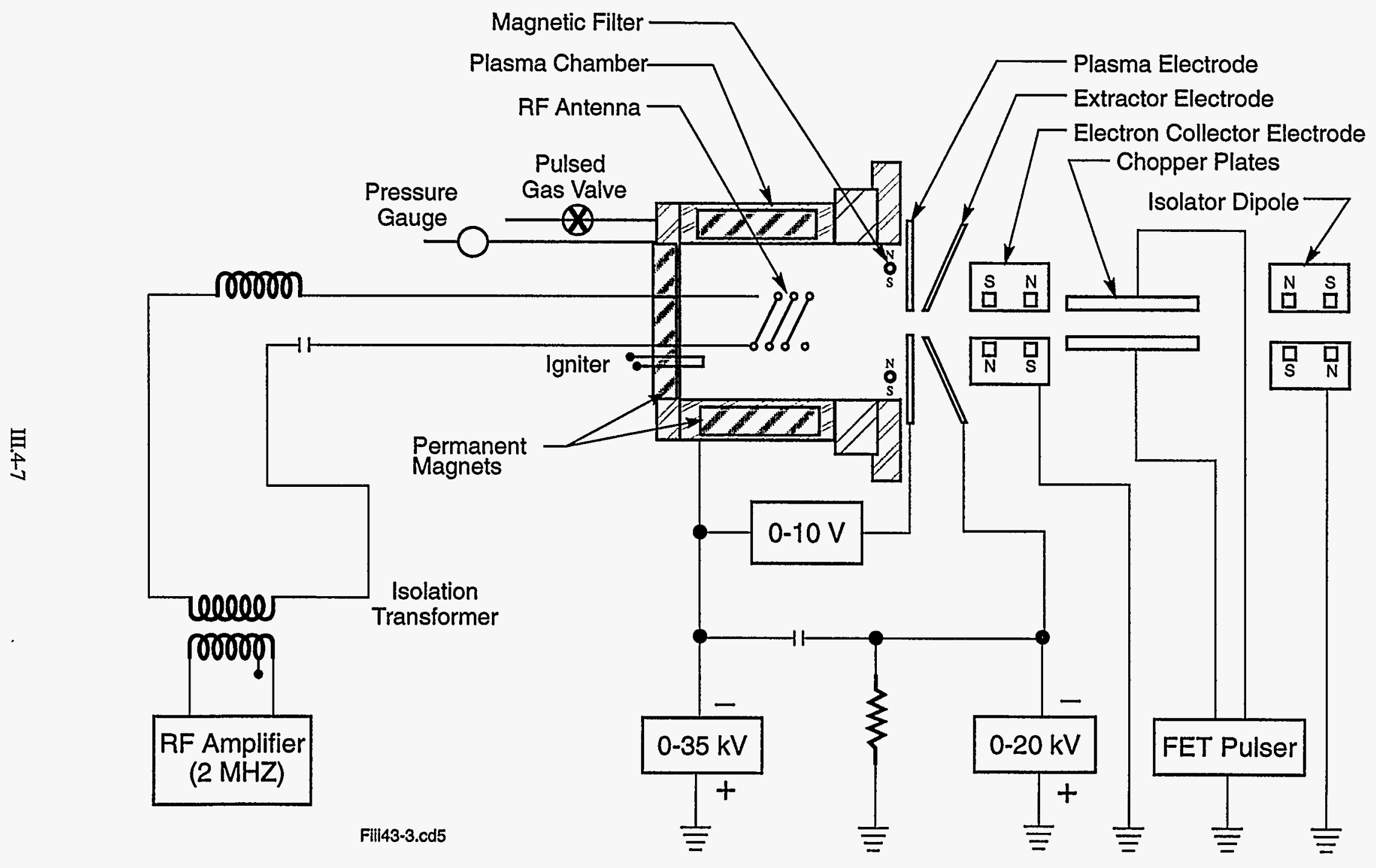

Figure III.4.3-3 Schematic of Ion Source Electrical Connections. 


\subsubsection{Radio-Frequency Quadrupole (RFQ)}

The RFQ resonator has four identical vane sections, machined from aluminum extrusions and bolted to the resonator wall. The if joint is independent of the vane mounting. This geometry minimizes the number of parts required and is rugged, easy to machine, and simple to assemble and tune. A continuous water cooling passage is provided along the vane at several locations, including one that is very near the narrow vane tip. These cooling channels allow the structure's temperature to be regulated to stabilize the resonant frequency, thereby eliminating the problem of differential expansion that results from the thermal isolation of two parts. A cross-sectional view of the RFQ is shown in Figure III.4.3-4.

The open geometry allows precise machining of the vane tips. Machining accuracy is readily checked before assembly by means of a coordinate measuring machine. Surfaces are precision-mated to ensure that the structure is accurately and reproducibly assembled without requiring critical adjustments. The mating surfaces are rigidly bolted together, thus minimizing the chance of relative movement of the pieces from vibration or thermal cycling. Because the RFQ is bolted together, it can be easily disassembled and reassembled to facilitate surface cleaning if it accidentally becomes contaminated during vacuum system servicing. The four vane tips can be initially located with enough accuracy to reduce the dipole fields to a level that they do not interfere with the main quadrupole accelerating field. Small tuners placed in openings along each quadrant and four rod tuners in each end wall are used to fine-tune the field components and resonant frequency. The thermal and mechanical stability of this RFQ has been demonstrated on a system at LANL. The RFQ operated at the design field for several months at a high duty factor. ${ }^{11}$ The parameters of the RFQ are given in Table III.4.3-2, and a photograph is shown in Figure III.4.3-5.

The entire RFQ is placed in a vacuum chamber with vacuum feedthroughs for the rf drive loop, water cooling, and rf pick-up loops. This configuration decouples the if and vacuum seals. The rigid vacuum system preserves internal alignment of the RFQ. A simple cylindrical vacuum chamber with a hinged cover provides easy access to the RFQ for alignment of the structure. This strategy, which provides more effective pumping than traditional designs in which the RFQ serves as the vacuum enclosure, speeds up conditioning and minimizes beam loss.

Resonant frequency control during operation is achieved by use of servo-driven tuning slugs with a range of $\pm 100 \mathrm{kHz}$. A single 3-1/8-in. coaxial drive loop couples the rf power into the RFQ at an operating frequency of $425 \mathrm{MHz}$.

\subsubsection{Ramped-Gradient Drift Tube Linac (RGDTL)}

The first tank immediately following the RFQ is the RGDTL, which is a short matching drift tube linac tank. The field in the RGDTL gaps is ramped to match the focal properties of the RFQ to the focal properties of the DTL. Closely coupling the RGDTL to the RFQ eliminates 


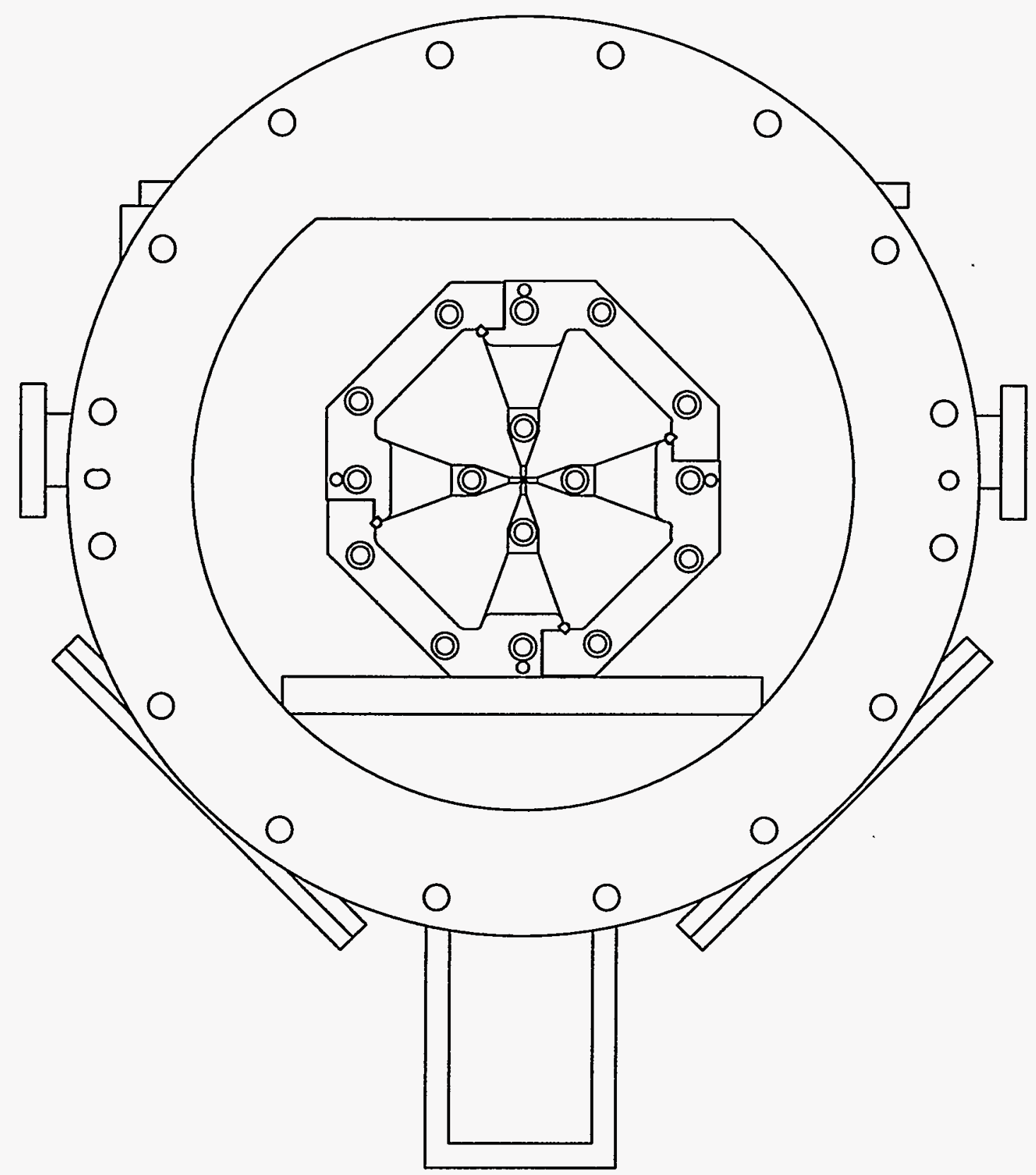

Figure III.4.3-4 Cross-Sectional View of the RFQ. 
Table III.4.3-2 Main RFQ Parameters

\begin{tabular}{lll}
\multicolumn{1}{c}{ Parameters } & Values & Units \\
\hline & & \\
\hline & & $\mathrm{MHz}$ \\
Operating frequency & 425 & $\mathrm{keV}$ \\
Injection energy & 35 & $\mathrm{MeV}$ \\
Final energy & 2 & $\mathrm{~mA}$ \\
Design input current & 50 & \\
& & $\mathrm{~mA}$ \\
Current limit & 100 & $\%$ \\
Transmission & 87 & $\pi \mathrm{cm}-\mathrm{mrad}$ \\
Input transverse emittance (normalized, rms) & 0.010 & $\pi \mathrm{cm}-\mathrm{mrad}$ \\
Output transverse emittance (normalized, $\mathrm{rms}$ ) & 0.012 & \\
& & \\
Vane tip radius ( $\rho$ ) & $1.8-2.7$ & $\mathrm{~mm}$ \\
Bore radius ( $\mathrm{r}_{\mathrm{o}}$ ) & $2.4-3.0$ & $\mathrm{~mm}$ \\
Peak radio-frequency field & $36 \mathrm{MV} / \mathrm{m}$ & $\left(1.8 \times \mathrm{E}_{\mathrm{kp}}\right.$ ) \\
Structure length & 2.16 & $\mathrm{~m}$ \\
& & \\
Peak structure power & 220 & $\mathrm{~kW}$ \\
Peak beam power & 80 & $\mathrm{~kW}$ \\
Peak radio-frequency input power & 300 & $\mathrm{~kW}$ \\
Average structure power & 6.8 & $\mathrm{~kW}$ \\
Coolant flow & 25 & $\mathrm{gal} / \mathrm{min}$ \\
\hline
\end{tabular}

a $\mathrm{rms}=$ root mean square. 


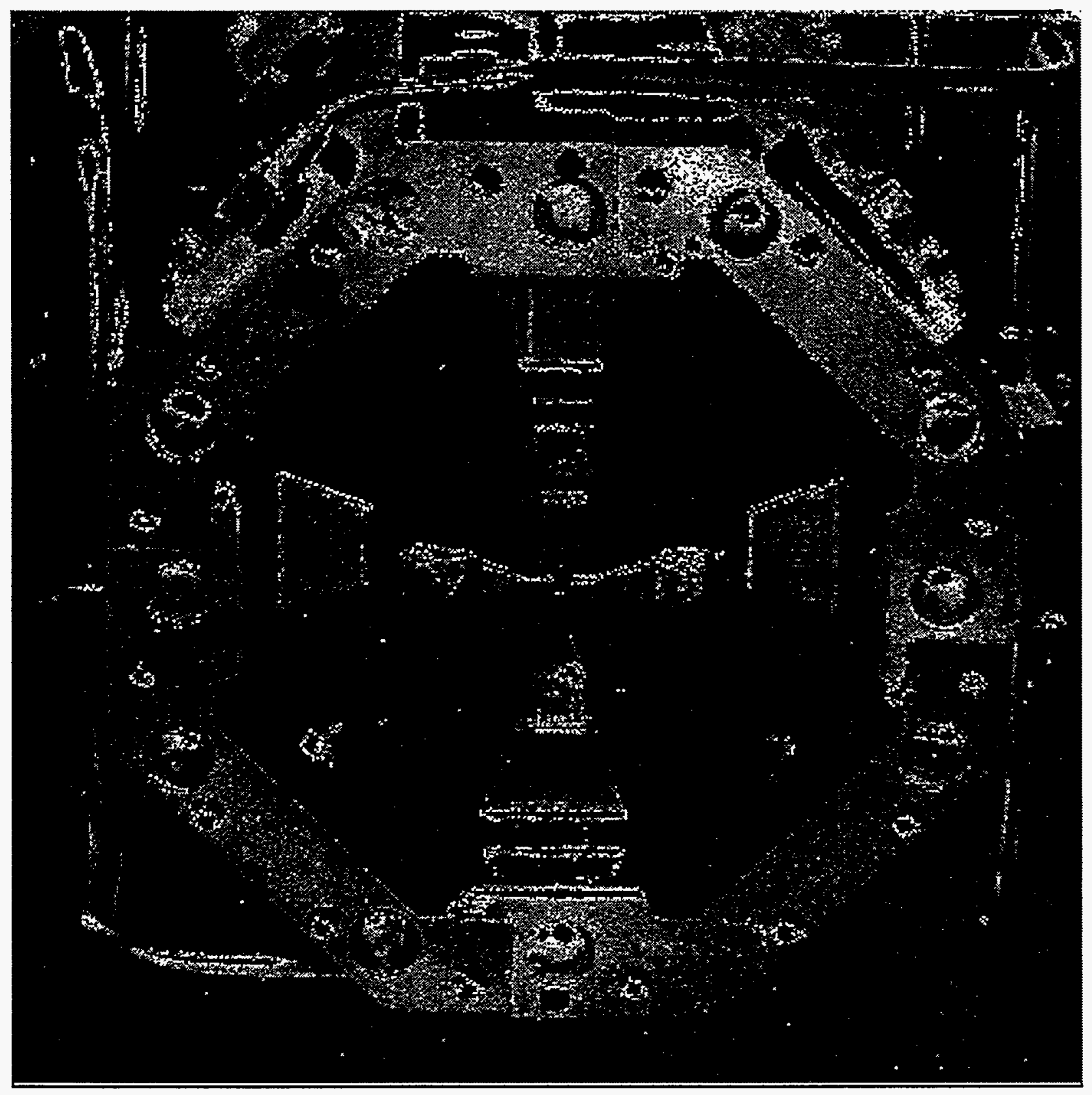

Figure III.4.3-5 Photograph of the RFQ. 
the need for a complex beam-matching section. Parameters of this tank are listed in Table III.4.3-3. The mechanical design of the RGDTL tank is described in Section III.4.4, along with the rest of the DTL.

\subsubsection{Radio-Frequency System for the RFQ and RGDTL}

The RFQ and the RGDTL tanks are driven by commercial, modular if power systems with wide-bandwidth amplitude and phase control. These amplifiers use 12 YU-176 planartriode tubes in a parallel self-balancing array as the final power stage. Special circuits eliminate the need to match tube characteristics to ensure equal power contributions. ${ }^{11}$ The YU-176 has large-area oxide-coated cathodes for efficient and reliable pulse operation. The peak power output is more than $1 \mathrm{~kW}$ per watt of heater power. Standard design features include load fault protection and dynamic amplitude and phase control to within $\pm 0.5 \%$ and $\pm 0.5^{\circ}$, respectively, with less than 3.0- $\mu \mathrm{sec}$ response time. The RFQ and RGDTL amplifiers operate at $425 \mathrm{MHz}$, with a peak power output of $360 \mathrm{~kW}$.

Figure III.4.3-6 is a block diagram of the if power amplifier system. Amplitude and frequency feedback control systems are provided. The driver amplifier is a solid-state unit capable of supplying $150 \mathrm{~W}$ at a $100 \%$ duty factor with an input drive power of $10 \mathrm{~mW}$. The intermediate power amplifier stages are tuned amplifiers. The first stage (IPA1) uses a single YU-176 tube and provides a 3.0-kW drive to the second stage. The second stage (IPA2) has two YU-176 triodes in parallel and provides $30-\mathrm{kW}$ output. Both stages are conservatively designed so they are not stressed in high-duty-factor operation. All of the control and tube protection circuits have been successfully implemented, and the tube output and cooling have been demonstrated at a $3 \%$ duty factor. ${ }^{12}$

The amplifier stages are operated Class-C for stability at high gain with a pulsed rf drive. The tubes are protected by electronic crowbar circuits that limit the fault energy dissipated in a tube to a safe value. These systems are specifically designed for operation with a high-Q resonant-cavity load, such as an if linear accelerator. The amplifier, transmission line, and resonant cavity load are tuned together for maximum performance. The tuned output circuit handles the high turn-on and turn-off VSWR without requiring expensive rf power isolators. Each system can handle the large dynamic change in load impedance that occurs in an accelerator when the beam is turned on and off.

The built-in amplitude, phase, and frequency control loops respond rapidly to changes in the load cavity fields. The output stage bandwidth is greater than $20 \mathrm{MHz}$. The amplitude control loop uses high-level cathode modulation of the power amplifier stage for fast response. The systems are high gain, requiring only $150-\mathrm{mW} \mathrm{CW}$ drive power, simplifying the distribution of the rf source power in multiple unit systems. The units are fully interlocked for personnel and equipment protection and fully shielded from electromagnetic interference (EMI). 
Table III.4.3-3 RGDTL Parameters

\begin{tabular}{lll}
\hline \multicolumn{1}{c}{ Parameters } & Values & Units \\
\hline $\begin{array}{l}\text { Operating frequency } \\
\text { Input beam energy }\end{array}$ & 425 & $\mathrm{MHz}$ \\
$\begin{array}{l}\text { Output beam energy } \\
\text { Length }\end{array}$ & 2 & $\mathrm{MeV}$ \\
& 4.2 & $\mathrm{MeV}$ \\
Number of drift tubes & 0.9 & $\mathrm{~m}$ \\
$\begin{array}{l}\text { Aperture radius } \\
\text { Electric field gradient }\end{array}$ & 16 & - \\
PMQ & & \\
& 0.5 & $\mathrm{~cm}$ \\
Peak structient & $2.4-4.2$ & $\mathrm{MV} / \mathrm{m}$ \\
$\begin{array}{l}\text { Peak beam power at } 40 \mathrm{~mA} \\
\text { Total peak radio-frequency power }\end{array}$ & 180 & $\mathrm{~T} / \mathrm{m}$ \\
Average structure dissipation & 254 & $\mathrm{~kW}$ \\
Cooling water flow & 5 & $\mathrm{~kW}$ \\
& 165 & $\mathrm{~kW}$ \\
\hline
\end{tabular}

a $P M Q=$ permanent magnet quadrupole. 


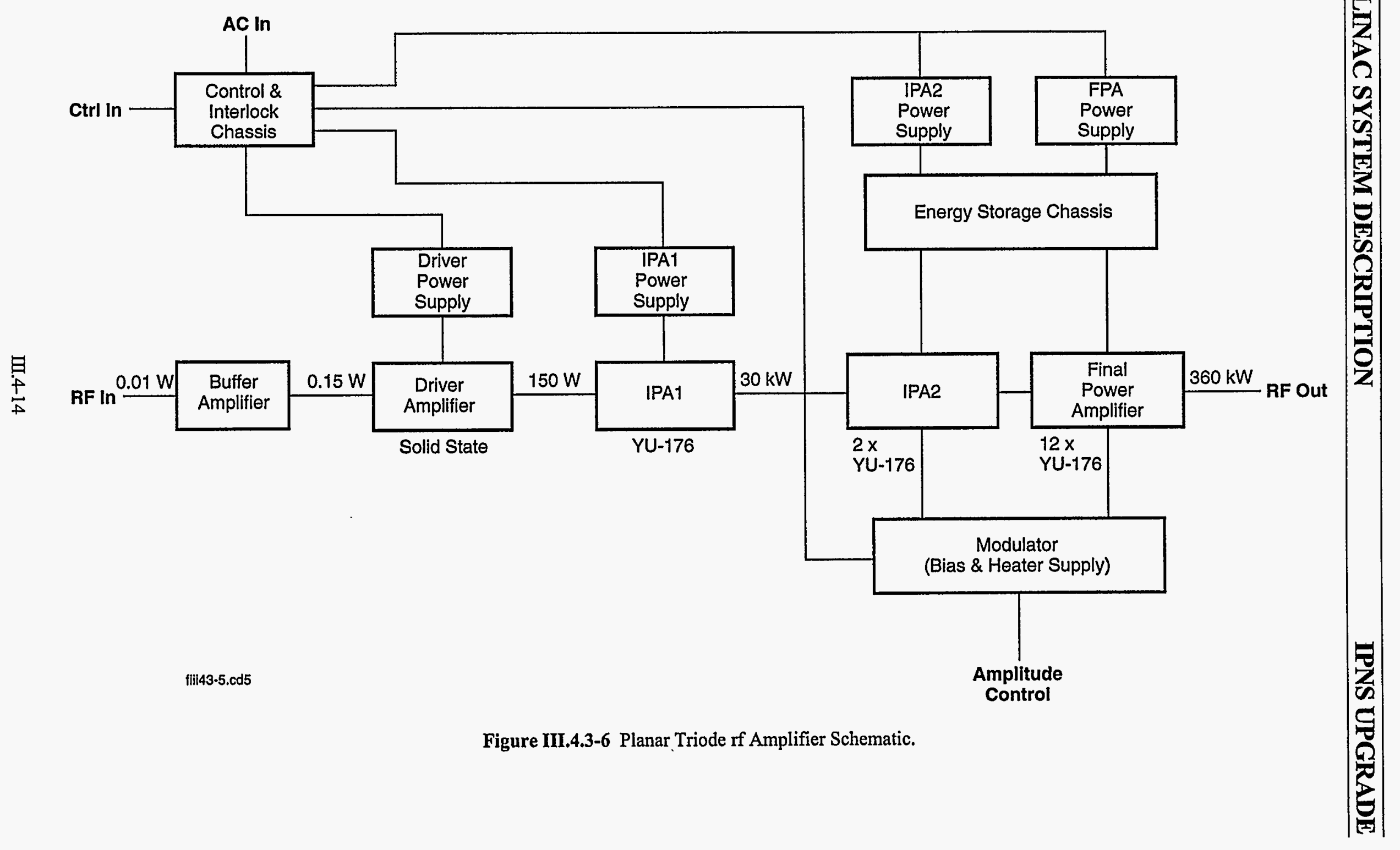




\subsubsection{Vacuum and Auxiliary Systems}

The ion injector has its own vacuum system, with an isolation valve for routine source maintenance. This system uses two 2,200-L/sec turbomolecular pumps to handle the high hydrogen gas load from the $\mathrm{H}^{-}$source. The vacuum is sufficient to prevent any sparking in the extraction gap and to reduce stripping losses. A vacuum gauge unit that uses two ion gauges and two thermocouple gauges monitors the pressure in the injector, beamline, and roughing lines. This unit provides local indication of pressure readings on its front panel, and it sends this information to the control system. The pressure in the injector vacuum chamber is typically 2 to $3 \times 10^{-5}$ Torr during operation.

A 2,200-L/sec turbomolecular pump mounted on the $\mathrm{RFQ}$ vacuum chamber maintains a $3 \times 10^{-7}$ Torr in the RFQ resonator. The restricted openings at the exit of the ion source mounting chamber and the entrance to the RFQ limit the gas flow between the ion source and the RFQ. The RGDTL uses a 500-L/sec ion pump to maintain a pressure of less than $1 \times 10^{-7}$ Torr. Some differential pumping of the RFQ is provided through the small bore of the half-drift-tube end of the RGDTL. Ion gauges are used to monitor the vacuum in both tanks.

A single commercial, packaged temperature controller with $\pm 1^{\circ} \mathrm{C}$ stability is used to maintain stable temperature for both the RFQ and the RGDTL. It is installed on the LIS support frame.

\subsection{DRIFT TUBE LINAC}

\subsubsection{Overview}

Three DTL tanks are used to accelerate the beam to $70 \mathrm{MeV}$. They are constant gradient tanks with one $\beta \lambda$ spacing. Permanent-magnet quadrupoles (PMQs) installed in each drift tube focus the beam through the structure and maintain beam emittance. The size of the beam aperture in the drift tubes is gradually increased along the DTL to minimize beam loss. Each gradient tank is powered by a 4-MW-peak, $425-\mathrm{MHz}$ klystron. Major parameters of the DTL tanks are shown in Table III.4.4-1.

The final operating frequency is achieved by using a hierarchy of tuning mechanisms. Tuning bars with a range of $\pm 2 \mathrm{MHz}$ are used for coarse-tuning of frequency during initial setup. Servo-driven tuning slugs with a range of $\pm 200 \mathrm{kHz}$ are used for fine-tuning during operation. In addition, the tank operating temperature can be varied to control the frequency over a range of $\pm 15 \mathrm{kHz}$.

A side view of a typical DTL tank is shown in Figure III.4.4-1, a cross-sectional view is shown in Figure III.4.4-2, and a photograph is shown in Figure II.4.4-3. The main components are the (1) rf and vacuum vessel, (2) drift tubes and post-stabilizer assemblies, (3) integral vacuum and cooling systems, and (4) support stand. Each of these components is described in the following subsections. 
Table III.4.4-1 DTL Parameters

\begin{tabular}{|c|c|c|c|c|}
\hline Parameters & Tank 1 & Tank 2 & Tank 3 & Units \\
\hline Operating frequency & 425 & 425 & 425 & $\mathrm{MHz}$ \\
\hline Input beam energy & 4.2 & 28.7 & 50.1 & MeV \\
\hline Output beam energy & 28.7 & 50.1 & 70 & $\mathrm{MeV}$ \\
\hline Length & 7.14 & 6.27 & 6.23 & m \\
\hline Number of drift tubes & 60 & 32 & 26 & - \\
\hline Aperture radius & $5-7$ & 8 & 9 & $\mathrm{~mm}$ \\
\hline Accelerating field gradient & 4.39 & 4.63 & 4.60 & $\mathrm{MV} / \mathrm{m}$ \\
\hline $\mathrm{PMQ}^{\mathrm{a}}$ gradient & $173-105$ & 100 & 95 & $T / m$ \\
\hline Peak structure power & 2.1 & 2.02 & 2.0 & MW \\
\hline Peak beam power at $40 \mathrm{~mA}$ & 0.98 & 0.86 & 0.8 & MW \\
\hline Total peak radio-frequency power & 3.1 & 2.9 & 2.8 & MW \\
\hline Average structure dissipation & 63 & 61 & 60 & $k W$ \\
\hline Cavity fill time & 35 & 35 & 35 & $\mu \mathrm{sec}$ \\
\hline
\end{tabular}

a $P M Q=$ permanent magnet quadrupole. 


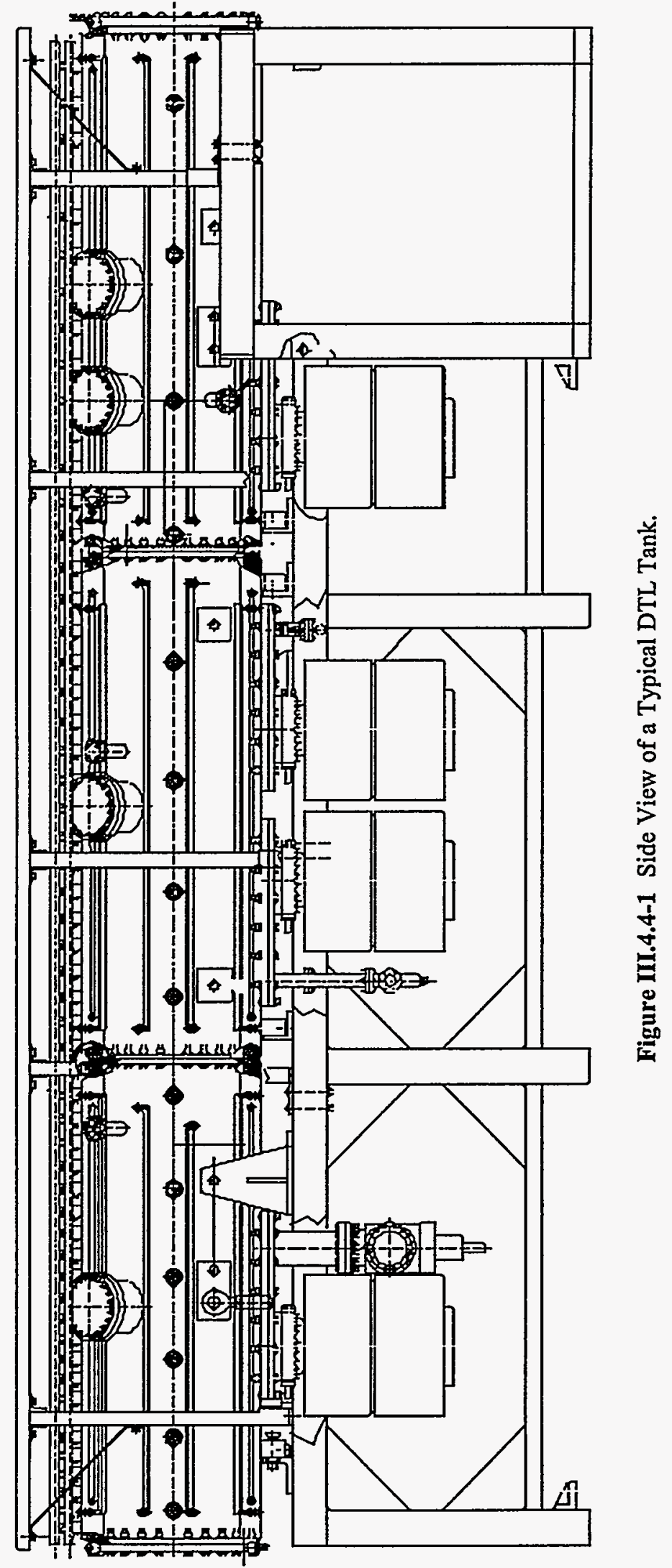




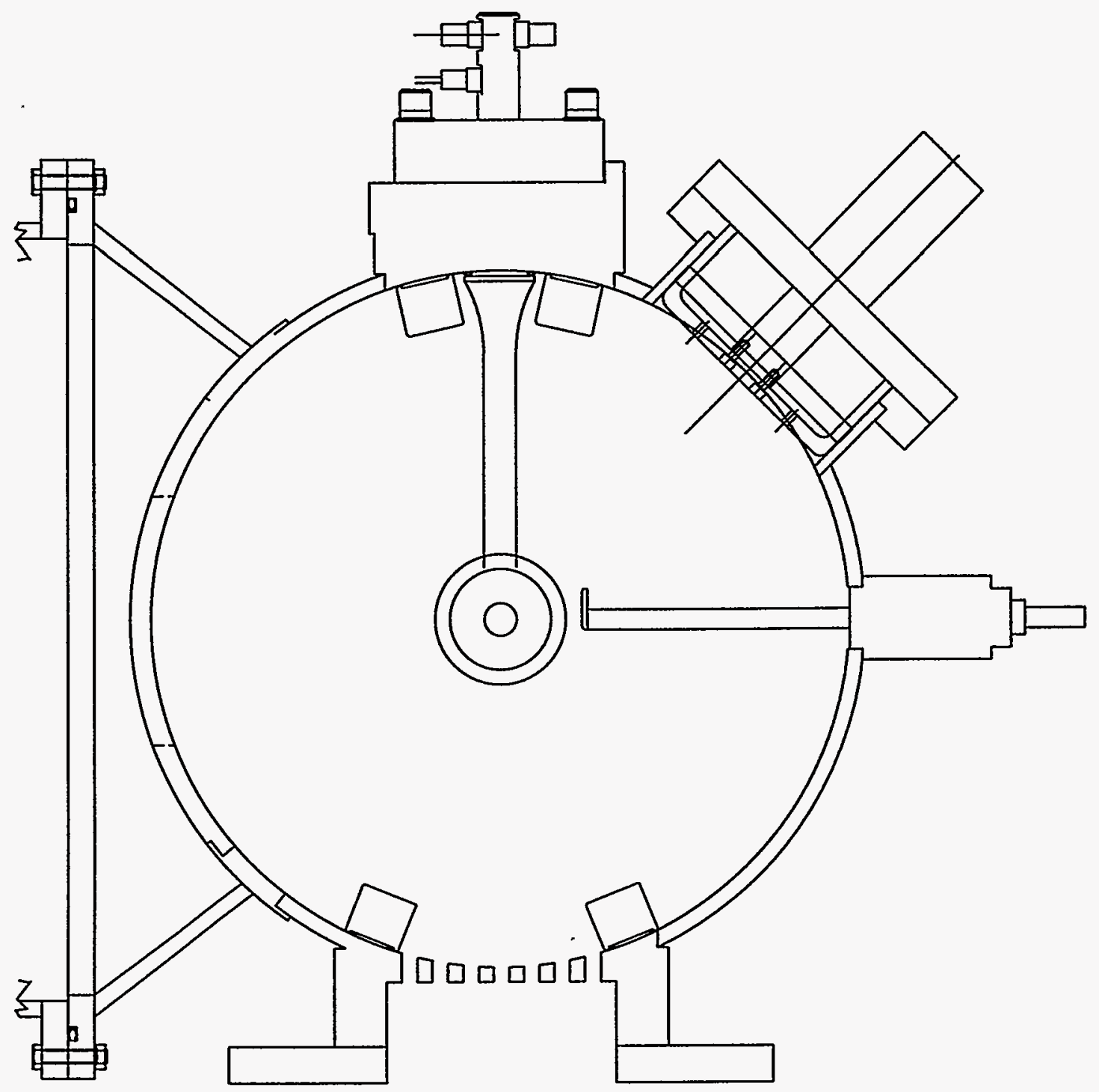

Figure III.4.4-2 Cross Section of a Typical DTL Tank. 


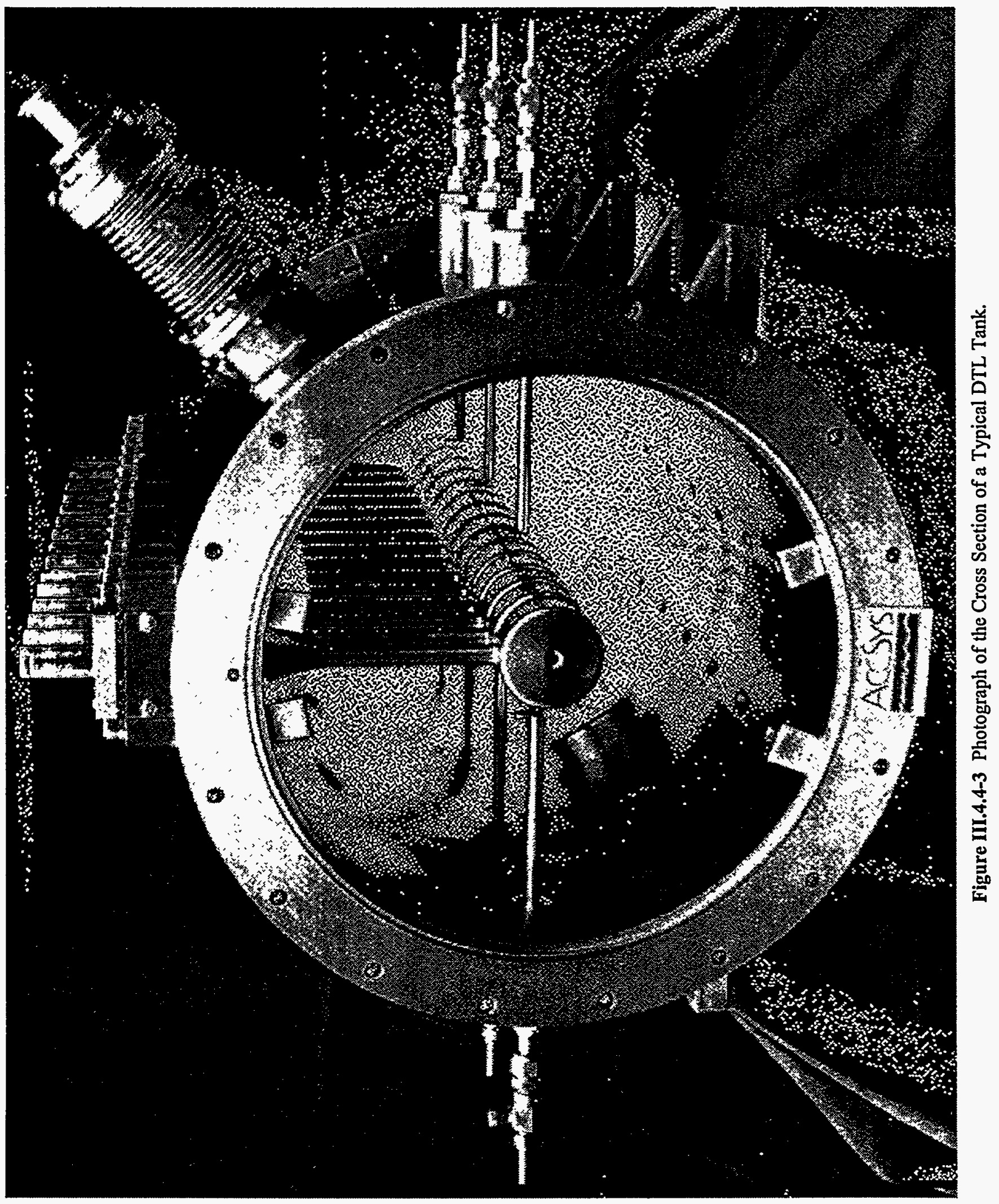




\subsubsection{Radio-Frequency and Vacuum Vessel}

Each tank is made in several sections for ease of handling and fabrication. The number of sections depends on the total length. These sections are made of steel for easy fabrication and are copper-plated for high conductivity. Each section is designed for maximum mechanical stability and consists of two rails, an upper and a lower, joined together by welding two circular wall sections between them. The upper rail supports the drift tubes as well as two tuning bars used for coarse frequency adjustments. Supporting the drift tubes on a rail that is integral to the main vessel eliminates the need for a large and difficult if and vacuum joint. The bottom rail increases structural stability while providing a mounting surface for additional tuning bars and vacuum pumps. The post-stabilizer mounts, slug tuner ports, and other vacuum penetrations are welded directly to the walls of the tank. Cooling channels brazed to the outer wall remove energy dissipated in the tank by the if fields.

The twin-rail design provides exceptional long-term dimensional stability. The tank is stress-relieved before plating and final machining to ensure that the drift tubes remain accurately aligned after installation. This design also simplifies fabrication by eliminating the need to accurately roll up a cylinder to the required inside diameter. The DTL tanks are separated by a short drift space to allow insertion of beam diagnostic instruments and magnetic optics correction magnets.

\subsubsection{Drift Tubes and Post-Stabilizer Assemblies}

The drift tube stems are made of stainless steel for strength and rigidity. They are copper-plated and brazed into the drift tube bodies. Compact permanent-magnet quadrupoles in the drift tubes maintain transverse beam focusing. Each copper drift tube is sealed to make it a vacuum-tight enclosure. The drift tube if surfaces are made from two copper shells that fit closely over the drift tube body and are electron-beam-welded to a copper ring. The drift tube bodies have internal cooling channels that are fed through the stem. The cooling channels are also sealed separately within the drift tubes.

The drift tubes are mounted to adjustable sockets on the upper rail and are rigidly aligned with little residual stress. Accurate positioning of the drift tubes is accomplished by using shims between reference surfaces on the drift tube stem and the support rail. This procedure provides precise incremental adjustment with long-term stability, eliminates expensive adjusting apparatus that is used only once in the life of the DTL, and allows the drift tubes to be removed and accurately reinstalled. It has been demonstrated that drift tube assemblies can be removed and reinstalled to less than $\pm 0.013 \mathrm{~mm}$ of their initial position. ${ }^{13}$

Resonant post-stabilizers are used to stabilize and fine-tune the on-axis electric field distribution of the DTL. The post-stabilizers greatly relax the tolerances on the drift tube dimensions and alignment. They also eliminate the need for continuous critical adjustment of if tuners to maintain the field distribution during operation. The post-stabilizers are in an adjustable mount that allows precise movement of the posts during tuning. The post-stabilizer 
mounting scheme allows accurate adjustment with a removable, reusable adjuster apparatus. The post-stabilizers are permanently locked into position after adjustment. The adjustment is made by using the tilt-sensitivity method developed at LANL for tuning the 100-MeV DTL tanks of its Meson Physics Facility accelerator. ${ }^{14}$ These adjustments are independent of the if and vacuum seals, thereby allowing the required electric field distribution to be easily achieved. A coaxial cooling channel removes heat generated by if fields.

This approach to designing and positioning drift tubes and post-stabilizers meets the key performance objectives of high beam transmission and optimal electric field distribution. It results in an operation that is accurate and stable for many years.

\subsubsection{Vacuum and Cooling}

The DTL tanks are maintained at a vacuum of $1 \times 10^{-7}$ Torr by 500-L/sec ion pumps connected to a port at the bottom of each section. Metal vacuum seals provide long-term reliability and radiation resistance.

A closed-cycle cooling system maintains the DTL's operating temperature. Temperature is stabilized in the tank by supplying temperature-controlled water through channels on the outside of the tank walls as well as through the drift tube assemblies and post-stabilizers. Individual commercial units are used for each tank.

\subsubsection{Support Stand}

The stand that supports the DTL is a weldment of square steel box beams. The weight of each tank rests on four adjustable supports. The DTL tank is supported on six adjustable struts for precise alignment.

Room underneath the tanks is sufficient to allow easy removal of the vacuum pumps. The frame also supports the cooling water manifold.

\subsection{MATCHING SECTION}

\subsubsection{Introduction}

The beam from the DTL is bunched at $425 \mathrm{MHz}$ and is matched into the CCL that operates at $1,275 \mathrm{MHz}$. The matching section transforms the beam from the DTL into the phase space volume required by the CCL. Two buncher cavities - short sections of side-coupled cavities that operate at $1,275 \mathrm{MHz}$ - and 10 quadrupole magnets are used to achieve this match. Matching section parameters are listed in Table III.4.5-1. 
Table III.4.5-1 Matching Section Parameters

\begin{tabular}{lll}
\hline \multicolumn{1}{c}{ Parameters } & \multicolumn{1}{c}{ Values } & Units \\
\hline & & \\
\hline $\begin{array}{l}\text { Length } \\
\text { Buncher type }\end{array}$ & 3.2 & $\mathrm{~m}$ \\
$\begin{array}{l}\text { Number of bunchers } \\
\text { Buncher frequency }\end{array}$ & 2 & - \\
& 1,275 & $\mathrm{MHz}$ \\
$\begin{array}{l}\text { Number of cells per buncher } \\
\text { Power required }\end{array}$ & 5 and 7 & - \\
$\begin{array}{l}\text { Number of quadrupole doublets } \\
\text { Number of quadrupole singlets }\end{array}$ & 500 and 700 & $\mathrm{~kW}$ \\
& 4 & - \\
$\begin{array}{l}\text { Quadrupole gradient } \\
\text { Quadrupole bore } \\
\text { Quadrupole length }\end{array}$ & 40 & - \\
Cavity fill time & 23 & $\mathrm{~T} / \mathrm{m}$ \\
& 40 & $\mathrm{~mm}$ \\
\hline
\end{tabular}




\subsection{COUPLED-CAVITY LINAC}

\subsubsection{Overview}

The CCL consists of 60 side-coupled-cavity tanks with 16 accelerating cavities per tank. There are 10 modules, each of which consists of six tanks and five bridge couplers. The tanks increase in length as beam energy increases. There are no bridge couplers between modules. $\mathrm{Rf}$ power is fed to each module by a WR650 waveguide connected to the center bridge coupler. The main parameters of the CCL are listed in Table III.4.6-1. A side view of three tanks and two bridge couplers is shown in Figure III.4.6-1.

\subsubsection{Radio-Frequency Structure Fabrication}

Half-cavities of the $1,275-\mathrm{MHz}$ if structures are machined from bar stock. The half cavities and cavity subassemblies are made with steps and matching counterbores to maintain alignment during brazing. The coupling cavities are also machined from solid stock and brazed together to form a subassembly. The post-stabilized bridge couplers consist of a cylinder with discs and end-cells brazed in place. They are equipped with lifting eyes at each end. The bridge couplers are connected to the tanks by means of rectangular brazed flanges. These flanges and their associated seals provide both an if and a vacuum joint. The same type of flange and rectangular vacuum seal are used for all of the CCL tanks and bridge couplers.

Integral cooling water passages, machined into the half-cavities before brazing, allow effective frequency stabilization of the if structure. Integral water passages are used in order to achieve superior thermal performance, simpler construction, and lower cost.

Slots are milled into the side walls of the tank and bridge coupler cavities to permit finetuning by dimpling the copper at the bottom of the slot. A vacuum nipple is brazed into each tank coupling cavity to allow access to the internal coupling cavity posts for tank tuning. The upper vacuum ports are capped; the lower ports are connected to the vacuum manifold by a bellows. A standard Conflat vacuum flange is brazed into the end of each tank for the inlet and outlet beamline.

The braze joint designs are important factors in the CCL tank fabrication process. The cavities are brazed by using washers of braze alloy to ensure complete joint wetting. The different alloys used for step-brazing the cavity components are selected because of their melting temperature, conductivity, braze reliability, and cost. Allowance is made for the braze alloy thickness remaining after brazing. Oxygen-free, high-conductivity (OFHC) copper is used for the accelerating and coupling cavities because of its high electrical conductivity and compatibility with brazing processes. The time-temperature profile of the brazing process is controlled and monitored to ensure good dimensional control and leak-tight joints. 
Table III.4.6-1 CCL Parameters

\begin{tabular}{|c|c|c|}
\hline Parameters & Values & Units \\
\hline Frequency & 1,275 & $\mathrm{MHz}$ \\
\hline Input energy & 70 & MeV \\
\hline Output energy & 400 & MeV \\
\hline Number of modules & 10 & - \\
\hline Number of tanks per module & 6 & - \\
\hline Number of cavities per tank & 16 & - \\
\hline Peak surface field & 28 & $\mathrm{MV} / \mathrm{m}$ \\
\hline Field gradient $\left(E_{0} T\right)$ & $5.9-5.5$ & $\mathrm{MV} / \mathrm{m}$ \\
\hline Synchronous phase & -30 to -25 & deg \\
\hline Peak structure power & 52.8 & MW \\
\hline Peak beam power at $40 \mathrm{~mA}$ & 13.2 & MW \\
\hline Total peak radio-frequency power & 66.0 & MW \\
\hline Magnet lattice & FODO & - \\
\hline Length & 92.1 & $\mathbf{m}$ \\
\hline Bore radius & $\geq 10$ & $\mathrm{~mm}$ \\
\hline First-neighbor coupling constant & 5 & $\%$ \\
\hline Intertank and intermodule spacing & 3.5 & $\beta \lambda$ \\
\hline Number of bridge couplers & 50 & \\
\hline Bridge coupler coupling constant & 10 & $\%$ \\
\hline Cavity fill time & 10 & $\mu \mathrm{sec}$ \\
\hline
\end{tabular}




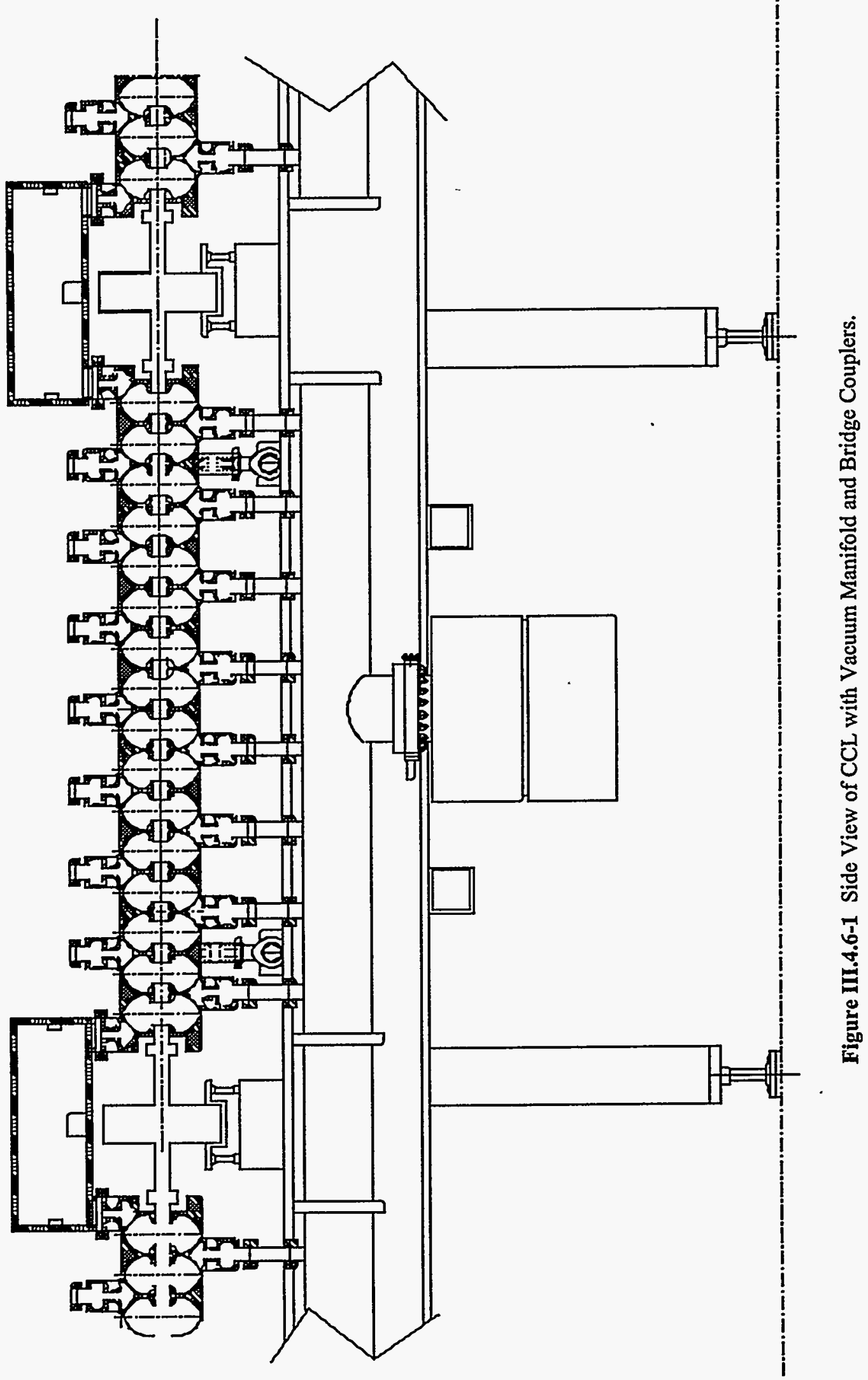


Four alignment target holders are mounted on the access aisle side of each tank. Steel-ball alignment targets are used to align the linac beam line to the required tolerance. There is a clear line of sight from the CCL alignment monuments to the floor monuments.

\subsubsection{Support Systems and Mechanical Design Features}

The if structure is supported by a series of six-strut support systems mounted on steel support stands. Each stand supports six tanks, five bridge couplers, a vacuum manifold, and associated water manifolding, as shown in Figure III.4.6-2. The intertank quadrupole magnet and diagnostics are also mounted on the support stand. Temporary legs and casters are mounted to the support stand to facilitate handling in the tuning area and linac tunnel. The stand - with its tanks, bridge couplers, and associated equipment - is lowered into the tunnel, wheeled into place, and installed. Then the temporary supports and casters are removed, and the stand is bolted to the tunnel floor. Adjustable legs allow rough alignment to the beamline, and fine adjustments are made with the six-strut support system.

The six-strut support system includes three vertical, one axial, and two horizontal struts. These six struts form an optimally constrained system. Each strut consists of a threaded body and two differentially threaded spherical rod ends. One full turn of the body changes the length of the strut by $1.06 \mathrm{~mm}$. The spherical strut joints allow the structure to expand and contract without binding or warping.

Individual tanks are supported on ledges machined into the tank support brackets. The brackets are bolted to threaded inserts in the tank walls. After a tank is bolted to a bracket, the bracket and tank are match-drilled for dowel pins. The tank support brackets, which are the attachment points for the six-strut support system, are fitted with lifting eyes. The longitudinal struts are located in the center of each module. A bellows is included with the intermodule diagnostics and beam steering to allow for longitudinal expansion and contraction.

The CCL tanks and bridge couplers are frequency-stabilized by pumping temperaturecontolled water through the cavity water passages. A temperature sensor located at each end of a tank senses the rf structure temperature and sends a signal to the temperature control unit (TCU). Stainless steel water manifolds distribute the cooling water to the if structure. One TCU is used per CCL module to control the cavity temperature within $\pm 0.1^{\circ} \mathrm{C}$.

\subsubsection{Vacuum System}

Attached to each CCL tank assembly are a vacuum manifold section and a 500-L/sec ion pump. The vacuum manifolds are bolted together during assembly to form a common vacuum manifold. The manifold is connected to the CCL tanks by demountable joints and bellows. The bellows allow for some misalignment between the manifold and the tanks and accommodate 


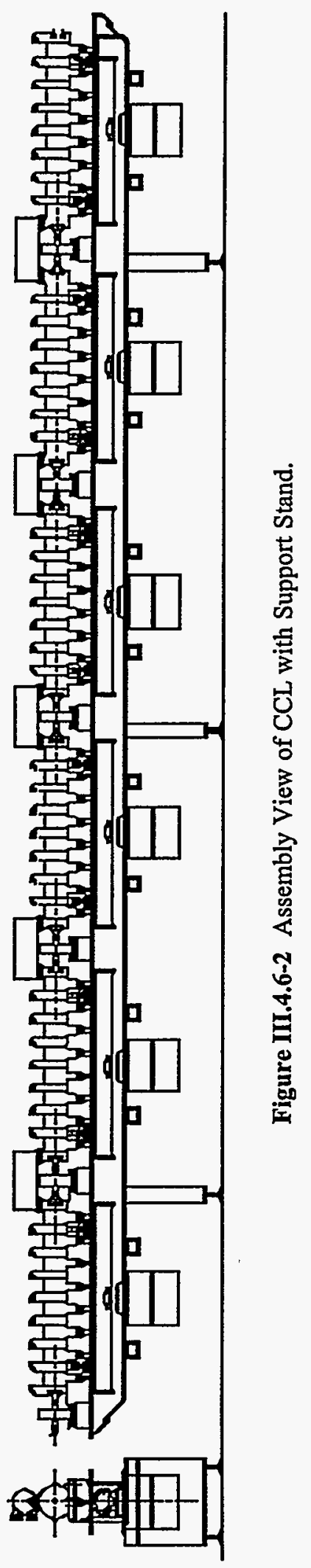


differential expansion and contraction. A gate valve between modules allows a module to be serviced without loss of vacuum in the rest of the linac beamline. Typical vacuum pressure is less than $1 \times 10^{-7}$ Torr.

\subsection{KLYSTRON POWER SYSTEMS}

\subsubsection{Overview}

The three large DTL tanks, matching section bunchers, and CCL are powered by klystrons driven by modulator systems containing the dc power supply, power switching tubes, and energy storage components. Table III.4.7-1 lists the operating parameters common to all these systems, and Table III.4.7-2 lists specific parameters for the different linac sections. The pulse width can be varied to allow for easier conditioning of the accelerator cavities.

The nominal VSWR is 1.6 with the CCL filled but no beam current. The klystron window is located at a point in the waveguide that is near the null position of the standing wave under these conditions. The VSWR decreases to a value of 1.1 at full beam current because of beam loading. The rf drive level is controlled to maintain a constant field level in the load cavity regardless of beam current.

\subsubsection{Klystrons}

Three different klystrons manufactured by Varian are used as the basis for estimating cost of the linac. A sixth cavity in the Varian tubes increases the If conversion efficiency from less than $50 \%$ to an estimated $63 \%$. This ensures that a high rf efficiency is achieved. Table III.4.7-3 lists the operating parameters of the selected klystrons.

Circulators are installed on all of the klystrons to protect them from high reflected power during cavity arcs and conditioning. Three-port circulators with a single load are used to protect the $425-\mathrm{MHz}$ klystrons. Four-port circulators with two loads are used to protect the 1,275-MHz klystrons.

The circulators and loads handle reflected power under four conditions: normal operating mode, cavity filling, conditioning, and cavity arcs. During cavity filling, there is a triangular spike of reflected power with an amplitude that is about half of the amplitude of the forward power. The total pulse duration is about a quarter of the cavity fill time. The fill time is $35 \mu \mathrm{sec}$ for the DTL and $10 \mu \mathrm{sec}$ for the matching section and CCL. The cavity coupling is adjusted so that with full beam current, the VSWR is $<1.1$. During conditioning, the nominal VSWR with the load cavity filled and no beam current is 1.6. The full power from the klystron is reflected during an arc. This condition persists until the klystron is crowbarred within $10 \mu \mathrm{sec}$. 
Table III.4.7-1 Klystron Operating Parameters Common to the Entire Injector

\begin{tabular}{|c|c|c|c|c|}
\hline Parameters & Maximum & Minimum & Nominal & Units \\
\hline Radio-frequency duty factor & 3 & 0.1 & 1.5 & $\%$ \\
\hline Pulse width - DTL & 550 & 100 & 535 & $\mu \mathrm{sec}$ \\
\hline Pulse width - CCL & 550 & 100 & 510 & $\mu \mathrm{sec}$ \\
\hline Pulse rate & 60 & 1 & 30 & $\mathrm{~Hz}$ \\
\hline Pulse-to-pulse amp. variation & \pm 0.2 & \pm 0.2 & \pm 0.2 & $\%$ \\
\hline Pulse-to-pulse time jitter & \pm 10 & \pm 6 & \pm 8 & nsec \\
\hline Pulse ripple, total & 0.5 & 0.5 & 0.5 & $\%$ \\
\hline Pulse droop & 3 & 1 & 3 & $\%$ \\
\hline Supply voltages & - & - & $480 \mathrm{~V}, 3 ø, 60 \mathrm{~Hz}$ & - \\
\hline
\end{tabular}


Table III.4.7-2 Klystron Operating Parameters for Linac Subsystems

\begin{tabular}{|c|c|c|c|c|}
\hline Parameters & DTL & MS & $\mathrm{CCL}$ & Units \\
\hline Operating frequency & 425 & 1,275 & 1,275 & $\mathrm{MHz}$ \\
\hline Peak output power & 4 & 1 & 9 & MW \\
\hline Radio-frequency output power, average & 120 & 30 & 270 & kW \\
\hline Radio-frequency output waveguide & WR1800 & WR650 & WR650 & - \\
\hline Number of amplifiers & 3 & 2 & 10 & - \\
\hline Peak power, VSWR < 1.1 (full beam) & 4 & 0.8 & 9 & MW \\
\hline Peak power, VSWR $=1.25$ & 3.6 & 0.72 & 8.1 & MW \\
\hline Peak power, VSWR = 1.6 (no beam) & 2.8 & 0.56 & 6.3 & MW \\
\hline
\end{tabular}


Table III.4.7-3 Varian Klystron System Parameters

\begin{tabular}{|c|c|c|c|c|}
\hline Parameters & DTL & MS & $\mathrm{CCL}$ & Units \\
\hline Tube type & VKP-8812 & TBD by Varian ${ }^{\mathrm{a}}$ & VKL-7796(M) & - \\
\hline Frequency & 425 & 1,275 & 1,275 & $\mathrm{MHz}$ \\
\hline Peak power output & 4 & 1 & 9 & MW \\
\hline Average power output & 120 & 30 & 270 & $k W$ \\
\hline Efficiency & 57 & 50 & 53 & $\%$ \\
\hline Beam voltage (maximum) & 130 & 70 & 140 & $k V$ \\
\hline Beam current (maximum) & 57 & 32 & 121 & A \\
\hline Beam power (maximum) & 7.0 & 2.0 & 16.9 & MW \\
\hline Modulation type & mod-anode & mod-anode & mod-anode & \\
\hline Mod-anode voltage, full beam & 130 & TBD & 140 & kV \\
\hline Mod-anode voltage, cut-off & -4 & TBD & -3 & $\mathrm{kV}$ \\
\hline Peak inverse voltage rating & 26 & TBD & 30 & $\mathrm{kV}$ \\
\hline Heater voltage & 24 & 25 & 25 & V \\
\hline Heater current & 75 & 12 & 30 & A \\
\hline Magnet voltage (maximum) & 200 & 200 & 200 & v \\
\hline Number of magnet coils & 6 & TBD & 9 & - \\
\hline Magnet power (maximum) & 6 & 2.5 & 10 & $\mathrm{~kW}$ \\
\hline Tube weight & 1,700 & TBD & 700 & lb \\
\hline Solenoid magnet weight & 2,400 & TBD & 1,400 & Ib \\
\hline High-voltage seal insulation & Oil & Oil & Oil & - \\
\hline Cooling water inlet temperature & $25 \pm 15$ & $25 \pm 15$ & $25 \pm 15$ & \\
\hline Collector water flow (minimum) & 120 & 20 & 200 & $\mathrm{gal} / \mathrm{min}$ \\
\hline Collector pressure drop & 60 & 55 & 55 & psic \\
\hline Body water flow (minimum) & 26 & TBD & 13 & $\mathrm{gal} / \mathrm{min}$ \\
\hline Body pressure drop & 60 & TBD & 70 & psi \\
\hline Output window coolant (medium) & $\mathrm{N}_{2}$ & TBD & TBD & - \\
\hline Output window coolant, flow & 200 & TBD & TBD & $\mathrm{ft}^{3} / \mathrm{min}$ \\
\hline Solenoid water flow & 5 & 5 & 5 & $\mathrm{gal} / \mathrm{min}$ \\
\hline Tube lifetime $\left(m t^{b f}\right)^{b}$ & 100,000 & 100,000 & 100,000 & $\mathrm{~h}$ \\
\hline Mounting position & $\begin{array}{l}\text { Vertical, } \\
\text { cathode down }\end{array}$ & $\begin{array}{l}\text { Vertical, } \\
\text { cathode down }\end{array}$ & $\begin{array}{l}\text { Vertical, } \\
\text { cathode down }\end{array}$ & - \\
\hline
\end{tabular}

\footnotetext{
a $\mathrm{TBD}=$ to be determined.

b $\mathrm{mtbf}=$ mean time before failure.

c psi $=$ pounds per square inch.
} 


\subsubsection{Modulators}

The modulator system supplies the operating voltages for the klystron gun, solenoids and filament, and mod-anode. Each modulator consists of the following:

- Regulator unit - SCR-controlled, 480 V, $3 \phi$ input; $240-528$ V, $3 \phi$ ac output; air cooled and insulated.

- Transformer-rectifier unit - high-voltage transformer $(140 \mathrm{kV})$, solid-state rectifier stack and filter choke; oil insulated, convectively cooled via finned tank structure.

- Crowbar and capacitor unit - energy storage capacitors and compensation circuits to minimize voltage droop during the pulse; high-voltage crowbar to dissipate stored energy and protect klystron in the event of a fault.

- Klystron tank - supports klystron, magnet, and associated equipment; contains isolation transformer, klystron heater supplies, modulator switch tubes, and related supplies; is oil filled.

- Controls - low-level controls and instrumentation, interlock logic, magnet power supplies, VME crate.

The first two components are located outside, adjacent to the klystron gallery. The other items are located inside. The modulators are energized from a $480 \mathrm{~V}, 3 \phi, 60-\mathrm{Hz}$ input. All cooling for the klystron is routed through the modulator. Table II.4.7-4 lists the specifications for the modulators for the different accelerator sections.

\subsection{BEAM DIAGNOSTICS}

\subsubsection{Overview}

Diagnostics are an important part of any accelerator, especially one with high average current. Typical parameters of interest are beam size, position in the beam tube, energy, phase, bunch length, and beam loss. Beam loss is particularly important. Excessive beam loss causes so much activation that hands-on maintenance is impossible. The microstrip beam diagnostics and the beam loss monitors are described below. 
Table III.4.7-4 Klystron Modulator Specifications by Subsystem

\begin{tabular}{lcccl}
\hline \multicolumn{1}{c}{ Parameters } & DTL & MS & CCL & Units \\
& & & & \\
\hline & 350 & 100 & 750 & $\mathrm{kVA}$ \\
Power, maximum load & 75 & 20 & 100 & $\mathrm{kVA}$ \\
Power, no load & 425 & 125 & 900 & $\mathrm{~A}$ \\
Current per phase, maximum load & 275 & 75 & 600 & $\mathrm{~kW}$ \\
Cooling required, maximum load & & & & \\
& 25 & 8 & 30 & $\mathrm{~kW}$ \\
Cooling required, no load & 160 & 80 & 220 & $\mathrm{gal} / \mathrm{min}$ \\
Coolant flow & 35 & 35 & 35 & ${ }^{\circ} \mathrm{C}$ \\
Coolant inlet temperature & 60 & 60 & 60 & $\mathrm{psi}^{\mathrm{a}}$ \\
Coolant pressure & & & & \\
\hline
\end{tabular}

a $p s i=$ pounds per square inch. 


\subsubsection{Microstrip Beam Diagnostics}

Microstrip beam diagnostics measure the centroids of the transverse beam positions, angles, synchronous phase, and energy. 15 Beam positions are measured by comparing the relative signal amplitudes from opposite sides of the microstrip probes. The beam centroid angle is calculated from the centroid position at two different probes that are some distance apart. Energy is derived from the time-of-flight measurement between two separated probes. Synchronous phase is determined by comparing the probe signal from the beam bunch centroid with the cavity field phase from a monitor loop in the cavity. The cavity field phase is corrected for the drift time to the probe derived from the energy measurement.

The probe is very compact and consists of four microstrip transmission lines that are connected to multilayer stripline transmission lines upstream and downstream. These striplines transport the signals through a vacuum flange to processing electronics. Image currents from the beam bunch induce electromagnetic signals onto the upstream and downstream striplines as the beam passes through the probe. The upstream signal is proportional to the beam intensity. Differences in the signal from opposite lobes of the probe are proportional to the centroid offset.

This diagnostic system has been extensively tested and characterized. 16 Processing electronics that interface with the VME standard and processing software that is compatible with the software chosen for this linac are available.

\subsubsection{Beam Loss Monitors}

Although the microstrip probes give information on the gross characteristics of the beam, they give no information on the beam halo. It is this halo that leads to beam loss and activation of the structure and equipment in the linac tunnel. Beam loss monitors measure the radiation induced when the beam halo strikes the beam tube. Beam loss measurement permits fine-tuning and optimization of the accelerator to minimize activation. Beam loss monitors provide prompt warning of catastrophic beam loss. This signal is used to provide fast shut-down to minimize damage to the structure and activation.

The beam-loss-monitor system consists of fast-ionization detectors and processing electronics that monitor the radiation level. 17 The ionization detectors are mounted close to the beamline to monitor the radiation from $(\mathrm{p}, \gamma)$ reactions. The system provides two trip points. The higher-level trip point is used to shut the accelerator down in $10 \mu \mathrm{sec}$ and protect against burnthrough from a major beam loss.

\subsubsection{Other Diagnostics}

Additional linac diagnostics include beam current toroids, segmented apertures, wire scanners, and Faraday cups. A slit and collector emittance unit is used for initial commissioning. 


\subsection{CONTROL SYSTEM}

\subsubsection{Overview}

This section includes the control system, data acquisition system, and the distribution systems for the rf master oscillator signals and accelerator timing.

\subsubsection{Data Acquisition and Control}

The linac data acquisition and control system is a distributed network consisting of operator, network, and hardware interfaces, as shown schematically in Figure III.4.9-1. EPICS is used to develop the front-end control software. ${ }^{8}$ Commercial and custom VXI modules are used to interface the control system to the linac hardware. These VXI modules are located in the racks of the systems to which they interface.

The operator interface is a Unix-based workstation with an X-Window graphical user interface (GUI). The workstation can be located at any point on the network and still maintain full functionality. The user can generate and alter control displays and can access interactive control programs, the archiver, the alarm handler, custom code, and various tools. Five operator workstations are associated with the linac; however, the linac can be controlled by any workstation on the network.

The network provides the communication path between the operator workstations, input and output crates, other hardware in the linac control system, and the controls for the rest of the facility. Transmission control protocol/Internet protocol (TCP/IP) is the underlying protocol for the network. A fiber-based Ethernet network is used for noise immunity.

The input/output controller (IOC) provides an input/output interface between the accelerator hardware and the rest of the control system. The standard crate uses the VXI standard and contains a network interface and various interfaces to the accelerator hardware. The crate controller is a Motorola 68040 -based processor. The VxWorks operating system provides the basis for real-time control, allowing the 68040 processor to run its software autonomously with respect to all other devices in the system.

Table III.4.9-1 lists the number of each type of data channel for each of the major subsystems, and Table III.4.9-2 lists the numbers of the various VXI components required for each subsystem.

The EPICS and the IPNS Upgrade accelerator control systems are described in Section II.8. The same control system is used to control the linac. 


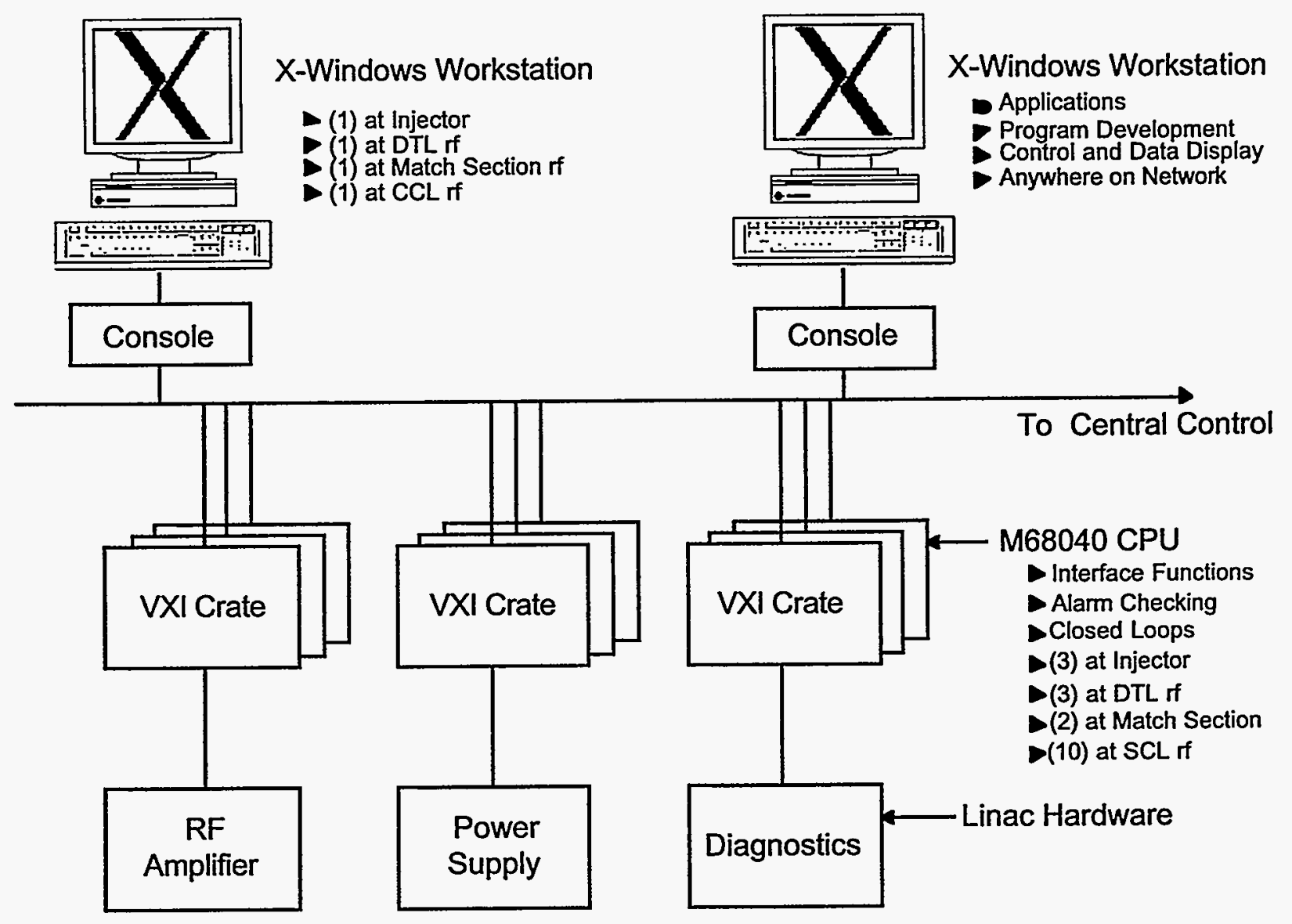

Figure III.4.9-1 Control System Diagram 
Table III.4.9-1 Number of Data Channels Required by Linac Subsystems

\begin{tabular}{lrrrrr}
\hline \multicolumn{1}{c}{ Channel Type } & LIS & DTL & MS & CCL & Total \\
\hline Digital input & 144 & 288 & 192 & 1,040 & 1,664 \\
Digital output & 104 & 156 & 120 & 600 & 980 \\
Slow analog input & 226 & 312 & 232 & 1,160 & 1,930 \\
& & & & & \\
Slow analog output & 72 & 84 & 72 & 360 & 588 \\
Counter/timer outputs & 13 & 36 & 24 & 120 & 193 \\
$\begin{array}{l}\text { Waveform inputs } \\
\text { Total }\end{array}$ & 24 & 36 & 36 & 120 & 216 \\
& & & & & 5,571 \\
\hline
\end{tabular}


Table III.4.9-2 Number of VXI Components Required by Linac Subsystems

\begin{tabular}{|c|c|c|c|c|c|}
\hline Component & LIS & DTL & MS & $\mathrm{CCL}$ & Total \\
\hline VXI crate & 3 & 3 & 2 & 10 & 18 \\
\hline Crate controller & 3 & 3 & 2 & 10 & 18 \\
\hline Network interface & 3 & 3 & 2 & 10 & 18 \\
\hline 32-bit digital in & 6 & 9 & 6 & 40 & 61 \\
\hline 32-bit digital out & 6 & 6 & 4 & 20 & 36 \\
\hline 64-channel analog input & 4 & 6 & 4 & 20 & 34 \\
\hline 16-channel analog output & 6 & 6 & 6 & 30 & 48 \\
\hline Counter/timer outputs & 4 & 9 & 6 & 30 & 49 \\
\hline 4-channel waveform digitizer & 7 & 9 & 9 & 30 & 55 \\
\hline Low-level radio-frequency & 2 & 3 & 2 & 10 & 17 \\
\hline
\end{tabular}




\subsubsection{Radio-Frequency Reference Signal Distribution and Low-Level System}

The RFQ and DTL operate at $425 \mathrm{MHz}$, and the matching section and CCL operate at $1,275 \mathrm{Mhz}$. These two frequencies are harmonically related. There are 17 amplifiers that power the linac, and precise frequency and phasing are maintained. The if reference generation system for the linac is based on one developed for the GTA at LANL. ${ }^{18}$

The rf reference generation system is configured as a star distribution network and provides coherent, phase-stable signals to the low-level rf subsystems for each amplifier and any other systems requiring these signals. The transport network maintains the phase error to $\pm 0.036^{\circ}$ over 50 meters of phase-stable cable.

The low-level rf provides the necessary amplitude and phase feedback control for the amplifiers. The VXI-based modules used in this linac are derived from ones developed at Fermilab. ${ }^{19}$ Phase and amplitude can be electronically adjusted. In addition, feed-forward control is available. In normal operation, the reference frequency signal comes from the reference signal distribution system. However, for cavity warm-up and conditioning, an internal VCO tracks the cavity frequency during warm-up.

\subsubsection{Timing Signal Distribution}

A dedicated timing system is required because of the asynchronous nature of TCP/IP Ethernet. This linac uses a system developed for the GTA at LANL. 20 A central master timing device generates the timing signals and distributes them to the various subsystems via optical fibers. The network is configured as a star with dedicated fibers for each subsystem IOC.

Four types of timing signal are provided: the system clock, system trigger, time tag clock, and time tag reset. The 5-MHz system clock is the reference for all timing events in the system. The system trigger synchronizes control and data acquisition. The time tag and time tag reset provide a unique event number and date/time stamp for recorded events.

This timing is implemented in VME modules, with EPICS used for programming. Ten channels with 200-nsec resolution are provided in each module. More channels per IOC can be provided by multiple modules. 


\subsection{DIMENSIONED FACILITY LAYOUT}

A cross-sectional view through the DTL is shown in Figure III.5.1-1. Figure III.5.1-2 is a plan view of the linac showing the linac tunnel and the klystron gallery. The locations of major utility requirements including electricity, cooling water, and gas cooling for the DTL if windows are shown on the drawing.

\subsection{UTILITY REQUIREMENTS}

Table III.5.2-1 lists the electricity and cooling water requirements for the $400 \mathrm{-MeV}$ linac. The values are the installed capacities and not the amounts actually consumed. All cooling for the klystrons is included in the numbers listed for the modulators. The water flow values given for the DTL load cooling system are for the water provided from the facility to the load heat exchangers. The 425-MHz loads use a 50:50 mixture of ethylene glycol and water from a closedloop system for cooling. The $1,275-\mathrm{MHz}$ loads are cooled by plain water because the if absorption is adequate at that frequency. 


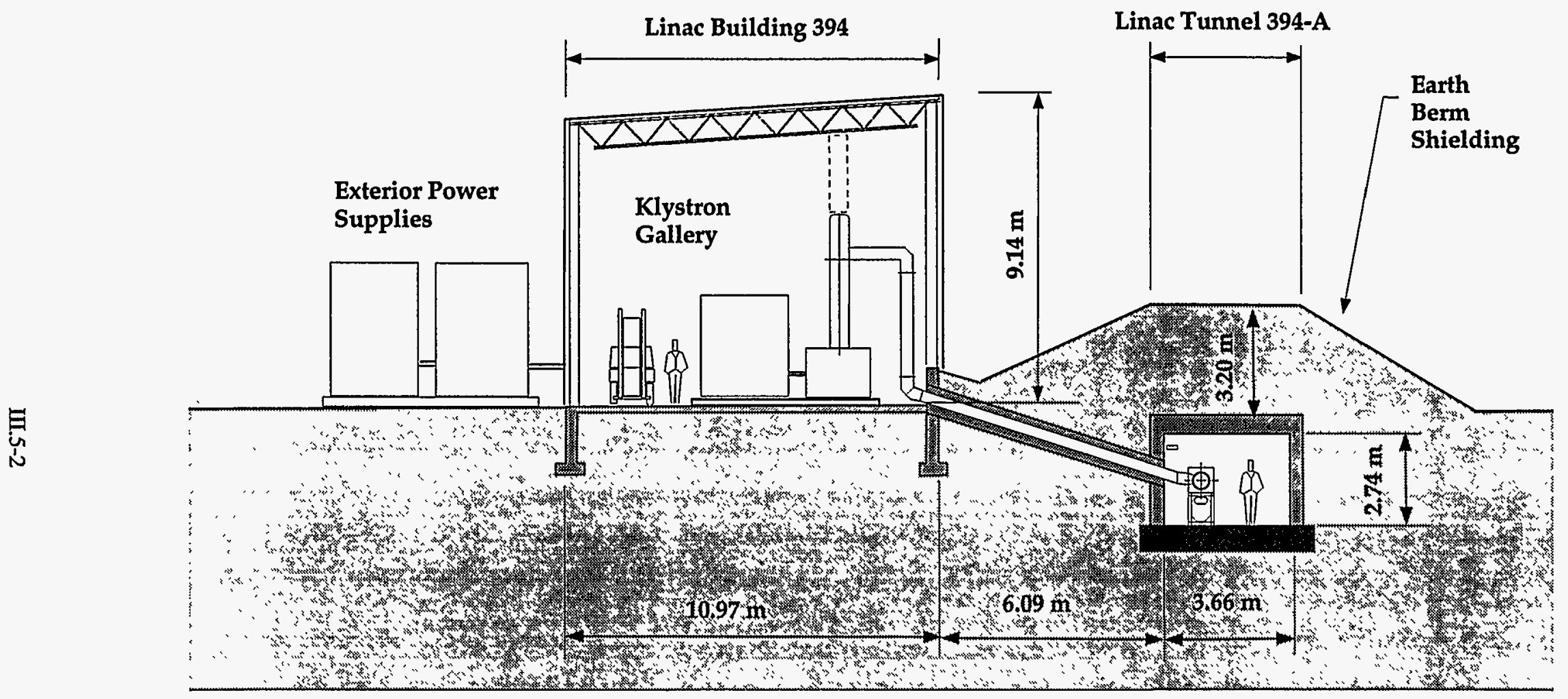




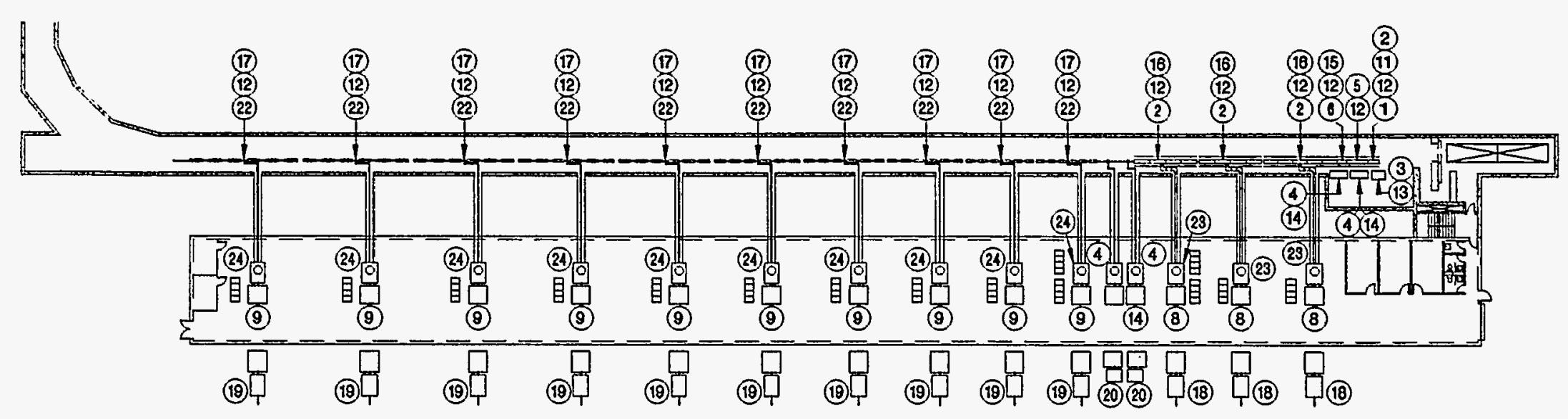

DI WATER, 20 deg. $C$ max. 60 psi min./120 psi max.

(1) $1.3 \mathrm{gpm}$
$6 \mathrm{gpm}$
(3) $01 W 7 \mathrm{gpm}$
(4) $12 \mathrm{gpm}$
(6) $3 \mathrm{gpm}$
$(8) 5 \mathrm{gpm}$
$60 \mathrm{gpm}$
(8) $180 \mathrm{gpm}$
(8) $220 \mathrm{gpm}$
(10) $100 \mathrm{gpm}$
(22) $150 \mathrm{gpm}$
(23) $35 \mathrm{gpm} 50 / 50$ ETHYLENE GLYCOL AND WATER
(24) $50 \mathrm{gpm}$

(11) $208 \mathrm{~V} \mathrm{AC/3} \mathrm{ph}$ AT $23 \mathrm{~A}$

(12) $110 V$ AC AT $3 A$

(13) $208 \mathrm{~V} \mathrm{AC/3}$ ph AT $100 \mathrm{~A}$

(14) $208 \mathrm{~V} \mathrm{AC/3}$ ph AT $80 \mathrm{~A}$

(15) $220 \mathrm{~V}$ AC/1 ph AT 15A

(18) $220 \mathrm{~V} \mathrm{AC/1}$ ph AT $30 \mathrm{~A}$

(17) $220 \mathrm{~V}$ AC/1 ph AT 45A

(18) $480 \mathrm{~V} \mathrm{AC/3}$ ph AT $180 \mathrm{~A}$

(18) $480 \mathrm{~V} \mathrm{AC} / 3$ ph AT $900 \mathrm{~A}$

(20) $480 \mathrm{~V} \mathrm{AC/3}$ ph AT $125 \mathrm{~A}$

(23) $35 \mathrm{gpm}$

(24) $50 \mathrm{gpm}$

PLAN WIEM OF THE LINAC SHOWMNG LOCATIONS OF UTILITY REOUIFIEMENTS 
Table III.5.2-1 Power and Water Cooling Requirements for the Injector Linac System

\begin{tabular}{|c|c|c|c|c|c|c|c|}
\hline \multirow[b]{2}{*}{ Description } & \multicolumn{2}{|c|}{ Maximum Water } & \multicolumn{5}{|c|}{ Installed Total A/Phase } \\
\hline & $\underset{(\mathrm{gal} / \mathrm{min})}{\mathrm{Q}_{\min }}$ & $q(k W)$ & $\begin{array}{c}1 \phi / \\
110 \mathrm{~V}\end{array}$ & $\begin{array}{c}1 \$ / \\
220 \mathrm{~V}\end{array}$ & $\begin{array}{c}3 \phi l \\
208 \mathrm{~V}\end{array}$ & $\begin{array}{c}3 \phi \prime \\
480 v\end{array}$ & $\begin{array}{c}\mathrm{N}_{2} \text { at } \\
25 \mathrm{psig} \\
\left(\mathrm{ft}^{3} / \mathrm{min}\right)\end{array}$ \\
\hline \multicolumn{8}{|l|}{ LIS } \\
\hline Ion source/beamline & $7(\mathrm{DI})$ & 13 & - & - & - & - & - \\
\hline Ion source electronics & - & - & - & - & 100 & - & - \\
\hline $2,200-\mathrm{L} / \mathrm{sec}$ turbopump (3) & 1 & 6 & - & 30 & - & - & - \\
\hline Roughing pumps (3) & - & - & - & 30 & - & - & - \\
\hline LEBT solenoids (2) & 6 & 13.2 & - & - & - & - & - \\
\hline RFQ amplifier & 12 (DI) & 6 & - & - & 40 & - & - \\
\hline RGDTL amplifier & 12 (DI) & 5 & - & - & 40 & - & - \\
\hline Cooling system & 7 & 7 & - & 9 & - & - & - \\
\hline \multicolumn{8}{|l|}{ DTL Tanks } \\
\hline Waveguide window & - & - & - & - & - & - & 600 \\
\hline Cooling system & 125 & 100 & - & 45 & - & - & - \\
\hline \multicolumn{8}{|l|}{ DTL RF System } \\
\hline Klystrons & \multicolumn{2}{|c|}{ incl. in modulator } & - & - & - & - & 600 \\
\hline Load cooling system ${ }^{a}$ & 25 & 15 & - & 30 & - & - & - \\
\hline Modulator/HVPS ${ }^{b}$ & 480 & 400 & - & - & - & 700 & $\cdot$ \\
\hline \multicolumn{8}{|l|}{ DTL Vacuum System } \\
\hline Ion pump controllers & - & - & - & 210 & - & - & - \\
\hline \multicolumn{8}{|l|}{ Matching Section } \\
\hline Klystrons & \multicolumn{2}{|c|}{ incl. in modulator } & - & - & - & - & - \\
\hline Modulator/HVPS & 100 & 75 & - & - & - & 140 & - \\
\hline Circulator and load & 30 & 2 & - & - & - & - & - \\
\hline Cavity cooling system & 30 & 25 & - & 15 & - & - & - \\
\hline Quadrupoles & 18 & 50 & - & - & - & - & - \\
\hline Quadrupole PS & - & - & - & - & 225 & - & - \\
\hline Ion pump controller & - & - & - & 30 & - & - & - \\
\hline \multicolumn{8}{|l|}{ CCL Module } \\
\hline Cooling systems & 1,000 & 900 & - & - & 60 & - & - \\
\hline Quadrupoles & 120 & 215 & - & - & - & - & - \\
\hline Quadrupole PS & - & - & - & - & 1,000 & - & - \\
\hline \multicolumn{8}{|l|}{ CCL Vacuum System } \\
\hline Ion gauge and controller & - & - & 30 & - & - & - & - \\
\hline lon pump controller & - & - & - & 900 & - & - & $\cdot$ \\
\hline \multicolumn{8}{|l|}{ CCL RF System } \\
\hline Klystrons & \multicolumn{2}{|c|}{ incl. in modulator } & - & - & - & - & - \\
\hline Modulator/HVPS & 2,200 & 2,500 & - & - & - & 4,500 & - \\
\hline Circulator and load & 300 & 60 & - & - & - & - & - \\
\hline
\end{tabular}

a Closed, recirculating loop of 50/50 ethylene glycol/water with heat exchange to facility water.

b HVPS = high-voltage power supply.

c $\mathrm{PS}=$ power supply. 


\subsection{REFERENCES}

1. L. Schroeder, K.N. Leung, and J. Alonso (editors), Proceedings of the Workshop on Ion Source Issues Relevant to a Pulsed Neutron Source, Lawrence Berkeley Laboratory Report No. LBL-36347 (Oct. 24-26, 1994).

2. J. DeBoers, SNOW - A Digital Computer Program for the Simulation of Ion Beam Devices, Sandia International Report No. SAND 79-1027 (1980).

3.' Documentation for TRACE: An Interactive Beam Transport Code, Los Alamos National Laboratory Report No. LA-10235-MS (1985).

4. PARMULT, Los Alamos Accelerator Code Group (Los Alamos National Laboratory, Los Alamos, N.M.).

5. G. Boicourt and J. Merson, PARMILA Users and Reference Manual (Los Alamos National Laboratory, Los Alamos, N.M., Jan. 10, 1990).

6. K.R. Crandall, TRACE 3-D Documentation, Los Alamos National Laboratory Report No. LA-11054-MS (Aug. 1987).

7. K.R. Crandall, private communication, as cited in F.W. Guy, in Proceedings of the 1993 Particle Accelerator Conference, p. 122 (1993).

8. L. Dalesio et al., "EPICS architecture," in Proceedings of the International Conference on Accelerator and Large Experimental Physics Control Systems, pp. 278-281 (KEK, Tsukaba, Japan, Nov. 11-15, 1991).

9. M.J. Barnes et al., "A variable duty factor beam chopper for a $300 \mathrm{keV}$ injection beamline," in EPAC Proceedings (1994).

10. J.G. Alessi, J.M. Brennan, and A. Kponou, "H' source and low energy transport for the BNL RFQ preinjector," in Proceedings of the Fifth International Symposium on Production and Neutralization of Negative Ions and Beams.

11. J.M. Potter, "A parallel planar triode array high power if system for accelerator applications," in EPAC 90 Proceedings, Editors Frontiers, p. 991 (1990).

12. R.E. Morgado et al., "The effects of proton beam quality on the production of gamma rays for nuclear resonance absorption in nitrogen," in SPIE Proceedings on Substance Detection Systems, Vol. 2092, p. 503 (1994).

13. U.S. Patent No. 5,179,350. 
14. J.H. Billen and A.H. Shapiro, "Post-coupler stabilization and tuning of a ramped gradient drift tube linac," presented at 1988 Linear Accelerator Conference, CEBAF Report No. 89-001, p. 128 (1989).

15. J.D. Gilpatrick et. al., Overview of the microstrip beam diagnostics, Los Alamos National Laboratory Report No. LA-CP-89-460 (1989).

16. S.P. Jachim et al., in Proceedings of the 1993 IEEE Particle Accelerator Conference, IEEE 93 CH 3279-7, p. 1154 (1993).

17. C.R. Rose, C.M. Fortgang, and J.P. Power, "GTA beamloss monitor system," in 1992 LAC Conference Proceedings, AECL-10728, p. 365 (1992).

18. A.H. Regan and P.M. Denney, "RF Reference Generation for the Ground Test Accelerator," in Proceedings of the 1991 Particle Accelerator Conference, p. 2946 (1991).

19. B.E. Chase and R.J. Pasquinelli, "VXI based low level RF system for Fermilab linac upgrade," in Proceedings of the 1993 Particle Accelerator Conference, p. 2355 (1993).

20. M. Stettler et al., "A distributed timing system for synchronizing control and data acquisition," in 1992 LAC Conference Proceedings, AECL-10728, p. 820 (1992). 
IV

Target Stations 



\subsection{GENERAL}

This chapter describes the target stations that produce the neutrons utilized by the neutron scattering instruments at IPNS Upgrade. A target station consists of the biological shield and hot cell and all the components they contain, including target and moderators, together with the necessary external cooling and ventilation systems. The pulses of protons from the accelerator impinge on the target, and the resulting spallation interactions produce large quantities of fast neutrons. The moderators slow these neutrons down to energies suitable for use by the neutron scattering instruments, and a portion of the neutrons pass through ports in the target station shielding into beam lines, which lead to the instruments.

The design for the target stations for IPNS Upgrade is based on a careful assessment of the requirements of the neutron scattering instruments. The IPNS Upgrade design is site-specific in that it uses existing buildings at Argonne. The target stations accept horizontal proton beams. from the accelerator and provide neutron beams that make optimal use of the existing experiment halls. Vertical injection of the proton beam into the target, which is another option, was rejected because it appeared to be more expensive than horizontal injection and offered too few advantages in view of the cost. This site-specific design results in significant cost savings while introducing few, if any, compromises in the performance of the facility.

Section IV.1.2 gives a brief overview of the target station components. These components are described and analyzed in detail in the remaining sections of this chapter.

Section IV.2 provides a detailed analysis of the power deposited in the target and of the cooling system required to extract this power. This includes a thermal-hydraulic analysis of the heat transfer from the target material to the coolant and calculations of thermally induced stresses in the target. This section also contains a discussion of metallurgy and corrosion questions that relate to the choice of machinable tungsten alloy as the target material.

Section IV.3 describes the moderators and reflectors and the associated cooling systems. Monte Carlo calculations of the power deposition in the different moderators are used to size the various water and cryogenic-liquid cooling systems. The neutron beam currents produced by the moderators are also calculated.

Section IV.4 provides details of the biological shield. The effectiveness of the shielding in reducing the radiation dose rate is also analyzed. Section IV.5 describes the remote handling facilities used to manipulate the activated target and moderator components in order to make repairs or to replace damaged components. Section IV.6 covers the various levels of containment designed to prevent accidental release of activity.

The controls and instrumentation required for safe and reliable operation of the target stations are discussed in Section IV.7.

Section IV.8 discusses the neutron beam ports and their interfaces to the portions of the neutron beam lines that are external to, and extend radially from, the target stations. These 
external beam lines are considered to be parts of the neutron scattering instruments and are discussed in Chapter V.

\subsection{OVERVIEW OF THE DESIGN}

IPNS Upgrade has two target stations, one operating at $10 \mathrm{~Hz}$ and one operating at $30 \mathrm{~Hz}$. The purpose of the target stations is to house and shield the primary-neutron-producing targets and the moderators that slow down the primary neutrons to useful energies. Neutron beam ports penetrate the shield to allow neutrons from the moderators to reach instruments outside the shield. The target stations also contain internal facilities for neutron activation and radiation-effects experiments. A switching system alternately directs pulses of $2-\mathrm{GeV}$ protons delivered at $30 \mathrm{~Hz}$ from the accelerator system to the two target stations. The $10-\mathrm{Hz}$ target station receives one pulse out of three. The $30-\mathrm{Hz}$ target station receives two pulses out of three in an iambic pattern, with an average frequency of $20 \mathrm{~Hz}$ under normal operating conditions, but it can efficiently utilize the full $1 \mathrm{MW}$ at $30 \mathrm{~Hz}$ if desired.

The target stations are housed in existing buildings to which slight modifications are made. Chapter VI describes the conventional facilities for IPNS Upgrade. The target stations are new construction. The two target stations are identical except for details of the moderators, which are differently optimized for the $10-\mathrm{Hz}$ and $30-\mathrm{Hz}$ instruments. This chapter describes the components of one of these target stations and, unless otherwise noted, all these components are duplicated for the second target station. Both targets and associated cooling systems are able to accept the full $1-\mathrm{MW}$ beam power. The $30-\mathrm{Hz}$ scattering instruments are designed to tolerate the $1 / 30$-s inter pulse interval, so when the $10-\mathrm{Hz}$ target is not operating, the $30-\mathrm{Hz}$ target can usefully accept the full-power $30-\mathrm{Hz}$ beam.

Figure IV.1.2-1 shows the two target stations located in Buildings 369 and 370. Each station provides neutron beams through 18 beam ports, derived from six individually tailored moderators optimized for the intended scattering instruments. This figure also shows a representative set of neutron scattering instruments located in appropriate positions on the neutron beam lines, as discussed in Chapter V. The inner portions of the target stations, consisting of the targets, moderators, and innermost reflectors, can move into the adjacent hot cells, equipped with shielding windows and manipulators, for modification and repair. The figure shows these components withdrawn into the servicing cells. Space is available within the experiment halls for other types of experimental capabilities, such as a pulsed-muon spinrotation facility. Appendix $\mathrm{C}$ describes this and other possible facilities that are not within the scope of this project. The outlying Buildings 367 and 371 and the vaults shown in Figure IV.1.2-1 house components of the cooling and containment systems.

Figure IV.1.2-2 schematically illustrates the target and moderator layout. The target is of the split-target or flux-trap design. This geometry allows the installation of four moderators in the tangentially viewed wing configuration and two flux-trap moderators. Each of the moderators provides three neutron beams from one viewed surface. The flux-trap moderators provide good 


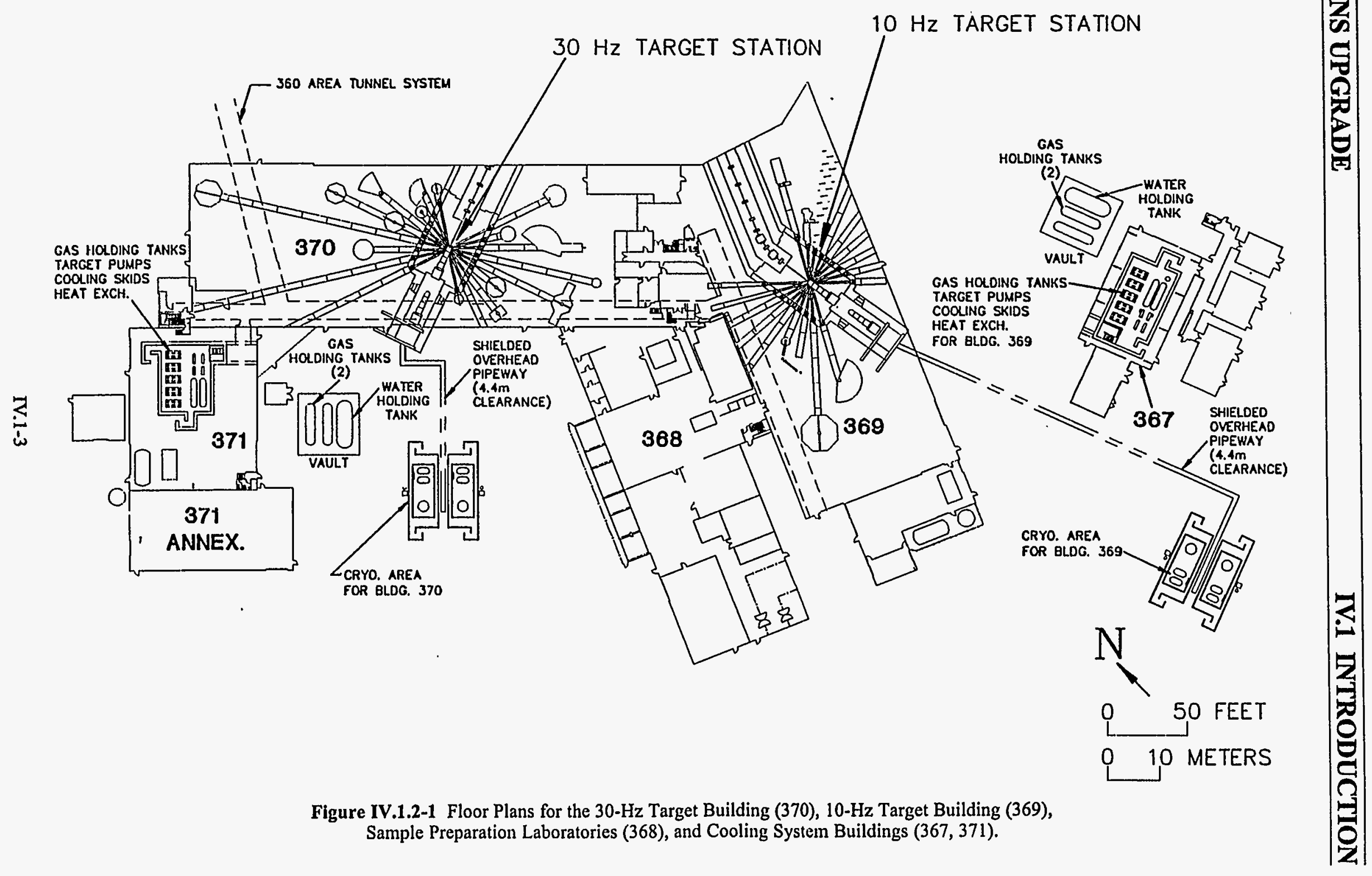




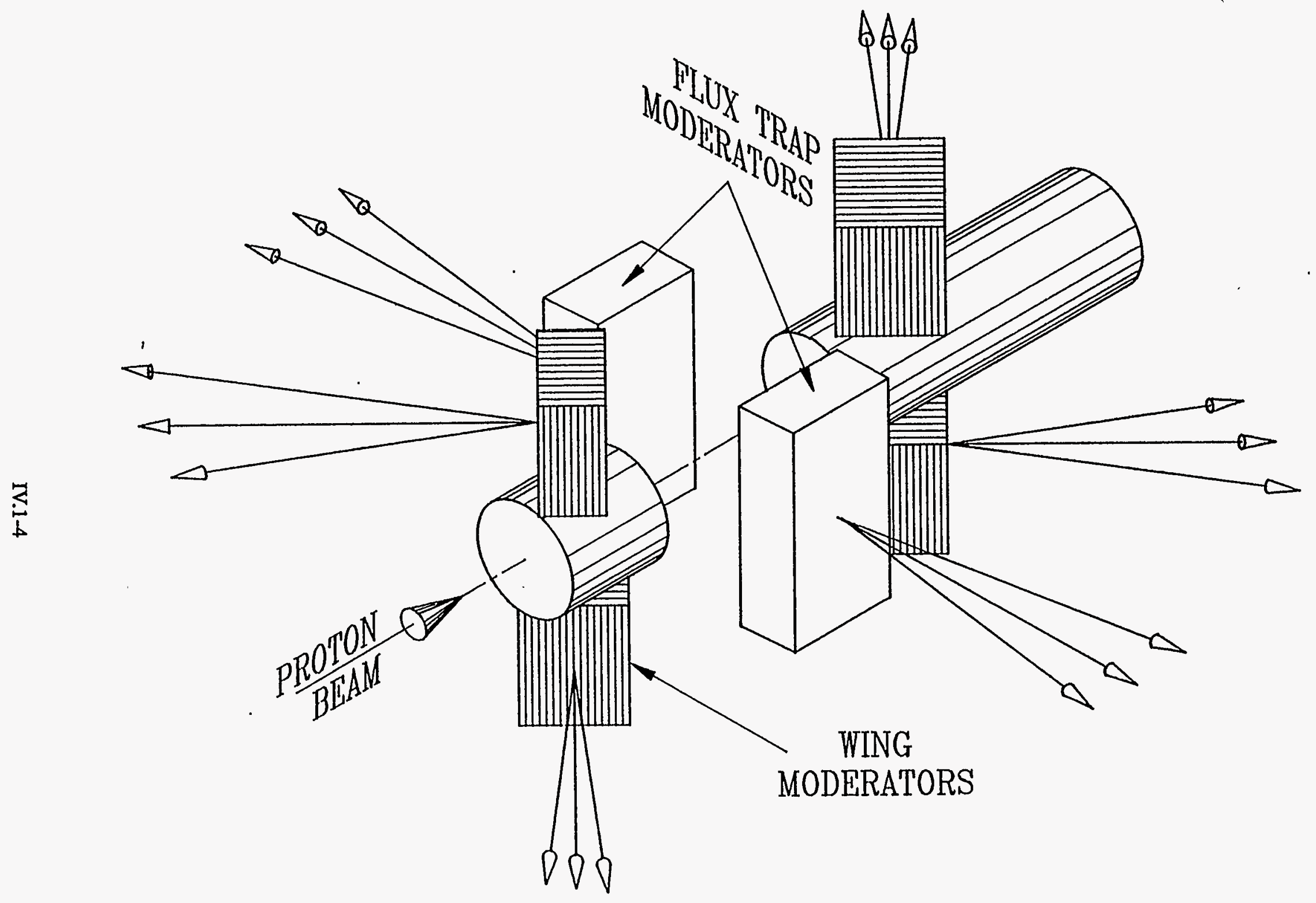

Figure IV.1.2-2 Schematic View of the Target and Moderator System. (Arrows indicate the directions of extracted neutron beams.). 
coupling to the primary neutron source. They are viewed at near $90^{\circ}$ to the proton beam direction, so the neutron beams do not view target material struck by the proton beam and there is little high-energy neutron contamination in the beams. In addition, because the flux-trap moderators are centered about the axis of the target, they are taller than the canonical wing moderator. This feature is advantageous for a number of scattering instruments and is easily accommodated with the horizontal proton beam geometry.

Figure IV.1.2-3 shows the design for the split target. This design is analyzed in detail in Section IV.2. The split is arranged so that neutrons are evenly shared between moderators in the upstream and downstream positions, while the gap is chosen in order to minimize losses in neutron production, maximize coupling to the flux-trap moderators, and avoid a direct view of the target by the neutron beam lines, thus reducing fast neutron backgrounds. The diameter of the target is selected to maximize neutron production and coupling to the moderators while minimizing proton leakage to the surroundings. The total target length exhausts the nuclear cascade and leaves little power in the downstream shielding. The proton beam profile used for target design is Gaussian with $5-\mathrm{cm}$ full width at half maximum, which is roughly the maximum size feasible from the point of view of proton beam transport. The two sections of the target share one common coolant flow. The local power densities in the target are low enough that the heat generated can be easily handled by conventional water cooling. The neutronic and thermal consequences of tighter focusing or more uniform spreading of the proton beam, to provide performance improvements or extend target lifetimes, are part of the preconstruction R\&D program.

The target material is a machinable tungsten alloy. A thin, plasma-sprayed titanium coating is applied for improved corrosion resistance. This target provides high atomic number and mass density for high neutron yield, high strength, and good properties at high temperature. Cooling is by light water that passes across the face of the target disks, guided by channels machined in the disk surfaces. Disk thicknesses in the upstream section are chosen to maintain single-phase (no boiling) convective heat transfer at the surface, while disk-center temperatures govern disk thickness in the back section. Disks are spring-loaded to take up dimensional changes due to thermal and radiation-induced growth. The front part of the target consists of circular disks with guide notches, clamped with four compression springs. The back part of the target consists of circular disks similar to those in the front, compressed by nested coil springs.

The target vessel is of welded stainless-steel construction, contains both front and back sections of the target, and provides water-flow plena that distribute cooling water to the disks and all parts of the vessel, including all springs. Cooling water is carried in and out from the downstream side of the target. The cooling plena located above and below the target disks allow the target cooling water in the plena to act as premoderator for the wing moderators. The cooling pipes clear the moderators and irradiation facilities and exit downstream of the biological shield, where they connect to the cooling system. The vessel and its attached piping constitute a single unit to facilitate handling and increase integrity. 


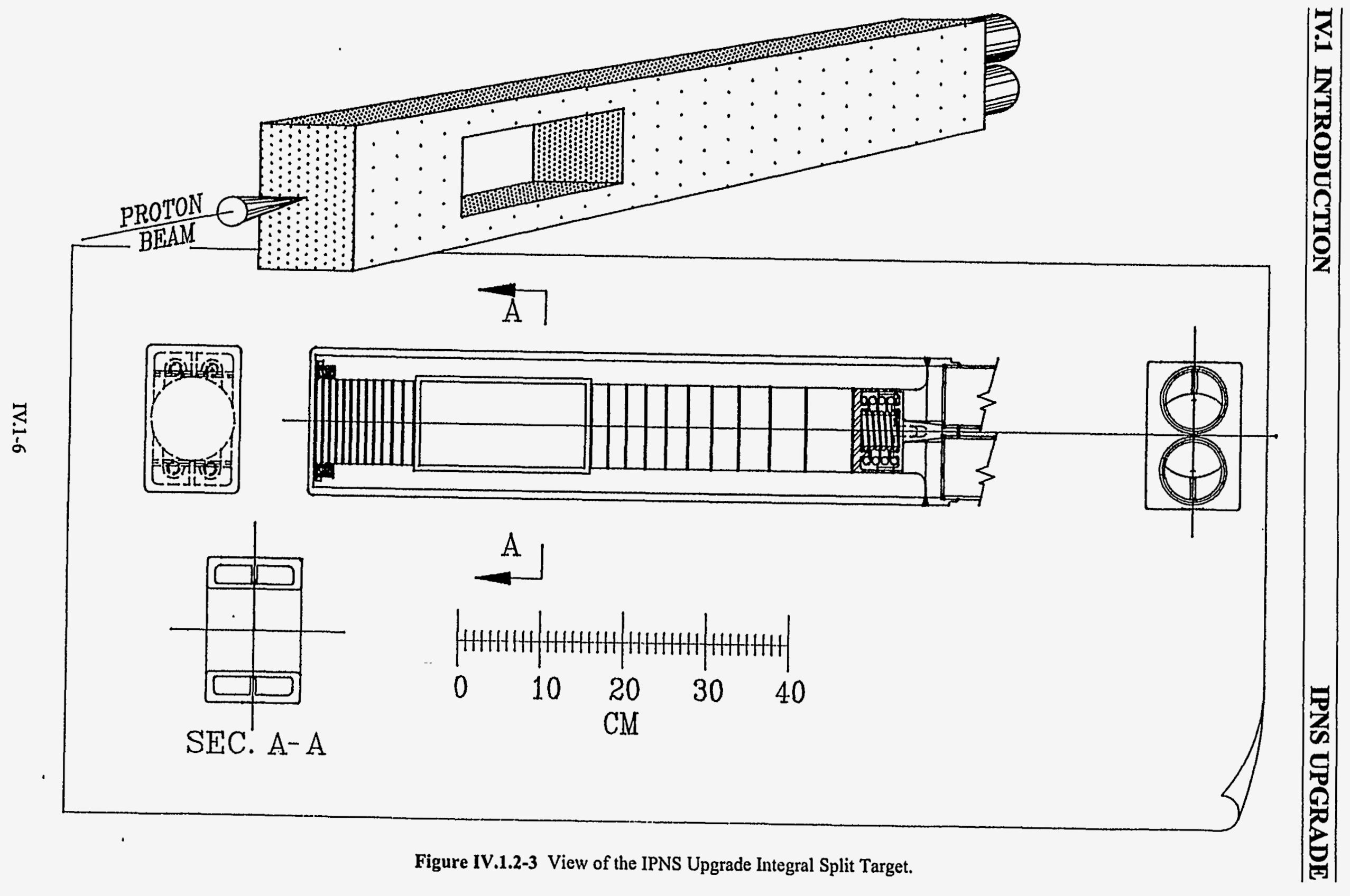


Moderators are of several types, optimized according to the intended applications for scattering instruments and arranged according to the requirements dictated by the surrounding scattering instruments. Three beams are extracted from each of the six viewed surfaces to provide a total of eighteen beams, nine beams on each of the two sides of each target station. The beams are separated by angles of $13^{\circ}$ and are about $1.4 \mathrm{~m}$ apart at the shield face, $6 \mathrm{~m}$ from the center. The moderators selected and placed as described in this design satisfy the requirements of the neutron scattering instruments as described in Chapter V.

Wing moderators are nominally $10 \times 10 \times 5 \mathrm{~cm}^{3}$, and flux-trap moderators are nominally $20 \times 10 \times 5 \mathrm{~cm}^{3}$. Moderating materials are light water, liquid methane, and liquid hydrogen. Premoderators significantly reduce the power that is removed at cryogenic temperatures and reduce the rate of radiation damage in the cryogenic media. Moderator vessels and housings are constructed of aluminum alloy. Figure IV.1.2-4 shows the six moderators in greater detail. Moderator and premoderator fluids flow in the attached piping, which consists of vacuuminsulated tubing. The exterior tubing is actively cooled. Each moderator and its associated piping constitute a single integrated unit to facilitate replacement and repair. Moderator piping emerges downstream of the biological shield where it connects to the cooling systems. Section IV.3 describes the moderators in more detail.

The cryogenic moderators of IPNS Upgrade rely heavily on the use of premoderators placed between the target and the portion of the moderator viewed by the neutron beams. This is a well-known concept, ${ }^{1}$ but one that has not yet been incorporated into any existing pulsed spallation neutron sources. Useful and informative neutronic tests have been performed at the Hokkaido Linac moderator testing facility. ${ }^{2}$ A solid methane moderator with a water premoderator is being installed in the 2-MW Russian IBR-2 pulsed reactor. ${ }^{3}$ Both of these will contribute important experience to the development of the premoderator concept. The function of premoderators is to slow down neutrons from high energies, so that most of the resulting heat is removed at ambient temperature. The ambient medium performs most of the thermalization, while the cryogenic material produces cold neutrons. Both water, and metal hydrides with higher proton densities than water, are possible premoderator materials. In the IPNS Upgrade cryogenic moderators, the face viewed by the neutron scattering instruments is cryogenic material, but the medium surrounding it on the other five faces is ambient-temperature water. This geometry is described in detail in Section IV.3.1. Only a few neutron mean-free-paths are required to thermalize the neutrons to the lower temperature in the cryogenic region.

Reflectors of beryllium, or of beryllium and heavy metal, are placed around the moderators to reflect neutrons back into the moderators. Considerable heat is generated in the reflectors due to the slowing down of neutrons and the absorption of gamma radiation. Active cooling removes this heat. The complete target-moderator-reflector assembly provides spaces for all the beam ports and coolant piping. Figure IV.1.2-5 shows the arrangement of the spaces in the bottom half of the reflector. 


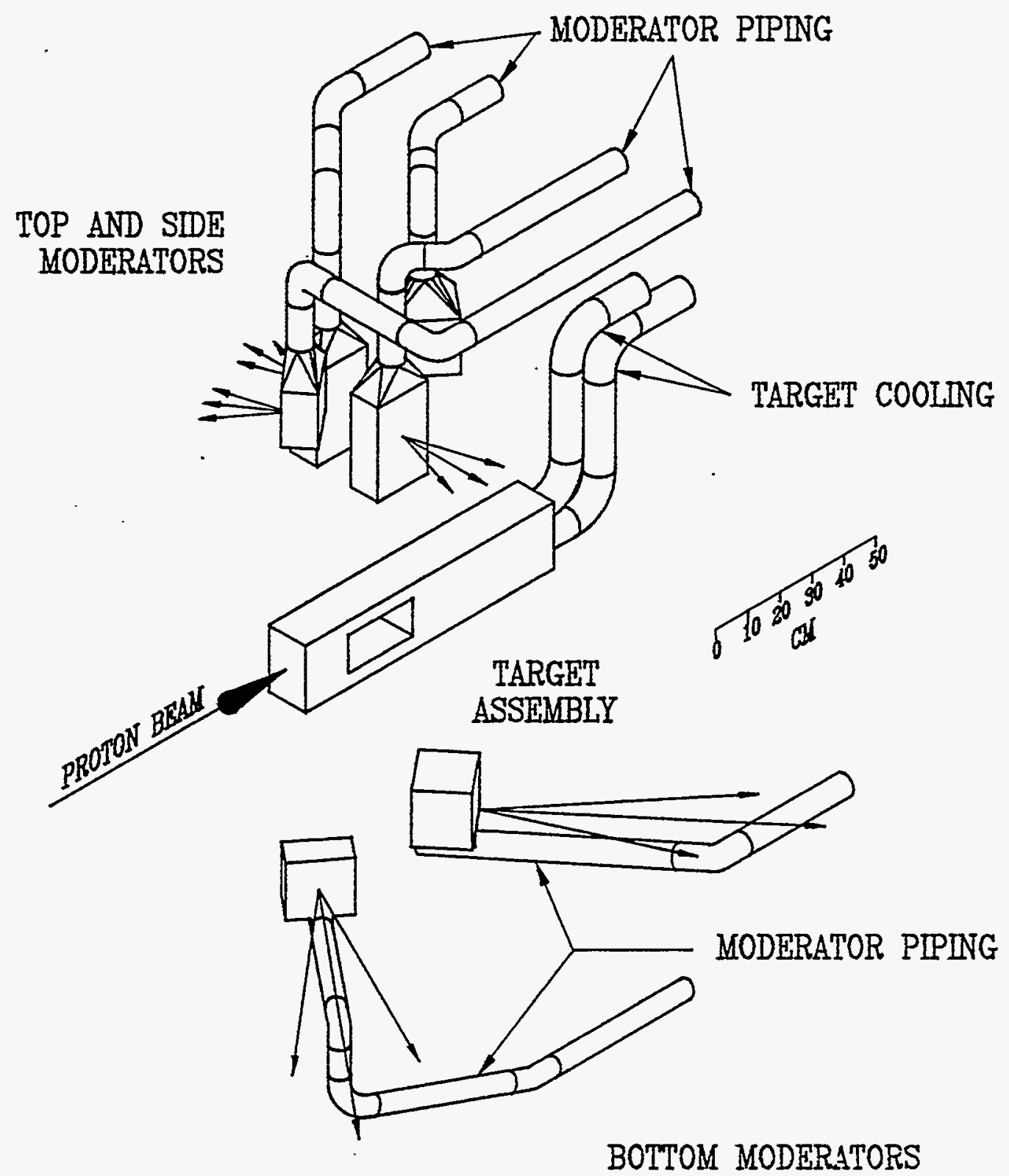

Figure IV.1.2-4 Six Moderators Arranged around the Target. (Exploded view; arrows emerging from the moderator faces represent the 18 neutron beams.) 


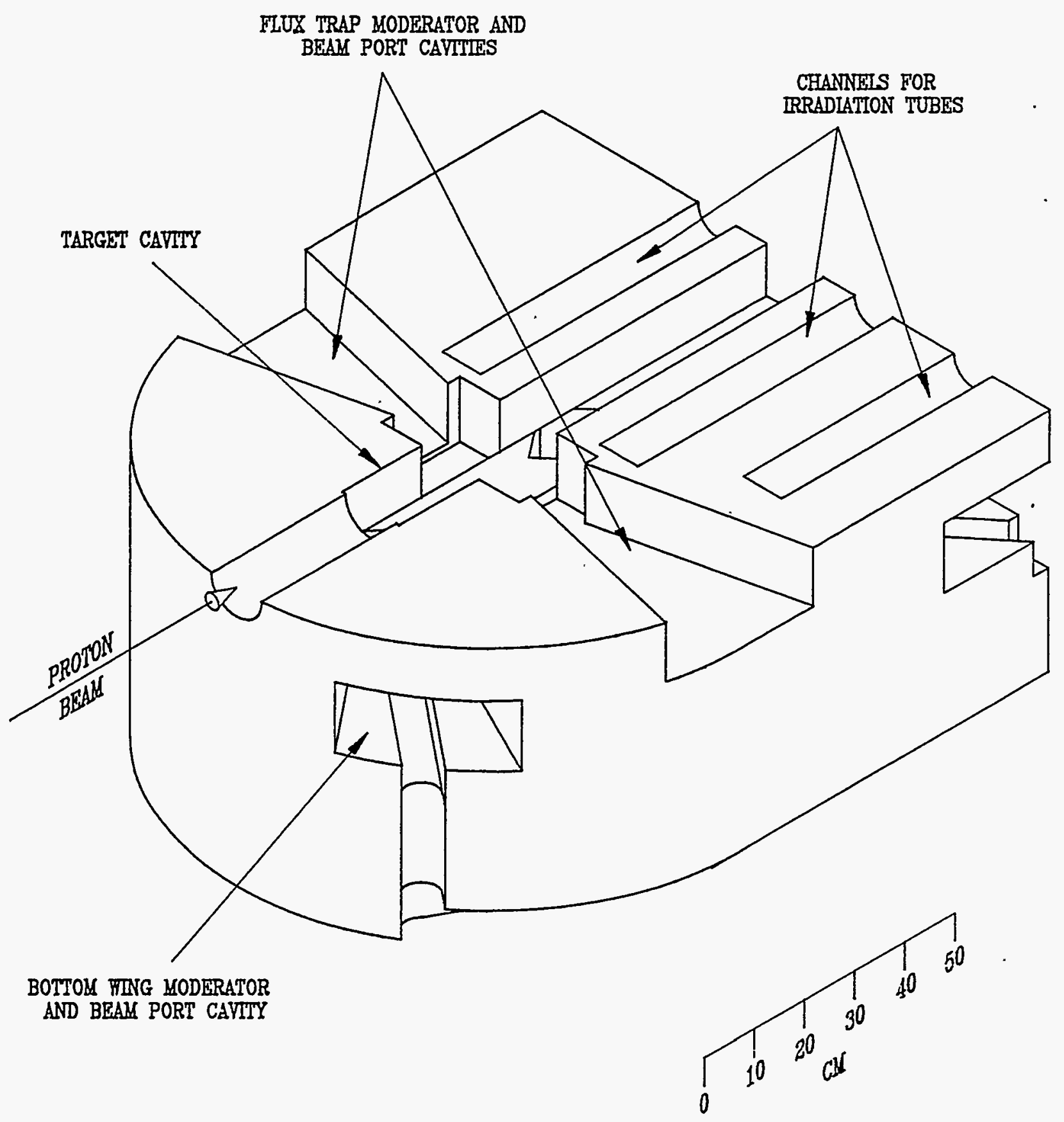

Figure IV.1.2-5 Bottom Reflector, Showing Neutron Beam Ports, Coolant Passages, Proton Duct, and Target and Moderator Cavities. 
Layers of neutron-absorbing material, or decouplers, are placed between the reflector and the moderators to preserve short pulses, because neutrons dwell longer in the reflector than in the moderators. These decouplers are transparent to neutrons with high energies, but opaque to neutrons at the lower energies of interest. The neutron beam ports require neutron-absorbing void liners, because the ports represent voids across which neutrons can fly back into the moderators after considerable delays. The reflector, decouplers, and void liners require active cooling because they are heated by scattered and absorbed neutrons and gamma rays. Watercooling systems remove this heat.

Three irradiation tubes provide close access to the target for neutron activation and neutron radiation-effects experiments. Samples can be inserted and withdrawn through the curved tubes from remote locations within and outside the remote-handling facility even while the target is in operation. These irradiation facilities are described in Section V.4. The irradiation tubes are gas-cooled to remove heat from irradiated samples and from the walls of the tubes.

The target-moderator-reflector assembly, portions of the piping for their respective cooling systems, portions of the irradiation tubes, and the massive steel shield blocks downstream from the target comprise an assembly called the target train. Figure IV.1.2-6 shows the target train, including the reflector system and the neutron beam ports. The target-moderatorreflector assembly arrangement allows the target and any of the moderators to be individually removed from the system without disturbing the other parts, which simplifies servicing and allows easy replacement of components.

During operation, the target train rests inside a sealed, helium-filled volume defined by a stationary liner inside the shield. This target tank is shown in Figure IV.1.2-7. The sealed volume provides containment of radioactive gases and solid residue produced in the targetmoderator-reflector assembly. The containment systems are described in Section IV.6. Neutron beams emerge from windows, which are thinned sections in the aluminum target tank. The downstream end of the target train carries a metal sheet that forms a seal with the target tank during operation, which can be disengaged to enable retraction of the target train. The upstream end of the target tank has a cavity for proton beam diagnostics. Figures IV.1.2-8a and IV.1.2-8b show the target-train assembly inserted into the target tank, and Section IV.5 details the steps involved in withdrawing the target-train assembly and servicing it in the hot cell. The target tank can also be removed into the hot cell and replaced.

The biological shield, illustrated in Figure IV.1.2-9, consists of an inner core of iron and an outer layer of concrete extending to a distance of about $6 \mathrm{~m}$ from the target in the shortest dimension that is $90^{\circ}$ from the proton beam direction. The shield is designed so that all parts of the hall housing the scattering instruments can be occupied during operation. The innermost portion of the shield receives active cooling to remove the heat. This shield is described in greater detail in Section IV.4. 


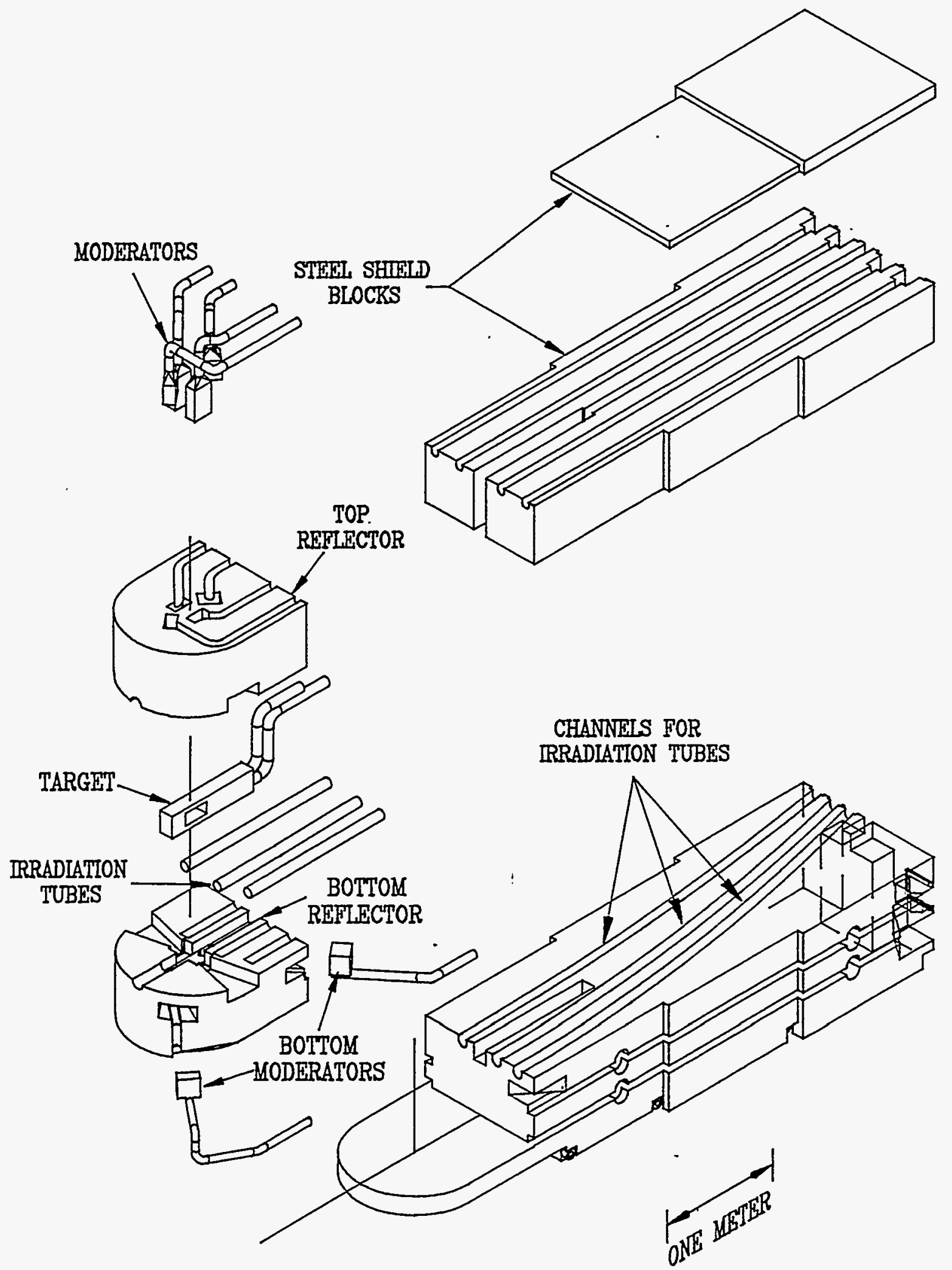

Figure IV.1.2-6 Exploded View of the Target-Train Assembly. 


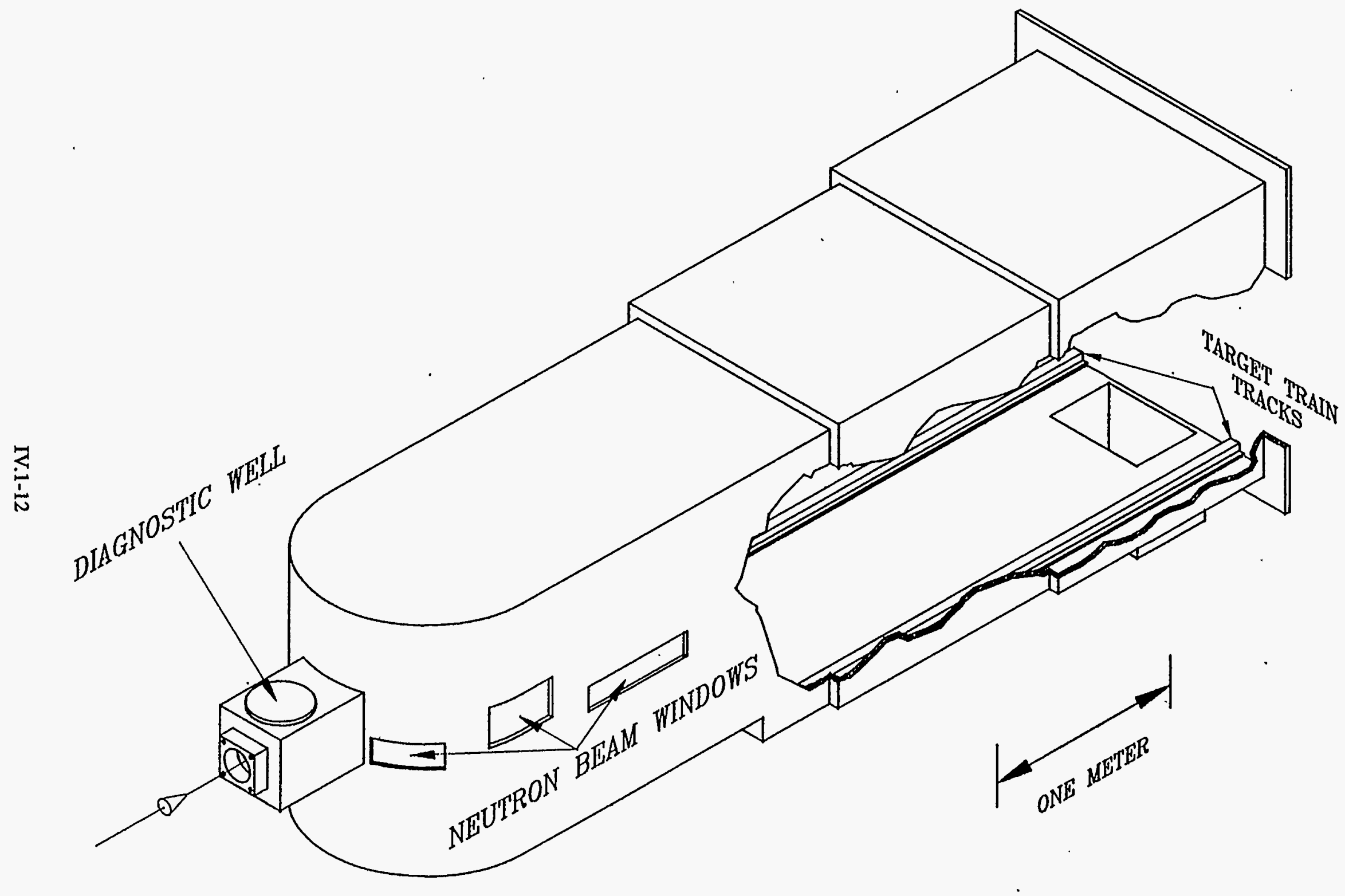

Figure IV.1.2-7 Sealed Target Tank into Which the Target Train Is Inserted for Operation. (Window sections are thinned to improve neutron beam transmission.). 

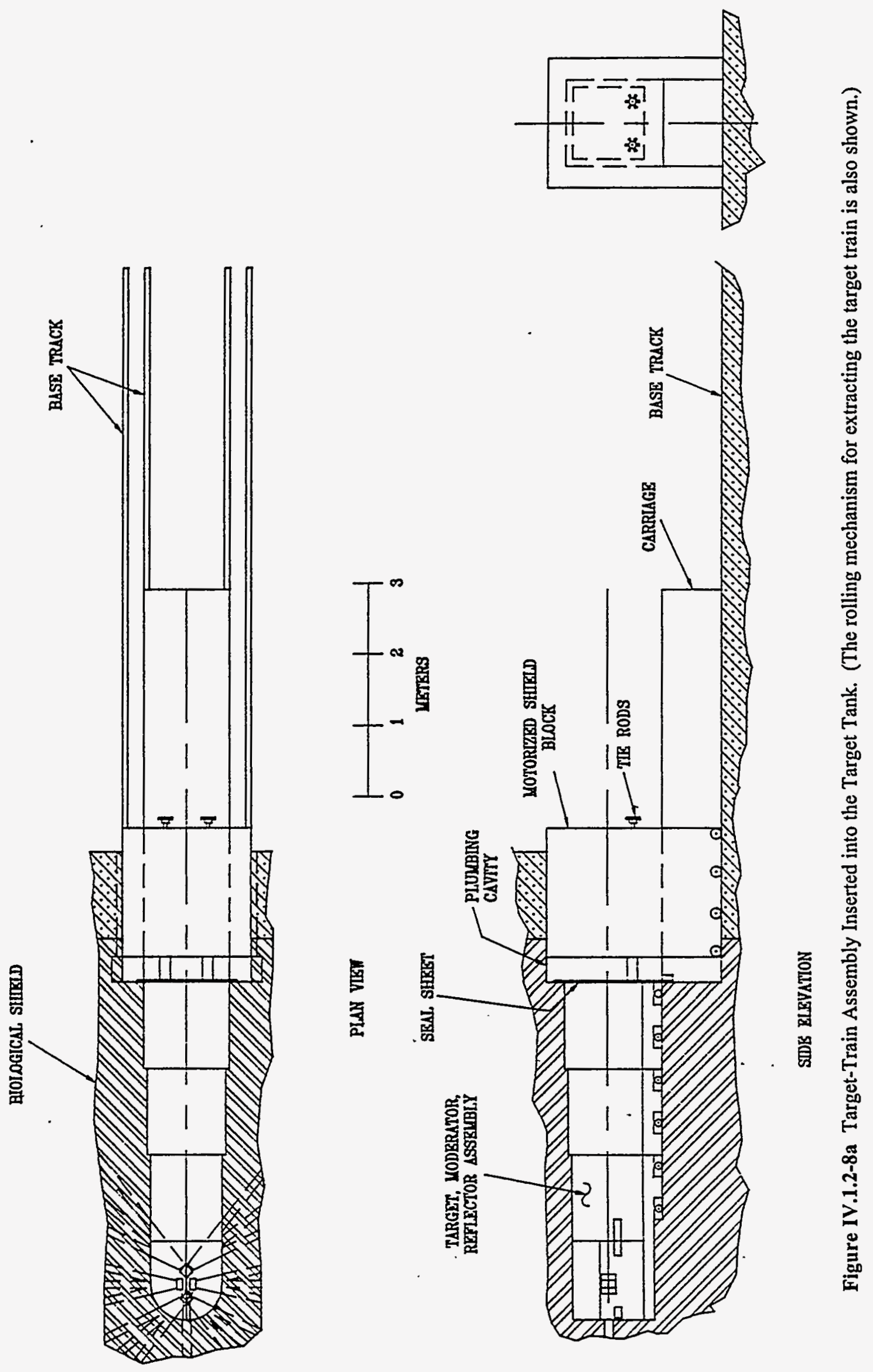


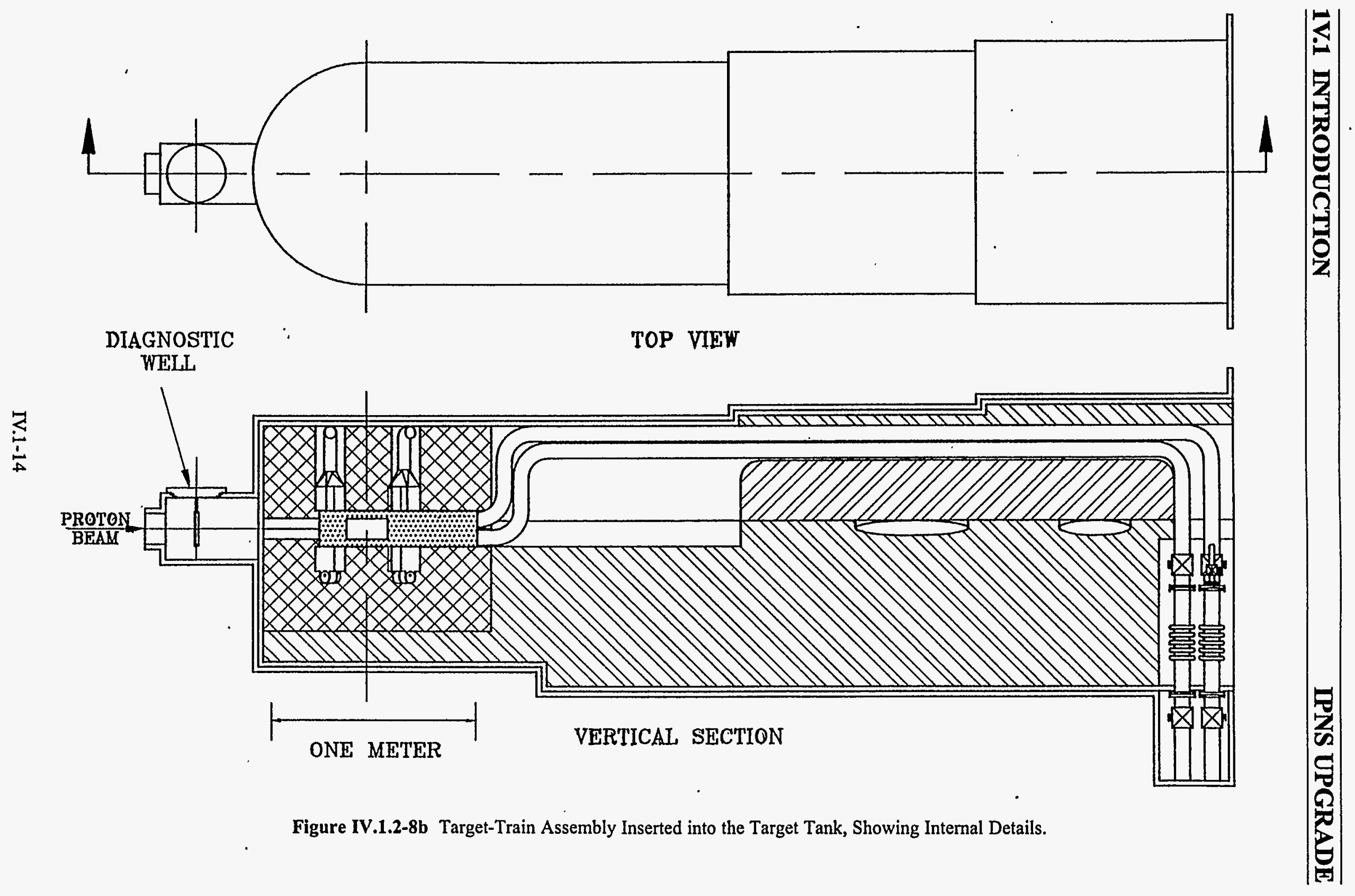




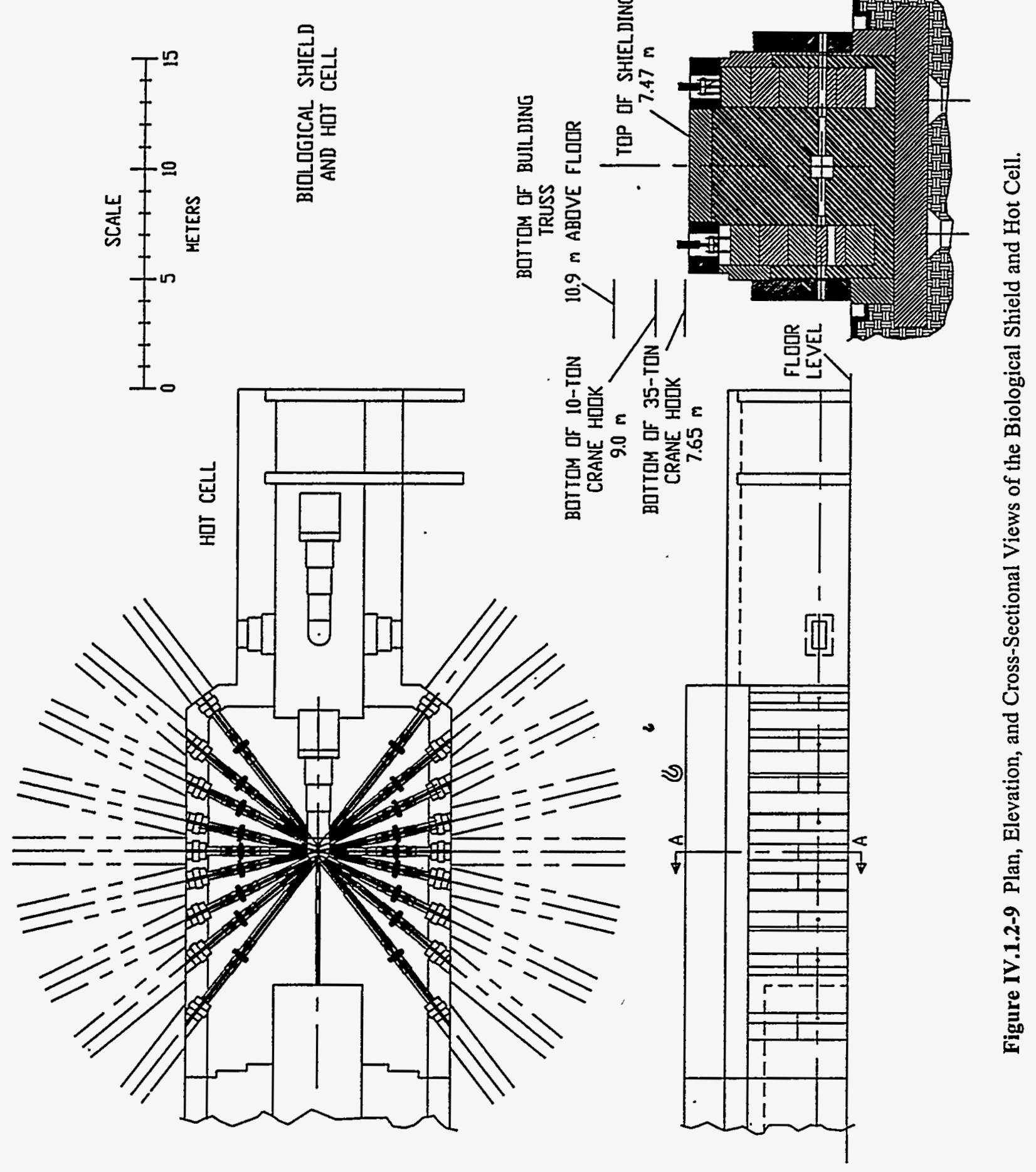


The biological shield rests on a newly-constructed reinforced-concrete pad. The entire structure weighs about 14,000 metric tons and is supported by columns extending to bedrock $24 \mathrm{~m}$ below. This provides long-term stability. A service trench surrounds the biological shield.

Neutrons stream to the instruments through beam ports containing collimating elements or neutron guide tubes. Hydraulically operated gates that can be raised and lowered in guided channels can be closed to attenuate the neutron beams to such a level as to permit hands-on changing of samples in the instruments. The beam ports and beam gates are described in Section IV.8.

Protons from the accelerator system enter the biological shield from the HET line through a water-cooled, double-walled, vacuum-tight window, which is shown in Figure IV.1.2-10. This window provides the interface between the vacuum of the proton beamline and the helium atmosphere of the target tank. Coolant water circulates through the annulus between the tube walls, cooling both the beam pipe and the window. Helical guides separate the inlet and outlet flows. The window has a lifetime of several years, ${ }^{4}$ so its design permits access and replacement. This proton beam port assembly also seals the helium atmospheres in the target tank and the shielding tank.

The proton beam port assembly passes through the shielding tank and extends to the target tank. A stainless-steel proton beam port tube, which has a circular cross section, is inserted in the opening and fastened by four threaded tie rods to the target tank. The insert tube has a series of square guides along its length to ensure alignment. The proton beam port tube is protected from the weight of the stacked steel shield by surrounding it with a steel tube with a square cross section. This steel tube is constructed of at least $1.25-\mathrm{cm}$-thick steel plate and is welded to the target tank housing and to a reinforced area on the shielding tank.

The proton beam port tube is sealed to the target tank with metal O-rings, making it part of the target tank containment barrier. O-rings and a flanged bellows mechanism seal the other end of this tube to the shielding tank, as shown in Figure IV.1.2-10. This provides a seal for the shielding tank atmosphere. The vacuum window insert is also sealed to the proton HET line vacuum chamber external to the shielding tank. 

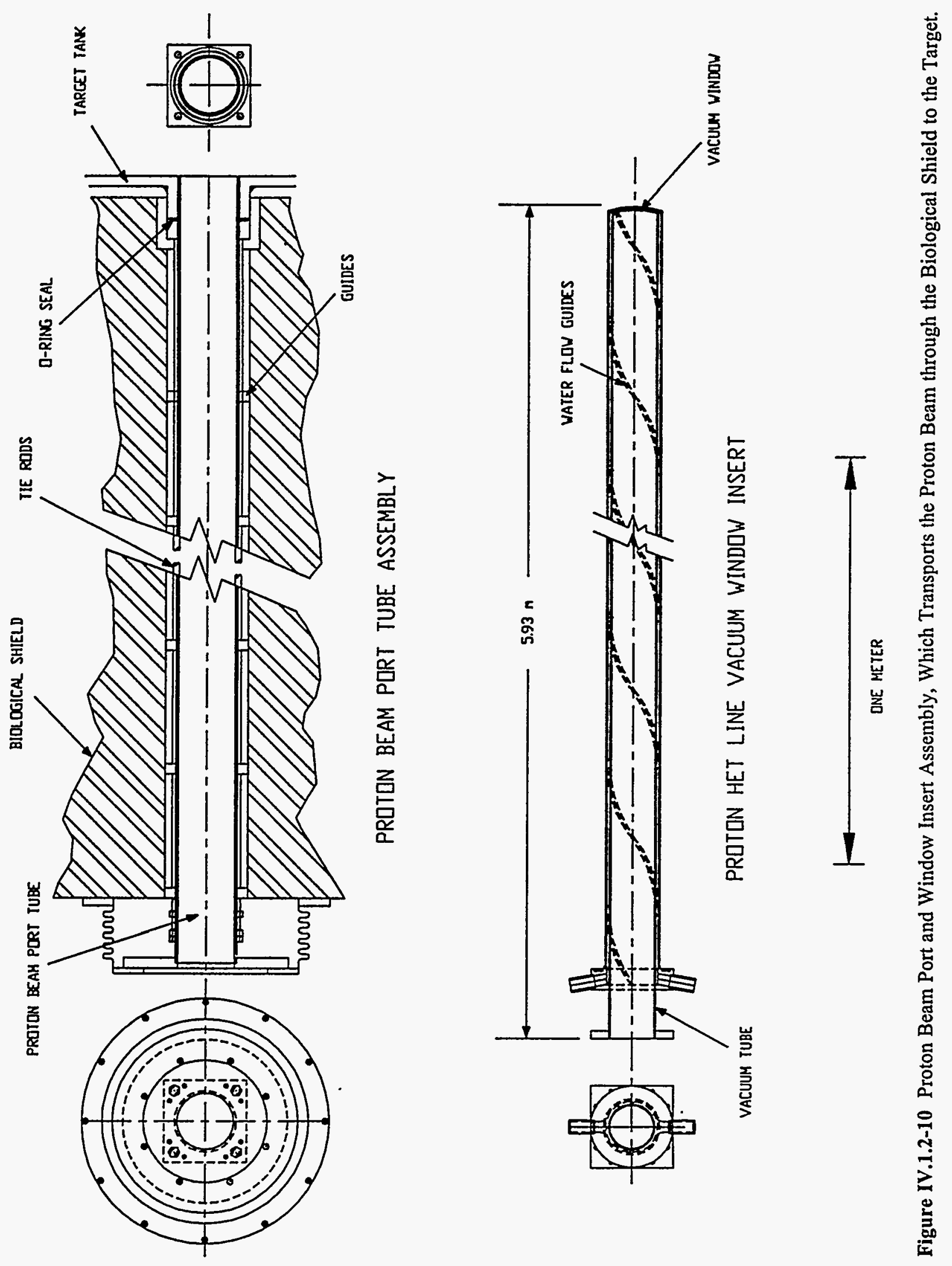


\subsection{REFERENCES}

1. N. Watanabe, Y. Kiyanagi, K. Inoue, M. Furusaka, S. Ikeda, M. Arai, and H. Iwasa, "Preliminary optimization experiments of coupled liquid hydrogen moderator for KENS-II," in Advanced Neutron Sources 1988, Proceedings of the 10th Meeting of the International Collaboration on Advanced Neutron Sources (ICANS X) (Los Alamos, N.M., Oct. 3-7, 1988); IOP Conference Series, No. 97 (Institute of Physics, New York, 1989), pp. 787-797 (1989).

2. Y. Kiyanagi, N. Watanabe, and H. Iwasa, "Experimental studies on neutronic performance of coupled liquid-hydrogen moderator for pulsed spallation neutron sources," Nucl. Instrum. Methods A312:561-570 (1992).

3. A.A. Beljakov, V.G. Ermilov, V.L. Lomidze, V.V. Melichov, and E.P. Shabalin, "First experience of cold moderator operation and solid methane irradiation at the IBR-2 pulsed reactor," in Proceedings of the 12th Meeting of the International Collaboration on Advanced Neutron Sources (ICANS XII) (Abingdon, U.K., May 24-28, 1993), Rutherford Appleton Laboratory Report No. 94-025, pp. T144-T155 (1994).

4. G.S. Bauer, presented at the Workshop on Materials for Spallation Neutron Sources, Los Alamos, N.M., Feb. 6-10, 1995 (unpublished). 
This section describes the target design and provides a detailed analysis of target performance and requirements. Section IV.2.1 gives an overview of the target geometry and of the selection of target material. Target geometry is determined by balancing the neutronic performance (Section IV.2.2) against the need to extract the deposited heat from the target. The relevant thermal-hydraulic analyses are presented in Section IV.2.3. The choice of target material depends on neutronic performance (Section IV.2.2), mechanical strength of the target relative to the induced stresses (Section IV.2.4), and the corrosion resistance and other nuclear and thermophysical properties of the material (Section IV.2.5).

Section IV.2.2 describes the Monte Carlo model used to calculate the detailed power deposition in the target and surroundings and to determine the neutronic output. The calculated power density distribution serves as input to the thermal-hydraulic calculations in Section IV.2.3. However, most of the discussion of neutronic performance is deferred until the moderators are discussed in Section IV.3.

Section IV.2.3 describes the thermal-hydraulic analyses related to the extraction of heat from the target. These analyses are used to refine the target geometry and to determine the target cooling capacity required. The target cooling system is discussed in Section IV.2.6. Section IV.2.4 presents analyses of the stresses induced in the target disks due to the temperature gradients calculated in Section IV.2.3.

The relevant materials properties data for candidate target materials are presented in Section IV.2.5. These data play an important role in the choice of target material, as discussed in Section IV.2.1.

Finally, Section IV.2.7 presents a brief discussion of potentially hazardous incidents. The preliminary assessments made here indicate no difficult safety-related problems. A detailed safety assessment will be performed once the project is underway.

\subsection{TARGET GEOMETRY AND MATERIAL}

Spallation neutron targets must be of a dense material with a high mass number in order to produce the greatest number of neutrons per proton in the spallation process. In addition, the material must have good thermal and corrosion properties, and it must be mechanically robust, easy to fabricate, and resistant to radiation damage. Targets made of tungsten, ${ }^{1}$ tantalum, ${ }^{2}$ depleted uranium, ${ }^{3-5}$ and enriched uranium ${ }^{6}$ have all seen extensive operation in pulsed neutron sources, and such other materials as lead, gold, and platinum are also candidates. The numbers of spallation neutrons produced per proton differ by only a small amount among all these materials. However, in the case of depleted uranium, additional neutrons are produced by fast-neutroninduced fissions, leading to an overall gain of a factor of $\sim 1.7$ over the nonfissioning target materials. Enriched-uranium targets operated as subcritical "boosters" can provide a considerably greater fission gain. 
A large fraction of the proton beam energy is deposited in the target via the spallation process in nonfissioning targets. This spallation energy deposition is also present in fissioning targets, but in these targets a large amount of energy is deposited by the fission process as well. Fission is a much less efficient process for the production of neutrons than is spallation, so the energy deposition per neutron produced is much greater in the fissioning targets than in the spallation-only targets. In the case of an enriched-uranium target, most of the neutrons are produced by fission, and heat removal becomes a major problem.

Because of heat-removal and materials-property problems, the use of an enriched-uranium target does not appear practical at the beam power levels of IPNS Upgrade. Depleted uranium is probably practical, but it must be clad in order to contain the fission products and to prevent corrosion. Fabrication difficulties, lifetime problems due to radiation damage, and problems in heat removal make this a very difficult material for use at the power levels being considered here. ${ }^{2}$ However, the gain of 1.7 is attractive, making it worthwhile to continue to investigate the possibility of using depleted-uranium targets at IPNS Upgrade. Experience at IPNS and ISIS with both depleted and enriched uranium targets, $\alpha$-phase Springfield alloy clad with zircaloy, indicates that these fail as a result of anisotropic growth due to irradiation and thermal cycling. Efforts are under way at both facilities to improve uranium target metallurgical design, but experience so far indicates that uranium targets cannot yet be counted upon to serve in 1-MW facilities.

Both tantalum-based and tungsten-based alloys were considered for the IPNS Upgrade targets. Assessment of the consequences of a Loss of Coolant Accident (LOCA) discussed in Section IV.2.7.1 indicated that the tantalum target temperature rose to very high values, short of melting but still potentially damaging to the surroundings. We also found that the afterheat in tungsten is much smaller than in tantalum (Section IV.2.2.4), so that LOCA temperatures are much lower. Moreover, we learned of recent Russian work ${ }^{7}$ in which plasma-sprayed titanium coatings on tungsten have been tested for corrosion resistance in an irradiated water environment and found to have very low corrosion rates. The combination of low afterheat and prospects for low corrosion rates led us to choose tungsten rather than tantalum. The neutronic calculations for tantalum apply with sufficient accuracy to tungsten as well, because densities and mass numbers of tantalum and tungsten are nearly identical. Therefore we have not repeated the calculations of neutron or power density distributions for tungsten.

A major problem in target design is the removal of heat from the target. Most pulsed spallation neutron source targets operated to date have utilized a "plate" design, with plates of target material oriented perpendicular to the beam and separated by thin coolant channels. Water (heavy water at ISIS) is used as the coolant in all such systems operated so far. This technology is well developed and understood. The analyses in the following sections indicate that this technology can be readily extended to the power levels at IPNS Upgrade, so this is the selected target geometry.

Russell ${ }^{1}$ developed the innovative idea of using a split target to provide a "flux-trap" gap along the proton beam axis between the two portions of the target. This creates a neutronically favorable position for the location of the flux trap moderators, avoiding a direct view of the 
target. It also extends the effective length of the target providing good positions for additional wing-geometry moderators. For these reasons, a split-target design is selected for the IPNS Upgrade targets.

The IPNS Upgrade target design consists of an upstream target section, the first struck by the proton beam, $\sim 10 \mathrm{~cm}$ in length and a downstream target section $\sim 30 \mathrm{~cm}$ in length mounted in the same housing (see Figure IV.1.2-3). Each of these target sections consists of stacks of disks of machinable tungsten. The disk shape provides for mechanical support of the disks and good target-moderator neutronic coupling. Figures IV.2.1-1 and IV.2.1-2 show details of the upstream and downstream sections of the target. Disk thicknesses increase from the upstream to the downstream end of each target section. The thicknesses are determined by cooling requirements and stress limitations (Sections IV.2.3 and IV.2.4). Cooling water flows through the 0.159-cmthick gaps between the disks and between the end disks and the target housing windows. Plena above and below the disks direct the cooling water to these gaps. In both target sections, the disk assemblies are spring-loaded in order to maintain this spacing and to compensate for expansion and contraction.

The two target sections are cooled by water in the common plena, reducing the external plumbing requirements and simplifying target replacement. Figure IV.1.2-4 shows the relative locations of the target, moderators, and their associated coolant pipes. Six different moderators are located in neutronically favorable positions, as shown in Figure IV.1.2-2, providing great flexibility in the selection of moderators to meet the requirements of the individual neutron scattering instruments. This arrangement also provides several locations for irradiation tubes close to the target, as shown in Figure IV.1.2-6. The two target stations are identical, although the choices of moderator materials will be different in the two cases.

\subsection{NEUTRONICS AND HEAT GENERATION}

\subsubsection{Calculational Model}

A Monte Carlo computing model was set up for the calculation of neutron beam characteristics and thermal power generation in the target system and its surroundings. The Los Alamos High Energy Transport (LAHET) code system ${ }^{8}$ was chosen for the calculations after an extensive search. The LAHET code system consists of a LANL version of the High Energy Transport Code (HETC) and the Monte Carlo code for Neutron and Photon transport (MCNP), with a few other associated codes. HETC calculates and transports high-energy particle cascades, including neutrons with energies above $20 \mathrm{MeV}$. The Bertini model was used for the intranuclear cascade calculation in HETC, as it is generally recognized to be acceptable up to a particle energy of $3.5 \mathrm{GeV}$. MCNP is used to calculate the transport of neutrons below $20 \mathrm{MeV}$ and of photons. For the photon cascade calculation, a hybrid model between the Troubetzkoy (E1) model and the spin-dependent model were used to provide the photon source distribution for MCNP. 


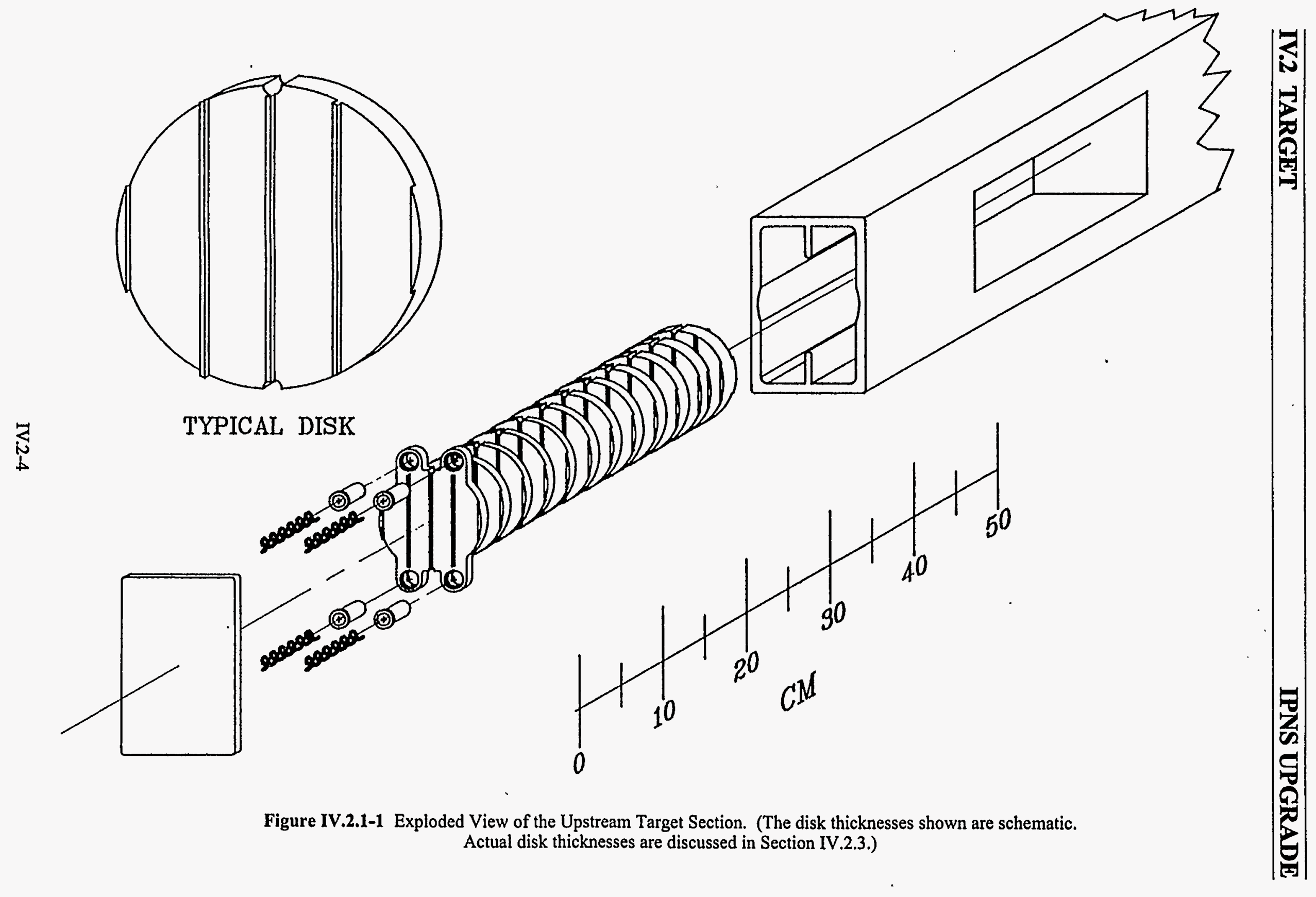




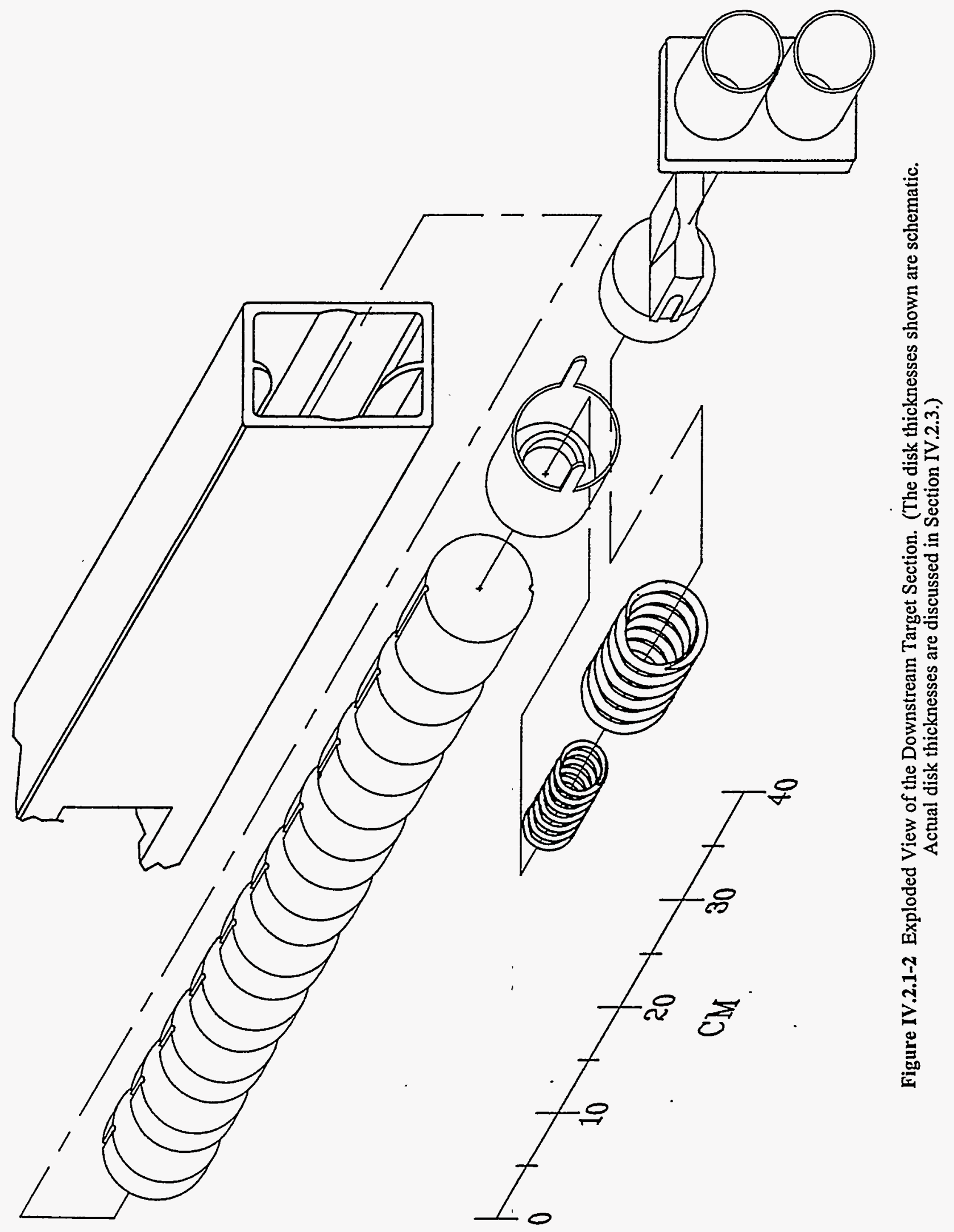


A validation of the LAHET code system and physical model was performed upon the installation of the code system. For this validation, a simple model consisting of a cylindrical target surrounded by a beryllium reflector was used. Figure IV.2.2-1 shows the calculated ratio of energy deposition in the target to the proton beam energy. The calculation was performed with tantalum and compared to other published results. ${ }^{9}$ Figure IV.2.2-1 confirms the validity of the LAHET code system and physical models used in the calculation. Figure IV.2.2-2 shows that the neutron production rate in the tantalum target is a little higher than that in a lead target. ${ }^{10}$

The LAHET code system uses a combinatorial surface geometry in which a system configuration is represented in terms of combinations of quadratic surfaces. The IPNS Upgrade target system model includes targets, moderators, neutron beamlines, and associated reflector and shield. Geometrical and material features of key components are listed in Table IV.2.2-1. In order to simplify the computational model, the shield has been truncated at a radius of $1 \mathrm{~m}$ from the target. This has no significant effect on the results for the target, moderator, and reflector regions. An isometric view of the modeled target station is shown in Figure IV.2.2-3, and in Figure IV.2.2-4 a plan view of the system is shown to illustrate the neutron beamlines. There are three neutron beamlines from each moderator, for a total of 18 beamlines in the target station. Figure IV.2.2-5 provides a detailed view of the target and moderator model and indicates the labels used to designate the six moderators. Figures IV.2.2-6 through IV.2.2-8 show the dimensions of the major components: Figure IV.2.2-6, the shield and reflector; Figure IV.2.2-7, the details of targets; and Figure IV.2.2-8, the details of moderators and the arrangement of neutron beamlines. As seen in Figure IV.2.2-8, the boron decoupler covers each neutron beamline in the reflector region and surrounds each moderator, except on the viewed surface. For most of the calculations, the model illustrated in Figures IV.2.2-3 through IV.2.2-8 was used. In some cases, slight modifications were made to this model; these modifications are described with the corresponding calculations.

The modeled system is somewhat simpler than the actual design of the target station. For instance, irradiation facilities and coolant pipes for moderators are not included in the model. However, the model is sufficiently accurate to provide the necessary parameters for the engineering design, such as heat deposition in the system and neutron beam characteristics from the moderators.

All calculations are based on a $2.2-\mathrm{GeV}$ proton beam with a Gaussian distribution of $5 \mathrm{~cm}$ full width at half maximum (fwhm) diameter and truncated at $10 \mathrm{~cm}$ diameter. The calculated results presented in subsequent sections are normalized results based on a 1-MW proton beam, unless otherwise noted.

\subsubsection{Heat Deposition in the Target and Surroundings}

The power deposited in the target and surroundings is summarized in Table IV.2.2-2. About $90 \%$ of the proton beam power is deposited in the modeled system. The remaining $10 \%$ 


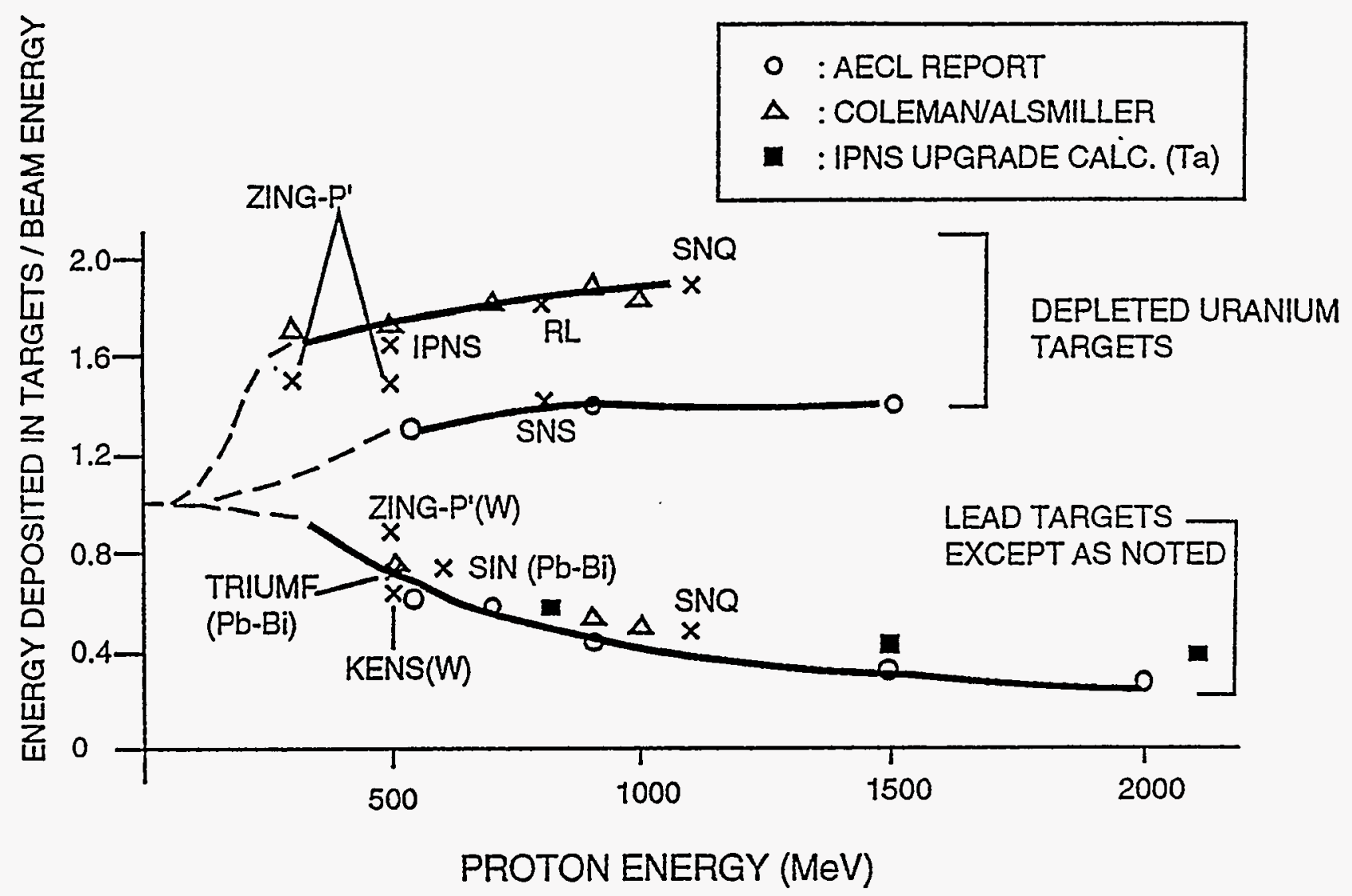

Figure IV.2.2-1 Validation of IPNS Upgrade Calculational Model for Heat Deposition in Targets. (Present calculations are compared with previous computed and experimental results. Legend: $\mathrm{X}$, measured values for sources and targets indicated; $O, A E C L$ report; $\triangle$, Coleman/Alsmiller;

$\square$, present IPNS Upgrade calculations for Ta.) (Source: Reference 9). 


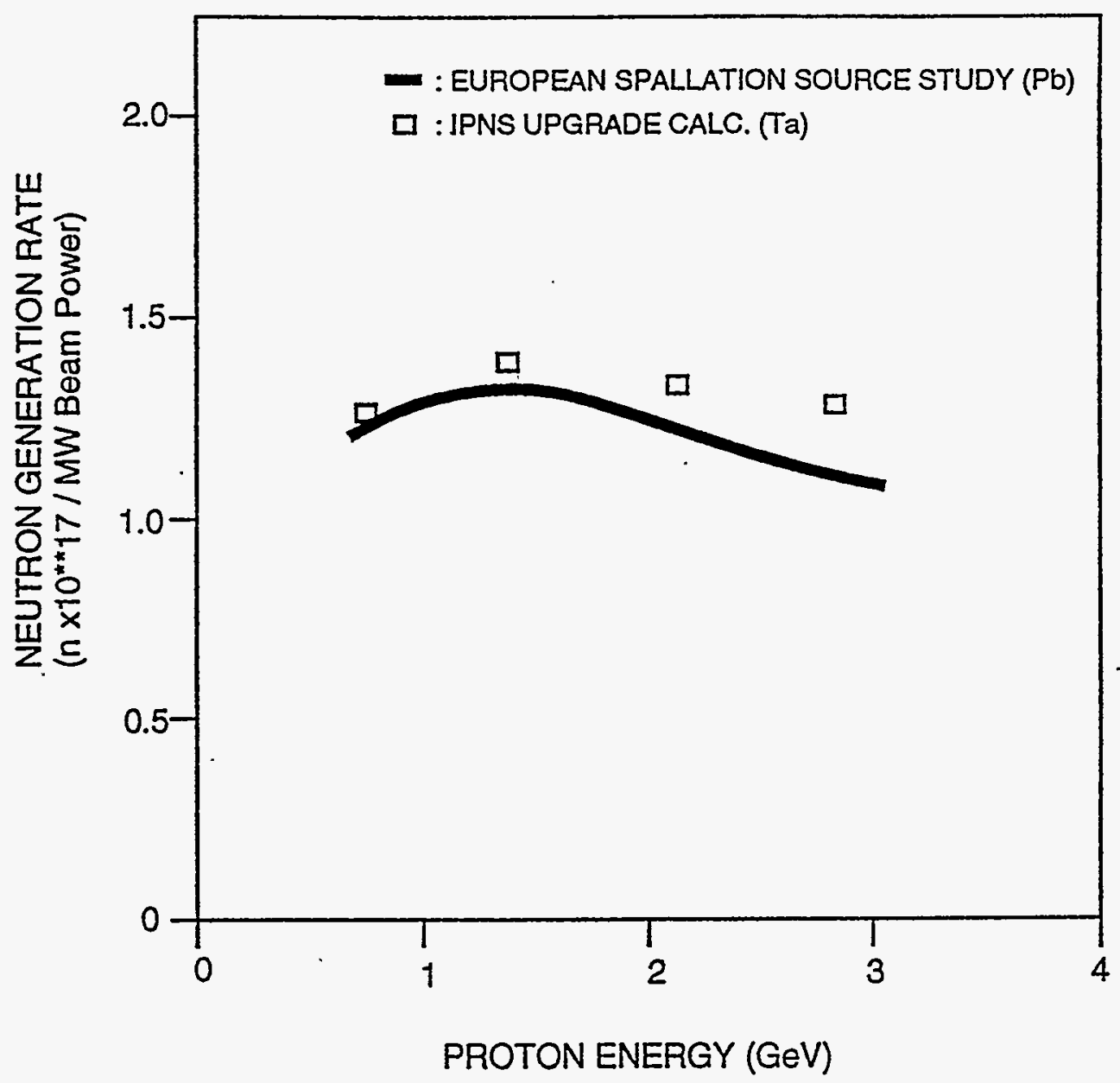

Figure IV.2.2-2 Validation of IPNS Upgrade Calculational Model for Neutron Generation Rate. (Present calculations are compared with previous computed results. Legend: -, European Spallation Source study [Pb]; $\square$, IPNS

Upgrade calculations for Ta.) (Source: Reference 10). 
Table IV.2.2-1 Geometrical and Material Features of the Target Station Model

\begin{tabular}{|c|c|c|}
\hline Component & Geometry and Dimensions ${ }^{a}$ & Material \\
\hline Target & $\begin{array}{l}\text { Two split targets with a flux-trap } \\
\text { region in-between }\end{array}$ & Tantalum \\
\hline Upstream section & $\begin{array}{l}\text { four elliptical disks with semiaxes of } \\
5.5 \text { and } 7.5 \mathrm{~cm} \text {, thickness } 2.5 \mathrm{~cm}\end{array}$ & \\
\hline Downstream section & $\begin{array}{l}\text { nine elliptical disks with semiaxes of } \\
5.5 \text { and } 7.5 \mathrm{~cm} \text {, thickness } 3.5 \mathrm{~cm}\end{array}$ & \\
\hline Housing & Rectangle with rounded side surfaces & $0.5-\mathrm{cm}$-thick stainless steel \\
\hline Upstream section & $18 \mathrm{~cm}$ wide, $12 \mathrm{~cm}$ high, $12 \mathrm{~cm}$ long & \\
\hline Downstream section & $18 \mathrm{~cm}$ wide, $12 \mathrm{~cm}$ high, $40 \mathrm{~cm}$ long & \\
\hline Flux Trap & $\begin{array}{l}\text { The 18-cm-long section between the } \\
\text { housings }\end{array}$ & Vacuum \\
\hline Coolant & $\begin{array}{l}\text { Coolant channel gap size between targel } \\
\text { disks: } 0.2 \mathrm{~cm}\end{array}$ & Water \\
\hline Moderator & & Water \\
\hline $\begin{array}{l}\text { Upstream moderators } \\
\text { (top and bottom) }\end{array}$ & $5 \mathrm{~cm}$ thick, $10 \mathrm{~cm}$ wide, $10 \mathrm{~cm}$ high & \\
\hline $\begin{array}{l}\text { Flux-trap moderators } \\
\text { (left and right) }\end{array}$ & $5 \mathrm{~cm}$ thick, $10 \mathrm{~cm}$ wide, $20 \mathrm{~cm}$ high & \\
\hline $\begin{array}{l}\text { Downstream } \\
\text { moderators } \\
\text { (top and bottom) }\end{array}$ & $10 \mathrm{~cm}$ thick, $10 \mathrm{~cm}$ wide, $10 \mathrm{~cm}$ high & \\
\hline Boron Decoupler & $\begin{array}{l}\text { 2-cm-thick cladding surrounding } \\
\text { moderators and neutron beamlines, } \\
\text { except the beamline face }\end{array}$ & $\begin{array}{l}20 \%{ }^{10} \mathrm{~B} \text { and } 80 \%{ }^{11} \mathrm{~B} \text { with } \\
3.3 \% \text { of natural boron } \\
\text { density } \mathrm{b}\end{array}$ \\
\hline Reflector & $\begin{array}{l}\text { Cylinder of } 54 \mathrm{~cm} \text { radius, } 100 \mathrm{~cm} \\
\text { height }\end{array}$ & Beryllium \\
\hline Shield & $\begin{array}{l}\text { Annulus of } 54 \mathrm{~cm} \text { inner radius, } \\
100 \mathrm{~cm} \text { outer radius, } 100 \mathrm{~cm} \text { height }\end{array}$ & Iron \\
\hline
\end{tabular}

a See Figures IV.2.2-3 through IV.2.2-7.

b This boron density is based on the neutron decoupling energy of $1.0 \mathrm{eV}$ for $1 / \mathrm{e}$ reduction of neutron flux across the decoupler. 


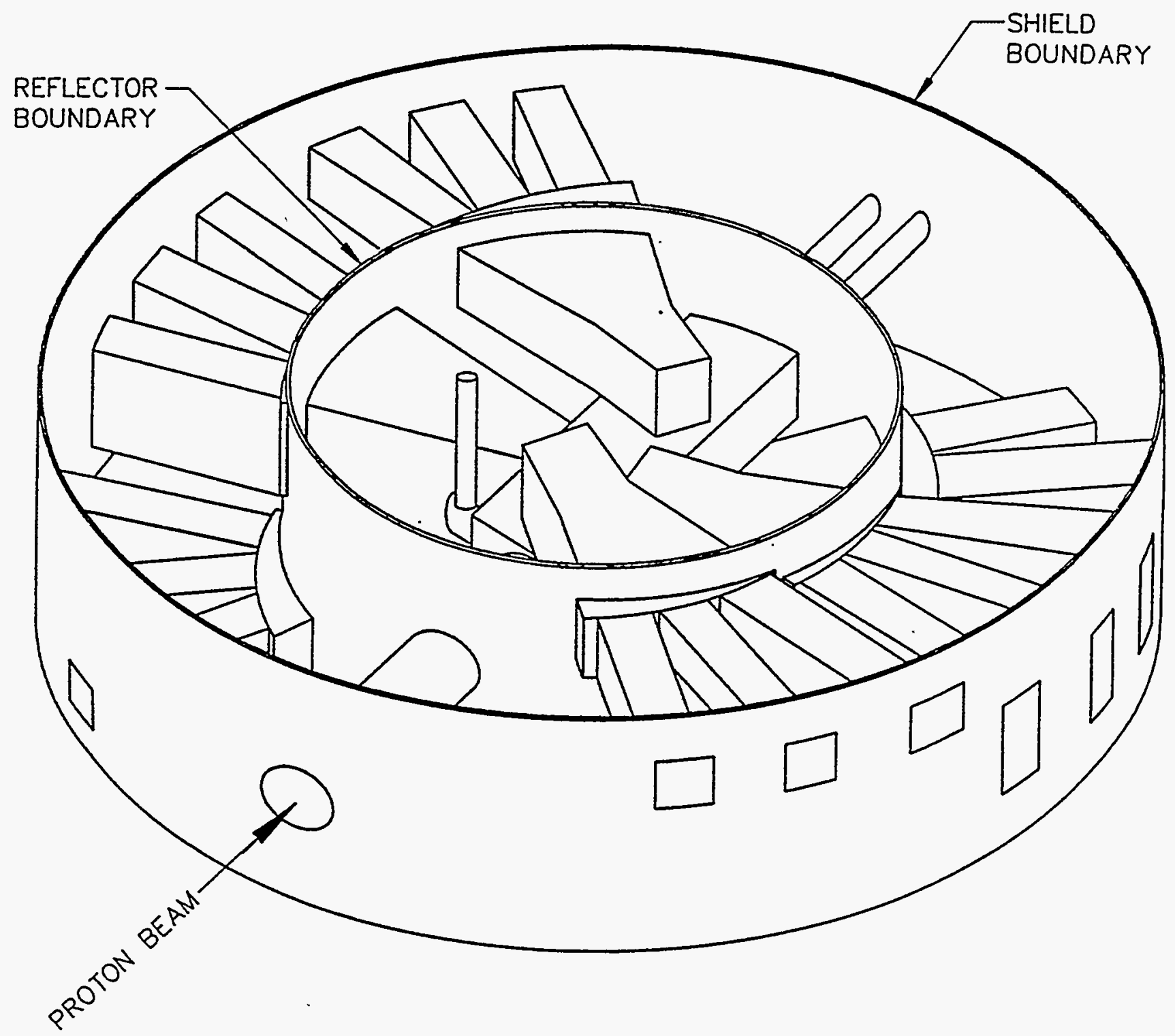

Figure IV.2.2-3 Isometric View of the Target Station Model. 


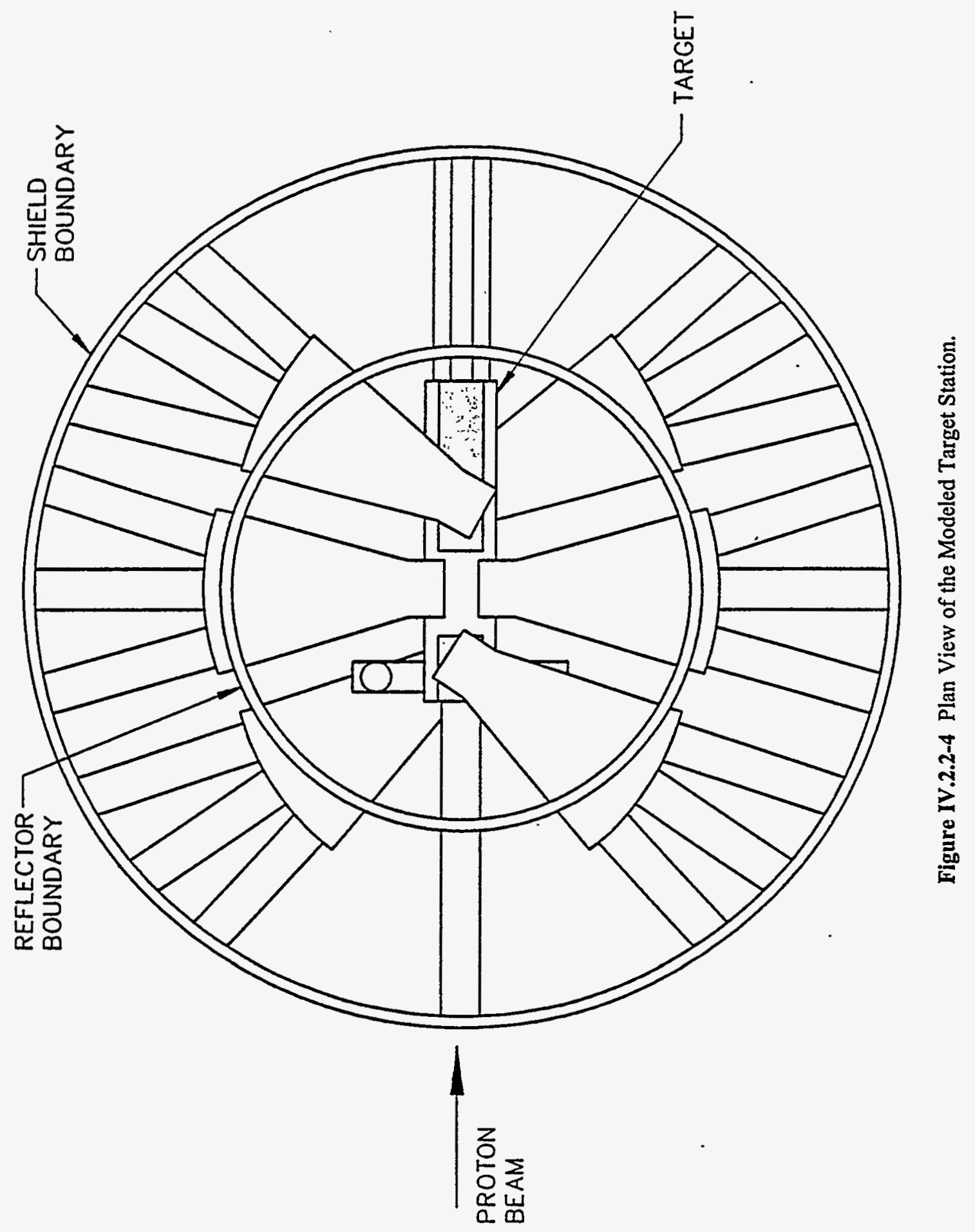




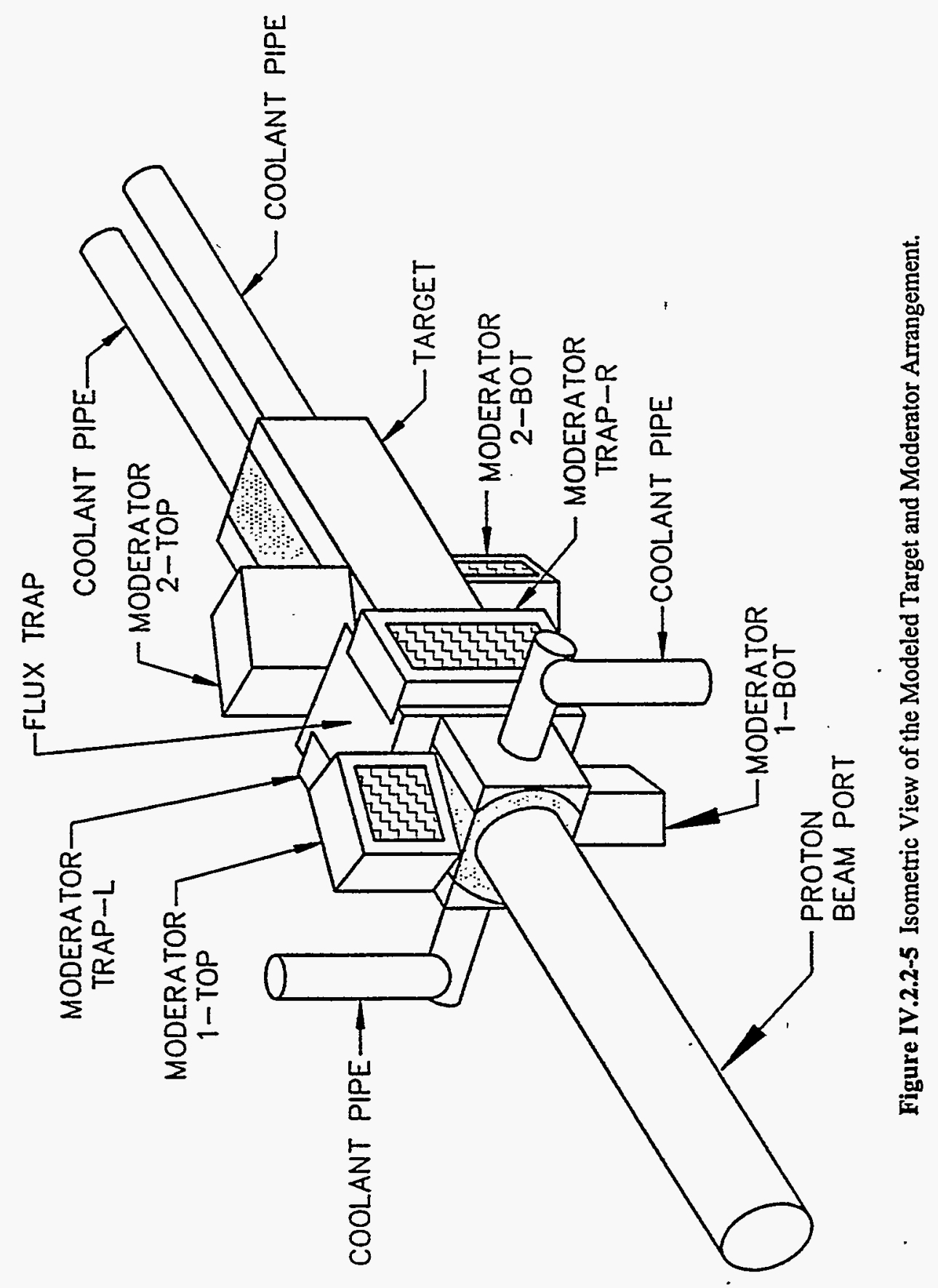




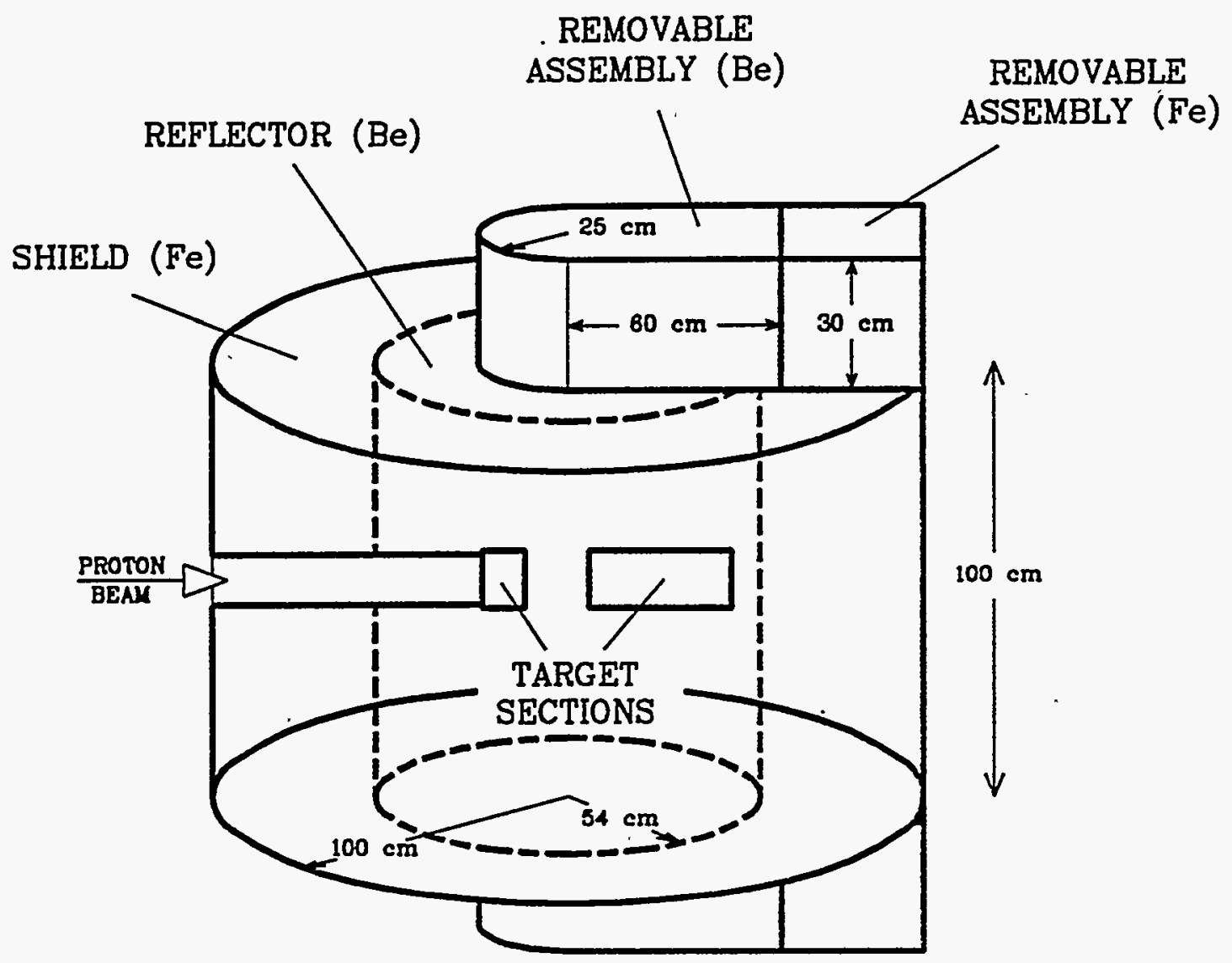

Figure IV.2.2-6 Modeled Configurations of the Reflector and Shield. 


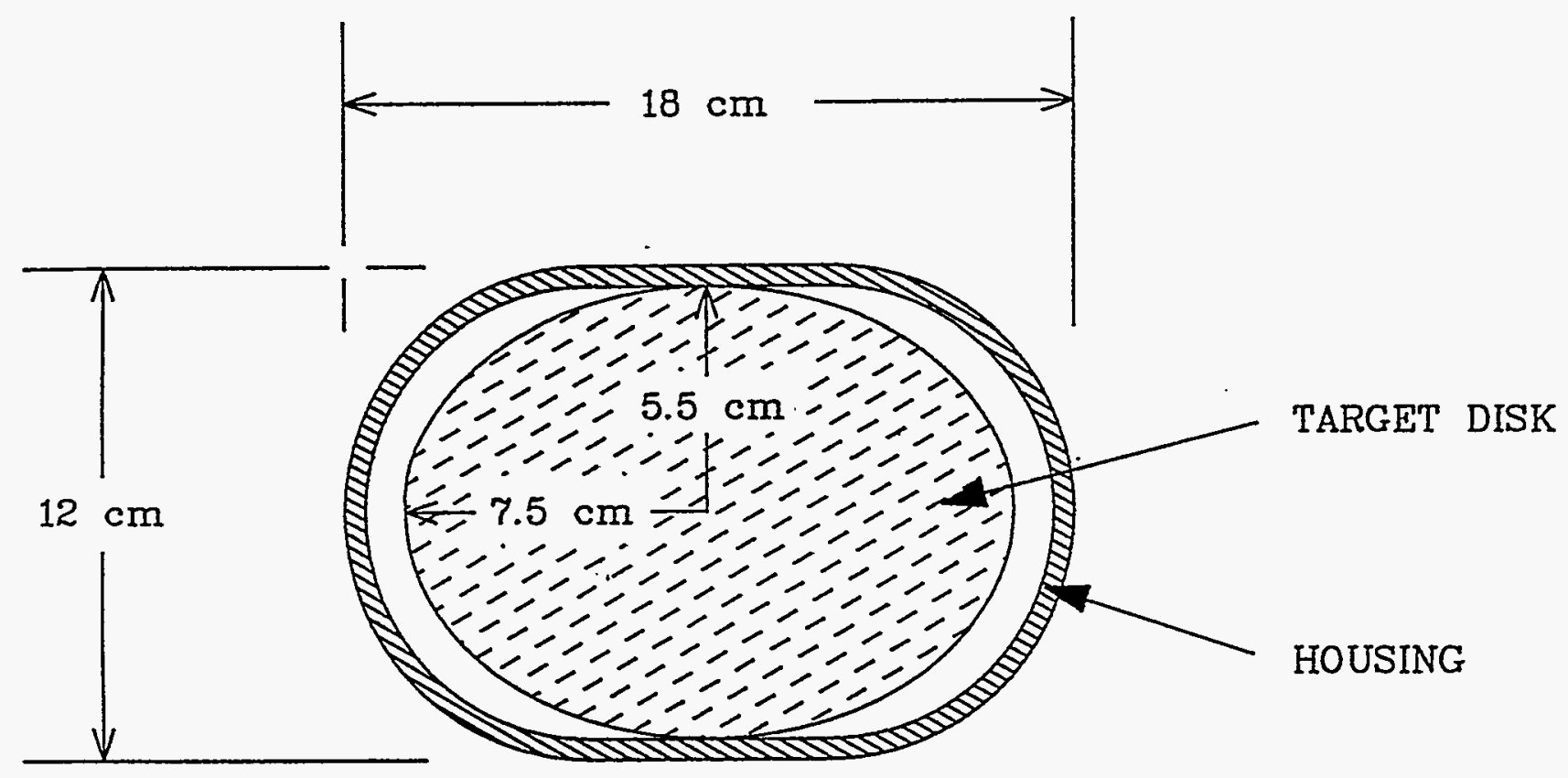

Figure IV.2.2-7 Cross Section of the Modeled Target Sections. (The target sections are modeled as a smaller number of thicker disks than is the actual case, in order to reduce computational time.) 

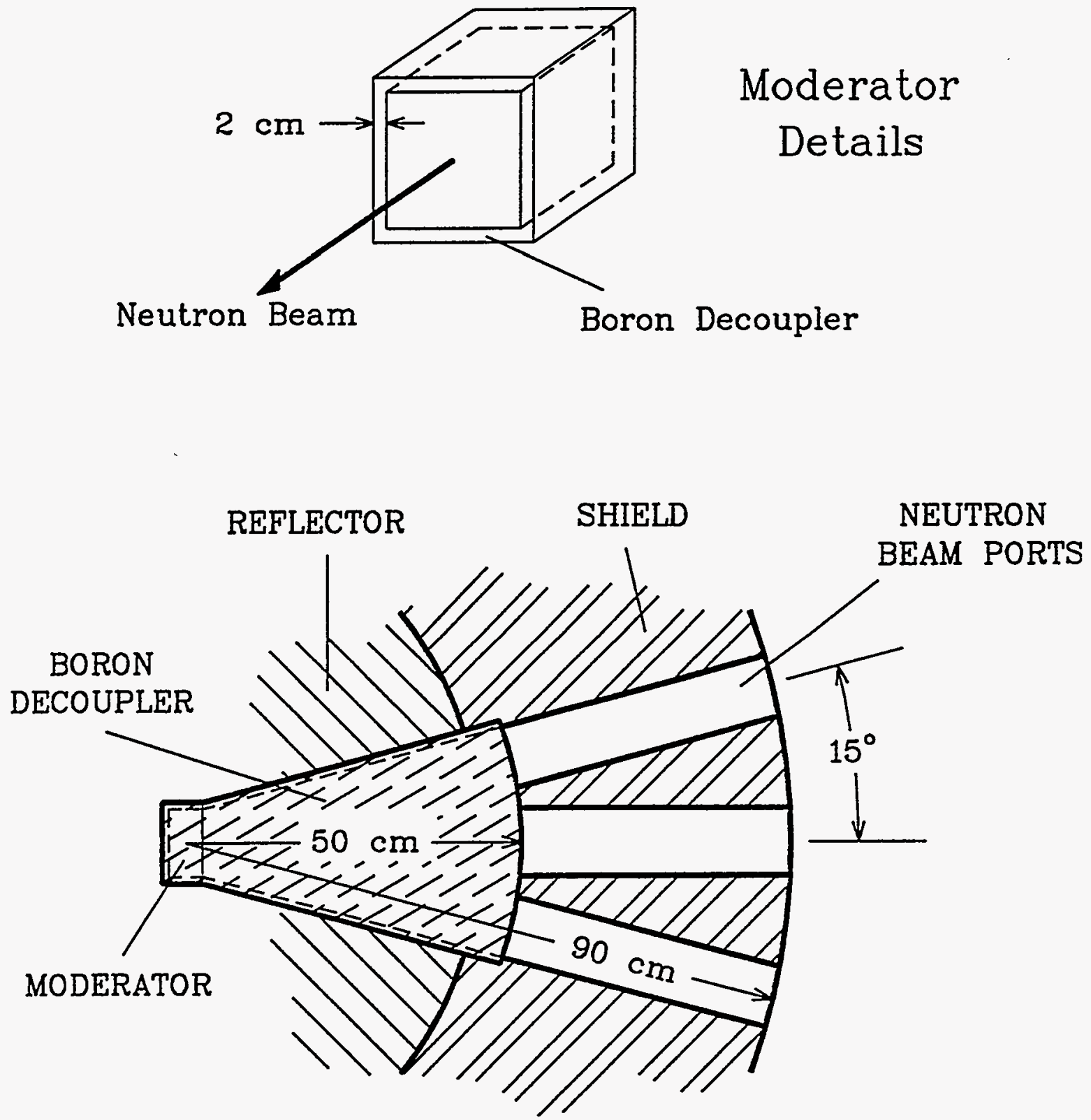

Figure IV.2.2-8 Modeled Configurations of Moderators and Neutron Beamlines. (Moderators are all $10 \mathrm{~cm}$ wide. Flux-trap moderators are $20 \mathrm{~cm}$ tall; others are $10 \mathrm{~cm}$ tall. Downstream moderators are $10 \mathrm{~cm}$ deep; others are $5 \mathrm{~cm}$ deep.) 
Table IV.2.2-2 Power Deposition in the Target Station for Proton Beam Power of $1 \mathrm{MW}$

\begin{tabular}{|c|c|c|}
\hline \multirow[b]{2}{*}{ Component } & \multicolumn{2}{|c|}{ Power Deposition (kW) } \\
\hline & Subcomponent & Total \\
\hline Target & - & 550.8 \\
\hline Coolant & - & 21.0 \\
\hline Structure Total & - & 34.2 \\
\hline Window & 9.40 & \\
\hline Upstream housing downstream plug & 6.62 & \\
\hline Downstream housing upstream plug & 4.49 & \\
\hline Downstream housing downstream plug & 0.45 & \\
\hline Upstream housing & 1.91 & \\
\hline Downstream housing & 6.69 & \\
\hline Spring & 4.66 & \\
\hline Moderator Total & & 7.6 \\
\hline Upstream top moderator & 0.78 & \\
\hline Upstream bottom moderator & 0.77 & \\
\hline Flux-trap moderator - 1 & 1.52 & \\
\hline Flux-trap moderator - 2 & 1.50 & \\
\hline Downstream top moderator & 1.58 & \\
\hline Downstream bottom moderator & 1.48 & \\
\hline Boron Decoupler & & 26.7 \\
\hline Reflector Total & & 126.6 \\
\hline Upstream & 29.75 & \\
\hline Middle & 28.12 & \\
\hline Downstream & 68.75 & \\
\hline Shield Total & & 128.0 \\
\hline Upstream & 17.02 & \\
\hline Middle & 10.28 & \\
\hline Downstream & 100.72 & \\
\hline Removable Assembly & & 7.0 \\
\hline Target Station Total & & 902.0 \\
\hline
\end{tabular}


either is in the form of binding energy resulting from the change of isotope composition during the cascade process or escapes through neutron and photon leakage. For instance, the number of neutrons escaping from the system in the model is about 9.7 neutrons per proton. If $8 \mathrm{MeV}$ is assumed for the neutron separation energy, this leakage constitutes about $35 \mathrm{~kW}$ per MW of beam power. The overall energy balance is satisfied in the calculation.

About $55 \%$ of the proton beam power is deposited in the target, as is indicated in Table IV.2.2-2. Table IV.2.2-3 and Figure IV.2.2-9 show the distribution of heat deposition, both in terms of total power and of power density, in each target disk along the proton beam direction. Table IV.2.2-4 indicates the radial distribution of power density and provides a fitted function that represents the radial power density in each of the four modeled disks in the upstream target section. Figure IV.2.2-10 illustrates the change in radial power density along the target axis. As expected, with increasing depth the profile flattens as a result of the spreading of the particle cascades and the widening of the proton beam due to collisions.

Figure IV.2.2-11 illustrates the spatial distribution of heat deposited in the shield and reflector, showing the total power in each modeled segment. The volumes of the upstream and downstream segments of the reflector are the same, while the middle segments are much smaller. The segmentation of the shield is similar to that of the reflector. Figure IV.2.2-11 indicates that the power deposited in the downstream segments of both shield and reflector is much greater than in the upstream segments. This difference arises because protons are still energetic even at the downstream end of the target (see Table IV.2.2-3) and the neutron distribution is forwarddirected. Negligible differences exist in power depositions in symmetrically-located moderator pairs (e.g., top and bottom upstream moderators), or in symmetric segments of the reflector or shield. These internal consistencies also confirm the soundness of the geometrical model used in the calculation.

\subsubsection{High-Energy Neutron Distribution around the Target}

To provide a basis for the shield design and to aid in understanding the power distribution in the reflector and shield shown in Figure IV.2.2-11, a simple calculation for the distribution of high-energy $(>20 \mathrm{MeV})$ neutrons around the target was performed. The much-simplified model used for this calculation includes a cylinder of tantalum having approximately the same dimensions as the actual target and a 3-m-radius evacuated sphere for tallying the neutrons. This model is sufficient for the calculation of the angular distribution of high-energy neutrons around the target, because these high-energy neutrons penetrate the reflector and shield and dominate the shielding requirements. The angular distribution of the high-energy neutrons is depicted in Figure IV.2.2-12, along with a schematic of the model. The neutron spectra are shown in Figure IV.2.2-13. Figure IV.2.2-12 shows that the total angular yield is forward-directed. The neutron spectrum is much harder (shifted to higher energies) in the forward direction, as shown in Figure IV.2.2-13. This anisotropic, forward-directed distribution of high-energy neutrons is due to the relatively high proton beam energy of $2.2 \mathrm{GeV}$. 
Table IV.2.2-3 Power Distribution in the Target Sections for Proton Beam Power of $1 \mathrm{MW}$

\begin{tabular}{lcc}
\hline Modeled Target Disk & $\begin{array}{c}\text { Total Power } \\
(\mathrm{kW})\end{array}$ & $\begin{array}{c}\text { Power Density } \\
\left(\mathrm{kW} / \mathrm{cm}^{3}\right)\end{array}$ \\
\hline Upstream Target Section & & \\
Disk 1 & & \\
Disk 2 & 88.5 & 0.273 \\
Disk 3 & 83.9 & 0.259 \\
Disk 4 & 72.0 & 0.222 \\
Downstream Target Section ${ }^{\mathrm{c}}$ & 62.2 & 0.192 \\
Disk 1 & & \\
Disk 2 & 53.8 & \\
Disk 3 & 47.4 & 0.119 \\
Disk 4 & 38.7 & 0.105 \\
Disk 5 & 29.9 & 0.085 \\
Disk 6 & 23.7 & 0.066 \\
Disk 7 & 18.5 & 0.041 \\
Disk 8 & 13.9 & 0.031 \\
Disk 9 & 10.5 & 0.023 \\
\hline
\end{tabular}

a Disk 1 is at the upstream end.

b The modeled upstream target disks are each $2.5 \mathrm{~cm}$ thick.

c The modeled downstream target disks are each $3.5 \mathrm{~cm}$ thick. 


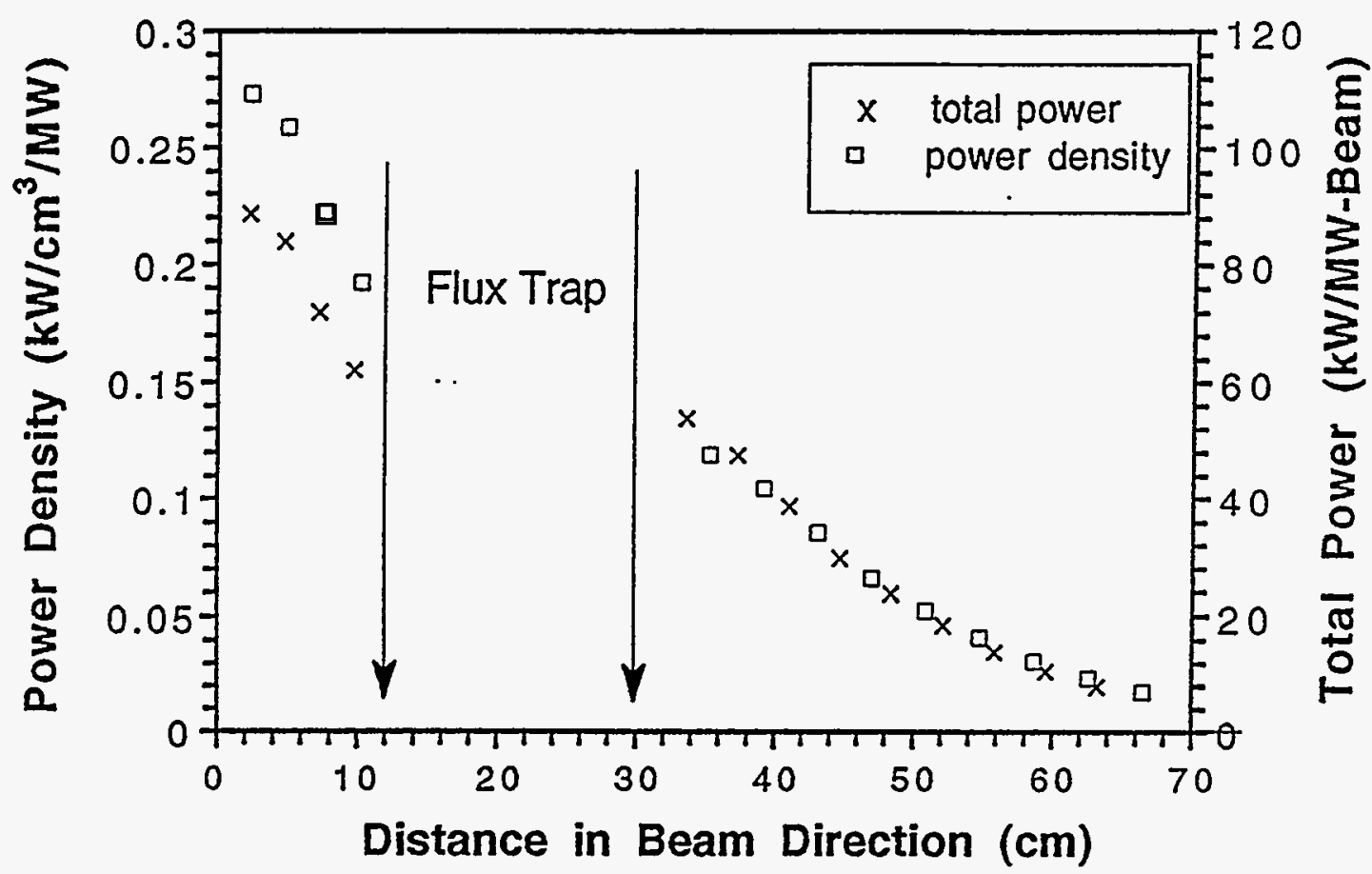

Figure IV.2.2-9 Power Density Distribution in the Target along the Proton Beam Direction. 
Table IV.2.2-4 Radial Power Distribution in the Disks of the Upstream Target Section for Proton Beam Power of $1 \mathrm{MW}$

\begin{tabular}{|c|c|c|c|}
\hline Region ${ }^{a, b}$ & $\begin{array}{c}\text { Mean Power } \\
\text { Density } \\
\left(\mathrm{kW} / \mathrm{cm}^{3}\right)\end{array}$ & $\begin{array}{c}\text { Standard } \\
\text { Error } \\
\sigma\left(\mathrm{kW} / \mathrm{cm}^{3}\right)\end{array}$ & $\begin{array}{l}\text { Radial Functional Form } \\
\qquad\left(\mathrm{kW} / \mathrm{cm}^{3}\right)\end{array}$ \\
\hline Disk 1 & & & $1.767 \exp \left(-0.1433 r^{2}\right)$ \\
\hline zone 1 & 1.650 & 0.073 & \\
\hline zone 2 & 0.988 & 0.022 & \\
\hline zone 3 & 0.339 & 0.010 & \\
\hline zone 4 & 0.090 & 0.005 & \\
\hline zone 5 & 0.019 & 0.001 & \\
\hline Disk 2 & & & $1.587 \exp \left(-0.1384 r^{2}\right)$ \\
\hline zone 1 & 1.444 & 0.065 & \\
\hline zone 2 & 0.944 & 0.022 & \\
\hline zone 3 & 0.314 & 0.009 & \\
\hline zone 4 & 0.091 & 0.004 & \\
\hline zone 5 & 0.025 & 0.001 & \\
\hline Disk 3 & & & $1.285 \exp \left(-0.1312 r^{2}\right)$ \\
\hline zone 1 & 1.199 & 0.055 & \\
\hline zone 2 & 0.748 & 0.018 & \\
\hline zone 3 & 0.286 & 0.008 & \\
\hline zone 4 & 0.084 & 0.004 & \\
\hline zone 5 & 0.030 & 0.002 & \\
\hline Disk 4 & & & $1.040 \exp \left(-0.1232 r^{2}\right)$ \\
\hline zone 1 & 0.954 & 0.047 & \\
\hline zone 2 & 0.646 & 0.017 & \\
\hline zone 3 & 0.245 & 0.007 & \\
\hline zone 4 & 0.082 & 0.003 & \\
\hline zone 5 & 0.028 & 0.001 & \\
\hline
\end{tabular}

a zone 1: $r \leq 1.0 \mathrm{~cm}$

zone 2: $1.0 \mathrm{~cm}<\mathrm{r} \leq 2.5 \mathrm{~cm}$

zone 3: $2.5 \mathrm{~cm}<r \leq 4.0 \mathrm{~cm}$

zone 4: $4.0 \mathrm{~cm}<\mathrm{r} \leq 5.0 \mathrm{~cm}$

zone 5: $5.0 \mathrm{~cm}<\mathrm{r} \leq$ ellipse of minor and major radii $5.5 \mathrm{~cm}$ and $7.5 \mathrm{~cm}$

b All zones are $2.5 \mathrm{~cm}$ thick. 


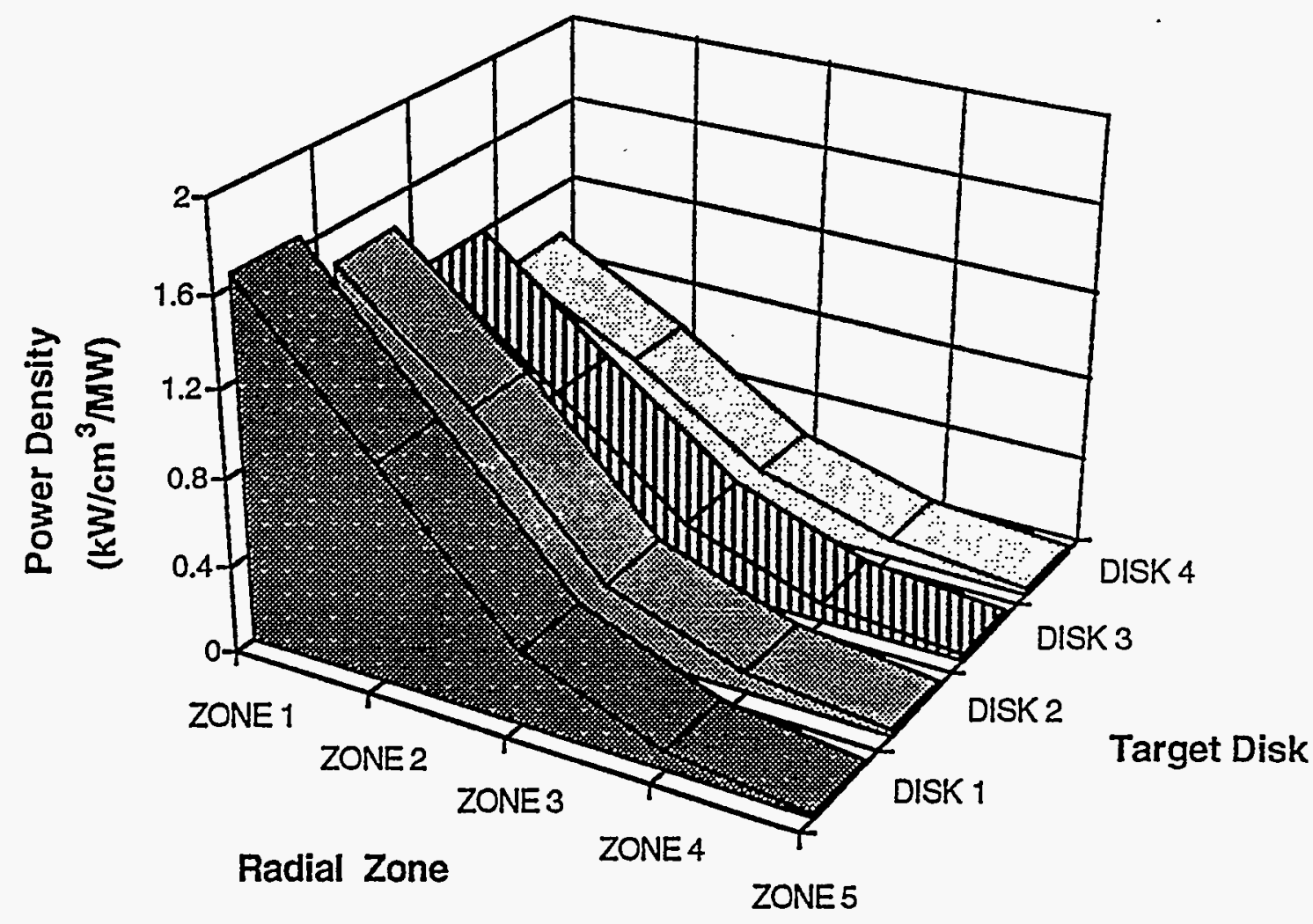

Figure W.2.2-10 Radial Power Distribution in the Disks in the Upstream Target Section. (See Table IV.2.2-4 for zone definitions.) 


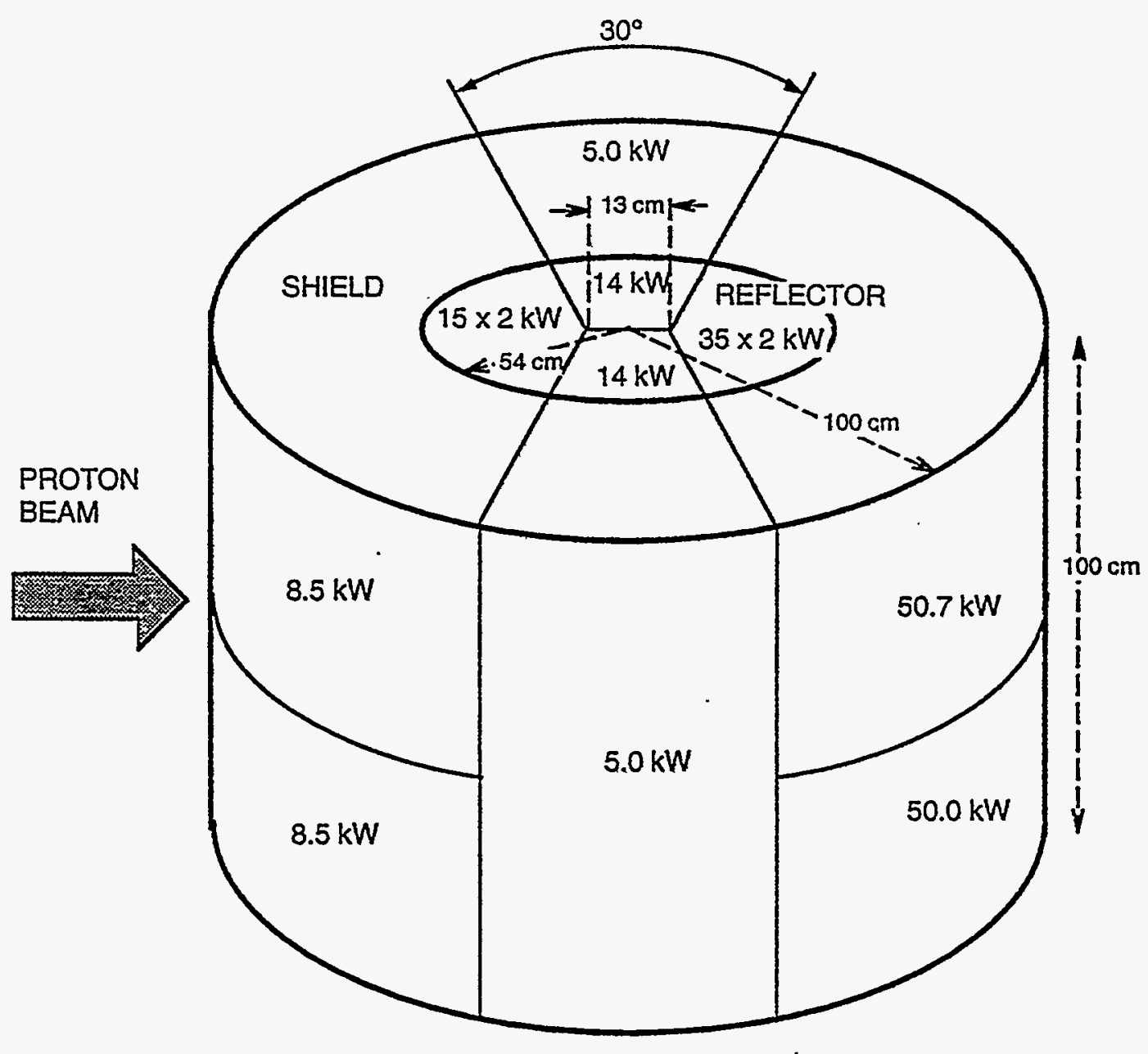

Figure IV.2.2-11 Power Distribution in the Reflector and Shield. 


\subsubsection{Activation Analyses}

Activation analyses ${ }^{11}$ were performed for a simplified, conservative model of IPNS Upgrade that provides upper-bound estimates of radioactivity and the buildup and decay of afterheat from decay of the induced activity in tantalum and tungsten targets. Tables IV.2.2-5 through IV.2.2-7 summarize the results. The detailed output for the activation calculations is available ${ }^{11}$ but is not presented here.

The calculations were performed using HETC, O5R, and ORIGEN, with a somewhat different geometrical model than that described above. The geometry for the HETC and O5R calculations was as follows:

- The target modeled was of elliptical cross section, with axes of $15 \mathrm{~cm}$ and $11 \mathrm{~cm}$.

- The upstream section of the target was $10 \mathrm{~cm}$ long and downstream section of the target was $30 \mathrm{~cm}$ long, the two sections being separated by $18 \mathrm{~cm}$. No coolant was included in the model.

- The target was enclosed in a solid beryllium reflector $20 \mathrm{~cm}$ thick, having a hole with the same cross section as the target to represent the proton beam pipe.

- The proton energy was $2.2 \mathrm{GeV}$ and the current was $500 \mu \mathrm{A}$. The proton beam distribution was Gaussian, with a full width at half maximum diameter of $2.5 \mathrm{~cm}$.

The low-energy-neutron transport in O5R has a cutoff at $1 \mathrm{eV}$, because it was judged that thermal neutron transport with no coolant present would be misleading. The results of these activation calculations thus include no contribution from interactions below $1 \mathrm{eV}$, but this should be a minor effect.

The production rates were used as input to a buildup and decay code, ORIGEN, used to calculate activity and afterheat. The calculation was performed for irradiation times of $1 \mathrm{~d}, 7 \mathrm{~d}$, $30 \mathrm{~d}$, and $1 \mathrm{yr}$; for each of these periods, the decay was calculated for the following cooling times: $0 \mathrm{~min}, 5 \mathrm{~min}, 10 \mathrm{~min}, 30 \mathrm{~min}, 1 \mathrm{~h}, 8 \mathrm{~h}, 1 \mathrm{~d}, 30 \mathrm{~d}$, and $1 \mathrm{yr}$.

Table IV.2.2-5 shows the buildup of activity and afterheat, which for continuous operation builds up most of the way in one year and saturates after about six years. Tables IV.2.2-6 and IV.2.2-7 show that for typical irradiation times of several weeks (or longer), the activity and afterheat decay rather slowly during cooldown periods. 


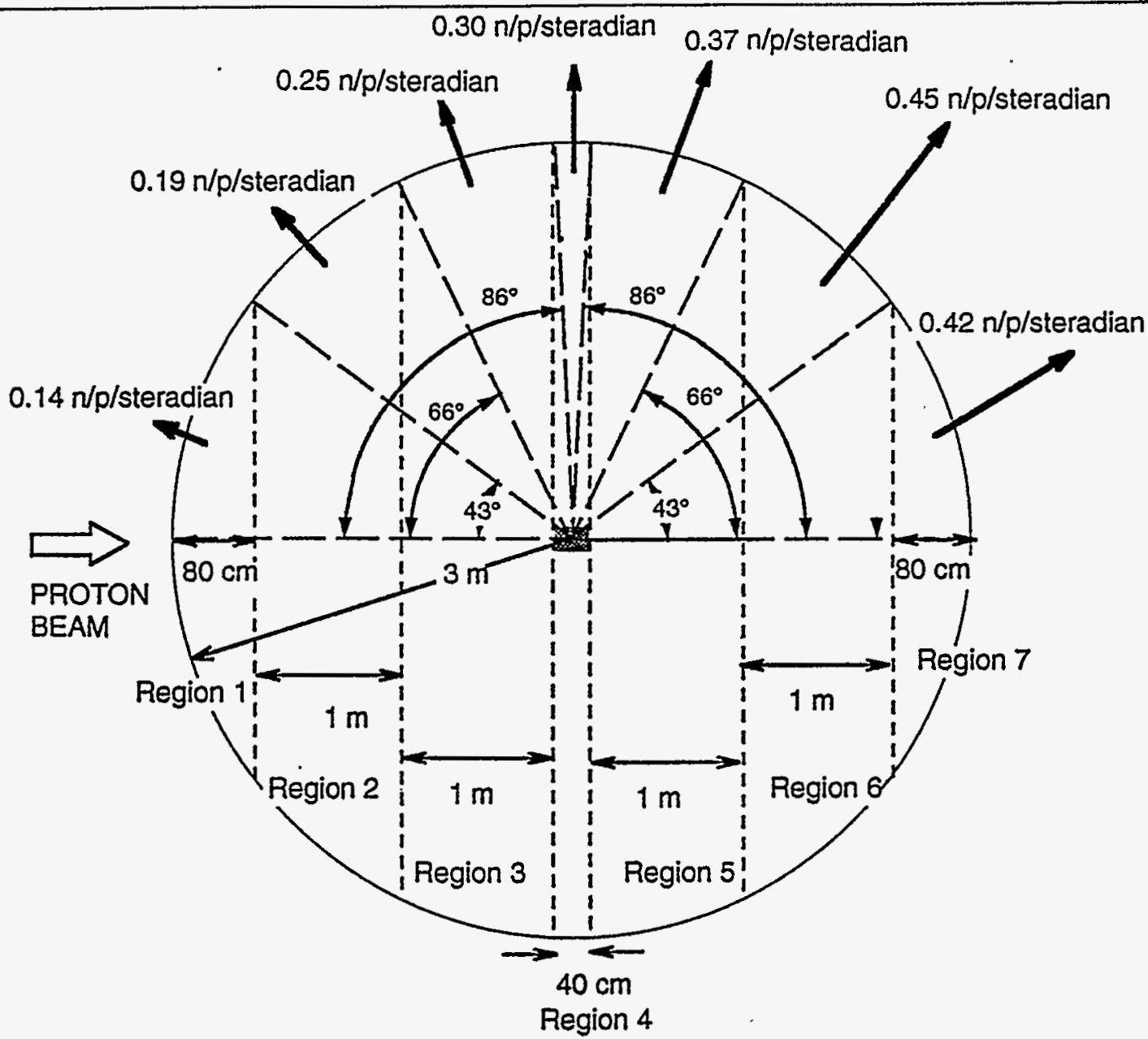

: TARGET ( $41.5 \mathrm{~cm}$ long $6.4 \mathrm{~cm}$ radius cylinder of tantalum)

Figure IV.2.2-12 High-Energy Neutron Yield around the Target. 


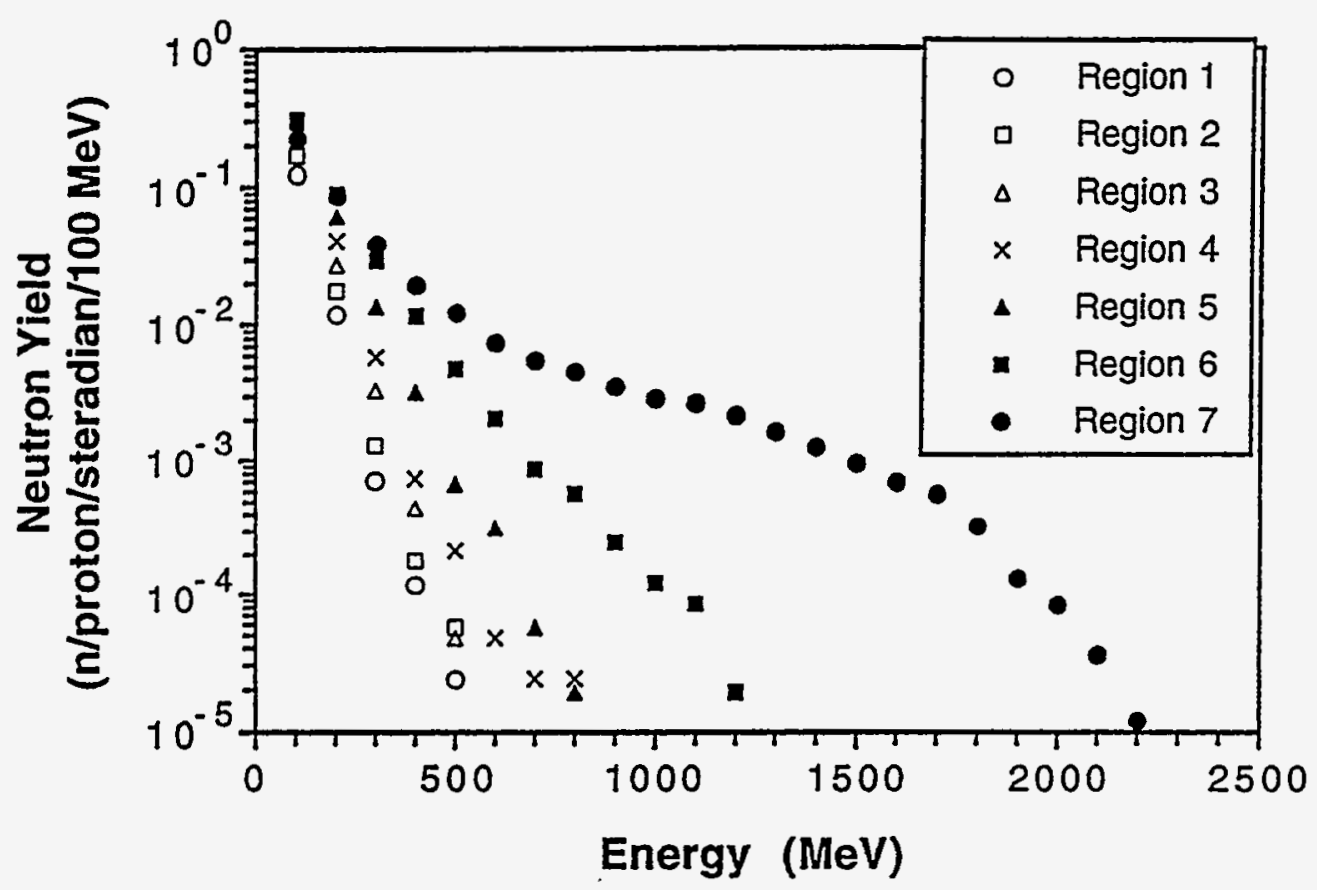

Figure IV.2.2-13 High-Energy Neutron Spectra around the Target. 
Table IV.2.2-5 Buildup of Activity and Afterheat for the IPNS Upgrade Target

\begin{tabular}{|c|c|c|c|c|}
\hline \multirow[b]{2}{*}{$\begin{array}{l}\text { Irradiation Time } \\
\text { (weeks) }\end{array}$} & \multicolumn{2}{|c|}{ Tantalum } & \multicolumn{2}{|c|}{ Tungsten } \\
\hline & $\begin{array}{l}\text { Activity } \\
\text { (TBq) }\end{array}$ & $\begin{array}{l}\text { Afterheat } \\
\text { (W) }\end{array}$ & $\begin{array}{l}\text { Activity } \\
\text { (TBq) }\end{array}$ & $\begin{array}{l}\text { Afterheat } \\
\text { (W) }\end{array}$ \\
\hline 1 & 21,800 & 3,290 & 24,700 & 3,230 \\
\hline 2 & 24,700 & 3,820 & 26,300 & 3,320 \\
\hline 3 & 26,900 & 4,280 & 27,300 & 3,360 \\
\hline 4 & 29,100 & 4,730 & 28,100 & 3,380 \\
\hline 6 & 32,900 & 5,530 & 29,000 & 3,410 \\
\hline 8 & 36,400 & 6,280 & 29,600 & 3,440 \\
\hline 10 & 39,500 & 6,760 & 30,700 & 3,460 \\
\hline 20 & 51,600 & 9,610 & 31,800 & 3,490 \\
\hline 30 & 59,500 & 11,400 & 35,100 & 3,600 \\
\hline 40 & 64,800 & 12,500 & 37,800 & 3,690 \\
\hline 60 & 70,700 & 13,700 & 39,000 & 3,750 \\
\hline 80 & 73,400 & 14,300 & 39,400 & 3,780 \\
\hline 100 & 74,700 & 14,500 & 39,800 & 3,800 \\
\hline 200 & 76,400 & 14,700 & 40,500 & 3,870 \\
\hline 300 & 77,000 & 14,800 & 40,800 & 3,900 \\
\hline 400 & 77,200 & 14,800 & 40,900 & 3,930 \\
\hline 500 & 77,400 & 14,800 & 41,000 & 3,940 \\
\hline 600 & 77,500 & 14,800 & 41,100 & 3,950 \\
\hline
\end{tabular}


Table IV.2.2-6 Decay of Afterheat Power for the IPNS Upgrade Target (Watts)

\begin{tabular}{|c|c|c|c|c|c|c|c|c|}
\hline \multirow{2}{*}{$\begin{array}{l}\text { Cooling } \\
\text { Time }\end{array}$} & \multicolumn{4}{|c|}{$\begin{array}{l}\text { Tantalum, } \\
\text { Irradiation Time (days) at } 1 \mathrm{MW}\end{array}$} & \multicolumn{4}{|c|}{$\begin{array}{l}\text { Tungsten, } \\
\text { Irradiation Time (days) at } 1 \mathrm{MW}\end{array}$} \\
\hline & 1 & 7 & 30 & 365 & 1 & 7 & 30 & 365 \\
\hline 0 & 2,410 & 3,280 & 4,840 & 13,300 & 2,410 & 3,230 & 3,380 & 3,720 \\
\hline $5 \mathrm{~min}$ & 858 & 1,390 & 3,030 & 11,500 & 1,880 & 2,710 & 2,860 & 3,200 \\
\hline $10 \mathrm{~min}$ & 822 & 1,350 & 3,000 & 11,500 & 1,750 & 2,560 & 2,720 & 3,070 \\
\hline $30 \mathrm{~min}$ & 738 & 1,290 & 2,910 & 11,300 & 1,500 & 2,310 & 2,460 & 2,800 \\
\hline $1 \mathrm{~h}$ & 671 & 1,240 & 2,830 & 11,300 & 1,310 & 2,120 & 2,260 & 2,610 \\
\hline $8 \mathrm{~h}$ & 387 & 966 & 2,500 & 11,000 & 750 & 1,410 & 1,550 & 1,900 \\
\hline $1 \mathrm{~d}$ & 196 & 732 & 2,240 & 10,600 & 390 & 850 & 982 & 1,320 \\
\hline $7 d$ & 77 & 494 & 1,910 & 10,000 & 21 & 84 & 175 & 500 \\
\hline $30 \mathrm{~d}$ & 59 & 405 & 1,620 & 8,700 & 3 & 22 & 81 & 353 \\
\hline $1 \mathrm{yr}$ & 8 & 54 & 218 & 1,200 & $<1$ & 2 & 9 & 76 \\
\hline
\end{tabular}


Table IV.2.2-7 Decay of Activity for the IPNS Upgrade Target (TBq)

\begin{tabular}{|c|c|c|c|c|c|c|c|c|}
\hline \multirow[b]{2}{*}{$\begin{array}{l}\text { Cooling } \\
\text { Time }\end{array}$} & \multicolumn{4}{|c|}{$\begin{array}{l}\text { Tantalum, } \\
\text { Irradiation Time (days) at } 1 \mathrm{MW}\end{array}$} & \multicolumn{4}{|c|}{$\begin{array}{l}\text { Tungsten, } \\
\text { Irradiation Time (days) at } 1 \mathrm{MW}\end{array}$} \\
\hline & 1 & 7 & 30 & 365 & 1 & 7 & 30 & 365 \\
\hline 0 & 16,500 & 21,800 & 29,700 & 68,900 & 17,780 & 24,730 & 28,270 & 38,690 \\
\hline $5 \min$ & 7,210 & 11,200 & 18,400 & 57,100 & 15,410 & 22,350 & 25,900 & 36,210 \\
\hline $10 \mathrm{~min}$ & 6,410 & 10,400 & 17,500 & 56,200 & 14,310 & 21,260 & 24,800 & 35,120 \\
\hline $30 \mathrm{~min}$ & 4,850 & 8,810 & 16,000 & 54,600 & 11,970 & 18,880 & 22,420 & 32,740 \\
\hline $1 \mathrm{~h}$ & 4,100 & 8,030 & 15,200 & 53,800 & 10,230 & 17,070 & 20,620 & 30,930 \\
\hline $8 \mathrm{~h}$ & 2,270 & 5,890 & 13,000 & 51,700 & 6,260 & 11,220 & 14,690 & 25,000 \\
\hline $1 d$ & 1,240 & 4,380 & 11,400 & 49,800 & 3,050 & 7,290 & 10,650 & 20,890 \\
\hline $7 d$ & 394 & 2,490 & 9,050 & 46,100 & 2,390 & 1,480 & 4,280 & 13,960 \\
\hline $30 \mathrm{~d}$ & 272 & 1,870 & 7,410 & 39,700 & 109 & 730 & 2,670 & 10,530 \\
\hline $1 \mathrm{yr}$ & 37 & 255 & 1,020 & 5,920 & 7 & 47 & 190 & 1,170 \\
\hline
\end{tabular}




\subsection{THERMAL-HYDRAULIC ANALYSES}

A thermal-hydraulic analysis of the IPNS Upgrade target was undertaken to determine maximum temperatures and to supply temperature distributions for use in the stress analyses. Two models that were developed using the THTB computer code ${ }^{12}$ formed the basis for the thermal analysis. One has three-dimensional rectangular geometry with continuous power input. The second is a two-dimensional version for transient studies.

The geometry for the first model is shown in Figure IV.2.3-1. Because of symmetry, only half of the width and half of the thickness for both the target and the coolant channel are modeled. Variations in the power input in the axial (y) direction (see Figure IV.2.3-1) are small and are neglected at this stage of analysis. Because of temperature rise in the coolant, the longitudinal ( $\mathrm{z}$ ) direction (direction of coolant flow, normal to the target axis) is modeled in its entirety. The radial and axial power distributions were obtained from the neutronics analysis described in Section IV.2.2. Each succeeding target disk has a lower power according to the calculated axial distribution. Heat-transfer coefficients were calculated using the Dittus-Boelter formulation with entrance length correction. A roughened surface was assumed, as discussed in Section IV.2.6. A continuous power input equivalent to an average over the actual pulsed operation was assumed, giving a steady-state temperature solution. The target disks are represented as being rectangular, which is not the case for the actual disks (Figures IV.2.1-1 and IV.2.1-2). However, because power levels are low at the edges of the disks, heat transfer there is minimal. Therefore, this geometrical simplification has negligible impact on the calculations.

A separate two-dimensional THTB model was developed to study the transient operation in more detail. This model, shown in Figure IV.2.3-2, used results from the first model to set coolant-temperature boundary conditions. Because the energy is deposited more or less uniformly through the thickness of the disks and then flows axially to the coolant, this model yields accurate results. This model includes a more detailed nodal structure in the radial and axial directions and a coordinate system compatible with the power input, which the first model does not have. A machinable tungsten material coated with a $10-\mu \mathrm{m}$ thickness of titanium was assumed for both models. The temperature-dependent conductivity for machinable tungsten was approximated by that for pure tungsten, decreased by the ratio of conductivities at room temperature (0.745). ${ }^{13}$

Results of the thermal-hydraulic analyses based on the first model are shown in Figures IV.2.3-3 through IV.2.3-12, and the transient studies with the second model are shown in Figures IV.2.3-13 through IV.2.3-16. Figure IV.2.3-3 shows the radial power distribution used for the upstream target section. Four axial positions are shown, corresponding to the calculations in the neutronics model. Parametric studies were performed for various disk thicknesses, coolant flow velocities, and disk materials, using the power distributions at these positions as input. The effect of coolant velocity on the centerline, surface, and outlet coolant temperatures for a $1-\mathrm{cm}$ thick tungsten target disk is shown in Figure IV.2.3-4. Figure IV.2.3-5 shows the effect of disk 


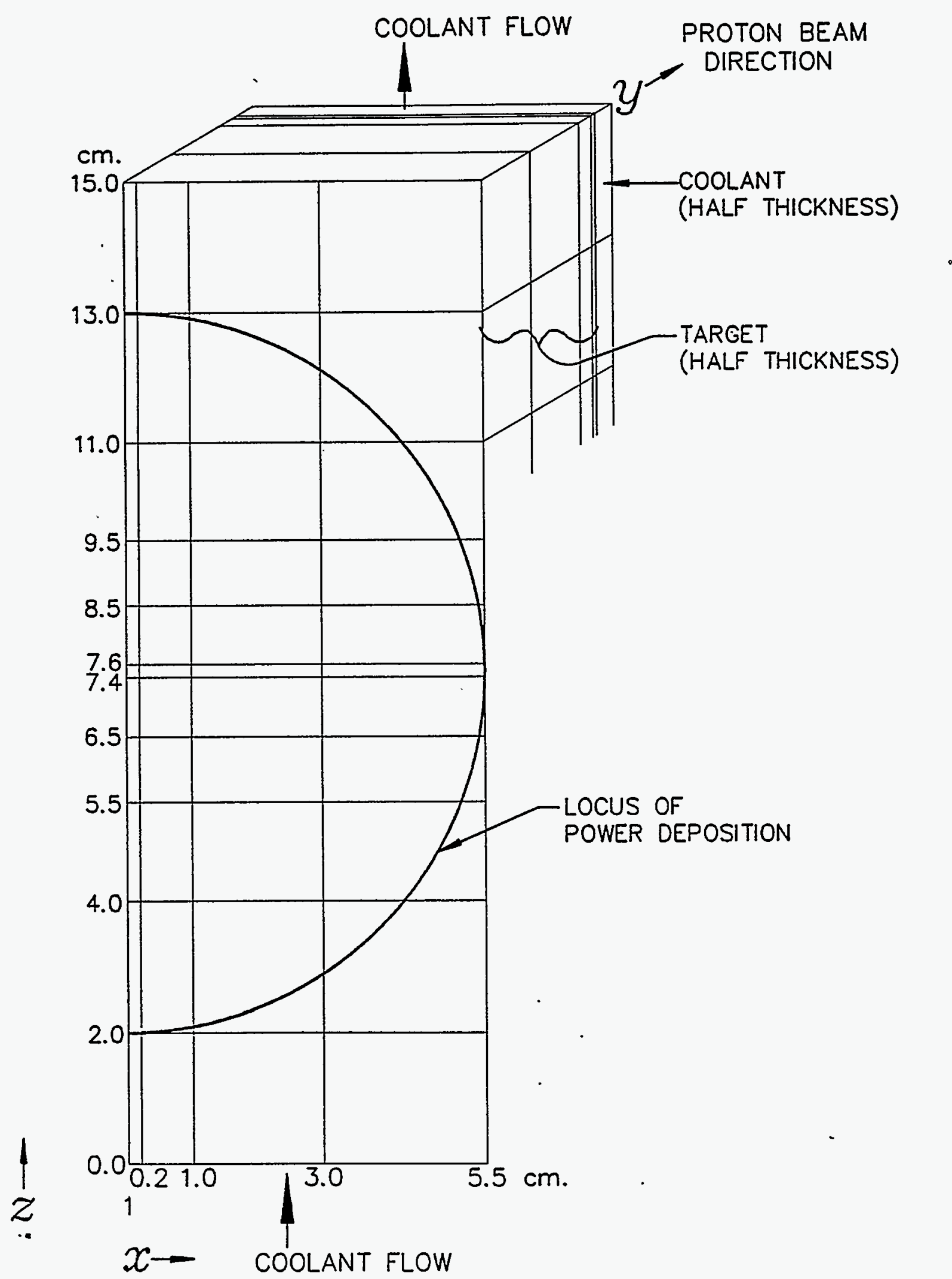

Figure IV.2.3-1 Geometry for Three-Dimensional Thermal-Hydraulic Model of IPNS Upgrade Target Disk. 


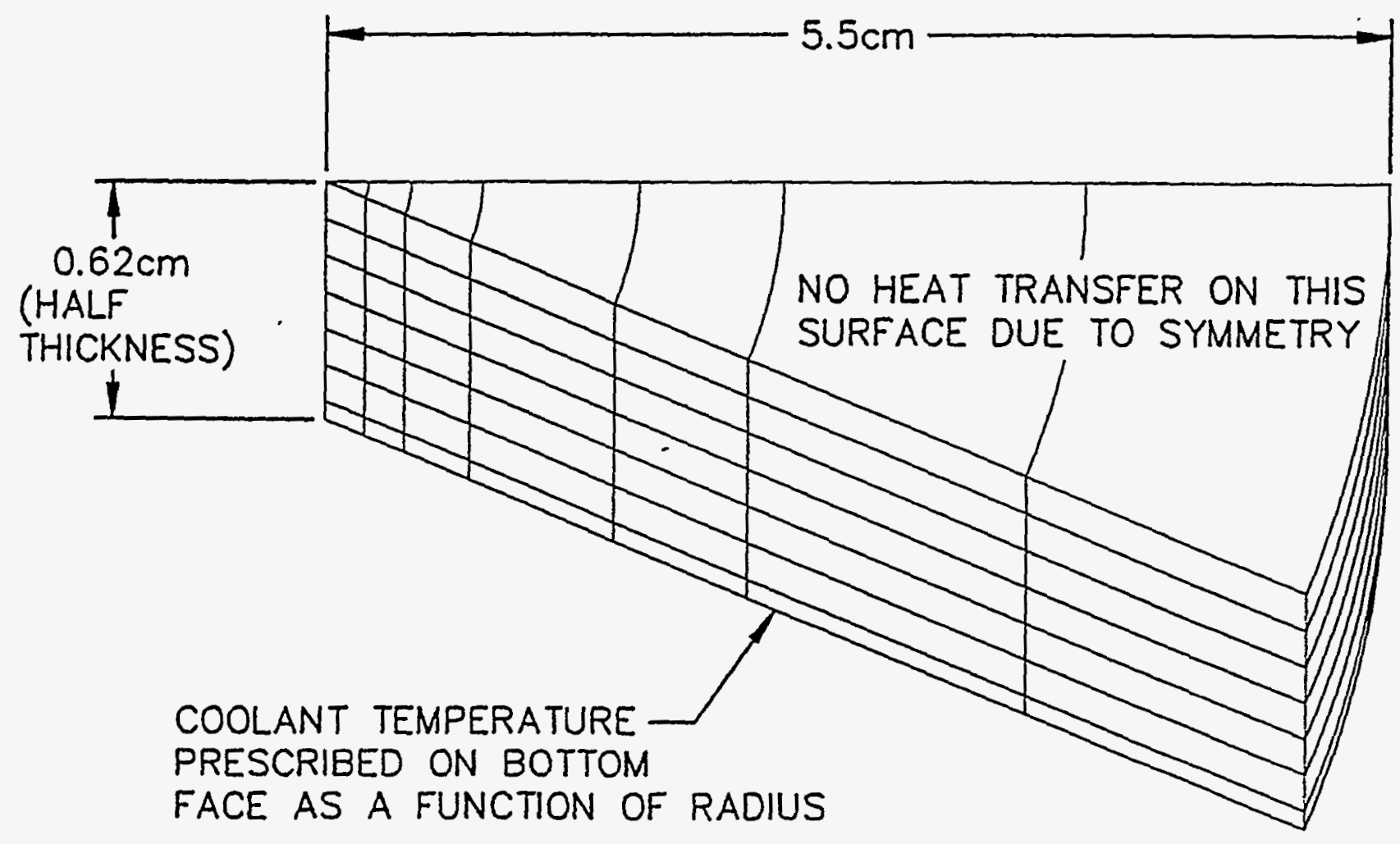

Figure IV.2.3-2 Geometry for Two-Dimensional Thermal-Hydraulic Model. (Note that axial and radial scales differ by a factor of 2 .) 


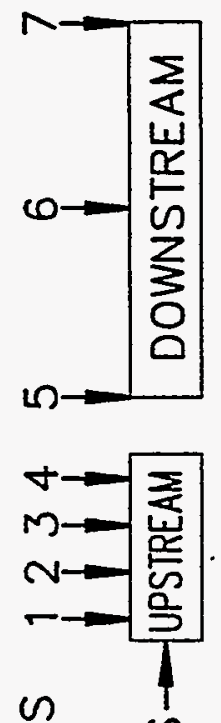

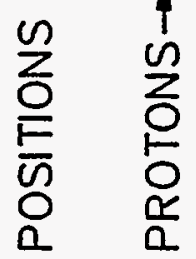
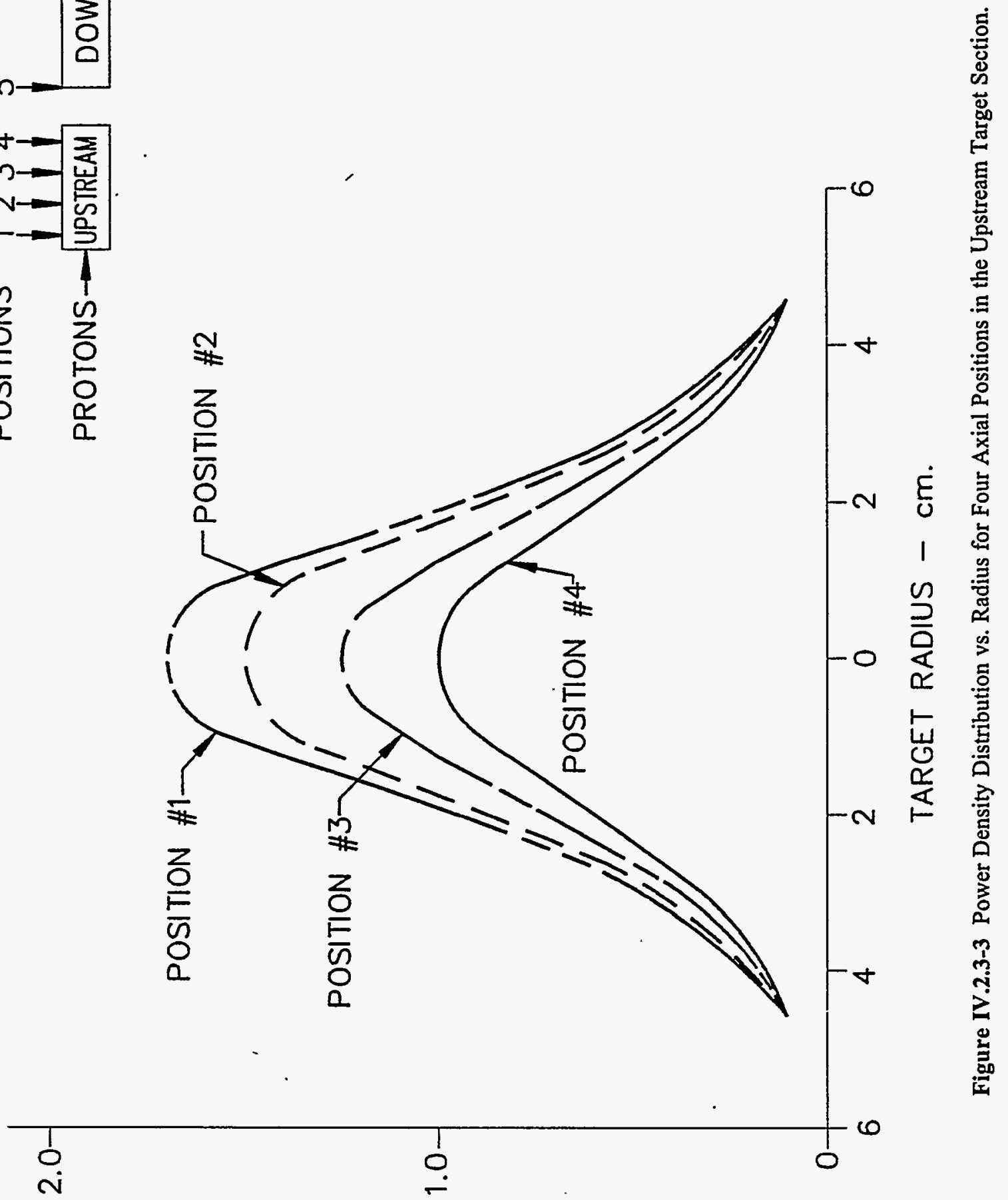

$W \forall \exists g \mathrm{MW} b / \varepsilon U \sigma / M H$ 
THICKNESS - DISK $-1 \mathrm{~cm}$. CHANNEL - $1.59 \mathrm{~mm}$

MATERIAL - TUNGSTEN

POWER LEVEL AND DISTRIBUTION

AS AT POSITION 3

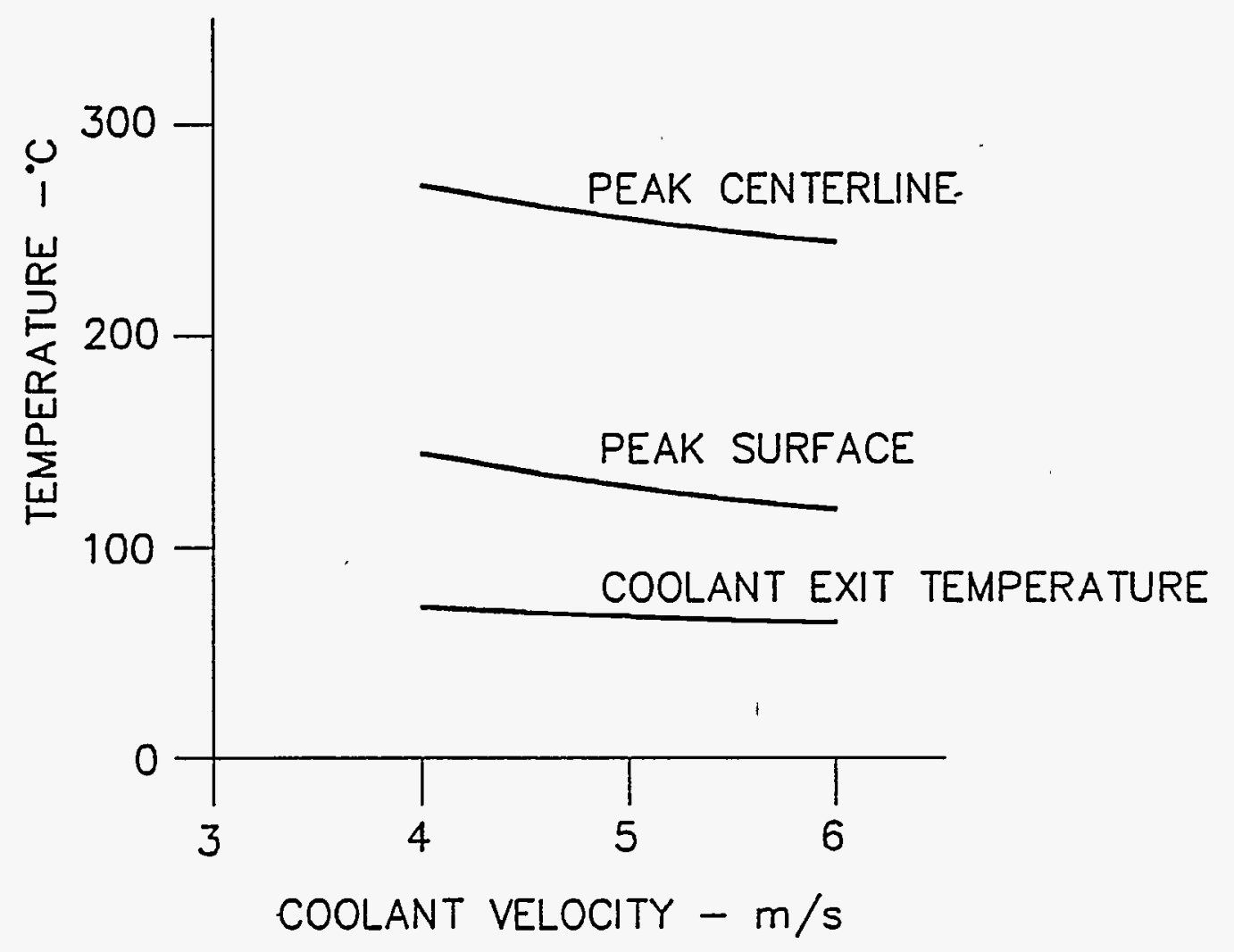

Figure IV.2.3-4 Centerline, Surface, and Coolant Exit Temperature vs. Coolant Velocity for Disk at Position 3. 


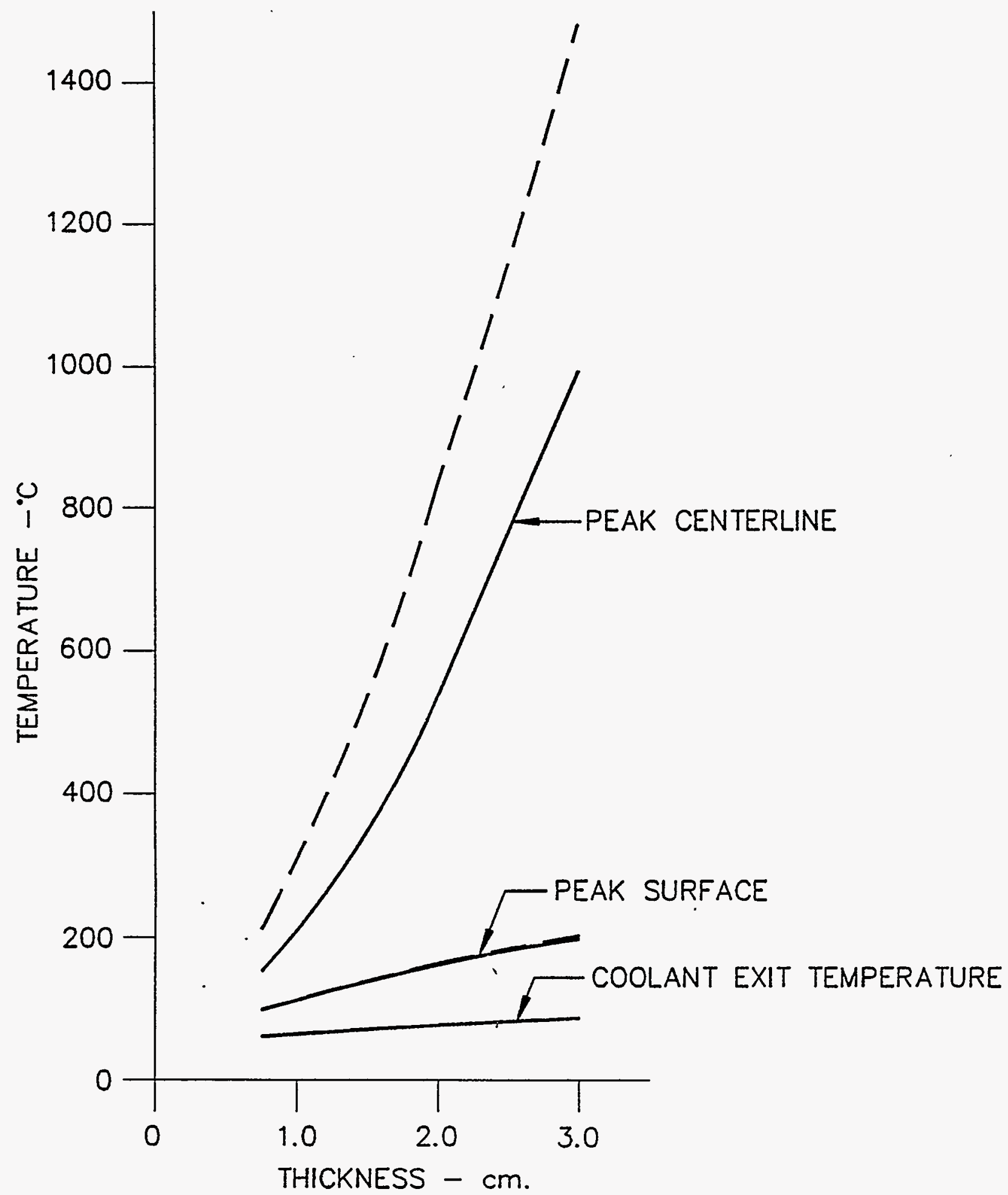

Figure IV.2.3-5 Centerline, Surface, and Coolant Exit Temperature vs. Disk Thickness for Disk at Position 1. 


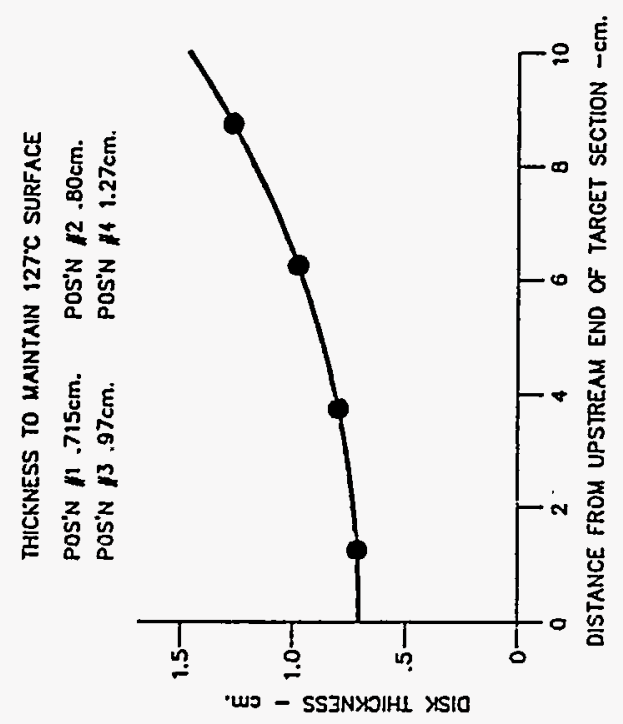

3NYOIHL YSIO 


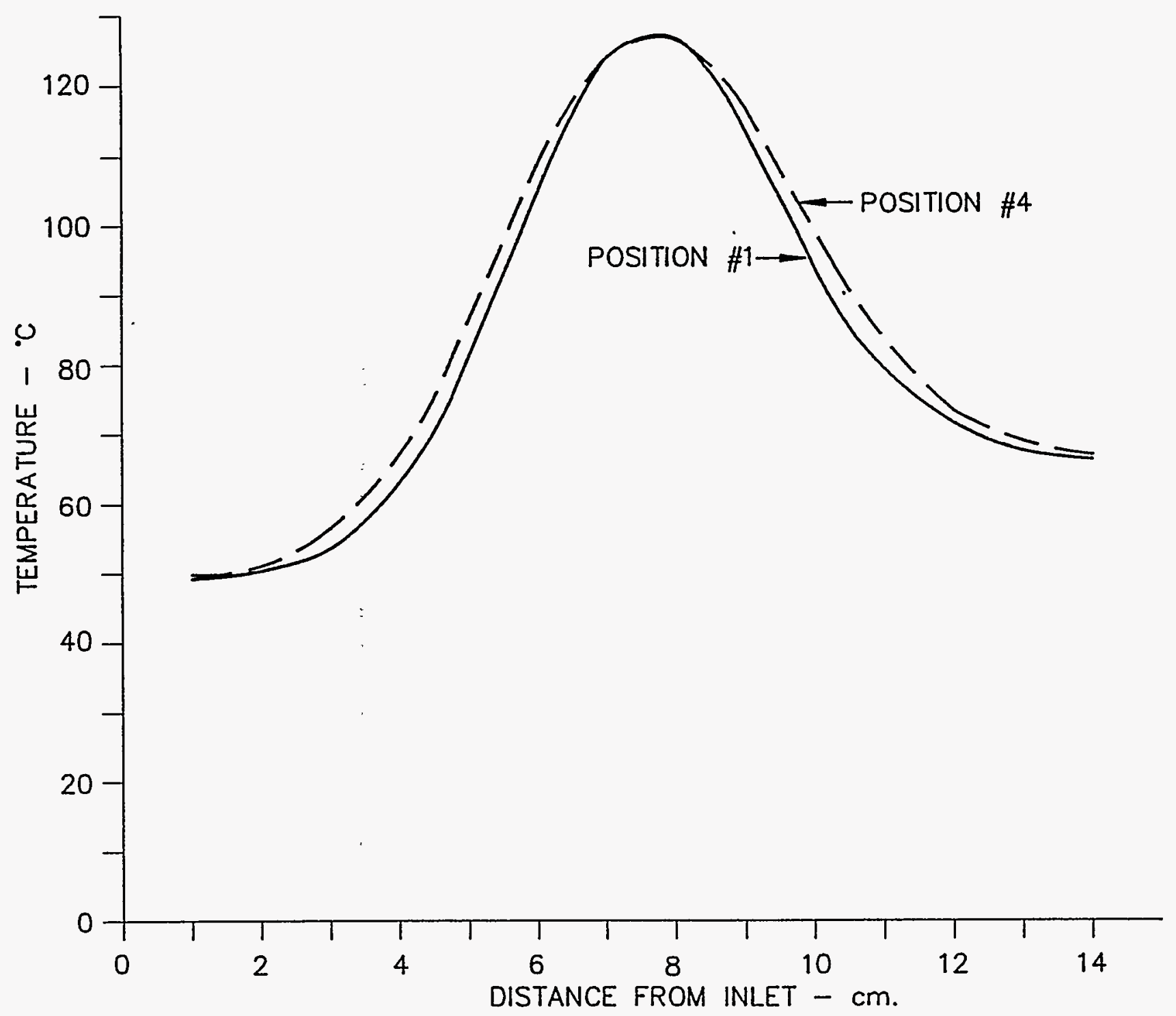

Figure IV.2.3-7 Surface-Temperature Distribution in Flow Direction for Two Axial Positions in the Upstream Target Section. 
ACTUAL-THICKNESS DISKS WITH

$1.59-\mathrm{mm}$ COOLING CHANNELS $(12.06 \mathrm{~cm}$ TOTAL)

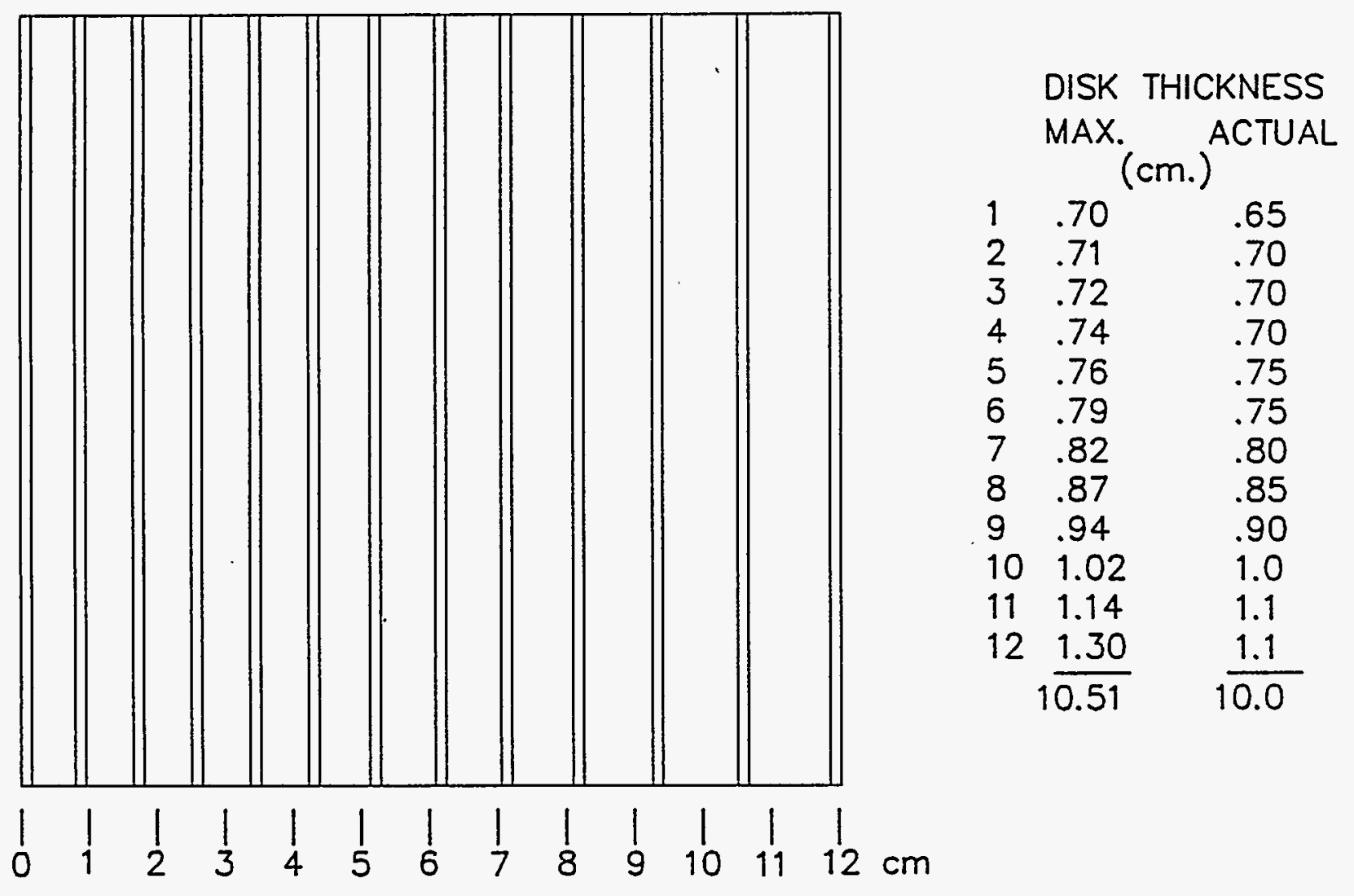

Figure IV.2.3-8 Upstream Target Section Configuration. 


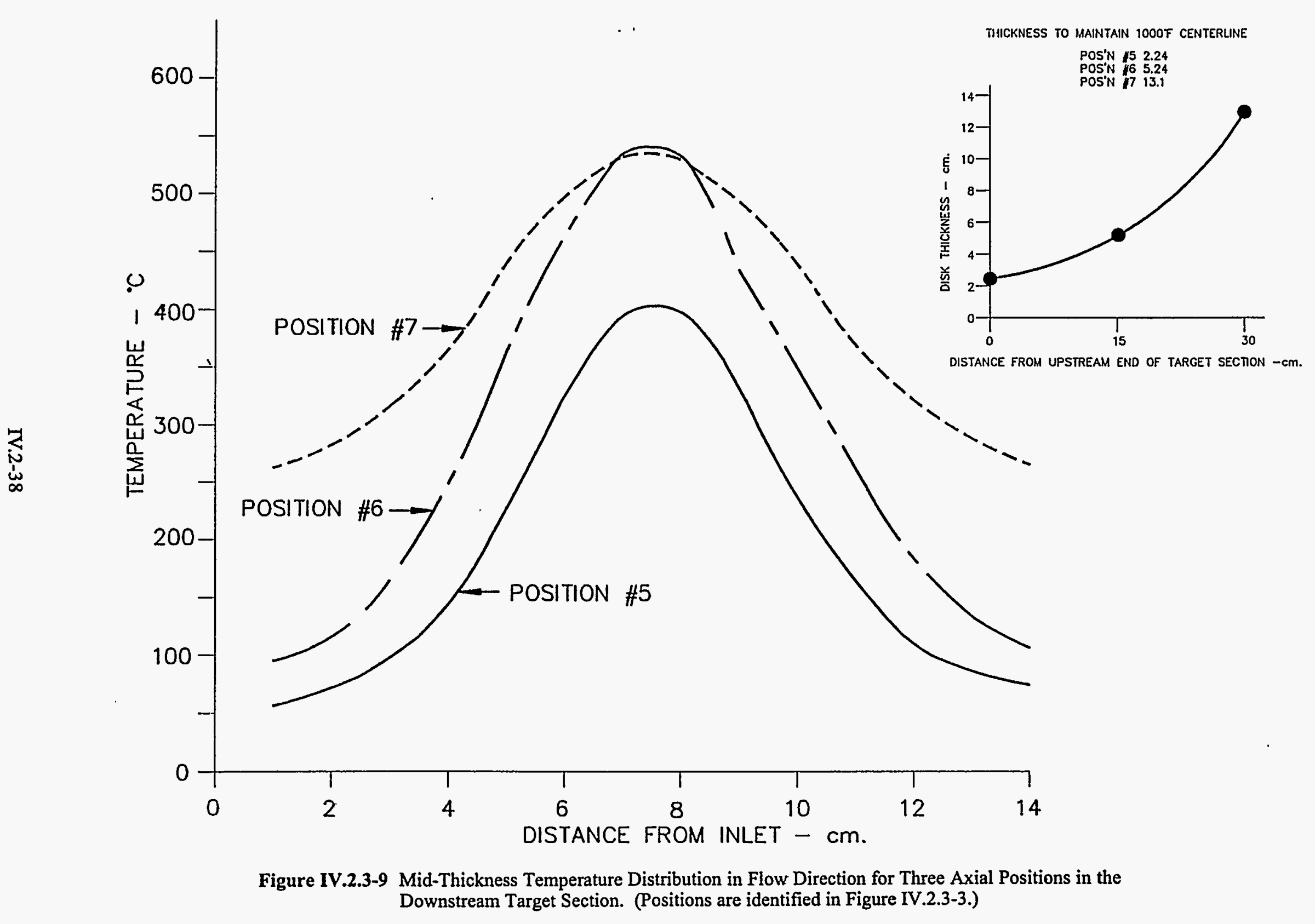




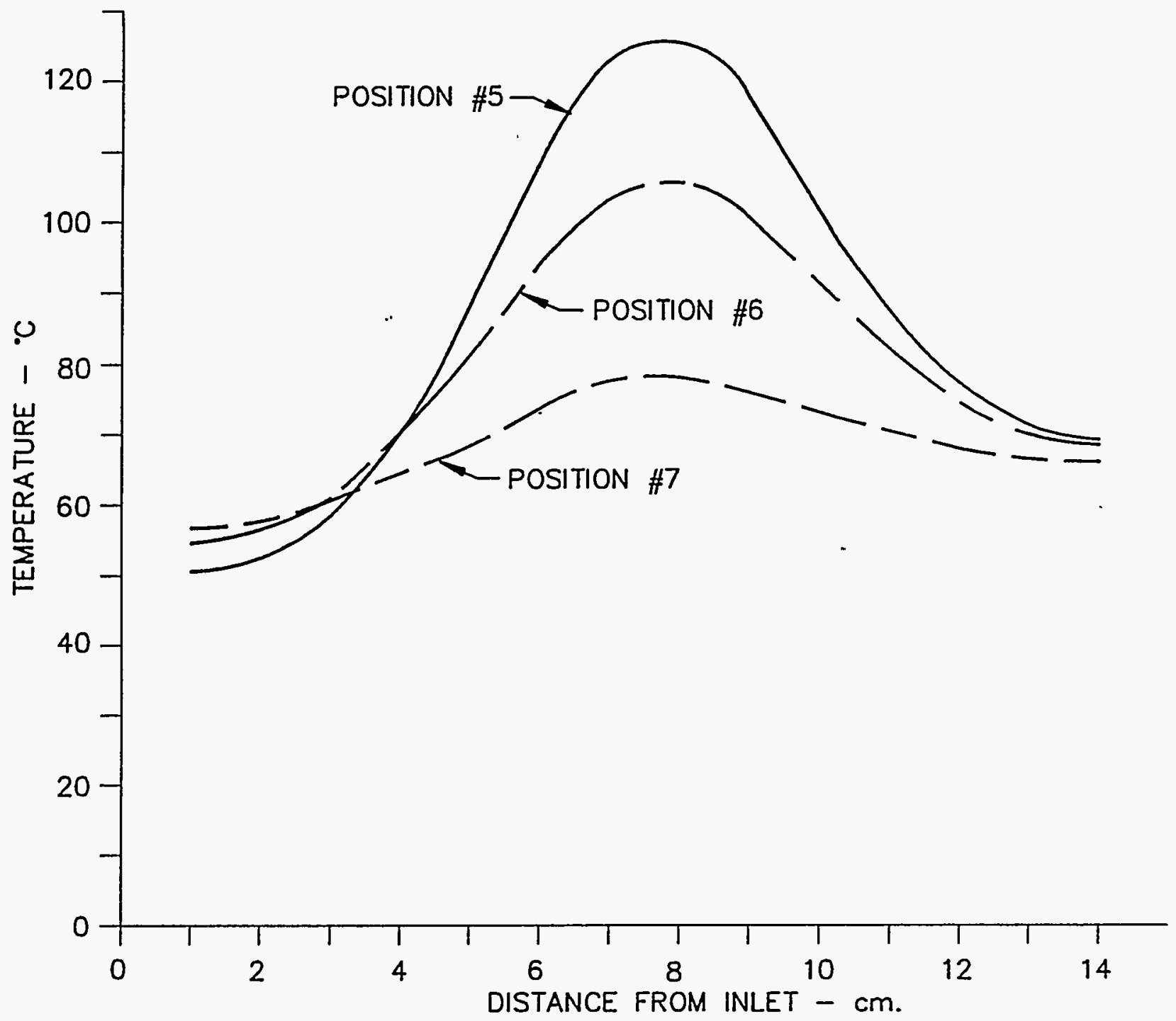

Figure IV.2.3-10 Surface Temperature Distribution in the Flow Direction for Three Axial Positions in the Downstream Target Section. 
ACTUAL-THICKNESS DISKS WITH

1.59- $\mathrm{mm}$ COOLING CHANNELS $(31.59 \mathrm{~cm}$ TOTAL)

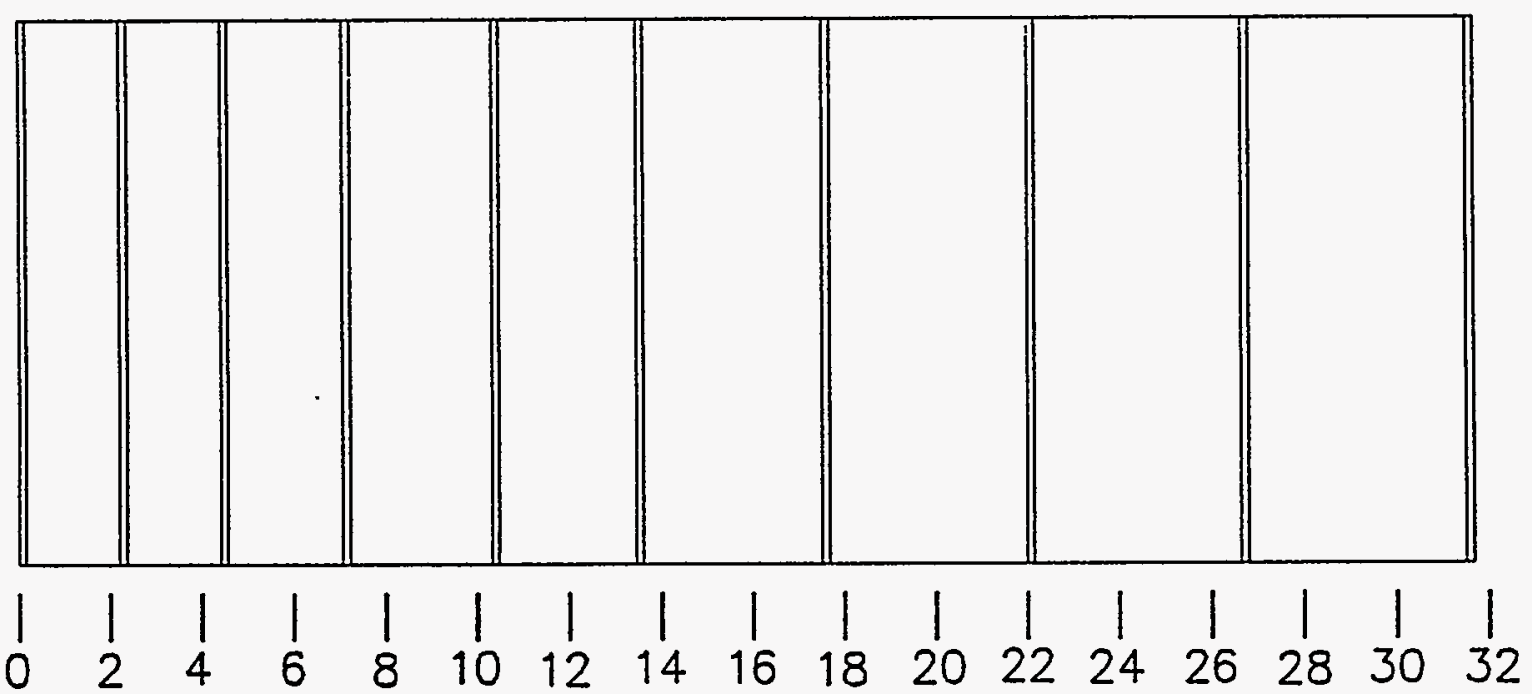

DISK THICKNESS

MAX. ACTUAL

\begin{tabular}{lll}
1 & 2.2 & (cm.) \\
2 & 2.4 & 2.0 \\
3 & 2.7 & 2.5 \\
4 & 3.2 & 3.0 \\
5 & 3.7 & 3.0 \\
6 & 4.4 & 4.0 \\
7 & 5.8 & 4.5 \\
8 & 7.7 & 4.5 \\
9 & 10.3 & 4.5 \\
\hline & $\frac{42.4}{30.0}$
\end{tabular}

Figure IV.2.3-11 Downstream Target Section Configuration. 
UPSTREAM TARGET SECTION

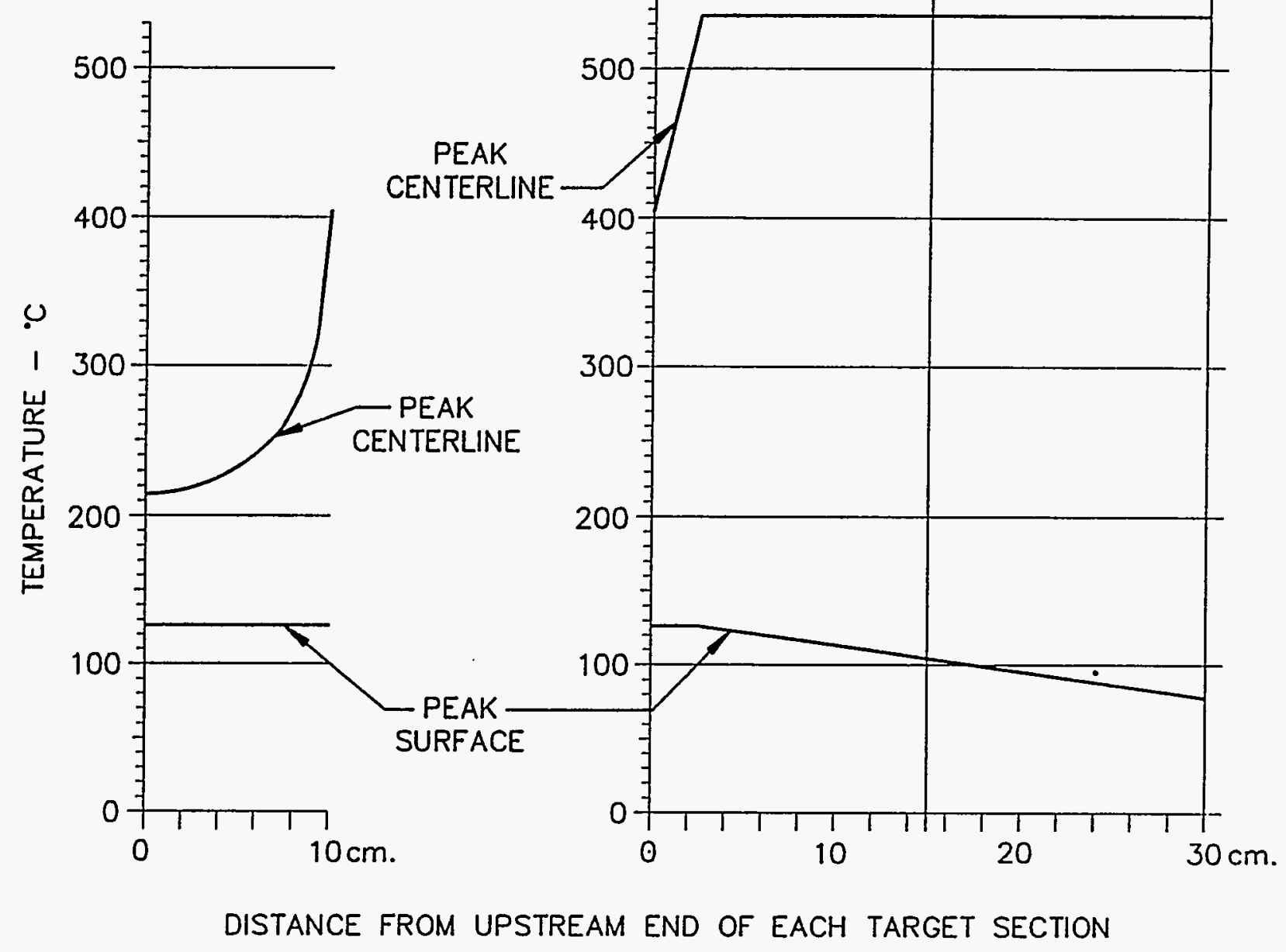

Figure IV.2.3-12 Peak Centerline and Surface Temperature vs. Axial Position, Upstream and Downstream Target Sections. 


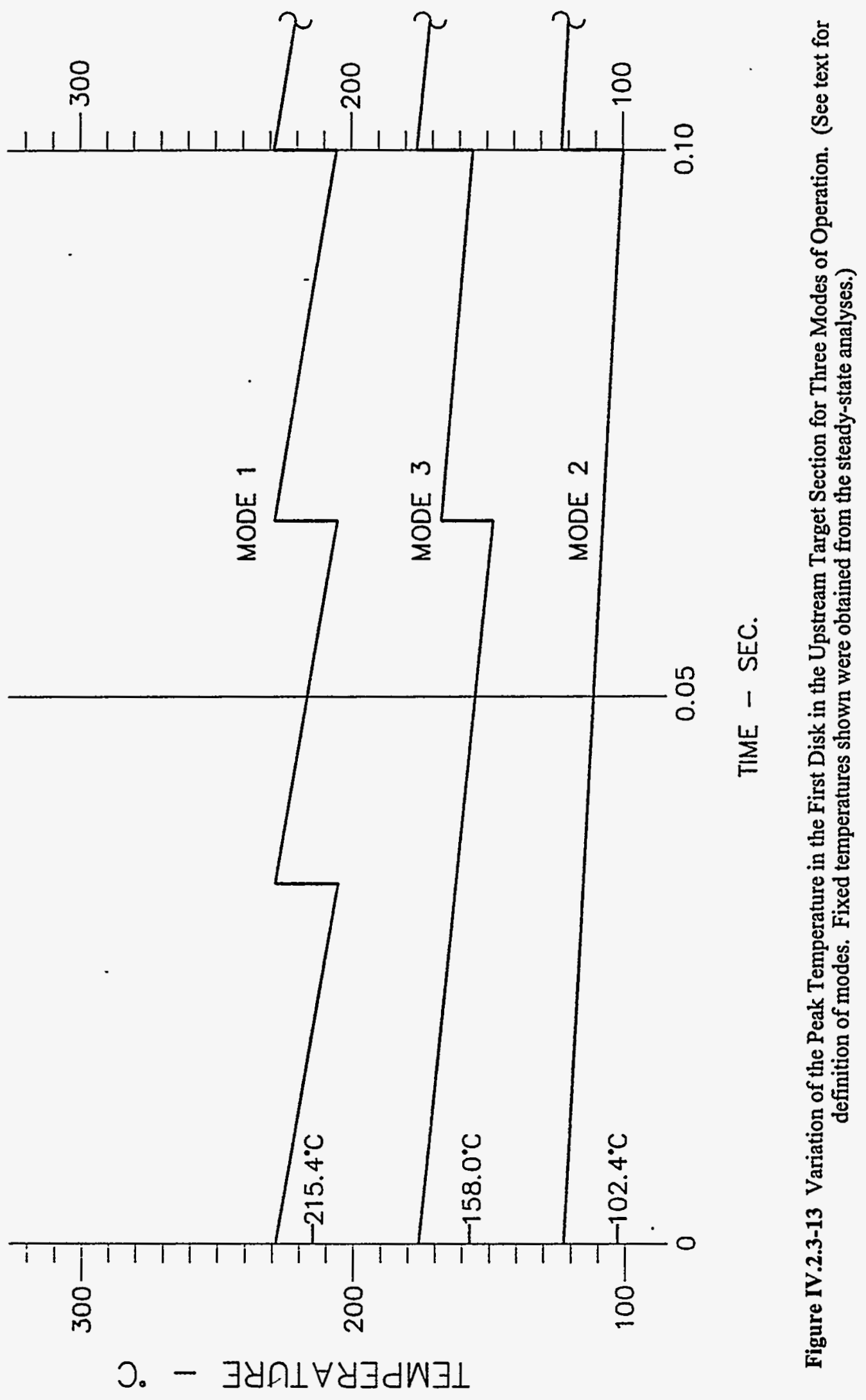




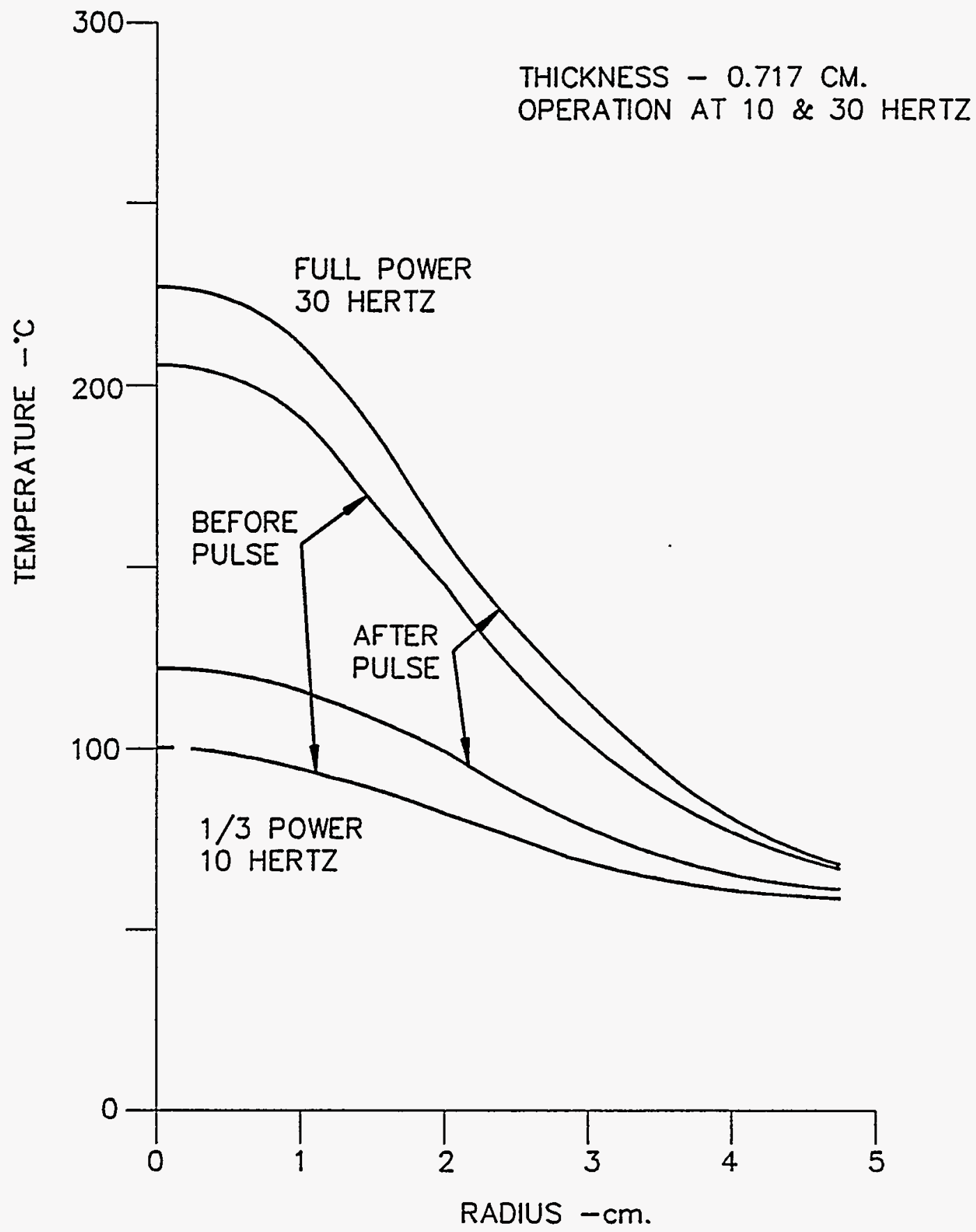

Figure IV.2.3-14 Radial Temperature-Time Variation in a Disk at Position 1 in the Upstream Target Section, Operated in Mode 1 or 2. 
THICKNESS $-0.717 \mathrm{~cm}$.

OPERATION - 2 PULSES ON, 1 OFF

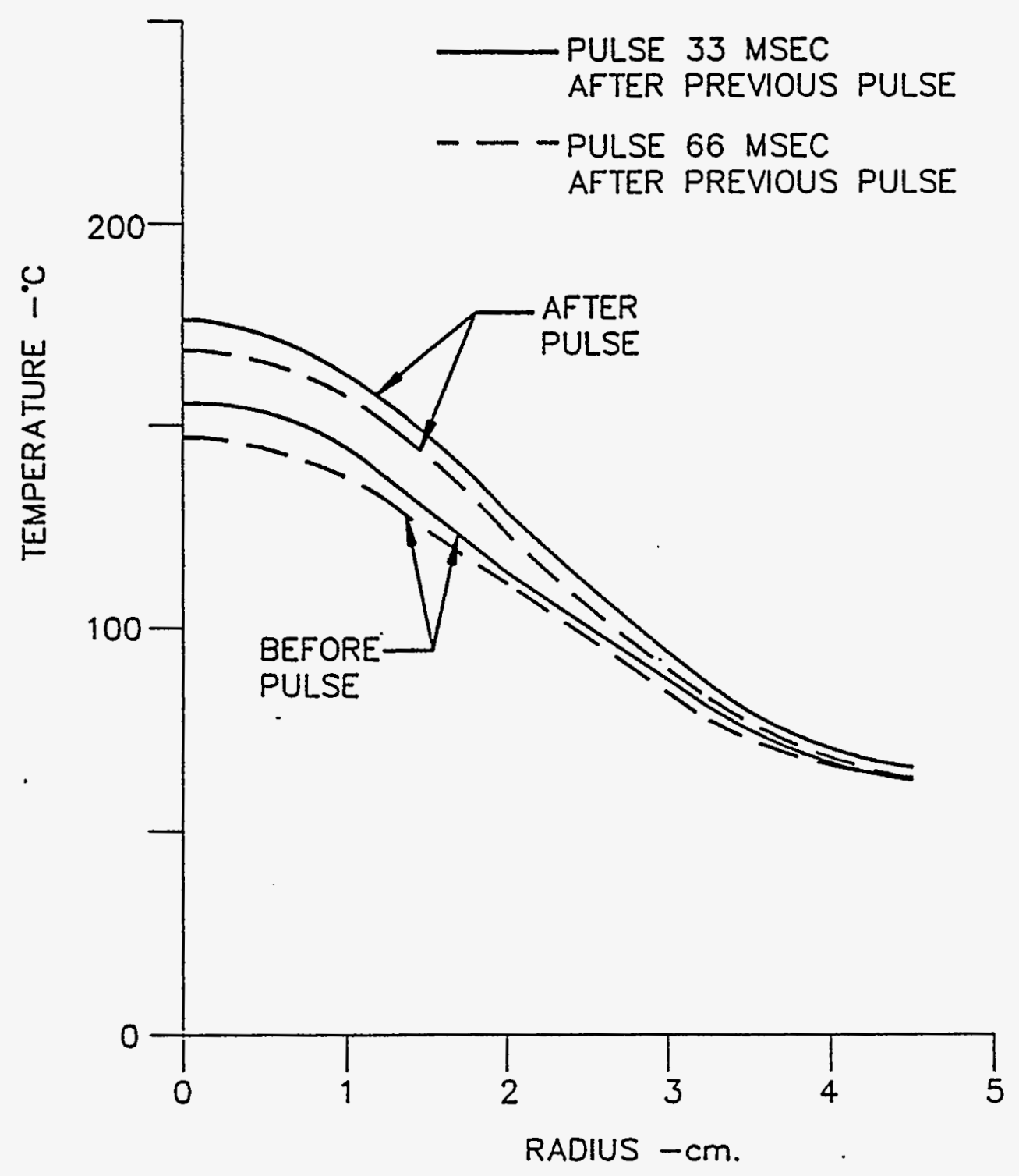

Figure IV.2.3-15 Radial Temperature-Time Variation in a Disk at Position 1 in the Upstream Target Section, Operated in Mode 3. 
THICKNESS $-1.24 \mathrm{~cm}$.

OPERATION = FULL POWER AT $30 \mathrm{~Hz}$.

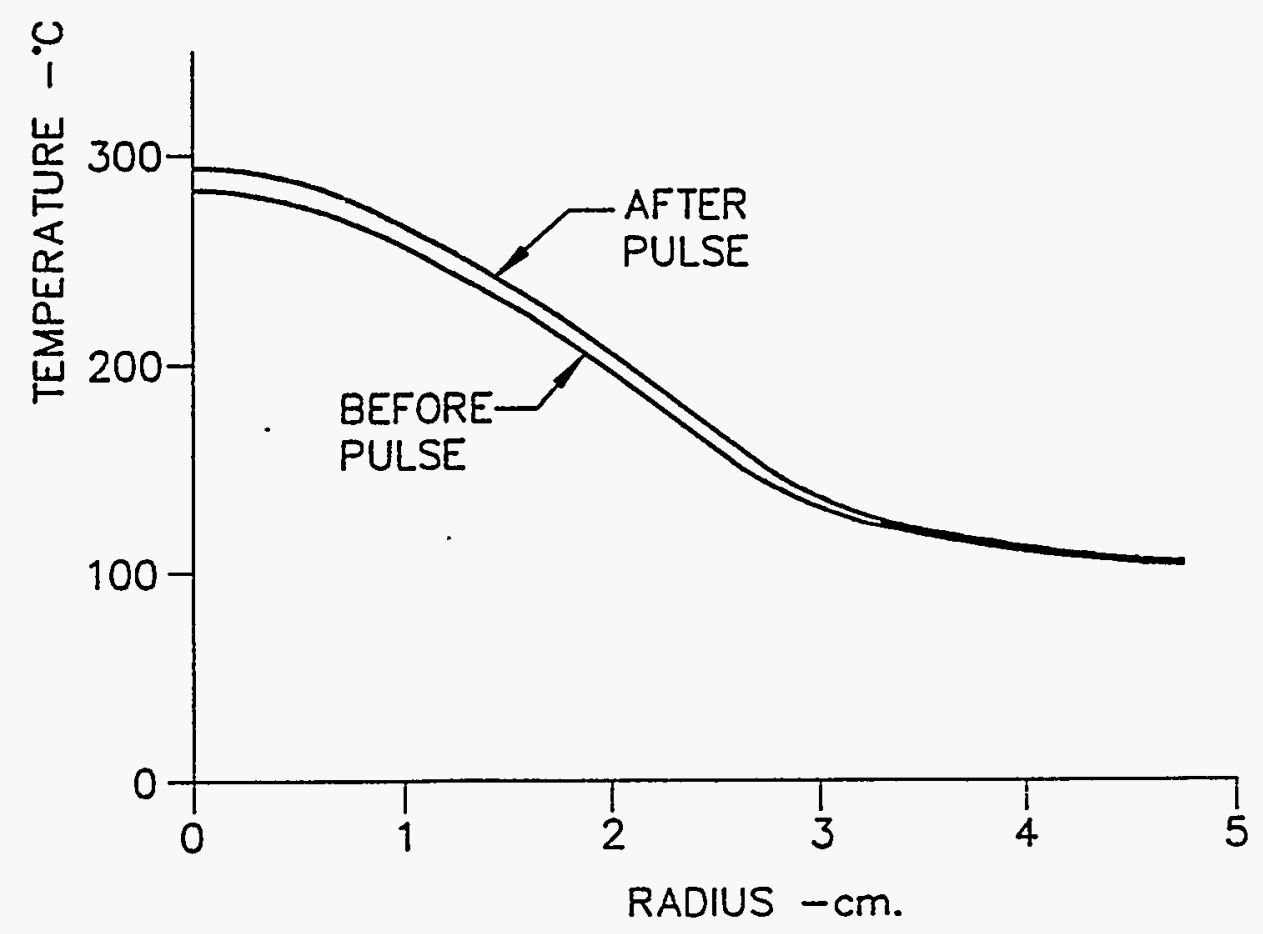

Figure IV.2.3-16 Radial Temperature-Time Variation in a Disk at Position 4 in the Upstream Target Section, Operated in Mode 1. 
thickness on disk and coolant temperatures for a coolant velocity of $5 \mathrm{~m} / \mathrm{s}$ for target disks fabricated from machinable tungsten and also from tantalum.

The peak disk temperature for these four power distributions is at the center of the disk, as shown in Figure IV.2.3-6. The maximum thickness of each disk was adjusted to limit the surface temperature to $127^{\circ} \mathrm{C}$. This maximum temperature occurs over a very local area at the peak of the energy input (center of the target disk), as seen in Figure IV.2.3-7. This surface temperature was chosen avoid local boiling at the design system pressure of $830 \mathrm{kPa}$. The maximum disk thicknesses at each of these four positions are plotted as a function of axial position in the small graph of Figure IV.2.3-6. Interpolations on this curve were used to determine the maximum thicknesses of all the disks in the upstream target section, under the requirement that the maximum surface temperature be $\leq 127^{\circ} \mathrm{C}$. Actual disk thicknesses were adjusted down from these maximum values to achieve the desired overall length of the target section. The resulting upstream target section consists of 12 disks, which vary in thickness from 0.65 to $1.1 \mathrm{~cm}$ as shown in Figure IV.2.3-8. This target section contains a total of $10-\mathrm{cm}$ thickness of target material, with $0.159-\mathrm{cm}$ coolant gaps between adjacent target disks and at the ends. Because the actual disk thicknesses are less than the maximum thicknesses, the peak surface temperatures are lower than $127^{\circ} \mathrm{C}$.

A similar analysis was carried out to determine the disk thicknesses in the downstream target section (Figures IV.2.3-9 to IV.2.3-11), which consists of nine elements and has a total of 30-cm thickness of target material. The radial power distribution in the downstream target section was extrapolated from the upstream target section, scaled to the calculated axial power distribution in the downstream target section. The resulting power distributions were used to determine the thicknesses of the disks in the downstream target section. This assumption is conservative because the actual power distributions are flatter. Beginning at a depth of $2.5 \mathrm{~cm}$, the disk thicknesses in the downstream target section are limited by centerline temperature rather than by surface temperature. The maximum disk thicknesses were chosen to keep the centerline temperature below $538^{\circ} \mathrm{C}$. Figure IV.2.3-11 shows the actual disk thicknesses, which were chosen to be less than the calculated maximum values. Figure IV.2.3-12 shows both the centerline and surface temperatures used to determine the thicknesses in both target sections.

The power input to the target is not constant with time, as was assumed in the first model. The time-power history is coordinated with the proton pulses, which occur at a $30-\mathrm{Hz}$ rate in the accelerator but are multiplexed between the two target stations. Thus, one target may receive a pulse every $1 / 30$ th of a second (Mode 1), every 1/10th of a second (Mode 2), or alternating between $1 / 30$ th and $1 / 15$ th of a second (Mode 3 ). These three modes of operation were analyzed with the second thermal model. Figure IV.2.3-13 shows the peak target-temperature response in a 0.717 -cm-thick disk at position 1 of the front target, along with the corresponding results for steady-state operation. It can be seen that the steady-state (continuous power input) temperatures accurately represent the average of the time-varying values. The radial temperature distributions before and after the pulse are shown in Figures IV.2.3-14 and IV.2.3-15 for the three modes of operation. Figure IV.2.3-16 shows these 
radial temperatures for position 4 in the upstream target section, calculated for the $30-\mathrm{Hz}$ operation.

Protons enter the target vessel through a stainless-steel window, which heats in a similar fashion to the target but is cooled on only one surface. Figure IV.2.3-17 shows the temperature distribution across the face of the window on both the cooled and uncooled window surfaces, calculated with the first model for a 0.5 -cm-thick window.

A high-density target can be easily fabricated using the parameters derived in this analysis. Maximum temperatures are within tolerable limits to prevent local boiling, so the flow is stable. Further refinement of the design may show some advantages in narrower flow channel widths and superior target materials.

\subsection{STRESS ANALYSIS}

A stress analysis performed on the target disks showed that all stresses are within acceptable limits. A thermal stress analysis of the target disks was performed by means of an analytical formulation 14 that uses a one-dimensional approximation to the temperature distribution. The analytical relations used are:

$$
\begin{aligned}
& \sigma_{r}=\alpha E\left[1 / R^{2} \int^{R} T\left(r^{\prime}\right) r^{\prime} d r^{\prime}-1 / r^{2} \int^{r} T\left(r^{\prime}\right) r^{\prime} d r^{\prime}\right], \\
& \sigma_{\mathrm{t}}=\alpha \mathrm{E}\left[-\mathrm{T}(\mathrm{r})+1 / \mathbb{R}^{2} \int^{\mathrm{R}} \mathrm{T}\left(\mathrm{r}^{\prime}\right) \mathrm{r}^{\prime} d \mathrm{r}^{\prime}+1 / \mathrm{r}^{2} \int^{\mathrm{r}} \mathrm{T}\left(\mathrm{r}^{\prime}\right) \mathrm{r}^{\prime} \mathrm{dr} \mathbf{r}^{\prime}\right] \text {, } \\
& \sigma_{a}=0, \\
& \mathrm{SI}=\operatorname{MAX}\left\{\left|\sigma_{\mathrm{r}}-\sigma_{\mathrm{t}},\right| \sigma_{\mathrm{r}}-\sigma_{\mathrm{d}}, \mid \sigma_{\mathrm{t}}-\sigma_{\mathrm{a}}\right\}
\end{aligned}
$$

and

where

$\sigma_{\mathrm{r}}, \sigma_{\mathrm{t}}$, and $\sigma_{\mathrm{a}}$ are radial, tangential, and axial stresses;

SI is the stress intensity;

$\alpha$ is the coefficient of thermal expansion;

$E$ is the modulus of elasticity; 


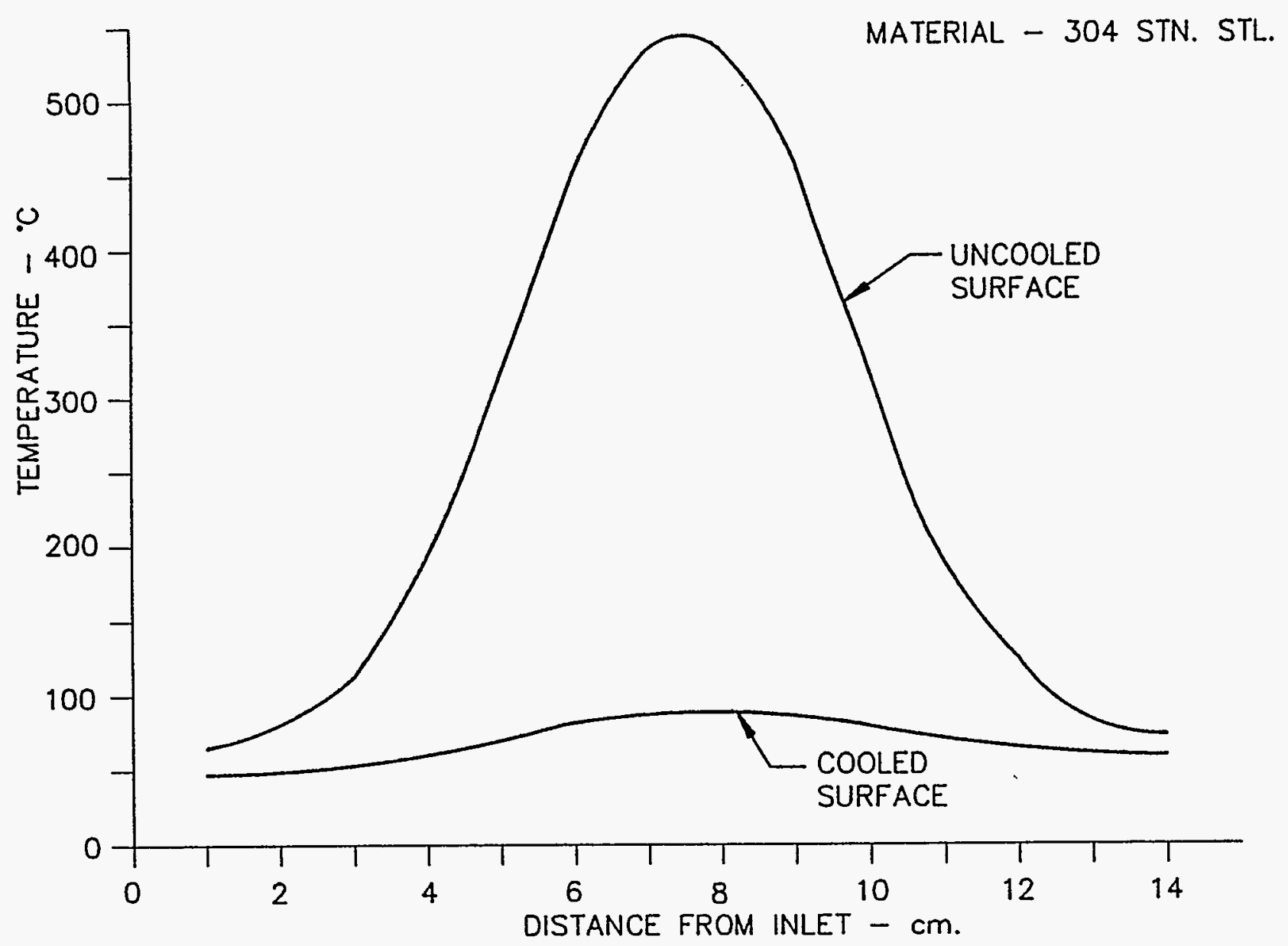

Figure IV.2.3-17 Surface Temperature of Target Vessel Window. 
$T(r)$ is the temperature at $r$ minus that at $R$; and

$\mathrm{R}$ and $\mathrm{r}$ are the full and local radii of the disk, respectively.

Equations IV.2.4-1 through IV.2.4-4 give the stress in a circular disk heated symmetrically about its center and uniformly through its thickness. The temperature is assumed to be a function of radius only.

Additionally, an ANSYS code ${ }^{15}$ model was used to obtain stresses from a twodimensional (radial and axial) temperature distribution for one disk. Figure IV.2.4-1 shows this model.

Figure IV.2.4-2 shows the ultimate strength for pure tungsten as a function of temperature. Also shown are room temperature values for yield and ultimate strength for machinable tungsten alloys (K1700 and K1850). ${ }^{13}$ The curve for pure tungsten shows the general trend for variation of ultimate strength for the machinable tungsten materials. For most materials yield strength follows ultimate strength at a somewhat lower value. The maximum calculated stresses at positions 1-4 in the upstream target section and positions 5-7 in the downstream section are also plotted in this figure. These stresses all fall well below the ultimate strength curve for pure tungsten, and this can be expected for the machinable tungsten alloys as well.

The distribution of stress intensity over the radius of the disks, on the basis of Equations IV.2.4-1-IV.2.4-4, is plotted in Figure IV.2.4-3 for positions 1 and 4 in the upstream target section and in Figure IV.2.4-4 for positions 5, 6, and 7 in the downstream target section. The stress intensities in the upstream target section are higher in the thicker disks located toward the downstream end of that section. However, these stresses are less than the ultimate stress', so major deformations or cracking should not result. The highest stress in either target section occurs in the middle of the downstream target section (Figure IV.2.4-4), with a maximum value of $265 \mathrm{MPa}$ at the center. This is still sufficiently below the alloy's yield stress to present no difficulty.

Table IV.2.4-1 lists the stresses for cyclic operation, which could cause fatigue damage to the disks. Stresses in the steady-power cases are also tabulated. We have not yet assessed the effect of fatigue on the life of the target.

A two-dimensional ANSYS model was used to assess the combined influence of axial and radial temperature gradients on the stress level. A temperature distribution corresponding to radial variation only (uniform axial) was input to the 2-D model for comparison to the analytical results. The radial and tangential stresses over the radius agreed with the 1-D model within about $5 \%$ for a $1.27 \mathrm{~cm}$ thick disk at position 4 of the upstream target section. This agreement supports the validity of the results obtained with 2-D temperature variation. Figure IV.2.4-5 shows the results of this computation. In this 2-D calculation, the radius of the 


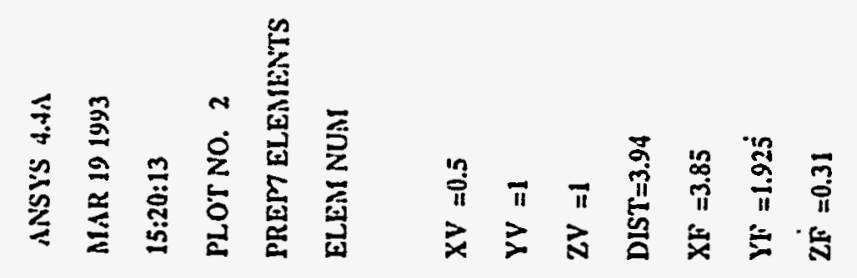

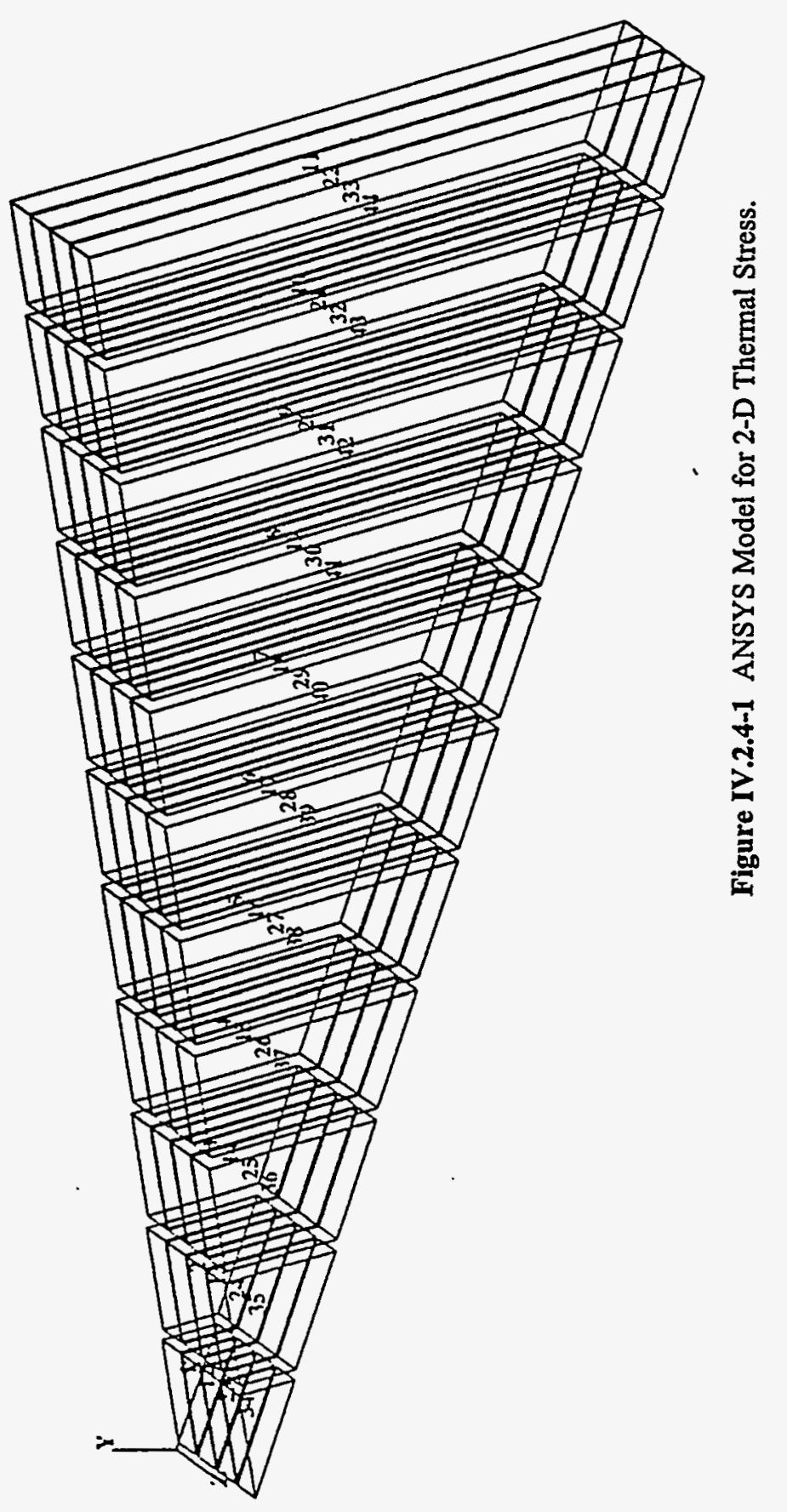




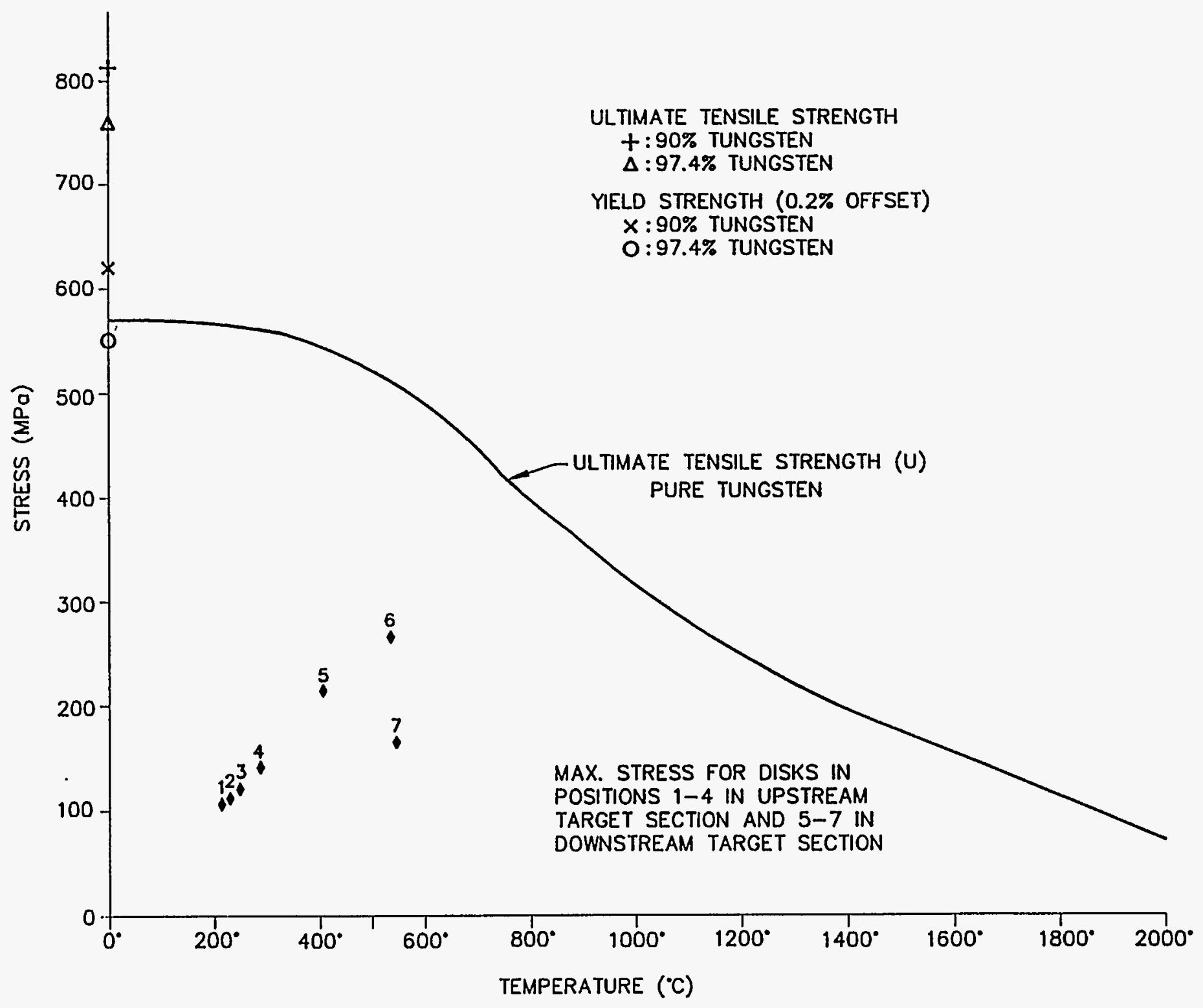

Figure IV.2.4-2 Mechanical Properties of Tungsten. 


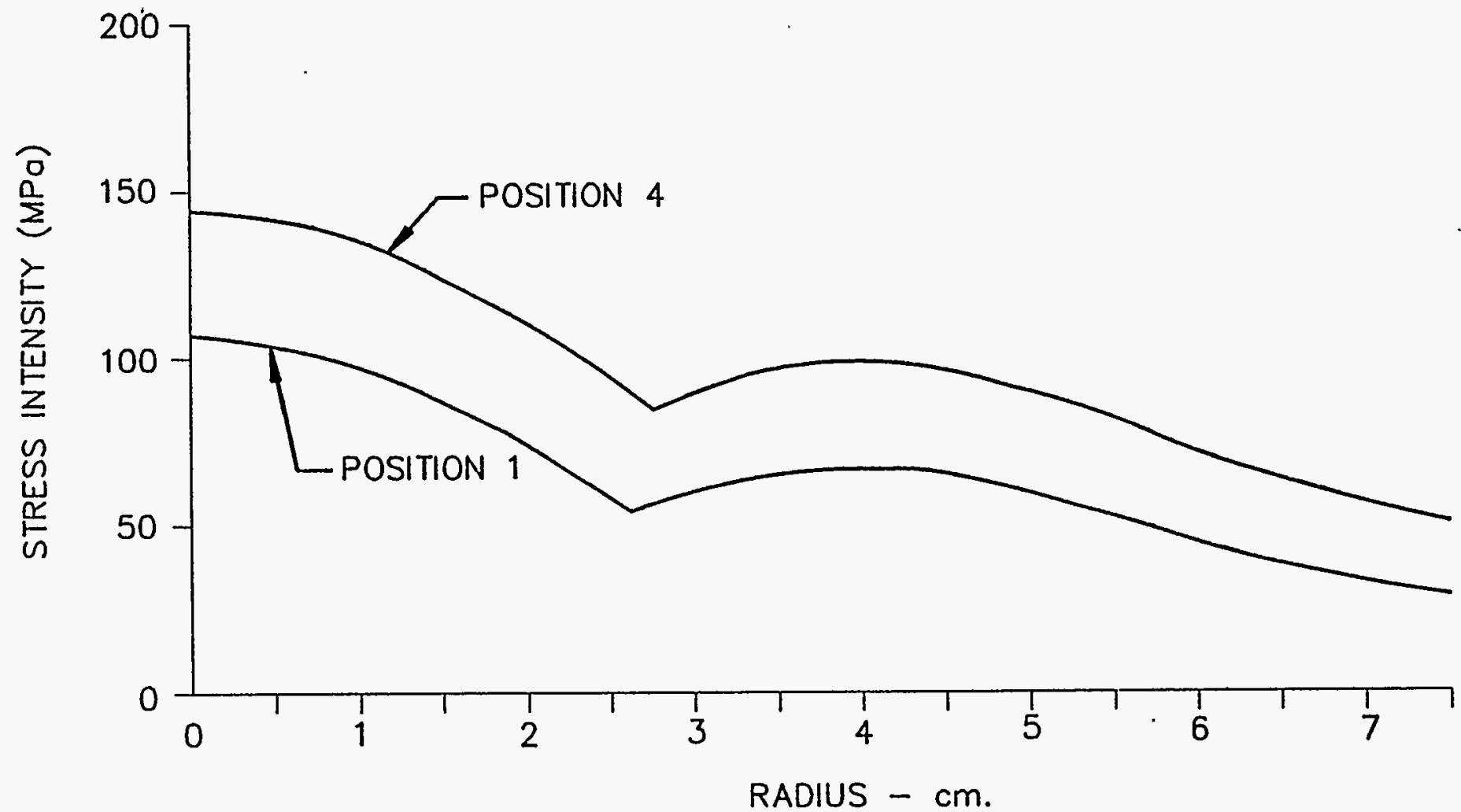

Figure IV.2.4-3 Stress Intensity in Disks at Two Positions in the Upstream Target Section Due to Radial Gradient Only. 


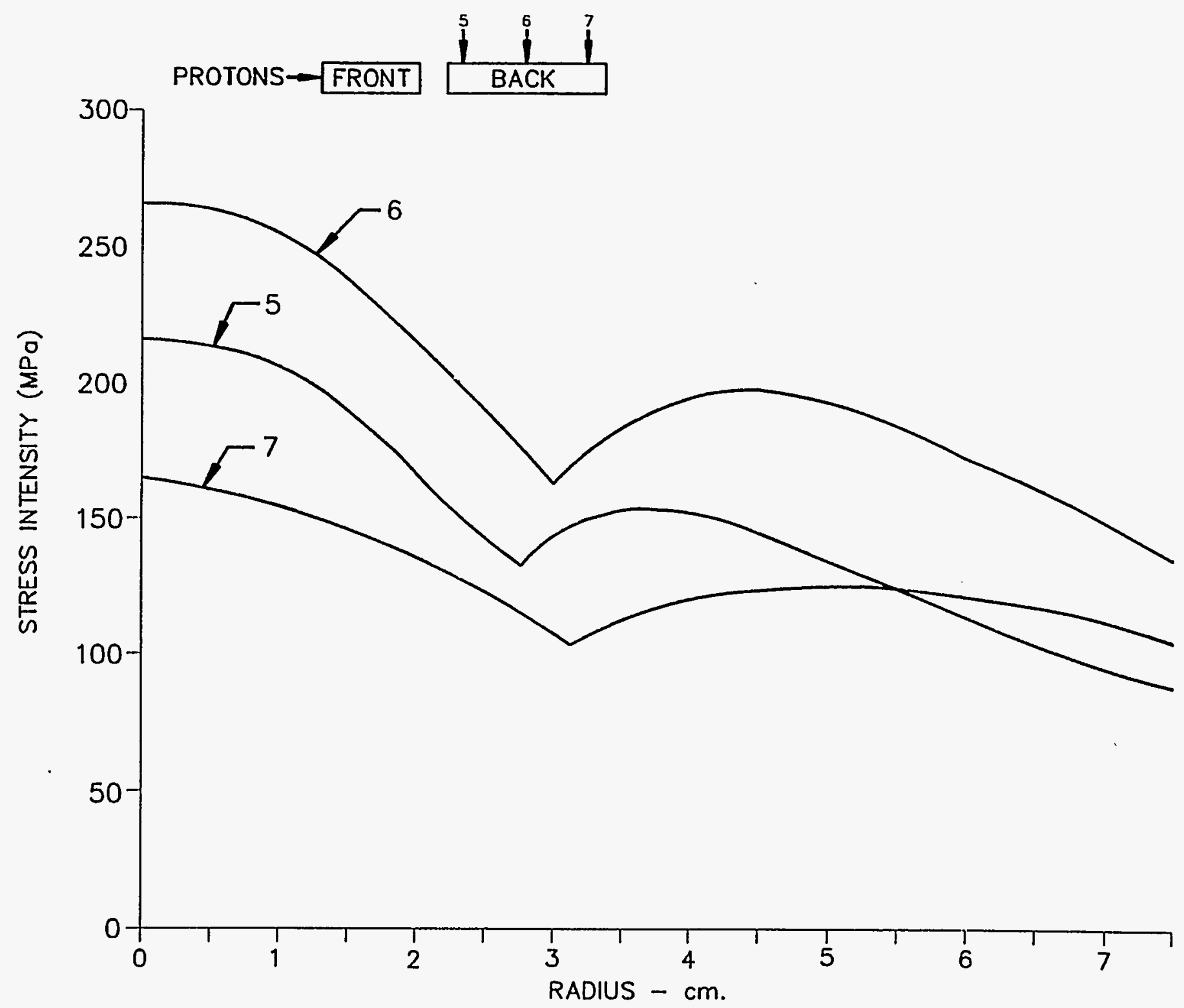

Figure IV.2.4-4 Stress Intensity in Disks at Three Positions in the Downstream Target Section Due to Radial Temperature Gradient Only. 
Table IV.2.4-1 Summary of Stresses

\begin{tabular}{|c|c|c|c|c|c|}
\hline $\begin{array}{l}\text { Target } \\
\text { Section }\end{array}$ & Position & Mode of Operation & $\begin{array}{l}\text { Power } \\
\text { Level }\end{array}$ & $\begin{array}{c}\text { Maximum } \\
\text { Temperature, }{ }^{\mathrm{a}} \\
{ }^{\circ} \mathrm{C}\end{array}$ & $\begin{array}{l}\text { Maximum Stress } \\
\text { Intensity } \\
\text { (MPa) }\end{array}$ \\
\hline Upstream & $\# 1$ & Steady power & Full & 214 & 96.4 \\
\hline Upstream & $\# 2$ & - & Full & 223. & 100.4 \\
\hline Upstream & \#3 & " & Full & 243. & 111.5 \\
\hline Upstream & $\# 4$ & $"$ & Full & 280. & 130.0 \\
\hline Downstream & $\# 5$ & " & Full & 403. & 215.9 \\
\hline Downstream & \# 6 & " & Full & 540. & 265.5 \\
\hline Downstream & $\# 7$ & " & Full & 540. & 165.0 \\
\hline Upstream & $\# 1$ & Mode 1, pre-pulse & Full & 206. & 83.1 \\
\hline Upstream & $\# 1$ & Mode 1, post-pulse & Full & 228. & 96.4 \\
\hline Upstream & $\# 1$ & Mode 2, pre-pulse & $1 / 3$ & 100. & 24.6 \\
\hline Upstream & $\# 1$ & Mode 2, post-pulse & $1 / 3$ & 122. & 36.7 \\
\hline Upstream & $\# 1$ & $\begin{array}{l}\text { Mode } 3 \text {, pre-pulse } \\
\text { after } 33 \mathrm{~ms} \text { off }\end{array}$ & $2 / 3$ & 156. & 55.3 \\
\hline Upstream & $\# 1$ & $\begin{array}{l}\text { Mode } 3 \text {, post-pulse } \\
\text { after } 33 \mathrm{~ms} \text { off }\end{array}$ & $2 / 3$ & 177. & 67.4 \\
\hline Upstream & $\# 1$ & $\begin{array}{l}\text { Mode } 3, \text { pre-pulse } \\
\text { after } 66 \mathrm{~ms} \text { off }\end{array}$ & $2 / 3$ & 148. & 50.8 \\
\hline Upstream & $\# 1$ & $\begin{array}{l}\text { Mode } 3 \text {, post-pulse } \\
\text { after } 66 \mathrm{~ms} \text { off }\end{array}$ & $2 / 3$ & 169. & 63.1 \\
\hline Upstream & $\# 4$ & Mode 1, pre-pulse & Full & 281. & 108.0 \\
\hline Upstream & \#4 & Mode 1 , post-pulse & Full & 294. & 114.6 \\
\hline
\end{tabular}

a Temperatures for the steady-power cases were calculated from a different model and hence are not directly comparable with those in Modes 1,2 , and 3. 
heated disk increases $26 \mu \mathrm{m}$, while both flat surfaces have maximum outward deformations of $8 \mu \mathrm{m}$ at their centers $($ at $\mathrm{r}=0.0 \mathrm{~cm})$.

This 2-D calculation gives peak tangential and radial stresses at $\mathrm{r}=0.0 \mathrm{~cm}$ that are about double those obtained above using the 1-D model. Other disks are expected to show similar increases in stress when a 2-D calculation is carried out. ANSYS calculations for a more representative geometrical model should not significantly change these stresses. The yield strength of tungsten alloy is substantially higher than stresses from the 2-D calculation. On the basis of the 1-D calculations, we expect that the stresses in all other disks will also be found to be below the yield strength. Because thermal stresses are secondary stresses, the ASME code limit is twice the yield strength giving an even greater margin of safety. Thus, all stresses appear to be within tolerable limits.

\subsection{TARGET METALLURGY AND CORROSION}

\subsubsection{Introduction}

The selection of feasible materials for the target for the 1-MW neutron spallation source was based on the criteria and the priorities listed in Table IV.2.5-1. These have been discussed in further detail in two engineering notes. ${ }^{16,17}$ The highest priority is that the target have a high mass number and density in order to produce the greatest number of neutrons per proton in the spallation process. The amount of afterheat produced by the target should be minimized to mitigate consequences of a loss of coolant accident (LOCA). The target material must have good mechanical, thermal, and corrosion properties, be resistant to radiation damage, resist surface damage due to cavitation, and not be subject to hydride formation. The target yield stress should be higher than the thermoelastic stress. High cycle fatigue could occur due to thermomechanical shock stresses because the beam is pulsed in times that are short compared to sound wave transit times. Table IV.2.5-1 summarizes the prioritization of material properties desired for a spallation target.

This prioritization of properties is a simplification because, for example, a candidate material could satisfy all of the priority 1-3 items but suffer severe cavitation damage under the water flow. The oxidation behavior of the candidate materials should be known. Formation of a protective oxide layer could be a positive factor, but on the negative side, incorporation of oxygen, especially as interstitials, could cause loss of ductility.

\subsubsection{Mechanical Properties}

Properties considered were strength, ductile-to-brittle transition temperature, fatigue resistance, ductility, and swelling. The mechanical properties of tungsten and tantalum and their 


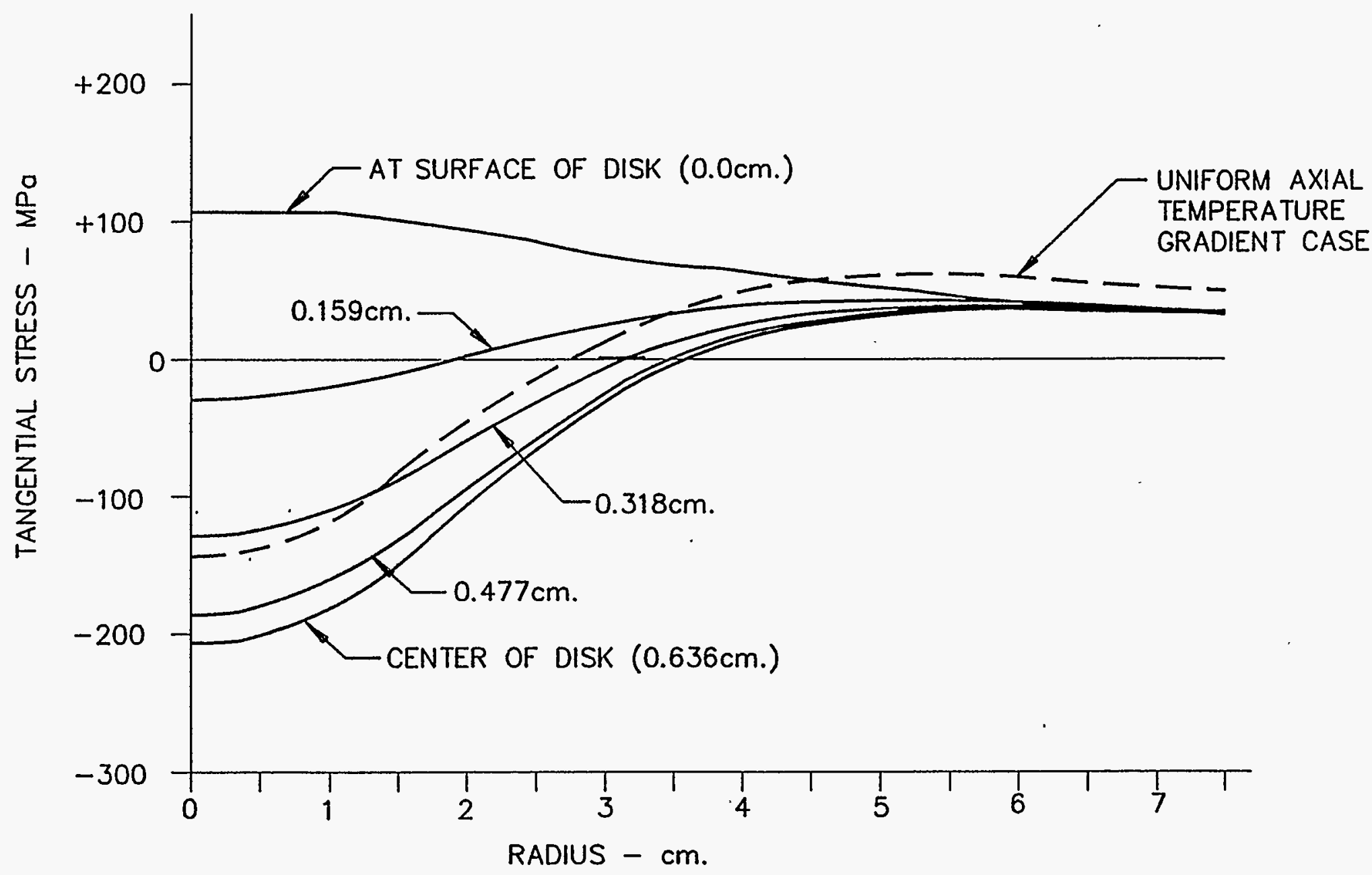

Figure IV.2.4-5 Tangential Stress Distribution in Last Disk of the Upstream Target Section Using 2-D Temperature Distribution. 
Table IV.2.5-1 Properties to Consider in Selection of Target Material

\begin{tabular}{lc}
\hline \multicolumn{1}{c}{ Property } & Priority \\
\hline & \\
\hline High atomic number & 1 \\
High density & 1 \\
Afterheat (LOCA consideration) & 1 \\
Corrosion resistance & 2 \\
Fatigue resistance & 2 \\
Hydrogen embrittlement and & 2 \\
hydride formation & 2 \\
Strength & 3 \\
Swelling and radiation resistance & 4 \\
Machinability and cost & 4 \\
Cavitation & 4 \\
Oxidation resistance & \\
\hline
\end{tabular}


alloys are favorable. It is generally true that the alloys, such as W-25\% Re or Ta-10\%W, are stronger than the elements alone, as shown in Figure IV.2.5-1.18-20 Additionally, alloying increases the ductile-to-brittle transition temperature and the fatigue resistance. Irradiation increases the yield stress, but it decreases the ductility, as is demonstrated for tungsten in Figure IV.2.5-2.21 Brittle fracture is not a problem as long as the thermoelastic stresses are less than the yield stress. Swelling of the tantalum-based alloys is quite low, 22 lower than pure tantalum. 20 Swelling of tungsten or its alloys has evidently not been measured.

\subsubsection{Corrosion}

The most extensive corrosion studies of high-density sintered tungsten and tungsten alloys showed that tungsten and all of the alloys tested undergo corrosion and that pure tungsten has the lowest corrosion rate of $\approx 0.5 \mu \mathrm{m} / \mathrm{yr}$ in a salt solution and $\approx 20 \mu \mathrm{m} / \mathrm{yr}$ in distilled water. ${ }^{23}$ The LANSCE target, a tungsten cermet with a $3 \% \mathrm{Ni}-\mathrm{Fe}$ binder phase, has been running at $60 \mathrm{~kW}$ with $800 \mathrm{MeV}$ protons for approximately 4 years. Fine particulate matter has been detected in the cooling water, ${ }^{24}$ and the results of preliminary analysis indicate that the particles contain tungsten. Recent investigations of the corrosion of titanium and tungsten in distilled water indicate that titanium corrodes at least a factor of ten less than tungsten at $200^{\circ} \mathrm{C} .7$ The corrosion rate at $200^{\circ} \mathrm{C}$ for titanium is $<0.2 \mu \mathrm{m} /$ year. This is negligible, so titanium is used as a corrosion-resistant coating.

\subsubsection{Hydrogen Embrittlement}

Hydrogen enters the target material during corrosion or by the accumulation of spallationproduced protons. The resulting formation of hydrides can cause significant embrittlement of the target material and is expected to be a major factor in determining target lifetime. Figure IV.2.5-3 shows the pronounced increased in brittleness with increasing hydrogen concentration in tantalum and a number of tantalum alloys. Embrittlement is expected to be important for tungsten as well, but comparable data are not yet available. Helium may also cause embrittlement, so it requires consideration.

\subsubsection{Afterheat}

Estimates of afterheat (radioactive decay heat) and activation have been obtained from numerical simulations for tantalum and tungsten (Section IV.2.2.4). The afterheat in tantalum results in very high temperatures under LOCA conditions (Section IV.2.7.1). Tungsten or its alloys, under similar conditions, have less than one-third of the afterheat power of tantalum or its alloys, and tungsten is therefore preferred. 


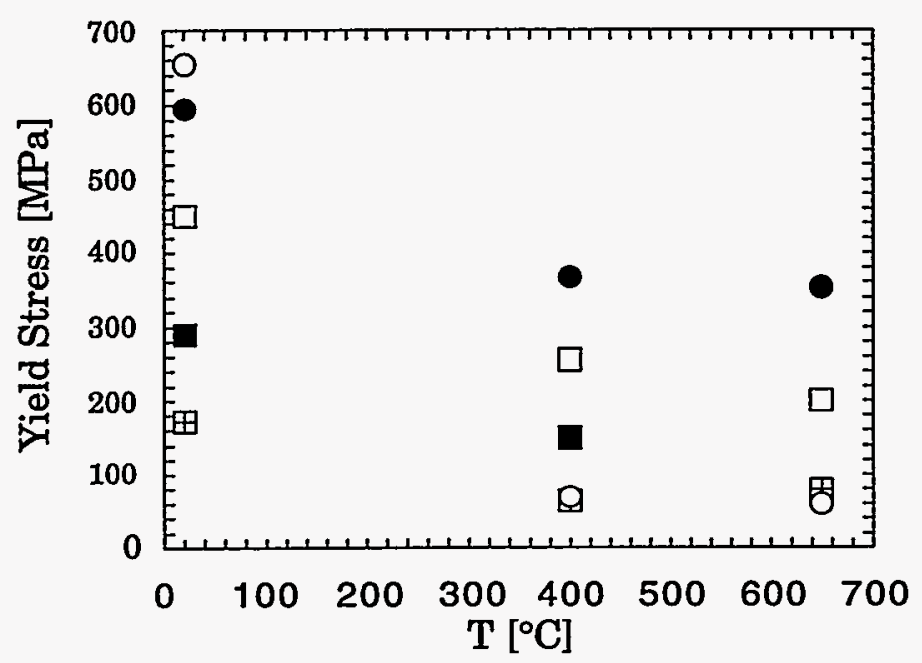

Figure IV.2.5-1 Yield Stress Measured at a Strain Rate, $\dot{\varepsilon} \approx 1 \times$ $10^{-4} \mathrm{~s}^{-1}$ (about the $\dot{\varepsilon}$ achieved during cool down) for Pure $\mathrm{Ta}$ (squares with cross from Ref. 18, filled squares from Ref. 19, and open squares from Ref. 20), Pure W (open circles from Ref. 19), and T-111 (filled circles from Ref. 18). ( $\mathrm{T}-111$ is the designation for a commercial alloy, Ta with $7.9 \% \mathrm{~W}$ and $2.3 \% \mathrm{Hf}$.). 


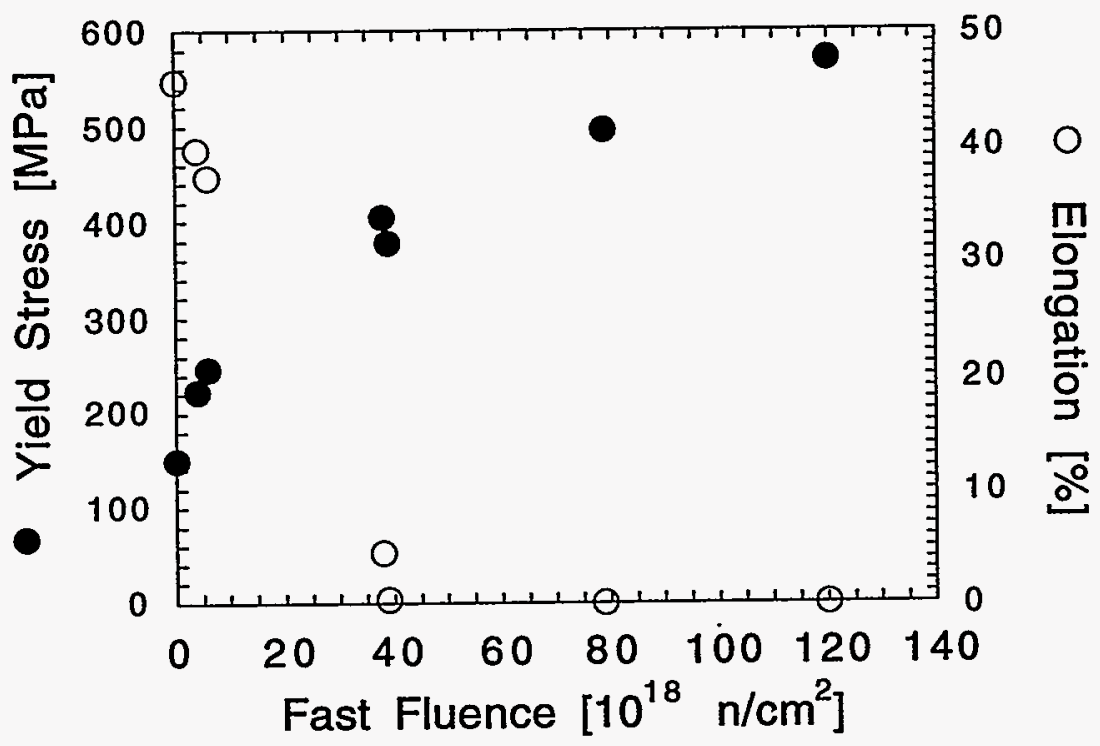

Figure IV.2.5-2 Variation of the Yield Stress and Percent Elongation at $400^{\circ} \mathrm{C}$ for Tungsten (Reference 21). 


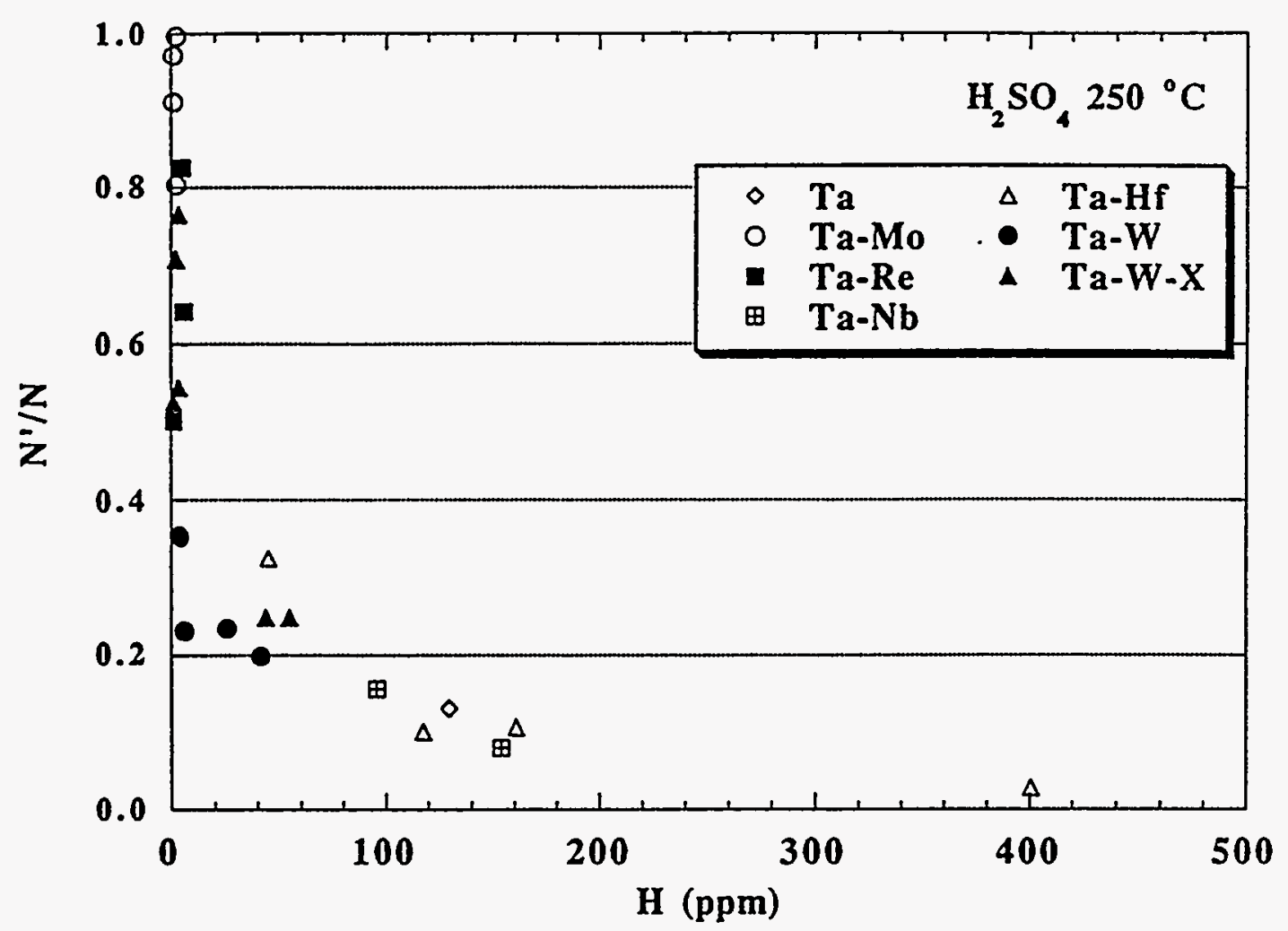

Figure IV.2.5-3 Variation of Brittleness with Hydrogen Concentration for Various Tantalum Alloys. (Brittleness is measured as the ratio of number of bends to fracture specimens with and without hydrogen $\left[N^{\prime} / N\right]$. Source: Reference 25.) 


\subsubsection{Other Properties}

Investigations of cavitation are usually performed at flow velocities about an order of magnitude higher than those expected in the IPNS Upgrade target, and under conditions of pulsating flow, to ensure that cavitation occurs. ${ }^{26}$ Therefore, it is anticipated that cavitation will not be a problem for the IPNS Upgrade target.

\subsubsection{Target Material Selection}

The available property data for the candidate target materials are compared to the prioritized properties listed in Table IV.2.5-1. Uranium and liquid metal targets have not been discussed, but they remain as options after further development. Table IV.2.5-2 presents a list of acceptable materials, presented in the order of preference for a particular property. On the basis of this assessment, tungsten, or a tungsten alloy, is the chosen target material.

\subsection{TARGET COOLING SYSTEM}

With proton beam power of $1 \mathrm{MW}$, a total heat load of approximately $610 \mathrm{~kW}$ is deposited in the two target sections, as described in Section IV.2.2.2 and Table IV.2.2-2. The upstream target section consists of 12 thin tungsten alloy disks with 13 coolant passages. The downstream target section has 9 thicker tungsten alloy disks and 10 cooling passages. The water velocity must be controlled through each of the cooling passages between disks.

A number of process-related assumptions are made that underlie the design of the water flow system for each target. The heat load corresponds to the full beam power of $1 \mathrm{MW}$, which is the maximum power that the $30-\mathrm{Hz}$ target receives. Both targets and their cooling systems are identical. This leads to savings in terms of design effort, procurement, maintenance, and the provision of spares.

The bulk water temperature adjacent to the convective film at the target surface in the direct path of the beam is approximately $60^{\circ} \mathrm{C}$, and the maximum target disk surface temperature is $127^{\circ} \mathrm{C}$ (Section IV.2.3.) The saturation temperature of water is $172^{\circ} \mathrm{C}$ at the minimum design water pressure of $830 \mathrm{kPa}$. Thus, there is a minimum margin to boiling of at least $45^{\circ} \mathrm{C}$. Some additional suppression of boiling due to convective flow occurs, so the temperature margin is even higher. The values calculated for the maximum surface heat flux from the first and last titanium-coated tungsten disks are $580 \mathrm{~W} / \mathrm{cm}^{2}$ and $530 \mathrm{~W} / \mathrm{cm}^{2}$, respectively. These flux values, and the design surface-to-bulk temperature difference of $67^{\circ} \mathrm{C}$, are used to calculate the necessary convective film coefficients for smooth surfaced target disks.

Smooth surfaces provide the least effective convective film heat transfer. Mechanical roughening of the surfaces is known to increase convective film coefficients by almost a factor of 
Table IV.2.5-2 Acceptable Candidate Materials in Order of Preference, Compared to the Desired Prioritized Properties of a Target Material

\begin{tabular}{|c|c|c|}
\hline Property & Acceptable Materials & $\begin{array}{c}\text { Unacceptable } \\
\text { or Borderline } \\
\text { Materials }\end{array}$ \\
\hline High atomic number & $\mathrm{Pt}, \mathrm{Ir}, \mathrm{Os}, \mathrm{Re}, \mathrm{W}, \mathrm{Ta}, \mathrm{Hf}, \mathrm{Au}$ & $\mathrm{Mo}, \mathrm{Nb}, \mathrm{V}, \mathrm{Zr}$ \\
\hline Afterheat & & \\
\hline Strength & $\begin{array}{l}\text { Re, T-111, ASTAR-811C, } \\
\text { W-(10-25\%)Re, Ir, } W^{a}{ }^{\text {Ta }} a^{a}\end{array}$ & $\mathrm{Pt}, \mathrm{Au}$ \\
\hline $\begin{array}{l}\text { Corrosion resistance } \\
\text { Fatigue resistance }\end{array}$ & $\begin{array}{l}\text { Pt, noble metals, } \mathrm{Ti}, \mathrm{Ta}-10 \% \mathrm{~W}, \mathrm{Ta}, \mathrm{W} \\
\mathrm{Au}, \mathrm{Ta}, \mathrm{W}, \mathrm{Ta}-10 \% \mathrm{~W}\end{array}$ & \\
\hline $\begin{array}{l}\text { Hydrogen embrittlement, } \\
\text { hydride formation }\end{array}$ & W, Ta-(2-10\%)W, Ta & \\
\hline Swelling and radiation resistance & $\mathrm{T}-111, \mathrm{Ta}-10 \% \mathrm{~W}$ & Ta, Mo \\
\hline Machinability and cost & Ta, T-111, ASTAR-811C, W-Re, W & \\
\hline Cavitation & No data available & \\
\hline Oxidation behavior & $\mathrm{Pt}, \mathrm{W}, \mathrm{Ta}$ & \\
\hline
\end{tabular}

a After some radiation hardening. 
two, albeit at the price of pressure loss. In order to assure a conservative cooling system design, these enhancements are not assumed. In the calculation of the required flow rates in the thermalhydraulic analysis reported in Section IV.2.3, such heat-transfer enhancements were considered. Therefore, the required flow rate indicated in Section IV.2.3 is substantially less than the one used in this section to size the cooling system. The reduced flow rate is used in the thermalhydraulic analysis because the lower flow rate causes a higher average bulk coolant temperature, and this introduces some conservatism in the thermal analysis of the target disks.

The respective required Reynolds numbers are calculated using the Dittus-Boelter convective film coefficient correlation and thermophysical properties of water evaluated at the assumed bulk temperature. These Reynolds numbers determine the required water velocities. Cooling the first disk requires a water velocity of $15.4 \mathrm{~m} / \mathrm{s}$, while cooling the last disk requires $13.4 \mathrm{~m} / \mathrm{s}$. The flow circuit provides for a uniform water velocity of $15.4 \mathrm{~m} / \mathrm{s}$ to give an extra cooling margin and to simplify the design. The rectangular flow passages between target disks are $0.159 \mathrm{~cm}$ thick, and the height is $9.0 \mathrm{~cm}$, so the required water flow rate for each target section is $28 \mathrm{~L} / \mathrm{s}$.

Pressure drops throughout various portions of the flow circuit are calculated by using standard Moody friction factors. The flow circuit is shown in Figure IV.2.6-1. Two $75 \mathrm{~kW}$ pumps $(28 \mathrm{~L} / \mathrm{s}$ each) supply the water to the target cooling circuit for normal operation. This arrangement allows flow reduction for the $10-\mathrm{Hz}$ target and provides some reduced cooling capability during a single pump failure. Two $0.75-\mathrm{kW}$ pumps are adequate for decay heat removal.

The design decay heat load of $5 \mathrm{~kW}$ (Section IV.2.2.4) is removed by means of a coolingwater flow rate of $0.2 \mathrm{~L} / \mathrm{s}$ with a bulk temperature rise of $20^{\circ} \mathrm{C}$. Pressure drop within the target cooling system is negligible for this small flow rate. A mobile cart having a small water pump, a small supply tank $(20 \mathrm{~L})$, and independent pressurization, level control, and gas safety relief systems provides cooling when targets are removed from the biological shield for servicing.

Each cooling circuit also has a control valve, flowmeter, heat exchanger, filter, and remote temperature and pressure transducers. The heat exchangers are located downstream of the pumps to remove power input by the pumps. The major portion of the piping for each flow circuit is nominally $8.9 \mathrm{~cm}$ diameter ( 3 in. Sch 40 ), while that portion of piping within the shielding is space-limited to $6.0 \mathrm{~cm}$ diameter (2 in. Sch 10). A single demineralizer is located in a side stream to the larger piping. The water supply tank has a capacity of $4,500 \mathrm{~L}$ to provide adequate water-storage volume and gas ullage space, and it includes a $\mathrm{H}_{2} / \mathrm{O}_{2}$ recombiner. A liquid-level gauge is used to maintain the water level in the tank at three-quarters full. A helium gas supply pressurizes the system initially to $690 \mathrm{kPa}$. It provides a gas bleed flow rate of $0.016 \mathrm{~L} / \mathrm{s}$ through a back-pressure control valve for purging the ullage of oxygen, hydrogen, and any activation products and for stabilizing the tank pressure level. A pressure relief valve and burst diaphragm and a large vent valve are included for rapid tank depressurization. Bleed and 


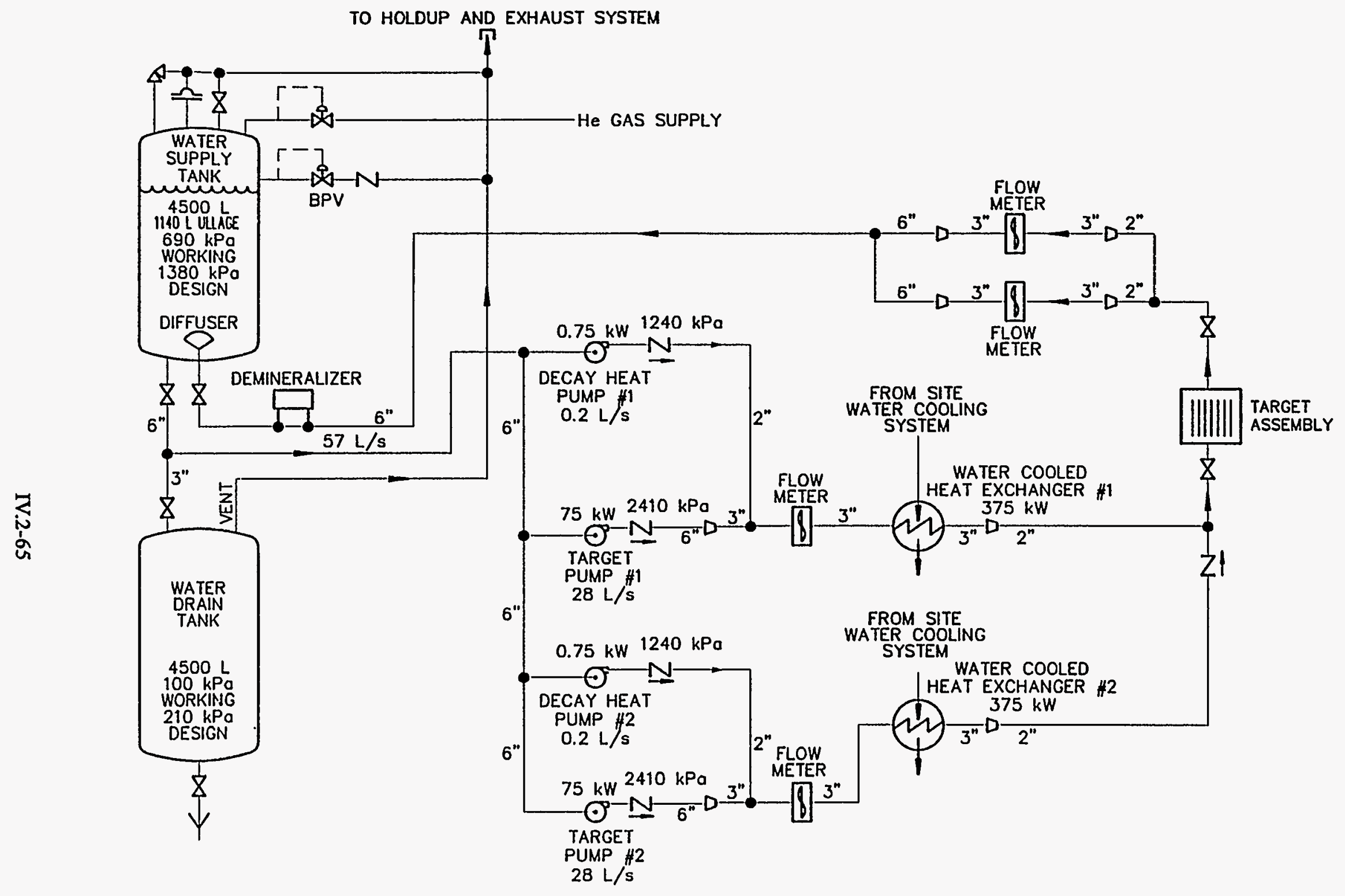


vent gases are routed to an 11,000-L holdup tank, which allows adequate time for radioactive species to decay. More details on the purge and vent gas holdup tank and exhaust system are given in Section IV.6.2.1.

The calculated total pressure drops through each target cooling-flow circuit are 1.7 MPa. The required pumping power in each circuit is $150 \mathrm{~kW}$, which allow $20 \%$ of the pressure drop for control and an assumed pump efficiency of 0.7 . The heat exchanger is sized to remove slightly more than $750 \mathrm{~kW}$.

Figure IV.2.6-2 shows the arrangement of the cooling-system components for the $30-\mathrm{Hz}$ target. Figure IV.2.6-2a is a sectional view through A-A of Figure IV.2.6-2. Those components associated with the target cooling system are highlighted in these figures. The supply and drain water tanks, target pumps, target decay-heat pumps, heat exchanger, and demineralizer are located in a concrete-shielded enclosure in Building 371. Piping from the shielded enclosure in 371 runs to the target biological shield in Building 370 .

Figure IV.2.6-3 shows the general arrangement of the cooling system components for the $10-\mathrm{Hz}$ target, with supply and drain water tanks, target pumps, target decay-heat pumps, heat exchanger and demineralizer located in a concrete shielded enclosure in Building 367 . These components are highlighted in the figure. Figure IV.2.6-3a shows a sectional view of the same area. Piping from Building 367 runs to the target biological shield in Building 369.

The supply water tank and water drain tank are approximately $1.4 \mathrm{~m}$ in diameter and $4 \mathrm{~m}$ long, constructed of 304SS. The tanks are mounted horizontally. The drain tank is located above the target pump suction to allow reuse of the drained water. The supply tank has two $16.8 \mathrm{~cm}$ (6 in. Sch. 40) connections for the target pump supply and cooling system return piping. The $16.8-\mathrm{cm}$ supply line also connects to the water drain tank so that water from the drain tank can be pumped back into the system.

The target cooling pumps are constructed of corrosion resistant materials. The installed motor power is $94 \mathrm{~kW}$ each. The pump outlet flow passes through a flowmeter and then through a water-cooled heat exchanger. The 304SS shell-and-tube heat exchanger is $3 \mathrm{~m}$ long with a $0.25-\mathrm{m}$ nominal diameter. It is mounted horizontally with $24-\mathrm{mm}$ flanged connections on both the shell and channel sides. Raw cooling water at $1,600 \mathrm{~L} / \mathrm{min}$ passes through the shell side of the heat exchanger. A de-ionized water supply is provided for initial system fill and make-up.

Insulation covering all cooling water lines limits heat transferred to the shielding enclosures.

\subsection{POTENTIALLY HAZARDOUS INCIDENTS}

Credible incidents have been examined for the IPNS Upgrade target stations. These incidents generally fall into the category of low-probability, low-consequence events. 


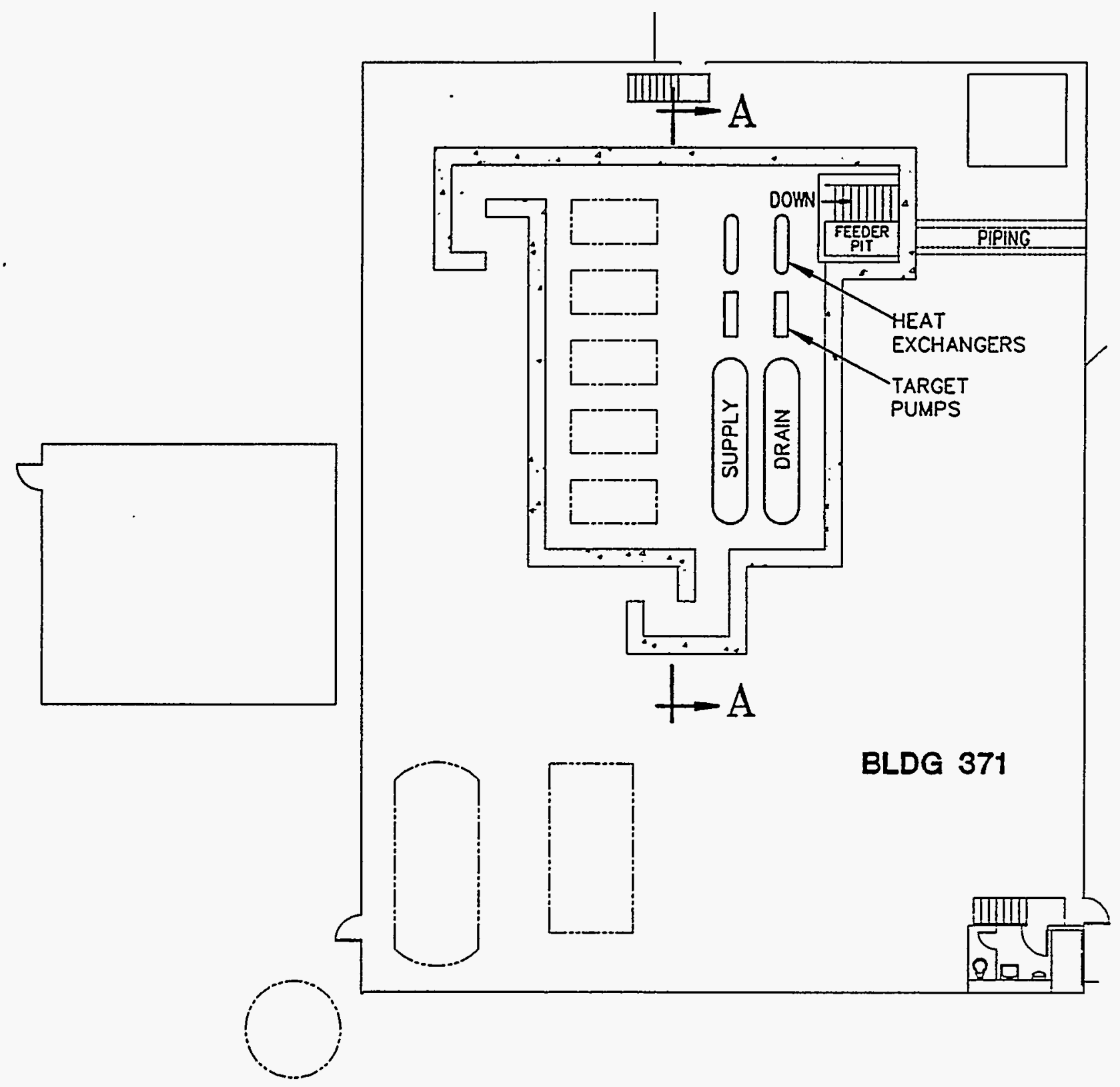

Figure IV.2.6-2 Arrangement of the 30-Hz Target Cooling System in Building 371 . 


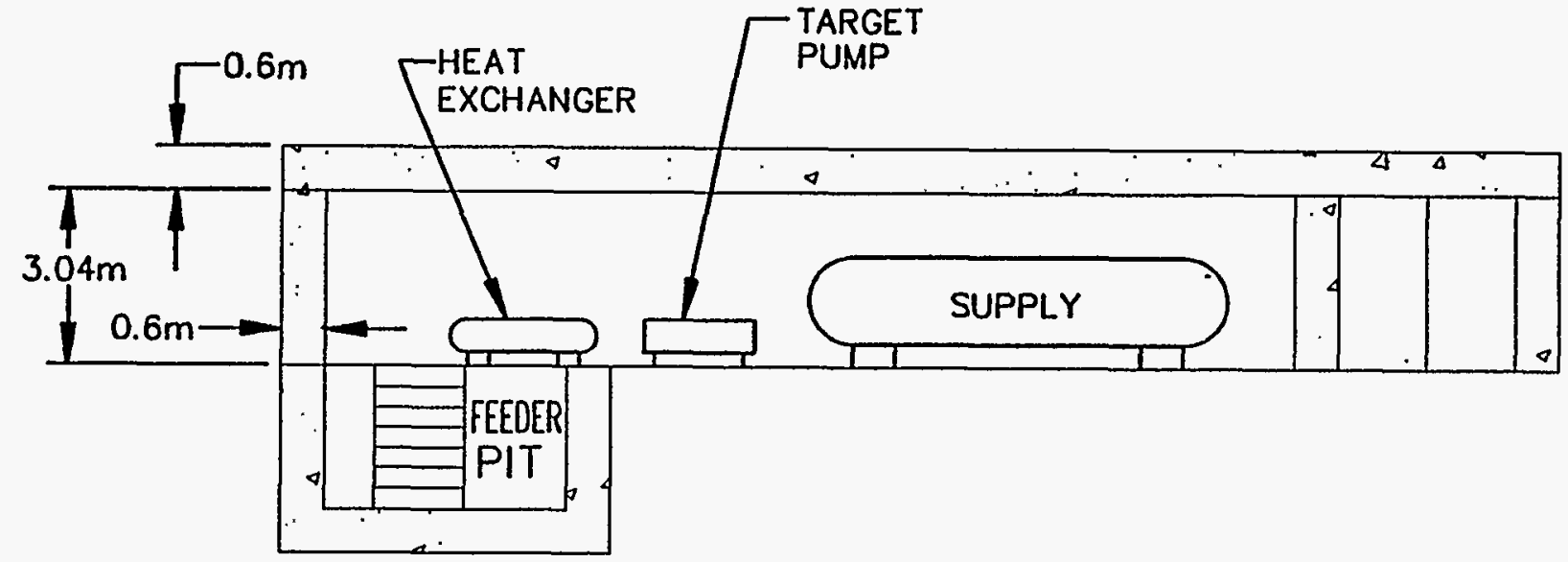

SECTION A-A

Figure IV.2.6-2a Sectional View of the 30-Hz Target Cooling System in Building. 371. 


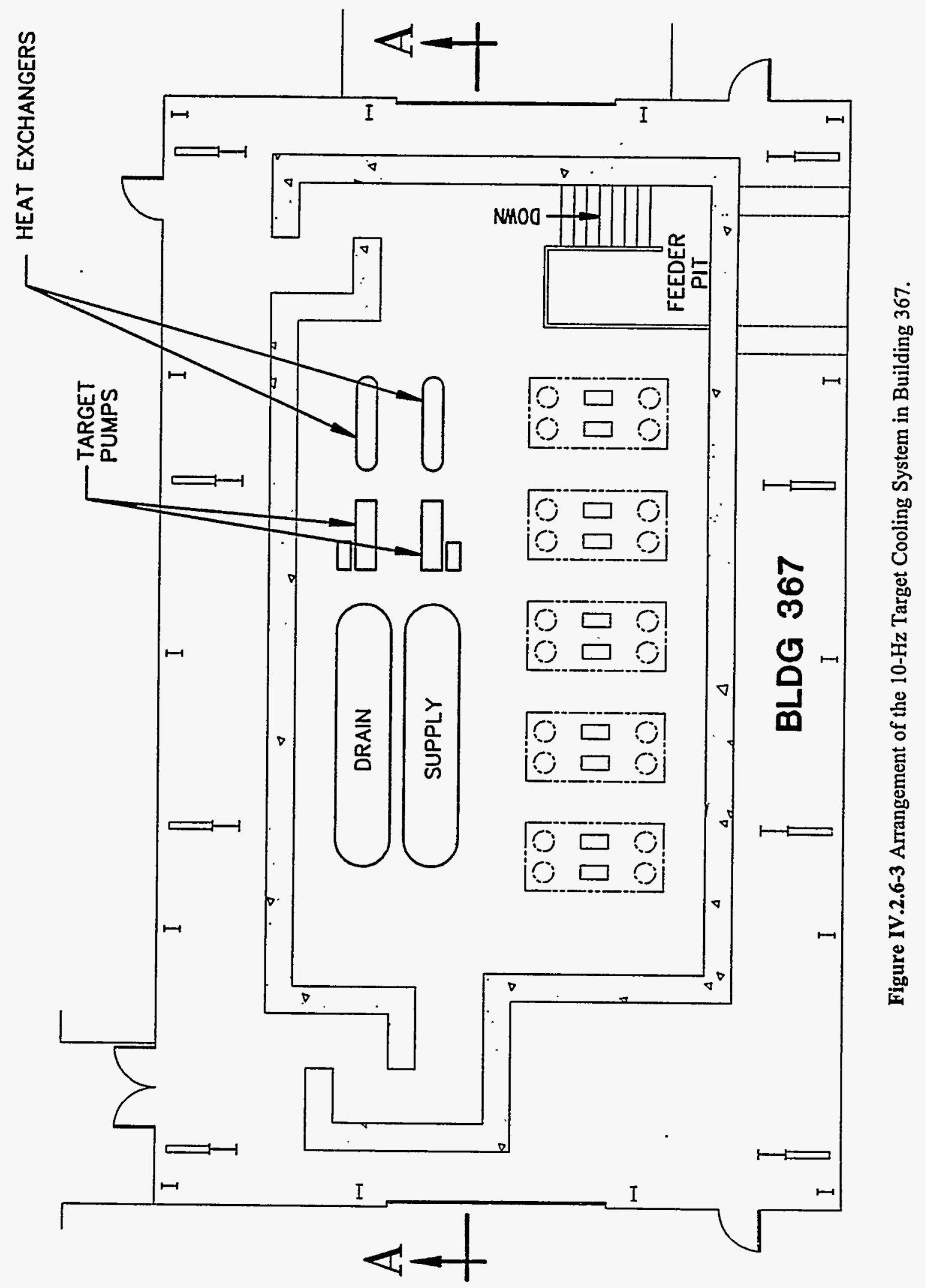




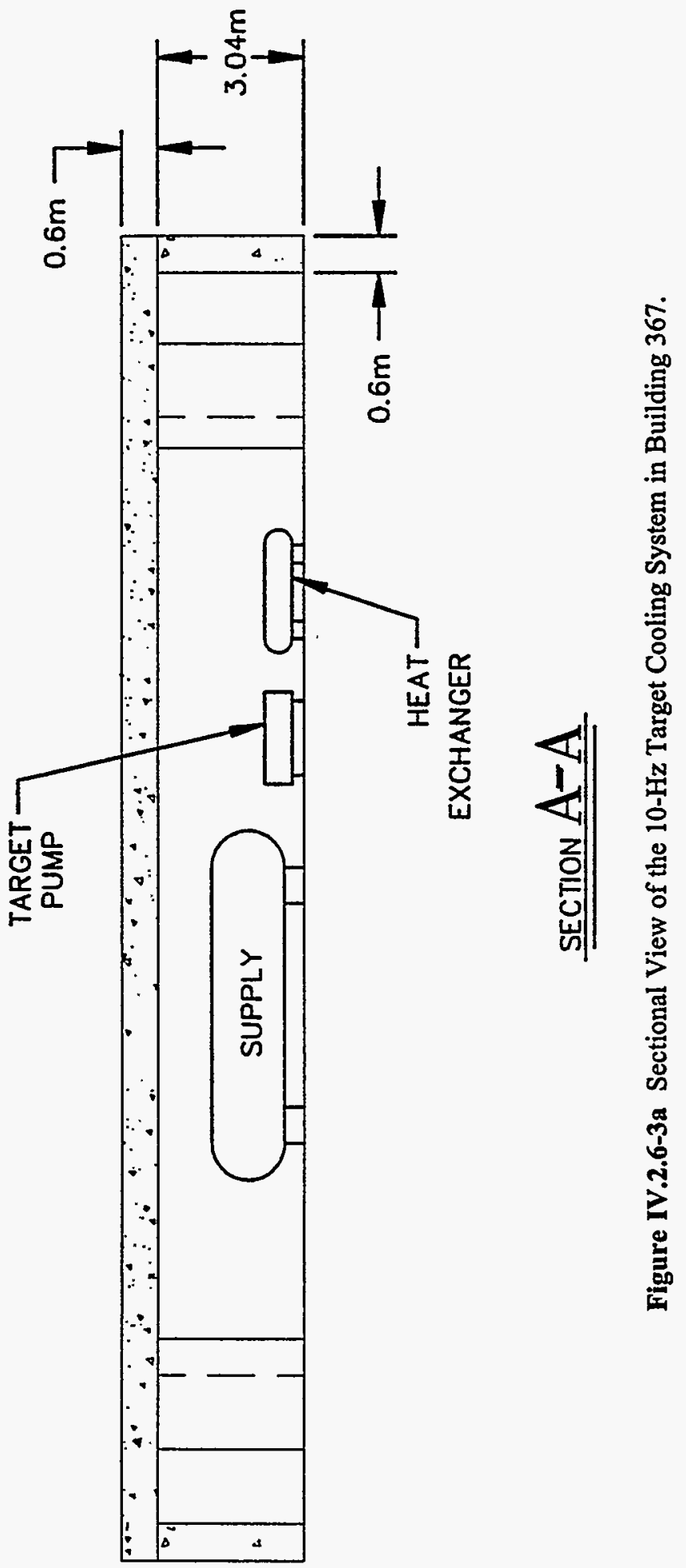


The IPNS Upgrade follows the standard practice of designing for defense in depth. This practice incorporates several barriers to the release of radioactive substances. The vast majority of the radioactive products are contained within the target. The target is enclosed in the primary water cooling system, which is a welded, pressure-rated, stainless-steel system. Next, a sealed helium-purged aluminum tank encloses the water system. The exhaust from this volume goes into a holdup tank and then through high-efficiency particulate air (HEPA) filters. Finally, the biological shield, a welded steel barrier, is also a helium-purged vented volume. The exhaust from the biological shield is also passed through HEPA filters. These numerous barriers provide in-depth containment and confinement of radioactive substances. In addition to these passive barriers to prevent activity release, the IPNS Upgrade also provides uninterruptible battery and diesel power supplies to provide electricity for coolant and ventilation systems.

Preliminary conceptual examination of credible incidents has been done. These will be studied in detail in the IPNS Upgrade Title II Design Safety Assessment Documents. The minimum list is:

1. Loss of target primary coolant flow;

2. Loss of target primary coolant;

3. Loss of vacuum surrounding the moderator cans;

4. Explosion of cryogenic fluids in moderator systems;

5. Loss of secondary coolant supply;

6. Tornado;

7. Earthquake; and

8. Facility fire.

DOE Order 6430.1A, titled "General Design Criteria," gives radiological siting guidelines for DOE nonreactor facilities. The postulated credible incidents listed above will have consequences less than the limits specified in DOE 6430.1A.

The IPNS Upgrade target is made of nonfissionable material. The reactor concerns involving prompt criticality and rapid power increases are not possible in spallation sources. Although reactors can be designed to safely control these processes, accelerator-driven spallation sources need not address them. 


\subsubsection{Loss of Coolant Analyses}

Consequences of a Loss of Coolant Accident (LOCA) were assessed by calculating the transient temperatures under LOCA conditions using a simple one-dimensional (radial) model. The target was represented as a cylinder of $5.5-\mathrm{cm}$ radius surrounded by a $0.5-\mathrm{cm}$-thick stainlesssteel vessel, separated from an annular $47.3-\mathrm{cm}$ thick beryllium reflector by a $3.5-\mathrm{cm}$ helium-filled gap. Cases included helium conduction; without helium conduction, to represent totally evacuated conditions; and without helium conduction but with the steel vessel maintained at $49^{\circ} \mathrm{C}$, as if by active cooling. In the ranges of temperature encountered, emissivities range from 0.2 to 0.5 for steel, 0.01 to 0.36 for tantalum, and 0.05 to 0.24 for tungsten. The emissivity for beryllium is 0.2 . The heat transfer is nonlinear because of radiative $\left(\mathrm{T}^{4}\right)$ heat transfer and the variation of the emissivities and the helium thermal conductivity with temperature. Therefore, the time to reach peak temperature varies with power input, reaching a maximum in about 30 minutes for tantalum and about one hour for tungsten at the higher powers. It always reaches maximum within less than five hours for the lower-power values studied. The calculations account for the decay of afterheat during the transient.

Afterheat powers of tantalum and tungsten are described in Section IV.2.2.4. Two scenarios were studied: first, for afterheat immediately following continuous irradiation for one year at $1 \mathrm{MW}$; second, for continuous irradiation at $2 / 3 \mathrm{MW}$ for one year, followed by continuous irradiation at $1 \mathrm{MW}$ for one month up to the beginning of the transient. Table IV.2.7-1 shows the resulting peak temperatures of the target and the vessel and the temperature of the reflector after 48 hours.

Tantalum, tungsten, and their practical alloys remain solid at the highest of these temperatures. Stainless steels melt at around $1425^{\circ} \mathrm{C}$, so the vessel would melt in either case if the target were of tantalum and the vessel were not actively cooled. The vessel retains its integrity without active cooling if the target is tungsten. Internal steel parts weaken or melt, however, even for tungsten-target material. Beryllium approaches its melting temperature $\left(1275^{\circ} \mathrm{C}\right)$ with tantalum, but not with tungsten in any of these cases. With a tungsten target, a LOCA would have no impact on the facility as a whole, but the target would have to be replaced. This is a tolerable consequence of a hypothetical loss of coolant accident. Tungsten is acceptable without any backup decay heat removal system, unlike the case for tantalum. Therefore, we choose tungsten or a practical tungsten alloy as the design basis target material and plan no decay heat removal systems for LOCA incidents. 
Table IV.2.7-1 Peak LOCA Transient Temperatures in the Target, Target Vessel, and Reflector ${ }^{a}$

\begin{tabular}{|c|c|c|c|c|c|c|c|c|c|}
\hline \multirow{3}{*}{$\begin{array}{c}\text { Material/ } \\
\text { Scenario }\end{array}$} & \multirow{3}{*}{$\begin{array}{c}\text { Power } \\
\text { (W) }\end{array}$} & \multicolumn{8}{|c|}{ Temperatures $\left({ }^{\circ} \mathrm{C}\right)$} \\
\hline & & \multicolumn{3}{|c|}{ Case $1^{b}$} & \multicolumn{3}{|c|}{ Case $2^{c}$} & \multicolumn{2}{|c|}{ Case $3^{\mathrm{d}}$} \\
\hline & & Target & Vessel & Reflector & Target & Vessel & Reflector & Target & Vessel \\
\hline \multicolumn{10}{|l|}{ Tantalum } \\
\hline Scenario 1 & 13300 & 2624 & 1700 & 1135 & 2737 & 1750 & 1135 & 2486 & 49 \\
\hline Scenario 2 & 9695 & 2118 & 1468 & 877 & 2232 & 1517 & 876 & 2049 & 49 \\
\hline \multicolumn{10}{|l|}{ Tungsten } \\
\hline Scenario 1 & 3720 & 1394 & 1067 & 372 & 1533 & 1129 & 371 & 1421 & 49 \\
\hline Scenario 2 & 3615 & 1371 & 1050 & 355 & 1513 & 1114 & 344 & 1403 & 49 \\
\hline
\end{tabular}

a Reflector temperatures are those after 48 hours, at which time they are still rising.

b With conduction in helium.

c No conduction in helium.

d No conduction in helium, cooled vessel. 


\subsection{REFERENCES}

1. G.J. Russell et al., "The LANSCE target system," in Proceedings of the 9th Meeting of the International Collaboration on Advanced Neutron Sources (ICANS IX) (Villigen, Switzerland, Sept. 22-26, 1986), Swiss Institute for Nuclear Research, ISBN 3-907998-01-4, pp. 177-244 (1987).

2. M. Holding and T.A. Broome, "The status of the ISIS targets," in Proceedings of the 11th Meeting of the International Collaboration on Advanced Neutron Sources (ICANS XI) (Tsukuba, Japan, Oct. 22-26, 1990), National Laboratory for High Energy Physics (KEK) Report No. 90-25, pp. 478-487 (1991).

3. J.M. Carpenter, "Status of Argonne's Intense Pulsed Neutron Source IPNS-I," in Proceedings of the 5th Meeting of the International Collaboration on Advanced Neutron Sources (ICANS V) (Jülich, Germany, June 22-26, 1981), Kernforschungsanlage Jülich Report No. Jül-Conf-45, pp. 33-52 (1981).

4. A. Carne et al., "The ISIS target," in Advanced Neutron Sources 1988, Proceedings of the 10th Meeting of the International Collaboration on Advanced Neutron Sources (ICANS X) (Los Alamos, N.M., Oct. 3-7, 1988), IOP Conference Series, No. 97 (Institute of Physics, New York, 1989), pp. 79-90 (1989).

5. Y. Ishikawa, "Present status and future project of KENS facility," in Proceedings of the 8th Meeting of the International Collaboration on Advanced Neutron Sources (ICANS VIII) (Oxford, U.K., July 8-12, 1985), Rutherford Appleton Laboratory Report No. RAL-85-110, pp. 17-43 (Chilton, U.K., 1985).

6. A.E. Knox et al., "Progress on the IPNS enriched uranium booster target," in Proceedings of the 9th Meeting of the International Collaboration on Advanced Neutron Sources (ICANS LX) (Villigen, Switzerland, Sept. 22-26, 1986), Swiss Institute for Nuclear Research, ISBN 3-907998-01-4, pp. 557-589 (1987).

7. A.V. Dementyev, S.F. Sidorkin, N.M. Sobolevsky, Y.Y. Stavissky, V.G. Miroschnichenko, I.I. Konovalov, A.A. Maslov, I.T. Tretakov, V.I. Trushkin, and A.D. Pogov, "The Moscow Meson Factory pulsed neutron source based on a uranium target," in Proceedings of the Second International Conference on Pulsed Advanced Neutron Sources (PANS II) (Dubna, Russia, June 1994).

8. R.E. Prael and H. Lichtenstein, User Guide to LCS: The LAHET Code System, Los Alamos National Laboratory Report No. LA-UR-89-3014 (Sept. 1989).

9. T.W. Armstrong et al., "Theoretical target physics studies for the SNQ spallation neutron source," Jul-Spez-120 (KfK Karlsruhe, Germany, July 1981). 
10. N. Watanabe et al., "Report of the moderator working group," presented at International Workshop on the Technology of Targets and Moderators for Medium to High Power Spallation Neutron Sources (Paul Scherrer Institute, Switzerland, Feb. 1992).

11. T.A. Broome, Calculations of Activity and Afterheat for the IPNS-II Tantalum and Tungsten Targets, Rutherford Appleton Laboratory Report No. ISIS/TS/N1/95 (Jan. 1995).

12. J. Kaganove, THTB at ANL, TM 281 (Sept. 1982).

13. Cabot Performance Materials, P.O. Box 1607, Boyertown, Pennsylvania.

14. R.J. Roark, Formulas for Stress and Strain (McGraw-Hill, New York, 1954).

15. ANSYS User Manual, DN-R144:44, Rev. 4.4, Swanson Analysis Systems, Inc. (May 1989).

16. J.L. Routbort, IPNS Upgrade NST Note 2 (1994).

17. J.M. Carpenter et al., IPNS Upgrade NST Note 3 (1993).

18. J.H. Bechtold, Acta Metall. 3:249 (1955).

19. Metals Handbook, Vol. 10 (ASM, Metals Park, Ohio, 1990).

20. F.W. Wiffen, "Defects and defect clusters in BCC metals and their alloys," in Nuclear Metallurgy 18, edited by R.J. Arsenault, p. 176 (NBS, Gaithersburg, Md., 1973).

21. R.C. Rau, J. Moteff, and R.L. Ladd, J. Nucl. Mater. 24:164 (1967).

22. M.L. Grossbeck and F.W. Wiffen, Space Nuclear Power Systems, 1985, Vol. 3, edited by M.S. El-Genk and M.D. Hoover, p. 85 (Orbit Book Co., Malabar, Fla., 1986).

23. J.J. Batten, I.G. McDonald, B.T.Y. Moore, and V.M. Silva, Corrosion of High-Density Sintered Tungsten Alloys, Part 1: Immersion Testing, Department of Defense Materials Research Laboratory Report No. MRL-R-1139 (Maribyrnong, Australia, 1988).

24. T. Brun; 1994 (private communication).

25. L.A. Gypen, M. Brabers, and A. Deruyttere, Werkstoffe und Korrosion 35:37 (1984).

25. F.G. Hammitt and M.K. De, Wear 52:243 (1979). 
This section discusses the moderators and the reflectors. Neutrons produced in the target have energies up to hundreds of $\mathrm{MeV}$, while the neutron scattering instruments require neutrons with energies less than a few tens of eV. It is the function of the moderators and the reflector to slow down the neutrons produced in the target to the energies required by the instruments and to direct as many as possible of these moderated neutrons down the beamlines to the instruments. In this process, the moderators and the reflector must not degrade the time-structure of the neutron pulses beyond limits imposed by the instruments, because this time structure is essential to the time-of-flight (TOF) energy analyses performed by the instruments.

Section IV.3.1 discusses the moderator geometry and materials and the importance of premoderators, decoupling, and poisoning. The neutronic requirements of the instruments, as discussed in Chapter V, determine the optimal moderator characteristics, but these must be tempered by such practical considerations as radiolysis of the moderator materials and moderator cooling requirements.

Section IV.3.2 discusses the Monte Carlo calculations of the moderator heat loads and the neutron beam currents from the moderators. The basic model for the Monte Carlo calculations is discussed in Section IV.2.2. Section IV.3.2 also makes use of known moderator performances at neutron energies of use to the instruments to estimate the energy spectra and pulse shapes for neutron beams from the various moderator types and positions. materials.

Section IV.3.3 discusses the problems associated with radiolysis of the moderator

Finally, Section IV.3.4 describes cooling systems sized to handle the cooling needs of the various room-temperature and cryogenic moderators. Other water cooling systems are also included in this section.

\subsection{MODERATOR AND REFLECTOR GEOMETRY AND MATERIALS}

As shown in Figure IV.1.2-2, each target station contains, six moderators, four in wing geometry and two in flux-trap geometry, and each of these moderators provides neutrons to three beam ports. Each moderator is independently tuned to optimize the trade-offs among neutron pulse-width, spectral temperature, and overall flux, as appropriate for the instruments to which it supplies neutrons. One possible set of instrument locations and the resulting roughly optimized selection of moderators is described in Section V.2.9.

We considered ambient-temperature water $\left(\mathrm{H}_{2} \mathrm{O}\right)$, liquid methane $\left(\mathrm{CH}_{4}\right)$ at $\sim 95 \mathrm{~K}$, and liquid hydrogen $\left(\mathrm{H}_{2}\right)$ at $\sim 20 \mathrm{~K}$ as moderator materials. These materials span a wide range of spectral temperatures for the moderated neutrons, which allows good matching to the very different requirements of the various instruments. All three of these materials have high hydrogen densities, making them very efficient moderators. Also, the heat deposition and radiolysis that occur in the high radiation field (at the moderator) are relatively well-understood, or at least empirically determined, for these materials. 
The liquid methane and liquid hydrogen moderators require cryogenic cooling systems for liquefying these gases and for extracting the heat deposited in the liquids during operation. The required cryogenic cooling capacity and also the problems associated with radiolysis can be significantly reduced by making the cryogenic moderator thin and surrounding it with a much larger ambient-temperature liquid water premoderator, except on the face through which the neutrons exit to the neutron beamlines. Such a premoderator-moderator combination can be optimized to provide essentially the same neutronic response as would be given by a much larger cryogenic moderator alone. All of the cryogenic moderators at IPNS Upgrade use such liquid water premoderators. A typical configuration is shown schematically in Figure IV.3.1-1. The liquid methane and liquid hydrogen moderators use continuous recirculation of the cooled moderator material to provide the necessary heat extraction. Each of the liquid water moderators and each of the liquid water premoderators also have a system to continuously recirculate and cool the water to extract the large amounts of heat deposited in these systems. The recirculating water and cryogenic-liquid systems require the attachment of several pipes to each of the moderators. These pipes are brought out from the tops or bottoms of the moderators through channels in the reflector, as shown in Figure IV.1.2-6. The associated cooling systems are described in Section IV.3.4.

An important part of any pulsed neutron source is the reflector, which directs fast neutrons back into the moderators. This can increase the moderated beam currents by as much as an order of magnitude relative to that from the same moderators with no reflector. ${ }^{1}$ At each of the IPNS Upgrade target stations, the targets and moderators are surrounded by a beryllium reflector, which provides at least $30 \mathrm{~cm}$ of reflector material in most directions. This reflector is made in two sections, as shown in Figure IV.1.2-6, to simplify remote servicing of the targets and moderators.

Other components of the moderator system are also varied to optimize the trade-off between pulse-width and intensity. These include the choice and location of the decoupler material and the poisoning material. The decoupler is a layer of material that absorbs low-energy neutrons but is transparent to higher-energy neutrons. This material is placed between the reflector and the moderator to prevent neutrons that undergo significant moderation in the reflector from leaking back into the moderator and, subsequently, out into the neutron beam. Because moderating times in the reflector are much longer than those in the moderator, such neutrons add a significant long-time tail onto the neutron pulse if they are not stopped. For most instruments, such a tail spoils the overall quality of the data; consequently, such instruments must view moderators that are decoupled. The decoupling material is cadmium in most cases, but a boron-loaded laminate can be used if higher decoupling energies are desired. ${ }^{2}$ The decoupler is located in intimate contact with the recirculating water of the moderator, in the case of liquid water moderators, or premoderator, in the case of cryogenic moderators, in order to remove the deposited heat. This geometry is indicated schematically in Figure IV.3.1-1. 


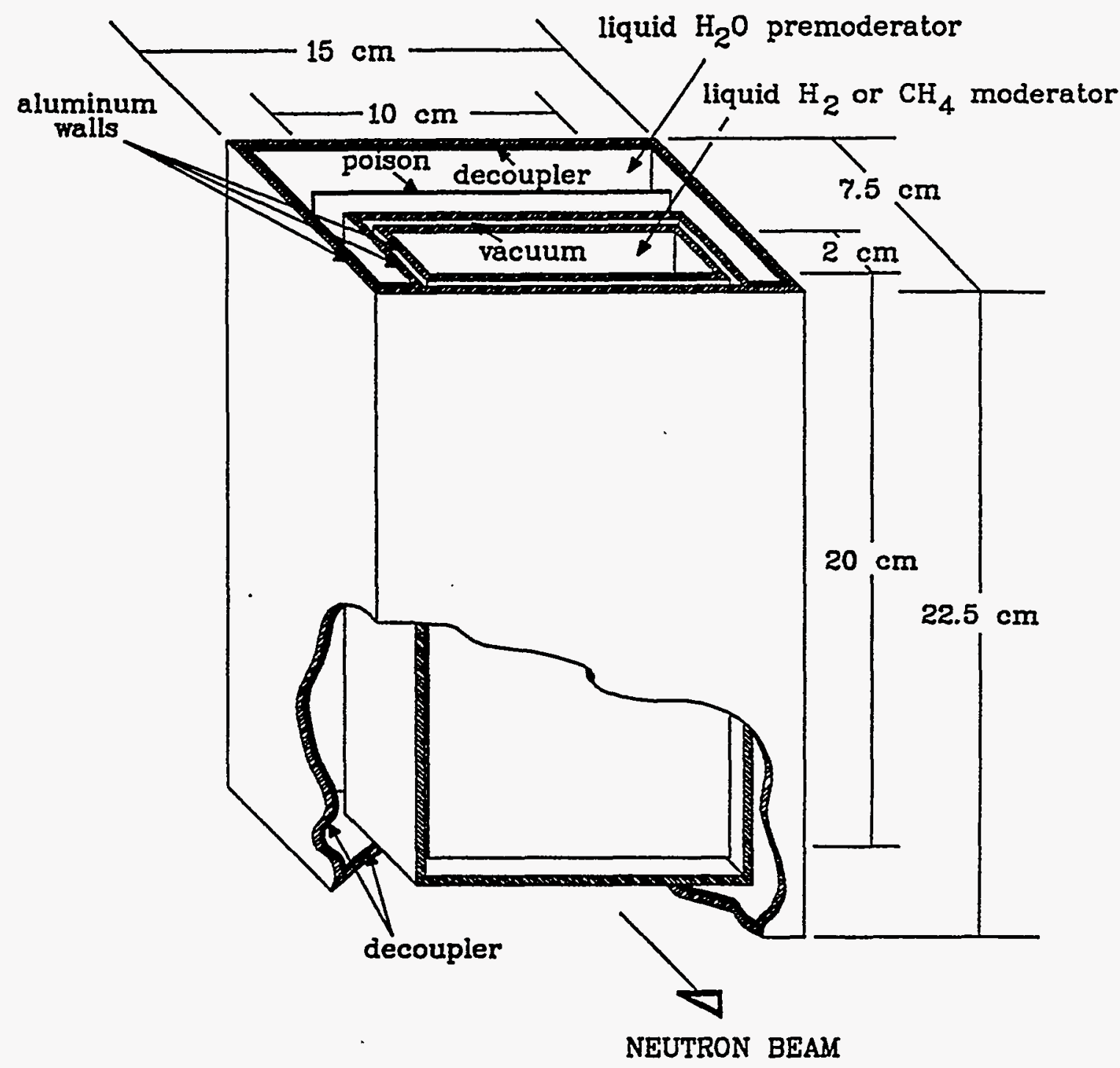

Figure IV.3.1-1 Schematic Representation of a Typical Cryogenic Moderator Assembly for IPNS Upgrade. (The moderator assembly is shown with the top and connecting plumbing removed. Dimensions are approximate and refer to one of the flux-trap moderator assemblies.

For wing moderators, the moderator height is reduced from the $20 \mathrm{~cm}$ shown to $10 \mathrm{~cm}$ with all other dimensions remaining the same.) 
The moderator poisoning is accomplished by placing within the moderator assembly a layer of material that absorbs low-energy neutrons but is transparent to high-energy neutrons. This absorber is usually gadolinium, either in foil or in other convenient form. This makes the moderator effectively thin, and hence provides a narrow neutron pulse-width, for low neutron energies, while providing much more intensity than would be possible if the moderator assembly were actually this thin. The location of the poison within the moderator assembly can be adjusted to provide the desired relationship between intensity and pulse-width. Because significant heat is deposited in the poisoning material, this material is located in the recirculating water of the moderator or premoderator, as shown in Figure IV.3.1-1.

Decoupling and poisoning can be applied to any of the moderators to achieve the desired performance. Both of these processes reduce the total neutron beam current emerging from the moderator and, consequently, are not applied unless necessary. For most of the intended scattering instruments, decoupling is necessary, but for many of these, poisoning may not be necessary. The highest neutron intensity is achieved when the decoupling material is omitted. The poisoning material is usually omitted in this case as well. Such a coupled moderator is used when there is little concern about the pulse-width, and in such cases the intensity can be increased even further by using a larger volume of moderating material, i.e., the moderator can be much thicker. Figure IV.3.1-2 shows typical dimensions of a coupled liquid hydrogen moderator in wing geometry at IPNS Upgrade. Such coupled moderators can provide up to about six times greater time-averaged neutron beam current than does a typical decoupled moderator. ${ }^{3}$

The performance of moderators is known for a few different types of poisoning and decoupling and for a few different geometries. Considerable development is required in order to provide the final optimization for the IPNS Upgrade moderators. Changes in the moderator systems will be made with some frequency, so the target-moderator-reflector assembly design allows easy replacement of moderators. This means that moderator parameters can be further optimized even after IPNS Upgrade is operational. Most of the basic principles underlying such optimization have been known for some time and have been reviewed in several places.1,4 Premoderators have not yet been used in conjunction with cryogenic moderators at any pulsedneutron source. A solid methane moderator with a liquid water premoderator is currently under construction for installation at the pulsed reactor at Dubna, ${ }^{5}$ and premoderated liquid hydrogen moderators have been proposed for use at KENS-II in Japan. ${ }^{6}$

\subsection{MODERATOR NEUTRONICS}

\subsubsection{Monte Carlo Calculations for Room-Temperature Water Moderators}

Some of the neutron beam characteristics were calculated with the model illustrated in Figures IV.2.2-3 through IV.2.2-8 in Section IV.2.2. Figure IV.3.2-1 shows the neutron beam spectra from the room-temperature water moderators. The data shown in this figure are neutron 


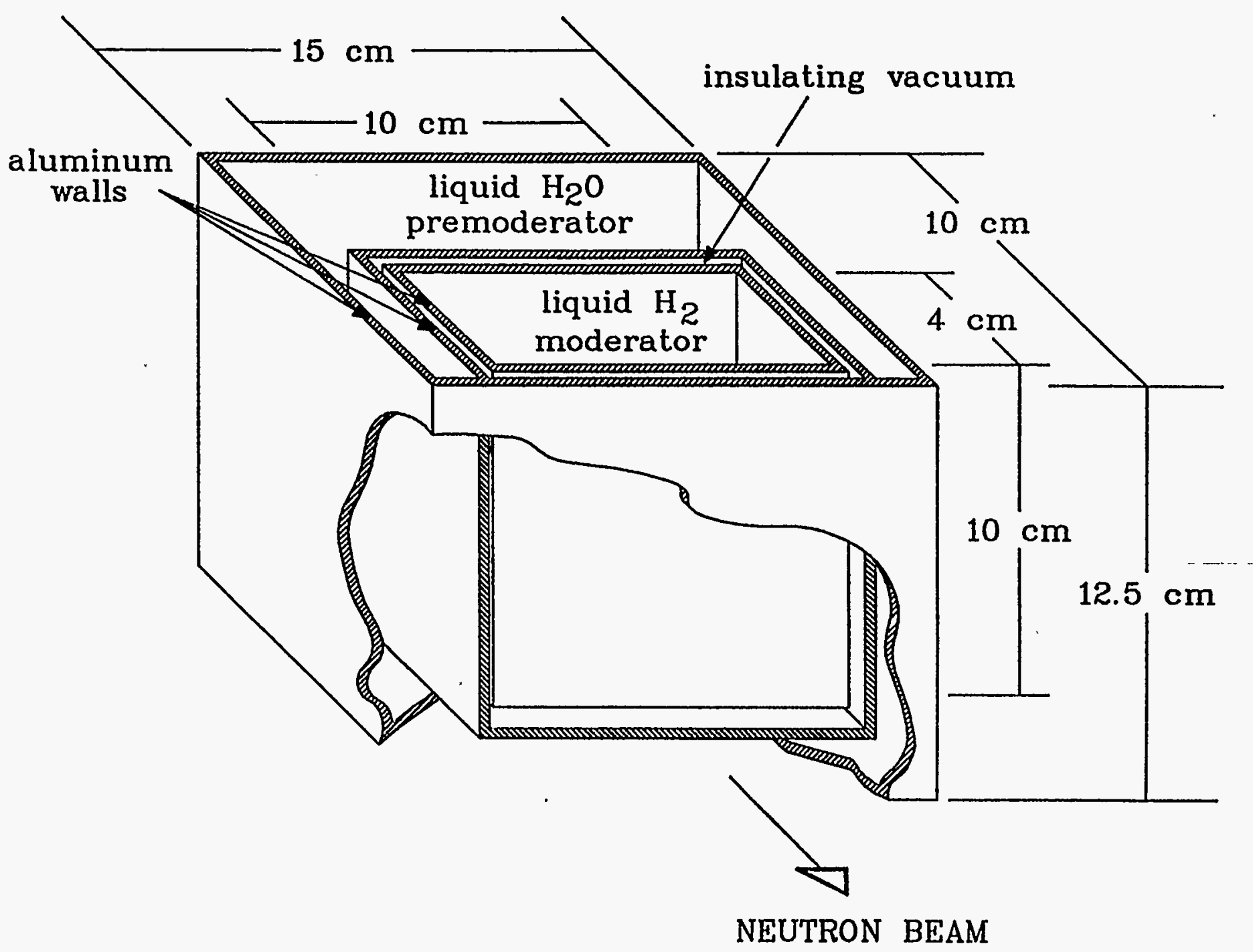

Figure IV.3.1-2 Typical Dimensions of a Coupled Liquid Hydrogen Moderator Assembly in Wing Geometry at IPNS Upgrade. 


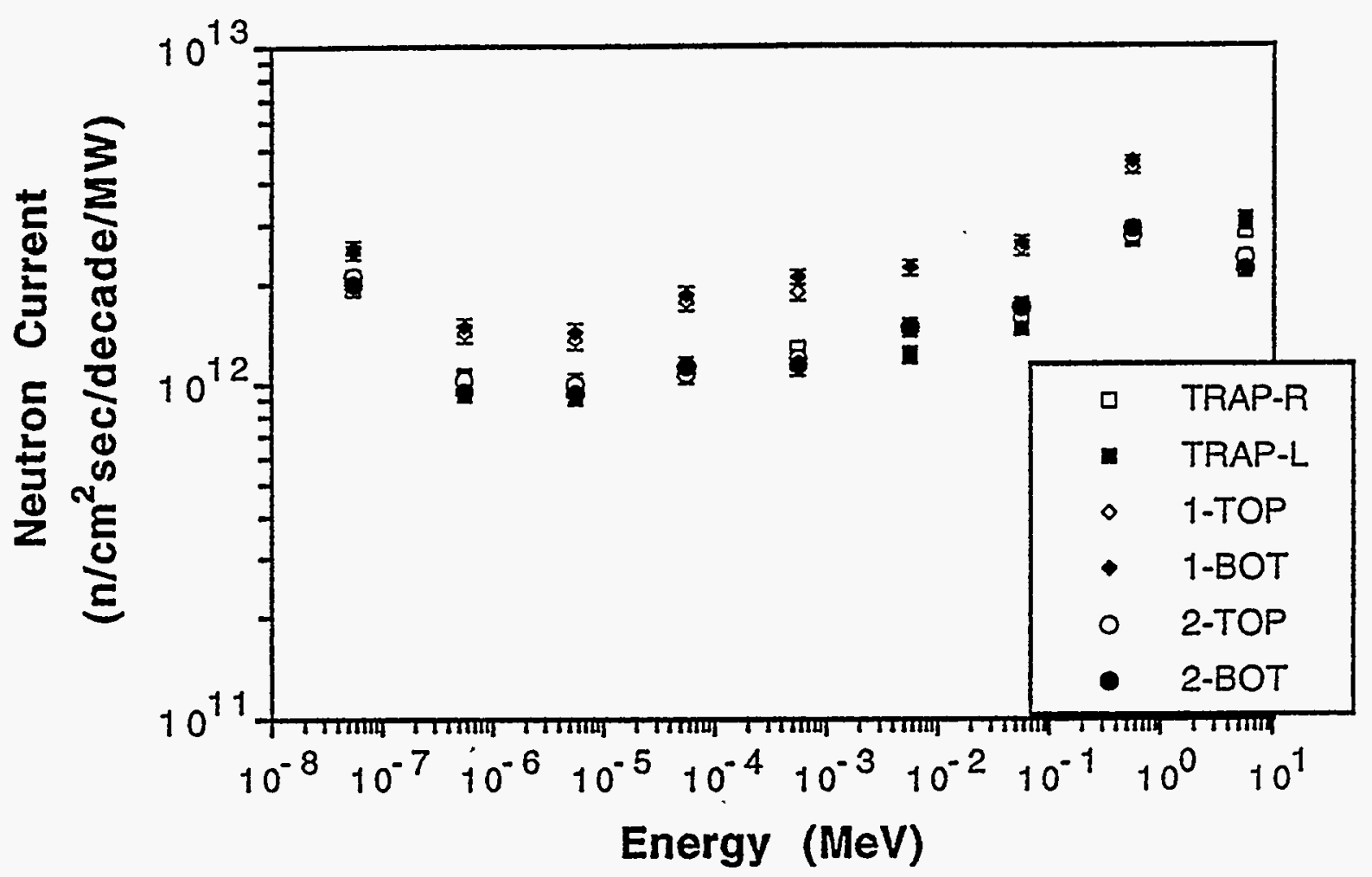

Figure IV.3.2-1 Neutron Beam Spectra at the Ambient Water Moderator Surfaces.

(Moderator locations are defined in Figure IV.2.2-5.) 
currents weighted by the cosine of the angle to the normal direction of the moderator surface and are tallied in decade bins (i.e., 0.1 to $1 \mathrm{MeV}, 1$ to $10 \mathrm{MeV}$, etc.). The labels of moderators shown in Figure IV.3.2-1 are defined in Figure IV.2.2-5. Figure IV.3.2-1 shows that in the range of epithermal energies, the shape of the neutron spectrum is almost the same for all six moderators and that the upstream moderators (1-TOP and 1-BOT; see Figure IV.2.2-5 for notation) yield the highest neutron current in all energy bins. The total number of neutrons produced in the target is 49 neutrons per proton, for $2.2 \mathrm{GeV}$ protons.

The moderator material has been modeled as ambient-temperature water. Small changes in the details of the moderator and poisoning materials produce significant differences in the details of the low-energy spectra, but the epithermal spectra, that is, those above about $1 \mathrm{eV}$, are insensitive to these differences. Thus the spectra calculated are valid at this energy and above.

\subsubsection{The Effects of Premoderators}

The full premoderator geometry indicated in Figures IV.3.1-1 and IV.3.1-2 was not modeled. However, a premoderator layer between the target and the cryogenic moderator was modeled, and the power distributions in slices within the moderators were calculated. For these calculations the shield region was omitted, and a premoderator region was attached to each moderator. Also, liquid hydrogen was used for the downstream-top (2-TOP) and one of the fluxtrap (TRAP-R) moderators. The downstream moderators were modeled without a boron decoupler.

The calculated power depositions for a $1 \mathrm{MW}$ proton beam are shown in Figure IV.3.2-2, along with a detailed illustration of the modeled moderator configuration. It is seen in Figure IV.3.2-2 that the power deposition in the directions parallel to the target is almost uniform, while the power deposition decreases rapidly with increasing distance from the target. The amount of heat eliminated by the premoderator depends on its dimensions. One conclusion drawn from Figure IV.3.2-2 is that much of the heat that would have been deposited in the cryogenic moderator can be eliminated by using a premoderator of a few centimeters thickness. For instance, $37 \%$ of the heat deposition occurs in the 2 -cm-thick premoderator, as seen in the upstream top moderator (1-TOP) of Figure IV.3.2-2, and almost $86 \%$ of the heat can be eliminated by the 3-cm thick premoderator, as seen in the flux-trap-right moderator (TRAP-R). Also, more than half of the heat is deposited in the premoderator region used with the liquid hydrogen moderator in the downistream top moderator position (2-TOP) in Figure IV.3.2-2.

The power deposition in liquid methane is assumed to be the same as that in liquid water because of the similarity in hydrogen densities. The total power deposition in a $2 \times 10 \times 10 \mathrm{~cm}^{3}$ moderator in the upstream moderator position is estimated to be $\sim 200 \mathrm{~W}$. For this case, $\sim 600 \mathrm{~W}$ is deposited in the premoderator (Figure IV.3.1-1). The power deposition in a $2 \times 10 \times 20 \mathrm{~cm}^{3}$ moderator in the flux-trap position is estimated to be $\sim 470 \mathrm{~W}$, while that in the corresponding premoderator is $\sim 1050 \mathrm{~W}$. 


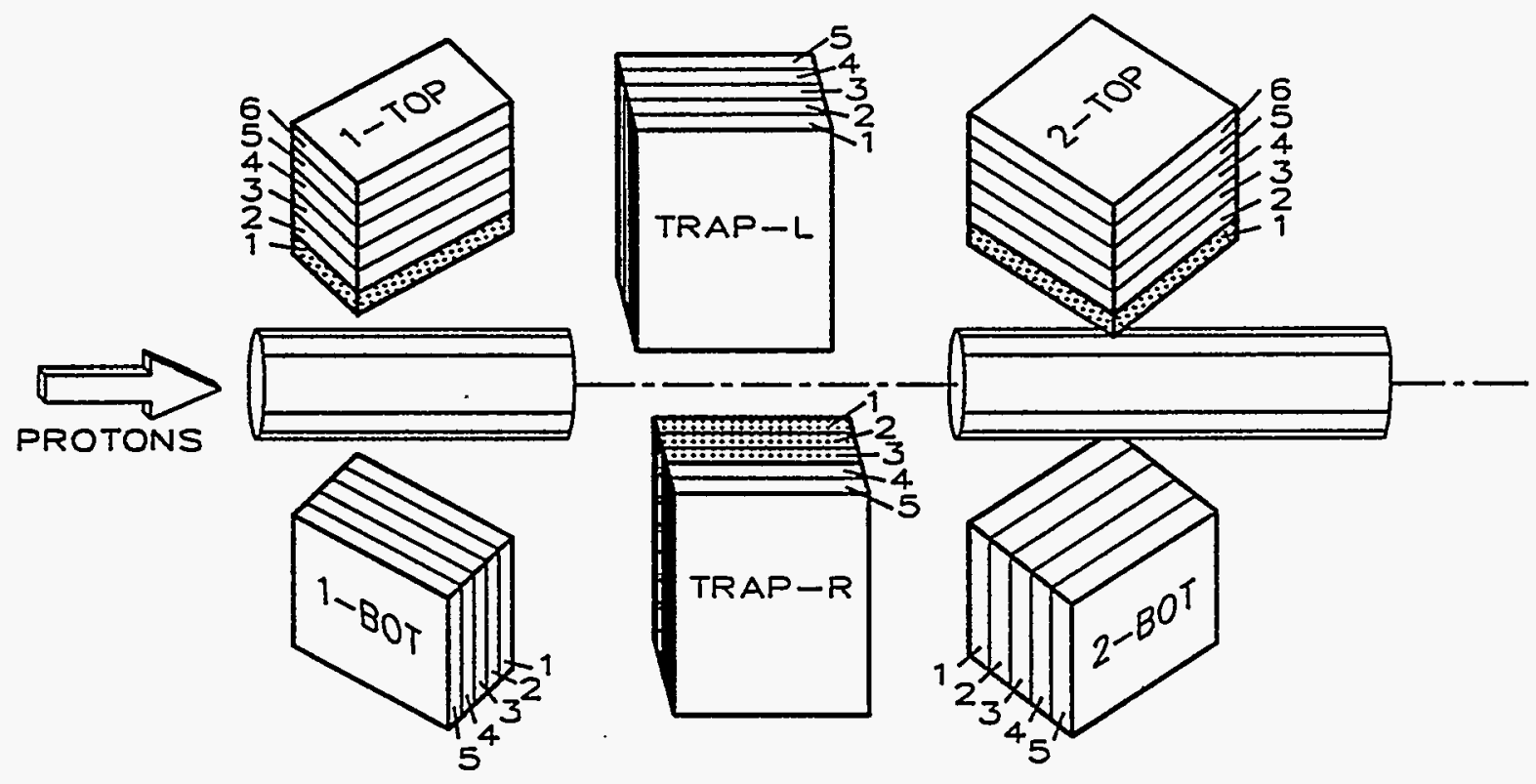

\begin{tabular}{llr}
\multicolumn{3}{c}{$1-$ TOP } \\
\multicolumn{3}{c}{$(10 \times 12 \times 5)$} \\
1 & $\mathrm{H}_{2} \mathrm{O}$ & 299 \\
2 & $\mathrm{H}_{2} \mathrm{O}$ & 188 \\
3 & $\mathrm{H}_{2} \mathrm{O}$ & 132 \\
4 & $\mathrm{H}_{2} \mathrm{O}$ & 86 \\
5 & $\mathrm{H}_{2} \mathrm{O}$ & 56 \\
6 & $\mathrm{H}_{2} \mathrm{O}$ & 45
\end{tabular}

\begin{tabular}{lll}
\multicolumn{3}{c}{ TRAP-L } \\
\multicolumn{3}{c}{$(1 \mathrm{O} \times 2 \mathrm{O} \times 5)$} \\
1 & $\mathrm{H}_{2} \mathrm{O}$ & 425 \\
2 & $\mathrm{H}_{2} \mathrm{O}$ & 338 \\
3 & $\mathrm{H}_{2} \mathrm{O}$ & 284 \\
4 & $\mathrm{H}_{2} \mathrm{O}$ & 268 \\
5 & $\mathrm{H}_{2} \mathrm{O}$ & 198
\end{tabular}

$\begin{array}{ccc}\text { 2-TOP } \\ (10 \times 12 \times 10-\text { coupl } \\ 1 & \mathrm{H}_{2} \mathrm{O} & 702 \\ 2 & \mathrm{H}_{2} & 193 \\ 3 & \mathrm{H}_{2} & 121 \\ 4 & \mathrm{H}_{2} & 94 \\ 5 & \mathrm{H}_{2} & 81 \\ 6 & \mathrm{H}_{2} & 65\end{array}$

$1-B O T$

$(10 \times 12 \times 5)$

$\begin{array}{lll}1 & \mathrm{H}_{2} \mathrm{O} & 153 \\ 2 & \mathrm{H}_{2} \mathrm{O} & 161 \\ 3 & \mathrm{H}_{2} \mathrm{O} & 152 \\ 4 & \mathrm{H}_{2} \mathrm{O} & 153 \\ 5 & \mathrm{H}_{2} \mathrm{O} & 149\end{array}$

TRAP-R $(10 \times 20 \times 5)$

$\begin{array}{llr}1 & \mathrm{H}_{2} \mathrm{O} & 444 \\ 2 & \mathrm{H}_{2} \mathrm{O} & 317 \\ 3 & \mathrm{H}_{2} \mathrm{O} & 253 \\ 4 & \mathrm{H}_{2} & 91 \\ 5 & \mathrm{H}_{2} & 79\end{array}$

2-BOT

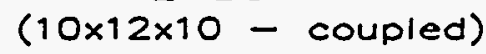

$\begin{array}{lll}1 & \mathrm{H}_{2} \mathrm{O} & 395 \\ 2 & \mathrm{H}_{2} \mathrm{O} & 388 \\ 3 & \mathrm{H}_{2} \mathrm{O} & 432 \\ 4 & \mathrm{H}_{2} \mathrm{O} & 370 \\ 5 & \mathrm{H}_{2} \mathrm{O} & 378\end{array}$

Figure IV.3.2-2 Calculated Power Distribution in Moderators and Premoderators. (The table indicates the composition in each slice and the power (W) deposited in that slice. Shaded slices are treated as premoderators. Moderator dimensions $(\mathrm{cm})$ are width $\times$ height $\times$ depth in direction of neutron beams. Moderators are decoupled unless indicated as coupled.) 
The power deposition in a $2 \times 10 \times 20 \mathrm{~cm}^{3}$ liquid hydrogen moderator in the flux-trap position is estimated to be $\sim 170 \mathrm{~W}$ with $1010 \mathrm{~W}$ in the premoderator. For a $4 \times 10 \times 10 \mathrm{~cm}^{3}$ liquid hydrogen moderator in the downstream position (Figure IV.3.1-2) the power deposition is estimated to be $\sim 220 \mathrm{~W}$, with $1460 \mathrm{~W}$ in the premoderator.

The power deposition density in the aluminum walls of the moderator containers is conservatively estimated to be the same as that calculated for water in similar locations: $\sim 1.01 \mathrm{~W} / \mathrm{cm}^{3}$ in the upstream position, $\sim 1.17 \mathrm{~W} / \mathrm{cm}^{3}$ in the flux-trap location, and $\sim 1.26 \mathrm{~W} / \mathrm{cm}^{3}$ in the downstream location.

The effects of the premoderator on the neutron current spectra from the moderator surfaces were also calculated. Neutron spectra with and without premoderators and decouplers are compared in Figures IV.3.2-3 through IV.3.2-7. Some of these calculations involved a model with no shield, but this difference in modeling is insignificant for the comparison of neutron spectra in moderators, as demonstrated in Figure IV.3.2-3. The comparison in Figure IV.3.2-3 is for a flux-trap moderator that has the same configuration and material in both models. Figure IV.3.2-4 shows that the thermal neutron spectrum is essentially unchanged by the presence of a premoderator. The fast-neutron spectrum decreases because the addition of a premoderator layer increases the water volume. In the comparison shown in Figure IV.3.2-4, water was used for both premoderator and moderator. If cryogenic material is used for the moderator and water is used for the premoderator, however, the neutron spectrum will be affected by the presence of a premoderator and by its dimensions. Further studies should be done for optimization of the premoderator geometry. Nevertheless, it is thought that the neutron spectrum from the cryogenic moderator will not be strongly affected by the water premoderator as long as the cryogenic moderator thickness represents several mean-free-paths of neutron scattering. Tests are under way at the moderator development facility at Hokkaido University to obtain more precise information.

The effects of the boron decoupler on the neutron spectrum are shown in Figure IV.3.2-5. The thermal spectrum is much higher in the moderator without the boron decoupler, as expected. A slight decrease of the fast-neutron spectrum in the moderator with no decoupler is due to the presence of the premoderator.

\subsubsection{Monte Carlo Calculations for Liquid Hydrogen Moderators}

As shown in Figure IV.3.2-2, two of the six moderators (TRAP-R and 2-TOP) were modeled as liquid hydrogen moderators with water premoderators. The premoderator in the model covers only one side of the moderator, rather than five sides as in Figures IV.3.1-1 and IV.3.1-2. The cross-section data of the hydrogen atom at room temperature $(300 \mathrm{~K})$ were used, but with the density set to a nominal liquid hydrogen density. 


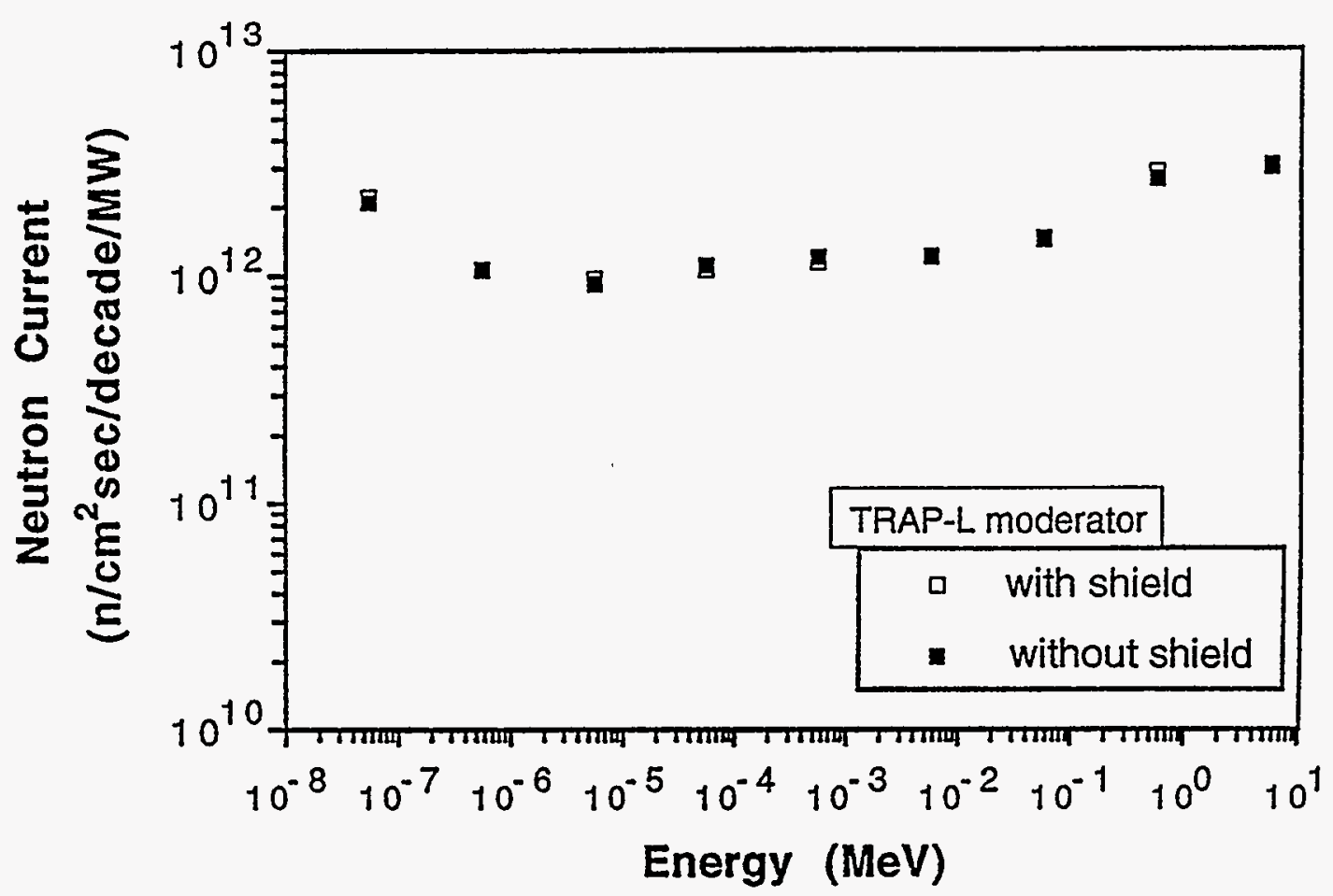

Figure IV.3.2-3 Comparison of Neutron Spectra from the Models with and without Shield. 


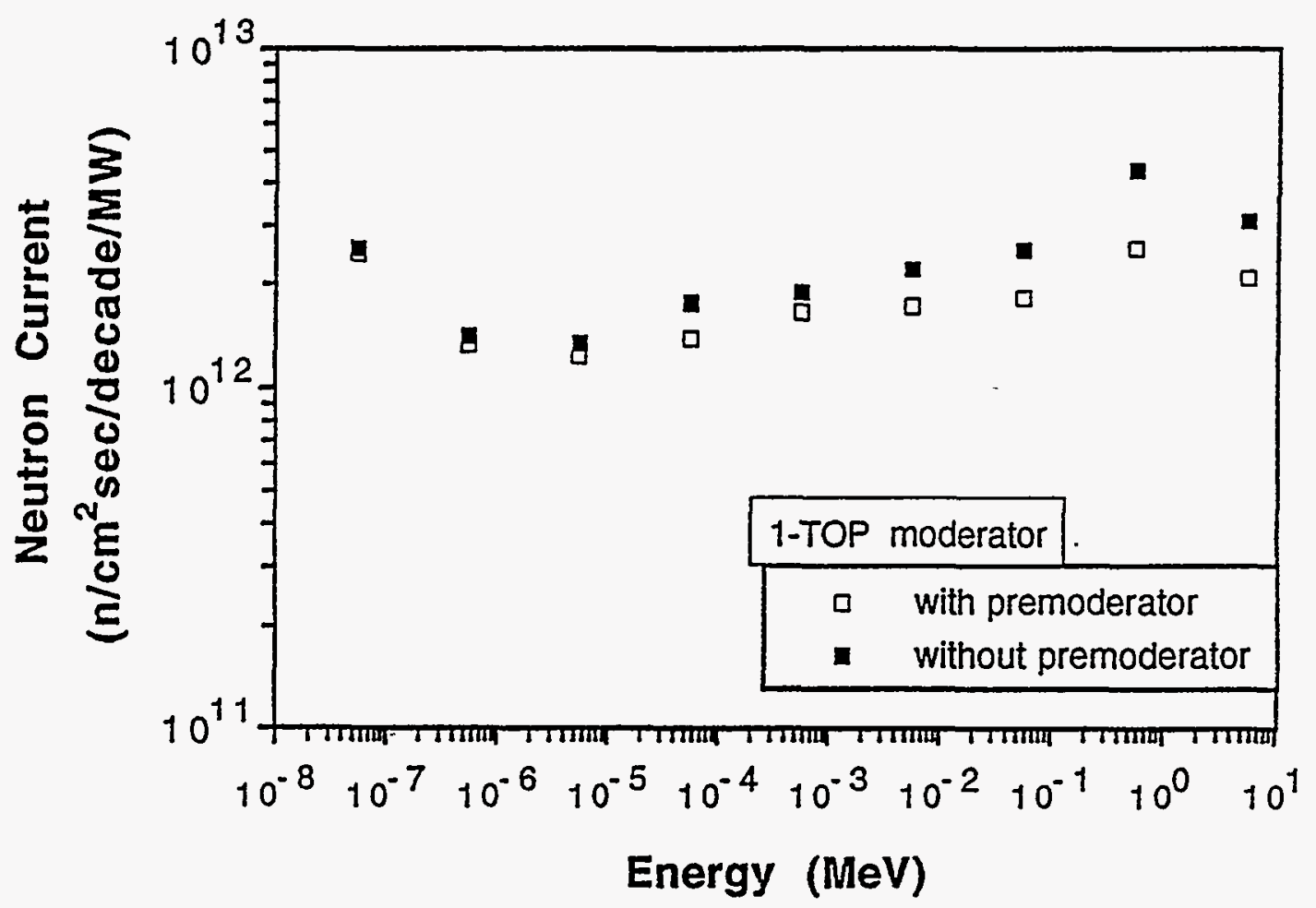

Figure IV.3.2-4 Effects of the Premoderator on the Neutron Beam Spectrum. 


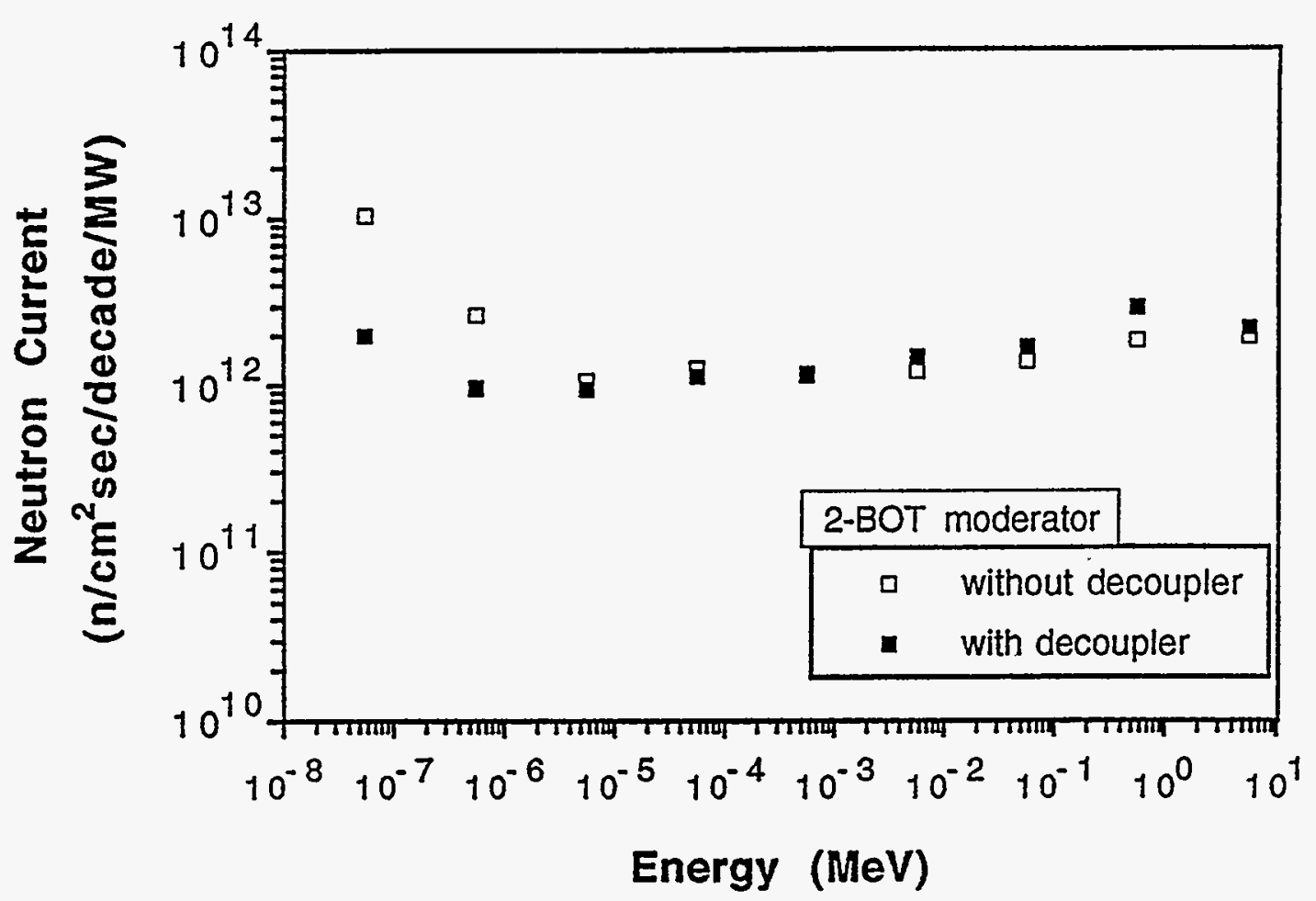

Figure IV.3.2-5 Effects of the Decoupler on the Neutron Beam Spectrum. 


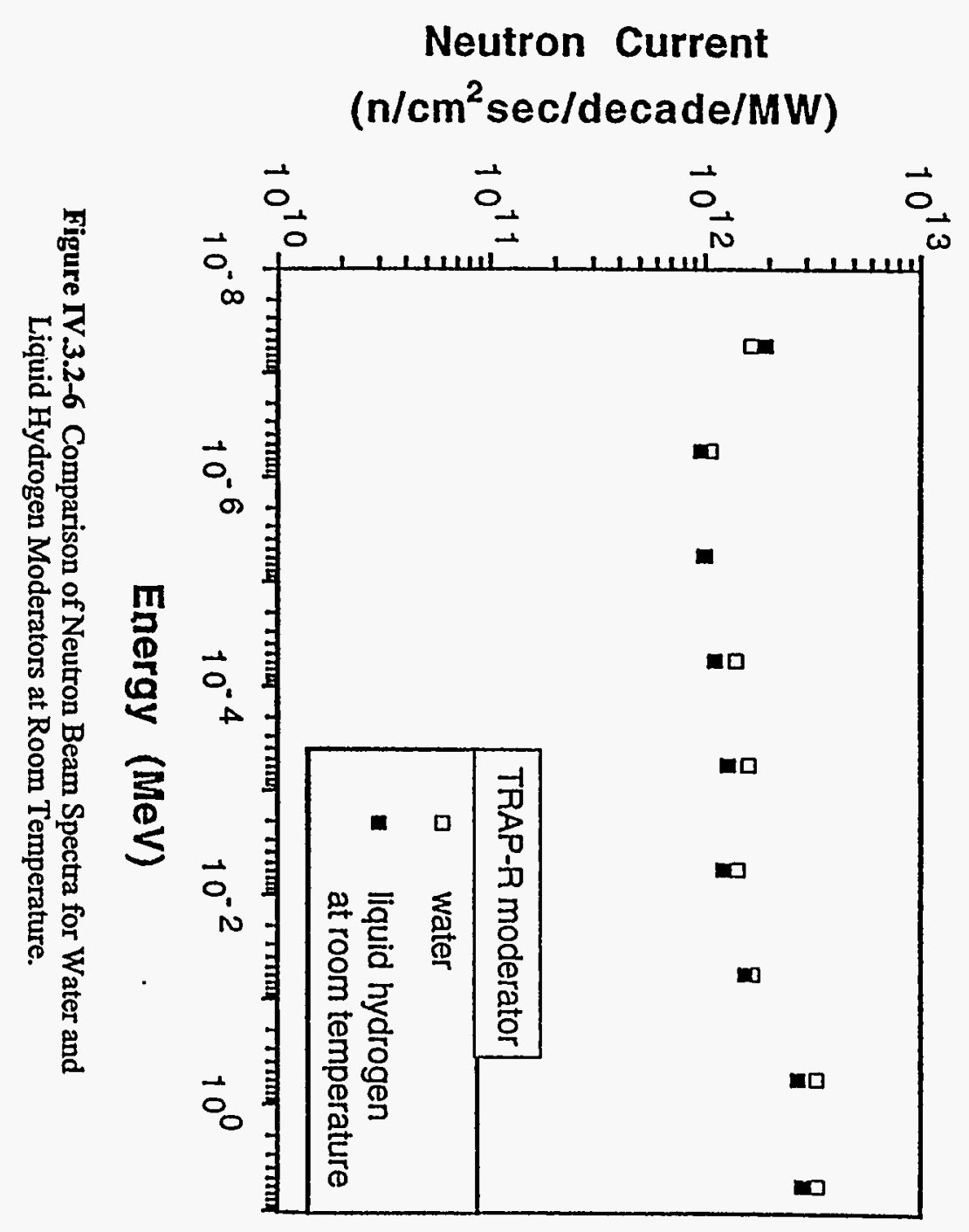




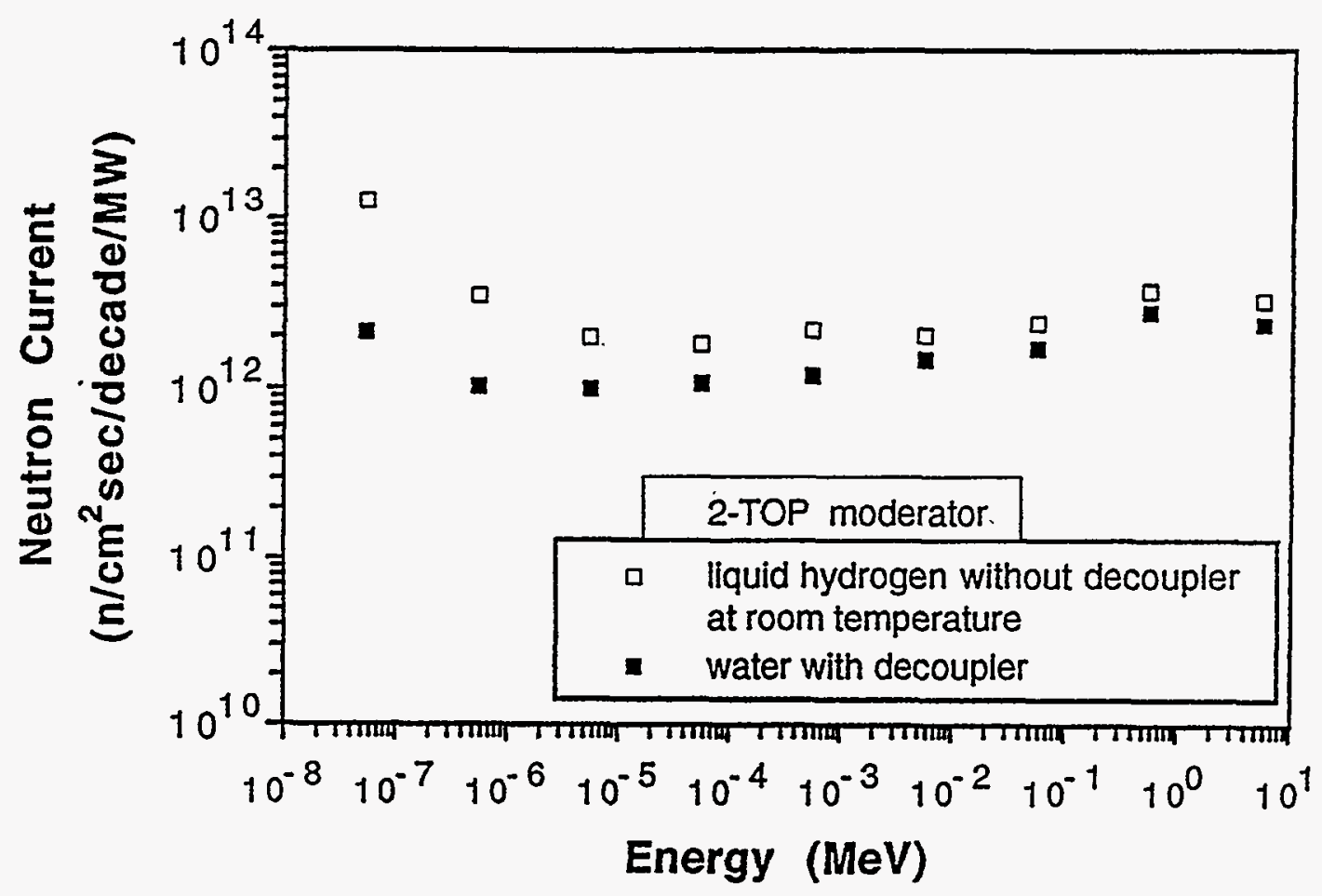

Figure IV.3.2-7 Effects of the Decoupler. 
Figure IV.3.2-6 shows that the neutron spectra for water and hydrogen moderators are almost identical. Figure IV.3.2-7 shows the effects of the neutron decoupler on the neutron-beam spectrum. The case without a decoupler was obtained with the hydrogen moderator, and the case with a decoupler was calculated with the water moderator. Considering that the calculated neutron spectra from hydrogen and water moderators are almost identical, as shown in Figure IV.3.2-6, the effect of the decoupler is thought to be the same for the hydrogen moderator as for the water moderator. Removing the decoupler causes the thermal spectrum to increase significantly, while only changing the fast-neutron spectrum slightly.

\subsubsection{Neutron Beam Currents at $1 \mathrm{eV}$}

The quantities calculated in the previous section are the total outgoing neutron currents $\mathbf{J}$ emerging from the moderator faces, integrated over the moderator face and over $2 \pi$ solid angle of emergence. The quantity relevant to the neutron beam instrumentation is the neutron beam current $I=d J / d \Omega$, which is the total current per unit solid angle emerging in a fixed direction near normal to the moderator face. ${ }^{1}$ The relationship between $\mathrm{J}$ and $\mathrm{I}$ for the planar geometry used here is the Milne problem, common in many applications of transport theory. ${ }^{7}$ The exact solution to the Milne problem, for I normal to the moderator surface is 7

$$
\mathrm{I}=0.41 \mathrm{~J} .
$$

The Monte Carlo results in the preceding section are tabulated as neutrons per decade of energy $\mathrm{E}$. The quantity $\mathrm{EI}(\mathrm{E})$ can be obtained by multiplying these tabulated results by $0.41 / \mathrm{n}_{\mathrm{e}} 10=0.18$. Using this conversion factor, the Monte Carlo calculations of Figures IV.3.2-3 through IV.3.2-7 give the results shown in Table IV.3.2-1. Beam currents at $1 \mathrm{eV}$ for the liquid methane moderator are assumed to be the same as for the liquid water moderator because hydrogen densities are roughly the same in both materials.

For the present IPNS with a depleted uranium target, the corresponding value ${ }^{8}$ for EI(E) at $1 \mathrm{eV}$ is $4.2 \times 10^{11} \mathrm{n} / \mathrm{sr}$-sec. The values in Table IV.3.2-1 range from $\sim 40$ to $\sim 110$ times the IPNS value. A representative estimate is that the time-averaged neutron beam currents at IPNS Upgrade, with the full 1-MW beam power on a single target, are $\sim 75$ times the present IPNS values. In normal operation, time-averaged beam currents at the $30-\mathrm{Hz}$ target station are two-thirds this value, or $\sim 50$ times those at IPNS, while at the $10-\mathrm{Hz}$ target station they are $\sim 25$ times those at IPNS. The actual beam currents will vary significantly from moderator to moderator, depending on the location of the moderator, choice of moderator material, decoupling and poisoning used, etc. However, these ratios of $50 \times$ IPNS and $25 \times$ IPNS should be realistic when comparing similar moderators at IPNS and IPNS Upgrade. It is likely that these ratios can be increased somewhat by further optimization of the IPNS Upgrade target-moderator-reflector geometries and materials. 
Table IV.3.2-1 Neutron Beam Current at $1 \mathrm{eV}$ from Moderator ${ }^{\mathrm{a}}$

\begin{tabular}{|c|c|c|c|c|c|}
\hline \multirow{2}{*}{$\begin{array}{l}\text { Moderator } \\
\text { Material }\end{array}$} & \multirow[b]{2}{*}{ Premoderator } & \multirow[b]{2}{*}{ Decoupler } & \multicolumn{3}{|c|}{$\begin{array}{l}\text { Neutron Beam Current, } \\
\text { El(E), at } 1 \mathrm{eV} \text { from Moderator } \\
\left(10^{13} \mathrm{n} / \mathrm{sr}-\mathrm{sec}\right)\end{array}$} \\
\hline & & & Upstream & Flux-Trap & Downstream \\
\hline Water & No & Yes & 2.4 & 3.5 & 1.7 \\
\hline Water & Yes & Yes & 2.3 & 3.5 & $-b$ \\
\hline Hydrogen & Yes & Yes & - & 3.5 & - \\
\hline Water & Yes & No & - & - & 3.1 \\
\hline Hydrogen & Yes & No & - & - & 4.7 \\
\hline
\end{tabular}

a Calculated for full 1-MW proton-beam power.

$b_{-}=$No calculations for this configuration. 


\subsubsection{Neutron Spectra and Pulse-Widths below $1 \mathrm{eV}$}

\subsubsection{Neutron Spectra}

The time-averaged neutron beam current I(E) from a moderator at a pulsed source, the number of neutrons per steradian per unit energy per unit time, is usually well-represented by a Maxwellian thermal spectrum joined to a nearly $1 / E$ epithermal spectrum as

$$
I(E)=I_{t h}\left[\frac{E}{E_{t}^{2}} e^{-E / E_{t}}\right]+I_{\text {epi }}\left[\frac{\Delta(E)}{E}\right]\left[\frac{E}{E_{\text {ref }}}\right]^{\alpha}
$$

In this equation, $E_{t}$ is a characteristic energy of the Maxwellian portion of the spectrum; $\Delta(E)$ is a joining function, which goes smoothly from 0 (for $\mathrm{E}$ below about $5 \mathrm{E}_{\mathrm{t}}$ ) to 1 (for $\mathrm{E}$ above about $\left.5 \mathrm{E}_{\mathrm{t}}\right) ; \mathrm{E}_{\text {Tef }}$ is a reference energy, typically taken to be $1 \mathrm{eV}$; and $\alpha$ is between 0 and 0.1 . $\mathrm{I}_{\text {th }}$ and $\mathrm{I}_{\mathrm{epi}}$ are scaling constants for the thermal and epithermal portions of the spectrum, respectively. We take a value of $1 \mathrm{eV}$ for $\mathrm{E}_{\text {ref }}$ so that the values of EI(E) at $1 \mathrm{eV}$ from Table IV.3.2-1 directly give $\mathrm{I}_{\mathrm{epi}}$ for some of the different moderators at IPNS Upgrade.

Because of the limited availability of scattering kernels for low-energy neutrons in real moderator materials, it is usual to estimate the thermal portion of the spectrum by empirical methods, rather than from Monte Carlo calculations. This is done by matching measured spectra with the calculated neutron beam currents at $1 \mathrm{eV}$, or by using empirical values of $E_{t}$ and $I_{t h} / I_{e p i}$ in Equation IV.3.2-2. Several empirical values are given in Table IV.3.2-2. The values in Table IV.3.2-2 should be taken as representative values only, because the decoupling energy, poisoning material and location, and moderator geometry can have significant effects on the performance of these moderators in the thermal energy range. Detailed calculations, tests, and interpolation among the empirical data in the literature will be required to provide the combinations of these parameters that optimize the moderator performance for the various sets of neutron scattering instruments.

The effective, time-averaged total thermal flux (reactor equivalent) $\phi_{\text {eff }}$ is given by

$$
\phi_{\mathrm{eff}}=4 \pi \mathrm{I}_{\mathrm{th}} / \mathrm{A},
$$

where $\mathrm{A}$ is the area of the face of the moderator. For the coupled liquid hydrogen moderator in the downstream position,

$$
\begin{aligned}
\phi_{\mathrm{eff}} & =4 \pi\left[\mathrm{EI}(\mathrm{E})_{1 \mathrm{eV}}\right] \times\left[\mathrm{I}_{\mathrm{th}} / \mathrm{I}_{\mathrm{epi}}\right] / \mathrm{A} \\
& \approx 4 \pi \times 4.7 \times 10^{13} \times 12 / 100=7.1 \times 10^{13} \mathrm{n} / \mathrm{cm}^{2}-\mathrm{s},
\end{aligned}
$$


Table IV.3.2-2 Maxwellian Parameters for Several Moderators

\begin{tabular}{lcc}
\hline \multicolumn{1}{c}{ Moderator Type } & $E_{\mathrm{t}}(\mathrm{MeV})$ & $\mathrm{I}_{\mathrm{th}} / \mathrm{I}_{\text {epi }}$ \\
\hline & & \\
Poisoned, decoupled, 320 K liquid water & $32^{\mathrm{a}}$ & $2.3^{\mathrm{a}}$ \\
Poisoned, decoupled, 95 K liquid methane & $11^{\mathrm{a}}$ & $2.1^{\mathrm{a}}$ \\
Decoupled, $18 \mathrm{~K}$ liquid hydrogen & $3.0^{\mathrm{b}}$ & $2.4^{\mathrm{b}}$ \\
Coupled, 320 K liquid water & $\sim 32^{\mathrm{c}}$ & $\sim 22^{\mathrm{c}}$ \\
Coupled, $18 \mathrm{~K}$ liquid hydrogen & $2.8^{\mathrm{b}}$ & $12^{\mathrm{b}}$ \\
& & \\
\hline
\end{tabular}

a ISIS moderator data; Source: Ref. 9.

b Based on the data of Kiyanagi et al. (Ref. 3); values are only approximate because liquid hydrogen spectra are not fit well by a Maxwellian function.

c Rough factor of 9.8 intensity increase over poisoned, decoupled water moderator is based on SNQ mock-up measurements (Ref. 10). 
and a similar calculation for the coupled liquid water moderator in the downstream position gives

$$
\phi_{\mathrm{eff}} \approx 8.6 \times 10^{13} \mathrm{n} / \mathrm{cm}^{2} \text {-s. }
$$

In normal operation, such moderators at the $30-\mathrm{Hz}$ target station produce two-thirds these equivalent flux values, while at the $10-\mathrm{Hz}$ station, they produce one-third these fluxes. It should be noted that $\phi_{\text {eff }}$ represents actual equivalent $4 \pi$ "perturbed" fluxes for the beams emerging from the moderators. In order to obtain a flux of this magnitude from a liquid hydrogen or deuterium cold moderator at a reactor, the nominal reactor flux would have to be significantly greater than $10^{14} \mathrm{n} / \mathrm{cm}^{2}$-s. For example, the high-flux reactor at ILL has a peak calculated unperturbed flux of $\sim 1.5 \times 10^{15} \mathrm{n} / \mathrm{cm}^{2}-\mathrm{s}$ in the ambient $\mathrm{D}_{2} \mathrm{O}$ moderator vessel. The calculated unperturbed flux ${ }^{11}$ at the location of the liquid deuterium cold moderator within this vessel is only $\sim 5 \times 10^{14} \mathrm{n} / \mathrm{cm}^{2}-\mathrm{s}$, and the actual perturbed flux at this location is even lower.

The Monte Carlo calculations indicate that these reactor-equivalent time-averaged fluxes can be increased significantly by placing the coupled moderators near the upstream target, rather than near the downstream target. It is likely that using larger moderators or premoderators in the coupled case will produce appreciable gains. Additional Monte Carlo studies are required to optimize such moderators in order to obtain the maximum possible neutron beam current for instruments that operate in the quasi-steady-state (QSS) mode.

\subsubsection{Neutron Pulse-Widths}

The neutron pulse shapes emerging from a moderator are highly energy dependent. The overall widths of these pulses can be changed dramatically by varying the poisoning and decoupling parameters, as well as by varying the moderator material. The premoderator geometry may also have an effect. Thus, there are a large number of degrees of freedom that can be varied in designing moderators optimized for the requirements of specific sets of instruments. Figure IV.3.2-8 shows the energy-dependent pulse-widths ( $\mathrm{fwhm}$ ) for the moderators specified in Table IV.3.2-2. These are representative values only, as small changes in the poisoning and/or decoupling give rise to variations of a factor of 2 in $I_{t h}$ and to correspondingly large variations in the pulse-width. Because of this great degree of tunability in moderator performance, moderator optimization must be studied in much greater detail before the final selections are made.

\subsection{RADIOLYSIS}

\subsubsection{Production of Hydrogen}

Irradiation of liquid water, methane, or hydrogen with fast neutrons causes the molecules to be radiolytically decomposed into lighter constituents, which can then recombine into new 


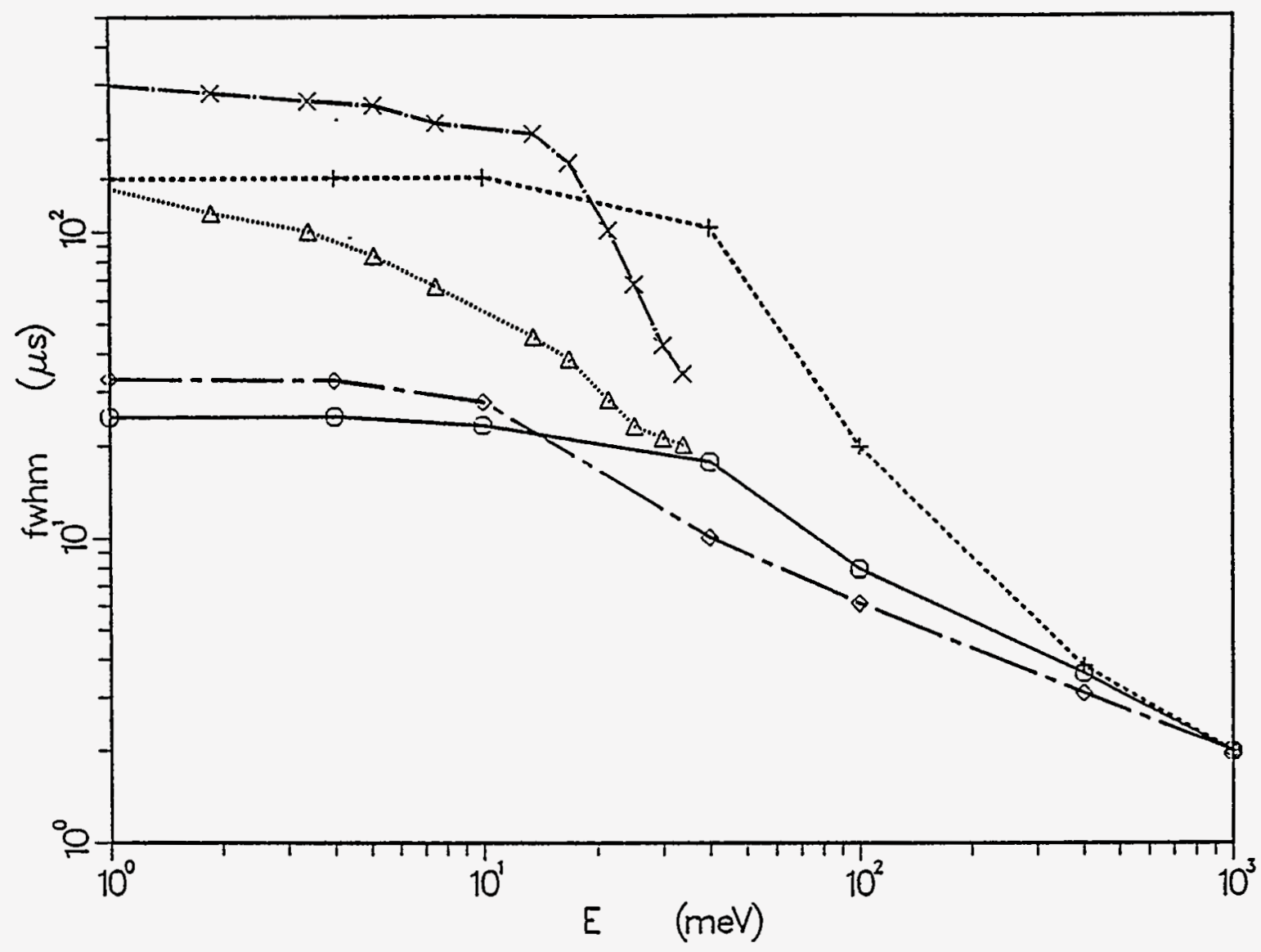

Figure IV.3.2-8 Pulse-Widths (fwhm) for the Moderators Listed in Table IV.3.2-2. (Circle = decoupled, poisoned liquid water; diamond = decoupled, poisoned liquid methane; triangle = decoupled liquid hydrogen; plus = coupled liquid water; cross = coupled liquid hydrogen. The lines are a guide to the eye only.). 
forms. Hydrogen moderator systems must be free of reactive contaminants to avoid reaction of the radiolytically formed hydrogen radicals to produce species that cause problems. Hydrogen at low temperatures gradually and spontaneously tends to the lowest-energy para state, which has significantly different total and differential inelastic scattering properties from the higher energy ortho state. Recombination of radiolytically decomposed hydrogen is into the statistically determined 3:1 ortho:para mixture, so that under irradiation the result is a mixture of ortho and para hydrogen that depends on the rate of radiolysis and on the time. The spectrum and emission time distributions may depend on the composition of the ortho:para mixture. Although this is not likely to have a major effect on moderator performance, the effect needs to be investigated.

The possible undesirable radiolytic products of water are hydrogen, oxygen, ozone, and hydrogen peroxide. Because several of these are gases under the conditions of operation of the water moderators and premoderators, they must be removed or recombined before they either displace a significant portion of the liquid water from the moderator container or reach potentially explosive concentrations. The moderators and premoderators are designed so that the resulting water flow patterns do not allow significant accumulation of gases in the moderator and premoderator regions. The recirculating systems for the IPNS Upgrade water moderators and premoderators contain provisions for recombining or purging the hydrogen and oxygen gases. Production rates for hydrogen and oxygen in the IPNS Upgrade liquid water moderators and premoderators are difficult to estimate, and some research and development effort is required in this area.

Possible undesirable radiolytic products of methane include hydrogen and virtually all higher hydrocarbons. The hydrogen is gaseous under the operating conditions of the liquid methane moderator and displaces methane from the moderator if it is allowed to accumulate. The methane flow pattern is designed to prevent accumulation of hydrogen gas in the moderators. The recirculating systems for the IPNS Upgrade liquid methane moderators contain provisions for removal of hydrogen gas produced. They also contain provisions for removal of some of the higher hydrocarbons from the recirculating mixture. However, not all of these higher hydrocarbons can be removed, which leads to an additional set of problems discussed in the following section.

\subsubsection{Buildup of Higher Hydrocarbons in the Liquid Methane Moderator}

No solid products result from radiolysis of water or hydrogen. The radiolysis of methane can form radicals that can recombine to form high-molecular weight hydrocarbons such as waxes or solids. These tend not to circulate with the liquid methane, but rather to deposit on the walls of the moderator container and associated piping, where they are subject to further radiation damage. Experience has shown that the resulting buildup, which cannot be flushed away with any practical solvents, eventually fills enough of the moderator container to degrade the moderator performance. This leads to a finite, predictable lifetime for liquid methane moderator assemblies. 
Since its startup in 1985, the ISIS pulsed-neutron facility has been operating a liquid methane moderator. By the end of 1990, the performance of this moderator had deteriorated to the extent that it had to be replaced. ${ }^{12}$ The moderator assembly that was removed was subsequently cut apart to assess the reasons for its failure. It was found that the moderator container was partially filled with a solid black residue, the result of the radiolytic decomposition of the methane and subsequent formation and deposition of higher hydrocarbons. This residue was determined by analysis to consist of highly cross-linked polymers. ${ }^{13}$ Tests of similar material collected from IPNS liquid methane moderators show that it is insoluble in any common solvent and refractory against thermal decomposition at reasonable temperatures. ${ }^{14}$

ISIS has since tried three different liquid methane moderators, with frequent replacement of the methane and with different flow configurations. ${ }^{13}$ However, during the same time the proton current at ISIS has been steadily increased up to the design goal of $200 \mu \mathrm{A}$ at $800 \mathrm{MeV}$. The net result is that the lifetime of each of these moderators has been shorter than that of the previous one, even when measured in terms of the integrated proton current. These lifetimes are summarized in Table IV.3.3-1, which is based on data from Broome. ${ }^{13}$ Also included in this table is an estimate of the lifetimes of liquid methane moderators at IPNS Upgrade, based on the assumptions that the flow conditions would be similar and that the correct scaling factor is the ratio (6.25) of the proton beam powers at IPNS Upgrade and ISIS.

Liquid methane moderator lifetimes can also be estimated in a somewhat different manner. At the design operating parameters of $200 \mu \mathrm{A}$ and $800 \mathrm{MeV}$, the power deposition in the ISIS liquid methane moderator was calculated to be somewhere between $283 \mathrm{~W}$ and $625 \mathrm{~W}$ when the depleted uranium target was used. ${ }^{15,16}$ For a tantalum or tungsten target, these estimates should be divided by $\sim 2$. The respective power deposition densities are $\sim 0.21 \mathrm{~W} / \mathrm{cm}^{3}$ and $\sim 0.48 \mathrm{~W} / \mathrm{cm}^{3}$. By comparison, the calculated power deposition density is $\sim 1.0-1.2 \mathrm{~W} / \mathrm{cm}^{3}$ in the liquid-methane portion of a premoderated liquid methane moderator at IPNS Upgrade operating at full power (see Section IV.3.2.2). Assuming that the rate of radiation damage and deposition of solid hydrocarbons scales with the power deposition density, this leads to projected liquid methane moderator lifetimes at IPNS Upgrade ranging from $\sim 340 \mathrm{~h}$ to $\sim 1,570 \mathrm{~h}$ at full power before the same degree of deterioration in performance is experienced that was observed at ISIS.

Both approaches suggest that operating lifetimes for liquid methane moderators at IPNS Upgrade could range from two weeks to two months. A two-month lifetime is marginally acceptable, but two-week lifetimes certainly are not acceptable. Thus, it is important that a development program be undertaken to provide better understanding of this hydrocarbon buildup and to develop procedures for its reduction, with the goal of eventually providing longer lifetimes for liquid methane moderators. A development program along these lines has already begun at ISIS. ${ }^{13}$ If liquid methane moderators with suitable lifetimes cannot be developed, it will still be possible to use either liquid hydrogen or water moderators in place of the liquid methane moderators. All of the instruments can still be operated, although in some cases there would be a reduction in performance if liquid methane were not available. 
Table IV.3.3-1 Lifetimes of Liquid Methane Moderators

\begin{tabular}{|c|c|c|c|c|}
\hline \multirow[b]{2}{*}{ Moderatora } & \multicolumn{2}{|c|}{$\begin{array}{c}\text { Effective Integrated } \\
\text { Proton Current }\end{array}$} & Estimate & \multirow{2}{*}{$\begin{array}{l}\text { d Lifetime } \\
\text { IPNS } \\
\text { Upgrade } \\
\text { (h) }\end{array}$} \\
\hline & $\begin{array}{c}\text { Depleted } \\
\text { U Target } \\
(\mathrm{mA-h})\end{array}$ & $\begin{array}{c}\text { Ta or } \\
\text { W Target } \\
(\mathrm{mA}-\mathrm{h})\end{array}$ & $\begin{array}{l}\text { ISISC }^{c} \\
\text { (h) }\end{array}$ & \\
\hline \#1 single foil & 747 & 1494 & 7470 & 1195 \\
\hline \#2 single foil & 575 & 1150 & 5750 & 920 \\
\hline $\begin{array}{l}\text { \#3 double foil, } \\
\text { reverse flow }\end{array}$ & 198 & 396 & 1980 & 317 \\
\hline $\begin{array}{l}\# 4 \text { single foil, } \\
\text { reverse flow }\end{array}$ & 164 & 328 & 1640 & 262 \\
\hline
\end{tabular}

a Single or double foil refers to the poisoning configuration within the moderator.

b Tantalum or tungsten produces roughly a factor of two fewer neutrons per proton than does depleted uranium, so a conversion factor of 2 has been used, as in Ref. 13.

c Lifetime with a tantalum or tungsten target.

d Projected lifetime, based on a tungsten target and scaled from ISIS by using the relative proton beam powers. 


\subsection{COOLING}

\subsubsection{Component Layouts for Water Cooling Systems}

This section describes the water cooling systems for moderators, reflectors, the biological shield, the target tank, and the proton beam window. Components for these cooling systems are located on separate skids in shielded areas in Buildings $367(10-\mathrm{Hz}$ target) and $371(30-\mathrm{Hz}$ target). Figures IV.3.4-1a and IV.3.4-1b show the layout of shielding and skid-mounted components in Building 367 for the 10- $\mathrm{Hz}$ target. Figures IV.3.4-2a and IV.3.4-2b show the layout of components in Building 371 for the $30-\mathrm{Hz}$ target.

The different water cooling systems are discussed in Sections IV.3.4.2, IV.3.4.5, IV.3.4.6, IV.3.4.7 and I.V.3.4.8.

\subsubsection{Room-Temperature Water Moderators and Premoderators}

Each target station has six moderators or premoderators, each of which contains water. The heat deposited in the water of a premoderator or moderator is within the range of one to several kilowatts. Also, the approximate 500-W nuclear heating that occurs in the vacuum jacket surrounding the cryogenic lines is absorbed by the same water cooling circuits. In all cases, a water flow rate of $0.021 \mathrm{~L} / \mathrm{sec}$ cools any water moderator and its attached piping with a relatively low increase in bulk temperature $(25 \mathrm{~K})$. This rise in bulk temperature does not approach saturation temperature $(445 \mathrm{~K})$, so boiling does not occur.

Water for each of the six water moderator circuits is supplied from a common manifold supplied by one of two redundant pumps, as depicted in Figure IV.3.4.3. The pumps are constructed of corrosion-resistant materials, primarily $316 \mathrm{SS}$. Installed motor power is $0.6 \mathrm{~kW}$. The active pump draws from a 100-L water supply tank at a pressure of $690 \mathrm{kPa}$ and discharges $0.13 \mathrm{~L} / \mathrm{sec}$ total water flow rate at a pressure of $790 \mathrm{kPa}$. The supply tank has an $\mathrm{H}_{2} / \mathrm{O}_{2}$ recombiner. A 100-L drain tank provides for local hold-up of the cooling water inventory. Check valves preclude reverse flow through the nonoperating pump. The pressure loss in the water supply and return lines is very small, so most of the head developed by the pump is due to pressure drop across the six identical, parallel flow-control orifices, each of which are $1.9 \mathrm{~mm}$ in diameter.

The tanks, pumps, heat exchanger, and demineralizer for the $10-\mathrm{Hz}$ target are located on a common skid in a shielded room in Building 367. A duplicate housed in Building 371 serves the 30-Hz target. This is shown in Figures IV.3.4-1a, IV.3.4-1b, IV.3.4-2a, and IV.3.4-2b. Supply and return piping between the cooling system components and the moderators and premoderators is shielded. All tanks and plumbing are insulated to reduce heat loads in the shielding and pump room. 


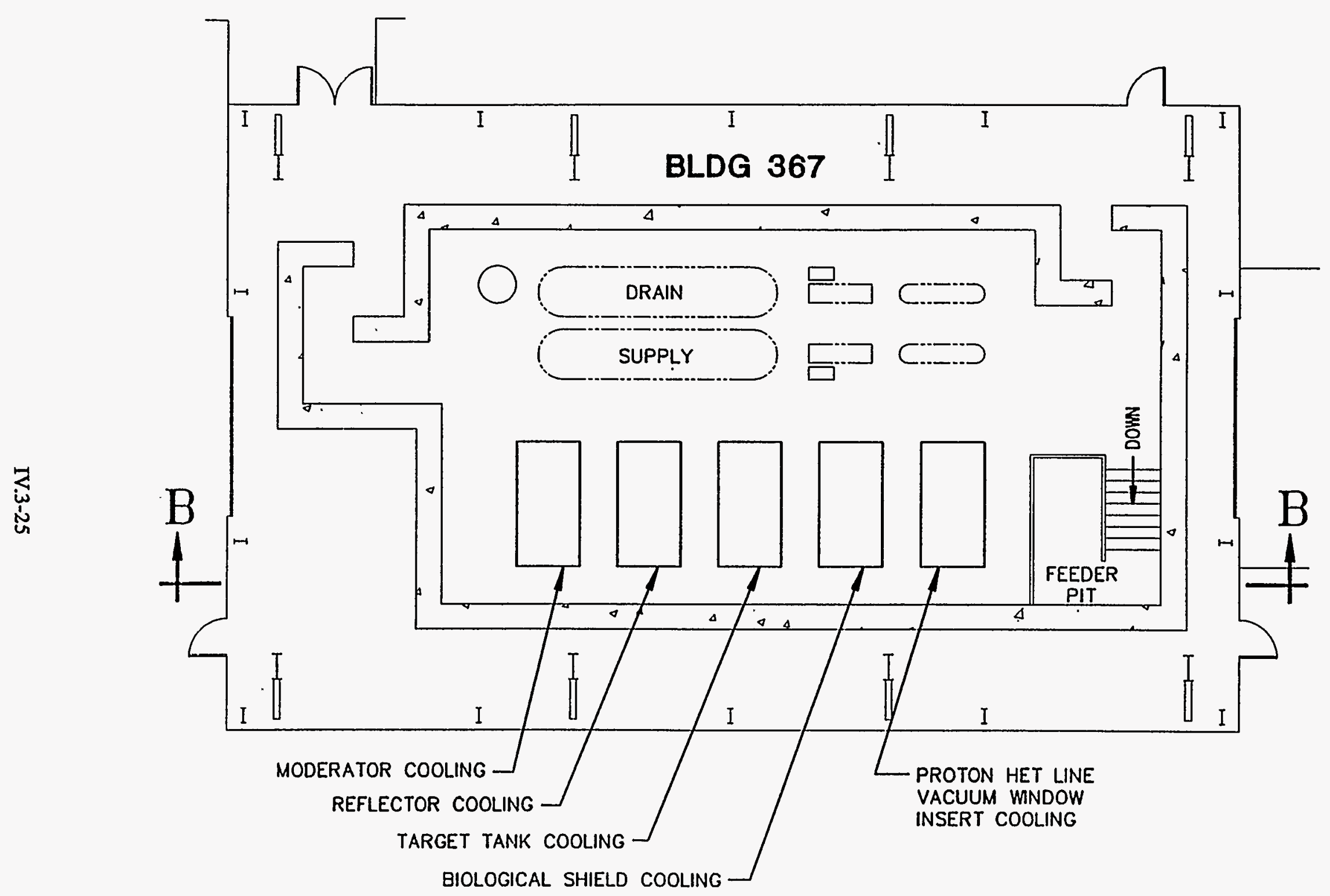

Figure IV.3.4-1a Layout of Skid-Mounted Cooling Systems in Building 367, for the 10-Hz Target. 


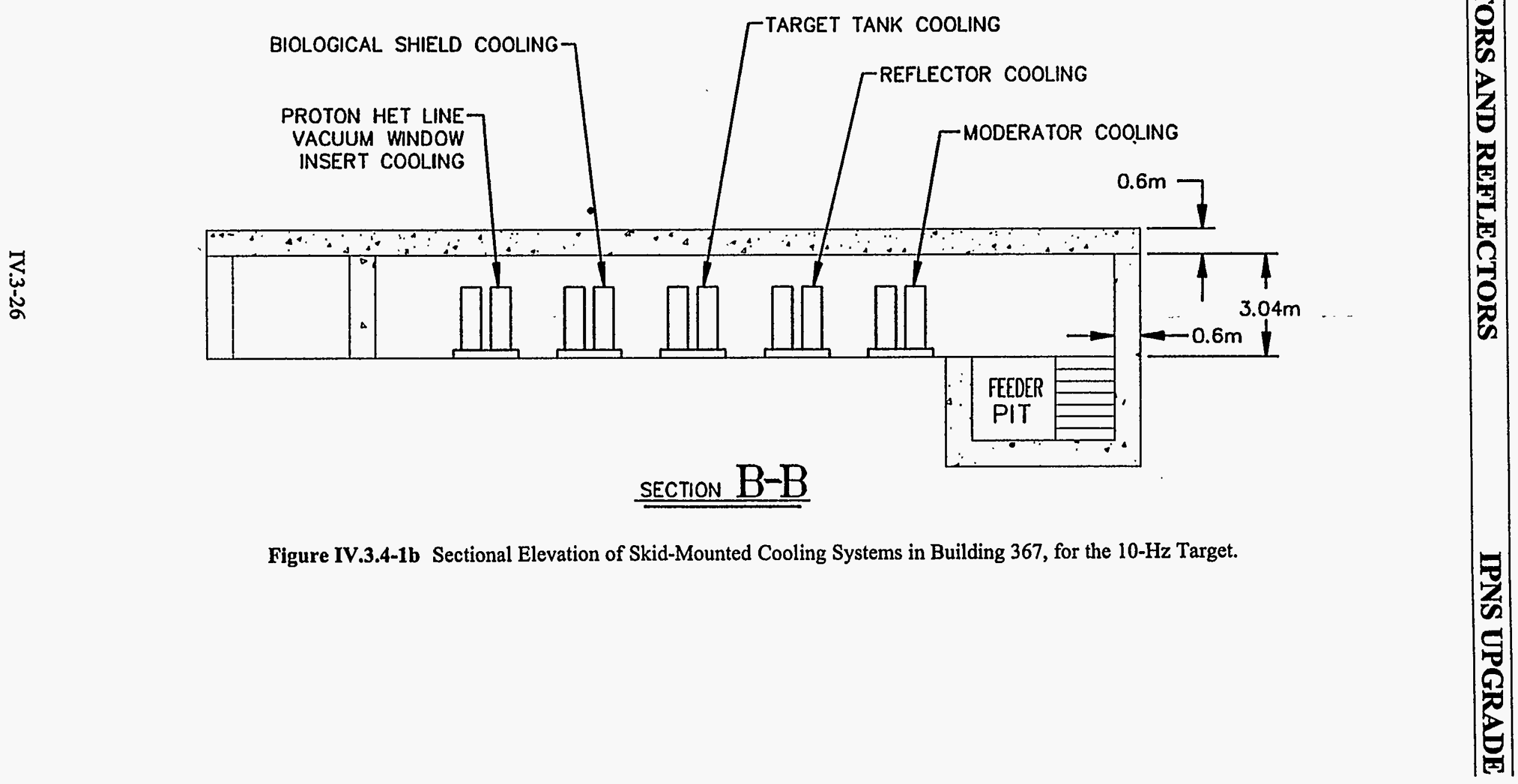




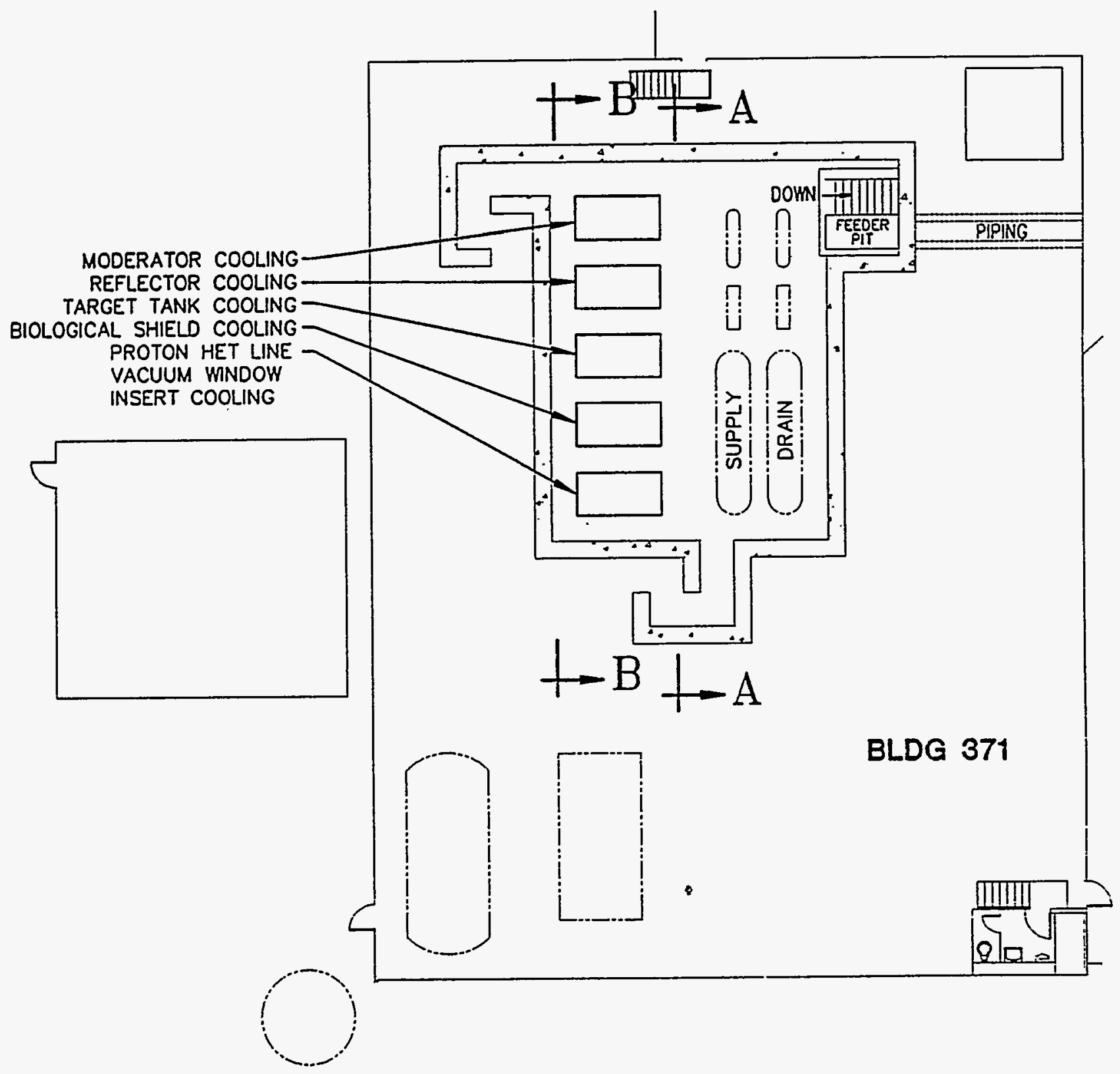

Figure IV.3.4-2a Layout of Skid-Mounted Cooling Systems in Building 371, for the 30-Hz Target. 


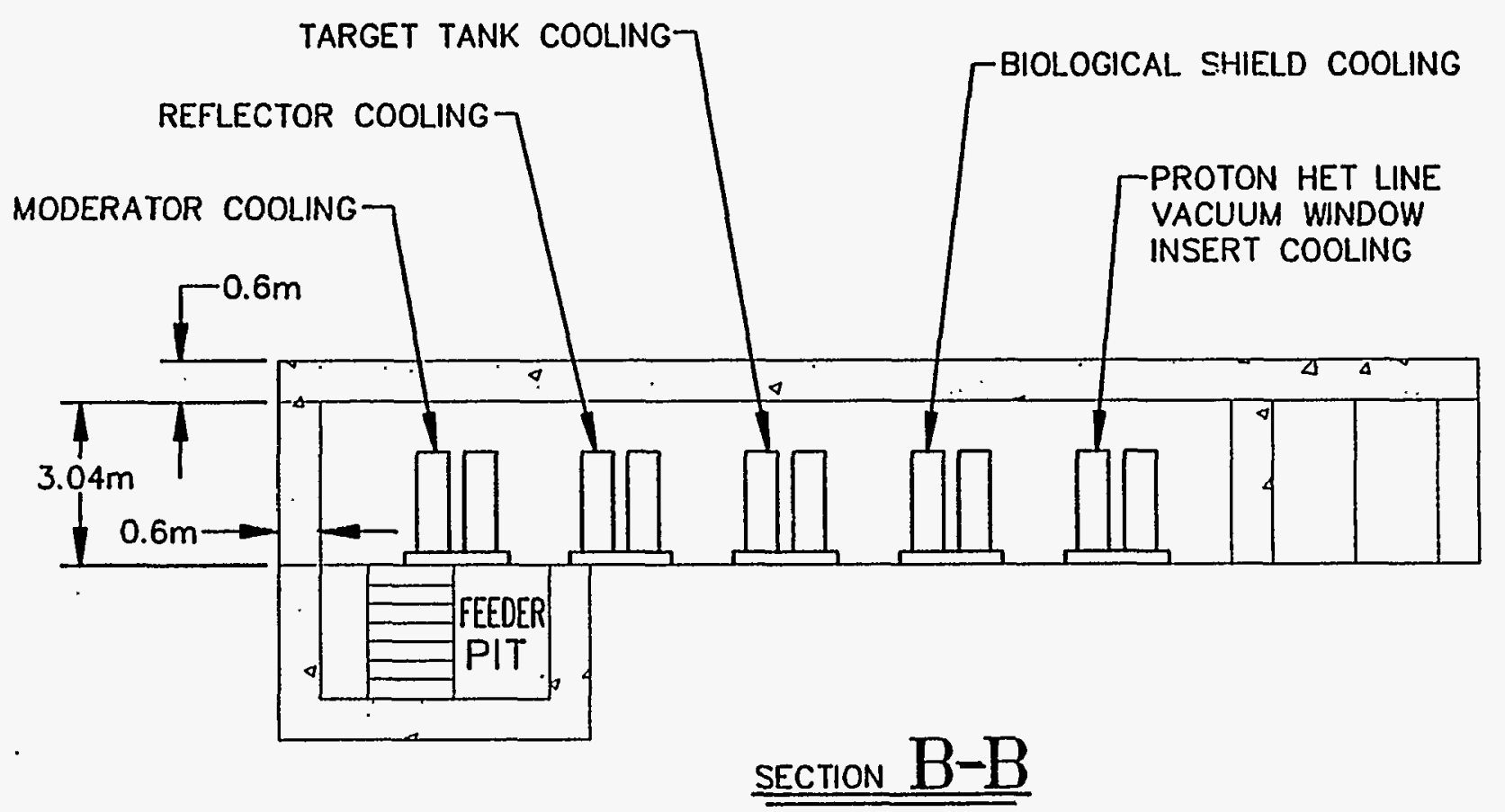

Figure IV.3.4-2b Sectional Elevation of Skid-Mounted Cooling Systems in Building 3.71, for the 30-Hz Target. 


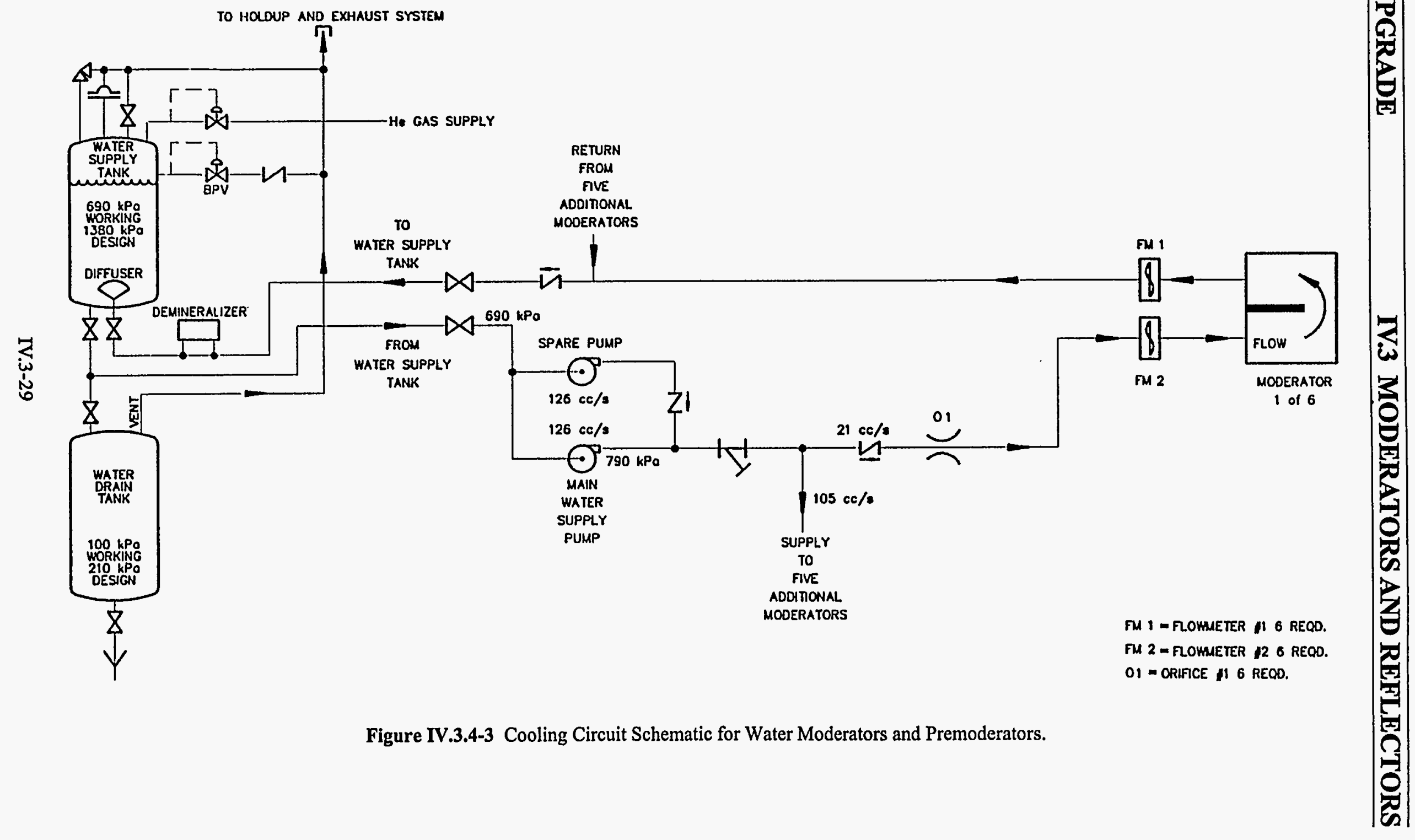




\subsubsection{Liquid Methane Moderators}

Each target station can have several liquid methane moderators. The methane moderators operate at approximately $90 \mathrm{~K}$, while the surrounding water premoderator is at a temperature near $300 \mathrm{~K}$. The methane is contained within an aluminum box with wall thickness of $1.5 \mathrm{~mm}$. This box is suspended by spring spacers within the vacuum space separating it from the aluminum premoderator box. This geometry is shown in Figures IV.3.1-1 and IV.3.1-2:

The heat loads estimated from the neutronics calculations of Sections IV.2.2 and IV.3.2 and the thermal analyses of preliminary moderator designs provide the basis for the analysis in this section. For simple spring spacer elements made of thin aluminum sheet, the thermal conduction loss is $11 \mathrm{~W}$. Thermal radiation from the water to the methane is $13 \mathrm{~W}$, assuming an emissivity of 0.8. Gamma and neutron heating in the walls of the methane container is $40 \mathrm{~W}$. Gamma and neutron heating in the bulk methane is $200 \mathrm{~W}$. All of these values are for a moderator in the upstream position and are twice as high for a liquid methane moderator in the flux-trap position. The heat loss in the vacuum-jacketed transfer line is $25 \mathrm{~W}$. The vacuum jacket absorbs approximately $500 \mathrm{~W}$ of gamma and neutron heating over a 1-m length near the targets. This heat is absorbed and removed by the water moderator/premoderator cooling circuits. The moderator heat load of $300-600 \mathrm{~W}$ is removed by flowing methane. An allowable temperature rise of $5-10 \mathrm{~K}$ in the methane is maintained by a volumetric flow rate of $36 \mathrm{~cm} / \mathrm{sec}$. A pressure drop of $34 \mathrm{kPa}$ is required to produce this flow in a tube with 13-mm OD by $0.9-\mathrm{mm}$ wall by $80-\mathrm{m}$ length. A pressure drop of $200 \mathrm{kPa}$ is allowed to provide for flow control and metering and extra parasitic losses. Pump power of $260 \mathrm{~W}$ provides a total flow rate of $108 \mathrm{~cm}^{3} / \mathrm{sec}$ at a pump efficiency of 0.1 (the efficiency of low-flow-rate cryogenic pumps is very low).

The long-chain hydrocarbons, oils, waxes, and tars that form from chemically active methane fragments (Section IV.3.3.2) must be removed from the flowing methane as they are formed to prevent plugging. Two pebble beds acting alternately are used to continuously clean the methane. One bed is regenerated while the other bed cleans the methane. The heavy hydrocarbons plate out on all surfaces, and since the pebble beds provide a much larger surface area the heavy hydrocarbons will tend to accumulate in the bed rather than on piping or the moderator-container surface.

The methane-moderator heat loads, transfer-line heat loads, pumping power, and other parasitic heat loads total approximately $500 \mathrm{~W}$ per moderator. All of this energy is deposited in a liquid nitrogen heat sink. This results in a liquid nitrogen boil-off rate of $4 \mathrm{~cm}^{3} / \mathrm{sec}$ per moderator. Liquid nitrogen kept at an absolute pressure of $350 \mathrm{kPa}$ boils above the freezing point of methane, so the system does not freeze and plug. Figure IV.3.4-4 shows the cooling circuit. All of the cryogenic hardware, with the exception of transfer-lines, is enclosed within a superinsulated vacuum cold box $1.5 \mathrm{~m}$ high and $2 \mathrm{~m}$ in diameter. A pressure vessel which is $1 \mathrm{~m}$ high $\times 1.2 \mathrm{~m}$ in diameter and is located off-axis inside the cold-box has a design pressure rating of $690 \mathrm{kPa}$ and contains liquid nitrogen maintained at $350 \mathrm{kPa}$ and a depth of $200 \mathrm{~mm}$. A coil of stainless-steel tubing that is $13 \mathrm{~mm} \mathrm{OD} \times 20 \mathrm{~m}$ long and is immersed in the liquid nitrogen serves 


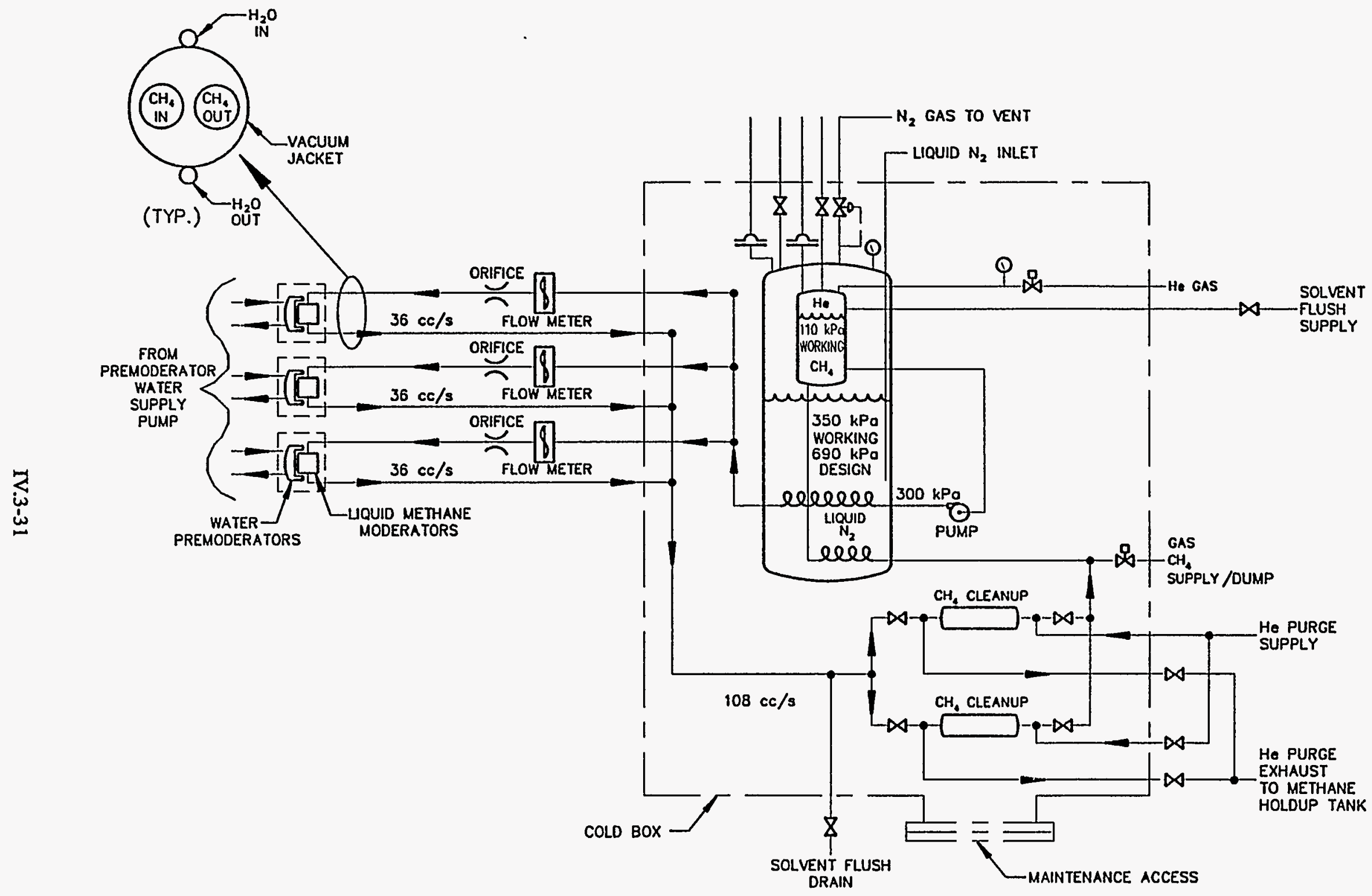

Figure IV.3.4-4 Liquid Methane Moderator Cooling Circuit Schematic, Shown for Three Moderators. 
as the methane-to-nitrogen heat exchanger. Suspended inside the liquid nitrogen vessel is another pressure vessel that is $250 \mathrm{~mm}$ in diameter $\times 300 \mathrm{~mm}$ long and contains helium-pressurized liquid methane at $110 \mathrm{kPa}$, maintained at a liquid depth of $200 \mathrm{~mm}$. A pump draws liquid methane from the suspended tank and pressurizes it to $300 \mathrm{kPa}$. The pressurized liquid methane travels through the heat exchanger to the outlet manifold, where control valves split it into equal flows for each of the liquid methane moderators. Each of the flows is continually monitored, and methane cleaners are regenerated when the pressure drop exceeds a certain level, yet to be determined.

The liquid methane moderator cooling-system components for the $30-\mathrm{Hz}$ target station are mainly located in a shielded area between Building 370 , Building 371 , and Building 368 . The liquid methane moderator cooling-system components for the $10-\mathrm{Hz}$ target station are mainly in a shielded area about $25 \mathrm{~m}$ from the Building 369 annex. The locations of these shielded areas are shown in Figure IV.1.2-1. The structure of these shielded areas and the layout of components within them are shown in Figures IV.3.4-5a, IV.3.4-5b, and IV.3.4-5c. The shielded areas for the methane moderator cooling-system components are adjacent to but separate from similar areas for the hydrogen moderator cooling-system components, as shown in the figures. All components containing methane, exclusive of the transfer piping and the moderator, are located inside a 15-cm-thick by 1.9-m-high shield wall. The shield labyrinth has no doors, and this allows natural ventilation. A lightweight lean-to roof covers the equipment but is supported separately from the walls. A $0.6-\mathrm{m}$ gap is provided between the shield and the roof for ventilation. Liquid methane flows to and from the moderators in vacuum-insulated piping in a shielded overhead pipeway, as shown in Figure IV.1.2-1.

All electrical equipment located in the methane moderator cooling-system meets the National Electrical Code, NFPA 70, requirements (or approved alternatives) for class I, division I, group B locations. The methane system design conforms to the applicable sections of the following:

- NFPA 54 National Fuel Gas Code; and

- NFPA 59A Standard for the Production, Storage, and Handling of Liquefied Natural Gas (LNG).

A 24,000-L liquid nitrogen tank outside Building 371 supplies liquid nitrogen to the methane cold box for the $30-\mathrm{Hz}$ target station. Nitrogen for the $10-\mathrm{Hz}$ target station is supplied from a $12,000-\mathrm{L}$ nitrogen tank outside the Building 369 annex.

\subsubsection{Liquid Hydrogen Moderators}

Several of the moderators on each target station are of liquid hydrogen. Each includes a water premoderator to slow neutrons and reduce the neutron thermalization and gamma ray heating of the cryogenic parts. Construction of the hydrogen moderator and water premoderator 


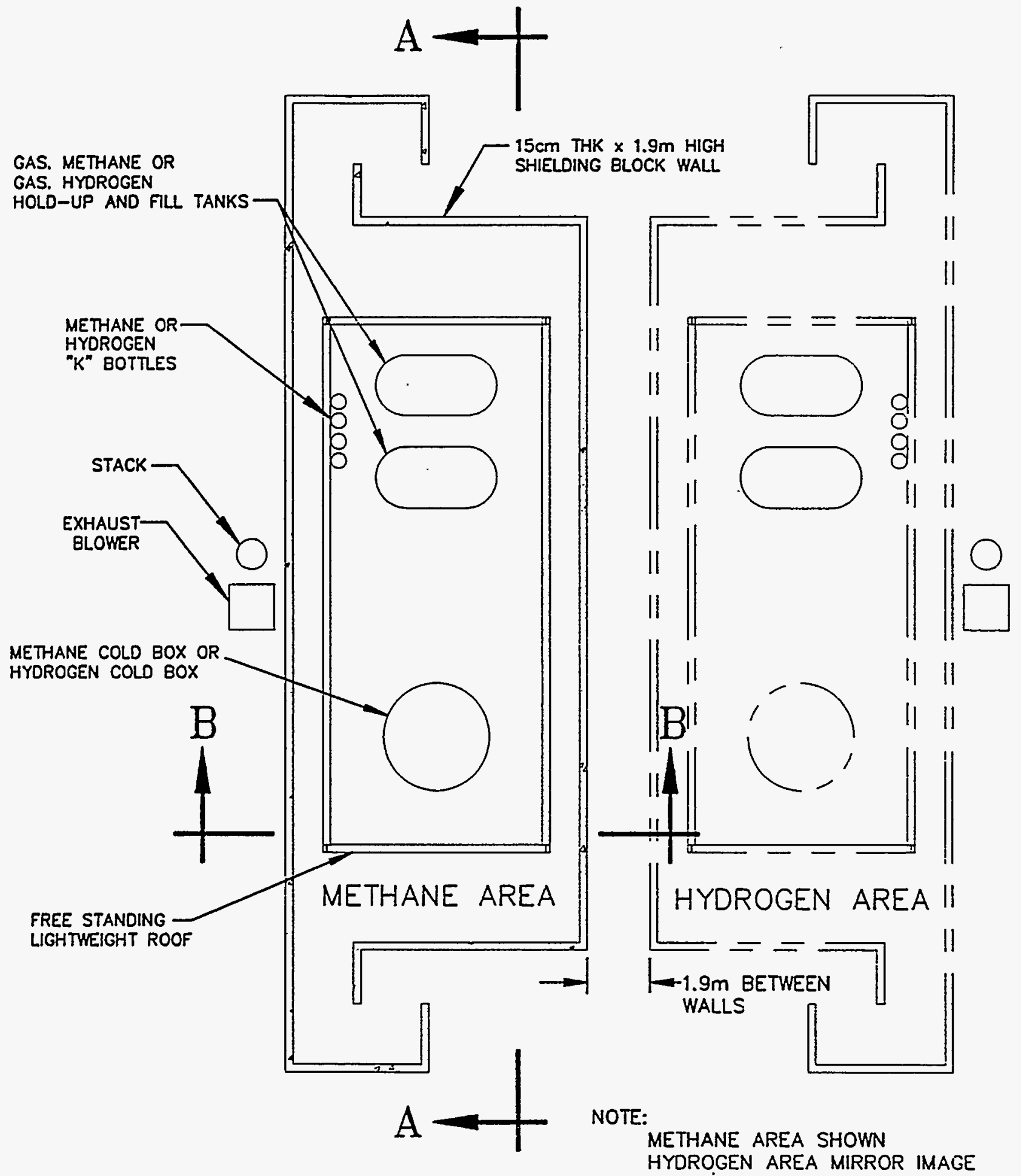

Figure IV.3.4-5a Plan View of One of the Shielded Areas Housing the Liquid Methane and Liquid Hydrogen Cooling Systems. 


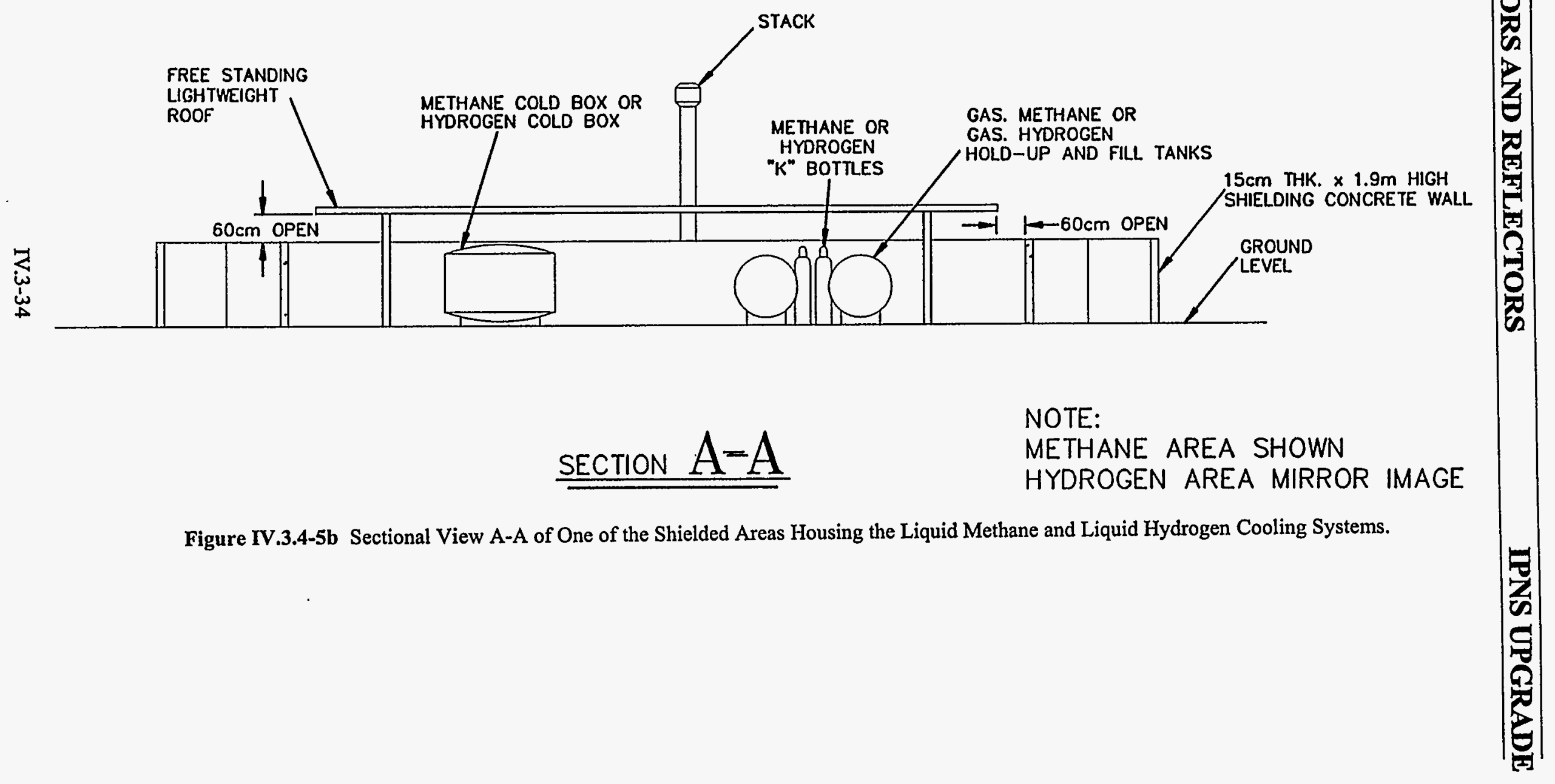




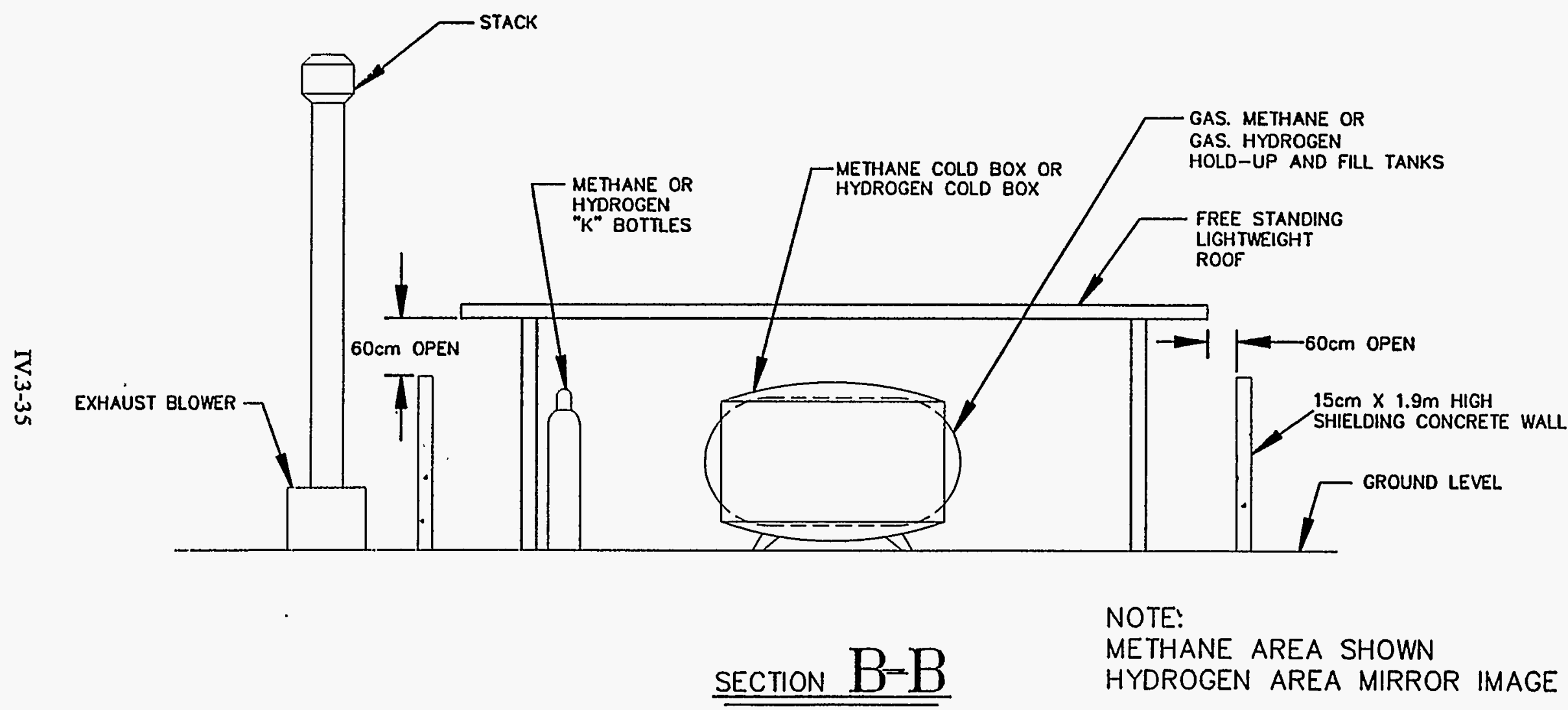

Figure IV.3.4-5c Sectional View B-B of One of the Shielded Areas Housing the Liquid Methane and Liquid Hydrogen Cooling Systems. 
containers is similar to that described for the methane and water systems; however, the dimensions are different. One of the hydrogen containers is $2 \times 10 \times 20 \mathrm{~cm}^{3}$ and is constructed of $1.5-\mathrm{mm} 6061$ aluminum.

The various heat loads are estimated using the neutronics calculations of Sections IV.2.2 and IV.3.2 and thermal analyses of the designs. The total heat load in a liquid hydrogen flux-trap moderator is $300 \mathrm{~W}$. Contributions are $19 \mathrm{~W}$ from thermal radiation, $21 \mathrm{~W}$ from conduction through the spacers, $170 \mathrm{~W}$ from gamma and neutron heating in the bulk hydrogen, and $90 \mathrm{~W}$ from gamma and neutron heating in the hydrogen container walls. The total heat load in a liquid hydrogen wing moderator is $314 \mathrm{~W}$. Contributions are $18 \mathrm{~W}$ from thermal radiation, $7 \mathrm{~W}$ from conduction through the spacers, $221 \mathrm{~W}$ from gamma and neutron heating in the bulk hydrogen, and $68 \mathrm{~W}$ from gamma and neutron heating of the container.

Liquid hydrogen is supplied to each moderator through vacuum-jacketed and superinsulated tubing. The vacuum jacket is $60 \mathrm{~mm}$ OD and has approximately $1 \mathrm{~W} / \mathrm{m}$ heat gain. Designing for a $5 \mathrm{~K}$ temperature rise in the hydrogen, from 18 to $23 \mathrm{~K}$, gives a total liquid hydrogen flow rate of $200 \mathrm{~cm}^{3} / \mathrm{sec}$. A pumping power of $70 \mathrm{~W}$ forces the hydrogen through 13-mm OD lines. Allowing for an additional 50-W heat gain in an evacuated, superinsulated cold box sets the requirement for a helium refrigeration system for two moderators to have $800 \mathrm{~W}$ capacity at $13 \mathrm{~K}$. Although heat loads in the hydrogen moderator system for the $10-\mathrm{Hz}$ target station are less, an identical helium refrigeration unit is used to cool the hydrogen moderators in the $10-\mathrm{Hz}$ target system as well.

The helium refrigerators, supplied as packaged units, use a liquid nitrogen heat sink for the cryogenic portion of the heat load. Figure IV.3.4-6 shows the process diagram for the hydrogen and helium cryogenics. A $250-\mathrm{kW}$ oil-flooded screw helium compressor with an electric motor running a direct drive at $3,600 \mathrm{rpm}$ is used. A water-cooled heat exchanger removes the compression energy from the helium. The helium travels through three stages of oil removal to reduce the oil contamination to less than 10 parts per billion (ppb). The helium is chilled to $80 \mathrm{~K}$ in a liquid-nitrogen-cooled heat exchanger and further cooled to approximately $25 \mathrm{~K}$ by counterflow exchange with helium returning from the hydrogen heat exchanger. Helium expanded in a multistage turbine to $13 \mathrm{~K}$ enters the hydrogen cooler at $14 \mathrm{~K}$. The helium flow rate and refrigeration capability vary according to the process requirements over a 3-to-1 range. Variable compressor inlet pressure and compressor bypass recycle flow accommodate this variation. This turndown capability allows the use of identical helium refrigeration systems for both the $10-\mathrm{Hz}$ and $30-\mathrm{Hz}$ target stations.

The liquid hydrogen moderator cooling-system components for the $30-\mathrm{Hz}$ target station are mainly located in a shielded area between Building 370 and Building 371 . The liquid hydrogen moderator cooling-system components for the $10-\mathrm{Hz}$ target station are mainly in a shielded area about $25 \mathrm{~m}$ from the Building 369 annex. The locations of these shielded areas are shown in Figure IV.1.2-1. The structure of these shielded areas and the layout of components within them are shown in Figures IV.3.4-5a, IV.3.4-5b, and IV.3.4-5c. The shielded areas for the hydrogen moderator cooling-system components are adjacent to but separate from similar areas for the 


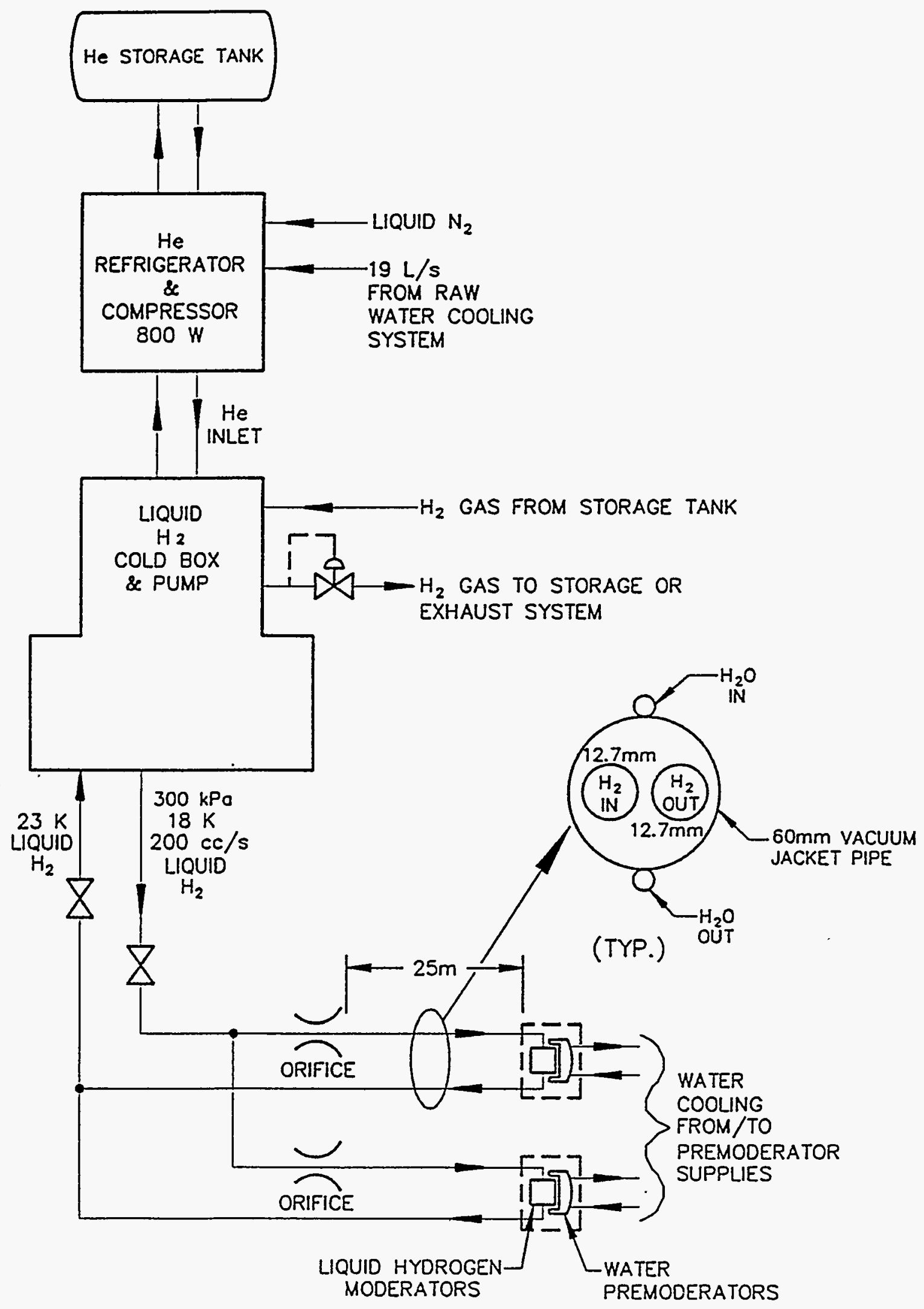

Figure IV.3.4-6 Schematic Diagram of the Liquid Hydrogen Moderator Cooling System, Shown for Two Hydrogen Moderators. 
methane moderator cooling-system components, as shown in the figures. All components containing hydrogen, exclusive of the transfer piping and the moderator, are located inside a 15-cm-thick by $1.9-\mathrm{m}$-high shield wall. The shield labyrinth has no doors, and this allows natural ventilation. A lightweight lean-to roof covers the equipment but is supported separately from the walls. A $0.6-\mathrm{m}$ gap is provided between the shield and the roof for ventilation. Liquid hydrogen flows to and from the moderators in vacuum-insulated piping in a shielded overhead pipeway, as shown in Figure IV.1.2-1. The vacuum jacket pressure relief-valve vents are monitored for hydrogen and are routed to the hydrogen vent stack. Figure IV.3.4-6 shows a typical sectional view of the vacuum-jacketed hydrogen piping.

All electrical equipment located in the hydrogen moderator cooling-system area meets the National Electrical Code, NFPA 70, requirements (or approved alternatives) for class I, division I, group B locations. The hydrogen system design conforms to the applicable sections of the following:

- 29 CFR Ch. XVII, Section No. 1910.103, "Hydrogen";

- NFPA 50A Standard for Gaseous Hydrogen Systems at Consumer Sites;

- NFPA 50B Standard for Liquefied Hydrogen Systems at Consumer Sites; and

- ANL ES\&H Manual, Chapter 4-8, "Hydrogen Safety."

A 24,000-L liquid nitrogen tank rests on a pad outside Building 371 and supplies the $30-\mathrm{Hz}$ target liquid methane and liquid hydrogen moderator cooling systems. The moderator cooling systems together require about 12,000-L storage capacity. The additional 12,000-L capacity provides general purpose liquid and gaseous nitrogen for both the $10-\mathrm{Hz}$ and $30-\mathrm{Hz}$ target areas.

The helium refrigerator and compressor system and the gaseous helium storage tank for the $30-\mathrm{Hz}$ target station are located in Building 371. The gaseous helium storage tank has a capacity of $15,500 \mathrm{~L}$ and a maximum working pressure rating of $1725 \mathrm{kPa}$. Helium gas from the compressor travels to the liquid hydrogen cold box in the shielded area outside Building 371. A vacuum pump skid outside the shielding services the liquid hydrogen cold box.

The liquid nitrogen supply for the $10-\mathrm{Hz}$ target systems is a $12,000-\mathrm{L}$ liquid storage tank located on a pad outside the annex to Building 369 . Vacuum-jacketed plumbing brings liquid from the nitrogen tank to the cold box in the shielded area outside the annex to Building 369 . This liquid nitrogen system serves only as a heat sink for the liquid methane and liquid hydrogen moderator cooling systems.

The annex to Building 369 houses the helium refrigerator and compressor system for the $10-\mathrm{Hz}$ target liquid hydrogen moderator cooling system. The 15,500-L gaseous helium storage tank is outside Building 369 . Helium gas from the compressor is routed to the liquid hydrogen cold box in the shielded area outside the Building 369 annex. The liquid hydrogen cold box is in 
the shielded area approximately $25 \mathrm{~m}$ from the Building 369 annex. Also located in this area are the hydrogen gas holdup tank and fill tank. Just outside the shielding are an exhaust fan, vent stack, and the vacuum pump skid to provide the cold box vacuum.

\subsubsection{Reflector}

Neutronic calculations described in Section IV.2.2 indicate that the heat load in the reflector is approximately $130 \mathrm{~kW}$. Figure IV.3.4-7 shows the reflector water-cooling system, which includes a 230-L stainless-steel storage-surge tank and has a working pressure of $690 \mathrm{kPa}$. The system also includes two redundant identical water pumps connected in parallel, check valves to preclude reversed flows, flowmeter, filter, water-to-water counterflow heat exchanger, demineralizer, and interconnecting piping. This water-cooling system is entirely separate from the other water-cooling systems to preclude the possible spread of beryllium contamination.

The water-supply tank has a regulated helium supply for the initial pressurization and for providing a continuous bleed flow rate of $8 \mathrm{~cm}^{3} / \mathrm{sec}$ to sweep radiolysis and activation products from the ullage space. The supply tank has an $\mathrm{H}_{2} / \mathrm{O}_{2}$ recombiner. The tank is vented through a remote valve. A pressure-relief valve and a backup burst diaphragm provide additional safety. The continuous helium bleed discharges through a back-pressure control valve. The vent gases are collected and held in the holdup tank described in Section IV.6.3. A 230-L drain tank allows local holdup of the system water inventory.

The water circuits for the top beryllium reflector and for the bottom beryllium reflector split downstream of the heat exchanger and rejoin ahead of the demineralizer. The major portion of each of these piping circuits consists of 2.54-cm-diameter stainless-steel tubing. Other piping is nominally $6.0-\mathrm{cm} \mathrm{OD}$. Tubing sections are joined by using standard flared fittings. Larger piping is welded. An 8.9-cm (3-in., schedule 10) outer pipe sheath supports and protects the four tubing runs within the target-train assembly that slides into the biological shield as shown in the inset in Figure IV.3.4-7.

The total calculated pressure drop around the water-flow circuits is $435 \mathrm{kPa}$. However, a pump-head capacity of $650 \mathrm{kPa}$ is used to permit flow control. Each of the redundant pumps requires a $3.75-\mathrm{kW}$ motor.

The pumps, tanks, demineralizer, and heat exchanger for the $10-\mathrm{Hz}$ target are located on a skid in the shielded room in Building 367. The shielded room in Building 371 contains an identical skid arrangement for the $30-\mathrm{Hz}$ target. These layouts are shown in Figures IV.3.4-1a, IV.3.4-1b, IV.3.4-2a, and IV.3.4-2b. 


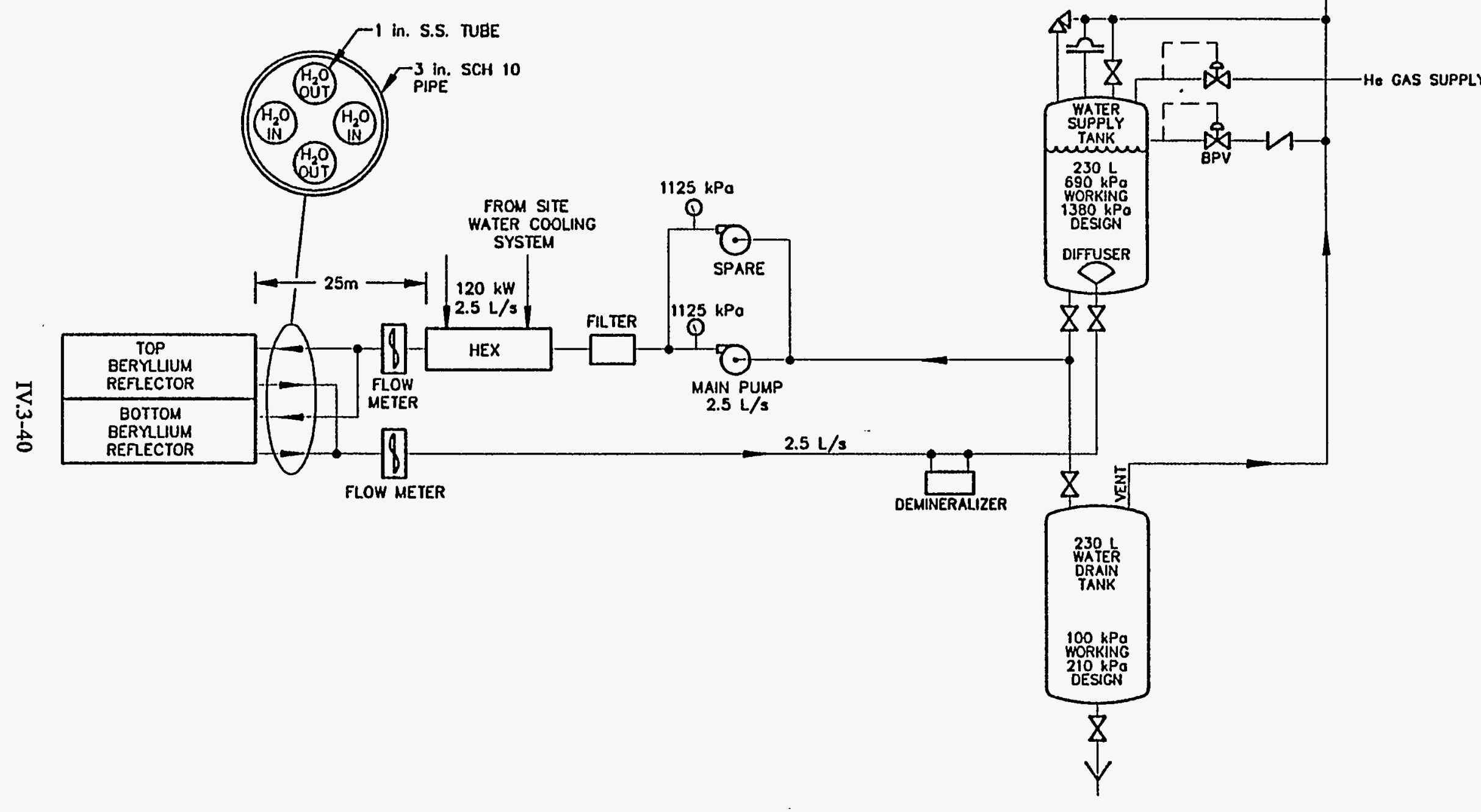

TO HOLOUP AND EXHAUST SYSTEM

Figure IV.3.4-7 Schematic Diagram of the Reflector Cooling System. 


\subsubsection{Biological Shield}

Neutronic calculations indicate that the gamma and neutron heat load in the shield region close to the reflector is approximately $130 \mathrm{~kW}$. The water-cooling system for the biological shield is shown in Figure IV.3.4-8. It is identical in configuration and capacity to the berylliumreflector cooling system, with the exception that a demineralizer is not required.

The shield adjacent to the reflector is iron. Cooling water flows in passages drilled into the iron blocks. The remainder of the water-cooling system also is constructed of iron to eliminate galvanic effects. As a further measure, buffered water is used to prevent corrosion. The 230-L water-supply tank has a helium pressurization, a $16 \mathrm{~cm}^{3} / \mathrm{sec}$ bleed purge system, and an $\mathrm{H}_{2} / \mathrm{O}_{2}$ recombiner. A liquid-level gauge is provided. Nominal tank pressurization is $690 \mathrm{kPa}$. Redundant $3.75-\mathrm{kW}$ motor pumps supply $2.5-\mathrm{L} / \mathrm{sec}$ water flow through 2 -in. Schedule 40 pipe to the parallel water-cooling passages in the shield. Orifices apportion water at equal flow rates to each cooling channel. Each of the vertical-axis cooling channels has a bayonet tube that extends down near the bottom of the cooling channel. The bayonet tube supplies water for reverse-flow back through the annular flow space between the coaxial tube and the drilled cooling channel. The cooling water is filtered before it is returned to the biological shield. A flowmeter monitors the total coolant flow rate. A counterflow water-to-water heat exchanger removes the steady heat load of approximately $130 \mathrm{~kW}$.

The tanks, pumps, filter, and heat exchanger for the 10-Hz target are mounted on a skid in the shielded area of Building 367. Building 371 houses an identical cooling system for the $30-\mathrm{Hz}$ target. These layouts are shown in Figures IV.3.4-1a, IV.3.4-1b, IV.3.4-2a, and IV.3.4-2b. Piping is routed from the cooling skids to the targets. All piping is insulated to limit heat loads on the shielded room and piping tunnel ventilation systems.

\subsubsection{Target Tank}

The target train fits within the target tank, which is described in Section IV.1.2. The target tank has its own cooling system, which is shown schematically in Figure IV.3.4-9. The system incorporates 240-L supply and drain tanks of 304SS construction, pressurized to $690 \mathrm{kPa}$. The supply tank has a $\mathrm{H}_{2} / \mathrm{O}_{2}$ recombiner. The main water supply pump, constructed of corrosion-resistant materials, draws water from the supply tank. Pump power is $0.6 \mathrm{~kW}$, which provides a differential pressure of $104 \mathrm{kPa}$. A spare pump is plumbed in parallel to the main pump.

The shielded room in Building 367 houses the target tank cooling system for the $10-\mathrm{Hz}$ target tank. The tanks, pumps, heat exchanger, and demineralizer share a common skid. Plumbing from the cooling system skid runs from Building 367 to Building 369 .

The target tank cooling system for the $30-\mathrm{Hz}$ target tank is located in the shielded room in Building 371. The tanks, pumps, heat exchanger, and demineralizer share a common skid. Plumbing from the cooling system skid runs from Building 371 to Building 370 . 


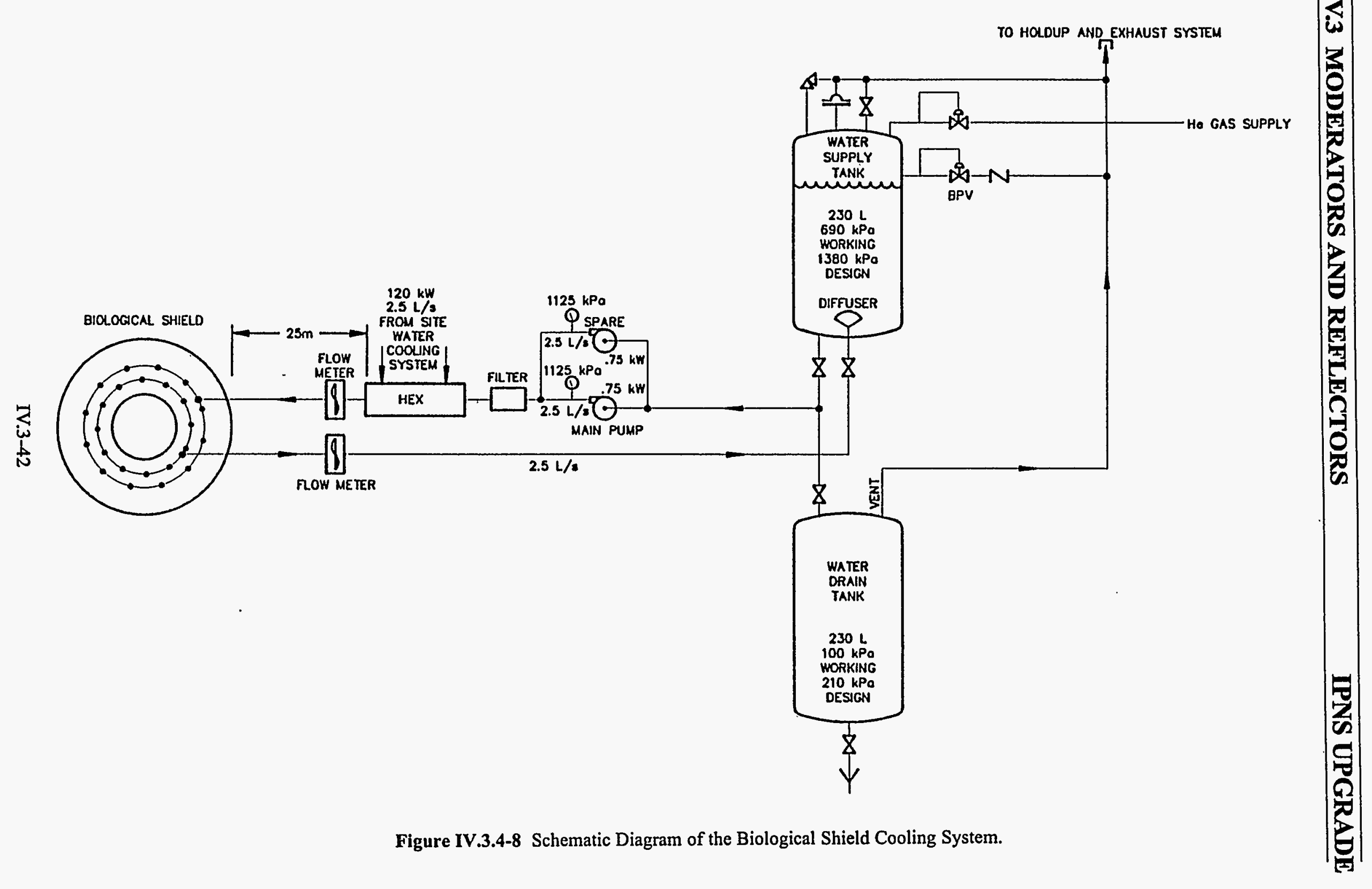




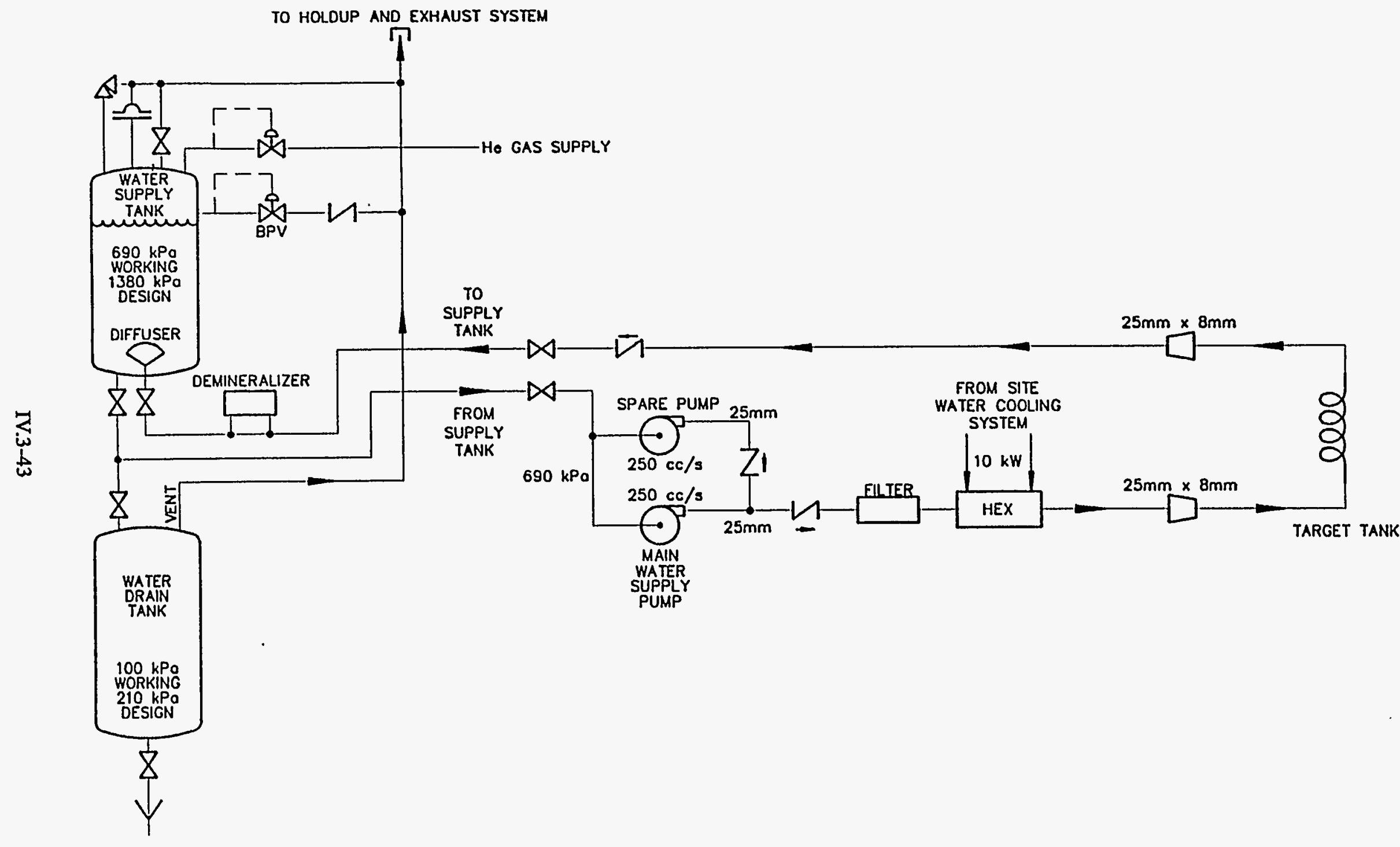

Figure IV.3.4-9 Schematic Diagram of the Target Tank Cooling System. 
All piping has insulation to reduce the heat loads and ventilation requirements.

\subsubsection{Proton HET Line Vacuum Window Insert}

Water circulates between the two walls of the proton HET line insert tube and across the window at the end, with helical guides separating the inlet and outlet flows, as shown in Figure IV.1.2-10. Calculations described in Section IV.2.2.2 (see Table IV.2.2-2) indicate that the proton beam deposits about $9 \mathrm{~kW}$ of heat in the proton beam line vacuum window. Figure IV.3.4-10 shows the proton HET line vacuum window insert cooling system. The system consists of a 240-L 304SS surge and supply tank with an $\mathrm{H}_{2} / \mathrm{O}_{2}$ recombiner, a similar drain tank, a pump, and a 10-kW heat exchanger. The pressure in the supply tank is $690 \mathrm{kPa}$. The supply tank feeds the main water supply pump. A spare pump is plumbed in parallel to the main pump. The pump differential pressure is $104 \mathrm{kPa}$. All plumbing components are stainless steel or other suitable corrosion-resistant materials.

The proton beamline vacuum window insert cooling system for the $10-\mathrm{Hz}$ target system is in the shielded room in Building 367. The tanks, pumps, heat exchanger, and demineralizer are located on a common skid. Plumbing from the cooling system skid runs from Building 367 to Building 369. The shielded room in Building 371 houses the proton HET line vacuum window insert cooling system for the $30-\mathrm{Hz}$ target system. The tanks, pumps, heat exchanger, and demineralizer are located on a common skid. Plumbing from the cooling system skid runs from Building 371 to Building 370. Component layouts are shown in Figures IV.3.4-1, IV.3.4-1a, IV.3.4-2, and IV.3.4-2a.

All system components have insulation to reduce the heat load and ventilation requirements in the shielded rooms. 


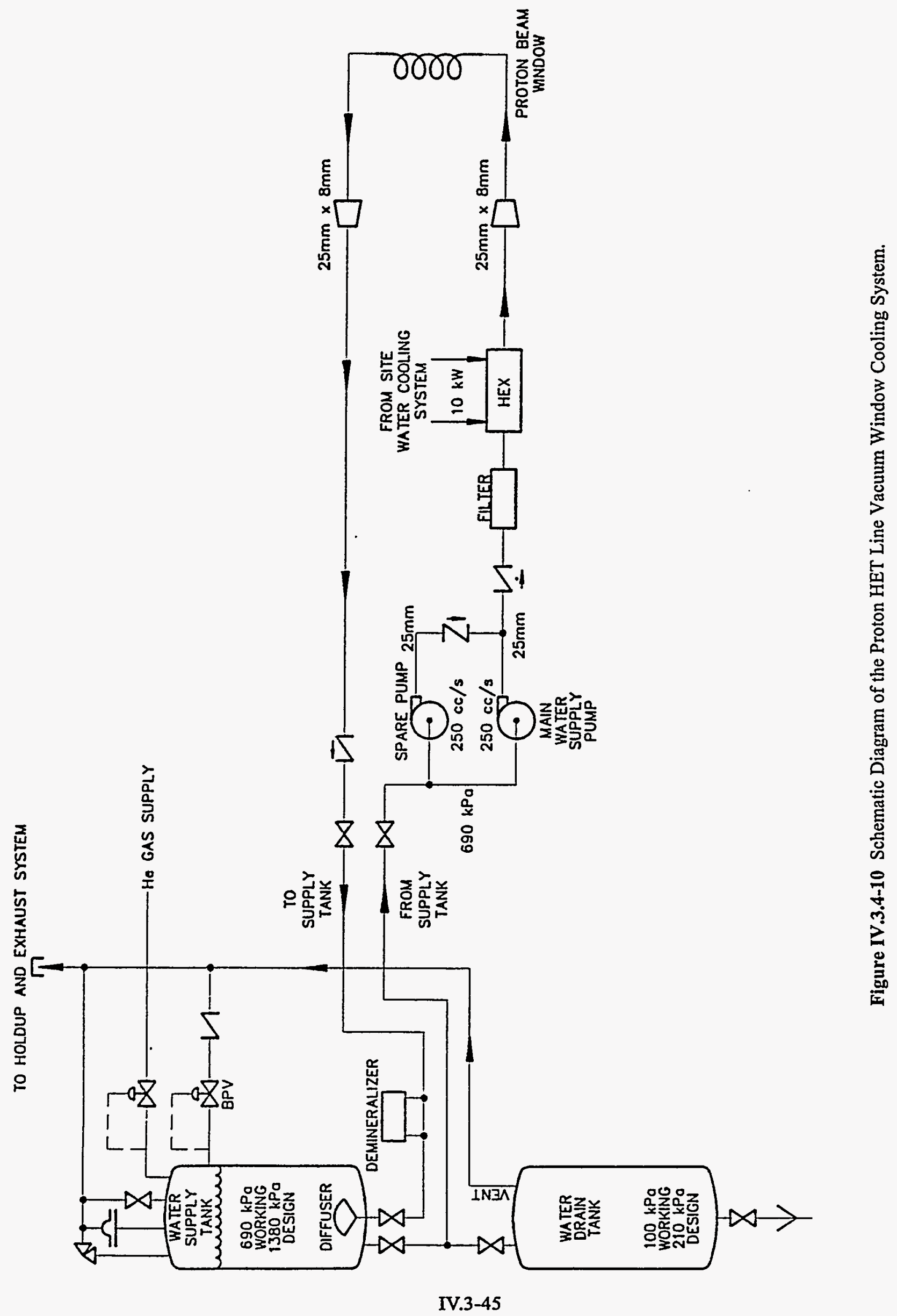




\subsection{REFERENCES}

1. J.M. Carpenter, "Pulsed spallation neutron sources for slow neutron scattering," Nucl. Instrum. Methods 145:91-113 (1977).

2. A. Carne, "Progress on the SNS target station," in Proceedings of the 6th Meeting of the International Collaboration on Advanced Neutron Sources (ICANS VI) (Argonne, Ill., June 28-July 2, 1982), Argonne National Laboratory Report No. ANL-82-80, pp. 315-326 (1982).

3. Y. Kiyanagi, N. Watanabe, and H. Iwasa, "Experimental studies on neutronic performance of coupled liquid-hydrogen moderator for pulsed spallation neutron sources," Nucl. Instrum. Methods A312:561-570 (1992).

4. J.M. Carpenter, and W.B. Yelon, "Neutron sources," in Methods of Experimental Physics, Volume 23, Neutron Scattering, Part A, edited by K. Sköld and D.L. Price, pp. 99-196 (Academic Press, New York, 1986).

5. E.P. Shabalin, "Pulsed neutron sources at DUBNA: Their past, present and future status," in Proceedings of the 11th Meeting of the International Collaboration on Advanced Neutron Sources (ICANS XI) (Tsukuba, Japan, Oct. 22-26, 1990), National Laboratory for High Energy Physics (KEK) Report No. 90-25, pp. 109-118 (1991).

6. N. Watanabe, "A design concept of target-moderator-reflector assemblies for KENS-II - A high-efficiency pulsed spallation neutron source," in Proceedings of the 11th Meeting of the International Collaboration on Advanced Neutron Sources (ICANS XI) (Tsukuba, Japan, Oct. 22-26, 1990), National Laboratory for High Energy Physics (KEK) Report No. 90-25, pp. 471-477 (1991).

7. A.M. Weinberg and E.P. Wigner, The Physical Theory of Neutron Chain Reactors, pp. 219-278 (The University of Chicago Press, Chicago, Ill., 1958).

8. J.M. Carpenter, C.W. Potts, and G.H. Lander, "Intense Pulsed Neutron Source (IPNS) at Argonne National Laboratory (ANL): A status report as of June, 1982," in Proceedings of the 6th Meeting of the International Collaboration on Advanced Neutron Sources (ICANS VI) (Argonne, Ill., June 28-July 2, 1982), Argonne National Laboratory Report No. ANL-82-80, pp. 77-101 (1983).

9. A.D. Taylor, "SNS moderator performance," in Proceedings of the 8th Meeting of the International Collaboration on Advanced Neutron Sources (ICANS VIII) (Oxford, U.K., July 8-12, 1985), Rutherford Appleton Laboratory Report No. RAL-85-110, pp. 319-328 (Chilton, U.K., 1985). 
10. G.S. Bauer et al., "Relative intensities and time structure of thermal neutron leakage from various moderator-decoupler systems for a spallation neutron source," in Proceedings of the 5th Meeting of the International Collaboration on Advanced Neutron Sources (ICANS V) (Jülich, Germany, June 22-26, 1981), Kernforschungsanlage Jülich Report No. Jül-Conf-45, pp. 417-444 (1981).

11. P. Ageron, "Les possibilités expérimentales généralités," Bulletin d'Informations Scientifiques et Techniques du Commissariat a l'Energie Atomique, No. 166 (Jan. 5-16, 1972).

12. ISIS Annual Report 1991, Vol. 1, edited by C. Wilson, Rutherford Appleton Laboratory Report No. RAL-91-050, pp. 3-4 (Chilton, U.K).

13. T.A. Broome, "The ISIS liquid methane moderator," in Proceedings of the Second International Seminar on Pulsed Advanced Neutron Sources, PANS-2 (Dubna, Russia, June 13-20, 1994) (in press).

14. J.M. Carpenter and T.L. Scott, Argonne National Laboratory, 1994 (unpublished information).

15. B. Boardman, Spallation Neutron Source: Description of Accelerator and Target, Rutherford Appleton Laboratory Report No. RL-82-006 (Chilton, U.K., 1982).

16. A.T. Lucas, "Cryogenic moderators for ISIS," Rutherford Appleton Laboratory Report No. ISIS/TS/N7/87, Issue 2 (Chilton, U.K., Jan. 16, 1992). 


\subsection{GEOMETRY AND COMPOSITION}

\subsubsection{Overview of Components}

The IPNS Upgrade biological shields provide the primary protection for personnel from direct radiation emitted from the target regions during operation and shutdown. During the operation of the facility, high-energy protons are directed to either or both of two target stations, where the protons interact with the targets to release neutrons that are utilized in research. Interaction of neutrons and protons with the target and shield materials also results in beta and gamma radiation. During a shutdown of the facility, this radiation is emitted in various intensities due to radioactive decay. The biological shields also provide structural support for the neutron facilities. They enclose components of the target systems and protect against physical damage from external forces.

The biological shields for both target stations are shown in their respective experiment halls in Figure IV.4.1-1. Figures IV.4.1-2 and IV.4.1-3 show one of these biological shields in greater detail. The shields for both target stations are identical rectangular structures of steel and concrete weighing approximately 14,000 metric tons (15,400 tons) each. These structures effectively limit the radiation dose rate at any location in the experiment areas to acceptable levels during operation and shutdown, as is described in Section IV.4.3. Each structure contains an aluminum target tank, as described in Section IV.1.2. For structural reasons, the target tank is enclosed by a steel target tank housing, which in turn is surrounded by cut and stacked steel plate required for shielding. This assembly of steel shielding is enveloped within a steel and reinforcedconcrete shielding tank. In addition, the shielding tank is covered with at least $1 \mathrm{~m}$ of poured-inplace concrete and concrete blocks. This steel and concrete assembly is the biological shield. The entire structure rests on a slab of reinforced concrete, which is supported by steel pilings that extend into Dolomite bedrock.

There are a total of 18 gated penetrations, 9 on each of two sides, extending radially from the target tank through the biological shield. These penetrations are the beam ports through which the neutrons travel to the neutron scattering instruments, and are described in detail in Section IV.8.

\subsubsection{Steel Shielding}

The purpose of the biological shielding, shown in Figures IV.4.1-2 and IV.4.1-3, is to attenuate high-energy and fast neutrons. It is a massive structure consisting of steel plate cut to fit into the shielding tank and around the target tank housing, beam port tubes, and other tubes and devices. Cracks and voids are filled with a dry grout consisting of steel shot or iron garnet. This grout is worked in by using a common vibration technique. Each biological shield requires 9,000 metric tons $(9,900$ tons) of steel for a geometry somewhat better optimized than that shown in the figures. 


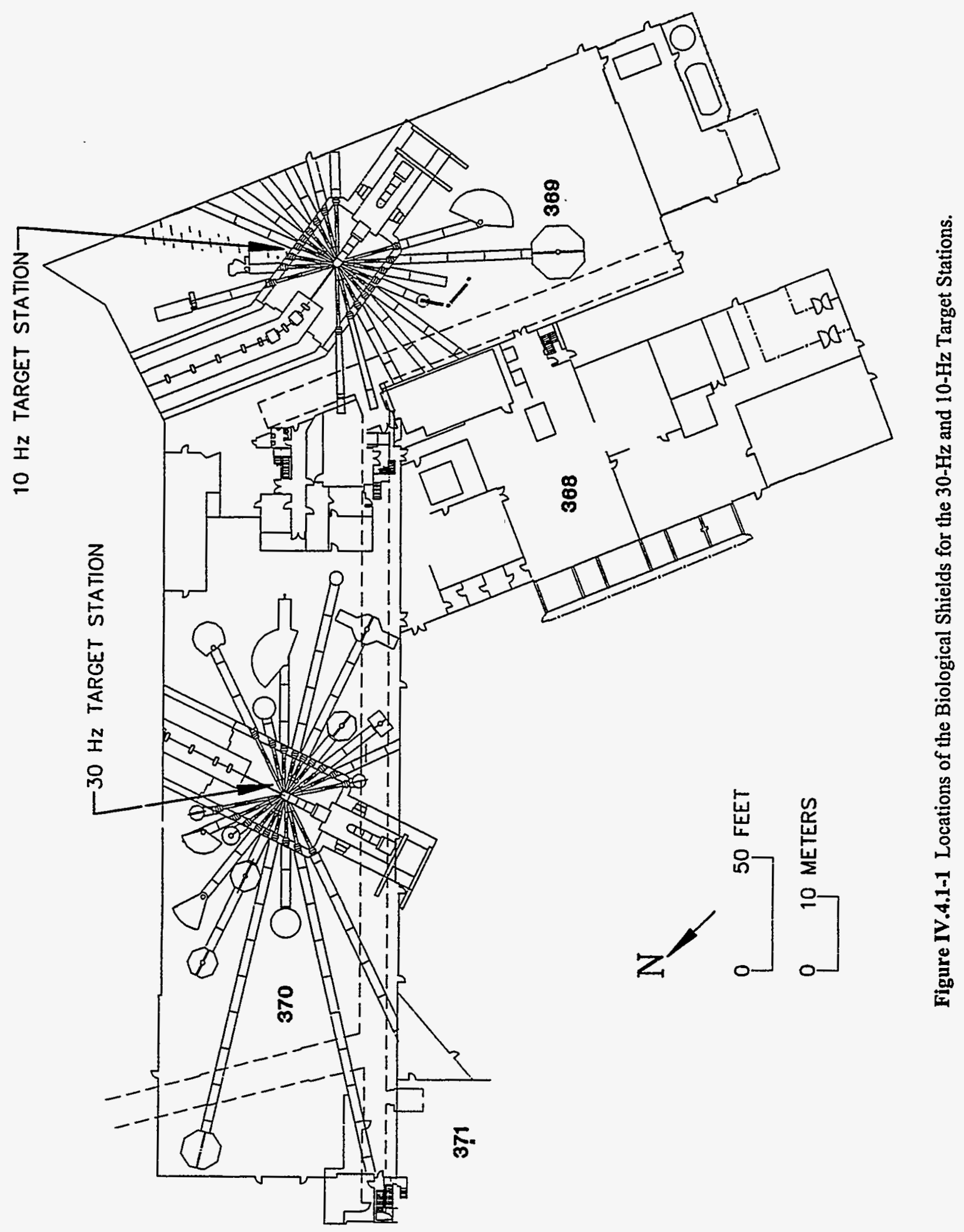




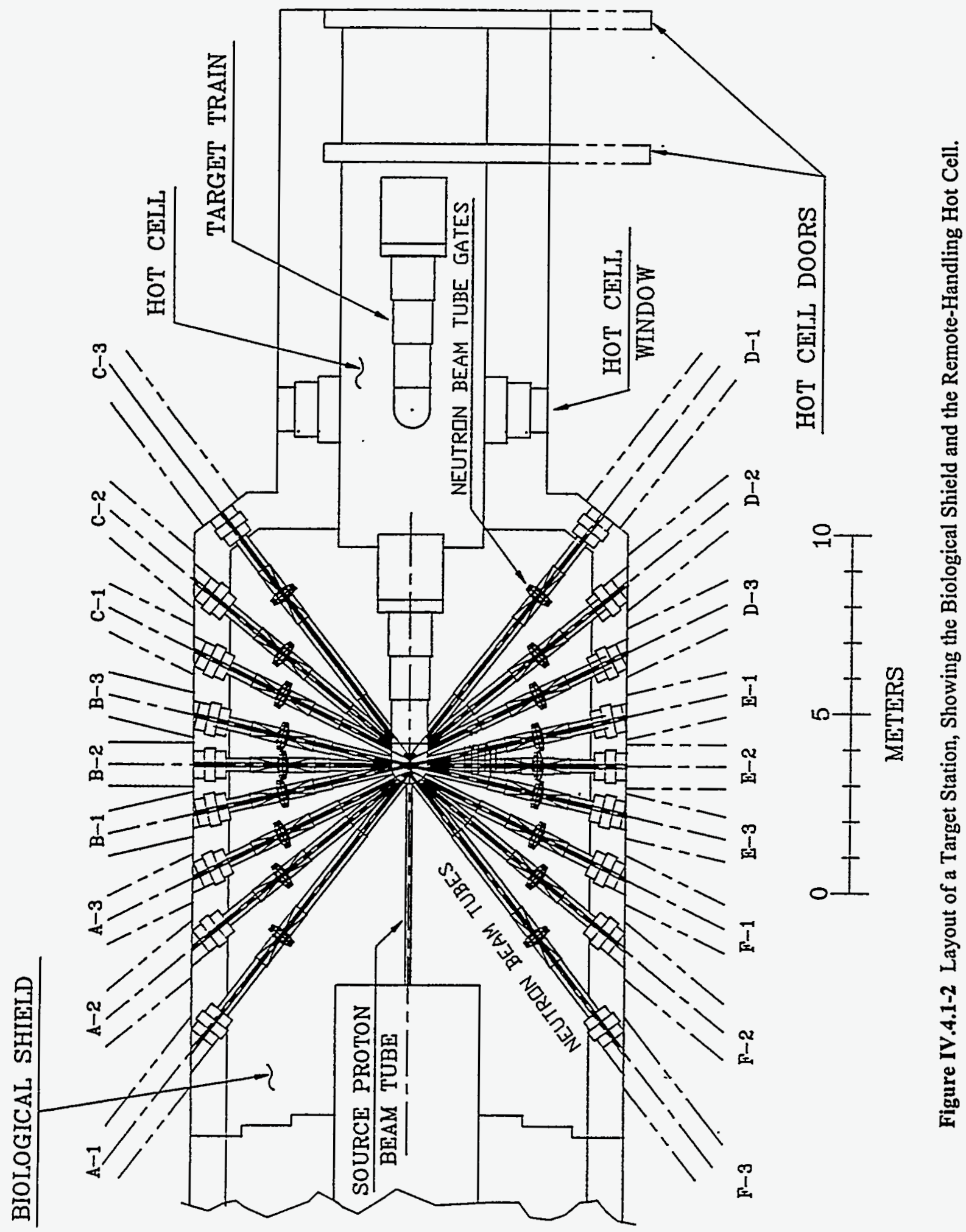




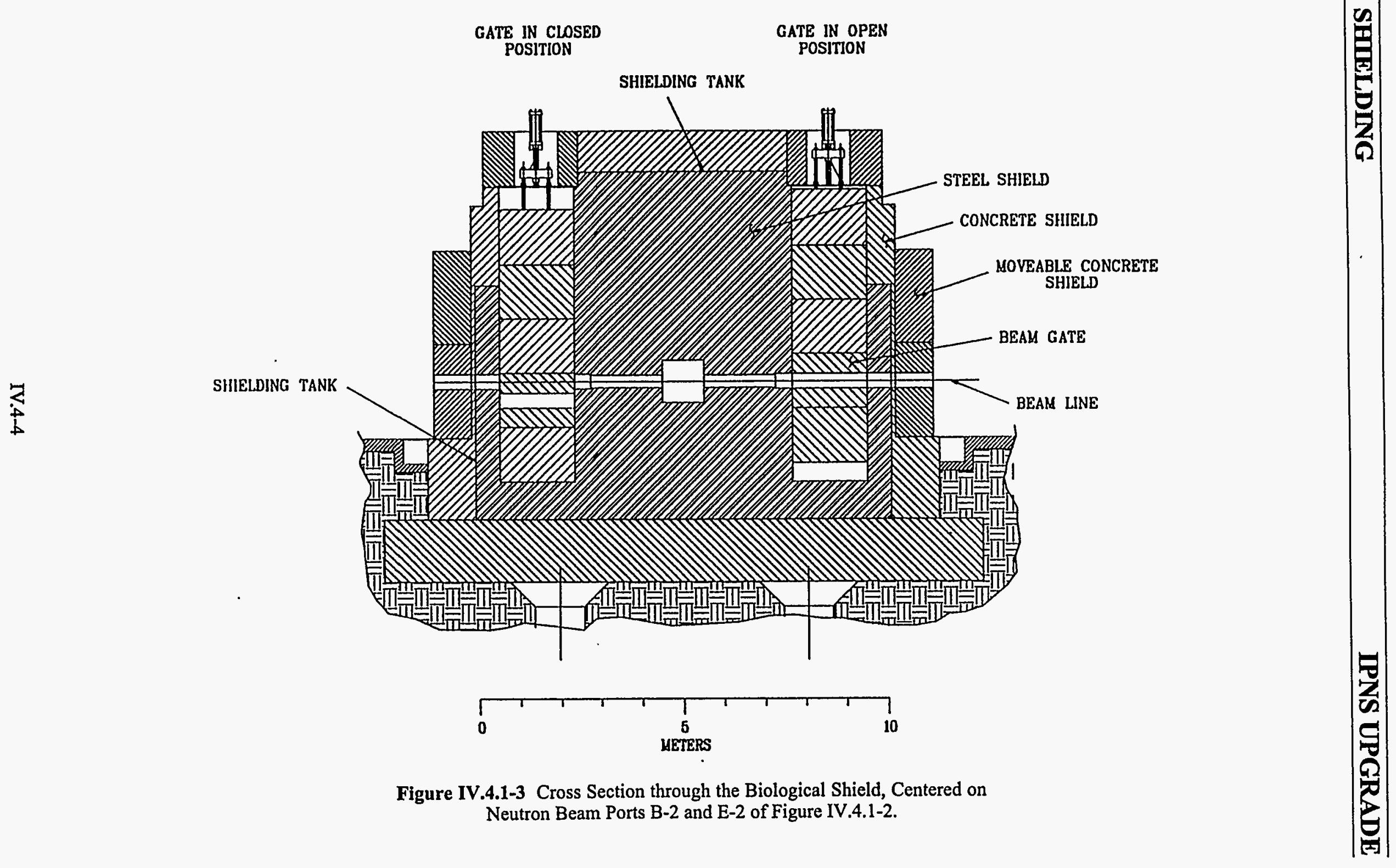


A target tank housing is located between the target tank and the stacked steel shielding in order to support the weight of the steel shield. This housing is water cooled and is made of 7.6-cm steel plate welded together to conform to the target tank shape within a tolerance of $6 \mathrm{~mm}$. Openings are made in the target tank housing for the neutron beam tubes and for the proton beam tube.

\subsubsection{Concrete Shielding}

The shield material around the outside of the shielding tank is concrete. At least $1 \mathrm{~m}$ of concrete is poured on all sides of the structure, including the top. The slab that supports the structure is at least $2 \mathrm{~m}$ thick to provide adequate shielding. Custom-made removable concrete blocks are used in the areas on the shielding tank where the neutron beams exit the structure, as discussed in Section IV.8.

\subsubsection{Hot Cell}

The hot cell, shown in Figure IV.4.1-2, is a rectangular sealed enclosure having dimensions of approximately $6.1 \mathrm{~m} \times 7.6 \mathrm{~m} \times 10.7 \mathrm{~m}$. It is located at the end of the biological shield and is composed of $2 \mathrm{~m}$ thick steel-reinforced concrete. The primary purpose of the hot cell is to provide for safe remote handling and maintenance of the target train. The operation of the hot cell and the assembly and disassembly of the target train are described in Section IV.5.

\subsection{SUPPORT STRUCTURE}

The large size of the IPNS Upgrade biological shields and their locations inside two existing buildings impose some limitations on the equipment and methods to be used for the installation of the foundation system. The requirements of the facility call for steel piles driven to bedrock some 30 to $33 \mathrm{~m}$ below existing grade.

The foundation system is illustrated in Figure IV.4.2-1. The existing concrete floor is saw-cut along the required perimeter and disposed of in an off-site landfill. The underlying material is excavated to the required depth and similarly disposed of. Any facility that is affected by the construction activities is rerouted in order to minimize disruption of those facilities to their users.

The foundation system under the biological shield structure consists of single steel piles on a square grid. The overhead clearance limitations inside the buildings dictate that each pile be driven in segments shorter than the standard mill lengths. Each segment is welded to the preceding one, and the assembly is driven into the ground. The process is repeated until the pile 


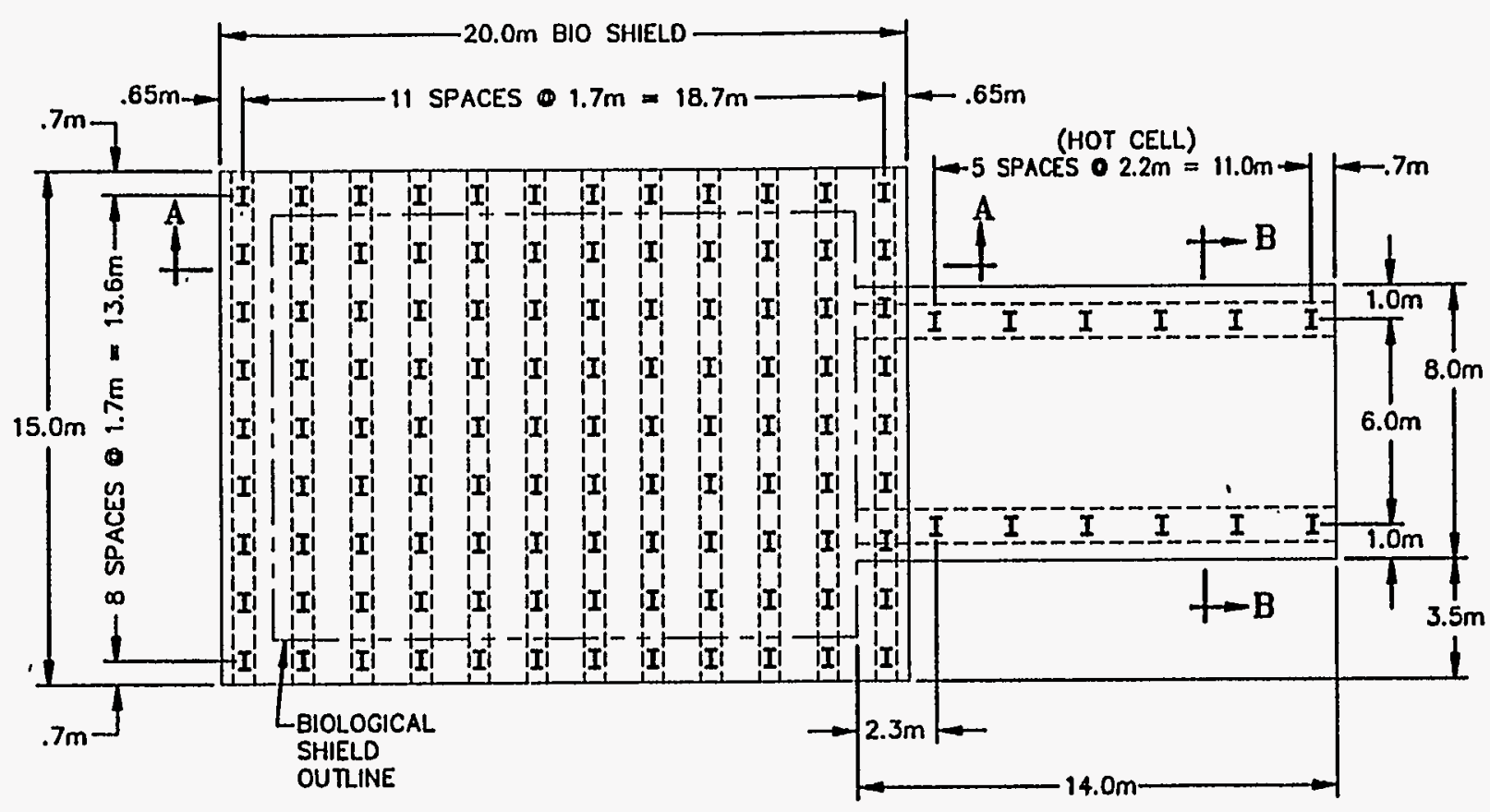

FOUNDATION PLAN

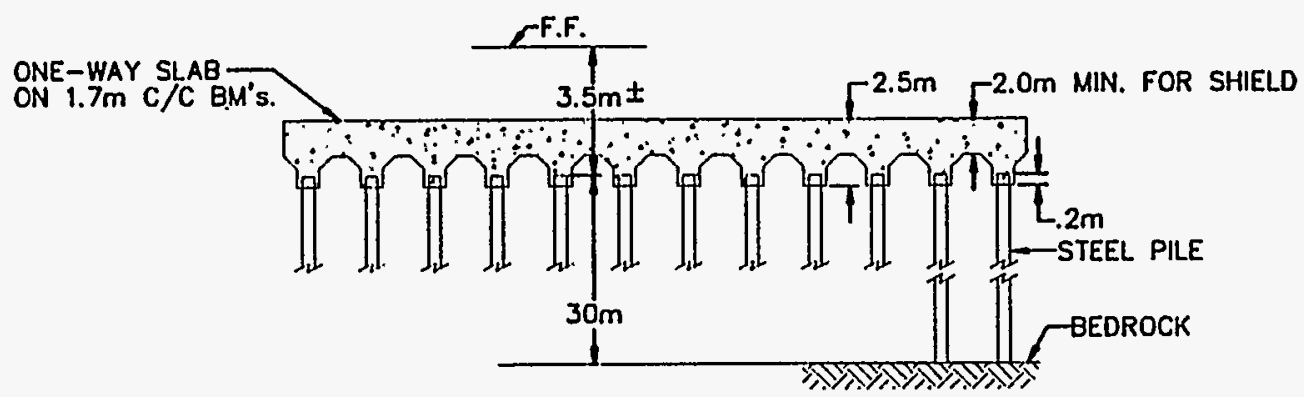

LONGITUDINAL SECTION A-A THROUGH BIO-SHIELD FOUNDATION

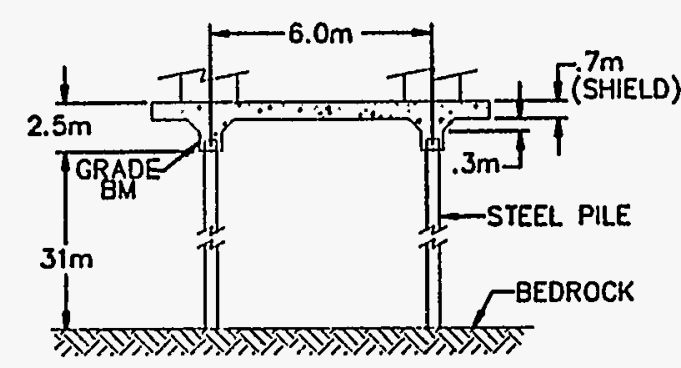

CROSS-SECTION B-B THROUGH HOT CELL FOUNDATION

Figure IV.4.2-1 Foundation Plan and Section Views. 
tip reaches bedrock, approximately 30 to $33 \mathrm{~m}$ below existing grade. The pile tops are then leveled and tied together in rows by cap beams, over which a structural slab is constructed. The slab thickness is $2 \mathrm{~m}$ to provide shielding. The load-bearing capacity of this structure is $64,000 \mathrm{~kg} / \mathrm{m}^{2}$, which is well above the loading from the biological shield.

The hot cell foundation is similarly constructed, except that two grids of piles are installed. The hot cell floor slab is tied to the biological shield floor slab structure with keys and shear-reinforcing steel to minimize any differential movement between the two structures. The slab thickness is at least $61 \mathrm{~cm}$ to provide shielding.

\subsection{SHIELDING EFFECTIVENESS}

\subsubsection{Design Objectives}

The biological shield complies with the design standards contained in Article 128 of DOE/EH-0256T. ${ }^{1}$ The design criterion is that the dose for an individual worker is less than $500 \mathrm{mrem} / \mathrm{yr}(5 \mathrm{mSv} / \mathrm{yr})$ and "As Low As Reasonably Achievable" (ALARA). The major contribution to the dose outside the main shield is expected to be due to neutrons, so the recommended neutron quality factor has been doubled for design purposes. The basis of the shielding design is a dose rate of $250 \mathrm{mrem} / \mathrm{yr}(2.5 \mathrm{mSv} / \mathrm{yr})$, conservatively augmented to ensure that radiation doses are ALARA. This augmentation accounts for such factors as differences between calculated and measured attenuations, local radiation leakage due to shield penetrations, and potential skyshine contributions at other on-site facilities. Personnel radiation exposures are maintained well below regulatory dose limits and reflect additional conservatism in that workers' exposure represents at maximum about 2000 hours per year.

\subsubsection{Shielding Parameters}

The shielding achieves the design goal at most accessible points on the outside of the biological shield during normal operations. To achieve a dose rate of $2.5 \mathrm{mSv} / \mathrm{yr}(250 \mathrm{mrem} / \mathrm{yr})$, the average dose rate is $<1.25 \mu \mathrm{Sv} / \mathrm{h}(<0.125 \mathrm{mrem} / \mathrm{h})$, based on an occupational year of $2000 \mathrm{~h}$. The calculations are based on a $500-\mu \mathrm{A}, 2.2-\mathrm{GeV}$ proton current. Iron shielding, backed up by concrete to attenuate the low-energy-neutron component, is the main shield material. At $90^{\circ}$ to the target, the thickness is nominally $6 \mathrm{~m}$, consisting of $5 \mathrm{~m}$ of iron and $1 \mathrm{~m}$ of concrete. 


\subsubsection{Shielding Methodology}

The shield effectiveness is determined by using the modified Moyer model approximation of Tesch and Zazula. ${ }^{2}$ The modified expression is

$$
H=\frac{H_{0} E_{p}^{0.8} e^{-d \rho / \lambda}}{r^{2}},
$$

where $H$ is the maximum dose equivalent per incident proton of energy $E_{p}(\mathrm{GeV}), \lambda$ is the attenuation coefficient for neutrons, $\rho$ is the shield density, $d$ is the shield thickness, and $r$ is the transverse distance to the beam. The suggested values for the other parameters for $E_{p}=2.2 \mathrm{GeV}$ with an iron shield and an iron target are $\mathrm{H}_{0} \mathrm{E}_{\mathrm{p}}{ }^{0.8}=4 \times 10^{-14} \mathrm{~Sv}-\mathrm{m}^{2} /$ proton (the dose per proton at $1 \mathrm{~m}$ ) and $\lambda=180 \mathrm{~g} / \mathrm{cm}^{2}$. Taking the ratio of total neutron production in tungsten to that in iron as 2.8 , the expected dose rate at $\mathrm{r}=6.3 \mathrm{~m}$ through a lateral iron shield $(5.0 \mathrm{~m})$ is then

$$
\dot{\mathrm{H}}(\mathrm{Fe})=\frac{2.364 \times 10^{11} \mathrm{e}^{-\mathrm{dp} / 180}}{\mathrm{r}^{2}}=19 \mu \mathrm{Sv} / \mathrm{h}(1.9 \mathrm{mrem} / \mathrm{h}) .
$$

The iron is backed up by $1 \mathrm{~m}$ of concrete to attenuate neutrons of approximately $25 \mathrm{keV}$ and $125 \mathrm{keV}$ that stream through the iron. The $1 \mathrm{~m}$ of concrete represents about eight attenuation lengths for the low-energy neutrons and effectively removes this component. The high-energy neutron component is further reduced by the $1 \mathrm{~m}$ of concrete according to

$$
\dot{\mathrm{H}}_{\text {Total }}=\dot{\mathrm{H}}(\mathrm{Fe}) \mathrm{e}^{-235 / 107}=19 \times 0.111=2.1 \mu \mathrm{Sv} / \mathrm{h}(0.21 \mathrm{mrem} / \mathrm{h}) .
$$

Here, the $107 \mathrm{~g} / \mathrm{cm}^{2}$ is the attenuation coefficient for the high-energy neutron component in concrete. The total dose rate exceeds the design goal of $1.25 \mu \mathrm{Sv} / \mathrm{h}(0.125 \mathrm{mrem} / \mathrm{h})$, allowing only about 24 hours of access per week at the face of the biological shield in the $90^{\circ}$ direction from the beam. This is administratively regulated.

\subsection{REFERENCES}

1. Radiological Control Manual, U.S. Department of Energy Report No. DOE/EH-0256T, rev. 1 (Washington, D.C., April 1994).

2. K. Tesch and J.M. Zazula, "Shielding properties of iron at high energy proton accelerators studied by a Monte Carlo code," Nucl. Instrum. Methods A300:179-187 (1991). 


\subsection{HOT CELL}

The target train can be withdrawn from its normal operating position in the target tank into a hot cell for servicing. To facilitate removal, the target train rests on a platform that also supports a stepped steel and concrete shield block. The entire train and shield block assembly moves on wheels between the operating position and the service position. Figure IV.5.1-1 indicates this arrangement, with the target train and shield shown in the operating position.

All of the components of the target-train assembly can be removed for exchange or servicing by means of remote-handling equipment in this hot cell, which is similar to the one in use for the ISIS target station. 1-3 $^{-3}$ The cell is attached to the downstream end of the biological shield, as shown in Figure IV.4.1-2. Two shielding doors provide an air-lock cavity in line behind the remote-handling cell. The air-lock cavity maintains the desired conditions within the remotehandling cell during transfer operations in and out of the cell. Two viewing ports are provided on opposite sides of the 1.7-m-thick shield walls. These 1-m-square, stepped windows are made of radiation attenuating lead glass, and are located in the same plane as the target-reflector sections. Panning television cameras are used to view all other sections of the remote-handling cell and the air-lock cell. Two remote manipulators, one located in the midplane of each window, have gripping capabilities as well as adapters for various tools such as cutoff saws for in-cell work. A third manipulator is located at the downstream end of the hot cell. This manipulator is used to install eyebolts for hoisting the shield blocks and to move piping sections attached to moderators or target sections into the air-lock or into shielded casks. A fourth manipulator is located in the air-lock cell for transfer of activated components into shielded casks. Two traveling bridge cranes ride on rails along each side of the remote-handling cell.

Access to the hot cell area is accomplished in two steps after appropriate environmental conditions are established. The first step is to open the outer, sliding main door between the hot cell air lock and the experimental area. The second step is to close the main door and open the door between the hot cell area and the air lock. This ensures that the hot cell atmosphere does not mix with the experimental-area atmosphere.

The hot cell is purged by a ventilation and exhaust system. The pressure of the cell is kept slightly below the local barometric pressure in order to assure confinement of the hot cell environment. The atmosphere of the cell is constantly monitored for radioactive contaminants prior to exhausting to the exhaust stack, as described in Section IV.6.

\subsection{PLUMBING CONNECTIONS}

Figure IV.5.2-1 shows the target train assembly with target, moderators, piping, and ducts through the shield plug. A seal sheet completes the containment enclosure when the target train is in its normal operating position. Just outside this sheet is the plumbing cavity, which allows hands-on servicing of the piping connections. All moderator fluid lines, three of the irradiation tubes, and the reflector water cooling lines pass through the seal sheet into this plumbing cavity. 


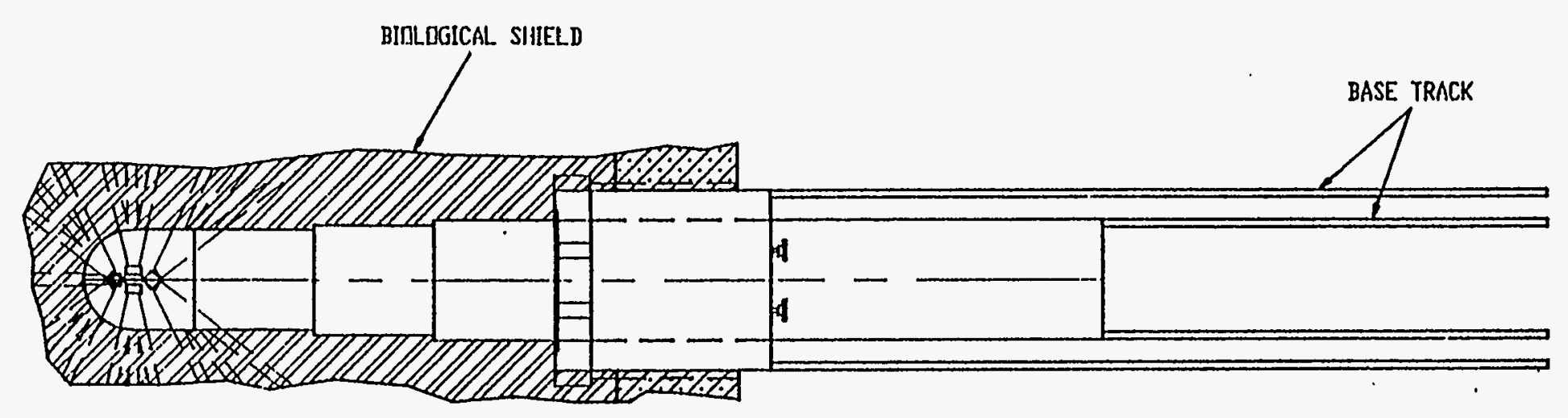

Figure IV.5.1-1 Target-Train Assembly Shown in the Operating Position. 


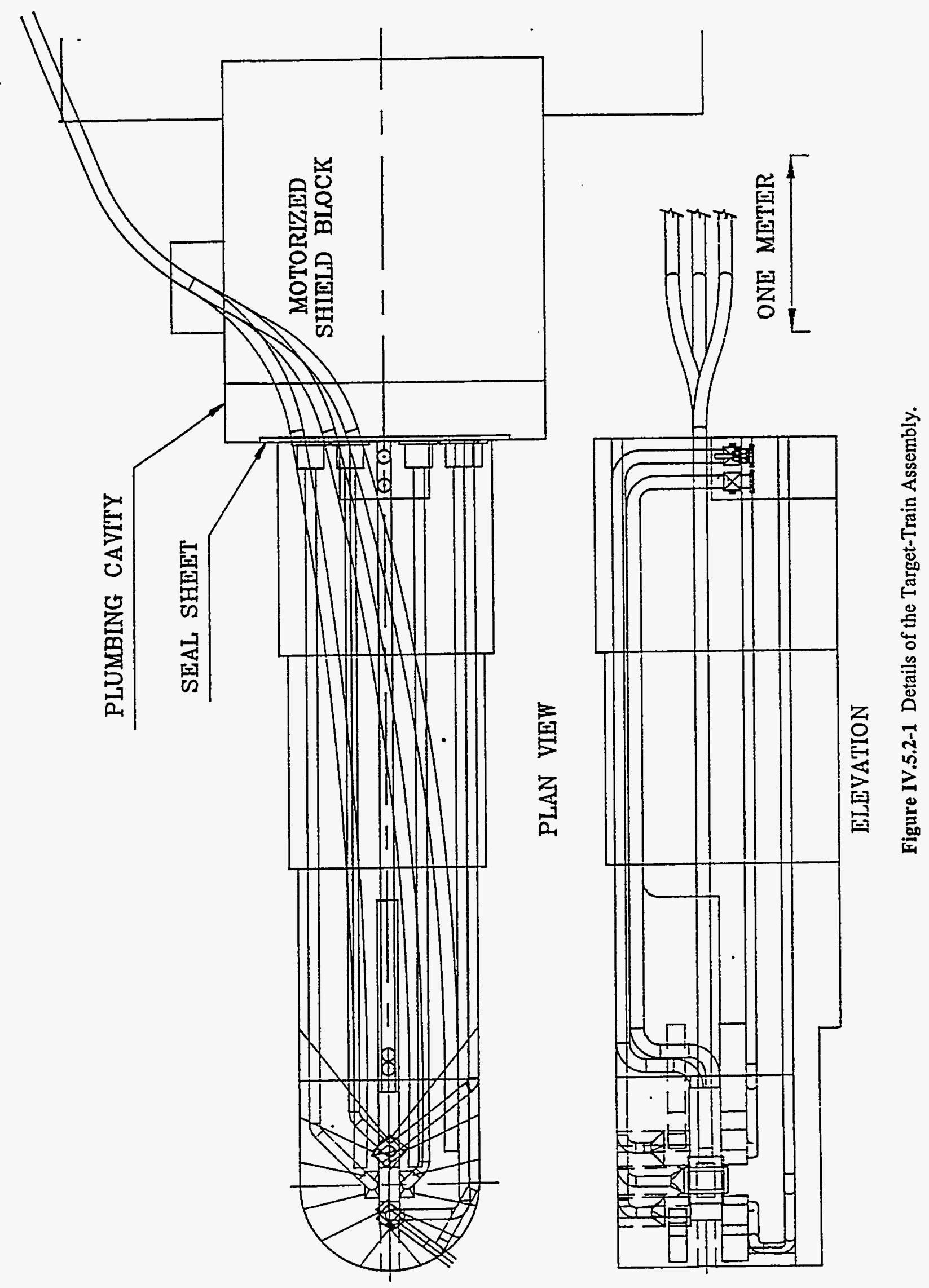

IV.5-3 
Figure IV.5.2-2 shows the arrangement of the cooling and moderator fluid connections in the plumbing cavity, and Figure IV.5.2-3 shows the seal sheet. Figure IV.5.2-4 shows the arrangement of the target cooling water connections. Figure IV.5.2-5 illustrates a typical junction box, showing the arrangement of the water cooling lines and the bayonet connections for the cryogenic lines.

\subsection{TARGET TRAIN REMOVAL AND DISASSEMBLY}

The sequence of operations necessary to access the plumbing cavity and manually disconnect lines is shown in Figure IV.5.3-1. The target sections are continuously cooled with flowing water because each can generate up to $5 \mathrm{~kW}$ of afterheat power. The plumbing cavity provides the means to manually change over from the main cooling circuits to a small-flow cooling circuit. The latter is mounted on a movable cart and is connected through flexible lines to the target cooling circuits. Small valves are connected by tee-joints to each of the target water coolant lines on the target side of the shutoff valves, as shown in Figure IV.5.2-2. These small valves are connected to the mobile-cart water cooling supply before shutting off the main water coolant supplies and returns. After the main valves are shut, water is drained from the main lines at the field joints on the opposite sides of the shutoff valves before these joints are opened.

An exploded view of the targets, moderators, reflectors, and shield blocks is shown in Figure IV.1.2-6. Following withdrawal of the entire target-train assembly into the remotehandling cell, the target and any of the moderators can be removed individually after the top shield block is dismounted. The lower wing moderators can be withdrawn horizontally. Irradiation tubes can be withdrawn backwards into the hot cell. There is room on the hot cell floor for temporary storage of components alongside the target-train assembly.

\subsection{HANDLING OF SPENT COMPONENTS}

The target sections remain very radioactive for a long time after irradiation, as shown in Table IV.2.2-6. Irradiated targets are temporarily stored in the hot-cell storage wells, before removal for permanent storage or disposal. The targets will be contained within heavy mobile shielding during transfers out of the cell.

There are eight storage wells in the hot cell, each capable of storing a target before its removal from the cell. The storage wells are fitted with a separate cooling circuit to remove decay heat. The targets are cooled at all times.

Water lines are cut near the target and disposed of in shielded containers. Less shielding is required because the water lines will not be activated to the same high levels.

Moderator containers and associated piping are not highly activated, and can be transferred out of the remote-handling cell in a manner similar to the target water lines. 

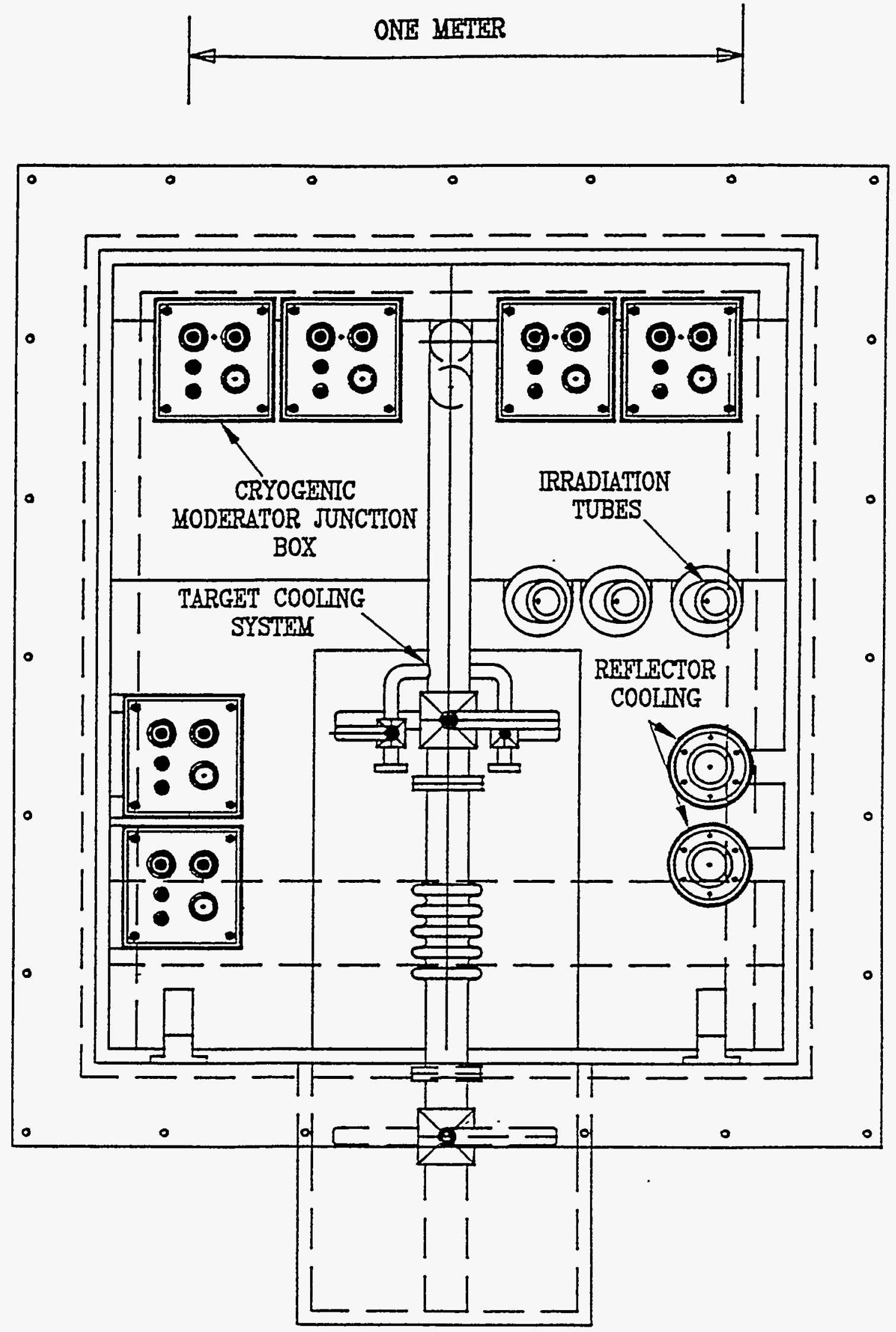

Figure IV.5.2-2 Arrangement of Target-Train Fluid Connections behind the Seal Sheet. 

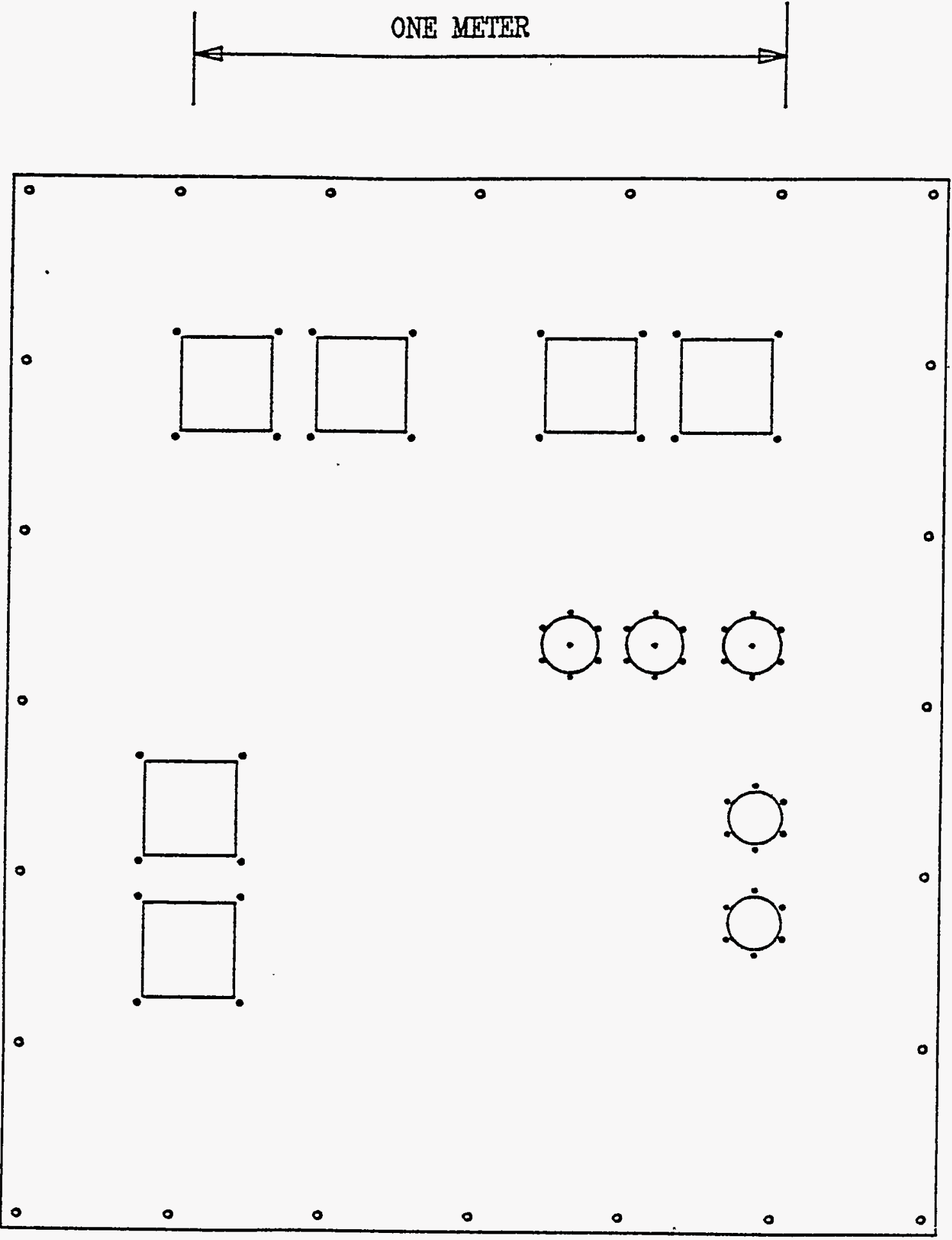

Figure IV.5.2-3 Outside View of the Target Seal Sheet. (Remove seals at each opening to release.) 

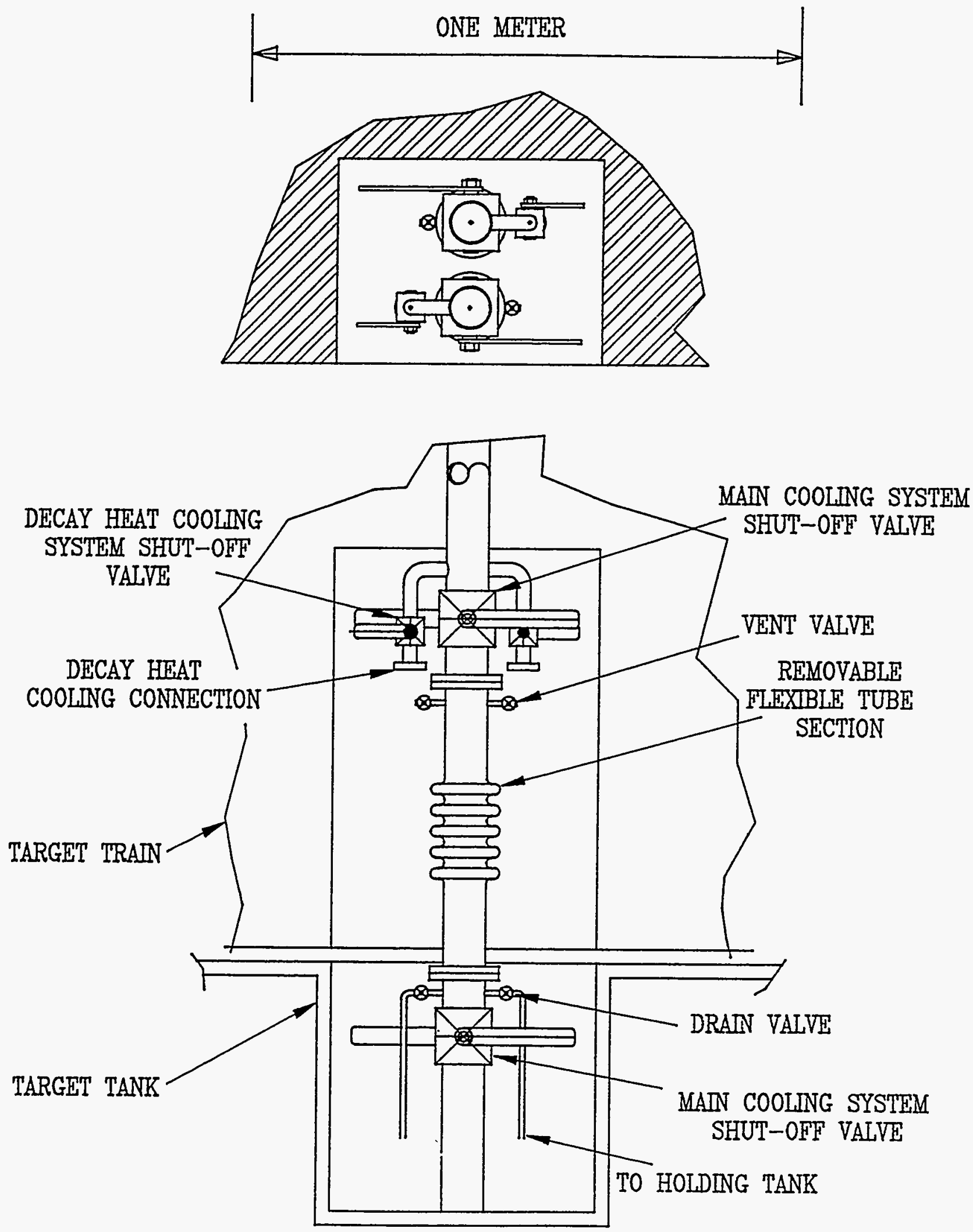

Figure IV.5.2-4 Arrangement of Target Cooling Water Connections behind the Seal Sheet. 


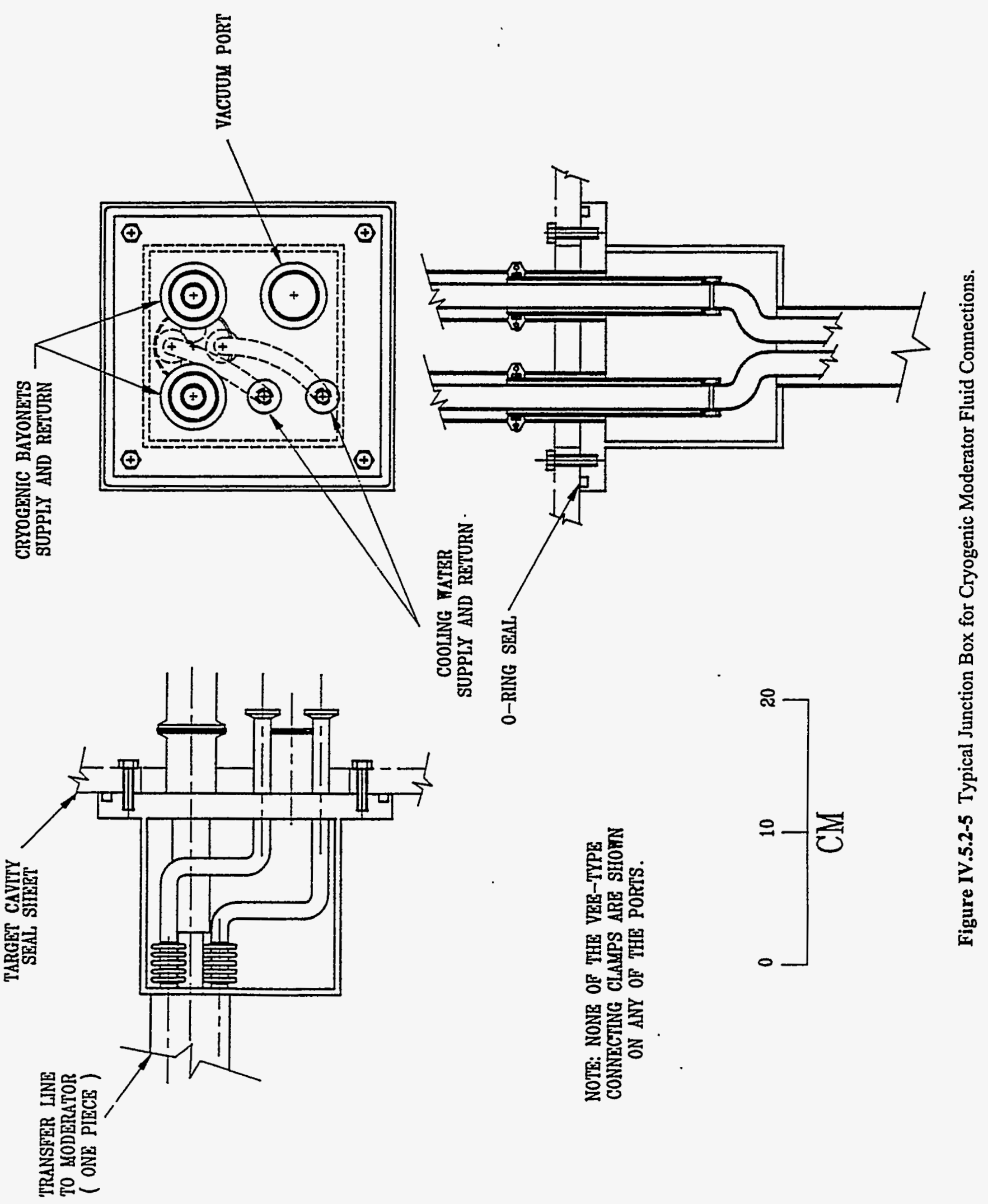



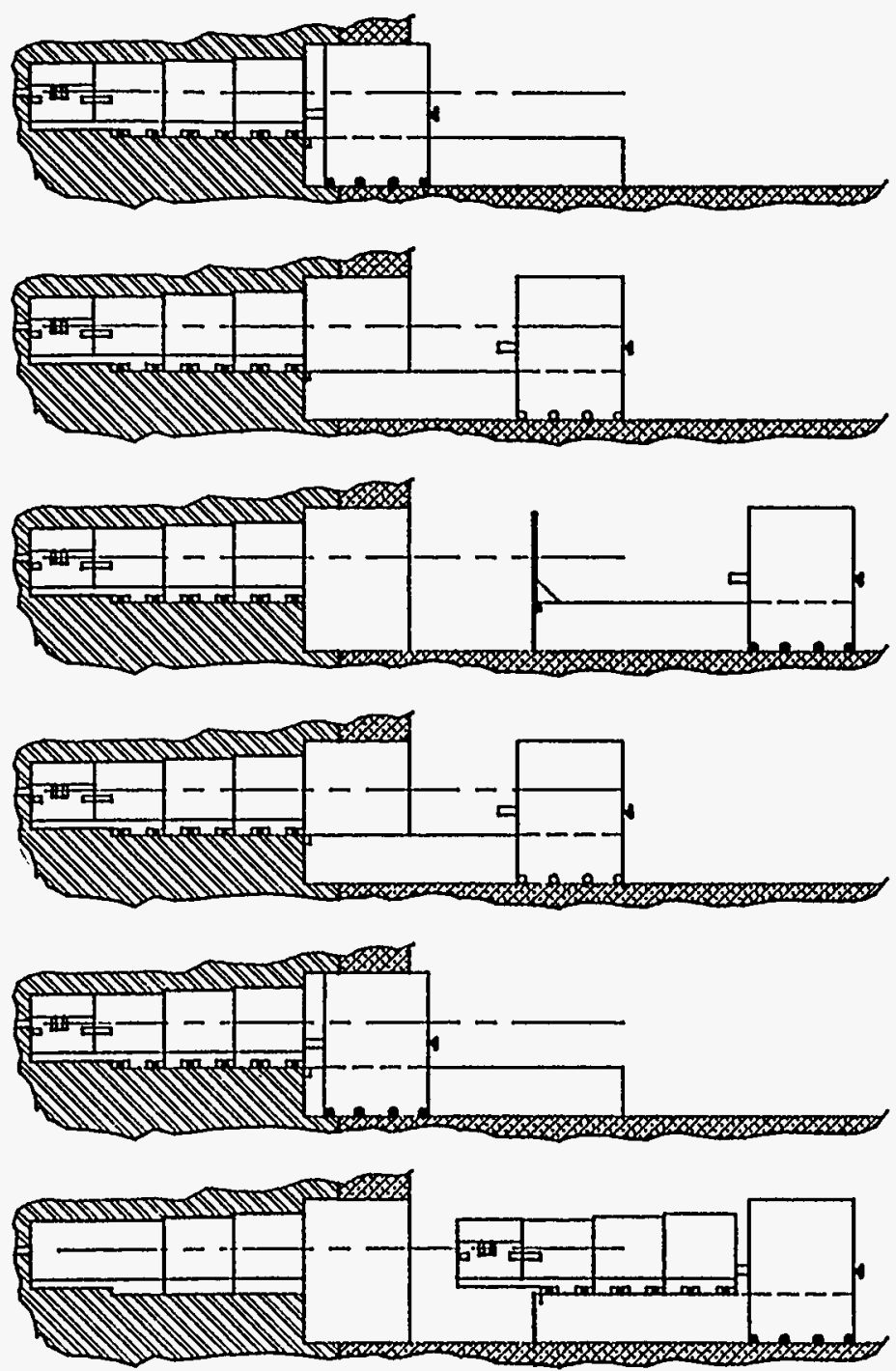

(1) In normal operation the carriage is fully to the left and anchored to the base track. The motorlzed shield is to the left and aganst the seal sheet.

(2) The first step $n$ the withdraval of the system after beam shutdown is to drive the motorized shleld block to the right end of the carriage. This allows access to the plumbing cavity and seal sheet The plumbing Jumpers between the seal sheet and the blological shield are removed ( hands on ). A stablizing fixture is mounted to the carrlage and the seal sheet. The seals between the plumbing and the seal sheet are unfastened. The seal sheet is disconnected fron the target tank.

(3) The carrlage is unlocked from the base track and locked onto the motorized shleld block. The motorized shield block is driven to the right with the seal sheet and the carriage attached. The seal sheet and the stabilizing fixture are removed from the carrlage by overhead crane.

(4) With the carrlage still locked to the motorized shield block, the carrlage and the shleld block are driven to the left. The carrlage is anchored to the base track and unlocked from the motorized shield block. The motorized shleld block' is driven to the right to clear the area. Flexible decay heat cooling lines are attached to the target assembly and flow established. The main target cooling lines are shut off and the removable flexible tube sections are drained and removed.

(5) The motorized shleld block is driven to the left until it contacts the target-moderator-reflector assembly. The the rods are fastened to the target-moderator- reflector assembly. With the carrlage still anchored to the base tracks, the motorized shleld block 15 driven to the right end of the carriage, pulling the target-moderator-reflector assembly onto the carrlage.

(6) The carriage is locked to the motorized sheld block and unlocked from the base track. The motorized shield block 15 driven to the right pulling the carriage and exposing the system.

* Left and right refer to the position in the drawng.

Figure IV.5.3-1 Sequence of Operations to Remove the Target-Train Assembly into the Remote-Handling Cell. 
The beryllium reflector pieces need to be handled safely for both radioactivity and beryllium toxicity. Radiation levels are not as high as those for the target sections. However, toxicity considerations dictate special handling procedures.

The irradiation tubes are not activated any more than the other piping leading in and out of the target area, and receive the same treatment as the other piping.

ANL does not have provisions for permanent storage of radioactive waste at its Illinois site. However, such facilities are available at Hanford, Washington. Transport of similar radioactive materials between sites is done routinely.

\subsection{REFERENCES}

1. T.A. Broome and M. Holding, "ISIS target station experience," presented at Argonne National Laboratory (Argonne, Ill., Nov. 23, 1992).

2. B. Boardman, Spallation Neutron Source: Description of Accelerator and Target, Rutherford Appleton Laboratory Report No. RL-82-006 (Chilton, U.K., March 1982).

3. B.H. Poulten, "Remote handling equipment for SNS," in Proceedings of the 6th Meeting of the International Collaboration on Advanced Neutron Sources (ICANS VI) (Argonne, Ill., June 28-July 2, 1982), Argonne National Laboratory Report No. ANL-82-80, Argonne, Ill., pp. 339-355 (1982). 


\subsection{CONTAINMENT BARRIERS}

Most of the radioactive products produced in the target are contained within the target material. The sealed target container and cooling system, described in Section IV.2, provide additional containment. Two secondary containment barriers, the target tank and the shielding tank are provided within the biological shield to prevent uncontrolled releases.

The moderator and premoderator boxes and associated piping are the primary containment for moderator and premoderator materials and their radioactive by-products. The vacuum jacketing required for insulation serves as an additional containment barrier in the cryogenic moderator systems. The target tank and the shielding tank are additional barriers against uncontrolled release of radioactive by-products.

Figure IV.1.2-7 shows the target tank, which encloses the target-reflector-moderator assembly. This tank is welded of $2.5 \mathrm{~cm}$-thick aluminum sheet. Portions of the target tank are machined thinner to provide windows for the neutrons to exit to the beam ports. The target tank is designed to sustain at least $3.4 \mathrm{kPa}$ of internal pressure and is able to withstand ordinary activities associated with moving heavy equipment. A seal sheet covers the open end of the target tank to complete the containment, as shown in Figure IV.5.2-1.

The target tank contains a helium atmosphere controlled at a positive pressure with respect to the local barometric pressure. This prevents air from entering the tank and avoids production of any of the air-associated activation products. A purge and exhaust ventilation system controls this atmosphere.

The shielding tank encloses the stacked steel portion of the biological shield, as shown in Figures IV.4.1-2 and IV.4.1-3. It is a welded helium-leak-tight container constructed of 2.5-cm-thick carbon steel plate. The shielding tank atmosphere is helium gas maintained at a slight positive pressure with respect to local barometric pressure. A purge and exhaust system controls this atmosphere. The shielding tank is designed to sustain an internal pressure of at least $3.4 \mathrm{kPa}$, and to withstand ordinary activities associated with moving heavy equipment.

The water cooling systems for the reflector and for the shield have low activity levels. Their plumbing is the primary containment for this activity. Secondary containment for the reflector system is provided by both the target tank and the shielding tank. The shield cooling water has much lower activity levels, and the shielding tank and shield cooling plumbing system provide adequate barriers against uncontrolled release of any of this activity.

All of these separate containment spaces, as well as the space inside the hot cell, have separate gas exhaust systems, discussed in Section IV.6.2. These feed into gas holdup tanks and a common HEPA filter and stack system, as discussed in Sections IV.6.3 and IV.6.4. Each of these spaces has a drain and retention system for containment of any liquids, as discussed in Section IV.6.5. 


\subsection{GAS EXHAUST SYSTEMS}

\subsubsection{Target Coolant Gases}

Figure IV.6.2-1 shows the exhaust system that handles the radioactive gases from the target cooling system. This same system also handles all gas exhausts discussed below, except those from the cryogenic moderators. Two methods are used to remove the oxygen, hydrogen and other radiolysis products generated in the target cooling water. The first method employs a heated catalyst pack, which recombines most of the hydrogen and oxygen in the water supply tank ullage space to form water. The resulting water condenses and remains within the tank. A continuous helium-gas purge of the ullage space removes the remaining gaseous products. The continuous helium bleed replenishes the ullage volume with clean gas within a time period of several hours. The purge gas is stored in a holdup tank where radioactive gaseous products are allowed to decay to safe levels before they are released through an exhaust system, as discussed in Section IV.6.3. HEPA filters capture particulate matter in the exhaust system, and the effluent is exhausted to atmosphere through a monitored stack.

\subsubsection{Moderator Gases}

The water premoderator and moderator radiolysis products are contained within the closed-circuit cooling system. The radiolysis products are handled with the other coolant gases, as discussed in Section IV.6.2.3.

The liquid hydrogen and liquid methane moderators must be periodically emptied and disconnected from their flow systems. The highly-flammable liquids are vaporized and the resulting gases are warmed to near-ambient temperatures. These gases are routed to separate holdup tanks, as discussed in Section IV.6.3. After sufficient decay time the gases are mixed with air so that the effluent mixture is nonflammable. This diluted mixture is exhausted to the atmosphere through HEPA filters and through a monitored stack.

\subsubsection{Shield, Reflector, and Other Coolant Gases}

The beryllium reflector system is separate to preclude beryllium contamination in the other cooling systems. This also permits the use of heavy water $\left(\mathrm{D}_{2} \mathrm{O}\right)$ for cooling, if desired. The shield cooling system is a buffered-water system, and is separate from the other water cooling systems. Radiolysis gases are produced at significantly lower rates in the reflector and shield than in the target cooling system. Consequently, a smaller helium purge into the ullage space of each supply tank sweeps the oxygen, hydrogen, and activation products away with the helium exhaust. The helium flow dilutes the oxygen and hydrogen gas mixture to a composition that is below combustion and detonation limits. These two gas streams are emptied into 
TO HEPA FILTERS AND STACK

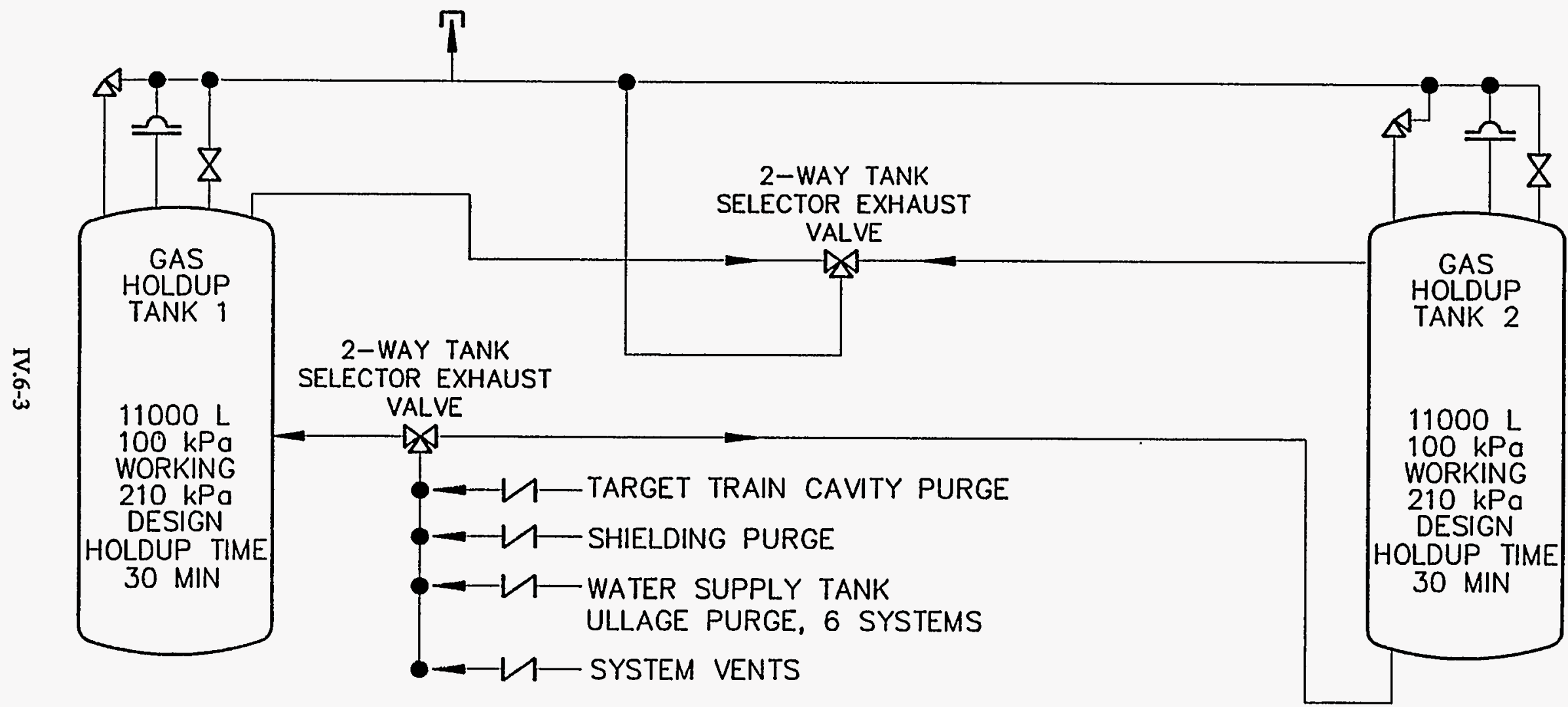

Figure IV.6.2-1 Common Holdup and Exhaust System Used for Most Gaseous Exhausts. 
the holdup tank to allow sufficient decay time for activation products, before being exhausted to atmosphere through HEPA filters and a monitored stack. This same design is used for the exhausts from the target tank cooling system and the proton HET line window cooling system.

\subsubsection{Neutron Beamline Exhaust}

The neutron beamlines that are not evacuated contain helium gas. The flowing helium purge-gas streams and the exhaust gases from the vacuum pumping systems are held for a period of time so that any radioactivity decays to a safe level. The common holdup tank system is used for this purpose.

\subsubsection{Irradiation Tube Exhaust}

The three horizontal irradiation tubes within the reflector and the fourth, vertical, irradiation tube are gas-cooled. The gases contained within the irradiation tubes are recirculated to prevent the spread of radioactive species. During operation, a hermetically sealed blower located outside the biological shield circulates gas to the ends of each tube, past the irradiation capsule, and back through the piping to a heat exchanger. Gases are exhausted from the tubes only when irradiation capsules are removed from the system. At these times, the gases are exhausted through the common holdup tank, HEPA filters, and the monitored stack. Gases from vents and pressure-relief safety valves are also exhausted through the common holdup tank, HEPA filters, and monitored stack.

\subsubsection{Purge and Exhaust Systems for Target Tank, Shielding Tank, and Hot Cell}

The purging systems for the target tank and the shielding tank consist of ventilation and exhaust systems as shown in Figure IV.6.2-2. Each space is continuously purged with at least $16 \mathrm{standard} \mathrm{cm}^{3} / \mathrm{sec}$ of helium gas, at a slight positive pressure. Each of these volumes has a separate supply and exhaust system. Two regulators operate in series to decrease the pressure to the level required. Appropriate pressure-relief devices prevent over-pressurization.

A volumetric pump employing differential pressure-control devices pressurizes the gases from the purged regions up to $100 \mathrm{kPa}$. The gases then flow to a common manifold and into the holdup tank which ensures an appropriate hold time prior to being exhausted to the atmosphere. These gases are continuously monitored for combustibles, such as hydrogen and methane.

The hot cell is maintained at a slight negative pressure to ensure that no leakage takes place from the cell into the target tank, shielding tank, or experiment area. Air through an inlet HEPA filter constantly purges the hot cell. Exiting gases from the hot cell purge system pass 


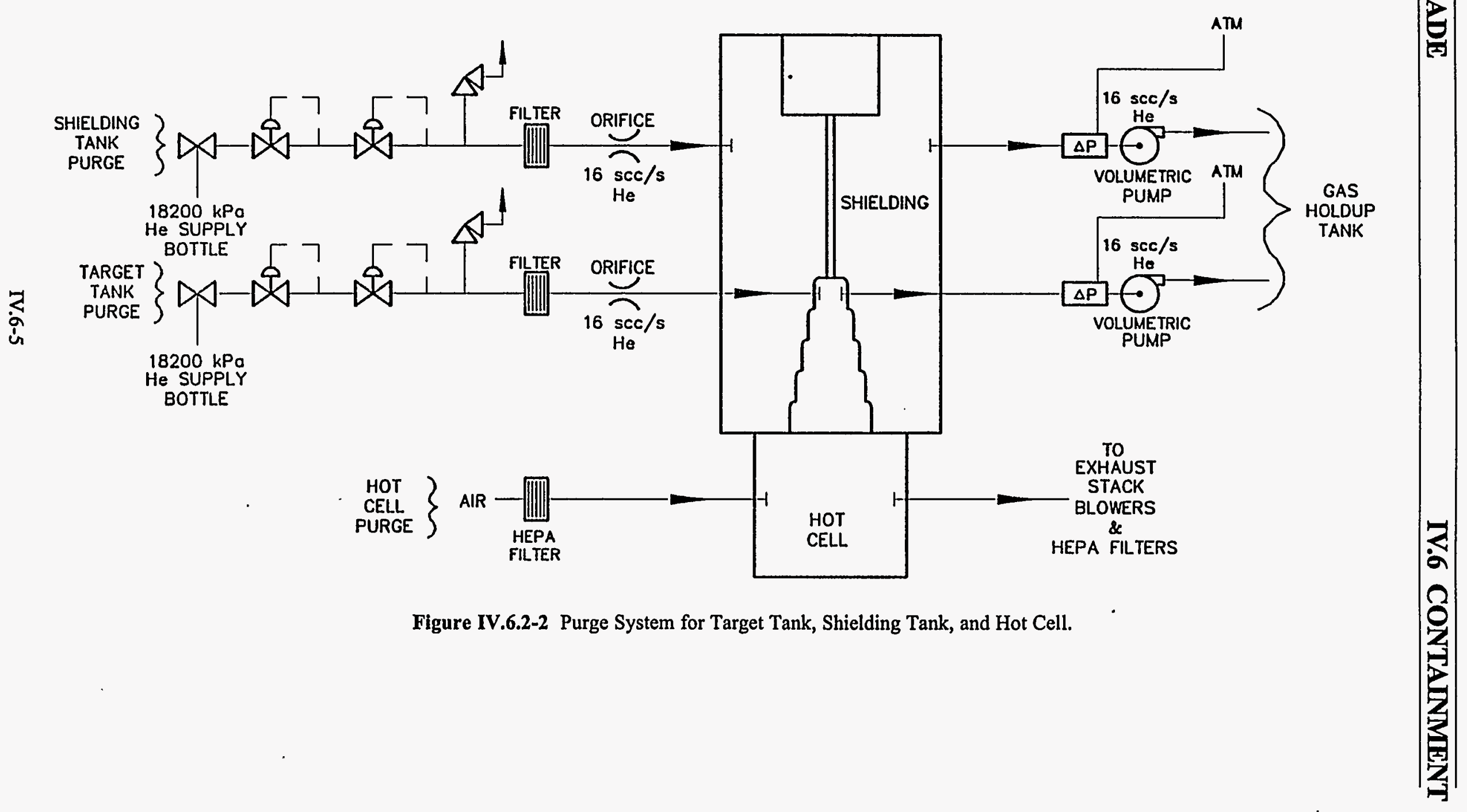


directly to the local building HEPA filter, exhaust blowers, and radiation-monitored exhaust stack system. The exhaust blower has $0.7 \mathrm{~m}^{3} / \mathrm{sec}$ capacity. The exhaust stack is a $46-\mathrm{cm}$-diameter stainless-steel single-wall stack, which is supported by the exterior building wall. The stack wall thickness is $1.22 \mathrm{~mm}$. The stack exhausts about five meters above the highest point of the building roof. Lightning protection for the stack is tied into the existing building lightning protection system.

Exhaust stacks are located on the south wall of Building 370 and on the east wall of Building 369.

\subsection{GAS HOLDUP TANKS}

As shown in Figure IV.6.2-1, exhaust gases from the target cooling system, the water moderator and premoderator cooling systems, the reflector cooling system, the shield cooling system, the biological shield purge, the neutron irradiation facilities, and the target area purge are retained in one of two holdup tanks to allow decay of radioactivity. Two 11,000-L tanks are used. One tank accumulates suspect gases, while the other tank statically holds gas for radioactive decay to safe levels. Each holdup tank is sized to hold a fast dump of all ullage gas in the target water supply tank, in addition to all of the purge gases from the various sources, for a time interval sufficient for adequate decay in the other tank. The exhaust-gas sources are connected to manifolds on the two holdup tanks by means of three-way valves which operate in unison. Each gas source is always connected to one of the holdup tanks. Each holdup tank has appropriate pressure-relief valves and a burst diaphragm to protect against over-pressurization. The effluents from relief valves and the vents are ducted through HEPA filters to the monitored stack.

Two additional 2,000-L tanks hold the hydrogen and methane moderator gases to permit decay of any radioactive products. These tanks are located in the shielded moderator components areas and are not interconnected. Each is dedicated to one of the two gases. Each tank is rated for service from vacuum to $180 \mathrm{kPa}$ pressure at ambient temperature. Safety relief and vent valves are provided. Vacuum pumps provide for initial evacuation.

The two 11,000-L gas holdup tanks for the $30-\mathrm{Hz}$ target system are in an underground vault located between Building 371 and Building 370 . An underground vault located between Building 367 and Building 369 houses the two 11,000-L gas holdup tanks for the $10-\mathrm{Hz}$ target system.

\subsection{HIGH-EFFICIENCY PARTICULATE AIR FILTERING}

None of the IPNS Upgrade buildings is designed as a containment building. The target systems, the remote-handling facilities, and hoods for work with radioactive materials require ventilation. Exhaust from these systems passes through HEPA filters before release to the environment. 
HEPA filtration assemblies precede all blowers that direct exhaust gases to the two target station stacks. All HEPA filtration assemblies include prefilters, testing provisions, and redundancy if required. They meet all required U.S. Department of Energy (DOE), federal, and state requirements.

The suspect exhaust ventilation systems in Buildings 369 and 370 consist of a series of ducts from the sources of possibly radioactive or chemically active gases described in this section to a single, airtight main exhaust duct header. Each system header carries the gases to a plenum manifold and from there, through a HEPA filter. A centrifugal vane blower exhausts this gas through a 45-cm-diameter exhaust stack, where the gases pass a stack radiation monitor before being exhausted to the outside atmosphere. Exhausts from pressurized areas have manual isolation valves. Two HEPA filter-blower combinations are installed in parallel on each main suspect exhaust system. One HEPA filter-blower combination operates and the other is in a standby condition. This standby unit starts automatically if the operating blower fails for any reason. Check dampers prevent back-flow through the standby blower. A pressure alarm sensor supplied from an independent power source reduces the likelihood of total system failure when power-source failures occur. Both blowers are connected to the area emergency power source. A tee in the blower suction line is equipped with a pressure control damper to mix outdoor air with the suspect exhaust stream to maintain a constant stack exit velocity. The stack terminates approximately $18 \mathrm{~m}$ above grade. A radiation monitor system installed in the stack provides a warning of any release of gaseous radioactive material to the ventilation system, as described in Section IV.7.1.1.

Each fan and filter in the ventilation system is designed to handle the gas flows independently under the worst credible emergency conditions.

Independent auxiliary ventilation exhaust systems, each with a separate HEPA filter and blower fan, are connected to the main exhaust stack to provide general ventilation and maintenance connections and serve hoods in Buildings 368, 369, and 370.

\subsection{LIQUID DRAIN AND RETENTION-TANK SYSTEM}

Each target station and associated remote-handling hot cell has its own drain system and retention tanks for suspect radioactive waste. This radioactive waste drain system collects liquid waste from points within the biological shield and remote-handling cell. It carries them by gravity to the main retention tank. Suspect wastes are characterized periodically and disposed of in a manner consistent with the hazard level, according to Laboratory procedures. The 16,000-L retention tank for the $10-\mathrm{Hz}$ target system is located in the underground concrete vault between Building 367 and Building 369, as shown in Figure IV.6.5-1. The underground concrete vault near Building 370 houses the $16,000-\mathrm{L}$ retention tank for the $30-\mathrm{Hz}$ target system. The main retention tank, drainage headers, and the drainage system of the remote-handling cell are vented to the vault ventilation system and out each vault stack. The 16,000-L volume of the main retention tank represents approximately $125 \%$ of the total liquid inventory of a target station. 

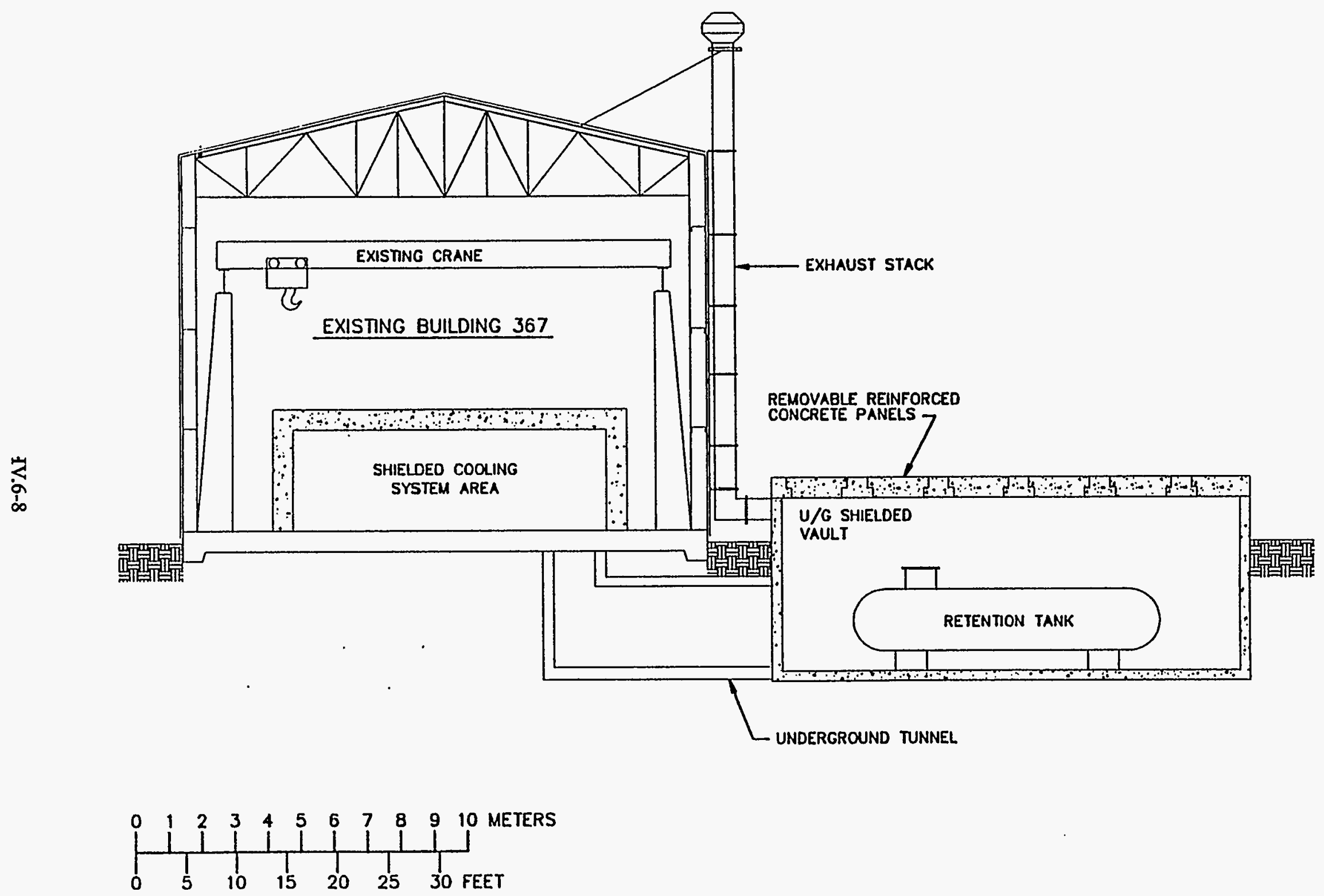

Figure IV.6.5-1 Radioactive Waste Retention Tank for the 10-Hz Target Station, Located in the Vault near Building 367. 
A separate drainage system and 400-L retention tank, sealed from the main drainage system by a remotely operated valve, serves each target tank and biological shielding tank system. This isolates the various gas spaces in each target station. Administrative control governs the opening of these valves.

All drainage systems use double-walled piping, and the vault floors and walls are sealed. These features provide an additional containment barrier for the liquid waste systems.

All tanks for suspect waste have redundant level sensors. These sensors alarm and shut down appropriate systems on a high-level alarm and bring into operation administrative procedures to handle accumulated suspect liquid waste.

The suspect waste drainage and retention system is primarily constructed of stainless steel, but it also uses other components compatible with the target station liquids are also used. The systems are of welded construction and are periodically leak-tested.

\subsection{RADIOACTIVE EFFLUENTS}

\subsubsection{Gaseous Effluents}

All airborne activity ends up in the ventilation system, where the particulate matter is captured by HEPA filters. The effluents are monitored for radioactivity before release to the atmosphere.

Spallation of air within the target station is predicted not to be a significant source of radioactive gaseous effluents.

The water spallation products $\mathrm{C}^{10}$ (19-second half life), $\mathrm{O}^{14}$ (70-second half life), $\mathrm{O}^{15}$ (2-minute half life) and $\mathrm{N}^{16}$ (7-second half life) also do not contribute significantly to the radioactive gaseous effluent. These isotopes are short-lived and are minimized by the exhaustline delay systems. The radioactive gaseous or airborne particulate products of significance are $\mathrm{Ar}^{41}, \mathrm{H}^{3}, \mathrm{Be}^{7}$, and $\mathrm{C}^{11}$. The $\mathrm{Ar}^{41}$ (1.8-hour half life) is produced via thermal neutron capture by $\mathrm{Ar}$ in any air in the controlled atmospheres of the target station. The $\mathrm{H}^{3}, \mathrm{Be}^{7}$, and $\mathrm{C}^{11}$ are water spallation products that are vented from the target cooling water system. The half lives of these isotopes are 12 years for $\mathrm{H}^{3}, 53$ days for $\mathrm{Be}^{7}$, and 20 minutes for $\mathrm{C}^{11}$. The half lives of both $\mathrm{H}^{3}$ and $\mathrm{Be}^{7}$ are long enough that they will build up continually.

A simple model was used to estimate the radioactive gaseous effluents from the 1-MW IPNS Upgrade target. The present IPNS enriched target effluent isotope quantities were taken as a benchmark. Since the power of the 1-MW IPNS Upgrade is a factor of 25 higher than the IPNS enriched target, all significant effluent radionuclides were multiplied by 25 and compared with the regulatory limits. In the case of the IPNS enriched target, the conversion of released activity to doses at the nearest ANL site boundary was done by using the AIRDOS-EPA/RADRISK Model 
and taking into account the annual probabilities of wind speed, wind direction, and wind stability class. Total doses for the effluent isotopes of $\mathrm{H}^{3}, \mathrm{Be}^{7}, \mathrm{Cl}^{11}$, and $\mathrm{Ar}^{41}$ were calculated and are listed in Table IV.6.6-1 for the 1-MW target station. The continuous-year-operations quantities are multiplied by 0.66 to account for normal downtime.

Title 40 of the Code of Federal Regulations, Part 61, subpart H, establishes standards for radioactive emissions from DOE facilities. The regulation limits radionuclide emissions based on an effective dose equivalent to the maximally exposed individual to $0.1 \mathrm{mSv} / \mathrm{yr}(10 \mathrm{mrem} / \mathrm{yr})$.

Table IV.6.6-1 shows that the predicted doses at IPNS Upgrade are less than the $10 \mathrm{mrem} / \mathrm{yr}$ radiation protection standards as given in DOE Order 5400.5, which follows the standards of 40 CFR 61 , subpart $H$.

\subsubsection{Target Cooling Water System Tritium Level}

The present IPNS enriched target cooling water system $\mathrm{H}^{3}$ concentration is estimated to be $0.056 \mu \mathrm{Ci} / \mathrm{cm}^{3}$ after five years of continuous operation. ${ }^{1}$ The IPNS Upgrade target power will be 25 times greater than the IPNS enriched target. Multiplication by this factor of 25 results in a predicted target cooling water $\mathrm{H}^{3}$ inventory of $1.4 \mu \mathrm{Ci} / \mathrm{cm}^{3}$ after five years.

The water $\mathrm{H}^{3}$ level is of concern during cooling water system maintenance and when it exceeds the level that can be safely released as liquid to the environment. Personnel protective gear and procedures make these predicted $\mathrm{H}^{3}$ water levels workable. The liquid to be disposed of is treated as radioactive liquid waste and handled according to Laboratory procedures.

\subsubsection{Cooling Water System Piping Dose Rates}

Operational data from the ISIS facility indicate that dose rates near pipework at ISIS reach levels as high as $0.5 \mathrm{mSv} / \mathrm{h}(50 \mathrm{mrem} / \mathrm{h}) .^{2}$ Much of this is due to $\mathrm{Be}^{7}$, which plates out on the inside of the pipes. IPNS Upgrade operates at a power six times greater than ISIS. This scaling factor of six predicts a dose rate around some pipework as high as $3 \mathrm{mSv} / \mathrm{h}(300 \mathrm{mrem} / \mathrm{h})$ for IPNS Upgrade. This dose rate causes problems for some maintenance and requires the use of extra shielding. This dose rate is greatly reduced by the addition of full flow filters and ion exchange columns and by carrying out periodic chemical pipe-flush operations. 
Table IV.6.6-1 Annual Predicted Dose from 1-MW IPNS

Upgrade to an Individual at the Nearest ANL Site Boundary

\begin{tabular}{lccc}
\hline Radionuclide & $\begin{array}{c}\text { Immersion } \\
\text { (mrem) }\end{array}$ & $\begin{array}{c}\text { Inhalation } \\
(\mathrm{mrem})\end{array}$ & $\begin{array}{c}\text { Ingestion } \\
\text { (mrem) }\end{array}$ \\
\hline $\mathrm{H}^{3}$ & $<0.025$ & $<0.025$ & $<0.025$ \\
$\mathrm{Be}^{7}$ & $<0.025$ & $<0.025$ & $<0.025$ \\
$\mathrm{C}^{11}$ & 10.95 & 0.975 & $<0.025$ \\
$\mathrm{Ar}^{41}$ & $<0.75$ & $<0.75$ & $<0.75$ \\
$\mathrm{Total}^{4}$ & $<11.75$ & $<1.78$ & $<0.825$ \\
& $\frac{\mathrm{x} 0.66}{7.75}$ & $\underline{\mathrm{x} 0.66}$ & $\mathbf{x} 0.66$ \\
& & 1.17 & 0.54 \\
\hline
\end{tabular}




\subsection{REFERENCES}

1. A.W. Schulke, Jr., A.V. Rauchas, A.E. Knox, J.L. Bailey, and T.R. Clayton, Final Safety Analysis Report (FSAR-II) for the Intense Pulsed Neutron Source Facility for Operation with the Enriched Uranium Booster Target, Argonne National Laboratory (March 1991).

2. T.A. Broome, Rutherford Appleton Laboratory, Chilton, U.K., May 1994 (private communication). 


\subsection{PERSONNEL AND FACILITY PROTECTION SYSTEMS}

\subsubsection{Overview}

IPNS Upgrade has a personnel protection system and a facility protection system, both of which are separate from the control system and employ redundant logic channels that provide independent means of inhibiting proton beam generation. The facility protection system receives inputs from all areas of the accelerator and target stations and initiates a proton beam shutdown upon detection of any out-of-limit conditions, for the purpose of component protection. As the primary safety system that ensures radiation containment boundaries, the facility protection system is important to public safety.

The personnel protection system shares some of the same shutdown devices, but otherwise is not associated with the facility protection system. The personnel protection system ensures that facility personnel are not exposed to unacceptable levels of radiation, high voltages, temperature extremes, pressure extremes, etc. This is accomplished primarily by shutting down the proton beam and closing neutron beam gates if personnel attempt to enter dangerous areas or if dangerous conditions develop.

This section discusses the target station and experimental area components interfaced to the facility protection and personnel protection systems. Interfacing of the accelerator to these protection systems is discussed in Chapter II.

\subsubsection{Facility Shutdown Interface}

Selected target systems must be in the beam permissive condition for proton beam generation and acceleration to be allowed. Similarly, the personnel protection system associated with the target station and its scattering instruments collects signals from various systems important to personnel protection, such as access gate switches, ventilation systems that provide confinement, and excess radiation monitors. It is required that all these systems be in the proton beam permissive condition for operation. Additionally, many of the personnel protection trip signals command neutron beam gate closures. All sensing signals are carried by dedicated wires carrying a fixed current (current loops). Open and short circuit and random signal protection is provided for all of these wires. Fail-safe outputs are provided by the individual systems, such that the outputs have to be energized to permit proton beam operation or keep neutron gates open. The shutdown time delay depends on the initiating device and the reason for shutdown, but it is less than a second in all cases for the proton beam and less than 30 seconds for a neutron beam gate.

The shutdown conditions for any operating mode are predetermined, and the summations and interlocking equations for all of the input signals are implemented redundantly within digital logic circuits. Each redundant circuit is powered by individual power supplies. The redundant outputs of the target station proton beam shutdown system are summed into the redundant 
facility protection system with other, accelerator-based shutdown summations through current loop interfaces. Any of the inputs into the personnel or facility protection systems are able to inhibit the generation of the proton beam. Due to the high power capability of the proton beam, the beam is inhibited at the point of lowest possible energy. Three independent devices are used. Two of these are physical devices, faraday cups, which have to be removed from the low-energy beam path for the beam to be accelerated. The other device is an electronic inhibitor that prevents beam generation in the ion source.

\subsubsection{Radiation}

The area radiation monitoring system uses microprocessor-based, multifunction ratemeters with dual channels and digital/analog dose rate display. Each unit supports both a neutron and a gamma probe and is capable of simultaneous detection. Separate, normally energized fail-safe contacts are provided. The ratemeter, which has a built-in diagnostic and failure alarm, utilizes a serial communication link for remote communications with a host computer. Lead acid batteries located within the unit provide a minimum of $8 \mathrm{~h}$ of operation. The gamma probe is an ion chamber with both steady-state $0.001-10 \mathrm{mGy} / \mathrm{h}(0.1-1000 \mathrm{mRad} / \mathrm{h})$ and satisfactory pulsed-radiation response capability, $10 \mathrm{~Gy} / \mathrm{h}(1000 \mathrm{Rad} / \mathrm{h})$ for $10-\mu \mathrm{sec}$ pulses at $100 \mathrm{~Hz}$. The neutron counter is a $\mathrm{BF}_{3}$ proportional counter, housed in an Anderson-Brauntype moderator, with a range of $0.001-10 \mathrm{mSv} / \mathrm{h}(0.1-1000 \mathrm{mrem} / \mathrm{h})$ and featuring gamma rejection up to $2.5 \mathrm{~Gy} / \mathrm{h}(250 \mathrm{Rad} / \mathrm{h})$. The detector has a $1-\mu \mathrm{sec}$ response time and performs satisfactorily in a pulsed field.

Gamma and neutron detectors are placed as necessary through the experiment areas. Radiation dose rates are displayed on the digital readout. The ratemeter is set for a warning-level alarm to indicate elevated radiation fields and a high-level alarm that initiates automatic shutdown of the proton beam. Additional detector units are placed along the neutron beamlines to measure radiation fields and to initiate proton beam shutdown and neutron beam gate closures.

\subsubsection{Cooling Systems}

An IPNS Upgrade target station has a number of individual cooling systems, each of which is important to the safety and operation of the facility. The consequences of a loss of cooling vary from system to system, but operation of the entire facility requires proper operation of all of the cooling systems.

The cooling systems and the sections in which they are discussed are listed here. The complement of instrumentation and control devices shown on the cooling system diagrams for these systems is the minimum needed to control the system and does not represent the entire complement needed for control, protection, and monitoring. 
1. Target cooling (Section IV.2.6)

2. Moderator and premoderator water cooling (Section IV.3.4.2)

3. Reflector cooling (Section IV.3.4.5)

4. Biological shield cooling (Section IV. 3.4.6)

5. Target tank assembly cooling (Section IV.3.4.7)

6. Proton HET line vacuum window cooling (Section IV.3.4.8)

7. Liquid methane moderator cooling (Section IV.3.4.3)

8. Liquid hydrogen moderator cooling (Section IV.3.4.4)

9. Neutron irradiation facilities cooling (Sections IV.6.2.5 and V.4.3)

The facility protection system monitors all cooling systems and carries out the ultimate protective actions should the other systems fail to work. The protection system's high reliability generally requires fully redundant systems to monitor critical parameters and to provide a protective action. The reliability required for the individual protection systems is commensurate with consequences of the failure of the monitored system. For instance, the failure of a target cooling system on the IPNS Upgrade requires an immediate proton beam shutdown to prevent the most significant radiological and economic consequences. Such a system requires the highest reliability of monitoring instruments and shutdown logic connecting this cooling system and the proton beam shutdown devices.

The protection system primarily relies on monitoring parameters such as flows, pressures and liquid levels that directly indicate a loss of cooling function, rather than relying on temperature measurements.

\subsubsection{Containment Systems}

The IPNS Upgrade containment and confinement systems described in Section IV.6 control the releases of radioactive gases, explosive gases, and radioactive liquids. This includes collecting all potentially radioactive or explosive gases and directing them to the stacks, minimizing activity at the stacks by delaying releases or by keeping air out of the biological shield purge gases, and collecting and storing all liquid leakages in sealed collection systems. 
Most of the facility or personnel protective actions associated with the containment and confinement systems require human intervention. Most systems allow sufficient time for operator reaction. Interlocks shut the facility down in the event of critical failures, such as failure of the main facility exhaust ventilation, overpressurization events, and coolant leakage events.

\subsubsection{Mechanical Systems}

A number of mechanical systems are critical to the safe operation of an IPNS Upgrade target station. These include neutron beam gates, hot-cell doors, personnel-entry safety interlocks, and irradiation facilities interlocks. These systems protect operating personnel, and must be in a permissive state to allow IPNS Upgrade operations.

Many systems associated with the neutron scattering beamlines also have interlocks that prevent their inadvertent operation or proton beam operation whenever their area is accessed. If a mechanical system ceases to be in a permissive state, there are a number of hard-wired shutdown and alarm systems that provide very reliable protective actions and alarms, as conditions warrant. For instance, if a person enters an interlock-protected area near a scattering instrument without prior required actions being taken, system logic shuts down the proton beam, closes appropriate neutron beam gates, and sounds local and control console alarms.

\subsection{CONTROL AND MONITORING SYSTEMS}

\subsubsection{Overview}

In addition to the protection system, there is also a control system, which provides the operator interface for normal facility operation. The IPNS Upgrade control system uses the Experimental Physics and Industrial Control System (EPICS), which has been developed by the Controls group at the Advanced Photon Source (APS) and the AT-8/GTA groups at Los Alamos National Laboratory. EPICS uses high-performance graphic workstations with an X-Window Graphical User Interface (GUI) at the operator level. It connects to VME/VXI-based microprocessors at the field level by means of high-performance networks. EPICS performs all of the control and monitoring of the accelerator systems and supervisory monitoring and control of the target stations. EPICS is described in more detail in Chapter II.

\subsubsection{Control System Interface}

Standardized interface modules are mounted in crates near the sources of the signals to be interfaced. The analog signals from target station devices are asynchronously converted to 12-bit binary form every $18 \mathrm{~ms}$, providing an accuracy of $0.025 \%$ of full scale. The digital input signals are handled by optically coupled input circuits to provide isolation from the transducer signal and conditioning devices. These binary signals are transmitted to the local microprocessors, where 
the raw data base is converted to appropriate engineering units and placed in a processed data base; in the latter, each signal is checked against preset levels. These levels, in descending algebraic order, are high out-of-transducer range, high transducer trip level, high transducer alarm level, low transducer alarm level, low transducer trip level, and low out-of-transducer range. Not all signals are checked for all of these conditions. The alarm level gives an opportunity for correcting the problem before shutdown occurs. The failure function calls attention to any type of channel malfunction, which causes the analog output of the channel to go to the limit opposite the alarm or shutdown level. The appropriate alarm/trip limits are predetermined for all operating modes and are preset into the microprocessor data base and regularly confirmed by the central control computer. All data from the field level microprocessor are transmitted to the GUI operator consoles for operator display. Trip conditions are also relayed to the personnel and facility protection systems as redundant control system inputs to these hard-wired shutdown systems.

\subsubsection{Radiation Monitoring}

\subsubsection{Exhaust Monitoring}

The stack sampling system is equipped with suitable detectors to quickly identify potentially serious incidents. Air emissions are surveyed for radioactivity with a microprocessor-controlled, continuous stack monitor located in a shielded enclosure downstream of the HEPA filters. The system includes the following components:

1. Particulate Monitor - A plastic scintillator counts beta emissions from particles collected on a cyclically moving, high-efficiency filter paper. A portion of the stack exhaust air is diverted through the filter, which is mounted close to the plastic scintillator. The system is used to document the adequacy of the HEPA filtering during normal operations and to detect increased levels of activity. Gamma spectrometry of exposed filter paper also identifies $\mathrm{Be}^{7}$ and other contributors.

2. Radionuclide Monitors - The diverted exhaust stream flows through a charcoal cartridge or other suitable collection medium. Two NaI(T1) crystals scan this medium. Different spectral regions, each corresponding to a specific photon energy (e.g., $1.3 \mathrm{MeV}$ for $\mathrm{Ar}^{41}$ and $1.12 \mathrm{MeV}$ for $\mathrm{Ta}^{182}$ ), are set up and the counts from these photons recorded.

3. Residual Gas Monitor - Another plastic scintillator is located in a sample chamber to monitor any gaseous radioactivity that has passed the filter paper and other collection media. This monitor indicates the presence of betaemitting radionuclides as the gas passes through a cylindrical chamber. These data are registered as a count rate and also are accumulated for use in estimating total stack releases. Grab samples obtained at IPNS with a 
$100-\mathrm{cm}^{3}$ sampler featuring a thin window indicated that the major activity is $C^{11}$.

The flow rate through the stack monitor system is dynamically controlled by the microprocessor to achieve isokinetic sampling of the stack environment. Stack flow rates are determined by periodic Pitot tube measurements. Remote readouts of the detector outputs and flow measurements are provided at the Control Room and in the Health Physics Office. The remote readouts are displayed, recorded, and alarmed for control and monitoring purposes only.

\subsubsection{Personnel Monitoring}

Argonne has an extensive radiation protection program, supported by the ESH Division. Details of the control requirements and procedures can be found in the ANL site-specific Radiological Control Manual. ${ }^{1}$ The IPNS Upgrade complies with the requirements of the manual. ESH supplies personal monitoring devices, collects them for analysis, processes the devices and analyzes the readouts to provide dose results. ESH maintains its own calibration facility with both gamma and neutron capability.

Buildings $367,369,370$ and 371 are radiologically controlled areas. Each building has hand and shoe monitors, each with a frisk detector, at the entrance and exit doors.

In addition to the personnel monitoring program, ESH provides monitoring and survey services and hazard analyses. These services include instrument surveys for alpha and betagamma contamination, as well as neutron and gamma fields, air samples of the ambient atmosphere, and surface and smear surveys for alpha and beta contamination.

\subsubsection{Cooling Systems}

Controllers monitor the cooling systems under the supervision of EPICS. EPICS provides setpoints and directly monitors critical cooling system parameters: usually flows, temperatures, pressures, and fluid levels. It also alarms or shuts down systems when out of normal operating range.

\subsubsection{Mechanical Systems}

Neutron beam choppers are located within a target station, but they are considered a part of the neutron scattering instrument they serve, as are most of their instrumentation and control systems. However, EPICS monitors their operation, and they are required to have interlocks that prevent their inadvertent operation or proton beam operation whenever their area is accessed.

Most of the mechanical systems, such as neutron beam gates and personnel entry safety interlocks, are locally controlled, but all are under the monitoring, alarming, and protective-action 
features of the IPNS Upgrade EPICS. EPICS software protective actions include proton beam shutdown, neutron beam gate closure, chopper shutdown, and local area alarms.

\subsection{CONTROL ROOM}

An rf-shielded control room enclosure that currently exists in Buildings 368 and 370 serves as the control room for both the $10-\mathrm{Hz}$ and $30-\mathrm{Hz}$ target stations. The raised floor area of this control room exists and covers about $100 \mathrm{~m}^{2}$. An adjacent office area, which is also rfshielded, covers an additional $100 \mathrm{~m}^{2}$. Modern fire protection systems exist in the control room.

\subsection{REFERENCE}

1. Environmental Safety and Health Manual, Argonne National Laboratory-East, Chapter 5 (April 1991). 


\subsection{BIOLOGICAL SHIELDING PENETRATIONS}

Each of the target stations has a total of 18 penetrations through the biological shield, 9 on each of two sides. These are the neutron beam ports through which neutrons are allowed to pass to the neutron scattering instruments. One or more neutron beams can be brought out through each beam port. Figure IV.4.1-2 shows the locations of these beam ports in the biological shield. Each of the six moderators in the target station is viewed by three such beam ports, with $\sim 13^{\circ}$ separation between beam ports in the horizontal plane.

Figure IV.8.1-1 shows a section through the biological shielding along the length of a representative beam port. The innermost end of the beam port closest to the moderator consists of a cutout section in the beryllium reflector. Here the three beam ports viewing that moderator converge, resulting in a single cutout in the reflector adjacent to each moderator, as shown in Figure IV.8.1-2. The cutouts for the different moderators are at different levels in the reflector, as can be seen in Figure IV.1.2-5. Each of these cutouts is lined with an absorbing void liner to prevent broadening of the neutron pulses by neutrons thermalized in the reflector. These cutouts allow each beam port to view the full $10 \times 10 \mathrm{~cm}^{2}$ or $10 \times 20 \mathrm{~cm}^{2}$ moderator face. The sealed aluminum target tank isolates this inner portion of the beam port from the outer portion in the biological shield. Helium gas fills this target tank, and hence also the reflector cutout portion of the beam port.

A separate steel beam port tube defines each beam port from the target tank to the shielding tank. Each tube is constructed of at least $1.25-\mathrm{cm}$-thick steel plate and has a cross section of $35 \times 35 \mathrm{~cm}^{2}$ at the inner end. The cross-section is increased in several steps along the tube length to prevent neutron streaming. The tubes vary in length from $3.65 \mathrm{~m}$ to $4.9 \mathrm{~m}$ and are welded to the shielding tank and to the target tank housing. Each of these tubes is also welded to a beam gate housing to allow a gate to block the beam, as shown in Figure IV.8.1-1. The atmosphere of these tubes is the same as the shielding tank atmosphere, because they are isolated from the target tank by the beam windows on the target tank. An O-ring and flange arrangement seals an aluminum window at the outer end of the beam port tube.

Outside the shielding tank, each beam port enlarges to provide a well-shielded chopper cavity that can accommodate neutron choppers or filters. The beam port then terminates in an interlocking transition to the external neutron beamline shielding. This external beamline shielding is considered to be part of the neutron scattering instruments, and is discussed in Chapter V.

\subsection{BEAM GATES}

The neutron beam gates are each $2 \mathrm{~m}$ long in the direction of the beam and $40 \mathrm{~cm}$ wide, and extend from $\sim 2.5 \mathrm{~m}$ below the beam ports to $\sim 4 \mathrm{~m}$ above the beam ports. These gates are made either of steel or of steel-shot-loaded concrete, and are made in sections to facilitate 


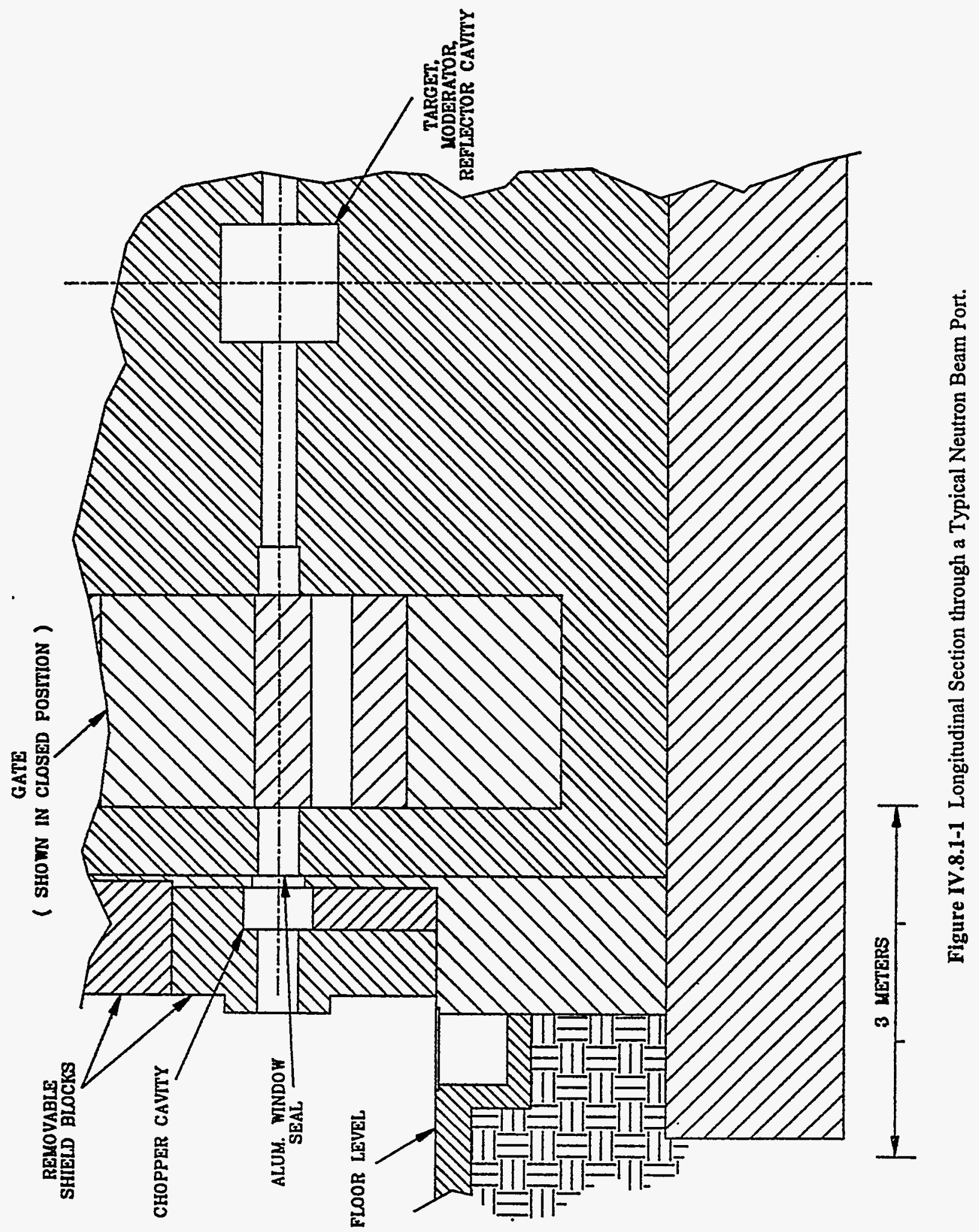




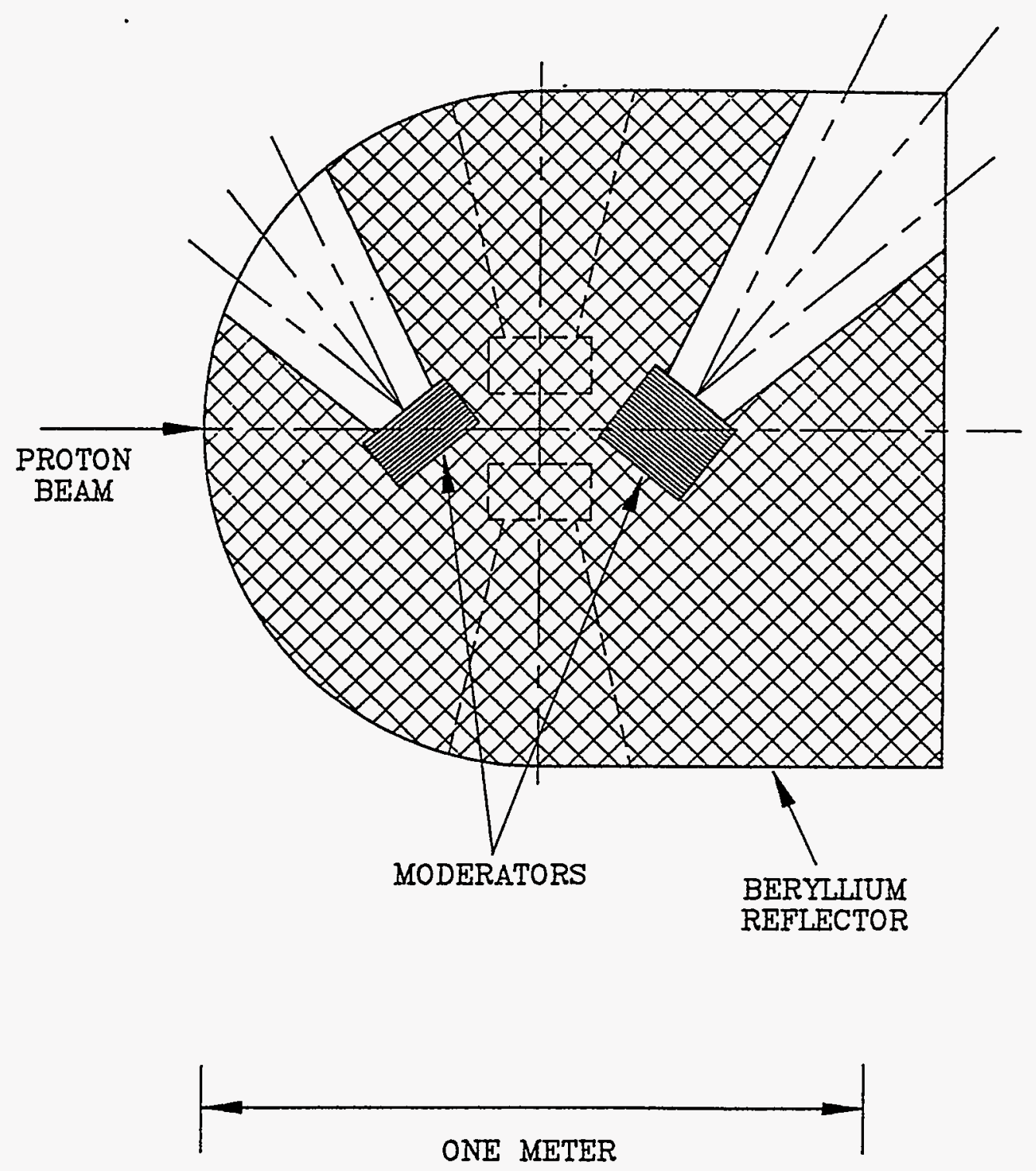

Figure IV.8.1-2 Horizontal Slice through the Reflector at the Midplane of the Upper Moderators, Showing the Cutouts for the Neutron Beams. 
installation. Hydraulic actuators move the gates vertically to either the gate-open or gate-closed positions. Each gate has an opening cut through it which is moved into alignment with the remainder of the beam port for the gate-open position. In the gate-closed position, this opening is moved some distance below the beam port, so the beam port is blocked by $2 \mathrm{~m}$ of gate material. The gate width is greater than the width of the beam port and is of stepped shape to prevent streaming of neutrons around the edges of the gate. The gate moves inside a steel housing which is welded to the beam port tube.

Figure IV.4.1-2 shows the locations of the gates in the biological shield and Figure IV.8.2-1 shows a cross-sectional view of two of the gates, one in the open position and one in the closed position. As indicated in Figure IV.4.1-2, all gates have their inner faces $2.5 \mathrm{~m}$ or more from the target, so heating of the gates is estimated to be less than $100 \mathrm{~W}$ per gate and no special cooling is required. This large separation from the target also minimizes activation of the gate materials and the beamline inserts.

The cutouts in the neutron beam gates contain neutron collimation elements or neutron guides to continue the definition and transport of the neutron beams appropriate for the specific neutron scattering instruments being served, whereas the remainder of the space in the gate is filled with neutron shielding. An individual hydraulic actuator at the top of each gate, shown in detail in Figures IV.8.2-1 and IV.8.2-2, drives that gate against adjustable stops to provide an aligned and reproducible open position. Adjustments on the actuator mount permit a small rotation of the gate about a vertical axis in order to optimize the angular alignment of the in-gate collimator or guide with the remainder of the beamline optical elements. A metal sheet seals the opening for the gate in the top of the shielding tank to complete the containment barrier.

The $2 \mathrm{~m}$ of shielding placed in a neutron beam when the gate is closed provides excellent biological shielding against any radiation resulting from residual activity inside the target station. This permits work in the external neutron beamline when the gate is closed and the accelerator is not running. However, when the accelerator is providing protons to this target station, this beam gate does not reduce the dose levels in the neutron beamline low enough to allow unrestricted access to the beamline. To make the gate sufficiently effective for this purpose requires a gate as thick as the remainder of the biological shielding, or approximately $6 \mathrm{~m}$, which is not practical. The gate does reduce external radiation in the beamline sufficiently to allow limited access to such beamline components as choppers or filters and to permit changing samples in the neutron scattering instrument while the accelerator is running. The gate is closed when these activities occur.

\subsection{NEUTRON BEAM GUIDES AND COLLIMATORS}

Some of the neutron scattering instruments use neutron guides to provide efficient transport of neutrons to the instrument. Section V.3.2 indicates which of the instruments require guides and also specifies parameters for these guides. In some cases the guide is required to extend to within $1-2 \mathrm{~m}$ of the moderator. This region is easily accessible with the present beam 


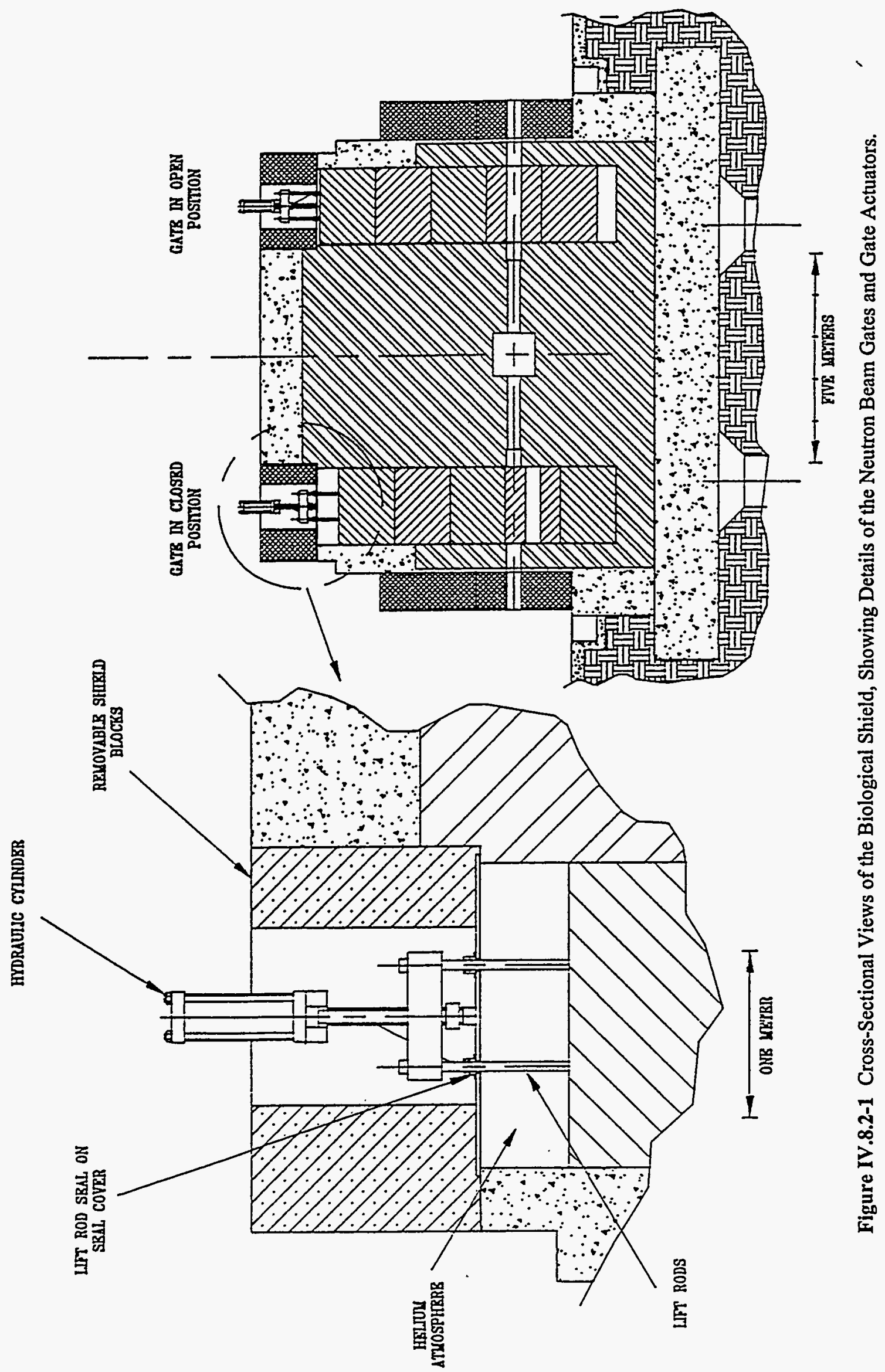




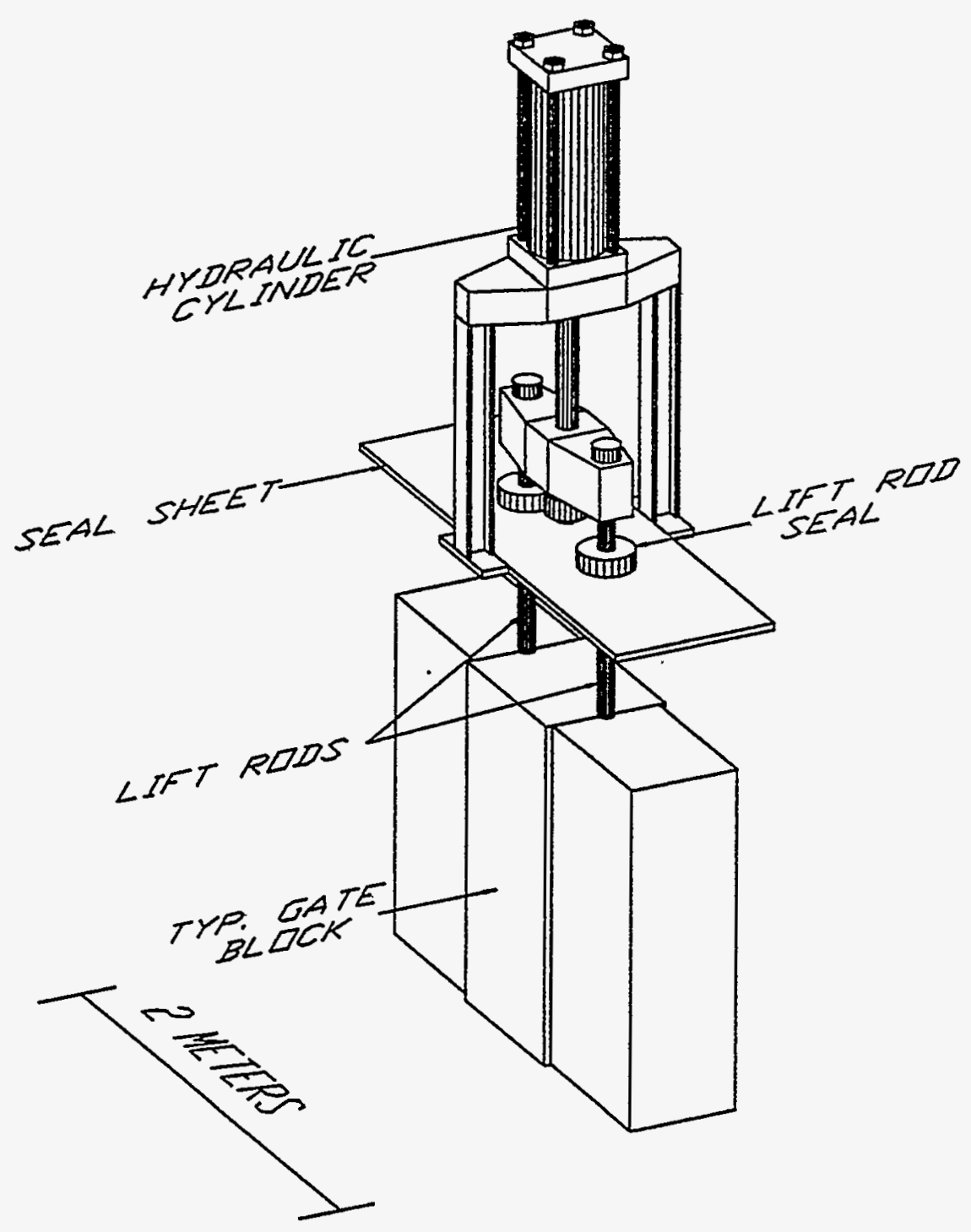

Figure IV.8.2-2 Details of a Neutron Beam Gate and Actuator. 
port design, as shown in Figure IV.8.1-1. Neutron guides are usually made of glass, with a thin coating of nickel to provide the reflective surface. However, at 1-2 $\mathrm{m}$ from the target, these guide materials deteriorate relatively rapidly due to radiation damage. At ISIS, the portions of guides that are in high radiation fields are made of polished steel, ${ }^{1}$ which has worked quite well with no deterioration yet observed. Those portions of the neutron guides that are in high radiation fields at IPNS Upgrade utilize similar construction. Construction of the remaining sections of the guides, including sections that must extend through the neutron gates, is also similar to that used at ISIS. 1,2 Any remaining space between the exterior of the guide and the walls of the beam port is filled with appropriate shielding material.

The interior of the remaining beam ports, including the portion that extends through the beam gate, is filled with shielding material arranged to leave a channel for the neutron beam. These beam port inserts are individually designed to the requirements of specific instruments. Neutron beam collimation is provided by a series of $\mathrm{B}_{4} \mathrm{C}$ irises, following standard practice. ${ }^{3}$

In some cases it is possible to bring out more than one neutron guide or collimated beamline within a single beam port. This is the case if there are two or more instruments that are very small and that require small beams from the same moderator, as is the case for the neutron reflectometers. Multiple guides from the same beamport can also be used for the QSS instruments, similar to the case at a neutron guide hall at a reactor. It is possible to bring out as many as three guides from a single beam port, where this is appropriate.

\subsection{CHOPPER CAVITIES FOR CHOPPERS AND FILTERS}

Many of the neutron scattering instruments require neutron choppers or filters in the beamline. Requirements for neutron choppers are discussed in detail in Section V.3.1. These devices scatter unwanted neutrons out of the beam before they reach the instrument, and hence introduce intense local radiation sources requiring massive shielding. Furthermore, it is desirable to locate most of these devices as close to the moderator as practical. For both of these reasons, each beam port includes a chopper cavity at the face of the biological shield, as shown in Figure IV.8.1-1, to accommodate choppers and filters. In this location, the biological shield provides most of the shielding required by the chopper or filter.

Removable shielding blocks above the cavity provide access. These extend to the top of the biological shielding, as shown in Figures IV.4.1-3 and IV.8.1-1. This design allows the chopper or filter to be serviced without disturbing the remainder of the instrument beamline or its shielding. Interlocks require that the beam gate be closed whenever these shielding pieces are removed. 


\subsection{INTERFACE TO NEUTRON BEAMLINE SHIELDING}

The shielding at the face of the chopper cavity interlocks with the shielding for the external portion of the neutron beamline. This design, shown schematically in Figure IV.8.1-1, prevents any streaming through cracks between shielding pieces.

\subsection{ATMOSPHERE CONTROL}

A helium gas atmosphere is maintained inside the target tank surrounding the targetmoderator-reflector region. A second, independent helium atmosphere is maintained throughout the portions of the biological shielding inside the shielding tank, including the beam port and beam gate regions. A thin aluminum window and O-ring flange assembly seals this atmosphere inside the beam port at the point where the beam port enters the chopper cavity (see Figure IV.8.1-1). Neutrons interact only minimally with gaseous helium, so this helium is satisfactory for most of the beamlines. When desired, an independently controlled helium atmosphere or a vacuum can be provided inside the neutron guide or collimator sections within the beam port. Channels in the biological shield at the beam gate and in the beam gate itself accommodate the necessary gas flow or vacuum lines for connection to such guide or collimator sections.

\subsection{REFERENCES}

1. J.C. Sutherland et al., "The performance of the IRIS and HRPD guides on the SNS," in Proceedings of the 8th Meeting of the International Collaboration on Advanced Neutron Sources (ICANS VIII) (Oxford, U.K., July 8-12, 1985), Rutherford Appleton Laboratory Report No. RAL-85-110, Chilton, U.K., pp. 771-776 (1985).

2. C.J. Carlile, M.W. Johnson, and W.G. Williams, Neutron Guides on Pulsed Sources, Rutherford Appleton Laboratory Report No. RL-79-084, Chilton, U.K. (1979).

3. A.D. Taylor, T.A. Broome, and D.J. Picton, "Neutron beam shielding at ISIS," in Proceedings of the 9th Meeting of the International Collaboration on Advanced Neutron Sources (CCANS LX) (Villigen, Switzerland, September 22-26, 1986), Swiss Institute for Nuclear Research, Villigen, Switzerland, ISBN 3-907998-01-4, pp. 519-528 (1987). 



\subsection{OVERVIEW OF NEUTRON BEAM INSTRUMENTATION}

In Sections V.2 and V.3, the experimental facilities that are located on neutron beamlines are discussed. Other types of experimental facilities that do not occupy one of the neutron beamlines are discussed in Section V.4.

\subsubsection{Instrument Discussions and Reference Set of Instruments}

A number of different neutron scattering instruments are discussed in sufficient detail in Section V.2 that their expected performances can be evaluated. A representative "reference set" is selected from among those instruments and is considered for the cost analysis in Chapter VII. The reference set of instruments is chosen to represent a plausible initial complement of instruments to be installed at IPNS Upgrade, under the assumption that 24 of the 36 available beam ports are to be instrumented initially. The set of instruments that will actually be selected for initial installation will be based on the requirements of the user community as assessed at the time of the construction project.

A number of very good neutron scattering instruments are currently operational at IPNS. IPNS Upgrade includes 10 of these instruments, in some cases with little change and in other cases with upgrading. The availability of these already-proven instruments makes it possible to have a large number of instruments fully operational at startup. Use of existing instruments also results in significant cost savings.

In most cases, the instrument data rates specified in this chapter for IPNS Upgrade are estimated by scaling from known rates for the same or similar instruments at IPNS, ISIS, or the Institut Laue-Langevin (ILL), taking into account differences such as path lengths, numbers of detectors, and types of moderators. This scaling is performed under the assumptions (1) that the proton beam is shared between the $30-\mathrm{Hz}$ and $10-\mathrm{Hz}$ targets in a $2: 1$ ratio and (2) that the timeaveraged current of neutrons per beam with a decoupled moderator at the $30-\mathrm{Hz}$ target station at IPNS Upgrade is $\sim 50$ times the corresponding current at IPNS with the depleted-uranium target, as described in Section IV.3.2.4. The time-averaged current per beam at the 10-Hz target station is one-half the amount at the $30-\mathrm{Hz}$ station. Additional factors of 4-6 in intensity are gained by using coupled moderators, where appropriate.

Most of the instruments discussed in this chapter use neutron time-of-flight (TOF) techniques, as is the case for all of the instruments currently operational at pulsed neutron sources. IPNS Upgrade has sufficient time-averaged intensity to make it practical to operate some of the beamlines in a quasi-steady-state (QSS) mode. In this mode, only the time-averaged flux from the moderator is of importance, and in principle, any instrument that works at a steady-state source (reactor) can be made to work in this QSS mode at IPNS Upgrade as well. The time-averaged flux from a moderator optimized for this type of operation at IPNS Upgrade is roughly equivalent to that at a $\sim 10^{14} \mathrm{n} / \mathrm{cm}^{2}-\mathrm{s}$ reactor, as discussed in Section IV.3.2.5. With many of these instruments the time-structure of the source can be used to advantage to reduce background contributions because of the reduced duty factor of 1:30 to 1:60, and to eliminate 
undesirable orders from monochromators and analyzers. Several instruments that work in this mode are discussed here. One of these, a cold-neutron triple-axis spectrometer, is included in the reference set of instruments.

\subsubsection{Details of Instrument Components}

Several critical types of instrument components common to many of the instruments are discussed in detail in Section V.3. Neutron chopper systems, neutron guides, beamline shielding, and detectors are included. Specific requirements in each of these areas are analyzed for the reference set of instruments.

\subsubsection{Utilization of the Remaining 12 Beam Ports}

Twelve beam ports remain available for future construction of instruments, by participating research teams and by IPNS Upgrade personnel, to support evolving needs of the user community.

\subsection{OVERVIEW OF OTHER EXPERIMENTAL FACILITIES}

The primary mission of IPNS Upgrade is neutron scattering. However, the various components of the facility provide capabilities that are extremely useful for other areas of science. Instrumentation to provide capabilities in radiation damage studies, neutron isotope production, and neutron activation analysis is included as part of this project. The facilities for these three areas are discussed in detail in Section V.4, and are included in the project cost and schedule.

IPNS Upgrade has potential capabilities in a number of other areas, including muon spin rotation. Some of these possibilities are discussed briefly in Appendix C. 


\subsection{POWDER DIFFRACTOMETERS}

\subsubsection{Transfer of Existing IPNS Instruments}

\subsubsection{SEPD — High-Intensity Powder Diffractometer}

The Special Environment Powder Diffractometer (SEPD) and the General Purpose Powder Diffractometer (GPPD) at IPNS have identical scattering flight paths, ${ }_{1}^{1}$ shown in Figure V.2.1-1. With minimal modification, the SEPD can be used as a high-intensity and specialenvironment powder diffractometer at IPNS Upgrade. The incident flight path is shortened from $14 \mathrm{~m}$ to $12 \mathrm{~m}$ to achieve a gain in intensity and an extension of the available d-spacing range at fixed scattering angle. This instrument provides high count rates while maintaining sufficient resolution in backscattering $\left(150^{\circ}\right)$ to obtain Rietveld-refinement-quality data. Added detectors approximately double the area of the original SEPD detector arrays to $\sim 0.2 \mathrm{sr}$ at both backscattering and $90^{\circ}$. This allows real-time studies with data-collection times on the order of 1-10 min for large samples. This instrument is also effective for the study of small samples. Rietveld-quality data for samples of $10-100 \mathrm{mg}$ are obtained in a few hours. The moderator is designed to give essentially the same resolution on the somewhat shorter flight path. The repetition rate of $30 \mathrm{~Hz}$ allows this instrument to provide a large d-spacing range for experiments done at a single scattering angle, although a bandpass-limiting/frame-overlap chopper may be required. Table V.2.1-1 summarizes the parameters of this upgraded SEPD.

\subsubsection{GPPD — Medium-Resolution Powder Diffractometer}

The incident flight path of the GPPD is lengthened from $20 \mathrm{~m}$ to $25 \mathrm{~m}$ to obtain higher resolution in backscattering. The $90^{\circ}$ detector arrays are moved to provide a much longer scattered flight path, as shown in Figure V.2.1-2, to maintain high resolution at $90^{\circ}$ for experiments in such special environments as pressure cells and controlled-atmosphere furnaces. The resolution $\Delta \mathrm{d} / \mathrm{d}$ at $90^{\circ}$ is $2 \times 10^{-3}$ full width at half maximum (fwhm), an improvement of 2.5 over the IPNS version. This resolution is achieved by lengthening the scattered flight path at $90^{\circ}$ to $\sim 4 \mathrm{~m}$ and by using a Soller collimator (approximately $\Delta \phi=0.08^{\circ}$ ) in the incident flight path to reduce the incident angular divergence. The desired resolution is achieved while still maintaining the ability to use samples as large as $1-\mathrm{cm}$ diameter $\times 5-\mathrm{cm}$ long. Removing the incident Soller collimator increases the count rate by a factor of 3 for experiments where high resolution at $90^{\circ}$ is not required, without degrading the resolution in backscattering. The $30-\mathrm{Hz}$ source repetition rate permits the collection of full data sets with the desired d-spacing range at a single scattering angle, although a bandpass-limiting chopper is required to eliminate frame-overlap neutrons. The backscattering detectors of this instrument provide a resolution of $2 \times 10^{-3}$, giving Rietveldquality data with data-collection times of a few minutes when the Soller collimators are removed. Table V.2.1-1 summarizes the parameters of this upgraded GPPD. 


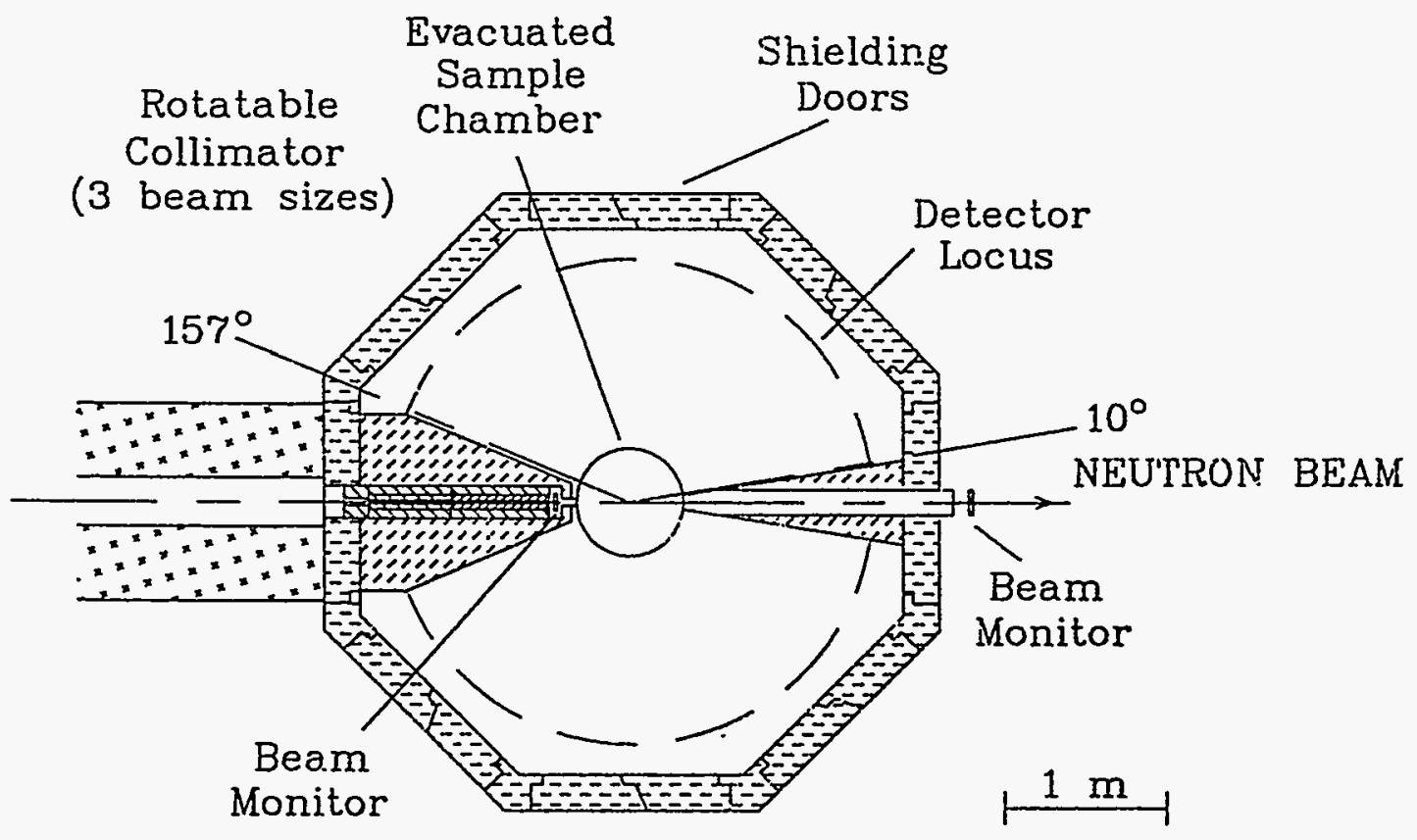

Figure V.2.1-1 Plan View of the SEPD Scattering Flight Path at IPNS Upgrade. 
Table V.2.1-1 SEPD and GPPD Parameters at IPNS Upgrade

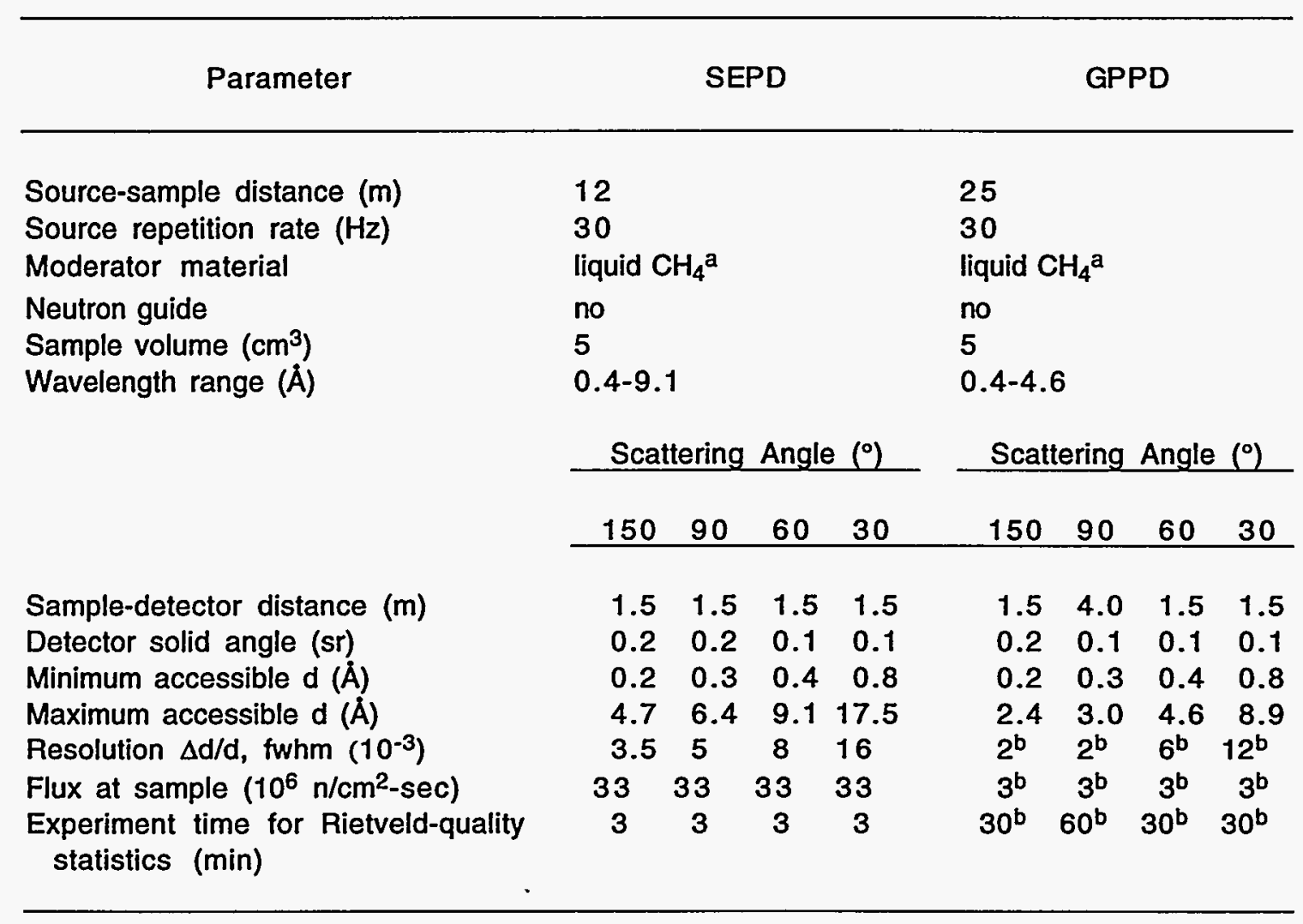

a Or room-temperature $\mathrm{H}_{2} \mathrm{O}$.

b With incident-beam Soller collimators. 


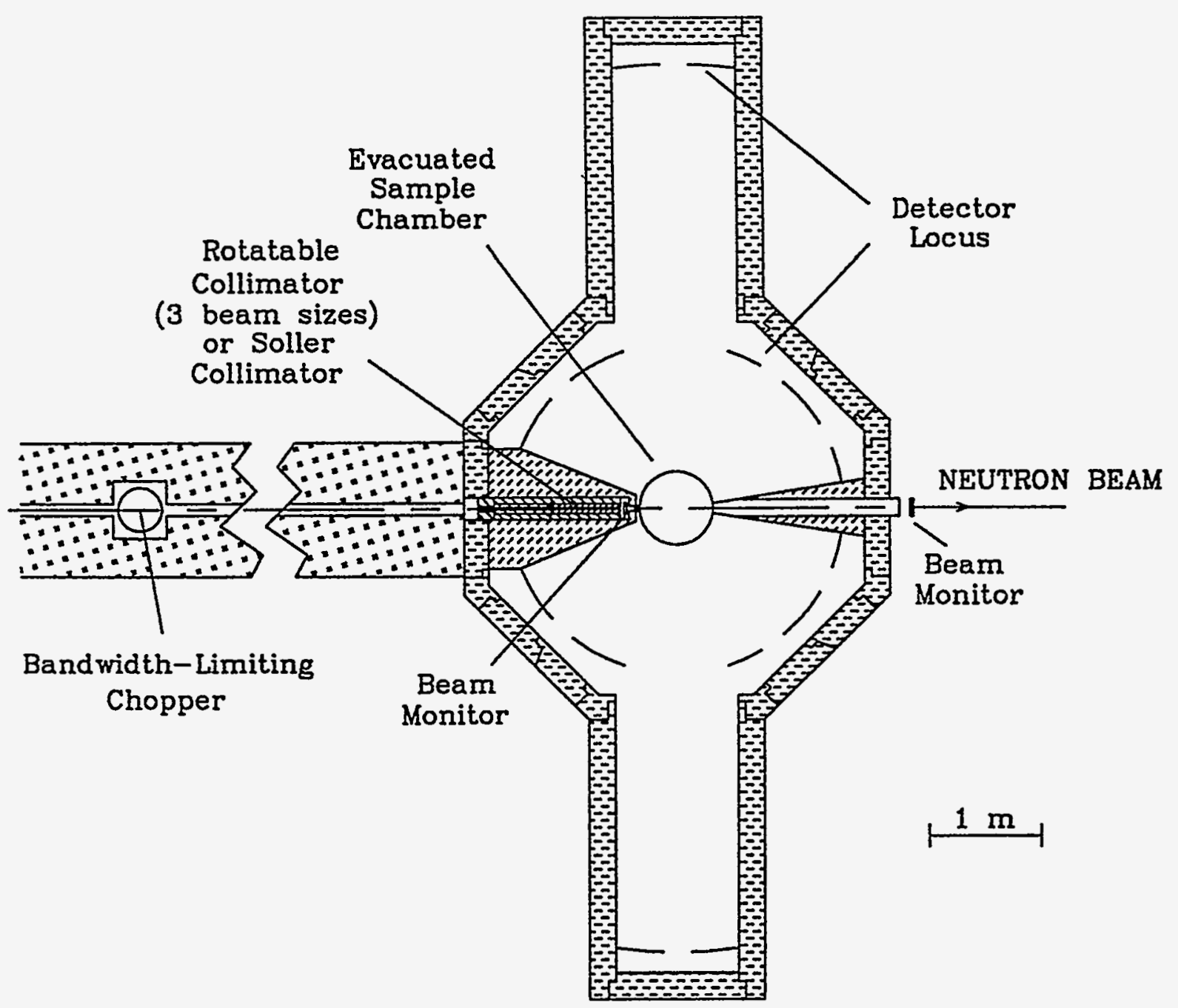

Figure V.2.1-2 GPPD Configuration at IPNS Upgrade, Showing Increased $90^{\circ}$ Scattering Path Length. 


\subsubsection{New Reference Set Instruments}

\subsubsection{HRPD — High-Resolution Powder Diffractometer}

The HRPD has an incident flight path of $50 \mathrm{~m}$ and is designed for state-of-the-art highresolution powder diffraction for Rietveld refinement. Instruments optimized for such work can be used for other purposes, such as the solution of unknown structures from powder data. The HRPD employs multiple detector arrays covering a wide range of scattering angles, ${ }^{2}$ so the sample-detector flight path looks like a somewhat larger version of the SEPD flight path shown in Figure V.2.1-1. The detectors are on a 2-m radius rather than $1.5 \mathrm{~m}$. The positions of the detectors around this 2-m radius are shown schematically in Figure V.2.1-3. Parameters for the instrument are given in Table V.2.1-2. The instrument is intended to be operated at a repetition rate of $30 \mathrm{~Hz}$, with choppers to select an appropriate band of wavelengths at the highestintensity part of the spectrum. A room-temperature moderator is advantageous because it gives a higher neutron flux over the relatively narrow wavelength range of interest.

Data from detector arrays at several different scattering angles are combined to provide full diffraction data. The resolution at each angle is adequate to resolve Bragg peaks for typical structures. The chopper phasing can be varied to access the desired d-spacings in backscattering, or at a larger scattering angle than for normal operation, if higher resolution at long $d$-spacing is required for a particular experiment. A guide tube is used to significantly increase the flux on the sample. It is important not to increase the horizontal angular divergence beyond certain limits, to maintain a reasonable resolution at lower scattering angles. Two approaches can be used to satisfy this criterion. A guide tube with only top and bottom surfaces can be employed. Alternatively, a four-sided guide tube can be used with Soller collimators before the sample. The latter approach has the advantage that the collimators can be removed for experiments where high flux is desired at the expense of resolution.

\subsubsection{RSD - Residual Stress Diffractometer}

A powder diffractometer dedicated to residual stress measurements satisfies strict design criteria directly related to the three main types of experiments: (1) bulk material measurements such as reactor-vessel weldments or sections of airplane wings, (2) temperature- or pressuredependent residual stress changes in small samples of composites, metal alloys, etc., and (3) triaxial stress-tensor measurements in complex mixed-phase materials. 


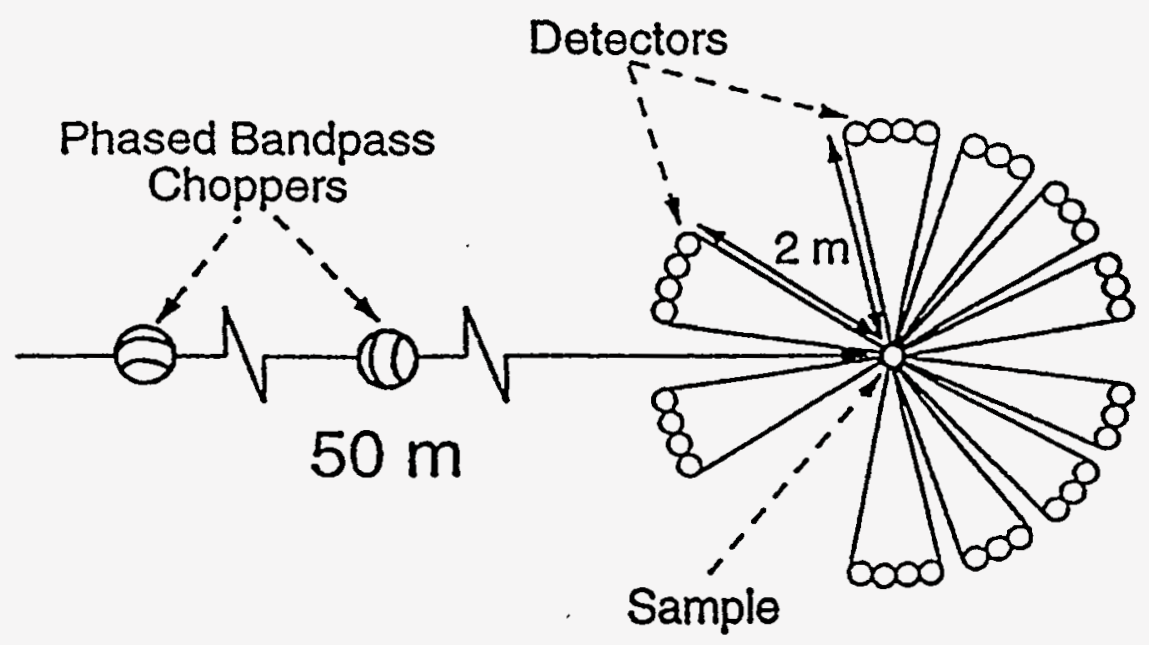

Figure V.2.1-3 Schematic Representation of the Detector Positioning for the HRPD. 
Table V.2.1-2 HRPD Parameters at IPNS Upgrade

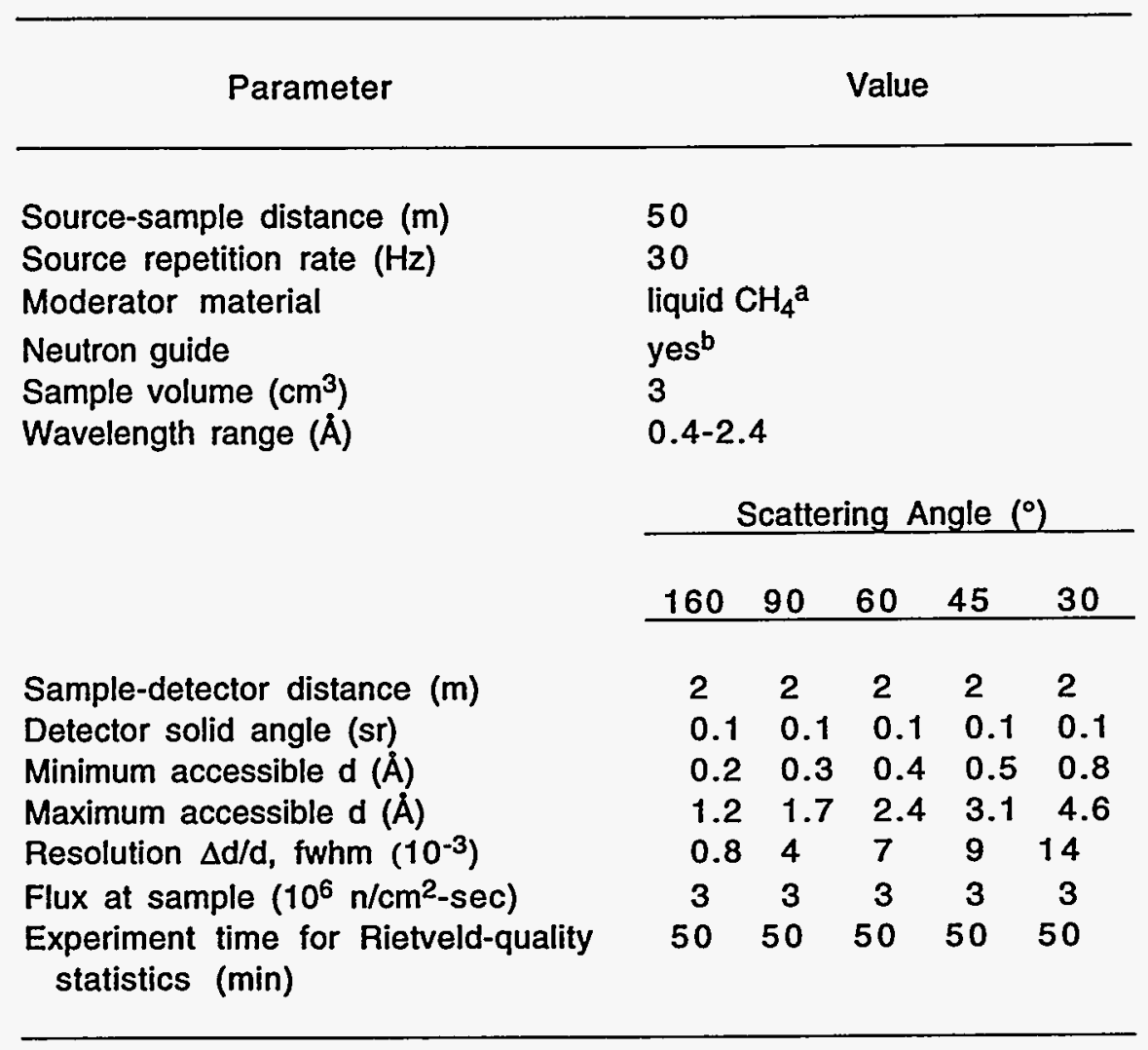

a Or room-temperature $\mathrm{H}_{2} \mathrm{O}$.

b With Soller collimators. 
Bulk material measurements involve sending large samples stepwise through the beam with accurate determination of lattice spacings at each step. Ideal sampling volumes are on the order of $2 \times 2 \times 2 \mathrm{~mm}^{3}$ for typical stress gradients. This requires a $2 \times 2-\mathrm{mm}^{2}$ incident beam, with a 2-mm defining slit in the scattered beam, or other comparable means of defining the sampling volumes. Neutron fluxes at the present pulsed sources are insufficient for this. For instance, residual stress measurements on the GPPD at IPNS require a $50.8 \times 2 \times 2-\mathrm{mm}^{3}$ volume, with the long axis in the direction of minimal gradient. The RSD has a 12-m source-sample distance and concentrates on $90^{\circ}$ scattering using a large, two-dimensional (2D) position-sensitive detector and Soller collimators in the scattered beam. This arrangement, shown schematically in Figure V.2.1-4, permits the detector to simultaneously sample a number of regularly shaped sampling volumes for all crystallographic reflections, thus giving a much more complete map of the stress distribution.

As is indicated in Table V.2.1-3, this instrument has the capability of collecting data sets from a number of $2 \times 2 \times 2-\mathrm{mm}^{3}$ sampling volumes in $\sim 15 \%$ of the time it now takes to collect data from a single sampling volume 25 times as large. The $\Delta \mathrm{d} / \mathrm{d}$ resolution is somewhat degraded relative to the current GPPD because of the shorter source-sample distance, but this resolution can be recovered by using incident-beam Soller collimators. If the incident-beam Soller collimators are used, data of comparable quality can be collected from a set of slightly larger sampling volumes $\left(3 \times 3 \times 3 \mathrm{~mm}^{3}\right)$ in roughly the same time.

The residual stress measurements on high-tech composites are quite different from those in bulk materials, and require the highest possible resolution at $\pm 150^{\circ}$ and $\pm 90^{\circ}$, without significant loss in turn-around time. The current reference standard is a $50.8 \times 6.4 \times 6.4-\mathrm{mm}^{3}$ metal-ceramic composite, for which $30 \mathrm{~min}$ is required on the GPPD at IPNS for good statistics. The RSD includes a second sample position at $25 \mathrm{~m}$ from the source, in order to provide the necessary $\pm 90^{\circ}$ resolution. Incident- and scattered-beam $\left( \pm 90^{\circ}\right)$ Soller collimators and banks of detectors at $\pm 150^{\circ}$ and $\pm 90^{\circ}$ are also required, as shown schematically in Figure V.2.1-4. This gives dramatically improved resolution of $3 \times 10^{-3}$ at $\pm 90^{\circ}$, while also decreasing the turn-around time to less than $10 \mathrm{~min}$.

Composites or other materials that are strongly textured or have stress states that are highly anisotropic require simultaneous measurement of the stresses in multiple, mutually orthogonal directions. Three 2D position-sensitive detectors are placed around the 25-m sample position at scattering angles of $70.5^{\circ}$, such that the sampled scattering vectors are mutually perpendicular (see Figure V.2.1-4). The performance characteristics are essentially the same as those indicated in Table V.2.1-3 for the conventional-detector configuration at the 25-m position.

This instrument is constructed to provide ready access for large industrial-component samples. 


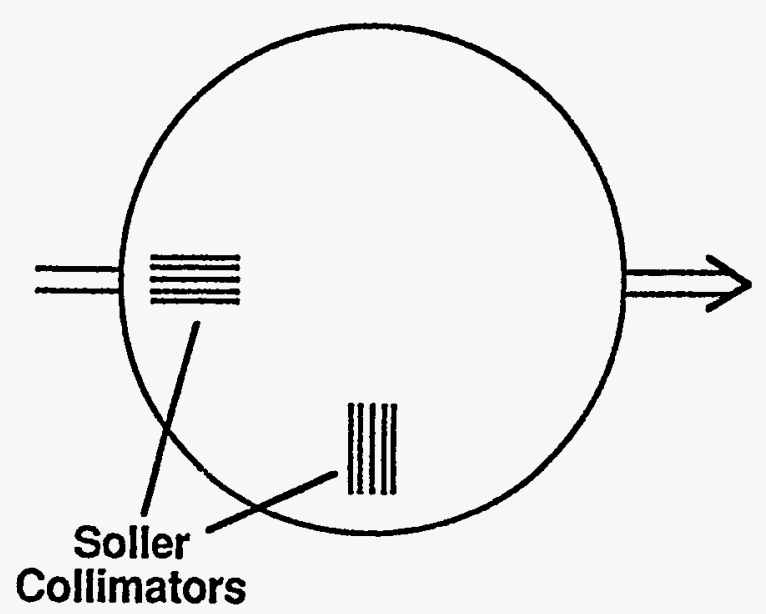

12 m Position

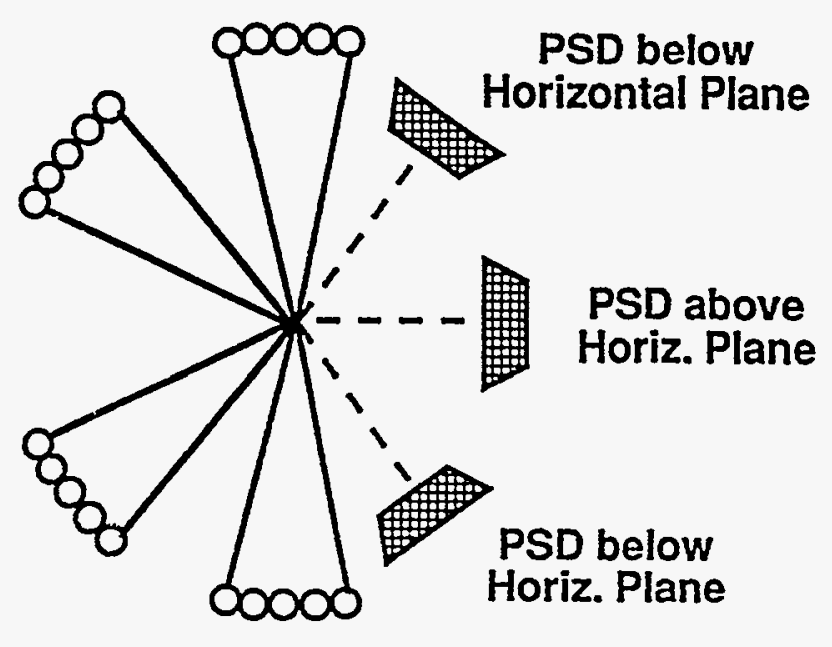

25 m Position

Figure V.2.1-4 Schematic Representation of the RSD, Showing the 12-m and 25-m Sample Positions. 
Table V.2.1-3 Parameters for the RSD

\begin{tabular}{|c|c|c|}
\hline \multirow[b]{2}{*}{ Parameter } & \multicolumn{2}{|c|}{ Source-Sample Distance $(\mathrm{m})$} \\
\hline & 12 & 25 \\
\hline $\begin{array}{l}\text { Source repetition rate }(\mathrm{Hz}) \\
\text { Moderator material }\end{array}$ & $\begin{array}{l}30 \\
\text { liquid } \mathrm{CH}_{4}{ }^{\mathrm{a}}\end{array}$ & $\begin{array}{l}30 \\
\text { liquid } \mathrm{CH}_{4}^{\mathrm{a}}\end{array}$ \\
\hline Sampling volume $\left(\mathrm{mm}^{3}\right)$ & $2 \times 2 \times 2$ & $2 \times 2 \times 2$ \\
\hline Wavelength range $(\AA)$ & 0.4-9 & $0.4-4.5$ \\
\hline Scattering angle $\left({ }^{\circ}\right)$ & 90 & $70.5, \pm 90$, and \pm 150 \\
\hline Sample-detector distance (m) & 1.5 & $1.5-3$ \\
\hline Resolution $\Delta \mathrm{d} / \mathrm{d}$, fwhm $\left(10^{-3}\right)$ & 5.5 & 3 \\
\hline Experiment time (min) & 50 & 9 \\
\hline
\end{tabular}

a Or room-temperature $\mathrm{H}_{2} \mathrm{O}$. 


\subsubsection{VSPD - Very-Small-Sample Powder Diffractometer}

The solid-state science community has been hampered by sample-size limitations. Typically, chemists produce materials in 10-100 mg quantities, which are too small for neutron scattering measurements at most facilities. The very-small-sample powder diffractometer addresses this situation. This instrument is located $12 \mathrm{~m}$ from the source, and has a $0.75-\mathrm{m}$ sample-to-detector distance and 0.635 -cm-diameter detectors. The secondary flight path is similar to a reduced-size version of the SEPD flight path shown in Figure V.2.1-1. The detectors are placed at $0.75 \mathrm{~m}$ to increase the solid angle by a factor of four compared to the current SEPD for the same area coverage of detectors. Some of the detectors are placed above and below the scattering plane on Debye-Scherrer cones in order to cover this solid angle. Focusing techniques in the incident beam provide further increases in data rate. The 0.635 -cm-diameter detectors match the 0.635-cm-diameter sample size. Monte Carlo simulations for this configuration indicate that the resolution at $\pm 150^{\circ}$ and $\pm 90^{\circ}$ is degraded less than $10 \%$ relative to that of the SEPD at IPNS Upgrade (see Table V.2.1-1). This combination of resolution and data rate allow Rietveld-quality data sets from 100-mg samples in $\sim 70 \mathrm{~min}$. Larger-diameter samples can also be used in this instrument, but the resolution is noticeably degraded, as much as $20-30 \%$ for 1.27-cm-diameter samples.

\subsubsection{Other Possibilities}

These instruments are not in the reference set and will probably not be among the initial complement at IPNS Upgrade. It is expected that instruments of these types will evolve and might be added at a later date.

\subsubsection{LFHRPD - Low-Frequency High-Resolution Powder Diffractometer}

A diffractometer having the same parameters as the HRPD can be located at the $10-\mathrm{Hz}$ target station rather than the $30-\mathrm{Hz}$ station. In this case, time-averaged incident fluxes are a factor of two lower than those indicated in Table V.2.1-2, but the maximum wavelength is tripled to $\sim 7 \AA$. Requirements for bandwidth-limiting choppers are considerably relaxed. This instrument can be considered either as an alternative to the HRPD or as a supplement to the HRPD if the demand is sufficiently great to support two instruments in this resolution range.

\subsubsection{LWPD — Long-Wavelength Powder Diffractometer}

This diffractometer is optimized to use cold neutrons with $\lambda>2 \AA$ to study magnetic structures and crystal structures with large unit cells over the range $2 \AA<d<10 \AA$ while benefiting from the high resolution afforded by backscattering. This instrument views a liquid

hydrogen moderator. It is designed for high count rates, fast enough for real-time studies in favorable cases such as real-time studies of absorption in zeolites. 


\subsubsection{PNPD — Polarized-Neutron Powder Diffractometer}

This diffractometer has parameters similar to the SEPD. It has a source-sample distance of $12 \mathrm{~m}$ and a sample-detector distance of $1.5 \mathrm{~m}$. It is outfitted with detectors at a number of scattering angles $\left( \pm 150^{\circ}, \pm 90^{\circ}, \pm 60^{\circ}\right.$, etc.), thus allowing access to similar ranges of d-spacing. In addition, either nickel guides with a critical angle of $0.1^{\circ}$ per $\AA$ or supermirrors with a critical angle of $0.3^{\circ}$ per $\AA$ are used to polarize the incident beam. If full polarization analysis is desired, supermirrors can be placed in the scattered beams as well. Soller collimators can be used to enhance resolution. Polarized neutrons and polarization analysis are useful in the study of magnetic structures. They can also be used to discriminate against incoherent scattering backgrounds from hydrogenous samples, considerably extending the types of samples that can be studied.

\subsubsection{UHPD — Ultra-High-Pressure Diffractometer}

This instrument utilizes a press to apply pressures in excess of $250 \mathrm{kbar}$ to sample sizes of a few cubic millimeters. Carefully designed gaskets between the press anvils allow neutrons in and out of the sample. Scattered neutrons can be detected at only a few discrete angles because of the collimation provided by the anvils, so this technique only works on a TOF instrument. Although the UHPD extends neutron powder diffraction capability to a previously inaccessible pressure regime, the small beams and limited solid angle for detection make this a low-data-rate instrument.

\subsubsection{UHRPD — Ultra-High-Resolution Powder Diffractometer}

This instrument is designed to achieve a backscattering resolution of $1-3 \times 10^{-4}$ and requires an incident flight path of $\sim 100 \mathrm{~m}$. Experience with the HRPD at ISIS has shown that such high resolution is advantageous for the investigation of many systems. ${ }^{3}$ During the initial stages of operation of that instrument many samples were not of sufficient quality to make use of its resolution. Scientists have now improved the quality of their samples so that many samples have intrinsic peak widths comparable with the ISIS-HRPD instrumental resolution. In such cases, structure refinements of an unmatched quality can be achieved, and signal-to-noise ratios are improved enough to allow the study of hydrogenous samples by powder techniques. Strains, particle-size distributions, stacking faults, and twin boundaries in samples can be studied by using such an instrument.

\subsubsection{Powder Diffractometer Summary}

The diffractometers described in Sections V.2.1.1 and V.2.1.2 are taken as a reference set that spans the range of capabilities required for most anticipated powder diffraction experiments. These are the "workhorse" instruments, and are almost certain to be included among the initial 24 instruments. Their capabilities are summarized in Table V.2.1-4. Particularly exciting are the 
Table V.2.1-4 Reference Set of TOF Powder Diffractometers at IPNS Upgrade

\begin{tabular}{llccc}
\hline Instrument & \multicolumn{1}{c}{ Application/Special Feature } & $\begin{array}{c}\text { Range for } \\
\mathrm{d}(\AA)\end{array}$ & $\begin{array}{c}\text { Best } \\
\Delta \mathrm{d} / \mathrm{d}(\%)\end{array}$ & $\begin{array}{c}\text { Measurement } \\
\text { Time (min) }\end{array}$ \\
\hline VSPD & Very small samples (10-100 mg) & $0.2-17$ & 0.35 & 70 \\
SEPDa & High intensity & $0.2-17$ & 0.35 & 3 \\
GPPD & Medium resolution (excellent at $\left.90^{\circ}\right)$ & $0.2-9$ & 0.2 & $10-60$ \\
HRPD & High resolution & $0.2-5$ & 0.08 & 50 \\
RSD & $\begin{array}{l}\text { Residual stress } \\
12-m \text { position }\end{array}$ & & & \\
& $25-m$ position & $0.2-3$ & 0.30 & 10 \\
\hline
\end{tabular}

a Transferred from IPNS with little change.

b Transferred from IPNS with flight-path modified. 
small-sample capabilities, the very high resolution available at $90^{\circ}$ which is essential for much of the work in specialized sample environments, and the short data-collection times, which make possible real-time studies of many types of systems.

A number of additional, more specialized, diffractometers are outlined in Section V.2.1.3. These instruments will probably not be among the initial complement at IPNS Upgrade, but might be added at a later date.

\subsection{SMALL-ANGLE DIFFRACTOMETERS}

\subsubsection{Transfer of Existing IPNS Instruments}

\subsubsection{SAND — General-Purpose Small-Angle Neutron Diffractometer}

The SAND is a new small-angle diffractometer at IPNS, shown in plan view in Figure V.2.2-1. As can be seen in the figure, the sample chamber is large, making it possible to accommodate a wide variety of ancillary equipment. Table V.2.2-1 lists parameters of the SAND at IPNS and IPNS Upgrade. The SAND has two automatically interchangeable sets of converging multiple-aperture collimators, so it can operate in either a low-resolution mode or a high-resolution mode to allow optimal trade-off between Q-range and intensity for a given experiment. It also has a cooled $\mathrm{MgO}$ filter to remove fast neutrons from the incident beam. This filter can be removed if a $t_{0}$ chopper, as described in Section V.3.1, is to be used. A chopper is also provided to eliminate most of the background caused by delayed neutrons. A $40 \times 40-\mathrm{cm}^{2}$ area detector and a bank of 65 linear-position-sensitive detectors (LPSDs) with a total active area $\sim 60 \times 70 \mathrm{~cm}^{2}$ are used.

An important feature of the SAND is the extremely wide Q-range it covers, even when restricted to wavelengths above the Bragg cutoff for crystalline samples. This entire Q-range can be measured with only one instrument setting. This is particularly important for the study of anisotropic scatterers and for kinetic or other time-dependent studies. The bank of LPSDs extends the maximum scattering angle up to $36^{\circ}$, making a huge improvement in the counting statistics in the higher-Q portion of the data. The SAND is exceptionally good for studies that can benefit from the wide Q-range. 


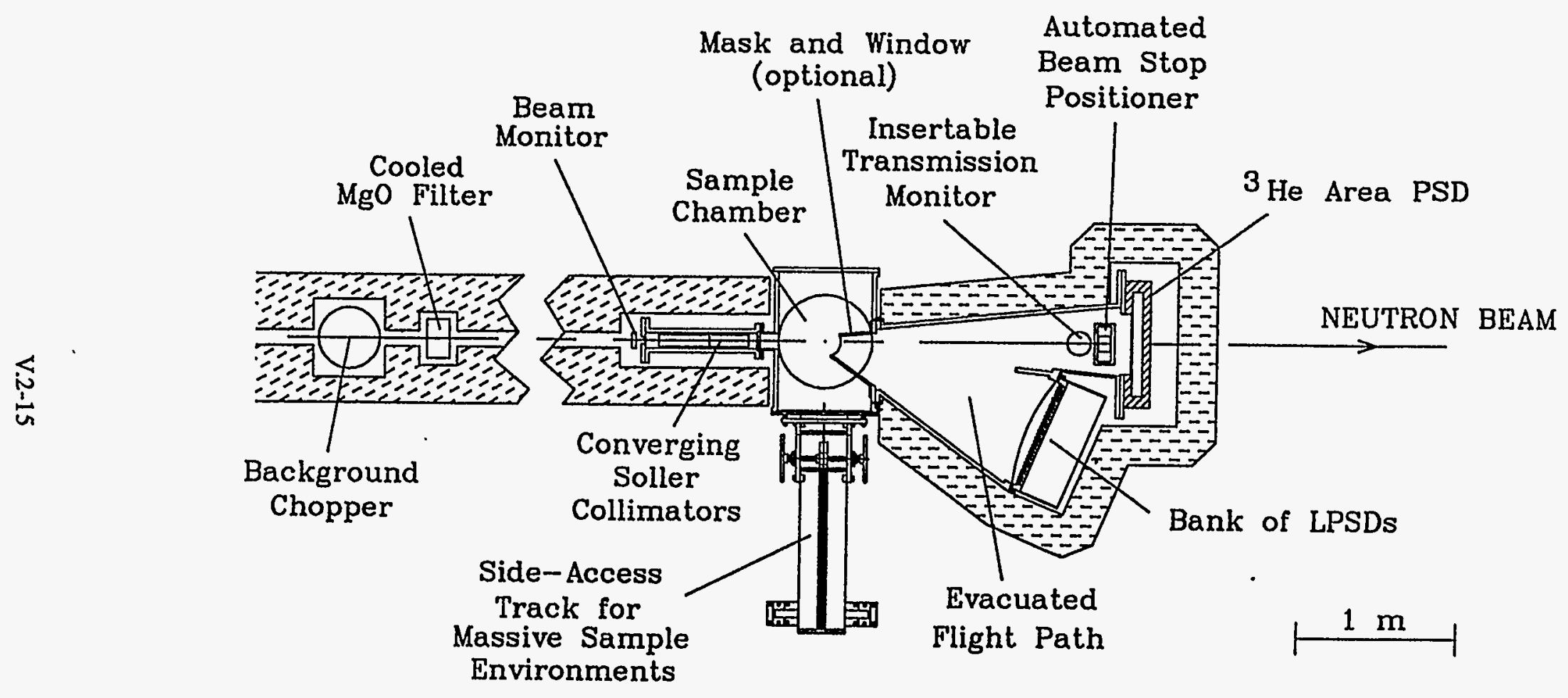

Figure V.2.2-1 Plan View of the SAND at IPNS Upgrade. 
Table V.2.2-1 Parameters for the SAND

\begin{tabular}{|c|c|c|}
\hline Parameter & IPNS & IPNS Upgrade \\
\hline Moderator & decoupled liquid $\mathrm{H}_{2}$ & coupled liquid $\mathrm{H}_{2}$ \\
\hline Source-to-sample distance & $9.0 \mathrm{~m}$ & $12 \mathrm{~m}$ \\
\hline Sample-to-area-detector distance & $2.0 \mathrm{~m}$ & \\
\hline Sample-to-LPSD distance & $1.524 \mathrm{~m}$ & \\
\hline Beam size at sample & $2.0-\mathrm{cm}$ diameter & $\leq 2$-cm diameter \\
\hline \multicolumn{3}{|l|}{ Area detector } \\
\hline active area & $40 \times 40 \mathrm{~cm}^{2}$ & $\mathbf{a}$ \\
\hline active thickness & $2.5 \mathrm{~cm}$ & $a$ \\
\hline resolution & 4-6 $\mathrm{mm}$ & a \\
\hline fill & $\begin{array}{l}2.6 \text { atm }{ }^{3} \mathrm{He} \text { plus } \\
1.4 \mathrm{~atm} \mathrm{CF}_{4}\end{array}$ & $\begin{array}{l}\mathbf{a} \\
\mathbf{a}\end{array}$ \\
\hline encoding & rise time, $256 \times 256$ & $\mathbf{a}$ \\
\hline \multicolumn{3}{|l|}{ LPSDs } \\
\hline active volume per detector & $1.1 \mathrm{~cm}$ diameter $\times 60 \mathrm{~cm}$ long & \\
\hline number of detectors & & \\
\hline resolution & $1-2 \mathrm{~cm}$ along detector & \\
\hline fill & $\sim 10 \mathrm{~atm}^{3} \mathrm{He}$ & \\
\hline encoding & $\begin{array}{l}\text { charge division, } 64 \text { segments/ } \\
\text { detector }\end{array}$ & \\
\hline max. scattering angle & $36^{\circ}$ & \\
\hline \multicolumn{3}{|l|}{$\begin{array}{l}\text { Focusing collimators, converging on } \\
\text { area detector }\end{array}$} \\
\hline coarse & $0.0034 \mathrm{rad}$ fwhm & \\
\hline fine & $0.0014 \mathrm{rad}$ fwhm & \\
\hline Wavelength range & $\begin{array}{l}\text { 1-14 } \AA \text { (5-14 } \AA \text { for } \\
\text { crystalline samples })\end{array}$ & $\begin{array}{c}-1-20 \AA \quad(-5-20 \AA \text { for } \\
\text { crystalline samples })\end{array}$ \\
\hline \multicolumn{3}{|r|}{ 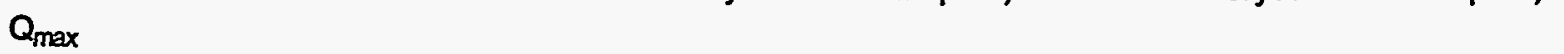 } \\
\hline$\lambda_{\min }=1.0 \AA$ (noncrystalline) & $>2.0 \AA^{-1}$ & \\
\hline$\lambda_{\min }=5.0 \AA$ (crystalline) & $\sim 0.6 \AA^{-1}$ & \\
\hline \multicolumn{3}{|l|}{$Q_{\min }$} \\
\hline coarse collimation & $\sim 0.004 \AA^{-1}$ & $-0.0028 \AA^{-1}$ \\
\hline fine collimation & $\sim 0.002 \AA^{-1}$ & $\sim 0.0014 \AA^{-1}$ \\
\hline \multicolumn{3}{|l|}{ Measurement time } \\
\hline coarse collimation & $0.5-17 \mathrm{~h}$ & $0.01-0.3 \mathrm{~h}$ \\
\hline fine collimation & $3-100 h$ & $0.06-2 \mathrm{~h}$ \\
\hline
\end{tabular}

a New detector with much faster encoding is required for the low-resolution mode. 
The SAND views a coupled liquid $\mathrm{H}_{2}$ moderator on the $10-\mathrm{Hz}$ target. The moderator-todetector distance is $\sim 12 \mathrm{~m}$. The useful wavelength is $\sim 1-20 \AA$, limited on the short-wavelength end by the incident-beam filter and on the long-wavelength end by the low neutron flux. Frame overlap occurs for wavelengths $>30 \AA$, so frame-overlap is not a significant background problem. Table V.2.2-1 indicates which instrument parameters are changed at IPNS Upgrade. The $300-400-\mu$ s pulse-width of coupled $\mathrm{H}_{2}$ moderators gives $\Delta \lambda / \lambda \leq 10 \%$ for the wavelength range of this instrument and so does not have a significant effect on the instrument resolution.

At IPNS Upgrade, the SAND count rate is increased by a factor of $\simeq 56$ due to a factor of 25 from the improvement in source current and a factor of 4 from the improved moderator, reduced by $(9 / 12)^{2}$ due to the increased flight path. It is possible to operate SAND with an even higher resolution and still obtain reasonable data rates at IPNS Upgrade.

Instantaneous count rates on the SAND area detector in the low-resolution mode are estimated to be as high as $\simeq 150,000$ counts/sec at IPNS Upgrade. The present SAND detector is not suitable at such high data rates. In the high-resolution mode, data rates are reduced by a factor of $\sim 6$, so this detector is adequate for use at IPNS Upgrade for most samples in this mode.

A higher-speed area detector will result in enhanced operation in the high-resolution mode in some cases and will permit operation in the low-resolution mode to obtain the higher data rates required for some kinetic studies. The development of a higher-resolution area detector with the same effective area coupled with the development of a tighter collimation system is necessary to allow the low-Q limit of the instrument to be significantly improved, so that larger structures can be studied.

\subsubsection{New Reference Set Instruments}

\subsubsection{HRSAND — High-Resolution Small-Angle Neutron Diffractometer}

This small-angle diffractometer provides a lower $Q_{\min }$ and better resolution than that given by the SAND. Based on an analysis of intensities, a $Q_{\min }$ of $\sim 0.0005 \AA^{-1}$ provides acceptable data rates, but this is essentially the limit to which the multiple-converging-aperture TOF small-angle-diffractometer concept can be pushed at IPNS Upgrade. A schematic layout of an instrument designed to achieve this $\mathrm{Q}_{\min }$ is shown in Figure V.2.2-2, and Table V.2.2-2 gives some of the parameters of this instrument. The sample chamber is identical to that on the SAND to provide easy interchange of ancillary equipment.

A gas-proportional-counter area detector is used because of the excellent background characteristics of such detectors. The sizes of gas detectors with high. position resolution are limited by technology. Current developments in microstrip gas detector technology 4,5 show promise of being able to provide the desired performance, but if they cannot, the detector size may have to be reduced in order to achieve the desired resolution. 


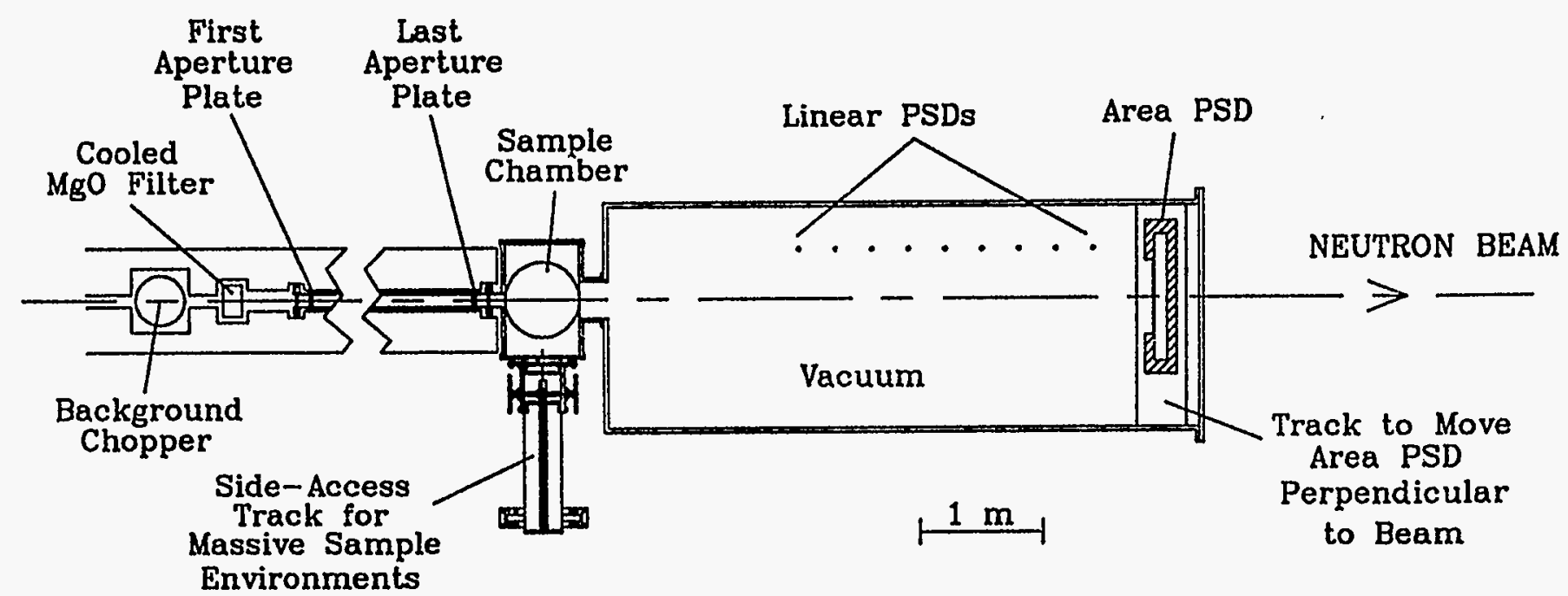

Figure V.2.2-2 Schematic Representation of the HRSAND. 
Table V.2.2-2 Parameters for the HRSAND

\begin{tabular}{|c|c|}
\hline Parameter & Value \\
\hline Moderator & coupled liquid $\mathrm{H}_{2}$ \\
\hline Source repetition rate $(\mathrm{Hz})$ & 10 \\
\hline Source-to-sample distance $(\mathrm{m})$ & 20 \\
\hline Collimation & converging multiple-aperture \\
\hline Source-to-first-collimator-aperture distance $(\mathrm{m})$ & 6 \\
\hline Diameter of first apertures $(\mathrm{cm})$ & 0.784 \\
\hline Source-to-last-collimator-aperture distance $(m)$ & 19.5 \\
\hline Diameter of last apertures $(\mathrm{cm})$ & 0.227 \\
\hline Number of converging aperture channels & $20-40$ \\
\hline Sample-to-area-detector distance (m) & 5 \\
\hline Beamstop diameter $(\mathrm{cm})$ & 0.84 \\
\hline Wavelength range $(\dot{A})$ & $1-15^{a}$ \\
\hline Beam diameter at sample $(\mathrm{cm})$ & 2.0 \\
\hline Required resolution of area detector $(\mathrm{mm})$ & -2 near beam ${ }^{b}$ \\
\hline Approximate size of area detector $(\mathrm{cm})$ & 60 \\
\hline \multicolumn{2}{|l|}{ Maximum scattering angle on area detector $\left({ }^{\circ}\right)$} \\
\hline on-axis & 3.4 \\
\hline off-axis & 6.8 \\
\hline Scattering angles for LPSDs $\left({ }^{\circ}\right)$ & $\sim 5-15$ \\
\hline$Q_{\min }\left(\AA^{-1}\right)$ & $\sim 0.0005$ \\
\hline \multicolumn{2}{|l|}{$Q_{\max }\left(\dot{A}^{-1}\right)$} \\
\hline area detector on-axis & $\sim 0.07-0.2$ \\
\hline area detector off-axis & $\sim 0.15-0.4$ \\
\hline Data rate & roughly like present SAD \\
\hline
\end{tabular}

a 5-15 $\AA$ for crystalline samples.

b Can be worse elsewhere. 
The incident multiple-aperture converging collimation system is designed to account for the gravitational drop of the longer-wavelength neutrons. The sample-detector distance is sufficiently short that the gravitational drop of the scattered neutrons is not a significant problem. A coarser collimator can be substituted when the full $\mathrm{Q}$ resolution is not required. A background-suppression chopper is provided. The maximum wavelength is $15.8 \AA$ before frameoverlap occurs for the $25-\mathrm{m}$ total distance on the $10-\mathrm{Hz}$ source. Wavelengths longer than this are removed by the background-suppression chopper. Because this instrument is intended to complement the SAND, no attempt is made to duplicate the extensive high-Q coverage obtainable with the latter instrument. However, many experiments focusing on the low- $\mathrm{Q}$ region can benefit from some coverage at higher Q, so a series of LPSDs is placed along one or both sides of the flight path to supplement the high-scattering-angle data, as shown schematically in Figure V.2.2-2.

\subsubsection{SPSAND - Special-Purpose Small-Angle Neutron Diffractometer}

One beamline viewing the coupled $\mathrm{H}_{2}$ moderator on the $10-\mathrm{Hz}$ source is reserved for special projects in small-angle diffraction. These can be either special experiments requiring specific instrument configurations not found on the other small-angle diffractometers or projects aimed at developing new small-angle scattering techniques. Among the possibilities are experiments using polarized neutrons, resonance small-angle scattering, ${ }^{6}$ the study of inelasticity effects in small-angle scattering, and the development of techniques for reaching even lower $Q_{\text {min. }}$.

This instrument is typically configured for one type of study for one or more months at a time, then reconfigured for a different study. It is not part of the user program in the usual sense but is open to proposals for extended use in specific configurations. Short experiments with a special configuration can be accommodated whenever that configuration is set up for other purposes.

The instrument is modular and very flexible. It includes shielded areas for choppers, a prompt-pulse-suppression chopper and other specialized choppers, a modular beamline and shielding, easily changeable collimation, one or more area detectors, and a large reserved section of floor space extending to $\sim 40 \mathrm{~m}$ from the source. Development of some components are deferred until the need arises.

\subsubsection{Other Possibilities}

These instruments are not in the reference set and will probably not be among the initial complement at IPNS Upgrade. It is expected that instruments of these types will evolve and might be added at a later date. 


\subsubsection{QSSAND - Quasi-Steady-State Small-Angle Neutron Diffractometer}

The high time-averaged cold-neutron flux available from the large coupled liquid $\mathrm{H}_{2}$ moderator on the $30-\mathrm{Hz}$ target station makes it practical to treat this as a QSS source of cold neutrons. A traditional reactor-type small-angle diffractometer can be placed on a beamline viewing this moderator. The $30-\mathrm{m}$ small-angle diffractometers ${ }^{7}$ operating at the National Institute of Standards and Technology (NIST) are state-of-the-art models for such an instrument. A velocity selector is used to define a variable wavelength band of $7-30 \% \Delta \lambda / \lambda$ that is centered at the desired wavelength. Removable sections of the incident-beam guide tube are used to vary the incident-beam divergence, and a large, movable area detector on a track is used to detect the scattered neutrons with the desired resolution. An ILL-type area-position-sensitive detector capable of $\sim 20,000$ counts/sec and having a resolution of $\sim 1 \mathrm{~cm}$ and an overall size of $\sim 65 \times 65 \mathrm{~cm}^{2}$ is used. The expected Q-range is 0.001 to $0.6 \AA^{-1}$. The pulses from this moderator are $\sim 1 \mathrm{~ms}$ long. In normal operation the counting electronics are gated on only during the time neutrons of the desired wavelength that are elastically scattered from the sample reach the detector: This provides discrimination against background and against most of the neutrons inelastically scattered from the sample. The electronics can also be set to count in broad timeslices over a much larger time window, to allow detailed concurrent measurement of both the elastically and inelastically scattered neutrons. This latter capability is useful in the study of samples containing hydrogen or deuterium, where inelastic scattering effects are known to be substantial. This energy-discrimination ability comes at essentially no cost in intensity, unlike the case of steady-state small-angle neutron scattering instruments. The pulsed nature of the source also makes possible measurements using pulsed sample environments synchronized to the data collection, with little or no loss in intensity.

\subsubsection{DCSAND - Double-Perfect-Crystal Bonse-Hart Small-Angle Neutron Diffractometer}

This instrument extends the minimum $Q$ that can be accessed in small-angle-scattering experiments down to $\sim 10^{-5} \AA^{-1}$ in some cases. It is constructed on one of the quasi-steady-state (QSS) beamlines, looking at a fully-coupled liquid $\mathrm{H}_{2}$ moderator on the $30-\mathrm{Hz}$ source. It can be easily placed upstream from the Cold-Neutron Quasi-Steady-State Triple-Axis Spectrometer (QSTAXC) (Section V.2.6.2.3) by using a premonochromator crystal to select a band of wavelengths from the guide serving the triple-axis spectrometer. The DCSAND is modeled after similar instruments at reactor sources, 8 and is shown schematically in Figure V.2.2-3. The perfect-crystal monochromator and analyzer select not only a very precise wavelength, but also a precise direction of the neutron beam. The intensity scattered at different angles is scanned by rotating the analyzer crystal through very small angles of typically less than one minute of arc.

Such instruments have been operated successfully at low-flux reactors, so there is no doubt that such an instrument can be successfully operated at IPNS Upgrade. The time-structure of this QSS source allows the detection electronics to be gated to minimize backgrounds (duty 


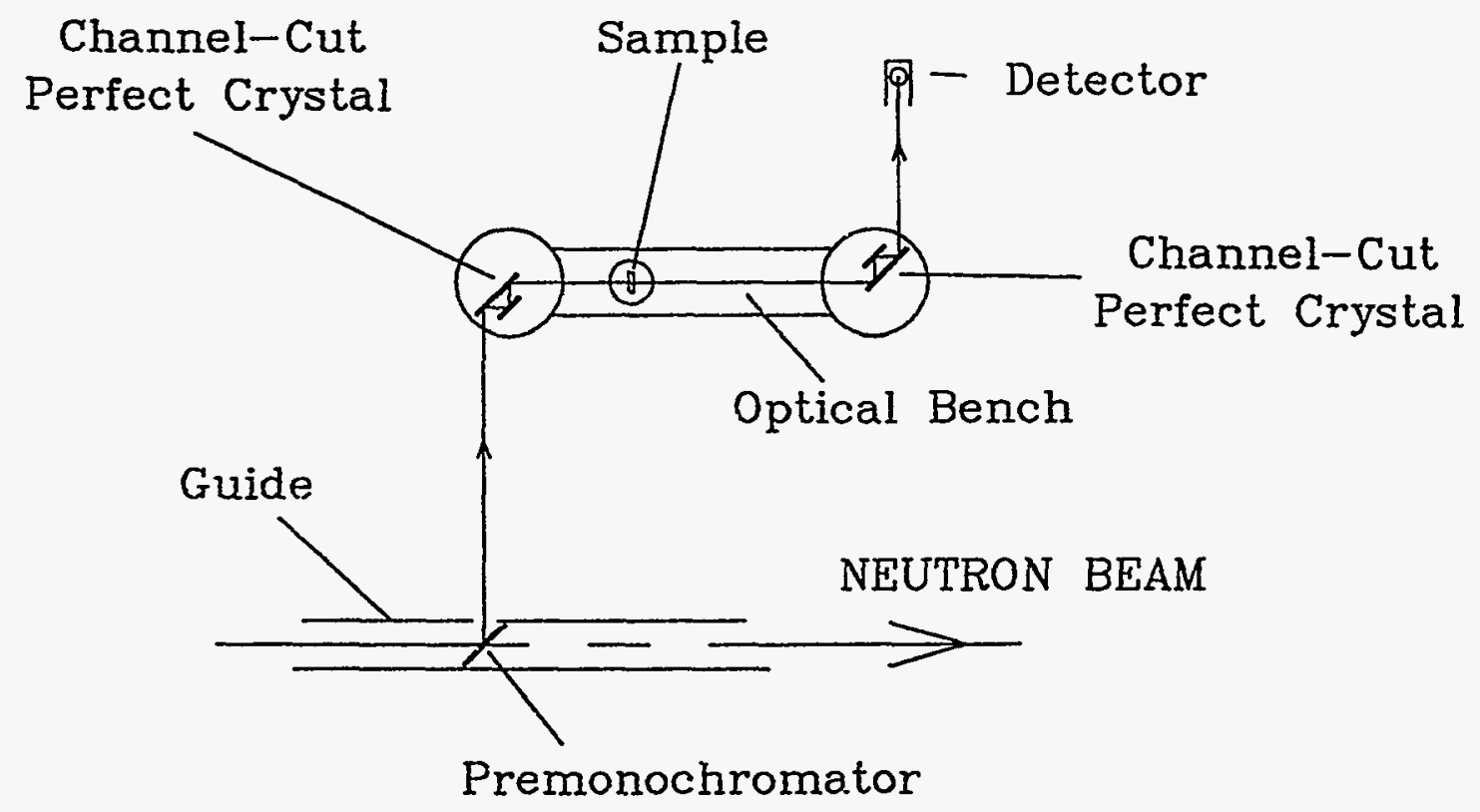

Figure V.2.2-3 Schematic Representation of the DCSAND. 
cycle of less than 1:30) and allows the separation of concurrently collected spectra from the different orders of the monochromator and analyzer crystals. Although the time-averaged flux obtained with the DCSAND is considerably below that of a similar instrument at a high-flux reactor, this instrument is a useful complement to the other small-angle diffractometers discussed here.

\subsubsection{Small-Angle Diffractometer Summary}

The very high data rates and broad Q-range of the SAND offer new opportunities in the study of small samples and in real-time studies of kinetic or other time-dependent effects in many types of materials. The HRSAND is a state-of-the-art instrument for the study of larger structures using lower $Q_{\min }$ than can be accessed on any other general-purpose small-angle neutron diffractometer in the United States. The flexibility offered by the SPSAND to be reconfigured for different types of experiments permits the exploration of totally new scientific areas, such as resonance small-angle scattering and the use of polarized neutrons in small-angle scattering experiments. The three small-angle neutron scattering instruments described in Sections V.2.2.1 and V.2.2.2 and summarized in Table V.2.2-3 form a reference set that covers a broad range of capabilities. They satisfy the requirements for virtually all types of current smallangle scattering experiments, and they also provide opportunities for many new types of experiments.

The two quasi-steady-state small-angle diffractometers provide the capability for extending to even smaller $Q_{\min }$ for some types of experiments. They might be better optimized for some other types of experiments, such as the study of inelastic effects in small-angle scattering, than are the three TOF small-angle diffractometers in the reference set. Instruments of these types, although probably not among the initial complement of instruments at IPNS Upgrade, will be considered in greater detail for possible inclusion at a later date.

\subsection{OTHER DIFFRACTOMETERS}

\subsubsection{Transfer of Existing IPNS Instruments}

\subsubsection{GLAD - Glasses, Liquids, and Amorphous Materials Diffractometer}

The GLAD is designed to study diffraction from liquids, glasses, and amorphous materials. ${ }^{9}$ It covers the Q-range of $\sim 0.05 \AA^{-1}$ to $\sim 50 \AA^{-1}$ using wavelengths from 0.1 to $4 \AA$. Measurements at each $Q$ value are made using the smallest scattering angles and shortest wavelengths practical, in order to minimize the inelasticity corrections required. Considerable attention has been paid to the shielding in the GLAD, so that the backgrounds are low even at wavelengths below $0.1 \AA$. Converging Soller collimators focused on the detector locus allow the 
Table V.2.2-3 Reference Set of TOF Small-Angle Diffractometers at IPNS Upgrade

\begin{tabular}{|c|c|c|c|c|}
\hline Instrument & Application/Special Feature & $Q_{\min }\left(\dot{A}^{-1}\right)$ & $Q_{\max }\left(\AA^{-1}\right)$ & $\begin{array}{l}\text { Measurement } \\
\text { Time (min) }\end{array}$ \\
\hline SAND ${ }^{a}$ & General purpose (wide Q-range) & 0.002 & 2 & $1-90$ \\
\hline HRSAND & High resolution & 0.0005 & 0.4 & $60-1800$ \\
\hline SPSAND & Reconfigurable for special purposes & - & variable & $-\cdots$ \\
\hline
\end{tabular}

a Transferred from IPNS with little change. 
use of relatively large samples with negligible sample-size contribution to the resolution. A large scattering solid angle is covered with LPSDs in order to achieve high data rates while maintaining good Q resolution. The GLAD is designed to utilize 408 such LPSDs, but only 235 are installed at present. Figure V.2.3-1 shows the GLAD design, and Table V.2.3-1 lists some of the performance parameters expected at IPNS Upgrade.

The GLAD can be moved to the $30-\mathrm{Hz}$ target station with essentially no changes, although it is desirable to increase the detector complement to the full 408 LPSDs and to develop faster position encoding for these detectors. The source-sample distance to the high-resolution sample position is $\sim 22 \mathrm{~m}$ at IPNS Upgrade. This provides greater time-of-flight separation between the prompt pulse and the 0.1- $\AA$ neutrons and allows more room for backgroundsuppression choppers. This increased distance also provide much easier access at the sides of the instrument. A cold moderator is selected because this helps to minimize the inelasticity corrections. The data-rate gain is a factor of $\sim 11$ relative to IPNS. Frame-overlap is not a problem, but a bandwidth-limiting chopper can be inserted if necessary. The GLAD is designed to allow the use of an energy-selecting Fermi chopper in the incident beamline in special cases where it is desired to perform a detailed separation of the elastic and inelastic scattering.

Maximum instantaneous data rates on the GLAD occur at the shortest wavelength used. For an intensely-scattering sample at $\lambda=0.1 \AA$ maximum instantaneous rates are $\sim 1,000$ counts/sec per LPSD at scattering angles above $\sim 10^{\circ}$. The 1,000-counts/sec rate with the present encoding time of $8 \mu \mathrm{sec}$ leads to dead-time losses of $0.8 \%$ at IPNS, which is acceptable. However, instantaneous rates at the IPNS Upgrade 30-Hz target station are a factor of 6 higher, so maximum dead-time losses reach $\sim 5 \%$ or more at the shortest wavelengths for intenselyscattering samples at IPNS Upgrade. Until faster encoding systems can be developed, the variable-aperture device available on the GLAD can be used to reduce the beam size for intensely-scattering samples if necessary.

\subsubsection{SCD - General-Purpose Single-Crystal Diffractometer}

The $\mathrm{SCD},{ }^{10}$ shown schematically in Figure V.2.3-2, is $10 \mathrm{~m}$ from the target at the $30-\mathrm{Hz}$ target station. A new area detector is required to cope with the data rates at IPNS Upgrade. The instrument is upgraded to permit sample-to-detector distances in the range of 30 to $60 \mathrm{~cm}$ and detector angles of $60^{\circ}, 90^{\circ}$, and $120^{\circ}$. A variable upstream collimator reduces the viewed portion of the moderator from 10-cm diameter to 5-cm diameter to improve the incident-beam collimation when desired. New shielding is required around the sample area. Some possible instrument configurations are described in Table V.2.3-2.

Configuration A is identical to the configuration of the SCD at IPNS. This configuration is used for small samples. The resolution required for background-to-background peak integration is five times $\Delta \mathrm{Q} / \mathrm{Q}$, or 0.045 , which permits measurement to $d_{\min }=1 \AA$ for a $22-\AA$ 


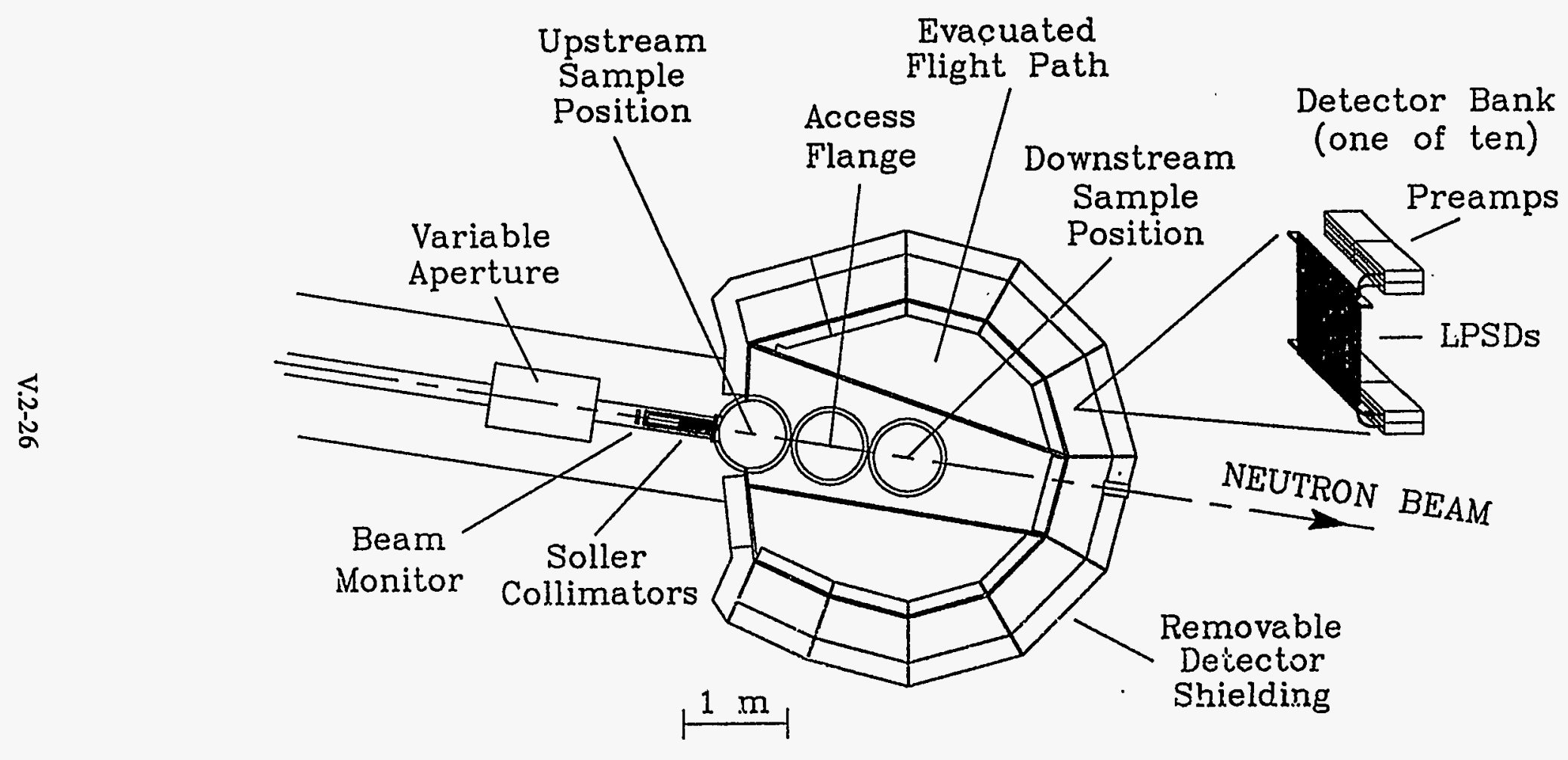

Figure V.2.3-1 Plan View of the GLAD. 
Table V.2.3-1 GLAD Parameters at IPNS Upgrade

\begin{tabular}{|c|c|c|}
\hline \multirow[b]{2}{*}{ Parameter } & \multicolumn{2}{|c|}{ Sample Position } \\
\hline & High Resolution & High Intensity \\
\hline Source-sample distance $(\mathrm{m})$ & 22 & 23.5 \\
\hline Source repetition rate $(\mathrm{Hz})$ & 30 & 30 \\
\hline Moderator material & liquid $\mathrm{CH}_{4}$ & liquid $\mathrm{CH}_{4}$ \\
\hline Sample volume $\left(\mathrm{cm}^{3}\right)$ & 3 & 3 \\
\hline Wavelength range $(\AA)$ & $0.1-4.0$ & $0.1-4.0$ \\
\hline Scattering angles $\left({ }^{\circ}\right)$ & $\sim 2.5-95$ & $\sim 5-150$ \\
\hline Sample-detector distance (m) & $\sim 3.0-1.0$ & -1.5 for all LPSDs \\
\hline LPSD diameter $(\mathrm{cm})$ & 1.1 & 1.1 \\
\hline LPSD length $(\mathrm{cm})$ & 60 & 60 \\
\hline LPSD ${ }^{3} \mathrm{He}$ fill pressure (atm) & 10 & 10 \\
\hline Collimation & converging Soller & converging Soller \\
\hline Beam size at sample $\left(\mathrm{cm}^{2}\right)$ & $1.2 \times 1.2$ & $0.6 \times 0.6$ \\
\hline Minimum accessible $Q\left(\dot{A}^{-1}\right)$ & 0.07 & 0.14 \\
\hline Maximum accessible $Q\left(\AA^{-1}\right)$ & 92 & 120 \\
\hline \multirow[t]{2}{*}{ Resolution $\Delta Q / Q$ (fwhm) } & $\sim 0.1$ at $Q=0.07$ & \\
\hline & $<0.012$ for $Q>5$ & \\
\hline \multirow{2}{*}{$\begin{array}{l}\text { Typical time to measure } \\
\text { one data set (min) }\end{array}$} & & \\
\hline & $20-50$ & $10-30$ \\
\hline
\end{tabular}




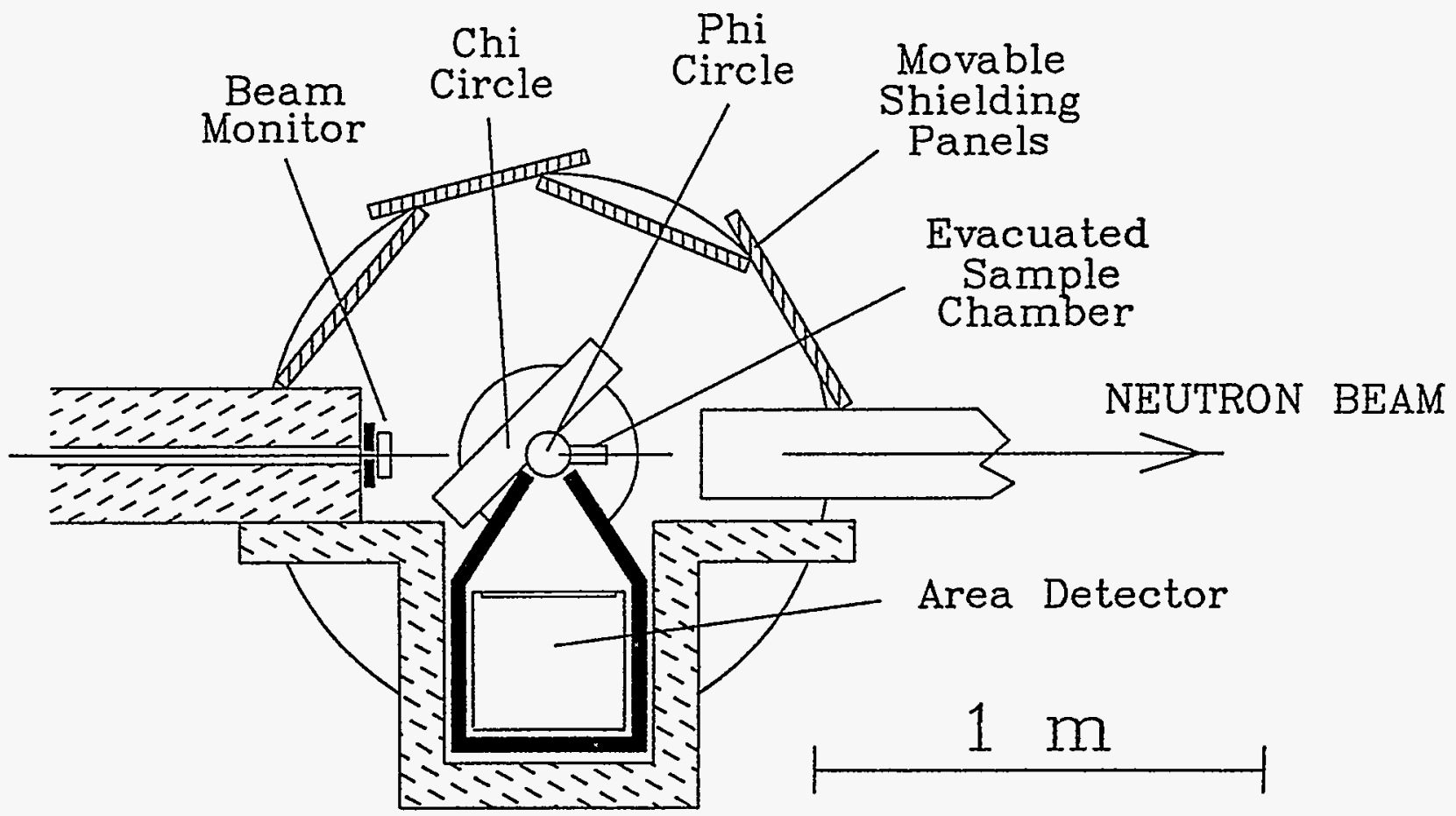

Figure V.2.3-2 Schematic Representation of the SCD. 
Table V.2.3-2 SCD Parameters at IPNS Upgrade

\begin{tabular}{|c|c|c|}
\hline \multirow[b]{2}{*}{ Parameter } & \multicolumn{2}{|c|}{ Configuration } \\
\hline & A & B \\
\hline Source-sample distance (m) & 10 & 10 \\
\hline Sample-detector distance $(\mathrm{cm})$ & 32 & 60 \\
\hline Moderator size $(\mathrm{cm})$ & 10 & 5 \\
\hline Detector size $\left(\mathrm{cm}^{2}\right)$ & $30 \times 30$ & $30 \times 30$ \\
\hline Detector type & Anger & Anger \\
\hline Detector resolution $(\mathrm{mm})$ & 3 & 3 \\
\hline Detector dead time ( $\mu$ sec) & $\sim 3$ & $\sim 3$ \\
\hline Wavelength range $(\ddot{A})$ & $\sim 0.7-10$ & $\sim 0.5-10$ \\
\hline Sample size $\left(\mathrm{mm}^{3}\right)$ & $1 \times 1 \times 1$ & $1 \times 1 \times 1$ \\
\hline Crystal mosaic $\left({ }^{\circ}\right)$ & 0.1 & 0.1 \\
\hline Total $\Delta 2 \theta\left(^{\circ}\right)$ & 0.8 & 0.5 \\
\hline Nominal detector angle $\left({ }^{\circ}\right)$ & 90 & 120 \\
\hline$\Delta t / t$ & 0.005 & 0.005 \\
\hline$\Delta Q / Q$ & 0.009 & 0.0055 \\
\hline Data-rate gain over IPNS & 40 & 3 \\
\hline
\end{tabular}


lattice repetition. Texture measurements, which currently require 1-2 days for each sample, can be obtained on 20 samples per day. This permits a much more precise analysis of the effects of small, systematic changes in processing parameters on the texture of samples, such as high- $T_{c}$ superconducting wires. For magnetic studies of single crystals, the detector can be rotated to the $60^{\circ}$ angle to obtain low- $Q$ data.

Configuration $B$ is optimized for high resolution and high $Q$. It permits background-tobackground peak integration of a $26-\AA$ lattice for $d_{\min }=1 \AA$. Alternatively, for a $10-\AA$ lattice, a minimum d-spacing of $0.3 \AA$ can be achieved with neutron wavelengths of $0.5 \AA$.

Frame-overlap occurs at $\sim 12 \AA$ for $30-\mathrm{Hz}$ operation at the $\sim 11-\mathrm{m}$ source-detector distance. Thus, there are no significant frame-overlap problems for this instrument.

At IPNS maximum dead-time losses are $\leq 1 \%$ for most samples on the SCD, with a few samples approaching $5 \%$. Scaling to IPNS Upgrade implies that detector dead-time is a problem in configuration A for many samples if the present Anger detector is retained for this instrument. Development of a much faster area detector having dead-time of $0.5 \mu \mathrm{sec}$ or less is necessary for full utilization of the capabilities of this instrument.

\subsubsection{New Reference Set Instruments}

\subsubsection{HQSCD — High-Q Single-Crystal Diffractometer}

This instrument is a higher-resolution, higher-Q-range complement to the SCD. Improving the resolution and extending the range to higher $\mathrm{Q}$ is important for many types of studies. Some examples are magnetostriction in $\mathrm{MnF}_{2}$ with a shift of $0.002 \AA$; conformational disorder in organic superconductors, such as $\beta$-(BETD-TTF $)_{2} \mathrm{I}_{3}$; analysis of anharmonic motion; resolution of the twin components of the low-temperature phase of high- $T_{c}$ superconductors; deformation density mapping in combination with high-Q $\mathrm{x}$-ray data; resolving satellite peaks near fundamental Bragg peaks; and Huang scattering. The improved resolution is also advantageous for diffuse scattering studies. Table V.2.3-3 gives some of the parameters for thisinstrument. The HQSCD has a geometry similar to the SCD shown in Figure V.2.3-2. The detector technology to be used is at present uncertain, since it is desirable to have a detector similar to the present SCD Anger detector but with a factor of two better resolution. If this is not possible, a larger detector placed farther from the sample can also be used. Data rates are down by at least a factor of 10 from those on SCD, so dead-time problems are not severe. 
Table V.2.3-3 Parameters for the HQSCD

\begin{tabular}{ll}
\hline \multicolumn{1}{c}{ Parameter } & \multicolumn{1}{c}{ Value } \\
& \\
\hline & liquid $\mathrm{CH}_{4}$ or liquid $\mathrm{H}_{2} \mathrm{O}$ \\
Moderator & 30 \\
Source frequency $(\mathrm{Hz})$ & 30 \\
Source-sample distance $(\mathrm{m})$ & $30(70)^{\mathrm{a}}$ \\
Sample-detector distance $(\mathrm{cm})$ & 10 \\
Moderator size $(\mathrm{cm})$ & $30 \times 30(\text { or } 4 \text { dets, each } 30 \times 30)^{\mathrm{a}}$ \\
Detector size $\left(\mathrm{cm}^{2}\right)$ & to be determined \\
Detector type & $1.5(3)^{\mathrm{a}}$ \\
Detector resolution $(\mathrm{mm})$ & $\sim 3$ \\
Detector dead time $(\mu \mathrm{sec})$ & $\sim 0.3-4$ \\
Wavelength range $(\tilde{A})$ & $1 \times 1 \times 1$ \\
Sample size $\left(\mathrm{mm}^{3}\right)$ & 0.1 \\
Crystal mosaic $\left({ }^{\circ}\right)$ & 0.8 \\
Total $\Delta 2 \theta\left(^{\circ}\right)$ & centered at $\sim 90$ (or $\sim 120)$ \\
Nominal detector angle $\left({ }^{\circ}\right)$ & 0.004 (or 0.003$)$ \\
$\Delta \mathrm{Q} / \mathrm{Q}$ & \\
\hline
\end{tabular}

a Depends on detector capabilities. One $30 \times 30 \mathrm{~cm}^{2}$ detector with $1.5-\mathrm{mm}$ resolution is desirable, but four $30 \times 30 \mathrm{~cm}^{2}$ detectors with $3-\mathrm{mm}$ resolution placed twice as far from the sample are roughly equivalent in data rate and resolution (but not in signal/background). 


\subsubsection{Other Possibilities}

This instrument is not in the reference set and will probably not be among the initial complement at IPNS Upgrade. It is expected that such an instrument might be added at a later date.

\subsubsection{PCSCD - Single-Crystal Diffractometer for Protein Crystallography}

Neutron diffraction from single crystals of proteins plays an important complementary role in structural biology with respect to x-ray diffraction and NMR. Neutron sources are inherently less intense than the newer synchrotron $x$-ray sources, but neutron diffraction permits accurate location of a protein structure's hydrogen atoms, which is difficult with $x$-ray methods. ${ }^{11,12}$ The chemistry of hydrogen dominates much of enzyme kinetics. Hydrogen structure is also crucial because such highly specific intermolecular recognition phenomena as DNA base pairing, genetic regulation, and expression require hydrogen bonding.

Calculations indicate that suitable data down to $\mathrm{d}_{\min }=1.5 \AA$ on small proteins can be measured in a few weeks. The neutron data are complementary to the x-ray structural data. In virtually all cases, high resolution $\left(\mathrm{d}_{\min }=1.2 \AA\right) \mathrm{x}$-ray data will exist, and this reduces the precision required of the neutron data to obtain suitable hydrogen structural results. This estimate assumes a crystal that is $50 \%$ deuterated, with unit cell axes of about $50 \AA$. It also assumes nearly $4 \pi$ detector coverage about the crystal. We propose to perform feasibility experiments on one or both of the single-crystal diffractometers (SCD or HQSCD) when IPNS Upgrade is operating. If the experiments confirm the calculations, the design and construction of an instrument optimized for protein crystallography can be initiated.

\subsubsection{Summary of Other Diffractometers}

The three instruments GLAD, SCD, and HQSCD form a reference set of instruments for the study of neutron diffraction from noncrystalline materials and from single crystals. These three instruments are summarized in Table V.2.3-4. 
Table V.2.3-4 Reference Set of Additional TOF Diffractometers at IPNS Upgrade

\begin{tabular}{llccc}
\hline Instrument & \multicolumn{1}{c}{$\begin{array}{c}\text { Application/ } \\
\text { Special Feature }\end{array}$} & $\begin{array}{c}\text { Range for } \\
\mathrm{Q}\left(\AA^{-1}\right)\end{array}$ & $\Delta \mathrm{Q} / \mathrm{Q}(\%)$ & $\begin{array}{c}\text { Measurement } \\
\text { Time (min) }\end{array}$ \\
\hline GLADa & $\begin{array}{l}\text { Glass, liquids, amorphous } \\
\text { materials }\end{array}$ & $0.07-120$ & $1.2-10$ & $10-50$ \\
$\mathrm{SCD}$ & $\begin{array}{l}\text { Single crystals, general } \\
\text { purpose }\end{array}$ & $0.9-17$ & $0.6-0.9$ & $20-200$ \\
$\mathrm{HQSCD}$ & $\begin{array}{l}\text { Single crystals, high real- } \\
\text { space resolution }\end{array}$ & $2-30$ & 0.4 & $\sim 200$ \\
\hline
\end{tabular}

a Transferred from IPNS with little change. 


\subsection{REFLECTOMETERS}

\subsubsection{Transfer of Existing IPNS}

\subsubsection{POSY-I — Polarized-Neutron Reflectometer}

At IPNS, the POSY-I and POSY-II reflectometers are located on separate beams brought out of the same beam port. A diagram of the present configuration is shown in Figure V.2.4-1. Since reflectometers use relatively small beams, one beam port at IPNS Upgrade also has separate beams supporting two separate reflectometers. At IPNS Upgrade these reflectometers are placed at a source-sample distance of $\sim 18 \mathrm{~m}$ at the $10-\mathrm{Hz}$ target station, so the two beams have much greater separation at the sample position than is the case at IPNS. The mounting table supporting the optical components of the two reflectometers must be redesigned. Because of the increased spatial separation between the instruments, a separate optical table is used for each of the two reflectometers in this case. One of these reflectometers is configured as the present POSY-I, the polarized neutron instrument, and uses the components from this instrument. ${ }^{13} \mathrm{It}$ has a vertical sample surface fed by a vertical-slit collimation system, includes a high-resolution Drabkin resonant spin flipper, and is equipped with complete polarization analysis. A background-suppression chopper can be used to remove the prompt pulse and frame-overlap neutrons or delayed neutrons if necessary. For typical samples, this instrument can measure polarization-dependent reflectivities down to $R=10^{-6}$ in $1-2 \mathrm{~h}$.

The second reflectometer sharing this beam port is a new high-data-rate reflectometer, which is discussed in Section V.2.4.2.1.

\subsubsection{POSY-II — General-Purpose Reflectometer}

A second beam port for reflectometers at the $10-\mathrm{Hz}$ target station provides three separate beams. One of these beams is configured essentially as the present POSY-II instrument, ${ }^{14}$ but it has a horizontal sample surface and horizontal-slit collimation so it can be used to study liquid surfaces as well. Although it uses many of the components of the present POSY-II, this instrument requires a new design. It is also located at a source-sample distance of $\sim 18 \mathrm{~m}$. A background-suppression chopper can be used to remove the prompt pulse and frame-overlap neutrons or delayed neutrons if necessary. This instrument is able to measure reflectivities down to $R=10^{-7}$ in $1-2 \mathrm{~h}$ from a wide variety of liquid and solid samples.

One of the other two beams sharing this beam port is devoted to a new reflectometer that is designed to study scattering at grazing incidence, as discussed below. The third beam is fed to an optical bench for experimentation to develop components and techniques. This is also discussed below. 


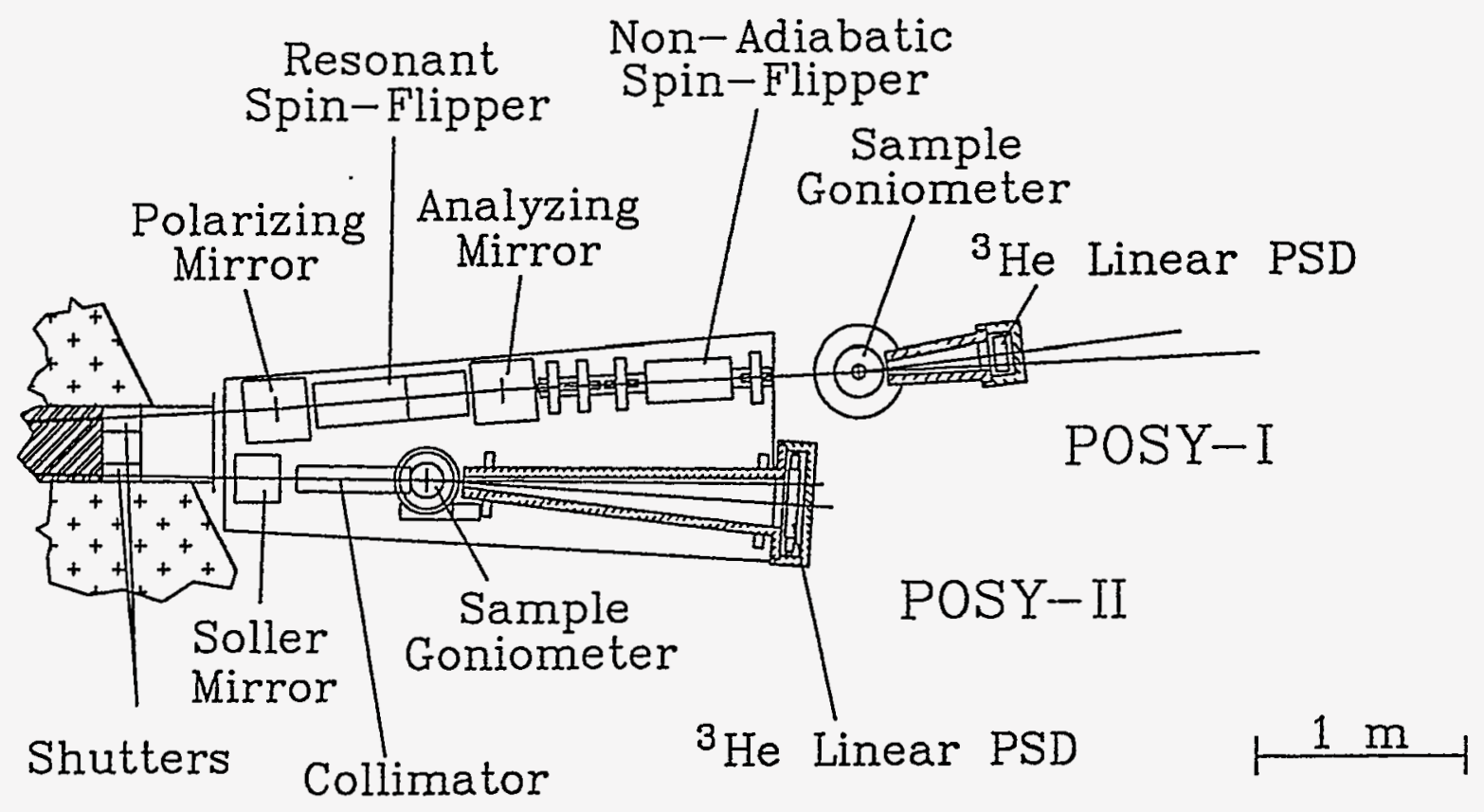

Figure V.2.4-1 Configurations of the POSY-I and POSY-II at IPNS. 


\subsubsection{New Reference Set Instruments}

\subsubsection{HIREF - High-Intensity Reflectometer}

This reflectometer is on a separate beam coming from the same beam port that supports the upgraded POSY-I reflectometer. It is located at a source-sample distance of $\sim 18 \mathrm{~m}$ and has a wide vertical slit, collimated horizontally so that beams of neutrons hit a horizontal sample surface at different angles, as shown schematically in Figure V.2.4-2. Table V.2.4-1 gives some of the parameters for this instrument. The higher-angle beams use curved guides in order to keep the total beam footprint small $(\sim 50 \mathrm{~mm})$. The maximum wavelength necessary varies from $3 \lambda_{\min }$ to $\lambda_{\min }$ over the incident-angle range $\theta \sim 0.5^{\circ}$ to $5.0^{\circ}$, in order for each of these reflections to overlap the $k$-space range covered by the next-lowest-angle beam. This assumes that the minimum wavelength $\lambda_{\min }$ is the same for all beams. In the reflectivity mode with no scattering, this instrument is very fast. If $\sim 20$ beams illuminate the sample, with angles ranging from $0.5^{\circ}$ to $5^{\circ}$ and with a relative divergence of 0.03 , a full reflectivity spectrum can be obtained in less than one minute. A background-suppression chopper can be used to remove the prompt pulse and frame-overlap neutrons or delayed neutrons if necessary. This instrument has the widest possible array of sample environments, including low and high temperature, vacuum, vaporsaturated or inert atmosphere, in order to study in situ the interfacial aspects of materials processing in real time.

\subsubsection{GREF - Reflectometer for Grazing-Incidence Scattering}

This instrument is located on one of the beams from the beam port shared by the rebuilt POSY-II, again at a source-sample distance of $\sim 18 \mathrm{~m}$. The GREF has a horizontal sample surface and horizontal-slit collimation. The collimation is variable in both directions. A 2D positionsensitive detector is used. A background-suppression chopper can be used to remove the prompt pulse and frame-overlap neutrons or delayed neutrons if necessary.

This instrument is able to measure specular reflectivity, forward small-angle off-specular scattering in the reflection plane, and small-angle off-specular scattering out of the reflection plane, as is shown schematically in Figure V.2.4-3. Table V.2.4-2 lists some parameters of this instrument.

\subsubsection{REFD — Reflectometry Development}

The beam for this instrument is round and comes from the same beam port that supports the rebuilt POSY-II. An optical bench permits a wide variety of configurations to be easily assembled and tested. This facility can be used, for example, for testing new optical components such as supermirrors and multilayers, or for assembly and testing of prototype instruments. 


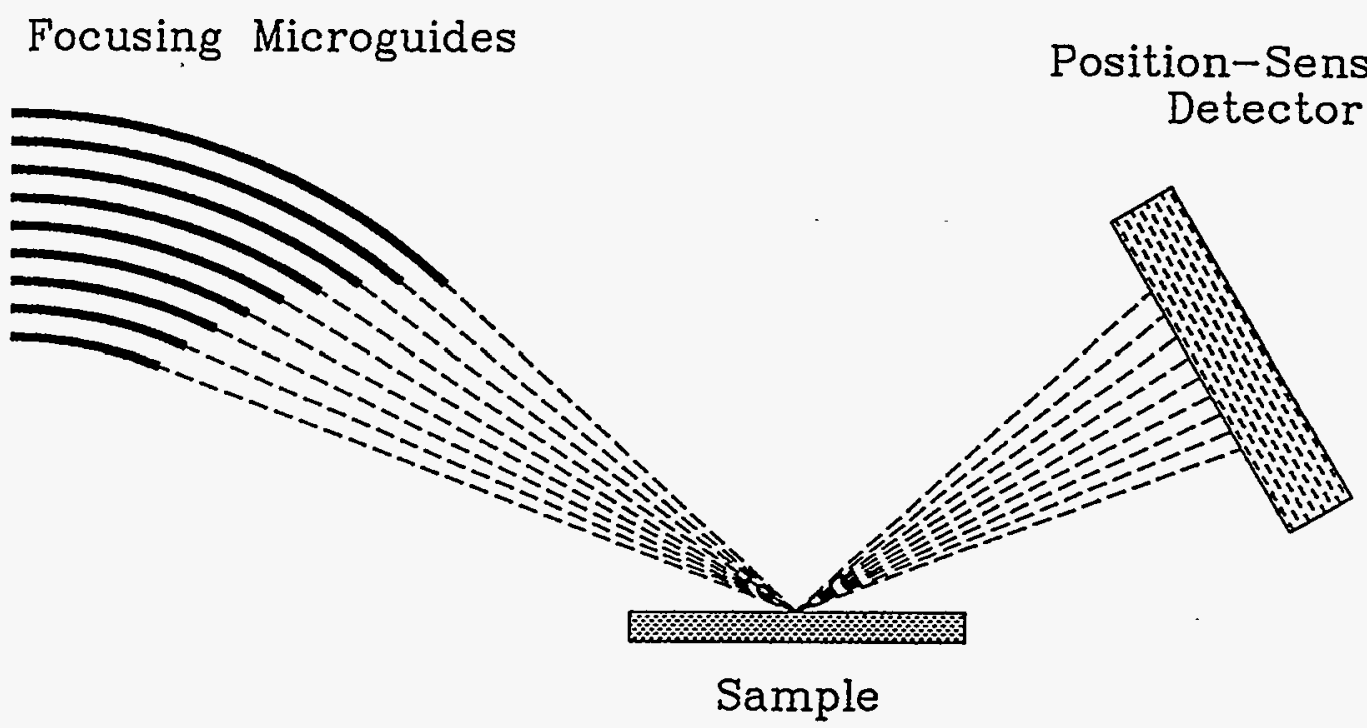

Figure V.2.4-2 Schematic Representation of the Multiple-Beam Geometry Used for the HIREF. 
Table V.2.4-1 Parameters for the HIREF

\begin{tabular}{|c|c|}
\hline Parameter & Value \\
\hline Moderator & decoupled liquid $\mathrm{H}_{2}$ \\
\hline Source repetition rate $(\mathrm{Hz})$ & 10 \\
\hline Source-to-sample distance (m) & 18 \\
\hline Number of incident beams & -20 \\
\hline Footprint of beam (sample size) $(\mathrm{mm})$ & 50 \\
\hline Range of reflection angles $\left({ }^{\circ}\right)$ & $2 \theta \sim 1-10$ \\
\hline \multicolumn{2}{|l|}{$\begin{array}{l}\text { Wavelength range for } 50 \% \text { overlap of } \\
\text { spectrum for adjacent beams }(\AA)\end{array}$} \\
\hline $1^{\circ}$ & $2.0-4.7$ \\
\hline $2^{\circ}$ & $2.0-4.0$ \\
\hline $5^{\circ}$ & $2.0-3.7$ \\
\hline Nominal range of reflectivity & $1 \geq R \geq 10^{-4}$ \\
\hline Time per measurement (min) & $\leq 1$ \\
\hline
\end{tabular}




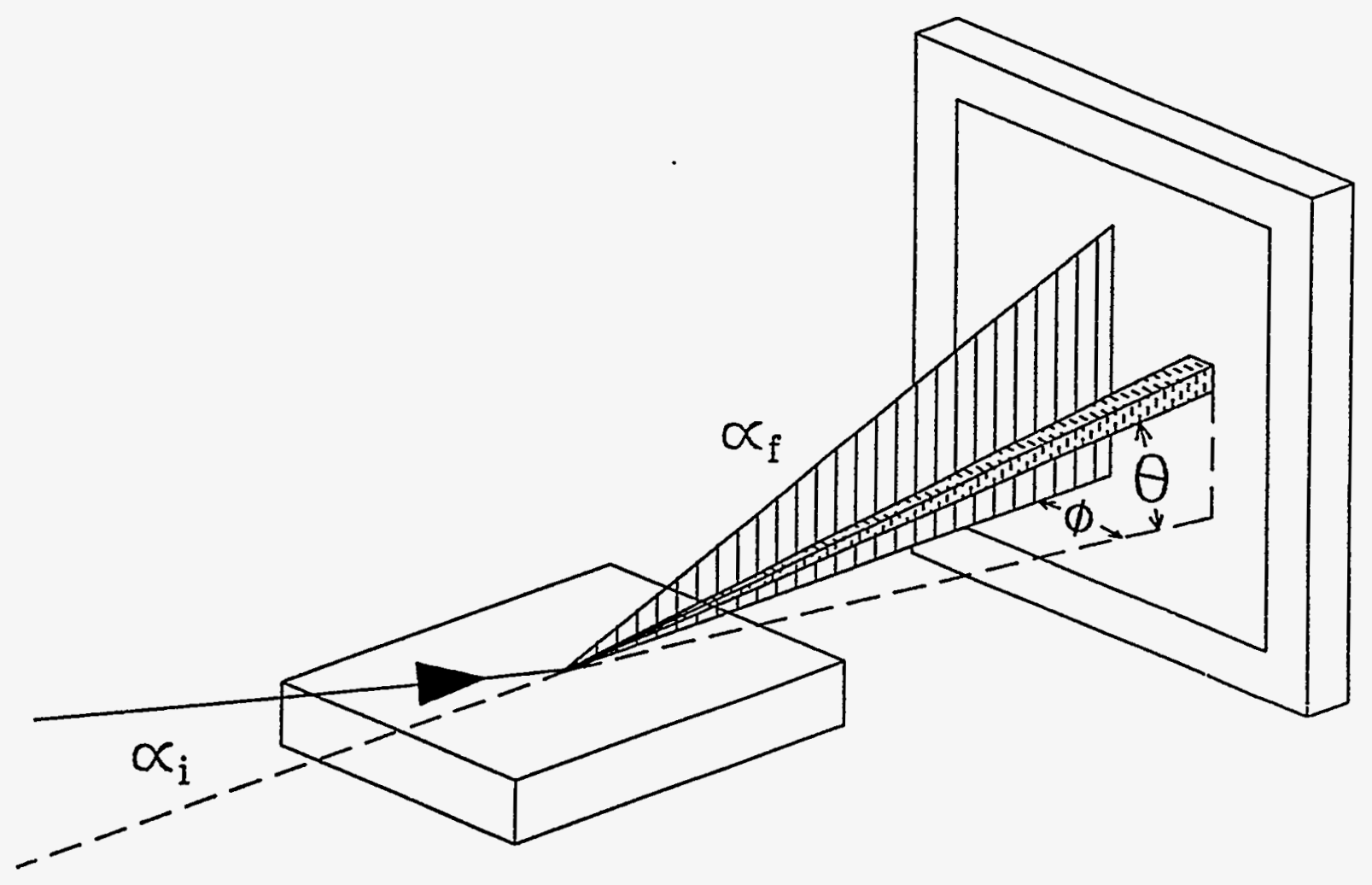

Figure V.2.4-3 Schematic Indication of the Geometry Used in the GREF Reflectometer. ( $\alpha_{i}$ and $\alpha_{f}$ are the incident and outgoing angles for neutrons in the plane defined by the incoming beam and the specular reflection. An out-of-plane scattered beam is shown with angles $\theta$ with respect to the sample surface and $\phi$ with respect to the reflection plane.) 
Table V.2.4-2 Parameters for the GREF

\begin{tabular}{ll}
\hline \multicolumn{1}{c}{ Parameter } & \multicolumn{1}{c}{ Value } \\
\hline & \\
Moderator & decoupled liquid $\mathrm{H}_{2}$ \\
Source repetition rate $(\mathrm{Hz})$ & 10 \\
Source-to-sample distance $(\mathrm{m})$ & 18 \\
Sample-to-detector distance $(m)$ & 5 \\
Maximum scattering or reflection angle $\left(^{\circ}\right)$ & 6 \\
Range in $Q$ for scattering $\left(\AA^{-1}\right)$ & $0.005 \leq Q \leq 1$ \\
Nominal range of reflectivity & $1 \geq R \geq 10^{-7}$ \\
Time per measurement $(h)$ & $1-2$ \\
& \\
\hline
\end{tabular}




\subsubsection{Reflectometer Summary}

The four reflectometers, POSY-I, POSY-II, HIREF, and GREF, discussed above form a set that provides a broad range of capabilities in this rapidly expanding field, and the associated development instrument REFD permits development of new or improved neutron optical components that can be used to enhance or expand these capabilities. Together, these five instruments are a reference set of reflectometers, and are summarized in Table V.2.4-3. These five instruments occupy only two beam ports.

\subsection{CHOPPER INELASTIC-SCATTERING INSTRUMENTS}

\subsubsection{Transfer of Existing IPNS Instruments}

\subsubsection{HRMECS - High-Resolution Chopper Spectrometer}

The HRMECS can be transferred from IPNS virtually unchanged to the $30-\mathrm{Hz}$ target station at IPNS Upgrade. It views a liquid $\mathrm{CH}_{4}$ moderator and has a source-sample distance of $\sim 18 \mathrm{~m}$. Figure V.2.5-1 shows the present HRMECS, and Table V.2.5-1 lists some of its parameters. ${ }^{15-17}$ At this distance, the HRMECS has a somewhat better resolution than at IPNS, particularly at low incident energies, and has a factor of $\sim 30$ increase in the data rate. This means that an experiment requiring a week at IPNS can be performed in a matter of hours at IPNS Upgrade. It also makes possible experiments on much smaller samples than are practical at present. With this higher flux, it is also practical to use tighter collimators and faster choppers to improve the resolution while still providing acceptable data rates.

The HRMECS is a very powerful and versatile instrument. However, some modifications make the HRMECS even more useful at IPNS Upgrade. One such modification is to change the forward-scattering portion of the vacuum flight path to allow a longer scattering flight path. This permits the use of position-sensitive detectors or an azimuthal array of short detectors to achieve much greater solid-angle coverage and better angular resolution at small scattering angles. A variable-aperture collimation system in the incident beam is required to optimize the use of these small scattering angles. Figure V.2.5-2 indicates this flight-path extension. Sufficient detectors are added to fill out the allowed detector complement on the HRMECS. 
Table V.2.4-3 Reference Set of TOF Reflectometers at IPNS Upgrade

\begin{tabular}{llllc}
\hline Instrument & $\begin{array}{c}\text { Application/ } \\
\text { Special Feature }\end{array}$ & Sample & $\begin{array}{c}\text { Minimum } \\
\text { Reflectivity }\end{array}$ & $\begin{array}{c}\text { Measurement } \\
\text { Time (min) }\end{array}$ \\
\hline POSY-Ia & Polarized neutrons & vertical & $10^{-6}$ & $60-120$ \\
POSY-II & General purpose & horizontal & $10^{-7}$ & $60-120$ \\
HIREF & High intensity & horizontal & $10^{-4}$ & $\leq 1$ \\
GREF & Grazing incidence & horizontal & $10^{-7}$ & $60-120$ \\
REFD & Development & - & - & - \\
\hline
\end{tabular}

a Rebuilt from components of IPNS instruments. 


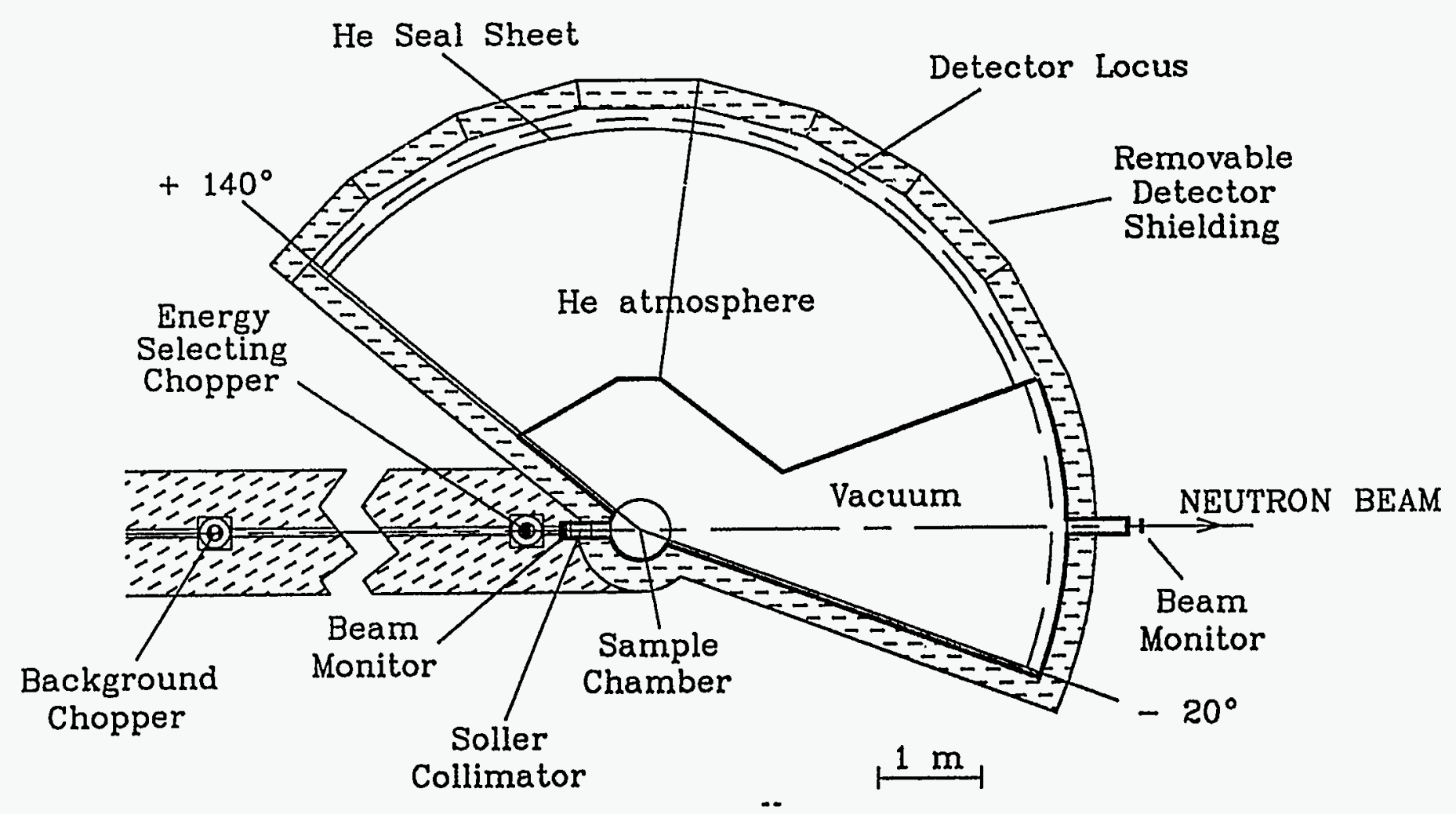

Figure V.2.5-1 Plan View of the HRMECS at IPNS. 
Table V.2.5-1 HRMECS and LRMECS Parameters at IPNS Upgrade

\begin{tabular}{|c|c|c|}
\hline Parameter & HRMECS & LRMECS \\
\hline Source-sample distance $(m)$ & 18 & 12 \\
\hline Source-chopper distance (m) & 17 & 11 \\
\hline Background-suppression chopper & yes $\sim 6 \mathrm{~m}$ from source & yes $-6 m$ from source \\
\hline Source repetition rate $(\mathrm{Hz})$ & & \\
\hline Moderator material & liquid $\mathrm{CH}_{4}$ & liquid $\mathrm{CH}_{4}$ \\
\hline Max. beam size at sample $\left(\mathrm{cm}^{2}\right)$ & $7.5 \times 10$ & $5 \times 10$ \\
\hline Incident energy range (meV) & $2-2000$ & $2-2000$ \\
\hline Scattering angles $\left({ }^{\circ}\right)$ & $(-20)-(-2.5), 2.5-140$ & $(-10)-(-2.5), 2.5-120$ \\
\hline Sample-detector distance $(\mathrm{m})$ & $\begin{array}{l}4.0 \text { ( } 8.0 \text { for low angle } \\
\text { extension) }\end{array}$ & $\sim 2.5$ \\
\hline Detector diameter $(\mathrm{cm})$ & 2.5 & 2.5 \\
\hline Detector length $(\mathrm{cm})$ & 42 (except at low angle) & 42 (except at low angle) \\
\hline Detector ${ }^{3} \mathrm{He}$ fill pressure (atm) & 6 & 6 \\
\hline Present number of detectors & $\sim 240$ & 160 \\
\hline Max. possible number of detectors ${ }^{a}$ & -800 & 200 \\
\hline Available Fermi choppers ${ }^{b}$ & \multicolumn{2}{|c|}{$\begin{array}{l}\text { Mechanical bearing, Be body, max. rotational speed } \\
\text { of } 270 \mathrm{~Hz} \text {, interchangeable between the two } \\
\text { instruments. Six choppers, optimized for } \\
\text { different energies and resolutions. }\end{array}$} \\
\hline Energy resolution $\Delta E / E_{\text {inc }}$ & $2-4 \%$ & $4-7 \%$ \\
\hline Typical time to measure one data set (h) & 2 & $<1$ \\
\hline
\end{tabular}

a Without desired modifications to flight paths.

b Faster, magnetic-bearing choppers are planned to provide higher resolutions than currently available. 


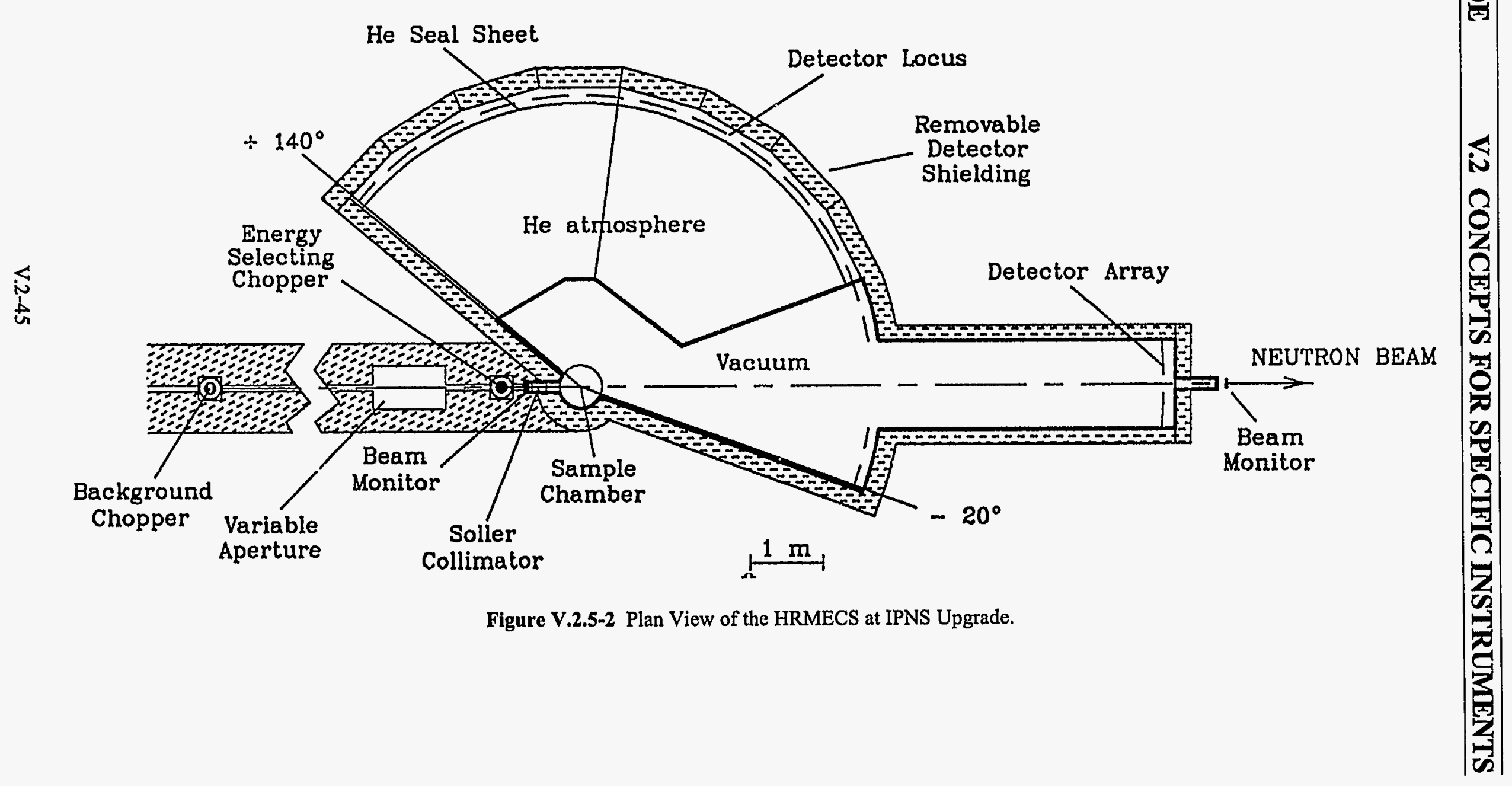




\subsubsection{LRMECS - High-Intensity General-Purpose Chopper Spectrometer}

The present LRMECS can also be transferred with virtually no changes to the $30-\mathrm{Hz}$ target station at IPNS Upgrade, where it views a liquid $\mathrm{CH}_{4}$ moderator with a source-sample distance of $\sim 12 \mathrm{~m}$. Figure V.2.5-3 shows the present LRMECS, and Table V.2.5-1 lists some of its parameters. ${ }^{15-17}$ At IPNS Upgrade, the LRMECS has a resolution similar to that at IPNS, with a nearly 20 -fold increase in data rates, so it is capable of performing modest-resolution experiments over a very wide region of Q-E space in a short time. Alternatively, similar experiments can be performed using small samples in a somewhat longer time.

Some improvements in LRMECS are also highly desirable. One improvement is modification of the entrance portion of the scattering flight path to accommodate larger sample apparatus. It is also desirable to modify the beam-exit portion of the scattering flight path to allow the installation of a detector or array of detectors providing azimuthal symmetry about the beam at small scattering angles.

\subsubsection{New Reference Set Instruments}

\subsubsection{CNCS - Cold-Neutron Chopper Spectrometer}

This spectrometer views a decoupled liquid $\mathrm{H}_{2}$ moderator on the $10-\mathrm{Hz}$ target station. The source-sample distance is $20 \mathrm{~m}$ and the sample-detector distance is $4 \mathrm{~m}$, so neutron wavelengths up to $\sim 16 \AA$, corresponding to incident energies down to $\sim 0.32 \mathrm{meV}$, can be used without frame-overlap. Fermi choppers provide the highest resolutions near the shortwavelength end of the wavelength range, while disk choppers provide the desired resolution, with somewhat higher intensities, at other wavelengths. Both types are available and interchangeable. A background-suppression chopper at $\sim 6 \mathrm{~m}$ from the source is required, and an additional chopper between this position and the $\mathrm{E}_{0}$ chopper may be required in order to suppress frameoverlap from successive $E_{0}$ chopper pulses. A neutron guide is used to enhance the intensity at the sample. The secondary flight path accommodates a row of detectors centered on the scattering plane and additional rows of detectors above and below that plane, for a total of $\sim 1000$ detectors. An area-position-sensitive detector or an array of linear-position-sensitive detectors is used in the forward scattering direction in order to provide better momentum resolution at small momentum transfers.

This set of instrument parameters are shown in Table V.2.5-2 and roughly duplicate the wavelength and resolution capabilities of the IN5 instrument ${ }^{18}$ at the ILL. With this choice of parameters, the resolution is at least as good as that of IN5 over the entire wavelength range (2-16 $\AA$ ) and is much better than that of IN5 over most of this range. The resolution and flux on sample for IN5 for an incident wavelength of $6 \AA$, corresponding to an incident energy $\mathrm{E}_{\mathrm{i}}$ of $2.3 \mathrm{meV}$, are $\Delta \mathrm{E} / \mathrm{E}_{\mathrm{i}} \sim 3.8 \%$ and flux $\sim 6 \times 10^{4} \mathrm{n} / \mathrm{cm}^{2}$-sec. The CNCS at this energy with the 


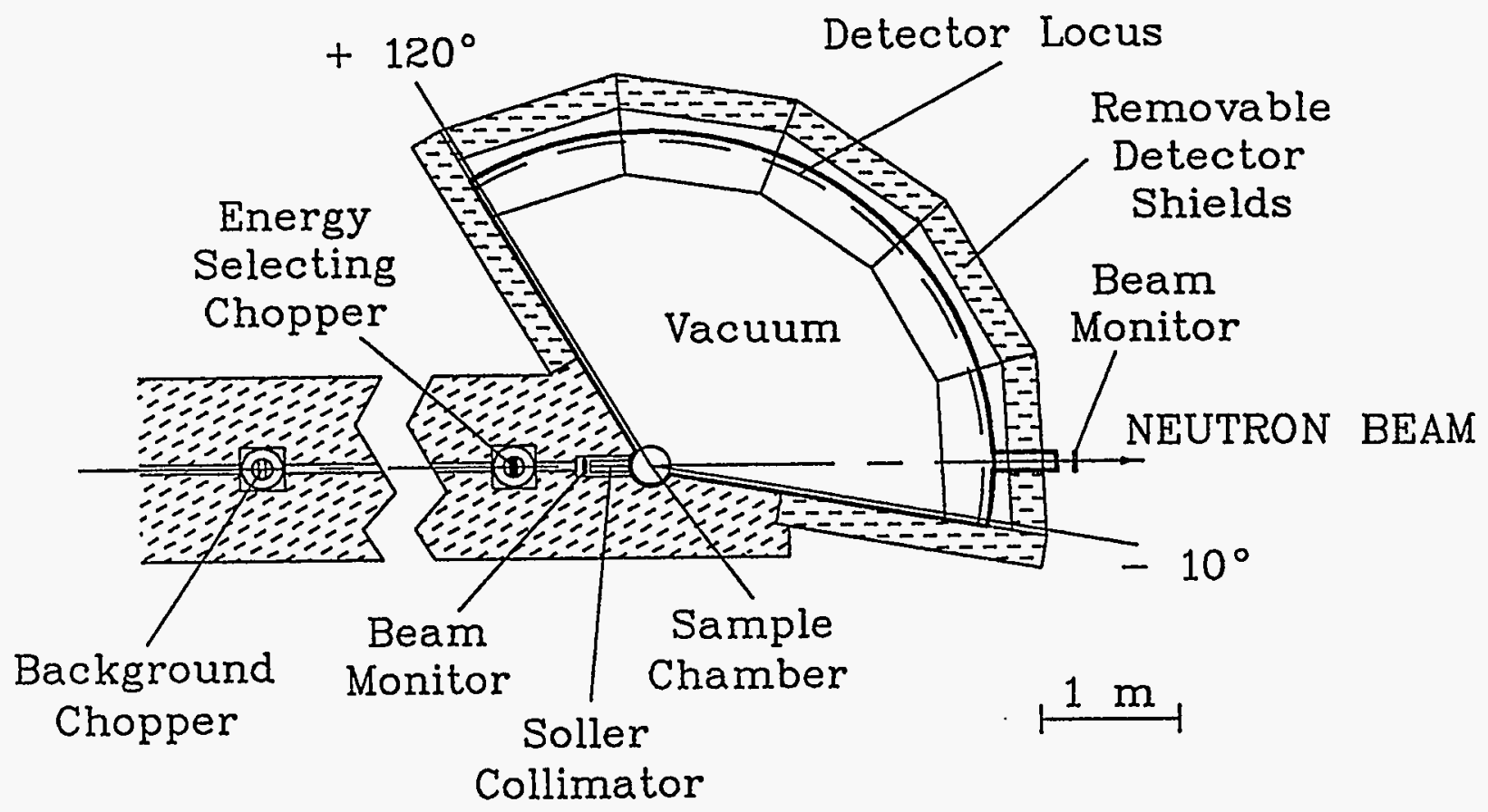

Figure V.2.5-3 Plan View of the LRMECS. 
Table V.2.5-2 CNCS Parameters at IPNS Upgrade

Parameter Value

Source-sample distance $(\mathrm{m})$

Source-chopper distance (m)

Background-suppression chopper

Source repetition rate $(\mathrm{Hz})$

Moderator material

Max. beam size at sample $\left(\mathrm{cm}^{2}\right)$

Incident energy range (meV)

Incident wavelength range $(\AA)$

Scattering angles $\left({ }^{\circ}\right)$

Sample-detector distance $(\mathrm{m})$

Detector diameter $(\mathrm{cm})$

Detector length $(\mathrm{cm})$

Detector ${ }^{3} \mathrm{He}$ fill pressure (atm)

Maximum possible number of detectors

$E_{0}$ chopper

Energy resolution $\Delta E / E_{\text {inc }}$

Typical time to measure one data set (h)
20

19

yes, $-6 \mathrm{~m}$ from source

10

liquid $\mathrm{H}_{2}$

$2 \times 10$

$0.3-20$

2-16

$\sim 5-140$, plus area detector or

LPSD array at lower angles

$\sim 4.0$

$1 \times 3.5^{\mathrm{a}}$

25

4

$\sim 1000$, plus area detector or $\sim 60$ LPSDs

Fermi or disk - interchangeable, depending on desired energy and resolution

$\leq 1 \%$ over entire energy range

$\sim 12-24$

a Uses flattened $2.54-\mathrm{cm}$-diameter cylindrical detectors to get good timing resolution. 
parameters given here has $\Delta \mathrm{E} / \mathrm{E}_{\mathrm{i}} \sim 1.0 \%$ and flux $\sim 1 \times 10^{4} \mathrm{n} / \mathrm{cm}^{2}$-sec, making it comparable to or somewhat better than $\mathbb{I N 5}$, which is an instrument much in demand for a broad range of scientific investigations. A more detailed optimization of this instrument, with careful attention to the interaction of guide and chopper parameters, ${ }^{19}$ may yield further improvements in performance.

\subsubsection{SCCS - Chopper Spectrometer for Excitations in Single Crystals and Low-Dimensional Systems}

Triple-axis spectrometers have long been used for the study of excitations in single crystals. However, the performance of triple-axis spectrometers begins to degrade rapidly as the neutron energy is increased, and only in special cases can they be used to study excitations with energies above $100 \mathrm{meV}$. Early work at IPNS 20 showed that chopper spectrometers at a pulsed neutron source can be used for the study of high-energy excitations in single crystals, and since then a number of experiments at ISIS 21,22 have shown that chopper spectrometers can perform extremely well for many such experiments. A new chopper spectrometer, the Multi-Angle Position-Sensitive Spectrometer (MAPS), optimized for and dedicated to the study of highenergy excitations such as magnetic excitations in single crystals, is being constructed ${ }^{23}$ at ISIS. This spectrometer is designed to provide a continuous detector array covering the scattering angles $-30^{\circ}$ to $+30^{\circ}$ at a distance of $4 \mathrm{~m}$ from the sample, with the necessary gap for the unscattered beam near $0^{\circ}$ made as small as possible. This detector array is made up of linearposition-sensitive gas proportional counters, each $2.5 \mathrm{~cm}$ in diameter $\times 1 \mathrm{~m}$ long and having position-encoding resolution of $\sim 2 \mathrm{~cm}$. A similar detector array covers scattering angles from $-20^{\circ}$ to $+20^{\circ}$ in the out-of-plane dimension. In all, $\sim 256$ LPSDs are required.

The SCCS instrument planned for IPNS Upgrade is similar to this MAPS instrument. However, since it will be constructed later than MAPS, its design will benefit from experience gained with MAPS. Figure V.2.5-4 shows schematically what this instrument might look like. The sample is located $\sim 16 \mathrm{~m}$ from a room-temperature $\mathrm{H}_{2} \mathrm{O}$ moderator on the $30-\mathrm{Hz}$ source. A high-speed Fermi chopper near the sample is used for incident-beam energy selection, with energy resolution $\Delta \mathrm{E} / \mathrm{E}_{\mathrm{i}} \sim 1 \%$. One or more additional choppers closer to the moderator provide background suppression. The continuous detector coverage ensures that all likely $\mathbf{Q}$ vectors of interest can be covered for energy transfers greater than $\sim 100 \mathrm{meV}$. The use of position-sensitive detectors permits the recording of neutrons in a fine-enough grid in terms of the $\mathbf{Q}$ vector and energy transfer to allow construction of scans in any $\mathbf{Q}$ direction and with good resolution in $\mathbf{Q}$ and energy transfer.

\subsubsection{Other Possibilities}

This instrument is not in the reference set and will probably not be among the initial complement at IPNS Upgrade. It is expected that instruments of this type will evolve and might be added at a later date. 


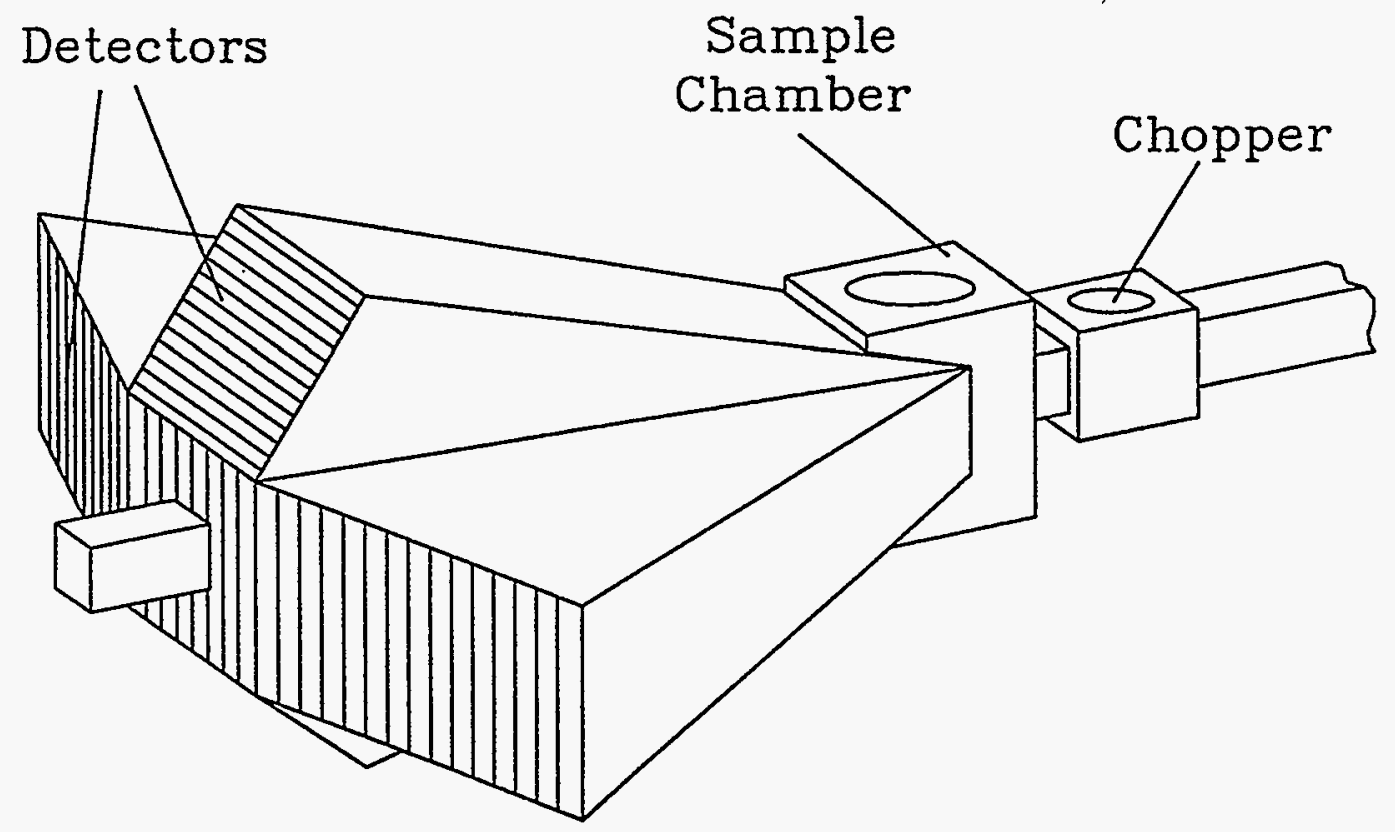

Figure V.2.5-4 Schematic Representation of the SCCS. 


\subsubsection{BSCS - Brillouin Scattering Chopper Spectrometer}

Neutron Brillouin scattering involves the measurement of excitations at small $Q$ values and moderate energy transfers. These are the requirements for the study of many of the collective excitations in liquids and glasses. Kinematic considerations require that such scattering is done with relatively high incident energies and small scattering angles. Robinson ${ }^{24}$ has analyzed these kinematic requirements, along with the resolution requirements for such an instrument. He found that an instrument having an incident flight path of $\sim 20 \mathrm{~m}$ and a scattering path of $\sim 10 \mathrm{~m}$ in the forward direction, with a minimum scattering angle of $\sim 0.4^{\circ}$ and an energy resolution $\Delta \mathrm{E} / \mathrm{E}_{\mathrm{i}} \sim 0.5 \%$, is adequate for many such measurements. These flight-path lengths are not too different from those already specified for the modified version of the HRMECS, and the HRMECS can provide nearly this energy resolution if high-speed choppers are used. Therefore, the HRMECS instrument will be used for the initial Brillouin scattering experiments at IPNS Upgrade. An instrument optimized for Brillouin scattering can be designed and constructed if demand warrants, after experience with such experiments is gained on the HRMECS.

\subsubsection{Chopper Spectrometer Summary}

The chopper spectrometers HRMECS and LRMECS are very versatile, general-purpose inelastic-scattering instruments. These two instruments offer considerably different tradeoffs between resolution and intensity, permitting the conditions to be optimized for a wide range of experiments. The cold-neutron spectrometer CNCS provides high-resolution spectroscopy in the very important low-energy range, and the single-crystal chopper spectrometer SCCS provides capabilities optimized for the study of high-energy excitations in single crystals. These four spectrometers are taken as a reference set of chopper spectrometers. Their capabilities are summarized in Table V.2.5-3.

\subsection{CRYSTAL-ANALYZER INELASTIC-SCATTERING INSTRUMENTS}

\subsubsection{Transfer of Existing IPNS Instruments}

\subsubsection{QENS - Medium-Resolution Crystal-Analyzer Spectrometer}

The QENS is a crystal-analyzer spectrometer optimized to provide $\sim 70-\mu \mathrm{eV}$ resolution for quasielastic scattering, but it also has very good resolution for chemical spectroscopy at excitation energies up to $150 \mathrm{meV}$. The polychromatic or white beam directly from the source is incident on the sample, so a separate detector can monitor diffraction from the sample. This allows a careful following of phase changes and in situ sample growth or modification, concurrently with the quasielastic- and inelastic-scattering measurements. The QENS has three 
Table V.2.5-3 Reference Set of TOF Chopper Spectrometers at IPNS Upgrade

\begin{tabular}{lllcr}
\hline Instrument & Application/Special Feature & $\begin{array}{l}\text { Range for } \\
\mathrm{E}_{\text {inc }}(\mathrm{meV})\end{array}$ & $\Delta \mathrm{E} / \mathrm{E}_{\mathbf{i}}(\%)$ & $\begin{array}{c}\text { Measurement } \\
\text { Time (h) }\end{array}$ \\
\hline HRMECSa & High resolution, general purpose & $4-2000$ & $2-4$ & 2 \\
LRMECS $^{\mathrm{b}}$ & Low resolution, general purpose & $3-2000$ & $4-7$ & $\leq 1$ \\
CNCS & High resolution, low energy & $0.3-20$ & $\leq 1$ & 12 \\
SCCS & Excitations in single crystals & $50-2000$ & -1 & -12 \\
\hline
\end{tabular}

a Transferred from IPNS with forward scattering path increased.

b Transferred from IPNS with little change. 
focused-crystal-analyzer arms mounted on a rotating table so that a wide range of scattering angles can be covered. Figure V.2.6-1 shows the schematic layout of the QENS, and Table V.2.6-1 lists some of its parameters. ${ }^{25}$ At IPNS Upgrade, the QENS spectrometer is placed on a beamline at the $30-\mathrm{Hz}$ target station, where it views either a liquid $\mathrm{CH}_{4}$ or a decoupled liquid $\mathrm{H}_{2}$ moderator. A liquid $\mathrm{H}_{2}$ moderator has a 3-5 times greater intensity at the long wavelengths used for most of the QENS experiments, but it also has a broader pulse so the total flight-path length must be increased in order to maintain the same resolution. Poisoning of the liquid $\mathrm{H}_{2}$ moderator might decrease the pulse-width, and guides can be used to minimize flux losses due to the greater source-sample distance. Until a detailed instrument design is considered and more information concerning the performance of poisoned liquid $\mathrm{H}_{2}$ moderators is available, it is not clear which moderator gives the better performance for QENS. If the liquid $\mathrm{CH}_{4}$ moderator is used, QENS is placed at a source-sample distance of $\sim 9 \mathrm{~m}$. In this location, count rates are increased by a factor of $\sim 40$ over those at the present IPNS, where it currently takes between $1 / 2$ day and 2 days to make a measurement on the QENS.

The QENS could be moved to IPNS Upgrade with essentially no changes, but it is considerably improved by incorporating a much greater solid angle of analyzer crystals and using a focusing guide system in the incident beam. A background-suppression chopper in the incident beam is also used. These changes result in an additional factor of 5 increase in instrument throughput. Such a redesign of the QENS is already in its initial stages at IPNS.

Neutron lenses made of capillary bundles or of silicon microguides are being developed at NIST and elsewhere. These offer the ability to focus a relatively large beam onto a sample of a few square millimeters or less in area.26,27 These lenses look very promising for providing a very-small-sample capability for QENS, where the increased angular divergence of the incident beam caused by such focusing is not a problem. Once such lenses are somewhat more developed, one can be designed to match the requirements of QENS and installed on the instrument. This will allow the use of very small samples, down to a few square millimeters in area, with only a small reduction in data rates, making this spectrometer very attractive for chemical spectroscopy in a much broader class of materials.

\subsubsection{New Reference Set Instruments}

\subsubsection{HRBS - High-Resolution Backscattering Spectrometer $(5 \mu \mathrm{eV})$}

This spectrometer has a resolution of $\sim 5 \mu \mathrm{eV}$ and is optimized for the study of quasielastic scattering and low-lying excitations. It views a poisoned, decoupled liquid $\mathrm{H}_{2}$ moderator at the $10-\mathrm{Hz}$ target station. This crystal-analyzer backscattering spectrometer is similar in concept to the IRIS spectrometer ${ }^{28}$ at ISIS, but with some significant differences. The spectrometer optimization is based on the use of near-back-reflection from the 004 planes of mica crystal analyzers. The $004 \mathrm{~d}$-spacing of mica is $\sim 5 \AA$, so $\sim 10-\AA(0.8-\mathrm{meV})$ neutrons are 


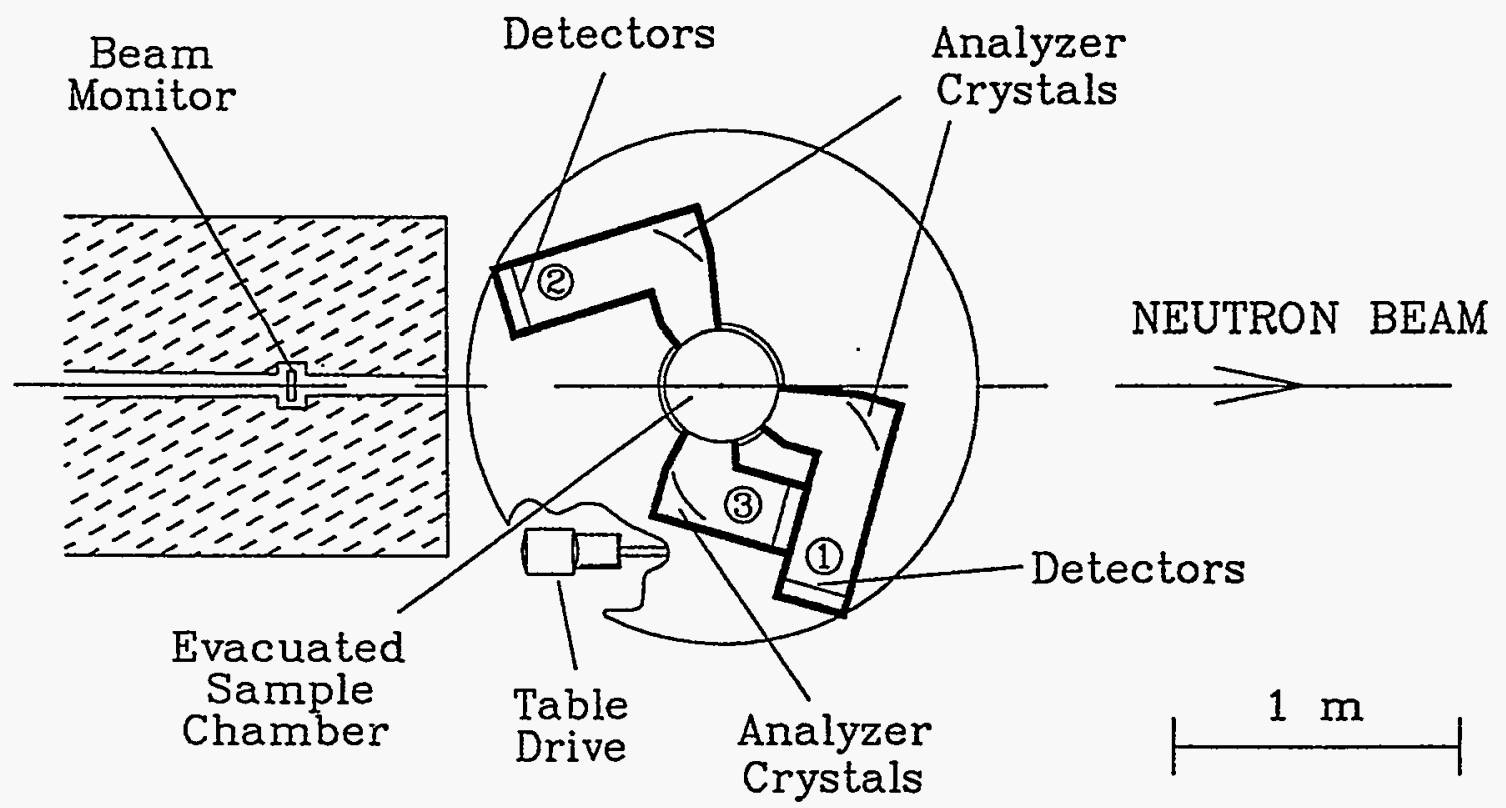

Figure V.2.6-1 Unmodified Configuration of the QENS. (Circled numbers identify the three analyzer arms.) 
Table V.2.6-1 QENS (unmodified) Parameters at IPNS Upgrade

\begin{tabular}{|c|c|c|c|}
\hline Parameter & Arm 1 & Arm 2 & Arm 3 \\
\hline Source-sample distance (m) & $9^{\mathbf{a}}$ & $9^{a}$ & $9^{a}$ \\
\hline Source repetition rate $(\mathrm{Hz})$ & 30 & 30 & 30 \\
\hline Moderator material & liquid $\mathrm{CH}_{4}^{\mathrm{a}}$ & liquid $\mathrm{CH}_{4}{ }^{\mathrm{a}}$ & liquid $\mathrm{CH}_{4}^{\mathrm{a}}$ \\
\hline Max. beam size at sample $\left(\mathrm{cm}^{2}\right)$ & $1.27 \times 10$ & $1.27 \times 10$ & $1.27 \times 10$ \\
\hline Angular acceptance $\left({ }^{\circ}\right)$ & 7 & 14 & 14 \\
\hline Range of scattering angles $\left({ }^{\circ}\right)$ & $25-55$ & $75-105$ & $115-155$ \\
\hline Analyzer crystals & graphite & graphite & graphite \\
\hline Analyzer energy (meV) & $-3.6^{\mathrm{b}}$ & $-3.6^{b}$ & $-3.6^{\mathrm{b}}$ \\
\hline Sample-crystal-detector distance (m) & 1.117 & 1.117 & 0.800 \\
\hline Detector diameter $(\mathrm{cm})$ & 0.64 & 0.64 & 0.64 \\
\hline Detector length (cm) & 10 & 10 & 10 \\
\hline Detector ${ }^{3} \mathrm{He}$ fill pressure (atm) & 10 & 10 & 10 \\
\hline Number of detectors & 13 & 13 & 9 \\
\hline \multicolumn{4}{|l|}{ Energy resolution $\Delta \mathrm{E}$ (meV) } \\
\hline energy transfer $=0 \mathrm{meV}$ & 0.07 & 0.07 & 0.08 \\
\hline energy transfer $=50 \mathrm{meV}$ & 1.57 & 1.57 & 1.57 \\
\hline Typical time to measure one data set (min) & - & $20-80$ & - \\
\hline
\end{tabular}

a Might use a poisoned, decoupled liquid $\mathrm{H}_{2}$ moderator, in which case the source-sample distance might have to be increased.

b Mosaic crystals are used, so there are a range of analyzer energies about this value. Each energy is focused on a different detector. 
back-reflected from the 004 planes. A resolution of $5 \mu \mathrm{eV}$ at this energy for elastic scattering means $\Delta \mathrm{E} / \mathrm{E}_{\mathrm{i}} \sim 0.6 \%$, which is evenly distributed in quadrature among the contributions from source pulse-width, path-length variations, and analyzer bandwidth. The pulse-width $\Delta \mathrm{t}_{\mathrm{s}}$ from the moderator is $\sim 150 \mu \mathrm{sec}$ at this wavelength, so a source-sample distance of $\sim 35 \mathrm{~m}$ is required to give $\Delta \mathrm{t}_{\mathrm{s}} / \mathrm{t}_{\mathrm{i}} \sim 0.17 \%$. The analyzer crystals are operated off backscattering by an amount that gives an analyzer wavelength band of $\Delta \lambda \lambda \sim 0.17 \%$. The analyzer Bragg angle used depends on the mosaic spread of the particular mica. On the basis of reported values, a Bragg angle of $\sim 82^{\circ}$ is required for the analyzer crystal arrays. The analyzer and detector arrays are designed to minimize path-length uncertainties so that sample sizes can be as large as practical within the constraint $\Delta \mathrm{L} / \mathrm{L}_{i} \sim 0.17 \%$.

The path lengths are chosen so that the portion of the TOF spectrum of interest is not obscured by the next prompt pulse from the source. Source-sample distance is reduced to $30 \mathrm{~m}$ to prevent this overlap. The resolution is still $\sim 5 \mu \mathrm{eV}$ for quasielastic scattering if the $\Delta \mathrm{L} / \mathrm{L}_{\mathrm{i}}$ is reduced to $\sim 0.14 \%$. The spectrometer is also designed to prevent overlap of inelastic spectra. Within a given pulse at the sample, neutrons that are up-scattered to an energy corresponding to a high-order analyzer reflection can arrive at the detectors after neutrons down-scattered to loworder planes. For example, at some point the neutron-energy-gain spectrum of the 004 analyzers overlaps the neutron-energy-loss spectrum of the 002 analyzer planes. This sets an ultimate limit on the incident bandwidth that can be allowed to reach the sample. It is desirable to maximize this bandwidth, and this requires maximizing the ratio of sample-analyzer-detector distance to source-sample distance. A practical limit for the sample-analyzer distance is $\sim 3 \mathrm{~m}$, or $\sim 6 \mathrm{~m}$ sample-analyzer-detector distance, so this ratio becomes 6:30. For this choice of flight paths, the separation in time of the 004 spectra from the 002 spectra requires that the incident bandwidth be restricted to $\Delta \lambda=1.9 \AA$. Separation of the 004 spectra from the 006 spectra requires $\Delta \lambda=0.6 \AA$. Larger-wavelength bands can be used if it is known that there is no neutronenergy-gain spectrum from the higher-order plane, as is the case if the sample is sufficiently cold. This bandpass can be centered at any wavelength simply by adjusting the phase of the bandpass chopper(s). In the most restrictive case with $\Delta \lambda=0.6 \AA$, this corresponds to an energy range of $\sim 100 \mu \mathrm{eV}$ centered on elastic scattering using the 004 plane. The same bandpass corresponds to an energy range of $\sim 375 \mu \mathrm{eV}$ when centered at $2 \mathrm{meV}$ and $\sim 4200 \mu \mathrm{eV}$ when centered at $10 \mathrm{meV}$.

Figure V.2.6-2 shows a schematic layout of this instrument, and Table V.2.6-2 summarizes some of its parameters. The instrument size is largely dictated by the moderator pulse width, so the final instrument configuration will not be fixed until the moderator performance has been optimized. The instrument uses a neutron guide over the incident flight path, with a converging supermirror section to focus the neutrons onto the sample. One or two choppers provide an adjustable bandpass. Either small ${ }^{3} \mathrm{He}$ detectors or an array of scintillation detectors based on the $\mathrm{ZnS}-\mathrm{LiF}$ scintillator can be used.

A major difference between this spectrometer and the related spectrometers IRIS ${ }^{28}$ at ISIS or LAM-80ET 29 at KENS is the large ratio of secondary to primary flight-path lengths. This permits a clean separation of the spectra from the various analyzer plane orders while 


\section{Section A-A}

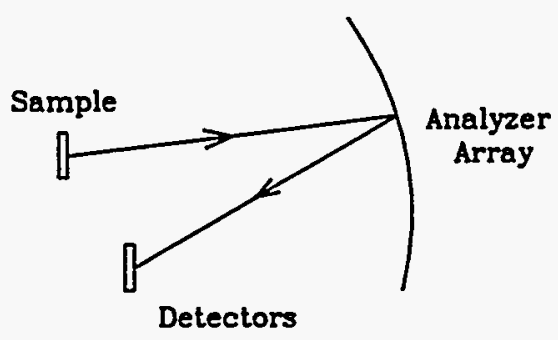

\section{typical}

sample-analyzer-detector

arrangement - not to scale

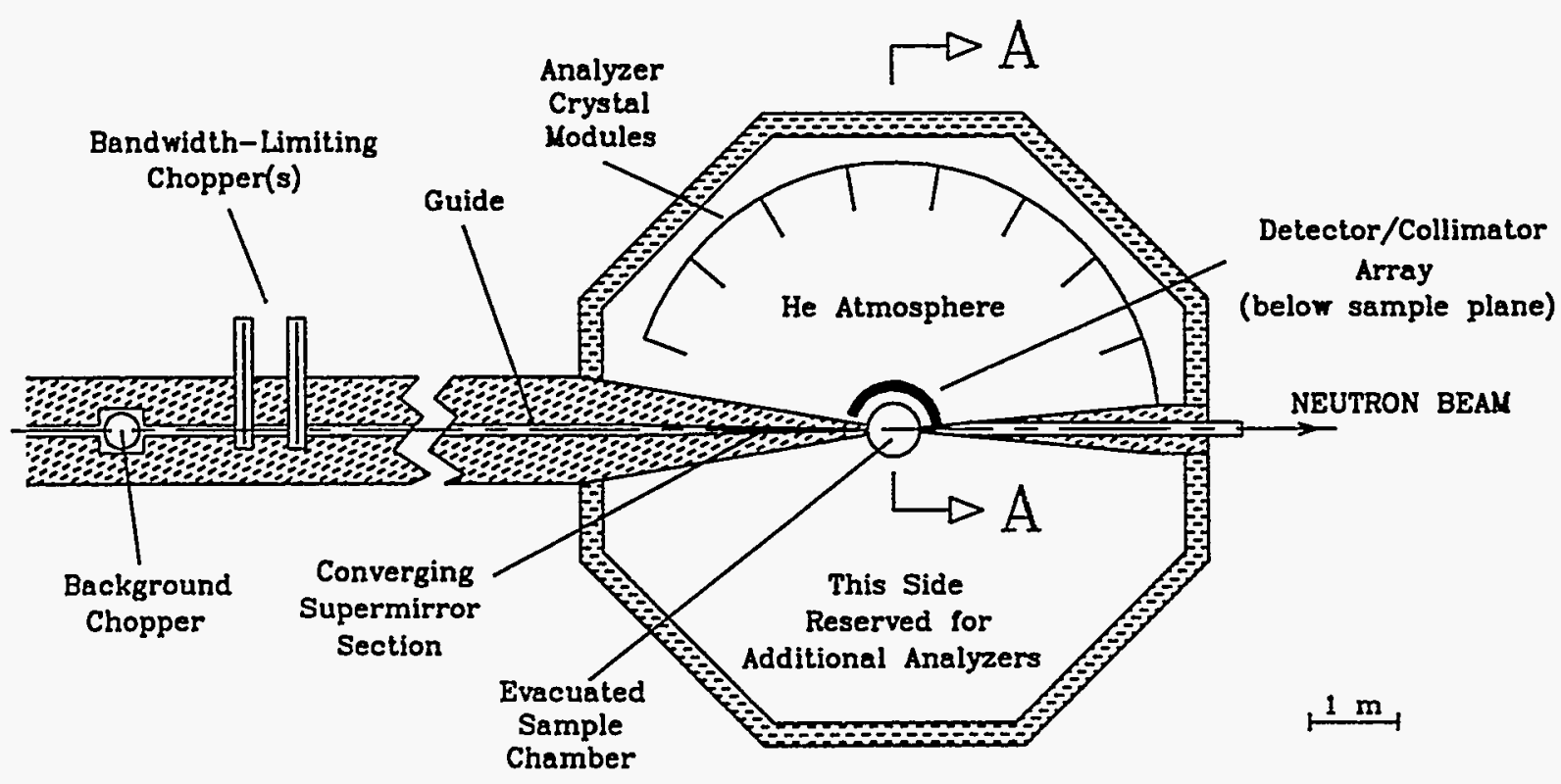

Figure V.2.6-2 Schematic Representation of the HRBS. 
Table V.2.6-2 Parameters for the HRBS

Parameter Value

Source-sample distance $(m)$

Source repetition rate $(\mathrm{Hz})$

Moderator material

Neutron guide

Bandwidth-limiting choppers

Background-suppression chopper

Max. beam size at sample $\left(\mathrm{cm}^{2}\right)$

Range of scattering angles $\left({ }^{\circ}\right)$

Analyzer crystals

Analyzer solid angle (sr)

Analyzer area $\left(\mathrm{m}^{2}\right)$

Sample-crystal-detector distance $(\mathrm{m})$

Detector type

Number of detectors $=$ number of scattering angles
30

10

poisoned, decoupled liquid $\mathrm{H}_{2}$

yes, with converging supermirror one or two

if needed

$\sim 2 \times 5$

$\sim$ 5-165

mica

$\sim 0.4$

$\sim 4$

$\sim 6$

to be determined

$\sim 150$

Analyzer Crystal Planes

\begin{tabular}{lll}
\multicolumn{1}{c}{002} & 004 & 0.06 \\
\hline 0.21 & 0.84 & 1.90 \\
& & \\
0.0012 & 0.0050 & 0.011 \\
0.013 & 0.017 & 0.023 \\
0.061 & 0.064 & 0.071
\end{tabular}

Analyzer energy (meV)

Energy resolution $\Delta E(\mathrm{meV})$

energy transfer $=0 \mathrm{meV}$

energy transfer $=2 \mathrm{meV}$

energy transfer $=10 \mathrm{meV}$

Typical time to measure one data set (min) alue

$\sim 80$ 
maintaining a relatively large energy range. This very large secondary flight path requires a large area of analyzer crystals in order to cover sufficient solid angle. Fortunately, mica is relatively inexpensive, so such a large-area analyzer array is feasible. The analyzer crystal array is made in modular sections to facilitate alignment. Initially, only one side of the flight path is filled with the mica analyzer array. The other side is left open for subsequent installation of a similar mica array to double the count rate, or of an array of other suitable analyzer crystals.

Although this instrument is optimized for the 004 planes of mica, cleanly separated spectra from the other planes $(002,006$, etc.) are also obtained just by changing the chopper phasing. In order to aid the in situ study of the time-evolution of samples, the spectrometer is also equipped with an array of detectors located to measure the diffraction pattern from the sample concurrently with the inelastic-scattering spectra. These features, coupled with the ability to easily and independently select both the incident energy and incident-energy bandwidth, make this an extremely powerful and flexible instrument for high-resolution spectroscopy.

\subsubsection{TFCA - Time-Focused Crystal-Analyzer Spectrometer for Chemical Spectroscopy}

The TFCA is a time-focused crystal-analyzer spectrometer similar in principle to the Time-Focused Crystal Analyser Spectrometer (TFXA) ${ }^{30}$ at ISIS. It provides a relatively high resolution for spectroscopy at energy transfers up to $1 \mathrm{eV}$ or more, considerably extending the range for spectroscopy provided by the QENS. For energy transfers within the range covered by the QENS, this spectrometer offers somewhat poorer resolution but significantly increased data rates, and so it is complementary to the QENS over this range as well. Figure V.2.6-3 shows a schematic representation of this instrument, and Table V.2.6-3 indicates some of its parameters.

A neutron lens similar to that discussed above for the QENS can also be installed on the TFCA to allow measurements on much smaller samples with little or no loss in data rate.

\subsubsection{QSTAXC - Cold-Neutron Quasi-Steady-State Triple-Axis Spectrometer}

The QSTAXC is a conventional triple-axis spectrometer located $\sim 17 \mathrm{~m}$ from a large, coupled liquid $\mathrm{H}_{2}$ moderator on the $30-\mathrm{Hz}$ target. A guide increases the cold neutron flux at the monochromator, and a focusing crystal monochromator is used. The spectrometer is modeled after successful reactor cold-neutron triple-axis spectrometers such as IN12 or IN14 at ILL.31 The main difference is that the data acquisition is gated synchronously with the arrival of neutrons that have the desired energy. No order-removal filters are required. Some evolution of the instrument is expected as experience is gained with its use in the pulsed-source environment. The design incorporates features that enhance the flexibility and future adaptability of this instrument. 


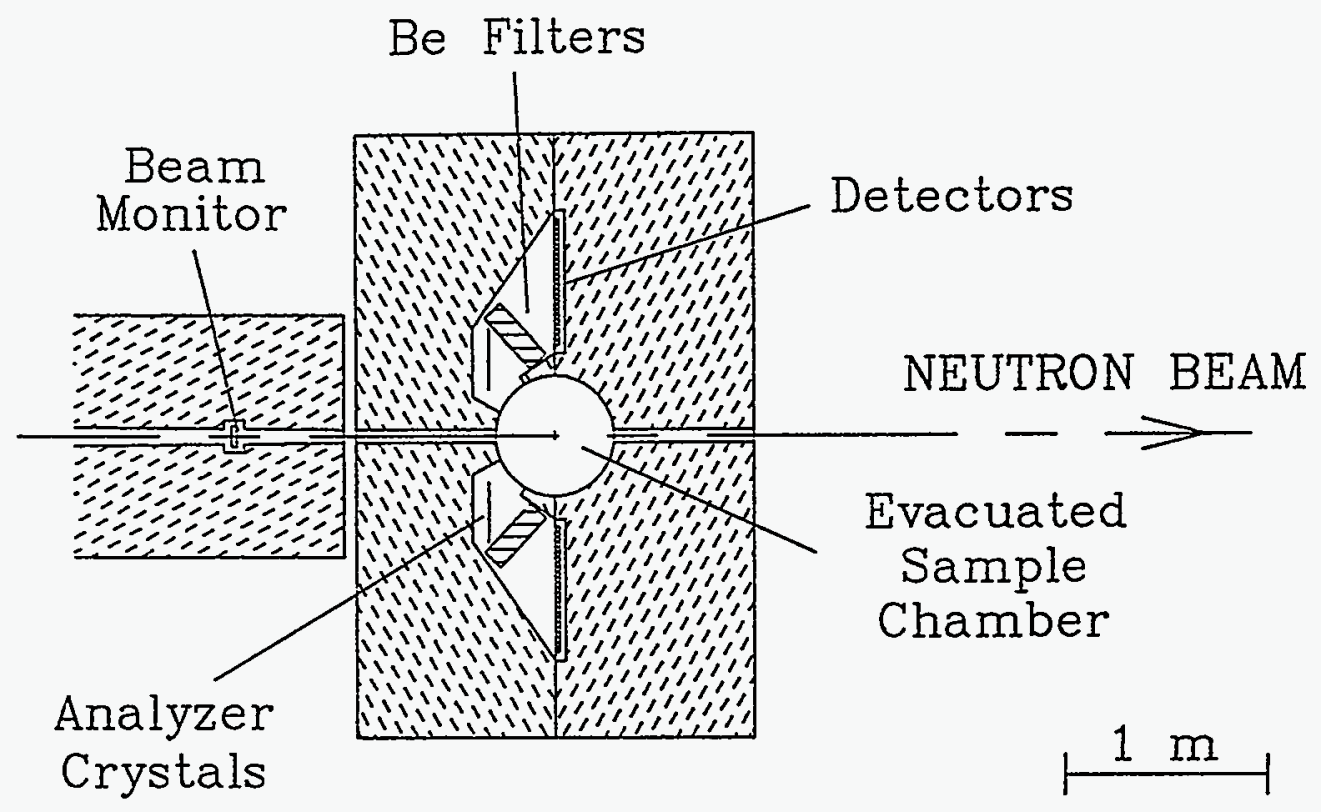

Figure V.2.6-3 Schematic Representation of the TFCA. 
Table V.2.6-3 Parameters for the TFCA

\begin{tabular}{ll}
\hline \multicolumn{1}{c}{ Parameter } & \multicolumn{1}{c}{ Value } \\
\hline & \\
Source-sample distance $(\mathrm{m})$ & 16 \\
Source repetition rate $(\mathrm{Hz})$ & 30 \\
Moderator material & poisoned liquid $\mathrm{CH}_{4}$ \\
Neutron guide & probably \\
Background-suppression chopper & if needed \\
Max. beam size at sample (cm $\left.{ }^{2}\right)$ & $\sim 2 \times 5$ \\
Analyzer crystals & pyrolytic graphite \\
Analyzer solid angle (sr) & $\sim 0.1-0.5$ \\
Detector type & $3 \mathrm{He}$ \\
Number of detectors & $\sim 200$ \\
Analyzer energy (meV) & $\sim 4$ \\
Energy resolution $\Delta \mathrm{E} / \mathrm{E}$ & $1-3 \%$ \\
Typical time to measure one data set (min) & $20-80$ \\
& \\
\hline
\end{tabular}


Section IV.3.2.5 shows that the time-averaged flux of cold neutrons on a sample is roughly the same as that expected from a cold source at a medium-flux reactor. This is sufficient to make the QSTAXC a very good instrument. The background-rejection capabilities of the QSTAXC make it unique for some types of experiments. It also forms a natural complement to other instruments at IPNS Upgrade.

\subsubsection{MICAS - Multiple-Independent-Crystal-Analyzer Spectrometer for Coherent Excitations}

The multiple-crystal-analyzer instruments $\mathrm{MAX}^{32}$ at KENS and PRISMA ${ }^{33}$ at ISIS allow (Q,E) scans along high symmetry directions in reciprocal space for mapping the coherent excitations in single-crystal samples. The sample is exposed to a polychromatic beam in these instruments, and the final energies of the scattered neutrons are determined by an array of analyzers and detectors. Incident energies are determined by time-of-flight. In typical operation, a high symmetry direction in the crystal is aligned parallel to the incident wavevector $\mathbf{k}_{\text {inc. }}$. Each analyzer selects a particular $\mathbf{k}_{\text {scatt, }}$ and $\left|\mathbf{k}_{\text {inc }}\right|$ is scanned by time-of-flight. All the analyzerdetector systems measure along the same line in reciprocal space if the Bragg angle $\theta_{B}$ of each analyzer is related to the scattering angle $\phi$ for that analyzer by the equation

$$
\mathrm{Q}_{\perp}=\pi \sin \theta_{\mathrm{B}} / \mathrm{d}_{\mathrm{B}} \sin \phi .
$$

Here $d_{B}$ is the spacing of the analyzer planes, and $Q \| l$ and $Q_{\perp}$ are the wavevector transfer components parallel and perpendicular to the incident beam, respectively. The scattering angle $\phi$ is different for each of the analyzer arms, so each arm measures a different energy transfer and wavevector transfer. The spectrometer performs a net of scans in the (Q\|l, E) plane. Some lines in reciprocal space are not accessible to such a spectrometer.

A large amount of development work has gone into PRISMA and MAX, and there is now considerable operating experience on both instruments. In favorable cases, PRISMA can collect a set of dispersion curves in a few hours. A major difficulty is mechanical interference between the independent analyzer arms, which limits the ranges of $\theta_{\mathrm{B}}$ that are accessible for some analyzers. In an effort to minimize this interference, shielding of the individual arms is kept to a minimum, and this leads to significant background problems in these instruments. Both of these problems can be significantly alleviated by the use of double analyzer crystals in each analyzer arm, so that the detector no longer needs to move about the analyzer position. Figure V.2.6-4 shows a schematic layout of a possible instrument configuration having 20 double-analyzer-crystal arms, each separated by $2^{\circ}$ in $\phi$. The entire bank of 20 analyzers can be rotated about the sample position, allowing coverage of $\phi$ roughly over the range $-120^{\circ}$ to $+120^{\circ}$. Graphite or germanium analyzer crystals, or a combination of the two, are used. The desirable moderator is liquid $\mathrm{CH}_{4}$ on the $30-\mathrm{Hz}$ source, and the incident flight path is on the order of $12 \mathrm{~m}$. 


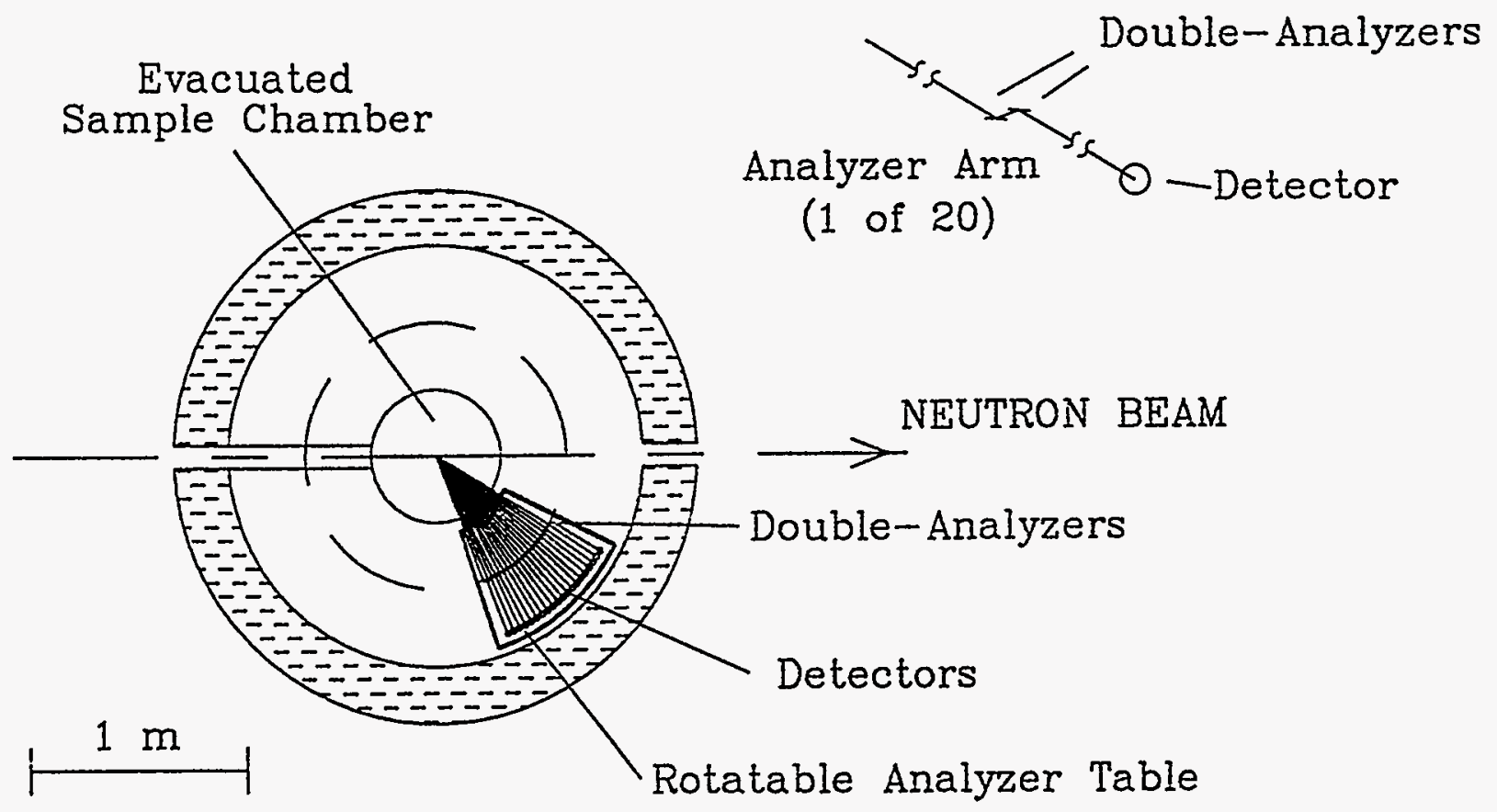

Figure V.2.6-4 Schematic Representation of the MICAS. 
A detailed analysis of the kinematic constraints and the resolution ellipsoids as functions of the different geometrical parameters is essential before final design parameters are fixed. It is desirable to provide analyzer arms capable of giving a finer angular coverage, down to $1^{\circ}$ separation between analyzers, if this can be done while still including adequate shielding for each analyzer. Such an instrument, optimized in light of the operating experience at MAX and PRISMA, can be a very useful survey instrument for mapping out coherent excitations in single crystals. In some cases, this might be followed by a detailed study of a more restricted region in reciprocal space using a triple-axis spectrometer, such as the QSTAXC or the QSTAXT (Section V.2.6.3.1).

\subsubsection{Other Possibilities}

This instrument is not in the reference set and will probably not be among the initial complement at IPNS Upgrade. It is expected that instruments of this type will evolve and might be added at a later date.

\subsubsection{QSTAXT - Thermal-Neutron Quasi-Steady-State Triple-Axis Spectrometer}

This is a complementary instrument to the cold-neutron triple-axis spectrometer QSTAXC. The QSTAXT is located $\sim 17 \mathrm{~m}$ from a large, room-temperature, coupled liquid $\mathrm{H}_{2} \mathrm{O}$ moderator on the $30-\mathrm{Hz}$ target. A guide provides some increase in the thermal neutron flux at the monochromator, and a focusing crystal monochromator is used. This instrument is physically similar to the QSTAXC, except for the changes in monochromator and analyzer crystals to optimize them for the thermal neutron range. As is the case for the QSTAXC, this instrument has very good background characteristics and rejects spurious peaks. Construction of the QSTAXT will be deferred until some experience is gained with the QSTAXC.

\subsubsection{Summary of Crystal-Analyzer Spectrometers}

The three instruments TFCA, QENS, and HRBS are optimized for chemical spectroscopy, and this group spans the full range of resolution and energy transfers practical for crystal-analyzer spectrometers at IPNS Upgrade. The high data rates on these instruments and their ability to work with small samples allows neutron chemical spectroscopy to be used for a broad range of samples for which it has previously been impractical. The two instruments QSTAXC and MICAS, optimized for the study of excitations along high-symmetry directions in single-crystals, cover the medium- and low-energy-transfer ranges much more efficiently than does the SCCS chopper spectrometer discussed above. The TFCA, QENS, HRBS, MICAS, and QSTAXC are taken as a reference set of crystal-analyzer spectrometers, and their performances are summarized in Table V.2.6-4. 
Table V.2.6-4 Reference Set of Crystal-Analyzer Spectrometers at IPNS Upgrade

\begin{tabular}{|c|c|c|c|c|}
\hline Instrument & Application/Special Feature & $\begin{array}{l}\text { Range for } \\
E \text { (meV) }\end{array}$ & $\Delta E(m e V)$ & $\begin{array}{l}\text { Measurement } \\
\text { Time (min) }\end{array}$ \\
\hline TFCA & General purpose (TOF) & $0-1000$ & $0.5-30$ & $20-80$ \\
\hline QENSa & Quasielastic, medium resolution (TOF) & $0-150$ & $0.05-3$ & $20-80$ \\
\hline HRBS & Microvolt resolution (TOF) & $0-10$ & $0.005-0.06$ & -80 \\
\hline MICAS & $\begin{array}{l}\text { Survey of single-crystal excitations } \\
\text { (TOF) }\end{array}$ & $0-20$ & -........ varies & ; --.---- \\
\hline QSTAXC & Cold-neutron triple axis (QSS) & - & - & - \\
\hline
\end{tabular}

a Transferred from IPNS with little change. 


\subsection{OTHER INELASTIC-SCATTERING INSTRUMENTS}

\subsubsection{New Reference Set Instruments}

\subsubsection{TOFNSE - Time-of-Flight Neutron Spin-Echo Spectrometer}

The spin-echo technique was first developed by Mezei and implemented on the IN11 spectrometer at ILL. ${ }^{34-37}$ In this technique, a neutron polarized perpendicular to the magneticfield direction travels through a precession field. The neutron rotates through an angle $\alpha$ during its traverse of the precession field region. The neutron spin is then flipped through an angle of $\pi$, before entering another precession field. There it rotates through an angle of $\alpha^{\prime}$ in the opposite sense to the first precession. The sample is placed before the $\pi$ spin flipper. If the fields are adjusted so that $\alpha^{\prime}=\alpha$ when there is no sample, then the phase shift $\alpha-\alpha^{\prime}$ is a measure of the energy change of the neutron upon scattering by the sample.

Mezei and Richter ${ }^{38}$ have analyzed the adaptation of this technique to a pulsed spallation neutron source instrument and have shown that the neutron spin-echo technique is particularly well-suited to time-of-flight applications. There is a gain of a factor of $\sim 8$ or more in useful data rate and a significant improvement in Q-resolution relative to a comparable instrument at a steady-state source having the same time-averaged flux. At the IN11 or the more recently constructed IN15 spin-echo spectrometers at ILL, only a single scattering angle is utilized at any given time, and the magnetic field is scanned to provide the intermediate scattering function $\mathrm{S}(\mathrm{Q}, \mathrm{t})$ for the sample. The TOFNSE instrument at IPNS Upgrade is based on the best features of IN11 and IN15, with time-of-flight electronics replacing the velocity selector for the wavelength determination. In this time-of-flight instrument, the wavelengths are scanned automatically to give concurrent data for a range of $Q$ values, but it is still necessary to perform the magnetic-field scan to determine $S(Q, t)$. The wavelength resolution, and hence $Q$ resolution, of the TOF measurement is inherently much better than the $\sim 20 \%$ wavelength resolution used on IN11. The accessible wavelength range, and hence $Q$ range, is also much greater than on IN11. Also, as shown by Mezei and Richter, this scan over a broad wavelength range leads to a much larger range for the $t$ variable in $S(Q, t)$ than on $\mathrm{IN} 11$.

Penfold and Williams ${ }^{39}$ proposed an instrument with multiple scattering angles. In this case the intermediate scattering function $S(Q, t)$ is directly determined by measurement of the phase shift as a function of scattering angle and TOF . The magnetic-field scan is unnecessary. However, such an instrument requires magnetic fields with a plane of symmetry. As a consequence, the field inhomogeneities are larger and are not correctable as are the inhomogeneities in the solenoidal fields. The resolution in the variable $t$ is considerably worse on such an instrument. 38 For this reason, and because the technology is further developed, the TOFNSE instrument is designed to use solenoidal fields, with components similar to those already developed for $\mathbb{I N 1 1}$ or $\mathbb{I N} 15$. The many magnetic fields used in this instrument require that there be no magnetic materials located within a few meters. Thus, it is placed on a relatively long beamline to provide the maximum separation from other instruments. A total moderator-to- 
detector distance of $25 \mathrm{~m}$ on the $10-\mathrm{Hz}$ source allows the use of wavelengths up to $15 \AA$ without frame-overlap. The intensity at wavelengths longer than this is not adequate for most experiments. A coupled liquid $\mathrm{H}_{2}$ moderator and a neutron guide provide the maximum flux of cold neutrons on the sample. Operation of this instrument is very similar to the operation of IN11. On the assumption that the gain in the effective use of neutrons is $\sim 8$, data rates are expected to be within a factor of $\sim 2$ of those on $\mathbb{N} 11$, while resolution and dynamical range are at least a factor of 10 better $^{38}$ than on IN11. Table V.2.7-1 summarizes the expected performance of this instrument and makes comparisons with $\mathrm{IN} 11$ where appropriate. Figure V.2.7-1 shows a schematic representation of the TOFNSE instrument.

\subsection{OTHER NEUTRON BEAMFACILITIES}

\subsubsection{New Reference Set Instrument}

\subsubsection{DEVEL — Instrument and Component Development}

Many of the instruments contemplated for IPNS Upgrade require development of one or more components in order to optimize performance. To assist in such development, one beamline is provided for general development work on neutron instrument components such as crystal analyzers and monochromators, detectors, collimators, and polarization devices. Such a development beamline requires appropriate collimation and shielding, a suitable work area, one or more detectors, and a data-acquisition system. This instrument is at the $10-\mathrm{Hz}$ target station.

\subsubsection{Other Possible Beamline Facilities}

These facilities are not included as part of the reference set, and will probably not be among the initial complement at IPNS Upgrade. They might be installed at a later date if demand warrants.

\subsubsection{RADIO — Neutron Radiography/Tomography}

The integrated thermal neutron beam current at the surface of a $10 \times 10-\mathrm{cm}^{2}$ coupled liquid $\mathrm{H}_{2}$ moderator on the $10-\mathrm{Hz}$ target is $\sim 2 \times 10^{14} \mathrm{n} / \mathrm{sr}$-sec. Cold neutrons make up $1 / 6$ of this flux. The total thermal flux at the end of a $25-\mathrm{m}$ beamline that views this moderator directly is $\sim 3 \times 10^{7} \mathrm{n} / \mathrm{cm}^{2}$-sec, and the corresponding cold neutron flux is $\sim 5 \times 10^{6} \mathrm{n} / \mathrm{cm}^{2}$-sec. These timeaveraged fluxes are adequate for a wide range of neutron radiography. ${ }^{41}$ A direct view of this moderator with no further collimation provides a length-to-diameter $(\mathrm{L} / \mathrm{D})$ ratio of 250 , which 
Table V.2.7-1 TOFNSE Parameters at IPNS Upgrade (comparison with IN11)

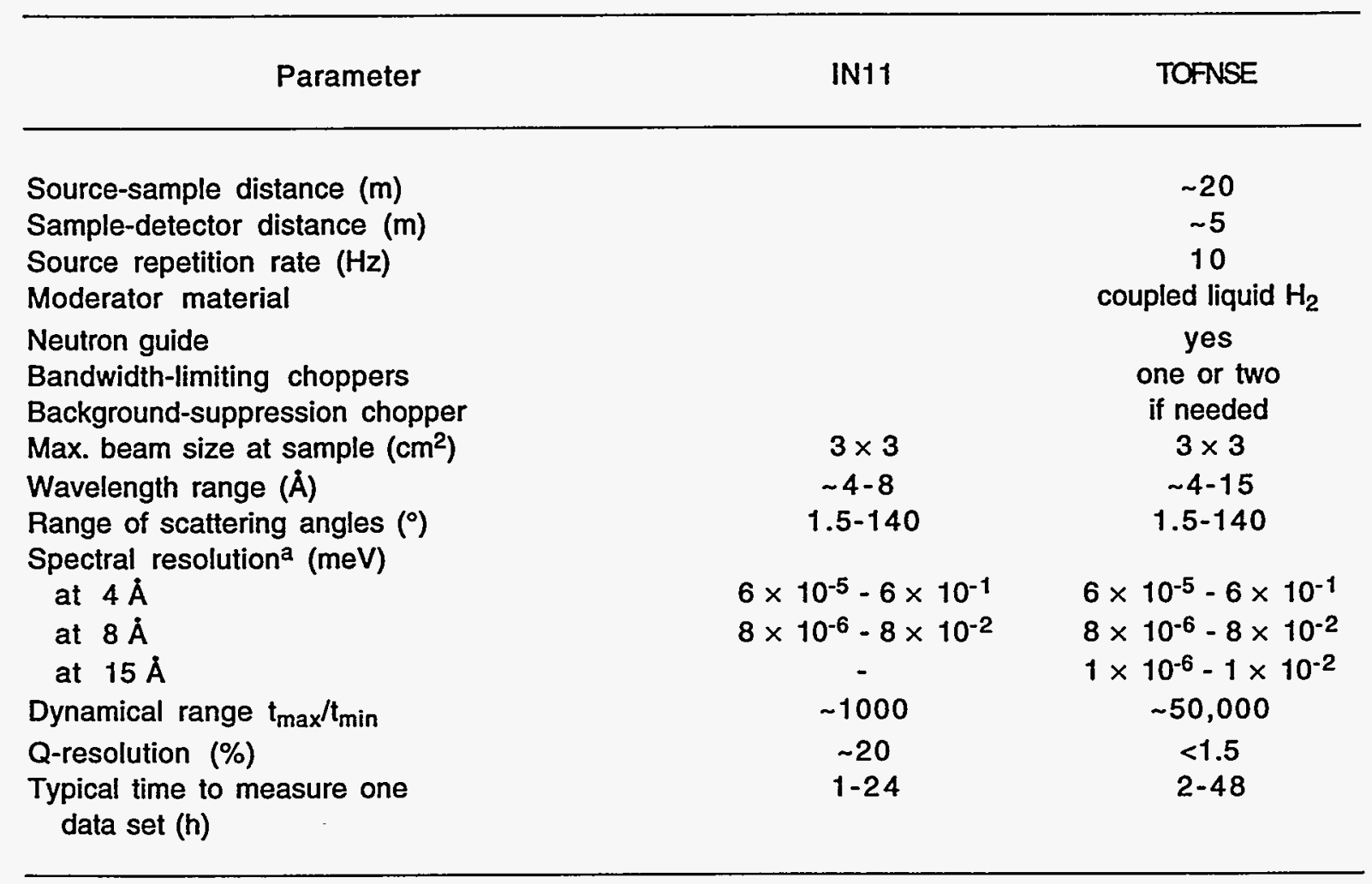

a The definition of "spectral resolution" differs from the definition of energy resolution on conventional spectrometers (see Reference 40). 


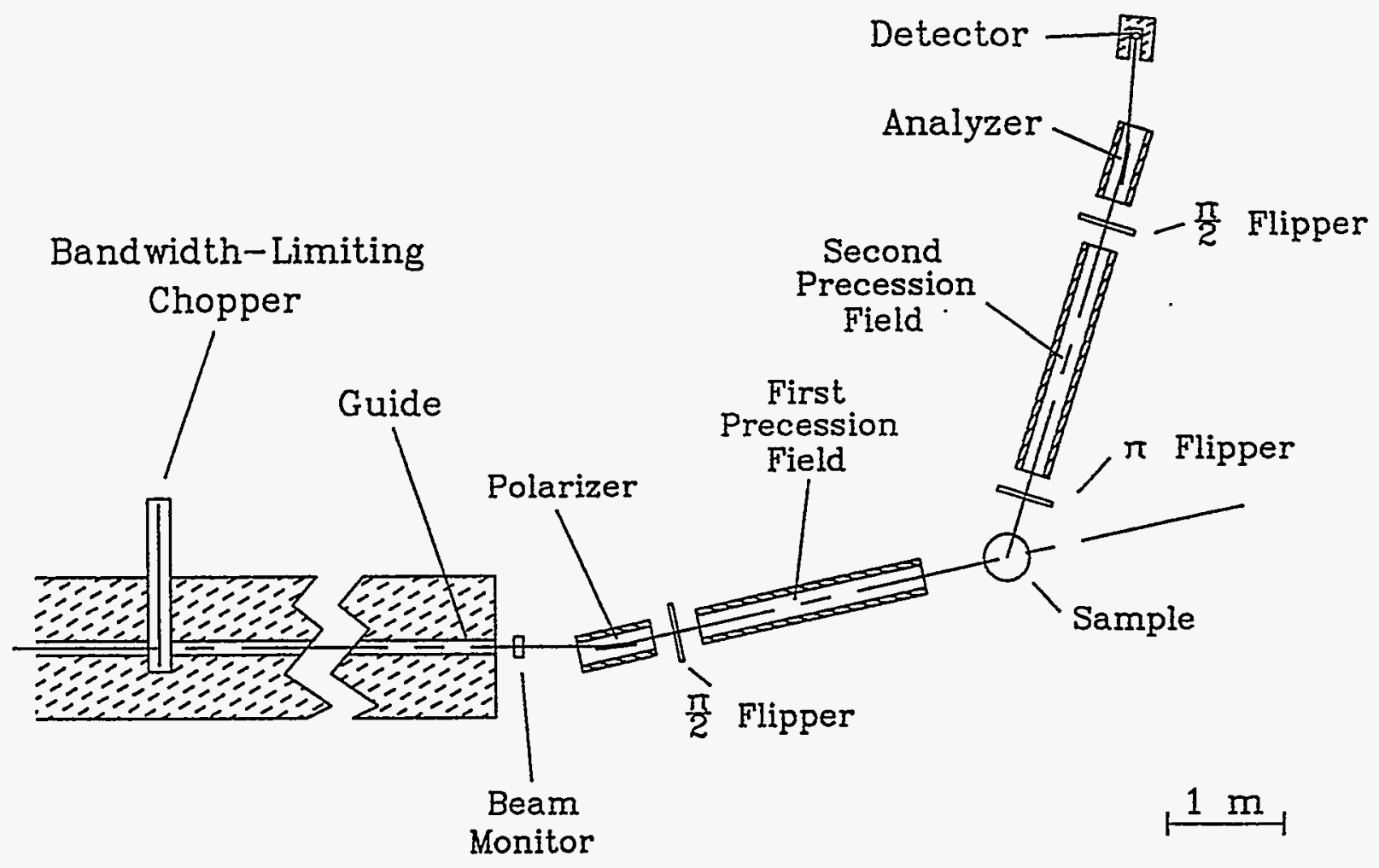

Figure V.2.7-1 Schematic Representation of the TOFNSE. 
gives a resolution of $1 \mathrm{~mm}$ for an object $25 \mathrm{~cm}$ from the detector plane. This resolution is adequate for viewing many large objects, but additional collimation can be inserted when necessary.

The RADIO can perform high-resolution time-integrated radiography using standard film or transfer-foil techniques and inserting various filters in the beam. Insertion of a Be filter into the beam allows cold-neutron radiography, while an $\mathrm{MgO}$ or sapphire filter limits the beam to thermal neutrons. A Cd or Gd filter can be used to remove the thermal neutrons, so fast-neutron radiography can also be performed on the same beamline.

A detector with timing capabilities can be used to collect the fast, thermal, and cold neutron radiographs concurrently. Resonance radiography and time-dependent radiography can be done on the same beamline. This facility can also be used for neutron tomography to provide a three-dimensional image of the specimen when desired.

\subsubsection{NDP/NAA — Neutron Depth Profiling and Neutron Activation Analysis}

The relatively high time-averaged flux of cold neutrons available at IPNS Upgrade make it possible to perform NDP and NAA, as well as they can be done at a $\sim 10^{14}-\mathrm{n} / \mathrm{cm}^{2}$-sec reactor. The time structure of the pulsed source appears to offer no pronounced advantages for these techniques.

\subsubsection{UCN - Ultra-Cold Neutron Facility}

The relatively high time-averaged flux of cold neutrons available at IPNS Upgrade make it possible to construct an ultra-cold neutron facility that functions as well as at a $\sim 10^{14}-\mathrm{n} / \mathrm{cm}^{2}$-sec reactor. The time structure of the pulsed source may offer some advantages for the production of ultra-cold neutrons.

\subsubsection{NINT - Neutron Interferometer}

The relatively high time-averaged flux of cold neutrons available at IPNS Upgrade make it possible to build a neutron interferometer that performs as well as a comparable interferometer at a $\sim 10^{14}-\mathrm{n} / \mathrm{cm}^{2}$-sec reactor. The time structure of the pulsed source appears to offer no pronounced advantage for most types of interferometry experiments.

\subsection{REFERENCE BEAM PORT ASSIGNMENTS}

Table V.2.9-1 lists the reference set of neutron scattering instruments identified in this chapter, separated according to $30-\mathrm{Hz}$ and $10-\mathrm{Hz}$ target stations. Also listed in the table are the 
flight-path lengths and moderator types required for these instruments. On the basis of these requirements, the instruments are assigned to beam ports on the two target stations, as indicated in the table. Table V.2.9-2 clearly indicates the types of moderators used in each of the six moderator locations on each target station and shows which instruments share each moderator.

Figure V.2.9-1 shows the locations of the instruments on their respective beamlines in the experiment halls. Buildings 369 and 370 accommodate all of the instruments in this reference set, with only the projected path that might be used for some configurations of the special-purpose small-angle neutron diffractometer SPSAND extending outside the buildings. If necessary, most of the remaining unused beamlines can be extended outside the buildings if some longer-flightpath instruments are desired in the future. 
Table V.2.9-1 Beamline Requirements for Instruments

\begin{tabular}{|c|c|c|c|c|c|c|}
\hline Instrument & Section & $L_{i}(m)$ & $L_{f}(m)$ & Moderator & Coupling $^{a}$ & Beam Port \\
\hline \multicolumn{7}{|l|}{ 30-Hz Target } \\
\hline *SEPD & 2.1 .1 .1 & 12 & 1.5 & $\mathrm{CH}_{4}{ }^{\mathrm{C}}$ & & H-B-3 \\
\hline *GPPD & 2.1 .1 .2 & 25 & $1.5-4$ & $\mathrm{CH}_{4}{ }^{\mathrm{C}}$ & & H-B-2 \\
\hline HRPD & 2.1.2.1 & 50 & 2 & $\mathrm{CH}_{4}{ }^{\mathrm{C}}$ & & $H-E-1$ \\
\hline RSD & 2.1.2.2 & 25 & $1.5-3$ & $\mathrm{CH}_{4}{ }^{\mathrm{C}}$ & & H-E-2 \\
\hline VSPD & 2.1.2.3 & 12 & 0.75 & $\mathrm{CH}_{4}{ }^{\mathrm{c}}$ & & $H-F-3$ \\
\hline${ }^{*}$ GLAD & 2.3.1.1 & $23.5^{d}$ & 1.5 & $\mathrm{CH}_{4}$ & & $\mathrm{H}-\mathrm{A}-1$ \\
\hline${ }^{*} \mathrm{SCD}$ & 2.3.1.2 & 10 & 0.6 & $\mathrm{CH}_{4}{ }^{\mathrm{C}}$ & & $\mathrm{H}-\mathrm{C}-3$ \\
\hline HQSCD & 2.3.2.1 & 30 & 0.7 & $\mathrm{CH}_{4}{ }^{\mathrm{C}}$ & & $\mathrm{H}-\mathrm{B}-1$ \\
\hline "HRMECS & 2.5.1.1 & 18 & 4 & $\mathrm{CH}_{4}$ & . & $H-A-3$ \\
\hline *LRMECS & 2.5.1.2 & 12 & 2.5 & $\mathrm{CH}_{4}$ & & $H-F-2$ \\
\hline $\operatorname{sccs}$ & 2.5.2.2 & 16 & 4 & $\mathrm{H}_{2} \mathrm{O}$ & & H-E-3 \\
\hline *QENS & 2.6 .1 .1 & 9 & $\leq 1$ & $\mathrm{CH}_{4}{ }^{\mathrm{e}}$ & & $\mathrm{H}-\mathrm{F}-1$ \\
\hline TFCA & 2.6.2.2 & 16 & $\leq 1.5$ & $\mathrm{CH}_{4}$ & & $\mathrm{H}-\mathrm{C}-1$ \\
\hline QSTAXC & 2.6.2.3 & 17 & $\leq 2$ & $\mathrm{H}_{2}$ & coupled & H-D-3 \\
\hline MICAS & 2.6.2.4 & 12 & 1.5 & $\mathrm{CH}_{4}$ & & $\mathrm{H}-\mathrm{A}-2$ \\
\hline $\begin{array}{l}\text { 10-Hz Target } \\
{ }^{*} \text { SAND }\end{array}$ & 2.2 .1 .1 & 12 & 2 & $\mathrm{H}_{2}$ & coupled & L-A-2 \\
\hline HRSAND & 2.2.2.1 & 20 & 5 & $\mathrm{H}_{2}$ & coupled & $L-A-1$ \\
\hline SPSAND & 2.2.2.2 & $\leq 40$ & & $\mathrm{H}_{2}$ & coupled & $L-A-3$ \\
\hline${ }^{\star}$ POSY-1 & 2.4.1.1 & 18 & $\leq 2$ & $\mathrm{H}_{2}$ & & L-F-2 \\
\hline HIREF & 2.4.2.1 & 18 & $\leq 2$ & $\mathrm{H}_{2}$ & & $L-F-2$ \\
\hline *POSY-II & 2.4.1.2 & 18 & $\leq 2$ & $\mathrm{H}_{2}$ & & L-F-3 \\
\hline GREF & 2.4.2.2 & 18 & 5 & $\mathrm{H}_{2}$ & & L-F-3 \\
\hline $\mathrm{PEDD}$ & 2.4.2.3 & 18 & & $\mathrm{H}_{2}$ & & L-F-3 \\
\hline CNCS & 2.5.2.1 & 20 & 4 & $\mathrm{H}_{2}$ & & L-D-1 \\
\hline HRBS & 2.6.2.1 & 30 & 3 & $\mathrm{H}_{2}$ & & L-D-2 \\
\hline TOFNSE & 2.7 .1 .1 & 16 & 4 & $\mathrm{H}_{2}$ & coupled & L-E-1 \\
\hline DEVEL & 2.8.1.1 & & & & & $L-F-1$ \\
\hline
\end{tabular}

- Instruments transferred from IPNS. All other instruments are new construction.

a All moderators are decoupled unless otherwise noted.

b Beam ports are numbered clockwise from the proton beam, as viewed from above, for each target station. Numbering scheme is target station $(\mathrm{L}$ or $\mathrm{H})$ - moderator (A thru F) and beam port on that moderator (1 thru 3). See Figure IV.4.1-2.

c Could be $\mathrm{H}_{2} \mathrm{O}$.

d To low-resolution sample position.

- Could be poisoned $\mathrm{H}_{2}$. 
Table V.2.9-2 Moderator Types

\begin{tabular}{|c|c|c|c|c|c|}
\hline Beam Port ${ }^{a}$ & Material & Instruments & Beam Porta & Material & Instruments \\
\hline 30-Hz target & station & & 10- $\mathrm{Hz}$ target & station & \\
\hline$H-A-1$ & liquid $\mathrm{CH}_{4}$ & GLAD & $L-A-1$ & coupled liq. $\mathrm{H}_{2}$ & HRSAND \\
\hline $\begin{array}{l}H-A-2 \\
H-A-3\end{array}$ & & $\begin{array}{l}\text { MICAS } \\
\text { HRMECS }\end{array}$ & $\begin{array}{l}\text { L-A-2 } \\
\text { L-A-3 }\end{array}$ & & $\begin{array}{l}\text { SAND } \\
\text { SPSAND }\end{array}$ \\
\hline H-B-1 & room temp. $\mathrm{H}_{2} \mathrm{O}$ & HQSCD & $L-B-1$ & $-b$ & - \\
\hline $\begin{array}{l}H-B-2 \\
H-B-3\end{array}$ & & $\begin{array}{l}\text { GPPD } \\
\text { SEPD }\end{array}$ & $\begin{array}{l}L-B-2 \\
L-B-3\end{array}$ & & - \\
\hline $\mathrm{H}-\mathrm{C}-1$ & liquid $\mathrm{CH}_{4}$ & TFCA & $L-C-1$ & - & - \\
\hline $\begin{array}{l}\mathrm{H}-\mathrm{C}-2 \\
\mathrm{H}-\mathrm{C}-3\end{array}$ & & $\overline{S C D}$ & $\begin{array}{l}L-C-2 \\
L-C-3\end{array}$ & & - \\
\hline$H-D-1$ & coupled liq. $\mathrm{H}_{2}$ & - & $L-D-1$ & poisoned liq. $\mathrm{H}_{2}$ & CNCS \\
\hline $\begin{array}{l}H-D-2 \\
H-D-3\end{array}$ & & QSTAXC & $\begin{array}{l}L-D-2 \\
L-D-3\end{array}$ & & HRBS \\
\hline$H-E-1$ & room temp. $\mathrm{H}_{2} \mathrm{O}$ & HRPD & $L-E-1$ & coupled liq. $\mathrm{H}_{2}$ & TOFNSE \\
\hline $\begin{array}{l}H-E-2 \\
H-E-3\end{array}$ & & $\begin{array}{l}\text { RSD } \\
\text { SCCS }\end{array}$ & $\begin{array}{l}\text { L-E-2 } \\
L-E-3\end{array}$ & & - \\
\hline$H-F-1$ & liquid $\mathrm{CH}_{4}$ & QENS & $L-F-1$ & liquid $\mathrm{H}_{2}$ & DEVEI \\
\hline $\begin{array}{l}H-F-2 \\
H-F-3\end{array}$ & & $\begin{array}{l}\text { LRMECS } \\
\text { VSPD }\end{array}$ & $\begin{array}{l}\text { L-F-2 } \\
\text { L-F-3 }\end{array}$ & & $\begin{array}{l}\text { POSY-I/HIREF } \\
\text { POSY-II/GREF/ } \\
\text { REFD }\end{array}$ \\
\hline
\end{tabular}

a Beam ports are numbered clockwise from the proton beam, as viewed from above, for each target station. Numbering scheme is target station $(\mathrm{L}$ or $\mathrm{H})$ moderator $(A$ thru $F)$ and beam port on that moderator (1 thru 3). See Figure IV.4.1-2.

b - = not used for initial instrument complement. 


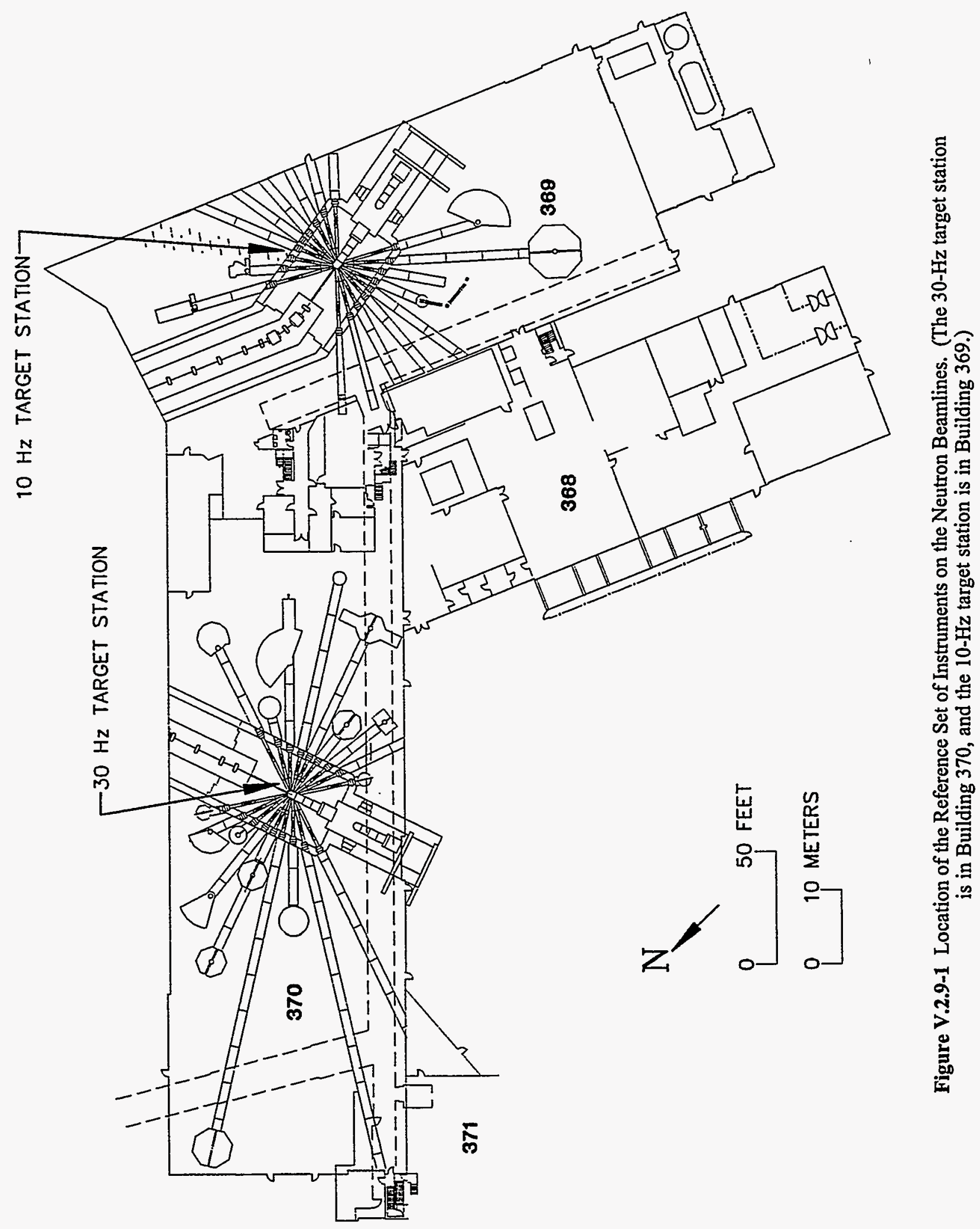




\subsection{REFERENCES}

1. J.D. Jorgensen et al., "Electronically focused time-of-flight powder diffractometers at the Intense Pulsed Neutron Source," J. Appl. Cryst. 22, 321-333 (1989).

2. J.D. Jorgensen, "Multiple-angle time-of-flight design concepts: Are we overlooking an order of magnitude in instrument performance for general structural studies?," in Proceedings of the 1986 Workshop on Advanced Time-of-Flight Neutron Powder Diffraction (Los Alamos, N.M., May 19-21, 1986), Los Alamos National Laboratory Report No. LA-10824-C, pp. $43-45$ (1986).

3. W.I.F. David, "Powder diffraction at ISIS," in Proceedings of the 11th Meeting of the International Collaboration on Advanced Neutron Sources (ICANS-XI) (Tsukuba, Japan, Oct. 22-26, 1990), National Laboratory for High Energy Physics (KEK) Report No. 90-25, pp. 1114-1121 (1991).

4. A. Oed, "Position-sensitive detector with microstrip anode for electron multiplication with gases," Nucl. Instrum. Methods A263, 351-359 (1988).

5. P. Geltenbort and A. Oed, "Features of microstrip proportional counters," in Proceedings of SPIE's 1992 International Symposium on Optical Applied Science and Engineering (San Diego, Calif., July 19-24, 1992), vol. 1737, pp. 289-293 (1992).

6. J.E. Epperson and P. Thiyagarajan, "Anomalous and resonance small angle scattering," J. Appl. Cryst. 21, 652-662 (1988).

7. B. Hammouda, S. Krueger, and C. Glinka, "Small angle neutron scattering at National Institute of Standards and Technology," NIST J. Res. (1991).

8. D. Schwahn et al., "Test of channel-cut perfect crystals for neutron small angle scattering experiments," Nucl. Instrum. Methods A239, 229-234 (1985).

9. R.K. Crawford et al., "Status of the new GLAD instrument at IPNS," in Proceedings of the 11th Meeting of the International Collaboration on Advanced Neutron Sources (ICANS-XI) (Tsukuba, Japan, Oct. 22-26, 1990), National Laboratory for High Energy Physics (KEK) Report No. 90-25, pp. 820-829 (1991).

10. A.J. Schultz, R.G. Teller, and J.M. Williams, "The IPNS time-of-flight single crystal diffractometer," in Proceedings of the 6th Meeting of the International Collaboration on Advanced Neutron Sources (ICANS-VI) (Argonne, Ill., June 28-July 2, 1982), Argonne National Laboratory Report No. ANL-82-80, pp. 116-124 (1982). 
11. B.P. Schoenborn and R. Diamond, "Neutron diffraction analysis of metmyoglobin," in Neutron Scattering for the Analysis of Biological Structures, Report of Symposium (June 2-6, 1975), edited by B.P. Schoenborn, Brookhaven Symposia in Biology No. 27, Brookhaven National Laboratory Report No. BNL 50453, pp. II-3-II-11 (1976).

12. B.P. Schoenborn, "A protein structure is only as good as the data," in Neutrons in Biology, edited by B.P. Schoenborn, pp. 261-279 (Plenum, New York, 1984).

13. G.P. Felcher et al., "Polarized neutron reflectometer: A new instrument to measure magnetic depth profiles," Rev. Sci: Instrum. 58, 609-619 (1987).

14. A. Karim et al., "An automated neutron reflectometer (POSY II) at the Intense Pulsed Neutron Source," Physica B 173, 17-24 (1991).

15. D.L. Price et al., "The IPNS-I chopper spectrometers," in Proceedings of the 6th Meeting of the International Collaboration on Advanced Neutron Sources (ICANS-VI) (Argonne, Ill., June 28-July 2, 1982), Argonne National Laboratory Report No. ANL-82-80, pp. 207-215 (1982).

16. C.-K. Loong, S. Ikeda, and J.M. Carpenter, "The resolution function of a pulsed-source neutron chopper spectrometer," Nucl. Instrum. Methods A260, 381-402 (1987).

17. J.M. Carpenter and C.-K. Loong, "IPNS chopper spectrometer improvements," in Proceedings of the 11th Meeting of the International Collaboration on Advanced Neutron Sources (ICANS-XI) (Tsukuba, Japan, Oct. 22-26, 1990), National Laboratory for High Energy Physics (KEK) Report No. 90-25, pp. 711-716 (1991).

18. Neutron Research Facilities at the ILL High Flux Reactor (Institut Laue-Langevin, Grenoble, France, 1983).

19. R.E. Lechner, "Optimization of a multi-disk chopper spectrometer for cold neutron scattering experiments," in Proceedings of the 11th. Meeting of the International Collaboration on Advanced Neutron Sources (ICANS-XI) (Tsukuba, Japan, Oct. 22-26, 1990), National Laboratory for High Energy Physics (KEK) Report No. 90-25, pp. 717-732 (1991).

20. C.-K. Loong et al., "Neutron scattering study of the magnetic excitations in ferromagnetic iron at high energy transfers," J. Appl. Phys. 55, 1895-1897 (1984).

21. S. Hayden et al., "High energy spin waves in $\mathrm{La}_{2} \mathrm{CuO}_{4}$," Phys. Rev. Lett. 67, 3622 (1991).

22. R.T. Heap et al., "High energy magnetic excitations in chromium," Physica B 174, 22 (1991).

23. D.McK. Paul and A. Boothroyd, "MAPS: A Multi-Angle Position Sensitive Spectrometer," Rutherford Appleton Laboratory, 1992 (unpublished). 
24. R.A Robinson, "On the kinematics and resolution of spectrometers for neutron Brillouin scattering," in Advanced Neutron Sources 1988, Proceedings of the 10th Meeting of the International Collaboration on Advanced Neutron Sources (ICANS-X) (Los Alamos, N.M., Oct. 3-7, 1988), Institute of Physics Conference Series No. 97, pp. 311-325 (Institute of Physics, New York, 1989).

25. K.F. Bradley et al., "The design and performance of QENS, a medium resolution, inverted geometry, TOF quasielastic and inelastic spectrometer at IPNS," Nucl. Instrum. Methods A270, 78-89 (1988).

26. H. Chen et al., "Cold neutron focusing using capillary optics," in Proceedings of SPIE's 1992 International Symposium on Optical Applied Science and Engineering (San Diego, Calif., July 19-24, 1992), Vol. 1738 (1992).

27. D.F.R. Mildner, A. Magerl, and U. Grüning, "Promise of thin silicon wafer microguides for future optical elements," in Proceedings of SPIE's 1992 International Symposium on Optical Applied Science and Engineering (San Diego, Calif., July 19-24, 1992), Vol. 1738 (1992).

28. C.J. Carlile et al., "Advances in crystal analyser spectroscopy at ISIS," in Proceedings of the 11th Meeting of the International Collaboration on Advanced Neutron Sources (ICANS-XI) (Tsukuba, Japan, Oct. 22-26, 1990), National Laboratory for High Energy Physics (KEK) Report No. 90-25, pp. 752-773 (1991).

29. S. Ikeda et al., "High resolution neutron spectrometer LAM-80ET and rotational tunnelling in 4-methylpyridine N-oxide," J. Phys. Soc. Japan 60, 3340-3350 (1991).

30. J. Penfold and J. Tomkinson, The ISIS Time Focused Crystal Analyser Spectrometer, TFXA, Rutherford Appleton Laboratory Report No. RAL-86-019 (1986).

31. W.G. Stirling and W. Kaiser, Preliminary Technical Specifications for Triple-Axis Spectrometer IN-14, Institut Laue-Langevin Technical Report No. 83ST24T (Grenoble, France, 1984).

32. Y. Todate et al., "Recent progress on MAX," in Proceedings of the 11th Meeting of the International Collaboration on Advanced Neutron Sources (ICANS-XI) (Tsukuba, Japan, Oct. 22-26, 1990), National Laboratory for High Energy Physics (KEK) Report No. 90-25, pp. 739-742 (1991).

33. U. Steigenberger et al., "Progress with the PRISMA spectrometer," in Proceedings of the 11th Meeting of the International Collaboration on Advanced Neutron Sources (ICANS-XI) (Tsukuba, Japan, Oct. 22-26, 1990), National Laboratory for High Energy Physics (KEK) Report No. 90-25, pp. 743-751 (1991).

34. F. Mezei, "The principles of neutron spin echo," in Neutron Spin Echo, edited by F. Mezei Lecture Notes in Physics, vol. 128, pp. 3-26 (Springer-Verlag, New York, 1980). 
35. O. Schärpf, "The polarized neutron technique of neutron spin echo," in Neutron Spin Echo, edited by F. Mezei, Lecture Notes in Physics, vol. 128, pp. 27-52 (Springer-Verlag, New York, 1980).

36. J. Hayter, "Theory of neutron spin-echo spectroscopy," in Neutron Spin Echo, edited by F. Mezei, Lecture Notes in Physics, vol. 128, pp. 53-65 (Springer-Verlag, New York, 1980).

37. P.A. Dagleish, J.B. Hayter, and F. Mezei, "The IN11 neutron spin echo spectrometer," in Neutron Spin Echo, edited by F. Mezei, Lecture Notes in Physics, vol. 128, pp. 66-71 (Springer-Verlag, New York, 1980).

38. F. Mezei and D. Richter, "Time-of-flight spin echo," in Proceedings of the Workshop on Neutron Scattering Instrumentation for SNQ (Maria Laach, Germany, Sept. 3-5, 1984), KFA Jülich Report No. 1954, pp. 368-377 (1984).

39. J. Penfold and W.G. Williams, Neutron Spin Echo Spectroscopy on the Spallation Neutron Source, Rutherford Appleton Laboratory Report No. RL-81-019 (1981).

40. A. Heidemann, W.S. Howells, and G. Jenkin, "Comparison of the performance of the backscattering spectrometer $\mathrm{IN} 10$ and the neutron spin echo spectrometer $\mathrm{IN} 11$ on the basis of experimental results," in Neutron Spin Echo, edited by F. Mezei, Lecture Notes in Physics, vol. 128, pp. 122-135 (Springer-Verlag, New York, 1980).

41. J.P. Barton and P. Von Der Hardt (editors), Neutron Radiography, proceedings of the First World Conference (San Diego, Calif., Dec. 7-10, 1981) (D. Reidel Publishing, Dordrecht, Holland, 1983). 


\subsection{CHOPPERS}

\subsubsection{Chopper Fundamentals}

Neutron choppers are devices that interrupt a neutron beam in a controlled fashion. Most neutron choppers are rotating mechanical devices. The basic equations for estimating the performance of such devices are summarized below; a much more detailed discussion of performance and optimization of rotating mechanical choppers is available in the book by Windsor. ${ }^{1}$ Alternatives to rotating mechanical choppers of the types discussed here have been used in some specialized applications. These alternatives include velocity selectors, statistical choppers, magnetic field choppers for polarized neutrons, oscillating or rotating Bragg-reflecting crystals, and nonrotating mechanical devices (linear motion, such as in piezoelectric, magnetic, or mechanical drives). However, since none of these alternative methods is required for use on the initial complement of instruments at IPNS Upgrade, the discussion here is restricted to the Fermi, drum, and disk types of rotating mechanical choppers.

The Fermi chopper is opaque to neutrons but contains a package of slits, usually curved, which transmit neutrons of certain speeds when the slits are lined up with the neutron beam. Such a chopper is shown schematically in Figure V.3.1-1a. Fermi choppers are rotated, usually at high rotational speeds, about an axis that is perpendicular to the neutron beam. Such choppers are capable of transmitting bursts of neutrons that have very narrow time-widths and hence are suitable for precise TOF measurements.

The time-width $\Delta t$ of the pulse of optimal-energy neutrons from such a Fermi chopper is given by

$$
\Delta t=\left[\left(\frac{\mathrm{d}}{2 \omega \mathrm{r}}\right)^{2}+\left(\frac{\Delta \alpha}{\omega}\right)^{2}\right]^{1 / 2},
$$

where the first term inside the square brackets is the square of the chopper pulse-width for a perfectly collimated beam and the second term gives the lighthouse effect resulting from the finite divergence $\Delta \alpha$ in the incident beam. Here $\omega$ is the angular frequency of the chopper, $d$ is the chopper slit-width, and $r$ is the chopper radius. The transmission of such a chopper is a maximum for neutrons of velocity

$$
\mathrm{v}_{\mathrm{o}}=2 \omega \mathrm{R},
$$

where $\mathrm{R}$ is the radius of curvature of the chopper slits. For neutron velocities $\mathrm{v}$ not too different in value from $v_{0}$, the transmission $T$ is given by

$$
T=X\left[1-\frac{8 \omega^{2} r^{4}}{3 d^{2}}\left(\frac{1}{v}-\frac{1}{v_{0}}\right)^{2}\right],
$$




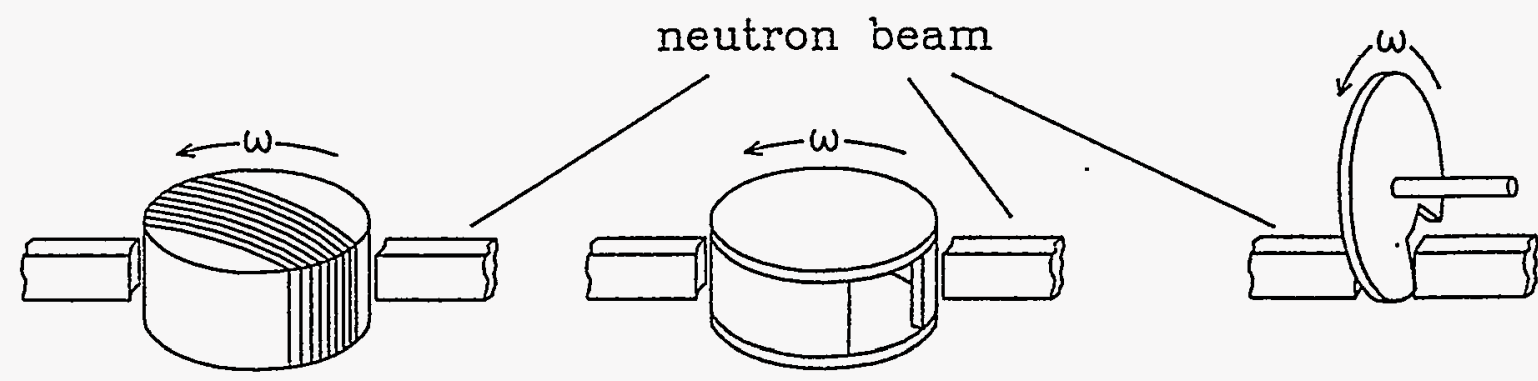

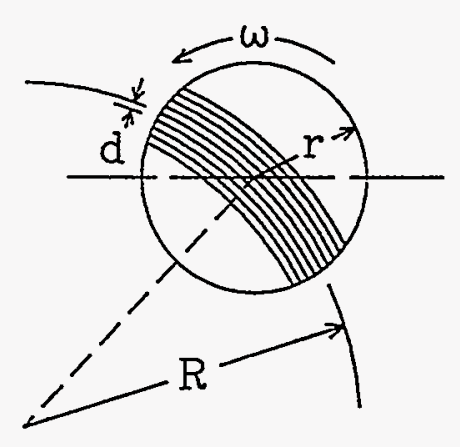

(a)

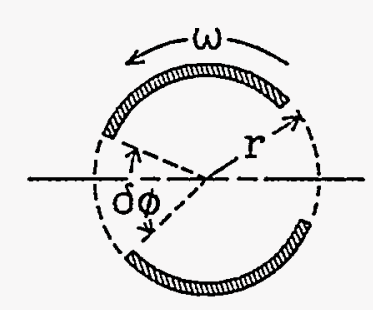

(b)

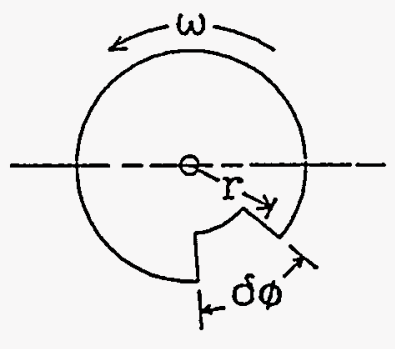

(c)

Figure V.3.1-1 Schematic Representations of (a) Fermi, (b) Drum, and (c) Disk Choppers.

(Symbols used in the text are defined here.) 
where $\mathrm{X}$ is the fraction of the cross-sectional area of the slit package that is transparent to neutrons.

Drum and disk choppers are shown schematically in Figures V.3.1-1b and V.3.1-1c, respectively. A drum chopper is rotated about an axis that is perpendicular to the neutron beam, while a disk chopper is rotated about an axis that is parallel to the neutron beam. These choppers are used to block the neutron beam for selected time intervals, such as when it is desired to limit the range of wavelengths that reach the sample or to prevent fast neutrons from the prompt $t_{o}$ pulse from reaching the sample. These types of choppers are generally not capable of producing sharp pulses of neutrons for precise TOF measurements except when very narrow beams of neutrons are being chopped.

The effective width $W$ of the neutron beam at the chopper is

$$
\mathrm{W}=\left[\mathrm{W}_{\mathrm{s}} \mathrm{L}_{\mathrm{c}}+\mathrm{W}_{\mathrm{m}}\left(\mathrm{L}_{\mathrm{s}}-\mathrm{L}_{\mathrm{c}}\right)\right] / \mathrm{L}_{\mathrm{s}},
$$

where $\mathrm{W}_{\mathrm{m}}$ and $\mathrm{W}_{\mathrm{s}}$ are the widths of the moderator and sample (or corresponding beam-defining apertures), respectively, and $\mathrm{L}_{\mathrm{c}}$ and $\mathrm{L}_{\mathrm{s}}$ are the moderator-to-chopper and moderator-to-sample distances, respectively. In order to go from fully open to fully closed, a disk chopper must rotate neutron absorber through the effective beam-width $\mathrm{W}$, while each side of a drum chopper only has to rotate absorber through half the beam. The required angular rotation $\delta \phi_{c}$ in the two cases is given by

$$
\begin{array}{ll}
\delta \phi_{c}=2 \sin ^{-1}(W / 2 r) & \text { disk } \\
\delta \phi_{c}=\sin ^{-1}(W / 2 r) & \text { drum , }
\end{array}
$$

where $r$ is the chopper radius. This definition of $\delta \phi_{c}$ allows the following equations to apply to both types of choppers.

The time $\delta \mathrm{t}_{\mathrm{c}}$ required for this rotation is

$$
\delta t_{c}=\delta \phi_{c} \omega \text {. }
$$

During this rotation, neutrons having a range of wavelengths $\delta \lambda_{c}$ are incident on, and partially transmitted by, the chopper:

$$
\delta \lambda_{c}=(\mathrm{h} / \mathrm{m}) \delta \phi_{d} \omega \mathrm{L}_{\mathrm{c}},
$$

where $\mathrm{h} / \mathrm{m}=3955.4 \AA-\mathrm{m} / \mathrm{sec}$ is Planck's constant divided by the neutron mass. 
If it is desired that a disk or drum chopper be fully open for neutrons with wavelengths between $\lambda_{\min }$ and $\lambda_{\max }$, then the chopper must be fully open between times $t_{\min }$ and $t_{\max }$. During this time interval, the chopper rotates through an angle $\delta \phi_{\mathrm{r}}$ given by

$$
\begin{aligned}
\delta \phi_{\mathrm{r}} & =\omega\left(\mathrm{t}_{\max }-\mathrm{t}_{\min }\right) \\
& =(\mathrm{m} / \mathrm{h}) \omega \mathrm{L}_{\mathrm{c}} \delta \lambda_{\mathrm{t}},
\end{aligned}
$$

where

$$
\delta \lambda_{t}=\lambda_{\max }-\lambda_{\min }
$$

The total chopper open angle $\delta \phi$ required in order to give the desired bandpass is

$$
\begin{array}{ll}
\delta \phi=\delta \phi_{\mathrm{r}}+\delta \phi_{\mathrm{c}} & \text { disk } \\
\delta \phi=\delta \phi_{\mathrm{r}}+2 \delta \phi_{\mathrm{c}} & \text { drum . }
\end{array}
$$

Drum choppers have the advantage that they open and close uniformly across the beam, while disk choppers open and close less rapidly for the portions of the beam nearer the disk axis. Thus, disk choppers are less satisfactory for large beams. Drum choppers can also incorporate large amounts of neutron absorber more easily than can disk choppers. However, on instruments with neutron guides, a disk chopper requires only one cut in the guide, while a drum chopper requires two. Furthermore, a pair of synchronized counter-rotating disk choppers can be made to open and close twice as fast as a single disk chopper of the same size and speed. In practice, the spatial constraints imposed by adjacent equipment may dictate which type of chopper is to be used.

If the moderator-sample-detector distance is $L_{d}$ and the source repetition rate is $f$, then the maximum wavelength of neutrons that can reach the detector before the next pulse occurs is $\lambda_{\text {frame, }}$ given by

$$
\lambda_{\text {frame }}=(\mathrm{h} / \mathrm{m}) / \mathrm{fL}_{\mathrm{d}} .
$$

The maximum wavelength that is transmitted before the chopper is fully closed is $\lambda_{\mathrm{cl}}$, given by

$$
\lambda_{\mathrm{cl}}=\lambda_{\max }+\delta \lambda_{\mathrm{c}}
$$

Neutrons of this wavelength arrive at the detector at the same time as neutrons having the wavelength $\lambda_{\text {fo }}$ originating from the following pulse, where

$$
\lambda_{\mathrm{fo}}=\lambda_{\mathrm{cl}}-\lambda_{\mathrm{frame}} .
$$


If $\lambda_{\mathrm{fo}}$ is negative, then none of the neutrons transmitted by the chopper arrive at the detectors during the following frame. However, if $\lambda_{\mathrm{fo}}$ is positive, the portion of the spectrum at wavelengths up to $\lambda_{\mathrm{fo}}$ is contaminated with such frame-overlap neutrons. If $\lambda_{\mathrm{fo}}$ is less than $\lambda_{\min }$, this is still not a problem; if $\lambda_{\mathrm{fo}}$ is greater than $\lambda_{\min }$, a frame-overlap condition exists, and the quality of the data may be degraded.

\subsubsection{Survey of Chopper Requirements}

Table V.3.1-1 lists the instruments that form the reference set and summarizes the requirements for choppers of various types. The sections of Chapter $\mathrm{V}$ where each of these instruments is discussed above are also indicated. Specific choppers to satisfy each of these requirements are discussed below.

\subsubsection{Specific Chopper Designs}

\subsubsection{Fermi $E_{0}$ Choppers}

The $E_{0}$ choppers are used to select the energy of the neutrons incident on the sample in chopper spectrometers and in specialized uses of some other instruments. At IPNS, several different choppers with transmissions optimized for different energies are available for use on the HRMECS and LRMECS. 2 These mechanical-bearing choppers, with relatively massive $\mathrm{Be}$ bodies and B/Al composite slit packages, are reliable and are very effective in stopping fast neutrons. They are designed to transmit relatively large beams, $5 \times 10 \mathrm{~cm}^{2}$ for the LRMECS and $7.5 \times 10 \mathrm{~cm}^{2}$ for the HRMECS, making it possible to take advantage of large samples. These choppers are used at IPNS Upgrade as well.

Additional choppers of this type are required in order to extend the energies for which optimized choppers are available and to avoid conflicts in scheduling the use of specific choppers for several instruments. These IPNS choppers are limited to a maximum rotational frequency of $270 \mathrm{~Hz}$. Figure V.3.1-2 shows such a chopper schematically. Figure V.3.1-3 shows the transmissions of the IPNS choppers at $270 \mathrm{~Hz}$ as functions of neutron energy, calculated using the full expression for the transmission function. ${ }^{3}$ This figure indicates that at least four, and perhaps five, different choppers are required to span the full range of incident energies useful on such instruments as the HRMECS and LRMECS.

Equation V.3.1-1 shows that the chopper pulse-width can be decreased, and hence the resolution improved, either by decreasing $d$ or by increasing the rotational speed $\omega$. There are practical limits to how small $\mathrm{d}$ can be made, so to provide better time resolution than that available from the IPNS choppers, $\omega$ must be increased. ISIS instruments operate with somewhat smaller choppers having $\mathrm{Al}$ bodies and $\mathrm{B} / \mathrm{Al}$ composite slit packages. These choppers 
Table V.3.1-1 Required Chopper Types

\begin{tabular}{|c|c|c|c|c|c|c|}
\hline \multirow[b]{2}{*}{ Instrument } & \multirow[b]{2}{*}{ Section } & \multirow[b]{2}{*}{$E_{0}$} & \multicolumn{3}{|c|}{$t_{0}$} & \multirow[b]{2}{*}{$\begin{array}{l}\text { Background/ } \\
\text { Bandwidth }^{\mathrm{a}}\end{array}$} \\
\hline & & & Broad & Narrow & $\begin{array}{l}\text { Pulse- } \\
\text { Eliminating }\end{array}$ & \\
\hline SEPD & 2.1.1.1 & & & & & bkgnd \\
\hline GPPD & 2.1 .1 .2 & & & & & $<0.4-4.6>$ \\
\hline HRPD & 2.1 .2 .1 & & yes? & & $<$ yes > & $<0.4-2.4>$ \\
\hline RSD & 2.1.2.2 & & & & & bkgnd \\
\hline VSPD & 2.1 .2 .3 & & & & & bkgnd \\
\hline SAND & 2.2 .1 .1 & yes? & yes? & & $?$ & $<b k g n d>$ \\
\hline HRSAND & 2.2.2.1 & & & & & <bkgnd> \\
\hline SPSAND & 2.2.2.2 & yes? & yes & & & bkgnd \\
\hline GLAD & 2.3 .1 .1 & yes & $?$ & yes & & bkgnd \\
\hline$S C D$ & 2.3.1.2 & & & & & bkgnd? \\
\hline HQSCD & 2.3.2.1 & & & & & bkgnd? \\
\hline POSY-I & 2.4 .1 .1 & & yes & & & $?$ \\
\hline POSY-11 & 2.4 .1 .2 & & yes & & & $?$ \\
\hline HIREF & 2.4.2.1 & & yes & & & $?$ \\
\hline GREF & 2.4.2.2 & & yes & & & $?$ \\
\hline REFD & 2.4.2.3 & & yes & & & $?$ \\
\hline HRMECS & 2.5.1.1 & $<$ yes $>$ & & $<$ yes $>$ & & \\
\hline LRMECS & 2.5 .1 .2 & <yes> & & <yes> & & \\
\hline CNCS & 2.5.2.1 & <yes> & & <yes> & & \\
\hline sccs & 2.5.2.2 & $<$ yes $>$ & & $<$ yes> & & \\
\hline QENS & 2.6 .1 .1 & & & & & bkgnd? \\
\hline HRBS & 2.6.2.1 & & & & & bands \\
\hline TFCA & 2.6.2.2 & & & & & bkgnd? \\
\hline QSTAXC & 2.6.2.3 & & & & & \\
\hline MICAS & 2.6.2.4 & & & & & bkgnd? \\
\hline TOFNSE & 2.7.1.1 & & & & & band? \\
\hline Total Essential & & 4 & 0 & 4 & 1 & 5 \\
\hline Total Potential & & 7 & 8 & 5 & 1 & 16 \\
\hline <> Essential & & & & & & \\
\hline
\end{tabular}

a Abbreviated as "bkgnd" and "band" here. 


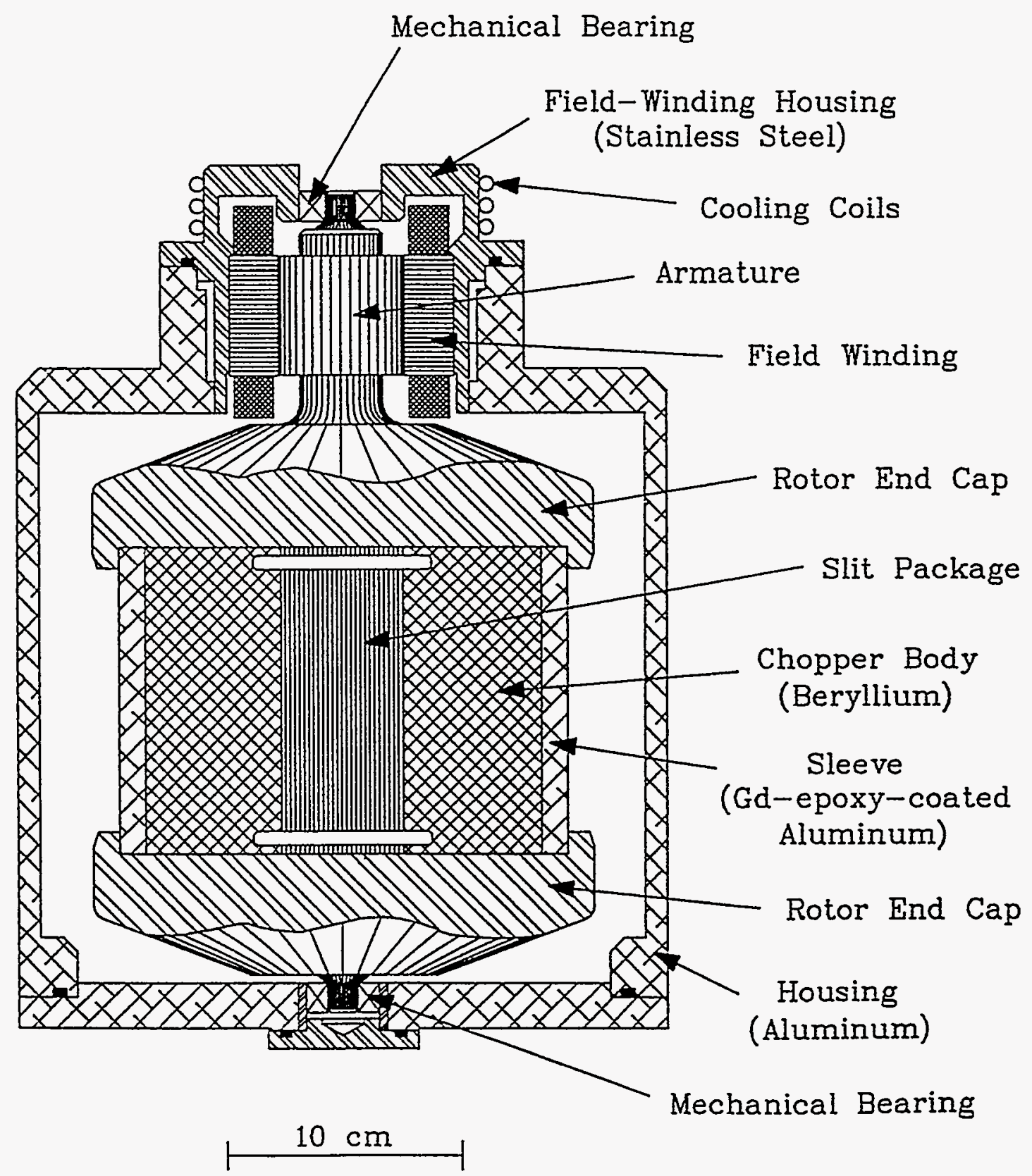

Figure V.3.1-2 The IPNS Fermi $\mathrm{E}_{0}$ Chopper Design. 


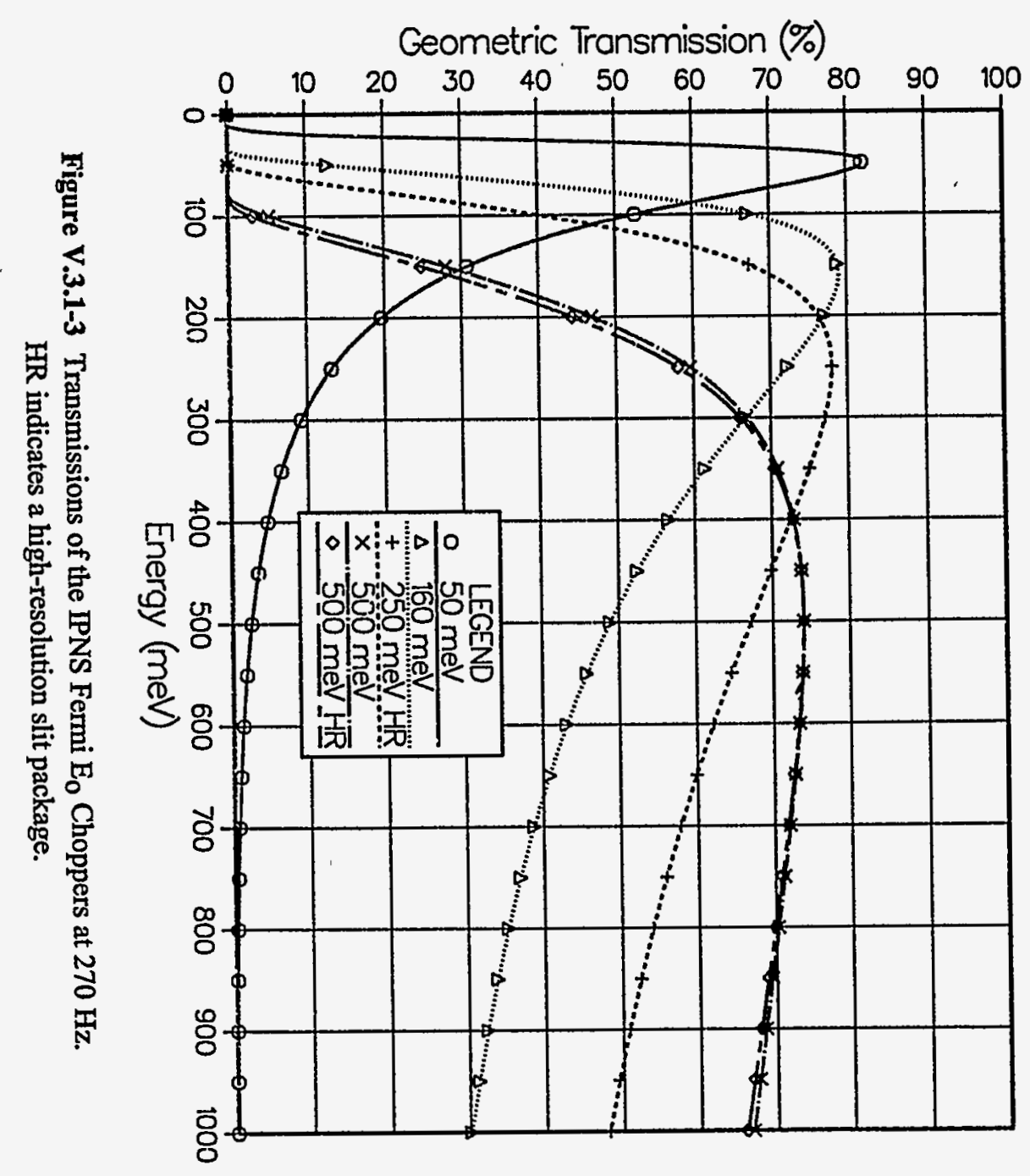


have magnetic bearings and can rotate at frequencies up to $600 \mathrm{~Hz} .^{4}$ However, because the choppers are smaller, they cannot transmit beams of such large cross section as can the IPNS choppers, and because they are made of $\mathrm{Al}$, they have little stopping power for fast neutrons. Thus, a narrow-band $t_{0}$ chopper must be used upstream from such an $E_{0}$ chopper in order to reduce the fast-neutron background. Although it is not essential to have such fast choppers, the flexibility of the instruments and the extremes in resolution will be enhanced if several such choppers, optimized for different energy ranges, are available to complement the IPNS-type $\mathrm{E}_{0}$ choppers. The technology developed at ISIS can be used for any such choppers designed for IPNS Upgrade instruments. This design can incorporate the same mounting fixtures used for the IPNS choppers, in order to facilitate the interchange of choppers on a given instrument and among instruments at IPNS Upgrade.

Table V.3.1-1 shows four instruments that require $E_{0}$ choppers and three other instruments for which they are useful in some applications. Each of the four chopper instruments needs to have access to a full set of $\sim 5-7 \mathrm{E}_{\mathrm{o}}$ choppers, optimized for different energies and resolutions. However, these choppers are interchangeable between instruments, so that somewhat fewer than 20-28 such choppers are required in total.

\subsubsection{Narrow-Band $t_{0}$ Choppers}

Narrow-band $t_{0}$ choppers are used for background suppression on chopper instruments, where the $E_{0}$ chopper passes only a narrow wavelength band $\Delta \lambda / \lambda$ of a few percent. In this case, the wavelength bandwidth passed by the $t_{0}$ chopper need be only slightly larger than the bandwidth passed by the $E_{0}$ chopper at this energy. The designation as a $t_{0}$ chopper implies that the chopper is neutronically opaque so that it significantly attenuates the fast neutrons in the prompt pulse at time $t_{o}$ when the chopper is closed. At IPNS, mechanical-bearing choppers made with Be bodies are used for this purpose. ${ }^{5}$ These choppers, shown schematically in Figure V.3.1-4, are similar in size and design to the IPNS $\mathrm{E}_{0}$ choppers but contain no slit package. The apertures in these choppers are sufficiently large to pass the full beam over the maximum bandwidth required by any of the $E_{o}$ choppers. These $t_{0}$ choppers can rotate at frequencies up to $150 \mathrm{~Hz}$. Such a chopper makes many revolutions, and hence is open many times, during one time frame. Its rotational speed and phasing must be carefully chosen so that openings of the $t_{0}$ chopper only coincide with openings of the $E_{o}$ chopper for neutrons having the desired wavelength. ${ }^{5}$ The $t_{0}$ choppers at IPNS are very effective. At least one such chopper is required for each chopper instrument at IPNS Upgrade, so 2-3 additional choppers must be fabricated in order to supply all the IPNS Upgrade instruments.

These choppers satisfy the drum chopper equations. Representative numbers for a $t_{0}$ chopper rotating at $150 \mathrm{~Hz}$ with $\mathrm{W}=6.5 \mathrm{~cm}, \delta \phi=41^{\circ}, \mathrm{r}=10 \mathrm{~cm}$, and $\mathrm{L}_{\mathrm{c}}=5.5 \mathrm{~m}$, which are the conditions on the present LRMECS, are $\delta_{\mathrm{tc}}=351 \mu \mathrm{sec}, \delta \lambda_{\mathrm{c}}=0.25 \AA$, and $\delta \lambda_{\mathrm{t}}=0.04 \AA$. If the chopper is phased to begin opening immediately after the prompt pulse, then it is fully open for neutron wavelengths of $0.25-0.29 \AA(1309-972 \mathrm{MeV})$. If it is necessary to have a chopper fully 


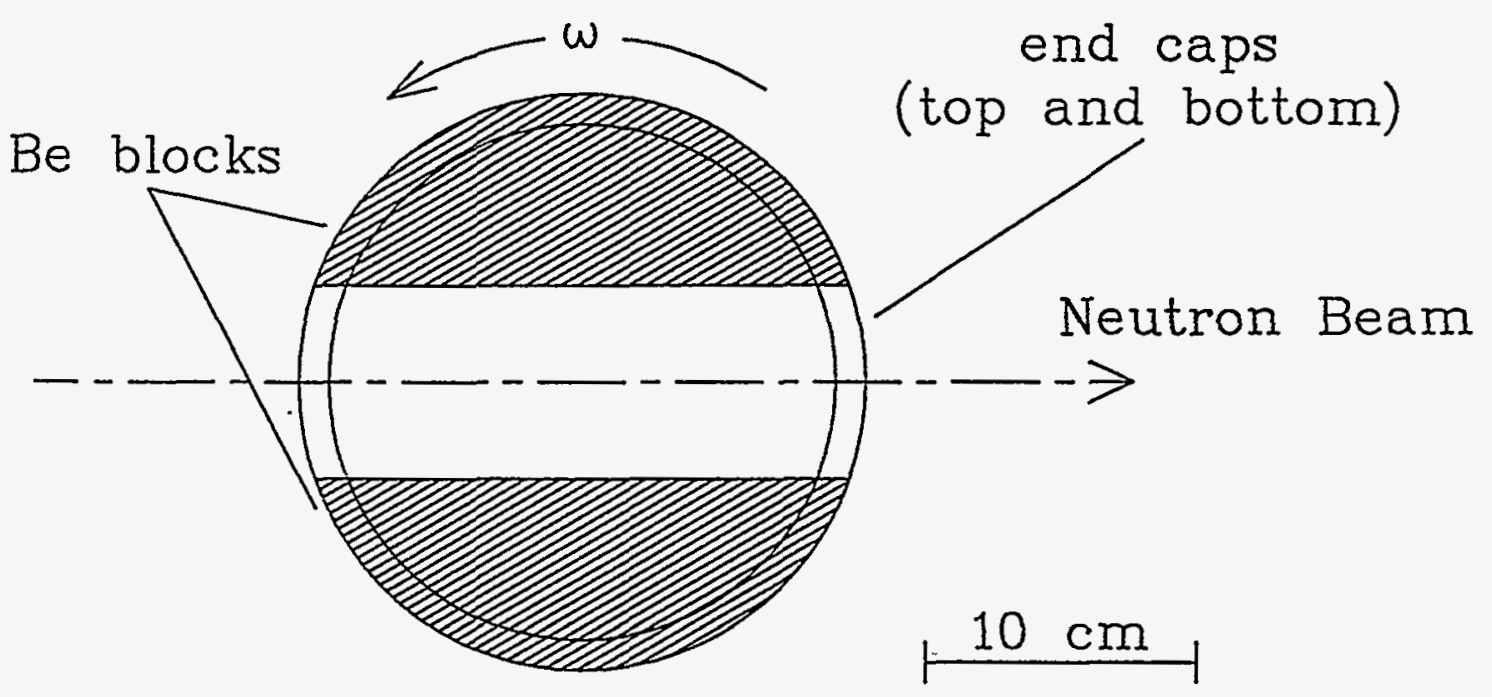

Figure V.3.1-4 The IPNS Narrow-Band to Choppers. 
closed at time $t_{0}$, that is fully open for shorter wavelengths, then either the beam-width at the chopper must be decreased, the distance of the chopper from the source must be increased, or the chopper radius or rotational frequency must be increased. The material properties determine the maximum peripheral velocity allowed for a particular chopper. For the IPNS $t_{0}$ chopper design, the combination of $150 \mathrm{~Hz}$ and $10-\mathrm{cm}$ radius gives close to the allowed peripheral velocity. The only options remaining for operating such a chopper at shorter wavelengths are to increase $L_{c}$ or decrease W.

\subsubsection{Broad-Band $t_{0}$ Choppers}

With the exception of the chopper instruments and a few crystal-monochromator instruments, all other TOF instruments rely on the use of a broad wavelength band of incident neutrons. However, in many of these instruments, backgrounds are improved if the fast neutrons from the prompt pulse are removed from the beam at a point far upstream from the sample. The narrow-band $t_{0}$ choppers discussed above cannot be used for this purpose, since they rotate through many revolutions in one time frame and transmit only a narrow bandwidth in each revolution. What is required is a slowly rotating chopper that places a neutronically opaque block in the beam for the duration of the prompt pulse and then moves this block out of the beam quickly and keeps it out for the remainder of the time frame of interest. Devices of this type are in operation at ISIS ${ }^{6}$ and the Los Alamos Neutron Scattering Center (LANSCE). The ISIS $t_{0}$ chopper is shown schematically in Figure V.3.1-5. Choppers of this type satisfy all the requirements for broad-band $t_{0}$ choppers indicated in Table V.3.1-1.

The equations for a disk chopper apply to a chopper of this type. If such a chopper of radius $25 \mathrm{~cm}$ is operated at a frequency of $90 \mathrm{~Hz}$ in a 7-cm-wide beam at a distance of $5.5 \mathrm{~m}$ from the source, then $\delta t_{c}=497 \mu$ s and $\delta \lambda_{c}=0.36 \AA$. A symmetrical chopper, such as that shown in Figure V.3.1-5, places neutron absorber in the beam twice per revolution. Such a chopper rotating at $90 \mathrm{~Hz}$ blocks the neutron beam every $5,556 \mu \mathrm{sec}$, and if the chopper is located $5.5 \mathrm{~m}$ from the source, this occurs at intervals of $4.00 \AA$ in the neutron spectrum. A chopper with the parameters given here operating at $90 \mathrm{~Hz}$ and having neutron absorber blocks as wide as the beam is fully open over the wavelength ranges $0.36-3.64 \AA, 4.36-7.64 \AA$, etc. If the same chopper instead operates at $15 \mathrm{~Hz}$ so that it blocks the beam at a $30-\mathrm{Hz}$ rate, then the corresponding open ranges are 2.16-21.84 $\AA, 26.16-45.84 \AA$, etc.

\subsubsection{Pulse-Eliminating $t_{0}$ Choppers}

For some of the instruments considered, the path-length and wavelength-band requirements are such that a second prompt pulse from the source occurs before the neutrons of interest reach the sample. The background at the sample is significantly reduced if this second prompt pulse is effectively blocked. It is desirable to block the fast neutrons from this second 


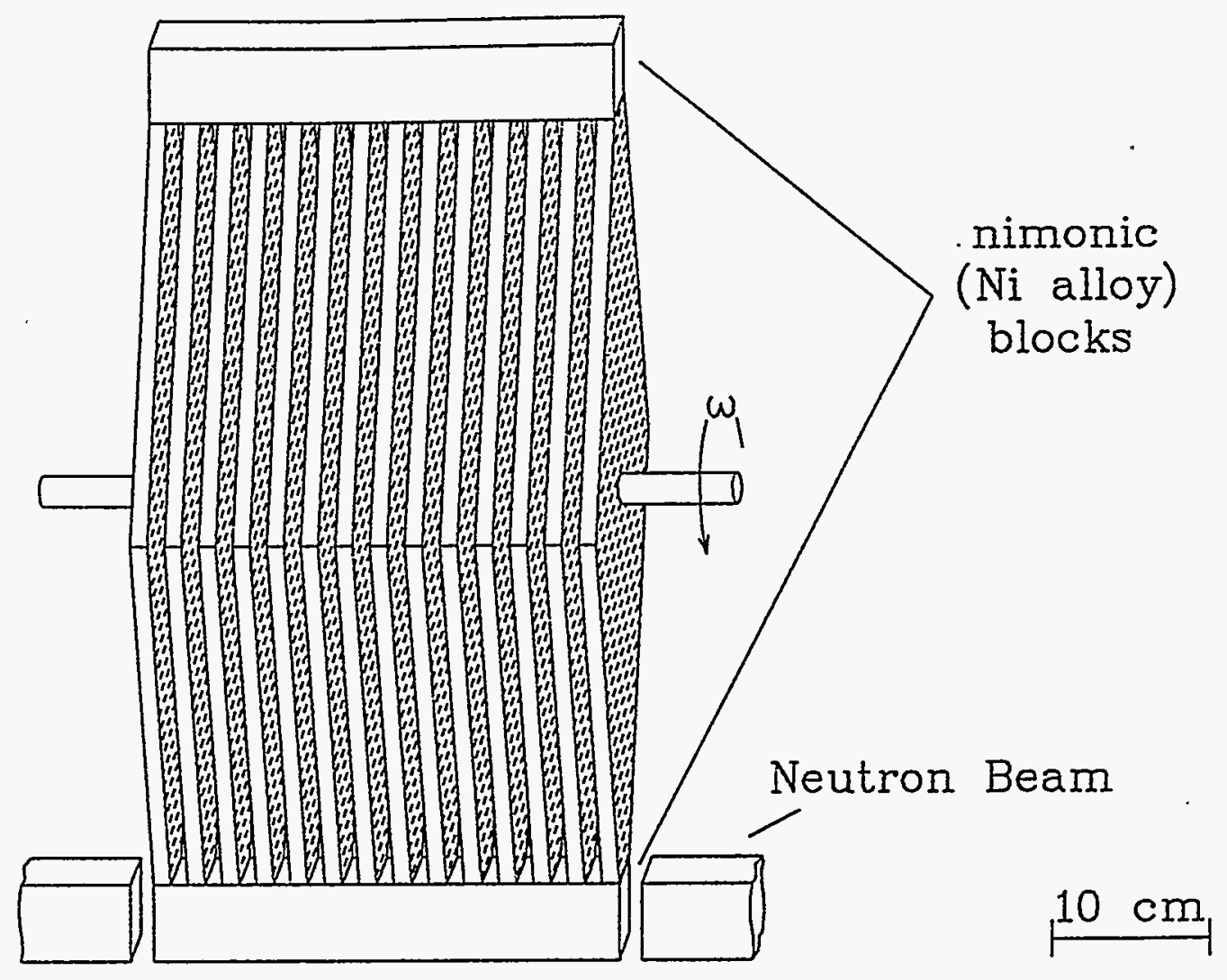

Figure V.3.1-5 The ISIS Broad-Band $t_{0}$ Chopper. 
prompt pulse and also to cut out somewhat slower neutrons. These requirements can be met by operating an ISIS-style broad-band $t_{0}$ chopper to eliminate the fast neutrons from every pulse, in conjunction with one of the bandwidth-limiting choppers.

\subsubsection{Bandwidth-Selecting Choppers}

Several instruments at IPNS (SEPD, GPPD, and SAND) use slowly rotating drum choppers. These are closed for more than half of the time frame but are open for all of the neutron wavelengths used by the instrument, typically up to the maximum wavelength allowed by frame-overlap. Such choppers are very effective in eliminating much of the background due to delayed neutrons from the moderator, but they are not sufficiently opaque to stop significant numbers of fast neutrons. ${ }^{7}$ Choppers of this type can also eliminate any background problems due to frame-overlap from longer-wavelength neutrons associated with the previous pulse. ${ }^{8}$ The choppers in use at IPNS are $20-40 \mathrm{~cm}$ in diameter, with a 1-cm-thick annulus of $\mathrm{B}_{4} \mathrm{C}$ as the stopping material and rotate at $15 \mathrm{~Hz}$. This type of chopper is designed for use at a specific distance from the source on an instrument having a specific flight-path length, so these instrument-specific choppers are not usable at IPNS Upgrade. However, choppers of this type are relatively inexpensive to fabricate and can easily be installed on any of the IPNS Upgrade instruments. Since the tungsten targets at IPNS Upgrade produce only very small numbers of delayed neutrons, choppers of this type may not provide significant benefits except where frame-overlap is a problem. Therefore, such choppers are not included in most of the initial instrument configurations at IPNS Upgrade. Instrument beamlines are designed to provide for possible later inclusion of such choppers.

A few of the instruments considered (GPPD, HRPD, and HRBS) require the selection of a specific and sometimes adjustable bandwidth from the incident beam. For this purpose either disk choppers or drum choppers can be used. ${ }^{7}$ If adjustability is required, properly phased pairs of such choppers can be used. Table V.3.1-2 indicates parameters appropriate for bandwidthlimiting choppers of either the drum or disk type on these instruments.

The data presented in the table indicate that serious frame-overlap problems occur for the GPPD and HRPD with the chopper parameters specified. In order to eliminate these problems, the choppers must be moved far enough from the source to reduce $\delta \lambda_{c}$ to the required value. For the 25-cm-radius disk choppers, this requires $\mathrm{L}_{\mathrm{c}}=19.3 \mathrm{~m}$ for the GPPD and $\mathrm{L}_{\mathrm{c}}=7.75 \mathrm{~m}$ for the HRPD. In both cases, it is desirable to have an additional chopper installed in the chopper cavity position described in Section IV.8 to remove most of the undesired neutrons, reducing the background generated by the downstream chopper.

For the HRBS, $\lambda_{\min }$ and $\lambda_{\max }$ are intended to be independently variable. This can be achieved by using two choppers and independently varying their phases relative to the source. If 
TABLE V.3.1-2 Parameters for Bandwidth-Limiting Choppers

\begin{tabular}{|c|c|c|c|c|c|c|c|c|c|c|c|c|}
\hline $\begin{array}{l}\text { Instrument, } \\
\text { by Chopper }\end{array}$ & $\begin{array}{l}L_{s} \\
(m)\end{array}$ & $\begin{array}{l}L_{d} \\
(m)\end{array}$ & $\begin{array}{c}W \\
(\mathrm{~cm})\end{array}$ & $\begin{array}{l}\lambda_{\min } \\
(\AA)\end{array}$ & $\begin{array}{c}\lambda_{\max } \\
(\AA)\end{array}$ & $\begin{array}{c}\lambda_{\text {trame }} \\
(\AA)\end{array}$ & $\begin{array}{r}\omega / 2 \pi \\
(H z)\end{array}$ & $\begin{array}{l}\delta \phi_{\mathrm{r}} \\
\left({ }^{\circ}\right)\end{array}$ & $\begin{array}{l}\delta \phi_{c} \\
\left({ }^{\circ}\right)\end{array}$ & $\begin{array}{c}\delta t_{c} \\
(\mu s)\end{array}$ & $\begin{array}{l}\delta \lambda_{c} \\
(\AA)\end{array}$ & $\begin{array}{l}\lambda_{f o} \\
(A)\end{array}$ \\
\hline \multicolumn{13}{|c|}{ 25-cm-radius disk chopper, $L_{c}=5.5 \mathrm{~m}$} \\
\hline GPPD & 25 & $\begin{array}{l}26.5 \\
29\end{array}$ & 8 & 0.4 & 4.6 & $\begin{array}{l}4.98 \\
4.55\end{array}$ & 30 & 63.1 & 18.4 & 1705 & 1.23 & $\begin{array}{l}0.85 \\
1.28\end{array}$ \\
\hline HRPD & 50 & 52 & 5 & 0.4 & 2.4 & 2.54 & 30 & 30.0 & 11.5 & 1063 & 0.76 & 0.62 \\
\hline HRBS & 30 & 36 & 5 & 2 & 10 & 10.99 & 10 & 40.1 & 11.5 & 3189 & 2.29 & 1.30 \\
\hline \multicolumn{13}{|c|}{$20-\mathrm{cm}$-radius drum chopper, $L_{c}=5.5 \mathrm{~m}$} \\
\hline GPPD & 25 & $\begin{array}{l}26.5 \\
29\end{array}$ & 8 & 0.4 & 4.6 & $\begin{array}{l}4.98 \\
4.55\end{array}$ & 15 & 31.5 & 11.5 & 2137 & 1.54 & $\begin{array}{l}1.16 \\
1.59\end{array}$ \\
\hline HRPD & 50 & 52 & 5 & 0.4 & 2.4 & 2.54 & 15 & 15.0 & 7.2 & 1330 & 0.96 & 0.82 \\
\hline HRBS & 30 & 36 & 5 & 2 & 10 & 10.99 & 5 & 20.0 & 7.2 & 3989 & 2.87 & 1.88 \\
\hline
\end{tabular}


a sharper wavelength cutoff or smaller $\delta \lambda_{c}$ is desired, then one of these choppers can be placed farther from the source. Dual choppers can also be used on the GPPD and HRPD to permit independent variation of $\lambda_{\min }$ and $\lambda_{\max }$ for these instruments, to increase flexibility.

\subsubsection{Chopper Control}

Neutron choppers operating at a pulsed neutron source require a system to drive the chopper motor and maintain a constant phase with respect to the pulsed proton beam. The drive system runs the chopper at the chosen speed, monitors chopper parameters, and shuts off chopper power in the event of a potentially damaging condition. Choppers at a pulsed source typically operate at a harmonic frequency of the source. Experience has shown that a wide range of energies can be achieved with a particular chopper by operating the chopper at different harmonic frequencies. This flexibility allows optimization of the transmission efficiency for a particular chopper at a particular energy. The drive system also provides a method of damping oscillations in the line frequency. A control system must monitor and adjust the chopperaccelerator phase difference to keep the difference constant even when the accelerator pulsing frequency varies slowly with time. A tolerance of $10-20 \%$ of the source burst time of a few microseconds is required.

The control system must provide a sufficiently user-friendly interface that even the novice user can select the desired energy and operating harmonic. The system must also allow for control and monitoring of all functions from a remote terminal. Provision must also be made for saving data on chopper parameters so that a time analysis of chopper problems can be performed.

The design of the chopper control systems for IPNS Upgrade draws on more than 10 years of experience $3,9,10$ in controlling a number of neutron choppers phased to the accelerator at IPNS. The IPNS Upgrade chopper control systems are based on closely coupled control modules that can be computer-controlled and monitored over a network, such as Ethernet. Each such system is located close to the chopper and instrument to which it belongs and is included as part of the data-acquisition system electronics. It is assumed that monitoring can be performed from any workstation on the network. The advantages of such a distributed chopper control system are minimized cabling, automated controls, similar configurations for all instruments and choppers, commercial availability of most modules, and remote monitoring capability.

The chopper control system is based on a standard instrumentation bus, such as the VXI bus. This structure combines the advantages of a large number of vendors supplying a wide variety of instruments, all of which are controlled by either their own on-board computer or a central crate computer. The central crate computer has the capability of communicating with and controlling each module in its crate. This computer is also the communication interface to the network for remote monitoring and control. A system can be configured with both commercial and custom modules that have the needed instrumentation to control and monitor the performance of one or more choppers on an instrument. 


\subsection{NEUTRON GUIDES}

\subsubsection{Important Considerations}

Neutron guides utilize the phenomenon of total reflection to transport thermal and cold neutrons over extended distances with minimum loss in beam intensity. Carlile, Johnson, and Williams ${ }^{11}$ provide an excellent review of the theories pertaining to the use of neutron guides at pulsed sources, as well as calculations and practical considerations related to the design of some of the guides for ISIS. The discussion presented here is based in part on their review.

The critical angle $\gamma_{c}$ for reflection of neutrons from a surface is directly proportional to the neutron wavelength. Among the readily available mirror materials, natural Ni has the largest value of $\gamma_{c}$, given by

$$
\gamma_{\mathrm{c}}=0.0017 \lambda
$$

for $\gamma_{c}$ in radians and $\lambda$ in $\AA$.

If losses are ignored, a guide can transmit all neutrons falling within a solid angle of $\sim 4 \gamma_{c}^{2}$ on the guide's entrance cross section. Full illumination of the guide by the moderator occurs when the projection of this maximum guide solid angle, from any point on the guide entrance to the plane of the moderator, is completely covered by the moderator area. For a fully illuminated guide, the differential flux $\Phi$ per unit solid angle at the guide entrance is uniform across this entrance and is equal to the differential flux $\Phi_{\mathrm{m}}$ per unit solid angle leaving the moderator in the direction of the guide. For a perfect, straight guide, the differential flux at the guide exit is also equal to $\Phi_{\mathrm{m}}$ and is uniform across the exit. Under conditions of full sample illumination by the guide, the differential flux at the sample is $\Phi_{\mathrm{m}}$ as well. Full illumination in the horizontal and vertical directions occurs under the conditions

$$
\begin{array}{ll}
\mathrm{L}_{\mathrm{i}} \gamma_{\mathrm{c}} \leq \frac{\left(\mathrm{M}_{\mathrm{h}}-\mathrm{g}_{\mathrm{h}}\right)}{2} & \text { horizontal } \\
\mathrm{L}_{\mathrm{i}} \gamma_{\mathrm{c}} \leq \frac{\left(\mathrm{M}_{\mathrm{v}}-\mathrm{g}_{\mathrm{v}}\right)}{2} & \text { vertical }
\end{array}
$$

Here $\mathrm{M}_{\mathrm{h}}$ and $\mathrm{M}_{\mathrm{V}}$ are the moderator's horizontal and vertical dimensions, the corresponding guide dimensions are $g_{h}$ and $g_{v}$, and the distance from the moderator to the guide entrance is $\mathrm{L}_{\mathrm{i}}$.

The practical gain $\mathrm{G}$ of a guide at a pulsed source with small moderators is defined as the ratio of the total neutron flux, integrated over solid angle at the sample, to the total flux at the same source-sample distance $L$ with no guide but viewing the entire moderator face. This gain is the product of the one-dimensional gains $R_{h}$ and $R_{v}$ :

$$
\mathrm{G}=\mathrm{R}_{\mathrm{h}} \mathrm{R}_{\mathrm{V}}
$$


With full illumination of guide and sample, the one-dimensional gains (indicated with the subscript $\mathrm{f}$ ) are given by

$$
\begin{array}{ll}
\mathrm{R}_{\mathrm{f}}=\frac{\mathrm{g}}{\mathrm{M}}+\frac{\left(\mathrm{L} \gamma_{\mathrm{c}}\right)^{2}}{\mathrm{gM}} & \mathrm{L} \gamma_{\mathrm{c}}<\mathrm{g} \\
\mathrm{R}_{\mathrm{f}}=\frac{2 \mathrm{~L} \gamma_{\mathrm{c}}}{\mathrm{M}} & \mathrm{L} \gamma_{\mathrm{c}}>\mathrm{g},
\end{array}
$$

where the subscript $h$ or $v$ is implied for $R_{f}, M$, and $g$. Gains of less than unity are possible at small values of $L \gamma_{c}$ if the guide is smaller than the moderator, because the guide can actually obscure the view of some of the moderator from the sample.

If the guide is not fully illuminated by the moderator in either the horizontal or vertical direction, then this gain must be multiplied by a factor $F_{m h}$ or $F_{m v}$, respectively. Under the conditions of uniform emission from the moderator face, the factors $\mathrm{F}$ are given approximately as

$$
\begin{array}{ll}
F_{m}=1 & L_{i} \gamma_{c}<\frac{(M-g)}{2} \\
F_{m}=\frac{g+M-L_{i} \gamma_{c}}{2 g}-\frac{(M-g)^{2}}{8 L_{i} \gamma_{c} g} & \frac{(M-g)}{2}<L_{i} \gamma_{c}<\frac{(M+g)}{2} \\
F_{m}=\frac{M}{2 L_{i} \gamma_{c}} & \frac{(M+g)}{2}<L_{i} \gamma_{c},
\end{array}
$$

where the subscript $h$ or $v$ is implied. The corrected one-dimensional gain is then

$$
\mathrm{R}=\mathbf{R}_{\mathrm{f}} \mathrm{F}_{\mathrm{m}} \text {. }
$$

At sufficiently long wavelength, the corrected gain saturates at the value

$$
\mathrm{R}_{\max }=\frac{\mathrm{L}}{\mathrm{L}_{\mathrm{i}}} \quad \mathrm{L}_{\mathrm{i}} \gamma_{\mathrm{c}}>\frac{(\mathrm{M}+\mathrm{g})}{2} .
$$

Similar conditions apply to the illumination of the sample by the guide. Full sample illumination occurs under the conditions

$$
\begin{array}{ll}
L_{e} \gamma_{c} \leq \frac{\left(g_{h}-S_{h}\right)}{2} & \text { horizontal } \\
L_{e} \gamma_{c} \leq \frac{\left(g_{v}-S_{v}\right)}{2} & \text { vertical }
\end{array}
$$


where $L_{e}$ is the distance from the guide exit to the sample and $S_{h}$ and $S_{v}$ are the horizontal and vertical sample dimensions.

If the sample is not fully illuminated, then the one-dimensional gains must be multiplied by the additional factor $F_{s h}$ or $F_{s v}$, given by

$$
\begin{array}{ll}
\mathrm{F}_{\mathrm{s}}=1 & \mathrm{~L}_{\mathrm{e}} \gamma_{\mathrm{c}}<\frac{(\mathrm{g}-\mathrm{S})}{2} \\
\mathrm{~F}_{\mathrm{s}}=\frac{\mathrm{g}+\mathrm{S}-\mathrm{L}_{\mathrm{e}} \gamma_{\mathrm{c}}}{2 \mathrm{~S}}-\frac{(\mathrm{g}-\mathrm{S})^{2}}{8 \mathrm{~L}_{\mathrm{e}} \gamma_{\mathrm{C}} \mathrm{S}} & \frac{(\mathrm{g}-\mathrm{S})}{2}<\mathrm{L}_{\mathrm{e}} \gamma_{\mathrm{c}}<\frac{(\mathrm{g}+\mathrm{S})}{2} \\
\mathrm{~F}_{\mathrm{S}}=\frac{\mathrm{g}}{2 \mathrm{~L}_{\mathrm{e}} \gamma_{\mathrm{c}}} & \frac{(\mathrm{g}+\mathrm{S})}{2}<\mathrm{L}_{\mathrm{e}} \gamma_{\mathrm{c}},
\end{array}
$$

where again the subscript $\mathrm{h}$ or $\mathrm{v}$ is implied.

The gain in flux at the sample due to the use of a guide is accomplished by increasing the angular divergence of the beam incident on the sample. This divergence of the incident beam can affect the energy or momentum resolution of the instrument, which is inappropriate for some instruments. If the maximum incident-beam divergence $2 \gamma_{c}\left(\lambda_{\max }\right)$ from the guide is greater than the maximum divergence $\Delta \alpha_{\max }$ appropriate to the instrument, then the instrument resolution is compromised. One solution is to increase $L_{e}$ or decrease $g$ beyond the value that gives full sample illumination. The maximum beam divergence $\Delta \alpha$ at the sample is then given by

$$
\begin{array}{ll}
\Delta \alpha=2(\mathrm{~g}+\mathrm{S}) \mathrm{L}_{\mathrm{e}} & \mathrm{g}+\mathrm{S}<\mathrm{L}_{\mathrm{e}} \gamma_{\mathrm{c}} \\
\Delta \alpha=2 \gamma_{\mathrm{c}} & \mathrm{g}+\mathrm{S}>\mathrm{L}_{\mathrm{e}} \gamma_{\mathrm{c}},
\end{array}
$$

and the guide gain is reduced by the factor $\mathrm{F}_{\mathrm{s}}$, given by Equation V.3.2-9. In some cases, this solves the beam divergence problem while still leaving an appreciable gain from the guide. In other cases, however, the required increase in $\mathrm{L}_{e}$ or decrease in $\mathrm{g}$ is so large that the guide is of little or no benefit and can be omitted.

Equations V.3.2-5 and V.3.2-9 place some constraints on the dimensions $g_{h}$ and $g_{v}$ of the guide, but they do not fully determine these quantities. Usually, physical constraints set lower allowable limits on $\mathrm{L}_{\mathrm{i}}$ and $\mathrm{L}_{\mathrm{e}}$. A practical approach is to use the minimum guide dimensions that allow full sample illumination with this value for $\mathrm{L}_{\mathrm{e}}$. Minimizing the guide cross-sectional area under these conditions minimizes the total number of neutrons brought to the instrument but does not affect the total number incident on the sample. This gives the best signal-to-background ratio possible. This approach must be modified if focusing is applied at the guide exit.

The neutron intensity on the sample can be increased by the use of a focusing guide section between the normal guide exit and the sample. If supermirror coatings are used on this 
focusing guide, the critical angle in this section can be made twice that of natural $\mathrm{Ni}$, or even higher. This allows the guide to focus most of the beam penumbra back into the umbra that strikes the sample. A twofold or greater increase in intensity on the sample results, at the price of still further increase in the angular divergence. It is of greatest benefit when small sample sizes must be used to minimize path-length differences for TOF measurements, or because of difficulties in sample preparation.

The guide tube gains determined from the above equations are based on perfect guides. Many factors can lower the actual gains observed. ${ }^{11}$ If good-quality, state-of-the-art fabrication techniques are used in producing the guide sections, then the most important sources of losses are due to imperfections in the mechanical installation of the guide sections. These can include the following:

1. Imperfect spatial alignment. If individual sections are misaligned by an amount $\Delta \mathrm{g}$, then the fractional loss $\Delta \mathrm{J} / \mathrm{I}$ per section in intensity is

$$
\Delta \mathrm{I} / \mathrm{I} \sim\left(\Delta \mathrm{g}_{\mathrm{h}} / \mathrm{g}_{\mathrm{h}}\right)\left(\Delta \mathrm{g}_{\mathrm{v}} / \mathrm{g}_{\mathrm{v}}\right) .
$$

2. Gaps between sections. These losses are proportional to the ratio of the gap length $\Delta \mathrm{L}$ to the distance between reflections,

$$
\Delta \mathrm{J} / \mathrm{I} \sim \gamma_{c} \Delta \mathrm{L} / \mathrm{g} .
$$

3. Imperfect angular alignment. Imperfections in alignment must be kept within the expected variation in surface waviness, which is $\sim 10^{-4}$ radians. There is no simple formula for predicting these effects, but they can be easily simulated in Monte Carlo calculations.

Guides can be fabricated and installed to meet these stringent requirements and give good performance. At ISIS, guides have been used for HRPD, a high-resolution powder diffractometer, and IRIS, a high-resolution crystal-analyzer spectrometer. The HRPD guide is a combination of curved and straight sections, $\sim 96 \mathrm{~m}$ total length, optimized for thermal neutrons. Measured transmissions ${ }^{12}$ of this guide, defined as the fraction of neutrons accepted by the guide that reach the guide exit, level off at $\sim 70 \%$ for wavelengths greater than $\sim 3 \AA$. The IRIS guide is also a combination of curved and straight sections, $\sim 28 \mathrm{~m}$ total length, but optimized for cold neutrons. Measured transmissions ${ }^{12}$ of this guide level off at $\sim 70 \%$ for wavelengths greater than $\sim 5 \AA$.

\subsubsection{Survey of Guide Requirements}

Table V.3.2-1 lists the instruments that form the reference set and presents a preliminary assessment of the benefits of a guide on each instrument. Cases for which $\Delta \alpha_{\max } \gtrsim \gamma_{c-m a x}$ and 
Table V.3.2-1 Neutron Guide Assessment

\begin{tabular}{|c|c|c|c|c|c|c|c|c|}
\hline Instrument & Section & $\underset{(m)}{L}$ & $\begin{array}{l}\lambda_{\max } \\
(\AA)\end{array}$ & $\begin{array}{c}\Delta \alpha_{\max }{ }^{a} \\
(\mathrm{rad})\end{array}$ & $\begin{array}{c}2 \gamma_{c-\max } \\
(\mathrm{rad})\end{array}$ & $\mathbf{R}_{\mathrm{f}-\max }{ }^{\mathrm{b}}$ & Guide $^{c}$ & Focus $^{d}$ \\
\hline SEPD & 2.1 .1 .1 & 12 & 9.1 & 0.012 & 0.031 & 3.7 & no & \\
\hline GPPD & 2.1 .1 .2 & 25 & 4.6 & 0.005 & 0.016 & 4 & no & \\
\hline HRPD & 2.1 .2 .1 & 50 & 2.4 & 0.009 & 0.008 & 4 & $?$ & \\
\hline \multirow{2}{*}{ RSD } & 2.1 .2 .2 & 25 & 4.5 & 0.004 & 0.015 & 3.8 & no & \\
\hline & & 12 & 9 & 0.012 & 0.031 & 3.7 & no & \\
\hline VSPD & 2.1 .2 .3 & 12 & 9.1 & 0.012 & 0.031 & 3.7 & no & \\
\hline SAND & 2.2 .1 .1 & 12 & 20 & speciale & 0.068 & 8.2 & $?$ & \\
\hline HRSAND & 2.2.2.1 & 20 & 14 & & 0.048 & 9.6 & no & \\
\hline SPSAND & 2.2.2.2 & $?$ & & & & & no & \\
\hline GLAD & 2.3.1.1 & 22 & 4 & 0.007 & 0.014 & 3.1 & $?$ & \\
\hline SCD & 2.3.1.2 & 10 & 10 & 0.009 & 0.034 & 3.4 & no & \\
\hline HQSCD & 2.3.2.1 & 30 & 4 & 0.005 & 0.014 & 4.2 & no & \\
\hline POSY-I & 2.4 .1 .1 & 18 & 18 & 0.003 & 0.061 & 11 & no & \\
\hline POSY-II & 2.4 .1 .2 & 18 & 18 & 0.004 & 0.061 & 11 & no & \\
\hline HIREF & 2.4 .2 .1 & 18 & 4.7 & speciale & 0.016 & 2.9 & $?$ & \\
\hline GREF & 2.4.2.2 & 18 & 18 & 0.004 & 0.061 & 11 & no & \\
\hline REFD & 2.4 .2 .3 & 18 & 18 & 0.004 & 0.061 & 11 & no & \\
\hline HRMECS & 2.5 .1 .1 & 18 & 6.0 & 0.012 & 0.020 & 3.6 & $?$ & \\
\hline LRMECS & 2.5.1.2 & 12 & 9.1 & 0.012 & 0.031 & 3.7 & no & \\
\hline CNCS & 2.5.2.1 & 20 & 16 & 0.013 & 0.054 & 11 & yes & \\
\hline sccs & 2.5.2.2 & 16 & -1 & 0.013 & 0.003 & $<1$ & no & \\
\hline QENS & 2.6 .1 .1 & 9 & 6.5 & 0.2 & 0.022 & 2 & yes & yes \\
\hline HRBS & 2.6 .2 .1 & 30 & 20 & 0.08 & 0.068 & 20 & yes & yes \\
\hline TFCA & 2.6.2.2 & 16 & 5 & 0.2 & 0.017 & 2.7 & yes & yes \\
\hline QSTAXC & 2.6.2.3 & 17 & & & & & yes & \\
\hline MICAS & 2.6.2.4 & 12 & & & & & no & \\
\hline TOFNSE & 2.7.1.1 & 16 & 15 & 0.017 & 0.051 & 8 & yes & \\
\hline
\end{tabular}

a Based on detector and sample sizes and separation, or on incident-beam collimators.

b Assumes 10-cm moderator.

c This column indicates whether a guide is potentially useful on a particular instrument. In most cases, this is determined from the requirements $\Delta \alpha_{\max } \geq \gamma_{c-\max }$ and $R_{f-\max } \gg 1$.

d This column indicates whether a focusing guide section would be useful.

e Used in conjunction with converging Soller collimator or microguide. 


\begin{tabular}{|c|c|c|c|c|c|c|c|c|c|c|c|c|c|c|c|}
\hline \multirow[b]{2}{*}{ Instrument } & \multirow[b]{2}{*}{$\begin{array}{c}M_{v}^{a} \\
(\mathrm{~cm})\end{array}$} & \multirow[b]{2}{*}{$\underset{(m)}{L}$} & \multirow[b]{2}{*}{$\begin{array}{l}\lambda_{\min } \\
(\AA)\end{array}$} & \multirow[b]{2}{*}{$\begin{array}{c}\lambda_{\max } \\
(\AA)\end{array}$} & \multirow[b]{2}{*}{$\begin{array}{c}S_{h} \\
(\mathrm{~cm})\end{array}$} & \multirow[b]{2}{*}{$\begin{array}{c}S_{v} \\
(\mathrm{~cm})\end{array}$} & \multirow[b]{2}{*}{$\begin{array}{c}g_{\mathrm{h}} \\
(\mathrm{cm})\end{array}$} & \multirow[b]{2}{*}{$\begin{array}{c}g_{v} \\
(\mathrm{~cm})\end{array}$} & \multirow[b]{2}{*}{$\begin{array}{c}L_{\theta-m a x}^{b} \\
(m)\end{array}$} & \multicolumn{3}{|c|}{$R_{h}\left(\lambda_{\max }\right)$} & \multicolumn{3}{|c|}{$R_{v}\left(\lambda_{\max }\right)$} \\
\hline & & & & & & & & & & $L_{i}=1^{c}$ & $L_{i}=2$ & $L_{i}=5$ & $L_{1}=1$ & $L_{i}=2$ & $L_{1}=5$ \\
\hline HRPD & 20 & 50 & 0.4 & 2.4 & 1.27 & 2.5 & 3 & 4.3 & 2.12 & 4.1 & 4.1 & 4.1 & 2.0 & 2.0 & 2.0 \\
\hline SAND & 10 & 12 & 1 & 20 & \multicolumn{2}{|c|}{ Note $d$} & & & & & & & & & \\
\hline GLAD & 10 & 22 & 0.1 & 4 & 2 & 2 & 5 & 5 & 2.14 & 3.1 & 3.1 & 3.0 & 3.1 & 3.1 & 3.0 \\
\hline HIREF & 10 & 18 & 2 & 4.7 & \multicolumn{2}{|c|}{ Note e } & & & & & & & & & \\
\hline HRMECS & 10 & 18 & 0.2 & 6.0 & 7.5 & 10 & 10 & 10 & $1.23^{f}$ & 3.5 & 3.3 & 2.7 & 3.4 & 3.1 & 2.6 \\
\hline CNCS & 10 & 20 & 2 & 16 & 2 & 10 & 8 & 10 & $0.92^{f}$ & 10.1 & 8.4 & 4.0 & 8.1 & 6.9 & 3.5 \\
\hline QENS & 10 & 9 & 0.7 & 6.5 & 0.65 & 10 & 5 & 10 & $1.97^{f}$ & 2.0 & 2.0 & 1.7 & 1.7 & 1.6 & 1.3 \\
\hline HRBS & 10 & 30 & 2 & 20 & 2 & 5 & 5 & 8 & 0.44 & 19.9 & 14.9 & 6.0 & 18.2 & 14.1 & 6.0 \\
\hline TFCA & 10 & 16 & 0.3 & 5 & 2 & 5 & 5 & 8 & 1.76 & 2.7 & 2.7 & 2.5 & 2.7 & 2.7 & 2.3 \\
\hline QSTAXC & 10 & 17 & & & \multicolumn{2}{|c|}{ Note $\mathrm{g}$} & & & & & & & & & \\
\hline TOFNSE & 20 & 16 & 4 & 15 & 3 & 3 & 5 & 5 & 0.39 & 8.2 & 7.1 & 3.2 & 4.1 & 4.1 & 3.2 \\
\hline
\end{tabular}

a $M_{h}$ is assumed to be $10 \mathrm{~cm}$ in all cases.

b $L_{\theta-m a x}$ is the maximum value of $L_{\theta}$ for full sample illumination at $\lambda_{\max }$ with the specified guide and sample dimensions. Guide dimensions are chosen to be no larger than the moderator and to produce reseasonable values for $L_{\theta}$.

c $L_{1}$ is specified in meters.

d Guide is used to illuminate converging Soller collimators. Gain must be assessed and parameters optimized using Monte Carlo codes.

- Guide is used to illuminate entrance to converging Soller collimator or converging microguide assembly. Gain must be assessed and parameters optimized using Monte Carlo codes.

Based on horizontal dimension only.

9 Guide sized to accommodate several quasi-steady-state instruments. 
$R_{f-m a x} \gg 1$ are indicated as possibly benefiting from guides. A few other instruments that violate these conditions but for which guides may still have some benefit are also indicated. Those instruments for which guides offer some advantage are analyzed in greater detail in Table V.3.2-2.

On the basis of this analysis, it appears that significant gains are provided by guides on the HRPD, GLAD, HRMECS, CNCS, QENS, HRBS, TFCA, and TOFNSE. In addition, a guide is used to transport the neutrons to the quasi-steady-state instrument QSTAXC so that it can be located in a region of low background some distance from the source. The use of guides on the SAND and HIREF, although not essential, might provide enough gain to be justified, but these specialized uses of the guides would have to be assessed by detailed Monte Carlo calculations.

The inner ends of the HRPD, GLAD, and TFCA guides can be terminated $\sim 5 \mathrm{~m}$ from the moderator without appreciable reduction in gain, but the HRMECS, CNCS, QENS, HRBS, and TOFNSE guides need to extend to within $\sim 2 \mathrm{~m}$ of the moderator. The CNCS, HRBS, and TOFNSE guides benefit even more if the end of the guide is $\sim 1 \mathrm{~m}$ from the moderator.

\subsection{BEAMLINE SHIELDING AND BEAMSTOPS}

\subsubsection{Beamline Shielding - Neutrons from Moderator}

The neutron beamlines transport the neutrons from the moderators through the beam ports to the face of the biological shield, and then on to the sample positions for their respective instruments. The biological shield provides the shielding for the inner portions of the beamlines, typically $-6 \mathrm{~m}$ long. Separate shielding must be provided for the portion of each beamline external to the biological shield. These external portions range in length from $\sim 1 \mathrm{~m}$ to $\sim 42 \mathrm{~m}$. The shielding thickness required around these external beamlines is assessed here. The shielding near the interface between the external beamlines and the biological shield has some additional requirements, which are addressed in Section V.3.3.2.

Each beamline is designed with apertures that remove small portions from the edges of the beam continuously along the beamline. These distributed-loss conditions are modeled as a line of isotropic point sources of neutrons within the beamline. This source produces $S(E)$ neutrons per unit beamline length per unit energy per second at energy $E$. This source term is estimated by assuming that all of the beam intercepted by the defining apertures is elastically scattered, isotropically, at the source points. The dose rate $\mathrm{D}$ at distance $\mathrm{r}$ from a beamline with cylindrical shield of radius $R$ is then

$$
D=\frac{1}{2 \pi r} \int[S(E) / F(E)] e^{-R / \Lambda(E)} d E
$$


where $F(E)$ is the conversion factor between dose rate and neutron flux and $\Lambda(E)$ is the neutron mean-free-path in the shielding material. Figure V.3.3-1 shows the values for $F(E)$ used here. The most difficult to stop are the high-energy $(\mathrm{E}>50 \mathrm{MeV})$ and fast $(1<\mathrm{E}<50 \mathrm{MeV})$ neutrons, so these energy ranges dominate the shielding considerations. Table V.3.3-1 lists meanfree-paths associated with these energy ranges for several common shielding materials.

At IPNS Upgrade, the neutron beamlines view moderators operated in wing geometry or in flux-trap geometry, as shown in Figure V.3.3-2, so the beamlines have no direct view of the target. Representative spectra for the neutron beams have been calculated in Chapter IV. A typical spectrum is shown in Figure V.3.3-3 in the form of EI(E), normalized to EI(E) $=1$ at $1 \mathrm{eV}$. Here $\mathrm{I}(\mathrm{E})$ is the time-averaged neutron beam current per unit solid angle per unit energy leaving the moderator face. Also shown in the figure is the dashed line $[\mathrm{EI}(\mathrm{E})]_{\mathrm{w}}$, which is an interpolated and extrapolated version of the calculated spectrum used for the shielding calculations here.

We assume a straight beam tube of internal cross-sectional area A. The fraction of the beam intercepted by the beam tube walls per unit length is

$$
\frac{d \Omega}{d L}=\frac{2 A}{L^{3}}
$$

The source term $\mathrm{S}(\mathrm{E})$ to be used in Equation V.3.3-1 is then given by

$$
S(E)=S_{o} \frac{2 A}{L^{3}} \frac{[E I(E)]_{W}}{E},
$$

where $S_{0}$ is the value of $\mathrm{EI}(\mathrm{E})$ at $1 \mathrm{eV}$ for the moderator in question. Equation V.3.3-1 then becomes

$$
\mathrm{D}=\mathrm{S}_{\mathrm{o}} \frac{2 \mathrm{~A}}{\mathrm{~L}^{3}} \frac{1}{2 \pi \mathrm{r}} \sum_{\mathrm{E}_{1}} \mathrm{e}^{-\mathrm{R} / \mathrm{A}\left(\mathrm{E}_{1}, \mathrm{E}_{2}\right)} \Delta \mathrm{I}\left(\mathrm{E}_{1}, \mathrm{E}_{2}\right),
$$

where $\Lambda\left(E_{1}, E_{2}\right)$ is assumed to be constant over the range $E_{1}$ to $E_{2}$, as indicated in Table V.3.3-1, and

$$
\begin{array}{rlrl}
\Delta \mathrm{II}\left(\mathrm{E}_{1}, \mathrm{E}_{2}\right) & =\int_{\mathrm{E}_{1}}^{\mathrm{E}_{2}} \frac{[\mathrm{EI}(\mathrm{E})]_{\mathrm{W}}}{\mathrm{EF}(\mathrm{E})} \mathrm{dE} & \\
& =0.66 \quad \text { for slow neutrons } & & \left(\mathrm{E}_{1}=0, \mathrm{E}_{2}=1 \mathrm{MeV}\right) \\
& =1.01 \quad \text { for fast neutrons } & & \left(\mathrm{E}_{1}=1, \mathrm{E}_{2}=50 \mathrm{MeV}\right) \\
& =0.0015 \quad \text { for high-energy neutrons } & & \left(\mathrm{E}_{1}=50, \mathrm{E}_{2}=2200 \mathrm{MeV}\right) .
\end{array}
$$




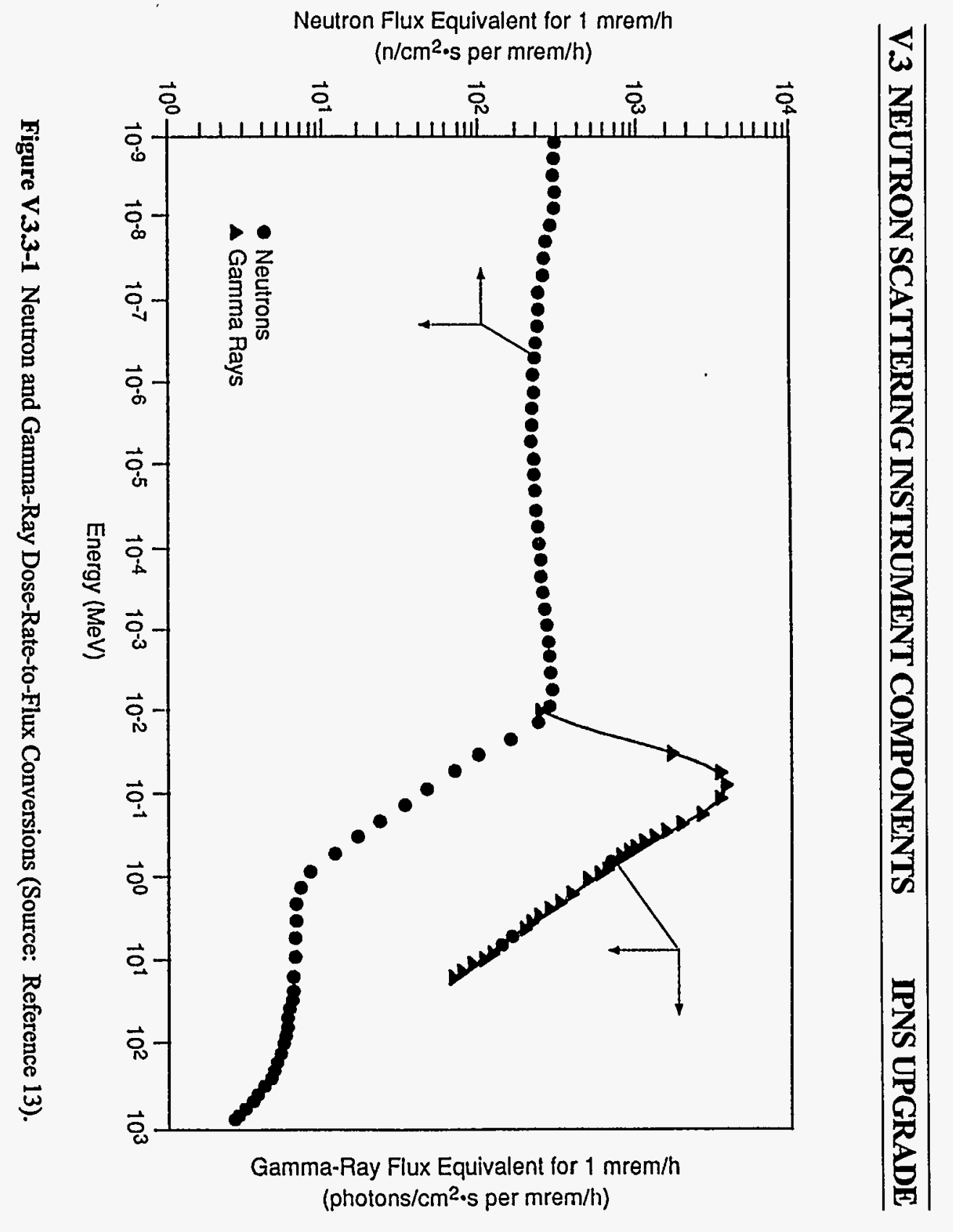


Table V.3.3-1 Neutron Mean-Free-Paths in Shielding Materials $(\mathrm{cm})$

\begin{tabular}{lcc}
\hline Material & $\Lambda, 1<\mathrm{E}<50 \mathrm{MeV}$ & $\Lambda, \mathrm{E}>50 \mathrm{MeV}$ \\
& & \\
\hline & & \\
Beryllium & 8.34 & 50. \\
Iron & 6.25 & 17.34 \\
Tungsten & 5.08 & 10.1 \\
Lead & $8.5^{\mathrm{a}}$ & 17.8 \\
Uranium & 5.97 & 11.1 \\
Concrete & 10.4 & 46.1 \\
HO & 9.62 & 90.25 \\
& & \\
\hline
\end{tabular}

a Using 3.5-barn removal cross section instead of Barbier ${ }^{14}$ value.

Source: Reference 14. 


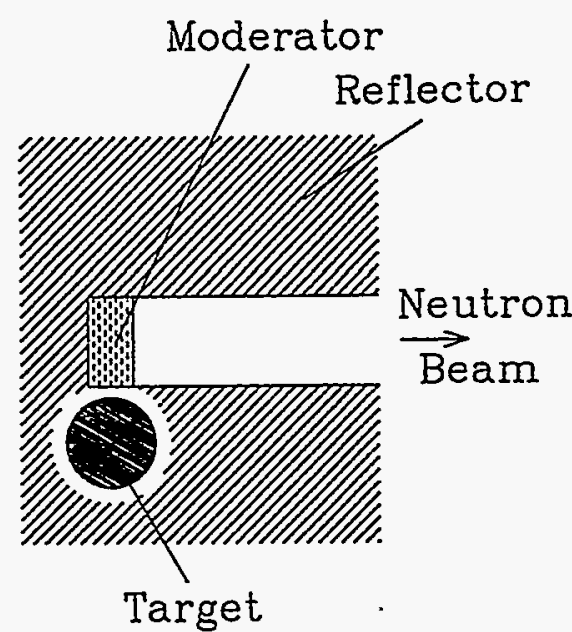

(a)
Split

Target

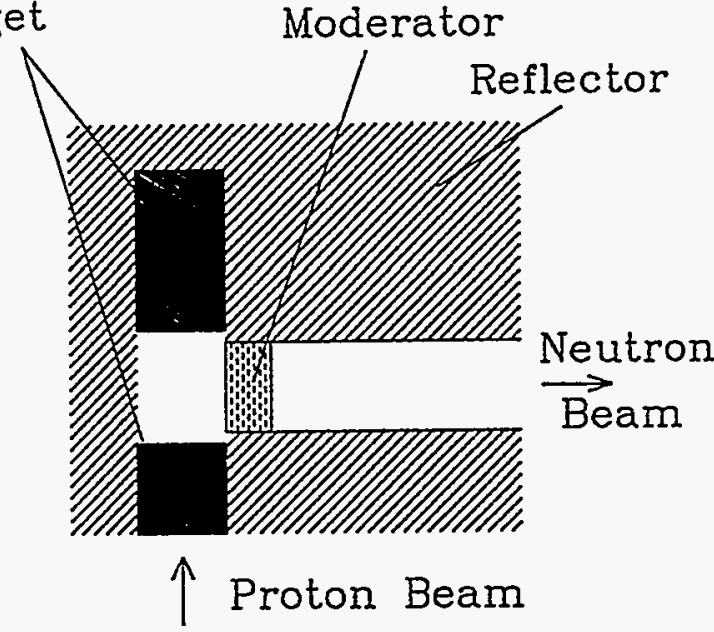

(b)

Figure V.3.3-2 Target-Moderator-Beamline Geometries: (a) Wing Geometry with Proton Beam Direction Normal to the Page and (b) Flux-Trap Geometry with Proton Beam Direction as Shown. 


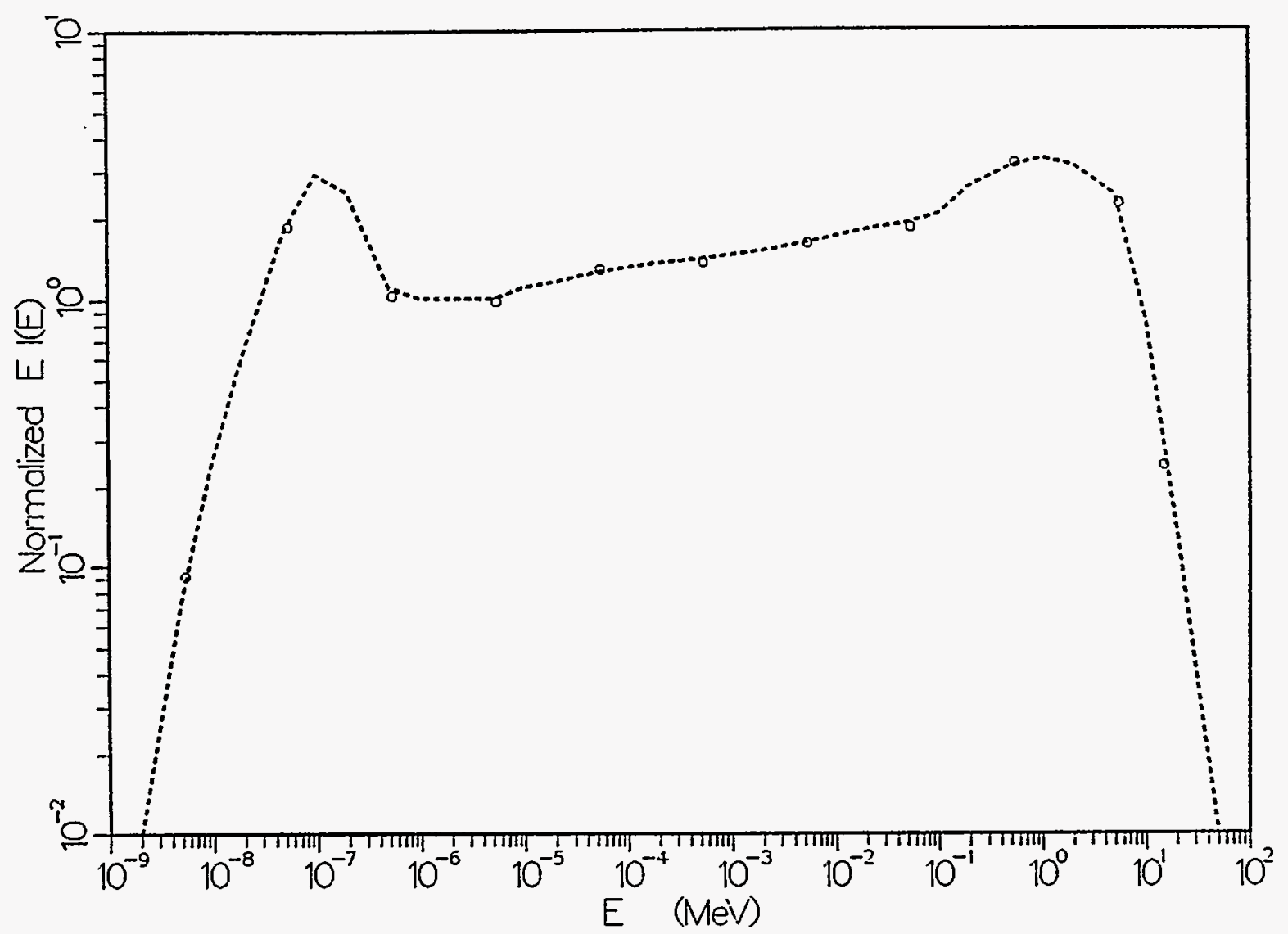

Figure V.3.3-3 Neutron-Beam Spectra from the IPNS Upgrade Moderators. (Circles indicate calculated values from Chapter IV for a representative moderator. The dashed line is the interpolated and extrapolated spectrum $[\mathrm{EI}(\mathrm{E})]_{\mathrm{w}}$ used for shielding calculations.) 
For IPNS Upgrade, we assume $S_{o}=4 \times 10^{13} \mathrm{n} / \mathrm{sr}-\mathrm{sec}$ on the basis of calculations in Chapter IV. This assumption is also consistent with scaling from measured IPNS values. ${ }^{15}$ The dose rate $\mathrm{D}$ at radius $\mathrm{R}+30 \mathrm{~cm}$ is calculated as a function of $\mathrm{R}$ for beamline shielding made of different materials. Figure V.3.3-4 shows some of the calculated values for iron shielding, which is the most effective practical material. These calculations assume a $10 \times 10 \mathrm{~cm}^{2}$ beam, and so it is assumed that the remaining portions of the beam port are filled with iron shielding. These calculations indicate that at $6 \mathrm{~m}$ from the moderator, $\sim 108 \mathrm{~cm}$ of iron is required to reduce the neutron dose rate to $0.125 \mathrm{mrem} / \mathrm{h}$ at $30 \mathrm{~cm}$ from the beamline shield surface. At this shield thickness the remaining dose rate is almost entirely due to the high-energy neutrons $(>50 \mathrm{MeV})$, even though there are very few in the original source term. Since scattering of high-energy neutrons is predominantly in the forward direction, this source term overestimates the required transverse shield thickness. An alternative is to base the shield thickness only on the fast neutrons. In that case only $\sim 81 \mathrm{~cm}$ of iron is required to reduce the dose rate to $0.125 \mathrm{mrem} / \mathrm{h}$. These two different calculations bracket the required shield thickness. Similar calculations show that the required iron-shielding thickness at $L=20 \mathrm{~m}$ from the moderator is between $\sim 86 \mathrm{~cm}$ and $\sim 73 \mathrm{~cm}$. An average thickness of $80 \mathrm{~cm}$ of iron shielding on all sides is used for the IPNS Upgrade neutron beamlines.

These shielding estimates are based on the use of iron shielding. The primary mechanisms for removal of high-energy and fast neutrons by the shielding involve (1) the scattering of the neutron to a lower energy and different direction or (2) an inelastic nuclear reaction, resulting in the production of one or more lower-energy neutrons proceeding in different directions. Some additional shielding material is required to slow down and absorb these remaining neutrons. This additional shielding material is also needed to absorb the $25 \mathrm{keV}$ and $125 \mathrm{keV}$ neutrons transmitted through the iron with little attenuation. The best material for slowing down neutrons is hydrogen, and the absorptions are best done by ${ }^{10} \mathrm{~B}$, because this reaction produces no highenergy gamma rays. Slow-neutron mean-free-paths in borated hydrogenous material such as borated polyethylene or wax are of the order of $1 \mathrm{~cm}$. Only a few centimeters of such material is required to slow the remaining neutrons down to an energy where the absorption cross-section of the boron becomes large. All of the beamline shielding needs to incorporate several centimeters of such material, in addition to the $80-\mathrm{cm}$ thickness of iron shielding. This borated hydrogenous shielding can be replaced with a larger thickness of concrete where space permits.

\subsubsection{Beamline Shielding - "Direct" Neutrons from the Target}

Some of the high-energy and fast neutrons directly from the target surface are also present in the beamline near the biological shield because of the relatively weak shielding between the target and beamline. Figure V.3.3-5a shows a sketch of the relevant geometry. Here it is again assumed that shielding apertures define the beam to a cross section of $10 \times 10 \mathrm{~cm}^{2}$. 


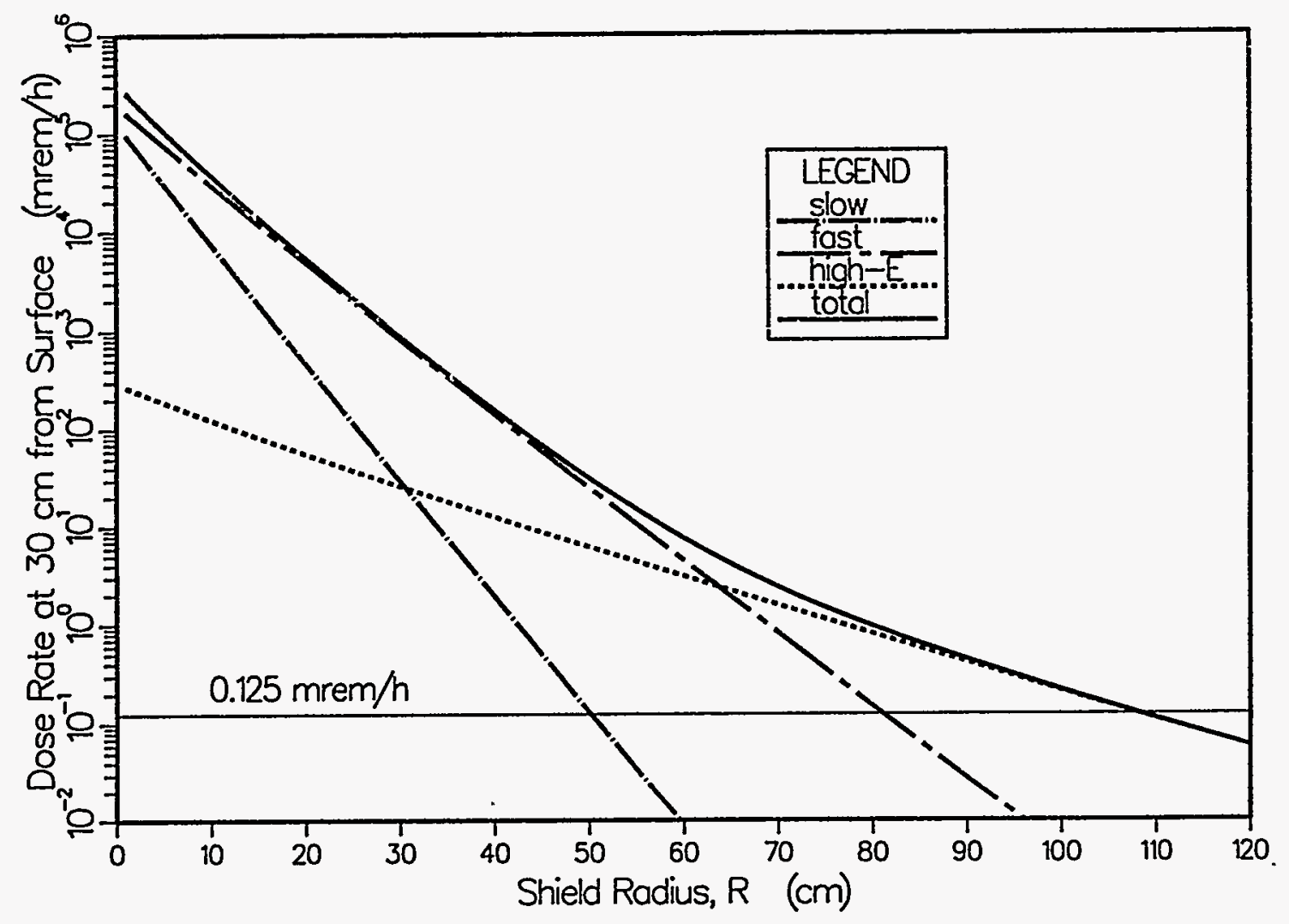

Figure V.3.3-4 Dose Rate $30 \mathrm{~cm}$ from the Surface of a Cylindrical Iron Beamline Shield of Radius $\mathrm{R}$, Calculated for a Distance of $6 \mathrm{~m}$ from the Moderator. 
The neutron path shown in the figure passes through lengths $L_{i}$ and $L_{c}$ of iron and concrete shielding. From the figure

$$
\begin{gathered}
\mathrm{L}_{\mathrm{i}}=(500+\mathrm{d}) \sec \alpha-10 \csc \alpha \\
\mathrm{L}_{\mathrm{c}}=100 \sec \alpha \\
\tan \alpha=\frac{\mathrm{R}+10}{600+\mathrm{d}}
\end{gathered}
$$

We consider the dose at a distance $30 \mathrm{~cm}$ from the point the path exits the shielding. This is at a total path distance $\mathrm{r}$ given by

$$
r=(600+d) \sec \alpha+30
$$

In Section IV.4.3 it was shown that the dose rate under these conditions is

$$
\mathrm{D}=\frac{2.364 \times 10^{15}}{\mathrm{r}^{2}} \mathrm{e}^{-0.0437 \mathrm{~L}_{\mathrm{i}}-0.0220 \mathrm{~L}_{\mathrm{c}}}
$$

for $\mathrm{L}_{\mathrm{i}}, \mathrm{L}_{\mathrm{c}}$, and $\mathrm{r}$ in $\mathrm{cm}$.

The radius of the external beamline iron shielding is $R=80 \mathrm{~cm}$. At this radius the dose rate due to neutrons directly from the target is reduced to $0.125 \mathrm{mrem} / \mathrm{h}$ at d $=77 \mathrm{~cm}$. At the face of the biological shield where $d=0$, a beamline shield radius of $R=216 \mathrm{~cm}$ is required to reduce the dose rate to $0.125 \mathrm{mrem} / \mathrm{h}$. A transition shield at the interface with the biological shield provides this additional shielding. This transition shield is shown schematically in Figure V.3.3-5b. Alternatively, access to regions near the biological shield can be administratively limited to less than 40 hours per week.

\subsubsection{Beamstops}

Each neutron beamline must have a beamstop beyond the scattering flight path to stop all the remaining beam. The dose rate at the sides of this beamstop is estimated by assuming a point source which isotropically scatters all the neutrons within the beamstop. The calculation then proceeds similarly to that for the beamline shielding radius.

$$
D=S_{o} \frac{A}{L^{2}} \frac{1}{4 \pi r^{2}} \sum_{E_{i}} e^{-R / \Lambda\left(E_{1} E_{2}\right)} \Delta I\left(E_{1} E_{2}\right)
$$

The shortest beamline has its beamstop at least $12 \mathrm{~m}$ from the moderator. For this maximum dose rate condition an $80 \mathrm{~cm}$ thickness of iron reduces the dose rate to below $0.125 \mathrm{mrem} / \mathrm{h}$ at $30 \mathrm{~cm}$ from the beamstop sides. 


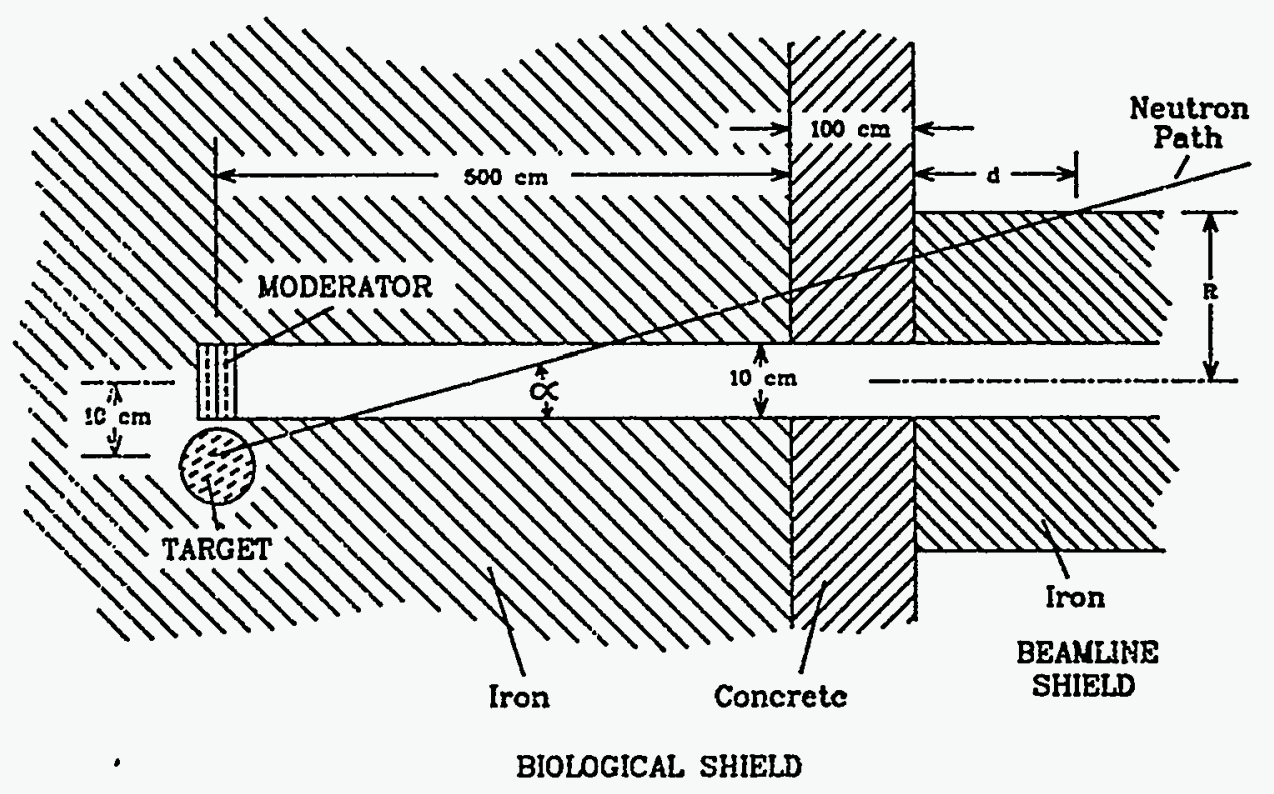

(a)

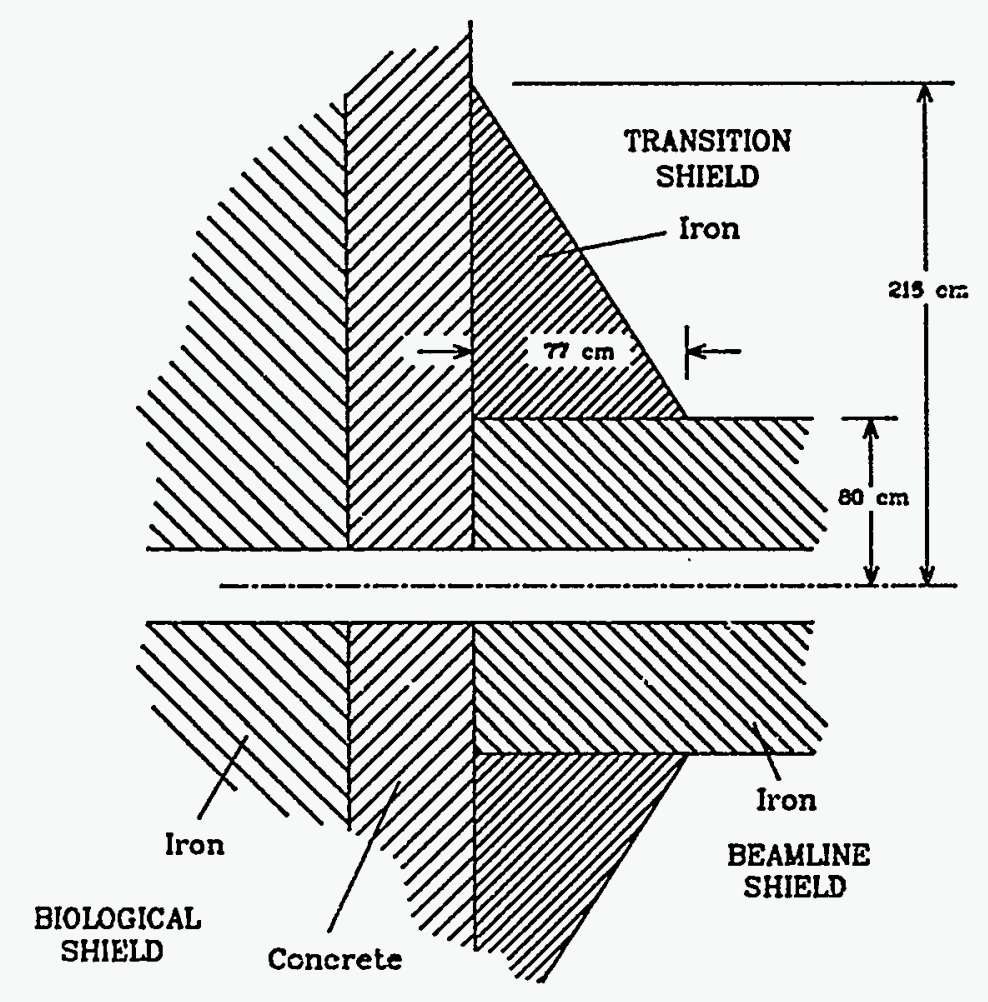

(b)

Figure V.3.3-5 (a) Geometry for Shielding against High-Energy Neutrons from the Target; (b) Transition Shield at the Beamline Interface with the Biological Shield. 
The high-energy neutrons in the beam proceed in the forward direction through the beamstop, so the dose rate at the end of the beamstop is given approximately by

$$
\begin{aligned}
D \simeq & \left.S_{0} \frac{A}{L^{2}} \frac{1}{4 \pi R_{f}^{2}}\left[e^{-R_{f} \Lambda(\text { slow })} \Delta \mathrm{r}(\text { slow })+e^{-R_{f} / \Lambda(\text { fast })} \Delta \mathrm{I} \text { (fast) }\right)\right] \\
& +S_{0} \frac{1}{L^{2}}\left[e^{-R_{f} / \Lambda(\text { high-energy })} \Delta \mathrm{I}(\text { high-energy })\right],
\end{aligned}
$$

where $R_{f}$ is the thickness of the beamstop shielding in the forward direction. The beamstop thickness required by the last term in Equation V.3.3-12 for a 0.125 -mrem/h dose rate in the forward direction from a beamstop $12 \mathrm{~m}$ from the moderator is

$$
\begin{aligned}
\mathrm{R}_{\mathrm{f}} & =-\Lambda \ln \left(\mathrm{L}^{2} \mathrm{D} / \mathrm{S}_{\mathrm{o}} \Delta \mathrm{I}\right) \\
& =220 \mathrm{~cm} .
\end{aligned}
$$

This value is much greater than the thickness required to shield against the slow and fast neutrons, so this high-energy-neutron term governs the beamstop shielding thickness in the forward direction.

As noted above for beamline shielding, the beamstops must also incorporate some borated hydrogenous material to perform the final slowing down and absorption of the neutrons.

\subsubsection{Shielding Interlocks}

The neutron dose rate in one of the neutron beams is estimated to be

$$
\begin{aligned}
D & \simeq S_{0} \frac{1}{L^{2}} \sum_{E_{1}} \Delta I\left(E_{1}, E_{2}\right) \\
& \simeq 7 \times 10^{13} / L^{2}
\end{aligned}
$$

for $L$ in $\mathrm{cm}$ and $\mathrm{D}$ in $\mathrm{mrem} / \mathrm{h}$. At a distance of $20 \mathrm{~m}$ from the moderator, this dose rate is $17,000 \mathrm{rem} / \mathrm{h}$. All movable shielding pieces, access to the sample area, and access to any other place in the beam are interlocked to the beam gate and to the accelerator. The interlocks prevent access to the beam while the beam gate is open and the accelerator is operating.

\subsubsection{Summary of Neutron Beamline Shielding Requirements}

Each neutron beamline is shielded on all sides by $80 \mathrm{~cm}$ of iron together with some hydrogenous and neutron absorbing materials. A larger-radius transition shield is used at the interface of each beamline with the biological shield. Each beamline has a beamstop that provides 
$80 \mathrm{~cm}$ of iron shielding on the sides and $220 \mathrm{~cm}$ of iron in the forward direction, and includes the necessary additional hydrogenous and neutron-absorbing materials.

\subsection{NEUTRON POLARIZATION}

Only two of the instruments in the reference set, the POSY-I and TOFNSE, are designed to operate with polarized neutrons. Both of these instruments work with cold neutrons and utilize limited angular ranges, so the neutron polarization can be done very well by reflection from polarizing supermirrors using current technology.

Other instruments that might be desired at IPNS Upgrade in the future require broad-band polarizers and analyzers that work at short wavelengths and over wide angular ranges. The technology to make satisfactory polarizers and analyzers of the type needed does not yet exist. Thus, although satisfactory solutions exist for the polarization requirements of the initial instrumentation of IPNS Upgrade, it is important to continue development of other types of polarization devices and techniques to provide for future expansion of the capabilities of IPNS Upgrade.

\subsection{DETECTORS}

\subsubsection{Discrete Detectors}

Table V.3.5-1 lists instruments from the reference set that use discrete detectors. The number and sizes of these detectors are also listed, and an estimate is made of the maximum instantaneous and time-averaged data rates per detector. All of these detectors are specified as ${ }^{3} \mathrm{He}$ gas proportional counters, but where background and geometrical considerations permit, scintillation counters can be used equally well.

Commercial cylindrical ${ }^{3} \mathrm{He}$ proportional counters and associated counting electronics typically have dead-times of $\sim 2 \mu \mathrm{sec}$. Excluding for the moment the chopper spectrometers, the maximum instantaneous count rate listed in Table V.3.5-1 is $5 \times 10^{4}$ counts/sec per detector for the SEPD. With 2- $\mu$ sec dead-time per detector, this gives dead-time losses of nearly $10 \%$ in the peaks of the spectrum. In the majority of cases instantaneous rates are significantly lower than those in Table V.3.5-1. Thus, it appears that ${ }^{3} \mathrm{He}$ proportional counters have adequate speed for all of the instruments in the table, excluding the chopper spectrometers operating in diffractometer mode, although dead-time corrections are needed in some cases.

The count-rate estimates for the chopper spectrometers listed in Table V.3.5-1 are made for the diffractometer mode, in which the choppers are removed from the beam and the instrument functions as a powder diffractometer. This mode is used only for instrument calibration and for rare cases when the growth or modification of samples in situ needs to be 
Table V.3.5-1 Discrete Detector Requirements ${ }^{a}$

\begin{tabular}{|c|c|c|c|c|c|c|}
\hline \multirow[b]{2}{*}{ Instrument ${ }^{b}$} & \multirow[b]{2}{*}{ Section } & \multirow[b]{2}{*}{$\begin{array}{l}\text { Number of } \\
\text { Detectors }\end{array}$} & \multicolumn{2}{|c|}{$\begin{array}{l}\text { Detector } \\
\text { Dimensions }\end{array}$} & \multicolumn{2}{|c|}{$\begin{array}{l}\text { Max. Rate per Detector } \\
\text { (counts/sec)c }^{\text {c }}\end{array}$} \\
\hline & & & $\begin{array}{l}\text { Dia. } \\
(\mathrm{cm})\end{array}$ & $\begin{array}{l}\text { Length } \\
(\mathrm{cm})\end{array}$ & Instantaneous & Time-Avg. \\
\hline SEPD & 2.1 .1 .1 & $\sim 280$ & 1.2 & 38 & $-5 \times 10^{4}$ & $\sim 5 \times 10^{3}$ \\
\hline GPPD & 2.1 .1 .2 & $\sim 520$ & 1.2 & 38 & $-5 \times 10^{3}$ & $-1 \times 10^{3}$ \\
\hline HRPD & 2.1 .2 .1 & $\sim 410$ & 1.2 & 38 & $-9 \times 10^{2}$ & $-2 \times 10^{2}$ \\
\hline RSD & 2.1 .2 .2 & -250 & 1.2 & 38 & $-4 \times 10^{3}$ & $-7 \times 10^{2}$ \\
\hline VSPD & 2.1 .2 .3 & $\sim 560$ & 0.6 & 19 & $-2 \times \cdot 10^{3}$ & $-3 \times 10^{2}$ \\
\hline HRMECS & 2.5.1.1 & -800 & 2.5 & 46 & $-2 \times 10^{5 d}$ & $-3 \times 10^{4 d}$ \\
\hline LRMECS & 2.5 .1 .2 & -160 & 2.5 & 46 & $-7 \times 10^{5 d}$ & $-5 \times 10^{4 d}$ \\
\hline CNCS & 2.5.2.1 & -1000 & $2.5^{e}$ & 25 & $\sim 4 \times 10^{4 d}$ & $-3 \times 10^{3 d}$ \\
\hline QENS & 2.6 .1 .1 & -100 & 0.6 & 10 & $-2 \times 10^{3}$ & $\sim 9 \times 10^{1}$ \\
\hline HRBS & 2.6.2.1 & $\sim 150$ & 0.6 & 10 & $-9 \times 10^{1}$ & $-9 \times 10^{0}$ \\
\hline TFCA & 2.6.2.2 & $\sim 300$ & 0.6 & 25 & $-2 \times 10^{3}$ & $-9 \times 10^{1}$ \\
\hline QSTAXC & 2.6 .2 .3 & $\sim 12$ & 0.6 & 10 & $-5 \times 10^{4}$ & $-7 \times 10^{2}$ \\
\hline MICAS & 2.6.2.4 & $\sim 20$ & 0.6 & 10 & $-5 \times 10^{4}$ & $-9 \times 10^{3}$ \\
\hline TOFNSE & 2.7 .1 .1 & $\sim 10$ & 0.6 & 10 & $-2 \times 10^{2}$ & $-2 \times 10^{1}$ \\
\hline
\end{tabular}

a Detectors are specified as cylindrical ${ }^{3} \mathrm{He}$ gas proportional counters, but in some cases scintillation detectors such as those used on some instruments at ISIS can be substituted.

b Instruments not listed do not use any discrete detectors.

c Scaled from IPNS rates where possible; otherwise, estimated on the assumption that the sample scatters $20 \%$ of the beam isotropically into a $4 \pi$ solid angle.

d Maximum rates are for diffractometer mode (choppers removed). Rates in normal operation are much lower.

e This instrument uses flattened detectors with thickness $\sim 1 \mathrm{~cm}$ and width $\sim 3.5 \mathrm{~cm}$. 
monitored. In the normal operating mode with choppers in place, data rates are significantly lower than those given in the table and pose no problem for the detectors. If necessary, an attenuator can be placed in the incident beam on the few occasions when the instrument is operated in diffractometer mode. Since this mode is used only rarely and data rates are high in this mode, the use of an attenuator in such cases will not appreciably affect the overall performance of the instrument.

All of the instruments listed in Table V.3.5-1 can utilize ${ }^{3} \mathrm{He}$ proportional counters without serious problems. The $\mathrm{ZnS}-\mathrm{LiF}$ scintillation detectors developed at ISIS ${ }^{16}$ have very good intrinsic backgrounds and discrimination against gamma radiation, but they are no faster than the ${ }^{3} \mathrm{He}$ counters and so offer no advantage in this respect. Other types of scintillation detectors, such as Li-glass, can be significantly faster but suffer from intrinsic background problems. ${ }^{17}$ These intrinsic background-contribution levels are not a problem for some of the instruments, and in these cases Li-glass scintillators can be used to achieve higher counting speeds than are feasible with the gas detectors.

\subsubsection{Position-Sensitive Detectors}

Table V.3.5-2 lists those instruments from the reference set that use position-sensitive detectors. The table indicates whether the required position encoding is one-dimensional (1D) or two-dimensional (2D). Some instruments use both 1D and 2D detectors, and some use a combination of position-sensitive and discrete detectors. The number of detectors required and the required sizes and position resolutions are also listed, and an estimate is made of the maximum instantaneous and time-averaged data rates per detector.

Most commercial 1D or $2 \mathrm{D}{ }^{3} \mathrm{He}$ position-sensitive proportional counters require several microseconds for the position encoding. A neutron arriving anywhere on the same detector during encoding either will not be counted or, worse, will cause position misencoding for one or both neutrons. ${ }^{18}$ Multi-electrode position-sensitive gas proportional counters available commercially work reliably at instantaneous rates up to $\sim 5 \times 10^{4}$, and such detectors are available at resolutions down to $1.5 \mathrm{~mm}$. Other types of encoding can be used for those instruments in the table for which instantaneous rates per detector are not expected to reach or exceed $\sim 10^{4}$ counts/sec an appreciable fraction of the time. This criterion implies that $1 \mathrm{D}$ gas proportional counters of the types currently in use at IPNS are satisfactory for use on the SAND, HRSAND, GLAD, POSY-I, and POSY-II at IPNS Upgrade. These detectors already provide the required position resolutions for these instruments. As discussed in Section V.2.2.1.1, the 2D position-sensitive detector in use on the SAND at IPNS is fast enough for use in the high-resolution mode on the SAND at IPNS Upgrade. It is clear from Table V.3.5-2, however, that it cannot be used in the low-resolution mode. One of the multielectrode proportional counters may be fast enough for this purpose. The multi-electrode detectors can also satisfy the requirements of the RSD and HIREF. A micro-channel-plate detector of the type used earlier on the POSY-I at IPNS ${ }^{19}$ is satisfactory for use on the REFD. 
The data rates estimated in the table for the chopper spectrometers are based on operation in the diffractometer mode. As noted in the previous section, this mode is used only a small fraction of the time, and the beam can be attenuated during this period. Thus, commercial ${ }^{3} \mathrm{He}$ gas proportional counters are appropriate for use as the 1D position-sensitive detectors on the chopper spectrometers HRMECS, LRMECS, CNCS, and SCCS.

Table V.3.5-3 summarizes the remaining position-sensitive detector requirements at IPNS Upgrade, for which no commercial solution currently exists. These detectors require development because of the data-rate limitations or because the required resolution is better than that usually found in a detector of the specified size, or both. The detector on the HQSCD is located close to the sample and needs to have high efficiency for short-wavelength neutrons, so gas-detector technology is inappropriate. All of these instruments operate in the TOF mode. Therefore, integrating detectors, which can provide good spatial resolution and are relatively unaffected by data rate, cannot be used. All of the instruments listed in this table can, if required, function using detectors at the limits of today's technology and still be extremely good instruments. For example, data-rate limitations can be overcome by using smaller samples or by partially attenuating the beam, and resolution limitations can be overcome by moving the detector farther from the sample or by using a smaller detector. However, these instruments will not reach their full potential at IPNS Upgrade without advances in detector technology. Emerging detector technologies ${ }^{20}$ show promise of solving one or more of these requirements, so it is likely that all of these detectors will be technologically possible by the time they are required at IPNS Upgrade.

\subsubsection{Beam Monitors}

Nearly all the instruments at IPNS use $\mathrm{BF}_{3}$ or ${ }^{3} \mathrm{He}$ pulsed ionization chambers as lowefficiency beam monitors ${ }^{19}$ to monitor the spectral shape and overall intensity of the incident neutron beams. This type of detector is stable and reliable, is relatively insensitive to gamma rays and fast neutrons, and samples the entire beam uniformly. The detection efficiency is inversely proportional to the neutron velocity over several orders of magnitude in velocity. The overall efficiency level can be varied by several orders of magnitude by changing the partial pressure of $\mathrm{BF}_{3}$ or ${ }^{3} \mathrm{He}$ in the fill gas. This technology is readily adaptable for use at IPNS Upgrade. At least one of these beam monitors is required for each instrument.

\subsection{DATA ACQUISITION}

The data-acquisition systems (DASs) for the instruments at IPNS Upgrade all use the same software and are based on a small number of standardized hardware modules. This philosophy has been followed at IPNS and has resulted in a large reduction in the effort required to maintain the data-acquisition systems and in minimal training requirements for users who utilize more than one instrument. The IPNS Upgrade system design draws on more than 10 years experience in operating TOF data acquisition systems at IPNS.21 


\begin{tabular}{|c|c|c|c|c|c|c|c|c|}
\hline \multirow[b]{2}{*}{ Instrumenta } & \multirow[b]{2}{*}{ Section } & \multirow[b]{2}{*}{ Dim. } & \multirow[b]{2}{*}{$\begin{array}{l}\text { Number of } \\
\text { Detectors }\end{array}$} & \multicolumn{2}{|c|}{$\begin{array}{l}\text { Dimensions at } \\
\text { Active Area }\end{array}$} & \multirow[b]{2}{*}{$\begin{array}{l}\text { Resolution } \\
\text { (mm) }\end{array}$} & \multicolumn{2}{|c|}{$\begin{array}{l}\text { Max. Rate per Detector } \\
\text { (counts/sec) }\end{array}$} \\
\hline & & & & $\begin{array}{l}\text { Width } \\
(\mathrm{cm})\end{array}$ & $\begin{array}{l}\text { Length } \\
(\mathrm{cm})\end{array}$ & & Instantaneous & Time-Avg. \\
\hline RSD & 2.1 .2 .2 & $2 D$ & 4 & 30 & 30 & 2 & $-1 \times 10^{5}$ & $-2 \times 10^{4}$ \\
\hline \multirow[t]{2}{*}{ SAND } & 2.2 .1 .1 & $2 D$ & 1 & 40 & 40 & 4 & $-2 \times 10^{5}$ & $\sim 7 \times 10^{4}$ \\
\hline & & 10 & 65 & 1.2 & 60 & 10 & $-9 \times 10^{3}$ & $\sim 3 \times 10^{3}$ \\
\hline \multirow[t]{2}{*}{ HRSAND } & 2.2.2.1 & $2 D$ & 1 & 60 & 60 & 2 & $-5 \times 10^{3}$ & $-2 \times 10^{3}$ \\
\hline & & 10 & -20 & 1.2 & 60 & 10 & $-5 \times 10^{2}$ & $-2 \times 10^{2}$ \\
\hline SPSAND & 2.2.2.2 & $2 D$ & 1 & 40 & 40 & 4 & $-5 \times 10^{4}$ & $-2 \times 10^{4}$ \\
\hline GLAD & 2.3.1.1 & 10 & $\sim 410$ & 1.2 & 60 & 10 & $\sim 7 \times 10^{3}$ & $-3 \times 10^{3}$ \\
\hline SCD & 2.3.1.2 & $2 D$ & 3 & 30 & 30 & 3 & $\sim 1 \times 10^{6}$ & $-1 \times 10^{5}$ \\
\hline HQSCD & 2.3.2.1 & $2 D$ & 1 & 30 & 30 & 1.5 & $\sim 1 \times 10^{5}$ & $\sim 1 \times 10^{4}$ \\
\hline POSY-I & 2.4.1.1 & 10 & 1 & 5 & 10 & 2 & $\sim 1 \times 10^{3}$ & $-4 \times 10^{2}$ \\
\hline POSY-II & 2.4 .1 .2 & $1 D$ & 1 & 5 & 20 & 2 & $-6 \times 10^{3}$ & $-2 \times 10^{3}$ \\
\hline HIREF & 2.4 .2 .1 & $1 D$ & 1 & 5 & 10 & 2 & $\sim 1 \times 10^{5}$ & $-3 \times 10^{4}$ \\
\hline GREF & 2.4.2.2 & $2 D$ & 1 & 60 & 60 & 2 & $-1 \times 10^{4}$ & $\sim 3 \times 10^{3}$ \\
\hline REFD & 2.4 .2 .3 & $2 D$ & 1 & 3 & 3 & 0.5 & $\sim 1 \times 10^{3}$ & $\sim 4 \times 10^{2}$ \\
\hline HRMECS & 2.5 .1 .1 & $1 D$ & $\sim 40$ & 2.5 & 100 & 20 & $\sim 1 \times 10^{5 c}$ & $\sim 2 \times 10^{4 c}$ \\
\hline LRMECS & 2.5.1.2 & $1 D$ & -30 & 2.5 & 46 & 20 & $-8 \times 10^{5 c}$ & $-6 \times 10^{4 c}$ \\
\hline CNCS & 2.5 .2 .1 & 10 & $\sim 30$ & $2.0^{d}$ & 60 & 20 & $-4 \times 10^{4 c}$ & $\sim 3 \times 10^{3 c}$ \\
\hline Sccs & 2.5.2.2 & $1 D$ & $\sim 256$ & 2.5 & 100 & 20 & $\sim 4 \times 10^{4 c}$ & $-8 \times 10^{3} c$ \\
\hline
\end{tabular}

a Instruments not listed do not use any position-sensitive detectors.

b Scaled from IPNS rates where possible; otherwise, estimated on the assumption that the sample scatters $20 \%$ of the beam isotropically into a $4 \pi$ solid angle.

c Maximum rates are for diffractometer mode (choppers removed). Rates in normal operation are much lower.

d This instrument uses flattened cylindrical linear-position-sensitive detectors with thickness $\sim 0.8 \mathrm{~cm}$ and width $\sim 2 \mathrm{~cm}$. 
TABLE V.3.5-3 Position-Sensitive Detectors Requiring Development

\begin{tabular}{|c|c|c|c|c|c|c|c|c|}
\hline \multirow[b]{3}{*}{ Instrument } & \multirow[b]{3}{*}{ Section } & \multirow[b]{3}{*}{ Dim. } & \multirow[b]{3}{*}{$\begin{array}{l}\text { Number of } \\
\text { Detectors }\end{array}$} & \multicolumn{2}{|c|}{$\begin{array}{l}\text { Dimensions at } \\
\text { Active Area }\end{array}$} & \multirow[b]{3}{*}{$\begin{array}{l}\text { Resolution } \\
\text { (mm) }\end{array}$} & \multirow{2}{*}{\multicolumn{2}{|c|}{$\begin{array}{l}\text { Max. Rate per Detector } \\
\text { (counts/sec) }\end{array}$}} \\
\hline & & & & & & & & \\
\hline & & & & $\begin{array}{l}\text { Width } \\
(\mathrm{cm})\end{array}$ & $\begin{array}{l}\text { Length } \\
(\mathrm{cm})\end{array}$ & & Instantaneous & Time-Avg. \\
\hline HASAND & 2.2.2.1 & 2D & 1 & 60 & 60 & 2 & $-5 \times 10^{3}$ & $-2 \times 10^{3}$ \\
\hline SCD & 2.3 .1 .2 & $2 \mathrm{D}$ & $3^{\prime}$ & 30 & 30 & 3 & $-1 \times 10^{6}$ & $-1 \times 10^{5}$ \\
\hline HQSCD & 2.3.2.1 & 2D & 1 & 30 & 30 & 1.5 & $-1 \times 10^{5}$ & $\sim 1 \times 10^{4}$ \\
\hline GREF & 2.4.2.2 & 2D & 1 & 60 & 60 & 2 & $-1 \times 10^{4}$ & $-3 \times 10^{3}$ \\
\hline
\end{tabular}


The DAS for IPNS Upgrade is based on a distributed processor system using a computer back-plane, such as VME, VXI, or Future Bus. Each crate in the system contains a local processor module, a crate control module, and as many digitizer modules as the crate accommodates. The detectors are located within $15 \mathrm{~m}$ of the digitizer modules and the preamps are within a few centimeters of the detectors. All connections to the digitizer modules are through the backplane to allow easy module removal and replacement. Each DAS has a workstation to serve as the user interface. Communications to the local processors and histogram memory are via fiber-optic local area networks, as indicated in Figure V.3.6-1.

Electronics capabilities change rapidly, so it is premature to choose a specific hardware implementation at this time. The following functionality must be provided:

1. Local processor module. The local processor module must provide for connection to a local area network (LAN), operate under a real-time kernel, and have a means of connection to a large, shared memory for accumulating histograms. The processor must also have a large local memory for execution software, as well as storage of large look-up tables. The software and look-up tables can be generated on a networked workstation and down-loaded to the processor at run time.

2. Crate control module. The crate control module contains the TOF clock generator, data gating circuitry, and data collection and buffer storage of raw data from within the crate. This module is custom-designed.

3. Digitizer modules. The digitizer modules are custom-designed and depend on the type of detector system. The number of detectors interfaced per module depends on the type of detector.

4. Workstation. The workstation serves as the main point of interaction between the user and the DAS. Each workstation communicates via network with the local processor in the data histograming system. A separate network connection provides the workstation with direct reading access to the live data accumulating in the histogram memory, as shown in Figure V.3.6-1. The choice of the type of workstation and operating system is based on an evaluation made at the time of detailed DAS design.

Each instrument has its own data-acquisition cabin to house all the data-acquisition and chopper-control electronics and local computers. Each cabin is a temperature-controlled modular room at least $3 \times 4 \mathrm{~m}^{2}$ in size and accommodates several electronics racks, a workstation, and a small desk. These cabins are located as close to their respective instruments as practical. 


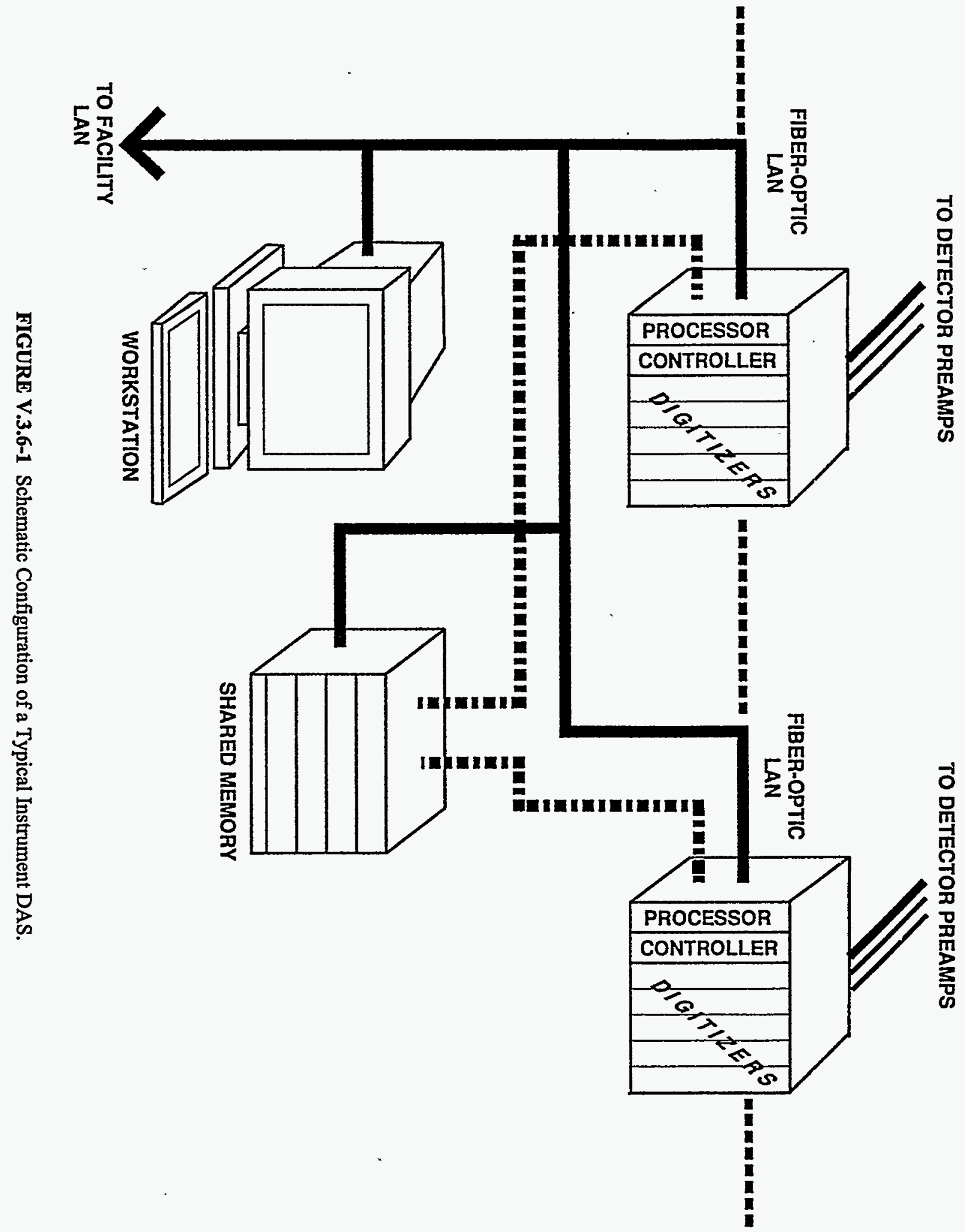




\subsection{DATA ANALYSIS}

Rapid preliminary analysis is required to make informed decisions regarding experiments in progress. Data display, manipulation, and scientific visualization software are required.

The central computing facility handles at least 80 users, has at least 20 gigabytes of online storage, and 200 gigabyte of near-line storage. Archiving and restoring of files are achieved with a minimum of human intervention through automation of the loading of the archival media. The archiving software makes the process transparent to the user. A high-speed fiber-optic network handles transfer of large quantities of data and graphic images. The central computing facility also provides backup capability for the individual workstations and provides data bases for tracking facility maintenance, proposal and experiment status, user proposals, and safety concerns.

\subsection{ANCILLARY EQUIPMENT}

\subsubsection{General-Use Items}

Standard equipment and standard components are used wherever possible. Most sampleenvironment equipment is under computer control, so that experiments can be set up in sequence and the sample-environment variable changed automatically between measurements. This automation includes both cryogenic and furnace temperature control, sample position and orientation, choice of sample, and magnetic field. Interfaces for such automation are standardized to facilitate the automation of additional devices.

Closed-cycle refrigerators, cryostats, or furnaces are required for most experiments. Data rates on some of the IPNS Upgrade instruments are sufficiently high that more time is spent changing and equilibrating the sample temperature than is spent on collecting the data. It is important to initiate a program to develop designs for cryogenic and furnace environments that permit much more rapid change and equilibration of sample temperature. It is desirable to provide automated sample changers or other means of rapid change of samples within the controlled-temperature environment. The results of this development program will be incorporated into any new furnaces, displexes, and cryostats procured for IPNS Upgrade.

\subsubsection{Specialized Items}

\subsubsection{Rotating-Crystal Insert for Diffractometers}

Two ways are used to obtain structural information on disordered crystals by diffraction experiments. One method is to perform a single-crystal-diffraction experiment, and the other method is to carry out a powder-diffraction experiment. Both types of experiment measure the 
diffuse scattering intensities, but both of these have drawbacks. The two-dimensional pair density function (2D-PDF) approach is a method in-between the single-crystal and powderdiffraction methods. In this approach, a single crystal is rotated around an axis while the scattering is recorded, providing a cylindrically averaged $\mathrm{S}\left(\mathrm{Q}_{\mathrm{z}}, \mathrm{Q}_{\mathrm{r}}\right)$. A $\mathrm{Q}_{z}$-resolved $2 \mathrm{D}-\mathrm{PDF}$ is obtained by a Bessel transformation. With this method, all the diffuse scattering intensities are collected, resolved by $\mathrm{Q}_{\mathrm{z}}$. By changing the rotating axis it is possible to reconstruct the 3D picture. This method has already been used in the study of quasicrystals by $x$-ray diffraction, 22 but it has not yet been applied to neutron diffraction.

At IPNS Upgrade, both the GLAD and SEPD are suitable for such measurements. An insert for these instruments is required to rotate the sample at low temperatures. Such an insert will be developed.

\subsubsection{Control of Sample Atmosphere}

An increasing number of experiments are designed to study the behavior of a sample in contact with a controlled atmosphere (e.g., controlled oxidizing or reducing atmosphere, vaporsaturated gases, gas mixture, etc.). The study may involve following the time-evolution of the sample as it interacts with this atmosphere, or the presence of the atmosphere may be required to stabilize the sample. Standardized gas-handling systems are provided for such experiments. These systems have the capability for computer control and monitoring of the gas parameters such as pressure, concentration, etc.

\subsubsection{Pulsed Sample Environments}

The detection electronics in the QSS instruments are gated on for only a small fraction of the total time frame. A sample environment variable such as pressure, uniaxial-stress, magnetic field, laser heating, electric field, etc. can be pulsed in phase with the time gating of the data acquisition, so that the peak pulsed environment is present for the full time that the neutrons of interest are at the sample. This makes more effective use of the neutrons than is the case on a true steady-state beam. This argument applies equally well to the chopper TOF instruments, but not to most of the white-beam TOF instruments. Some techniques and equipment for pulsing sample variables exist, and others will be developed. 


\subsection{UTILITIES}

\subsubsection{Electrical Power}

Each instrument at IPNS Upgrade has an installed capacity of $30 \mathrm{~kW}$ utility power and $10 \mathrm{~kW}$ clean power. Most of the clean power is installed in the data-acquisition cabins for the instruments, and the remaining power is installed in easily accessible distribution panels located on each of the instruments. Table V.3.9-1 summarizes expected utility usage by the instruments at IPNS Upgrade.

\subsubsection{Other Utilities}

Manifolds for $\mathrm{N}_{2}$ gas, He gas, experiment water $\left(32^{\circ} \mathrm{C}\right)$, lab chilled water $\left(7^{\circ} \mathrm{C}\right)$, and compressed air $(620 \mathrm{kPa})$ are available at easily accessible manifolds located on each of the instruments. For the IPNS instruments, monthly usage of $\mathrm{N}_{2}$ gas and He gas are $\sim 9 \times 10^{4} \mathrm{~L}$ and $6 \times 10^{4} \mathrm{~L}$ per instrument, respectively, and for compressed air, usage is $9 \times 10^{4} \mathrm{~L}$ at standard conditions per instrument. About $2 \times 10^{6} \mathrm{~L}$ of water, half of it chilled, is used per instrument per month. At IPNS Upgrade, these capacities per instrument are doubled, leading to the values given in Table V.3.9-1.

\subsection{EXPERIMENTAL SUPPORT FACILITIES}

A number of support facilities are required to maintain the instruments and to service the day-to-day needs of the experimental program. These include:

1. A small machine shop with a minimum of $\sim 1,000 \mathrm{ft}^{2}\left(90 \mathrm{~m}^{2}\right)$.

2. An electronics shop with a minimum of $\sim 1,000 \mathrm{ft}^{2}\left(90 \mathrm{~m}^{2}\right)$.

3. Laboratory space for development and testing of ancillary equipment and instrument components, having a minimum of $\sim 2,000 \mathrm{ft}^{2}\left(180 \mathrm{~m}^{2}\right)$.

4. Laboratory space for development and testing of detectors, having a minimum of $\sim 1,000 \mathrm{ft}^{2}\left(90 \mathrm{~m}^{2}\right)$.

5. Several well-equipped laboratories for sample preparation and handling. These must include hoods, chemical handling and storage facilities, and normal laboratory services and equipment. At least one of these must be a controlled area for handling of samples that have been activated. A minimum of $~ 3,000 \mathrm{ft}^{2}\left(270 \mathrm{~m}^{2}\right)$ is required for these laboratories. 
Table V.3.9-1 Utility Requirements for the Instruments at IPNS Upgrade

\begin{tabular}{lll}
\hline \multicolumn{1}{c}{ Utility } & $\begin{array}{c}\text { Required Capacity } \\
\text { per Instrument }\end{array}$ & $\begin{array}{c}\text { Monthly Usage } \\
\text { per Instrument }\end{array}$ \\
\hline Electricity (normal) & & \\
Electricity (clean) & $\sim 30 \mathrm{~kW}$ & $\sim 15 \mathrm{MWh}$ \\
$\mathrm{N}_{2}$ gas (low pressure) & $\sim 10 \mathrm{~kW}$ & $\sim 5 \mathrm{MWh}$ \\
He gas (low pressure) & & $\sim 2 \times 10^{5} \mathrm{~L}$ \\
Compressed air (at $\sim 90 \mathrm{psig})$ & & $\sim 1.5 \times 10^{5} \mathrm{~L}$ \\
Water $\left(-80^{\circ} \mathrm{F}\right)$ & $45 \mathrm{~L}$ /min & $\sim 2 \times 10^{5} \mathrm{~L}$ \\
Water $\left(-45^{\circ} \mathrm{F}\right)$ & $45 \mathrm{~L} / \mathrm{min}$ & $\sim 2 \times 10^{6} \mathrm{~L}$ \\
& & \\
\hline
\end{tabular}


6. Data-handling room, to contain a number of hard-copy devices, workstations, and terminals, as well as desks and tables for temporary use by the users, having a minimum of $\sim 2,000 \mathrm{ft}^{2}\left(180 \mathrm{~m}^{2}\right)$.

Most of these spaces are in Building 368, between the $10-\mathrm{Hz}$ and $30-\mathrm{Hz}$ target buildings, to facilitate transport of equipment and samples back and forth to the instruments and for ease of access by the users. Additional facilities that are required, such as a site for the remainder of the shared computer facilities and laboratories for sample synthesis need not be contiguous to the experiment halls.

\subsection{REFERENCES}

1. C.G. Windsor, Pulsed Neutron Scattering, pp. 172-188 and 296-302 (Halsted Press, New York, 1981).

2. C.-K. Loong, S. Ikeda, and J.M. Carpenter, "The resolution function of a pulsed-source neutron chopper spectrometer," Nucl. Instrum. Methods A260:381-402 (1987).

3. G.E. Ostrowski et al., "The IPNS chopper control and accelerator interface system," in Proceedings of the 8th Meeting of the International Collaboration on Advanced Neutron Sources (ICANS VIII) (Oxford, U.K., July 8-12, 1985), Rutherford Appleton Laboratory Report No. RAL-85-110, pp. 676-688 (1985).

4. T.L.J. Jones et al., "Experience with the KFA/IGV (Jülich) magnetic bearing systems on an SNS neutron chopper," in Proceedings of the 8th Meeting of the International Collaboration on Advanced Neutron Sources (ICANS VIII) (Oxford, U.K., July 8-12, 1985), Rutherford Appleton Laboratory Report No. RAL-85-110, pp. 707-716 (1985).

5. J.M. Carpenter and C.-K. Loong, "IPNS chopper spectrometer improvements," in Proceedings of the 11th Meeting of the International Collaboration on Advanced Neutron Sources (ICANS XI) (Tsukuba, Japan, Oct. 22-26, 1990), National Laboratory for High Energy Physics (KEK) Report No. 90-25, pp. 711-716 (1991).

6. T.L.J. Jones et al., "The HET background suppressing chopper," in Proceedings of the 9th Meeting of the International Collaboration on Advanced Neutron Sources (ICANS IX) (Villigen, Switzerland, Sept. 22-26, 1986), Swiss Institute for Nuclear Research, ISBN 3-907998-01-4, pp. 529-533 (1987).

7. R.K. Crawford, Argonne National Laboratory, 1986 (unpublished information).

8. R.K. Crawford, Argonne National Laboratory, 1990 (unpublished information). 
9. A.V. Rauchas et al., "IPNS accelerator system and neutron chopper synchronization," in Proceedings of the 7th Meeting of the International Collaboration on Advanced Neutron Sources (ICANS VII) (Chalk River, Canada, Sept. 13-16, 1983), Atomic Energy of Canada Limited Report No. AECL-8488, pp. 157-161 (1984).

10. L. Donley, "Phase locking the IPNS neutron chopper to the $60-\mathrm{Hz}$ power line," in Proceedings of the 8th Meeting of the International Collaboration on Advanced Neutron Sources (ICANS VIII) (Oxford, U.K., July 8-12, 1985), Rutherford Appleton Laboratory Report No. RAL-85-110, pp. 689-696 (1985).

11. C.J. Carlile, M.W. Johnson, and W.G. Williams, Neutron Guides on Pulsed Sources, Rutherford Appleton Laboratory Report No. RL-79-084 (1979).

12. J.C. Sutherland et al., "The performance of the IRIS and HRPD guides on the SNS," in Proceedings of the 8th Meeting of the International Collaboration on Advanced Neutron Sources (ICANS VIII) (Oxford, U.K., July 8-12, 1985), Rutherford Appleton Laboratory Report No. RAL-85-110, pp. 771-776 (1985).

13. G.J. Russell et al., "Shielding concerns at a spallation source," Advanced Neutron Sources 1988, Proceedings of the 10th Meeting of the International Collaboration on Advanced Neutron Sources (ICANS X) (Los Alamos, N.M., Oct. 3-7, 1988), Institute of Physics Conference Series Number 97, Institute of Physics, New York, pp. 123-132 (1989).

14. M. Barbier, Shielding and Activation Study for the Intense Pulsed Neutron Source at Argonne National Laboratory, MITRE Technical Report No. MTR-6998 (MITRE Corp., McLean, Va., 1975).

15. J.M. Carpenter, C.W. Potts, and G.H. Lander, "Intense Pulsed Neutron Source (IPNS) at Argonne National Laboratory (ANL): A status report as of June, 1982," in Proceedings of the 6th Meeting of the International Collaboration on Advanced Neutron Sources (ICANS VI) (Argonne, Ill., June 28-July 2, 1982), Argonne National Laboratory Report No. ANL-82-80, pp. 77-101 (1983).

16. P.L. Davidson, M.W. Johnson, and N.J. Rhodes, "An Update on ISIS detectors," in Proceedings of the 11th Meeting of the International Collaboration on Advanced Neutron Sources (ICANS XI) (Tsukuba, Japan, Oct. 22-26, 1990), National Laboratory for High Energy Physics (KEK) Report No. 90-25, pp. 957-970 (1991).

17. P.L. Davidson et al., "Detector development at the ISIS facility," in Advanced Neutron Sources 1988, Proceedings of the 10th Meeting of the International Collaboration on Advanced Neutron Sources (ICANS X) (Los Alamos, N.M., Oct. 3-7, 1988), Institute of Physics Conference Series Number 97, Institute of Physics, New York, pp. 523-528 (1989). 
18. D.G. Montague et al., "Use of a semi-opaque beamstop for monitoring sample transmissions at GLAD," in Proceedings of the 11th Meeting of the International Collaboration on Advanced Neutron Sources (ICANS XI) (Tsukuba, Japan, Oct. 22-26, 1990), National Laboratory for High Energy Physics (KEK) Report No. 90-25, pp. 830839 (1991).

19. R.K. Crawford, J.R. Haumann, and G.E. Ostrowski, "Neutron detectors at IPNS," in Proceedings of the 11th Meeting of the International Collaboration on Advanced Neutron Sources (ICANS XI) (Tsukuba, Japan, Oct. 22-26, 1990), National Laboratory for High Energy Physics (KEK) Report No. 90-25, pp. 943-956 (1991).

20. P. Geltenbort and A. Oed, "Features of microstrip proportional counters," in Proceedings of SPIE's International Symposium on Optical Applied Science and Engineering (San Diego, Calif., July 19-24, 1992), vol. 1737, pp. 289-293 (1992).

21. R.K. Crawford et al., "The data acquisition system for the neutron scattering instruments at the Intense Pulsed Neutron Source," IEEE Trans. Nucl. Sci. NS-28, 3692-3700 (1981).

22. T. Egami, University of Pennsylvania, Philadelphia, 1992 (private communication). 


\subsection{INTRODUCTION}

IPNS Upgrade includes neutron-irradiation facilities for the purpose of radiation damage studies, production of isotopes, and activation analysis. The anticipated uses of these facilities are discussed in Section 4.2, the details of the irradiation facilities are described in Section 4.3, and the external facilities to be used in support of these activities are described in Section 4.4.

The IPNS Upgrade neutron-irradiation facilities include four irradiation tubes for each of the two target stations. These tubes are designed to be used either for fast-neutron radiation damage measurements or for slow-neutron activation and isotope production. The tubes are located at different positions within the volumes surrounding each target. The integrated proton currents to these target stations differ by a factor of two, so the integrated neutron dose rate differs by a factor of two at the two stations. Irradiation positions at different distances from the target and at different angles with respect to the proton beam provide different neutron energy spectra, flux levels, and sample heat loads. These differences provide the opportunity to optimize the irradiation conditions for different purposes. The hot cells used to service targetsystem components, also serve as sample-handling facilities supporting these irradiation facilities. Radioactive samples and materials are transferred from the irradiation positions to inhouse experimental facilities or to containers for shipment off-site.

\subsection{ANTICIPATED USES OF THE FACILITIES}

\subsubsection{Radiation Effects}

Samples are irradiated in the irradiation tubes to produce atomic defects that alter the structure and properties of the irradiated material. Investigation of these defects and their properties is an aspect of materials science research. Basic research in neutron radiation effects requires well-controlled and well-instrumented experimental facilities. A spallation neutron source has the advantages of ease of control, access, and instrumentation; low gamma flux; and constancy of neutron energy spectrum. The pulsed nature of the neutron flux offers additional scientific opportunities.

\subsubsection{Isotope Production}

The irradiation tubes provide available space in the neutron flux to produce various isotopes. Numerous isotopes can be produced, including iodine 125, molybdenum 99, xenon 133 , dysprosium 165 , tungsten 188 , rhenium 186 , samarium 153 , and lutetium 177 . These isotopes serve such fields as medicine, geology, biology, agriculture, manufacturing, and industrial calibration. 


\subsubsection{Activation Analysis}

In activation analysis, traces of various elements can be identified and measured by analyzing the gamma rays they emit after neutron irradiation. Pneumatic capsules quickly transfer the irradiated activated samples to an automatic counting chamber. A computerized neutron cross-section and gamma-energy library provides accurate information on isotope quantities. Activation analysis is widely used in such fields as chemistry, biology, geology, forensics, and medicine.

\subsection{OVERVIEW OF THE IRRADIATION FACILITIES}

The irradiation tubes are located in the reflector material between the neutron beamlines in each station, allowing operation of the neutron scattering instruments and experiments on activation and radiation effects to proceed simultaneously. Figure V.4.3-1 shows a horizontal cutaway view through the midplane of the target and reflector assembly, indicating the locations of these tubes. Two irradiation tubes, labeled IT-1 and IT-2, are symmetrically placed on either side of and parallel to the long axis of the downstream section of the target in each target assembly. These tubes are located in the highest neutron flux and provide access to positions with intense fluxes of neutrons with energies up to $2 \mathrm{GeV}$. The highest-energy neutrons are found in the downstream direction, as illustrated in Figure IV.2.2-13. One irradiation tube, labeled IT-3, is also located in the beryllium reflector region, but is somewhat farther from the second target section than are IT-1 and IT-2. This tube provides a high neutron flux and low sample heating, with low fluxes of high-energy neutrons and scattered or secondary protons. These three tubes all have diameters of $5 \mathrm{~cm}$ and are designed for rapid insertion and removal of specimen capsules through use of a pneumatic system. Alternatively, the tube can be used for irradiation of an instrumented in situ experimental assembly. Figure IV.1.2-6 shows the arrangement of the pneumatic access tubes. A fourth, $10-\mathrm{cm}$ diameter, vertically accessed tube (IT-4) is located between neutron beamlines in the iron shielding adjacent to the beryllium reflector, upstream from the target. The upstream position reduces the flux of very-high-energy neutrons and scattered protons. The thermal neutron flux is also lower in this tube.

Neutron fluxes and heat loads have been estimated at several positions in the IPNS Upgrade target assembly through the use of the High Energy Transport Code (HETC) and the low-energy Monte Carlo code for neutron and photon transport (MCNP). The computer model of the target is described in Section IV.2.2.1. Heat deposition was calculated for iron samples in the irradiation tubes. The neutron fluxes calculated for tubes IT- 1 through IT- 4 are given in Table V.4.3-1. The time-averaged available neutron flux, integrated over energies above $0.1 \mathrm{MeV}$, in thimble IT-1 or IT-2 is about $2 \times 10^{13} \mathrm{n} / \mathrm{cm}^{2}-\mathrm{sec}$ for $1 \mathrm{MW}$ beam power.

The heat loads estimated for all tubes are sufficiently small that accurate temperature control can be maintained independent of variations in the proton current. Since experimental control is decoupled from neutron production, a spallation neutron source allows control of irradiation conditions superior to that available at any other neutron source. 

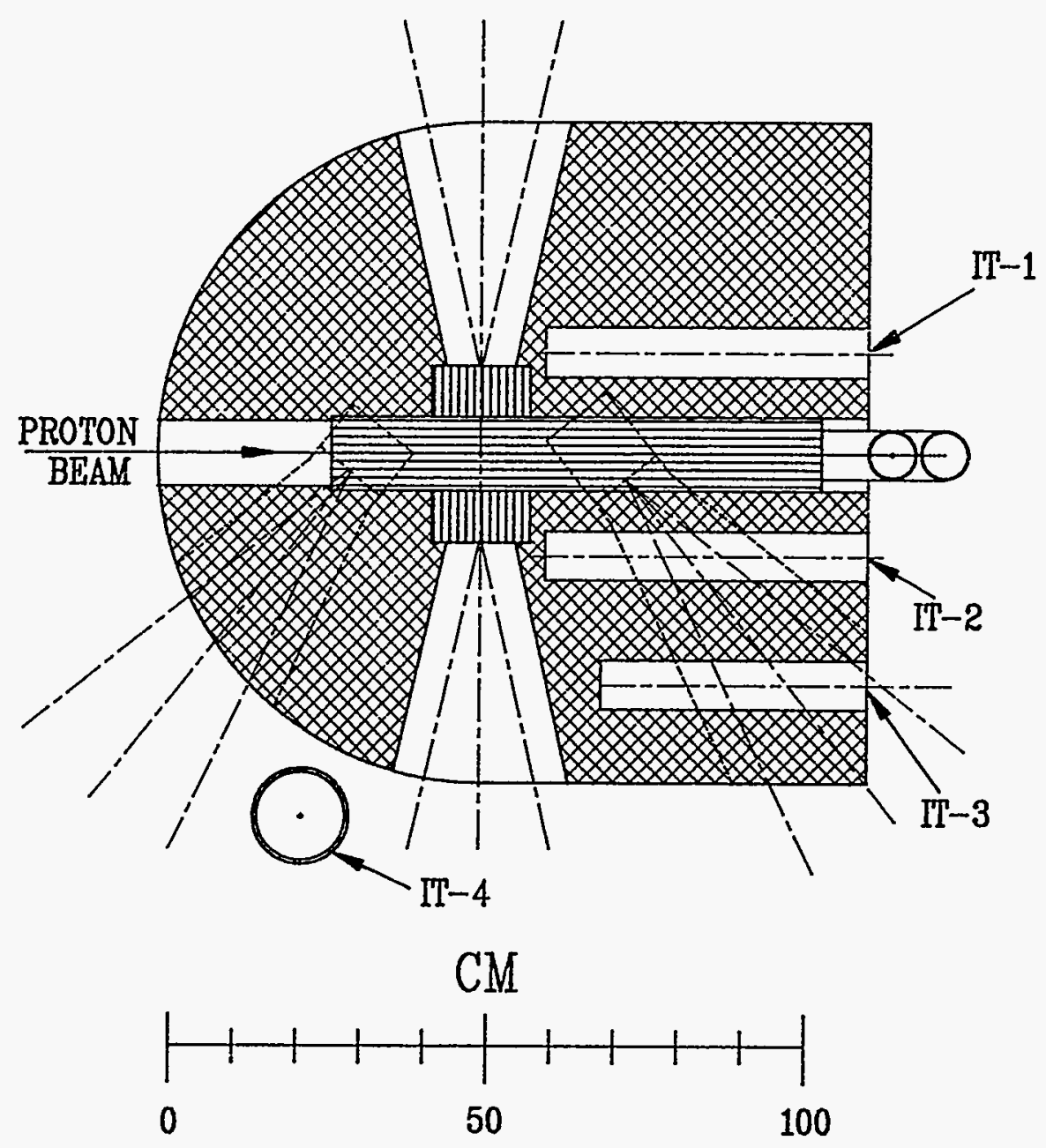

Figure V.4.3-1 Locations of the Irradiation Tubes. 
Table V.4.3-1 Characteristics of IPNS Upgrade Neutron Irradiation Tubes at 1-MW Proton Beam Power

\begin{tabular}{llll}
\hline \multicolumn{1}{c}{ Item } & IT-1 or IT-2 & IT-3 & IT-4 \\
\hline Total neutron flux $\left(10^{12} \mathrm{n} / \mathrm{cm}^{2}-\mathrm{sec}\right)$ & 60.6 & 14.1 & 3.8 \\
Neutron flux $>0.1 \mathrm{MeV}\left(10^{12} \mathrm{n} / \mathrm{cm}^{2}-\mathrm{sec}\right)$ & 17.8 & 2.8 & 1.5 \\
\% neutrons with energy $>20 \mathrm{MeV}$ & 3 & 1.5 & $<1$ \\
Heat load $(\mathrm{W} / \mathrm{g}$ of Fe) & 0.33 & 0.087 & 0.007 \\
Diameter (cm) & 5 & 5 & 10 \\
\hline
\end{tabular}


All irradiation tubes in IPNS Upgrade permit penetration of electrical leads into the irradiation volumes for continuous specimen monitoring or experimental control during in situ experiments. The tubes are vacuum-tight, both for containment of radioactive gases or materials that will be produced during irradiation and for establishment of the desired controlled irradiation conditions. Each irradiation tube has an independent gas-handling system to allow purging before irradiation and removal of radioactive gases after irradiation. Because of the thermal heat load, each tube has an independent flowing-helium-gas cooling system that uses a coaxial piping design. Elevated irradiation temperatures are achieved by resistive-heater inserts and regulation of internal tube pressure.

For uninstrumented irradiations, such as for isotope production or activation analysis, the pneumatic tubes are accessed by pneumatic capsules, which have various sending and receiving stations. One receiving station is inside the hot cell. Radioactive specimens can be unloaded from the capsules and repackaged within the cell. Other unloading stations permit direct transfer of the capsule to small transfer shields or to other appropriate facilities for specialized handling. These other sending and receiving stations provide containment to preclude releases of airborne activity.

The vertical tube (IT-4) is accessed via stepped concrete and steel shield plugs that are slotted to accommodate instrumentation and control conduits. These shield plugs are lifted and set aside by a crane located in the roof-truss region of the building. A shielding cask is available to accommodate the removal of experiments.

\subsection{IRRADIATION SUPPORT FACILITIES}

\subsubsection{Radiation Effects}

In-house facilities external to the irradiation tubes include neutron scattering for atomic structure determination, transmission and scanning electron microscopy (TEM and SEM) for microstructure characterization, and gamma spectroscopy for isotope identification. Mechanical testing of irradiated specimens is performed within the hot cell facility. Local characterization facilities at Argonne include $x$-ray scattering at the Advanced Photon Source (APS) for study of atomic structure and microstructure. In addition, the radiation effects produced by the neutron irradiations at IPNS Upgrade will be studied at the Argonne electron microscopy/ion accelerator facility.

\subsubsection{Isotope Production}

Irradiated samples are loaded into shielded casks within the hot cell. These casks are ready for transport to the site at which the isotopes are to be used. The IPNS Upgrade facility has no additional facilities for preparation, characterization, or certification of the isotopes produced in such samples. 


\subsubsection{Activation Analysis}

The samples irradiated for activation analysis are quickly transported to a shielded room equipped for gamma spectroscopy, where the gamma spectra produced by the induced activation is measured. This transport is by pneumatic capsule or by shielded cask. If a shielded cask is used, the samples are loaded into this cask within the hot cell. The shielded counting room is located nearby in one of the laboratory areas at IPNS Upgrade. 
VI

Conventional Facilities 


\subsection{OVERVIEW}

Argonne National Laboratory (ANL) is sited on 688 hectares (ha) in southern DuPage County, Illinois. The site is $43 \mathrm{~km}$ southwest of downtown Chicago and $38 \mathrm{~km}$ west of Lake Michigan. It is just north of the Des Plaines River valley, immediately south of Interstate Highway I-55, and west of Illinois Highway 83. Argonne's existing campus, as shown in Figure VI.1.1-1, houses several major research facilities, including the existing Intense Pulsed Neutron Source (IPNS) in the 360 area. Buildings associated with the existing IPNS are listed in Figure VI.1.1-2.

The IPNS Upgrade strategy is to reuse existing structures in the 360 area. There are $61,785 \mathrm{~m}^{2}$ of existing facilities in this area that were completed in the early 1960 s to house the 12.5-GeV Zero Gradient Synchrotron (ZGS). The ZGS site accommodates the IPNS Upgrade and has potential for adaptability and future expansion. The IPNS Upgrade reuses $51,370 \mathrm{~m}^{2}$ of the existing space in its operation phase. An additional $3,700 \mathrm{~m}^{2}$ of space is needed for the new linac facility, other buildings, and mechanical system modifications for a total of $55,200 \mathrm{~m}^{2}$. The 360 area contains approximately 26.2 hectares of land with significant existing infrastructure, including $4.26 \mathrm{~km}$ of paved roads and a comparable amount of buried site utilities. The site utility study projects only minor amounts of new utilities and roads needed for the IPNS Upgrade.

The IPNS Upgrade consists of a 400-MeV linac and a 2-GeV Rapid Cycling Synchrotron (RCS), producing a 1-MW neutron source at $30 \mathrm{~Hz}$. There are two target stations. One operates at $30 \mathrm{~Hz}$, and the other operates at $10 \mathrm{~Hz}$. The linac is housed in a new facility that is connected by the Low-Energy Transport (LET) tunnel to the existing ZGS Ring Building (Building 365) where the new RCS is installed. The existing ZGS Ring Building is an annular concrete tunnel with an inner diameter of $22.6 \mathrm{~m}$, an outer diameter of $32.9 \mathrm{~m}$, and an overall height of $12.8 \mathrm{~m}$. Building 369 houses the $10-\mathrm{Hz}$ target, and target support facilities are located in Building 367 . Building 370 houses the $30-\mathrm{Hz}$ target, and its support facilities are housed in Building 371 . The two targets can accommodate 36 instrument lines. Sample preparation laboratories are located in Building 368 adjacent to the target facilities. The existing control room and office areas in Building 360 are used. Building 362 serves as a central laboratory and office facility for staff and users.

The Center Building (Building 364) and Power Supply Building (Building 376) both house mechanical and power systems for the RCS, High-Energy Transport (HET), and the LET. Site Facility 377 contains cooling towers and associated support facilities. Target coolant retention tanks are located in new buried vaults adjacent to the target cooling buildings. The site includes roads, parking, docks, facility support, and staging areas. Available utilities in the 360 area include water systems, cooling towers, power mains, transformer systems, sewers, special scientific services, general infrastructure, and miscellaneous amenities. Existing utility tunnels in the ZGS area are retained. An existing tunnel in Building 370 is relocated to allow construction of the pile foundation for the target biological shield. 


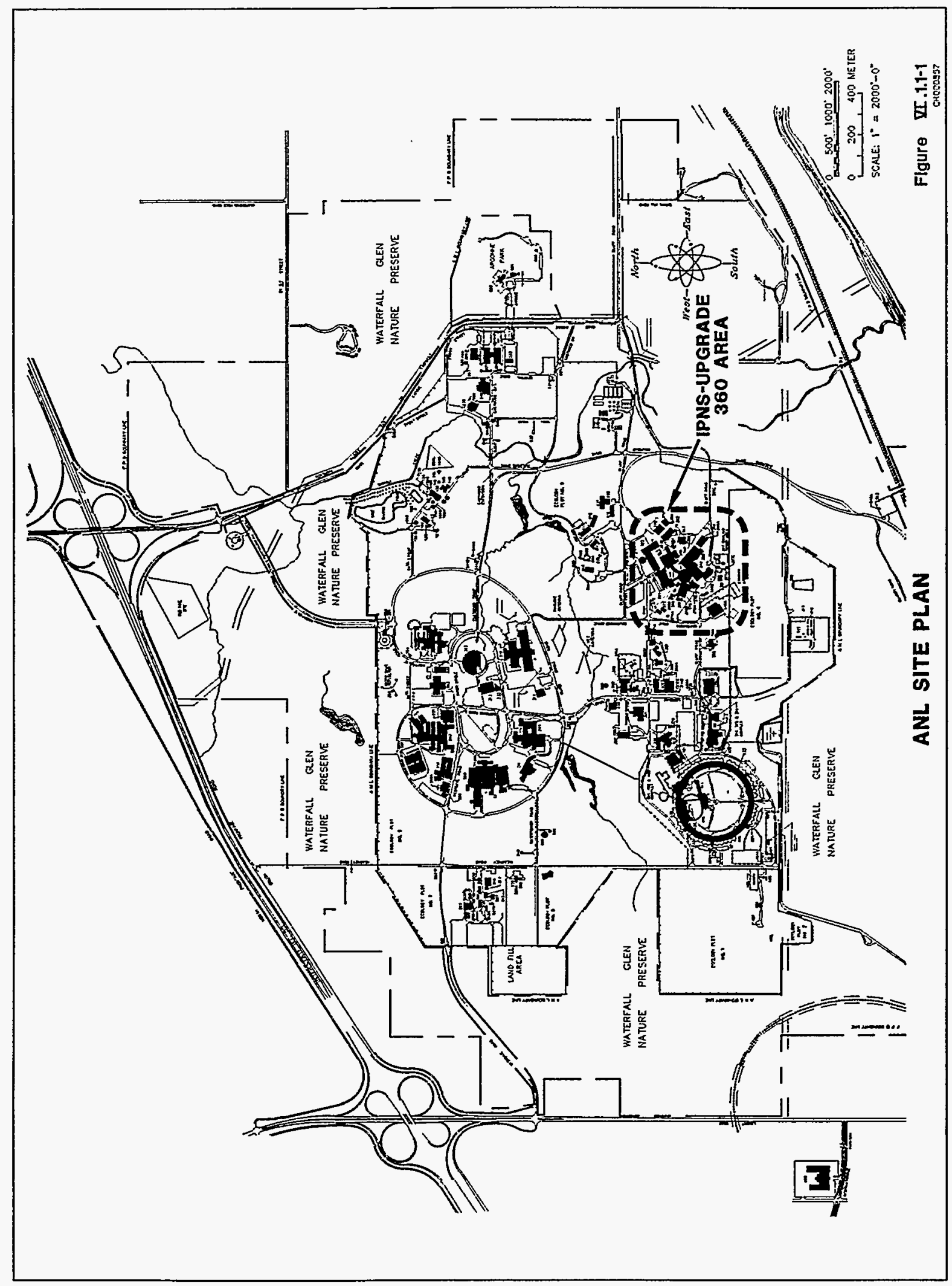




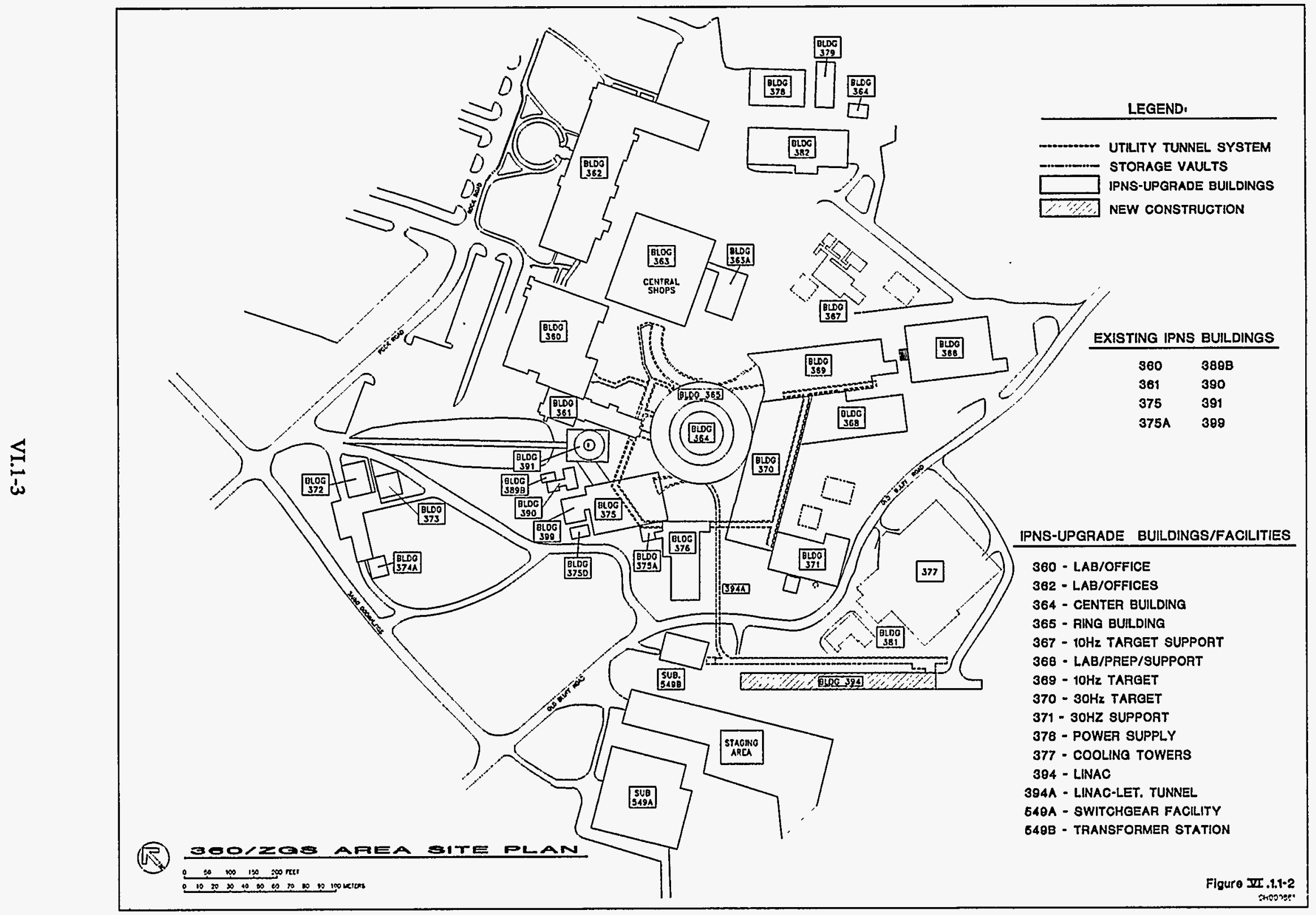


The above-mentioned buildings are shown as hatched areas in Figure VI.1.1-2. The new linac building, Building 394, is also shown in crosshatch in Figure VI.1.1-2. Figure VI.1.1-3 is a photograph of the existing site with an overlay of the new linac and LET tunnel. The IPNS Upgrade conventional facilities are illustrated in Figure VI.1.1-4, the IPNS Upgrade Area Plan.

The adaptive reuse of these existing facilities addresses the usability and operational efficiency of the overall project. Engineering design provisions follow U.S. Department of Energy (DOE) Order 6430.1A, dated April 6, 1989, entitled "General Design Criteria Manual." The following significant criteria are considered in developing the siting, configuration, and building systems selections:

- Existing buildings are large enough to house the proposed technical facility.

- New technical components can be constructed within the existing buildings at reasonable cost.

Building rehabilitation, as described in this report, meets the above two criteria. 


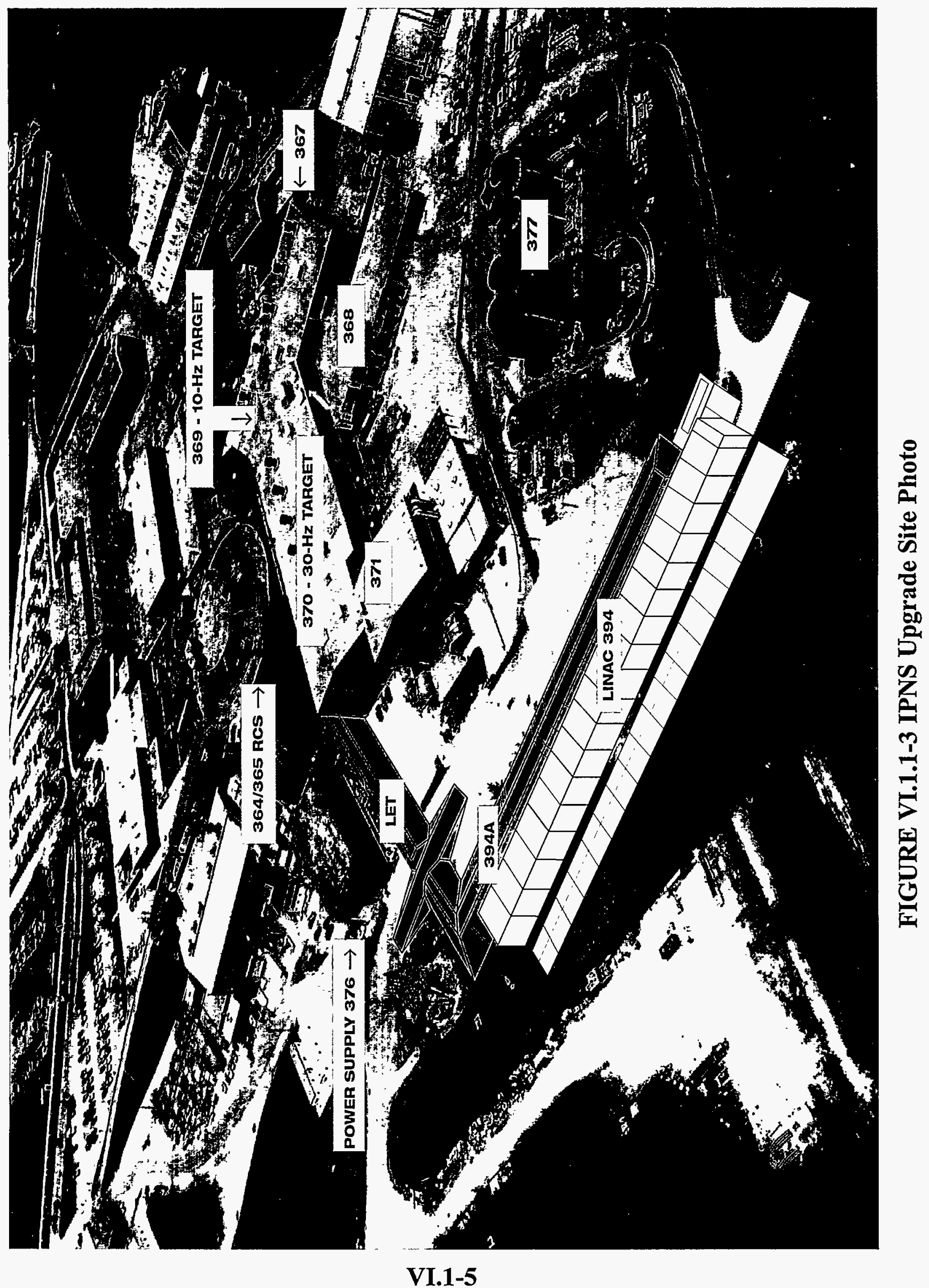




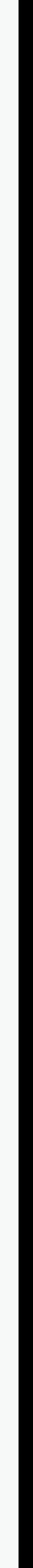




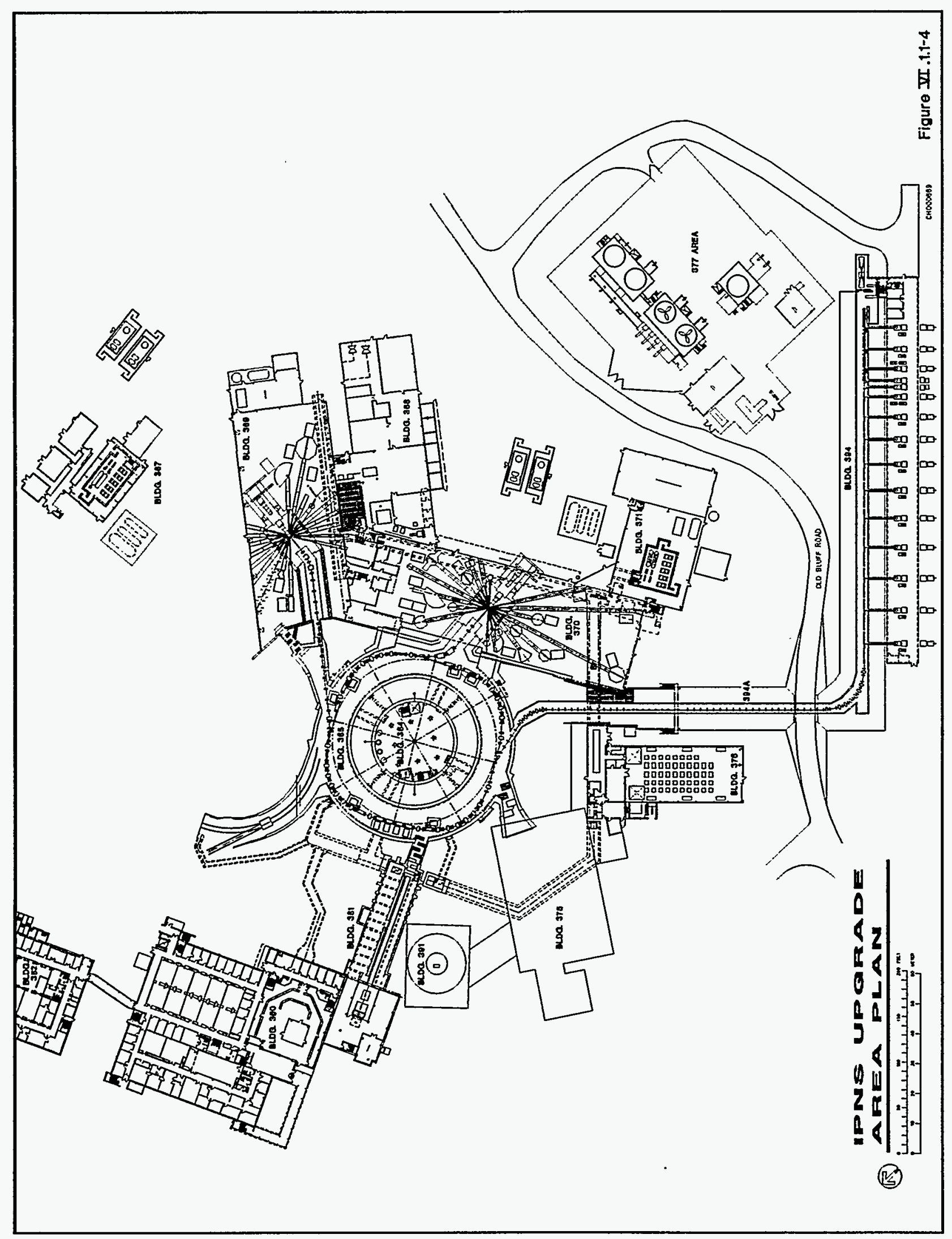




\subsection{APPLICABLE CODES AND STANDARDS}

All systems will be designed to meet the latest editions of the applicable codes, standards, and guides published by the organizations as listed below at the time the Title-II Design is begun. The design described in this report meets current codes, standards, and guides.

\subsubsection{Architectural/Structural Codes and Standards}

- U.S. Department of Energy, DOE 6430.1A, "General Design Criteria Manual"

- International Conference of Building Officials, "Uniform Building Code" (UBC)

- American Concrete Institute (AC), ACI 318, "Building Code Requirements for Reinforced Concrete"

- American National Standards Institute (ANSI)/American Concrete Institute (ACI) standards:

ANSI/ACI 302, "Practice for Concrete Floor and Slab Construction"

ANSI/ACI 531, "Building Code Requirements for Concrete Masonry Structures"

- Prestressed Concrete Institute Standards

- American National Standards Institute (ANSI)/National Bureau of Standards (NBS)

ANSI/NBS 211, "Building Code Requirements for Masonry"

ANSI/NBS Handbook H74, "Building Code Requirements for Reinforced Masonry"

- Structural Clay Products Institute, "Recommended Building Code Requirements for Engineered Brick Masonry"

- National Concrete Masonry Association, "Specifications for the Design and Construction of Load Bearing Concrete Masonry"

- American Institute of Steel Construction, "Specification for Design, Fabrication, and Erection of Structural Steel for Buildings"

- Steel Joist Institute, "Standard Specifications, Load Tables and Weight Tables for Steel Joists and Joist Girders" 
- Steel Deck Institute, "Steel Deck Design Manual for Composite Decks, Form Decks and Roof Decks"

- Steel Deck Institute, "Steel Deck Institute Diaphragm Design Manual"

- American Iron and Steel Institute, "Specifications for the Design of Light Gauge ColdFormed Steel Structural Members"

- American Welding Society (AWS), AWS D1.1, "Structural Welding Code"

- American National Standards Institute (ANSI), ANSI A58.1, "Building Code Requirements for Minimum Design Loads in Buildings and Other Structures"

- Metal Building Manufacturers Association, "Metal Building Systems Manual"

- U.S. Department of the Army, TM-5-809-10, "Seismic Design for Buildings" (lateral force design methodologies in this technical manual can be used effectively for wind design as well as seismic design)

- Building Officials and Code Administrators, International, Inc. (BOCA), "National Building Code"

- National Fire Protection Association (NFPA), NFPA-101, "Life Safety Code"

\subsubsection{Heating, Ventilation, and Air Conditioning (HVAC) Codes and Standards}

- U.S. Department of Energy, DOE 6430.1A, "General Design Criteria Manual," Chapter 5

- American National Standards Institute (ANSI), "Code Requirements"

- American Society of Mechanical Engineers (ASME), "Boiler and Pressure Vessel Code Requirements"

- National Fire Protection Association (NFPA), "National Fire Codes" and Standard 90A, "Air Conditioning and Ventilation Systems"

- International Association of Plumbing and Mechanical Officials, "Uniform Mechanical Code"

- Associated Air Balance Council (AABC)

- Air Moving and Conditioning Association (AMCA) 
- American National Standards Institute (ANSI)

- Air-Conditioning and Refrigeration Institute (ARD)

- American Society of Heating, Refrigerating and Air Conditioning Engineers, Inc. (ASHRAE), "Handbook of Fundamentals" and standards:

90A, "Energy Conservation in New Building Design"

15-1994, "Safety Code for Mechanical Refrigeration"

62-1989, "Ventilation for Acceptable Air Quality"

- Construction Specifications Institute (CSI)

- Cooling Tower Institute (CTI)

- Hydronics Institute (HI)

- National Environmental Balancing Bureau (NEBB)

- National Electric Manufacturers Association (NEMA)

- Sheet Metal and Air Conditioning Contractors National Association, Inc. (SMACNA)

- Underwriters' Laboratories, Inc. (UL)

- American Conference of Government Industrial Hygienists, "Industrial Ventilation Manual"

- General Services Administration/Public Buildings Service (GSA/PBS) Publication, "Energy Conservation Design Guidelines for New Office Buildings"

- Federal Construction Council (FCC), "Federal Construction Guide Specifications"

\subsubsection{Plumbing Codes and Standards}

- U.S. Department of Energy, DOE 6430.1A, "General Design Criteria Manual" and DOE 5480.4, "Environmental Protection, Safety, and Health Protection Standards"

- "National Standard Plumbing Code"

- "Illinois State Plumbing Code" 


\subsubsection{Fire Protection Codes and Standards}

- U.S. Department of Energy, DOE 6430.1A, "General Design Criteria Manual" and DOE 5480.4, "Environmental Protection, Safety, and Health Protection Standards"

- National Fire Protection Association

NFPA 10, "Portable Fire Extinguishers"

NFPA 13, "Installation of Sprinkler Systems"

NFPA 24, "Installation of Private Fire Service Mains and their Appurtenances"

NFPA 101, "Life Safety Code"

\subsubsection{Electrical}

- American National Standards Institute (ANSI)

- American Society for Testing Materials (ASTM)

- Certified Ballast Manufacturer (CBM)

- Electrical Testing Laboratories (ETL)

- Institute of Electrical and Electronics Engineers (IEEE)

- Insulated Power Cable Engineers Association (IPCEA)

- National Electric Code (NEC)

- National Electrical Manufacturers Association (NEMA)

- National Electrical Safety Code (NESC)

- National Fire Protection Association (NFPA)

- Occupational Safety and Health Act (OSHA)

- Underwriters Laboratories, Inc. (UL)

- Joint Industrial Council (JC) 


\subsection{UTILITIES}

Argonne National Laboratory owns and operates the following systems at the ANL-East site:

- Steam generation and distribution

- Natural gas distribution

- Domestic, laboratory, and canal water treatment and distribution

- Collection and treatment of sanitary waste

- Generation and distribution of chilled water

- Electric power distribution

- Telecommunications system.

Vendors and public utilities supply natural gas, oil, coal, electric power, and telephone service to ANL-East.

\subsubsection{Steam}

Most of the steam required by the Laboratory is generated in the Central Heating Plant (CHP) and distributed by an extensive piping network to most of the buildings. Steam is used primarily for central heating.

\subsubsection{Natural Gas}

Natural gas is purchased from the Defense Fuel Supply Center (operated by the Defense Logistics Administration). Northern Illinois Gas Co. delivers gas through its distribution grid to the Laboratory. The Central Heating Plant is the major user of natural gas.

\subsubsection{General Water Supply}

The general water supply of ANL-East is pumped from municipal water systems of adjacent communities and from four ANL wells to the Water Treatment Plant. After softening, the water is pumped into the storage tanks then into the general distribution system, which provides for domestic and laboratory process use and for fire protection. 


\subsubsection{Canal Water}

The canal water system consists of a water-treatment facility, storage tanks, and a distribution system. Nonpotable canal water is used on-site, primarily in cooling towers and, to a lesser degree, for a variety of other cooling needs.

\subsubsection{Storm Sewers}

Roof drains, downspouts, and certain noncontact cooling water discharges are connected to storm sewers at most permanent buildings. Storm sewers are discharged into the nearest suitable outfall point, usually a creek or a well-drained low area.

\subsubsection{Sanitary Sewers}

Sanitary sewage from various buildings is conveyed by underground sewers to the Sanitary Wastewater Treatment Plant (SWTP).

\subsubsection{Chilled Water Systems}

Chilled water for process and comfort cooling is provided by three separate chilled water systems, serving many of the major buildings on-site: (1) the 360-Area Chilled Water System serving the IPNS Upgrade, (2) the APS Chilled Water System, and (3) the Central Chilled Water System.

\subsubsection{Electric Power Distribution}

Electric power for the ANL-East site is purchased from the Commonwealth Edison Company (CECo) at $138 \mathrm{kV}$. The CECo transmission consists of two lines, fed by the Joliet and McCook generating stations and routed along the Atchison, Topeka, and Santa Fe Railroad in the Des Plaines River valley south of the site.

\subsubsection{Telecommunication Systems}

An InteCom IBX S/80 digital private branch exchange (PBX) serves the ANL-East site. The PBX supports standard analog telephones, InteCom-manufactured digital stations for voice, data, or simultaneous voice and data service, and LANmark local area networking. 
This section describes the buildings and facilities that support the various technical components of the IPNS Upgrade, including the linac, RCS, $10-\mathrm{Hz}$ and $30-\mathrm{Hz}$ targets, and experiment instrument lines. Each target station supports 18 neutron beam lines and scattering experiments. The existing structures in the 360 area are all surveyed and verified against existing as-built construction documents. Machine design and component layout are optimized to fit into the existing structures.

The existing buildings in the 360 area are at least 30-years old, but they have been included in ANL ongoing maintenance programs and have undergone various upgrades. All roofs in the 360 area were replaced in 1990 with new Inverted Roof Membrane Assembly (IRMA) systems. Electrical systems were upgraded on a building-by-building basis. Mechanical systems were also refurbished and updated on a per-building basis. Life-safety surveys were completed in 1992. Fire suppression systems and emergency exits were reviewed, and upgrades are in progress. Air conditioning refurbishing and replacement is on a per-building basis. Existing utility/control tunnels are in satisfactory condition.

Radiation-safe entrances to the linac tunnel, transfer line tunnel, and to the RCS are provided by shielded mazes. Access to the RCS is provided through a curved tunnel in Building 363 and through labyrinths in Buildings $361,364,369,375$, and 394A.

\subsection{LINAC BUILDING (BUILDINGS 394 AND 394A)}

The Linac Building is the only new building for the IPNS Upgrade. All other facilities are the adapted reuse of existing ZGS area buildings and facilities with minor modifications.

The new Linac Building, Building 394, is located in the southwest sector of the ZGS campus, southwest of existing Building 376 and parallel to Old Bluff Road, as shown in Figure VI.2.1-1.

Linac tunnel construction utilizes a "cut and cover" method that was successfully used at Fermilab. The top of the linac tunnel enclosure is at grade (Elevation $711.0 \mathrm{ft}$ ), with the tunnel floor $3.04 \mathrm{~m}$ below grade (Elevation $701.0 \mathrm{ft}$ ). Earth shielding is added to the grade above the tunnel, creating a tapered berm $3.20 \mathrm{~m}$ high. The linac tunnel cross section is $3.66 \mathrm{~m}$ wide $\times$ $2.74 \mathrm{~m}$ high and the LET tunnel is $3.04 \mathrm{~m}$ wide $\times 2.74 \mathrm{~m}$ high, as shown in Figures VI.2.1-2 and VI.2.1-3. The linac tunnel is $154.8 \mathrm{~m}$ long, and the transfer tunnel to the RCS is $118.9 \mathrm{~m}$ long. Tunnel segments are precast-concrete, inverted-U-shaped elements $2.44 \mathrm{~m}$ in length with 0.3-m-thick walls and roof, which are erected on a cast-in-place 0.6 -m-thick concrete slab that has perimeter drains.

Linac construction generates minimal conflicts with existing site utilities. The two 475-mm-diameter main chiller lines are lowered by $1 \mathrm{~m}$ to pass under the LET tunnel (394A) and avoid interference, as shown in Figure VI.2.1-2, Section A. Existing Old Bluff Road crosses over 


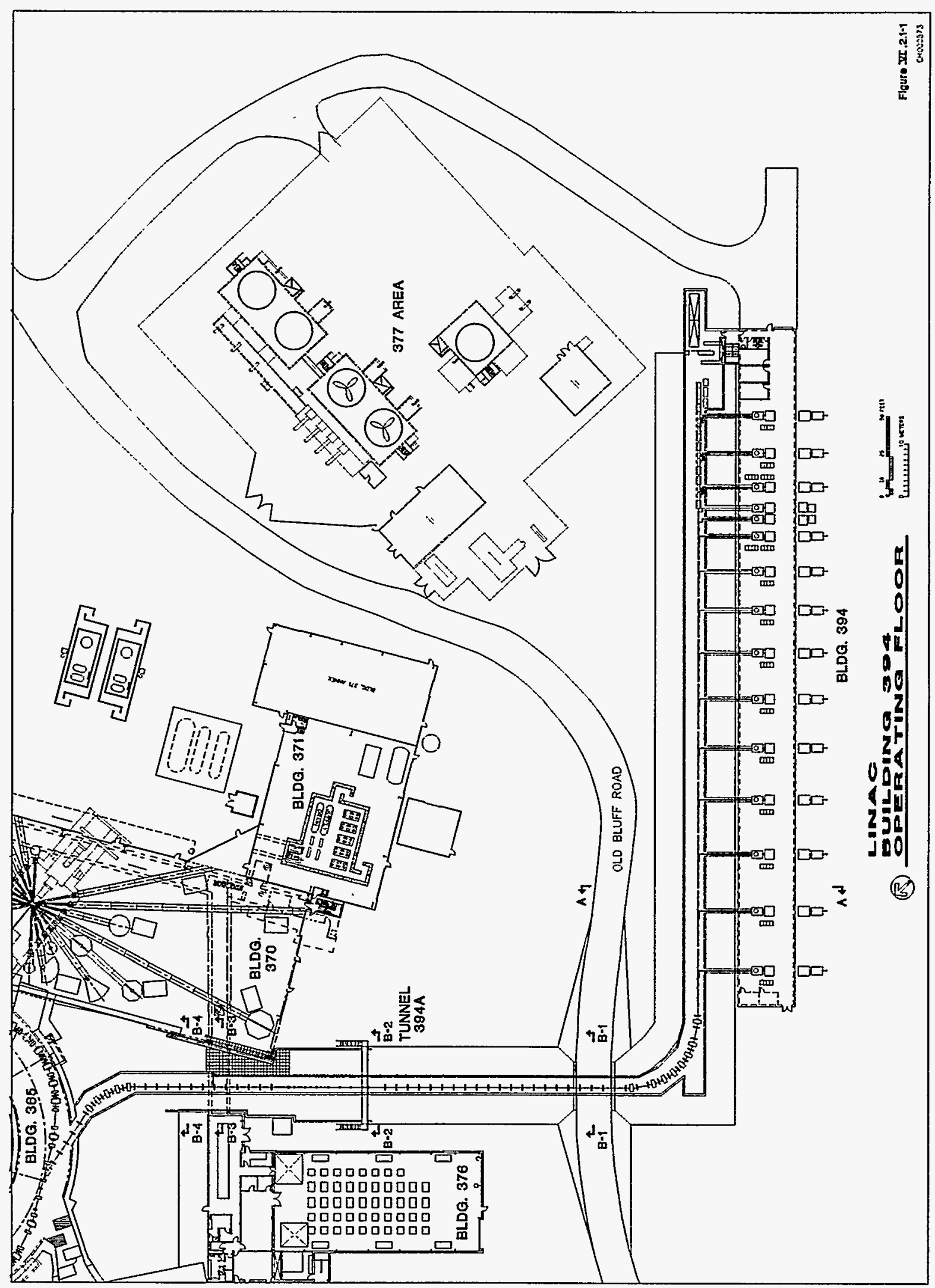




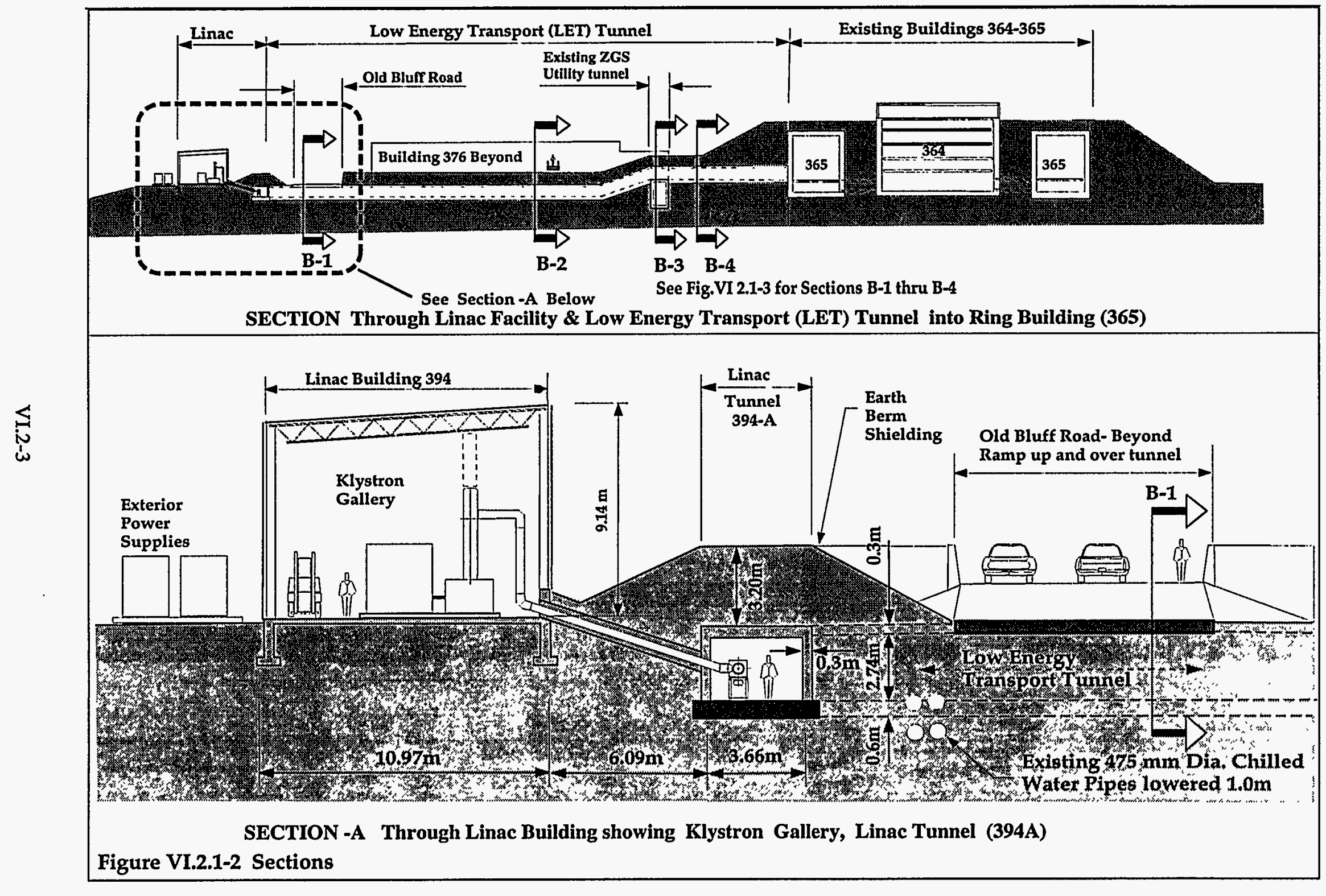




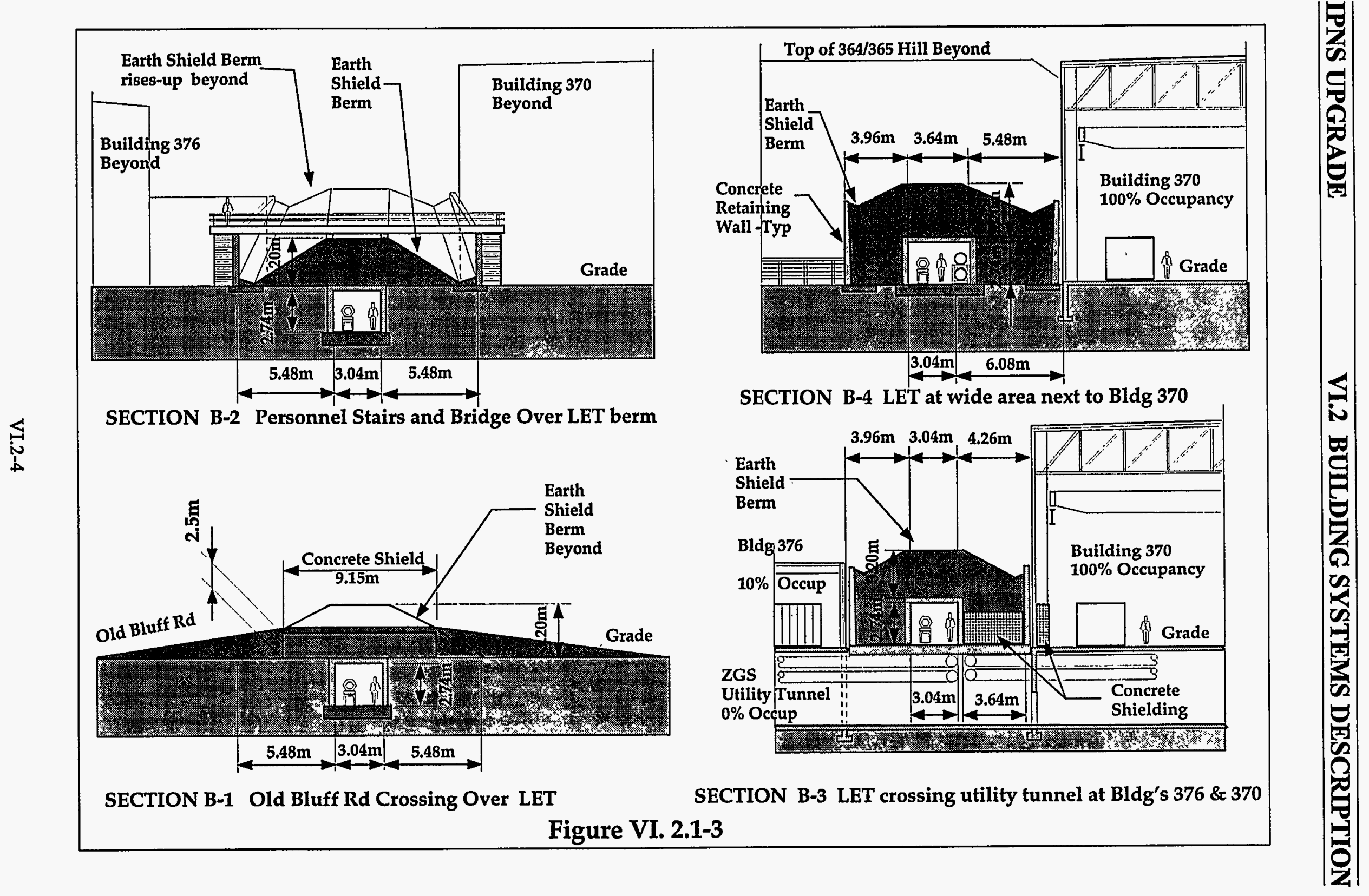


the top of the LET tunnel southwest of Building 376. The LET tunnel roof is thickened and reinforced to carry vehicle loads and to provide the required shielding at the roadway, as shown in Figure VI.2.1-3, Section B-1.

The klystron gallery in the Linac Building (Building 394) is $10.97 \mathrm{~m}$ wide $\times 9.14 \mathrm{~m}$ high $\times$ $129.5 \mathrm{~m}$ long and has a gross floor area of $2,509 \mathrm{~m}^{2}$. It houses the 15 klystrons and their pulse modulators. Other associated electrical equipment and power supplies are located on 15 concrete slabs outside of, but next to, the exterior west wall. There are three $3.04 \mathrm{~m} \times 4.88 \mathrm{~m}$ rooms, two $2.0 \mathrm{~m} \times 2.0 \mathrm{~m}$ handicapped-accessible toilets, and a janitor room, located inside the building at the south end. Space over this area has a floor for mechanical equipment and is accessed by adjacent stairs. These stairs also allow access to the linac tunnel (394A), which is below grade. The end of the linac tunnel has a $2 \mathrm{~m} \times 12 \mathrm{~m}$ access hatch to allow installation of linac components.

\subsubsection{Architecture (Buildings 394 and 394A)}

The interior of the Linac Building is a single open space to house technical components along the length of the linac. The building can be entered through personnel doors at either the north or the south end. The south end has vehicle access for movement of major components into klystron and tunnel areas. Conduit, piping, duct-work and the exposed underside of the roof deck are unpainted. The floors are exposed, surface-hardened concrete.

Partitions are gypsum drywall on metal studs with painted finish. The south end contains office, shop, toilets, and technical-support rooms. The area above these rooms has a floor for mechanical equipment and is accessible by means of a stairway. Only these rooms have suspended acoustical tile ceilings, with recessed lighting and HVAC diffusers, concealing the electrical and mechanical distribution systems.

The Linac Building has 9.14-m-high, windowless facades with minimal architectural elements and is clad with 0.1-m-thick insulated metal building panels having a rating of R-19+. The vertical insulated metal panels are coated with a durable epoxy-type finish. Louvers and other accessories match the finish of the wall-panel system.

The roof has a side slope with roof scuppers and downspouts discharging at grade. The IRMA roof system meets existing Argonne standards. Insulated metal exterior doors are used for personnel access. Vehicle access is provided by a sectional overhead door of insulated metal panels.

\subsubsection{Structure (Buildings 394 and 394A)}

The structural system for the Linac Building consists of a steel beam-and-girder roof system supporting a welded-steel deck with wide-flange columns on spread footings. The frame has horizontal steel girts, which support vertical insulated-metal wall panels. A 0.2 -m-thick 
reinforced-concrete floor slab rests on $0.15-\mathrm{m}$-thick compacted granular backfill. The roof has a steel deck with an IRMA system, which consists of a single-ply roof membrane, protection board, and 7-cm-thick rigid foam insulation bonded to 1-cm-thick cement exterior board.

There are $273.7 \mathrm{~m}$ of tunnel associated with the linac and the LET. Basic tunnel construction is of precast concrete elements erected on a concrete base slab. The base slab is $0.6 \mathrm{~m}$ thick and runs the continuous length of the tunnel. Perimeter drains are located at the bottom edge of each side. There are weld plates embedded in the bottom exterior edge of the precast elements and cast-in-place base slab. The precast elements are joined every $2.44 \mathrm{~m}$. The tunnel is covered with a multicomponent waterproofing system that consists of a $25-\mathrm{mm}$ diameter backer rod with bentonite sealant, multi-ply exterior sheet overlaid with a 3-mm-thick protection board. The tunnel is provided with a $0.3-\mathrm{m}$-thick layer of gravel backfill to allow water to flow around it to the continuous perimeter drains, which are connected to ejector pumps.

Unistrut channels are cast into the tunnel segments for mounting mechanical and technical components. The west wall of the linac tunnel has 15 openings for waveguide connections, as shown in Figures VI.2.1-1 and VI.2.1-2, Section A.

The top of the LET tunnel under the roadway is thickened and reinforced for shielding and vehicle support. The LET tunnel rises vertically and crosses over the existing main utility tunnel between Buildings 376 and 370, as shown in Figure VI.2.1-2. Shielding configurations in this area allow maximum exterior access to the existing facilities and provide for proper shielding performance. Concrete is used in the constricted locations between Buildings 376 and 370 , as shown in Figures VI.2.1-1 and VI.2.1-3, Section B-3.

\subsubsection{Mechanical Systems (Buildings 394 and 394A)}

Heating, Ventilating, and Air Conditioning (HVAC). The klystron gallery is provided with one 30,000-cfm (cubic feet per minute) air-handling unit (AHU), with chilled-water cooling and steam-heating coils and return/exhaust fans. The temperature during summer does not exceed $32^{\circ} \mathrm{C}$. In winter, 12 steam unit heaters with integral thermostats maintain the building to at least $16^{\circ} \mathrm{C}$.

The linac and LET tunnels are furnished with $100 \%$ outside air. The AHU is located in the enclosure at ground level, near the intersection of the linac and LET tunnels. Capacity of the unit is approximately $20,000 \mathrm{cfm}$, and it contains chilled-water cooling and steam-heating coils. The tunnel conditions are maintained such that temperatures do not exceed $32^{\circ} \mathrm{C}$ in summer and do not fall below $16^{\circ} \mathrm{C}$ in winter. There are two exhaust fans located at the northernmost end of the LET tunnel and the southernmost end of the linac. 
The Linac Building has fully automated direct digital controls (DDCs) for control of HVAC equipment. Winter humidification is provided; however, no summer dehumidification is provided.

Plumbing, Process Piping, and Fire Protection. Plumbing systems for the Linac Building and the LET consist of floor drains, domestic cold water, and sanitary waste and vent systems. Process piping systems supply compressed air and process water at $32^{\circ} \mathrm{C}$ as required for specific equipment and functions. Fire protection consists of a wet-pipe automatic-sprinkler system. Hand-operated fire extinguishers are located as required near electronic components. Two chilled-water pipes are routed from the utility tunnel through the LET tunnel, to the service floor of Building 365, as shown in Figure VI.2.1-3, Section B4.

\subsubsection{Electrical Systems (Buildings 394 and 394A)}

Electrical power is distributed at $480 \mathrm{~V}$ from substations west of the Linac Building. Electrical power to injector system components is supplied from three 480-V substations, as shown in Figure VI.2.1-4. These substations are located outside the Linac Building. Each substation consists of a 13.2-kV dual-primary-switch, 2000-kVA transformer, and associated $480-\mathrm{V}$ switchgear. The 480-V switchgear feeds power distribution panels located inside the Linac Building. Power at $13.2 \mathrm{kV}$ is supplied from Site Electrical Facility 549.

The linac and LET tunnels are lighted with two-lamp, surface-mounted fluorescent fixtures. Illuminated emergency exit signs are installed. Emergency lighting is provided by $1.5-\mathrm{h}$ battery-powered units with built-in chargers. The klystron gallery is lighted with high-bay, metal-halide fixtures to an average of 30 foot-candles of illumination. Convenience receptacles and power and welding receptacles are provided at various locations.

A motor control center is provided to feed power to building mechanical equipment and other installed motors rated at $0.5 \mathrm{hp}$ and above.

An equipment grounding system is provided. Each piece of equipment is connected to grounding conductors that run together with all power feeders. Lightning protection is provided by copper rods mounted around the roof perimeter. These rods are connected by copper cables Cadwelded to the building structural steel. The building ground system uses bare copper Cadwelded to the building structural steel, which is connected to ground rods spaced around the building. In addition, all computer and related equipment is provided with a separate quiet grounding system that is isolated from the other grounding systems and is connected to a separate counterpoise and ground rod system. 


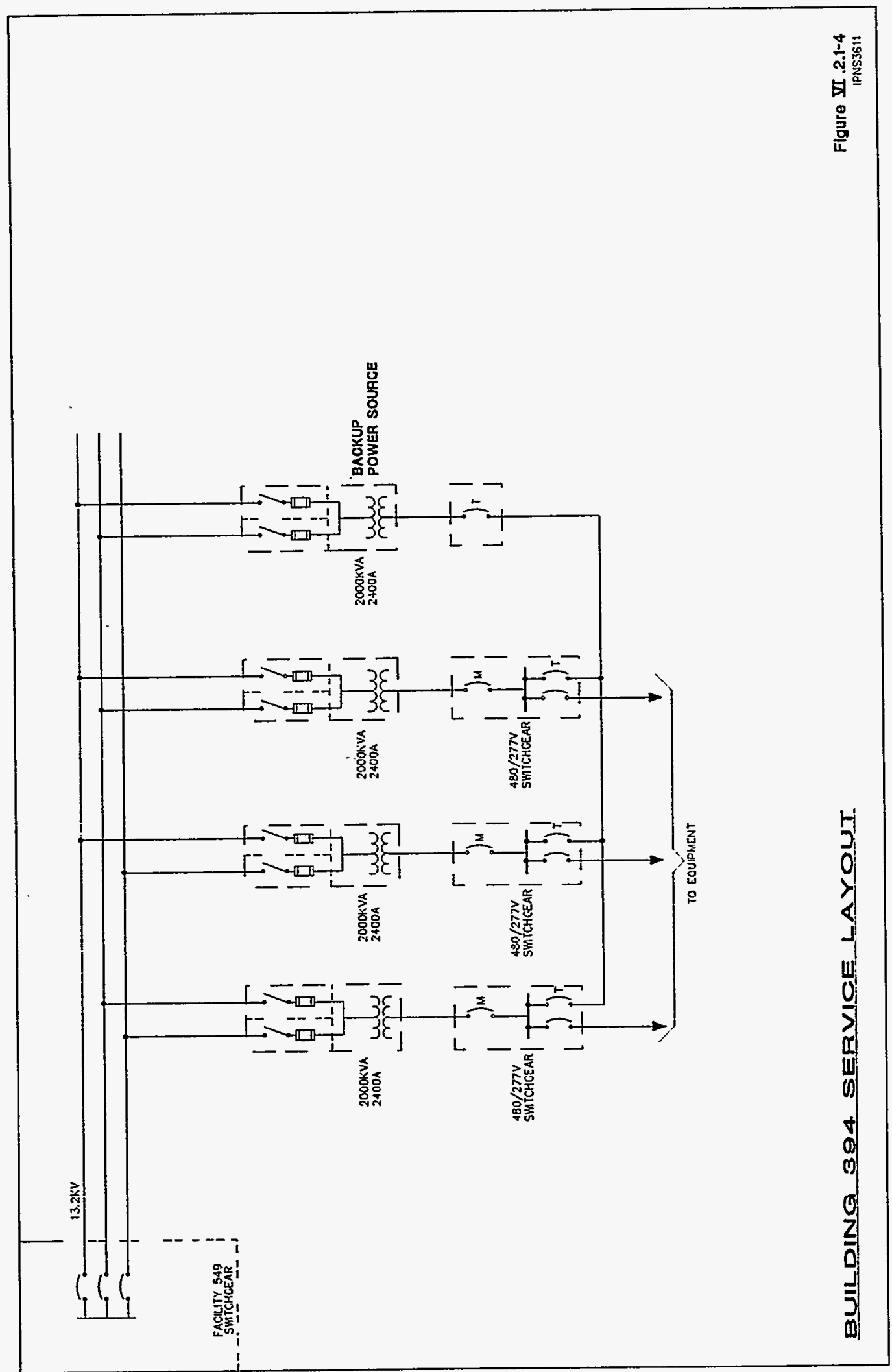


The fire alarm system consists of manual pull stations located at building exits and flow and tamper switches in the sprinkler system. Smoke and heat detectors are installed throughout the building and in the HVAC system. The alarm system is annunciated at the Argonne Fire Station and in the IPNS Upgrade main control room.

Telecommunications are from a branch from the PBX in Building 360, with a telephone terminal in the Linac Building. Telephone communications equipment includes a new terminal cabinet, telephone outlets, and telephone cables. The paging system has new speakers, conduit, and wires and is tied to the existing system.

Clean power requirements are met by the use of $480-\mathrm{V} / 480-\mathrm{V}$ isolation transformers. Emergency power is provided from an existing diesel generator rated at $250 \mathrm{kVA}$ located near Building 376.

\subsection{RING BUILDING (EXISTING BUILDING 365)}

Building 365 was completed in 1962 to house the $12.5-\mathrm{GeV}$ Zero Gradient Synchrotron. It has two floor levels and a gross floor area of $7,014 \mathrm{~m}^{2}$. The upper level is the operating floor for the RCS, and there is a service floor below.

This building houses the IPNS Upgrade RCS, and it is totally surrounded by an existing earth berm. The Ring Building is donut-shaped, with an interior radius of $22.6 \mathrm{~m}$ and an outer radius of $32.9 \mathrm{~m}$, and with an overall height of $12.8 \mathrm{~m}$. It encircles the cylindrical-shaped Center Building 364. There are $7 \mathrm{~m}$ of earth shielding between these buildings. Building 365 is connected to Buildings $361,363,364,369,370,375$, and 394A. Figure VI.2.2-1 is a vertical cross section of the Ring Building and Center Building showing the original ZGS. Figure VI.2.2-2 is a plan view of the Ring Building operating floor and includes the RCS layout.

\subsubsection{Architecture (Building 365)}

This building is a two-level reinforced-concrete circular tunnel, with a clear floor width of $10.3 \mathrm{~m}$, a floor-to-ceiling height of $9.9 \mathrm{~m}$ for the upper level, a floor-to-ceiling height of $2.9 \mathrm{~m}$ in the lower level, and a typical concrete wall thickness of $1.07 \mathrm{~m}$. Personnel access to the Ring Building is provided through shielded labyrinths from Buildings $361,364,369,375,394 \mathrm{~A}$, and the utility tunnel system. Equipment access is provided through a tunnel from Building 363 having a rail-mounted, movable shield assembly. The lower-level service floor of Building 365 houses support equipment for the RCS. Four access stairways connect the operating floor and service floor.

Building modifications include new floor construction at the operating level, and stairway and life-safety exit relocation. 


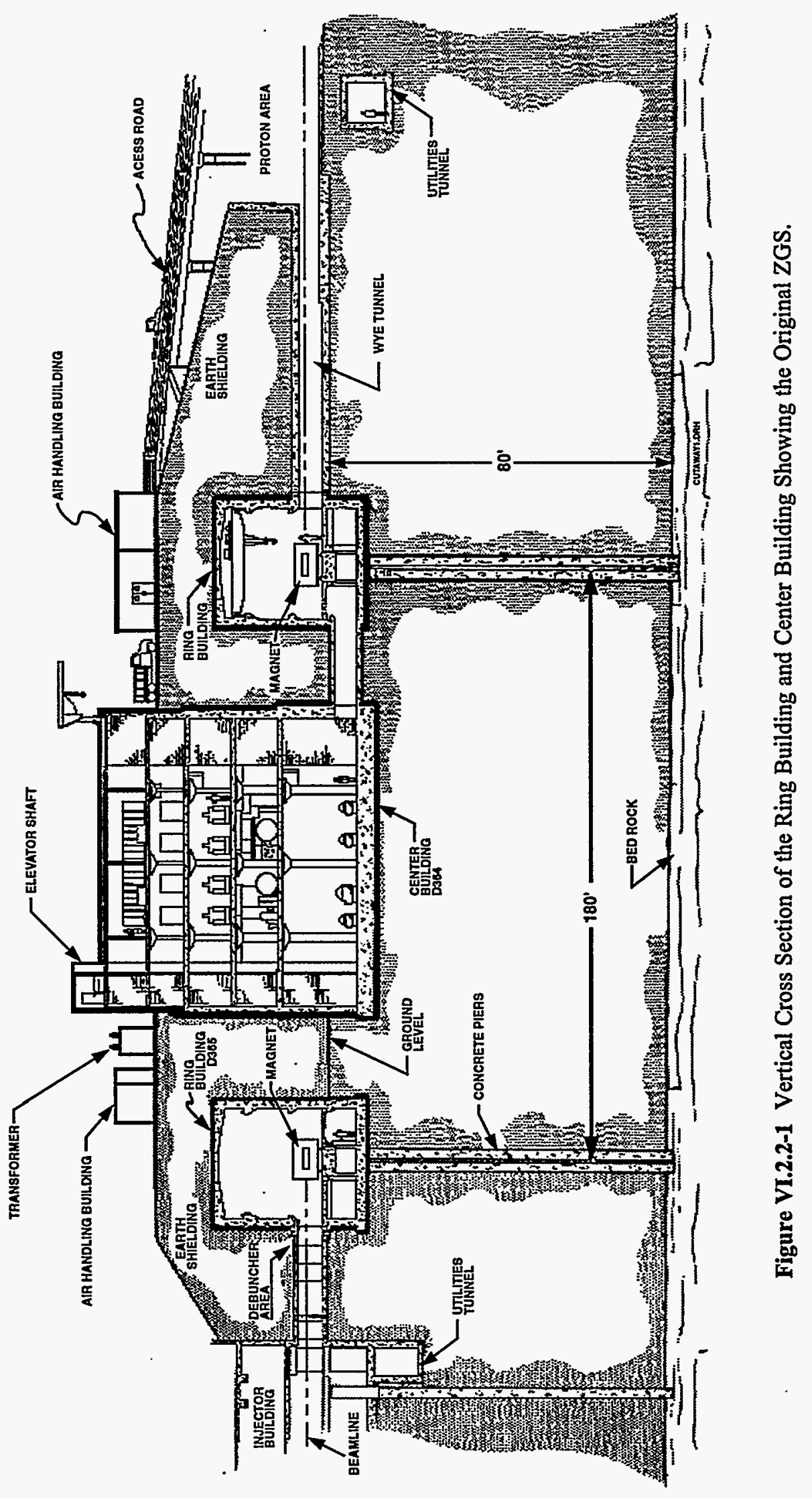




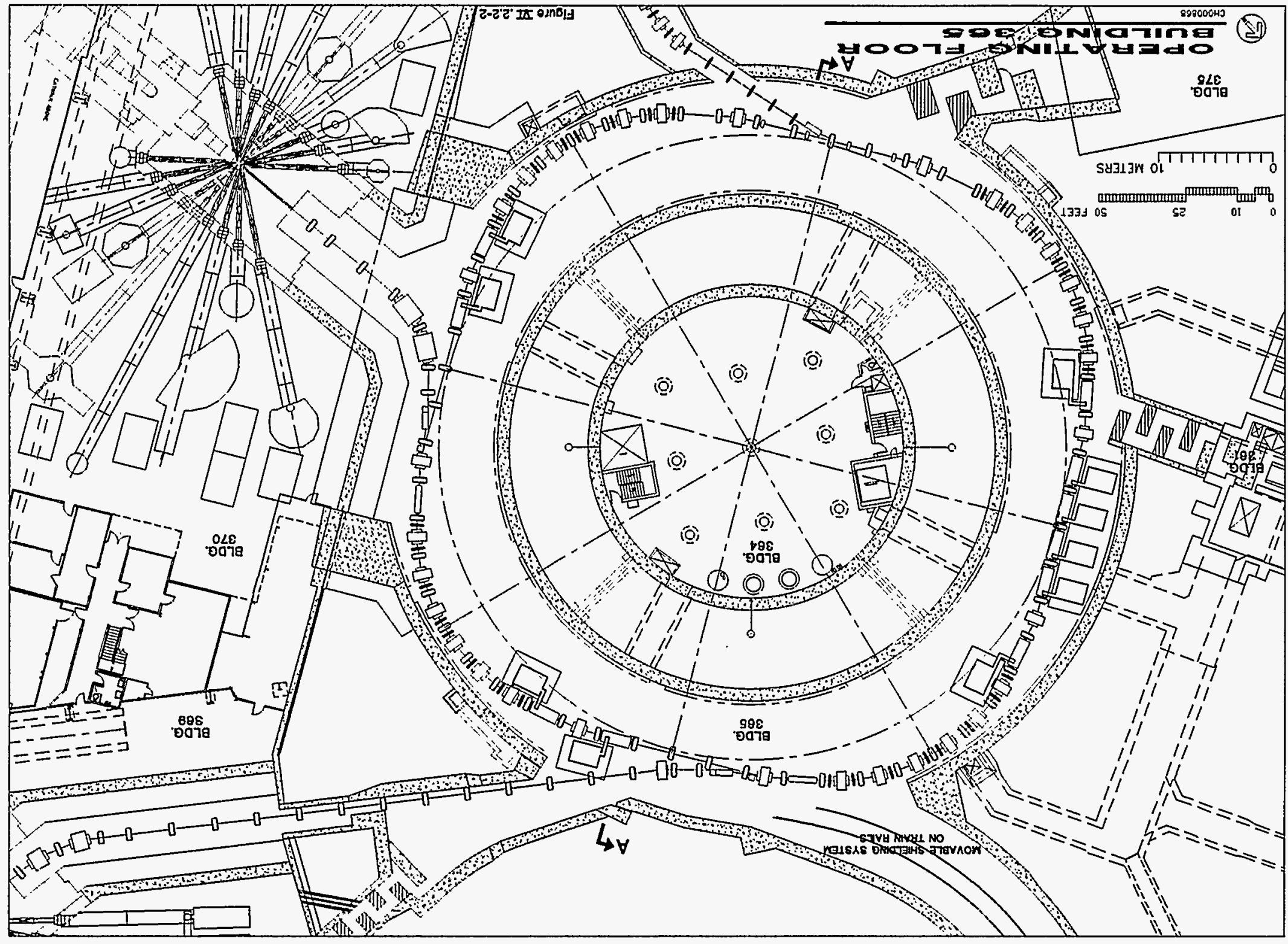




\subsubsection{Structure (Building 365)}

The building has eight 1-m-thick equipment piers supported on 24 concrete caissons that extend $24 \mathrm{~m}$ down to bedrock. The operating floor structural system ties into these pier supports, concrete columns, and outer walls. A new 0.3-m-thick concrete floor is constructed on a steel and girder subframe connected to the existing concrete substructure. The floor load capacity is $1000 \mathrm{lb} / \mathrm{ft}^{2}$.

The outer and inner walls are poured-in-place reinforced concrete and are $1.07 \mathrm{~m}$ thick. The service level has a 1.07-m-thick reinforced concrete floor, with a floor load capacity of $2000 \mathrm{lb} / \mathrm{ft}^{2}$.

Cranes and Hoists. Three overhead bridge cranes span the 10.3- $\mathrm{m}$ width and are capable of circling the tunnel in either direction. All three bridge cranes have a 20-ton capacity, and one crane also has a 2-ton auxiliary hoist. These cranes run on continuous circular rails that are supported by cast-in-place concrete wall brackets $7.5 \mathrm{~m}$ above the floor.

\subsubsection{Mechanical Systems (Building 365)}

Central Plant and Piping. No central cooling plant equipment, such as chillers, and no deionized water (DIW) plant equipment is located in the building. Chilled water and steam to the exterior perimeter AHUs are piped from Building 364. Deteriorated underground chilled-water and steam supply-and-return piping to exterior AHUs is replaced as required.

Air Distribution. Three existing, exterior 25,000-cfm AHUs are refurbished. Coils, motors, bearings, filters, and controls are replaced. New variable frequency drives (VFDs) are installed. Housing and fans are reused. A new fourth unit of similar capacity and configuration is installed on an available pad. The pad supports are also refurbished. The final total capacity is approximately $100,000 \mathrm{cfm}$.

Three of the AHUs serve the circular duct that runs at the underside of the ceiling slab. The fourth unit supplies air to the two HET tunnels, one of which connects Building 365 to 370 and the other of which connects Building 365 to 369 . This double-wall ductwork runs outside and is insulated. The air is returned from Building 365 at the lower floor through the four existing return registers and air shafts as shown in Figure VI.2.2-3.

Existing circular ductwork is cleaned and utilized. Drainage traps are installed at points of main-duct tie-ins into the circular ductwork. Supply duct circular nozzles are modified to provide proper air distribution within the ring.

Existing unit heaters at the building entrance remain. 


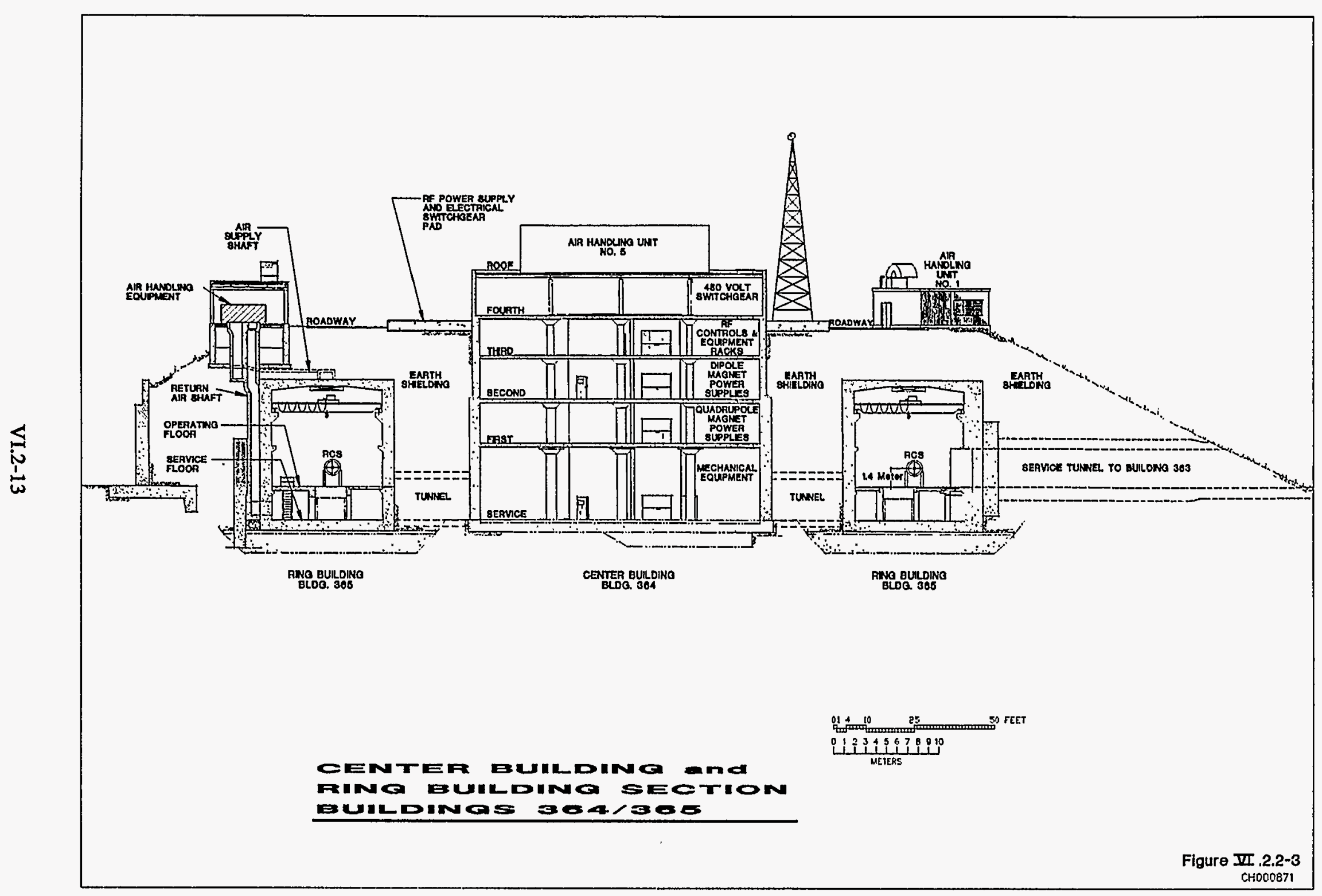


Plumbing and Fire Protection. Existing building facilities and plumbing systems are used. Floor drains are provided at each of the concrete return-air shafts. New piping connects these floor drains to the existing building sewers. equipment.

Existing fire protection systems are upgraded as necessary to provide coverage for new

\subsubsection{Electrical Systems (Building 365)}

Electrical power to the RCS is supplied from Site Facility 549 by five 13.2-kV lines. Four of these lines feed 2,500-kVA, 480-V transformers and switchgear. Each of the 480-V switchgear units can be energized from either of two different $13.2-\mathrm{kV}$ lines by using separate $15-\mathrm{kV}$ switches. The secondary of the transformer is connected via bus duct to indoor $480-\mathrm{V}$ switchgear located on the fourth floor. A one-line diagram is shown in Figure VI.2.2-4. A sixth 2,500-kVA, 480-V transformer provides backup power service. The backup power source is brought into service by disconnecting the $15-\mathrm{kV}$ switchgear of the damaged transformer and toggling the 480-V switch from $M$ to $T$, as shown in Figure VI.2.2-4. Power to the rf equipment is supplied by a fifth $13.2-\mathrm{kV}$ line. This is done to minimize interactions between the if amplifiers and the rest of the equipment.

The RCS components in Building 365 receive their ac power from 480-V switchgear located on the fourth floor of the Center Building 364. There are 480-V power-distribution panel boards located near the equipment.

Cleaned, repaired, and relamped existing lighting fixtures provide adequate lighting. Lighting fixture circuit wiring is new to improve reliability. New breakers are provided for existing power and lighting panel board interiors.

Telephone, paging, and fire alarm systems are used with only minor modifications.

New illuminated emergency exit signs are installed. Additional emergency lighting is provided by 1.5 - $\mathrm{h}$ battery-powered units with built-in chargers. 


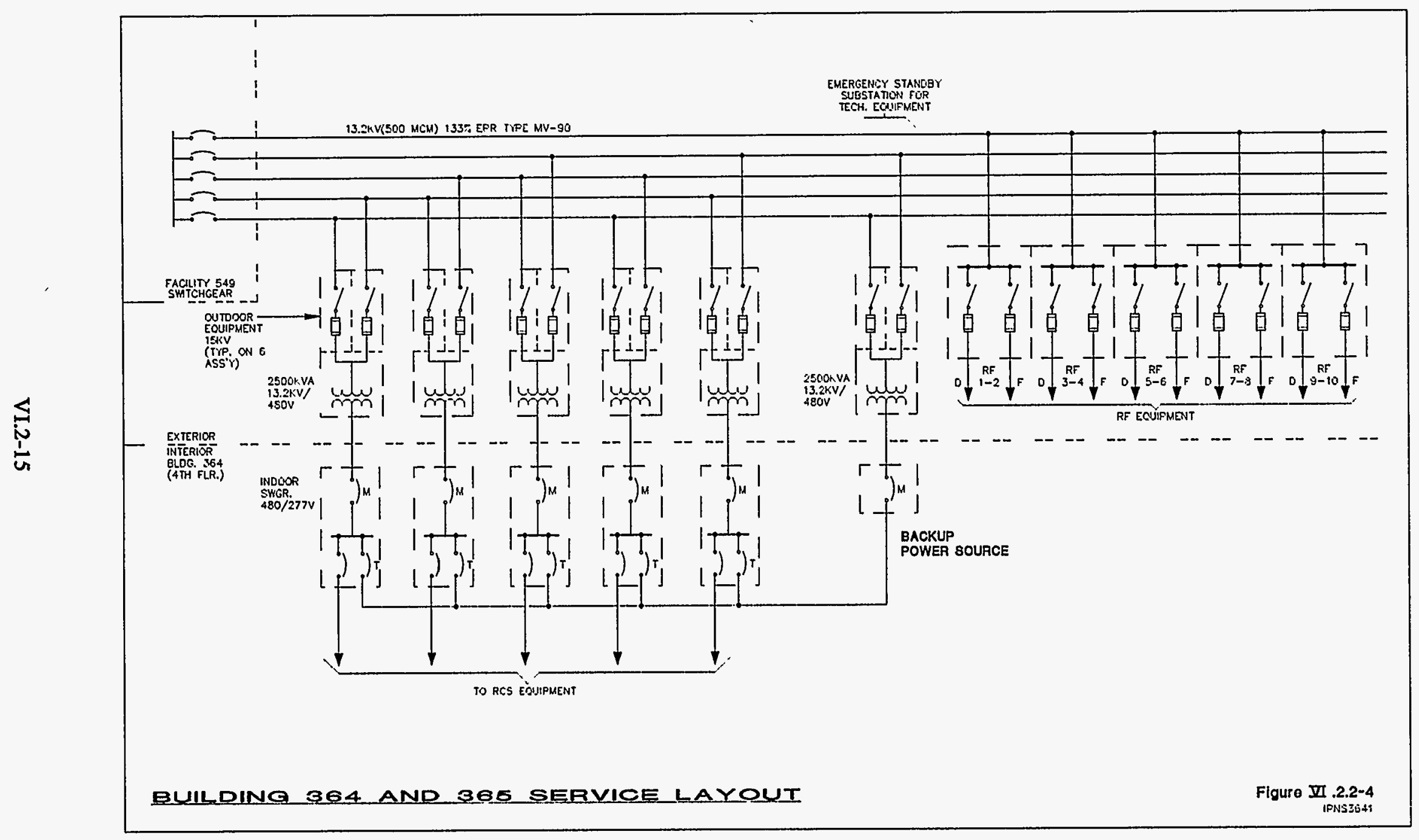




\subsection{CENTER BUILDING (EXISTING BUILDING 364)}

The Center Building (Building 364) is a five-story cylindrical building, located inside the Ring Building. The plan view of Buildings 364 and 365 is shown in Figure VI.2.2-2, and a cross section through them is shown in Figure VI.2.2-3. This building was completed in 1962 to house equipment for the ZGS located in Building 365 . Building 364 has a gross floor area of $3,196 \mathrm{~m}^{2}$. The main mechanical distribution center for the ZGS area is located in this facility.

The function of this facility is similar to its original use. The service floor continues to provide the ZGS area mechanical systems support, with water chillers and a deionized-water plant. The first floor houses quadrupole magnet power supplies, and the second floor houses the dipole magnet power supply system for the RCS. The third floor houses controls and equipment for the rf amplifiers located in the Ring Building. The fourth floor contains electrical switchgear supplying power to all floors and also to Building 365 . The dc power supplies and transformers for the if amplifiers are located on the fourth-floor roadway level of the Center Building. The ring road around the fourth floor provides access to $\mathrm{rf}$ power supplies and electrical switchgear. A new air-handling unit located on the roof of the Center Building supplies conditioned air to all floors.

\subsubsection{Architecture (Building 364)}

The lower four floors of this five-story, concrete, cylindrical building are surrounded by earth shielding. This shield consists of $7 \mathrm{~m}$ of earth and a 1.07-m-thick reinforced concrete wall between the Ring Building (Building 365) and the Center Building (Building 364). There is an access/ring road at the building's fourth-floor level, as shown in Figure VI.2.2-3. There is a toilet room on each of the five floors. Vertical transportation with access to all levels is provided by a 2-ton capacity service elevator and two stairways.

Service Floor. This floor plan is circularly shaped, with an inside diameter of $26.8 \mathrm{~m}$ and a ceiling height of $7 \mathrm{~m}$. The service floor is below the Ring Building operating floor. Mechanical equipment and piping for the 360 area are located on this floor, including three existing chillers and a new water-deionization plant. Three mechanical service tunnels and one labyrinth pedestrian tunnel connect this floor to the Ring Building (365) service floor. Figure VI.2.3-1 shows the service tunnel access and locations of chillers and deionization equipment on the service floor.

First Floor. This floor plan is circularly shaped, with an inside diameter of $26.8 \mathrm{~m}$ and a ceiling height of $3.96 \mathrm{~m}$. There are 14 power supplies for the RCS, seven each for the focusing and defocusing quadrupole magnets located on this floor, as shown in Figure VI.2.3-2.

Second Floor. This floor plan is circularly shaped, with an inside diameter of $26.8 \mathrm{~m}$ and a ceiling height of $3.96 \mathrm{~m}$. The 16 power supplies for the RCS dipole magnet power supply system are located on this floor, as shown in Figure VI.2.3-3. 


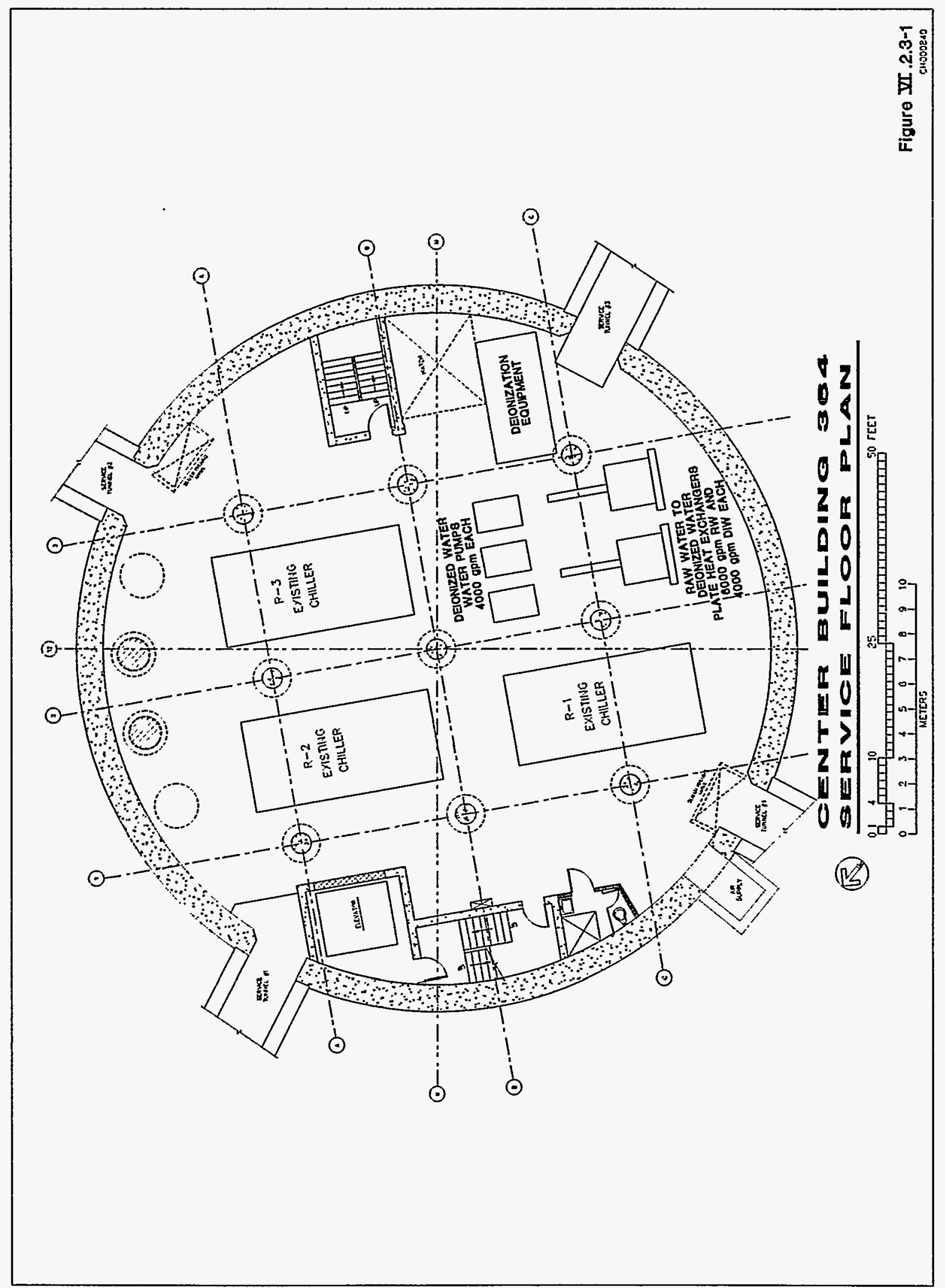




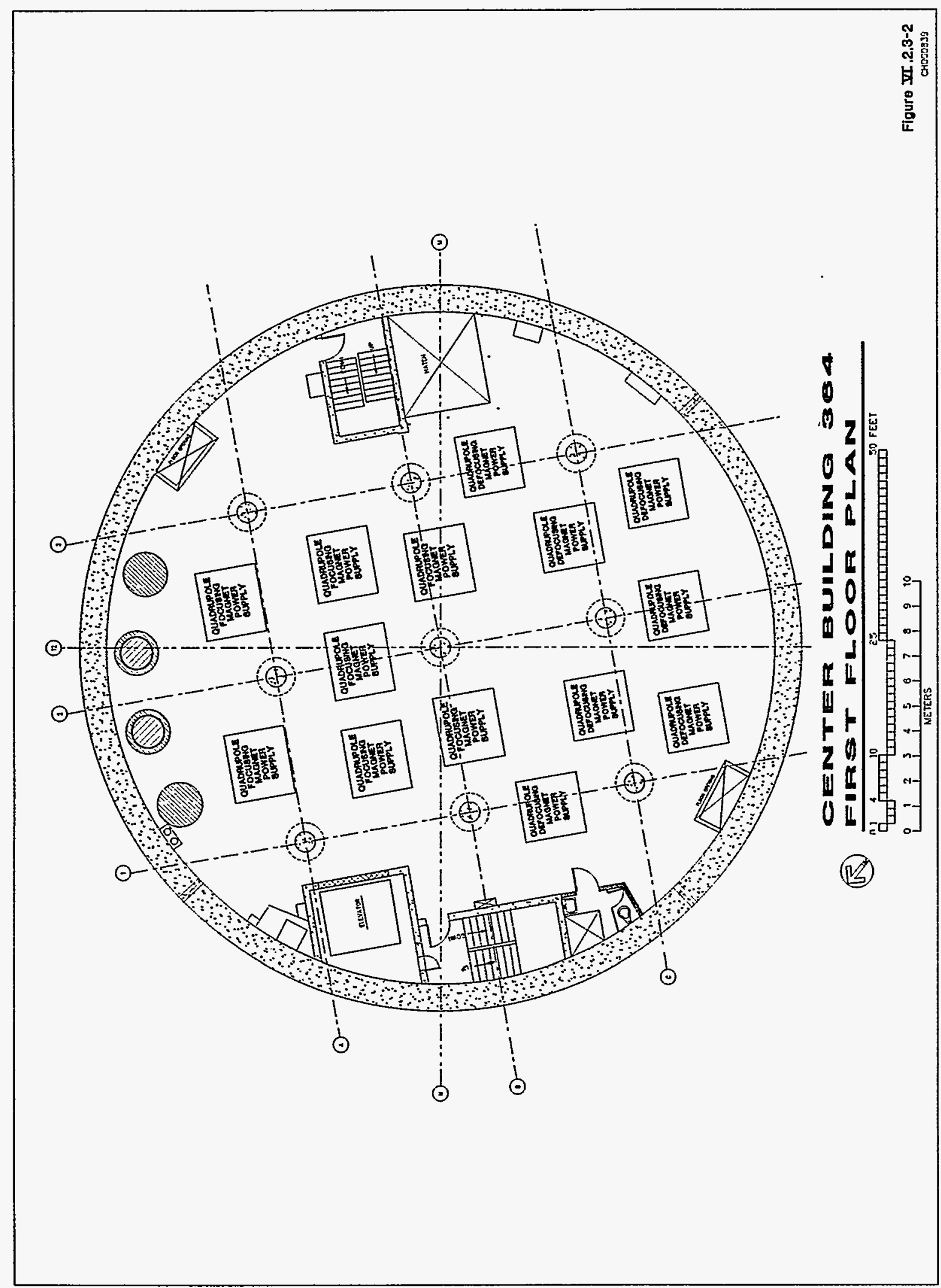




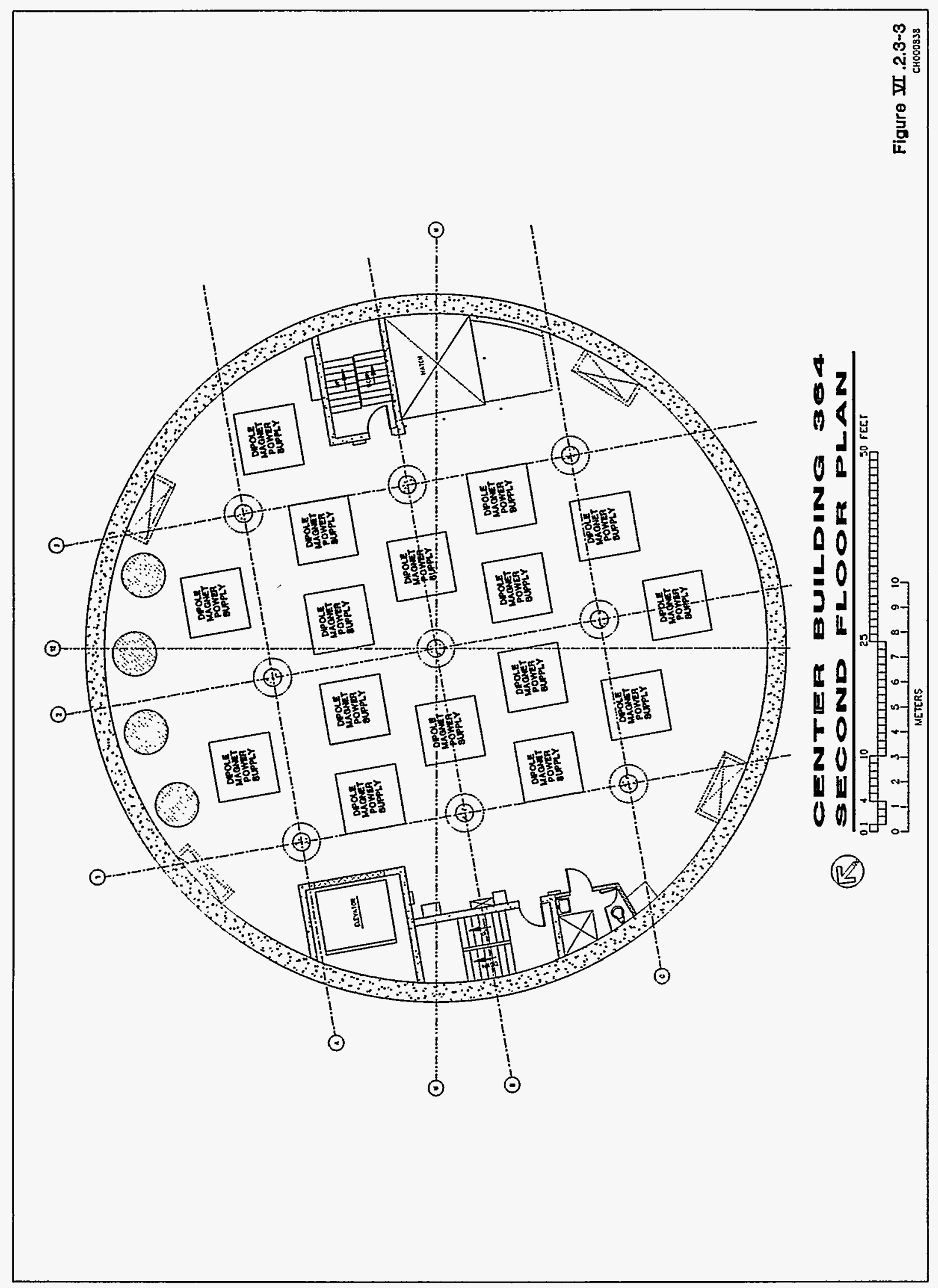


Third Floor. This floor plan is circularly shaped, with an inside diameter of $26.8 \mathrm{~m}$ and a ceiling height of $3.65 \mathrm{~m}$. The if amplifier controls and equipment are located on this floor, as shown in Figure VI.2.3-4.

Fourth Floor. This floor plan is circularly shaped, with an inside diameter of $27.7 \mathrm{~m}$ and a ceiling height of 3.12 meters. On this floor there is a loading dock with direct access to the freight elevator. There is also a dock with a jib-crane to lower equipment to each floor through a hatch system. The fourth floor is $16.6 \mathrm{~m}$ above the Ring Building operating floor. This is the only floor without earth shielding surrounding it. The $480-\mathrm{V}$ switchgear that provides power for the entire building is located on this floor, as shown in Figure VI.2.3-5.

Road Surrounding Fourth-Floor Level. A new road and new concrete transformer pads are located outside on the fourth-floor level, as shown in Figure VI.2.3-6. This road is accessed from a ramped roadway and bridge at the northwest side.

Roofing. In 1990, an IRMA roofing system with 5-cm insulation board and 1-cm cement topping was installed over the existing roof structure.

Building Modifications/Refurbishments. Minor modifications are made as necessary to bring the buildings into compliance with current life safety codes. Outdated areas, typically stairways and toilet rooms, are refurbished.

\subsubsection{Structure (Building 364)}

The cylinder-shaped Center Building is constructed of poured-in-place concrete with five floors and a roof. The floors below the fourth floor are all surrounded by the earth berm shield; their outer wall thicknesses vary from floor to floor. The outer concrete-wall thickness of the service and first floors is $1.07 \mathrm{~m}$, that of the second and third floors is $0.8 \mathrm{~m}$, and that of the fourth floor is $0.3 \mathrm{~m}$.

The service floor is a 0.3 -m-thick slab-on-grade, poured on a 1-m-thick concrete sub-base, and has a $1,000-1 \mathrm{~b} / \mathrm{ft}^{2}$ floor load capacity.

The four floors above the service floor are all $0.3-\mathrm{m}$ thick and are supported on nine round concrete columns with bell-shaped tops. The diameter of the columns varies from floor to floor. The first floor load capacity is $250 \mathrm{lb} / \mathrm{ft}^{2}$; second floor, $200 \mathrm{lb} / \mathrm{ft}^{2}$; third floor, $150 \mathrm{lb} / \mathrm{ft}^{2}$; and fourth floor, $100 \mathrm{lb} / \mathrm{ft}^{2}$.

The roof structure is a steel-beam and girder frame with metal deck and concrete topping, supported on nine steel columns. The roof deck slab is $0.15-\mathrm{m}$ thick, and the roof load capacity is $50 \mathrm{lb} / \mathrm{ft}^{2}$. There is a five-ton jib-crane at roof level with access to each floor through a roof hatch. 


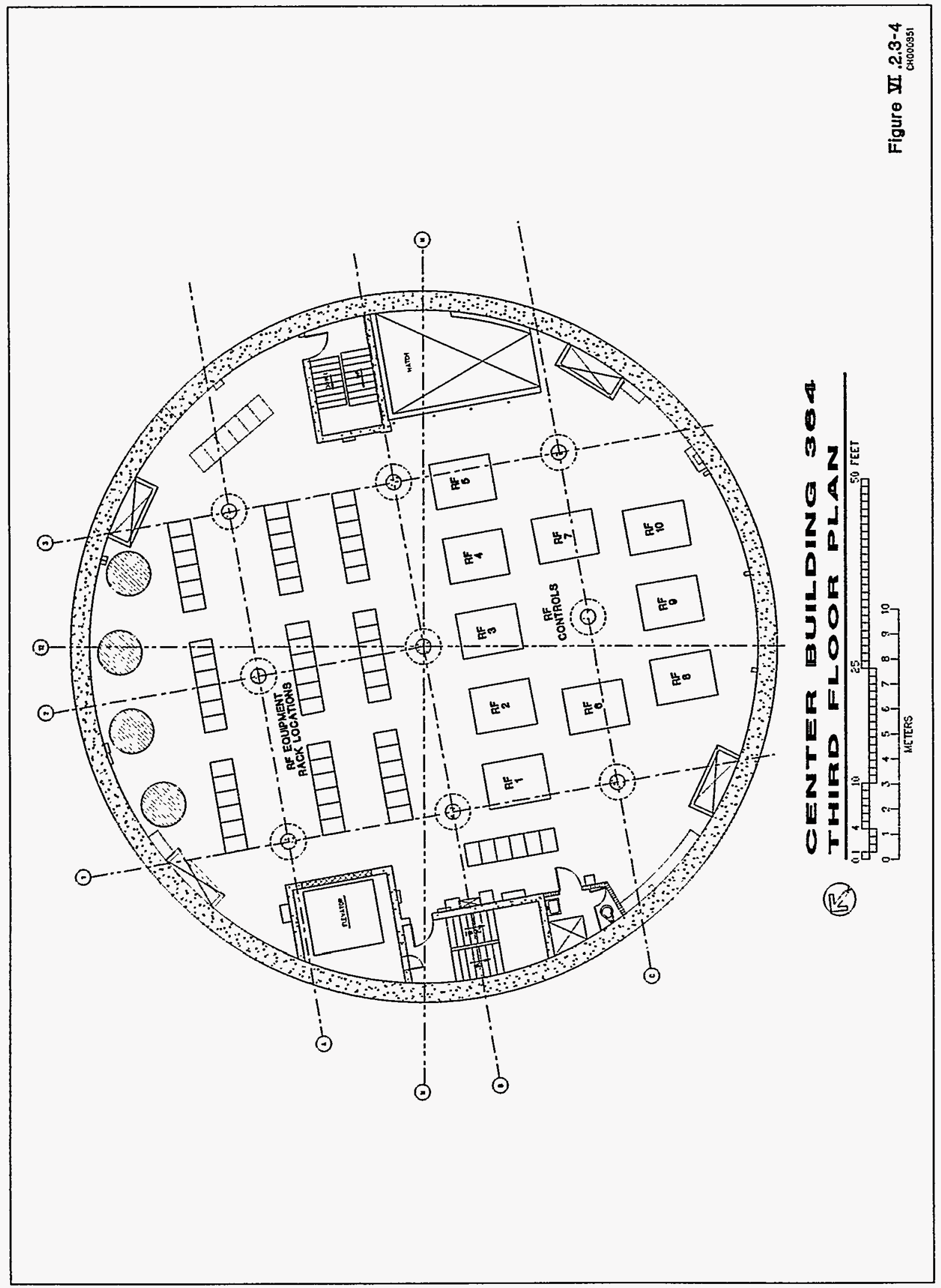




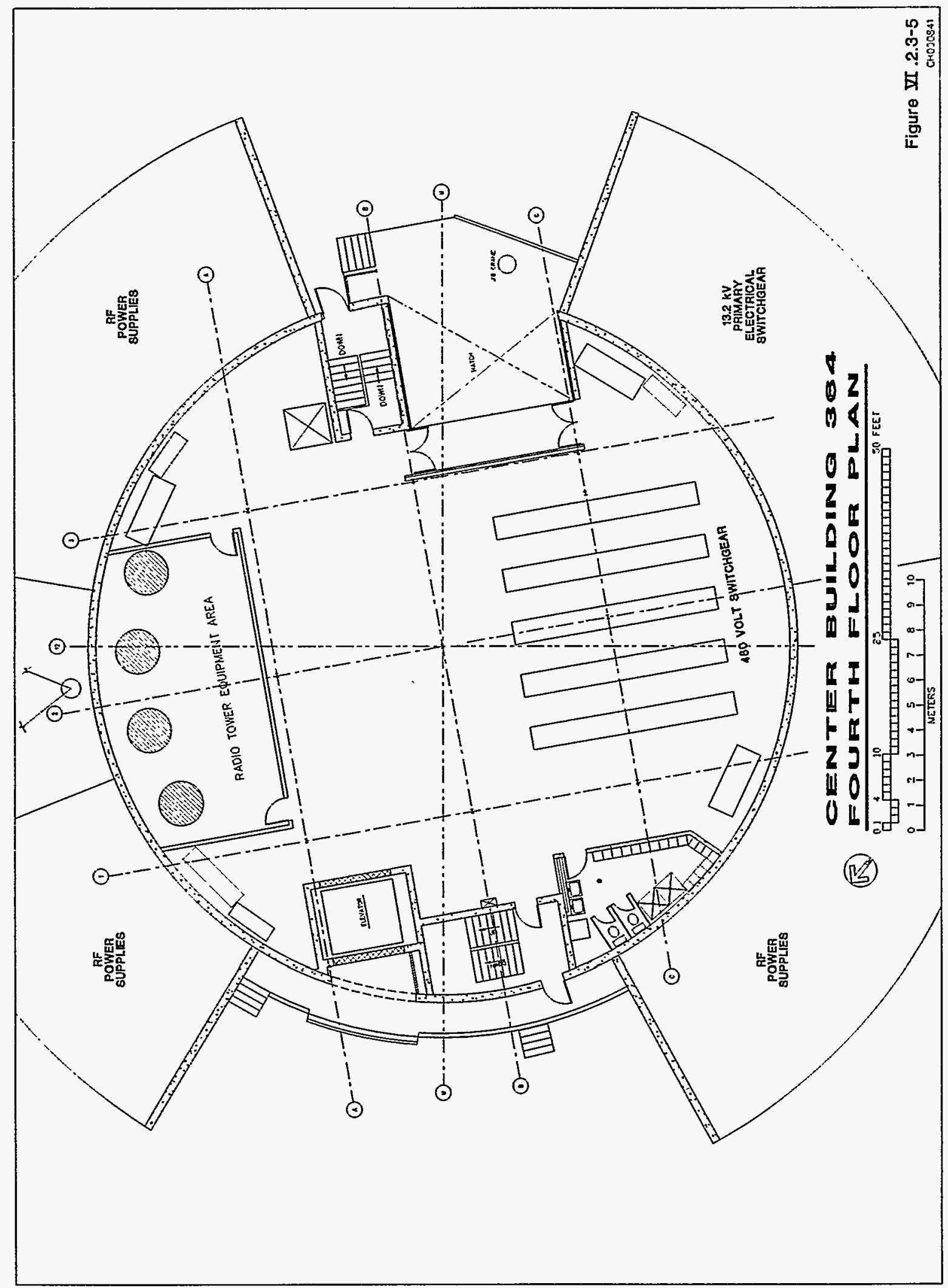




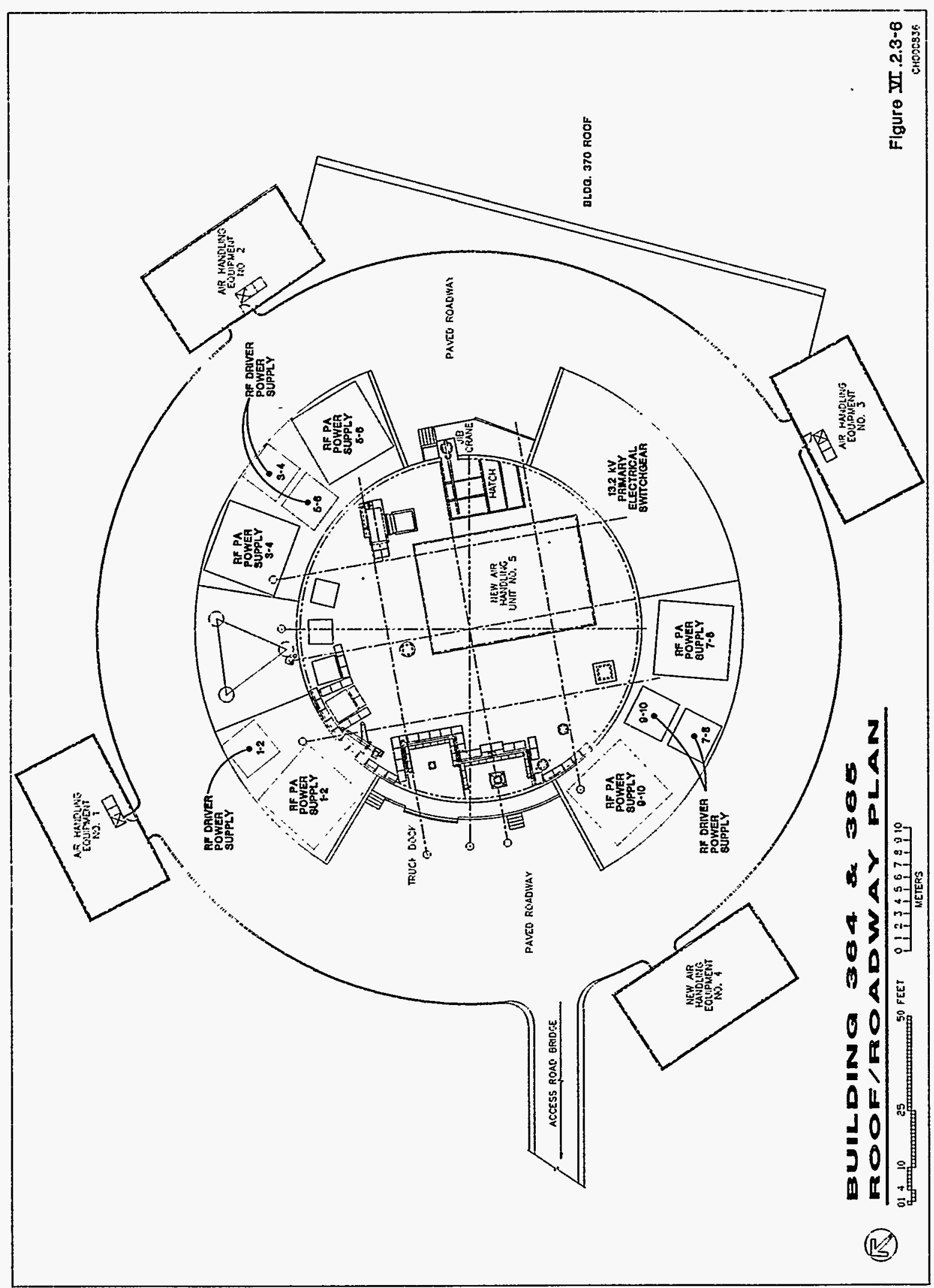




\subsubsection{Mechanical Systems (Building 364)}

Demolition. The majority of existing mechanical systems and equipment in the service floor mechanical room remain, with minor demolition. Suspended fan-coil AHUs presently utilized for cooling of the first, second, and third floors are removed, along with the fourth floor AHU and associated piping. Miscellaneous piping at the service floor is relocated to permit installation and connection of new equipment.

Central Plant Equipment and Piping. A new deionized-water (DIW) plant for the IPNS Upgrade is located at the service floor of Building 364. The DIW plant incorporates two new, plate-type raw-water (RW) to DIW heat exchangers, each with an RW capacity of $6,000 \mathrm{gal} / \mathrm{min}$ and a DIW capacity of $4,000 \mathrm{gal} / \mathrm{min}$.

Raw water to the new DIW plant is fed from the existing 750-mm-diameter RW main, which runs from the utility tunnel, through the LET tunnel, and across the service floor of Building 365 to this building.

DIW from the plant ties into the existing 350-mm-diameter piping in Building 365 for the RCS, as well as into a new piping loop that provides DIW to the linac facility.

The DIW supply (S) and return (R) risers are installed to serve equipment located on the first, second, and third floors of the building. At each of the floors, DIW S/R loops run along the wall. Piping is provided with capped valve-stubs for equipment connection.

The service floor of Building 364 houses three existing chillers, two with capacities of $5,275 \mathrm{~kW}$ (cooling output) and one with a capacity of $3,500 \mathrm{~kW}$ (cooling output). Chilled water (CW) is supplied to a new AHU located on the roof of this building (see Figure VI.2.2-3) and to four AHU buildings located along the access road, as shown in Figures VI.2.2-3 and VI.2.3-6. These AHUs supply conditioned air to Building 365 . Piping and ductwork risers to the new rooftop AHU run through the building. CW piping branches out from the piping risers to the four perimeter AHUs that serve Building 365 .

Steam and condensate piping are connected to the existing systems in the building. Existing steam unit heaters at the fourth floor are retained, with piping modifications.

Air Distribution. The new AHU located on the roof of Building 364 has 70,000-cfm capacity and has a return/exhaust fan with variable-speed drives to supply air to the first, second, third, and fourth floors. This unit consists of a CW cooling coil, low-pressure-steam heating coils, and two supply blowers.

Each of the equipment floors is served by cooling-only variable-air volume (VAV) boxes, ductwork, and diffusers. Air is returned to the return air duct risers through registers.

Plumbing and Fire Protection. Existing building toilet facilities and plumbing systems are used. Existing fire protection systems are modified to provide coverage for new equipment. 


\subsubsection{Electrical Systems (Building 364)}

Electrical power to the RCS is supplied from Site Facility 549 by five 13.2-kV lines. Four of these lines feed 2,500-kVA, 480-V transformers and switchgear. Each of the 480-V switchgear units can be energized from either of two different $13.2-\mathrm{kV}$ lines by using separate $15-\mathrm{kV}$ switches. The secondary of the transformer is connected via bus duct to indoor $480-\mathrm{V}$ switchgear located on the fourth floor. A one-line diagram is shown in Figure VI.2.2-4. A sixth 2,500-kVA, 480-V transformer provides backup power service.

Lighting fixture circuit wiring is new to improve reliability. Cleaned, repaired, and relamped existing fixtures provide adequate illumination. New breakers are provided for existing power and lighting panel board interiors.

New outdoor, high-pressure sodium fixtures are installed to provide perimeter lighting. New illuminated emergency exit signs are installed. Additional emergency lighting is provided by 1.5 -h battery-powered units with built-in chargers.

The fire alarm system is upgraded as necessary to provide coverage for new equipment.

Additional telephone outlets are provided where required. A new amplifier is installed for the paging system, but existing speakers and wiring are retained.

\subsection{POWER SUPPLY BUILDING (EXISTING BUILDING 376)}

Building 376 is a one-story, high-bay building that was completed in 1961 and has a gross floor area of $1,978 \mathrm{~m}^{2}$. The eight RCS sextupole supplies, 40 magnet power supplies for the transport lines, and a CW plant are located in this building. This building has access to the lowerlevel service floor and utility tunnel. Figure VI.2.4-1 shows the service floor with the CW processing plant, and Figure VI.2.4-2 shows the first floor with magnet power supplies.

\subsubsection{Architecture (Building 376)}

The existing building is approximately $18.9 \mathrm{~m}$ wide and $50.1 \mathrm{~m}$ long. The service level has utility tunnel connections to Building 370 . The building exterior is constructed of insulated, corrugated-aluminum panels supported on a steel frame structure. The building facade has 2-m-high window bands on the long sides. The first floor has a 7.9-m high bay equipped with a 10-ton crane.

The existing building is in an ideal location to accept power from Site Electrical Facility 549 and water from Site Cooling Tower Facility 377 . Utilities and magnet power supply cables from Building 376 are directly routed to the existing utility tunnels and LET tunnel. 


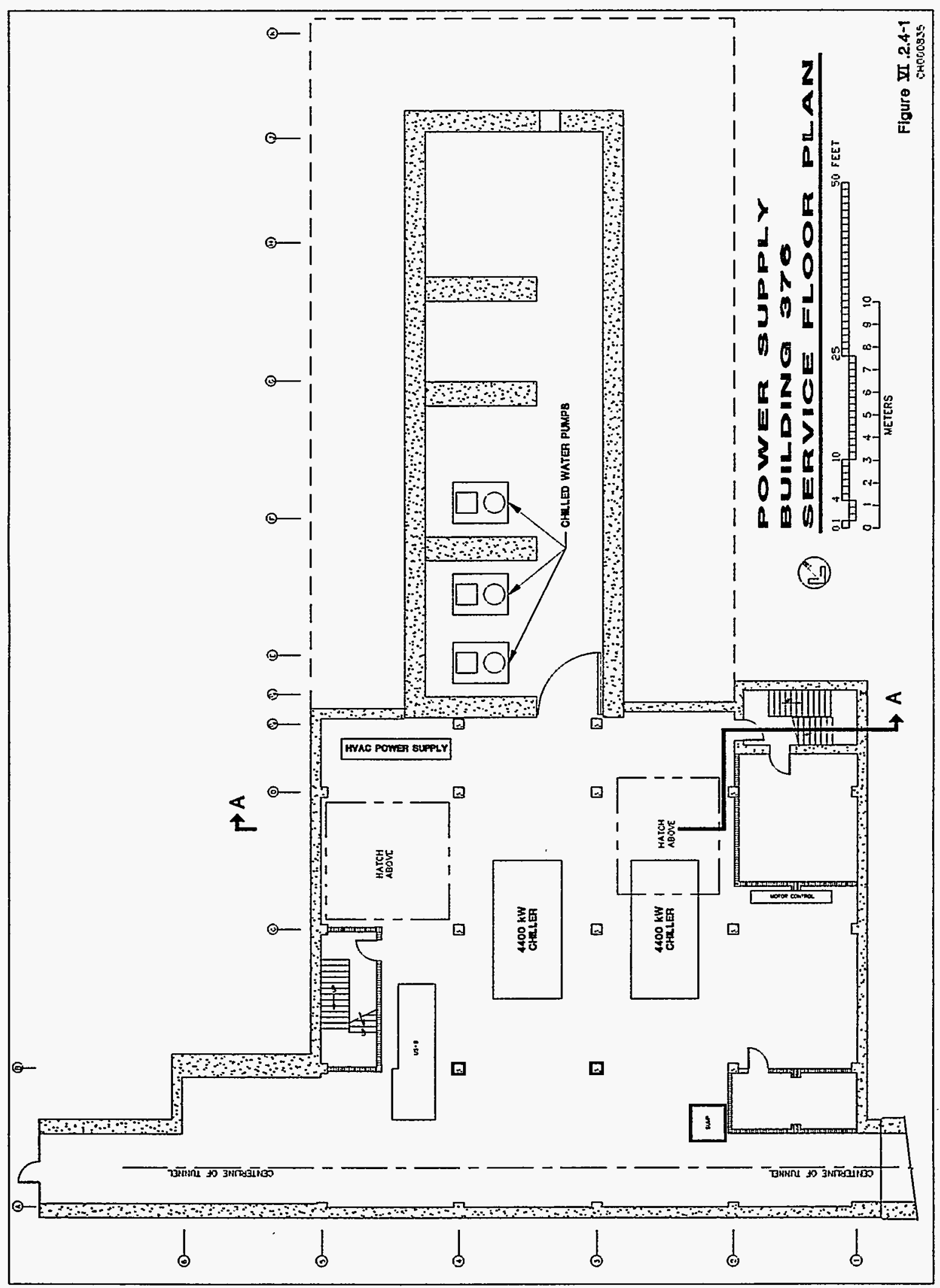




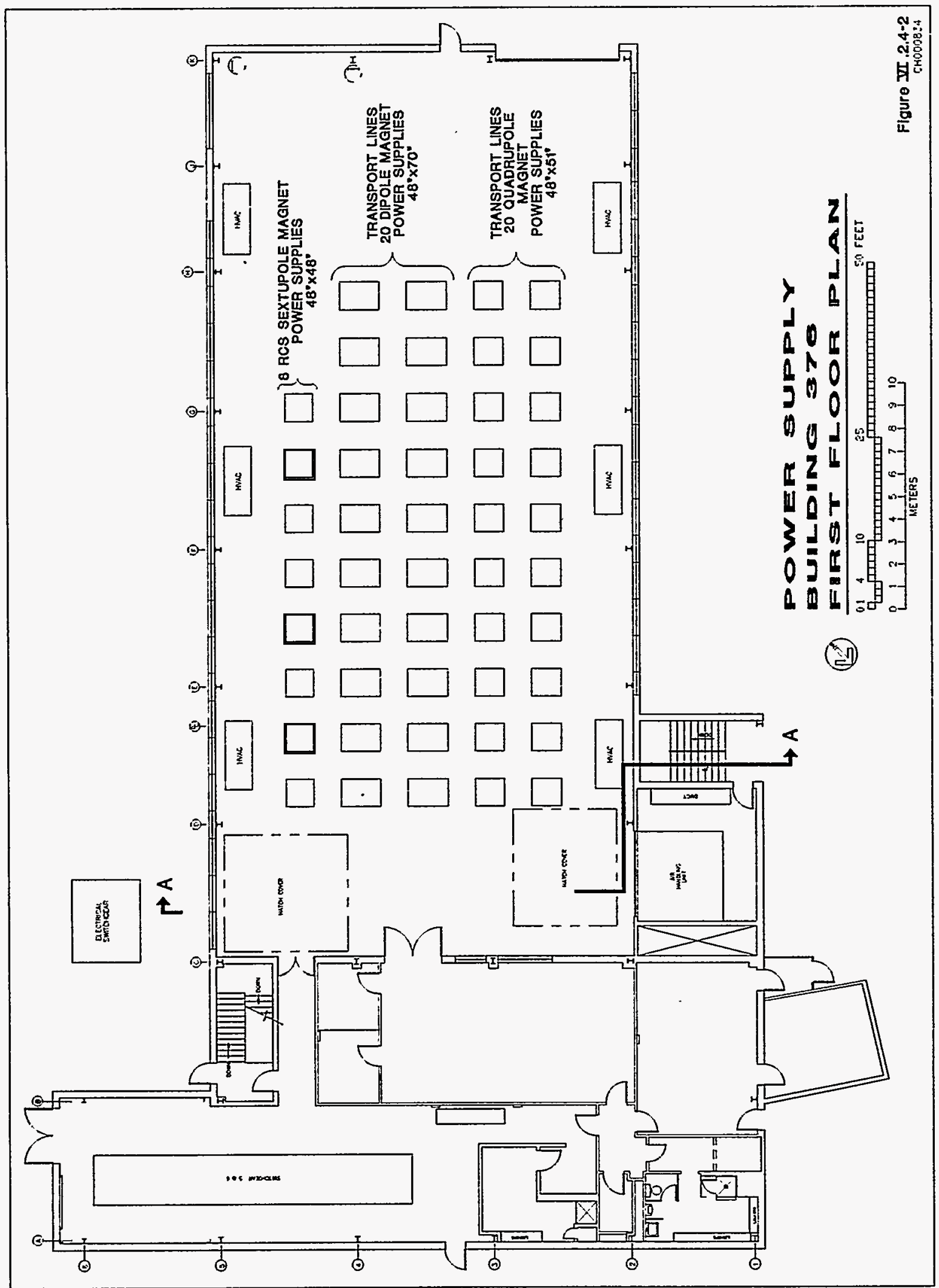


There are facility and support offices, toilet and locker facilities, and electrical rooms on the first floor. Existing walls and partitions are painted concrete-masonry units. Suspended acoustical tile ceilings with lay-in-type fluorescent lighting fixtures are used in office areas.

Access into the building is through five exterior personnel doors at the grade level. Access to the first floor is also possible by two stairwells from the service level, as well as through Building 375. A vehicle access door permits trucks to enter the building high bay. Vehicle unloading is accomplished with the 10-ton overhead crane. Vehicle parking areas are located adjacent to the building.

Roofing. In 1990, an IRMA roofing system with 5-cm-thick insulation board and 1-cmthick cement topping was installed over the existing roof structure.

Utility Tunnel. The northeast section of the basement is part of the utility tunnel system. These tunnels are used for routing of utilities and controls. The average tunnel is $3.04 \mathrm{~m}$ wide and $4.26 \mathrm{~m}$ high.

Building refurbishing includes painting, minor exterior siding, and window, door, and partition repair work.

\subsubsection{Structure (Building 376)}

The existing high-bay superstructure consists of a concrete foundation with steel columns supporting a long-span truss over the high bay area. The rest of the building has a steel-beam and girder framing system, which supports a welded-metal deck roof.

The first floor is a combination of cast-in-place, slab-on-grade, and precast concrete. Most of the first floor is poured-in-place reinforced concrete that is $0.15 \mathrm{~m}$ thick and has a floor load capacity of $800 \mathrm{lb} / \mathrm{ft}^{2}$. The rest of the floor has areas of precast-concrete panels. Two hatches allow crane access from the high bay to equipment on the service floor and the utility tunnel. Section A-A through Building 376, indicated in Figures VI.2.4-1 and VI.2.4-2, is shown in Figure VI.2.4-3.

Crane. An existing 10-ton, top-running bridge crane in the high bay provides full coverage for the high-bay area.

Structural building modifications consist of minor steel work for mechanical, electrical, and plumbing supports.

\subsubsection{Mechanical Systems (Building 376)}

Demolition. The existing, deteriorated AHU located in the mezzanine above the office area is removed, along with other unused mechanical equipment. 


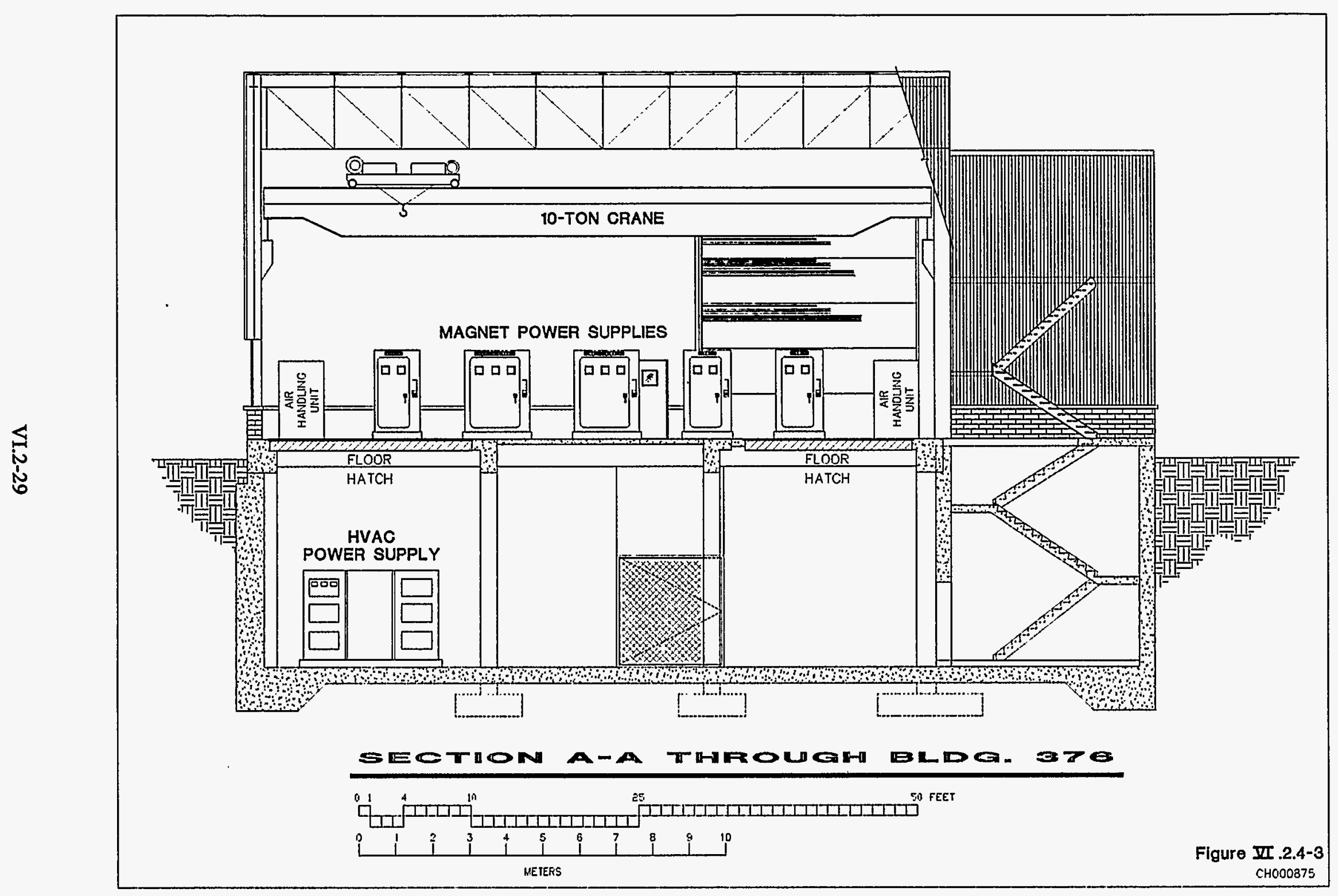


Central Plant Equipment and Piping. A new chiller plant comprising two 4,400-kW chillers and three chilled-water pumps is housed on the service floor.

Chiller condensers are connected to existing RW piping in the tunnel. CWS and CWR piping from the chillers is routed through the utility tunnels to Buildings $364,365,369$, and 370 .

DIW for this building is supplied via the utility tunnel system. DIW S/R mains run along one wall of the high bay. The piping is equipped with capped valve-stubs to facilitate connection to user equipment. The following are routed through, and accessible in, the tunnels: Fire protection water, raw water, chilled water, domestic cold water, eight wall-mounted electric and communications cable trays, electric power, compressed air, and a mechanical service water piping system.

Existing unit heaters are retained and are relocated as required.

Air Distribution. Cooling for the high bay is provided by six new, self-contained packaged units, with a cooling capacity of $34 \mathrm{~kW}$ each. These units provide local air cooling of component equipment, distributed by integral overhead ductwork. Chilled water is piped to the six HVAC units in the high bay from the service floor, and coil condensate drains back down to the service floor. The location of the six HVAC units is indicated in Figure VI.2.4-2. Existing exhaust fans, unit heaters, and the switchgear room ventilation unit are refurbished.

A new AHU with ducts and diffusers serves offices on the first floor. The unit has CW cooling coil and steam preheat coils.

The service floor is furnished with a new ventilation system.

Plumbing and Fire Protection. Existing building toilet facilities and plumbing systems are used. Existing fire protection systems are used, with upgrades to provide coverage for new equipment. New sprinkler piping is provided on the service floor.

\subsubsection{Electrical Systems (Building 376)}

Electrical power to technical equipment is provided by two $13.2-\mathrm{kV}$ to $480-\mathrm{V}$ switchable feeds, as shown in Figure VI.2.4-4. The substation consists of two $13.2-\mathrm{kV}$ incoming-line switches, two 2,000-kVA transformers, and a 480-V switchgear line-up. The 13.2-kV incoming switches and transformers are located outside the building and are supplied from switchgear located at Site Electrical Facility 549. Six 480-V power distribution panels are located inside the building on the service floor. These panel boards feed individual pieces of technical equipment.

A $250-\mathrm{kW}$ diesel generator located near Building 376 provides part of the emergency backup power to the 360 area. 


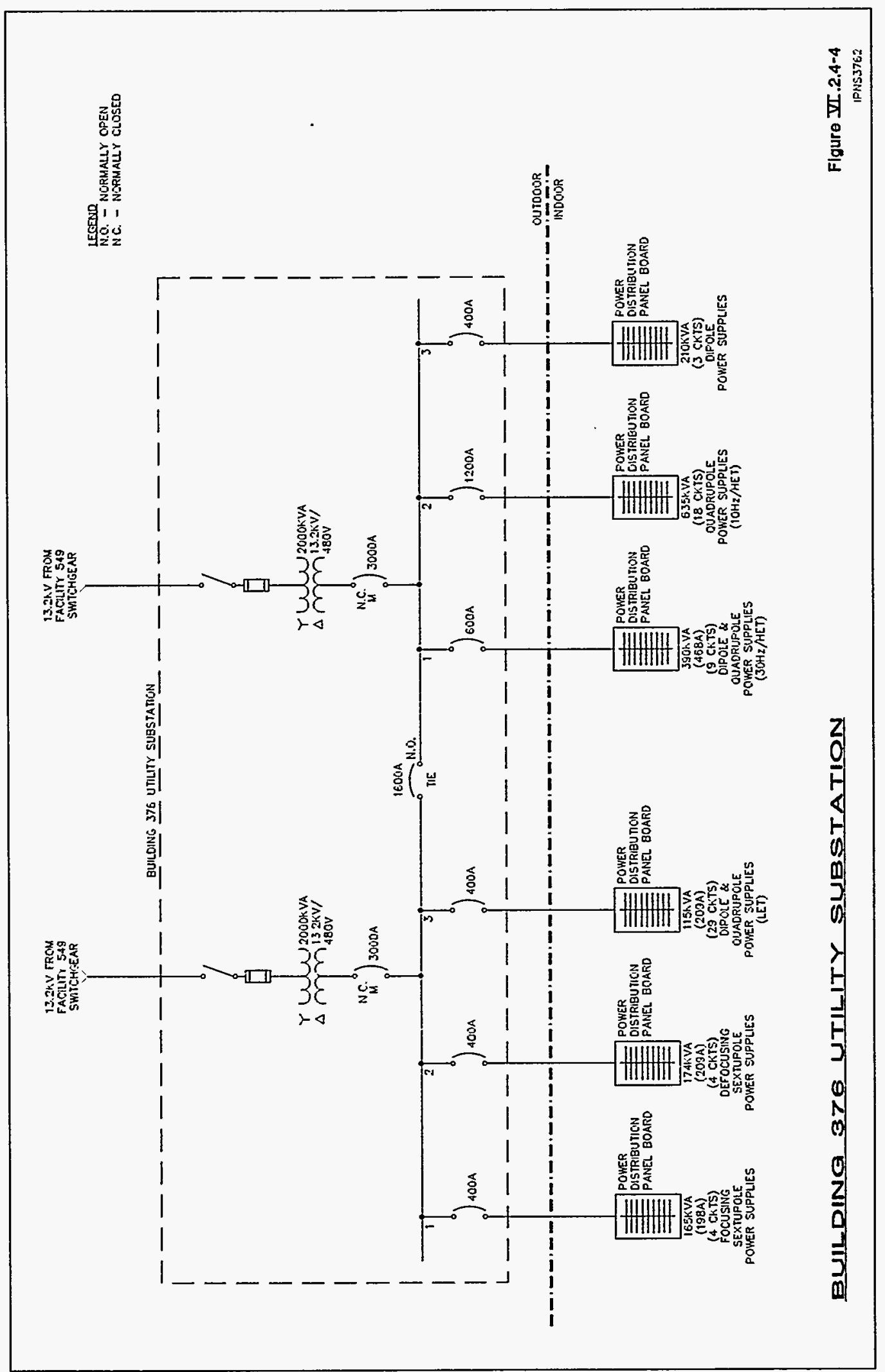


Cleaned, refurbished, and relamped lighting fixtures provide adequate lighting levels. Lighting fixture circuit wiring is new for improved reliability. New breakers are provided for existing power and lighting panel board interiors.

Special electrical systems, such as telephone, paging, and fire alarm, are satisfactory to meet requirements.

\subsection{0-Hz TARGET BUILDING (EXISTING BUILDING 369)}

Building 369 , a one-story high-bay building, $32.4 \mathrm{~m}$ wide $\times 73.1 \mathrm{~m}$ long, houses the $10-\mathrm{Hz}$ target and 18 instrument lines. An adjacent annex is $24.2 \mathrm{~m}$ long $\times 9.2 \mathrm{~m}$ wide. Completed in 1963, this high-bay building has a gross floor area of $2,471 \mathrm{~m}^{2}$ and a clear height of $10.9 \mathrm{~m}$ to the bottom of the roof trusses. Building 369 adjoins Buildings 365,368 , and 370 . Figure VI.2.5-1 shows the first-floor plan and 10-Hz target, and Figure VI.2.5-2 shows a section through the building.

\subsubsection{Architecture (Building 369)}

The exterior walls are insulated metal panels, supported on a steel-framed structure. The north wall is a cast-in-place concrete wall that is designed to hold in place the earth shielding separating the Target Building from the Ring Building. The earth shielding thickness varies from $6 \mathrm{~m}$ to $18 \mathrm{~m}$.

\subsubsection{Structure (Building 369)}

The structural system, supported by a concrete foundation, is composed of steel columns and steel roof trusses with a welded-metal roof deck. The exterior walls, bridge crane, and roof are supported by steel structural system. The concrete floor is 0.15 -m-thick slab-on-grade with $800-1 \mathrm{~b} / \mathrm{ft}^{2}$ capacity. The welded-metal roof deck is protected by a PVC-IRMA roof system installed in 1990.

The targets, shields, and foundations are described in Chapter IV.

Existing first-floor trenches in Building 369 are eliminated.

Tunnel. The existing utility tunnel system covers the 360 area and carries utilities, power, and controls. Personnel access to these buildings is possible via the tunnels. An existing $60.95-\mathrm{m}$-long tunnel under Building 369 is $3.04-\mathrm{m}$ wide $\times 2.62-\mathrm{m}$ high and is used as a tornado shelter.

Cranes and Hoists. The high bay has a 35-ton, top-running bridge-crane with an auxiliary 10 -ton hoist, which provides full high-bay coverage. 


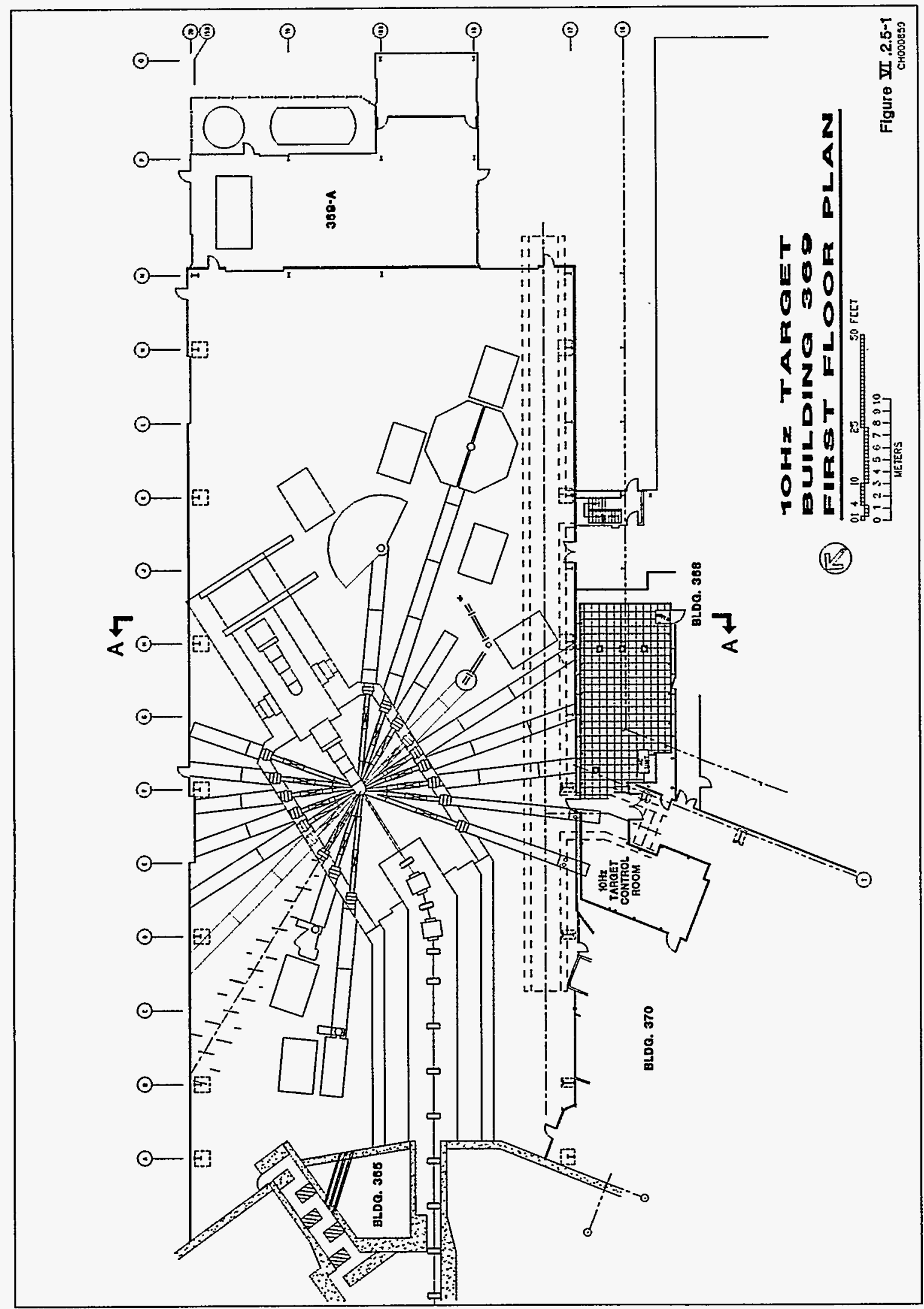




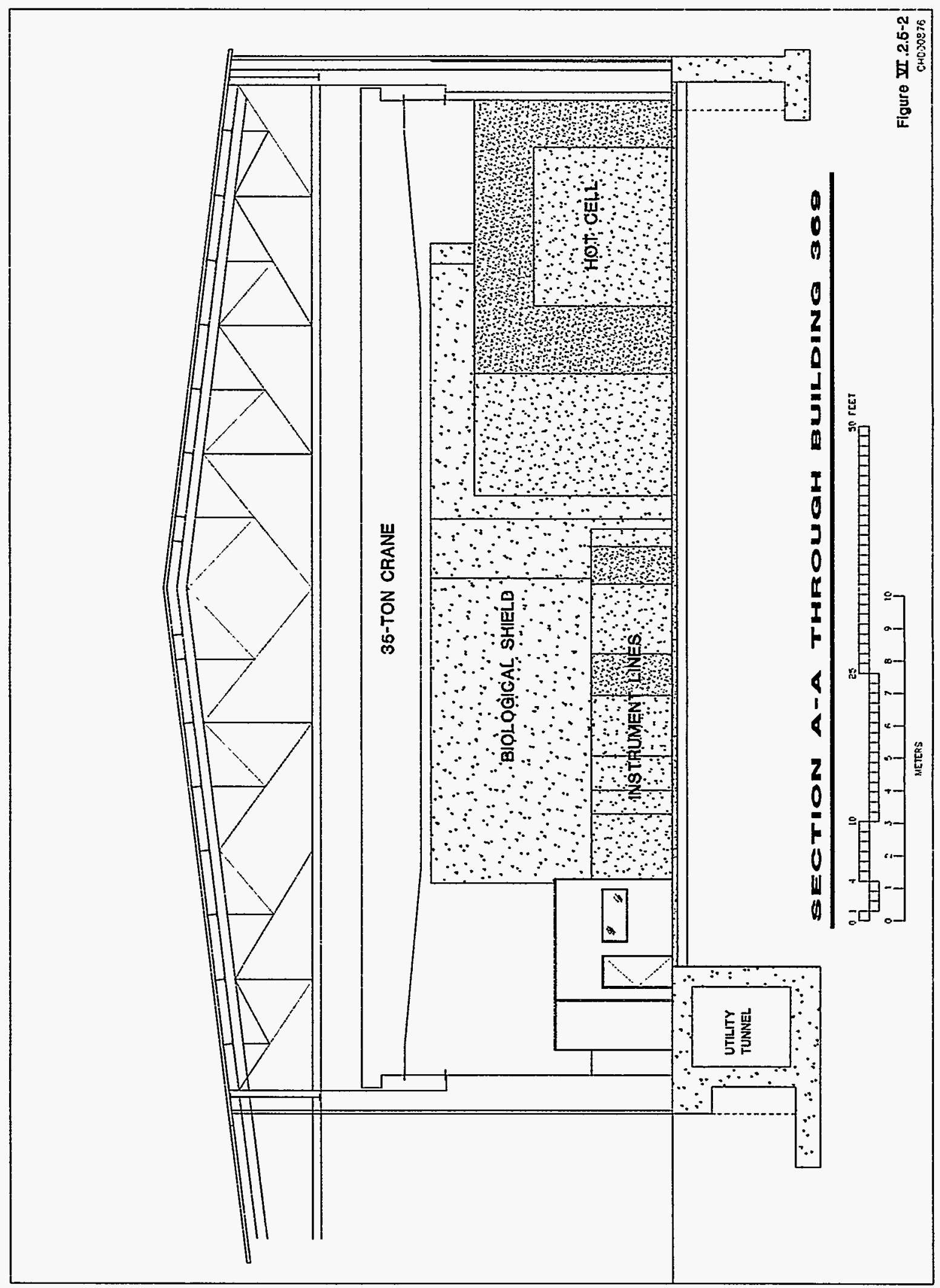




\subsubsection{Mechanical Systems (Building 369)}

Demolition. Miscellaneous mechanical systems and piping are relocated to facilitate construction of target biological shielding.

The existing tunnel under the southwest part of the building provides the following services: Fire protection water piping, high-pressure steam supply and condensate return piping, $\mathrm{RW}, \mathrm{CW}$, sanitary drain, wall-mounted electric cable trays, and electric power. This utility tunnel has an automatic sprinkler system, fire detection system, floor drains, and access panels to the building above; it also is used as a tornado shelter.

Central Plant Equipment and Piping. No DIW is required for the equipment in this building. Equipment utilizing DIW is located in Building 367. DIW piping runs from the utility tunnel through the building and feeds Building 367 .

Existing CW piping presently utilized at the three existing AHU cooling coils is retained. New CW plant piping required for local cooling at the instrument lines is tied into the existing $\mathrm{CW}$ piping in the tunnel.

Existing unit heaters are retained. Heaters and piping are modified and moved as required to accommodate new equipment.

Air Distribution. The building is presently air-conditioned by three truss-mounted air handling units of 40,000-cfm capacity each. These units are refurbished and used, and new controls are provided. Existing diffusers and ductwork are retained.

The five existing 89,000-cfm hydrogen-evacuation exhaust fans on the roof are refurbished and used.

Plumbing and Fire Protection. Existing building plumbing systems are used. Existing fire protection systems are used, with upgrades to provide coverage for new equipment.

\subsubsection{Electrical Systems (Building 369)}

The existing 1,000-kVA transformer and 480-V switchboard are used to provide power for conventional facilities. The switchboard is modified to accommodate new equipment. A new motor control center provides power to building mechanical equipment and other installed motors that are rated at more than $0.5 \mathrm{hp}$.

The $10-\mathrm{Hz}$ target equipment is fed from a $480-\mathrm{V}$ double-ended substation, as shown in Figure VI.2.5-3. This substation has two $13.2-\mathrm{kV}$ incoming line switches, two 2,000-kVA transformers, and associated $480-\mathrm{V}$ switchgear. The $13.2-\mathrm{kV}$ switchgear is located outdoors near Building 369 , and power is supplied to it at $13.2 \mathrm{kV}$ from switchgear located at Site Electrical 


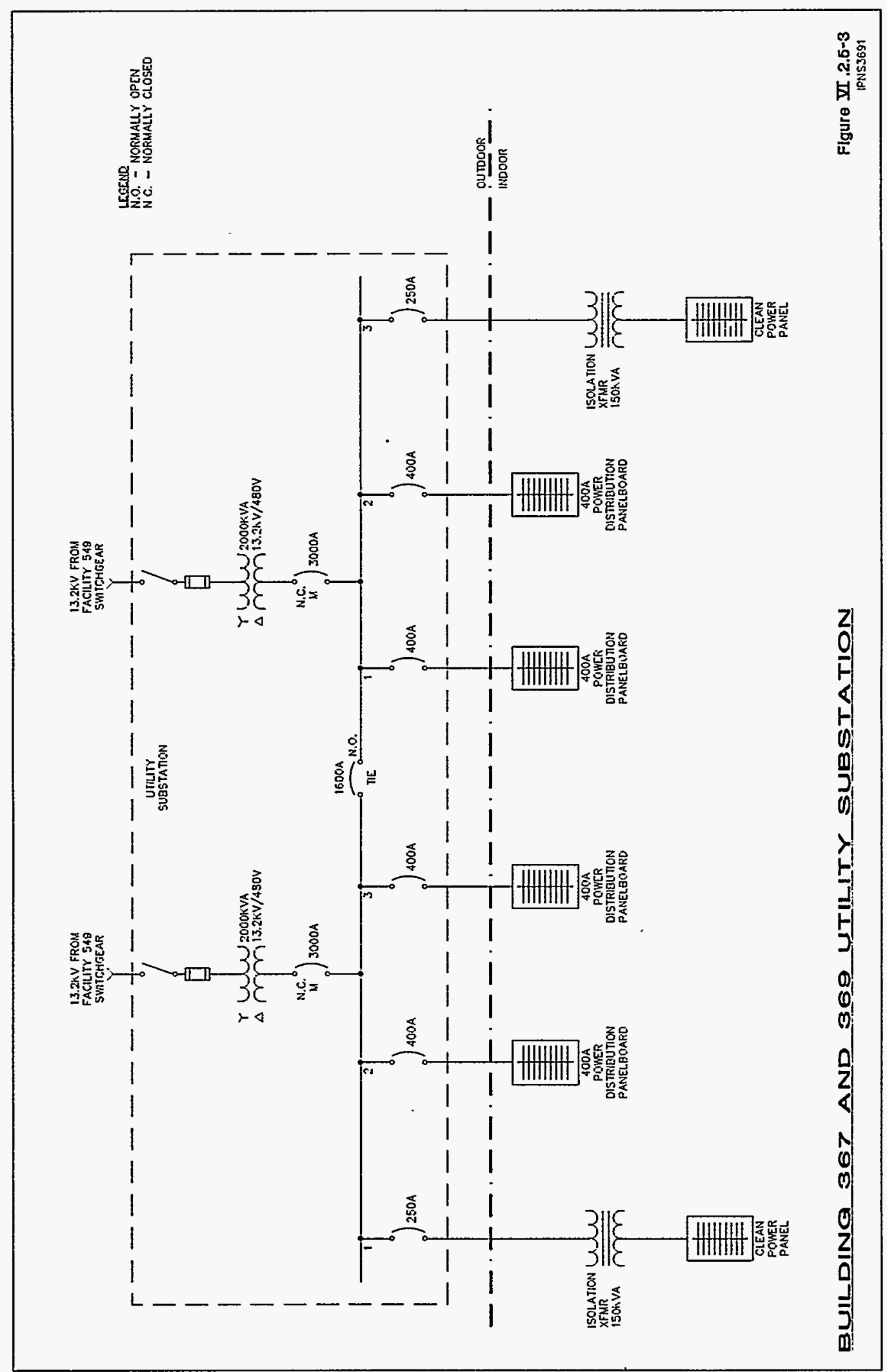


Facility 549. The 480-V switchgear is located inside Building 369 and feeds power to distribution panel boards located near the technical equipment. Individual equipment is powered from these panels.

Clean power requirements are met by using $480-\mathrm{V}$ to $480-\mathrm{V}$ isolation transformers, as shown in Figure VI.2.5-3.

Cleaned, repaired, and relamped existing lighting fixtures provide adequate illumination. Lighting fixture circuit wiring is new to improve reliability. New breakers are provided for existing power and lighting panel board interiors.

Special systems, such as telephone, paging, and fire alarms, are satisfactory to meet requirements.

\subsection{0-Hz TARGET SUPPORT BUILDING (EXISTING BUILDING 367)}

Building 367 is a single-story high-bay building with three low-story areas connected by a common corridor, as shown in Figure VI.2.6-1. The high bay is $15.2 \mathrm{~m}$ long, $24.4 \mathrm{~m}$ wide, and $10.0 \mathrm{~m}$ high, with a $7-\mathrm{m}$-wide $\times 12$-m-long annex to the south. This facility, completed in 1968 , has a gross floor area of $677 \mathrm{~m}^{2}$. The high-bay area is equipped with a 35-ton crane. A new shielded cooling system for the 10-Hz target in Building 369 is located in the high bay, as shown in Figure VI.2.6-2. Building 369 is approximately $27.4 \mathrm{~m}$ to the west of Building 367 . The target cooling system is self-contained and the cooling system area is shielded, because the coolant is potentially radioactive. The building has monitored limited access. Target cooling water pipes are double-walled and are direct-buried between Buildings 367 and 369. Target cooling is discussed in detail in Chapter IV.

\subsubsection{Architecture (Building 367)}

The exterior walls consist of vertically corrugated, insulated metal panels on horizontal steel girts. Exterior access to the high bay is provided by three personnel doors and a vehicle door. Vehicle parking is available adjacent to the building.

The self-contained target cooling system housed in this building falls within the scope of technical components and is described in Chapter IV.

Roofing. In 1990, an IRMA roofing system with 5-cm-thick insulation board and 1-cmthick cement topping was installed over the existing roof structure.

Building refurbishments include minor repairs to the wall-panel system, as well as general painting, sealing and caulking. 


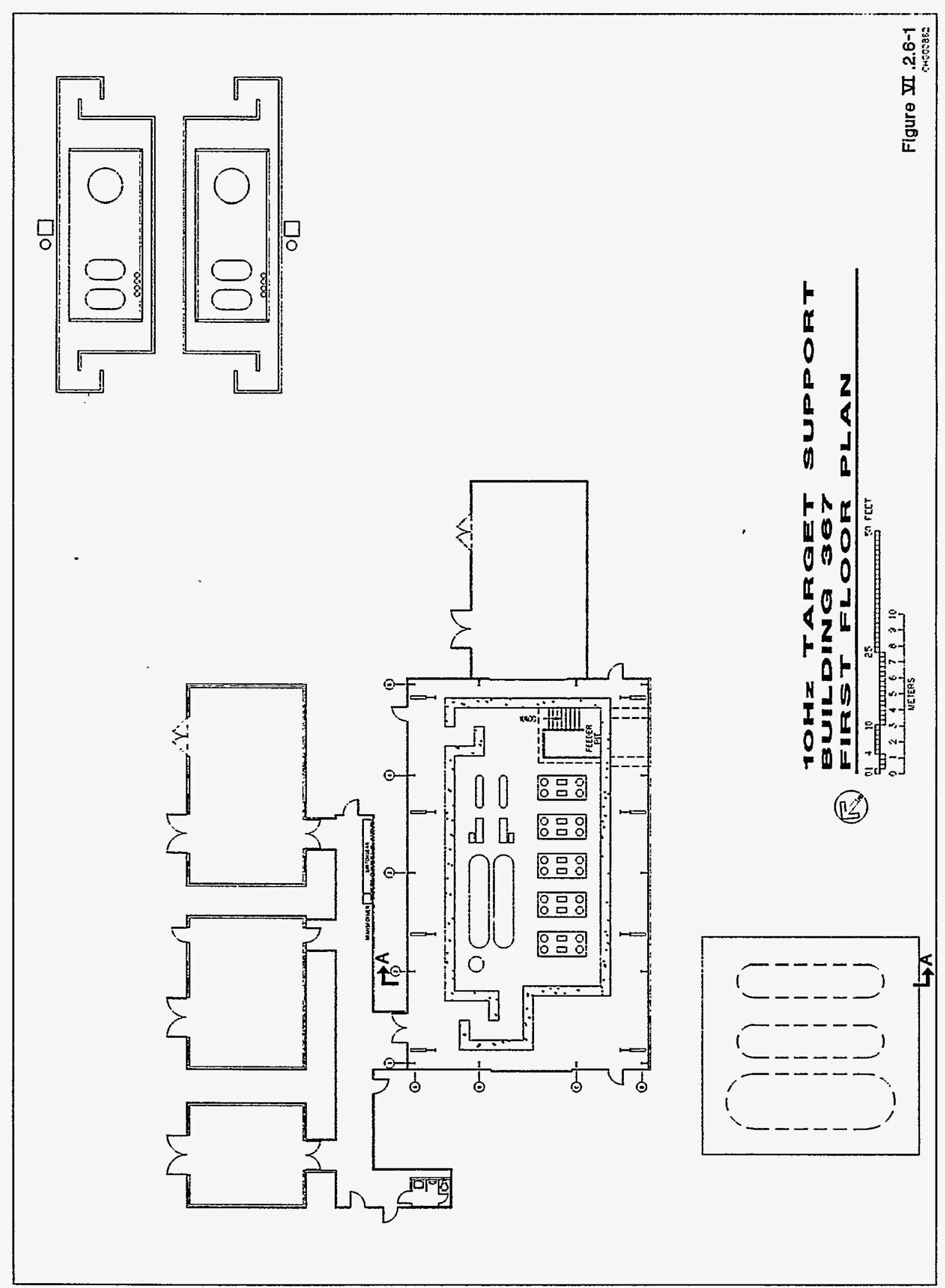




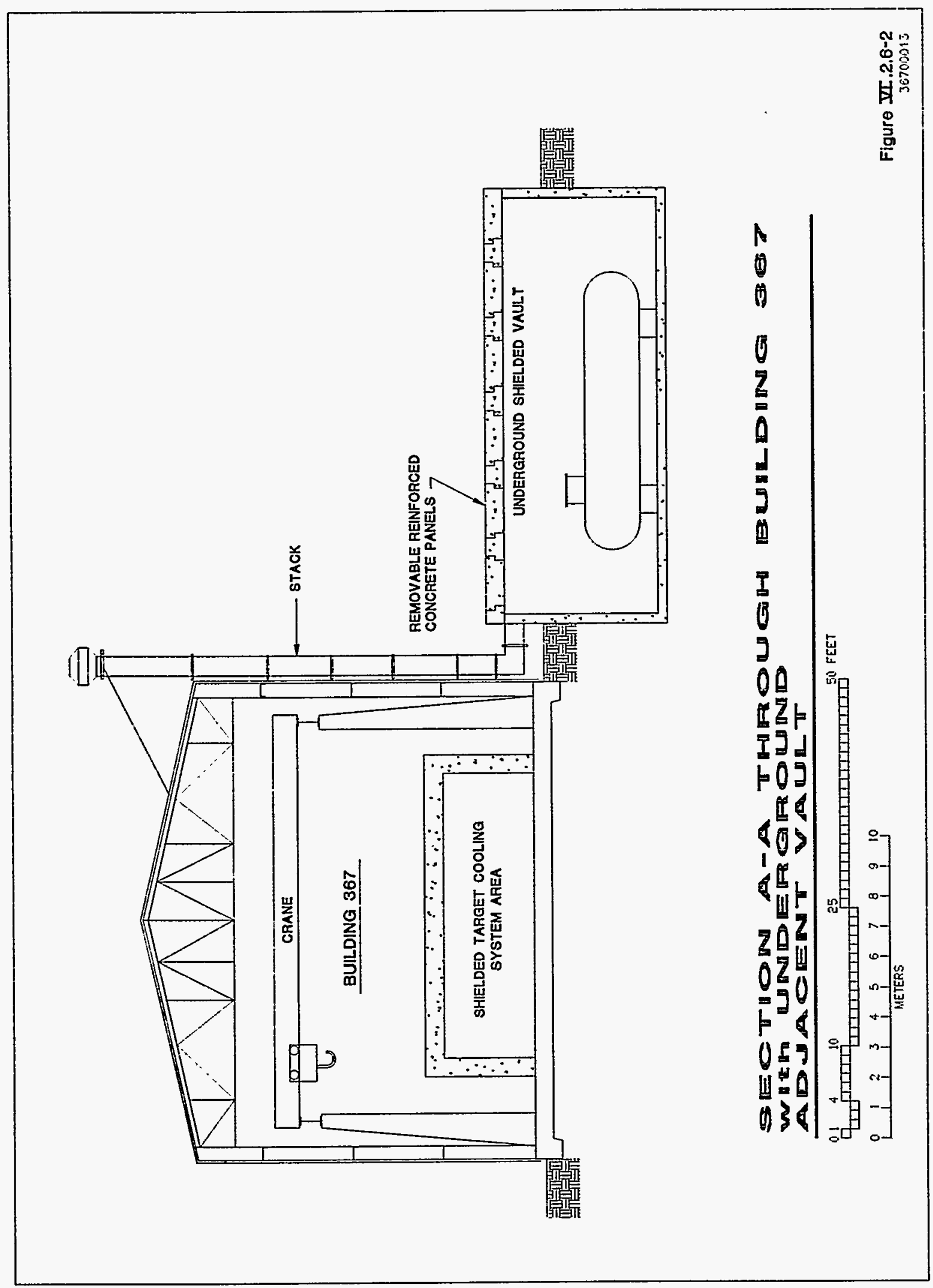




\subsubsection{Structure (Building 367)}

The existing building structural system consists of steel columns supporting a beam and girder framing system with a welded-metal deck roof. Structural steel bracing in the exterior wall provides resistance against lateral loads. Foundations are conventional reinforced-concrete spread footings. The floor is slab-on-grade reinforced concrete and is $0.45 \mathrm{~m}$ thick, with a floor load capacity of $3,000 \mathrm{lb} / \mathrm{ft}^{2}$. The high bay is equipped with a top-running 35 -ton crane, bridgesupported on an independent structure.

There are no structural modifications to Building 367 except for the addition of minor supports associated with the new technical, mechanical, and electrical equipment installations.

\subsubsection{Mechanical Systems (Building 367)}

Existing mechanical systems in the building are retained. Mechanical systems associated with the target cooling and coolant retention systems are described in Chapter IV.

Central Plant Equipment and Piping. There are no chillers, DIW plants, or other plant facilities cooling equipment located in the building.

DIW is provided from Building 369. DIW S/R runs along the high-bay wall. Piping is provided with capped valve-stubs to facilitate connection to user equipment.

Existing unit heaters are retained and relocated.

Air Distribution. The building uses existing, refurbished exhaust fans and louvers for ventilation. No air conditioning and no additional AHUs are provided.

Plumbing and Fire Protection. Existing building toilet facilities and plumbing systems are used. Existing fire protection systems are used, with upgrades to provide coverage for new equipment.

\subsubsection{Electrical Systems (Building 367)}

Electrical power to Building 367 is delivered from $480-\mathrm{V}$ switchgear located in Building 369, as shown in Figure VI.2.5-3. This switchgear feeds new 480-V power distribution panel boards.

Lighting fixture circuit wiring is new to improve reliability. Cleaned, repaired, and relamped fixtures provide adequate illumination. New breakers are provided for existing power and lighting panel board interiors. 

lighting.

New outdoor, high-pressure sodium lighting fixtures are installed to provide perimeter

Emergency lighting is provided by 1.5 -h battery-pack units. Special systems, such as telephone, paging, and fire alarms, are adequate to meet requirements.

\subsection{0-Hz TARGET BUILDING (EXISTING BUILDING 370)}

Building 370, a one-story high-bay building, $31.4 \mathrm{~m}$ wide $\times 97.5 \mathrm{~m}$ long, houses the $30-\mathrm{Hz}$ target and 18 instrument lines. The high-bay building, completed in 1963, has a gross floor area of $3,679 \mathrm{~m}^{2}$ and a clear height of $10.9 \mathrm{~m}$ to the bottom of the roof trusses. Building 370 adjoins Buildings $365,368,369$, and 371. Figure VI.2.7-1 shows the first-floor plan and $30-\mathrm{Hz}$ target, and Figure VI.2.7-2 shows a section through the building.

\subsubsection{Architecture (Building 370)}

The three exterior walls are insulated metal panels on horizontal girts. The north wall is the common concrete and earth shield wall between the Ring Building and the $30-\mathrm{Hz}$ Target Building. It has a 3.6-m-wide $\times 4.6-\mathrm{m}$-high cast-in-place concrete girder that provides an opening $28.8 \mathrm{~m}$ wide $\times 2.9 \mathrm{~m}$ high between Buildings 365 and 370 for the high-energy transfer line to the 30- $\mathrm{Hz}$ target. A section through this area is shown in Figure VI.2.7-2.

The east wall is a common wall between the $10-\mathrm{Hz}$ Target Building and the $30-\mathrm{Hz}$ Target Building.

The existing control rooms at the east end of Building 370 are used.

Roofing. In 1990, an IRMA roofing system with 5-cm-thick insulation board and 1-cmthick cement topping was installed over the existing roof structure.

\subsubsection{Structure (Building 370)}

The structural system is composed of steel columns on a concrete foundation supporting steel trusses and a welded-steel roof deck.

The slab-on-grade concrete floor is $0.15 \mathrm{~m}$ thick, with a floor load capacity of $800 \mathrm{lb} / \mathrm{ft}^{2}$.

The targets, shields, and associated foundations are described in Chapter IV.

Existing floor trenches in Building 370 are eliminated. 


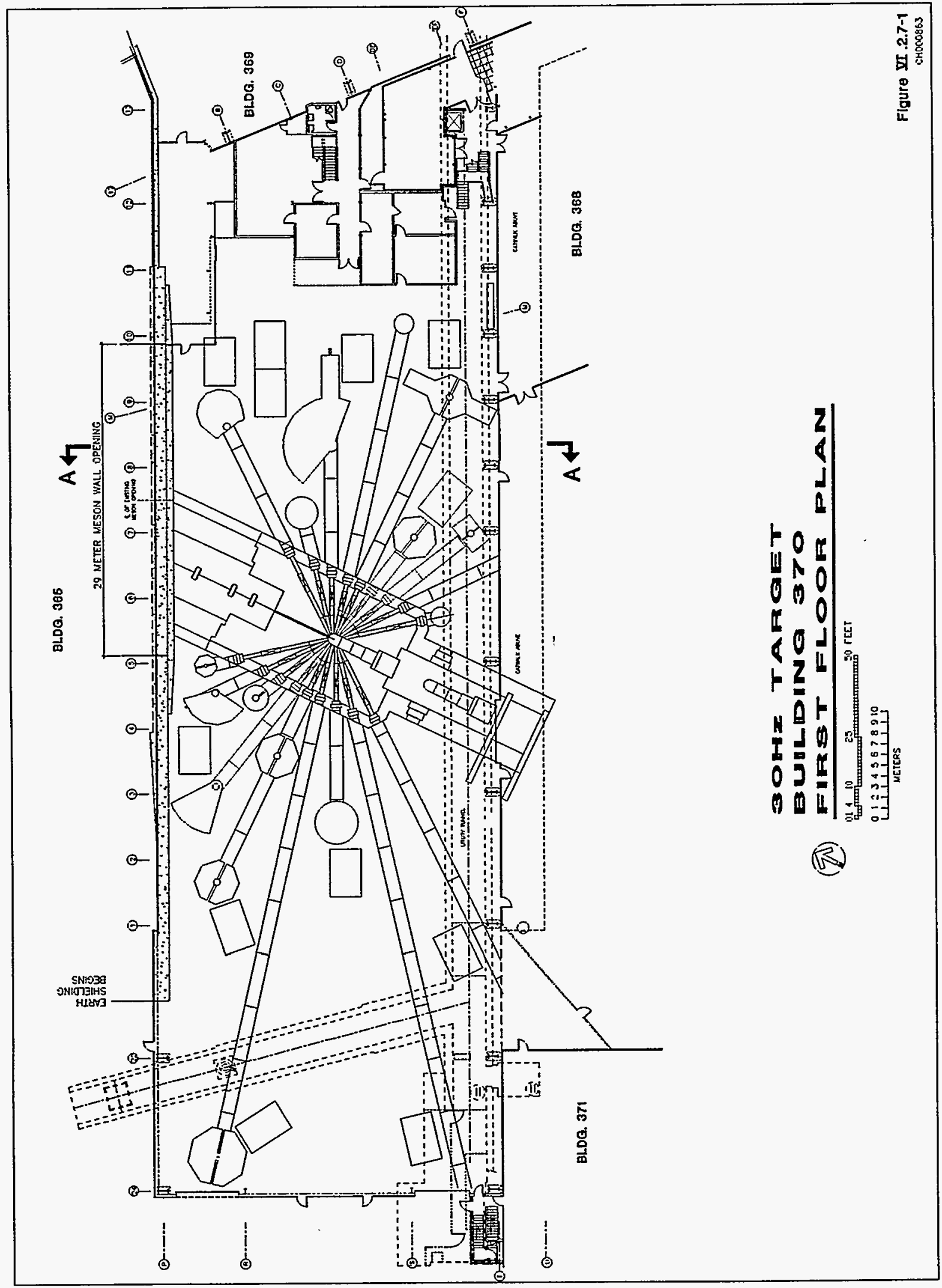




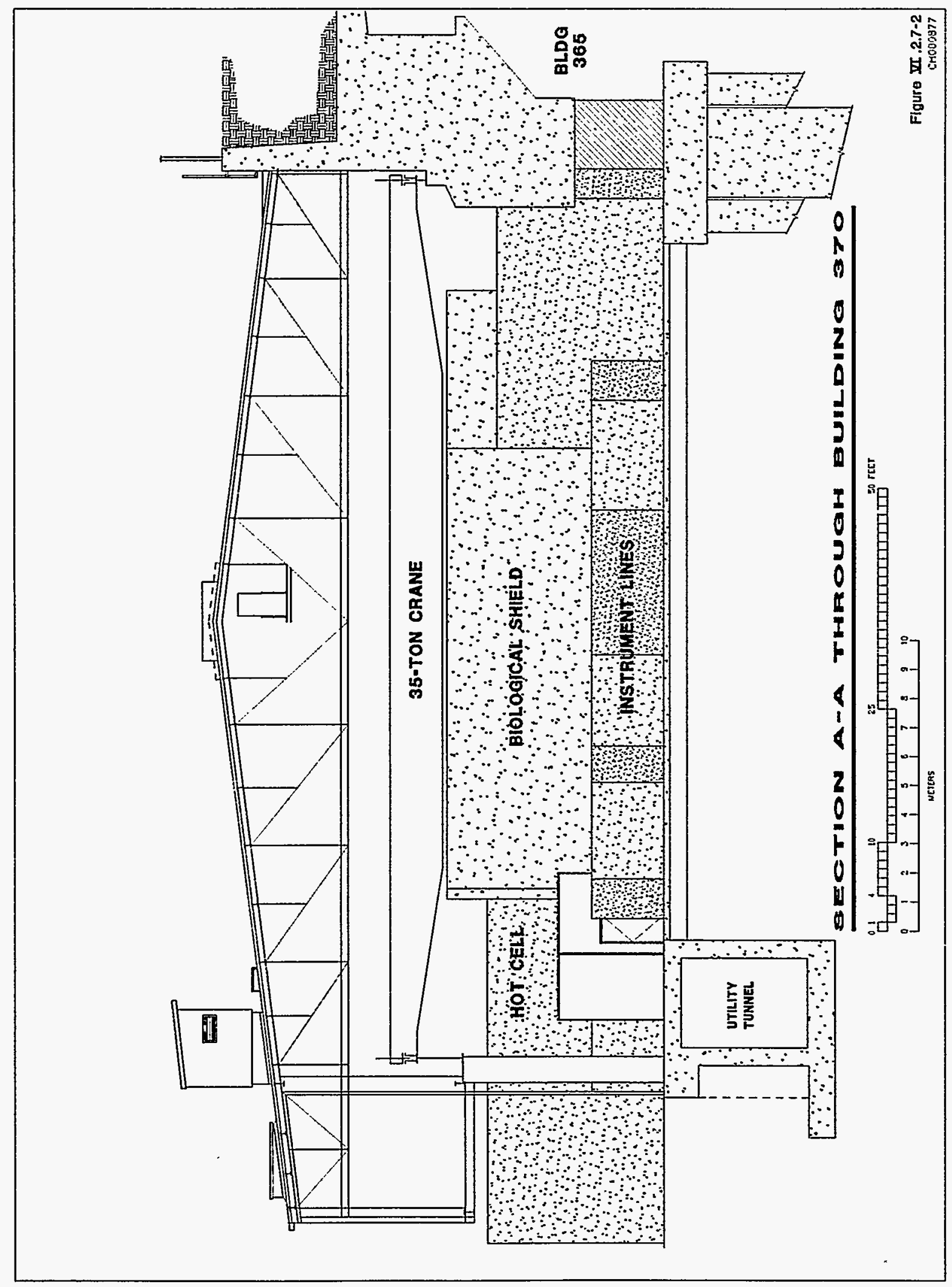


Tunnel. An existing tunnel system through the entire 360 area carries utilities, power, and controls and provides personnel access to these buildings. A 115.8-m-long utility tunnel runs under the south and west areas of the first floor of Building 370; this tunnel is $4.27-\mathrm{m}$ high $\times$ 3.04-m wide.

Cranes and Hoists. The high bay is equipped with a 35-ton, top-running bridge crane with full high-bay coverage, plus an auxiliary 10-ton hoist.

\subsubsection{Mechanical Systems (Building 370)}

Demolition. Miscellaneous mechanical systems and piping are relocated as necessary to facilitate construction of target biological shielding. A section of the utility tunnel passing under this building is filled with concrete.

Mechanical systems associated with target cooling and coolant retention systems are described in Chapter IV.

Central Plant and Piping. No DIW is required for the equipment in this building. Equipment utilizing DIW is located in Building 371. Existing CW piping is used for local cooling. New CW plant piping for local cooling is tied into the existing CW piping in the tunnel. Existing unit heaters are used. Heaters and piping are modified or moved to accommodate new equipment.

The following services are provided in the utility tunnel: Fire protection water, steam, $\mathrm{RW}$, hot water, $\mathrm{CW}$, condensate return, domestic cold water, two mechanical water systems, sanitary drain, DIW, ten wall-mounted cable trays, and electric power. The tunnel system has an automatic sprinkler system, fire-detection system, floor drains, and access panels to the building above. It is also used as a tornado shelter.

Air Distribution. The building is presently ventilated by four refurbished, truss-mounted air-handling units of $40,000 \mathrm{cfm}$ capacity each. Existing $\mathrm{CW}$ cooling coils that were not connected to $\mathrm{CW}$ piping are furnished with chilled water. New controls for all units are provided.

Existing diffusers and ductwork remain.

The six existing 89,000 -cfm hydrogen-evacuation fans on the roof are refurbished and used.

Plumbing and Fire Protection. Existing building plumbing systems are used. Existing fire protection systems are used, with upgrades to provide coverage for new equipment. 


\subsubsection{Electrical Systems (Building 370)}

The $30-\mathrm{Hz}$ target equipment is fed from a $480-\mathrm{V}$ double-ended substation, as shown in Figure VI.2.7-3. This substation has two $13.2-\mathrm{kV}$ incoming line switches, two 2,000-kVA transformers, and associated $480-\mathrm{V}$ switchgear. The $13.2-\mathrm{kV}$ switchgear is located outdoors near Building 370, and power is supplied to it by switchgear located at Site Electrical Facility 549. The 480-V switchgear is located inside Building 370 and feeds power to distribution panel boards located near the technical equipment. Individual equipment is powered from these panels. Clean power requirements are met by using $480-\mathrm{V}$ to $480-\mathrm{V}$ isolation transformers.

A separate instrumentation grounding system for all computers and computer-related equipment is furnished.

Cleaned, repaired, and relamped existing lighting fixtures provide adequate illumination. Lighting fixture circuit wiring is new to improve reliability. New breakers are provided for existing power and lighting panel board interiors.

Special systems, such as telephone, paging, and fire alarms, are adequate to meet requirements. 


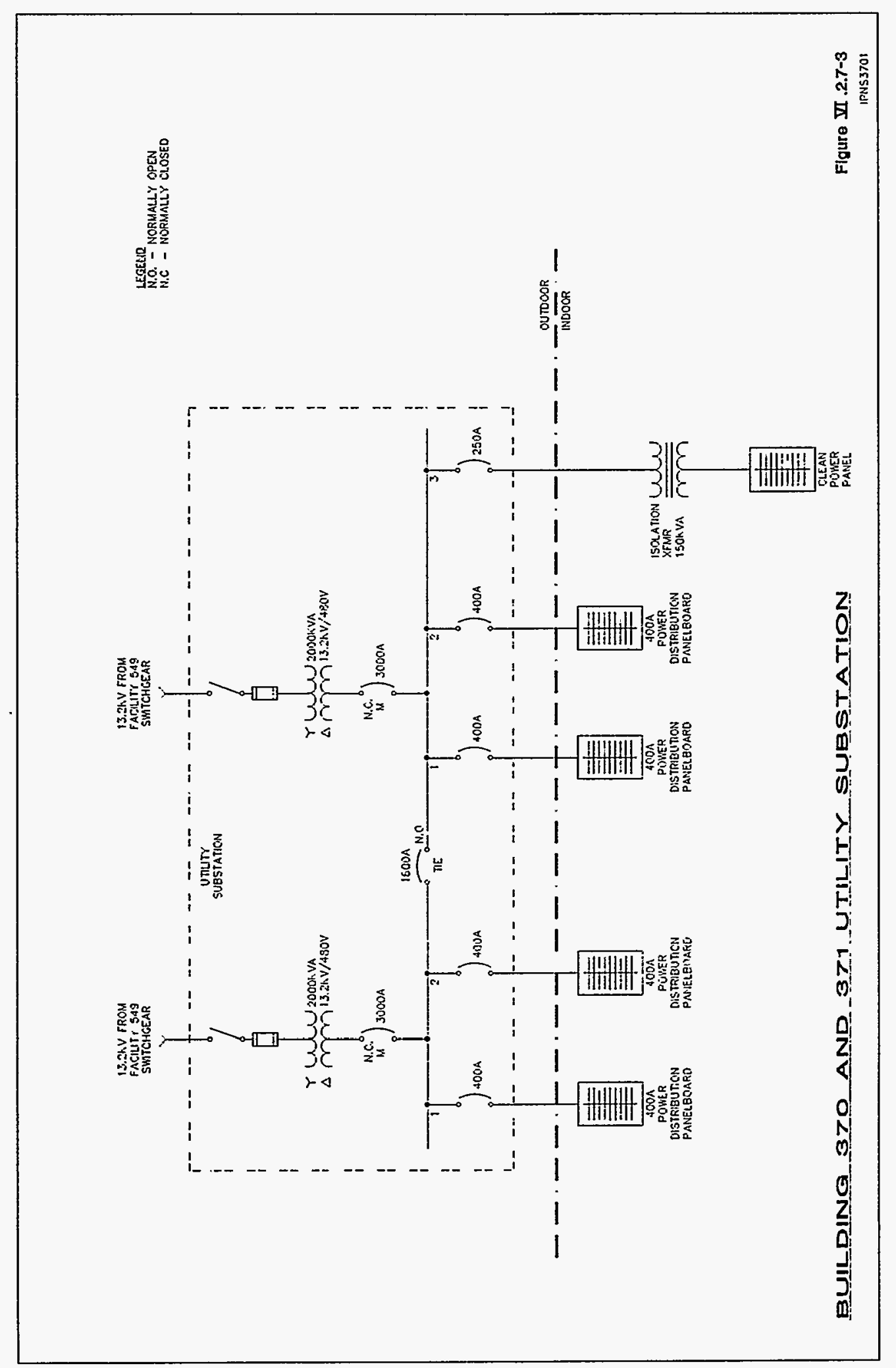




\subsection{0-Hz TARGET SUPPORT BUILDING (EXISTING BUILDING 371)}

Building 371 is a single-story high-bay building. It houses the cooling system for the $30-\mathrm{Hz}$ target located in Building 370 . The high bay is approximately $25 \mathrm{~m}$ wide $\times 31.7 \mathrm{~m}$ long, with a clear height of $12.4 \mathrm{~m}$ to the bottom of the roof trusses. An annex, Building 371A, adjacent to Building 371 is $32.7 \mathrm{~m}$ long $\times 16.0 \mathrm{~m}$ wide. A 35-ton crane serves the building. This building was completed in 1961 and, together with its annex, has a gross floor area of $1,361 \mathrm{~m}^{2}$. A new shielded cooling system, shown in Figure VI.2.8-1, is located in the high bay for the $30-\mathrm{Hz}$ target in Building 370. The target cooling system is self-contained and the cooling system area is shielded, because the coolant is potentially radioactive. Target cooling water pipes are double-walled construction and are direct-buried between Buildings 371 and 370 . Cross sections of Building 371 and of the adjacent underground vault are shown in Figure VI.2.8-2. Target cooling is discussed in detail in Chapter IV.

\subsubsection{Architecture (Building 371)}

Exterior Facade and Entrances. The exterior walls are constructed of vertical corrugated insulated metal panels supported on a steel frame structure. Exterior access to the high-bay area is provided by three personnel doors and two vehicle access doors. Vehicle parking is available adjacent to the building.

The only direct connection to the underground utility tunnel system is through an access pit at the north corner of the building.

The self-contained target-cooling system housed in this building falls within the scope of technical components and is described in Chapter IV.

Building refurbishments include minor repairs to the wall-panel system, as well as general painting, sealing, and caulking.

\subsubsection{Structure (Building 371)}

The building structural system consists of concrete foundations and steel columns supporting a truss-and-girder roof. Building column framing has structural steel bracing for the exterior metal-panel walls, providing resistance against lateral wind loads. Foundation walls are supported by conventional, reinforced-concrete spread footings. The floor is reinforced-concrete slab-on-grade construction and is $0.3 \mathrm{~m}$ thick, with a floor load capacity of $3,000 \mathrm{lb} / \mathrm{ft}^{2}$.

Cranes and Hoists. The high bay is equipped with a 35-ton, top-running bridge crane that provides high-bay coverage. A 10 -ton auxiliary hoist is also available.

Building Modifications. There are no modifications to Building 371, except for the addition of minor structural supports associated with the new installations. 


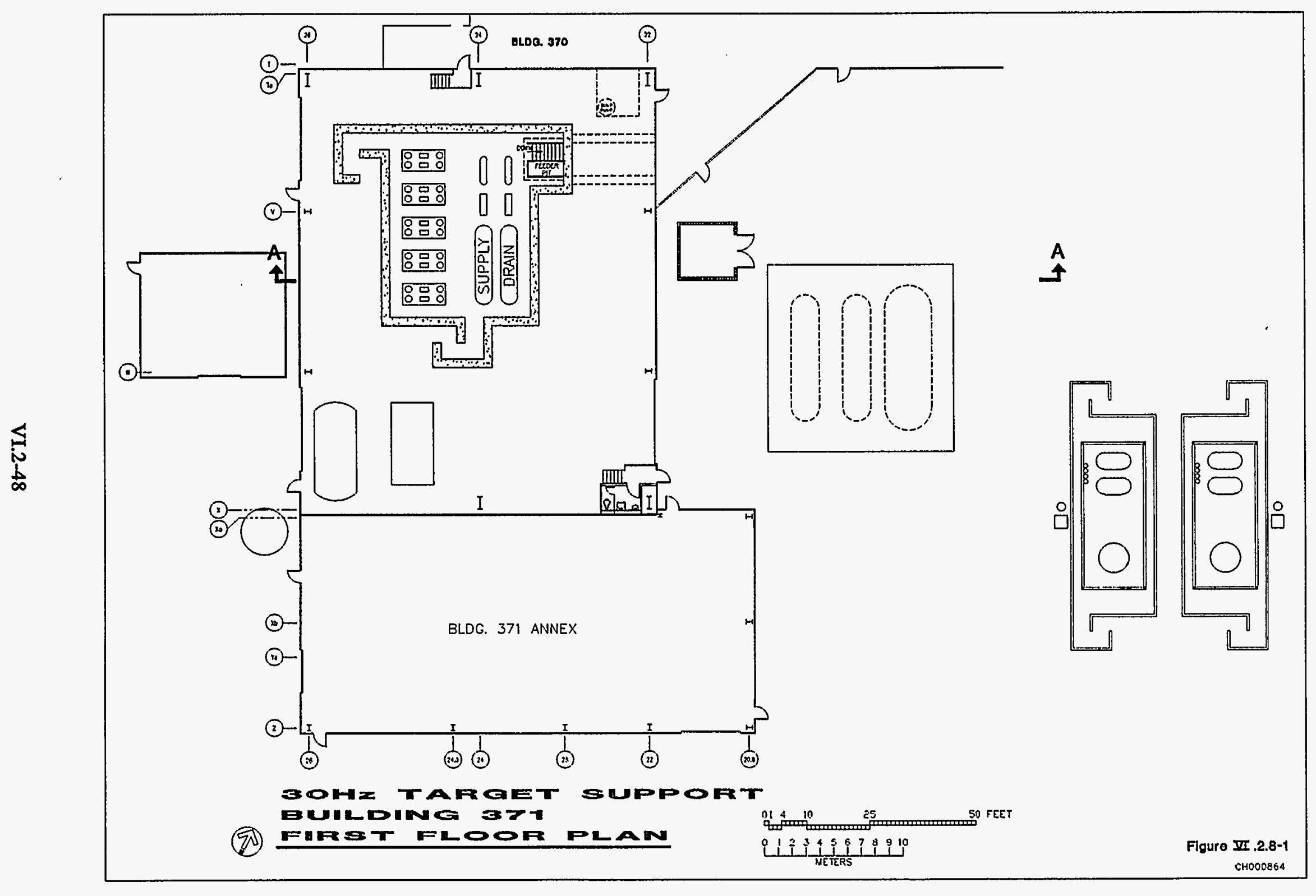




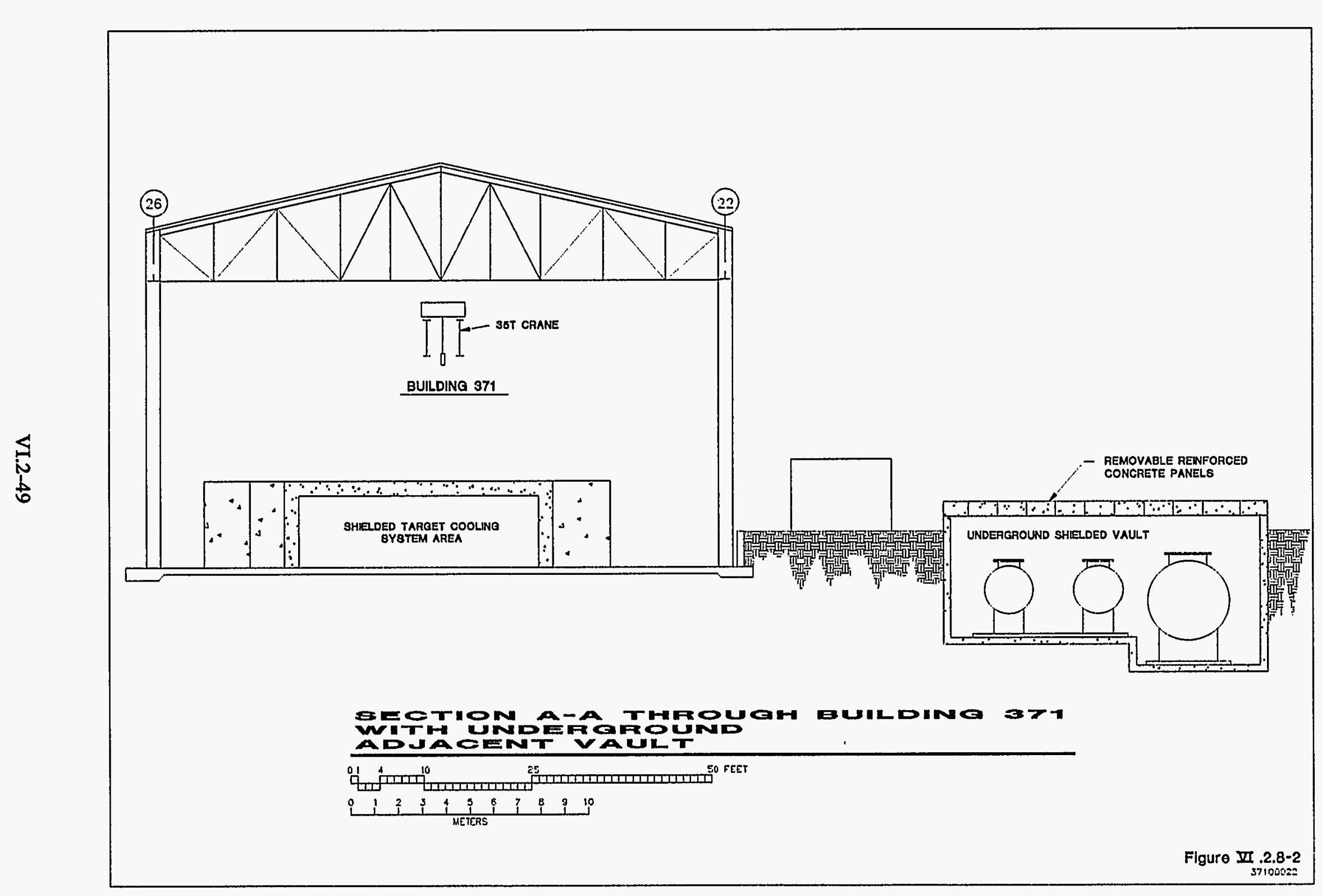




\subsubsection{Mechanical Systems (Building 371)}

Existing mechanical systems in the building are retained. Mechanical systems associated with the target cooling and coolant-retention systems are described in Chapter IV.

Central Plant Equipment and Piping. No central cooling plant equipment is located in the high bay. A previously inactive DIW plant in the adjacent Building $371 \mathrm{~A}$ is restored to its original capacity to furnish the required pressure and flow. Piping in the high bay is provided with capped valve-stubs to facilitate connection to user equipment.

Existing unit heaters are retained and are relocated as necessary.

Air Distribution. Two refurbished, truss-mounted heating and ventilating AHUs each provide $20,000 \mathrm{cfm}$ capacity. Six existing $89,000-\mathrm{cfm}$ hydrogen-evacuation fans on the roof are refurbished.

Plumbing and Fire Protection. Existing building toilet facilities and plumbing systems are used. Existing fire protection systems are used, with upgrades to provide coverage for new equipment.

\subsubsection{Electrical Systems (Building 371)}

Electrical power to Building 371 is delivered from $480-\mathrm{V}$ switchgear located in Building 370, as shown in Figure VI.2.7-3. This switchgear feeds $480-\mathrm{V}$ power distribution panel boards.

Lighting fixture circuit wiring is new to improve reliability. Cleaned, repaired, and relamped existing lighting fixtures provide adequate illumination. New breakers are provided for existing power and lighting panel board interiors.

Outdoor, high-pressure sodium fixtures provide perimeter lighting.

Emergency lighting is provided by 1.5 -h battery-powered units. Special systems, such as telephone, paging, and fire alarms, are adequate.

\subsection{PREPARATION LABORATORIES (EXISTING BUILDING 368)}

Building 368 houses preparation laboratories for the two target facilities. The building is $23 \mathrm{~m}$ wide $\times 67 \mathrm{~m}$ long $\times 4.6 \mathrm{~m}$ high. It was originally designed for heavy electrical distribution and has an experimental testing facility addition. This building, completed in 1963, has a gross floor area of $1,822 \mathrm{~m}^{2}$. 
A portion of the south wall of Building 370 forms the north wall of Building 368 , and part of the Building 369 west wall forms Building 368's east wall. The layout of Building 368, with adjacent areas of 369 and 370, is shown in Figure VI.2.9-1. The control room for the $10-\mathrm{Hz}$ target is located in the northeast corner of Building 368. The south half of this facility is used as a convenient laboratory and experiment preparation area. It has direct access to the two target buildings, as shown in Figure VI.2.9-1.

\subsubsection{Architecture (Building 368)}

The three exterior building walls are constructed of vertically corrugated, insulated metal panels on horizontal structural girts. Entrance to the building is provided by six personnel doors and two vehicle-access doors.

Roofing. In 1990, an IRMA roofing system with 5-cm-thick insulation board and 1-cmthick cement topping was installed over the existing roof structure.

Building Modifications. There are no changes in the building function. Therefore, only minor modifications, refurbishing, and painting are required.

\subsubsection{Structure (Building 368)}

The existing building structural system consists of concrete foundations and steel columns supporting a beam-and-girder framing system. Foundation walls are supported by conventional spread footings. The floor is reinforced-concrete, 0.15 -m-thick slab-on-grade with a floor load capacity of $800 \mathrm{lb} / \mathrm{ft}^{2}$.

Structural Modifications. Building function is unchanged, so no structural modifications are required.

\subsubsection{Mechanical Systems (Building 368)}

Demolition. The existing DIW plant, which consists of a double-barrel shell-and-tube heat exchanger, two DIW pumps, and DIW equipment with tank, is removed.

Central Plant Equipment and Piping. A new 3,000-gpm-capacity DIW plant, which consists of a plate heat exchanger, two DIW pumps, DIW equipment, and tanks, is installed. The new DIW plant is connected to existing RW and DIW in the adjacent utility tunnel system under Building 370 .

Existing unit heaters are reused and relocated as necessary. 


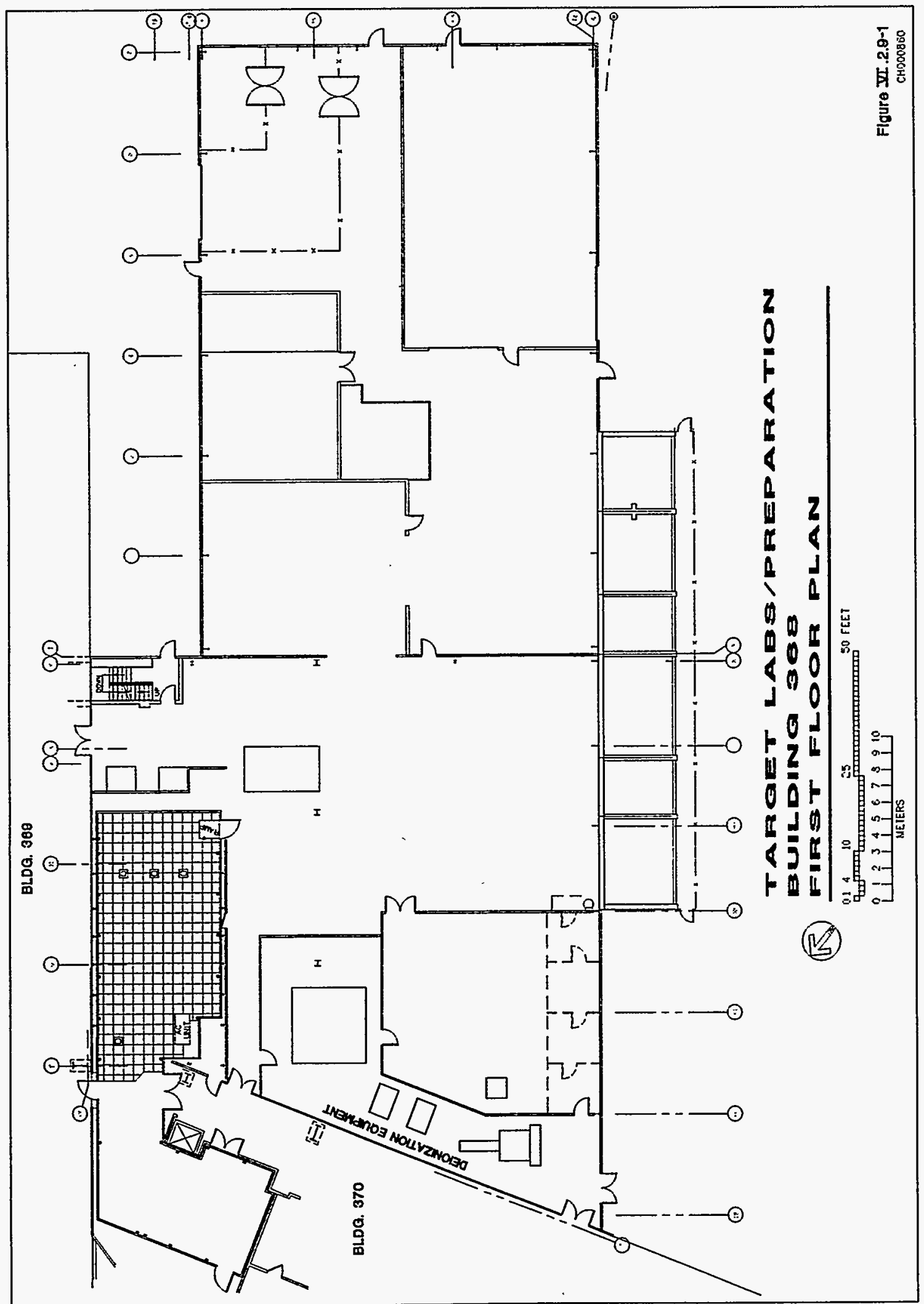


Air Distribution. The building uses existing, refurbished exhaust fans and louvers for ventilation. No additional air conditioning and no additional AHUs are provided. A refurbished AHU serves the transformer room.

Plumbing and Fire Protection. Existing building plumbing systems are used. Existing fire protection systems are used, with upgrades to provide coverage for new equipment.

\subsubsection{Electrical Systems (Building 368)} equipment.

Minor modifications are made to the electrical power system to feed mechanical

An existing 250-kW diesel-powered emergency generator is located in this building.

\subsection{LABORATORY AND OFFICE BUILDING I (EXISTING BUILDING 360)}

Building 360, a two-story laboratory and office building completed in 1961, houses the main accelerator control room, 22 laboratories, and 90 offices. The building is $56.4 \mathrm{~m}$ wide $\times$ $62.5 \mathrm{~m}$ long and has a gross floor area of $9,179 \mathrm{~m}^{2}$. It has a roof fan room and a service floor. The layout of the first floor is shown in Figure VI.2.10-1.

\subsubsection{Architecture (Building 360)}

The building exterior is constructed of brick and insulated metal-sandwich panels supported on a structural-steel framing system.

The two main entrances are located on the north and west facades. The west entry has an L-shaped covered walkway. Other entrances make connections to Buildings 361,362 , and 363. The service floor of Building 360 is connected by a utility tunnel to the service floors of Buildings 362 and 365 and to the utility tunnel system.

Typical rooms and corridors in this building consist of tiled floor, dropped acoustical ceiling with fluorescent lighting, and either stud-and-panel or concrete-block walls.

Vehicle parking areas are located to the north of the building and across Rock Road.

Specialty Rooms. The existing IPNS accelerator control room is located on the first floor, between administrative offices and experimental facilities. The existing control room is equipped with floor-mounted control consoles, which have direct raceway connections to the cable trays at the service floor below and to the rest of the IPNS complex. These cable trays and raceways are used to route all signal, control, power, and fiber-optic cables for the IPNS Upgrade accelerators. 


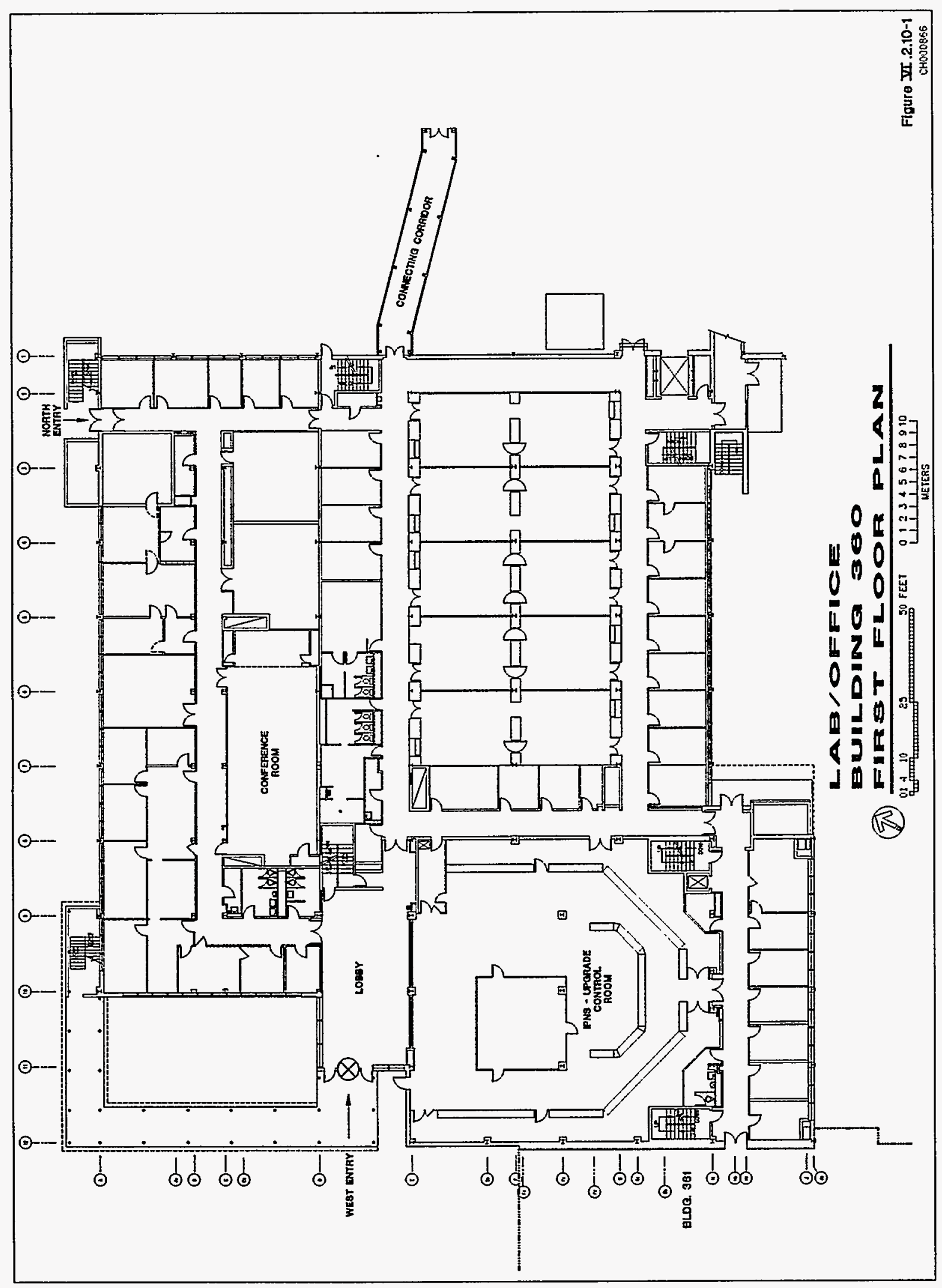


A 120-seat-capacity, theater-style conference room with a raised stage is located on the first floor. The $\mathbf{3 6 0}$ area main maintenance shop is located on the service floor. Building maintenance mechanics support all 360 area buildings from this shop.

Existing asbestos contamination, which is restricted to floor tiles and mechanical systems, is continually monitored by ANL Industrial Hygiene and PFS personnel.

Roofing. In 1990, an IRMA roofing system with 5-cm-thick insulation board and 1-cmthick cement topping was installed over the existing roof structure.

\subsubsection{Structure (Building 360)}

The structural system for Building 360 consists of steel columns supporting a beam-andgirder framing system. Building framing is supported on a reinforced-concrete foundation. The steel framing system supports reinforced-concrete floor and roof slabs.

Elevators and Hoists. A five-ton hydraulic service elevator serves the basement, first floor, second floor, and roof. The first-floor stop also opens to an exterior loading-dock area. A 0.75-ton hydraulic elevator stops in the basement and on the first floor. The basement maintenance shop has a one-ton monorail electric hoist.

Modifications. No architectural, structural, mechanical, or electrical alterations are required in Building 360 .

\subsubsection{Mechanical Systems (Building 360)}

Existing toilet facilities and plumbing systems are used. Existing fire protection systems are in satisfactory condition.

\subsubsection{Electrical Systems (Building 360)}

The existing electrical system is adequate to provide power to the required equipment. Lighting, telephone, paging, and fire alarm systems are adequate.

\subsection{LABORATORY AND OFFICE BUILDING II (EXISTING BUILDING 362)}

Building 362, a three-story laboratory and office building, houses a high-bay experimental assembly area, a 298-seat auditorium, library, 177 offices, and 84 laboratory spaces. This threestory building has a lower-level service floor. Completed in 1965, it has a gross floor area of $19,400 \mathrm{~m}^{2}$. Figure VI.2.11-1 shows the first-floor plan. 


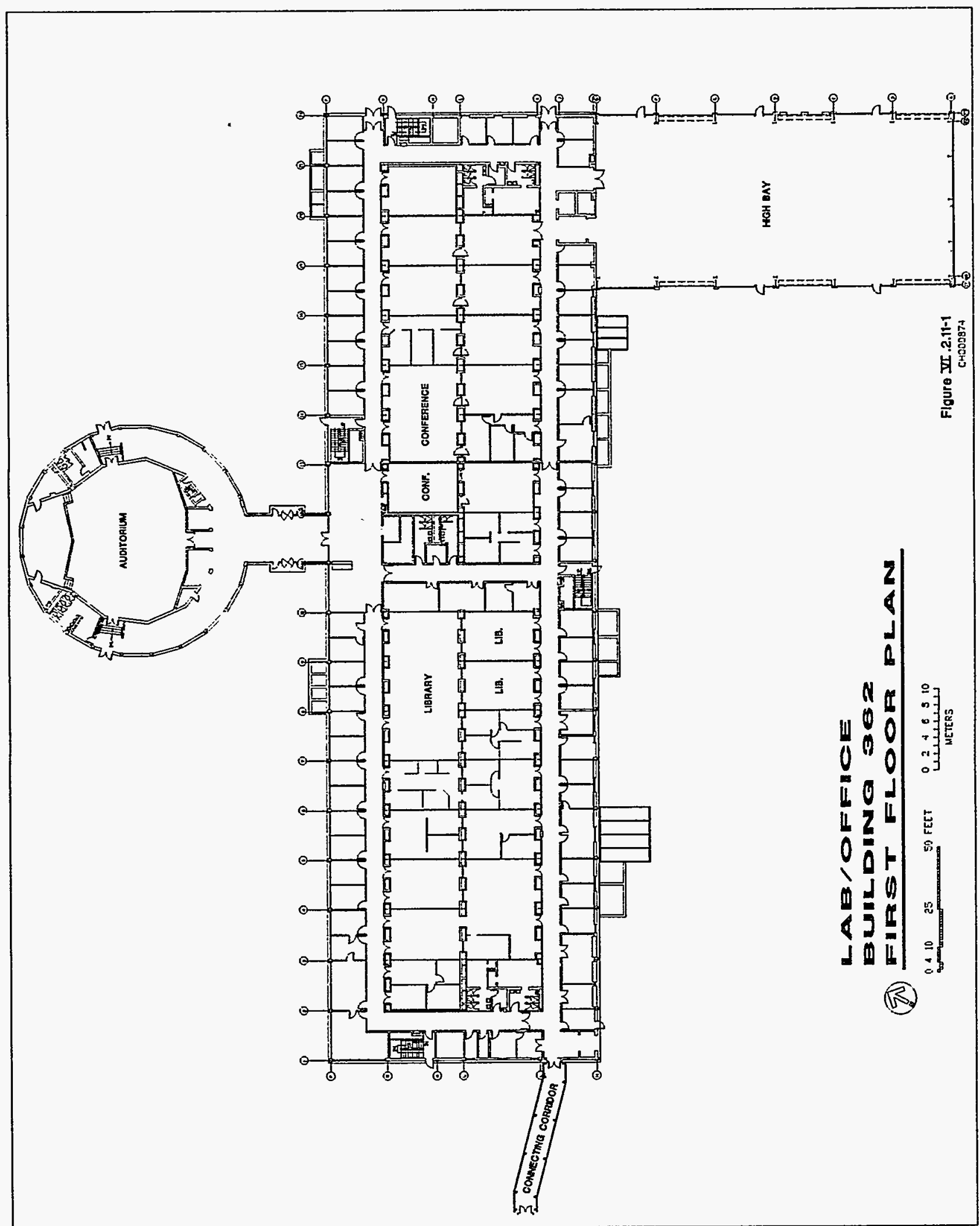


The building is $34.1 \mathrm{~m}$ wide $\times 112.8 \mathrm{~m}$ long and has a floor-to-floor height of $4.5 \mathrm{~m}$. A 28.0-m-diameter circular brick building containing the 298-seat auditorium is connected to Building 362 by a common-entry vestibule. The $21.5-\mathrm{m} \times 44.0-\mathrm{m}$ high-bay experimental and assembly area is located at the southeast corner of Building 362 . It has a 20-ton overhead crane that provides full coverage of the high-bay area. Parking for 300 cars is provided in three lots to the north of the building.

\subsubsection{Architecture (Building 362)}

The building exterior is composed of brick and vertical metal wall panels. The first floor facade has large horizontal windows with low-height brick sill walls. The second and third floors are faced with vertical metal sandwich panels with vertical window elements. There is a service floor below and a fan room at the roof level.

Primary access to the building and auditorium is provided by two entrances at the connecting entry vestibule to the auditorium. A two-level corridor connects Building 362 to Building 360 , with a tunnel below at the service floor level.

Typical rooms and corridors in this building consist of tiled floor, dropped acoustical ceiling with fluorescent lighting, and either stud-and-panel or concrete-block walls.

Specialty Rooms. A 298-seat auditorium adjacent to the central research laboratory and office area provides a facility for seminars, lecture activities, and other assemblies. Additional conference rooms are located within the building, providing facilities for 30, 40, and 70 people.

Roofing. In 1990, an IRMA roofing system with 5-cm-thick insulation board and $1-\mathrm{cm}$-thick cement topping was installed over the existing roof structure.

\subsubsection{Structure (Building 362)}

The Building 362 structural system consists of steel columns supporting a beam-andgirder framing system. Building framing is supported on a reinforced-concrete foundation. The steel framing system supports reinforced-concrete floor and roof slabs.

The spray-on fireproofing used to protect the structural-steel framing system contained asbestos. The original floor tile and mastic used in Building 362 also contain asbestos. A complete asbestos survey of the building was performed, and floor tiles in the first- and secondfloor corridors were replaced. Remaining areas with asbestos-containing materials are monitored and maintained by Industrial Hygiene and PFS personnel. Existing asbestos is not considered a major problem.

Cranes and Elevators. A 20-ton, top-running bridge crane in the high bay provides full coverage for the experimental assembly area. Building 362 is equipped with four elevators, the 
largest of which is a four-ton-capacity cable-hoist service elevator. The other three elevators are 1.25-ton-capacity cable-hoist elevators.

\subsubsection{Mechanical Systems (Building 362)}

No improvements or alterations to the existing mechanical systems are required. Existing toilet facilities and plumbing systems are in satisfactory condition. The existing fire protection system is in satisfactory condition.

\subsubsection{Electrical Systems (Building 362)}

Existing power distribution systems, lighting, telephone, PBX, paging, and fire alarm systems are adequate for IPNS Upgrade program requirements. Two $13.2-\mathrm{kV}$ to $480-\mathrm{V}$ electrical substations, one with a capacity of $2,500 \mathrm{kVA}$ and the other with a capacity of $1,000 \mathrm{kVA}$, were installed during 1993.

\subsection{UTILITIES AND SITE WORK}

The existing ZGS/360 site is over 30 years old and has all of the required utilities to support the IPNS Upgrade. Only minor modifications are required to connect the existing mechanical utilities to new equipment. Site modifications for the 10- and $30-\mathrm{Hz}$ target cooling systems, buried vaults, and cryogenic support facilities are described in Chapter IV.

\subsubsection{Site Mechanical Systems}

The existing site mechanical systems are refurbished and utilized wherever feasible. A new, modern chiller plant utilizing centrifugal chillers with CFC-free refrigerant is constructed at the service floor of Building 376. The plant includes new pumps, piping, and associated auxiliary equipment. The new chiller plant is connected to the existing Cooling Tower Facility 377, as shown in the Mechanical Site Plan in Figure VI.2.12-1. This cooling tower is completely rebuilt and is furnished with new condenser water pumps, as shown in the 377 Area Cooling Tower Plan in Figure VI.2.12-2. New cooling towers are not required for the IPNS Upgrade. The existing piping in the site utility tunnels, including steam and condensate along with $\mathrm{CW}$, condenser, and DIW piping, is retained and modified as necessary to accommodate increased utility service demands associated with the IPNS Upgrade.

A new DIW plant is located in Building 364. The existing DIW plant in Building 371A is upgraded, and the existing DIW plant in Building 368 is replaced. The 360/ZGS Mechanical Site Plan is shown in Figure VI.2.12-1. New DIW piping and fittings are constructed of stainless steel. The existing shell-and-tube RW-to-DIW heat exchanger and associated pumps in Building $371 \mathrm{~A}$ are reactivated. 


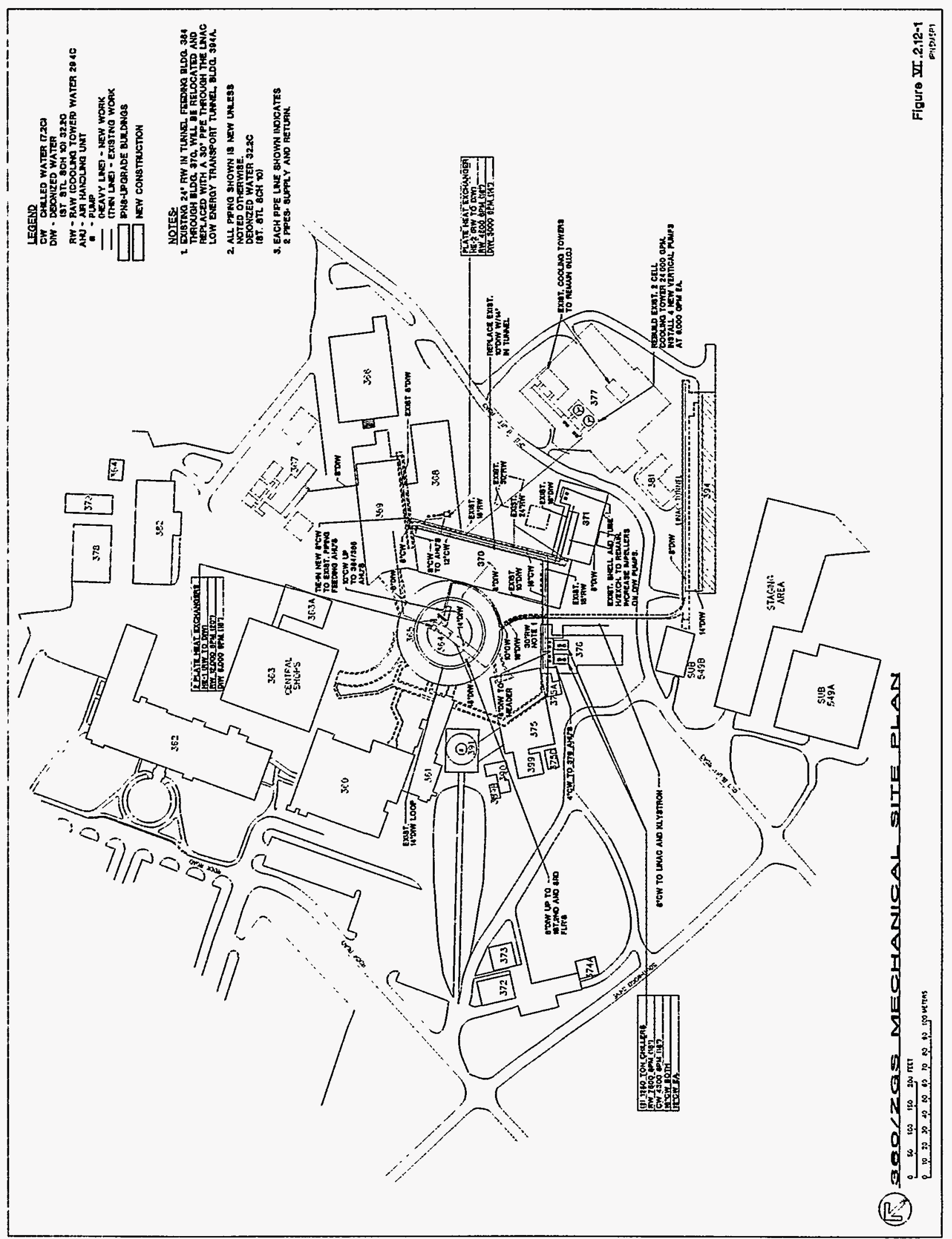




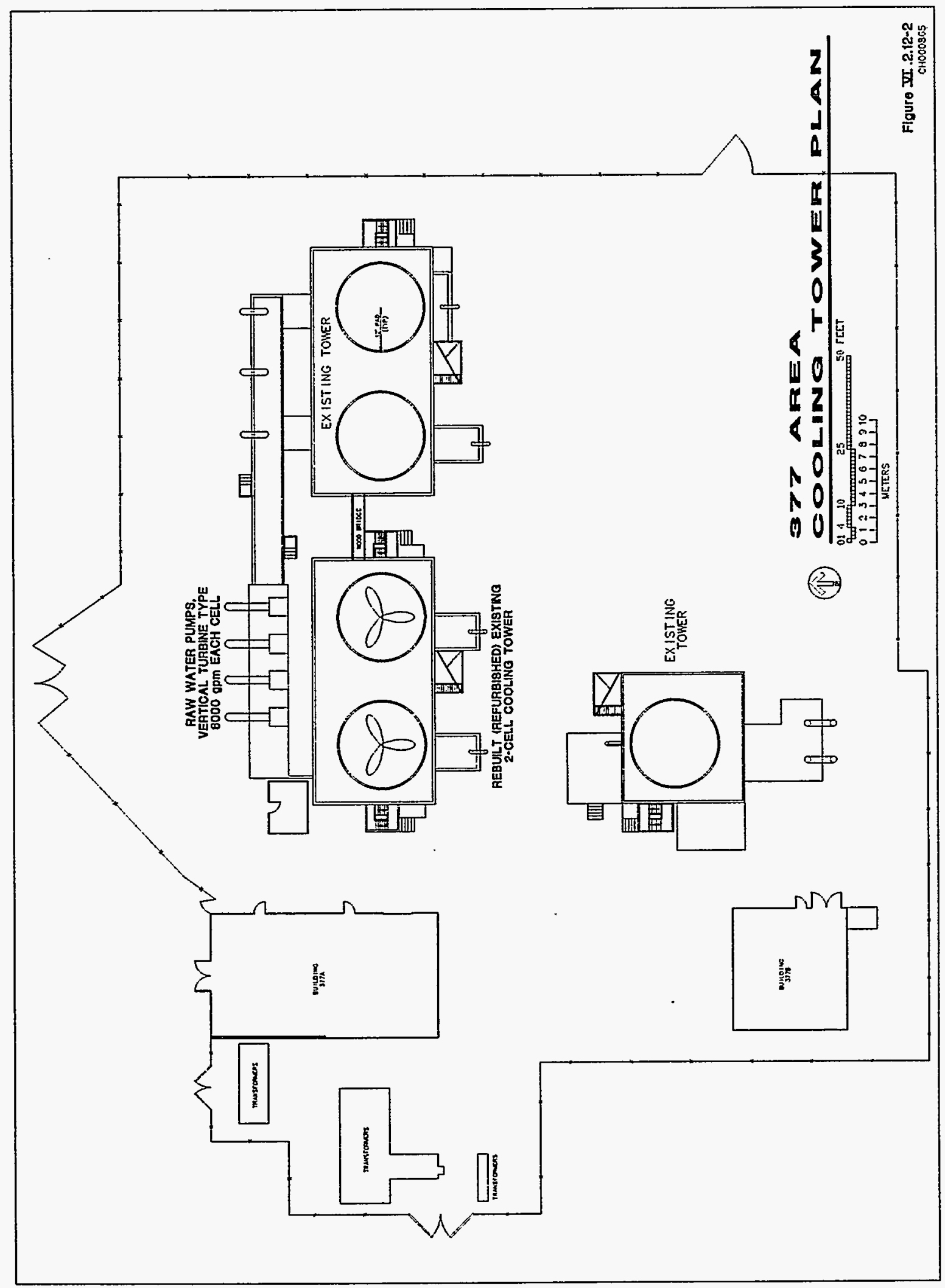


Existing site steam and condensate return piping systems furnished by the Argonne central boiler plant are utilized. The piping is modified and extended to accommodate a small number of new AHUs. Some of the underground steam piping is replaced.

Existing site plumbing systems are reused. No major modifications are anticipated. Existing site fire protection systems, including sprinkler piping, are used with minor modifications to accommodate new equipment in accordance with applicable standards.

\subsubsection{Site Power Distribution System}

The existing 360 site receives electrical power from site Electrical Facility 549A. A new 13.2-kV switchgear line-up in a prefabricated building at Facility 549 provides power to new technical equipment in various buildings, as shown on Figure VI.2.12-3, the 360/ZGS Electrical Site Plan. New $13.2-\mathrm{kV}$ cable is routed to these buildings via underground duct banks and manholes. This switchgear line-up receives power from existing $138-\mathrm{kV}$ to $13.2-\mathrm{kV}$ transformers located at this facility. Building utility substations and diesel emergency power generators for the area are shown on Figure VI.2.12-3, the 360/ZGS Electrical Site Plan.

\subsubsection{Site Work}

The existing $\mathrm{ZGS} / 360$ area requires a minimum of site work to facilitate the installation of the IPNS Upgrade. The only major new site development is the construction of the new Linac Building and earth-bermed Linac and LET tunnel, as shown in Figure VI.1.1-2. Site work associated with additional concrete shielding over the LET tunnel results in the rebuilding of Old Bluff Road, as shown in section B-1 in Figure VI.2.1-3. Two existing, 475-mm chilled water pipes are lowered $1 \mathrm{~m}$ so they pass under the LET tunnel, to eliminate interference. This is shown in Figure VI.2.1-2, Section through Linac Facility.

The buried Linac and LET tunnels use earth berms for shielding. At the narrow area between Buildings 376 and 370, the LET changes elevation to cross over the existing ZGS utility tunnel, and the berm is contained with concrete retaining walls. A steel-grating stair allows pedestrian access over the LET berm, as shown in section B-2 in Figure VI.2.1-3.

Excavation and shoring of the existing earth berm at the RCS Ring Building 365 are required at the LET tunnel connection area. New buried piping between the target Buildings 369 and 370 and target cooling systems in Buildings 367 and 371 require minor trenching and replacement of pavement areas along piping lines shown in Figure VI.2.12-1, 360/ZGS Mechanical Site Area. 


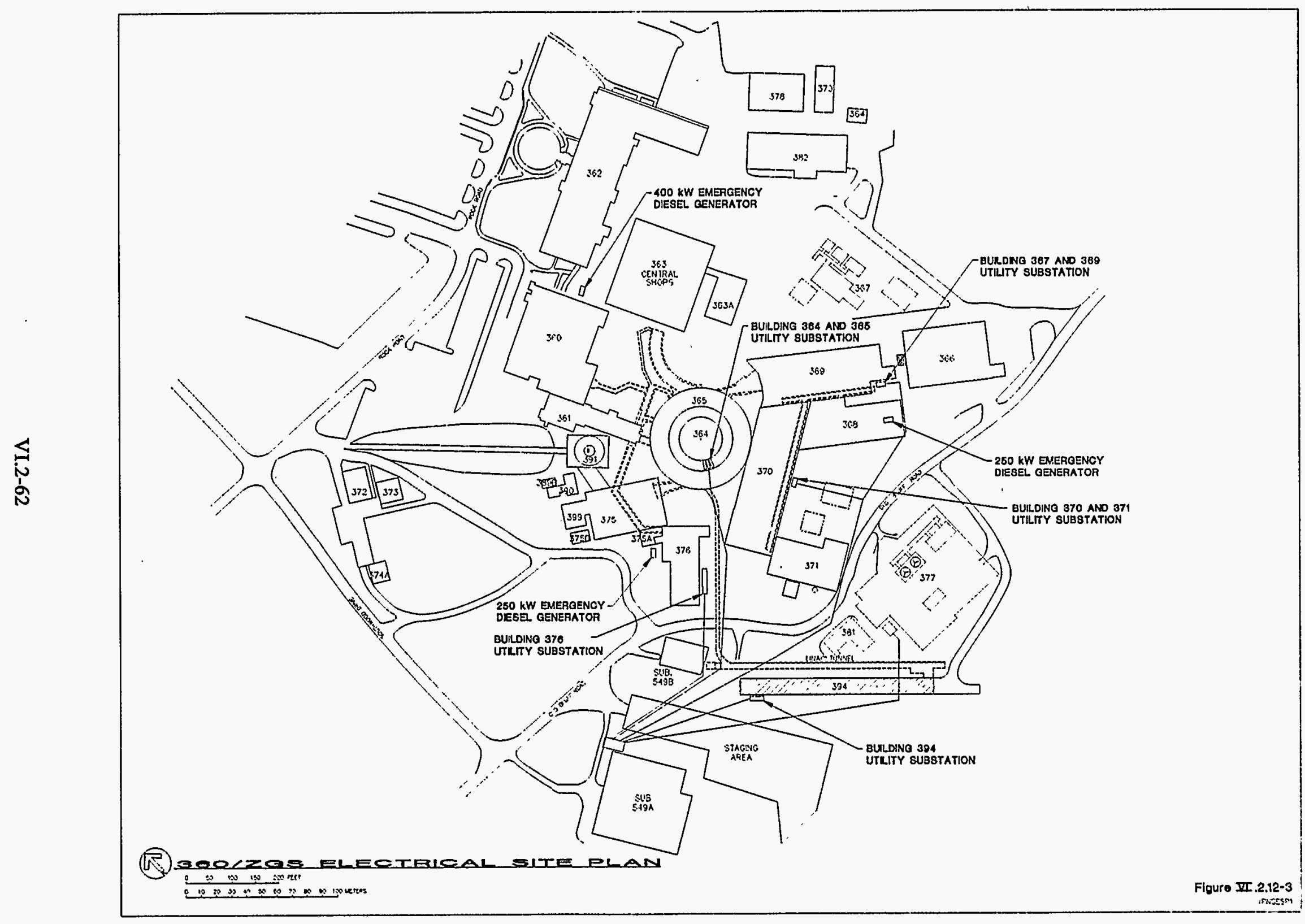


Cost and Schedule 
All costs for the IPNS Upgrade Project are developed from the work breakdown structure (WBS) by using the same method as that used for the Advanced Photon Source (APS) Project. The WBS encompasses all elements of the construction phase, from design to end of construction. The accelerator and neutron-generating target facilities' technical components and the initial set of beamlines and instrumentation were designed and cost-estimated by ANL personnel. The linac was designed and cost-estimated by AccSys Technology, Inc. The conventional facility designs associated with modifications to the existing buildings and with new construction were developed by personnel from ANL's Plant Facilities and Services Division (PFS) and by APS staff; these designs were cost-estimated by the construction consulting firm of Hanscomb Associates, Inc. This firm did the cost estimate for the APS project.

The WBS in Table VII.1-1 shows the project at Level 1. Level 2, the responsibility and functionality level, groups functionally identifiable items of the project. Also included in this level are project management, contingency, and miscellaneous costs, such as Laboratory overhead and materials and service expenses of the Laboratory staff. Level 3 in the WBS identifies major components of the facility, such as the linac, synchrotron, and target stations. The engineering, design, and inspection (ED\&I) costs for each Level 2 item are shown in Level 3.

The cost estimates include all costs incurred after project approval. The project scope includes the accelerator facility, two neutron-generating target stations, an initial complement of 27 instruments, conventional facilities, and associated ED\&I and project management. Preconstruction research and development $(R \& D)$ and preoperational activities required for commissioning of the facility and/or for the scientific research program are not included in the estimate.

The time estimates for engineering, design, and construction of the conventional facilities and the beneficial occupancy date of each building were generated by the APS and PFS staff. The beneficial occupancy dates are used to set schedules for activities related to the technical components. The schedule assumes availability of fiscal year (FY) 1996 preconstruction R\&D funds. 
Table VII.1-1 Complete Work Breakdown Structure

\begin{tabular}{|c|c|}
\hline WBS Code & Element Description \\
\hline 1 & IPNS UPGRADE (1 MW) \\
\hline 1.1 & Project Management \\
\hline 1.1 .1 & Project Direction \\
\hline 1.1 .2 & Project Direction Support \\
\hline 1.2 & Accelerator Systems \\
\hline 1.2 .1 & Injector System \\
\hline 1.2.1.1 & Linac Injector System \\
\hline 1.2 .1 .2 & DTL \\
\hline 1.2 .1 .3 & Matching Section \\
\hline 1.2 .1 .4 & $\mathrm{SCL}$ \\
\hline 1.2.1.5 & DTL if Systems \\
\hline 1.2 .1 .6 & Matching Section rf Systems \\
\hline 1.2.1.7 & SCL if Systems \\
\hline 1.2 .1 .8 & Beam Diagnostics \\
\hline 1.2 .1 .9 & Control Systems \\
\hline 1.2 .1 .10 & Documentation and Training \\
\hline 1.2 .1 .11 & Installation \\
\hline 1.2.1.12 & ANL Support \\
\hline 1.2 .2 & Main Accelerator System \\
\hline 1.2.2.1 & Synchrotron \\
\hline 1.2.2.1.1 & Magnets \\
\hline 1.2.2.1.1.1 & Dipole Magnet \\
\hline 1.2.2.1.1.2 & Quadrupole Magnet \\
\hline 1.2.2.1.1.3 & Sextupole Magnet \\
\hline 1.2.2.1.1.4 & Corrector Magnets \\
\hline 1.2.2.1.1.5 & Injection (Inj.) Magnets \\
\hline 1.2 .2 .1 .1 .5 .1 & Inj. Septum A \\
\hline 1.2 .2 .1 .1 .5 .2 & Inj. Septum B \\
\hline 1.2 .2 .1 .1 .5 .3 & Inj. Bumper \\
\hline 1.2 .2 .1 .1 .6 & Extraction (Extr.) Magnets \\
\hline 1.2.2.1.1.6.1 & Extr. Kicker A \\
\hline 1.2.2.1.1.6.2 & Extr. Kicker B \\
\hline 1.2.2.1.1.6.3 & Extr. Kicker C \\
\hline 1.2.2.1.1.6.4 & Extr. Septum C \\
\hline 1.2.2.1.1.6.5 & Extr. Septum D \\
\hline 1.2 .2 .1 .1 .6 .6 & Extr. Septum E \\
\hline 1.2 .2 .1 .1 .7 & Supports \\
\hline 1.2.2.1.1.8 & Installation \\
\hline 1.2.2.1.1.9 & Magnet ED\& $\left.\right|^{a}$ \\
\hline 1.2 .2 .1 .2 & Power Supplies \\
\hline 1.2.2.1.2.1 & Power Supply Dipole \\
\hline 1.2 .2 .1 .2 .1 .1 & Power Supply Dipole \\
\hline 1.2 .2 .1 .2 .1 .2 & Installation and Wiring \\
\hline 1.2.2.1.2.2 & Power Supply Quadrupole \\
\hline
\end{tabular}


Table VII.1-1 (Cont)

WBS Code Element Description

1.2.2.1.2.2.1

1.2.2.1.2.2.2

1.2.2.1.2.3

1.2.2.1.2.3.1

1.2.2.1.2.3.2

1.2.2.1.2.4

1.2.2.1.2.4.1

1.2.2.1.2.4.1.1

1.2.2.1.2.4.1.2

1.2.2.1.2.4.2

1.2.2.1.2.4.2.1

1.2.2.1.2.4.2.2

$1.2 .2 .1 \cdot 2.4 .3$

1.2.2.1.2.4.3.1

1.2.2.1.2.4.3.2

1.2.2.1.2.5

1.2.2.1.2.5.1

1.2.2.1.2.5.1.1

1.2.2.1.2.5.1.2

1.2.2.1.2.5.2

1.2.2.1.2.5.2.1

1.2.2.1.2.5.2.2

1.2.2.1.2.5.3

1.2.2.1.2.5.3.1

1.2.2.1.2.5.3.2

1.2.2.1.2.6

1.2.2.1.2.6.1

1.2.2.1.2.6.2

1.2.2.1.3

1.2.2.1.3.1

1.2.2.1.3.2

1.2.2.1.3.3

1.2.2.1.3.4

1.2.2.1.3.5

1.2.2.1.3.6

1.2.2.1.4

1.2.2.1.4.1

1.2.2.1.4.2

1.2.2.1.4.3

1.2.2.1.4.4

1.2.2.1.5

1.2.2.1.5.1

1.2 .2 .1 .5 .2

1.2.2.1.5.3

1.2 .2 .1 .5 .4

1.2.2.1.5.5
Power Supply Quadrupole

Installation and Wiring

Power Supply Sextupole

Power Supply Sextupole

Installation and Wiring

Injection Magnets

Power Supply Inj. Bumper

Power Supply Inj. Bumper

Installation and Wiring

Power Supply Inj. Septum

Power Supply Inj. Septum

Installation and Wiring

Power Supply Inj. Thick Septum

Power Supply Inj. Thick Septum

Installation and Wiring

Extraction Magnets

Power Supply Extr. Kicker

Power Supply Extr. Kicker

Installation and Wiring

Power Supply Extr. Thin Septum

Power Supply Extr. Thin Septum

Installation and Wiring

Power Supply Extr. Thick Septum

Power Supply Extr. Thick Septum

Installation and Wiring

Power Supply H/V Corrector

Power Supply H/V Corrector

Installation and Wiring

Vacuum

Vacuum Chambers

Pumping

Monitoring

Valves

Bellows

Misc. Vacuum Hardware

if

If Cavities

Power Amplifiers

Low-Level System

if Effort

Diagnostics

Current Monitors

Position Monitors

Beam Loss System

Segmented Faraday Cups

Beam Scraper System 
Table VII.1-1 (Cont.)

\begin{tabular}{|c|c|}
\hline WBS Code & Element Description \\
\hline 1.2.2.1.5.6 & Beam Excitation System \\
\hline 1.2.2.1.5.7 & ED\&I Effort \\
\hline 1.2.2.1.6 & Controls/Interlocks \\
\hline 1.2.2.1.6.1 & Network Infrastructure \\
\hline 1.2.2.1.6.2 & Diagnostics Node \\
\hline 1.2.2.1.6.3 & if Node \\
\hline 1.2.2.1.6.4 & Synchrotron Vacuum, Power Supply Node \\
\hline 1.2.2.1.6.5 & Injector Systems Computer \\
\hline 1.2.2.1.6.6 & Timing Node \\
\hline 1.2.2.1.6.7 & Cable X-Bar Plant \\
\hline 1.2.2.1.6.8 & Local Consoles \\
\hline 1.2.2.1.7 & Personnel Access Control System \\
\hline 1.2 .3 & Beam Transports \\
\hline 1.2.3.1 & Low-Energy Beam Transport \\
\hline 1.2.3.1.1 & Magnets \\
\hline 1.2.3.1.1.1 & Dipole Magnet \\
\hline 1.2.3.1.1.2 & Quadrupole Magnet \\
\hline 1.2.3.1.1.3 & Correction Magnet \\
\hline 1.2.3.1.1.4 & Supports \\
\hline 1.2.3.1.1.5 & Installation \\
\hline 1.2.3.1.1.6 & Magnet ED\&I \\
\hline 1.2.3.1.2 & Power Supplies \\
\hline 1.2.3.1.2.1 & Power Supply Dipole \\
\hline 1.2.3.1.2.1.1 & Power Supply Dipole \\
\hline 1.2.3.1.2.1.2 & Installation and Wiring \\
\hline 1.2.3.1.2.2 & Power Supply Quadrupole \\
\hline 1.2.3.1.2.2.1 & Power Supply Quadrupole \\
\hline 1.2.3.1.2.2.2 & Installation and Wiring \\
\hline 1.2.3.1.2.3 & Power Supply Horizontal/Vertical (HV) Corrector \\
\hline 1.2.3.1.2.3.1 & Power Supply HN Corrector \\
\hline 1.2.3.1.2.3.2 & Installation and Wiring \\
\hline 1.2.3.1.3 & Vacuum \\
\hline 1.2.3.1.3.1 & Vacuum Chambers \\
\hline 1.2.3.1.3.2 & Pumping \\
\hline 1.2.3.1.3.3 & Monitoring \\
\hline 1.2.3.1.3.4 & Valves \\
\hline 1.2.3.1.3.5 & Bellows \\
\hline 1.2.3.1.3.6 & Misc. Vacuum Hardware \\
\hline 1.2.3.1.5 & Diagnostics \\
\hline 1.2.3.1.5.1 & Current Monitors \\
\hline 1.2.3.1.5.2 & Position Monitors \\
\hline 1.2.3.1.5.3 & Beam Loss System \\
\hline 1.2.3.1.5.4 & Profile Monitors \\
\hline 1.2.3.1.5.7 & ED\&I Effort \\
\hline 1.2.3.2 & High-Energy Beam Transport \\
\hline
\end{tabular}


Table VII.1-1 (Cont.)

\begin{tabular}{|c|c|}
\hline WBS Code & Element Description \\
\hline 1.2.3.2.1 & Magnets \\
\hline 1.2.3.2.1.1 & Dipole Magnet \\
\hline 1.2.3.2.1.2 & Quadrupole Magnet \\
\hline 1.2.3.2.1.3 & Correction Magnet \\
\hline 1.2.3.2.1.4 & Supports \\
\hline 1.2.3.2.1.5 & Installation \\
\hline 1.2.3.2.1.6 & Magnet ED\&I \\
\hline 1.2.3.2.2 & Power Supplies \\
\hline 1.2.3.2.2.1 & Power Supply Dipole (10-Hz transport) \\
\hline 1.2.3.2.2.1.1 & Power Supply Dipole (10-Hz transport) \\
\hline 1.2.3.2.2.1.2 & Installation and Wiring \\
\hline 1.2.3.2.2.2 & Power Supply Quadrupole (10-Hz transport) \\
\hline 1.2.3.2.2.2.1 & Power Supply Quadrupole (10-Hz transport) \\
\hline 1.2.3.2.2.2.2 & Installation and Wiring \\
\hline 1.2.3.2.2.3 & Power Supply H/V Corrector (10-Hz transport) \\
\hline 1.2.3.2.2.3.1 & Power Supply HIV Corrector (10-Hz transport) \\
\hline 1.2.3.2.2.3.2 & Installation and Wiring \\
\hline 1.2.3.3.2.4 & Power Supply Dipole (30-Hz transport) \\
\hline 1.2.3.3.2.4.1 & Power Supply Dipole (30-Hz transport) \\
\hline 1.2.3.3.2.4.2 & Installation and Wiring \\
\hline 1.2.3.3.2.5 & Power Supply Quadrupole (30-Hz transport) \\
\hline 1.2.3.3.2.5.1 & Power Supply Quadrupole (30-Hz transport) \\
\hline 1.2.3.3.2.5.2 & Installation and Wiring \\
\hline 1.2.3.3.2.6 & Power Supply $\mathrm{H} N$ Corrector $(30-\mathrm{Hz}$ transport) \\
\hline 1.2.3.3.2.6.1 & Power Supply $\mathrm{H} / \mathrm{V}$ Corrector $(30-\mathrm{Hz}$ transport) \\
\hline 1.2.3.3.2.6.2 & Installation and Wiring \\
\hline 1.2.3.2.3 & Vacuum \\
\hline 1.2.3.2.3.1 & Vacuum Chambers \\
\hline 1.2.3.2.3.2 & Pumping \\
\hline 1.2.3.2.3.3 & Monitoring \\
\hline 1.2.3.2.3.4 & Valves \\
\hline 1.2.3.2.3.5 & Bellows \\
\hline 1.2.3.2.3.6 & Misc. Vacuum Hardware \\
\hline 1.2.3.2.5 & Diagnostics \\
\hline 1.2.3.2.5.1 & Current Monitors \\
\hline 1.2.3.2.5.2 & Position Monitors \\
\hline 1.2.3.2.5.3 & Beam Loss System \\
\hline 1.2.3.2.5.4 & Profile Monitors \\
\hline 1.2.3.2.5.5 & Target Protection Monitor \\
\hline 1.2.3.2.5.7 & ED\&I Effort \\
\hline 1.2 .4 & Technical Support Systems \\
\hline 1.2 .4 .1 & Central Control System \\
\hline 1.2.4.1.1 & Central Control Computer System \\
\hline 1.2.4.1.1.1 & File Server and Host Computer \\
\hline 1.2.4.1.1.2 & Disk Drives \\
\hline 1.2.4.1.1.3 & Printers \\
\hline
\end{tabular}


Table VII.1-1 (Cont.)

\section{WBS Code}

Element Description

1.2.4.1.1.4

1.2.4.1.1.5

1.2.4.1.1.6

1.2.4.1.1.7

1.2.4.1.1.8

1.2.4.1.1.9

1.2.4.1.2

1.2.4.1.2.1

1.2.4.1.2.2

1.2.4.1.2.3

1.2.4.1.2.4

1.2.4.1.2.5

1.2.4.1.2.6

1.2.4.1.2.7

1.2:4.1.2.8

1.2.4.1.2.9

1.2.4.1.3

1.2.4.1.3.1

1.2.4.1.3.2

1.2.4.1.3.3

1.2.4.1.3.4

1.2.4.1.4

1.2.4.1.4.1

1.2.4.1.4.2

1.2.4.2

1.2.4.2.1

1.2.4.2.2

1.2.4.2.3

1.2.4.2.4

1.2.4.3

1.2.4.3.1

1.3

1.3.1

1.3.1.1

1.3.1.1.1

1.3.1.1.1.1

1.3.1.1.1.2

1.3.1.1.1.3

1.3.1.1.1.4

1.3.1.1.1.5

1.3.1.1.1.6

1.3.1.1.1.7

1.3.1.1.2

1.3.1.1.2.1

1.3.1.1.2.1.1
Tape Drives

$X$ Terminals

Central Network Equipment

Terminal Server Interface

Software Licenses

Operator Consoles

Control Consoles

Console Computers

Network Equipment

Engineering Development Computer

Software Licenses

Video Processors

Video Displays

Main Control Room Tables and Racks

Signal Display Systems

Cable Plant

Ancillary Control and Monitoring Equipment

Signal Analysis instrumentation

Signal Oscilloscopes

Video Displays

Video Support Equipment

Networks

Network Infrastructure

Network Analysis Instrumentation

Survey and Alignment

Synchrotron Survey and Alignment

Injector and LET Survey and Alignment

HET Survey and Alignment

Survey and Alignment Hardware

Magnet Measurement

Magnet Measurement

Targets and Instruments

Target Stations

30- $\mathrm{Hz}$ Target Station

Target System

Target Assembly

Target Cooling System

Target Steel-Shield Plug

Target Sealed Tank

Target-Train Housing

Target Tank Cooling

Proton Beamline Vacuum Window Insert Cooling System

Moderators

Moderator Assemblies

Room Temperature Moderator Assembly 
Table VII.1-1 (Cont.)

WBS Code Element Description

1.3.1.1.2.1.2

1.3.1.1.2.1.3

1.3.1.1.2.1.4

1.3.1.1.2.1.5

1.3.1.1.2.2

1.3.1.1.2.2.1

1.3.1.1.2.2.2

1.3.1.1.2.2.3

1.3.1.1.3

1.3.1.1.3.1

1.3.1.1.3.2

1.3.1.1.4

1.3.1.1.4.1

1.3.1.1.4.2

1.3.1.1.4.3

1.3.1.1.4.4

1.3.1.1.5

1.3.1.1.5.1

1.3.1.1.5.2

1.3.1.1.6

1.3.1.1.6.1

1.3.1.1.6.1.1

1.3.1.1.6.1.2

1.3.1.1.6.1.3

1.3.1.1.6.2

1.3.1.1.6.2.1

1.3.1.1.6.2.2

1.3.1.1.6.2.3

1.3.1.1.6.2.4

1.3.1.1.6.3

1.3.1.1.6.4

1.3.1.1.6.5

1.3.1.1.6.6

1.3.1.1.6.7

1.3.1.1.7

1.3.1.1.7.1

1.3.1.1.7.2

1.3.1.1.8

1.3.1.1.8.1

1.3.1.1.8.2

1.3.1.1.8.3

1.3.1.1.10

1.3.1.1.11

1.3.1.1.12

1.3.1.1.13
Liquid H2 Moderator Assembly

Liquid H2 Premoderator Assembly

Liquid $\mathrm{CH} 4$ Moderator Assembly

Liquid $\mathrm{CH} 4$ Premoderator Assembly

Moderator Cooling

Premoderator and Room Temperature Moderator Cooling

Liquid H2 Moderator Cooling

Liquid CH4 Moderator Cooling

Reflector

Reflector Assembly

Reflector Cooling

Containment Systems

Bulk Shield Sealed Tank

Holdup System

He Supply System

HEPA Filter and Exhaust System

Remote Handling System

Hot Cell Accessories

Long-Term Storage Enclosures

Instrumentation and Control (I\&C)

Target Monitoring System

Target Temperature Monitoring

Target Physical Parameter Monitoring

Target Cooling System Monitoring

Moderator Monitoring and Control

Moderator Temperature Monitoring

Moderator Coolant Flow Monitoring

Moderator Vacuum Monitoring

Moderator Composition Monitoring

Reflector Monitoring

Bulk Shield Monitoring

Radiation Monitoring

Accelerator I\&C Interface

Accelerator Shutdown Interface

Beamlines (in bulk shield)

Beamline Assemblies

Beam Gates

Bulk Shielding

Biological Shield Assembly

Shield Cooling

Shield Structural Support

Piping Supports, Insulation, and Misc.

Liquid Nitrogen System

Pumproom Biological Shield

Relocate Existing 370 Tunnel Piping and Utilities 
Table VII.1-1 (Cont.)

WBS Code Element Description
1.3.1.1.14
1.3.1.1.15
1.3.1.1.16
1.3.1.2
1.3.1.2.1
1.3.1.2.1.1
1.3.1.2.1.2
1.3.1.2.1.3
1.3.1.1.1.4
1.3.1.2.1.5
1.3.1.2.1.6
1.3.1.2.1.7
1.3.1.2.2
1.3.1.2.2.1
1.3.1.2.2.1.1
1.3.1.2.2.1.2
1.3.1.2.2.1.3
1.3.1.2.2.1.4
1.3.1.2.2.1.5
1.3.1.2.2.2
1.3.1.2.2.2.1
1.3.1.2.2.2.2
1.3.1.2.2.2.3
1.3.1.2.3
1.3.1.2.3.1
1.3.1.2.3.2
1.3.1.2.4
1.3.1.2.4.1
1.3.1.2.4.2
1.3.1.2.4.3
1.3.1.2.4.4
1.3.1.2.5
1.3.1.2.5.1
1.3.1.2.5.2
1.3.1.2.6
1.3.1.2.6.1
1.3.1.2.6.1.1
1.3.1.2.6.1.2
1.3.1.2.6.1.3
1.3.1.2.6.2
1.3.1.2.6.2.1
1.3.1.2.6.2.2
1.3.1.2.6.2.3
1.3.1.2.6.2.4
1.3.1.2.6.3
1.3.1.2.6.4

Inside 370 Sub-Floor Piping Work

Outside 370 Below-Ground Piping

Target Group Management

10- $\mathrm{Hz}$ Target Station

Target System

Target Assembly

Target Cooling System

Target Steel-Shield Plug

Target Sealed Tank

Target-Train Housing

Target Tank Cooling

Proton Beamline Vacuum Window Insert Cooling System

Moderators

Moderator Assemblies

Room Temperature Moderator Assembly

Liquid $\mathrm{H} 2$ Moderator Assembly

Liquid H2 Premoderator Assembly

Liquid $\mathrm{CH} 4$ Moderator Assembly

Liquid $\mathrm{CH} 4$ Premoderator Assembly

Moderator Cooling

Premoderator and Room Temperature Moderator Cooling

Liquid H2 Moderator Cooling

Liquid $\mathrm{CH} 4$ Moderator Cooling

Reflector

Reflector Assembly

Reflector Cooling

Containment Systems

Bulk Shield Sealed Tank

Holdup System

He Supply System

HEPA Filter and Exhaust System

Remote Handling System

Hot Cell Accessories

Long-Term Storage Enclosures

Control and Instrumentation

Target Monitoring System

Target Temperature Monitoring

Target Physical Parameter Monitoring

Target Cooling System Monitoring

Moderator Monitoring and Control

Moderator Temperature Monitoring

Moderator Coolant Flow Monitoring

Moderator Vacuum Monitoring

Moderator Composition Monitoring

Reflector Monitoring

Bulk Shield Monitoring 
1.3.1.2.6.5

1.3.1.2.6.6

1.3.1.2.6.7

1.3.1.2.7

1.3.1.2.7.1

1.3.1.2.7.2

1.3.1.2.8

1.3.1.2.8.1

1.3.1.2.8.2

1.3.1.2.8.3

1.3.1.2.10

1.3.1.2.11

1.3.1.2.12

1.3.1.2.13

1.3.1.2.14

1.3.1.2.15

1.3 .2

1.3.2.1

1.3.2.1.1

1.3.2.1.2

1.3.2.1.3

1.3.2.1.4

1.3.2.1.5

1.3.2.2

1.3.2.2.1

1.3.2.2.2

1.3.2.2.3

1.3.2.3

1.3.2.3.1

1.3.2.3.2

1.3.2.3.3

1.3.2.4

1.3.2.4.1

1.3.2.4.2

1.3.2.4.3

1.3.2.4.4

1.3.2.4.5

1.3.2.5

1.3.2.5.1

1.3.2.5.2

1.3.2.5.3

1.3.2.5.4

1.3.2.6
Radiation Monitoring

Accelerator I\&C Interface

Accelerator Shutdown Interface

Beamlines (in bulk shield)

Beamline Assemblies

Beam Gates

Bulk Shielding

Biological Shield Assembly

Shield Cooling

Shield Structural Support

Piping Supports, Insulation, and Misc.

Liquid Nitrogen System

Pumproom Biological Shield

Inside 369 Methane Piping and Shielding

Outside 369 Below-Ground Piping

Target Group Management

Instruments and Support

Powder Diffractometers

SEPD - High Intensity Powder Diffractometer

GPPD - Medium-Resolution Powder Diffractometer

HRPD - High-Resolution Powder Diffractometer

RSD - Residual Stress Diffractometer

VSPD - Very-Small-Sample Powder Diffractometer

Small-Angle Diffractometers

SAND - General-Purpose Small-Angle Diffractometer

HRSAND - High-Resolution Small-Angle Diffractometer

SPSAND - Special-Purpose Small-Angle Diffractometer

Other Diffractometers

GLAD - Liquids and Amorphous Materials Diffractometer

SCD - General-Purpose Single-Crystal Diffractometer

HQSCD - High-Q Single-Crystal Diffractometer

Reflectometers

POSY-I - Polarized-Neutron Reflectometer

POSY-II - General-Purpose Reflectometer

HIREF - High-Intensity Reflectometer

GREF - Reflectometer for Grazing-Incidence Scattering

REFD - Reflectometry Development Beam

Inelastic Scattering Instruments

HRMECS - High-Resolution Chopper Spectrometer

LRMECS - General-Purpose Chopper Spectrometer

CNCS - Cold-Neutron Chopper Spectrometer

SCCS - Chopper Spectrometer for Single Crystals

Crystal-Analyzer Inelastic Scattering Instruments 
Table VII.1-1 (Cont.)

\section{WBS Code Element Description}

1.3.2.6.1

1.3.2.6.2

1.3.2.6.3

1.3.2.6.4

1.3.2.6.5

1.3.2.7

1.3.2.7.1

1.3.2.8

1.3.2.8.1

1.3.2.9

1.3.2.9.1

1.3.2.9.1.1

1.3.2.9.1.2

1.3.2.9.2

1.3.2.9.2.1

1.3.2.9.2.2

1.3.2.9.3

1.3.2.10

1.3.2.11

1.3.2.12

1.3.2.13

1.3.2.14

1.4

1.4.1

1.4.1.1

1.4.1.1.1

1.4.1.1.1.1

1.4.1.1.1.2

1.4.1.1.1.3

1.4.1.1.1.4

1.4.1.1.1.5

1.4.1.1.1.6

1.4.1.1.1.7

1.4.1.1.1.8

1.4.1.1.2

1.4.1.1.2.1

1.4.1.1.2.2

1.4.1.1.2.3

1.4.1.1.2.4

1.4.1.1.3

1.4.1.1.3.1

1.4.1.1.3.2
QENS - Medium-Resolution Crystal-Analyzer Spectrometer

HRBS - High-Resolution Backscattering Spectrometer

TFCA - Time-Focused Crystal Analyzer Spectrometer

QSTAXC - Cold-Neutron Quasi-Steady-State Triple-Axis Spectr.

MICAS - Multiple-Independent-Crystal-Analyzer Spectrometer

Other Inelastic Scattering Instruments

TOFNSE - Time-of-Flight Neutron Spin-Echo Spectrometer

Other Neutron Beam Facilities

DEVEL - Instrument/Component Development Beamline

Neutron Irradiation Facility

30- $\mathrm{Hz}$ Target Neutron Irradiation Facility

Irradiation Facility Sampling, Handling, and Preparation System

Irradiation Facility Sampling and Monitoring System

10-Hz Target Neutron Irradiation Facility

Irradiation Facility Sampling, Handling, and Preparation System

Irradiation Facility Sampling and Monitoring System

Activation Analysis Counting System

Data Analysis Systems

Ancillary Equipment

Sample Preparation Area

Hot Cell Handling Area

Instrumentation Group Management

Conventional Facilities

Existing Building Rehabilitation

Ring Building (365)

Architectural/Structural

General Demolition

Substructure

Superstructure

Exterior Closure

Roofing

Partitions and Doors

Finishes

Specialties

Mechanical

Mechanical Demolition

Plumbing

Heating, Ventilation, and Air Conditioning

Fire Protection

Electrical

Electrical Demolition

Service and Distribution 
Table VII.1-1 (Cont.)

\begin{tabular}{|c|c|}
\hline WBS Code & Element Description \\
\hline 1.4.1.1.3.3 & Lighting and Power \\
\hline 1.4 .1 .1 .3 .4 & Special Systems \\
\hline 1.4.1.1.4 & Civil \\
\hline 1.4.1.1.4.1 & Site Works \\
\hline 1.4.1.1.5 & Shielding \\
\hline 1.4 .1 .2 & Center Building (364) \\
\hline 1.4.1.2.1 & Architectural/Structural \\
\hline 1.4.1.2.1.1 & General Demolition \\
\hline 1.4.1.2.1.2 & Substructure \\
\hline 1.4.1.2.1.3 & Superstructure \\
\hline 1.4.1.2.1.4 & Exterior Closure \\
\hline 1.4.1.2.1.5 & Roofing \\
\hline 1.4.1.2.1.6 & Partitions and Doors \\
\hline 1.4.1.2.1.7 & Finishes \\
\hline 1.4.1.2.1.8 & Specialties \\
\hline 1.4.1.2.2 & Mechanical \\
\hline 1.4.1.2.2.1 & Mechanical Demolition \\
\hline 1.4.1.2.2.2 & Plumbing \\
\hline 1.4.1.2.2.3 & Heating, Ventilation, and Air Conditioning \\
\hline 1.4.1.2.2.4 & Fire Protection \\
\hline 1.4.1.2.3 & Electrical \\
\hline 1.4.1.2.3.1 & Electrical Demolition \\
\hline 1.4.1.2.3.2 & Service and Distribution \\
\hline 1.4.1.2.3.3 & Lighting and Power \\
\hline 1.4.1.2.3.4 & Special Systems \\
\hline 1.4.1.2.4 & Civil \\
\hline 1.4.1.2.4.1 & Site Works \\
\hline 1.4.1.3 & Power Supply Building (376) \\
\hline 1.4.1.3.1 & Architectural/Structural \\
\hline 1.4.1.3.1.1 & General Demolition \\
\hline 1.4.1.3.1.2 & Substructure \\
\hline 1.4.1.3.1.3 & Superstructure \\
\hline 1.4.1.3.1.4 & Exterior Closure \\
\hline 1.4.1.3.1.5 & Roofing \\
\hline 1.4.1.3.1.6 & Partitions and Doors \\
\hline 1.4.1.3.1.7 & Finishes \\
\hline 1.4.1.3.1.8 & Specialties \\
\hline 1.4.1.3.2 & Mechanical \\
\hline 1.4.1.3.2.1 & Mechanical Demolition \\
\hline 1.4.1.3.2.2 & Plumbing \\
\hline 1.4.1.3.2.3 & Heating, Ventilation, and Air Conditioning \\
\hline 1.4.1.3.2.4 & Fire Protection \\
\hline 1.4.1.3.3 & Electrical \\
\hline 1.4.1.3.3.1 & Electrical Demolition \\
\hline 1.4.1.3.3.2 & Service and Distribution \\
\hline
\end{tabular}


Table VII.1-1 (Cont.)

\begin{tabular}{|c|c|}
\hline WBS Code & Element Description \\
\hline 1.4.1.3.3.3 & Lighting and Power \\
\hline 1.4.1.3.3.4 & Special Systems \\
\hline 1.4.1.3.4 & Civil \\
\hline 1.4.1.3.4.1 & Site Works \\
\hline 1.4.1.4 & 10-Hz Target Building (369) \\
\hline 1.4.1.4.1 & Architectural/Structural \\
\hline 1.4.1.4.1.1 & General Demolition \\
\hline 1.4.1.4.1.2 & Substructure \\
\hline 1.4.1.4.1.3 & Superstructure \\
\hline 1.4.1.4.1.4 & Exterior Closure \\
\hline 1.4.1.4.1.5 & Roofing \\
\hline 1.4.1.4.1.6 & Partitions and Doors \\
\hline 1.4.1.4.1.7 & Finishes \\
\hline 1.4.1.4.1.8 & Specialties \\
\hline 1.4.1.4.2 & Mechanical \\
\hline 1.4.1.4.2.1 & Mechanical Demolition \\
\hline 1.4.1.4.2.2 & Plumbing \\
\hline 1.4.1.4.2.3 & Heating, Ventilation, and Air Conditioning \\
\hline 1.4.1.4.2.4 & Fire Protection \\
\hline 1.4.1.4.3 & Electrical \\
\hline 1.4.1.4.3.1 & Electrical Demolition \\
\hline 1.4.1.4.3.2 & Service and Distribution \\
\hline 1.4.1.4.3.3 & Lighting and Power \\
\hline 1.4.1.4.3.4 & Special Systems \\
\hline 1.4.1.4.4 & Civil \\
\hline 1.4.1.4.4.1 & Site Works \\
\hline 1.4.1.4.5 & Shielding \\
\hline 1.4.1.5 & 10- $\mathrm{Hz}$ Target Support Building (367) \\
\hline 1.4.1.5.1 & Architectural/Structural \\
\hline 1.4.1.5.1.1 & General Demolition \\
\hline 1.4.1.5.1.2 & Substructure \\
\hline 1.4.1.5.1.3 & Superstructure \\
\hline 1.4.1.5.1.4 & Exterior Closure \\
\hline 1.4.1.5.1.5 & Roofing \\
\hline 1.4.1.5.1.6 & Partitions and Doors \\
\hline 1.4.1.5.1.7 & Finishes \\
\hline 1.4.1.5.1.8 & Specialties \\
\hline 1.4.1.5.2 & Mechanical \\
\hline 1.4.1.5.2.1 & Mechanical Demolition \\
\hline 1.4.1.5.2.2 & Plumbing \\
\hline 1.4.1.5.2.3 & Heating, Ventilation, and Air Conditioning \\
\hline 1.4.1.5.2.4 & Fire Protection \\
\hline 1.4.1.5.3 & Electrical \\
\hline 1.4.1.5.3.1 & Electrical Demolition \\
\hline 1.4.1.5.3.2 & Service and Distribution \\
\hline
\end{tabular}


Table VII.1-1 (Cont.)

\begin{tabular}{|c|c|}
\hline WBS Code & Element Description \\
\hline 1.4.1.5.3.3 & Lighting and Power \\
\hline 1.4.1.5.3.4 & Special Systems \\
\hline 1.4.1.5.4 & Civil \\
\hline 1.4.1.5.4.1 & Site Works \\
\hline 1.4.1.5.5 & Shielding \\
\hline 1.4.1.6 & $30-\mathrm{Hz}$ Target Building (370) \\
\hline 1.4.1.6.1 & Architectural/Structural \\
\hline 1.4.1.6.1.1 & General Demolition : \\
\hline 1.4.1.6.1.2 & Substructure \\
\hline 1.4.1.6.1.3 & Superstructure \\
\hline 1.4.1.6.1.4 & Exterior Closure \\
\hline 1.4.1.6.1.5 & Roofing \\
\hline 1.4.1.6.1.6 & Partitions and Doors \\
\hline 1.4.1.6.1.7 & Finishes \\
\hline 1.4.1.6.1.8 & Specialties \\
\hline 1.4.1.6.2 & Mechanical \\
\hline 1.4.1.6.2.1 & Mechanical Demolition \\
\hline 1.4.1.6.2.2 & Plumbing \\
\hline 1.4.1.6.2.3 & Heating, Ventilation, and Air Conditioning \\
\hline 1.4.1.6.2.4 & Fire Protection \\
\hline 1.4.1.6.3 & Electrical \\
\hline 1.4.1.6.3.1 & Electrical Demolition \\
\hline 1.4.1.6.3.2 & Service and Distribution \\
\hline 1.4.1.6.3.3 & Lighting and Power \\
\hline 1.4:1.6.3.4 & Special Systems \\
\hline 1.4.1.6.4 & Civil \\
\hline 1.4.1.6.4.1 & Site Works \\
\hline 1.4.1.6.5 & Shielding \\
\hline 1.4.1.7 & 30-Hz Target Support Building (371) \\
\hline 1.4.1.7.1 & Architectural/Structural \\
\hline 1.4.1.7.1.1 & General Demolition \\
\hline 1.4.1.7.1.2 & Substructure \\
\hline 1.4.1.7.1.3 & Superstructure \\
\hline 1.4.1.7.1.4 & Exterior Closure \\
\hline 1.4.1.7.1.5 & Roofing \\
\hline 1.4.1.7.1.6 & Partitions and Doors \\
\hline 1.4.1.7.1.7 & Finishes \\
\hline 1.4.1.7.1.8 & Specialties \\
\hline 1.4.1.7.2 & Mechanical \\
\hline 1.4.1.7.2.1 & Mechanical Demolition \\
\hline 1.4.1.7.2.2 & Plumbing \\
\hline 1.4.1.7.2.3 & Heating, Ventilation, and Air Conditioning \\
\hline 1.4.1.7.2.4 & Fire Protection \\
\hline 1.4.1.7.3 & Electrical \\
\hline 1.4.1.7.3.1 & Electrical Demolition \\
\hline
\end{tabular}


Table VII.1-1 (Cont.)

WBS Code

Element Description

1.4.1.7.3.2

1.4.1.7.3.3

1.4.1.7.3.4

1.4.1.7.4

1.4.1.7.4.1

1.4.1.7.5

1.4.1.8

1.4.1.8.1

1.4.1.8.1.1

1.4.1.8.1.2

1.4.1.8.1.3

1.4.1.8.1.4

1.4.1.8.1.5

1.4.1.8.1.6

1.4.1.8.1.7

1.4.1.8.1.8

1.4.1.8.2

1.4.1.8.2.1

1.4.1.8.2.2

1.4.1.8.2.3

1.4.1.8.2.4

1.4.1.8.3

1.4.1.8.3.1

1.4.1.8.3.2

1.4.1.8.3.3

1.4.1.8.3.4

1.4.1.8.4

1.4.1.8.4.1

1.4.1.8.5

1.4.1.9

1.4.1.9.1

1.4.1.9.1.1

1.4.1.9.1.2

1.4.1.9.1.3

1.4.1.9.1.4

1.4.1.9.1.5

1.4.1.9.1.6

1.4.1.9.1.7

1.4.1.9.1.8

1.4.1.9.2

1.4.1.9.2.1

1.4.1.9.2.2

1.4.1.9.2.3

1.4.1.9.2.4

1.4.1.9.3
Service and Distribution

Lighting and Power

Special Systems

Civil

Site Works

Shielding

Preparation Laboratories Building (368)

Architectural/Structural

General Demolition

Substructure

Superstructure

Exterior Closure

Roofing

Partitions and Doors

Finishes

Specialties

Mechanical

Mechanical Demolition

Plumbing

Heating, Ventilation, and Air Conditioning

Fire Protection

Electrical

Electrical Demolition

Service and Distribution

Lighting and Power

Special Systems

Civil

Site Works

Shielding

Laboratory and Office Building I (360)

Architectural/Structural

General Demolition

Substructure

Superstructure

Exterior Closure

Roofing

Partitions and Doors

Finishes

Specialties

Mechanical

Mechanical Demolition

Plumbing

Heating, Ventilation, and Air Conditioning

Fire Protection

Electrical 
Table VII.1-1 (Cont.)

\begin{tabular}{|c|c|}
\hline WBS Code & Element Description \\
\hline 1.4.1.9.3.1 & Electrical Demolition \\
\hline 1.4.1.9.3.2 & Service and Distribution \\
\hline 1.4.1.9.3.3 & Lighting and Power \\
\hline 1.4.1.9.3.4 & Special Systems \\
\hline 1.4.1.9.4 & Civil \\
\hline 1.4.1.9.4.1 & Site Works \\
\hline 1.4.1.10 & Laboratory and Office Building II (362) \\
\hline 1.4.1.10.1 & Architectural/Structural \\
\hline 1.4.1.10.1.1 & General Demolition \\
\hline 1.4.1.10.1.2 & Substructure \\
\hline 1.4.1.10.1.3 & Superstructure \\
\hline 1.4.1.10.1.4 & Exterior Closure \\
\hline 1.4.1.10.1.5 & Roofing \\
\hline 1.4.1.10.1.6 & Partitions and Doors \\
\hline 1.4.1.10.1.7 & Finishes \\
\hline 1.4.1.10.1.8 & Specialties \\
\hline 1.4.1.10.2 & Mechanical \\
\hline 1.4.1.10.2.1 & Mechanical Demolition \\
\hline 1.4.1.10.2.2 & Plumbing \\
\hline 1.4.1.10.2.3 & Heating, Ventilation, and Air Conditioning \\
\hline 1.4.1.10.2.4 & Fire Protection \\
\hline 1.4.1.10.3 & Electrical \\
\hline 1.4.1.10.3.1 & Electrical Demolition \\
\hline 1.4.1.10.3.2 & Service and Distribution \\
\hline 1.4.1.10.3.3 & Lighting and Power \\
\hline 1.4.1.10.3.4 & Special Systems \\
\hline 1.4.1.10.4 & Civil \\
\hline 1.4.1.10.4.1 & Site Works \\
\hline 1.4 .2 & New Construction \\
\hline 1.4.2.1 & Injector - Linac Building, Tunnel, and LET (394 and 394-A) \\
\hline 1.4.2.1.1 & Architectural/Structural \\
\hline 1.4.2.1.1.1 & General Demolition \\
\hline 1.4.2.1.1.2 & Substructure \\
\hline 1.4.2.1.1.3 & Superstructure \\
\hline 1.4.2.1.1.4 & Exterior Closure \\
\hline 1.4.2.1.1.5 & Roofing \\
\hline 1.4.2.1.1.6 & Partitions and Doors \\
\hline 1.4.2.1.1.7 & Finishes \\
\hline 1.4.2.1.1.8 & Specialties \\
\hline 1.4.2.1.2 & Mechanical \\
\hline 1.4.2.1.2.1 & Mechanical Demolition \\
\hline 1.4.2.1.2.2 & Plumbing \\
\hline 1.4.2.1.2.3 & Heating, Ventilation, and Air Conditioning \\
\hline 1.4.2.1.2.4 & Fire Protection \\
\hline 1.4.2.1.3 & Electrical \\
\hline
\end{tabular}


Table VII.1-1 (Cont.)

\begin{tabular}{|c|c|}
\hline WBS Code & Element Description \\
\hline 1.4.2.1.3.1 & Electrical Demolition \\
\hline 1.4.2.1.3.2 & Service and Distribution \\
\hline 1.4.2.1.3.3 & Lighting and Power \\
\hline 1.4.2.1.3.4 & Special Systems \\
\hline 1.4.2.1.4 & Civil \\
\hline 1.4.2.1.4.1 & Site Works \\
\hline 1.4.2.1.5 & Shielding \\
\hline 1.4.3 & Utilities \\
\hline 1.4.3.1 & Site Work \\
\hline 1.4.3.2 & Mechanical Site Utilities \\
\hline 1.4.3.3 & Electrical Site Utilities \\
\hline 1.4 .4 & Conventional Facilities EDI\&A ${ }^{b}$ \\
\hline 1.5 & Shielding Structures \\
\hline 1.5 .1 & High-Energy Proton Transport Shielding \\
\hline 1.5.1.1 & Low-Frequency PTS Shielding \\
\hline 1.5.1.2 & High-Frequency PTS Shielding \\
\hline 1.5 .2 & $30-\mathrm{Hz}$ Target Shielding \\
\hline 1.5.2.1 & Biological Shield Assembly \\
\hline 1.5.2.2 & Biological Shield Structural Support \\
\hline 1.5 .3 & 10-Hz Target Shielding \\
\hline 1.5.3.1 & Biological Shield Assembly \\
\hline 1.5.3.2 & Biological Shield Structural Support \\
\hline 1.8 & Misc. Costs \\
\hline 1.8 .1 & Overheads \\
\hline 1.8.1.1 & Materials and Subcontracts (@6.2\%) \\
\hline 1.8.1.2 & Common Support (@19.2\%) \\
\hline 1.8.1.3 & General and Adm. (@1.9\%) \\
\hline 1.8 .2 & Unproductive Time \\
\hline 1.9 & Contingency \\
\hline
\end{tabular}

a $E D \& I$ = engineering, design, and inspection.

b EDI\&A = engineering, design, inspection, and administration. 
All cost estimates include engineering, design, and inspection (ED\&I) and hardware deliverables. Fiscal year 1995 dollars are used throughout. Crafts needed for construction are grouped into three categories, and details of the crafts used and their rates are shown in Table VII.2-1. Contingency is addressed at the estimating stage and is discussed later in this section. Escalation is added after the budget profile is applied. The escalation rates used for this purpose come from a collection of DOE rates provided annually by DOE-Chicago and accumulated by the APS project; these rates are shown in Table VII.2-2.

The project cost detail corresponding to each element of the WBS list shown in Table VII.1-1 is appended in two separate books, Supplement A for technical components and Supplement B for conventional facilities. The project cost summary, rolled up from these books, is shown in Table VII.2-3 in FY 1995 dollars. The costs are broken down into three categories: (a) ED\&I cost, (b) construction cost, and (c) contingency. Each category is further subdivided to provide detail corresponding to Level 3 of the WBS. The costs for technical components, excluding the injector linac, are rolled up from detailed WBS sheets generated by the ANL staff that show all hardware and effort costs for each item, from design through installation and checkout. The costs for the injector linac and its associated technical components were estimated by the industrial firm AccSys Technology, Inc., of Pleasanton, California. One of the options under consideration is a turn-key procurement of the injector system from industry. The costs for conventional facilities are estimates generated by the construction consulting firm of Hanscomb Associates, Inc. (see Supplement B), and are based on designs by ANL staff from APS and PFS.

The project management costs shown in Table VII.2-3 consist of four parts:

1. Project Management Costs. These are the costs of personnel involved in project direction and coordination, together with the costs associated with compliance with environment, safety, and health (ES\&H) requirements.

2. Laboratory Overhead. The Laboratory recovers its indirect expenses, which are the costs associated with administration (functions such as personnel, accounting, and procurement) and maintenance of physical facilities, by means of charges against the funds received for operations, which are commonly known as operating funds - in contrast to (1) capital equipment funds, which are used for equipment fabrication or purchase, and (2) plant funds, which are used for the construction project. Indirect Laboratory costs were previously recovered through a charge of $26.9 \%$ applied to all in-house direct operating costs. Construction projects, which usually add only incrementally to indirect expenses, contribute a smaller amount to cover indirect expenses. Under the old Laboratory policy, this cost recovery was achieved by a charge of $26.9 \%$ that was applied only to the in-house Laboratory project management and ED\&I effort costs and not to other costs. The overhead for the APS Project under this rule was $22 \%$, which was applied only to the in-house ED\&I and 
Table VII.2-1 Craft Code Rate for 1995

\begin{tabular}{|c|c|c|}
\hline Item & $\begin{array}{l}\text { Craft } \\
\text { Code }\end{array}$ & $\begin{array}{c}\text { Rate } \\
\text { (\$/hour) }\end{array}$ \\
\hline \multicolumn{3}{|l|}{ Manufacturing/Fabrication/Assembly/Test ${ }^{a}$} \\
\hline Technician (assembly, test, etc.), relatively unskilled & T1 & 25.00 \\
\hline Technician (assembly, test, etc.), experienced/skilled & T2 & 33.00 \\
\hline Shops (average capability machinists, fab, etc.) & s1 & 50.00 \\
\hline Shops (specialized, precision, high tech, etc.) & S2 & 55.00 \\
\hline Factory support (parts handling, inspections, $Q A$, etc.) & F1 & 45.00 \\
\hline Factory support (superv., managers, prod. engineers, etc.) & $\mathrm{F} 2$ & 55.00 \\
\hline \multicolumn{3}{|l|}{ Installation ("Davis-Bacon")a } \\
\hline Plumber, steam fitter, sheet metal worker, pipefitter & IP & 56.00 \\
\hline Electrician & IE & 54.00 \\
\hline Carpenter, 'painter, rigger, crane operator & IC & 51.00 \\
\hline Laborer & IL & 42.00 \\
\hline Technician & IT & 54.00 \\
\hline \multicolumn{3}{|l|}{ Technical Engineering, Design, and Inspection (ED\&l) } \\
\hline Physicist & $\mathrm{PH}$ & 49.00 \\
\hline Engineer (mechanical and electrical) & EN & 49.00 \\
\hline Computer software designer & SW & 49.00 \\
\hline Designer/coordinator & $\mathrm{DC}$ & 39.00 \\
\hline Drafter & DR & 30.00 \\
\hline \multicolumn{3}{|l|}{ Management $t^{b}$} \\
\hline Manager & MN & 49.00 \\
\hline Secretary & ss & 31.00 \\
\hline
\end{tabular}

a The rate in this category includes hourly base pay, employee benefits and overhead, and profit for the outside vendor or contractor.

b The rate in this category includes hourly base pay and employee benefits, but it does not include laboratory overhead, which is included in a separate category. 
Table VII.2-2 Escalation Rates

\begin{tabular}{ccc}
\hline Fiscal Year & Inflation (\%) & Scaling Factor \\
\hline & & \\
1995 & - & 1.000 \\
1996 & 3.8 & 1.038 \\
1997 & 3.9 & 1.078 \\
1998 & 3.8 & 1.119 \\
1999 & 3.6 & 1.159 \\
2000 & 3.6 & 1.201 \\
2001 & 3.6 & 1.244 \\
\hline
\end{tabular}


Table VII.2-3 Project Cost Breakdown by Major Categories (FY 1995 k\$)

A. Engineering, Design, and Inspection

43,158

1. Technical Components

a. Injector System

b. Synchrotron

c. Beam Transports

d. Technical Support Systems

e. Target Stations

f. Instrumentation and Support

g. Shielding Structures

2. Conventional Facilities (EDI\&A)

5,115

B. Construction Costs

$\begin{array}{rr}16 \% & \\ 7 \% & 3,965 \\ 22 \% & 13,377 \\ 20 \% & 2,744 \\ 34 \% & 2,503 \\ 31 \% & 6,394 \\ 14 \% & 7,619 \\ 5 \% & 1,442\end{array}$

$13 \%$

3,377

2,744

2,503

6,394

1,442

38,043

356,815

1. Technical Components
a. Injector System
b. Synchrotron
c. Beam Transports
d. Technical Support Systems
e. Target Stations
f. Instrumentation and Support
g. Shielding Structures

2. Conventional Facilities

a. Existing Bldg. Rehabilitation

b. New Construction

c. Utilities

3. Project Management
a. Management Cost
b. Laboratory Overhead Cost
c. Unproductive Time
d. M\&S for Staff

58,098

62,186

13,579

7,330

20,616

52,562

28,090

242,461

24,824

7,393

8,703

40,921

10,102

44,527

8,059

10,745

73,433

78,066

$22 \%$

9,559

$21 \%$

51,950

$21 \%$

8,775

3. Conventional Facilities

$11 \%$

7,782 
project-management effort costs. As of FY 1995, the new DOE policy is to collect indirect costs by using a graduated scale. The Laboratory now charges $6.2 \%$ for direct materials and subcontracts procurement and $19.2 \%$ for all other common support expenses. In addition to these charges of $6.2 \%$ and $19.2 \%$, there is a charge of $1.9 \%$ of all costs for all general and administrative costs.

The indirect charge to be borne by this construction project under the new policy is $\$ 44.5$ million in FY 1995 dollars. This number is to be compared with $\$ 14.2$ million under the old policy.

3. Materials and Services Costs. These are the M\&S costs of the Laboratory direct personnel in the project and are estimated to be $20 \%$ of the effort costs.

4. Unproductive Time Costs for ANL Staff. Under new DOE policy, vacation and holiday time accrued by the project personnel must be accounted for separately. The budgeted amount for this category is calculated by allocating $15.0 \%$ of the direct personnel effort costs.

It should be noted that inclusion of the last three items is necessitated by the fact that the craft code and craft rate shown in Table VII.2-1 for engineering, design, inspection, and administration (EDI\&A) personnel only takes into account the hourly base pay and benefits.

The contingency costs are applied to the rate shown in Table VII.2-3. The rates shown for ED\&I and technical components are averages for individual components and reflect the completeness of the design, uncertainties, and variability of market conditions. For example, a relatively high rate of $35 \%$ is assigned to some of the neutron-generating target components because the design of the target is somewhat uncertain at this time. The rate for conventional facilities is based on estimates by Hanscomb Associates, Inc.

The contents of Table VII.2-3 can be rearranged to show the project cost profile by the major cost components. This is shown in Table VII.2-4.

The cost schedule that takes into account escalation and budget time profile is discussed in Section VII.4. 
Table VII.2-4 Project Cost Breakdown by WBS Code

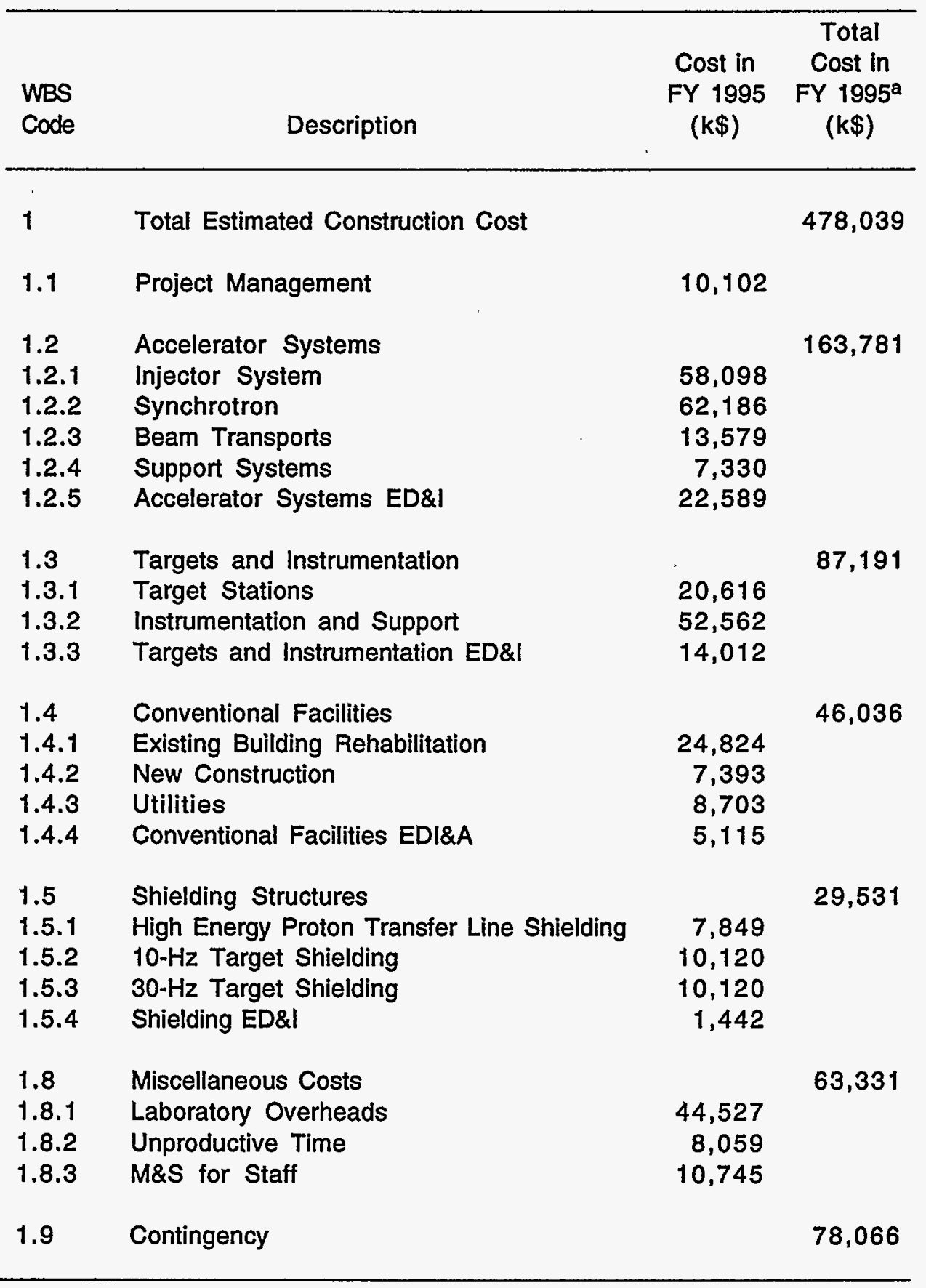

a Numbers are spreadsheet-generated, and costs may not exactly add up to totals because of rounding. 
The schedule is based on the assumptions that (1) the construction project starts in FY 1997 and (2) there are sufficient funds to carry out preconstruction R\&D and construction planning, including environmental assessment between FY 1996 and FY 1998. The preconstruction R\&D activities are described in Appendix B. In addition, the following concepts have been incorporated in scheduling the construction:

- DOE Headquarters Key Decision (KD) milestones, $\mathrm{KD} 0$ and $\mathrm{KD} 1$, are met at the middle of fiscal years 1995 and 1996, respectively.

- With the KD 0 milestone, the environmental assessment process commences. National Environmental Protection Act (NEPA) and other permit requests are submitted soon after $\mathrm{KD} 0$.

- For optimal project efficiency, front loading of the project is exercised wherever possible.

- Design, construction, and installation periods for technical components used in the scheduling are based on experiences of accelerator personnel in this Laboratory, particularly at the APS; at other laboratories, such as the European Synchrotron Radiation Facility (ESRF); and on vendor quotations.

- All technical components of a subsystem to be installed in a building will be assembled and tested beforehand to maintain an optimal installation schedule with a relatively uniform level of effort.

- The existence of technical component assembly areas used to support the APS construction and of technical component support facilities, such as the magnet measuring facility and survey and alignment facility, has been taken into account in making the accelerator schedule.

- The conventional construction (rehabilitation) schedule of beneficial occupancy provided by PFS and APS conventional facilities personnel is used to determine the accelerator and target station schedules.

- Sequential commissioning of two major accelerators is planned. The first accelerator to be commissioned is the linac system. Six months after the linac is commissioned, the RCS is scheduled to be commissioned. Another six months after the synchrotron is commissioned, the commissioning of the neutron-generating target stations takes place.

Figure VII.3-1 shows the conventional facilities schedule, and Figures VII.3-2a through VII.3-2k show the technical component schedules. These figures also show the key technical component R\&D schedules that are phased into the construction schedule. Figure VII.3-3 is the project critical path diagram for the major components of the project at Level 3 of the WBS. As the project design progresses, a more detailed critical path analysis at Level 4 and below will be 
established and will be used to monitor the progress of the project. As shown in Figure VII.3-3, the commissioning of the synchrotron begins at the start of FY 2000, with neutron beam lines in routine operation beginning in the third quarter of FY 2001. 


\begin{tabular}{|c|c|c|c|c|c|c|c|}
\hline CONVENTIONAL FACILITIES SCHEDULE & & & & & & & \\
\hline Fiscal Year & FY95 & FY96 & FY97 & FY98 & FY99 & FYOO & FY01 \\
\hline KD 0 Milestone & $\$$ & & & & & & \\
\hline KD I Milestone & & \& & & & & & \\
\hline E/A & & T1 & & & & & \\
\hline NEPA and Other Permits & & & & & & & \\
\hline Construction Start Milestone & & & & & & & \\
\hline DESIGN & & & & & & & \\
\hline Title I & & & & & & & \\
\hline Final Design & & & & & & & \\
\hline CONSTRUCTION & & & & & & & \\
\hline Linac, Klystron Gallery and LET Tunne & & & & & & & \\
\hline (New Construction for Linac) & & & & & & & \\
\hline Center Building and Ring Building & & & & & A & & \\
\hline (Buildings 364 and 365 ) & & & & & & & \\
\hline & & & & & & & \\
\hline Magnet Power Supplies Building & & & & & $\mathbb{Z}$ 菌 & & \\
\hline (Building 376) & & & & & & & \\
\hline Targat Buildinges & & & $\mathbb{W}$ & & & & \\
\hline (Buildings 369 and 370 ) & & & & & & & \\
\hline & & & & & & & \\
\hline Target Support Buildings & & & & IIIT & & & \\
\hline (Buildings 367 and 371 ) & & & & & & & \\
\hline & & & & & & & \\
\hline Utilities & & & & & & & \\
\hline & & & & & & & \\
\hline All Other Buildings Rehab and Site & & & & & & & \\
\hline Commissioning Starts Milestone & & & & & & & \\
\hline Commissioning Ends Milestone & & & & & & & 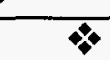 \\
\hline
\end{tabular}

Figure VII.3-1 Conventional Facilities Schedule. 


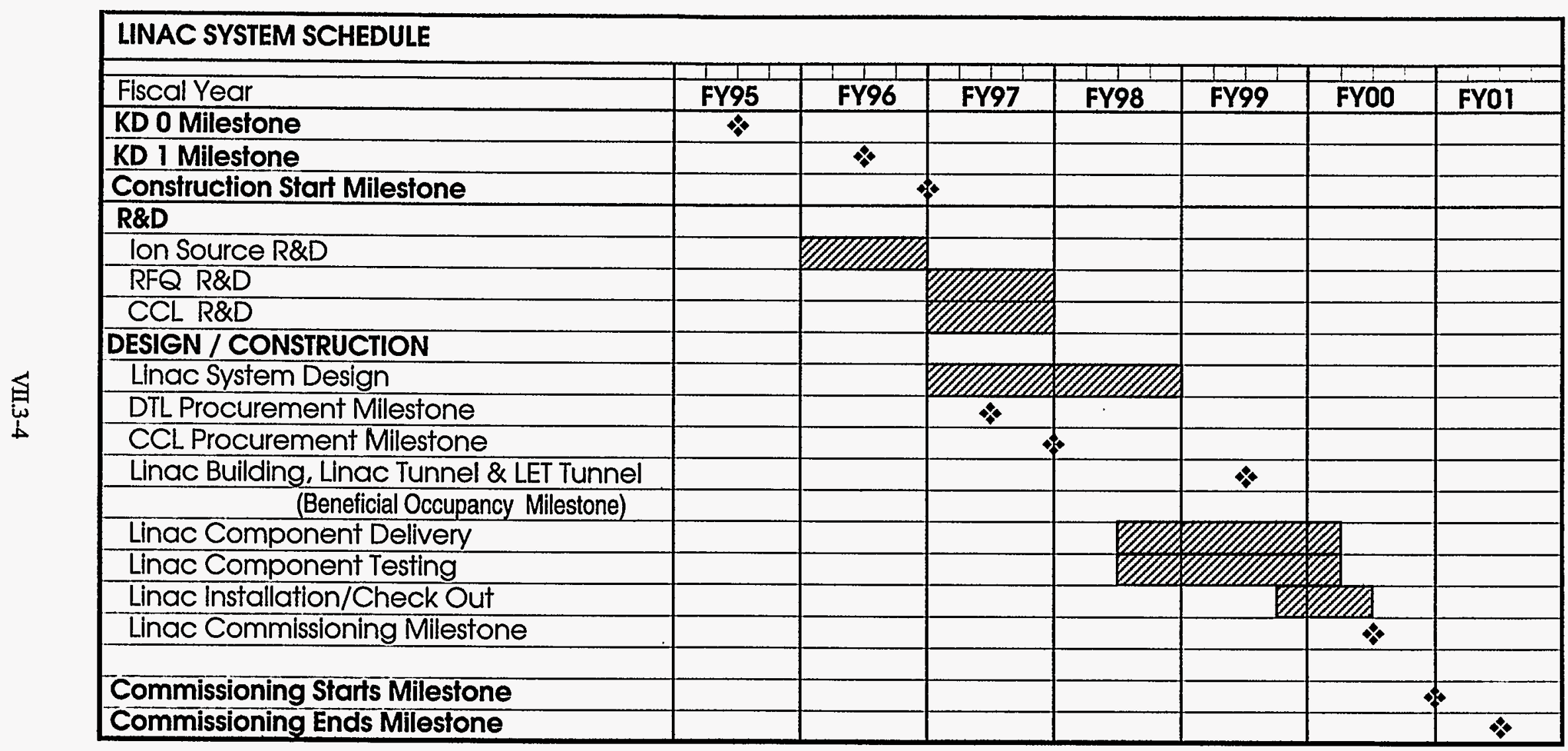

Figure VII.3-2a Linac System Schedule. 


\begin{tabular}{|c|c|c|c|c|c|c|c|}
\hline \multicolumn{8}{|l|}{ SYNCHROTRON MAGNET SCHEDULE } \\
\hline Fiscal Year & FY95 & FY96 & FY97 & FY98 & FY99 & FYOO & FY01 \\
\hline KD O Milestone & 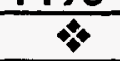 & & & & & & \\
\hline KD I Milestone & & $\star$ & & & & & \\
\hline Construction Start Milestone & & & & & & & \\
\hline R\&D & & & & & & & \\
\hline Magnets Performance Design R\&D & T/1/ & & & & & & \\
\hline DESIGN / CONSTRUCTION & & & & & & & \\
\hline Magnets Engineering Design & & & & & & & \\
\hline & & & & & & & \\
\hline Magnets Procurement Milestone & & & & & & & \\
\hline First Magnets Dellvery Milestone & & & & 4 & & & \\
\hline Magnets Production Starts Milestone & & & & & & & \\
\hline Magnets Dellvery & & & & & & & \\
\hline Magnets Measurement & & & & & & & \\
\hline Ring Building (Beneficial Occupancy Milestone) & & & & & $\$$ & & \\
\hline Magnets Installation & & & & & & & \\
\hline & & & & & & & \\
\hline Commissioning Starts Milestone & & & & & & & \\
\hline Commissioning Ends Milestone & & & & & & & $\$$ \\
\hline
\end{tabular}

Figure VII.3-2b Synchrotron Magnet Schedule. 


\begin{tabular}{|c|c|c|c|c|c|c|c|}
\hline \multicolumn{8}{|c|}{ SYNCHROTRON MAGNETS POWER SUPPLIES SCHEDULE } \\
\hline Fiscal Year & FY95 & $\frac{1}{\text { FY96 }}$ & $\frac{1}{\text { FY97 }}$ & FY98 & FY99 & FYOO & FYOI \\
\hline KD O Milestone & 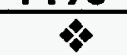 & & & & & & \\
\hline KD I Milestone & & $\$$ & & & & & \\
\hline Construction Start Milestone & & & & & & & \\
\hline \multicolumn{8}{|l|}{ R\&D } \\
\hline \multicolumn{8}{|l|}{ Dual Frequency Model R\&D } \\
\hline \multicolumn{8}{|l|}{ DESIGN / CONSTRUCTION } \\
\hline \multicolumn{8}{|l|}{ Power Supplies Design } \\
\hline Power Supplies Procurement Milestone & & & \multicolumn{2}{|c|}{ 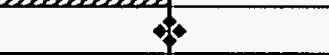 } & & & \\
\hline First Supply Delivery Milestone & & & & $\$$ & & & \\
\hline \multicolumn{8}{|l|}{ Power Supplies Delivery } \\
\hline \multicolumn{8}{|l|}{ Power Dummy Load Test } \\
\hline \multicolumn{2}{|l|}{ Power Supply Building (Beneficial Occupancy Milestone) } & & & & $\$$ & & \\
\hline & & & & & & & \\
\hline \multicolumn{8}{|l|}{ Power Supplies installation and Connection } \\
\hline & & & & & & & \\
\hline Commissioning Starts Milestone & & & & & & & \\
\hline Commissioning Ends Milestone & & & & & & & $\%$ \\
\hline
\end{tabular}

Figure VII.3-2c Synchrotron Magnets - Power Supplies Schedule. 


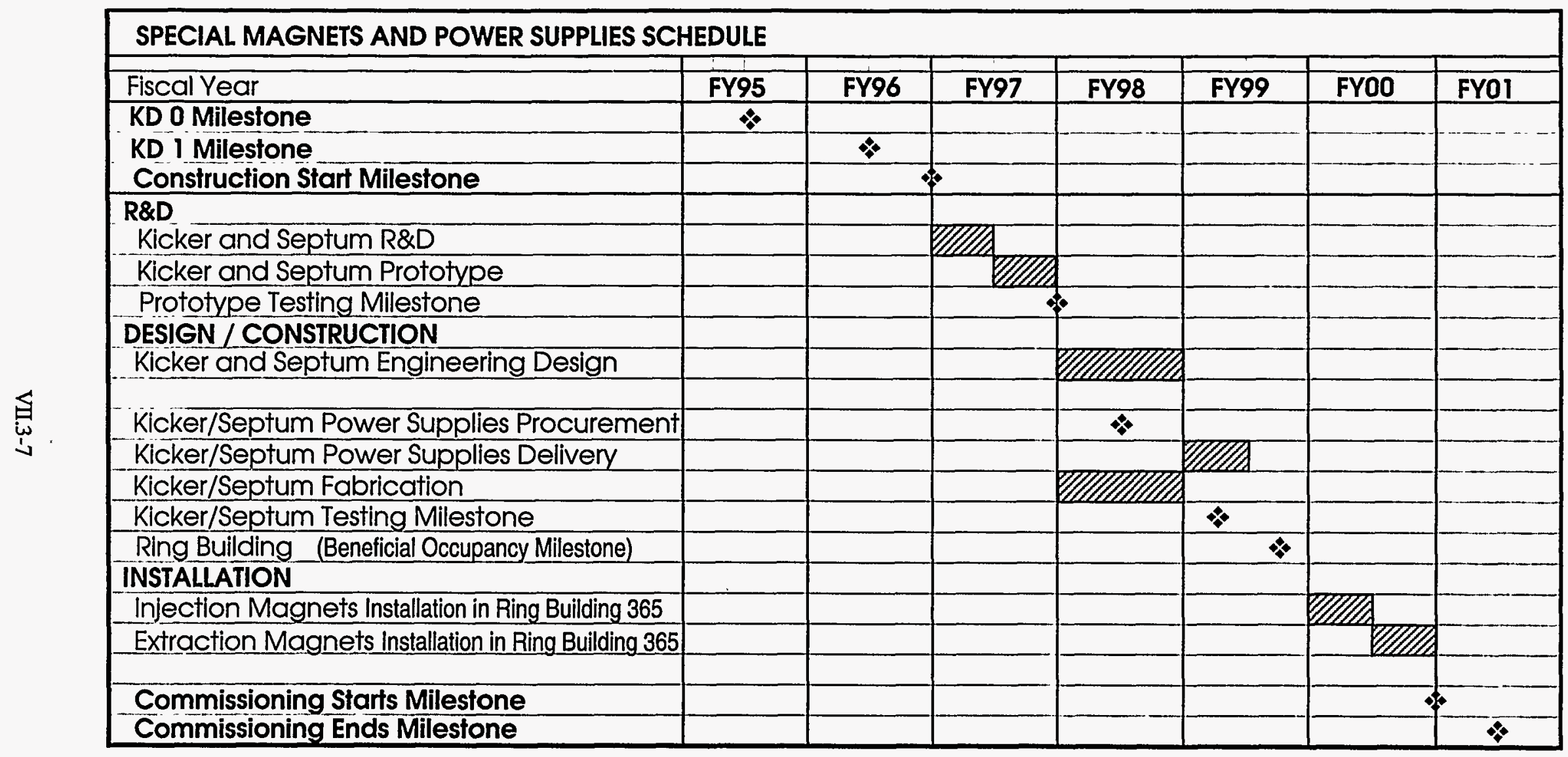

Figure VII.3-2d Special Magnets and Power Supplies Schedule. 


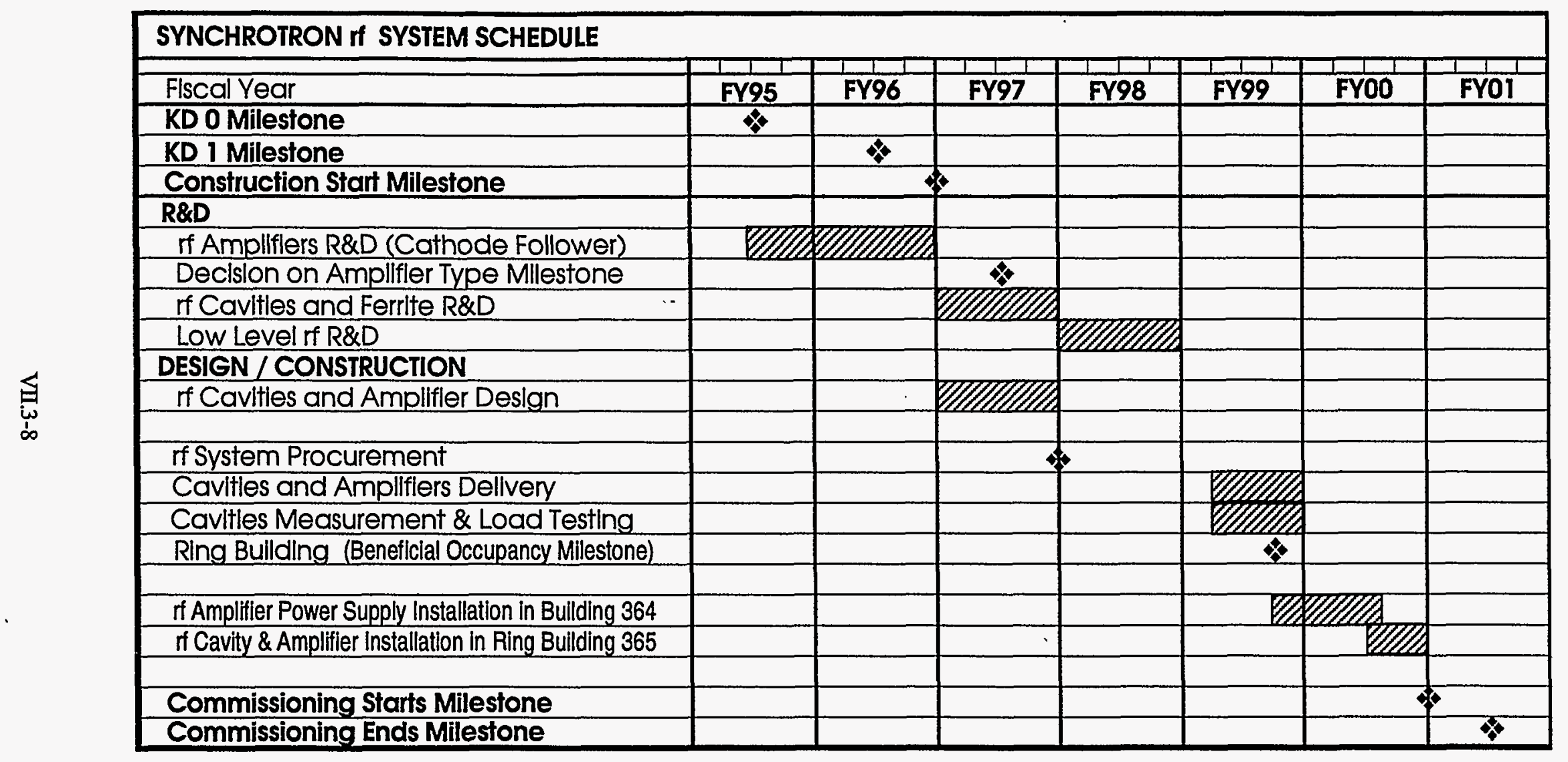

Figure VII.3-2e Synchrotron if System Schedule. 


\begin{tabular}{|c|c|c|c|c|c|c|c|}
\hline \multicolumn{8}{|l|}{ SYNCHROTRON VACUUM SYSTEM SCHEDULE } \\
\hline Fiscal Year & FY95 & FY96 & FY97 & FY98 & FY99 & FYOO & FYO1 \\
\hline KD 0 Milestone & $\$$ & & & & & & \\
\hline KD 1 Milestone & & $\%$ & & & & & \\
\hline Construction Stant Milestone & & & & & & & \\
\hline \multicolumn{8}{|l|}{ R\&D } \\
\hline \multicolumn{8}{|l|}{ Ceramic Chamber Prototype R\&D } \\
\hline \multirow{2}{*}{\multicolumn{8}{|c|}{$\begin{array}{l}\text { Chamber Feed Through R\&D } \\
\text { Chamber if Shield R\&D }\end{array}$}} \\
\hline \multirow{2}{*}{\multicolumn{8}{|c|}{$\begin{array}{l}\text { Chamber I ShIela RED } \\
\text { DESIGN / CONSTRUCTION }\end{array}$}} \\
\hline & & & & & & & \\
\hline \multicolumn{8}{|l|}{ Chamber System Design } \\
\hline & & & \multirow{2}{*}{\multicolumn{2}{|c|}{ te }} & & & \\
\hline Chamber System Procurement & & & & & & & \\
\hline Pumping System Procurement & & & & $\$$ & & & \\
\hline \multicolumn{8}{|l|}{$\begin{array}{l}\text { Chamber Delivery } \\
\text { ff Shield Svstem Installation }\end{array}$} \\
\hline If Shield System Installation & & & & & & T & \\
\hline \multirow{2}{*}{\multicolumn{8}{|c|}{ Ring Building (Beneficial Occupancy Milestone) }} \\
\hline & & & & & & & \\
\hline \multicolumn{8}{|l|}{ Chamber Installation in Building 365} \\
\hline \multicolumn{8}{|l|}{ Pumping Down Sectors } \\
\hline & & & & & & & \\
\hline Commissioning Starts Milestone & & & & & & 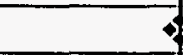 & \\
\hline Commissioning Ends Milestone & & & & & & & \$ \\
\hline
\end{tabular}

Figure VII.3-2f Synchrotron Vacuum System Schedule. 


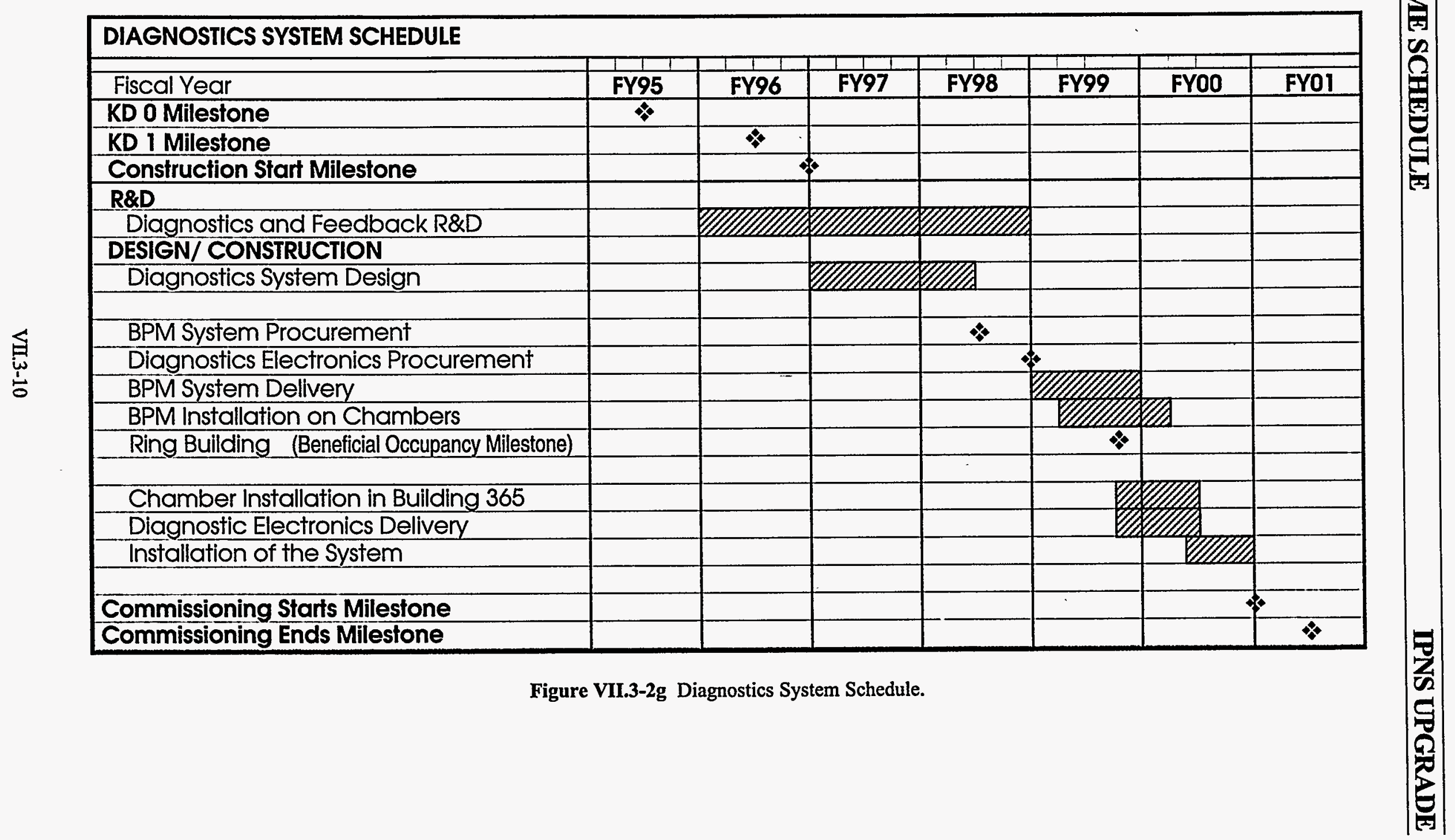




\begin{tabular}{|c|c|c|c|c|c|c|c|}
\hline \multicolumn{8}{|c|}{ CONTROLS AND COMPUTING SYSTEM SCHEDULE } \\
\hline KD O Milestone & 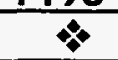 & & & & & & \\
\hline Construction Start Milestone & & & & & & & \\
\hline R\&D & & & & & & & \\
\hline Control System R\&D -Adapt from APS & & & & & & & \\
\hline Control System Design & & & E & & & & \\
\hline Integration of Subsystems Control Design & & & & & & & \\
\hline CONSTRUCTION & & & & & & & \\
\hline Write Control Software & & & & & & & \\
\hline Control System Electronics Procurement & & & & $\star$ & & & \\
\hline Control System Computers Procurement & & & & & & & \\
\hline Subsystem Testing & & & & & 8 & ד्र & \\
\hline Installation of the System & & & & & & & \\
\hline & & & & & & & \\
\hline Data System Computer Procurement & & & & & $\%$ & & \\
\hline Data System Computer Dellvery & & & & & & $*$ & \\
\hline & & & & & & & \\
\hline Commissioning Starts Milestone & & & & & & & 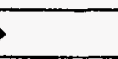 \\
\hline Commissioning Ends Milestone & & & & & & & 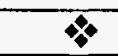 \\
\hline
\end{tabular}

Figure VII.3-2h Controls and Computing System Schedule. 


\begin{tabular}{|c|c|c|c|c|c|c|c|}
\hline TRANSFER LINES SCHEDULE & & & & & & & \\
\hline Fiscal Year & FY95 & FY96 & FY97 & FY98 & FY99 & FYOO & FY01 \\
\hline KD O Milestone & 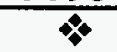 & & & & & & \\
\hline R\&D & & & & & & & \\
\hline Performance Specifications & & & WII & & & & \\
\hline DESIGN / CONSTRUCTION & & & & & & & \\
\hline Low Energy Transfer Line Delivery & & & & & & & \\
\hline Low Energy Transfer Line Measurements & & & & & 牳 & & \\
\hline Ring Building (Beneficial Occupancy Milestone) & & & & & \& & & \\
\hline & & & & & & & \\
\hline Low Energy Transfer Line Installation & & & & & & Z & \\
\hline Linac Commisioning Milestone & & & & & & $\$$ & \\
\hline High Energy Transfer Line Installation & & & & & & & \\
\hline & & & & & & & \\
\hline Commissioning Starts Milestone & & & & & & & \\
\hline Commissioning Ends Milestone & & & & & & & $\$$ \\
\hline
\end{tabular}

Figure VII.3-2i Transfer Lines Schedule. 


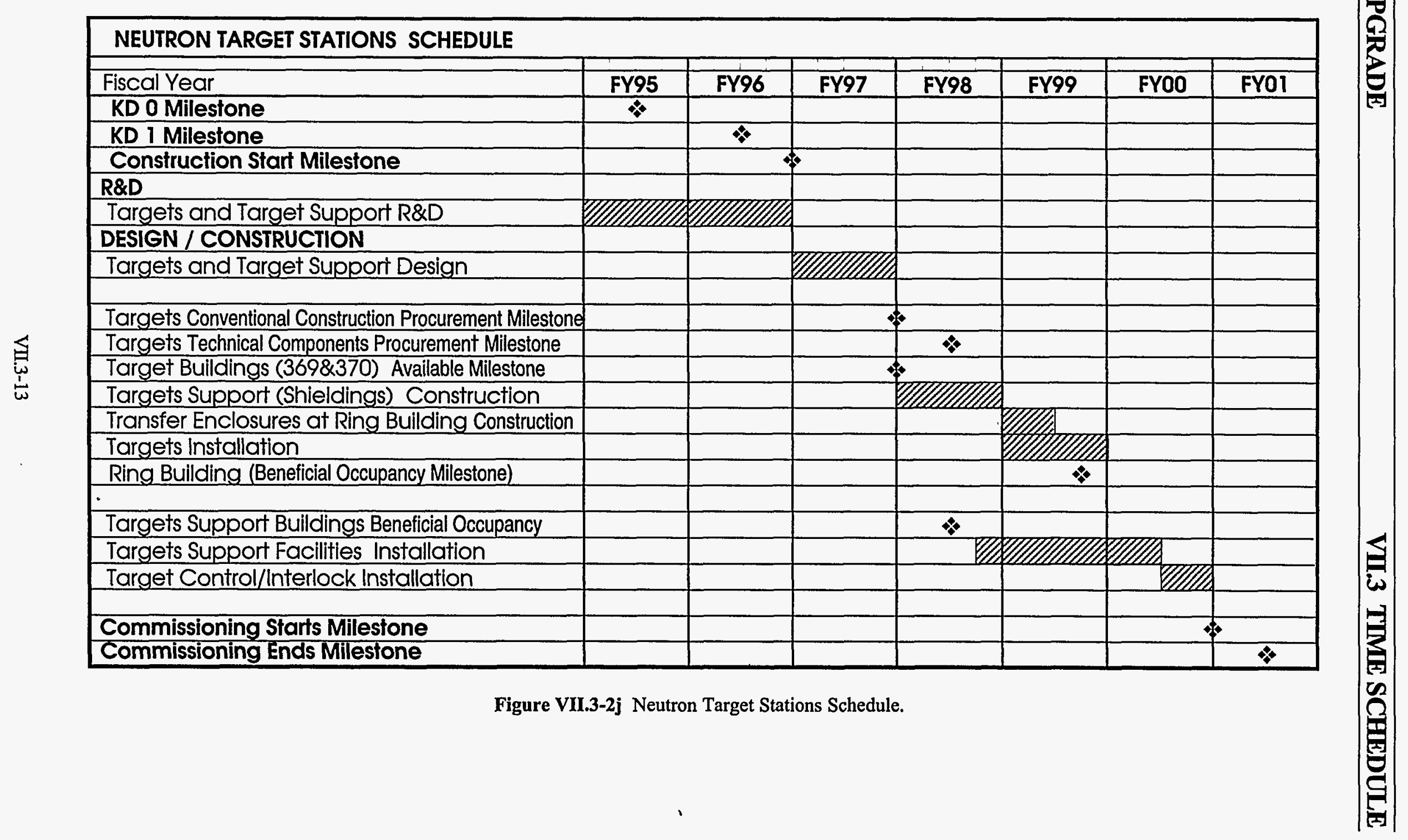




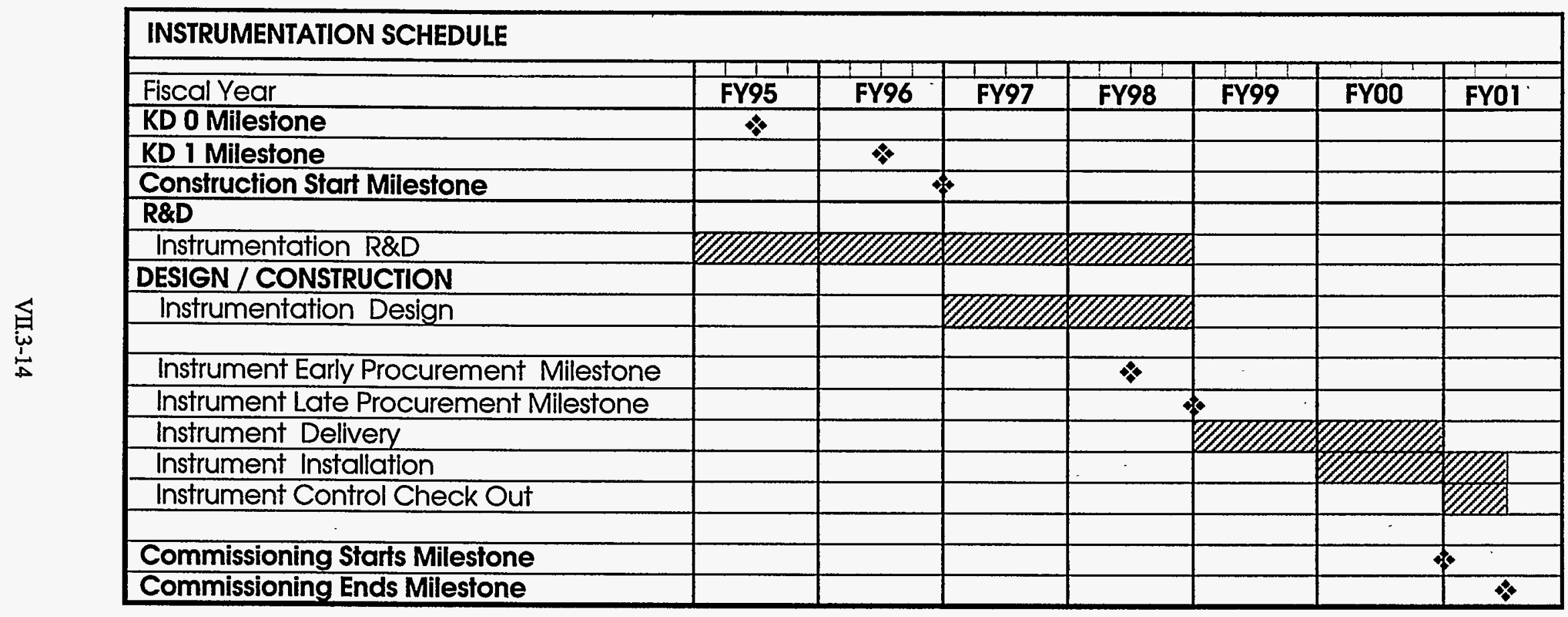

Figure VII.3-2k Instrumentation Schedule. 


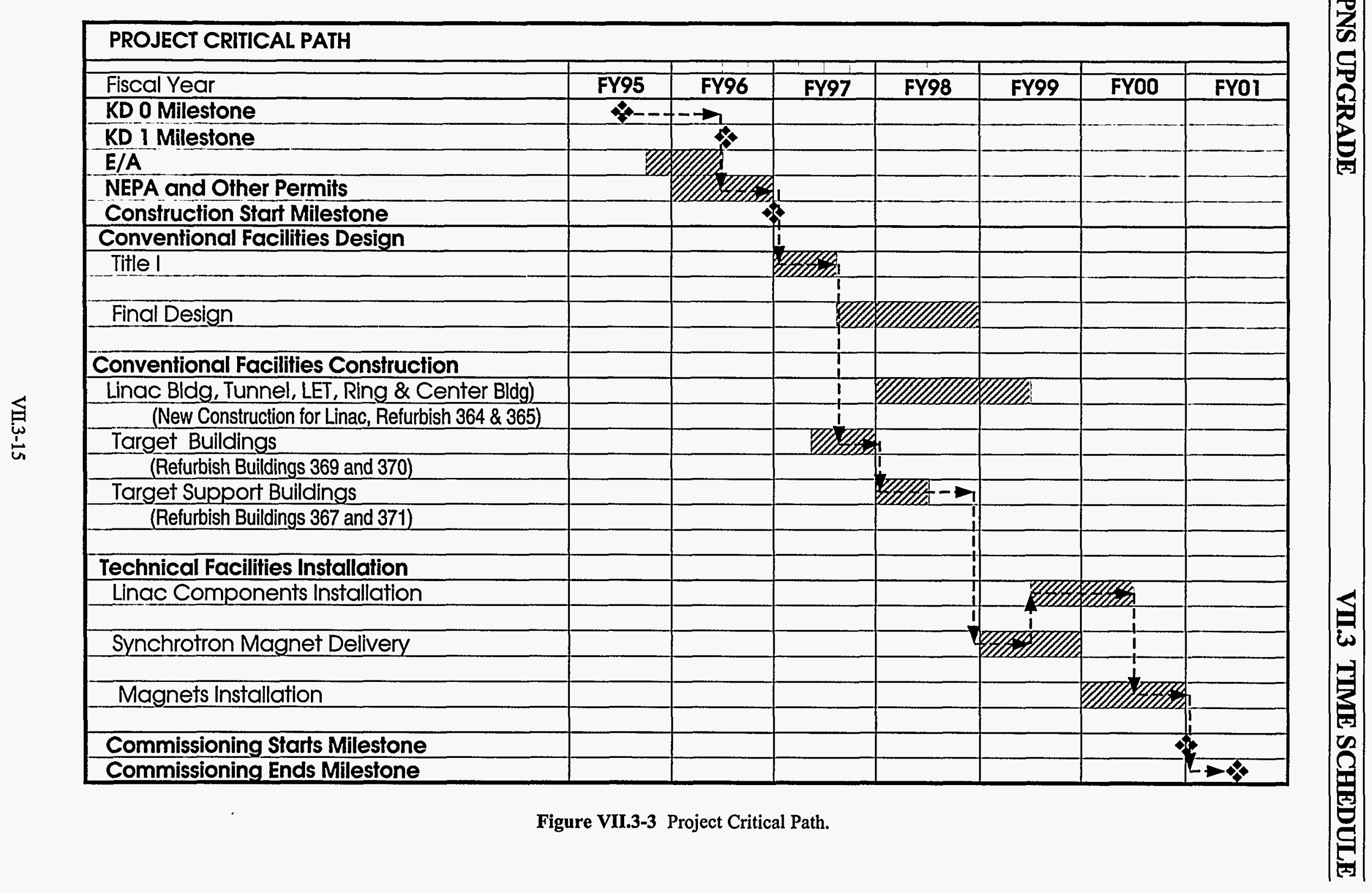


The cost schedule is obtained by adding the time-based person-power costs to the timebased materials costs. On the basis of the detailed WBS-based cost estimates (which contain the cost of materials and labor of various crafts for each of the components), together with the technical components construction schedule described in the previous section, technical component person-power needs per quarter year have been estimated as a function of time. The effort costs per year are shown in Figure VII.4-1. Materials costs based on the procurement schedule, and conventional facility construction costs based on the $\mathrm{A} / \mathrm{E}$ schedule, are similarly projected for each quarter year.

The budget profile of the project is obtained from

- The manpower distribution (Figure VII.4-1),

- Materials costs distribution,

- Addition of contingency proportional to the part of the task or activities not completed, and

- Incorporation of the escalation shown in Table VII.2-2.

Table VII.4.1 shows the budget authority (BA) profile for high-level WBS elements. Integration of the schedule into the BA distribution can be seen in this table. Figure VII.4-2 shows the BA distribution, and Figure VII.4-3 shows the cumulative BA distribution that extends from FY 1997 to FY 2001.

This schedule projects that the total estimated cost (TEC) of the construction is $\$ 558.9$ million. The schedule results in an escalation of $\$ 80.9$ million, which is shown as the difference between the TEC of $\$ 558.9$ million and the FY 1995 construction cost estimate of $\$ 478.0$ million shown in Table VII.2-3. The TEC, broken down into (1) plant and capital equipment (PACE) construction costs and (2) ED\&I costs, together with the escalation and contingency, is shown in Table VII.4-2. The breakdown follows the guidelines established by the Engineering, Design and Inspection Steering Committee of the U.S. Department of Energy (DOE) Committee for Cost Methods Development and described in a report dated August 23, 1985, and in DOE Order 4700.1.

The cash flow distribution, the budget outlay (BO), for high-level WBS elements is shown in Table VII.4-3. Figure VII.4-4 shows the comparison between the cumulative BA and the BO. 


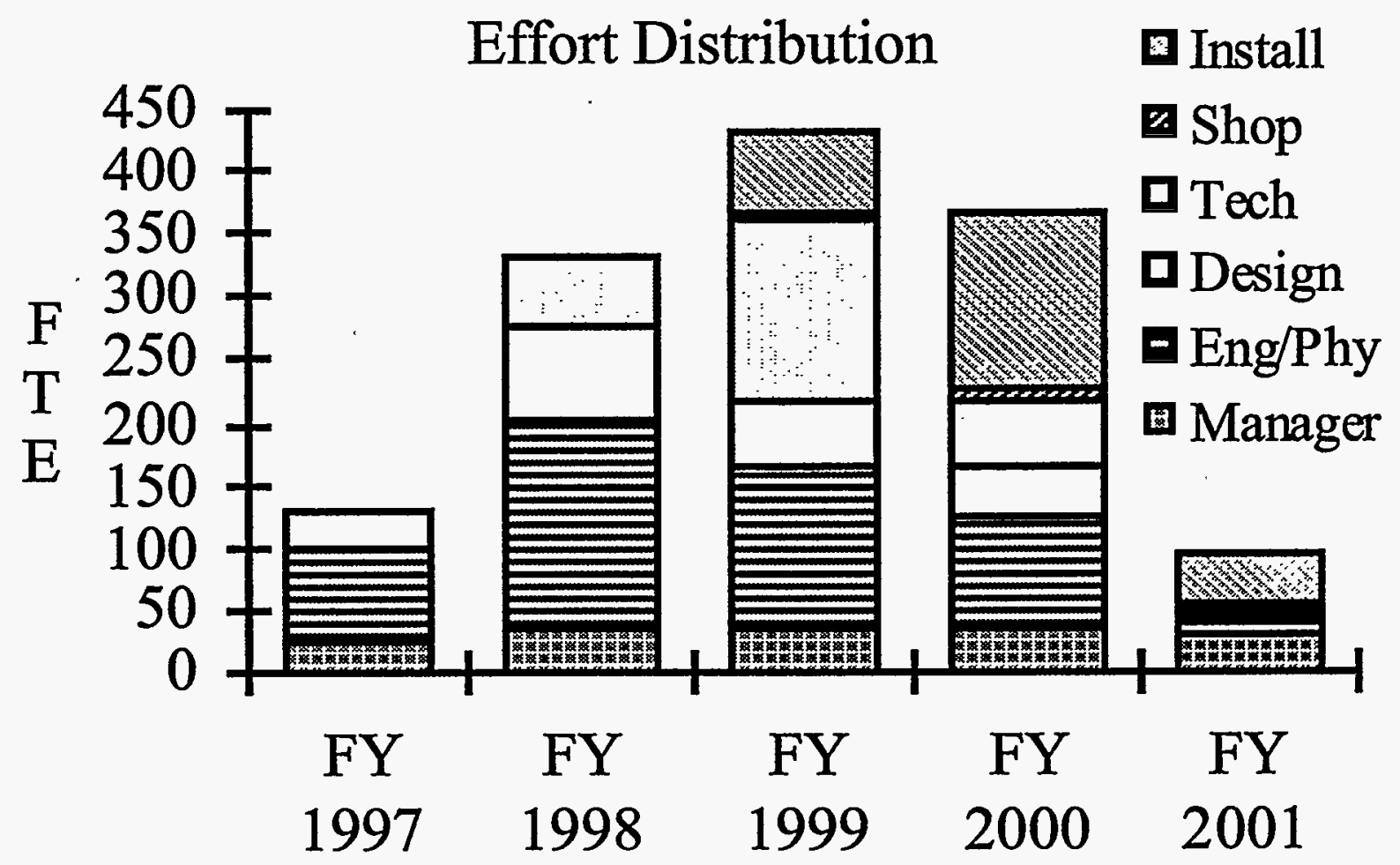

Figure VII.4-1 Construction Project Person-Effort Distribution. 
Table VII.4-1 Budget Authority (BA) Distribution (Actual Year k\$) ${ }^{\mathrm{a}}$

\begin{tabular}{|c|c|c|c|c|c|c|c|}
\hline $\begin{array}{l}\text { WBS } \\
\text { Code }\end{array}$ & Description & FY1997 & FY1998 & FY1999 & FY2000 & FY2001 & Total \\
\hline \multicolumn{2}{|c|}{ Escalation \% from FY $1995 \$$} & $7.8 \%$ & $3.8 \%$ & $3.6 \%$ & $3.6 \%$ & $3.6 \%$ & \\
\hline \multicolumn{2}{|c|}{ Escalation Factor } & 1.078 & 1.119 & 1.159 & 1.201 & 1.244 & \\
\hline 1 & IPNS UPGRADE (1 MW) & 16839 & 95053 & 207294 & 205966 & 33786 & 558938 \\
\hline 1.1 & Project Management & 2695 & 2238 & 2393 & 2283 & 2037 & 11645 \\
\hline 1.2 & Accelerator Systems & 6145 & 41411 & 100223 & 41576 & 0 & 189354 \\
\hline 1.2 .1 & Injector System & 2911 & 17273 & 39994 & 11322 & 0 & 71500 \\
\hline 1.2 .2 & Synchrotron & 3234 & 19281 & 46821 & 17945 & 0 & 87281 \\
\hline 1.2 .3 & Beam Transports & 0 & 4353 & 9350 & 5243 & 0 & 18947 \\
\hline 1.2 .4 & Technical Support Systems & 0 & 504 & 4057 & 7065 & 0 & 11626 \\
\hline 1.3 & Targets and Instruments & 1617 & 10686 & 14426 & 56717 & 20494 & 103940 \\
\hline 1.3 .1 & Target Stations & 1078 & 4867 & 6311 & 13439 & 6253 & 31949 \\
\hline 1.3 .2 & Instruments and Support & 539 & 5819 & 8115 & 43279 & 14241 & 71991 \\
\hline 1.4 & Conventional Facilities & 1078 & 11749 & 19030 & 20957 & 834 & 53648 \\
\hline 1.4 .1 & Existing Building Rehabilitation & 0 & 5595 & 10707 & 12263 & 470 & 29034 \\
\hline 1.4 .2 & New Construction & 0 & 2238 & 3892 & 2445 & 0 & 8575 \\
\hline 1.4 .3 & Utilities & 0 & 1119 & 3478 & 5648 & 0 & 10245 \\
\hline 1.4 .4 & Conventional Facilities EDI\&A & 1078 & 2797 & 953 & 600 & 365 & 5793 \\
\hline 1.5 & Shielding Structures & 323 & 854 & 9908 & 23511 & 428 & 35024 \\
\hline 1.5 .1 & HE Transport Shielding & 108 & 182 & 2721 & 6608 & 0 & 9619 \\
\hline 1.5 .2 & 30-Hz Target Shielding & 108 & 336 & 3594 & 8452 & 214 & 12703 \\
\hline 1.5 .3 & 10-Hz Target Shielding & 108 & 336 & 3594 & 8452 & 214 & 12703 \\
\hline 1.8 & Misc. Costs & 2231 & 12593 & 27462 & 27287 & 4476 & 74049 \\
\hline 1.8 .1 & Overheads & 1568 & 8854 & 19308 & 19185 & 3147 & 52062 \\
\hline 1.8 .2 & Unproductive Time & 284 & 1602 & 3495 & 3472 & 570 & 9423 \\
\hline 1.8 .3 & M\&S for Staff & 378 & 2137 & 4659 & 4630 & 759 & 12563 \\
\hline 1.9 & Contingency & 2750 & 15523 & 33852 & 33635 & 5518 & 91278 \\
\hline
\end{tabular}

a Numbers are spreadsheet-generated, and costs may not exactly add up to totals because of rounding. 


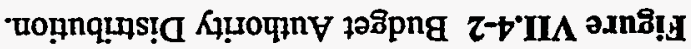

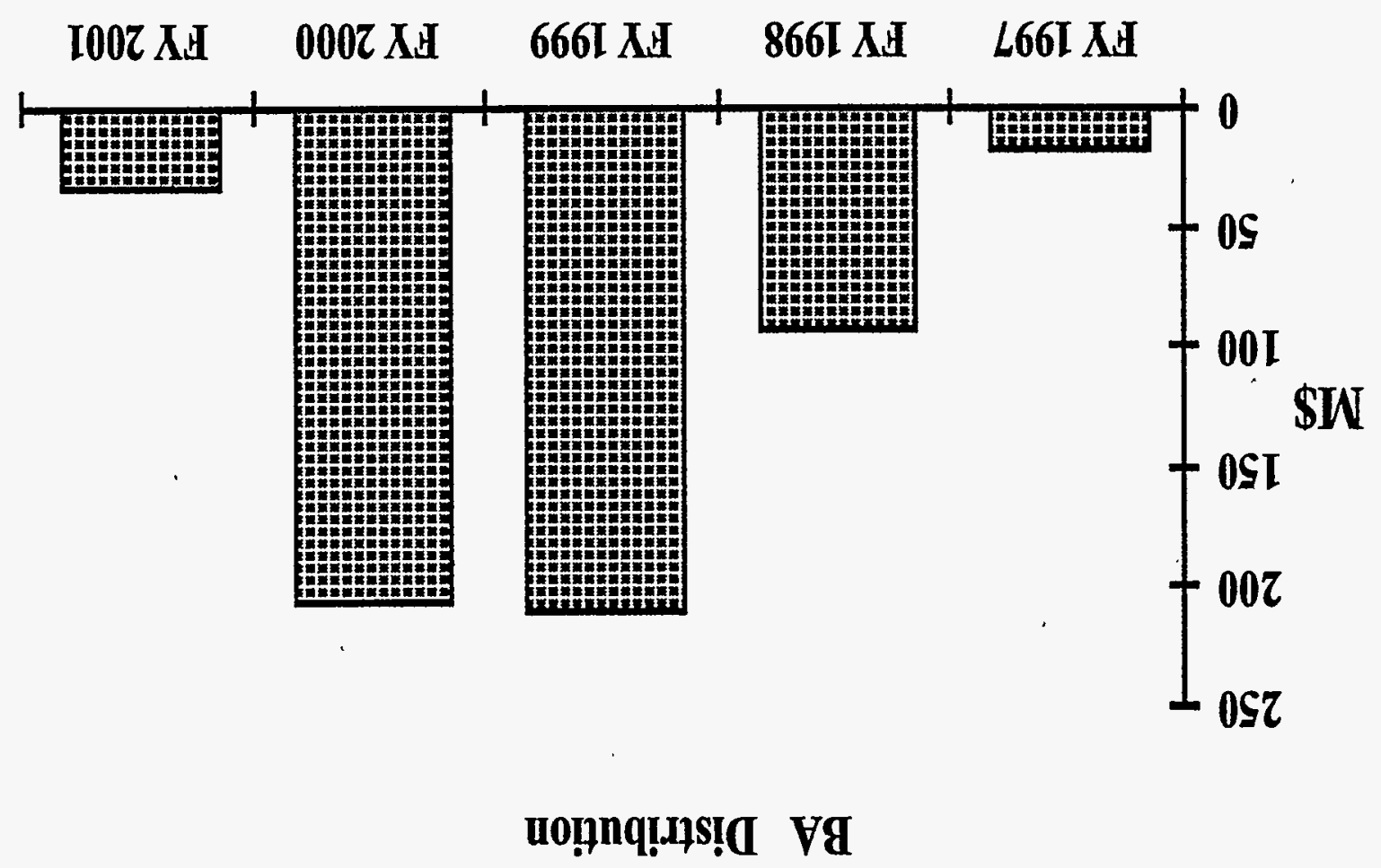




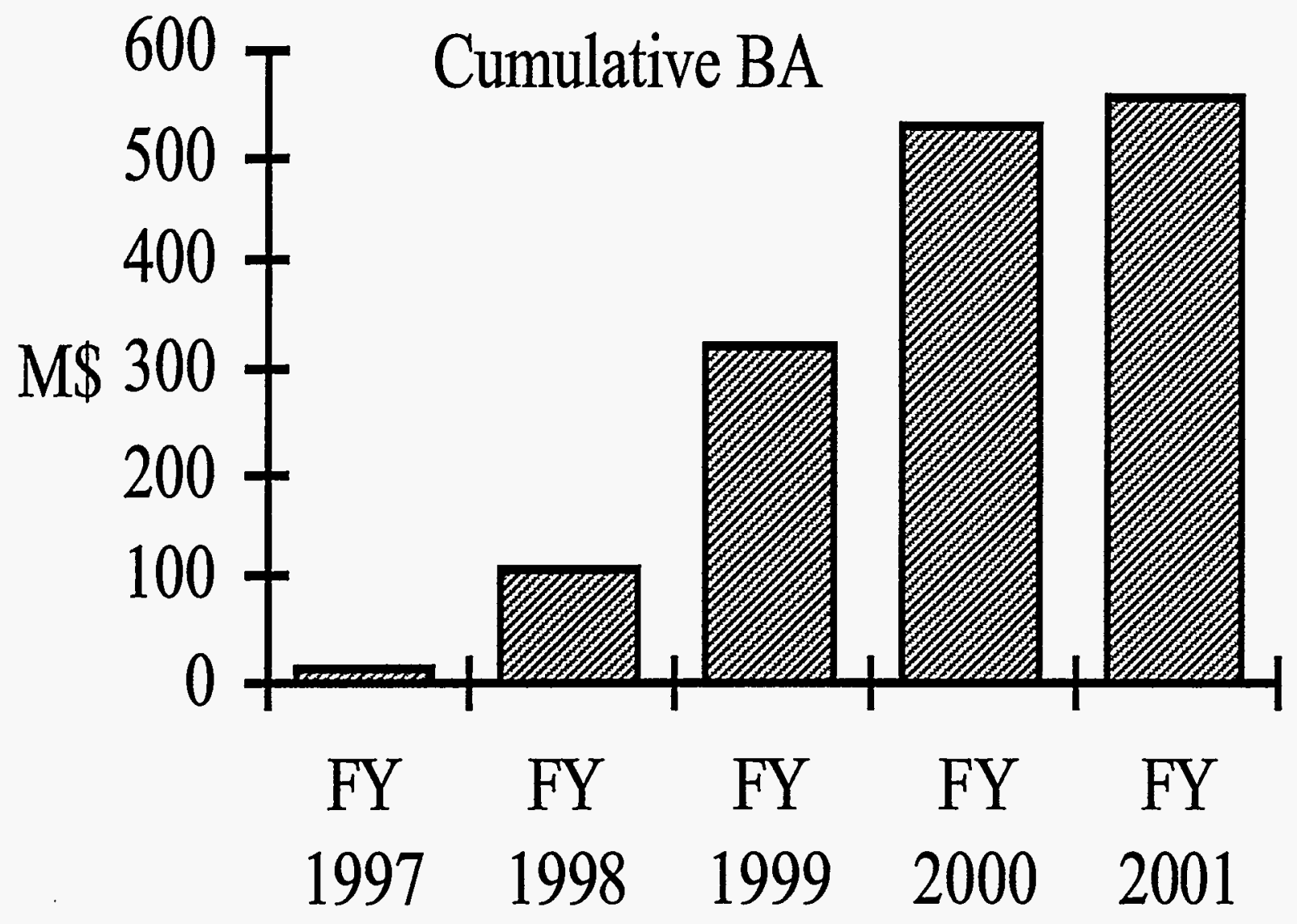

Figure VII.4-3 Cumulative BA Distribution. 
Table VII.4-2 TEC Breakdown by Major Category

(\$ million estimated in FY 1995)

Engineering, Design, and Inspection Construction

Contingency

Escalation

TEC
43.158

356.815

78.066

80.899

558.938 
Table VII.4-3 Budget Outlay (BO) Distribution (Actual Fiscal Year k\$) ${ }^{\mathrm{a}}$

\begin{tabular}{|c|c|c|c|c|c|c|c|}
\hline $\begin{array}{l}\text { WBS } \\
\text { Code }\end{array}$ & Description & FY1997 & FY1998 & FY1999 & FY2000 & FY2001 & Total \\
\hline 1 & IPNS UPGRADE (1 MW) & 16838 & 69853 & 175759 & 228869 & 67620 & 558938 \\
\hline 1.1 & Project Management & 2695 & 2238 & 2393 & 2283 & 2037 & 11645 \\
\hline 1.2 & Accelerator Systems & 6145 & 23611 & 79023 & 80576 & 0 & 189354 \\
\hline 1.2 .1 & Injector System & 2911 & 9273 & 27994 & 31322 & 0 & 71500 \\
\hline 1.2 .2 & Synchrotron & 3234 & 11281 & 35821 & 36945 & 0 & 87281 \\
\hline 1.2 .3 & Beam Transports & 0 & 2553 & 11150 & 5243 & 0 & 18947 \\
\hline 1.2 .4 & Technical Support Systems & 0 & 504 & 4057 & 7065 & 0 & 11626 \\
\hline 1.3 & Targets and Instruments & 1617 & 7686 & 12426 & 37717 & 44494 & 103940 \\
\hline 1.3 .1 & Target Stations & 1078 & 2867 & 6311 & 11439 & 10253 & 31949 \\
\hline 1.3 .2 & Instruments and Support & 539 & 4819 & 6115 & 26279 & 34241 & 71991 \\
\hline 1.4 & Conventional Facilities & 1078 & 7349 & 14196 & 25359 & 5666 & 53648 \\
\hline 1.4 .1 & Existing Building Rehabilitation & 0 & 2994 & 8628 & 14611 & 2802 & 29034 \\
\hline 1.4 .2 & New Construction & 0 & 1238 & 3137 & 3700 & 500 & 8575 \\
\hline 1.4 .3 & Utilities & 0 & 319 & 1478 & 6448 & 2000 & 10245 \\
\hline 1.4 .4 & Conventional Facilities EDI\&A & 1078 & 2797 & 953 & 600 & 365 & 5793 \\
\hline 1.5 & Shielding Structures & 323 & 854 & 6408 & 22011 & 5428 & 35024 \\
\hline 1.5 .1 & HE Transport Shielding & 108 & 182 & 2221 & 7108 & 0 & 9619 \\
\hline 1.5 .2 & 30-Hz Target Shielding & 108 & 336 & 2094 & 7452 & 2714 & 12703 \\
\hline 1.5 .3 & 10-Hz Target Shielding & 108 & 336 & 2094 & 7452 & 2714 & 12703 \\
\hline 1.8 & Misc. Costs & 2230 & 12593 & 27462 & 27287 & 4477 & 74049 \\
\hline 1.8 .1 & Overhead & 1568 & 8854 & 19308 & 19185 & 3147 & 52062 \\
\hline 1.8 .2 & Unproductive Time & 284 & 1602 & 3495 & 3472 & 570 & 9423 \\
\hline 1.8 .3 & M\&S for Staff & 378 & 2137 & 4659 & 4630 & 759 & 12563 \\
\hline 1.9 & Contingency & 2750 & 15523 & 33852 & 33635 & 5518 & 91278 \\
\hline
\end{tabular}

a Numbers are spreadsheet-generated, and costs may not exactly add up to totals because of rounding. 


\section{Cumulative BA and BO}

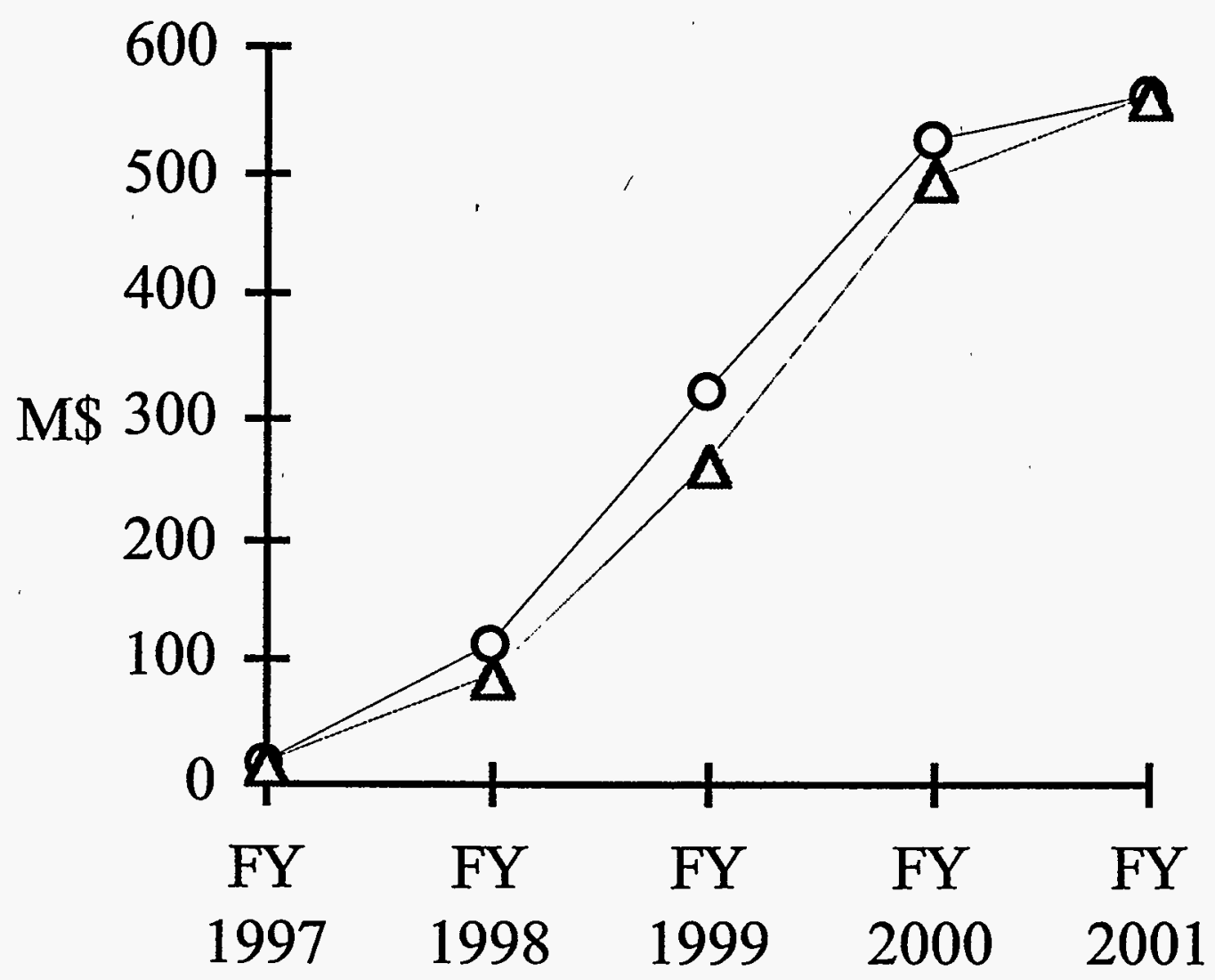

Figure VII.4-4 Comparison between Cumulative BA and BO.

(The top curve is BA, and the bottom curve is $\mathrm{BO}$.) 


\section{Appendix A}

Parameter List 
All parameters are at nominal operating conditions, unless otherwise noted.

\section{RCS Performance Parameters}

Beam energy

Injection

Extraction

Maximum design energy

Beam repetition rate

Beam average current

Beam power

Number of circulating protons

Beam emittance

Horizontal

Vertical

Longitudinal

Momentum spread, $\Delta \mathrm{p} / \mathrm{p}$, fwhm

Injection

Extraction

Kinetic energy spread, $\Delta \mathrm{T} / \mathrm{T}$, fwhm

Injection

Extraction

Bunch length, baseline

Injection

Extraction

Bunching factor, peak current/average current

Injection

Extraction

$\begin{array}{ll}400 & \mathrm{MeV} \\ 2.0 & \mathrm{GeV} \\ 2.2 & \mathrm{GeV} \\ 30 & \mathrm{~Hz} \\ 0.5 & \mathrm{~mA} \\ 1.0 & \mathrm{MW} \\ 1.04 \times 10^{14} & \\ & \\ 375 \pi & \mathrm{mm}-\mathrm{mrad} \\ 375 \pi & \text { mm-mrad } \\ 7.3 & \text { eV sec } \\ & \\ 1.0 & \% \\ 0.8 & \%\end{array}$

$1.7 \quad \%$

$1.1 \quad \%$

$0.8 \times 190.4 \mathrm{~m}$

$0.3 \times 190.4 \mathrm{~m}$

0.38

0.16

\section{RCS Lattice and Orbit Parameters}

Circumference

Super-periodicity

Revolution time

Injection

Extraction

Harmonic number

Radio frequency

Injection

Extraction

190.4

4

$890.1 \quad$ nsec

$665.1 \quad$ nsec

Lattice structure

Normal FODO cells per quadrant

Missing magnet FODO cells per quadrant

1

Empty FODO cells per quadrant

$1.1235 \quad \mathrm{MHz}$

$1.5035 \mathrm{MHz}$


Mean radius

Bending field

Injection

0.48

Extraction

Maximum design value

Magnetic lengths in a normal FODO cell

$(\mathrm{D}=$ drift, $\mathrm{B}=$ bend, $\mathrm{Q}=$ quad, $\mathrm{S}=$ sext)

0.25

0.30

m

D1

0.20

$\mathrm{m}$

SD

0.30

m

D1

1.30

$\mathbf{m}$

B

0.80

m

D2

0.50

m

QF

0.30

$\mathbf{m}$

D1

0.20

m

SF

0.30

m

D1

1.30

m

B

0.80

\section{D2}

0.25

$\mathbf{m}$

QD

6.821

5.731

Vertical

Synchrotron frequency

Injection

3.0

$\mathrm{kHz}$

Extraction

0.9

$\mathrm{kHz}$

Natural chromaticity, $\xi=(\Delta v / v) /(\Delta p / p)$

Horizontal

$-1.06$

Vertical

$-1.20$

Beta functions

Maximum horizontal

10.59

2.25

Minimum horizontal

11.98

Maximum vertical

Minimum vertical

2.53

m

m

Dispersion function

Maximum horizontal

Minimum horizontal

Momentum compaction

Transition Gamma, $\gamma_{t}$

$2.16 \mathrm{~m}$

$-0.06$

$\mathbf{m}$

$3.42 \times 10^{-2}$

5.40 


\section{RCS Tolerances, rms}

Quadrupole placement $2.0 \times 10^{-4} \quad \mathrm{~m}$

Sextupole placement $2.0 \times 10^{-4} \quad \mathrm{~m}$

Random dipole roll-angle error

$1.0 \times 10^{-3} \mathrm{rad}$

Dipole tracking error

$1.0 \times 10^{-3}$

Quadrupole tracking error

$2.5 \times 10^{-4}$

\section{RCS Hardware}

a. Magnets and power supplies

Dipoles

Number $\quad 32$

Number of independent power supplies $\quad 24$

Magnetic length $\quad 1.3$

Bending radius $\quad 6.62$

Dipole field at $2 \mathrm{GeV}$

1.40

m

Beam-stay-clear

Entrance, max

Horizontal

136

Vertical

156

$\mathrm{mm}$

Center, max

Horizontal

160

Vertical

134

$\mathbf{m}$

it, $\max$

Horizontal

Vertical

$\mathrm{mm}$

Quadrupoles

Number, QF, QD

QF family

QD family

Number of independent power supplies, QF, QD 42

QF family

QD family

Magnetic length

Maximum gradient at $2 \mathrm{GeV}$

7.73

m

Beam-stay-clear

QF family, max

Horizontal

Vertical

D family, $\max$

Horizontal

$\mathrm{mm}$

Vertical

190

$\mathrm{mm}$

\section{Sextupoles}

Number, SF, SD, S1 
SF family $\quad 16$

SD family $\quad 16$

S1 family 8

Number of independent power supplies, SF, SD, S1 40

SF family

16

SD family

S1 family

16

8

Magnetic length

Maximum strength at $2 \mathrm{GeV}$

0.20

52

m

Beam-stay-clear

SF family, max

Horizontal

202

Vertical

102

mm

SD family, max

Horizontal

124

Vertical

168

$\mathrm{mm}$

S1 family, max

Horizontal

Vertical

100

174

$\mathrm{mm}$

Correction dipoles

Horizontal correctors

Number

Independent power supplies

28

Magnetic length

0.15

m

Peak field at $2 \mathrm{GeV}$

0.07

$\mathrm{T}$

Beam stay clear, max

Horizontal

222

$\mathrm{mm}$

Vertical

94

$\mathrm{mm}$

Vertical correctors

Number

28

Independent power supplies

28

Magnetic length

0.15

m

Peak field at $2 \mathrm{GeV}$

0.07

$\mathrm{T}$

Beam stay clear, max

Horizontal

114

$\mathrm{mm}$

Vertical

182

$\mathrm{mm}$

b. RF system

Frequency

Injection

Extraction

1.1235

$\mathrm{MHz}$

1.5035

$\mathrm{MHz}$

Number of cavities

10

Harmonic number

Length (inside)

1

m 
Peak voltage

Cavity

Total rf system

18

180

$\mathrm{kV}$

Cavity Q

Injection

33.2

Extraction

63.3

Cavity shunt impedance

Injection

Extraction

Total peak $\mathrm{rf}$ power

$\begin{array}{ll}5 & \mathrm{k} \Omega \\ 8.1 & \mathrm{k} \Omega \\ 2.62 & \mathrm{MW}\end{array}$

c. Injection equipment

Injection Septa

Number

2

Magnetic length

Septum A

Septum B

Bending angle

Septum A

Septum B

Maximum design field at $400 \mathrm{MeV}$

Septum A

Septum B

2.62

$\mathrm{kV}$

Field index

Septum A

Septum B

Gap height

Septum A

Septum B

1.2

0.3

m

m

$228 \quad \mathrm{mrad}$

$24 \mathrm{mrad}$

$0.61 \quad \mathrm{~T}$

$0.52 \quad \mathrm{~T}$

Gap width

Septum A

Septum B

$-20$

0

Minimum septum thickness

Septum A

Septum B

20

$\mathrm{mm}$

$24 \mathrm{~mm}$

$51 \quad \mathrm{~mm}$

$40 \quad \mathrm{~mm}$

$33.4 \quad \mathrm{~mm}$

$3.0 \quad \mathrm{~mm}$

Bumper magnets

Number

4

Magnetic length

Bending angle

Peak central field at $400 \mathrm{MeV}$

Gap height

0.3

20

0.212

241.5

min

Gap width

300

m

$\operatorname{mrad}$

$\mathrm{T}$

$\mathrm{mm}$

$\mathrm{mm}$ 
d. Extraction equipment

Extraction septa

Number

Magnetic length

Septum C

Septum D

Septum E

Bending angle

Septum C

Septum D

Septum E

Peak central field at $2.0 \mathrm{GeV}$

Septum C

Septum D

Septum E

Gap height

Septum C

Septum D

Septum E

Gap width

Septum C

Septum D

Septum E

Minimum septum thickness

Septum C

Septum D

Septum E

Kicker magnets

Number

Magnetic length

Kicker A

Kicker B

Kicker C

Bending angle

Kicker A

Kicker B

Kicker C

Peak central field at $2.0 \mathrm{GeV}$

Kicker A

Kicker B

Kicker C

$\begin{array}{ll}0.5 & \mathrm{~m} \\ 1.5 & \mathrm{~m} \\ 0.9 & \mathrm{~m}\end{array}$

55

210

126

m

$\mathbf{m}$

$\mathrm{m}$

$1.02 \quad \mathrm{~T}$

$1.3 \quad \mathrm{~T}$

$1.3 \mathrm{~T}$

70

$\mathrm{mm}$

90

$\mathrm{mm}$

110

mm

122

$\mathrm{mm}$

102

$\mathrm{mm}$

70

$\mathrm{mm}$

6.0

$\mathrm{mm}$

57.1

227.7

$\mathrm{mm}$

3

0.65

0.87

m

1.105

$\mathrm{m}$

1.105

m

4.37

4.79

$\operatorname{mrad}$

4.84

mrad

4.84

mrad

Gap height

Kicker A

0.0618

0.0512

$\mathrm{T}$

0.0408

$\mathrm{T}$

$\mathrm{T}$

Kicker B

132.4

$\mathrm{mm}$

159.2

$\mathrm{mm}$

Kicker C

200.0

$\mathrm{mm}$ 
Gap width

Kicker A

220.0

195.0

mm

Kicker B

170.0

$\mathrm{mm}$

Kicker C

$\mathrm{mm}$

e. Beam diagnostics (number of each type)

2-Stripline BPMs

Horizontal

28

Vertical

28

Current transformer

2

Loss monitor

Pingers

Horizontal

Vertical

Segmented Faraday cup

4

Horizontal

2

Vertical

Solid first-turn Faraday cup

\section{Injector Linacs}

a. General

Accelerated ion

Output kinetic energy

Pulsed beam current

Beam duty factor

Beam pulse length

Beam repetition rate

Time-averaged current

Radio-frequency duty factor

Total rf power

Peak

Average

Transverse emittance, normalized, rms

Output from linac

Input to RCS, required

Output energy spread, fwhm

Length

b. Ion source

Output energy

Output current

Output transverse emittance, normalized, rms

Length
$\mathrm{H}^{-}$

400

35

1.5

500

30

0.5

3

104

4.85

$0.2 \pi$

$1.0 \pi$

2.5

120

35

60

$0.1 \pi$

0.5
$\mathrm{MeV}$

$\mathrm{mA}$

$\%$

$\mu \mathrm{sec}$

$\mathrm{Hz}$

$\mathrm{mA}$

$\%$

MW

MW

mm - mrad

mm-mrad

$\mathrm{MeV}$

m

$\mathrm{keV}$

$\mathrm{mA}$

mm-mrad

m 
c. RFQ

Input energy

Output energy

Output current

Output transverse emittance, normalized, rms

Operating frequency

Bore radius

Synchronous phase

Peak structure power

Number of tanks/modules

Structure length

Peak beam power at $40 \mathrm{~mA}$
35

2.0

35

$0.12 \pi$

425

2.4-3.0

$-30$

0.22

1

2.16

0.08

2.0

70.0

35

$0.135 \pi$

425

2.4-4.6

$5.0-9.0$

$-30$

6.28

4

20.54

2.72

70.0

70.0

35

Output current

Output transverse emittance, normalized, rms

Operating frequency

Accelerating gradient, $\mathrm{E}_{0} \mathrm{~T}$

Bore radius

Synchronous phase

Peak structure power

Number of tanks/modules

Structure length

Peak beam power at $40 \mathrm{~mA}$
$0.151 \pi$

1,275

$\overline{10.0}$

$\overline{1.2}$

2

3.2

$-$

70.0

400.0

35
$\mathrm{keV}$

$\mathrm{MeV}$

$\mathrm{mA}$

mm-mrad

$\mathrm{MHz}$

$\mathrm{mm}$

deg

MW

m

MW

$\mathrm{MeV}$

$\mathrm{MeV}$

$\mathrm{mA}$

mm - mrad

$\mathrm{MHz}$

$\mathrm{MV} / \mathrm{m}$

$\mathrm{mm}$

deg

MW

m

MW

$\mathrm{MeV}$

$\mathrm{MeV}$

$\mathrm{mA}$

mm - mrad

$\mathrm{MHz}$

$\mathrm{MV} / \mathrm{m}$

$\mathrm{mm}$

deg

MW

m

MW

$\mathrm{MeV}$

$\mathrm{MeV}$

$\mathrm{mA}$ 
Output transverse emittance, normalized, rms

$0.194 \pi$

Operating frequency

Accelerating gradient, $\mathrm{E}_{0} \mathrm{~T}$

Bore radius

Synchronous phase

Peak structure power

Number of tanks/modules

Structure length

Peak beam power at $40 \mathrm{~mA}$
1,275

5.9-5.5

$10.0-12.0$

-30 to -25

52.8

$6 / 10$

92.1

13.2 mm- mrad

$\mathrm{MHz}$

$\mathrm{MV} / \mathrm{m}$

$\mathrm{mm}$

deg

MW

m

MW

\section{Transport Line between Linac and RCS}

a. Magnets

Dipoles, A

Number

15

No. of independent power supplies

3

Magnetic length

Peak field at $400 \mathrm{MeV}$

Bending angle at $400 \mathrm{MeV}$

Gap

Height

Width

Quadrupoles, A

Number

1.0
0.91

m

286

$\mathrm{T}$

40

$\operatorname{mrad}$

No. of independent power supplies

$\mathrm{mm}$

Magnetic length

Peak strength at $400 \mathrm{MeV}$

Gap

Quadrupoles, B

Number

No. of independent power supplies

Magnetic length

Peak strength at $400 \mathrm{MeV}$

Gap

Correction dipoles

62

62

0.2

14.0

m

53

$\mathrm{T} / \mathrm{m}$

$\mathrm{mm}$

Number

3

3

0.2

12.0

m

72

$\mathrm{T} / \mathrm{m}$

16

No. of independent power supplies

16

Magnetic length

Peak field at $400 \mathrm{MeV}$

Bending angle at $400 \mathrm{MeV}$

0.1

0.064

2.0

$\mathrm{mm}$

Gap

Height

53

Width

m

$\mathrm{T}$

mrad

$\mathrm{mm}$

$\mathrm{mm}$ 
b. Beam diagnostics, number of each type

4-Stripline BPM

Current transformer

Profile detectors

Loss monitor

\section{Transport Line between RCS and Target}

a. Magnets, $10 \mathrm{~Hz}, 30 \mathrm{~Hz}$

Dipoles, B

Number

No. of independent power supplies

Magnetic length

Peak field at $2.0 \mathrm{GeV}$

Bending angle at $2.0 \mathrm{GeV}$

Gap

Height

Width

$\operatorname{mrad}$

Dipoles, C

450

mm

Number

2

No. of independent power supplies

2

Magnetic length

Peak field at $2.0 \mathrm{GeV}$

Bending angle at $2.0 \mathrm{GeV}$

Gap

Height

182

Width

450

mo

Dipoles, D

2

Number

2

Magnetic length

Peak field at $2.0 \mathrm{GeV}$

Bending angle at $2.0 \mathrm{GeV}$

Gap

Height

Width

Quadrupoles, C

Number

2.8

1.36

410

m

$\mathrm{T}$

$\operatorname{mrad}$

182

$\mathrm{mm}$

450

$\mathrm{mm}$

17

17

0.5

9.1

170

$\mathrm{T} / \mathrm{m}$

Gap

$\mathrm{mm}$

Quadrupoles, D

Number

No. of independent power supplies 
Magnetic length

Peak strength at $2.0 \mathrm{GeV}$

Gap

Correction dipoles

Number

No. of independent power supplies

Magnetic length

Peak field at $2.0 \mathrm{GeV}$

Bending angle

Gap

Height

Width

$\mathrm{m}$

$\mathrm{mm}$

16

16

0.15

$\mathrm{m}$

0.127

$\mathrm{T}$

2

$\operatorname{mrad}$

300

$\mathrm{mm}$

300

$\mathrm{mm}$

b. Beam diagnostics, number of each type

10-Hz transport line

4-Stripline BPM

4

Current transformer

3

Profile detectors

Loss monitor

4

Target protection monitor

1

1

$30-\mathrm{Hz}$ transport line

4-Stripline BPM

6

Current transformer

Profile detector

4

Loss monitor

1

Target protection monitor

1 


\section{Appendix B}

\section{Research and Development Plan}




\section{B.1 INTRODUCTION}

Preconstruction research and development (R\&D) work, when properly executed, can save construction costs and shorten the construction period. Therefore, a vigorous R\&D program is planned as soon as funds for such activities are available. The R\&D work in support of construction can be categorized in two parts. The first part is associated with support of hardware design activities to obtain the best possible level of performance, and the second part is associated with reliability of the hardware. Both categories of $R \& D$ will be pursued according to the plan described in this section. The assumption has been made that construction of the facility will start in FY 1997.

Accelerator-related $R \& D$ is described in Sections B.2 through B.10. $R \& D$ related to the target and instruments is discussed in Sections B.11 and B.12. Funding required to carry out this $R \& D$ program is described in Section B.13.

An accelerator with very high beam power, such as this rapid cycling synchrotron (RCS), must have beam losses less than about $0.1 \%$ in order to permit hands-on maintenance, as was discussed in Chapter II. Beam loss refers to those particles that are lost during the injection, capture, acceleration, and extraction processes. Understanding of the loss mechanisms and preventing the loss processes are the accelerator physics R\&D topics.

The R\&D plan covers the following:

- Accelerator physics - theoretical;

- Accelerator physics - experimental;

- $\mathrm{H}^{-}$ion source reliability testing;

- Radio-frequency (rf) power source prototyping and testing;

- Ceramic vacuum chamber prototyping and testing; and

- Dual-harmonic ring magnet power supply prototyping and testing.

Preconstruction R\&D is scheduled so that it blends in smoothly with the initiation of the construction phase in FY 1997. Decision-making milestones are scheduled for key R\&D items and are phased appropriately with the construction schedule.

The schedule for R\&D activities is presented in Figure B.1-1, a bar chart that indicates the duration of the activities and the associated milestones. 


\section{PRECONSTRUCTION R\&D SCHEDULE}

\begin{tabular}{|c|c|c|c|c|c|c|c|}
\hline Fiscal Year & FY95 & FY96 & FY97 & FY98 & FY99 & FYOO & FY01 \\
\hline KD O Milestone & 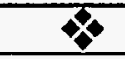 & & & & & & \\
\hline KD 1 Milestone & & 4 & & & & & \\
\hline Construction Start Milestone & & & & & & & \\
\hline Commissioning Starts Milestone & & & & & & & \\
\hline Commissioning Ends Milestone & & & & & & & $\%$ \\
\hline Accelerator Physics & & & & & & & \\
\hline If Amplifiers R\&D (Cathode Follower) & & & \& & & & & \\
\hline Decision on Amplifier Type Milestone & & & 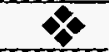 & & & & \\
\hline If Cavities and Ferrite R\&D & & & & & & & \\
\hline Low Level if R\&D & & & & & & & \\
\hline Magnets Performance Design R\&D & & & & & & & \\
\hline Dual Frequency Model Power Supply R\&D & & & & & & & \\
\hline Ceramic Chamber Prototype R\&D & & & & & & & \\
\hline Chamber Feed Through R\&D & & & & & & & \\
\hline Chamber if Shield R\&D & & & & & & & \\
\hline lon Source R\&D & & & & & & & \\
\hline$R F Q R \& D$ & & & & & & & \\
\hline$C C L R \& D$ & & & & & & & \\
\hline Kicker and Septum R\&D & & & & & & & \\
\hline Kicker \& Septum Prototype & & & & & & & \\
\hline Prototype Testing Milestone & & & & & & & \\
\hline Diägnostics and Feedback R\&D & & & & & & & \\
\hline Control system R\&D-Adapt from APS & & & & & & & \\
\hline $\begin{array}{l}\text { Prototype Control Computer R\&D Procurement } \\
\text { Targets and Control Support R\&D } \\
\text { Instrumentation R\&D }\end{array}$ & & & & & & & \\
\hline
\end{tabular}

Figure B.1-1 Preconstruction R\&D Schedule. 


\section{B.2 ACCELERATOR PHYSICS}

\section{B.2.1 Theoretical - Beam Dynamics and Beam Loss}

For the past several years, extensive beam dynamics studies have been carried out by personnel associated with this feasibility study. Beam loss conditions during the injection, capture, and acceleration processes have been simulated. The results are discussed in Chapter II. Work performed to date includes studies of longitudinal and transverse beam dynamics during the injection, capture, and acceleration processes. The effect of motion in the transverse plane on the dynamic aperture was studied. Magnet and beam position monitor placement tolerances and magnetic multipole components arising from fabrication tolerances were studied for synchrotron magnets under nonaccelerating conditions.

The results of these studies are incorporated into this feasibility report and cover (1) the need for chopping the linac beam to eliminate capture losses, (2) the specification of the linac beam momentum spread to eliminate acceleration losses and to meet Keil-Schnell longitudinal stability criteria, and (3) specifications for the alignment and multipole tolerances.

The plan for the coming years is to further refine the tracking study to include all relevant physical phenomena in the simulation, both individually and combined. After these detailed studies have been performed for the Intense Pulsed Neutron Source (IPNS) Upgrade lattice, particle tracking simulations will be performed with equal thoroughness for the existing IPNS accelerator. This procedure provides a way to experimentally check the calculations by using an actual accelerator.

\section{B.2.2 Experimental - Measurements at Existing Synchrotrons}

The longitudinal tracking studies performed to date have provided us with an understanding of many phenomena associated with beam losses. Many of these phenomena can be measured experimentally at existing fast cycling synchrotron facilities. Machine experiments will be performed at the existing IPNS accelerators. Planned studies are described below.

\section{B.2.2.1 Chopped Beam Injection Studies}

Chopped beam injection will be done to study the bunch to bucket beam transfer. A traveling wave chopper will be placed between the $750-\mathrm{kV}$ preinjector and the drift tube linac (DTL) linac at IPNS. The injection and capture processes were already simulated by Monte Carlo tracking calculations, so experiments will be performed with the synchrotron to check the validity of the predictions. 


\section{B.2.2.2 rf Voltage Programming}

The rf voltage programming affects various beam parameters and beam loss conditions. Several accelerator experiments are planned. One accelerator experiment verifies the if voltage program in the later part of the acceleration cycle. At the end of acceleration, a high synchrotron frequency must be maintained to permit the bunches to follow rapid changes in the synchronous phase angle, as was discussed Chapter II.

It is foreseen that both theoretical and experimental accelerator physics activities will be carried out throughout the construction period in support of this facility. Personnel associated with those activities will participate in commissioning and operations-related work as the schedule progresses.

\section{B.3 rf SYSTEM}

The rf system is a key hardware element and requires early $R \& D$ work in three areas.

\section{B.3.1 Cathode-Follower rf Amplifier}

The cathode-follower if amplifier system receives particular emphasis in the if R\&D studies. In theory, the system has a very small impedance, and it can automatically compensate for beam loading. That is an advantage of the cathode-follower when compared with the conventional amplifier system described in this report. A cathode-follower system would be built and tested as part of the proposed R\&D in order to demonstrate its possible use in the IPNS Upgrade. Figure B.1-1 shows that the planned duration of this R\&D activity is two years, after which the decision on amplifier type, shown as a milestone in the figure, must be made. Actual construction of the amplifier begins in the second quarter of FY 1997.

\section{B.3.2 rf Cavities and Ferrite}

There are $10 \mathrm{rf}$ cavities in the design, for a total of $180 \mathrm{kV}$, and the resonance frequency varies from 1.1 to $1.5 \mathrm{MHz}$. The tuning is achieved by adjusting the biased magnetic field of ferrite rings. It is important to choose proper ferrite for this and to cool the ferrite to remove heat from rf losses. The duration of this $R \& D$ is about a year, and the effort is scheduled to be complete in FY 1997.

\section{B.3.3 Low-Level rf System}

Amplifier stability in the presence of the heavy beam loading in the RCS is achieved in part through low-level feedback loops. It is important to model and test as many of these systems in advance of construction as possible to ensure a successful design. The amplitude and 
phase low-level feedback and feedforward loops are all studied in this R\&D program. The planned duration of this activity is about a year.

\section{B.4 MAGNET PERFORMANCE DESIGN}

Synchrotron ring magnet procurement may take some time to accomplish. One way to expedite it is to select a vendor or vendors to produce the mechanical engineering designs on the basis of performance specifications provided by this facility. This method of procurement has been used at the APS and was used extensively at the European Synchrotron Radiation Facility (ESRF). This option for magnet procurement requires that a detailed performance specification be worked out. This activity is planned to start this year and be completed by next year so that the procurement can be initiated soon after the start of the project.

\section{B.5 DUAL-HARMONIC POWER SUPPLY FOR THE RING MAGNETS}

It is common practice to energize a rapid cycling synchrotron by using a resonant circuit composed of a capacitor bank and two inductive elements: a string of magnets and a choke. The stored energy in the circuit oscillates back and forth between the two inductive components. The oscillation frequency of the stored energy is the repetition rate of the synchrotron. One advanced feature of the IPNS Upgrade design is the use of a dual-frequency system to power the magnet system. The magnets are excited to the peak field with a $20-\mathrm{Hz}$ sine wave, and are de-energized with a $60-\mathrm{Hz}$ cosine wave, resulting in an average $30-\mathrm{Hz}$ repetition rate. The R\&D plan for this topic is to build and test a scale model of the power supply in FY 1996 so that power supply design engineering can commence in FY 1997.

\section{B.6 VACUUM CHAMBER}

\section{B.6.1 Ceramic Chamber Prototype}

Two types of vacuum chambers are commonly used for rapid cycling proton synchrotrons. In the first type, the magnet steel and the coils are encased inside vacuum-tight casings. That is used at the Fermilab booster and at the present IPNS 500-MeV machine. The other scheme is the ceramic chamber system, which is used at the ISIS facility. In recent years, TRIUMF personnel have constructed prototypes of these chambers for the proposed KAON facility. The R\&D plan is to carry out a literature search and construct prototypes through FY 1997, as shown in Figure B.1-1. 


\section{B.6.2 Ceramic Chamber Feedthrough}

The chamber system requires a large number of feedthroughs to extract diagnostic signals from the inside of the chamber. To design and construct a reliable chamber system, plans are to pursue an R\&D program on feedthroughs in FY 1997. The vacuum chamber prototyping program started in FY 1996, and the resulting prototype chamber is used in the feedthrough study, as shown in Figure B.1-1.

\section{B.6.3 Chamber $\mathrm{rf}$ Shields}

The rf shield is a wire cage following the beam envelope. It shields the beam from induced If signals and minimizes the coupling impedance between the beam and its surroundings. Such a shielding system has been operational at ISIS for many years. After chamber prototyping is complete, an R\&D program for the shield will commence, as shown in Figure B.1-1. The shield must be mechanically rigid and have suitable if properties.

\section{B.7 LINAC SYSTEM}

One procurement option is to obtain the linac from industry in a turn-key contract. The rationale for this is to directly use Superconducting Super Collider (SSC) industry-developed technology. In this case, the plan is to develop strong in-house capability in linac operations and improvements. The main emphasis is to be placed on operational reliability.

\section{B.7.1 Ion Source}

The source current and duty factor requirements for the IPNS Upgrade are satisfied by the existing Penning source of ISIS. In the Workshop on Ion Source Issues Relevant to a Pulsed Spallation Neutron Source held on October 24-26, 1994, at Lawrence Berkeley Laboratory, it was concluded that that extensive ion source $R \& D$ is required for all other pulsed source concepts. The IPNS Upgrade plan is to construct a test stand for reliability and serviceability testing of an ion source.

The plan is to obtain a Penning source and a volume source in FY 1996 and to construct a test stand on which a source can run 24 hours a day and 7 days a week for reliability testing. After this study, the source could be connected to the RFQ for further reliability testing. The linac-related schedules are shown in the bar charts in Figure B.1-1.

\section{B.7.2 RFQ}

The RFQ is added to the $\mathrm{H}^{-}$ion source test stand after the $\mathrm{H}^{-}$ion source has been studied for a year. The combined system of ion source and RFQ is tested starting in FY 1997. 
The purpose of this program is to gain operational experience on serviceability and maintainability of the combined system.

The beam chopping requirement described in Chapter II is tested during this period by using a chopper that is placed between the ion source and the RFQ.

\section{B.7.3 CCL}

Argonne personnel must gain experience with the industrially produced linac, especially the CCL part. The plan is to participate in the early testing phase of the linac, develop operational expertise, and make operational and commissioning plans that will be ready when the linac is delivered to ANL. These activities are planned for FY 1997-1998.

\section{B.8 KICKERS AND SEPTUM MAGNETS}

These special magnets are pulsed at a $30-\mathrm{Hz}$ repetition rate. They require prototyping at the earliest possible time to understand and implement a reliable system. Past experience shows that the time required to develop a reliable system is 1 to 2 years. This work is scheduled to start in the third quarter of 1997, as shown in Figure B.1-1.

\section{B.9 DIAGNOSTICS}

The Target Protection Monitor System monitors beam size and shape; its input is interpreted, along with focusing magnet setpoint and readback. values, to provide a beam shutdown signal when required.

Research and development is required to choose suitable components and to ensure reliability, since this system functions in a very-high-radiation environment.

As fast electronics and digital technology improve, beam diagnostics and beam feedback technology improve accordingly. Therefore, diagnostics R\&D is planned throughout the construction period to take advantage of these technology improvements, as shown in Figure B.1-1.

\section{B.10 CONTROL SYSTEM}

The APS project has developed the EPICS control system, as described in Chapter II. The IPNS Upgrade control system is adapted from the APS system. Minimal work is required for this task before the start of construction, as shown in Figure B.1-1. One notable requirement is that a control computer must be procured in FY 1998 before the control software is transferred from the APS. 


\section{B.11 RESEARCH AND DEVELOPMENT FOR TARGET STATIONS}

The IPNS Upgrade has two target stations. The target stations include targets, moderators, and reflectors and their cooling and support systems, biological shields, hot cells, irradiation tubes, and other items. Both targets are identical and are able to accept the full 1-MW beam power. This power level lies considerably beyond current experience, although our studies indicate that systems for this performance are feasible. Some of the components can be designed on the basis of available information. Some components require design optimization, and some require hardware testing to provide a facility that is safe, effective, reliable, and efficient. This R\&D program provides the needed information to support the IPNS Upgrade design.

\section{B.11.1 Neutronic Calculations}

Two categories of neutronic calculations are needed. The first and straightforward category is the calculation of power density distributions, radiation damage rates, isotope production, afterheat, high-energy neutron production, etc., to support the design of targets, moderators, reflectors, premoderators, and decouplers, and their respective cooling and support systems. The second is the optimization of these systems to provide optimal characteristics for the neutron beams. For both purposes, computer code packages such as HETC/NMTC, ORIHET, and ORIGEN suffice without significant modification. The tasks are substantial, however, since the codes and the geometrical models are complex and disparate. This work is planned for FY 1996-1997.

\section{B.11.2 Metallurgy and Tests}

Both targets consist of water-cooled tungsten alloy plates. Feasibility studies verify that such plates withstand temperatures and stresses that occur during normal operation and in some abnormal circumstances. Evidence is scant, however, to provide for understanding the likely mechanism of failure and to support estimates of lifetime against failure (targets will certainly have a short lifetime when compared with the facility lifetime) and estimates of corrosion and erosion rates. A program of research and testing is required to provide a basis for establishing a servicing strategy, designing against transport of radioactive materials in the coolant systems, and analyzing accident conditions. So far, feasibility studies have revealed that radiation-induced embrittlement due to hydrogen and helium accumulation in target material most likely represents the life-limiting effect. Materials surveys may identify candidate materials resistant to this embrittlement. Calculations of static and dynamic stresses will reveal methods to extend the lifetime of brittle-designed materials, possibly leading to specifications for proton beam spreading mechanisms in the high-energy proton transfer line upstream of the target. Bench-testing by using rapid-flowing coolant is performed to reveal the extent of cavitation and erosion in the real system. Testing in irradiated coolant provides corrosion rate data and identifies methods for controlling corrosion; in this effort, we expect close collaboration with British (ISIS), European Union (ESS), and Russian (Dubna, Troitsk) groups with shared interests. This work is planned for FY 1996-1997. 


\section{B.11.3 Thermal Hydraulics}

Measurements in instrumented, electrically heated model target flow channels are intended to verify calculations of heat transfer and coolant pressure drops. This work is planned for FY 1996-1997.

\section{B.11.4 Handling and Changing}

Mockups of targets, moderators, reflectors, and shielding are constructed to test and refine methods for handling and changing these components. This work, which improves the reliability and availability of the target and moderator systems, is planned for FY 1996-1997.

\section{B.11.5 Cryogenic Systems and Controls}

Cryogenic moderators require attention on several fronts. The behavior of cryogenic moderator materials under irradiation presents numerous questions. Radiolysis in liquid methane produces heavy hydrocarbons that accumulate, restricting flow and diminishing neutronic performance. Methods need to be identified to extend the lifetime of liquid methane moderator systems, by reducing radiation damage through placement of premoderators, by removing hydrocarbon precursors of accumulating solids, or by removing accumulated solids. For example, ozone flushing may remove accumulated solids. Liquid hydrogen goes into the para phase at low temperatures but reverts to an ortho-para mixture under irradiation; the two phases have significantly different neutron thermalization properties, so some instability in the performance of liquid hydrogen moderators can be expected under intense irradiation, which is not understood. These effects - radiolysis of methane and para-to-normal conversion of hydrogen - are to be investigated experimentally in a reactor test being constructed for other, related purposes at the Penn State TRIGA reactor. If liquid methane cannot be made to perform satisfactorily, composite moderators of water or metal hydride and liquid hydrogen may provide the needed neutronic performance; development and neutronic measurements of these composite systems is occurring at the Hokkaido electron linac. Members of the R\&D team are to participate in this program as part an already-existing collaboration. Mixed protonated/deuterated materials have the potential for better performance than pure protonated materials, and these are to be investigated both experimentally and theoretically. Neutronic measurements are coordinated with calculations. Big gains in performance and reliability are possible with further moderator system developments. Final moderator design depends on the outcome of these R\&D efforts, which are planned for completion in FY 1998.

\section{B.11.6 Shielding and Radiation Monitoring}

Optimization of the shielding composition for the main biological shield, beamline shielding, beam stops, and special-purpose local shielding leads to more efficient shield design. This work, which is mostly confined to calculations of candidate material compositions, relies in 
part on consulting others who have experience at existing spallation source installations. Some development and testing of radiation monitoring devices for area personnel are needed to assure efficient design and effective performance in pulsed radiation fields. The biological shield studies are planned for completion in FY 1996. The instrument shielding studies are planned for FY 1997-1998.

\section{B.12 NEUTRON BEAM INSTRUMENTATION}

The $R \& D$ required to provide the initial complement of neutron beam instrumentation at a pulsed neutron source falls naturally into two categories: R\&D supporting the selection of instruments to be built, and R\&D on instrument components. For the most part, the first category of R\&D is done by the appropriate instrument scientists, with the involvement of the user community. The second category requires the heavy involvement of other types of specialists, including engineers, computer scientists and programmers, detector experts, or experts in neutron optics and surface deposition techniques. It may also include the considerable involvement of current or potential component vendors.

\section{B.12.1 Selection of Neutron Beam Instruments}

The suggested process is outlined in the following steps:

1. Identification of Instrument Concepts. Small workshops are convened to discuss concepts for instruments. The goal is to outline concepts for all instruments that could conceivably be among the initial set to be built. Such instruments amount to considerably more than would actually be built; they might add up to as many as 50 instruments. At these workshops, preliminary assessments of the appropriate mixes of instruments for various broad classes of science are made, such as structures at the atomic scale, large-scale structures, and inelastic scattering.

Results from the workshop on scientific opportunities held at Argonne in May 1993, the instrumentation sections of the IPNS Upgrade feasibility study, and corresponding instrumentation considerations from LANSCE serve as input to this process. Reports from a number of earlier workshops (Shelter Island, Maria Laach, Rapallo, and the ESS instrumentation workshop at ISIS) are also relevant.

2. Initial Assessment of Performance and Source Requirements for Each Instrument. These instrument concepts are analyzed to determine instrument performance and source requirements for each of the potential instruments identified in step 1 . This step 2 has already been accomplished for the 27 instruments considered as part of the IPNS Upgrade reference set; probably no more than $20-25$ additional instrument concepts have to be 
considered at this stage. Such analyses also include a moderately detailed sketch of the instrument concept, assessment of the usefulness of existing instruments versus new construction to fill the specified purpose, and indication of the component development required to optimize instrument performance.

3. Selection of Prioritized Package of Instruments for Construction. A steering committee or another group that represents the user community and includes a few technical experts uses the performance analyses of step 2 to recommend a selection of instruments to cover the types of science desired. This procedure may involve a redefinition of instrument concepts and a repetition of step $2-$ to include some additional instruments to fill a gap or to combine the functions of two or more instruments, for example. The instrument selection is to be completed in FY 1997.

4. Complete Conceptual Design of the Selected Set of Instruments. This step represents a complete instrument conceptual design and optimization, involving extensive use of Monte Carlo design codes. Since staffing will not be adequate to carry out all these conceptual designs in parallel, the first step will be to establish an order of priority for the instruments in the selected set, based on the optimal timetable for beginning the detailed design for each of these instruments. This work is to begin in FY 1997 and continue through FY 1999.

\section{B.12.2 Specific Beamline Instrument Components}

A large number of instrument components that are improved in either performance or cost by $R \& D$ can be identified. The relative priorities of $R \& D$ in each of these areas depend in part on the ability of current state-of-the-art components to meet the requirements developed in the instrument analyses discussed above. Many of the instruments can be built with existing technology, but almost all benefit from improvements in one or more of these areas.

It is necessary to prioritize the component R\&D. Criteria include the following: (1) one or more of the desired instruments cannot be built without improvement in the state-of-the-art for such components or (2) significant performance gains or savings in instrument cost result from improvements in such components. A further criterion in both cases is that an R\&D program of reasonable scope and with a high probability of success can be defined. The workshop suggested in step 1 of the instrument selection process addressed the prioritization of these various component $R \& D$ programs, although these priorities may change as the process proceeds. The component $R \& D$ effort continues throughout the project. 
For areas in which instrument component $R \& D$ is required or desired, several avenues are explored:

- Support of individual development efforts at universities or national laboratories. (For example, many of the data acquisition, visualization, and verification problems and the shielding questions can be handled in this manner.)

- Award of development contracts to current or potential component suppliers. (Development of detectors, some types of beamline optics, some aspects of choppers, and sample environment equipment can be handled in this manner.)

- Participation in European or Japanese development efforts. (The European Community is currently funding a two-year development program for some aspects of neutron beam instrumentation, and the Japanese have a very active program in the development of white-beam neutron polarizers.)

R\&D programs for instrument components include the following:

1. Detector Development. Detectors represent a large portion (30-50\%) of the cost of time-of-flight neutron scattering instruments. Several of the instruments at the IPNS Upgrade require position-sensitive detectors (PSDs) and the associated encoding electronics, which provide better resolution or higher speed than that available with current technology. We will work with the PSD manufacturers and detector development groups at various laboratories worldwide to extend existing technologies or develop new technologies to meet these requirements.

2. Data Acquisition and Chopper Control Electronics. This program involves the efforts of engineers and technicians to develop a complete set of prototype data acquisition and chopper control electronics and includes the design and testing of system components.

3. Data Verification, Manipulation, and Visualization Software. This effort involves software design engineering and programming, under the supervision of one or more of the neutron scattering scientists. The object is to develop general-purpose code packages for data verification, manipulation, and visualization at each step of the manipulation. Commercial software packages are used to the extent practical, and much of the additional work is done with the support of and in collaboration with faculty and students at university computer science departments. Coordination and collaboration with ongoing efforts in these areas at various centers are important.

4. Collimator Development. Multiple-aperture converging collimating systems must be developed to provide the maximum intensity on sample and provide 
the tight, clean collimation required for some instruments. Development of these and other specialized collimators requires a combination of analysis, fabrication, and testing of prototypes. This work requires an available beam at an operating neutron source. The required effort involves overall technical direction by one or more scientists and some involvement of a mechanical designer or engineer, but other technical staff, technicians, and students do most of the fabrication and testing of prototypes.

5. Beamline Optics. Developments aimed at the improvement of neutron guides and other optical components that depend on the reflection of neutrons from mirrors or supermirrors are already underway at various laboratories and companies. Therefore, until specific device requirements are identified by the instrument analyses, all that is necessary is to maintain an awareness of the current status of and the performances achieved by these ongoing efforts.

6. Polarization Devices. Supermirror devices for the polarization and analysis of cold neutrons are readily available, and performances are continually being improved by some of the development efforts noted in program 5 . However, suitable polarization devices for higher-energy neutrons and for wide-angle applications are not yet available. Despite recent progress in developing polarized ${ }^{3} \mathrm{He}$ filters for neutron polarization, continued long-term, large-scale development is required before any satisfactory devices are produced. We plan to participate in one of the ongoing development efforts, such as the program in Japan.

7. Sample Environment Equipment. The widest-possible range of modern automated sample environment equipment is essential for the effective utilization of IPNS Upgrade. Continuing development in this area is important. This involves efforts by engineers, designers, and technicians to develop and evaluate additional ancillary equipment such as furnaces, pressure cells, sample changers for furnaces, cryostats, displex refrigerators, and automated control systems. In some cases, scientific staff are also required to evaluate the performance of a given piece of ancillary equipment in a neutron instrument. A workshop is planned to assess and prioritize these needs.

\section{B.13 COSTS FOR PRECONSTRUCTION RESEARCH AND DEVELOPMENT PROGRAM}

The funds needed to accomplish the preconstruction R\&D work are based on the R\&D items identified in this Appendix B. The budget profile for this work takes into account the construction schedule described in Chapter VII of this report. One of the most important considerations in developing the schedule is the need for making a smooth transition from the R\&D phase to the construction phase while maintaining the schedule for the key project milestones. 
Adequate operating funds (not listed here) are needed to support the precommissioning activities necessary to make the transition to the commissioning phase as various subsystems of the facility are ready for trial operation. The precommissioning activities are scheduled to start in FY 1999. The costs for precommissioning activities are to be developed at a later date.

Costs for the preconstruction R\&D effort, direct materials, and service are shown in Table B.13-1 in actual year's dollars. 
Table B.13-1 Costs for Preconstruction R\&D

\begin{tabular}{|c|c|c|c|c|c|c|c|}
\hline \multirow[b]{2}{*}{ Cost Item } & \multicolumn{7}{|c|}{ Fiscal Year } \\
\hline & FY95 & FY96 & FY97 & FY98 & FY99 & FYOO & FY01 \\
\hline KD 0 Milestone & $\boldsymbol{\bullet}$ & - & - & - & - & - & - \\
\hline KD 1 Milestone & - & 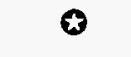 & - & - & - & - & - \\
\hline Construction Start Milestone & - & - & 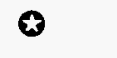 & - & - & - & - \\
\hline Commissioning Start Milestone & - & - & - & - & - & - & $\boldsymbol{\theta}$ \\
\hline Commissioning Ends Milestone & - & - & - & - & - & - & $\bullet$ \\
\hline Accelerator $R \& D$ & 6 & 21 & 35 & 27 & 8 & 3 & - \\
\hline Accelerator Physics & 3 & 4 & 6 & 6 & 5 & 3 & - \\
\hline if Amplifier R\&D (Cathode Follower) & 1 & 4 & 2 & - & - & - & - \\
\hline Decision on Amplifier Type Milestone & - & - & 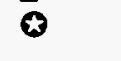 & - & - & - & - \\
\hline if Cavity and Ferrite R\&D & - & - & 4 & - & - & - & - \\
\hline Low-Level if R\&D & - & - & - & 4 & - & - & - \\
\hline Magnet Performance R\&D & 1 & 2 & - & - & - & - & - \\
\hline Dual Frequency Model Power Supply & - & 3 & - & - & - & - & - \\
\hline Ceramic Chamber Prototype R\&D & 1 & 3 & 4 & - & - & - & - \\
\hline Chamber Feedthrough & - & - & 3 & - & - & - & - \\
\hline Chamber of Shield R\&D & - & - & - & 5 & - & - & - \\
\hline Ion Source R\&D & - & 3 & $\mathbf{3}$ & - & - & - & - \\
\hline RFQR\&D & - & - & 3 & 3 & - & - & - \\
\hline CCL R\&D & - & - & 2 & 2 & - & - & - \\
\hline Kickers, Septum Magnets R\&D & - & - & 2 & - & - & - & - \\
\hline Prototype Kicker and Septum & - & - & 3 & 4 & - & - & - \\
\hline Prototype Kicker Test Milestone & - & - & - & $\boldsymbol{\oplus}$ & - & - & - \\
\hline Diagnostics and Feedback R\&D & - & 2 & 2 & 3 & 3 & - & - \\
\hline Control System R\&D - Import APS & - & - & 1 & - & - & - & - \\
\hline Prototype Control Computer Procure & - & - & - & $\boldsymbol{\oplus}$ & - & - & - \\
\hline Target and Moderator R\&D & 1 & 12 & 16 & 7 & 3 & - & - \\
\hline \multicolumn{8}{|l|}{ Target Systems } \\
\hline Neutronic Calculations & 0.2 & 0.5 & 0.5 & 0.5 & 0.5 & - & - \\
\hline Metallurgy and Tests & 0.2 & 2 & 5 & 2 & - & - & - \\
\hline Safety & - & 0.5 & 0.5 & - & - & - & - \\
\hline Thermal-Hydraulics & 0.1 & 0.5 & 1.5 & - & - & - & - \\
\hline Shielding & - & 0.5 & 0.5 & - & - & - & - \\
\hline Monitoring & - & 0.5 & - & - & - & - & - \\
\hline Handling/Changing & 0.1 & 0.5 & 1.5 & 0.5 & - & - & - \\
\hline \multicolumn{8}{|l|}{ Moderator Systems } \\
\hline Handling/Changing & 0.1 & 0.5 & 0.5 & 0.5 & - & - & - \\
\hline Safety & - & 0.5 & - & - & - & - & - \\
\hline $\begin{array}{l}\text { Neutronic Calculations and } \\
\text { Benchmarking }\end{array}$ & 0.1 & 1.5 & 1.5 & 0.5 & 0.5 & - & - \\
\hline Tests & 0.1 & 3 & 3 & 1 & 1 & - & - \\
\hline Cryogenic Systems and Control & 0.1 & 1 & 1 & 2 & 1 & - & - \\
\hline Radiation Monitoring & - & 0.5 & 0.5 & - & - & - & - \\
\hline
\end{tabular}


Table B.13-1 (Cont.)

Fiscal Year

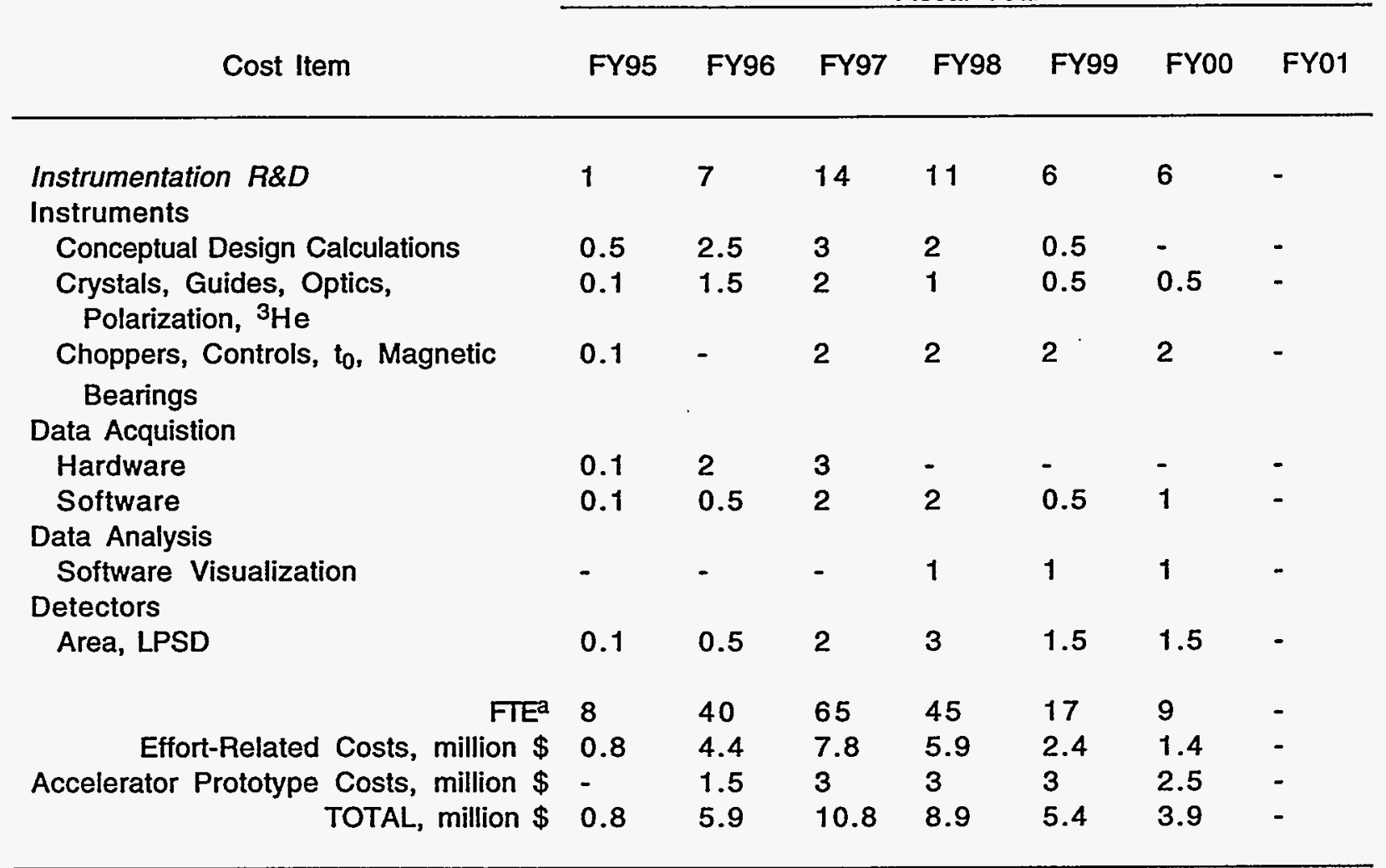

a Full-time equivalent. 


\section{Appendix C}

\section{Possible Future}

Experimental Facilities 

Although the primary mission of the Intense Pulsed Neutron Source (IPNS) Upgrade is neutron scattering, the various components of the facility provide capabilities that are extremely useful for other areas of science. Instrumentation to provide modest capabilities in three of these areas - radiation damage studies, neutron isotope production, and neutron activation analysis is included as part of this project. The facilities for these three areas of investigation are discussed in detail in Section V.4. The project cost and schedule reflects commitment to build these facilities.

The IPNS Upgrade has potential capabilities in pulsed muon spin rotation and neutrino physics. These are discussed briefly in this appendix. The discussion of potential applications is not intended to be exhaustive, but rather to provide an indication of some of the possibilities. Costs of these potential future applications are not included within the scope of this project.

\section{PULSED MUON SPIN ROTATION}

Positive muons are an important probe for the study of condensed matter. They are used in the technique known as $\mu S R$, which stands for muon spin rotation, relaxation, or resonance, depending on the particular variation of the technique. This technique involves the implantation of initially spin-polarized muons into a sample and the monitoring of their subsequent decay. This is not a scattering technique; the muons are actually stopped in the sample. In most experiments, the muon behaves chemically as a light-weight proton, having a factor of $\sim 9$ smaller mass. It can bind an electron to form muonium, in analogy to hydrogen, and the subsequent decay is used to probe hydrogen chemistry in a wide variety of systems. The spin of the muon also serves as a very sensitive probe of local magnetic fields and electronic configurations. An extensive review of the types of science studied with $\mu$ SR has been provided by Cox. ${ }^{1}$ Timing of the decays of the muons is required in most of the muon spin relaxation techniques. A pulsed muon facility operating at a relatively low frequency provides this timing naturally, and so can deliver at least two orders of magnitude more useful muons on the sample than can steady-state sources. Relative advantages of pulsed muon sources for various types of $\mu$ SR experiments have been discussed in great detail by Cox. ${ }^{1}$

Negative muons behave as very heavy electrons. These can be substituted for atomic electrons to form exotic atoms. Because of the muon's higher mass, its orbitals are much smaller in size, this being most pronounced for the exotic analogs of hydrogen, deuterium, and tritium. When a molecule is formed with a combination of exotic hydrogen analogs and normal hydrogen isotopes, the much smaller size of the exotic analogs allows close approaches of the nuclei, resulting in muon-catalyzed nuclear fusion. This is an area of active study. ${ }^{2}$

Fundamental experiments on the spectroscopy of free muonium are in progress at ISIS, where pulsed laser spectroscopy has been phased to the pulsing of the source to provide a significant advantage. ${ }^{3}$ A relatively low-intensity pulsed muon facility is located at the KEK laboratory in Japan, and a high-intensity pulsed muon facility has been included from the beginning 4,5 as part of the ISIS pulsed neutron facility in the United Kingdom. This latter facility, which can be tuned to provide either positive or negative muons, is heavily 
oversubscribed. Recently, this facility has been expanded and more muon beamlines have been added. ${ }^{6}$

Figure C.1-1 shows the configuration of the ISIS pulsed muon facility. An intermediate target is located in the proton beam upstream from the neutron production target. A few percent of the protons interact with this thin target, producing pions, which come to rest in the target. These pions decay with a 26-ns lifetime into muons and neutrinos, the muons being extracted at $90^{\circ}$ to the proton beamline. For most experiments, the muon beamline optics are tuned to accept only surface muons (i.e., those produced by pions at rest near the surface of the target) that are fully polarized parallel to their flight direction. The muon optics are designed to eliminate muons of the wrong energy or charge and to eliminate other particles, such as positrons, that might interfere with the measurements. These optics also direct the muon beam onto the sample, where the muon is thermalized and then precesses about the local magnetic field. The muon decays with a 2.2- $\mu$ s lifetime into a positron and two neutrinos, the positron being preferentially emitted along the muon spin direction. Fast positron detectors are used to detect the positrons, and the data-acquisition electronics forms a histogram of the number of detected positrons as a function of time after the proton pulse. This signal is then analyzed to determine the Larmor precession frequency and relaxation of the muon spin polarization with time.

Although few U.S. investigators make use of pulsed muon techniques at present, experience at ISIS suggests that demand for such a facility is likely to develop. No pulsed muon facility is included as part of the initial construction of the IPNS Upgrade, but such a facility, modeled on the one at ISIS, can easily be added after the IPNS Upgrade is operational. It is expected that any perturbation of the neutron scattering program resulting from such an addition would be minor, and losses in neutron production would be minimal.

Space has been reserved for a pulsed muon facility in one of the IPNS Upgrade experiment halls; Figure C.1-2 shows one possible location. 
Figure C.1-1 Layout of the ISIS Pulsed Muon Facilities (Source: Reference 6) 


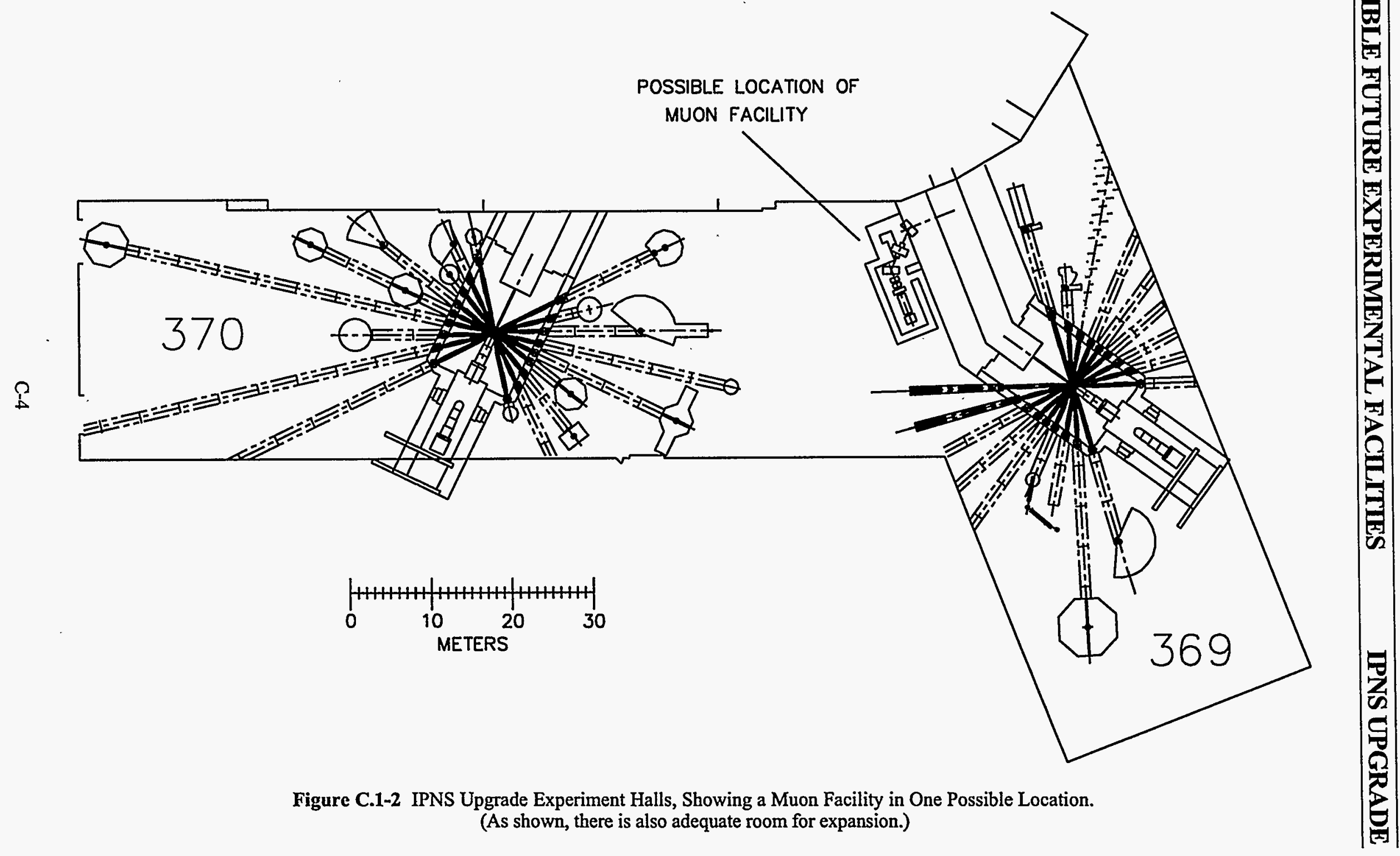




\section{NEUTRINO PHYSICS}

In addition to the neutrons produced in the IPNS Upgrade neutron scattering targets, the interactions of the $2-\mathrm{GeV}$ proton beam with the targets also produce intense fluxes of pions. These pions decay according to

$$
\begin{array}{ll}
\pi^{0} \rightarrow 2 \gamma & \text { (half life } \sim 10^{-16} \mathrm{~s} \text { ), } \\
\pi^{+} \rightarrow \mu^{+}+v_{\mu} & \text { (half life } \left.\sim 2.6 \times 10^{-8} \mathrm{~s}\right),
\end{array}
$$

and

$$
\pi \rightarrow \mu^{-}+\bar{v}_{\mu} \quad \text { (half life } \sim 2.6 \times 10^{-8} \mathrm{~s} \text { ), }
$$

and the resulting muons decay according to

$$
\mu^{+} \rightarrow \mathrm{e}^{+}+v_{\mathrm{e}}+\bar{v}_{\mu} \quad \text { (half life } \sim 2.2 \times 10^{-6} \mathrm{~s} \text { ) }
$$

and

$$
\mu^{-} \rightarrow \mathrm{e}^{-}+\bar{v}_{\mathrm{e}}+v_{\mu} \quad \text { (half life } \sim 2.2 \times 10^{-6} \mathrm{~s} \text { ). }
$$

However, most of the $\pi$ are captured by the nuclei of the target material before they can decay according to this scheme (Equation C.2-3). Consequently, the neutrino production consists predominantly of $v_{\mu}, v_{e}$, and $\bar{v}_{\mu}$ in equal numbers. The time-structure of the source and the fact that both electron and muon neutrinos are produced provide some unique advantages for neutrino studies with the IPNS Upgrade. Time separation can also be used to distinguish between the $v_{\mu}$, which are produced promptly, and the $v_{\mathrm{e}}$ and $\bar{v}_{\mu}$, which are produced over the muon lifetime. These advantages have been recognized at the ISIS facility, where a major British-German collaboration has constructed the 300-ton KARMEN detector for neutrino interaction studies. ${ }^{7}$ This detector has recently undergone commissioning and has begun its experimental program, which is expected to last at least five years. ${ }^{8}$ The IPNS Upgrade will provide at least six times more neutrino flux than ISIS because of the higher energy and current at the IPNS Upgrade facility.

It is not clear at present whether there will be sufficient demand for a similar detector, or for a different type of detector, for neutrino studies at the IPNS Upgrade facility. It is not proposed at present to pursue this possibility, but merely to alert the physics community to the fact that it will be possible to carry out various types of fundamental physics studies using the neutrinos produced at the IPNS Upgrade facility. 


\section{REFERENCES}

1. S.F.J. Cox, "Implanted muon studies in condensed matter science," J. Phys. C: Solid State Phys. 20:3187-3319 (1987).

2. ISIS Annual Report 1991, Vol. 1, edited by C. Wilson, Rutherford Appleton Laboratory Report No. RAL-91-050, p. 42 (1991); see also Vol. 2, pages A300-A330, for experimental reports indicating the types of pulsed muon experiments recently performed at this facility.

3. ISIS Annual Report 1989, edited by A. Taylor and C. Wilson, Rutherford Appleton Laboratory Report No. RAL-89-050, p. 39 (1989).

4. G.H. Eaton et al., "A pulsed surface muon beam/pion beam for the Rutherford Appleton Laboratory Spallation Neutron Source," Nucl. Instrum. Methods 214:151-167 (1983).

5. G.H. Eaton et al., "Commissioning of the Rutherford Appleton Laboratory Pulsed Muon Facility," Nucl. Instrum. Methods A269:483-491 (1988).

6. C.C. Wilson, "A guided tour of ISIS - the U.K. spallation neutron source," Neutron News 6(2):27-34 (1995).

7. ISIS Annual Report 1986, edited by S. Biggin, Rutherford Appleton Laboratory Report No. RAL-86-061, pp. 67-69 (1986).

8. ISIS Annual Report 1991, Vol. 1, edited by C. Wilson, Rutherford Appleton Laboratory Report No. RAL-91-050, pp. $43-44$ (1991). 


\section{Appendix D}

Greenfield Option 



\section{D.1 INTRODUCTION}

The IPNS Upgrade strategy to reuse the existing 360 area conventional facilities has a potential cost savings of approximately $\$ 150$ million. For comparison, a design for a totally new facility on an alternate site (i.e., the "Greenfield Option") was also developed. Several existing sites that had prior Argonne National Laboratory (ANL) support facilities built on them were reviewed. The 800 area was selected as the IPNS Upgrade Greenfield site.

The 800 area is one of the oldest sites on the Argonne campus and has been used for a variety of functions since 1946. The 800 area buildings were constructed around 1950 and contain $3,950 \mathrm{~m}^{2}$ of existing structures. Most of these buildings are World-War-II-era "quonset-hut" structures. Approximately one-third of the structures on the site have been demolished; the remaining buildings are scheduled for demolition as funding becomes available for replacement facilities. There are approximately $900 \mathrm{~m}$ of paved roads and buried site utilities in the 800 area.

The 800 area site is located on the west side of the ANL campus, as shown in Figure D.1.1-1. It is $650 \mathrm{~m}$ north of the Advanced Photon Source (APS) and approximately $620 \mathrm{~m}$ northwest of the APS User Residence Facility. The north side of the 800 area is defined by Westgate Road and the west entry to ANL. The west side of the 800 area is defined by the ANL site fence and the ANL landfill area. The east side of the 800 area is defined by Kearney Road. The south side is defined by an east-west line parallel to and approximately $600 \mathrm{~m}$ south of Westgate Road.

The land area of the 800 site is 23.4 ha, or about $10 \%$ smaller than the 360 site. The plot of land containing 11+ ha southwest of the 800 area was used for landfill disposal of ANL solid wastes. The landfill was closed in 1994; however, existing truck access to it through the 800 area will be maintained.

The design philosophy employed in the Greenfield study was to replicate the functionality of the 360 area buildings in the 800 area and to arrange the structures to suit the geography and the required building function. The total gross floor area of the 800 site buildings is the same as the 360 site buildings and is $55,200 \mathrm{~m}^{2}$.

The cost estimators used the existing 360 area buildings' "as-built" construction documents for architectural, structural, mechanical, and electrical systems but assumed current construction techniques. Optimization of the Greenfield buildings requires detailed design development; however, use of the existing building documents enables the costs to be estimated to within $30 \%$ of the costs of a totally new design.

The new Linac Building (394) and tunnel (394A) conceptual design drawings were used to estimate this facility. The IPNS Upgrade 360 area site plan is shown in Figure VI.1.1-2. The 360 IPNS Upgrade buildings are shown in Figure VI.1.1-4. The Greenfield buildings are shown in Figure D.1.1-2. 


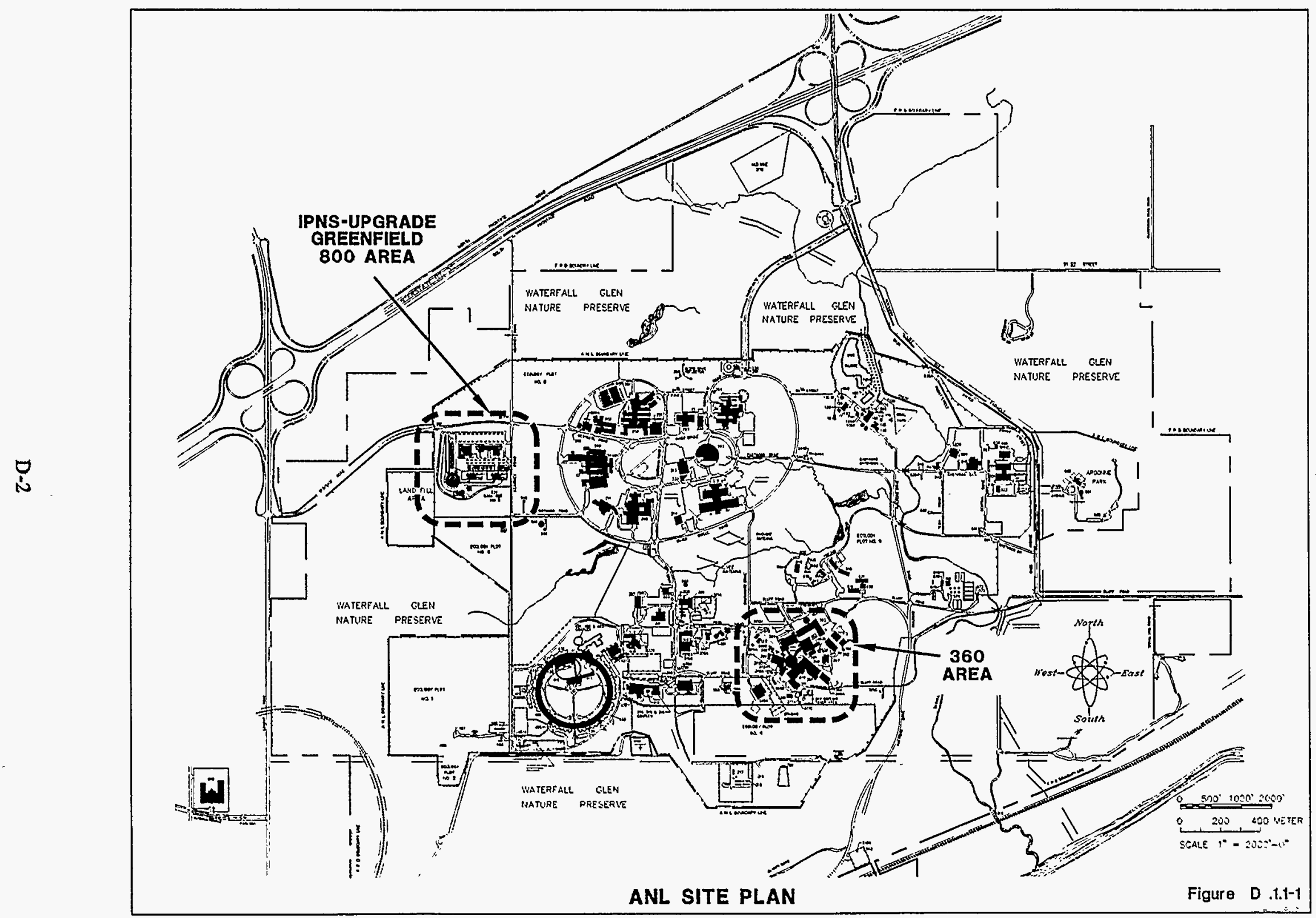




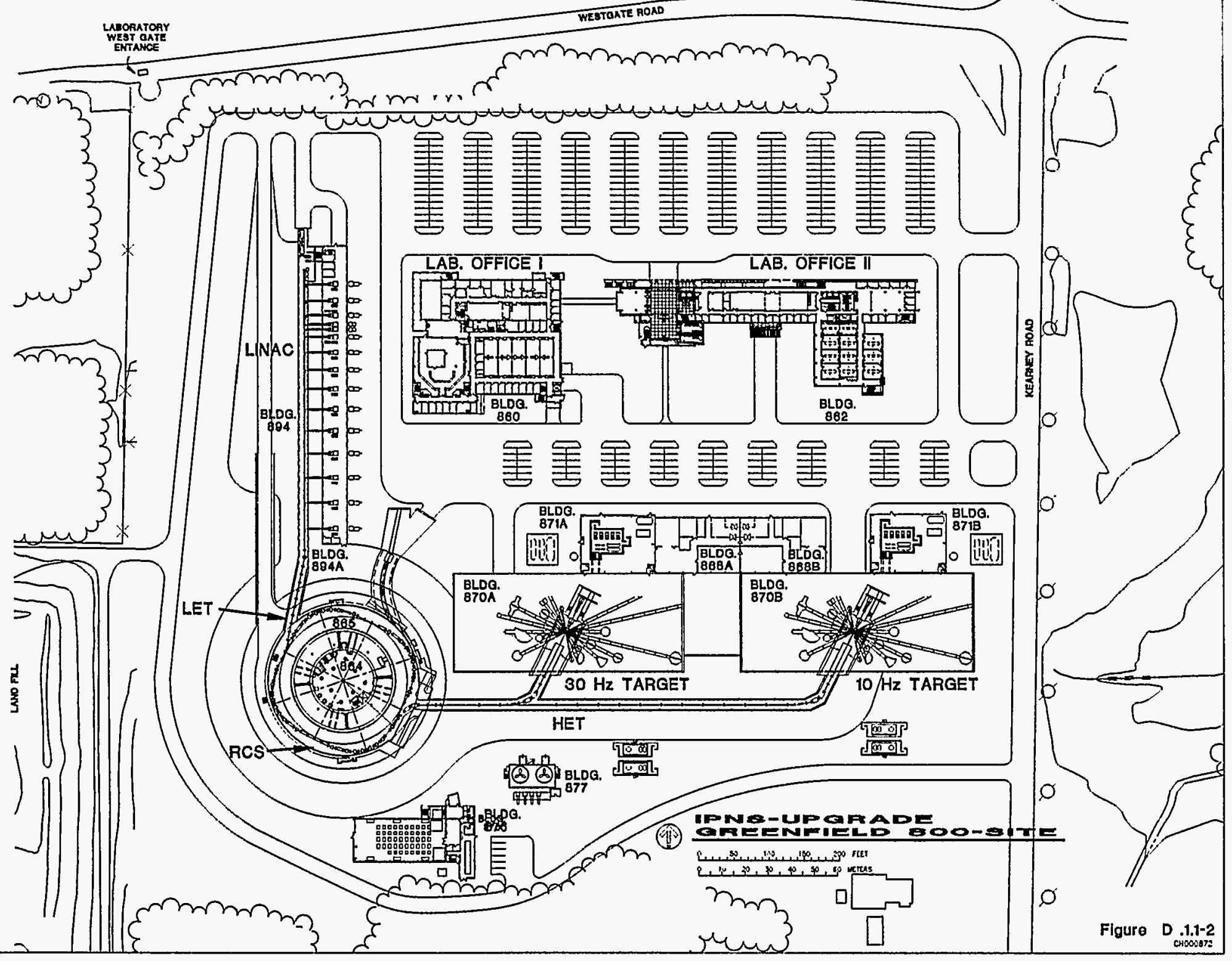




\section{D.2 BULDINGS}

Costs of the buildings and facilities that support the various technical components of the IPNS Upgrade in the 800 area were estimated by using existing IPNS and APS facilities as models. Chapter VI includes complete building descriptions and plan details. Table D.2-1 lists the 360 site buildings and their counterparts in the 800 area. Except for two cases, the 300 number designation became the 800 site equivalent; for example, existing Building 364 became new Building 864.

\section{D.3 FACILITY DESCRIPTION}

The Linac Building 894 and low-energy transport (LET) Tunnel 894A are placed on a north-to-south line parallel to the ANL west fence line, with the linac source at the north end. The operating floors of all 800 area buildings are at grade. The LET and high-energy transport (HET) tunnels are also at grade. The vehicle access ramp and bridge to the top of the Ring Building are $7 \mathrm{~m}$ west of the linac and parallel to it. The Ring and Center Buildings are at the south end of the linac tunnel, as shown in Figure D.1.1-2. The extraction point for the HET line is at the east side of the Ring Building. The HET tunnel and earth shielding run due east.

The 10-Hz and 30-Hz Target Buildings are identical in floor plan and cross section. Their south walls are constructed of cast-in-place concrete and function as retaining walls to hold in place the $8 \mathrm{~m}$ of HET tunnel earth shielding.

Preparation Laboratory Buildings $868 \mathrm{~A}$ and $868 \mathrm{~B}$ share the common wall of the Target Buildings at the northeast corner of Building $870 \mathrm{~A}$ and the northwest corner of Building $870 \mathrm{~B}$. The two Target Support Buildings $871 \mathrm{~A}$ and $871 \mathrm{~B}$ also share the north wall of Buildings $870 \mathrm{~A}$ and $870 \mathrm{~B}$ and are located to the east and west of the Preparation Laboratory Building. Two vaults containing coolant retention tanks are buried to the east and west of Buildings $870 \mathrm{~A}$ and $870 \mathrm{~B}$.

Located on an east-to-west line parallel and north of the Target Buildings are Laboratory Office Buildings $I$ (860) and II (862). These two buildings are connected by a tunnel at the service level and a corridor at grade and second floors. A site utility tunnel system connects all of the buildings.

The area to the south of the Laboratory Office Buildings and north of the Target Buildings contains a staging area with truck access and parking for 100 automobiles. The area to the north of the Laboratory Office Buildings serves as a main building entrance and parking area for 300 automobiles, as shown in Figure D.1.1-2. 
Table D.2-1 Relationships between 360 Area and 800 Area Buildings

\begin{tabular}{|c|c|c|c|}
\hline Building Function & 360 Area & 800 Area & Comments \\
\hline New Linac Building & 394 & 894 & - \\
\hline New Linac and LET Tunnel & $394 \mathrm{~A}$ & $894 \mathrm{~A}$ & - \\
\hline Ring Building & 365 & 865 & - \\
\hline Center Building & 364 & 864 & - \\
\hline Power Supply Building & 376 & 876 & - \\
\hline 10- $\mathrm{Hz}$ Target Building & 369 & $870 B$ & $\begin{array}{l}\text { Building } 370 \text { was used as a } \\
\text { typical design. }\end{array}$ \\
\hline 10-Hz Target Support Building & 367 & $871 \mathrm{~B}$ & $\begin{array}{l}\text { Building } 371 \text { was used as a } \\
\text { typical design. }\end{array}$ \\
\hline 30-Hz Target Building & 370 & $870 \mathrm{~A}$ & - \\
\hline 30-Hz Target Support Building & 371 & $871 \mathrm{~A}$ & - \\
\hline $\begin{array}{l}\text { Target Laboratories and } \\
\text { Preparation Areas }\end{array}$ & 368 & $868 \mathrm{~A}$ and $868 \mathrm{~B}$ & $\begin{array}{l}\text { Half of Building } 368 \text { was used as } \\
\text { a typical design for both. }\end{array}$ \\
\hline Laboratory/Office 1 & 360 & 860 & - \\
\hline Laboratory/Office II & 401 & 862 & See exception $1 .^{a}$ \\
\hline Cooling Towers & 377 & 877 & - \\
\hline Exterior HET Tunnel & None & $\begin{array}{l}\text { Exterior HET Tunnel } \\
\text { in earth-bermed } \\
\text { shielding }\end{array}$ & See exception $2 . \mathbf{b}$ \\
\hline
\end{tabular}

a Exception 1. For Building 862 , which is the 800 area Laboratory Office building II, data from the APS CLO and Building 401 were used for costing purposes. Building 401 is an office building with a laboratory wing and a gross floor area of $19,400 \mathrm{~m}^{2}$ that is currently under construction. It is equal in size and function to Building 362 and meets the current DOE standards for office space planning and design. The structure is four stories high with a service floor.

b Exception 2. The 800 area option has a long exterior high-energy transport (HET) line that branches off to the $10-\mathrm{Hz}$ and $30-\mathrm{Hz}$ targets. The tunnel cross section is 3.04-m wide by $2.74-\mathrm{m}$ high, the same as that of the linac low-energy transport (LET) tunnel, but the earth berm shield requirement increases from $3.2 \mathrm{~m}$ to $8 \mathrm{~m}$. This structure has no counterpart in the 360 area. 


\section{D.4 SITE UTILITIES AT THE 800 AREA}

New site utilities are included in the 800 area site development. A new steam line connects to the existing ANL central steam line $400 \mathrm{~m}$ to the east. Site electricity connects to existing ANL Site Electrical Facility 549A.

\section{D.5 SUMMARY}

The total estimated cost for the Greenfield Option conventional facilities and site development is summarized as $\$ 208.6$ million in supplement $\mathrm{C}$. This cost does not include EDI\&A of $\$ 21.6$ million that, added to the estimate, would make a grand total of $\$ 230.2$ million.

The total estimated cost to refurbish and reuse the existing 360 area conventional facilities is listed in Supplement B as $\$ 49.5$ million. This cost does not include EDI\&A of $\$ 5.1$ million, for a grand total of $\$ 54.6$ million.

Therefore, refurbishing and reusing the existing 360 area for the IPNS Upgrade should save approximately $\$ 175.6$ million with respect to the cost of constructing entirely new conventional facilities and associated site development.

It should be noted that an actual new IPNS design would not be the Greenfield Option illustrated in this appendix. No value engineering or trade-off studies were performed on the Greenfield Option. The basis of the Greenfield Option was only to ascertain the replacement value of the existing 360 area facility. A new IPNS design would not replicate the 360 area; instead, a specific design for a new facility would take into account current programming and planning guidelines and would incorporate the latest construction techniques. A specific design program would be developed first, which would define the many building components and functional relationships. Designs of various elements would be evaluated and optimized through value engineering studies. With a specific program and design, the new IPNS conventional facility could cost 20 to $25 \%$ less than the projected $\$ 230.2$ million.

The detailed Greenfield estimate is described in Supplement C. 


\section{Appendix E}

Notation 
1D one-dimensional

2D two-dimensional

2D-PDF two-dimensional pair diversity function

$\mathrm{A} / \mathrm{E} \quad$ architect/engineer

AABC Associated Air Balance Council

ac

alternating current

ACI

American Concrete Institute

ADC analog-to-digital converter

AHU air-handling unit

ai

ALARA

analog input

AMCA

as low as reasonably achievable

ANL

ANS

ANSI

ao

APS

ARI Air Moving and Conditioning Association Argonne National Laboratory Advanced Neutron Source

ASHRAE American National Standards Institute analog output Advanced Photon Source

ASME Air-Conditioning and Refrigeration Institute

ASTM

ATM

atm

AWS American Society of Heating, Refrigerating and Air Conditioning Engineers, Inc. American Society of Mechanical Engineers American Society for Testing and Materials asynchronous transfer mode atmosphere (pressure) American Welding Society

\begin{tabular}{|c|c|}
\hline BA & budget authority \\
\hline BNL & Brookhaven National Laboratory \\
\hline BO & budget outlay \\
\hline BOCA & Building Officials and Code Administrators Inter \\
\hline BPM & Beam Position Monitor \\
\hline BSC & beam-stay-clear \\
\hline BSCS & Brillouin Scattering Chopper Spectrometer \\
\hline BURT & backup and restore tool \\
\hline CBM & Certified Ballast Manufacturer \\
\hline cc & cubic centimeter(s) \\
\hline CCL & coupled cavity linac \\
\hline CEBAF & Continuous Electron Beam Accelerator Facility \\
\hline CECo & Commonwealth Edison Company \\
\hline $\mathrm{cfm}$ & cubic foot (feet) per minute \\
\hline CHP & central heating plant \\
\hline CNCS & Cold-Neutron Chopper Spectrometer \\
\hline COD & closed-orbit distortion \\
\hline CPU & central processing unit \\
\hline
\end{tabular}


CSI Construction Specifications Institute

CTI Cooling Tower Institute

CW chilled water

CW continuous wave

CWR chilled-water return

CWS chilled-water supply

DAC digital-to-analog converter

DAS data-acquisition system

dc direct current

DCSAND Double-Perfect-Crystal Bonse-Hart Small-Angle Neutron Diffractometer

DCT database configuration tool

DDC direct digital controls

DESY Deutsches Electronen Synchrotron

DEVEL Instrument and Component Development

DIW deionized water

DOE U.S. Department of Energy

DTL drift tube linac

ED\&I engineering, design, and inspection

EDI\&A engineering, design, inspection, and administration

EDM

EMI

EPB1

electronic distance measurement

electromagnetic interference

EPICS

Building 369

ES\&H

ESRF

ESS

Experimental Physics and Industrial Control System

environment, safety, and health

ETL

European Synchrotron Radiation Facility

European Spallation Source

Electrical Testing Laboratories

FCC

FDDI

Federal Construction Council

FEL

fiber-distributed data interface

FET

Free Electron Laser

FFAG

field effect transistor

FODO

fixed field alternating gradient

fwhm

FY

focus drift defocus drift; focusing/defocusing

full width at half maximum

fiscal year

GDCT

GIF

graphical database configuration tool

GLAD

graphic interface format

GPIB

Glasses, Liquids, and Amorphous Materials Diffractometer

general-purpose interface bus

gpm

gallon(s) per minute 


$\begin{array}{ll}\text { GPPD } & \text { General Purpose Powder Diffractometer } \\ \text { GREF } & \text { Reflectometer for Grazing-Incidence Scattering } \\ \text { GSA/PBS } & \begin{array}{l}\text { General Services Administration/Public Buildings Service } \\ \text { graphical user interface }\end{array} \\ \text { GUI } & \text { high-efficiency particulate air } \\ \text { HEPA } & \text { high-energy transport; high-energy transport line } \\ \text { HET } & \text { High Energy Transport Code } \\ \text { HETC } & \text { Hydronics Institute } \\ \text { HI } & \text { High-Intensity Reflectometer } \\ \text { HIREF } & \text { high-level radio-frequency } \\ \text { HLRF } & \text { High-Q Single-Crystal Diffractometer } \\ \text { HQSCD } & \text { High-Resolution Backscattering Spectrometer } \\ \text { HRBS } & \text { High-Resolution Chopper Spectrometer } \\ \text { HRMECS } & \text { High-Resolution Powder Diffractometer } \\ \text { HRPD } & \text { High-Resolution Small-Angle Neutron Diffractometer } \\ \text { HRSAND } & \text { heating, ventilating, and air conditioning } \\ \text { HVAC } & \text { high-voltage direct current } \\ \text { HVDC } & \text { high-voltage power supply } \\ \text { HVPS } & \text { input/output } \\ \text { I/O } & \text { International Collaboration on Advanced Neutron Sources } \\ \text { ICANS } & \text { inner diameter; inner dimension } \\ \text { ID } & \text { Institute of Electrical and Electronics Engineers } \\ \text { IEEE } & \text { insulated-gate bipolar transistor } \\ \text { IGBT } & \text { Institut Laue-Langevin } \\ \text { ILL } & \text { input/output controller } \\ \text { IOC } & \text { Insulated Power Cable Engineers Association } \\ \text { IPCEA } & \text { Intense Pulsed Neutron Source } \\ \text { IPNS } & \text { Inverted Roof Membrane Assembly } \\ \text { IRMA } & \text { irradiation tubes } \\ \text { IT } & \text { Joint Industrial Council } \\ \text { JIC } & \text { Kapchinskij-Vladimirskij } \\ \text { K-V } & \text { key decision } \\ \text { KD } & \text { knob manager } \\ \text { KM } & \text { thousand pounds per square inch } \\ \text { ksi } & \text { liter(s) } \\ \text { L } & \text { length-to-diameter ratio } \\ \text { L/D } & \text { local area network } \\ \text { LAN } & \text { Los Alamos Neutron Scattering Center } \\ \text { LANCSE } & \text { Los Alamos National Laboratory } \\ \text { LANL } & \\ & \text { ind }\end{array}$


LBL Lawrence Berkeley Laboratory

LEBT low-energy beam transport

LET low-energy transport; low-energy transport line

LFHRPD Low-Frequency High-Resolution Powder Diffractometer

LIS linac injector system

LLRF low-level radio-frequency

LNPI Leningrad Nuclear Physics Institute

LOCA loss of coolant accident

LOTO lockout/tagout

LPSD linear-position-sensitive detector

LRMECS High-Intensity General-Purpose Chopper Spectrometer

LWPD Long-Wavelength Powder Diffractometer

MAPS Multi-Angle Positron-Sensitive Spectrometer

MCNP Monte Carlo code for neutron and photon transport

MCR main control room

MEDM Motif-based editor display/manager tool

MHD magnetohydrodynamics

MICAS Multiple-Independent-Crystal-Analyzer Spectrometer for Coherent Excitations

MIT

MS

mtbf Massachusetts Institute of Technology

matching section

mean time before failure

NBS

National Bureau of Standards

NDP/NAA Neutron Depth Profiling and Neutron Activation Analysis

NEBB

NEC

NEMA

NEPA

NESC

NFPA

NINT

NIST

National Environmental Balancing Bureau

National Electric Code

National Electric Manufacturers Association

National Environmental Protection Act

National Electrical Safety Code

National Fire Protection Association

Neutron Interferometer

National Institute of Standards and Technology

OD outer diameter; outer dimension

OFHC oxygen-free high-conductivity

OPI

OSHA

operator interface

PACE plant and capital equipment

PBS private branch exchange

PCSCD Single-Crystal Diffractometer for Protein Crystallography

PFN pulse-forming network

PFS

Plant Facilities and Services Division (ANL) 
PID proportional integral derivative

PMQ permanent magnet quadrupole

PNPD Polarized-Neutron Powder Diffractometer

POSY-I Polarized-Neutron Reflectometer

POSY-II General-Purpose Reflectometer

ppm part(s) per minute

PROM programmable read-only memory

PRT participating research team

PS

PSF

psi

PSI

PSS

pv

PVC

power supply

pound(s) per square foot

pound(s) per square inch

Paul Scherrer Institut

pulsed spallation source

process variable

polyvinyl chloride

PWM pulse-width-modulated

QENS Medium-Resolution Crystal-Analyzer Spectrometer

QSS quasi-steady state

QSSAND Quasi-Steady-State Small-Angle Neutron Diffractometer

QSTAXC Cold-Neutron Quasi-Steady-State Triple-Axis Spectrometer

QSTAXT Thermal-Neutron Quasi-Steady-State Triple-Axis Spectrometer

$\mathrm{R} \quad$ return

R\&D research and development

RADIO Neutron Radiography/Tomography

RCS Rapid Cycling Sychrotron

REFD Reflectometry Development

If

RFQ

radio-frequency

RGDTL ramped-gradient drift tube linac

rms

RSD

RW

root mean square

Residual Stress Diffractometer

raw water

$S$

supply

SAND General-Purpose Small-Angle Neutron Diffractometer

SCCS

Chopper Spectrometer for Excitations in Single Crystals and Low-Dimensional

Systems

SCD General-Purpose Single-Crystal Diffractometer

SCL side-coupled linac

SD defocusing sextupole

sec second(s)

SEM scanning electron microscopy; secondary emission monitor 
SEPD Special Environment Powder Diffractometer

$\mathrm{SF}$

SLAC focusing sextupole

SMACNA

SNQ

Stanford Linear Accelerator Center

SPSAND

Sheet Metal and Air Conditioning Contractors National Association, Inc.

sr

Spallations Neutronenquelle

SSC

Sv

Special-Purpose Small-Angle Neutron Diffractometer

SWTP

steradian(s)

Superconducting Super Collider

Sievert(s)

$\mathrm{T}$

TCP/IP

TCU

TEC

Sanitary Wastewater Treatment Plant

TEM

TFCA

Tesla(s)

TFXA

TOF

transmission control protocol//nternet protocol

temperature control unit

total estimated cost

transmission electron microscopy

Time-Focused Crystal-Analyzer Spectrometer for Chemical Spectroscopy

TOFNSE Time-of-Flight Neutron Spin-Echo Spectrometer

TPM

Time-Focused Crystal Analyzer Spectrometer

time-of-flight

target protection monitor

UBC

Uniform Building Code

UCN

Ultra-Cold Neutron Facility

UHPD

Ultra-High-Pressure Diffractometer

UHRPD

Ultra-High-Resolution Powder Diffractometer

UL

Underwriters' Laboratories, Inc.

VAV

variable air volume

VCO

voltage-controlled oscillator

VFD

variable frequency drives

VSPD Very-Small-Sample Powder Diffractometer

VSWR voltage standing wave ratio

W/G

waveguide

WBS

work breakdown structure

yr year(s)

ZGS Zero Gradient Synchrotron

ZING-P ZGS Intense Neutron Generator Prototype 